\title{
Compendium of Material Composition Data for Radiation Transport Modeling
}

\section{Revision 1}
RJ McConn Jr
CJ Gesh
RT Pagh
RA Rucker
RG Williams III

March 4, 2011

Pacific Northwest

NATIONAL LABORATORY

Proudly Operated by Battelle Since 1965 


\title{
DISCLAIMER
}

This report was prepared as an account of work sponsored by an agency of the United States Government. Neither the United States Government nor any agency thereof, nor Battelle Memorial Institute, nor any of their employees, makes any warranty, express or implied, or assumes any legal liability or responsibility for the accuracy, completeness, or usefulness of any information, apparatus, product, or process disclosed, or represents that its use would not infringe privately owned rights. Reference herein to any specific commercial product, process, or service by trade name, trademark, manufacturer, or otherwise does not necessarily constitute or imply its endorsement, recommendation, or favoring by the United States Government or any agency thereof, or Battelle Memorial Institute. The views and opinions of authors expressed herein do not necessarily state or reflect those of the United States Government or any agency thereof.

\author{
PACIFIC NORTHWEST NATIONAL LABORATORY \\ operated by \\ BATTELLE \\ for the \\ UNITED STATES DEPARTMENT OF ENERGY \\ under Contract DE-AC05-76RL01830
}

Printed in the United States of America
Available to DOE and DOE contractors from the Office of Scientific and Technical Information,
P.O. Box 62, Oak Ridge, TN 37831-0062;
ph: (865) 576-8401
fax: $(865) 576-5728$
email: reports@adonis.osti.gov

Available to the public from the National Technical Information Service

5301 Shawnee Rd., Alexandria, VA 22312

ph: (800) 553-NTIS (6847)

email: orders@ntis.gov $<$ http://www.ntis.gov/about/form.aspx $>$

Online ordering: http:/www.ntis.gov 


\title{
Radiation Portal Monitor Project
}

\section{Compendium of Material Composition Data for Radiation Transport Modeling}

\author{
RJ McConn Jr \\ CJ Gesh \\ RT Pagh \\ RA Rucker \\ RG Williams III
}

March 4, 2011

Prepared for the U.S. Department of Homeland Security U.S. Customs and Border Protection and Domestic Nuclear Detection Office under U.S. Department of Energy Contract DE-AC05-76RL01830

Pacific Northwest National Laboratory

Richland, Washington 99352 


\section{Revision Log and Approvals}

\section{Compendium of Material Composition Data for Radiation Transport Modeling}

\begin{tabular}{|c|c|l|c|}
\hline Rev. No. & Date & \multicolumn{1}{|c|}{ Describe Changes } & Pages Changed \\
\hline 0 & $10 / 31 / 2006$ & $\begin{array}{l}\text { Original document was published under PNNL-15870 } \\
\text { only, not as an RPMP document. Therefore, Revision 1 } \\
\text { is the first version of this document to be issued under } \\
\text { PIET-43741-TM-963. }\end{array}$ & NA \\
\hline 1 & $03 / 04 / 11$ & $\begin{array}{l}\text { Revised to correct errors or inconsistencies in the data for } \\
\text { the original materials, as well as to increase the number } \\
\text { of materials listed. }\end{array}$ & All \\
\hline
\end{tabular}

\begin{tabular}{|l|c|c|}
\hline \multicolumn{1}{|c|}{ Name and Title } & Approvals & Date \\
\hline $\begin{array}{l}\text { Ron McConn Jr., } \\
\text { Subtask Manager }\end{array}$ & Approved via email & $02 / 29 / 11$ \\
\hline $\begin{array}{l}\text { Richard Pagh for Joel Hoyt, } \\
\text { Science and Technology Task } \\
\text { Manager }\end{array}$ & Approved via email & $03 / 01 / 11$ \\
\hline $\begin{array}{l}\text { Daniel Stephens, } \\
\text { Principal Investigator }\end{array}$ & Approved via email & $03 / 04 / 11$ \\
\hline $\begin{array}{l}\text { Daniel Stephens, } \\
\text { RPMP Manager }\end{array}$ & Approved via email & $03 / 04 / 11$ \\
\hline
\end{tabular}

Note: Document approvals that were received via email are retained in the project records.

RADS ADC Identifier: fuIUdJ 


\section{Acronyms and Abbreviations}

ASTAR

CEPXS/ONELD

ICRP

ICRU

KENO

MCNP

NCRP

NIST

PNNL

PSTAR

RSICC

RSICC

SCALE

\author{
Alpha Stopping-Power and Range \\ Coupled Electron-Photon One-Dimensional Discrete Ordinates (code) \\ International Commission on Radiological Protection \\ International Commission on Radiation Units and Measurements \\ A Monte Carlo criticality code that is maintained by Oak Ridge National \\ Laboratory (ORNL) \\ Monte-Carlo-N-Particle (code) \\ National Council on Radiation Protection and Measurements Definition \\ National Institute of Standards and Technology \\ Pacific Northwest National Laboratory \\ Proton Stopping-Power and Range \\ Radiation Safety Information Computational Center \\ Radiation Safety Information Computational Center \\ Standardized Computer Analysis for Licensing Evaluation
}




\section{Contents}

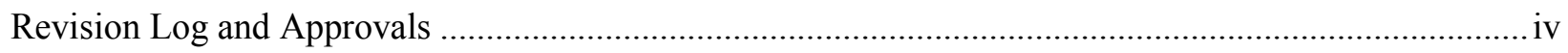

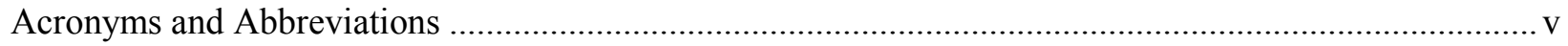

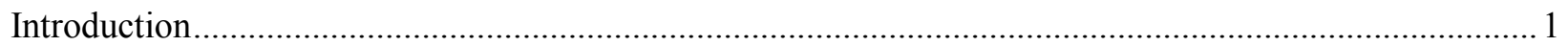

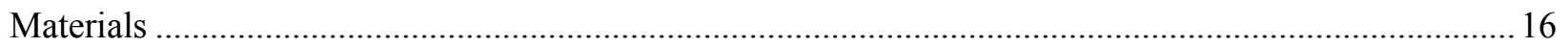

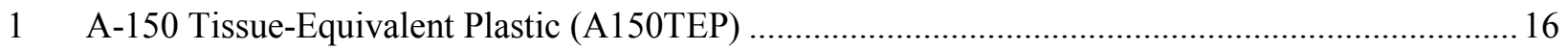

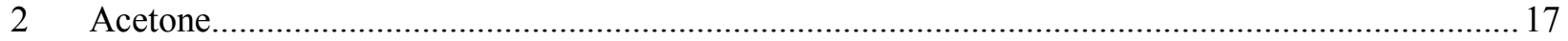

3 Acetylene

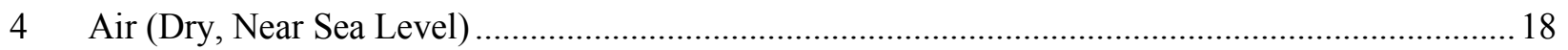

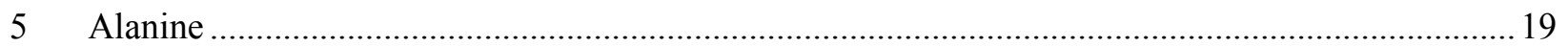

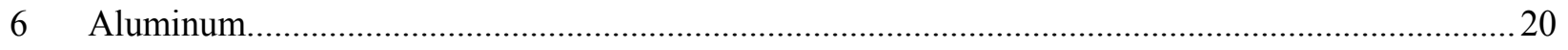

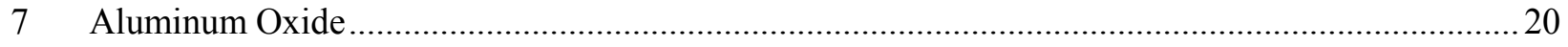

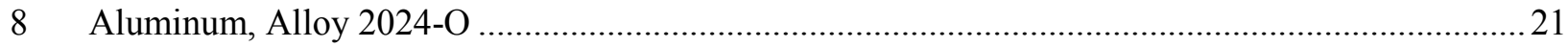

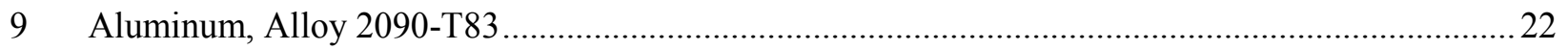

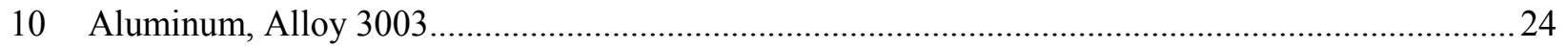

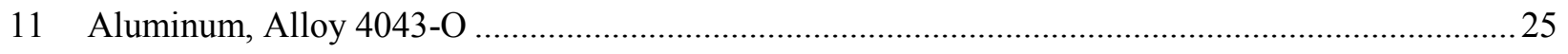

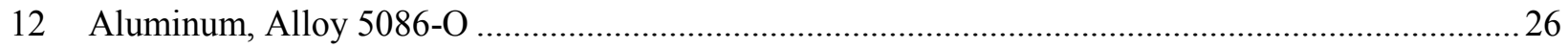

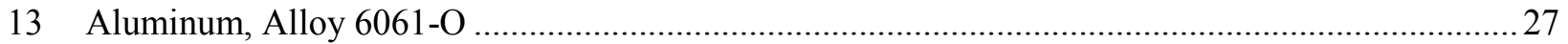

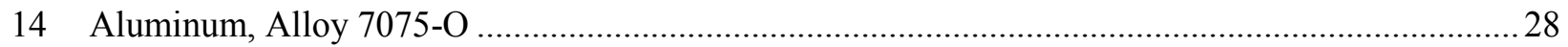

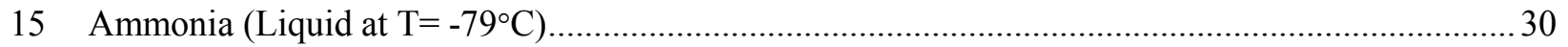

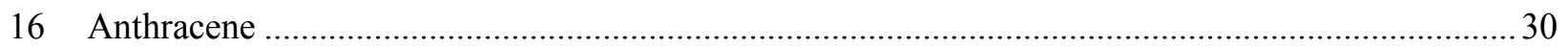

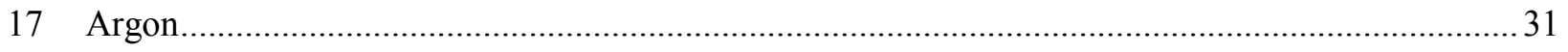

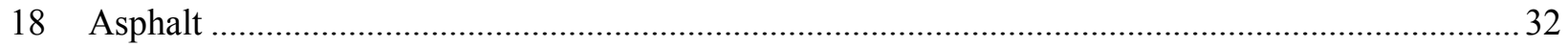

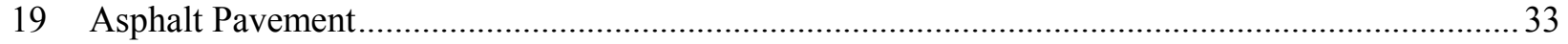

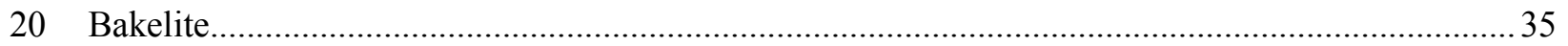

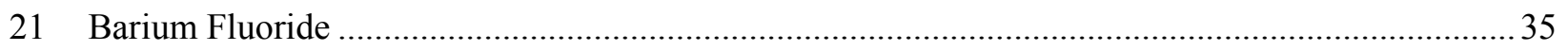

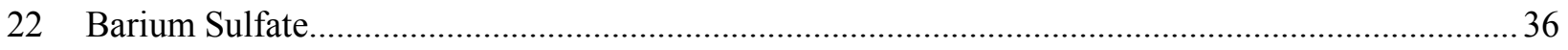

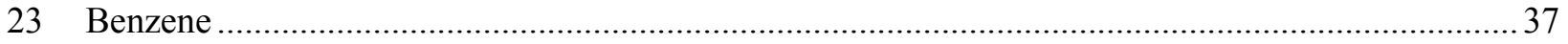

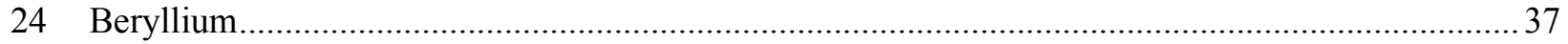

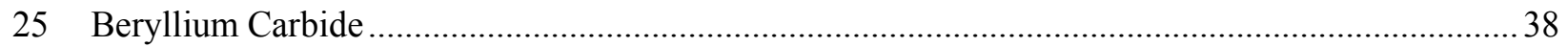

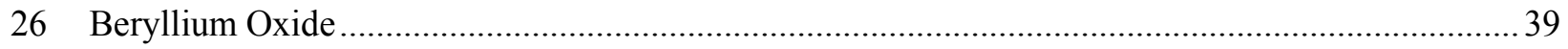




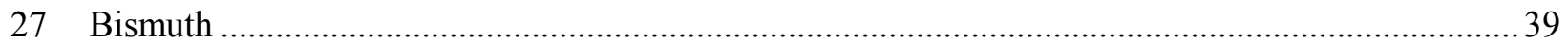

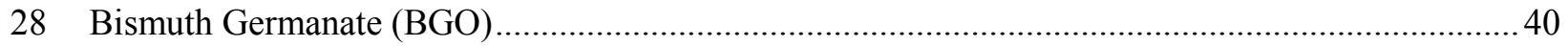

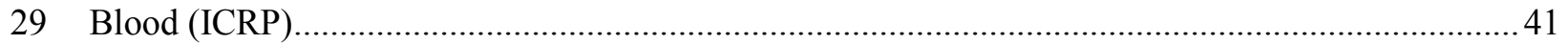

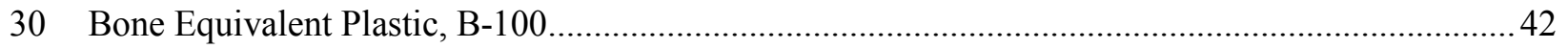

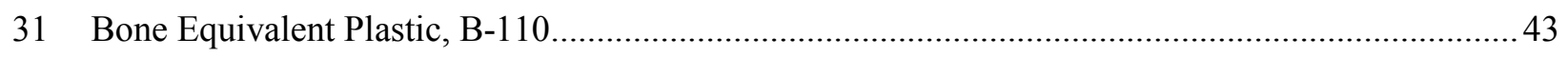

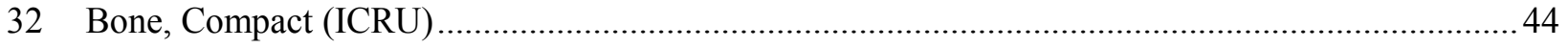

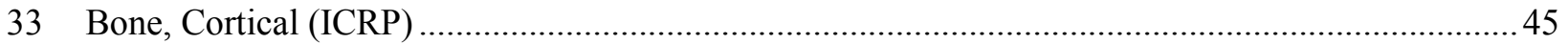

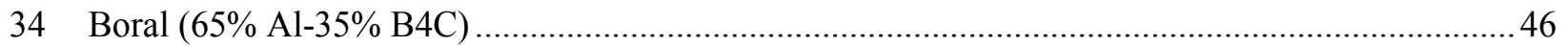

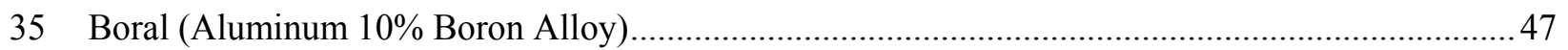

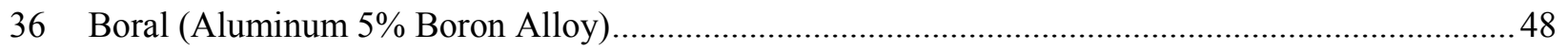

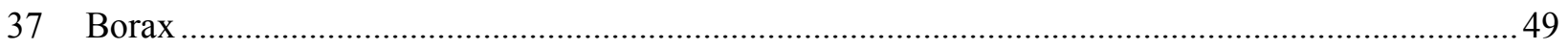

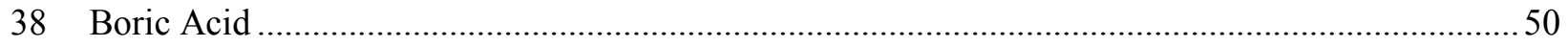

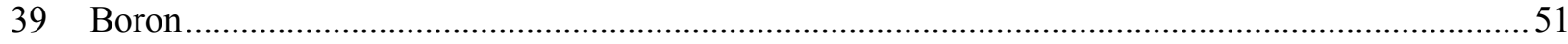

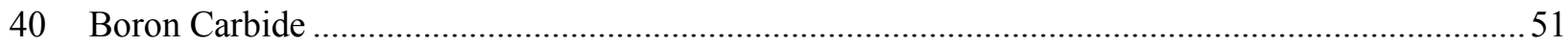

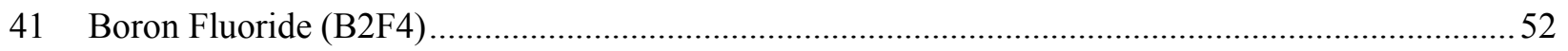

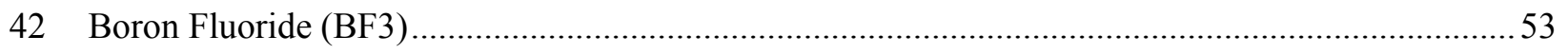

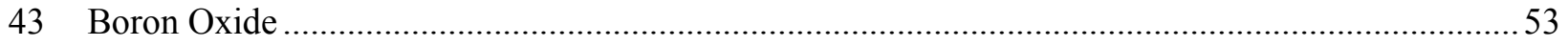

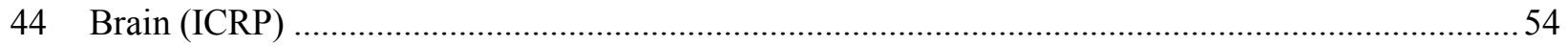

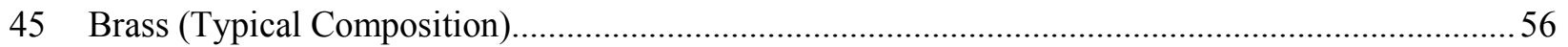

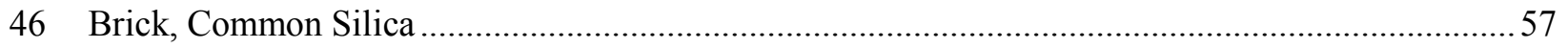

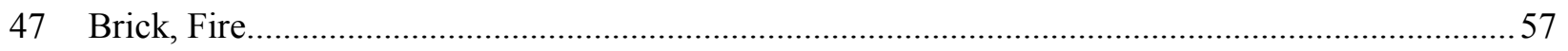

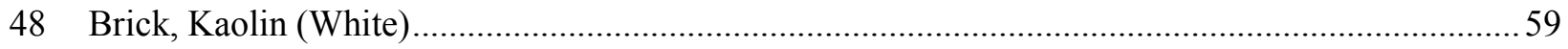

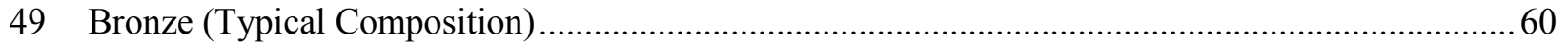

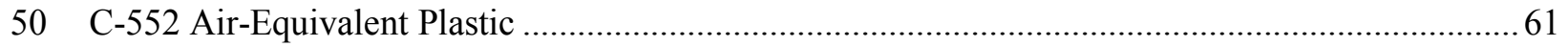

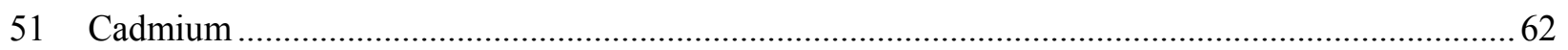

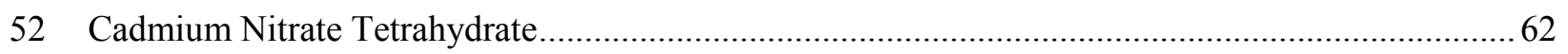

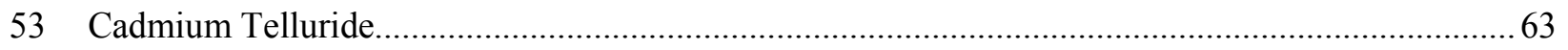

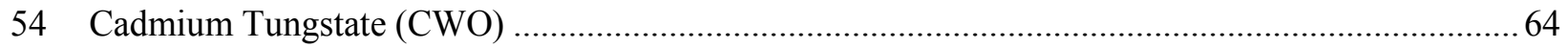

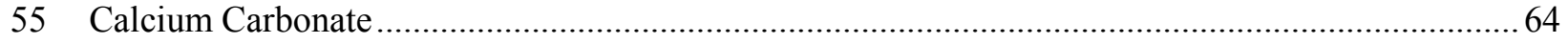

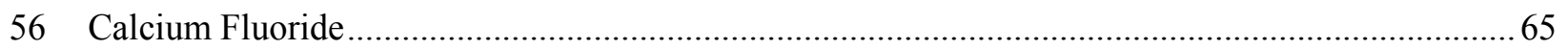

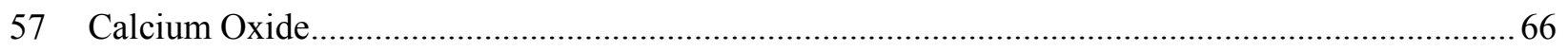

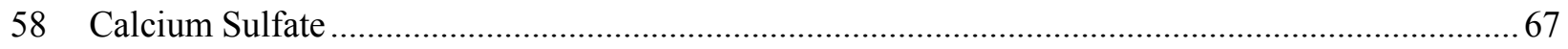




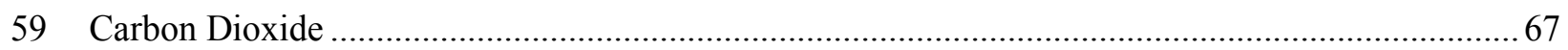

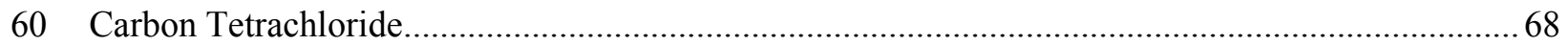

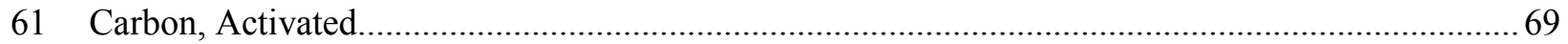

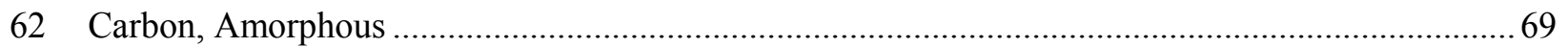

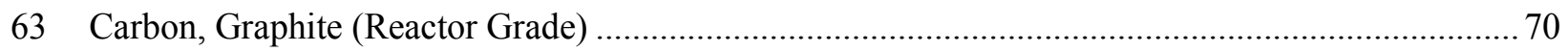

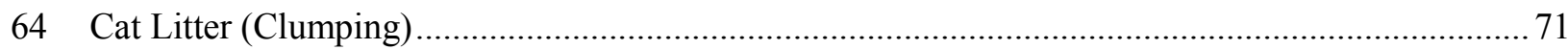

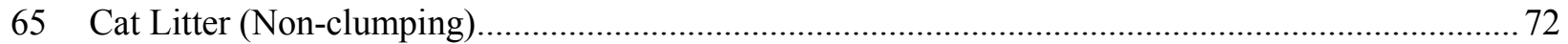

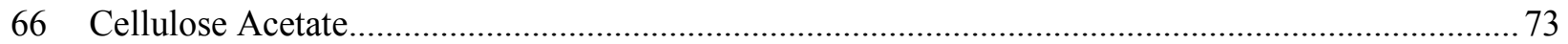

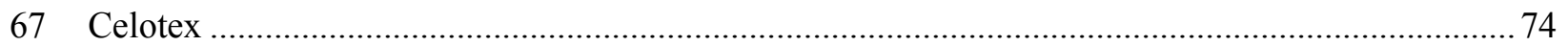

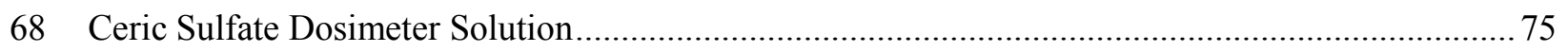

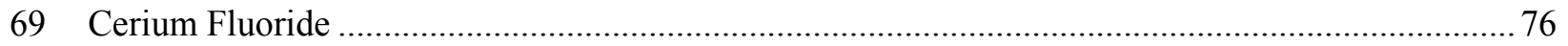

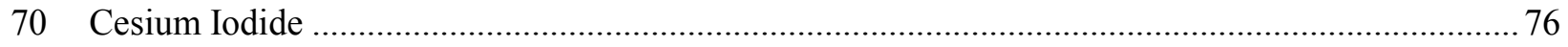

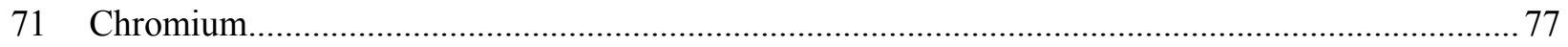

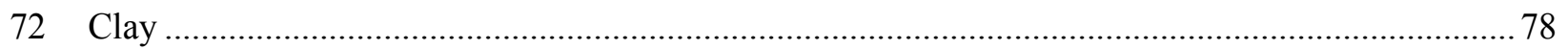

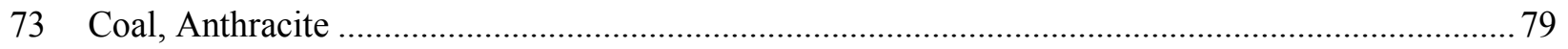

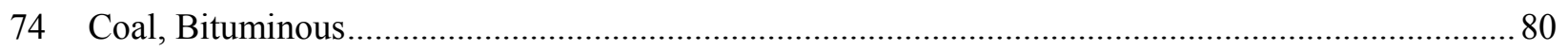

75 Coal, Lignite

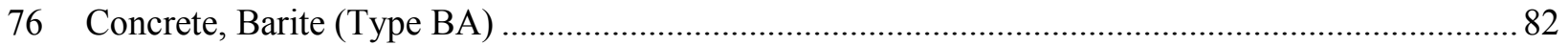

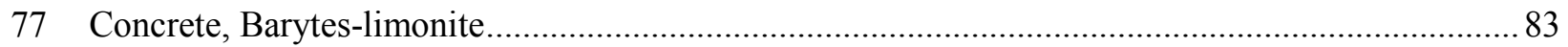

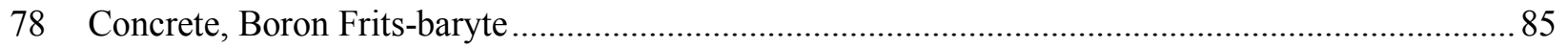

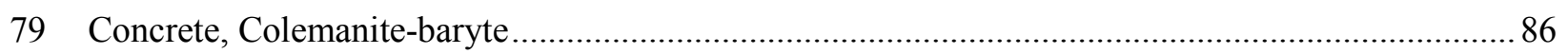

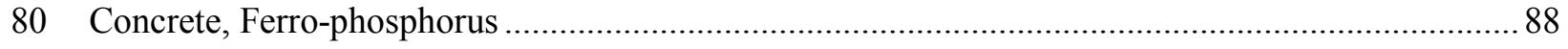

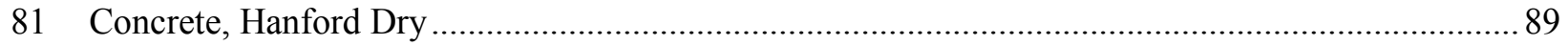

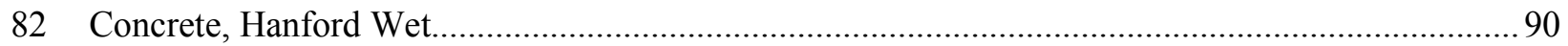

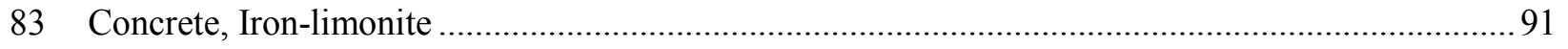

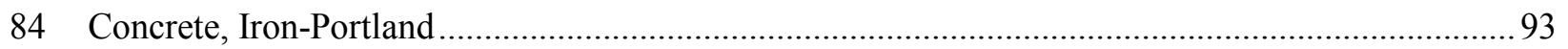

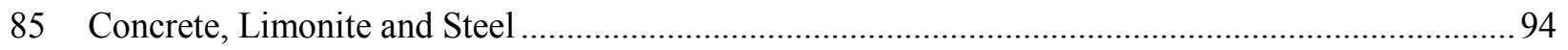

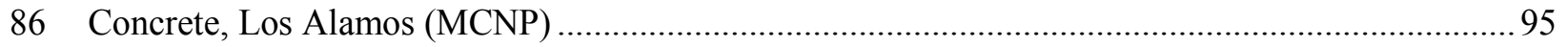

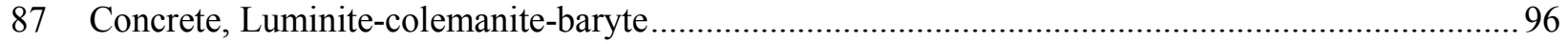

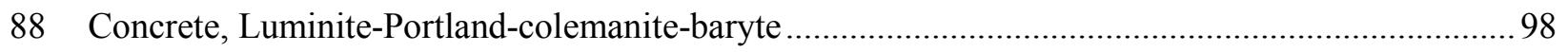

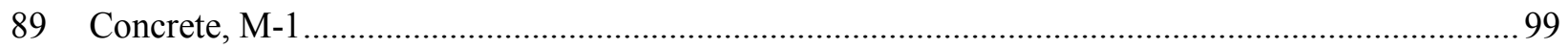

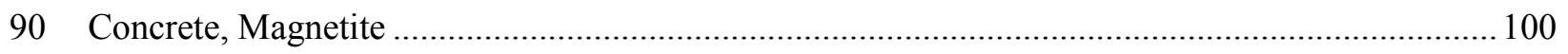




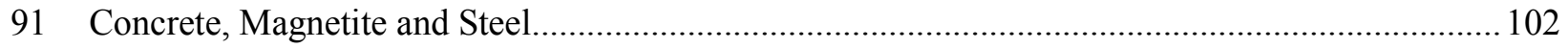

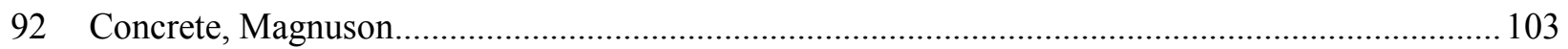

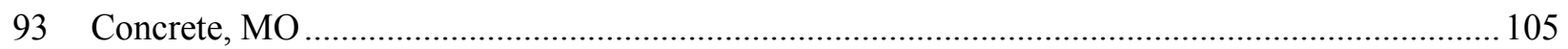

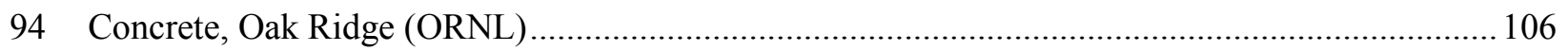

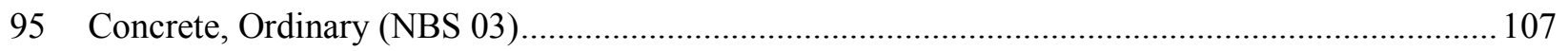

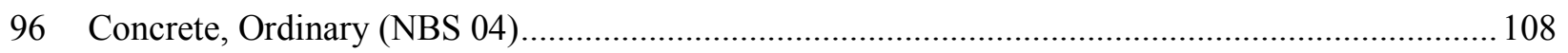

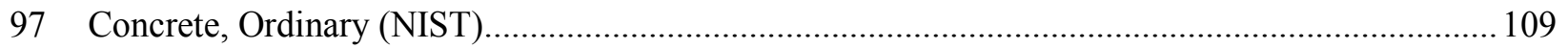

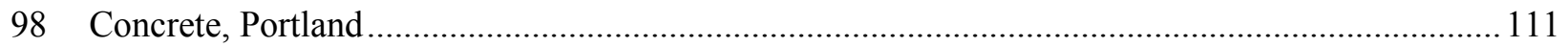

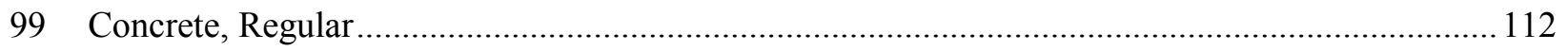

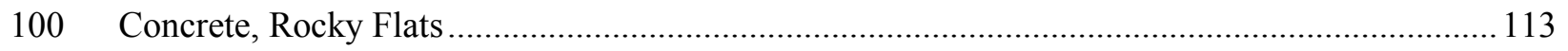

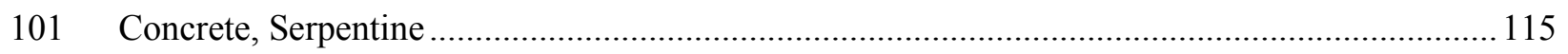

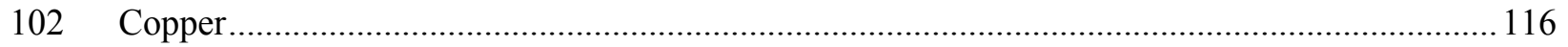

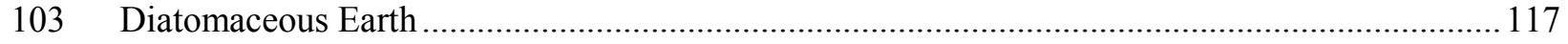

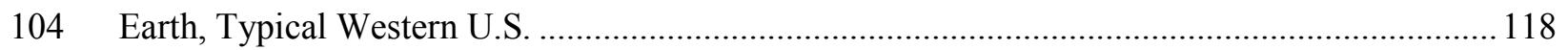

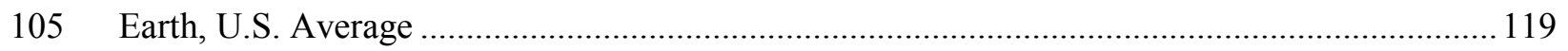

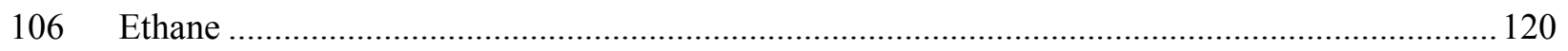

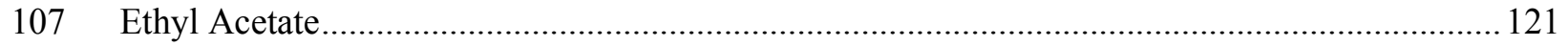

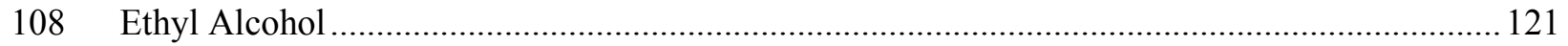

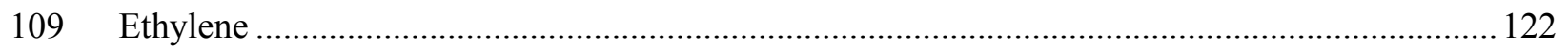

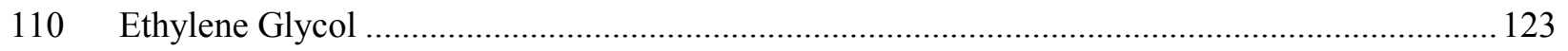

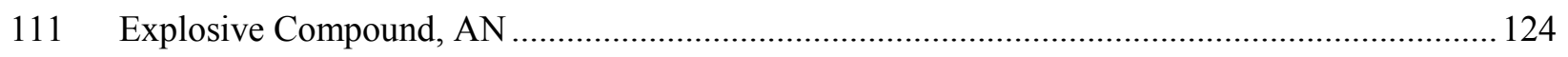

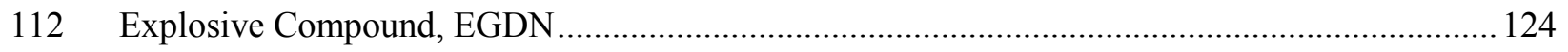

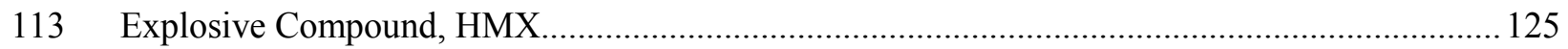

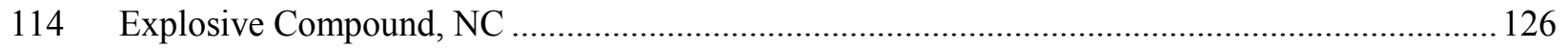

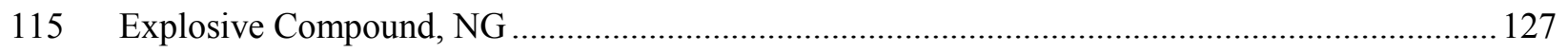

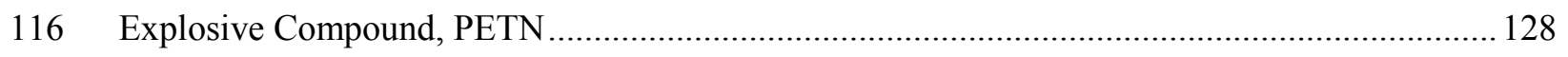

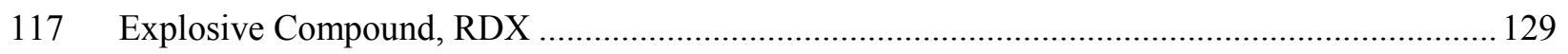

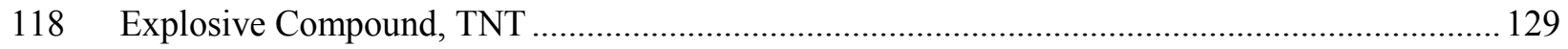

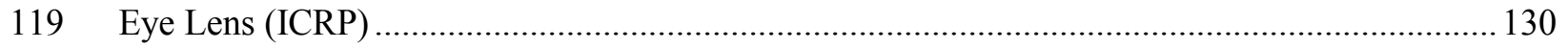

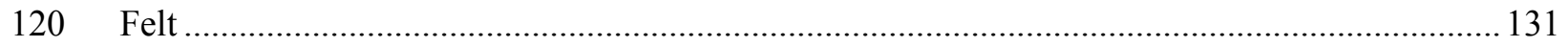

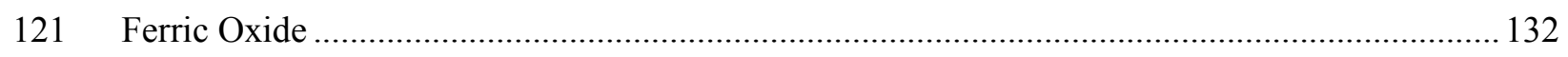

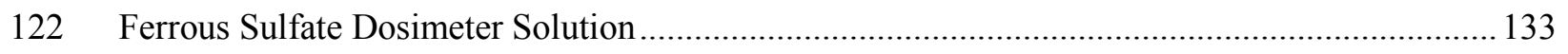




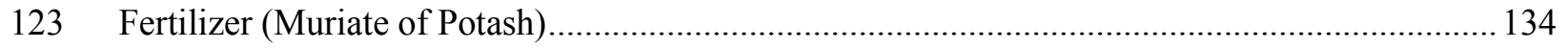

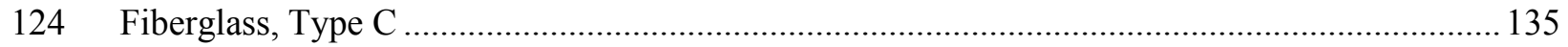

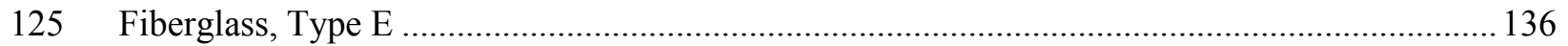

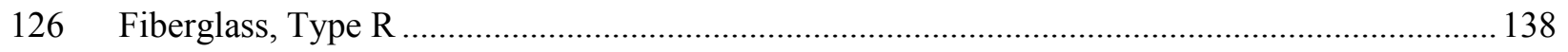

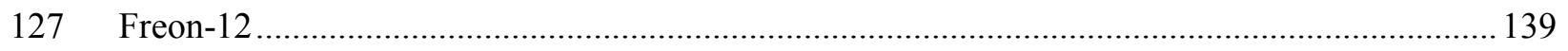

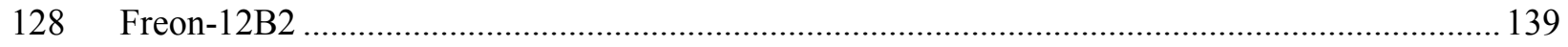

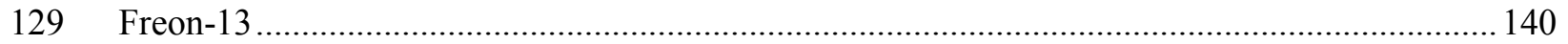

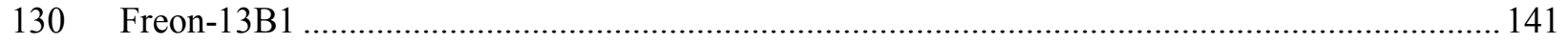

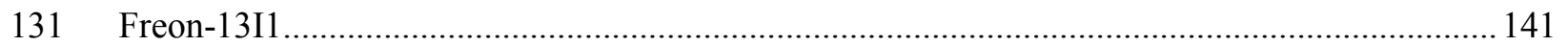

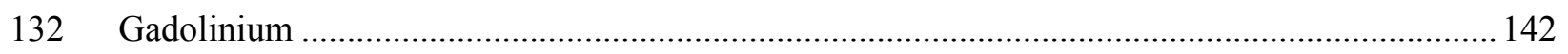

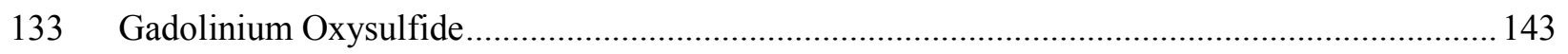

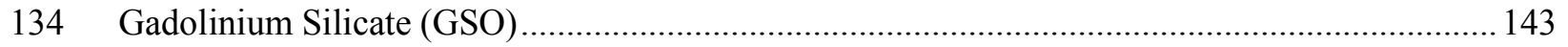

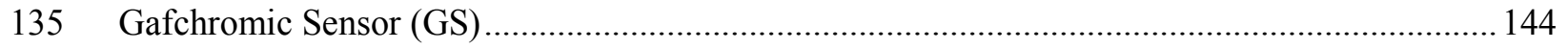

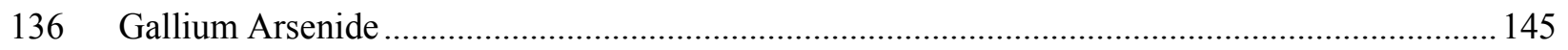

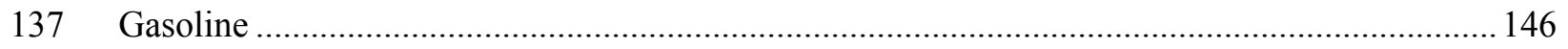

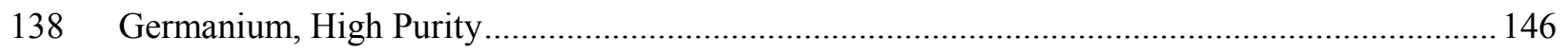

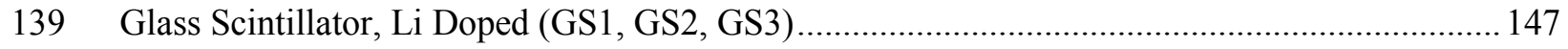

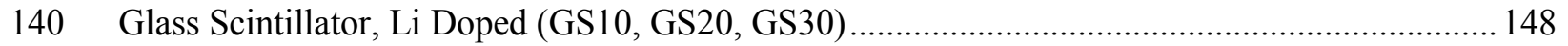

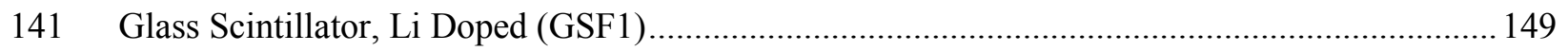

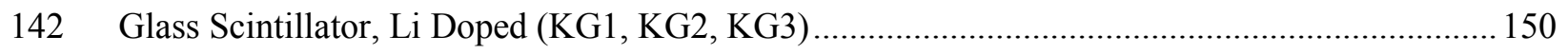

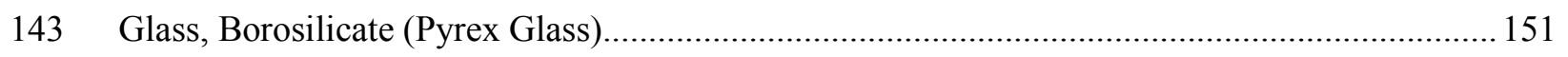

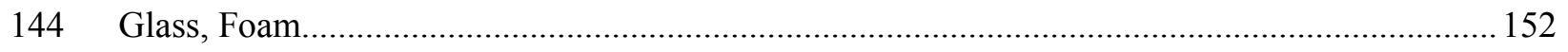

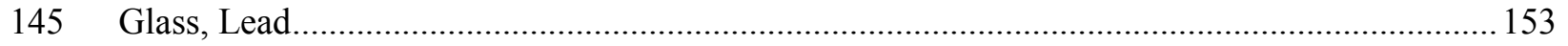

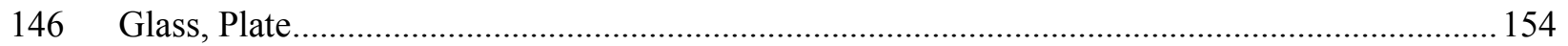

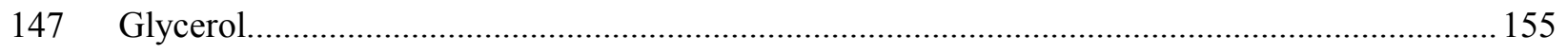

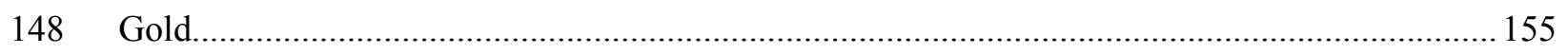

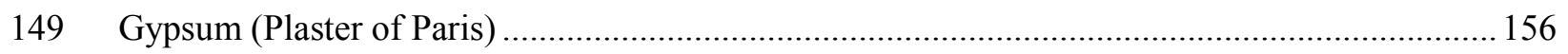

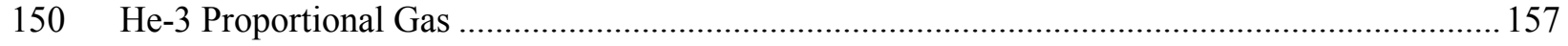

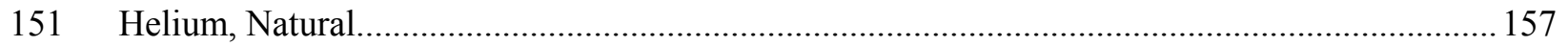

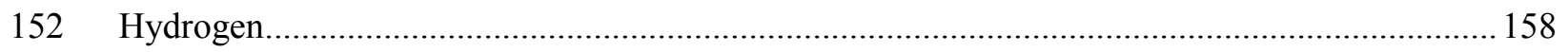

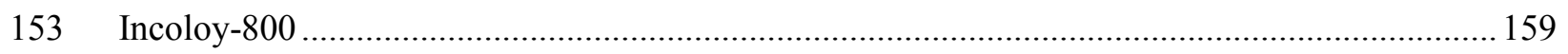

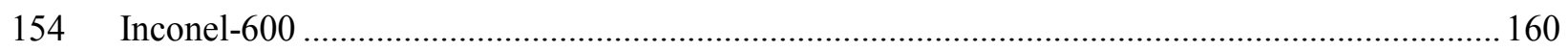




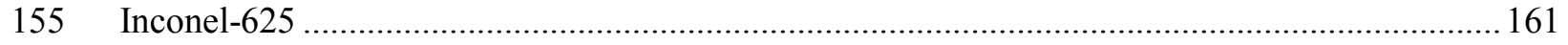

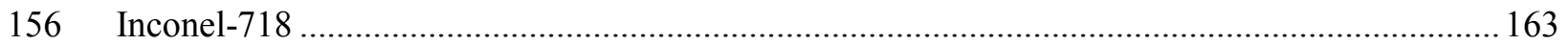

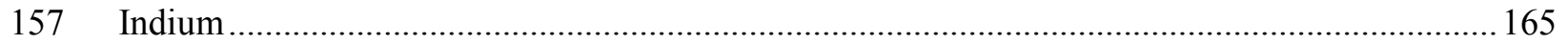

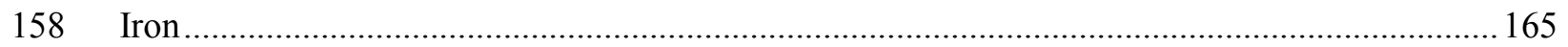

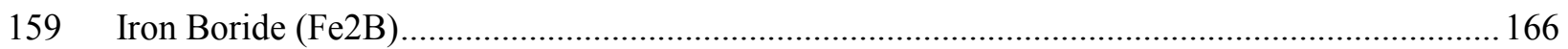

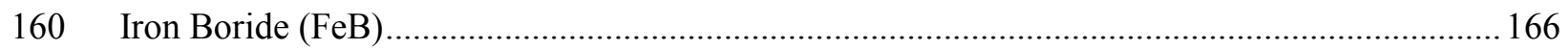

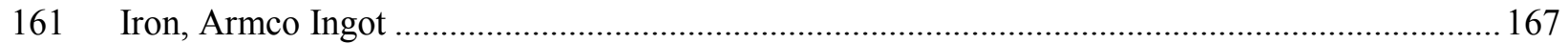

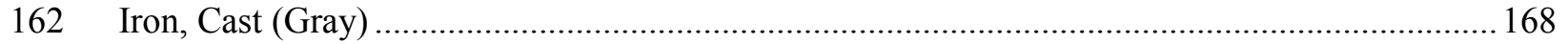

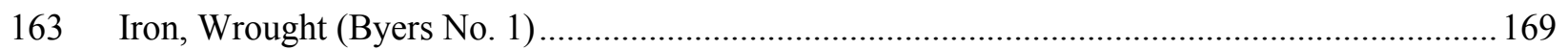

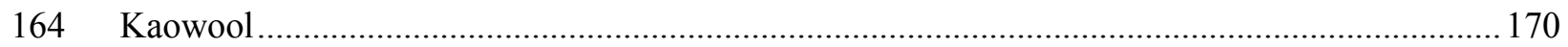

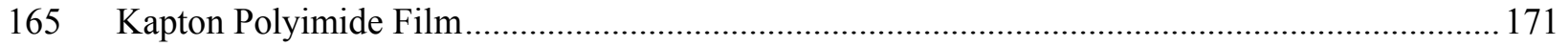

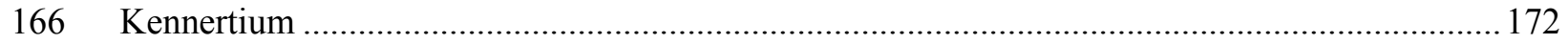

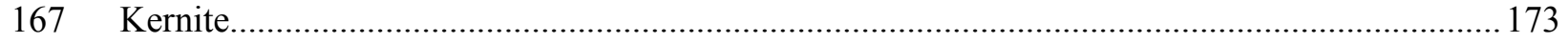

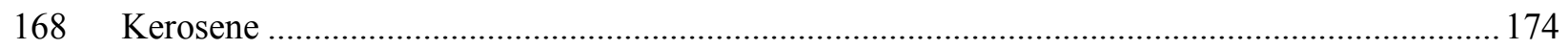

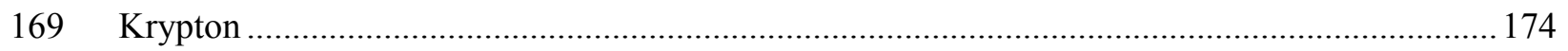

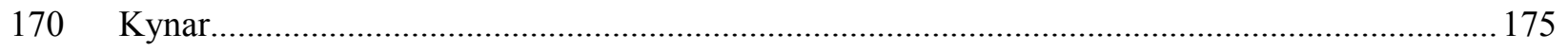

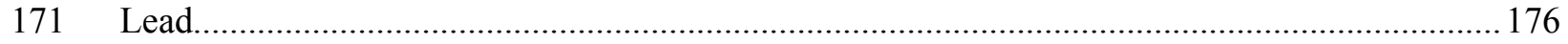

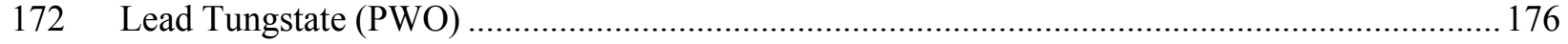

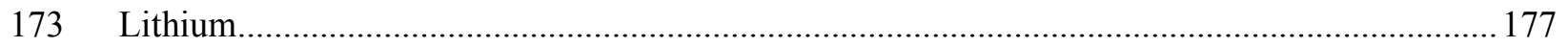

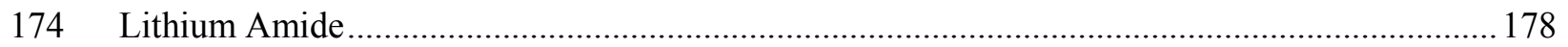

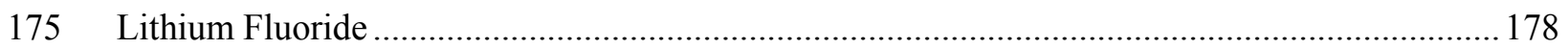

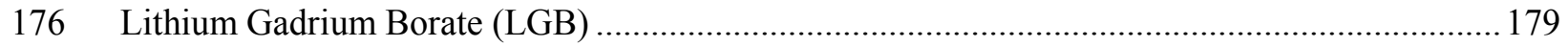

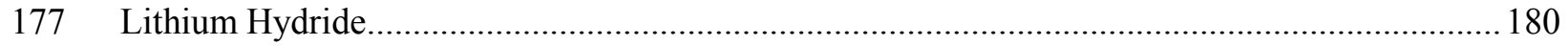

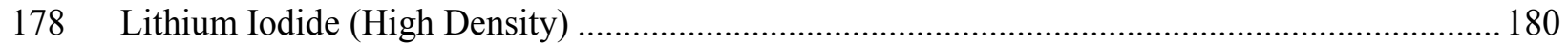

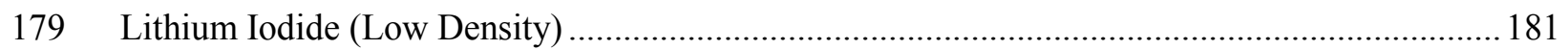

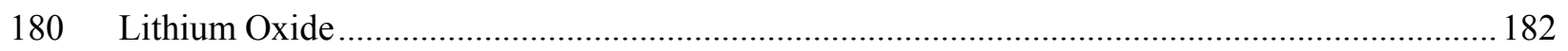

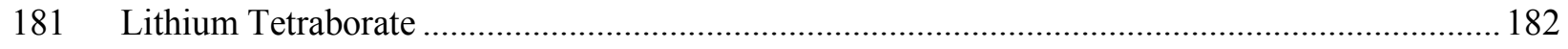

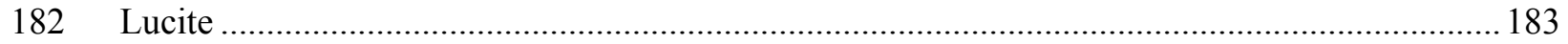

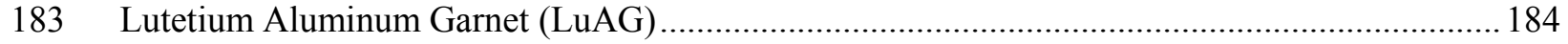

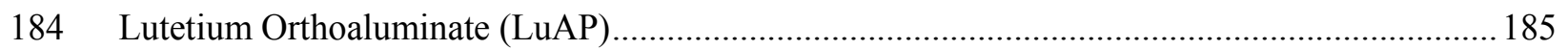

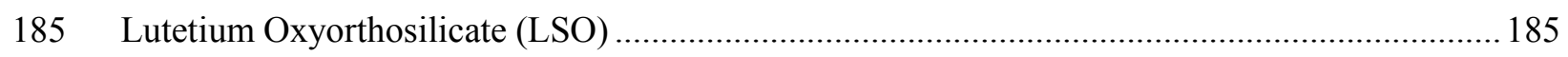

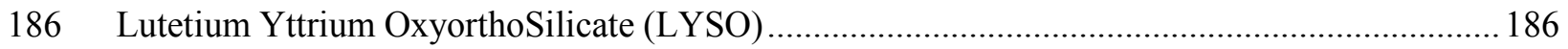




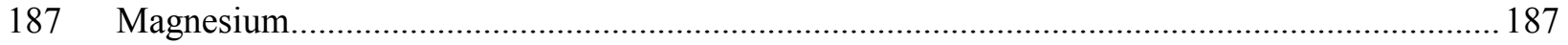

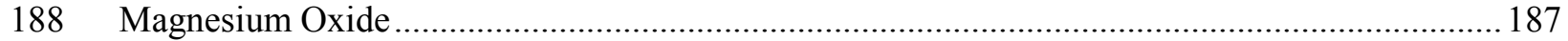

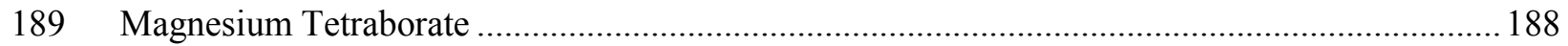

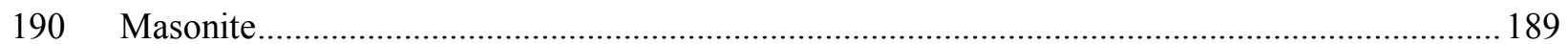

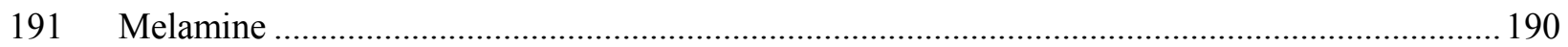

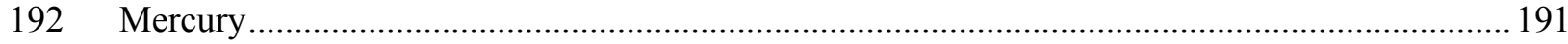

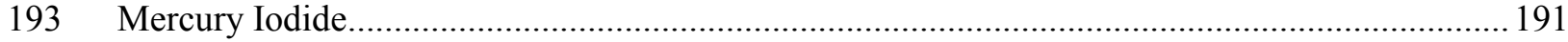

194 Methane

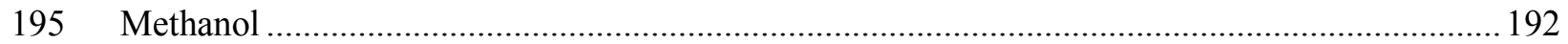

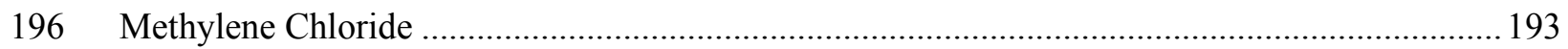

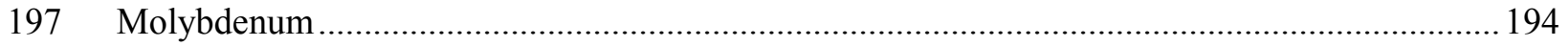

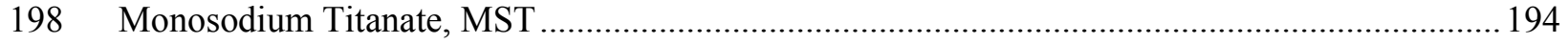

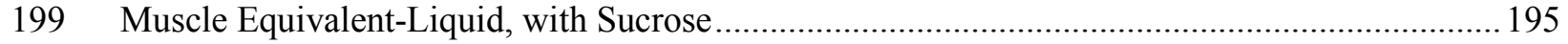

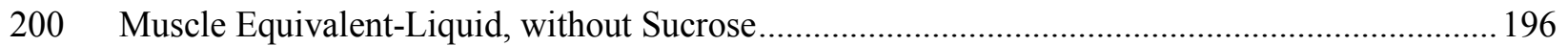

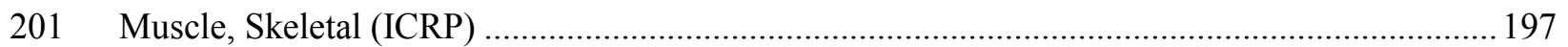

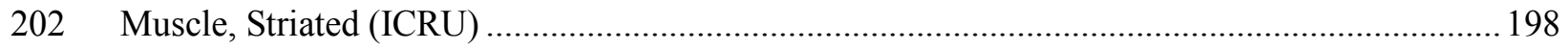

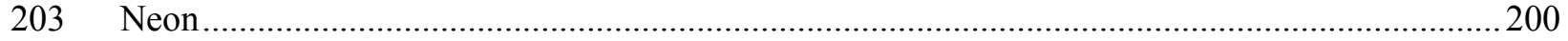

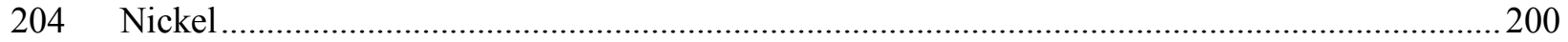

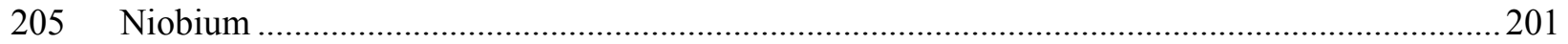

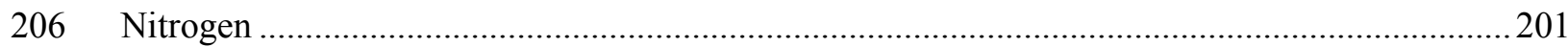

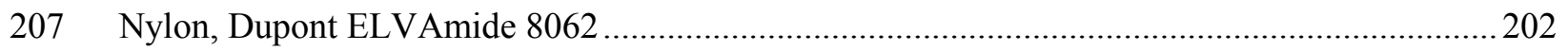

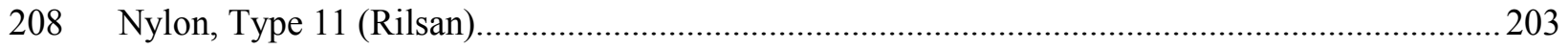

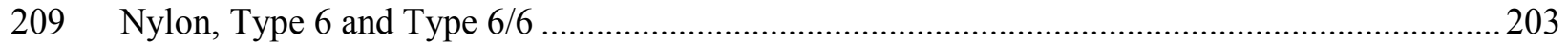

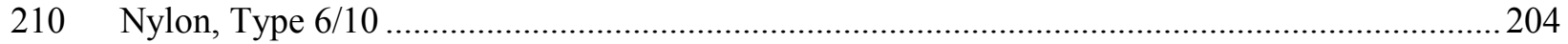

211 Oil, Crude (Heavy, Cold Lake, Canada) ....................................................................................... 205

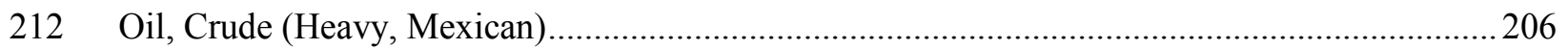

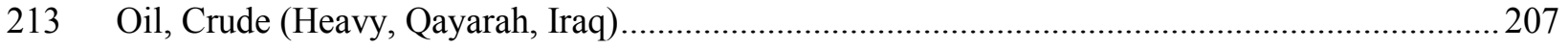

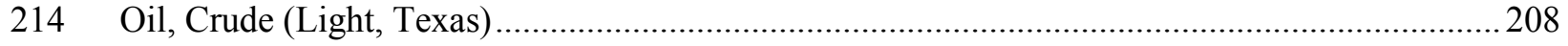

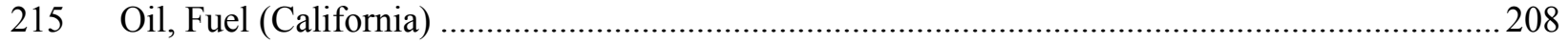

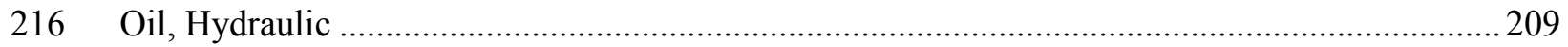

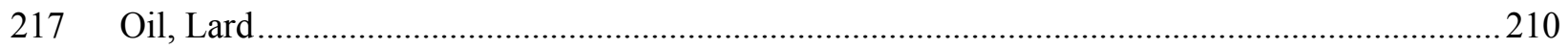

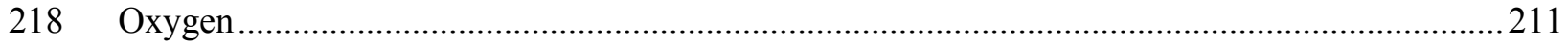


222 Photographic Emulsion, Gel in 213

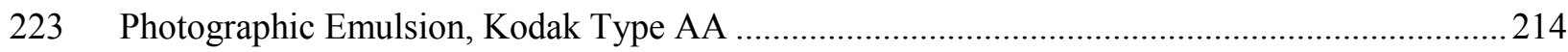

224 Photographic Emulsion, Standard Nuclear .............................................................................. 215

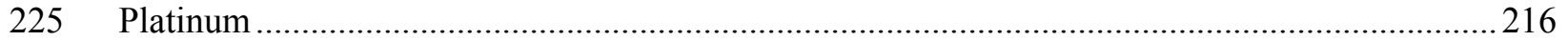

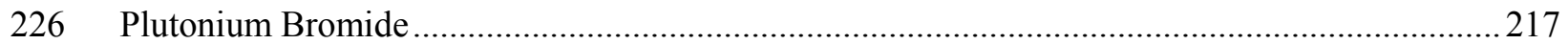

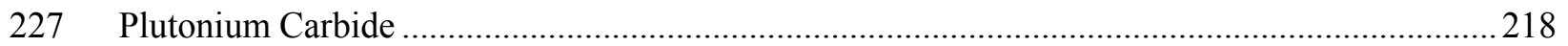

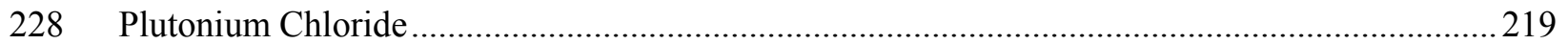

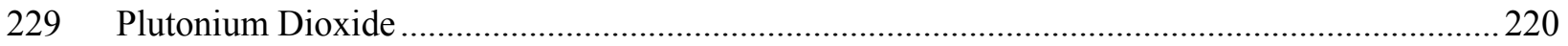

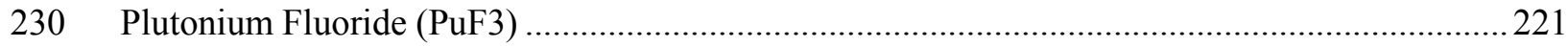

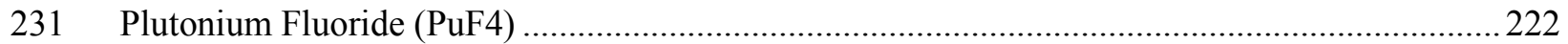

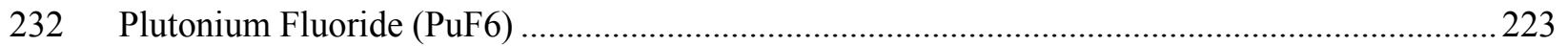

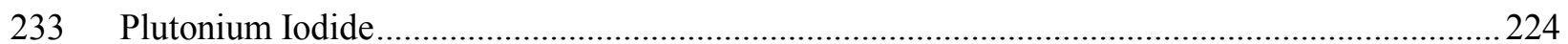

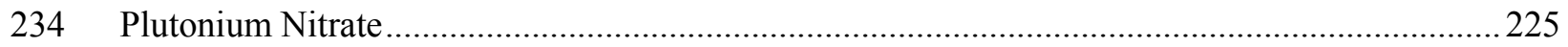

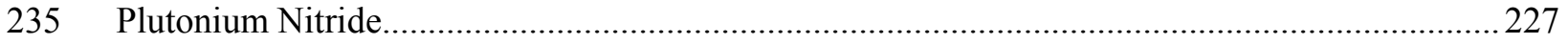

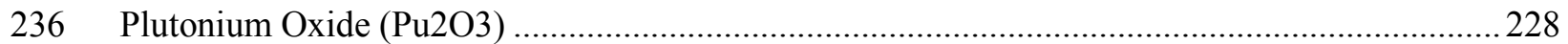

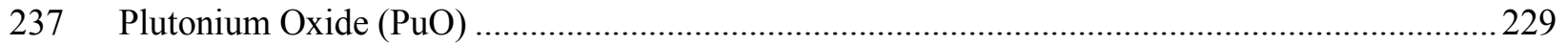

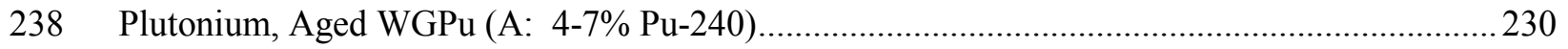

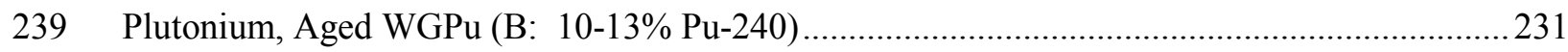

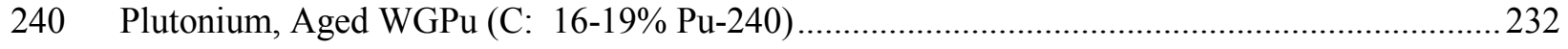

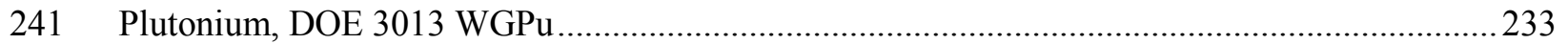

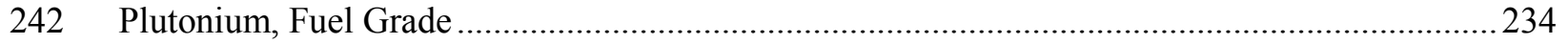

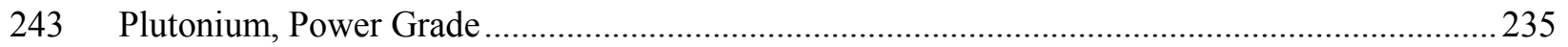

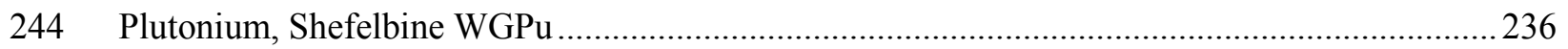

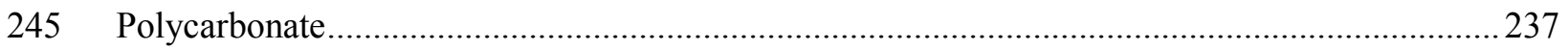

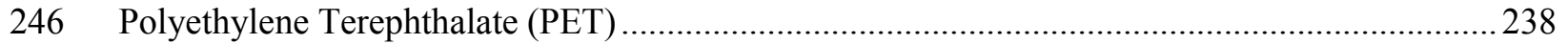

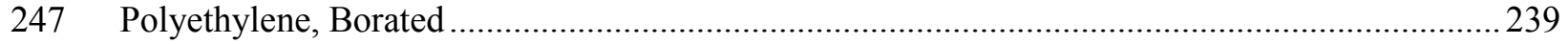

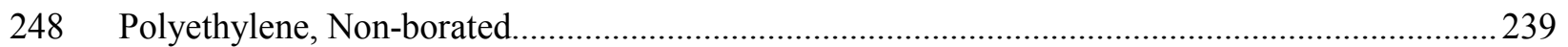

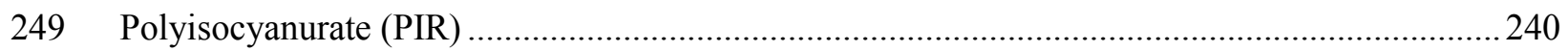

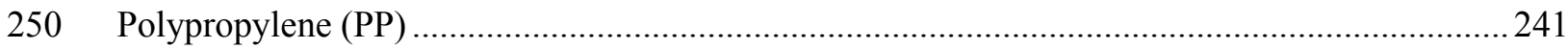




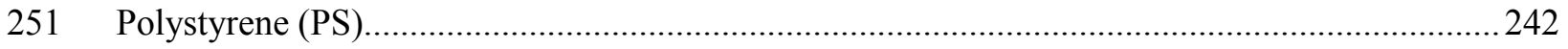

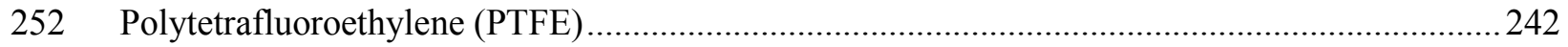

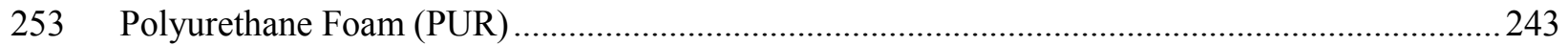

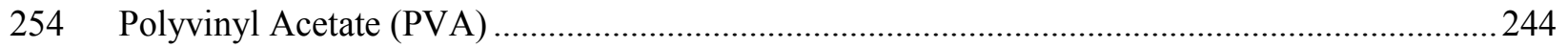

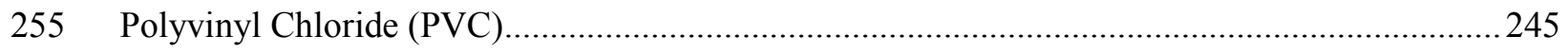

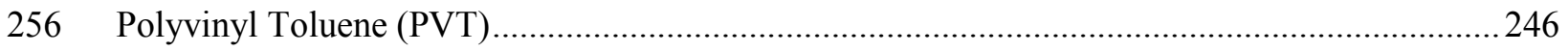

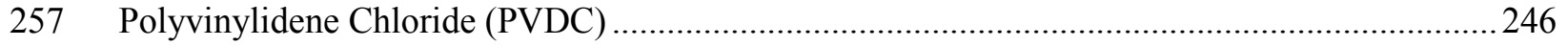

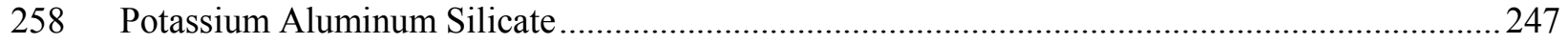

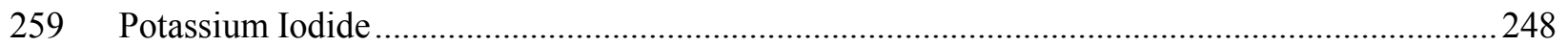

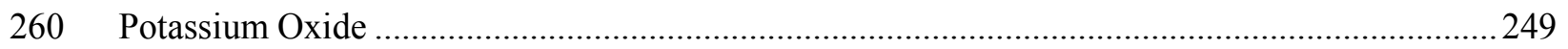

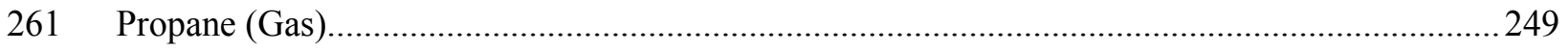

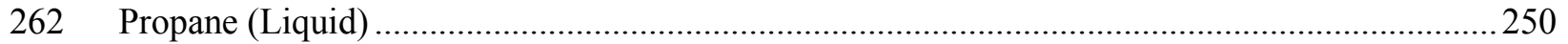

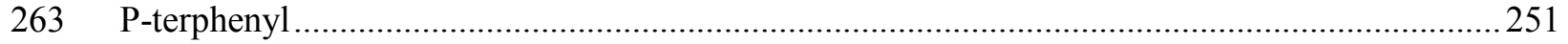

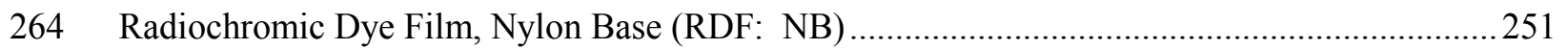

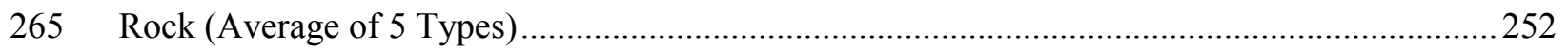

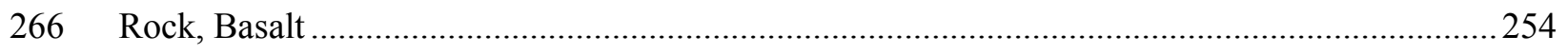

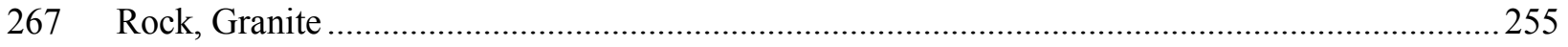

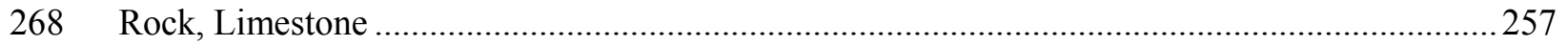

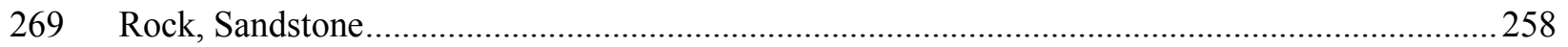

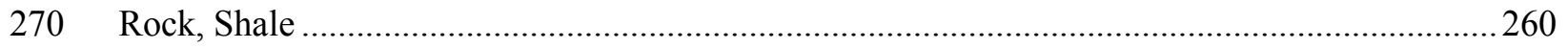

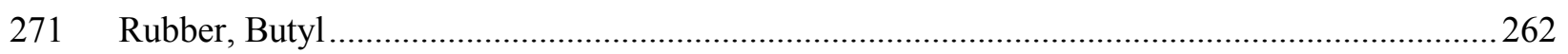

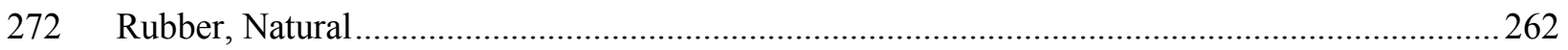

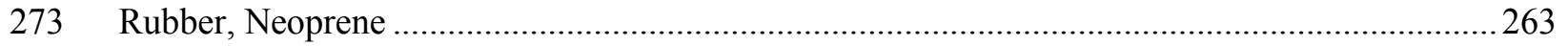

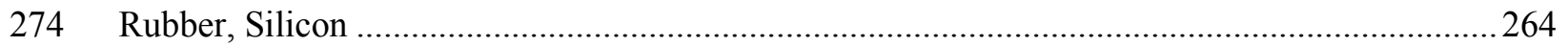

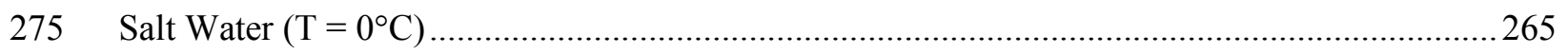

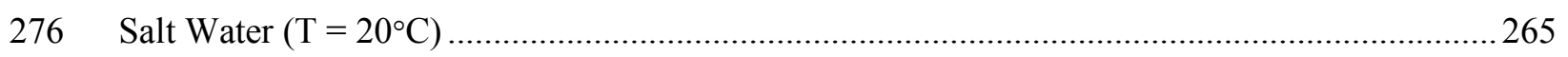

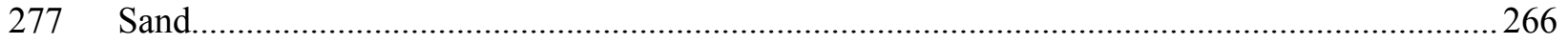

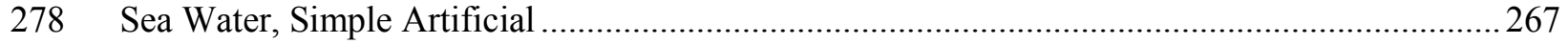

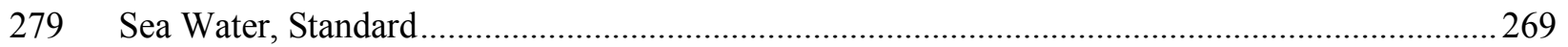

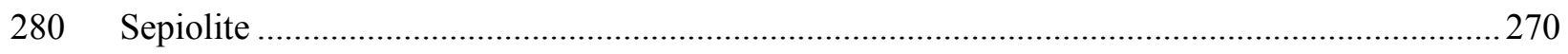

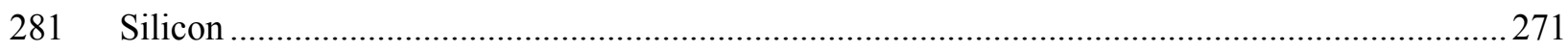

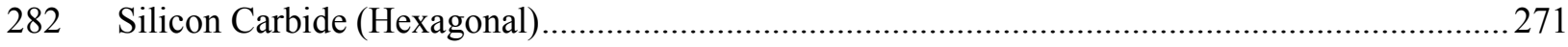




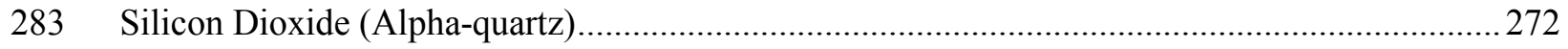

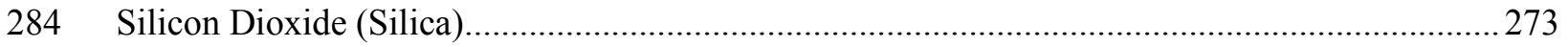

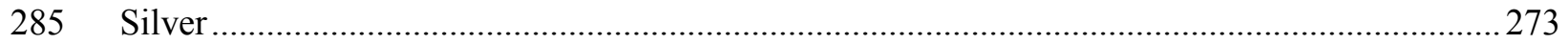

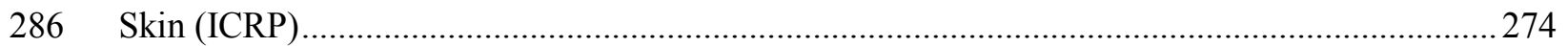

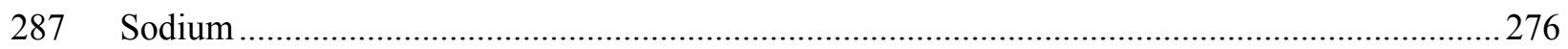

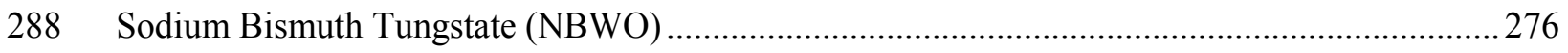

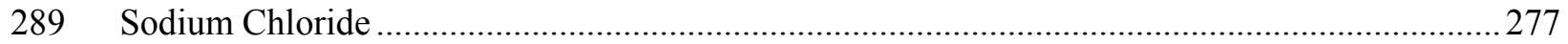

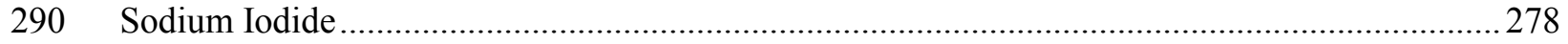

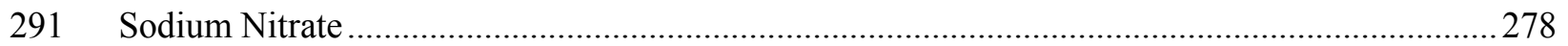

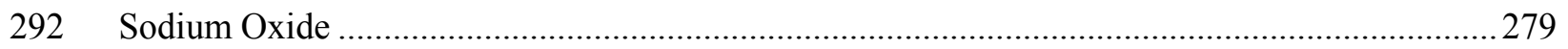

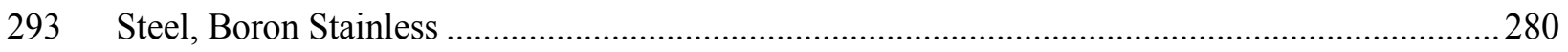

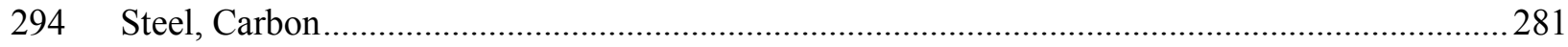

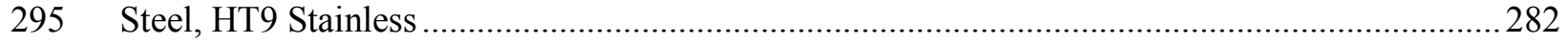

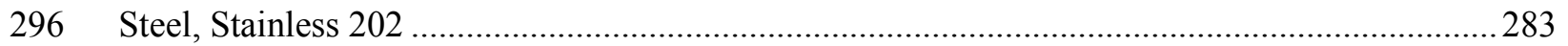

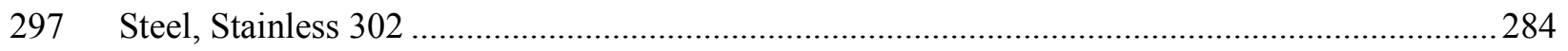

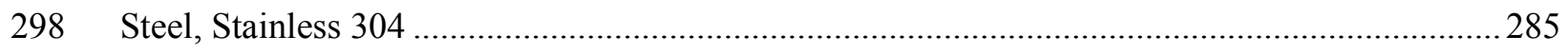

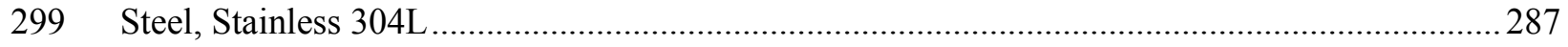

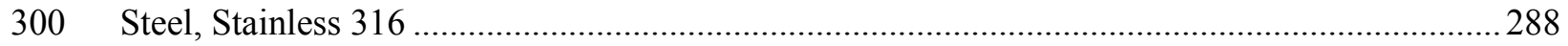

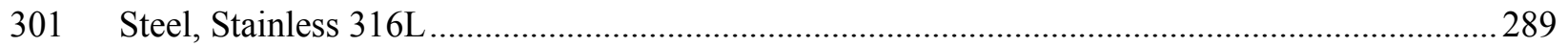

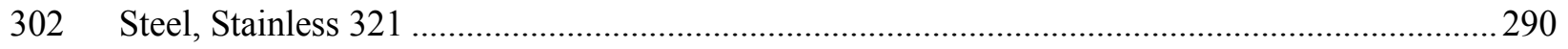

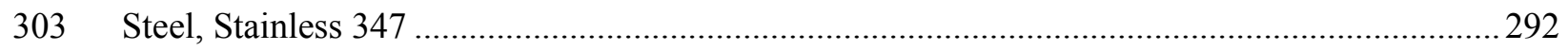

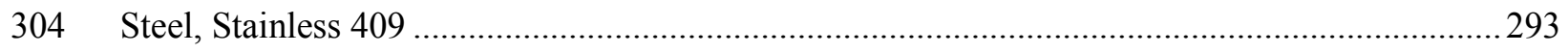

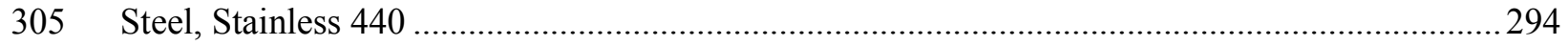

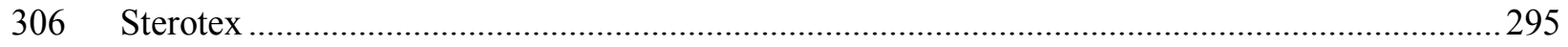

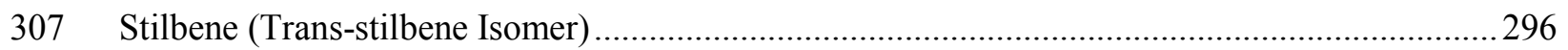

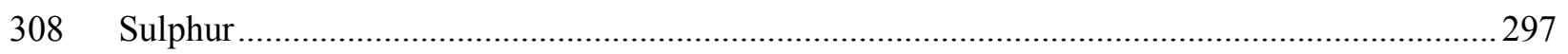

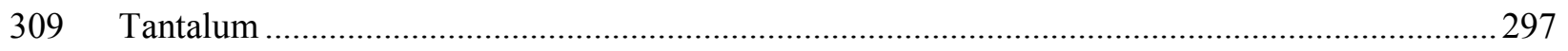

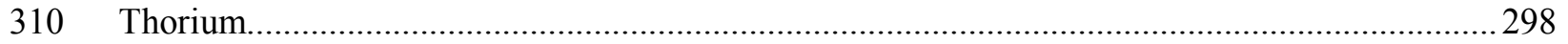

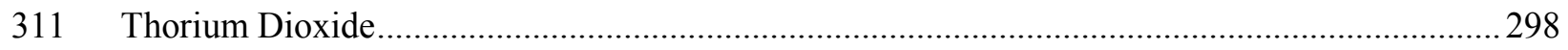

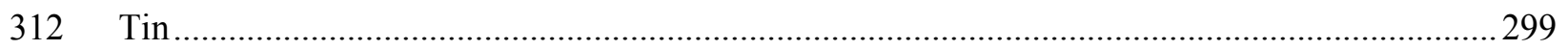

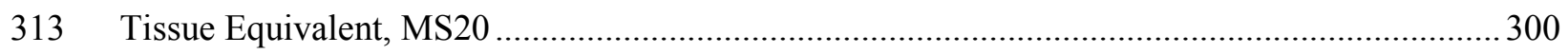

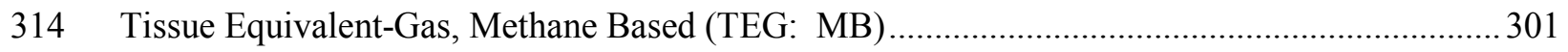




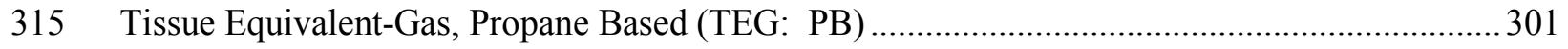

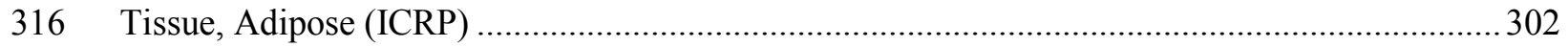

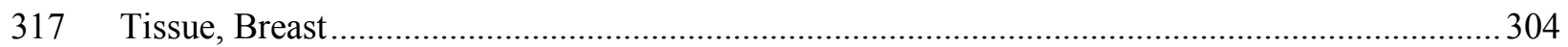

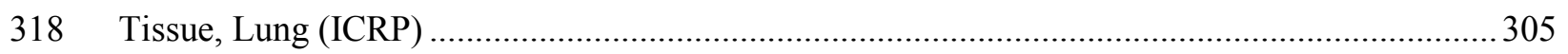

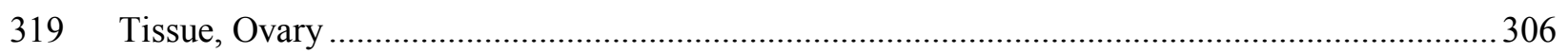

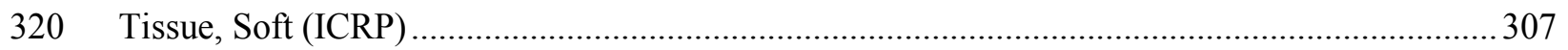

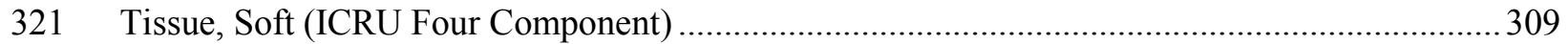

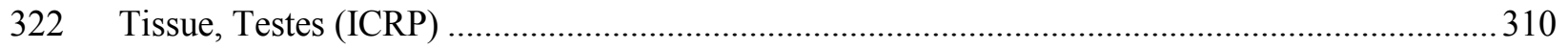

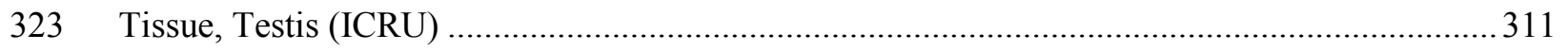

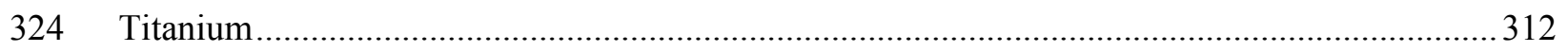

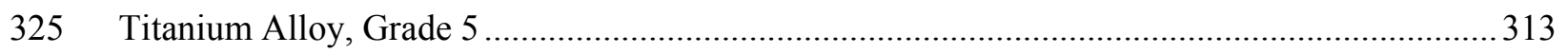

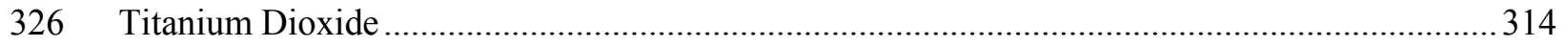

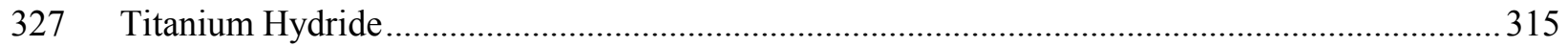

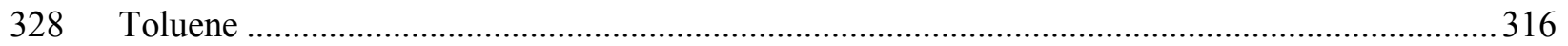

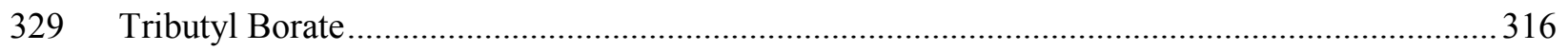

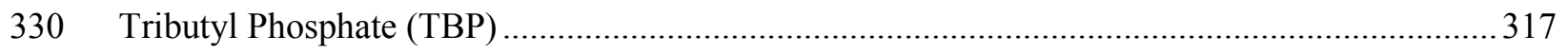

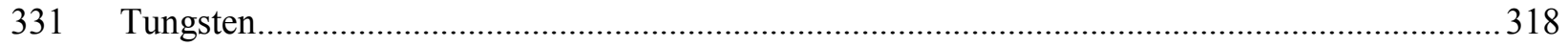

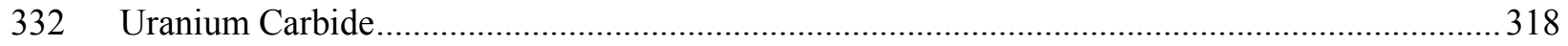

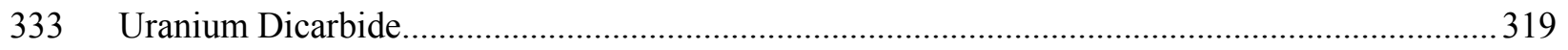

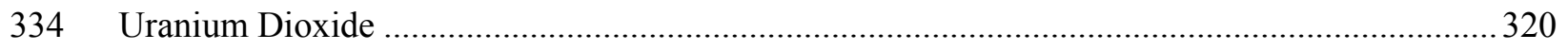

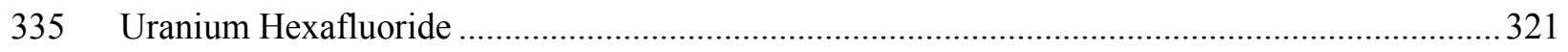

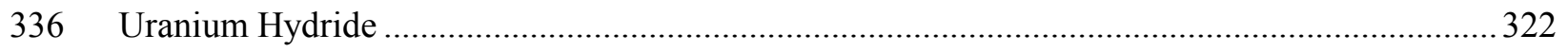

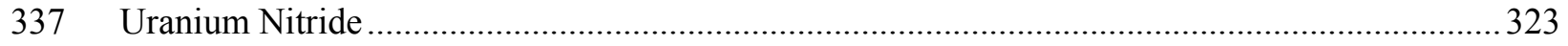

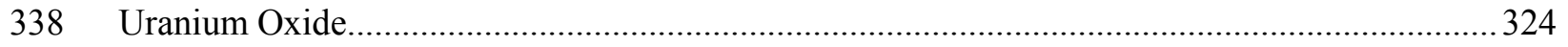

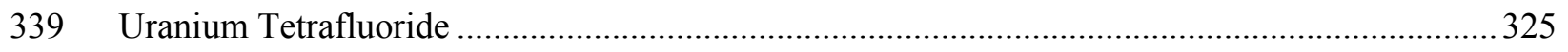

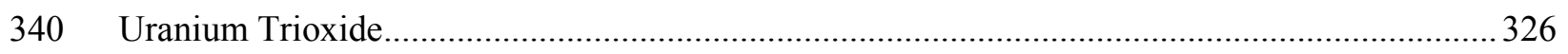

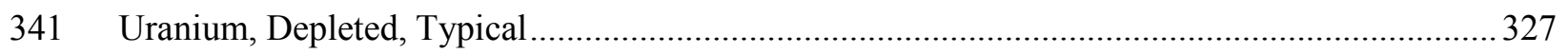

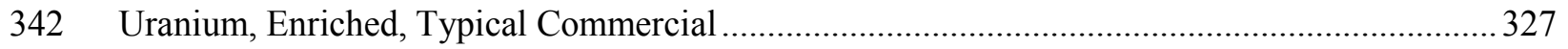

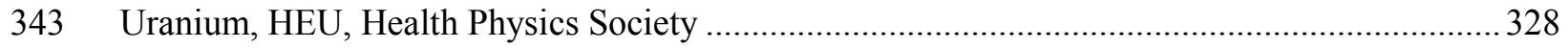

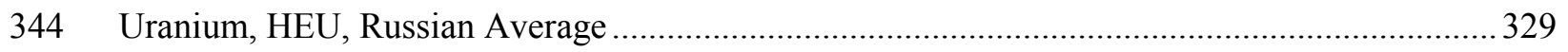

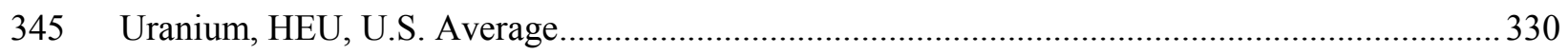

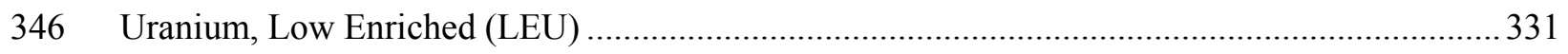




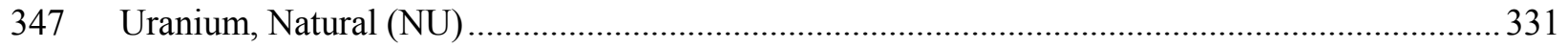

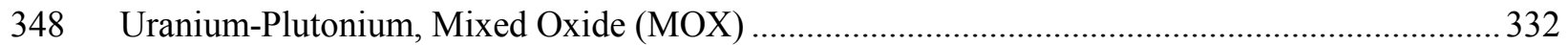

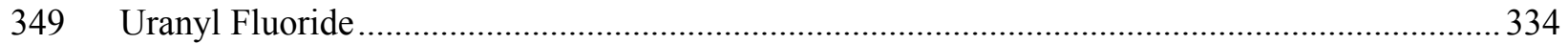

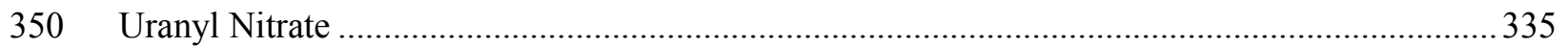

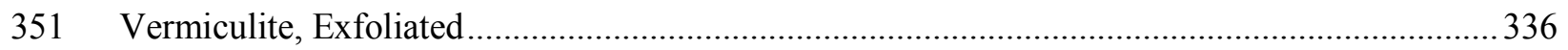

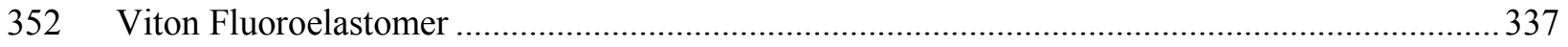

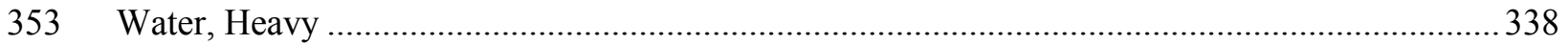

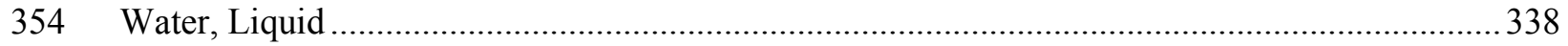

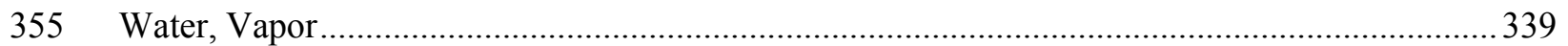

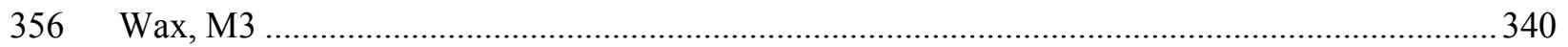

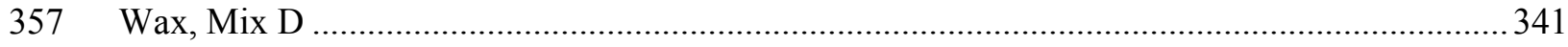

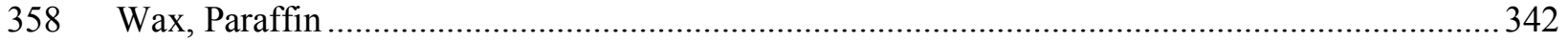

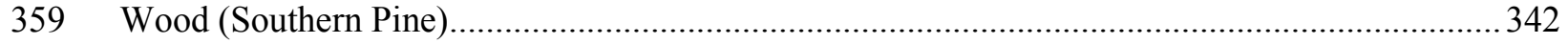

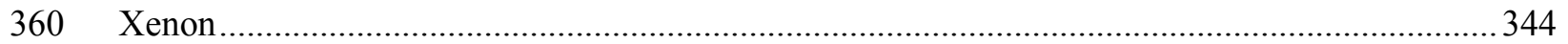

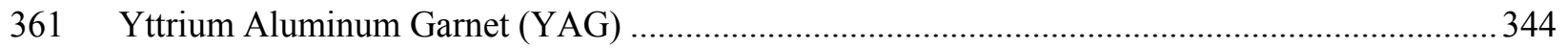

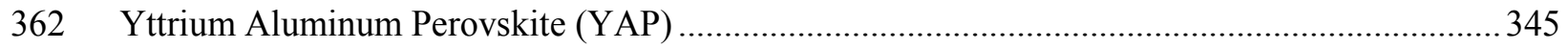

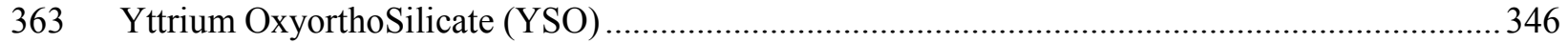

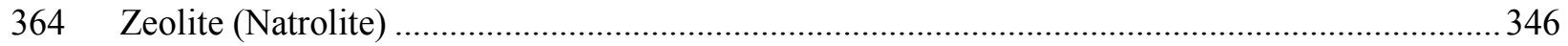

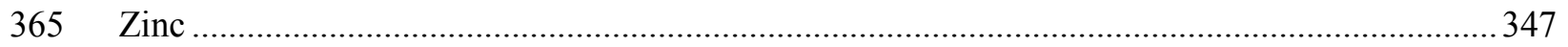

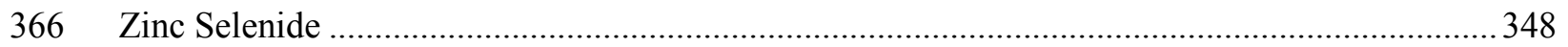

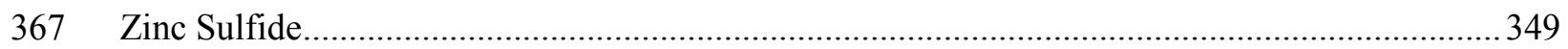

368 Zircaloy-2

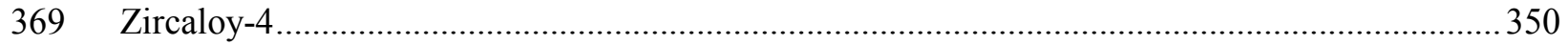

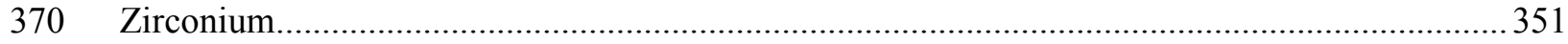

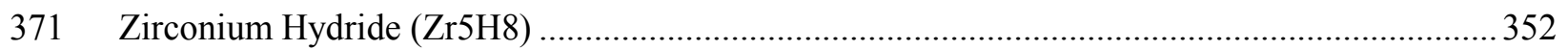

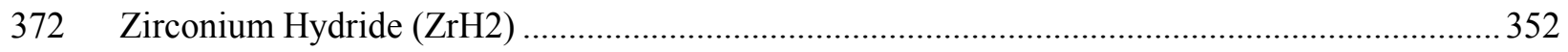

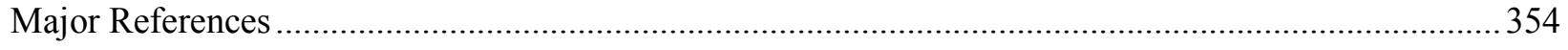


PIET-43741-TM-963

PNNL-15870 Rev. 1

\section{Introduction}

Meaningful simulations of radiation transport applications require realistic definitions of material composition and densities. When seeking that information for applications in fields such as homeland security, radiation shielding and protection, and criticality safety, researchers usually encounter a variety of materials for which elemental compositions are not readily available or densities are not defined. Publication of the Compendium of Material Composition Data for Radiation Transport Modeling, Revision 0, in 2006 was the first step toward mitigating this problem. Revision 0 of this document listed 121 materials, selected mostly from the combined personal libraries of staff at the Pacific Northwest National Laboratory (PNNL), and thus had a scope that was recognized at the time to be limited. Nevertheless, its creation did provide a well-referenced source of some unique or hard-to-define material data in a format that could be used directly in radiation transport calculations being performed at PNNL. Moreover, having a single common set of material definitions also helped to standardize at least one aspect of the various modeling efforts across the laboratory by providing separate researchers the ability to compare different model results using a common basis of materials.

The authors of the 2006 compendium understood that, depending on its use and feedback, the compendium would need to be revised to correct errors or inconsistencies in the data for the original 121 materials, as well as to increase (per users suggestions) the number of materials listed. This 2010 revision of the compendium has accomplished both of those objectives. The most obvious change is the increased number of materials from 121 to 372. The not-so-obvious change is the mechanism used to produce the data listed here. The data listed in the 2006 document were compiled, evaluated, entered, and errorchecked by a group of individuals essentially by hand, providing no library file or mechanism for revising the data in a consistent and traceable manner. The authors of this revision have addressed that problem by first compiling all of the information (i.e., numbers and references) for all the materials into a single database, maintained at PNNL, that was then used as the basis for this document.

The 372 materials included in this document are shown in Table 1. They were selected based on their inclusion in the following references:

- Compendium of Material Composition Data for Radiation Transport Modeling, Revision 0 (Williams III et al. 2006)

- Criticality Calculations with MCNP5: A Primer, Appendix B, pp. 131-140 (Brewer 2009)

- "Standard Composition Library" for the SCALE/KENO code system in SCALE Ver 4.4: A Modular Code System for Performing Standardized Computer Analyses for Licensing Evaluation -- Functional Models F9 - F11 (Petrie et al. 2000)

- Critical Dimensions of Systems Containing ${ }^{235} U,{ }^{239} \mathrm{Pu}$, and ${ }^{233} \mathrm{U}: 1986$ Revision, Appendix, pp 200201 (Paxton and Pruvost 1986)

- Radiation Detection and Measurement (Knoll 2000)

- "Materials: Volume 1" in Reactor Handbook (Hungerford 1960)

- Criticality Handbook, Volume 1, pp II.F.1-1 to 1-8 (Carter et al. 1968)

- "Shielding Materials" in Volume 2 of Engineering Compendium on Radiation Shielding (Jaeger et al. 1975) 
PIET-43741-TM-963

PNNL-15870 Rev. 1

- Radiation Protection for Particle Accelerator Facilities (NCRP 2003)

- Principles of Radiation Shielding (Chilton et al. 1984)

- Radiation Shielding (Shultis and Faw 1996)

- Reactor Shielding for Nuclear Engineers (Schaeffer 1973)

- Materials that are on both of the National Institute of Standards and Technology (NIST) PSTAR and ASTAR lists at http://physics.nist.gov/PhysRefData/Star/Text/table2.html (NIST 1998)

- Materials listed in the NIST X-Ray Mass Attenuation Coefficients (Table 2), at http://physics.nist.gov/PhysRefData/XrayMassCoef/tab2.html (NIST 1996)

As with the first version of this compendium, the data are listed in formats suitable for use in the Monte Carlo N-Particle code, $\mathrm{MCNP}^{1}$, and in the coupled electron-photon one-dimensional discrete ordinates code, CEPXS/ONELD ${ }^{2}$. Unlike the first version, however, the data for each material are divided into four blocks instead of three: 1) the base information block, 2) the MCNP card block, 3) the CEPXS card block, and 4) the comments and reference block. The base information block contains the elemental composition of the material listed using standard elemental symbols. The elements are listed by weight fraction and atom fraction, both normalized to unity. The elements are also listed by atom density (atoms per barn-cm) individually and summed, based on the provided density.

It should be noted that the density of a material can vary widely from typical or average values, especially for foams and insulating/shock absorbing materials. Project-specific density values should always be used, if available, instead of the density values used here. In particular, users should be careful to use the appropriate type of density, i.e., theoretical density vs. bulk density, for their particular application. Bulk densities are sometimes discussed in the comments and reference block. Sources for bulk densities include the following:

- Engineering Resources - Bulk Density Chart at www.powderandbulk.com/resources/bulk_density/material_bulk_density_chart_a.htm (Powder and Bulk Dot Com 2010

- Density of bulk materials chart at http://www.simetric.co.uk/si_materials.htm (Walker 2009)

- MatWeb material property database at http://www.matweb.com/search/search.aspx (Automation Creations 2010)

- Table 6.1.5 of Mark's Standard Handbook for Mechanical Engineers (Avallone and Baumeister III 1996)

- Table 51.65 of Hungerford (1960).

\footnotetext{
${ }^{1}$ MCNP is a trademark of Los Alamos National Security, LLC, Los Alamos National Laboratory. The MCNP code and manuals can be obtained from the Radiation Safety Information Computational Center (RSICC), P.O. Box 2008, Oak Ridge, TN, 37831-6362, (ID\#: C00740MNYCP02, RSICC\#: CCC-740).

${ }^{2}$ CEPXS is part of the one-dimensional coupled electron-photon multigroup discrete ordinates code system, CEPXS/ONELD. CEPXS generates cross sections to be input to the ONELD code. ONELD is a one-dimensional coupled electron-photon transport code. This package is also distributed by RSICC. (ID\#: C00544MNYCP02, RSIC\#: CCC-544).
} 
The MCNP card block provides the material definitions according to the format required by MCNP. Separate lists of those data are given for neutrons and photons. The neutron data should be used for neutron or coupled neutron-photon transport calculations. The photon data should be used for photon transport calculations. Users may choose to input the material data using values of weight fractions, atom fractions, or atom densities. As required for MCNP input, the weight fractions are listed as negative values. Note, however, that Volume 2, Chapter 3, of the MCNP manual (X-5 Monte Carlo Team 2003) recommends that the atom densities be used because the code will convert input weight fractions to atom fractions based on atomic weight values internal to the code, which may not match the values used to calculate atom fractions or atom densities in this compendium. While the difference between the atom fraction listed in this compendium and that calculated by MCNP may be small, it provides a potential uncertainty calculations.

The MCNP user should also note that in the base information block, the total atom density is listed, ready for use in the cell definition cards and, depending on type, in some tally cards. Cross-section information is given in each row of weight fraction, atom fraction, and atom density value as the element $(\mathrm{Z})$ and isotope (A) portion of the ZAID cross-section identification number as provided in Volume 1, Appendix G, of the MCNP manual (X-5 Monte Carlo Team 2005). They appear as a string of integers for each element. For example, 1001 specifies the neutron cross section for hydrogen. The neutron ZA numbers for certain elements ( $\mathrm{Li}, \mathrm{B}, \mathrm{Ge}, \mathrm{Se}, \mathrm{Br}, \mathrm{Kr}, \mathrm{Sr}, \mathrm{Pd}, \mathrm{Ba}, \mathrm{Ce}$, and $\mathrm{Lu}$ ) are omitted, as indicated by a dash, in the MCNP card block. The dash indicates that users should input different ZA numbers for each of the isotopes that are present in that element. Users must also apportion the weight fraction/atom fraction/atom density by the isotopic fraction of each of those isotopes.

The ZA values and the values of weight fraction, atom fraction, or atom density for each element are formatted in this compendium so they can be pasted directly into an MCNP input file using a text editor. After pasting, however, users must replace any resulting tabs with spaces. If the user does not want the MCNP default cross-sections in the data, then the user needs to specify the ID fractional part of the complete ZAID number (e.g., by adding ".50c" to 1001 for hydrogen).

The CEPXS material card block provides the data according to the format required by the cross-section generation part of the CEPXS/ONELD package. The first section of the format block is the material composition. The word "material" is followed by a listing of elements defined by the standard elemental symbols followed by values defining the weight fraction of that element. CEPXS requires that the weight fraction of the elements sum, within a small tolerance, to unity. The second section of the format block is the material name. This is the name for this material in the cross-section file generated by CEPXS. The third section of the format block contains the density information. Using the density provided in this document, CEPXS will generate macroscopic cross sections for use in radiation transport codes. To generate microscopic cross sections, this density value will need to be modified to an appropriate value. Note that the word "gas" must be located below the density in the CEPXS material card block for a material that is a gas, but it is not included in this document. Therefore, for a material that is a gas, the word "gas" must be inserted below the word "density" in a CEPXS input file.

Weight fractions for about $10 \%$ of the 372 materials in this compendium were adjusted so they would sum to unity. The process of normalizing the weight fractions usually was done by dividing the weight fraction for each element in a material by the sum of the non-normalized values. For small changes, normalization occasionally was achieved by adjusting the largest weight fraction so that the weight fractions of all elements in the material would sum to unity. 
Finally, users are cautioned regarding the precision of the values listed here. The calculated weight fractions, atom fractions, and atom densities are all formatted in scientific notation using a fixed format that keeps five digits to the right of the decimal point. Do not infer from this convention that these values are all significant, since in almost every case, the input density has a much larger uncertainty than this. For example, the density used for wood $\left(0.65 \mathrm{~g} / \mathrm{cm}^{3}\right)$ is for southern pine, which generally has a range of 0.61 to $0.67 \mathrm{~g} / \mathrm{cm}^{3}$, but other types of pine can range from 0.43 to $0.71 \mathrm{~g} / \mathrm{cm}^{3}$, and other types of wood can range from 0.11 to $1.33 \mathrm{~g} / \mathrm{cm}^{3}$. But, the weight fractions, atom fractions, and atom densities for wood are calculated using the input density of $0.65 \mathrm{~g} / \mathrm{cm}^{3}$ as though it is an exact value. Users ultimately must take into account the effect of uncertainties in the material density and composition.

Comments regarding this document or suggestions for materials to be included in possible future revisions may be submitted to RJ McConn, Jr. (mailto: ronald.mcconn@pnl.gov.). 
Table 1. Materials Included in This Compendium

\begin{tabular}{|c|c|}
\hline Material Names, Abbreviations, and Alternate Names & Material Number \\
\hline A-150 Tissue-Equivalent Plastic (A150TEP) & 1 \\
\hline Acetone & 2 \\
\hline Acetylene & 3 \\
\hline \multicolumn{2}{|l|}{ Acrylic (see Lucite) } \\
\hline \multicolumn{2}{|l|}{ Acrylic Glass (see Lucite) } \\
\hline \multicolumn{2}{|l|}{ Acrylite (see Lucite) } \\
\hline \multicolumn{2}{|l|}{ Adipose Tissue (see Tissue, Adipose) } \\
\hline \multicolumn{2}{|l|}{ Aggregate (see Rock, Average of 5 Types) } \\
\hline Air (Dry, Near Sea Level) & 4 \\
\hline \multicolumn{2}{|l|}{ Air-Equivalent Plastic (see C-552 Air-Equivalent Plastic) } \\
\hline Alanine & 5 \\
\hline \multicolumn{2}{|l|}{ Alumina (see Aluminum Oxide) } \\
\hline Aluminum & 6 \\
\hline Aluminum Oxide & 7 \\
\hline Aluminum, Alloy 2024-O & 8 \\
\hline Aluminum, Alloy 2090-T83 & 9 \\
\hline Aluminum, Alloy 3003 & 10 \\
\hline Aluminum, Alloy 4043-O & 11 \\
\hline Aluminum, Alloy 5086-O & 12 \\
\hline Aluminum, Alloy 6061-O & 13 \\
\hline Aluminum, Alloy 7075-O & 14 \\
\hline Ammonia (Liquid at $\mathrm{T}=-79^{\circ} \mathrm{C}$ ) & 15 \\
\hline \multicolumn{2}{|l|}{ Ammonium Nitrate (see Explosive Compound, AN) } \\
\hline \multicolumn{2}{|l|}{ AN (see Explosive Compound, AN) } \\
\hline Anthracene & 16 \\
\hline Argon & 17 \\
\hline Asphalt & 18 \\
\hline Asphalt Pavement & 19 \\
\hline \multicolumn{2}{|l|}{ B-100 (see Bone Equivalent Plastic, B-100) } \\
\hline \multicolumn{2}{|l|}{ B-110 (see Bone Equivalent Plastic, B-110) } \\
\hline Bakelite & 20 \\
\hline Barium Fluoride & 21 \\
\hline Barium Sulfate & 22 \\
\hline Benzene & 23 \\
\hline Beryllium & 24 \\
\hline Beryllium Carbide & 25 \\
\hline Beryllium Oxide & 26 \\
\hline \multicolumn{2}{|l|}{ BGO (see Bismuth Germanate) } \\
\hline Bismuth & 27 \\
\hline Bismuth Germanate (BGO) & 28 \\
\hline \multicolumn{2}{|l|}{ Bitumen (see Asphalt) } \\
\hline \multicolumn{2}{|l|}{ Blacktop (see Asphalt Pavement) } \\
\hline Blood (ICRP) & 29 \\
\hline Bone Equivalent Plastic, B-100 & 30 \\
\hline Bone Equivalent Plastic, B-110 & 31 \\
\hline Bone, Compact (ICRU) & 32 \\
\hline
\end{tabular}


PIET-43741-TM-963

PNNL-15870 Rev. 1

\begin{tabular}{|c|c|}
\hline Material Names, Abbreviations, and Alternate Names & Material Number \\
\hline Bone, Cortical (ICRP) & 33 \\
\hline Boral (65\% Al-35\% B4C) & 34 \\
\hline Boral (Aluminum 10\% Boron Alloy) & 35 \\
\hline Boral (Aluminum 5\% Boron Alloy) & 36 \\
\hline Borax & 37 \\
\hline Boric Acid & 38 \\
\hline Boron & 39 \\
\hline Boron Carbide & 40 \\
\hline Boron Fluoride (B2F4) & 41 \\
\hline Boron Fluoride (BF3) & 42 \\
\hline Boron Oxide & 43 \\
\hline Brain (ICRP) & 44 \\
\hline Brass (Typical Composition) & 45 \\
\hline \multicolumn{2}{|l|}{ Breast Tissue (see Tissue, Breast) } \\
\hline Brick, Common Silica & 46 \\
\hline Brick, Fire & 47 \\
\hline Brick, Kaolin (White) & 48 \\
\hline Bronze (Typical Composition) & 49 \\
\hline C-552 Air-Equivalent Plastic & 50 \\
\hline Cadmium & 51 \\
\hline Cadmium Nitrate Tetrahydrate & 52 \\
\hline Cadmium Telluride & 53 \\
\hline \multicolumn{2}{|l|}{ Cadmium Tungstanate (see Cadmium Tungstate) } \\
\hline Cadmium Tungstate (CWO) & 54 \\
\hline \multicolumn{2}{|l|}{ Calcite (see Calcium Carbonate) } \\
\hline Calcium Carbonate & 55 \\
\hline Calcium Fluoride & 56 \\
\hline Calcium Oxide & 57 \\
\hline Calcium Sulfate & 58 \\
\hline Carbon Dioxide & 59 \\
\hline Carbon Tetrachloride & 60 \\
\hline Carbon, Activated & 61 \\
\hline Carbon, Amorphous & 62 \\
\hline Carbon, Graphite (Reactor Grade) & 63 \\
\hline \multicolumn{2}{|l|}{ Carborundum (see Silicon Carbide) } \\
\hline Cat Litter (Clumping) & 64 \\
\hline Cat Litter (Non-clumping) & 65 \\
\hline \multicolumn{2}{|l|}{ Cellophane (see Cellulose Acetate) } \\
\hline \multicolumn{2}{|l|}{ Cellulose (see Celotex and Masonite) } \\
\hline Cellulose Acetate & 66 \\
\hline \multicolumn{2}{|l|}{ Cellulose Nitrate (see Explosive Compound, NC) } \\
\hline Celotex & 67 \\
\hline Ceric Sulfate Dosimeter Solution & 68 \\
\hline Cerium Fluoride & 69 \\
\hline Cesium Iodide & 70 \\
\hline Chromium & 71 \\
\hline Clay & 72 \\
\hline Coal, Anthracite & 73 \\
\hline
\end{tabular}


PIET-43741-TM-963

PNNL-15870 Rev. 1

\begin{tabular}{|c|c|}
\hline Material Names, Abbreviations, and Alternate Names & Material Number \\
\hline Coal, Bituminous & 74 \\
\hline Coal, Lignite & 75 \\
\hline Concrete, Barite (Type BA) & 76 \\
\hline Concrete, Barytes-limonite & 77 \\
\hline Concrete, Boron Frits-baryte & 78 \\
\hline Concrete, Colemanite-baryte & 79 \\
\hline Concrete, Ferro-phosphorus & 80 \\
\hline Concrete, Hanford Dry & 81 \\
\hline Concrete, Hanford Wet & 82 \\
\hline Concrete, Iron-limonite & 83 \\
\hline Concrete, Iron-Portland & 84 \\
\hline Concrete, Limonite and Steel & 85 \\
\hline Concrete, Los Alamos (MCNP) & 86 \\
\hline Concrete, Luminite-colemanite-baryte & 87 \\
\hline Concrete, Luminite-Portland-colemanite-baryte & 88 \\
\hline Concrete, M-1 & 89 \\
\hline Concrete, Magnetite & 90 \\
\hline Concrete, Magnetite and Steel & 91 \\
\hline Concrete, Magnuson & 92 \\
\hline Concrete, $\mathrm{MO}$ & 93 \\
\hline Concrete, Oak Ridge (ORNL) & 94 \\
\hline Concrete, Ordinary (NBS 03) & 95 \\
\hline Concrete, Ordinary (NBS 04) & 96 \\
\hline Concrete, Ordinary (NIST) & 97 \\
\hline Concrete, Portland & 98 \\
\hline Concrete, Regular & 99 \\
\hline Concrete, Rocky Flats & 100 \\
\hline Concrete, Serpentine & 101 \\
\hline Copper & 102 \\
\hline \multicolumn{2}{|l|}{ Corundum (see Aluminum Oxide) } \\
\hline \multicolumn{2}{|l|}{ CWO (see Cadmium Tungstate) } \\
\hline \multicolumn{2}{|l|}{ Dacron (see Polyethylene Terephthalate) } \\
\hline \multicolumn{2}{|l|}{ DE (see Diatomaceous Earth) } \\
\hline \multicolumn{2}{|l|}{ Deuterium Oxide (see Water, Heavy) } \\
\hline Diatomaceous Earth & 103 \\
\hline \multicolumn{2}{|l|}{ Diatomite (see Diatomaceous Earth) } \\
\hline \multicolumn{2}{|l|}{ Dichloromethane (see Methylene Chloride) } \\
\hline \multicolumn{2}{|c|}{ Dirt (see Earth) } \\
\hline \multicolumn{2}{|c|}{$\begin{array}{l}\text { Dosimeter Solution (see Ceric Sulfate Dosimeter Solution or Ferrous Sulfate } \\
\text { Dosimeter Solution) }\end{array}$} \\
\hline \multicolumn{2}{|c|}{ Drywall (see Gypsum) } \\
\hline Earth, Typical Western U.S. & 104 \\
\hline Earth, U.S. Average & 105 \\
\hline \multicolumn{2}{|l|}{ EGDN (see Explosive Compound, EGDN) } \\
\hline Ethane & 106 \\
\hline \multicolumn{2}{|l|}{ Ethanol (see Ethyl Alcohol) } \\
\hline Ethyl Acetate & 107 \\
\hline Ethyl Alcohol & 108 \\
\hline
\end{tabular}


PIET-43741-TM-963

PNNL-15870 Rev. 1

\begin{tabular}{|c|c|}
\hline Material Names, Abbreviations, and Alternate Names & Material Number \\
\hline Ethylene & 109 \\
\hline Ethylene Glycol & 110 \\
\hline \multicolumn{2}{|l|}{ Ethylene Glycol Dinitrate (see Explosive Compound, EGDN) } \\
\hline Explosive Compound, AN & 111 \\
\hline Explosive Compound, EGDN & 112 \\
\hline Explosive Compound, HMX & 113 \\
\hline Explosive Compound, NC & 114 \\
\hline Explosive Compound, NG & 115 \\
\hline Explosive Compound, PETN & 116 \\
\hline Explosive Compound, RDX & 117 \\
\hline Explosive Compound, TNT & 118 \\
\hline Eye Lens (ICRP) & 119 \\
\hline \multicolumn{2}{|l|}{ F1063 (see Toluene) } \\
\hline Felt & 120 \\
\hline Ferric Oxide & 121 \\
\hline \multicolumn{2}{|l|}{ Ferro Boron (see Iron Boride) } \\
\hline \multicolumn{2}{|l|}{ Ferroboride (see Iron Boride) } \\
\hline Ferrous Sulfate Dosimeter Solution & 122 \\
\hline Fertilizer (Muriate of Potash) & 123 \\
\hline \multicolumn{2}{|l|}{ Fiberboard (see Celotex) } \\
\hline Fiberglass, Type C & 124 \\
\hline Fiberglass, Type E & 125 \\
\hline Fiberglass, Type R & 126 \\
\hline \multicolumn{2}{|l|}{ Formica (see Melamine) } \\
\hline Freon-12 & 127 \\
\hline Freon-12B2 & 128 \\
\hline Freon-13 & 129 \\
\hline Freon-13B1 & 130 \\
\hline Freon-13I1 & 131 \\
\hline \multicolumn{2}{|l|}{ Fricke (see Ferrous Sulfate Dosimeter Solution) } \\
\hline Gadolinium & 132 \\
\hline Gadolinium Oxysulfide & 133 \\
\hline Gadolinium Silicate (GSO) & 134 \\
\hline \multicolumn{2}{|l|}{ Gadolinium Sulfoxylate (see Gadolinium Oxysulfide) } \\
\hline Gafchromic Sensor (GS) & 135 \\
\hline Gallium Arsenide & 136 \\
\hline Gasoline & 137 \\
\hline Germanium, High Purity & 138 \\
\hline Glass Scintillator, Li Doped (GS1, GS2, GS3) & 139 \\
\hline Glass Scintillator, Li Doped (GS10, GS20, GS30) & 140 \\
\hline Glass Scintillator, Li Doped (GSF1) & 141 \\
\hline Glass Scintillator, Li Doped (KG1, KG2, KG3) & 142 \\
\hline Glass, Borosilicate (Pyrex Glass) & 143 \\
\hline Glass, Foam & 144 \\
\hline Glass, Lead & 145 \\
\hline Glass, Plate & 146 \\
\hline \multicolumn{2}{|l|}{ Glycerin (see Glycerol) } \\
\hline Glycerol & 147 \\
\hline
\end{tabular}


PIET-43741-TM-963

PNNL-15870 Rev. 1

\begin{tabular}{|c|c|}
\hline Material Names, Abbreviations, and Alternate Names & Material Number \\
\hline \multicolumn{2}{|l|}{ Glycerol Trinitrate (see Explosive Compound, NC) } \\
\hline Gold & 148 \\
\hline \multicolumn{2}{|l|}{ GOS (see Gadolinium Oxysulfide) } \\
\hline \multicolumn{2}{|l|}{ Graphite (see Carbon, Graphite) } \\
\hline \multicolumn{2}{|l|}{ Gravel (see Rock, Average of 5 Types) } \\
\hline \multicolumn{2}{|l|}{ GS (see Gafchromic Sensor) } \\
\hline \multicolumn{2}{|l|}{ GSO (see Gadolinium Silicate) } \\
\hline Gypsum (Plaster of Paris) & 149 \\
\hline \multicolumn{2}{|l|}{ Hardboard (see Masonite) } \\
\hline He-3 Proportional Gas & 150 \\
\hline \multicolumn{2}{|l|}{ Heavy Oil (see Oil, Crude) } \\
\hline Helium, Natural & 151 \\
\hline \multicolumn{2}{|l|}{ HEU (see Uranium, HEU) } \\
\hline \multicolumn{2}{|l|}{ HMX (see Explosive compound, HMX) } \\
\hline Hydrogen & 152 \\
\hline Incoloy-800 & 153 \\
\hline Inconel-600 & 154 \\
\hline Inconel-625 & 155 \\
\hline Inconel-718 & 156 \\
\hline Indium & 157 \\
\hline Iron & 158 \\
\hline Iron Boride $(\mathrm{Fe} 2 \mathrm{~B})$ & 159 \\
\hline Iron Boride $(\mathrm{FeB})$ & 160 \\
\hline \multicolumn{2}{|l|}{ Iron Oxide (see Ferric Oxide) } \\
\hline Iron, Armco Ingot & 161 \\
\hline Iron, Cast (Gray) & 162 \\
\hline Iron, Wrought (Byers No. 1) & 163 \\
\hline \multicolumn{2}{|l|}{ ISO (see Polyisocyanurate) } \\
\hline \multicolumn{2}{|l|}{ Isocyanurate (see Polyisocyanurate) } \\
\hline \multicolumn{2}{|l|}{ Kaolinite (see Kaowool) } \\
\hline Kaowool & 164 \\
\hline Kapton Polyimide Film & 165 \\
\hline Kennertium & 166 \\
\hline Kernite & 167 \\
\hline Kerosene & 168 \\
\hline \multicolumn{2}{|l|}{ Kitty Litter (see Cat Litter) } \\
\hline Krypton & 169 \\
\hline Kynar & 170 \\
\hline \multicolumn{2}{|l|}{ Lard (see Oil, Lard) } \\
\hline Lead & 171 \\
\hline Lead Tungstate (PWO) & 172 \\
\hline \multicolumn{2}{|l|}{ LEU (see Uranium, Low Enriched) } \\
\hline \multicolumn{2}{|l|}{ Lexan (see Polycarbonate) } \\
\hline \multicolumn{2}{|l|}{ LGB (see Lithium Gadrium Borate) } \\
\hline \multicolumn{2}{|l|}{ Li Doped Glass Scintillator (see Glass Scintillator, Li Doped) } \\
\hline \multicolumn{2}{|l|}{ Limestone (see Calcium Carbonate) } \\
\hline Lithium & 173 \\
\hline Lithium Amide & 174 \\
\hline
\end{tabular}


PIET-43741-TM-963

PNNL-15870 Rev. 1

\begin{tabular}{|c|c|}
\hline Material Names, Abbreviations, and Alternate Names & Material Number \\
\hline Lithium Fluoride & 175 \\
\hline Lithium Gadrium Borate (LGB) & 176 \\
\hline Lithium Hydride & 177 \\
\hline Lithium Iodide (High Density) & 178 \\
\hline Lithium Iodide (Low Density) & 179 \\
\hline Lithium Oxide & 180 \\
\hline Lithium Tetraborate & 181 \\
\hline \multicolumn{2}{|l|}{ LSO (see Lutetium Oxyorthosilicate) } \\
\hline \multicolumn{2}{|l|}{ LuAG (see Lutetium Aluminum Garnet) } \\
\hline \multicolumn{2}{|l|}{ LuAP (see Lutetium Orthoaluminate) } \\
\hline Lucite & 182 \\
\hline \multicolumn{2}{|l|}{ Lung Tissue (see Tissue, Lung) } \\
\hline Lutetium Aluminum Garnet (LuAG) & 183 \\
\hline Lutetium Orthoaluminate (LuAP) & 184 \\
\hline Lutetium Oxyorthosilicate (LSO) & 185 \\
\hline Lutetium Yttrium OxyorthoSilicate (LYSO) & 186 \\
\hline \multicolumn{2}{|l|}{ LYSO (see Lutetium Yttrium OxyorthoSilicate) } \\
\hline \multicolumn{2}{|l|}{ M3 Wax (see Wax, M3) } \\
\hline Magnesium & 187 \\
\hline \multicolumn{2}{|l|}{ Magnesium Borate (see Magnesium Tetraborate) } \\
\hline Magnesium Oxide & 188 \\
\hline Magnesium Tetraborate & 189 \\
\hline \multicolumn{2}{|l|}{ Makrolon (see Polycarbonate) } \\
\hline \multicolumn{2}{|l|}{ Marble (see Calcium Carbonate) } \\
\hline Masonite & 190 \\
\hline Melamine & 191 \\
\hline Mercury & 192 \\
\hline Mercury Iodide & 193 \\
\hline Methane & 194 \\
\hline Methanol & 195 \\
\hline \multicolumn{2}{|l|}{ Methyl Alcohol (see Methanol) } \\
\hline \multicolumn{2}{|l|}{ Methylbenzene (see Toluene) } \\
\hline Methylene Chloride & 196 \\
\hline Molybdenum & 197 \\
\hline Monosodium Titanate, MST & 198 \\
\hline \multicolumn{2}{|l|}{ MOX (see Uranium-Plutonium, Mixed Oxide) } \\
\hline \multicolumn{2}{|l|}{ MS20 (see Tissue Equivalent, MS20) } \\
\hline \multicolumn{2}{|l|}{ MST (see Monosodium Titanate) } \\
\hline \multicolumn{2}{|l|}{ Muriate of Potash (see Fertilizer) } \\
\hline Muscle-Equivalent Liquid with Sucrose & 199 \\
\hline Muscle-Equivalent Liquid without Sucrose & 200 \\
\hline Muscle, Skeletal (ICRP) & 201 \\
\hline Muscle, Striated (ICRU) & 202 \\
\hline \multicolumn{2}{|l|}{ Mylar (see Polyethylene Terephthalate) } \\
\hline \multicolumn{2}{|l|}{ Natrolite (see Zeolite) } \\
\hline \multicolumn{2}{|l|}{ NBWO (see Sodium Bismuth Tungstate) } \\
\hline \multicolumn{2}{|l|}{ NC (see Explosive Compound, NC) } \\
\hline Neon & 203 \\
\hline
\end{tabular}


PIET-43741-TM-963

PNNL-15870 Rev. 1

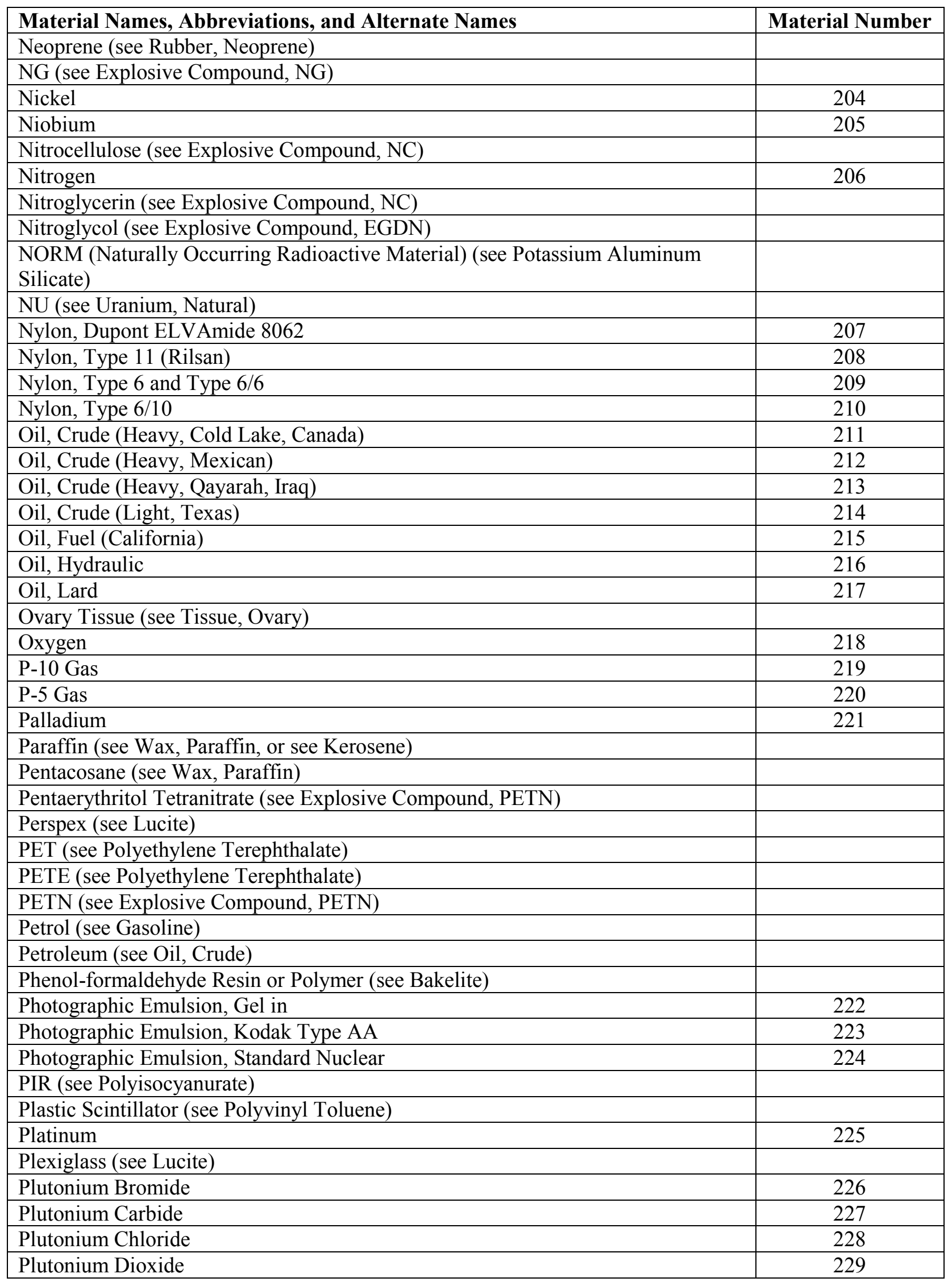


PIET-43741-TM-963

PNNL-15870 Rev. 1

\begin{tabular}{|c|c|}
\hline Material Names, Abbreviations, and Alternate Names & Material Number \\
\hline Plutonium Fluoride (PuF3) & 230 \\
\hline Plutonium Fluoride (PuF4) & 231 \\
\hline Plutonium Fluoride (PuF6) & 232 \\
\hline Plutonium Iodide & 233 \\
\hline Plutonium Nitrate & 234 \\
\hline Plutonium Nitride & 235 \\
\hline Plutonium Oxide (Pu2O3) & 236 \\
\hline Plutonium Oxide $(\mathrm{PuO})$ & 237 \\
\hline Plutonium, Aged WGPu (A: 4-7\% Pu-240) & 238 \\
\hline Plutonium, Aged WGPu (B: $10-13 \% \mathrm{Pu}-240)$ & 239 \\
\hline Plutonium, Aged WGPu (C: 16-19\% Pu-240) & 240 \\
\hline Plutonium, DOE $3013 \mathrm{WGPu}$ & 241 \\
\hline Plutonium, Fuel Grade & 242 \\
\hline Plutonium, Power Grade & 243 \\
\hline Plutonium, Shefelbine WGPu & 244 \\
\hline \multicolumn{2}{|l|}{ Plywood (see Wood) } \\
\hline \multicolumn{2}{|l|}{ PMMA (see Lucite) } \\
\hline \multicolumn{2}{|l|}{ Polyamide (see Nylon, Type 6) } \\
\hline Polycarbonate & 245 \\
\hline \multicolumn{2}{|l|}{ Polychloroprene (see Rubber, Neoprene) } \\
\hline \multicolumn{2}{|l|}{ Polyester (see Polyethylene Terephthalate) } \\
\hline Polyethylene Terephthalate (PET) & 246 \\
\hline Polyethylene, Borated & 247 \\
\hline Polyethylene, Non-borated & 248 \\
\hline \multicolumn{2}{|l|}{ Polyimide Film (see Kapton Polyimide Film) } \\
\hline \multicolumn{2}{|l|}{ Polyiso (see Polyisocyanurate) } \\
\hline \multicolumn{2}{|l|}{ Polyisobutylene (see Rubber, Butyl) } \\
\hline Polyisocyanurate (PIR) & 249 \\
\hline \multicolumn{2}{|l|}{ Polymethyl Methacrylate (see Lucite) } \\
\hline Polypropylene (PP) & 250 \\
\hline Polystyrene (PS) & 251 \\
\hline Polytetrafluoroethylene (PTFE) & 252 \\
\hline Polyurethane Foam (PUR) & 253 \\
\hline Polyvinyl Acetate (PVA) & 254 \\
\hline Polyvinyl Chloride (PVC) & 255 \\
\hline Polyvinyl Toluene (PVT) & 256 \\
\hline Polyvinylidene Chloride (PVDC) & 257 \\
\hline \multicolumn{2}{|l|}{ Polyvinylidene Fluoride (see Kynar) } \\
\hline Potassium Aluminum Silicate & 258 \\
\hline Potassium Iodide & 259 \\
\hline Potassium Oxide & 260 \\
\hline \multicolumn{2}{|l|}{ PP (see Polypropylene) } \\
\hline Propane (Gas) & 261 \\
\hline Propane (Liquid) & 262 \\
\hline \multicolumn{2}{|l|}{ PS (see Polystyrene) } \\
\hline P-terphenyl & 263 \\
\hline \multicolumn{2}{|l|}{ PTFE (see Polytetrafluoroethylene) } \\
\hline PU (see Plutonium or Polyurethane Foam) & \\
\hline
\end{tabular}


PIET-43741-TM-963

PNNL-15870 Rev. 1

\begin{tabular}{|c|c|}
\hline Material Names, Abbreviations, and Alternate Names & Material Number \\
\hline \multicolumn{2}{|l|}{ PUR (see Polyurethane Foam) } \\
\hline \multicolumn{2}{|l|}{ PVA (see Polyvinyl Acetate) } \\
\hline \multicolumn{2}{|l|}{ PVC (see Polyvinyl Chloride) } \\
\hline \multicolumn{2}{|l|}{ PVDF (see Kynar) } \\
\hline \multicolumn{2}{|l|}{ PVT (see Polyvinyl Toluene) } \\
\hline \multicolumn{2}{|l|}{ PWO (see Lead Tungstate) } \\
\hline \multicolumn{2}{|l|}{ Pyrex Glass (see Glass, Borosilicate) } \\
\hline \multicolumn{2}{|l|}{ Quartz (see Silicon Dioxide [Alpha-quartz]) } \\
\hline Radiochromic Dye Film, Nylon Base (RDF: NB) & 264 \\
\hline \multicolumn{2}{|l|}{ RDX (see Explosive Compound, RDX) } \\
\hline Rock (Average of 5 Types) & 265 \\
\hline Rock, Basalt & 266 \\
\hline Rock, Granite & 267 \\
\hline Rock, Limestone & 268 \\
\hline Rock, Sandstone & 269 \\
\hline Rock, Shale & 270 \\
\hline \multicolumn{2}{|l|}{ Rock Salt (see Sodium Chloride) } \\
\hline Rubber, Butyl & 271 \\
\hline Rubber, Natural & 272 \\
\hline Rubber, Neoprene & 273 \\
\hline Rubber, Silicon & 274 \\
\hline \multicolumn{2}{|l|}{ Salt (see Sodium Chloride) } \\
\hline Salt Water $\left(\mathrm{T}=0^{\circ} \mathrm{C}\right)$ & 275 \\
\hline Salt Water $\left(\mathrm{T}=20^{\circ} \mathrm{C}\right)$ & 276 \\
\hline Sand & 277 \\
\hline \multicolumn{2}{|l|}{ Saran (see Polyvinylidene Chloride) } \\
\hline \multicolumn{2}{|l|}{ Scintillator (see Polyvinyl Toluene) } \\
\hline Sea Water, Simple Artificial & 278 \\
\hline Sea Water, Standard & 279 \\
\hline Sepiolite & 280 \\
\hline \multicolumn{2}{|l|}{ Silica (see Silicon Dioxide [Silica]) } \\
\hline Silicon & 281 \\
\hline Silicon Carbide (Hexagonal) & 282 \\
\hline Silicon Dioxide (Alpha-quartz) & 283 \\
\hline Silicon Dioxide (Silica) & 284 \\
\hline Silver & 285 \\
\hline Skin (ICRP) & 286 \\
\hline Sodium & 287 \\
\hline Sodium Bismuth Tungstate (NBWO) & 288 \\
\hline Sodium Chloride & 289 \\
\hline Sodium Iodide & 290 \\
\hline Sodium Nitrate & 291 \\
\hline Sodium Oxide & 292 \\
\hline \multicolumn{2}{|l|}{ Soft Tissue (see Tissue, Soft) } \\
\hline \multicolumn{2}{|l|}{ Soil (see Earth) } \\
\hline \multicolumn{2}{|l|}{ Stainless Steel (see Steel) } \\
\hline \multicolumn{2}{|l|}{ Standard Fricke (see Ferrous Sulfate Dosimeter Solution) } \\
\hline Steel, Boron Stainless & 293 \\
\hline
\end{tabular}


PIET-43741-TM-963

PNNL-15870 Rev. 1

\begin{tabular}{|c|c|}
\hline Material Names, Abbreviations, and Alternate Names & Material Number \\
\hline Steel, Carbon & 294 \\
\hline Steel, HT9 Stainless & 295 \\
\hline Steel, Stainless 202 & 296 \\
\hline Steel, Stainless 302 & 297 \\
\hline Steel, Stainless 304 & 298 \\
\hline Steel, Stainless 304L & 299 \\
\hline Steel, Stainless 316 & 300 \\
\hline Steel, Stainless 316L & 301 \\
\hline Steel, Stainless 321 & 302 \\
\hline Steel, Stainless 347 & 303 \\
\hline Steel, Stainless 409 & 304 \\
\hline Steel, Stainless 440 & 305 \\
\hline Sterotex & 306 \\
\hline Stilbene (Trans-stilbene Isomer) & 307 \\
\hline \multicolumn{2}{|l|}{ Styrofoam (see Polystyrene) } \\
\hline Sulphur & 308 \\
\hline Tantalum & 309 \\
\hline \multicolumn{2}{|l|}{ TBP (see Tributyl Phosphate) } \\
\hline \multicolumn{2}{|l|}{ Teflon (see Polytetrafluoroethylene) } \\
\hline \multicolumn{2}{|l|}{ TEG (see Tissue-Equivalent Gas) } \\
\hline \multicolumn{2}{|l|}{ Testes Tissue (see Tissue, Testes) } \\
\hline Thorium & 310 \\
\hline Thorium Dioxide & 311 \\
\hline Tin & 312 \\
\hline Tissue Equivalent, MS20 & 313 \\
\hline Tissue-Equivalent Gas, Methane Based (TEG: MB) & 314 \\
\hline Tissue-Equivalent Gas, Propane Based (TEG: PB) & 315 \\
\hline Tissue, Adipose (ICRP) & 316 \\
\hline Tissue, Breast & 317 \\
\hline Tissue, Lung (ICRP) & 318 \\
\hline Tissue, Ovary & 319 \\
\hline Tissue, Soft (ICRP) & 320 \\
\hline Tissue, Soft (ICRU Four Component) & 321 \\
\hline Tissue, Testes (ICRP) & 322 \\
\hline Tissue, Testis (ICRU) & 323 \\
\hline Titanium & 324 \\
\hline Titanium Alloy, Grade 5 & 325 \\
\hline Titanium Dioxide & 326 \\
\hline Titanium Hydride & 327 \\
\hline \multicolumn{2}{|l|}{ TNT (see Explosive Compound, TNT) } \\
\hline Toluene & 328 \\
\hline \multicolumn{2}{|l|}{ Trans-stilbene (see Stilbene) } \\
\hline Tributyl Borate & 329 \\
\hline Tributyl Phosphate (TBP) & 330 \\
\hline \multicolumn{2}{|l|}{ Trinitroglycerol (see Explosive Compound, NC) } \\
\hline \multicolumn{2}{|l|}{ Trinitrotoluene (see Explosive Compound, TNT) } \\
\hline Tungsten & 331 \\
\hline Uranium Carbide & 332 \\
\hline
\end{tabular}


PIET-43741-TM-963

PNNL-15870 Rev. 1

\begin{tabular}{|c|c|}
\hline Material Names, Abbreviations, and Alternate Names & Material Number \\
\hline Uranium Dicarbide & 333 \\
\hline Uranium Dioxide & 334 \\
\hline Uranium Hexafluoride & 335 \\
\hline Uranium Hydride & 336 \\
\hline Uranium Nitride & 337 \\
\hline Uranium Oxide & 338 \\
\hline Uranium Tetrafluoride & 339 \\
\hline Uranium Trioxide & 340 \\
\hline Uranium, Depleted, Typical & 341 \\
\hline Uranium, Enriched, Typical Commercial & 342 \\
\hline Uranium, HEU, Health Physics Society & 343 \\
\hline Uranium, HEU, Russian Average & 344 \\
\hline Uranium, HEU, U.S. Average & 345 \\
\hline Uranium, Low Enriched (LEU) & 346 \\
\hline Uranium, Natural (NU) & 347 \\
\hline Uranium-Plutonium, Mixed Oxide (MOX) & 348 \\
\hline Uranyl Fluoride & 349 \\
\hline Uranyl Nitrate & 350 \\
\hline Vermiculite, Exfoliated & 351 \\
\hline \multicolumn{2}{|l|}{ Vinyltoluene (see Polyvinyl Toluene) } \\
\hline Viton Fluoroelastomer & 352 \\
\hline \multicolumn{2}{|l|}{ Wallboard (see Gypsum) } \\
\hline Water, Heavy & 353 \\
\hline Water, Liquid & 354 \\
\hline Water, Vapor & 355 \\
\hline Wax, M3 & 356 \\
\hline Wax, Mix D & 357 \\
\hline Wax, Paraffin & 358 \\
\hline \multicolumn{2}{|l|}{ Weapons Grade Plutonium (see Plutonium, Aged WGPu) } \\
\hline Wood (Southern Pine) & 359 \\
\hline Xenon & 360 \\
\hline \multicolumn{2}{|l|}{ YAG (see Yttrium Aluminum Garnet) } \\
\hline \multicolumn{2}{|l|}{ YAP (see Yttrium Aluminum Perovskite) } \\
\hline \multicolumn{2}{|l|}{ YSO (see Yttrium OxyorthoSilicate) } \\
\hline Yttrium Aluminum Garnet (YAG) & 361 \\
\hline Yttrium Aluminum Perovskite (YAP) & 362 \\
\hline Yttrium OxyorthoSilicate (YSO) & 363 \\
\hline Zeolite (Natrolite) & 364 \\
\hline Zinc & 365 \\
\hline Zinc Selenide & 366 \\
\hline Zinc Sulfide & 367 \\
\hline Zircaloy-2 & 368 \\
\hline Zircaloy-4 & 369 \\
\hline Zirconium & 370 \\
\hline Zirconium Hydride (Zr5H8) & 371 \\
\hline Zirconium Hydride (ZrH2) & 372 \\
\hline
\end{tabular}




\section{Materials}

\section{$1 \quad$ A-150 Tissue-Equivalent Plastic (A150TEP)}

\begin{tabular}{llll}
\hline Formula $=$ & - & Molecular weight $(\mathrm{g} / \mathrm{mole})=$ \\
Density $(\mathrm{g} / \mathrm{cm} 3)=$ & 1.127000 & Total atom density $($ atoms $/ \mathrm{b}-\mathrm{cm})=$ & - \\
\hline & $1.169 \mathrm{E}-01$
\end{tabular}

The above density is estimated to be accurate to 4 significant digits. Uncertainties are not addressed.

The following data were calculated from the input weight fractions.

\begin{tabular}{|c|c|c|c|c|c|c|}
\hline Element & Neutron ZA & Photon ZA & $\begin{array}{l}\text { Weight } \\
\text { Fraction }\end{array}$ & $\begin{array}{l}\text { Atom } \\
\text { Fraction }\end{array}$ & $\begin{array}{l}\text { Atom } \\
\text { Density }\end{array}$ & \\
\hline $\mathrm{H}$ & 1001 & 1000 & 0.101327 & 0.583640 & 0.068228 & \\
\hline $\mathrm{C}$ & 6000 & 6000 & 0.775501 & 0.374859 & 0.043822 & \\
\hline $\mathrm{N}$ & 7014 & 7000 & 0.035057 & 0.014531 & 0.001699 & \\
\hline $\mathrm{O}$ & 8016 & 8000 & 0.052316 & 0.018984 & 0.002219 & \\
\hline $\mathrm{F}$ & 9019 & 9000 & 0.017422 & 0.005324 & 0.000622 & \\
\hline $\mathrm{Ca}$ & 20000 & 20000 & 0.018378 & 0.002662 & 0.000311 & \\
\hline Total & & & 1.000001 & 1.000000 & 0.116902 & \\
\hline MCNP Form & \multicolumn{2}{|c|}{ Weight Fractions } & \multicolumn{2}{|c|}{ Atom Fractions } & \multicolumn{2}{|c|}{ Atom Densities } \\
\hline \multirow[t]{6}{*}{ Neutrons } & 1001 & -0.101327 & 1001 & 0.583640 & 1001 & 0.068228 \\
\hline & 6000 & -0.775501 & 6000 & 0.374859 & 6000 & 0.043822 \\
\hline & 7014 & -0.035057 & 7014 & 0.014531 & 7014 & 0.001699 \\
\hline & 8016 & -0.052316 & 8016 & 0.018984 & 8016 & 0.002219 \\
\hline & 9019 & -0.017422 & 9019 & 0.005324 & 9019 & 0.000622 \\
\hline & 20000 & -0.018378 & 20000 & 0.002662 & 20000 & 0.000311 \\
\hline \multirow[t]{6}{*}{ Photons } & 1000 & -0.101327 & 1000 & 0.583640 & 1000 & 0.068228 \\
\hline & 6000 & -0.775501 & 6000 & 0.374859 & 6000 & 0.043822 \\
\hline & 7000 & -0.035057 & 7000 & 0.014531 & 7000 & 0.001699 \\
\hline & 8000 & -0.052316 & 8000 & 0.018984 & 8000 & 0.002219 \\
\hline & 9000 & -0.017422 & 9000 & 0.005324 & 9000 & 0.000622 \\
\hline & 20000 & -0.018378 & 20000 & 0.002662 & 20000 & 0.000311 \\
\hline \multirow[t]{7}{*}{ CEPXS Form: } & material & $\mathrm{H}$ & 0.101327 & & & \\
\hline & & C & 0.775501 & & & \\
\hline & & $\mathrm{N}$ & 0.035057 & & & \\
\hline & & 0 & 0.052316 & & & \\
\hline & & $\mathrm{F}$ & 0.017422 & & & \\
\hline & & $\mathrm{Ca}$ & 0.018378 & & & \\
\hline & $\begin{array}{l}\text { matname } \\
\text { density }\end{array}$ & \multicolumn{3}{|c|}{$\begin{array}{l}\text { A-150 Tissue-Equivalent Plastic (A150TEP) } \\
1.127000\end{array}$} & & \\
\hline
\end{tabular}




\section{Acetone}

\begin{tabular}{llll}
\hline Formula $=$ & C3H6O & Molecular weight $(\mathrm{g} / \mathrm{mole})=$ & 58.07914 \\
Density $(\mathrm{g} / \mathrm{cm} 3)=$ & 0.789900 & Total atom density $($ atoms $/ \mathrm{b}-\mathrm{cm})=$ & $8.190 \mathrm{E}-02$
\end{tabular}

The above density is estimated to be accurate to 4 significant digits. Uncertainties are not addressed.

The following data were calculated from the input weight fractions.

\begin{tabular}{|c|c|c|c|c|c|}
\hline Element & Neutron ZA & Photon ZA & $\begin{array}{l}\text { Weight } \\
\text { Fraction }\end{array}$ & $\begin{array}{c}\text { Atom } \\
\text { Fraction }\end{array}$ & $\begin{array}{c}\text { Atom } \\
\text { Density }\end{array}$ \\
\hline $\mathrm{H}$ & 1001 & 1000 & 0.104122 & 0.599985 & 0.049140 \\
\hline C & 6000 & 6000 & 0.620405 & 0.300013 & 0.024571 \\
\hline 0 & 8016 & 8000 & 0.275473 & 0.100002 & 0.008190 \\
\hline
\end{tabular}

Total

$1.000000 \quad 1.000000 \quad 0.081901$

\begin{tabular}{|c|c|c|c|c|c|c|}
\hline MCNP Form & \multicolumn{2}{|c|}{ Weight Fractions } & \multicolumn{2}{|c|}{ Atom Fractions } & \multicolumn{2}{|c|}{ Atom Densities } \\
\hline \multirow[t]{3}{*}{ Neutrons } & 1001 & -0.104122 & 1001 & 0.599985 & 1001 & 0.049140 \\
\hline & 6000 & -0.620405 & 6000 & 0.300013 & 6000 & 0.024571 \\
\hline & 8016 & -0.275473 & 8016 & 0.100002 & 8016 & 0.008190 \\
\hline \multirow[t]{3}{*}{ Photons } & 1000 & -0.104122 & 1000 & 0.599985 & 1000 & 0.049140 \\
\hline & 6000 & -0.620405 & 6000 & 0.300013 & 6000 & 0.024571 \\
\hline & 8000 & -0.275473 & 8000 & 0.100002 & 8000 & 0.008190 \\
\hline \multirow[t]{4}{*}{ CEPXS Form: } & material & $\mathrm{H}$ & 0.104122 & & & \\
\hline & & C & 0.620405 & & & \\
\hline & & $\mathrm{O}$ & 0.275473 & & & \\
\hline & $\begin{array}{c}\text { matname } \\
\text { density }\end{array}$ & $\begin{array}{l}\text { Acetone } \\
0.789900\end{array}$ & & & & \\
\hline \multicolumn{7}{|c|}{$\begin{array}{l}\text { Comments and References } \\
\text { Density and weight fractions from http://physics.nist.gov/cgi-bin/Star/compos.pl?matno=100 (NIST 1998). } \\
\text { Formula from } \\
\text { http://www.matweb.com/search/DataSheet.aspx?MatGUID=1f9fd4a5357e428f9a82e750f4fbbf0e } \\
\text { (Automation Creations } 2010) \text {. } \\
\text { Formula and density }=0.7845 \text { in Lide (2008), pgs } 3-4 \text {. }\end{array}$} \\
\hline
\end{tabular}

\section{Acetylene}

$\begin{array}{llll}\text { Formula }= & \mathrm{C} 2 \mathrm{H} 2 & \text { Molecular weight }(\mathrm{g} / \mathrm{mole})= & 26.03728 \\ \text { Density }(\mathrm{g} / \mathrm{cm} 3)= & 0.001097 & \text { Total atom density }(\text { atoms } / \mathrm{b}-\mathrm{cm})= & 1.015 \mathrm{E}-04\end{array}$

The above density is estimated to be accurate to 4 significant digits. Uncertainties are not addressed.

The following data were calculated from the input weight fractions.

\begin{tabular}{|c|c|c|c|c|c|}
\hline lement & Neutron ZA & Photon ZA & $\begin{array}{l}\text { Weight } \\
\text { Fraction }\end{array}$ & $\begin{array}{c}\text { Atom } \\
\text { Fraction }\end{array}$ & $\begin{array}{l}\text { Atom } \\
\text { Density }\end{array}$ \\
\hline $\mathrm{H}$ & 1001 & 1000 & 0.077418 & 0.499983 & 0.000051 \\
\hline
\end{tabular}


PIET-43741-TM-963

PNNL-15870 Rev. 1

\begin{tabular}{|c|c|c|c|c|c|c|}
\hline C & 6000 & 6000 & 0.922582 & 0.500017 & \multicolumn{2}{|c|}{0.000051} \\
\hline \multicolumn{3}{|l|}{ Total } & 1.000000 & 1.000000 & \multicolumn{2}{|c|}{0.000101} \\
\hline MCNP Form & \multicolumn{2}{|c|}{ Weight Fractions } & \multicolumn{2}{|c|}{ Atom Fractions } & \multicolumn{2}{|c|}{ Atom Densities } \\
\hline \multirow[t]{2}{*}{ Neutrons } & 1001 & -0.077418 & 1001 & 0.499983 & 1001 & 0.000051 \\
\hline & 6000 & -0.922582 & 6000 & 0.500017 & 6000 & 0.000051 \\
\hline \multirow[t]{2}{*}{ Photons } & 1000 & -0.077418 & 1000 & 0.499983 & 1000 & 0.000051 \\
\hline & 6000 & -0.922582 & 6000 & 0.500017 & 6000 & 0.000051 \\
\hline \multirow[t]{3}{*}{ CEPXS Form: } & material & $\mathrm{H}$ & 0.077418 & & & \\
\hline & & C & 0.922582 & & & \\
\hline & $\begin{array}{c}\text { matname } \\
\text { density }\end{array}$ & $\begin{array}{l}\text { Acetylene } \\
0.001097\end{array}$ & & & & \\
\hline \multicolumn{7}{|c|}{$\begin{array}{l}\text { Comments and References } \\
\text { Density and weight fractions from http://physics.nist.gov/cgi-bin/Star/compos.pl?matno=101 (NIST 1998). } \\
\text { Formula from Lide (2008), pgs } 3-6 \text {. }\end{array}$} \\
\hline
\end{tabular}

\section{$4 \quad$ Air (Dry, Near Sea Level)}

\begin{tabular}{llll}
\hline Formula $=$ & - & Molecular weight $(\mathrm{g} / \mathrm{mole})=$ \\
Density $(\mathrm{g} / \mathrm{cm} 3)=$ & 0.001205 & Total atom density $($ atoms $/ \mathrm{b}-\mathrm{cm})=\begin{array}{l}- \\
\text { The }\end{array} \quad$ es8E-05
\end{tabular}

The above density is estimated to be accurate to 4 significant digits. Uncertainties are not addressed.

The following data were calculated from the input weight fractions.

\begin{tabular}{|c|c|c|c|c|c|c|}
\hline Element & Neutron ZA & Photon ZA & $\begin{array}{l}\text { Weight } \\
\text { Fraction }\end{array}$ & $\begin{array}{c}\text { Atom } \\
\text { Fraction }\end{array}$ & $\begin{array}{l}\text { Atom } \\
\text { Density }\end{array}$ & \\
\hline $\begin{array}{l}C \\
N\end{array}$ & $\begin{array}{l}6000 \\
7014\end{array}$ & $\begin{array}{l}6000 \\
7000\end{array}$ & $\begin{array}{l}0.0001<4 \\
0755268\end{array}$ & $\begin{array}{l}0.000150 \\
0784431\end{array}$ & 0.000039 & \\
\hline 0 & 8016 & 8000 & 0.231781 & 0.210748 & 0.000011 & \\
\hline $\mathrm{Ar}$ & 18000 & 18000 & 0.012827 & 0.004671 & 0.000000 & \\
\hline Total & & & 1.000000 & 1.000000 & 0.000050 & \\
\hline MCNP Form & \multicolumn{2}{|c|}{ Weight Fractions } & \multicolumn{2}{|c|}{ Atom Fractions } & \multicolumn{2}{|c|}{ Atom Densities } \\
\hline \multirow[t]{4}{*}{ Neutrons } & 6000 & -0.000124 & 6000 & 0.000150 & 6000 & 0.000000 \\
\hline & 7014 & -0.755268 & 7014 & 0.784431 & 7014 & 0.000039 \\
\hline & 8016 & -0.231781 & 8016 & 0.210748 & 8016 & 0.000011 \\
\hline & 18000 & -0.012827 & 18000 & 0.004671 & 18000 & 0.000000 \\
\hline \multirow[t]{4}{*}{ Photons } & 6000 & -0.000124 & 6000 & 0.000150 & 6000 & 0.000000 \\
\hline & 7000 & -0.755268 & 7000 & 0.784431 & 7000 & 0.000039 \\
\hline & 8000 & -0.231781 & 8000 & 0.210748 & 8000 & 0.000011 \\
\hline & 18000 & -0.012827 & 18000 & 0.004671 & 18000 & 0.000000 \\
\hline
\end{tabular}




\begin{tabular}{|c|c|c|c|}
\hline \multirow[t]{4}{*}{ CEPXS Form: } & \multirow[t]{4}{*}{ material } & C & 0.000124 \\
\hline & & $\mathrm{N}$ & 0.755268 \\
\hline & & $\mathrm{O}$ & 0.231781 \\
\hline & & $\mathrm{Ar}$ & 0.012827 \\
\hline
\end{tabular}

matname Air (Dry, Near Sea Level) density $\quad 0.001205$

\section{Comments and References}

Density and weight fractions from http://physics.nist.gov/PhysRefData/XrayMassCoef/tab2.html (NIST 1996).

Note: This NIST data yields a CO2 content in air of about $299 \mathrm{ppm}$ by volume, whereas measurements at the Mauna Loa Observatory in 2004 indicate an average CO2 content of 377.38 ppm (Lide 2009, pgs 14 - 28).

\begin{tabular}{|c|c|c|c|c|c|c|}
\hline Alanine & & & & & & \\
\hline Formula $=$ & - & & Molecular & ght (g/mole) & & \\
\hline Density (g/cm3) & $=\quad 1.42000$ & & Total aton & nsity (atoms & $m)=$ & E-01 \\
\hline $\begin{array}{l}\text { The above dens } \\
\text { The following da }\end{array}$ & $\begin{array}{l}\text { ty is estimated } \\
\text { ta were calcula }\end{array}$ & $\begin{array}{l}\text { to be accurat } \\
\text { ted from the ir }\end{array}$ & $\begin{array}{l}3 \text { significa } \\
t \text { weight fra }\end{array}$ & $\begin{array}{l}\text { gits. Uncert } \\
\text { Is. }\end{array}$ & ies are no & essed. \\
\hline & & & Weight & Atom & Atom & \\
\hline Element & Neutron ZA & Photon ZA & Fraction & Fraction & Density & \\
\hline $\mathrm{H}$ & 1001 & 1000 & $\overline{0.079190}$ & $\overline{0.538450}$ & 0.06718 & \\
\hline $\mathrm{C}$ & 6000 & 6000 & 0.404439 & 0.230778 & 0.02879 & \\
\hline $\mathrm{N}$ & 7014 & 7000 & 0.157213 & 0.076924 & 0.00959 & \\
\hline $\mathrm{O}$ & 8016 & 8000 & 0.359159 & 0.153848 & 0.01919 & \\
\hline Total & & & 1.000001 & 1.000000 & 0.12477 & \\
\hline MCNP Form & Weight $\mathrm{F}$ & ractions & Atom & ctions & Ator & sities \\
\hline Neutrons & 1001 & -0.079190 & 1001 & 0.538450 & 1001 & 0.067185 \\
\hline & 6000 & -0.404439 & 6000 & 0.230778 & 6000 & 0.028795 \\
\hline & 7014 & -0.157213 & 7014 & 0.076924 & 7014 & 0.009598 \\
\hline & 8016 & -0.359159 & 8016 & 0.153848 & 8016 & 0.019197 \\
\hline Photons & 1000 & -0.079190 & 1000 & 0.538450 & 1000 & 0.067185 \\
\hline & 6000 & -0.404439 & 6000 & 0.230778 & 6000 & 0.028795 \\
\hline & 7000 & -0.157213 & 7000 & 0.076924 & 7000 & 0.009598 \\
\hline & 8000 & -0.359159 & 8000 & 0.153848 & 8000 & 0.019197 \\
\hline CEPXS Form: & material & $\mathrm{H}$ & 0.079190 & & & \\
\hline & & C & 0.404439 & & & \\
\hline & & $\mathrm{N}$ & 0.157213 & & & \\
\hline & & O & 0.359159 & & & \\
\hline & $\begin{array}{c}\text { matname } \\
\text { density }\end{array}$ & $\begin{array}{l}\text { Alanine } \\
1.420000\end{array}$ & & & & \\
\hline
\end{tabular}


PIET-43741-TM-963

PNNL-15870 Rev. 1

Comments and References

Density and weight fractions from http://physics.nist.gov/cgi-bin/Star/compos.pl?matno=105 (NIST 1998).

\section{$6 \quad$ Aluminum}

$\begin{array}{llll}\text { Formula }= & \mathrm{Al} & \text { Molecular weight }(\mathrm{g} / \mathrm{mole})= & 26.981538 \\ \text { Density }(\mathrm{g} / \mathrm{cm} 3)= & 2.698900 & \text { Total atom density }(\text { atoms } / \mathrm{b}-\mathrm{cm})= & 6.024 \mathrm{E}-02\end{array}$

The above density is estimated to be accurate to 4 significant digits. Uncertainties are not addressed.

The following data was calculated from the input formula.

$\begin{array}{cccccc}\frac{\text { Element }}{\mathrm{Al}} & \frac{\text { Neutron ZA }}{13027} & \frac{\text { Photon ZA }}{13000} & \frac{\begin{array}{c}\text { Weight } \\ \text { Fraction }\end{array}}{1.000000} & \frac{\begin{array}{c}\text { Atom } \\ \text { Fraction }\end{array}}{1.000000} & \begin{array}{c}\text { Atom } \\ \text { Density }\end{array} \\ \text { Total } & & & 1.000000 & 1.000000 & 0.060238\end{array}$

\begin{tabular}{|c|cc|cc|cr|}
\hline MCNP Form & \multicolumn{2}{c|}{ Weight Fractions } & \multicolumn{2}{c|}{ Atom Fractions } & \multicolumn{2}{c|}{ Atom Densities } \\
\hline Neutrons & 13027 & -1.000000 & 13027 & 1.000000 & 13027 & 0.060238 \\
& & & & & & 13000 \\
Photons & 13000 & -1.000000 & 13000 & 1.000000 & 0.060238
\end{tabular}

CEPXS Form: material $\quad \mathrm{Al} \quad 1.000000$

matname Aluminum

density 2.698900

Comments and References

Density from http://physics.nist.gov/cgi-bin/Star/compos.pl?matno=013 (NIST 1998).

\section{$7 \quad$ Aluminum Oxide}

\begin{tabular}{llll}
\hline Formula $=$ & Al2O3 & Molecular weight $(\mathrm{g} / \mathrm{mole})=$ & 101.961276 \\
Density $(\mathrm{g} / \mathrm{cm} 3)=$ & 3.970000 & Total atom density $($ atoms $/ \mathrm{b}-\mathrm{cm})=$ & $1.172 \mathrm{E}-01$
\end{tabular}

The above density is estimated to be accurate to 3 significant digits. Uncertainties are not addressed.

The following data were calculated from the input weight fractions.

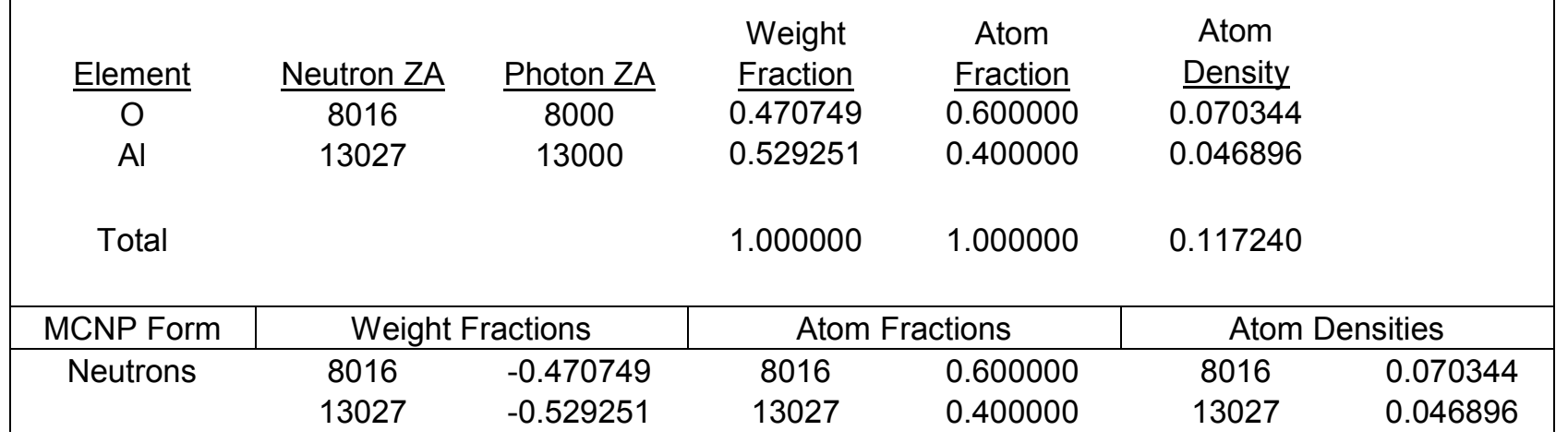


PIET-43741-TM-963

PNNL-15870 Rev. 1

\begin{tabular}{|c|c|c|c|c|c|c|}
\hline Photons & $\begin{array}{c}8000 \\
13000\end{array}$ & $\begin{array}{l}-0.470749 \\
-0.529251\end{array}$ & $\begin{array}{c}8000 \\
13000\end{array}$ & $\begin{array}{l}0.600000 \\
0.400000\end{array}$ & $\begin{array}{c}8000 \\
13000\end{array}$ & $\begin{array}{l}0.070344 \\
0.046896\end{array}$ \\
\hline CEPXS Form: & $\begin{array}{c}\text { matname } \\
\text { density }\end{array}$ & $\begin{array}{l}\text { O } \\
\text { Al } \\
\text { Aluminum O } \\
3.970000\end{array}$ & $\begin{array}{l}0.470749 \\
0.529251\end{array}$ & & & \\
\hline \multicolumn{7}{|c|}{$\begin{array}{l}\text { Comments and References } \\
\text { Density and weight fractions from http://physics.nist.gov/cgi-bin/Star/compos.pl?matno=106 (NIST 1998). } \\
\text { Formula from Lide (2008), pgs } 4-45 \text {. } \\
\text { Also called alumina or corundum (http://en.wikipedia.org/wiki/Aluminum_oxide). Bulk density for alumina } \\
\text { is } 0.64 \text { at http://www.powderandbulk.com/resources/bulk_density/material_bulk_density_chart_a.htm } \\
\text { (Powder and Bulk Dot Com 2010). }\end{array}$} \\
\hline
\end{tabular}

\section{Aluminum, Alloy 2024-O}

\begin{tabular}{llll}
\hline Formula $=$ & - & Molecular weight $(\mathrm{g} / \mathrm{mole})=$ & - \\
Density $(\mathrm{g} / \mathrm{cm} 3)=$ & 2.780000 & Total atom density $($ atoms $/ \mathrm{b}-\mathrm{cm})=$ & $6.022 \mathrm{E}-02$
\end{tabular}

The above density is estimated to be accurate to 3 significant digits. Uncertainties are not addressed. The following data were calculated from the input weight fractions.

\begin{tabular}{|c|c|c|c|c|c|c|}
\hline Element & Neutron ZA & Photon ZA & $\begin{array}{l}\text { Weight } \\
\text { Fraction }\end{array}$ & $\begin{array}{c}\text { Atom } \\
\text { Fraction }\end{array}$ & $\begin{array}{c}\text { Atom } \\
\text { Density }\end{array}$ & \\
\hline $\mathrm{Mg}$ & 12000 & 12000 & 0.015000 & 0.017158 & 0.001033 & \\
\hline $\mathrm{Al}$ & 13027 & 13000 & 0.927000 & 0.955163 & 0.057519 & \\
\hline Si & 14000 & 14000 & 0.002830 & 0.002801 & 0.000169 & \\
\hline $\mathrm{Ti}$ & 22000 & 22000 & 0.000850 & 0.000494 & 0.000030 & \\
\hline $\mathrm{Cr}$ & 24000 & 24000 & 0.000570 & 0.000305 & 0.000018 & \\
\hline $\mathrm{Mn}$ & 25055 & 25000 & 0.006000 & 0.003036 & 0.000183 & \\
\hline $\mathrm{Fe}$ & 26000 & 26000 & 0.002830 & 0.001409 & 0.000085 & \\
\hline $\mathrm{Cu}$ & 29000 & 29000 & 0.043500 & 0.019031 & 0.001146 & \\
\hline $\mathrm{Zn}$ & 30000 & 30000 & 0.001420 & 0.000604 & 0.000036 & \\
\hline Total & & & 1.000000 & 1.000000 & 0.060219 & \\
\hline MCNP Form & \multicolumn{2}{|c|}{ Weight Fractions } & \multicolumn{2}{|c|}{ Atom Fractions } & \multicolumn{2}{|c|}{ Atom Densities } \\
\hline \multirow[t]{9}{*}{ Neutrons } & 12000 & -0.015000 & 12000 & 0.017158 & 12000 & 0.001033 \\
\hline & 13027 & -0.927000 & 13027 & 0.955163 & 13027 & 0.057519 \\
\hline & 14000 & -0.002830 & 14000 & 0.002801 & 14000 & 0.000169 \\
\hline & 22000 & -0.000850 & 22000 & 0.000494 & 22000 & 0.000030 \\
\hline & 24000 & -0.000570 & 24000 & 0.000305 & 24000 & 0.000018 \\
\hline & 25055 & -0.006000 & 25055 & 0.003036 & 25055 & 0.000183 \\
\hline & 26000 & -0.002830 & 26000 & 0.001409 & 26000 & 0.000085 \\
\hline & 29000 & -0.043500 & 29000 & 0.019031 & 29000 & 0.001146 \\
\hline & 30000 & -0.001420 & 30000 & 0.000604 & 30000 & 0.000036 \\
\hline
\end{tabular}


PIET-43741-TM-963

PNNL-15870 Rev. 1

\begin{tabular}{|c|c|c|c|c|c|c|}
\hline \multirow[t]{9}{*}{ Photons } & 12000 & -0.015000 & 12000 & 0.017158 & 12000 & 0.001033 \\
\hline & 13000 & -0.927000 & 13000 & 0.955163 & 13000 & 0.057519 \\
\hline & 14000 & -0.002830 & 14000 & 0.002801 & 14000 & 0.000169 \\
\hline & 22000 & -0.000850 & 22000 & 0.000494 & 22000 & 0.000030 \\
\hline & 24000 & -0.000570 & 24000 & 0.000305 & 24000 & 0.000018 \\
\hline & 25000 & -0.006000 & 25000 & 0.003036 & 25000 & 0.000183 \\
\hline & 26000 & -0.002830 & 26000 & 0.001409 & 26000 & 0.000085 \\
\hline & 29000 & -0.043500 & 29000 & 0.019031 & 29000 & 0.001146 \\
\hline & 30000 & -0.001420 & 30000 & 0.000604 & 30000 & 0.000036 \\
\hline \multirow[t]{10}{*}{ CEPXS Form: } & material & $\mathrm{Mg}$ & 0.015000 & & & \\
\hline & & $\mathrm{Al}$ & 0.927000 & & & \\
\hline & & $\mathrm{Si}$ & 0.002830 & & & \\
\hline & & $\mathrm{Ti}$ & 0.000850 & & & \\
\hline & & $\mathrm{Cr}$ & 0.000570 & & & \\
\hline & & $\mathrm{Mn}$ & 0.006000 & & & \\
\hline & & $\mathrm{Fe}$ & 0.002830 & & & \\
\hline & & $\mathrm{Cu}$ & 0.043500 & & & \\
\hline & & $\mathrm{Zn}$ & 0.001420 & & & \\
\hline & $\begin{array}{l}\text { matname } \\
\text { density }\end{array}$ & \multicolumn{2}{|c|}{$\begin{array}{l}\text { Aluminum, Alloy 2024-O } \\
2.780000\end{array}$} & & & \\
\hline \multicolumn{7}{|c|}{$\begin{array}{l}\text { Comments and References } \\
\text { Density }=2.78 \mathrm{~g} / \mathrm{cm} 3 \text { and weight fractions from } \\
\text { http://www.matweb.com/search/DataSheet.aspx?MatGUID=642e240585794f0ab91428aa78c27b4e } \\
\text { (Automation Creations } 2010 \text { ). } \\
\text { Weight fractions for } \mathrm{Mg}, \mathrm{Al}, \mathrm{Mn} \text { and Cu set at the average of the allowed range. Weight fractions for Si, Ti, } \\
\mathrm{Cr}, \mathrm{Fe} \text {, and } \mathrm{Zn} \text { were set at } 56.7 \% \text { of their upper limits to allow the total to sum to unity. }\end{array}$} \\
\hline
\end{tabular}

\section{Aluminum, Alloy 2090-T83}

\begin{tabular}{llll}
\hline Formula $=$ & - & Molecular weight $(\mathrm{g} / \mathrm{mole})=$ & - \\
Density $(\mathrm{g} / \mathrm{cm} 3)=$ & 2.590000 & Total atom density $($ atoms $/ \mathrm{b}-\mathrm{cm})=$ & $6.054 \mathrm{E}-02$
\end{tabular}

The above density is estimated to be accurate to 3 significant digits. Uncertainties are not addressed.

The following data were calculated from the input weight fractions.

\begin{tabular}{|c|c|c|c|c|c|}
\hline Element & Neutron ZA & Photon ZA & $\begin{array}{l}\text { Weight } \\
\text { Fraction }\end{array}$ & $\begin{array}{c}\text { Atom } \\
\text { Fraction }\end{array}$ & $\begin{array}{l}\text { Atom } \\
\text { Density }\end{array}$ \\
\hline $\mathrm{Li}$ & - & 3000 & 0.022500 & 0.083519 & 0.005056 \\
\hline $\mathrm{Mg}$ & 12000 & 12000 & 0.001630 & 0.001728 & 0.000105 \\
\hline $\mathrm{Al}$ & 13027 & 13000 & 0.944000 & 0.901423 & 0.054570 \\
\hline $\mathrm{Si}$ & 14000 & 14000 & 0.000650 & 0.000596 & 0.000036 \\
\hline $\mathrm{Ti}$ & 22000 & 22000 & 0.000980 & 0.000527 & 0.000032 \\
\hline $\mathrm{Cr}$ & 24000 & 24000 & 0.000330 & 0.000164 & 0.000010 \\
\hline $\mathrm{Mn}$ & 25055 & 25000 & 0.000330 & 0.000155 & 0.000009 \\
\hline $\mathrm{Fe}$ & 26000 & 26000 & 0.000780 & 0.000360 & 0.000022 \\
\hline $\mathrm{Cu}$ & 29000 & 29000 & 0.027000 & 0.010947 & 0.000663 \\
\hline $\mathrm{Zn}$ & 30000 & 30000 & 0.000650 & 0.000256 & 0.000015 \\
\hline
\end{tabular}


PIET-43741-TM-963

PNNL-15870 Rev. 1

\begin{tabular}{|c|c|c|c|c|c|c|}
\hline $\mathrm{Zr}$ & 40000 & 40000 & 0.001150 & 0.000325 & \multicolumn{2}{|l|}{0.000020} \\
\hline \multicolumn{3}{|l|}{ Total } & 1.000000 & 1.000000 & \multicolumn{2}{|l|}{0.060538} \\
\hline MCNP Form & \multicolumn{2}{|c|}{ Weight Fractions } & \multicolumn{2}{|c|}{ Atom Fractions } & \multicolumn{2}{|c|}{ Atom Densities } \\
\hline \multirow[t]{11}{*}{ Neutrons } & - & -0.022500 & - & 0.083519 & - & 0.005056 \\
\hline & 12000 & -0.001630 & 12000 & 0.001728 & 12000 & 0.000105 \\
\hline & 13027 & -0.944000 & 13027 & 0.901423 & 13027 & 0.054570 \\
\hline & 14000 & -0.000650 & 14000 & 0.000596 & 14000 & 0.000036 \\
\hline & 22000 & -0.000980 & 22000 & 0.000527 & 22000 & 0.000032 \\
\hline & 24000 & -0.000330 & 24000 & 0.000164 & 24000 & 0.000010 \\
\hline & 25055 & -0.000330 & 25055 & 0.000155 & 25055 & 0.000009 \\
\hline & 26000 & -0.000780 & 26000 & 0.000360 & 26000 & 0.000022 \\
\hline & 29000 & -0.027000 & 29000 & 0.010947 & 29000 & 0.000663 \\
\hline & 30000 & -0.000650 & 30000 & 0.000256 & 30000 & 0.000015 \\
\hline & 40000 & -0.001150 & 40000 & 0.000325 & 40000 & 0.000020 \\
\hline \multirow[t]{11}{*}{ Photons } & 3000 & -0.022500 & 3000 & 0.083519 & 3000 & 0.005056 \\
\hline & 12000 & -0.001630 & 12000 & 0.001728 & 12000 & 0.000105 \\
\hline & 13000 & -0.944000 & 13000 & 0.901423 & 13000 & 0.054570 \\
\hline & 14000 & -0.000650 & 14000 & 0.000596 & 14000 & 0.000036 \\
\hline & 22000 & -0.000980 & 22000 & 0.000527 & 22000 & 0.000032 \\
\hline & 24000 & -0.000330 & 24000 & 0.000164 & 24000 & 0.000010 \\
\hline & 25000 & -0.000330 & 25000 & 0.000155 & 25000 & 0.000009 \\
\hline & 26000 & -0.000780 & 26000 & 0.000360 & 26000 & 0.000022 \\
\hline & 29000 & -0.027000 & 29000 & 0.010947 & 29000 & 0.000663 \\
\hline & 30000 & -0.000650 & 30000 & 0.000256 & 30000 & 0.000015 \\
\hline & 40000 & -0.001150 & 40000 & 0.000325 & 40000 & 0.000020 \\
\hline \multirow[t]{12}{*}{ CEPXS Form: } & material & $\mathrm{Li}$ & 0.022500 & & & \\
\hline & & $\mathrm{Mg}$ & 0.001630 & & & \\
\hline & & $\mathrm{Al}$ & 0.944000 & & & \\
\hline & & $\mathrm{Si}$ & 0.000650 & & & \\
\hline & & $\mathrm{Ti}$ & 0.000980 & & & \\
\hline & & $\mathrm{Cr}$ & 0.000330 & & & \\
\hline & & $\mathrm{Mn}$ & 0.000330 & & & \\
\hline & & $\mathrm{Fe}$ & 0.000780 & & & \\
\hline & & $\mathrm{Cu}$ & 0.027000 & & & \\
\hline & & $\mathrm{Zn}$ & 0.000650 & & & \\
\hline & & $\mathrm{Zr}$ & 0.001150 & & & \\
\hline & $\begin{array}{l}\text { matname } \\
\text { density }\end{array}$ & \multicolumn{3}{|c|}{$\begin{array}{l}\text { Aluminum, Alloy 2090-T83 } \\
2.590000\end{array}$} & & \\
\hline \multicolumn{7}{|c|}{$\begin{array}{l}\text { Comments and References } \\
\text { Density = } 2.59 \mathrm{~g} / \mathrm{cm} 3 \text { and weight fractions from www.alcoa.com/mill_products/catalog/pdf/alloy2090- } \\
\text { t83techsheet.pdf (ALCOA n.d.) and } \\
\text { http://www.matweb.com/search/DataSheet.aspx?MatGUID=a79a000ba9314c8d90fe } 75 d c 76 e f c c 8 a \\
\text { (Automation Creations } 2010) \text {. } \\
\text { Weight fractions for } \mathrm{Li}, \mathrm{Al}, \mathrm{Cu} \text {, and } \mathrm{Zr} \text { set at the average of the allowed range. Weight fractions for Mg, Si, } \\
\text { Ti, Cr, Mn, Fe, and Zn were set at } 65.2 \% \text { of their upper limits to allow the total to sum to unity. }\end{array}$} \\
\hline
\end{tabular}




\section{Aluminum, Alloy 3003}

\begin{tabular}{llll}
\hline Formula $=$ & - & Molecular weight $(\mathrm{g} / \mathrm{mole})=$ & - \\
Density $(\mathrm{g} / \mathrm{cm} 3)=$ & 2.730000 & Total atom density $($ atoms $/ \mathrm{b}-\mathrm{cm})=$ & $6.035 \mathrm{E}-02$
\end{tabular}

The above density is estimated to be accurate to 3 significant digits. Uncertainties are not addressed.

The following data were calculated from the input weight fractions.

\begin{tabular}{|c|c|c|c|c|c|}
\hline Element & Neutron ZA & Photon ZA & $\begin{array}{l}\text { Weight } \\
\text { Fraction }\end{array}$ & $\begin{array}{c}\text { Atom } \\
\text { Fraction }\end{array}$ & $\begin{array}{l}\text { Atom } \\
\text { Density }\end{array}$ \\
\hline $\mathrm{Al}$ & 13027 & 13000 & 0.978500 & $\overline{0.987924}$ & 0.059622 \\
\hline $\mathrm{Si}$ & 14000 & 14000 & 0.003320 & 0.003220 & 0.000194 \\
\hline $\mathrm{Mn}$ & 25055 & 25000 & 0.012500 & 0.006198 & 0.000374 \\
\hline $\mathrm{Fe}$ & 26000 & 26000 & 0.003880 & 0.001893 & 0.000114 \\
\hline $\mathrm{Cu}$ & 29000 & 29000 & 0.001250 & 0.000536 & 0.000032 \\
\hline $\mathrm{Zn}$ & 30000 & 30000 & 0.000550 & 0.000229 & 0.000014 \\
\hline Total & & & 1.000000 & 1.000000 & 0.060351 \\
\hline
\end{tabular}

\begin{tabular}{|c|cc|cccr|}
\hline MCNP Form & \multicolumn{2}{c|}{ Weight Fractions } & \multicolumn{2}{c|}{ Atom Fractions } & \multicolumn{2}{c|}{ Atom Densities } \\
\hline Neutrons & 13027 & -0.978500 & 13027 & 0.987924 & 13027 & 0.059622 \\
& 14000 & -0.003320 & 14000 & 0.003220 & 14000 & 0.000194 \\
& 25055 & -0.012500 & 25055 & 0.006198 & 25055 & 0.000374 \\
& 26000 & -0.003880 & 26000 & 0.001893 & 26000 & 0.000114 \\
& 29000 & -0.001250 & 29000 & 0.000536 & 29000 & 0.000032 \\
& 30000 & -0.000550 & 30000 & 0.000229 & 30000 & 0.000014 \\
Photons & & & & & & \\
& 13000 & -0.978500 & 13000 & 0.987924 & 13000 & 0.059622 \\
& 14000 & -0.003320 & 14000 & 0.003220 & 14000 & 0.000194 \\
& 25000 & -0.012500 & 25000 & 0.006198 & 25000 & 0.000374 \\
& 26000 & -0.003880 & 26000 & 0.001893 & 26000 & 0.000114 \\
& 29000 & -0.001250 & 29000 & 0.000536 & 29000 & 0.000032 \\
& 30000 & -0.000550 & 30000 & 0.000229 & 30000 & 0.000014
\end{tabular}

$\begin{array}{ccc}\text { CEPXS Form: } & \mathrm{Al} & 0.978500 \\ & \mathrm{Si} & 0.003320 \\ \mathrm{Mn} & 0.012500 \\ & \mathrm{Fe} & 0.003880 \\ & \mathrm{Cu} & 0.001250 \\ & \mathrm{Zn} & 0.000550\end{array}$

matname Aluminum, Alloy 3003

density 2.730000

\section{Comments and References}

Density $=2.73 \mathrm{~g} / \mathrm{cm} 3$ and weight fractions from

http://www.matweb.com/search/DataSheet.aspx?MatGUID=fd4a40f87d3f4912925e5e6eab1fbc40

(Automation Creations 2010).

Weight fractions for $\mathrm{Al}, \mathrm{Mn}$, and $\mathrm{Cu}$ set at the average of the allowed range.

Weight fractions for $\mathrm{Si}, \mathrm{Fe}$, and $\mathrm{Zn}$ were set at $55.4 \%$ of their upper limits to allow the total to sum to unity. 


\section{Aluminum, Alloy $4043-0$}

\begin{tabular}{llll}
\hline Formula $=$ & - & Molecular weight $(\mathrm{g} / \mathrm{mole})=$ & - \\
Density $(\mathrm{g} / \mathrm{cm} 3)=$ & 2.690000 & Total atom density $($ atoms $/ \mathrm{b}-\mathrm{cm})=$ & $5.966 \mathrm{E}-02$
\end{tabular}

The above density is estimated to be accurate to 3 significant digits. Uncertainties are not addressed.

The following data were calculated from the input weight fractions.

\begin{tabular}{|c|c|c|c|c|c|c|}
\hline Element & Neutron ZA & Photon ZA & $\begin{array}{l}\text { Weight } \\
\text { Fraction }\end{array}$ & $\begin{array}{c}\text { Atom } \\
\text { Fraction }\end{array}$ & $\begin{array}{l}\text { Atom } \\
\text { Density }\end{array}$ & \\
\hline $\mathrm{Be}$ & 4009 & 4000 & 0.000005 & 0.000015 & 0.000001 & \\
\hline $\mathrm{Mg}$ & 12000 & 12000 & 0.000280 & 0.000313 & 0.000019 & \\
\hline $\mathrm{Al}$ & 13027 & 13000 & 0.939000 & 0.944970 & 0.056377 & \\
\hline $\mathrm{Si}$ & 14000 & 14000 & 0.052500 & 0.050757 & 0.003028 & \\
\hline $\mathrm{Ti}$ & 22000 & 22000 & 0.001130 & 0.000641 & 0.000038 & \\
\hline $\mathrm{Mn}$ & 25055 & 25000 & 0.000280 & 0.000138 & 0.000008 & \\
\hline $\mathrm{Fe}$ & 26000 & 26000 & 0.004530 & 0.002203 & 0.000131 & \\
\hline $\mathrm{Cu}$ & 29000 & 29000 & 0.001700 & 0.000726 & 0.000043 & \\
\hline $\mathrm{Zn}$ & 30000 & 30000 & 0.000570 & 0.000237 & 0.000014 & \\
\hline Total & & & 0.999995 & 1.000000 & 0.059660 & \\
\hline MCNP Form & \multicolumn{2}{|c|}{ Weight Fractions } & \multicolumn{2}{|c|}{ Atom Fractions } & \multicolumn{2}{|c|}{ Atom Densities } \\
\hline \multirow[t]{9}{*}{ Neutrons } & 4009 & -0.000005 & 4009 & 0.000015 & 4009 & 0.000001 \\
\hline & 12000 & -0.000280 & 12000 & 0.000313 & 12000 & 0.000019 \\
\hline & 13027 & -0.939000 & 13027 & 0.944970 & 13027 & 0.056377 \\
\hline & 14000 & -0.052500 & 14000 & 0.050757 & 14000 & 0.003028 \\
\hline & 22000 & -0.001130 & 22000 & 0.000641 & 22000 & 0.000038 \\
\hline & 25055 & -0.000280 & 25055 & 0.000138 & 25055 & 0.000008 \\
\hline & 26000 & -0.004530 & 26000 & 0.002203 & 26000 & 0.000131 \\
\hline & 29000 & -0.001700 & 29000 & 0.000726 & 29000 & 0.000043 \\
\hline & 30000 & -0.000570 & 30000 & 0.000237 & 30000 & 0.000014 \\
\hline \multirow[t]{9}{*}{ Photons } & 4000 & -0.000005 & 4000 & 0.000015 & 4000 & 0.000001 \\
\hline & 12000 & -0.000280 & 12000 & 0.000313 & 12000 & 0.000019 \\
\hline & 13000 & -0.939000 & 13000 & 0.944970 & 13000 & 0.056377 \\
\hline & 14000 & -0.052500 & 14000 & 0.050757 & 14000 & 0.003028 \\
\hline & 22000 & -0.001130 & 22000 & 0.000641 & 22000 & 0.000038 \\
\hline & 25000 & -0.000280 & 25000 & 0.000138 & 25000 & 0.000008 \\
\hline & 26000 & -0.004530 & 26000 & 0.002203 & 26000 & 0.000131 \\
\hline & 29000 & -0.001700 & 29000 & 0.000726 & 29000 & 0.000043 \\
\hline & 30000 & -0.000570 & 30000 & 0.000237 & 30000 & 0.000014 \\
\hline \multirow[t]{5}{*}{ CEPXS Form: } & material & $\mathrm{Be}$ & 0.000005 & & & \\
\hline & & $\mathrm{Mg}$ & 0.000280 & & & \\
\hline & & $\mathrm{Al}$ & 0.939000 & & & \\
\hline & & $\mathrm{Si}$ & 0.052500 & & & \\
\hline & & $\mathrm{Ti}$ & 0.001130 & & & \\
\hline
\end{tabular}




\begin{tabular}{|ll}
$\mathrm{Mn}$ & 0.000280 \\
$\mathrm{Fe}$ & 0.004530 \\
$\mathrm{Cu}$ & 0.001700 \\
$\mathrm{Zn}$ & 0.000570
\end{tabular}

matname Aluminum, Alloy 4043-O

density 2.690000

\section{Comments and References}

Density $=2.69 \mathrm{~g} / \mathrm{cm} 3$ and weight fractions from

http://www.matweb.com/search/DataSheet.aspx?MatGUID=febb330c9c0548b39ed4105628912ffd

(Automation Creations 2010).

Weight fractions for $\mathrm{Al}$ and $\mathrm{Si}$ set at the average of the allowed range. Weight fractions for $\mathrm{Be}, \mathrm{Mg}, \mathrm{Ti}, \mathrm{Mn}$, $\mathrm{Fe}, \mathrm{Cu}$, and $\mathrm{Zn}$ were set at $56.6 \%$ of their upper limits to allow the total to sum to unity.

\section{Aluminum, Alloy 5086-0}

$\begin{array}{llll}\text { Formula }= & - & \text { Molecular weight }(\mathrm{g} / \mathrm{mole})= & - \\ \text { Density }(\mathrm{g} / \mathrm{cm} 3)= & 2.660000 & \text { Total atom density }(\text { atoms } / \mathrm{b}-\mathrm{cm})= & 5.928 \mathrm{E}-02\end{array}$

The above density is estimated to be accurate to 3 significant digits. Uncertainties are not addressed.

The following data were calculated from the input weight fractions.

\begin{tabular}{|c|c|c|c|c|c|c|}
\hline Element & Neutron ZA & Photon ZA & $\begin{array}{l}\text { Weight } \\
\text { Fraction }\end{array}$ & $\begin{array}{c}\text { Atom } \\
\text { Fraction }\end{array}$ & $\begin{array}{l}\text { Atom } \\
\text { Density }\end{array}$ & \\
\hline $\mathrm{Mg}$ & 12000 & 12000 & 0.040000 & 0.044473 & 0.002636 & \\
\hline $\mathrm{Al}$ & 13027 & 13000 & 0.946500 & 0.947944 & 0.056194 & \\
\hline $\mathrm{Si}$ & 14000 & 14000 & 0.002140 & 0.002059 & 0.000122 & \\
\hline $\mathrm{Ti}$ & 22000 & 22000 & 0.000800 & 0.000452 & 0.000027 & \\
\hline $\mathrm{Cr}$ & 24000 & 24000 & 0.001500 & 0.000780 & 0.000046 & \\
\hline $\mathrm{Mn}$ & 25055 & 25000 & 0.004500 & 0.002213 & 0.000131 & \\
\hline $\mathrm{Fe}$ & 26000 & 26000 & 0.002680 & 0.001297 & 0.000077 & \\
\hline $\mathrm{Cu}$ & 29000 & 29000 & 0.000540 & 0.000230 & 0.000014 & \\
\hline $\mathrm{Zn}$ & 30000 & 30000 & 0.001340 & 0.000554 & 0.000033 & \\
\hline Total & & & 1.000000 & 1.000000 & 0.059279 & \\
\hline MCNP Form & \multicolumn{2}{|c|}{ Weight Fractions } & \multicolumn{2}{|c|}{ Atom Fractions } & \multicolumn{2}{|c|}{ Atom Densities } \\
\hline \multirow[t]{9}{*}{ Neutrons } & 12000 & -0.040000 & 12000 & 0.044473 & 12000 & 0.002636 \\
\hline & 13027 & -0.946500 & 13027 & 0.947944 & 13027 & 0.056194 \\
\hline & 14000 & -0.002140 & 14000 & 0.002059 & 14000 & 0.000122 \\
\hline & 22000 & -0.000800 & 22000 & 0.000452 & 22000 & 0.000027 \\
\hline & 24000 & -0.001500 & 24000 & 0.000780 & 24000 & 0.000046 \\
\hline & 25055 & -0.004500 & 25055 & 0.002213 & 25055 & 0.000131 \\
\hline & 26000 & -0.002680 & 26000 & 0.001297 & 26000 & 0.000077 \\
\hline & 29000 & -0.000540 & 29000 & 0.000230 & 29000 & 0.000014 \\
\hline & 30000 & -0.001340 & 30000 & 0.000554 & 30000 & 0.000033 \\
\hline \multirow[t]{2}{*}{ Photons } & 12000 & -0.040000 & 12000 & 0.044473 & 12000 & 0.002636 \\
\hline & 13000 & -0.946500 & 13000 & 0.947944 & 13000 & 0.056194 \\
\hline
\end{tabular}


PIET-43741-TM-963

PNNL-15870 Rev. 1

\begin{tabular}{|c|c|c|c|c|c|c|}
\hline & 14000 & -0.002140 & 14000 & 0.002059 & 14000 & 0.000122 \\
\hline & 22000 & -0.000800 & 22000 & 0.000452 & 22000 & 0.000027 \\
\hline & 24000 & -0.001500 & 24000 & 0.000780 & 24000 & 0.000046 \\
\hline & 25000 & -0.004500 & 25000 & 0.002213 & 25000 & 0.000131 \\
\hline & 26000 & -0.002680 & 26000 & 0.001297 & 26000 & 0.000077 \\
\hline & 29000 & -0.000540 & 29000 & 0.000230 & 29000 & 0.000014 \\
\hline & 30000 & -0.001340 & 30000 & 0.000554 & 30000 & 0.000033 \\
\hline \multirow[t]{10}{*}{ CEPXS Form: } & material & $\mathrm{Mg}$ & 0.040000 & & & \\
\hline & & $\mathrm{Al}$ & 0.946500 & & & \\
\hline & & $\mathrm{Si}$ & 0.002140 & & & \\
\hline & & $\mathrm{Ti}$ & 0.000800 & & & \\
\hline & & $\mathrm{Cr}$ & 0.001500 & & & \\
\hline & & $\mathrm{Mn}$ & 0.004500 & & & \\
\hline & & $\mathrm{Fe}$ & 0.002680 & & & \\
\hline & & $\mathrm{Cu}$ & 0.000540 & & & \\
\hline & & $\mathrm{Zn}$ & 0.001340 & & & \\
\hline & $\begin{array}{c}\text { matname } \\
\text { density }\end{array}$ & \multicolumn{2}{|c|}{$\begin{array}{l}\text { Aluminum, Alloy } 5086-O \\
2.660000\end{array}$} & & & \\
\hline \multicolumn{7}{|c|}{$\begin{array}{l}\text { Comments and References } \\
\text { Density = } 2.66 \mathrm{~g} / \mathrm{cm} 3 \text { and weight fractions from } \\
\text { http://www.matweb.com/search/DataSheet.aspx?MatGUID=d0271cf3b5f84d63a17e328d02419587 } \\
\text { (Automation Creations } 2010 \text { ). } \\
\text { Weight fractions for Mg, Al, Cr, and Mn set at the average of the allowed range. Weight fractions for Si, } \\
\mathrm{Ti}, \mathrm{Fe}, \mathrm{Cu} \text {, and } \mathrm{Zn} \text { were set at } 53.6 \% \text { of their upper limits to allow the total to sum to unity. }\end{array}$} \\
\hline
\end{tabular}

\section{Aluminum, Alloy 6061-0}

\begin{tabular}{llll}
\hline Formula $=$ & - & Molecular weight $(\mathrm{g} / \mathrm{mole})=$ & - \\
Density $(\mathrm{g} / \mathrm{cm} 3)=$ & 2.700000 & Total atom density $($ atoms $/ \mathrm{b}-\mathrm{cm})=$ & $5.993 \mathrm{E}-02$
\end{tabular}

The above density is estimated to be accurate to 3 significant digits. Uncertainties are not addressed.

The following data were calculated from the input weight fractions.

\begin{tabular}{|c|c|c|c|c|c|}
\hline Element & Neutron ZA & Photon ZA & $\begin{array}{l}\text { Weight } \\
\text { Fraction }\end{array}$ & $\begin{array}{c}\text { Atom } \\
\text { Fraction }\end{array}$ & $\begin{array}{l}\text { Atom } \\
\text { Density }\end{array}$ \\
\hline $\mathrm{Mg}$ & 12000 & 12000 & 0.010000 & 0.011162 & 0.000669 \\
\hline $\mathrm{Al}$ & 13027 & 13000 & 0.972000 & 0.977325 & 0.058575 \\
\hline $\mathrm{Si}$ & 14000 & 14000 & 0.006000 & 0.005796 & 0.000347 \\
\hline $\mathrm{Ti}$ & 22000 & 22000 & 0.000880 & 0.000499 & 0.000030 \\
\hline $\mathrm{Cr}$ & 24000 & 24000 & 0.001950 & 0.001017 & 0.000061 \\
\hline $\mathrm{Mn}$ & 25055 & 25000 & 0.000880 & 0.000435 & 0.000026 \\
\hline $\mathrm{Fe}$ & 26000 & 26000 & 0.004090 & 0.001987 & 0.000119 \\
\hline $\mathrm{Cu}$ & 29000 & 29000 & 0.002750 & 0.001174 & 0.000070 \\
\hline $\mathrm{Zn}$ & 30000 & 30000 & 0.001460 & 0.000606 & 0.000036 \\
\hline Total & & & 1.000010 & 1.000000 & 0.059934 \\
\hline
\end{tabular}


PIET-43741-TM-963

PNNL-15870 Rev. 1

\begin{tabular}{|c|c|c|c|c|c|c|}
\hline MCNP Form & \multicolumn{2}{|c|}{ Weight Fractions } & \multicolumn{2}{|c|}{ Atom Fractions } & \multicolumn{2}{|c|}{ Atom Densities } \\
\hline \multirow[t]{9}{*}{ Neutrons } & 12000 & -0.010000 & 12000 & 0.011162 & 12000 & 0.000669 \\
\hline & 13027 & -0.972000 & 13027 & 0.977325 & 13027 & 0.058575 \\
\hline & 14000 & -0.006000 & 14000 & 0.005796 & 14000 & 0.000347 \\
\hline & 22000 & -0.000880 & 22000 & 0.000499 & 22000 & 0.000030 \\
\hline & 24000 & -0.001950 & 24000 & 0.001017 & 24000 & 0.000061 \\
\hline & 25055 & -0.000880 & 25055 & 0.000435 & 25055 & 0.000026 \\
\hline & 26000 & -0.004090 & 26000 & 0.001987 & 26000 & 0.000119 \\
\hline & 29000 & -0.002750 & 29000 & 0.001174 & 29000 & 0.000070 \\
\hline & 30000 & -0.001460 & 30000 & 0.000606 & 30000 & 0.000036 \\
\hline \multirow[t]{9}{*}{ Photons } & 12000 & -0.010000 & 12000 & 0.011162 & 12000 & 0.000669 \\
\hline & 13000 & -0.972000 & 13000 & 0.977325 & 13000 & 0.058575 \\
\hline & 14000 & -0.006000 & 14000 & 0.005796 & 14000 & 0.000347 \\
\hline & 22000 & -0.000880 & 22000 & 0.000499 & 22000 & 0.000030 \\
\hline & 24000 & -0.001950 & 24000 & 0.001017 & 24000 & 0.000061 \\
\hline & 25000 & -0.000880 & 25000 & 0.000435 & 25000 & 0.000026 \\
\hline & 26000 & -0.004090 & 26000 & 0.001987 & 26000 & 0.000119 \\
\hline & 29000 & -0.002750 & 29000 & 0.001174 & 29000 & 0.000070 \\
\hline & 30000 & -0.001460 & 30000 & 0.000606 & 30000 & 0.000036 \\
\hline \multirow[t]{10}{*}{ CEPXS Form: } & material & $\mathrm{Mg}$ & 0.010000 & & & \\
\hline & & $\mathrm{Al}$ & 0.972000 & & & \\
\hline & & $\mathrm{Si}$ & 0.006000 & & & \\
\hline & & $\mathrm{Ti}$ & 0.000880 & & & \\
\hline & & $\mathrm{Cr}$ & 0.001950 & & & \\
\hline & & $\mathrm{Mn}$ & 0.000880 & & & \\
\hline & & $\mathrm{Fe}$ & 0.004090 & & & \\
\hline & & $\mathrm{Cu}$ & 0.002750 & & & \\
\hline & & $\mathrm{Zn}$ & 0.001460 & & & \\
\hline & $\begin{array}{c}\text { matname } \\
\text { density }\end{array}$ & $\begin{array}{c}\text { Aluminum, } \\
2.700000\end{array}$ & 6061-O & & & \\
\hline \multicolumn{7}{|c|}{$\begin{array}{l}\text { Comments and References } \\
\text { Density = } 2.70 \mathrm{~g} / \mathrm{cm} 3 \text { and weight fractions from } \\
\text { http://www.matweb.com/search/DataSheet.aspx?MatGUID=626ec8cdca604f1994be4fc2bc6f7f63 } \\
\text { (Automation Creations } 2010 \text { ). } \\
\text { Weight fractions for } \mathrm{Mg}, \mathrm{Al}, \mathrm{Si}, \mathrm{Cr} \text {, and Cu set at the average of the allowed range. Weight fractions for Ti, } \\
\text { Mn, Fe, and Zn were set at } 58.4 \% \text { of their upper limits to allow the total to sum to unity. }\end{array}$} \\
\hline
\end{tabular}

\section{Aluminum, Alloy 7075-0}

\begin{tabular}{llll}
\hline Formula $=$ & - & Molecular weight $(\mathrm{g} / \mathrm{mole})=$ & - \\
Density $(\mathrm{g} / \mathrm{cm} 3)=$ & 2.810000 & Total atom density $($ atoms $/ \mathrm{b}-\mathrm{cm})=$ & $5.999 \mathrm{E}-02$ \\
The above density is estimated to be accurate to 3 significant digits. Uncertainties are not addressed. & \\
The following data were calculated from the input weight fractions.
\end{tabular}




\begin{tabular}{|c|c|c|c|c|c|c|}
\hline Element & Neutron ZA & $\underline{\text { Photon ZA }}$ & $\begin{array}{l}\text { Weight } \\
\text { Fraction }\end{array}$ & $\begin{array}{l}\text { Atom } \\
\text { Fraction }\end{array}$ & $\begin{array}{l}\text { Atom } \\
\text { Density }\end{array}$ & \\
\hline $\mathrm{Mg}$ & 12000 & 12000 & 0.025000 & 0.029014 & 0.001741 & \\
\hline $\mathrm{Al}$ & 13027 & 13000 & 0.892500 & 0.933062 & 0.055976 & \\
\hline $\mathrm{Si}$ & 14000 & 14000 & 0.002340 & 0.002350 & 0.000141 & \\
\hline $\mathrm{Ti}$ & 22000 & 22000 & 0.001170 & 0.000689 & 0.000041 & \\
\hline $\mathrm{Cr}$ & 24000 & 24000 & 0.002300 & 0.001248 & 0.000075 & \\
\hline $\mathrm{Mn}$ & 25055 & 25000 & 0.001760 & 0.000904 & 0.000054 & \\
\hline $\mathrm{Fe}$ & 26000 & 26000 & 0.002930 & 0.001480 & 0.000089 & \\
\hline $\mathrm{Cu}$ & 29000 & 29000 & 0.016000 & 0.007102 & 0.000426 & \\
\hline $\mathrm{Zn}$ & 30000 & 30000 & 0.056000 & 0.024150 & 0.001449 & \\
\hline Total & & & 1.000000 & 1.000000 & 0.059991 & \\
\hline MCNP Form & \multicolumn{2}{|c|}{ Weight Fractions } & \multicolumn{2}{|c|}{ Atom Fractions } & \multicolumn{2}{|c|}{ Atom Densities } \\
\hline \multirow[t]{9}{*}{ Neutrons } & 12000 & -0.025000 & 12000 & 0.029014 & 12000 & 0.001741 \\
\hline & 13027 & -0.892500 & 13027 & 0.933062 & 13027 & 0.055976 \\
\hline & 14000 & -0.002340 & 14000 & 0.002350 & 14000 & 0.000141 \\
\hline & 22000 & -0.001170 & 22000 & 0.000689 & 22000 & 0.000041 \\
\hline & 24000 & -0.002300 & 24000 & 0.001248 & 24000 & 0.000075 \\
\hline & 25055 & -0.001760 & 25055 & 0.000904 & 25055 & 0.000054 \\
\hline & 26000 & -0.002930 & 26000 & 0.001480 & 26000 & 0.000089 \\
\hline & 29000 & -0.016000 & 29000 & 0.007102 & 29000 & 0.000426 \\
\hline & 30000 & -0.056000 & 30000 & 0.024150 & 30000 & 0.001449 \\
\hline \multirow[t]{9}{*}{ Photons } & 12000 & -0.025000 & 12000 & 0.029014 & 12000 & 0.001741 \\
\hline & 13000 & -0.892500 & 13000 & 0.933062 & 13000 & 0.055976 \\
\hline & 14000 & -0.002340 & 14000 & 0.002350 & 14000 & 0.000141 \\
\hline & 22000 & -0.001170 & 22000 & 0.000689 & 22000 & 0.000041 \\
\hline & 24000 & -0.002300 & 24000 & 0.001248 & 24000 & 0.000075 \\
\hline & 25000 & -0.001760 & 25000 & 0.000904 & 25000 & 0.000054 \\
\hline & 26000 & -0.002930 & 26000 & 0.001480 & 26000 & 0.000089 \\
\hline & 29000 & -0.016000 & 29000 & 0.007102 & 29000 & 0.000426 \\
\hline & 30000 & -0.056000 & 30000 & 0.024150 & 30000 & 0.001449 \\
\hline \multirow[t]{10}{*}{ CEPXS Form: } & material & $\mathrm{Mg}$ & 0.025000 & & & \\
\hline & & $\mathrm{Al}$ & 0.892500 & & & \\
\hline & & $\mathrm{Si}$ & 0.002340 & & & \\
\hline & & $\mathrm{Ti}$ & 0.001170 & & & \\
\hline & & $\mathrm{Cr}$ & 0.002300 & & & \\
\hline & & $\mathrm{Mn}$ & 0.001760 & & & \\
\hline & & $\mathrm{Fe}$ & 0.002930 & & & \\
\hline & & $\mathrm{Cu}$ & 0.016000 & & & \\
\hline & & $\mathrm{Zn}$ & 0.056000 & & & \\
\hline & $\begin{array}{l}\text { matname } \\
\text { density }\end{array}$ & \multicolumn{2}{|c|}{$\begin{array}{l}\text { Aluminum, Alloy } 7075-\mathrm{O} \\
2.810000\end{array}$} & & & \\
\hline
\end{tabular}


(Automation Creations 2010).

Weight fractions for $\mathrm{Mg}, \mathrm{Al}, \mathrm{Cr}, \mathrm{Cu}$, and $\mathrm{Zn}$ set at the average of the allowed range. Weight fractions for $\mathrm{Si}, \mathrm{Ti}, \mathrm{Mn}$, and $\mathrm{Fe}$ were set at $58.6 \%$ of their upper limits to allow the total to sum to unity.

\section{Ammonia (Liquid at $\mathrm{T}=-79^{\circ} \mathrm{C}$ )}

\begin{tabular}{|c|c|c|c|}
\hline Formula $=$ & $\mathrm{NH} 3$ & Molecular weight $(\mathrm{g} / \mathrm{mole})=$ & 17.03052 \\
\hline Density $(\mathrm{g} / \mathrm{cm} 3)=$ & 0.771000 & Total atom density $($ atoms $/ \mathrm{b}-\mathrm{cm})=$ & 1.091E-01 \\
\hline
\end{tabular}

\begin{tabular}{|c|c|c|c|c|c|c|}
\hline Element & Neutron ZA & Photon ZA & $\begin{array}{l}\text { Weight } \\
\text { Fraction }\end{array}$ & $\begin{array}{l}\text { Atom } \\
\text { Fraction }\end{array}$ & $\begin{array}{l}\text { Atom } \\
\text { Density }\end{array}$ & \\
\hline $\mathrm{H}$ & 1001 & 1000 & $\overline{0.177547}$ & $\overline{0.749992}$ & 0.081787 & \\
\hline $\mathrm{N}$ & 7014 & 7000 & 0.822453 & 0.250008 & 0.027263 & \\
\hline Total & & & 1.000000 & 1.000000 & 0.109050 & \\
\hline MCNP Form & \multicolumn{2}{|c|}{ Weight Fractions } & \multicolumn{2}{|c|}{ Atom Fractions } & \multicolumn{2}{|c|}{ Atom Densities } \\
\hline \multirow[t]{2}{*}{ Neutrons } & 1001 & -0.177547 & 1001 & 0.749992 & 1001 & 0.081787 \\
\hline & 7014 & -0.822453 & 7014 & 0.250008 & 7014 & 0.027263 \\
\hline \multirow[t]{2}{*}{ Photons } & 1000 & -0.177547 & 1000 & 0.749992 & 1000 & 0.081787 \\
\hline & 7000 & -0.822453 & 7000 & 0.250008 & 7000 & 0.027263 \\
\hline \multirow[t]{3}{*}{ CEPXS Form: } & material & $\mathrm{H}$ & 0.177547 & & & \\
\hline & & $N$ & 0.822453 & & & \\
\hline & $\begin{array}{l}\text { matname } \\
\text { density }\end{array}$ & \multicolumn{3}{|c|}{$\begin{array}{l}\text { Ammonia (Liquid at } \mathrm{T}=-79^{\circ} \mathrm{C} \text { ) } \\
0.771000\end{array}$} & & \\
\hline \multicolumn{7}{|c|}{$\begin{array}{l}\text { Comments and References } \\
\text { Weight fractions from http://physics.nist.gov/cgi-bin/Star/compos.pl?matno=108 (NIST 1998). } \\
\text { Density }=0.771 \text { at } \mathrm{T}=-79^{\circ} \mathrm{C} \text { from Table } 51.11 \text { of }(\text { Hungerford 1960). } \\
\text { At room temperature, ammonia is a gas with density }=8.26019 \mathrm{e}-04 \text { (http://physics.nist.gov/cgi- } \\
\text { bin/Star/compos.pl?matno=108) (NIST 1998). }\end{array}$} \\
\hline
\end{tabular}

\section{Anthracene}

$\begin{array}{llll}\text { Formula }= & \mathrm{C} 14 \mathrm{H} 10 & \text { Molecular weight }(\mathrm{g} / \mathrm{mole})= & 178.2292 \\ \text { Density }(\mathrm{g} / \mathrm{cm} 3)= & 1.250000 & \text { Total atom density }(\text { atoms } / \mathrm{b}-\mathrm{cm})= & 1.014 \mathrm{E}-01\end{array}$

The above density is estimated to be accurate to 3 significant digits. Uncertainties are not addressed. The following data was calculated from the input formula.

$\begin{array}{llllll}\text { Element } & \frac{\text { Neutron ZA }}{\mathrm{H}} & \frac{\text { Photon ZA }}{1000} & \frac{\begin{array}{c}\text { Weight } \\ \text { Fraction }\end{array}}{0.056553} & \begin{array}{c}\text { Atom } \\ \text { Fraction }\end{array} & \begin{array}{c}\text { Atom } \\ \text { Density }\end{array} \\ 0.416667 & 0.042236\end{array}$


PIET-43741-TM-963

PNNL-15870 Rev. 1

\begin{tabular}{|c|c|c|c|c|c|c|}
\hline C & 6000 & 6000 & 0.943447 & 0.583333 & 0.05913 & \\
\hline Total & & & 1.000000 & 1.000000 & 0.10136 & \\
\hline MCNP Form & Weigh & ractions & Atom & ctions & Ato & sities \\
\hline Neutrons & 1001 & -0.056553 & 1001 & 0.416667 & 1001 & 0.042236 \\
\hline & 6000 & -0.943447 & 6000 & 0.583333 & 6000 & 0.059130 \\
\hline Photons & 1000 & -0.056553 & 1000 & 0.416667 & 1000 & 0.042236 \\
\hline & 6000 & -0.943447 & 6000 & 0.583333 & 6000 & 0.059130 \\
\hline CEPXS Form: & material & $\mathrm{H}$ & 0.056553 & & & \\
\hline & & C & 0.943447 & & & \\
\hline & $\begin{array}{c}\text { matname } \\
\text { density }\end{array}$ & $\begin{array}{c}\text { Anthracene } \\
1.250000\end{array}$ & & & & \\
\hline $\begin{array}{l}\text { Comments a } \\
\text { http://www.ap }\end{array}$ & $\begin{array}{l}\text { erences } \\
\text { ence.co }\end{array}$ & 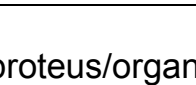 & & 2009). & & \\
\hline
\end{tabular}

\section{Argon}

\begin{tabular}{llll}
\hline Formula $=$ & $\mathrm{Ar}$ & Molecular weight $(\mathrm{g} / \mathrm{mole})=$ & 39.948 \\
Density $(\mathrm{g} / \mathrm{cm} 3)=$ & 0.001662 & Total atom density $($ atoms $/ \mathrm{b}-\mathrm{cm})=$ & $2.505 \mathrm{E}-05$
\end{tabular}

The above density is estimated to be accurate to 4 significant digits. Uncertainties are not addressed.

The following data was calculated from the input formula.

\begin{tabular}{|c|c|c|c|c|c|c|}
\hline$\frac{\text { Element }}{\mathrm{Ar}}$ & $\frac{\text { Neutron ZA }}{18000}$ & $\frac{\text { Photon ZA }}{18000}$ & $\begin{array}{l}\text { Weight } \\
\frac{\text { Fraction }}{1.000000}\end{array}$ & $\begin{array}{c}\begin{array}{c}\text { Atom } \\
\text { Fraction }\end{array} \\
1.000000\end{array}$ & $\begin{array}{c}\text { Atom } \\
\text { Density } \\
0.000025\end{array}$ & \\
\hline Total & & & 1.000000 & 1.000000 & 0.000025 & \\
\hline MCNP Form & \multicolumn{2}{|c|}{ Weight Fractions } & \multicolumn{2}{|c|}{ Atom Fractions } & \multicolumn{2}{|c|}{ Atom Densities } \\
\hline Neutrons & 18000 & -1.000000 & 18000 & 1.000000 & 18000 & 0.000025 \\
\hline Photons & 18000 & -1.000000 & 18000 & 1.000000 & 18000 & 0.000025 \\
\hline CEPXS Form: & $\begin{array}{l}\text { material } \\
\text { matname } \\
\text { density }\end{array}$ & $\begin{array}{c}\text { Ar } \\
\text { Argon } \\
0.001662\end{array}$ & 1.000000 & & & \\
\hline
\end{tabular}




\section{Asphalt}

\section{Formula $=$}

Density $(\mathrm{g} / \mathrm{cm} 3)=1.300000$

The above density

The following data were calculated from the input weight fractions.

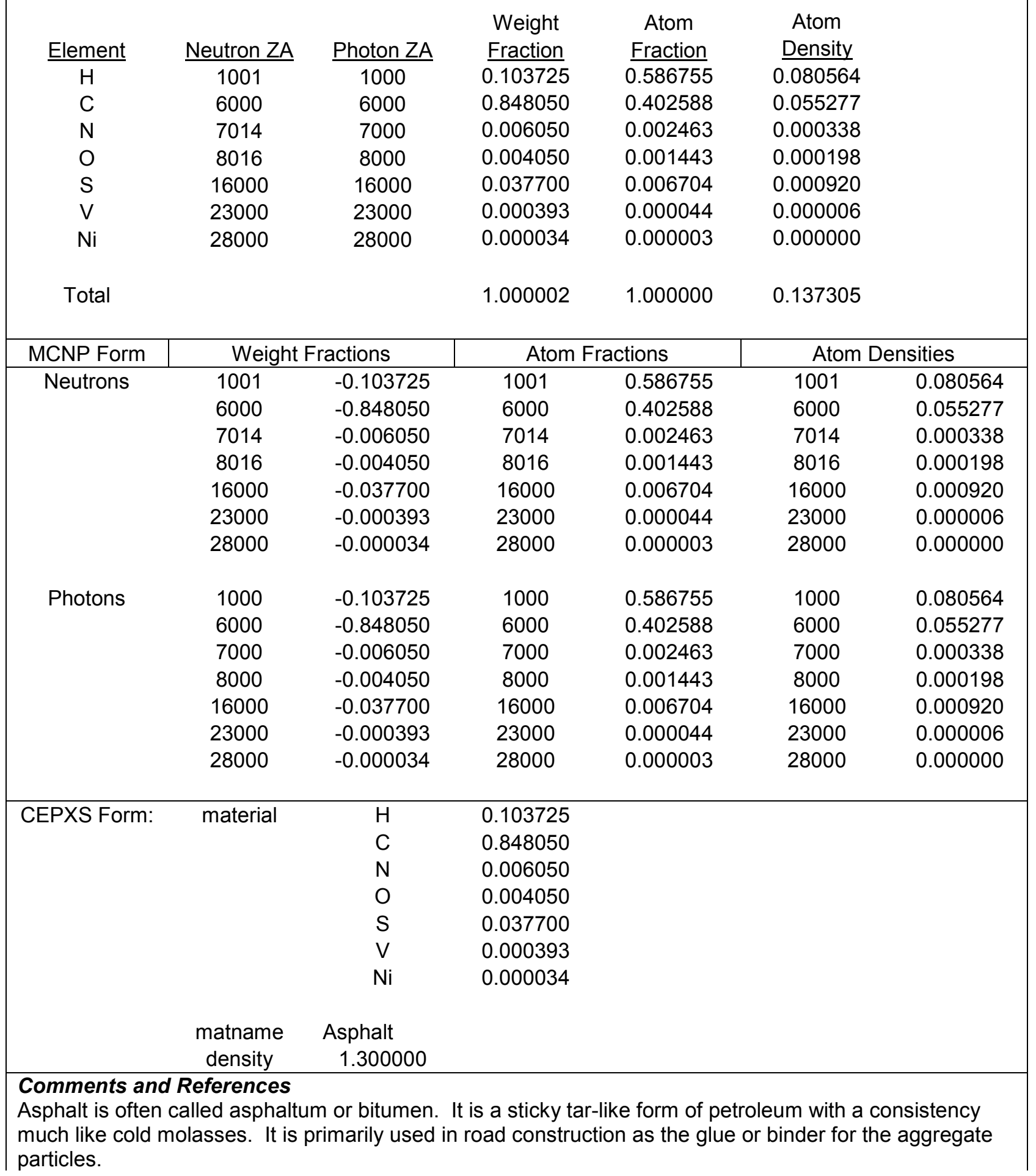


The weight fractions are an average of the 4 asphalt compositions from different crude sources from Table 2 of Wess et al. (2004) at http://www.inchem.org/documents/cicads/cicads/cicad59.htm.

Density $=1.1$ to $1.5 \mathrm{~g} / \mathrm{cm} 3$ in Table 6.1 .5 of Avallone and Baumeister III (1996). Also in Table $2-120$ of Green and Perry (2008).

\section{Asphalt Pavement}

\begin{tabular}{llll}
\hline Formula $=$ & - & Molecular weight $(\mathrm{g} / \mathrm{mole})=$ & - \\
Density $(\mathrm{g} / \mathrm{cm} 3)=$ & 2.578400 & Total atom density $($ atoms $/ \mathrm{b}-\mathrm{cm})=$ & $8.943 \mathrm{E}-02$
\end{tabular}

The above density is estimated to be accurate to 3 significant digits. Uncertainties are not addressed.

The following data were calculated from the input weight fractions.

\begin{tabular}{|c|c|c|c|c|c|c|}
\hline Element & Neutron ZA & Photon ZA & $\begin{array}{l}\text { Weight } \\
\text { Fraction }\end{array}$ & $\begin{array}{c}\text { Atom } \\
\text { Fraction }\end{array}$ & $\begin{array}{l}\text { Atom } \\
\text { Density }\end{array}$ & \\
\hline $\mathrm{H}$ & 1001 & 1000 & 0.007781 & 0.134043 & 0.011988 & \\
\hline C & 6000 & 6000 & 0.076175 & 0.110118 & 0.009848 & \\
\hline $\mathrm{N}$ & 7014 & 7000 & 0.000363 & 0.000450 & 0.000040 & \\
\hline $\mathrm{O}$ & 8016 & 8000 & 0.459103 & 0.498220 & 0.044556 & \\
\hline $\mathrm{Na}$ & 11023 & 11000 & 0.011659 & 0.008805 & 0.000787 & \\
\hline $\mathrm{Mg}$ & 12000 & 12000 & 0.021757 & 0.015543 & 0.001390 & \\
\hline $\mathrm{Al}$ & 13027 & 13000 & 0.051009 & 0.032824 & 0.002935 & \\
\hline $\mathrm{Si}$ & 14000 & 14000 & 0.231474 & 0.143098 & 0.012797 & \\
\hline $\mathrm{S}$ & 16000 & 16000 & 0.002804 & 0.001519 & 0.000136 & \\
\hline $\mathrm{K}$ & 19000 & 19000 & 0.017058 & 0.007575 & 0.000677 & \\
\hline $\mathrm{Ca}$ & 20000 & 20000 & 0.084471 & 0.036595 & 0.003273 & \\
\hline $\mathrm{Ti}$ & 22000 & 22000 & 0.003403 & 0.001235 & 0.000110 & \\
\hline $\mathrm{V}$ & 23000 & 23000 & 0.000024 & 0.000008 & 0.000001 & \\
\hline $\mathrm{Mn}$ & 25055 & 25000 & 0.000362 & 0.000115 & 0.000010 & \\
\hline $\mathrm{Fe}$ & 26000 & 26000 & 0.031375 & 0.009755 & 0.000872 & \\
\hline $\mathrm{Ni}$ & 28000 & 28000 & 0.000002 & 0.000001 & 0.000000 & \\
\hline $\mathrm{Pb}$ & 82000 & 82000 & 0.001179 & 0.000099 & 0.000009 & \\
\hline Total & & & 1.000000 & 1.000000 & 0.089431 & \\
\hline MCNP Form & \multicolumn{2}{|c|}{ Weight Fractions } & \multicolumn{2}{|c|}{ Atom Fractions } & \multicolumn{2}{|c|}{ Atom Densities } \\
\hline \multirow[t]{13}{*}{ Neutrons } & 1001 & -0.007781 & 1001 & 0.134043 & 1001 & 0.011988 \\
\hline & 6000 & -0.076175 & 6000 & 0.110118 & 6000 & 0.009848 \\
\hline & 7014 & -0.000363 & 7014 & 0.000450 & 7014 & 0.000040 \\
\hline & 8016 & -0.459103 & 8016 & 0.498220 & 8016 & 0.044556 \\
\hline & 11023 & -0.011659 & 11023 & 0.008805 & 11023 & 0.000787 \\
\hline & 12000 & -0.021757 & 12000 & 0.015543 & 12000 & 0.001390 \\
\hline & 13027 & -0.051009 & 13027 & 0.032824 & 13027 & 0.002935 \\
\hline & 14000 & -0.231474 & 14000 & 0.143098 & 14000 & 0.012797 \\
\hline & 16000 & -0.002804 & 16000 & 0.001519 & 16000 & 0.000136 \\
\hline & 19000 & -0.017058 & 19000 & 0.007575 & 19000 & 0.000677 \\
\hline & 20000 & -0.084471 & 20000 & 0.036595 & 20000 & 0.003273 \\
\hline & 22000 & -0.003403 & 22000 & 0.001235 & 22000 & 0.000110 \\
\hline & 23000 & -0.000024 & 23000 & 0.000008 & 23000 & 0.000001 \\
\hline
\end{tabular}


PIET-43741-TM-963

PNNL-15870 Rev. 1

\begin{tabular}{|c|c|c|c|c|c|c|}
\hline & 25055 & -0.000362 & 25055 & 0.000115 & 25055 & 0.000010 \\
\hline & 26000 & -0.031375 & 26000 & 0.009755 & 26000 & 0.000872 \\
\hline & 28000 & -0.000002 & 28000 & 0.000001 & 28000 & 0.000000 \\
\hline & 82000 & -0.001179 & 82000 & 0.000099 & 82000 & 0.000009 \\
\hline Photons & 1000 & -0.007781 & 1000 & 0.134043 & 1000 & 0.011988 \\
\hline & 6000 & -0.076175 & 6000 & 0.110118 & 6000 & 0.009848 \\
\hline & 7000 & -0.000363 & 7000 & 0.000450 & 7000 & 0.000040 \\
\hline & 8000 & -0.459103 & 8000 & 0.498220 & 8000 & 0.044556 \\
\hline & 11000 & -0.011659 & 11000 & 0.008805 & 11000 & 0.000787 \\
\hline & 12000 & -0.021757 & 12000 & 0.015543 & 12000 & 0.001390 \\
\hline & 13000 & -0.051009 & 13000 & 0.032824 & 13000 & 0.002935 \\
\hline & 14000 & -0.231474 & 14000 & 0.143098 & 14000 & 0.012797 \\
\hline & 16000 & -0.002804 & 16000 & 0.001519 & 16000 & 0.000136 \\
\hline & 19000 & -0.017058 & 19000 & 0.007575 & 19000 & 0.000677 \\
\hline & 20000 & -0.084471 & 20000 & 0.036595 & 20000 & 0.003273 \\
\hline & 22000 & -0.003403 & 22000 & 0.001235 & 22000 & 0.000110 \\
\hline & 23000 & -0.000024 & 23000 & 0.000008 & 23000 & 0.000001 \\
\hline & 25000 & -0.000362 & 25000 & 0.000115 & 25000 & 0.000010 \\
\hline & 26000 & -0.031375 & 26000 & 0.009755 & 26000 & 0.000872 \\
\hline & 28000 & -0.000002 & 28000 & 0.000001 & 28000 & 0.000000 \\
\hline & 82000 & -0.001179 & 82000 & 0.000099 & 82000 & 0.000009 \\
\hline CEPXS Form: & material & $\mathrm{H}$ & 0.007781 & & & \\
\hline & & C & 0.076175 & & & \\
\hline & & $\mathrm{N}$ & 0.000363 & & & \\
\hline & & 0 & 0.459103 & & & \\
\hline & & $\mathrm{Na}$ & 0.011659 & & & \\
\hline & & $\mathrm{Mg}$ & 0.021757 & & & \\
\hline & & $\mathrm{Al}$ & 0.051009 & & & \\
\hline & & $\mathrm{Si}$ & 0.231474 & & & \\
\hline & & $S$ & 0.002804 & & & \\
\hline & & $\mathrm{K}$ & 0.017058 & & & \\
\hline & & $\mathrm{Ca}$ & 0.084471 & & & \\
\hline & & $\mathrm{Ti}$ & 0.003403 & & & \\
\hline & & $\mathrm{V}$ & 0.000024 & & & \\
\hline & & $\mathrm{Mn}$ & 0.000362 & & & \\
\hline & & $\mathrm{Fe}$ & 0.031375 & & & \\
\hline & & $\mathrm{Ni}$ & 0.000002 & & & \\
\hline & & $\mathrm{Pb}$ & 0.001179 & & & \\
\hline & $\begin{array}{c}\text { matname } \\
\text { density }\end{array}$ & $\begin{array}{l}\text { Asphalt Pave } \\
2.578400\end{array}$ & & & & \\
\hline $\begin{array}{l}\text { Comments anc } \\
\text { Asphalt paveme } \\
\text { asphalt (as disc } \\
\text { Density and we } \\
\text { It may also be }\end{array}$ & $\begin{array}{l}\text { Peferences } \\
\text { can also b } \\
\text { sed above) } \\
\text { t fractions } \\
\text { essary to a }\end{array}$ & $\begin{array}{l}\text { lled asphalt } \\
\text { aggregate, } \\
\text { based on a } \\
\text { for void. }\end{array}$ & $\begin{array}{l}\text { crete, asph } \\
\text { d can also } \\
\text { ure of } 6 \mathrm{wt} \\
\text { ggregate is }\end{array}$ & $\begin{array}{l}\text { avement, } \\
\text { y be calle } \\
\text { sphalt and } \\
\text { ed on "Ro }\end{array}$ & $\begin{array}{l}\text { ktop. It } \\
\text { alt. } \\
\text { ggrega } \\
\text { rage o }\end{array}$ & $\begin{array}{l}\text { ixture of } \\
\text { es)". }\end{array}$ \\
\hline
\end{tabular}




\section{Bakelite}

$\begin{array}{llll}\text { Formula }= & - & \text { Molecular weight }(\mathrm{g} / \mathrm{mole})= & - \\ \text { Density }(\mathrm{g} / \mathrm{cm} 3)= & 1.250000 & \text { Total atom density }(\text { atoms } / \mathrm{b}-\mathrm{cm})= & 9.935 \mathrm{E}-02\end{array}$

The above density is estimated to be accurate to 3 significant digits. Uncertainties are not addressed.

The following data were calculated from the input weight fractions.

\begin{tabular}{|c|c|c|c|c|c|c|}
\hline Element & Neutron ZA & Photon ZA & $\begin{array}{l}\text { Weight } \\
\text { Fraction }\end{array}$ & $\begin{array}{c}\text { Atom } \\
\text { Fraction }\end{array}$ & $\begin{array}{l}\text { Atom } \\
\text { Density }\end{array}$ & \\
\hline $\mathrm{H}$ & 1001 & 1000 & 0.057444 & 0.431814 & 0.042901 & \\
\hline $\mathrm{C}$ & 6000 & 6000 & 0.774589 & 0.488641 & 0.048547 & \\
\hline $\mathrm{O}$ & 8016 & 8000 & 0.167968 & 0.079544 & 0.007903 & \\
\hline Total & & & 1.000001 & 1.000000 & 0.099351 & \\
\hline MCNP Form & \multicolumn{2}{|c|}{ Weight Fractions } & \multicolumn{2}{|c|}{ Atom Fractions } & \multicolumn{2}{|c|}{ Atom Densities } \\
\hline \multirow[t]{3}{*}{ Neutrons } & 1001 & -0.057444 & 1001 & 0.431814 & 1001 & 0.042901 \\
\hline & 6000 & -0.774589 & 6000 & 0.488641 & 6000 & 0.048547 \\
\hline & 8016 & -0.167968 & 8016 & 0.079544 & 8016 & 0.007903 \\
\hline \multirow[t]{3}{*}{ Photons } & 1000 & -0.057444 & 1000 & 0.431814 & 1000 & 0.042901 \\
\hline & 6000 & -0.774589 & 6000 & 0.488641 & 6000 & 0.048547 \\
\hline & 8000 & -0.167968 & 8000 & 0.079544 & 8000 & 0.007903 \\
\hline \multirow[t]{4}{*}{ CEPXS Form: } & material & $\mathrm{H}$ & 0.057444 & & & \\
\hline & & C & 0.774589 & & & \\
\hline & & $\mathrm{O}$ & 0.167968 & & & \\
\hline & $\begin{array}{c}\text { matname } \\
\text { density }\end{array}$ & $\begin{array}{l}\text { Bakelite } \\
1.250000\end{array}$ & & & & \\
\hline & Peferences & & & & & \\
\hline
\end{tabular}

\section{Barium Fluoride}

\begin{tabular}{llll}
\hline Formula $=$ & BaF2 & Molecular weight $(\mathrm{g} / \mathrm{mole})=$ & 175.3238064 \\
Density $(\mathrm{g} / \mathrm{cm} 3)=$ & 4.890000 & Total atom density $($ atoms $/ \mathrm{b}-\mathrm{cm})=$ & $5.039 \mathrm{E}-02$
\end{tabular}

The above density is estimated to be accurate to 3 significant digits. Uncertainties are not addressed.

The following data were calculated from the input weight fractions.

\begin{tabular}{|c|c|c|c|c|c|}
\hline Element & Neutron ZA & Photon ZA & $\begin{array}{l}\text { Weight } \\
\text { Fraction }\end{array}$ & $\begin{array}{c}\text { Atom } \\
\text { Fraction }\end{array}$ & $\begin{array}{c}\text { Atom } \\
\text { Density }\end{array}$ \\
\hline$F$ & 9019 & 9000 & 0.216720 & $\overline{0.666662}$ & 0.033592 \\
\hline $\mathrm{Ba}$ & - & 56000 & 0.783280 & 0.333338 & 0.016797 \\
\hline Total & & & 1.000000 & 1.000000 & 0.050389 \\
\hline
\end{tabular}


PIET-43741-TM-963

PNNL-15870 Rev. 1

\begin{tabular}{|c|c|c|c|c|c|c|}
\hline MCNP Form & \multicolumn{2}{|c|}{ Weight Fractions } & \multicolumn{2}{|c|}{ Atom Fractions } & \multicolumn{2}{|c|}{ Atom Densities } \\
\hline \multirow[t]{2}{*}{ Neutrons } & 9019 & -0.216720 & 9019 & 0.666662 & 9019 & 0.033592 \\
\hline & - & -0.783280 & - & 0.333338 & - & 0.016797 \\
\hline \multirow[t]{2}{*}{ Photons } & 9000 & -0.216720 & 9000 & 0.666662 & 9000 & 0.033592 \\
\hline & 56000 & -0.783280 & 56000 & 0.333338 & 56000 & 0.016797 \\
\hline \multirow[t]{3}{*}{ CEPXS Form: } & material & $\mathrm{F}$ & 0.216720 & & & \\
\hline & & $\mathrm{Ba}$ & 0.783280 & & & \\
\hline & $\begin{array}{c}\text { matname } \\
\text { density }\end{array}$ & $\begin{array}{c}\text { Barium Fluo } \\
4.890000\end{array}$ & & & & \\
\hline \multicolumn{7}{|c|}{$\begin{array}{l}\text { Comments and References } \\
\text { Density }=4.89 \mathrm{~g} / \mathrm{cm} 3 \text { and weight fractions from http://physics.nist.gov/cgi-bin/Star/compos.pl?matno=113 } \\
\text { (NIST } 1998) . \\
\text { Density }=4.89 \mathrm{~g} / \mathrm{cm} 3 \text { and formula from pg } 235 \text { of Knoll }(2000) .\end{array}$} \\
\hline
\end{tabular}

\section{Barium Sulfate}

\begin{tabular}{llll}
\hline Formula $=$ & BaSO4 & Molecular weight $(\mathrm{g} / \mathrm{mole})=$ & 233.3896 \\
Density $(\mathrm{g} / \mathrm{cm} 3)=$ & 4.500000 & Total atom density $($ atoms $/ \mathrm{b}-\mathrm{cm})=$ & $6.967 \mathrm{E}-02$
\end{tabular}

The above density is estimated to be accurate to 3 significant digits. Uncertainties are not addressed.

The following data were calculated from the input weight fractions.

\begin{tabular}{|c|c|c|c|c|c|c|}
\hline Element & Neutron ZA & Photon ZA & $\begin{array}{l}\text { Weight } \\
\text { Fraction }\end{array}$ & $\begin{array}{c}\text { Atom } \\
\text { Fraction }\end{array}$ & $\begin{array}{l}\text { Atom } \\
\text { Density }\end{array}$ & \\
\hline 0 & 8016 & 8000 & 0.274212 & 0.666682 & 0.046446 & \\
\hline$S$ & 16000 & 16000 & 0.137368 & 0.166644 & 0.011610 & \\
\hline $\mathrm{Ba}$ & - & 56000 & 0.588420 & 0.166674 & 0.011612 & \\
\hline Total & & & 1.000000 & 1.000000 & 0.069667 & \\
\hline MCNP Form & \multicolumn{2}{|c|}{ Weight Fractions } & \multicolumn{2}{|c|}{ Atom Fractions } & \multicolumn{2}{|c|}{ Atom Densities } \\
\hline \multirow[t]{3}{*}{ Neutrons } & 8016 & -0.274212 & 8016 & 0.666682 & 8016 & 0.046446 \\
\hline & 16000 & -0.137368 & 16000 & 0.166644 & 16000 & 0.011610 \\
\hline & - & -0.588420 & - & 0.166674 & - & 0.011612 \\
\hline \multirow[t]{3}{*}{ Photons } & 8000 & -0.274212 & 8000 & 0.666682 & 8000 & 0.046446 \\
\hline & 16000 & -0.137368 & 16000 & 0.166644 & 16000 & 0.011610 \\
\hline & 56000 & -0.588420 & 56000 & 0.166674 & 56000 & 0.011612 \\
\hline \multirow[t]{4}{*}{ CEPXS Form: } & material & $\mathrm{O}$ & 0.274212 & & & \\
\hline & & $S$ & 0.137368 & & & \\
\hline & & $\mathrm{Ba}$ & 0.588420 & & & \\
\hline & $\begin{array}{l}\text { matname } \\
\text { density }\end{array}$ & $\begin{array}{l}\text { Barium Sulfate } \\
4.500000\end{array}$ & & & & \\
\hline
\end{tabular}


Comments and References

Density and weight fractions from http://physics.nist.gov/cgi-bin/Star/compos.pl?matno=114 (NIST 1998).

Formula from Lide (2008), pgs 4 - 51.

\section{Benzene}

\begin{tabular}{llll}
\hline Formula $=$ & $\mathrm{C} 6 \mathrm{H} 6$ & Molecular weight $(\mathrm{g} / \mathrm{mole})=$ & 78.11184 \\
Density $(\mathrm{g} / \mathrm{cm} 3)=$ & 0.876500 & Total atom density $($ atoms $/ \mathrm{b}-\mathrm{cm})=$ & $8.109 \mathrm{E}-02$
\end{tabular}

The above density is estimated to be accurate to 4 significant digits. Uncertainties are not addressed.

The following data were calculated from the input weight fractions.

\begin{tabular}{|c|c|c|c|c|c|c|}
\hline Element & Neutron ZA & Photon ZA & $\begin{array}{l}\text { Weight } \\
\text { Fraction }\end{array}$ & $\begin{array}{l}\text { Atom } \\
\text { Fraction }\end{array}$ & \multicolumn{2}{|l|}{$\begin{array}{l}\text { Atom } \\
\text { Density }\end{array}$} \\
\hline $\mathrm{H}$ & 1001 & 1000 & $\overline{0.077418}$ & $\overline{0.499983}$ & \multicolumn{2}{|l|}{0.040542} \\
\hline C & 6000 & 6000 & 0.922582 & 0.500017 & \multicolumn{2}{|l|}{0.040545} \\
\hline Total & & & 1.000000 & 1.000000 & \multicolumn{2}{|l|}{0.081088} \\
\hline MCNP Form & \multicolumn{2}{|c|}{ Weight Fractions } & \multicolumn{2}{|c|}{ Atom Fractions } & \multicolumn{2}{|c|}{ Atom Densities } \\
\hline \multirow{2}{*}{ Neutrons } & 1001 & -0.077418 & 1001 & 0.499983 & 1001 & 0.040542 \\
\hline & 6000 & -0.922582 & 6000 & 0.500017 & 6000 & 0.040545 \\
\hline \multirow[t]{2}{*}{ Photons } & 1000 & -0.077418 & 1000 & 0.499983 & 1000 & 0.040542 \\
\hline & 6000 & -0.922582 & 6000 & 0.500017 & 6000 & 0.040545 \\
\hline \multirow[t]{3}{*}{ CEPXS Form: } & material & $\mathrm{H}$ & 0.077418 & & & \\
\hline & & C & 0.922582 & & & \\
\hline & $\begin{array}{c}\text { matname } \\
\text { density }\end{array}$ & $\begin{array}{l}\text { Benzene } \\
0.876500\end{array}$ & & & & \\
\hline \multicolumn{7}{|c|}{$\begin{array}{l}\text { Comments and References } \\
\text { Density and weight fractions from http://physics.nist.gov/cgi-bin/Star/compos.pl?matno=115 (NIST 1998). } \\
\text { Same density in Lide (2008), pgs } 3-32 \text {, but NIST density = 0.87865. } \\
\text { Formula from } \\
\text { http://www.matweb.com/search/DataSheet.aspx?MatGUID=e6a3974d195942d4941514c285151f10 } \\
\text { (Automation Creations 2010). }\end{array}$} \\
\hline
\end{tabular}

\section{Beryllium}

\begin{tabular}{llll}
\hline Formula $=$ & Be & Molecular weight $(\mathrm{g} / \mathrm{mole})=$ & 9.012182 \\
Density $(\mathrm{g} / \mathrm{cm} 3)=$ & 1.848000 & Total atom density $($ atoms $/ \mathrm{b}-\mathrm{cm})=$ & $1.235 \mathrm{E}-01$
\end{tabular}

The above density is estimated to be accurate to 4 significant digits. Uncertainties are not addressed.

The following data was calculated from the input formula. 


\begin{tabular}{|c|c|c|c|c|c|c|}
\hline$\frac{\text { Element }}{\mathrm{Be}}$ & $\frac{\text { Neutron ZA }}{4009}$ & $\frac{\text { Photon ZA }}{4000}$ & $\begin{array}{l}\text { Weight } \\
\text { Fraction } \\
1.000000\end{array}$ & $\begin{array}{c}\text { Atom } \\
\frac{\text { Fraction }}{1.000000}\end{array}$ & $\begin{array}{c}\text { Atom } \\
\text { Density } \\
0.123487\end{array}$ & \\
\hline Total & & & 1.000000 & 1.000000 & 0.123487 & \\
\hline MCNP Form & \multicolumn{2}{|c|}{ Weight Fractions } & \multicolumn{2}{|c|}{ Atom Fractions } & \multicolumn{2}{|c|}{ Atom Densities } \\
\hline Neutrons & 4009 & -1.000000 & 4009 & 1.000000 & 4009 & 0.123487 \\
\hline Photons & 4000 & -1.000000 & 4000 & 1.000000 & 4000 & 0.123487 \\
\hline CEPXS Form: & $\begin{array}{l}\text { material } \\
\text { matname } \\
\text { density }\end{array}$ & $\begin{array}{c}\text { Be } \\
\text { Beryllium } \\
1.848000\end{array}$ & 1.000000 & & & \\
\hline $\begin{array}{l}\text { Comments an } \\
\text { Density from h }\end{array}$ & $\begin{array}{l}\text { eferences } \\
\text { /physics.nis }\end{array}$ & 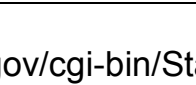 & & & & \\
\hline
\end{tabular}

\section{Beryllium Carbide}

$\begin{array}{llll}\text { Formula }= & \text { Be2C } & \text { Molecular weight }(\mathrm{g} / \mathrm{mole})= & 30.035064 \\ \text { Density }(\mathrm{g} / \mathrm{cm} 3)= & 1.900000 & \text { Total atom density }(\text { atoms } / \mathrm{b}-\mathrm{cm})= & 1.143 \mathrm{E}-01\end{array}$

The above density is estimated to be accurate to 3 significant digits. Uncertainties are not addressed. The following data was calculated from the input formula.

\begin{tabular}{|c|c|c|c|c|c|c|}
\hline $\begin{array}{c}\text { Element } \\
\mathrm{Be} \\
\mathrm{C}\end{array}$ & $\begin{array}{c}\text { Neutron ZA } \\
4009 \\
6000\end{array}$ & $\begin{array}{c}\text { Photon ZA } \\
4000 \\
6000\end{array}$ & $\begin{array}{c}\text { Weight } \\
\text { Fraction } \\
0.600111 \\
0.399889\end{array}$ & $\begin{array}{c}\text { Atom } \\
\text { Fraction } \\
0.666667 \\
0.333333\end{array}$ & $\begin{array}{c}\begin{array}{c}\text { Atom } \\
\text { Density }\end{array} \\
0.076191 \\
0.038096\end{array}$ & \\
\hline Total & & & 1.000000 & 1.000000 & 0.114287 & \\
\hline MCNP Form & \multicolumn{2}{|c|}{ Weight Fractions } & \multicolumn{2}{|c|}{ Atom Fractions } & \multicolumn{2}{|c|}{ Atom Densities } \\
\hline Neutrons & $\begin{array}{l}4009 \\
6000\end{array}$ & $\begin{array}{l}-0.600111 \\
-0.399889\end{array}$ & $\begin{array}{l}4009 \\
6000\end{array}$ & $\begin{array}{l}0.666667 \\
0.333333\end{array}$ & $\begin{array}{l}4009 \\
6000\end{array}$ & $\begin{array}{l}0.076191 \\
0.038096\end{array}$ \\
\hline Photons & $\begin{array}{l}4000 \\
6000\end{array}$ & $\begin{array}{l}-0.600111 \\
-0.399889\end{array}$ & $\begin{array}{l}4000 \\
6000\end{array}$ & $\begin{array}{l}0.666667 \\
0.333333\end{array}$ & $\begin{array}{l}4000 \\
6000\end{array}$ & $\begin{array}{l}0.076191 \\
0.038096\end{array}$ \\
\hline CEPXS Form: & $\begin{array}{c}\text { matname } \\
\text { density }\end{array}$ & $\begin{array}{c}\text { Be } \\
\text { C } \\
\text { Beryllium Ca } \\
1.900000\end{array}$ & $\begin{array}{l}0.600111 \\
0.399889\end{array}$ & & & \\
\hline $\begin{array}{l}\text { Comments an } \\
\text { Formula and de } \\
\text { Density also in }\end{array}$ & $\begin{array}{l}\text { References } \\
\text { sity from Lide } \\
\text { able } 51.14 \text { of }\end{array}$ & $\begin{array}{l}\text { 2008), pgs } 4 \\
\text { ungerford (19 }\end{array}$ & & & & \\
\hline
\end{tabular}




\section{Beryllium Oxide}

\begin{tabular}{lllc}
\hline Formula $=$ & $\mathrm{BeO}$ & Molecular weight $(\mathrm{g} / \mathrm{mole})=$ & 25.011582 \\
Density $(\mathrm{g} / \mathrm{cm} 3)=$ & 3.010000 & Total atom density $($ atoms $/ \mathrm{b}-\mathrm{cm})=$ & $1.449 \mathrm{E}-01$ \\
The above density is estimated to be accurate to & 3 significant digits. Uncertainties are not addressed. \\
The following data were calculated from the input weight fractions.
\end{tabular}

\begin{tabular}{|c|c|c|c|c|c|c|}
\hline Element & Neutron ZA & Photon ZA & $\begin{array}{l}\text { Weight } \\
\text { Fraction }\end{array}$ & $\begin{array}{l}\text { Atom } \\
\text { Fraction }\end{array}$ & $\begin{array}{l}\text { Atom } \\
\text { Density }\end{array}$ & \\
\hline $\mathrm{Be}$ & 4009 & 4000 & 0.360320 & 0.500000 & 0.072473 & \\
\hline $\mathrm{O}$ & 8016 & 8000 & 0.639680 & 0.500000 & 0.072473 & \\
\hline Total & & & 1.000000 & 1.000000 & 0.144946 & \\
\hline MCNP Form & \multicolumn{2}{|c|}{ Weight Fractions } & \multicolumn{2}{|c|}{ Atom Fractions } & \multicolumn{2}{|c|}{ Atom Densities } \\
\hline \multirow[t]{2}{*}{ Neutrons } & 4009 & -0.360320 & 4009 & 0.500000 & 4009 & 0.072473 \\
\hline & 8016 & -0.639680 & 8016 & 0.500000 & 8016 & 0.072473 \\
\hline \multirow[t]{2}{*}{ Photons } & 4000 & -0.360320 & 4000 & 0.500000 & 4000 & 0.072473 \\
\hline & 8000 & -0.639680 & 8000 & 0.500000 & 8000 & 0.072473 \\
\hline \multirow[t]{3}{*}{ CEPXS Form: } & material & $\mathrm{Be}$ & 0.360320 & & & \\
\hline & & O & 0.639680 & & & \\
\hline & $\begin{array}{l}\text { matname } \\
\text { density }\end{array}$ & $\begin{array}{l}\text { Beryllium Oxide } \\
3.010000\end{array}$ & & & & \\
\hline
\end{tabular}

\section{Comments and References}

Density and weight fractions from http://physics.nist.gov/cgi-bin/Star/compos.pl?matno=116 (NIST 1998). Formula from Lide (2008), pgs 4 - 51.

Density =- $2.3 \mathrm{~g} / \mathrm{cm} 3$ for hot-pressed blocks, Table 51.14 of Hungerford (1960).

\section{Bismuth}

$\begin{array}{llll}\text { Formula }= & \mathrm{Bi} & \text { Molecular weight }(\mathrm{g} / \mathrm{mole})= & 208.98038 \\ \text { Density }(\mathrm{g} / \mathrm{cm} 3)= & 9.747000 & \text { Total atom density }(\text { atoms } / \mathrm{b}-\mathrm{cm})= & 2.809 \mathrm{E}-02\end{array}$

The above density is estimated to be accurate to 4 significant digits. Uncertainties are not addressed.

The following data was calculated from the input formula.

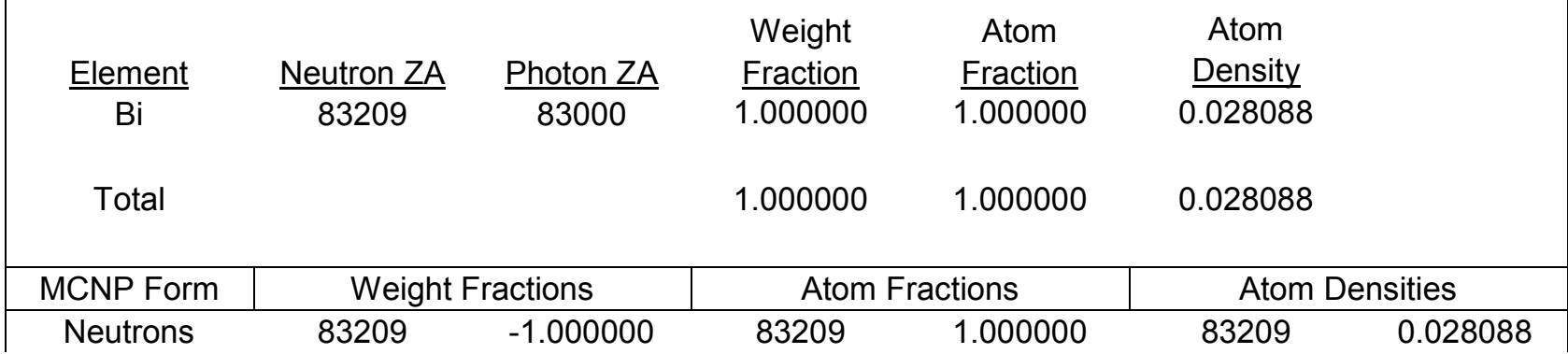


PIET-43741-TM-963

PNNL-15870 Rev. 1

\begin{tabular}{|c|c|c|c|c|c|c|}
\hline Photons & 83000 & -1.000000 & 83000 & 1.000000 & 83000 & 0.028088 \\
\hline CEPXS Form: & $\begin{array}{l}\text { material } \\
\text { matname } \\
\text { density }\end{array}$ & $\begin{array}{c}\text { Bi } \\
\text { Bismuth } \\
9.747000\end{array}$ & 1.000000 & & & \\
\hline $\begin{array}{l}\text { Comments an } \\
\text { Density from h }\end{array}$ & $\begin{array}{l}\text { References } \\
\text { //physics.ni }\end{array}$ & ov/cgi-bin/S & mpos.p & $\mathrm{O}=083(\mathrm{NI}$ & & \\
\hline
\end{tabular}

\section{Bismuth Germanate (BGO)}

\begin{tabular}{llll}
\hline Formula $=$ & Bi4Ge3O12 & Molecular weight $(\mathrm{g} / \mathrm{mole})=$ & 1245.83432 \\
Density $(\mathrm{g} / \mathrm{cm} 3)=$ & 7.130000 & Total atom density $($ atoms $/ \mathrm{b}-\mathrm{cm})=$ & $6.548 \mathrm{E}-02$
\end{tabular}

The above density is estimated to be accurate to 3 significant digits. Uncertainties are not addressed.

The following data were calculated from the input weight fractions.

\begin{tabular}{|c|c|c|c|c|c|c|}
\hline Element & Neutron ZA & Photon ZA & $\begin{array}{l}\text { Weight } \\
\text { Fraction }\end{array}$ & $\begin{array}{l}\text { Atom } \\
\text { Fraction }\end{array}$ & $\begin{array}{l}\text { Atom } \\
\text { Density }\end{array}$ & \\
\hline 0 & 8016 & 8000 & $\overline{0.154126}$ & 0.631647 & 0.041363 & \\
\hline $\mathrm{Ge}$ & - & 32000 & 0.174820 & 0.157804 & 0.010334 & \\
\hline $\mathrm{Bi}$ & 83209 & 83000 & 0.671054 & 0.210549 & 0.013788 & \\
\hline Total & & & 1.000000 & 1.000000 & 0.065484 & \\
\hline MCNP Form & \multicolumn{2}{|c|}{ Weight Fractions } & \multicolumn{2}{|c|}{ Atom Fractions } & \multicolumn{2}{|c|}{ Atom Densities } \\
\hline \multirow[t]{3}{*}{ Neutrons } & 8016 & -0.154126 & 8016 & 0.631647 & 8016 & 0.041363 \\
\hline & - & -0.174820 & - & 0.157804 & - & 0.010334 \\
\hline & 83209 & -0.671054 & 83209 & 0.210549 & 83209 & 0.013788 \\
\hline \multirow[t]{3}{*}{ Photons } & 8000 & -0.154126 & 8000 & 0.631647 & 8000 & 0.041363 \\
\hline & 32000 & -0.174820 & 32000 & 0.157804 & 32000 & 0.010334 \\
\hline & 83000 & -0.671054 & 83000 & 0.210549 & 83000 & 0.013788 \\
\hline \multirow[t]{4}{*}{ CEPXS Form: } & material & $\mathrm{O}$ & 0.154126 & & & \\
\hline & & $\mathrm{Ge}$ & 0.174820 & & & \\
\hline & & $\mathrm{Bi}$ & 0.671054 & & & \\
\hline & $\begin{array}{l}\text { matname } \\
\text { density }\end{array}$ & \multicolumn{2}{|c|}{$\begin{array}{l}\text { Bismuth Germanate (BGO) } \\
7.130000\end{array}$} & & & \\
\hline \multicolumn{7}{|c|}{$\begin{array}{l}\text { Comments and References } \\
\text { Density and weight fractions from http://physics.nist.gov/cgi-bin/Star/compos.pl?matno=117 (NIST 1998), } \\
\text { where it is called Bismuth Germanium Oxide. Same density given on pg } 235 \text { of Knoll (2000). }\end{array}$} \\
\hline
\end{tabular}




\section{Blood (ICRP)}

\begin{tabular}{|c|c|c|c|}
\hline Formula $=$ & - & Molecular weight ( $\mathrm{g} / \mathrm{mole})=$ & - \\
\hline Density $(\mathrm{g} / \mathrm{cm} 3)=$ & 1.060000 & Total atom density $($ atoms $/ \mathrm{b}-\mathrm{cm})=$ & 1.017E-01 \\
\hline
\end{tabular}

\begin{tabular}{|c|c|c|c|c|c|c|}
\hline Element & Neutron ZA & Photon ZA & $\begin{array}{l}\text { Weight } \\
\text { Fraction }\end{array}$ & $\begin{array}{c}\text { Atom } \\
\text { Fraction }\end{array}$ & $\begin{array}{l}\text { Atom } \\
\text { Density }\end{array}$ & \\
\hline $\mathrm{H}$ & 1001 & 1000 & $\overline{0.101866}$ & $\overline{0.634604}$ & 0.064514 & \\
\hline C & 6000 & 6000 & 0.100020 & 0.052291 & 0.005316 & \\
\hline $\mathrm{N}$ & 7014 & 7000 & 0.029640 & 0.013288 & 0.001351 & \\
\hline 0 & 8016 & 8000 & 0.759414 & 0.298046 & 0.030299 & \\
\hline $\mathrm{Na}$ & 11023 & 11000 & 0.001850 & 0.000505 & 0.000051 & \\
\hline $\mathrm{Mg}$ & 12000 & 12000 & 0.000040 & 0.000010 & 0.000001 & \\
\hline $\mathrm{Si}$ & 14000 & 14000 & 0.000030 & 0.000007 & 0.000001 & \\
\hline $\mathrm{P}$ & 15031 & 15000 & 0.000350 & 0.000071 & 0.000007 & \\
\hline$S$ & 16000 & 16000 & 0.001850 & 0.000362 & 0.000037 & \\
\hline $\mathrm{Cl}$ & 17000 & 17000 & 0.002780 & 0.000492 & 0.000050 & \\
\hline $\mathrm{K}$ & 19000 & 19000 & 0.001630 & 0.000262 & 0.000027 & \\
\hline $\mathrm{Ca}$ & 20000 & 20000 & 0.000060 & 0.000009 & 0.000001 & \\
\hline $\mathrm{Fe}$ & 26000 & 26000 & 0.000460 & 0.000052 & 0.000005 & \\
\hline $\mathrm{Zn}$ & 30000 & 30000 & 0.000010 & 0.000001 & 0.000000 & \\
\hline Total & & & 1.000000 & 1.000000 & 0.101660 & \\
\hline MCNP Form & \multicolumn{2}{|c|}{ Weight Fractions } & \multicolumn{2}{|c|}{ Atom Fractions } & \multicolumn{2}{|c|}{ Atom Densities } \\
\hline \multirow[t]{14}{*}{ Neutrons } & 1001 & -0.101866 & 1001 & 0.634604 & 1001 & 0.064514 \\
\hline & 6000 & -0.100020 & 6000 & 0.052291 & 6000 & 0.005316 \\
\hline & 7014 & -0.029640 & 7014 & 0.013288 & 7014 & 0.001351 \\
\hline & 8016 & -0.759414 & 8016 & 0.298046 & 8016 & 0.030299 \\
\hline & 11023 & -0.001850 & 11023 & 0.000505 & 11023 & 0.000051 \\
\hline & 12000 & -0.000040 & 12000 & 0.000010 & 12000 & 0.000001 \\
\hline & 14000 & -0.000030 & 14000 & 0.000007 & 14000 & 0.000001 \\
\hline & 15031 & -0.000350 & 15031 & 0.000071 & 15031 & 0.000007 \\
\hline & 16000 & -0.001850 & 16000 & 0.000362 & 16000 & 0.000037 \\
\hline & 17000 & -0.002780 & 17000 & 0.000492 & 17000 & 0.000050 \\
\hline & 19000 & -0.001630 & 19000 & 0.000262 & 19000 & 0.000027 \\
\hline & 20000 & -0.000060 & 20000 & 0.000009 & 20000 & 0.000001 \\
\hline & 26000 & -0.000460 & 26000 & 0.000052 & 26000 & 0.000005 \\
\hline & 30000 & -0.000010 & 30000 & 0.000001 & 30000 & 0.000000 \\
\hline \multirow[t]{8}{*}{ Photons } & 1000 & -0.101866 & 1000 & 0.634604 & 1000 & 0.064514 \\
\hline & 6000 & -0.100020 & 6000 & 0.052291 & 6000 & 0.005316 \\
\hline & 7000 & -0.029640 & 7000 & 0.013288 & 7000 & 0.001351 \\
\hline & 8000 & -0.759414 & 8000 & 0.298046 & 8000 & 0.030299 \\
\hline & 11000 & -0.001850 & 11000 & 0.000505 & 11000 & 0.000051 \\
\hline & 12000 & -0.000040 & 12000 & 0.000010 & 12000 & 0.000001 \\
\hline & 14000 & -0.000030 & 14000 & 0.000007 & 14000 & 0.000001 \\
\hline & 15000 & -0.000350 & 15000 & 0.000071 & 15000 & 0.000007 \\
\hline
\end{tabular}


PIET-43741-TM-963

PNNL-15870 Rev. 1

\begin{tabular}{|c|c|c|c|c|c|c|}
\hline & 16000 & -0.001850 & 16000 & 0.000362 & 16000 & 0.000037 \\
\hline & 17000 & -0.002780 & 17000 & 0.000492 & 17000 & 0.000050 \\
\hline & 19000 & -0.001630 & 19000 & 0.000262 & 19000 & 0.000027 \\
\hline & 20000 & -0.000060 & 20000 & 0.000009 & 20000 & 0.000001 \\
\hline & 26000 & -0.000460 & 26000 & 0.000052 & 26000 & 0.000005 \\
\hline & 30000 & -0.000010 & 30000 & 0.000001 & 30000 & 0.000000 \\
\hline \multirow[t]{16}{*}{ CEPXS Form: } & material & $\mathrm{H}$ & 0.101866 & & & \\
\hline & & $\mathrm{C}$ & 0.100020 & & & \\
\hline & & $\mathrm{N}$ & 0.029640 & & & \\
\hline & & $\mathrm{O}$ & 0.759414 & & & \\
\hline & & $\mathrm{Na}$ & 0.001850 & & & \\
\hline & & $\mathrm{Mg}$ & 0.000040 & & & \\
\hline & & $\mathrm{Si}$ & 0.000030 & & & \\
\hline & & $\mathrm{P}$ & 0.000350 & & & \\
\hline & & $S$ & 0.001850 & & & \\
\hline & & $\mathrm{Cl}$ & 0.002780 & & & \\
\hline & & $\mathrm{K}$ & 0.001630 & & & \\
\hline & & $\mathrm{Ca}$ & 0.000060 & & & \\
\hline & & $\mathrm{Fe}$ & 0.000460 & & & \\
\hline & & $\mathrm{Zn}$ & 0.000010 & & & \\
\hline & matname & Blood (ICRP) & & & & \\
\hline & density & 1.060000 & & & & \\
\hline
\end{tabular}

\section{Bone Equivalent Plastic, B-100}

\begin{tabular}{llll}
\hline Formula $=$ & - & Molecular weight $(\mathrm{g} / \mathrm{mole})=$ & - \\
Density $(\mathrm{g} / \mathrm{cm} 3)=$ & 1.450000 & Total atom density $($ atoms $/ \mathrm{b}-\mathrm{cm})=$ & $1.104 \mathrm{E}-01$
\end{tabular}

The above density is estimated to be accurate to 3 significant digits. Uncertainties are not addressed.

The following data were calculated from the input weight fractions.

\begin{tabular}{|c|c|c|c|c|c|c|}
\hline Element & Neutron ZA & Photon ZA & $\begin{array}{l}\text { Weight } \\
\text { Fraction }\end{array}$ & $\begin{array}{c}\text { Atom } \\
\text { Fraction }\end{array}$ & $\begin{array}{l}\text { Atom } \\
\text { Density }\end{array}$ & \\
\hline $\mathrm{H}$ & 1001 & 1000 & $\overline{0.065471}$ & $\overline{0.513809}$ & 0.056720 & \\
\hline $\mathrm{C}$ & 6000 & 6000 & 0.536945 & 0.353630 & 0.039037 & \\
\hline $\mathrm{N}$ & 7014 & 7000 & 0.021500 & 0.012142 & 0.001340 & \\
\hline $\mathrm{O}$ & 8016 & 8000 & 0.032085 & 0.015863 & 0.001751 & \\
\hline $\mathrm{F}$ & 9019 & 9000 & 0.167411 & 0.069703 & 0.007695 & \\
\hline $\mathrm{Ca}$ & 20000 & 20000 & 0.176589 & 0.034853 & 0.003847 & \\
\hline Total & & & 1.000001 & 1.000000 & 0.110391 & \\
\hline MCNP Form & \multicolumn{2}{|c|}{ Weight Fractions } & \multicolumn{2}{|c|}{ Atom Fractions } & Atom & sities \\
\hline Neutrons & 1001 & $\begin{array}{l}-0.065471 \\
-0.536945\end{array}$ & 1001 & 0.513809 & 1001 & 0.056720 \\
\hline
\end{tabular}


PIET-43741-TM-963

PNNL-15870 Rev. 1

\begin{tabular}{|c|c|c|c|c|c|c|}
\hline & 7014 & -0.021500 & 7014 & 0.012142 & 7014 & 0.001340 \\
\hline & 8016 & -0.032085 & 8016 & 0.015863 & 8016 & 0.001751 \\
\hline & 9019 & -0.167411 & 9019 & 0.069703 & 9019 & 0.007695 \\
\hline & 20000 & -0.176589 & 20000 & 0.034853 & 20000 & 0.003847 \\
\hline Photons & 1000 & -0.065471 & 1000 & 0.513809 & 1000 & 0.056720 \\
\hline & 6000 & -0.536945 & 6000 & 0.353630 & 6000 & 0.039037 \\
\hline & 7000 & -0.021500 & 7000 & 0.012142 & 7000 & 0.001340 \\
\hline & 8000 & -0.032085 & 8000 & 0.015863 & 8000 & 0.001751 \\
\hline & 9000 & -0.167411 & 9000 & 0.069703 & 9000 & 0.007695 \\
\hline & 20000 & -0.176589 & 20000 & 0.034853 & 20000 & 0.003847 \\
\hline CEPXS Form: & material & $\mathrm{H}$ & 0.065471 & & & \\
\hline & & $\mathrm{C}$ & 0.536945 & & & \\
\hline & & $\mathrm{N}$ & 0.021500 & & & \\
\hline & & $\mathrm{O}$ & 0.032085 & & & \\
\hline & & $\mathrm{F}$ & 0.167411 & & & \\
\hline & & $\mathrm{Ca}$ & 0.176589 & & & \\
\hline & $\begin{array}{c}\text { matname } \\
\text { density }\end{array}$ & $\begin{array}{c}\text { Bone Equiv } \\
1.450000 \\
\end{array}$ & Plastic, B & & & \\
\hline
\end{tabular}

\section{Bone Equivalent Plastic, B-110}

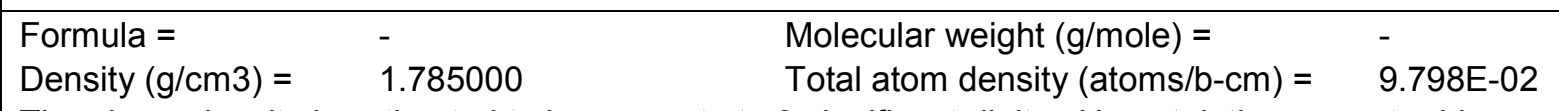

The above density is estimated to be accurate to 3 significant digits. Uncertainties are not addressed.

The following data were calculated from the input weight fractions.

\begin{tabular}{|c|c|c|c|c|c|c|}
\hline Element & Neutron ZA & Photon ZA & $\begin{array}{l}\text { Weight } \\
\text { Fraction }\end{array}$ & $\begin{array}{c}\text { Atom } \\
\text { Fraction }\end{array}$ & $\begin{array}{l}\text { Atom } \\
\text { Density }\end{array}$ & \\
\hline $\mathrm{H}$ & 1001 & 1000 & 0.035500 & $\overline{0.386404}$ & 0.03786 & \\
\hline C & 6000 & 6000 & 0.367300 & 0.335506 & 0.03287 & \\
\hline $\mathrm{N}$ & 7014 & 7000 & 0.039700 & 0.031096 & 0.00304 & \\
\hline O & 8016 & 8000 & 0.045300 & 0.031063 & 0.00304 & \\
\hline$F$ & 9019 & 9000 & 0.249300 & 0.143964 & 0.01410 & \\
\hline $\mathrm{Ca}$ & 20000 & 20000 & 0.262900 & 0.071967 & 0.00705 & \\
\hline Total & & & 1.000000 & 1.000000 & 0.09798 & \\
\hline MCNP Form & \multicolumn{2}{|c|}{ Weight Fractions } & \multicolumn{2}{|c|}{ Atom Fractions } & \multicolumn{2}{|c|}{ Atom Densities } \\
\hline \multirow[t]{5}{*}{ Neutrons } & 1001 & -0.035500 & 1001 & 0.386404 & 1001 & 0.037860 \\
\hline & 6000 & -0.367300 & 6000 & 0.335506 & 6000 & 0.032873 \\
\hline & 7014 & -0.039700 & 7014 & 0.031096 & 7014 & 0.003047 \\
\hline & 8016 & -0.045300 & 8016 & 0.031063 & 8016 & 0.003044 \\
\hline & 9019 & -0.249300 & 9019 & 0.143964 & 9019 & 0.014106 \\
\hline
\end{tabular}


PIET-43741-TM-963

PNNL-15870 Rev. 1

\begin{tabular}{|c|c|c|c|c|c|c|}
\hline & 20000 & -0.262900 & 20000 & 0.071967 & 20000 & 0.007051 \\
\hline \multirow[t]{6}{*}{ Photons } & 1000 & -0.035500 & 1000 & 0.386404 & 1000 & 0.037860 \\
\hline & 6000 & -0.367300 & 6000 & 0.335506 & 6000 & 0.032873 \\
\hline & 7000 & -0.039700 & 7000 & 0.031096 & 7000 & 0.003047 \\
\hline & 8000 & -0.045300 & 8000 & 0.031063 & 8000 & 0.003044 \\
\hline & 9000 & -0.249300 & 9000 & 0.143964 & 9000 & 0.014106 \\
\hline & 20000 & -0.262900 & 20000 & 0.071967 & 20000 & 0.007051 \\
\hline \multirow[t]{7}{*}{ CEPXS Form: } & material & $\mathrm{H}$ & 0.035500 & & & \\
\hline & & C & 0.367300 & & & \\
\hline & & $\mathrm{N}$ & 0.039700 & & & \\
\hline & & $\mathrm{O}$ & 0.045300 & & & \\
\hline & & $\mathrm{F}$ & 0.249300 & & & \\
\hline & & $\mathrm{Ca}$ & 0.262900 & & & \\
\hline & $\begin{array}{l}\text { matname } \\
\text { density }\end{array}$ & \multicolumn{5}{|c|}{$\begin{array}{l}\text { Bone Equivalent Plastic, B-110 } \\
1.785000\end{array}$} \\
\hline \multicolumn{7}{|c|}{$\begin{array}{l}\text { Comments and References } \\
\text { Density and weight fractions in Spokas and White (1982) at http://www.iop.org/EJ/article/0031- } \\
\text { 9155/27/1/012/pbv27i1p115.pdf. }\end{array}$} \\
\hline
\end{tabular}

\section{Bone, Compact (ICRU)}

\begin{tabular}{llll}
\hline Formula $=$ & - & Molecular weight $(\mathrm{g} / \mathrm{mole})=$ & - \\
Density $(\mathrm{g} / \mathrm{cm} 3)=$ & 1.850000 & Total atom density $($ atoms $/ \mathrm{b}-\mathrm{cm})=$ & $1.340 \mathrm{E}-01$
\end{tabular}

The above density is estimated to be accurate to 3 significant digits. Uncertainties are not addressed.

The following data were calculated from the input weight fractions.

\begin{tabular}{|c|c|c|c|c|c|c|}
\hline Element & Neutron ZA & Photon ZA & $\begin{array}{l}\text { Weight } \\
\text { Fraction }\end{array}$ & $\begin{array}{c}\text { Atom } \\
\text { Fraction }\end{array}$ & $\begin{array}{l}\text { Atom } \\
\text { Density }\end{array}$ & \\
\hline $\mathrm{H}$ & 1001 & 1000 & $\overline{0.063984}$ & $\overline{0.527886}$ & 0.070723 & \\
\hline C & 6000 & 6000 & 0.278000 & 0.192478 & 0.025787 & \\
\hline $\mathrm{N}$ & 7014 & 7000 & 0.027000 & 0.016030 & 0.002148 & \\
\hline $\mathrm{O}$ & 8016 & 8000 & 0.410016 & 0.213109 & 0.028551 & \\
\hline $\mathrm{Mg}$ & 12000 & 12000 & 0.002000 & 0.000684 & 0.000092 & \\
\hline $\mathrm{P}$ & 15031 & 15000 & 0.070000 & 0.018794 & 0.002518 & \\
\hline$S$ & 16000 & 16000 & 0.002000 & 0.000519 & 0.000069 & \\
\hline $\mathrm{Ca}$ & 20000 & 20000 & 0.147000 & 0.030501 & 0.004086 & \\
\hline Total & & & 1.000000 & 1.000000 & 0.133974 & \\
\hline MCNP Form & \multicolumn{2}{|c|}{ Weight Fractions } & \multicolumn{2}{|c|}{ Atom Fractions } & \multicolumn{2}{|c|}{ Atom Densities } \\
\hline \multirow[t]{5}{*}{ Neutrons } & 1001 & -0.063984 & 1001 & 0.527886 & 1001 & 0.070723 \\
\hline & 6000 & -0.278000 & 6000 & 0.192478 & 6000 & 0.025787 \\
\hline & 7014 & -0.027000 & 7014 & 0.016030 & 7014 & 0.002148 \\
\hline & 8016 & -0.410016 & 8016 & 0.213109 & 8016 & 0.028551 \\
\hline & 12000 & -0.002000 & 12000 & 0.000684 & 12000 & 0.000092 \\
\hline
\end{tabular}


PIET-43741-TM-963

PNNL-15870 Rev. 1

\begin{tabular}{|c|c|c|c|c|c|c|}
\hline & 15031 & -0.070000 & 15031 & 0.018794 & 15031 & 0.002518 \\
\hline & 16000 & -0.002000 & 16000 & 0.000519 & 16000 & 0.000069 \\
\hline & 20000 & -0.147000 & 20000 & 0.030501 & 20000 & 0.004086 \\
\hline Photons & 1000 & -0.063984 & 1000 & 0.527886 & 1000 & 0.070723 \\
\hline & 6000 & -0.278000 & 6000 & 0.192478 & 6000 & 0.025787 \\
\hline & 7000 & -0.027000 & 7000 & 0.016030 & 7000 & 0.002148 \\
\hline & 8000 & -0.410016 & 8000 & 0.213109 & 8000 & 0.028551 \\
\hline & 12000 & -0.002000 & 12000 & 0.000684 & 12000 & 0.000092 \\
\hline & 15000 & -0.070000 & 15000 & 0.018794 & 15000 & 0.002518 \\
\hline & 16000 & -0.002000 & 16000 & 0.000519 & 16000 & 0.000069 \\
\hline & 20000 & -0.147000 & 20000 & 0.030501 & 20000 & 0.004086 \\
\hline CEPXS Form: & material & $\mathrm{H}$ & 0.063984 & & & \\
\hline & & C & 0.278000 & & & \\
\hline & & $\mathrm{N}$ & 0.027000 & & & \\
\hline & & $\mathrm{O}$ & 0.410016 & & & \\
\hline & & $\mathrm{Mg}$ & 0.002000 & & & \\
\hline & & $\mathrm{P}$ & 0.070000 & & & \\
\hline & & $S$ & 0.002000 & & & \\
\hline & & $\mathrm{Ca}$ & 0.147000 & & & \\
\hline & $\begin{array}{c}\text { matname } \\
\text { density }\end{array}$ & $\begin{array}{c}\text { Bone, Comp } \\
1.850000\end{array}$ & (ICRU) & & & \\
\hline
\end{tabular}

\section{Bone, Cortical (ICRP)}

\begin{tabular}{|c|c|c|c|}
\hline & - & Molecular weight $(\mathrm{g} / \mathrm{mole})=$ & \\
\hline Density $(\mathrm{g} / \mathrm{cm} 3)=$ & 1.850000 & Total atom density $($ atoms $/ \mathrm{b}-\mathrm{cm})=$ & 1.098E-01 \\
\hline
\end{tabular}

The above density is estimated to be accurate to 3 significant digits. Uncertainties are not addressed.

The following data were calculated from the input weight fractions.

\begin{tabular}{|c|c|c|c|c|c|}
\hline Element & Neutron ZA & Photon ZA & $\begin{array}{l}\text { Weight } \\
\text { Fraction }\end{array}$ & $\begin{array}{c}\text { Atom } \\
\text { Fraction }\end{array}$ & $\begin{array}{c}\text { Atom } \\
\text { Density }\end{array}$ \\
\hline $\mathrm{H}$ & 1001 & 1000 & 0.047234 & 0.475389 & 0.052209 \\
\hline C & 6000 & 6000 & 0.144330 & 0.121904 & 0.013388 \\
\hline$N$ & 7014 & 7000 & 0.041990 & 0.030412 & 0.003340 \\
\hline 0 & 8016 & 8000 & 0.446096 & 0.282848 & 0.031063 \\
\hline $\mathrm{Mg}$ & 12000 & 12000 & 0.002200 & 0.000918 & 0.000101 \\
\hline $\mathrm{P}$ & 15031 & 15000 & 0.104970 & 0.034380 & 0.003776 \\
\hline$S$ & 16000 & 16000 & 0.003150 & 0.000997 & 0.000109 \\
\hline $\mathrm{Ca}$ & 20000 & 20000 & 0.209930 & 0.053137 & 0.005836 \\
\hline $\mathrm{Zn}$ & 30000 & 30000 & 0.000100 & 0.000016 & 0.000002 \\
\hline Total & & & 1.000000 & 1.000000 & 0.109823 \\
\hline
\end{tabular}


PIET-43741-TM-963

PNNL-15870 Rev. 1

\begin{tabular}{|c|c|c|c|c|c|c|}
\hline MCNP Form & \multicolumn{2}{|c|}{ Weight Fractions } & \multicolumn{2}{|c|}{ Atom Fractions } & \multicolumn{2}{|c|}{ Atom Densities } \\
\hline \multirow[t]{9}{*}{ Neutrons } & 1001 & -0.047234 & 1001 & 0.475389 & 1001 & 0.052209 \\
\hline & 6000 & -0.144330 & 6000 & 0.121904 & 6000 & 0.013388 \\
\hline & 7014 & -0.041990 & 7014 & 0.030412 & 7014 & 0.003340 \\
\hline & 8016 & -0.446096 & 8016 & 0.282848 & 8016 & 0.031063 \\
\hline & 12000 & -0.002200 & 12000 & 0.000918 & 12000 & 0.000101 \\
\hline & 15031 & -0.104970 & 15031 & 0.034380 & 15031 & 0.003776 \\
\hline & 16000 & -0.003150 & 16000 & 0.000997 & 16000 & 0.000109 \\
\hline & 20000 & -0.209930 & 20000 & 0.053137 & 20000 & 0.005836 \\
\hline & 30000 & -0.000100 & 30000 & 0.000016 & 30000 & 0.000002 \\
\hline \multirow[t]{9}{*}{ Photons } & 1000 & -0.047234 & 1000 & 0.475389 & 1000 & 0.052209 \\
\hline & 6000 & -0.144330 & 6000 & 0.121904 & 6000 & 0.013388 \\
\hline & 7000 & -0.041990 & 7000 & 0.030412 & 7000 & 0.003340 \\
\hline & 8000 & -0.446096 & 8000 & 0.282848 & 8000 & 0.031063 \\
\hline & 12000 & -0.002200 & 12000 & 0.000918 & 12000 & 0.000101 \\
\hline & 15000 & -0.104970 & 15000 & 0.034380 & 15000 & 0.003776 \\
\hline & 16000 & -0.003150 & 16000 & 0.000997 & 16000 & 0.000109 \\
\hline & 20000 & -0.209930 & 20000 & 0.053137 & 20000 & 0.005836 \\
\hline & 30000 & -0.000100 & 30000 & 0.000016 & 30000 & 0.000002 \\
\hline \multirow[t]{10}{*}{ CEPXS Form: } & material & $\mathrm{H}$ & 0.047234 & & & \\
\hline & & C & 0.144330 & & & \\
\hline & & $\mathrm{N}$ & 0.041990 & & & \\
\hline & & 0 & 0.446096 & & & \\
\hline & & $\mathrm{Mg}$ & 0.002200 & & & \\
\hline & & $P$ & 0.104970 & & & \\
\hline & & $s$ & 0.003150 & & & \\
\hline & & $\mathrm{Ca}$ & 0.209930 & & & \\
\hline & & $\mathrm{Zn}$ & 0.000100 & & & \\
\hline & $\begin{array}{c}\text { matname } \\
\text { density }\end{array}$ & \multicolumn{2}{|c|}{ Bone, Cortical (ICRP) } & & & \\
\hline
\end{tabular}

\section{Boral (65\% Al-35\% B4C)}

\begin{tabular}{llll}
\hline Formula $=$ & - & Molecular weight $(\mathrm{g} / \mathrm{mole})=$ \\
Density $(\mathrm{g} / \mathrm{cm} 3)=$ & 2.530000 & Total atom density $($ atoms $/ \mathrm{b}-\mathrm{cm})=$ & - \\
\hline & & $3.496 \mathrm{E}-02$
\end{tabular}

The above density is estimated to be accurate to 3 significant digits. Uncertainties are not addressed.

The following data were calculated from the input weight fractions.

\begin{tabular}{|c|c|c|c|c|c|}
\hline ment & Neutron ZA & Photon ZA & $\begin{array}{l}\text { Weight } \\
\text { Fraction }\end{array}$ & $\begin{array}{c}\text { Atom } \\
\text { Fraction }\end{array}$ & $\begin{array}{l}\text { Atom } \\
\text { Density }\end{array}$ \\
\hline B & - & 5000 & 0.274000 & 0.454507 & 0.038615 \\
\hline C & 6000 & 6000 & 0.076000 & 0.113475 & 0.009641 \\
\hline
\end{tabular}


PIET-43741-TM-963

PNNL-15870 Rev. 1

\begin{tabular}{|c|c|c|c|c|c|c|}
\hline $\mathrm{Al}$ & 13027 & 13000 & 0.650000 & 0.432018 & \multicolumn{2}{|c|}{0.036704} \\
\hline Total & & & 1.000000 & 1.000000 & \multicolumn{2}{|c|}{0.084960} \\
\hline MCNP Form & \multicolumn{2}{|c|}{ Weight Fractions } & \multicolumn{2}{|c|}{ Atom Fractions } & \multicolumn{2}{|c|}{ Atom Densities } \\
\hline \multirow[t]{3}{*}{ Neutrons } & - & -0.274000 & - & 0.454507 & - & 0.038615 \\
\hline & 6000 & -0.076000 & 6000 & 0.113475 & 6000 & 0.009641 \\
\hline & 13027 & -0.650000 & 13027 & 0.432018 & 13027 & 0.036704 \\
\hline \multirow[t]{3}{*}{ Photons } & 5000 & -0.274000 & 5000 & 0.454507 & 5000 & 0.038615 \\
\hline & 6000 & -0.076000 & 6000 & 0.113475 & 6000 & 0.009641 \\
\hline & 13000 & -0.650000 & 13000 & 0.432018 & 13000 & 0.036704 \\
\hline \multirow[t]{4}{*}{ CEPXS Form: } & material & B & 0.274000 & & & \\
\hline & & C & 0.076000 & & & \\
\hline & & $\mathrm{Al}$ & 0.650000 & & & \\
\hline & $\begin{array}{c}\text { matname } \\
\text { density }\end{array}$ & \multicolumn{2}{|c|}{$\begin{array}{l}\text { Boral (65\% Al-35\% B4C) } \\
2.530000\end{array}$} & & & \\
\hline \multicolumn{7}{|c|}{$\begin{array}{l}\text { Comments and References } \\
\text { Without aluminum clad. Density and weight fractions from Brewer (2009). This data evidently came from } \\
\text { pg II.F.1-1 of Carter et al. (1968). }\end{array}$} \\
\hline
\end{tabular}

\section{Boral (Aluminum 10\% Boron Alloy)}

\begin{tabular}{llll}
\hline Formula $=$ & - & Molecular weight $(\mathrm{g} / \mathrm{mole})=$ \\
Density $(\mathrm{g} / \mathrm{cm} 3)=$ & 2.600000 & Total atom density $($ atoms $/ \mathrm{b}-\mathrm{cm})=$ & - \\
The.647E-02
\end{tabular}

The above density is estimated to be accurate to 2 significant digits. Uncertainties are not addressed.

The following data were calculated from the input weight fractions.

\begin{tabular}{|c|c|c|c|c|c|c|}
\hline Element & Neutron ZA & Photon ZA & $\begin{array}{l}\text { Weight } \\
\text { Fraction }\end{array}$ & $\begin{array}{c}\text { Atom } \\
\text { Fraction }\end{array}$ & $\begin{array}{c}\text { Atom } \\
\text { Density }\end{array}$ & \\
\hline$B$ & - & 5000 & $\overline{0.100000}$ & 0.217879 & 0.014483 & \\
\hline $\mathrm{Na}$ & 11023 & 11000 & 0.005000 & 0.005123 & 0.000341 & \\
\hline $\mathrm{Al}$ & 13027 & 13000 & 0.879000 & 0.767366 & 0.051009 & \\
\hline Si & 14000 & 14000 & 0.002500 & 0.002097 & 0.000139 & \\
\hline $\mathrm{K}$ & 19000 & 19000 & 0.010000 & 0.006025 & 0.000400 & \\
\hline $\mathrm{Ti}$ & 22000 & 22000 & 0.000500 & 0.000246 & 0.000016 & \\
\hline $\mathrm{Fe}$ & 26000 & 26000 & 0.003000 & 0.001265 & 0.000084 & \\
\hline Total & & & 1.000000 & 1.000000 & 0.066473 & \\
\hline MCNP Form & \multicolumn{2}{|c|}{ Weight Fractions } & \multicolumn{2}{|c|}{ Atom Fractions } & \multicolumn{2}{|c|}{ Atom Densities } \\
\hline \multirow[t]{4}{*}{ Neutrons } & - & -0.100000 & - & 0.217879 & - & 0.014483 \\
\hline & 11023 & -0.005000 & 11023 & 0.005123 & 11023 & 0.000341 \\
\hline & 13027 & -0.879000 & 13027 & 0.767366 & 13027 & 0.051009 \\
\hline & 14000 & -0.002500 & 14000 & 0.002097 & 14000 & 0.000139 \\
\hline
\end{tabular}


PIET-43741-TM-963

PNNL-15870 Rev. 1

\begin{tabular}{|c|c|c|c|c|c|c|}
\hline & 19000 & -0.010000 & 19000 & 0.006025 & 19000 & 0.000400 \\
\hline & 22000 & -0.000500 & 22000 & 0.000246 & 22000 & 0.000016 \\
\hline & 26000 & -0.003000 & 26000 & 0.001265 & 26000 & 0.000084 \\
\hline Photons & 5000 & -0.100000 & 5000 & 0.217879 & 5000 & 0.014483 \\
\hline & 11000 & -0.005000 & 11000 & 0.005123 & 11000 & 0.000341 \\
\hline & 13000 & -0.879000 & 13000 & 0.767366 & 13000 & 0.051009 \\
\hline & 14000 & -0.002500 & 14000 & 0.002097 & 14000 & 0.000139 \\
\hline & 19000 & -0.010000 & 19000 & 0.006025 & 19000 & 0.000400 \\
\hline & 22000 & -0.000500 & 22000 & 0.000246 & 22000 & 0.000016 \\
\hline & 26000 & -0.003000 & 26000 & 0.001265 & 26000 & 0.000084 \\
\hline CEPXS Form: & material & $B$ & 0.100000 & & & \\
\hline & & $\mathrm{Na}$ & 0.005000 & & & \\
\hline & & $\mathrm{Al}$ & 0.879000 & & & \\
\hline & & $\mathrm{Si}$ & 0.002500 & & & \\
\hline & & $\mathrm{K}$ & 0.010000 & & & \\
\hline & & $\mathrm{Ti}$ & 0.000500 & & & \\
\hline & & $\mathrm{Fe}$ & 0.003000 & & & \\
\hline & $\begin{array}{c}\text { matname } \\
\text { density }\end{array}$ & $\begin{array}{c}\text { Boral (Alumi } \\
2.600000\end{array}$ & $10 \%$ Boro & loy) & & \\
\hline $\begin{array}{l}\text { Comments anc } \\
\text { The compositior } \\
\text { http://www.matv } \\
\text { (Automation Cre } \\
\text { A reference for }\end{array}$ & $\begin{array}{l}\text { References } \\
\text { s for } 10.0 \text { u } \\
\text { b.com/sear } \\
\text { tions 2010) } \\
\text { e density cc }\end{array}$ & $\begin{array}{l}\text { b boron in an } \\
\text { /DataSheet.as } \\
\text { The boron con } \\
\text { d not be foun }\end{array}$ & $\begin{array}{l}\text { ninum-boro } \\
\text { MatGUID= } \\
\text { ts at MatWe } \\
2.6 \mathrm{~g} / \mathrm{cm} 3\end{array}$ & $\begin{array}{l}\text { oy from KB } \\
68 \mathrm{e} 906 \mathrm{fb} 74 \\
\text { clude } 3 \%, \\
\text { s assumed. }\end{array}$ & $\begin{array}{l}\text { listed a } \\
21 \mathrm{fdebac} \\
\%, 8 \%, \mathrm{a}\end{array}$ & $\begin{array}{l}94 d \\
\% .\end{array}$ \\
\hline
\end{tabular}

\section{Boral (Aluminum 5\% Boron Alloy)}

Formula $=$

Density $(\mathrm{g} / \mathrm{cm} 3)=2.600000$
Molecular weight $(\mathrm{g} / \mathrm{mole})=$

Total atom density $($ atoms $/ \mathrm{b}-\mathrm{cm})=6.213 \mathrm{E}-02$

The above density is estimated to be accurate to 2 significant digits. Uncertainties are not addressed.

The following data were calculated from the input weight fractions.

\begin{tabular}{cccccc} 
Element & Neutron ZA & Photon ZA & $\begin{array}{c}\text { Weight } \\
\text { Fraction }\end{array}$ & $\begin{array}{c}\text { Atom } \\
\text { Fraction }\end{array}$ & $\begin{array}{c}\text { Atom } \\
\text { Density }\end{array}$ \\
\cline { 5 - 7 } $\mathrm{B}$ & - & 5000 & 0.050000 & 0.116547 & 0.007241 \\
$\mathrm{Na}$ & 11023 & 11000 & 0.005000 & 0.005481 & 0.000341 \\
$\mathrm{Al}$ & 13027 & 13000 & 0.929500 & 0.868116 & 0.053940 \\
$\mathrm{Si}$ & 14000 & 14000 & 0.002000 & 0.001794 & 0.000111 \\
$\mathrm{~K}$ & 19000 & 19000 & 0.010000 & 0.006445 & 0.000400 \\
$\mathrm{Ti}$ & 22000 & 22000 & 0.000500 & 0.000263 & 0.000016 \\
$\mathrm{Fe}$ & 26000 & 26000 & 0.003000 & 0.001354 & 0.000084 \\
Total & & & & & \\
& & & 1.000000 & 1.000000 & 0.062134
\end{tabular}


PIET-43741-TM-963

PNNL-15870 Rev. 1

\begin{tabular}{|c|c|c|c|c|c|c|}
\hline MCNP Form & \multicolumn{2}{|c|}{ Weight Fractions } & \multicolumn{2}{|c|}{ Atom Fractions } & \multicolumn{2}{|c|}{ Atom Densities } \\
\hline \multirow[t]{7}{*}{ Neutrons } & - & -0.050000 & - & 0.116547 & - & 0.007241 \\
\hline & 11023 & -0.005000 & 11023 & 0.005481 & 11023 & 0.000341 \\
\hline & 13027 & -0.929500 & 13027 & 0.868116 & 13027 & 0.053940 \\
\hline & 14000 & -0.002000 & 14000 & 0.001794 & 14000 & 0.000111 \\
\hline & 19000 & -0.010000 & 19000 & 0.006445 & 19000 & 0.000400 \\
\hline & 22000 & -0.000500 & 22000 & 0.000263 & 22000 & 0.000016 \\
\hline & 26000 & -0.003000 & 26000 & 0.001354 & 26000 & 0.000084 \\
\hline \multirow[t]{7}{*}{ Photons } & 5000 & -0.050000 & 5000 & 0.116547 & 5000 & 0.007241 \\
\hline & 11000 & -0.005000 & 11000 & 0.005481 & 11000 & 0.000341 \\
\hline & 13000 & -0.929500 & 13000 & 0.868116 & 13000 & 0.053940 \\
\hline & 14000 & -0.002000 & 14000 & 0.001794 & 14000 & 0.000111 \\
\hline & 19000 & -0.010000 & 19000 & 0.006445 & 19000 & 0.000400 \\
\hline & 22000 & -0.000500 & 22000 & 0.000263 & 22000 & 0.000016 \\
\hline & 26000 & -0.003000 & 26000 & 0.001354 & 26000 & 0.000084 \\
\hline \multirow[t]{8}{*}{ CEPXS Form: } & material & $B$ & 0.050000 & & & \\
\hline & & $\mathrm{Na}$ & 0.005000 & & & \\
\hline & & $\mathrm{Al}$ & 0.929500 & & & \\
\hline & & $\mathrm{Si}$ & 0.002000 & & & \\
\hline & & $\mathrm{K}$ & 0.010000 & & & \\
\hline & & $\mathrm{Ti}$ & 0.000500 & & & \\
\hline & & $\mathrm{Fe}$ & 0.003000 & & & \\
\hline & $\begin{array}{c}\text { matname } \\
\text { density }\end{array}$ & $\begin{array}{c}\text { Boral (Alumi } \\
2.600000\end{array}$ & n $5 \%$ Boror & & & \\
\hline \multicolumn{7}{|c|}{$\begin{array}{l}\text { Comments and References } \\
\text { The composition is for } 5.0 \mathrm{wt} \% \text { boron in an aluminum-boron alloy from KB alloys listed at } \\
\text { http://www.matweb.com/search/DataSheet.aspx?MatGUID }=2 \mathrm{~d} 8 \mathrm{cc} 1 \mathrm{~b} 6 \mathrm{af} 7 \mathrm{f} 4747 \mathrm{aec} 9 \mathrm{dfdd} 65 \mathrm{~d} 4 \mathrm{f} 97 \mathrm{a} \\
\text { (Automation Creations } 2010 \text { ). The boron contents at MatWeb include } 3 \%, 4 \%, 5 \%, 8 \% \text {, and } 10 \% \text {. } \\
\text { A reference for the density could not be found so } 2.6 \mathrm{~g} / \mathrm{cm} 3 \text { was assumed. }\end{array}$} \\
\hline
\end{tabular}

\section{Borax}

Formula $=$ NA2B4O7-10(H2O) Molecular weight $(\mathrm{g} / \mathrm{mole})=$

Density $(\mathrm{g} / \mathrm{cm} 3)=1.730000 \quad$ Total atom density $($ atoms $/ \mathrm{b}-\mathrm{cm})=1.175 \mathrm{E}-01$ 381.37214 The above density is estimated to be accurate to 3 significant digits. Uncertainties are not addressed. The following data was calculated from the input formula.

\begin{tabular}{|c|c|c|c|c|c|}
\hline Element & Neutron ZA & Photon ZA & $\begin{array}{l}\text { Weight } \\
\text { Fraction }\end{array}$ & $\begin{array}{c}\text { Atom } \\
\text { Fraction }\end{array}$ & $\begin{array}{l}\text { Atom } \\
\text { Density }\end{array}$ \\
\hline $\mathrm{H}$ & 1001 & 1000 & $\overline{0.052859}$ & $\overline{0.465116}$ & 0.054636 \\
\hline$B$ & - & 5000 & 0.113391 & 0.093023 & 0.010927 \\
\hline 0 & 8016 & 8000 & 0.713187 & 0.395349 & 0.046441 \\
\hline $\mathrm{Na}$ & 11023 & 11000 & 0.120563 & 0.046512 & 0.005464 \\
\hline Total & & & 1.000000 & 1.000000 & 0.117467 \\
\hline
\end{tabular}


PIET-43741-TM-963

PNNL-15870 Rev. 1

\begin{tabular}{|c|c|c|c|c|c|c|}
\hline MCNP Form & \multicolumn{2}{|c|}{ Weight Fractions } & \multicolumn{2}{|c|}{ Atom Fractions } & \multicolumn{2}{|c|}{ Atom Densities } \\
\hline \multirow{4}{*}{ Neutrons } & 1001 & -0.052859 & 1001 & 0.465116 & 1001 & 0.054636 \\
\hline & - & -0.113391 & - & 0.093023 & - & 0.010927 \\
\hline & 8016 & -0.713187 & 8016 & 0.395349 & 8016 & 0.046441 \\
\hline & 11023 & -0.120563 & 11023 & 0.046512 & 11023 & 0.005464 \\
\hline \multirow[t]{4}{*}{ Photons } & 1000 & -0.052859 & 1000 & 0.465116 & 1000 & 0.054636 \\
\hline & 5000 & -0.113391 & 5000 & 0.093023 & 5000 & 0.010927 \\
\hline & 8000 & -0.713187 & 8000 & 0.395349 & 8000 & 0.046441 \\
\hline & 11000 & -0.120563 & 11000 & 0.046512 & 11000 & 0.005464 \\
\hline \multirow[t]{5}{*}{ CEPXS Form: } & material & $\mathrm{H}$ & 0.052859 & & & \\
\hline & & B & 0.113391 & & & \\
\hline & & O & 0.713187 & & & \\
\hline & & $\mathrm{Na}$ & 0.120563 & & & \\
\hline & $\begin{array}{c}\text { matname } \\
\text { density }\end{array}$ & $\begin{array}{l}\text { Borax } \\
1.730000\end{array}$ & & & & \\
\hline \multicolumn{7}{|c|}{$\begin{array}{l}\text { Comments and References } \\
\text { Density and formula from Lide (2008), pgs } 4 \text { - 91, for sodium tetraborate decahydrate. Also listed in } \\
\text { Brewer (2009), pg II.F.1-1 of Carter et al. (1968), and Automation Creations (2010). }\end{array}$} \\
\hline
\end{tabular}

\section{Boric Acid}

\begin{tabular}{llll}
\hline Formula $=$ & $\mathrm{H} 3 \mathrm{BO} 3$ & Molecular weight $(\mathrm{g} / \mathrm{mole})=$ & 61.83302 \\
Density $(\mathrm{g} / \mathrm{cm} 3)=$ & 1.500000 & Total atom density $($ atoms $/ \mathrm{b}-\mathrm{cm})=$ & $1.023 \mathrm{E}-01$
\end{tabular}

The above density is estimated to be accurate to 3 significant digits. Uncertainties are not addressed.

The following data was calculated from the input formula.

\begin{tabular}{|c|c|c|c|c|c|c|}
\hline Element & Neutron ZA & Photon ZA & $\begin{array}{l}\text { Weight } \\
\text { Fraction }\end{array}$ & $\begin{array}{c}\text { Atom } \\
\text { Fraction }\end{array}$ & $\begin{array}{l}\text { Atom } \\
\text { Density }\end{array}$ & \\
\hline $\mathrm{H}$ & 1001 & 1000 & 0.048903 & 0.428571 & 0.043827 & \\
\hline$B$ & - & 5000 & 0.174842 & 0.142857 & 0.014609 & \\
\hline $\mathrm{O}$ & 8016 & 8000 & 0.776255 & 0.428571 & 0.043827 & \\
\hline Total & & & 1.000000 & 1.000000 & 0.102263 & \\
\hline MCNP Form & \multicolumn{2}{|c|}{ Weight Fractions } & \multicolumn{2}{|c|}{ Atom Fractions } & \multicolumn{2}{|c|}{ Atom Densities } \\
\hline \multirow[t]{3}{*}{ Neutrons } & 1001 & -0.048903 & 1001 & 0.428571 & 1001 & 0.043827 \\
\hline & - & -0.174842 & - & 0.142857 & - & 0.014609 \\
\hline & 8016 & -0.776255 & 8016 & 0.428571 & 8016 & 0.043827 \\
\hline \multirow[t]{3}{*}{ Photons } & 1000 & -0.048903 & 1000 & 0.428571 & 1000 & 0.043827 \\
\hline & 5000 & -0.174842 & 5000 & 0.142857 & 5000 & 0.014609 \\
\hline & 8000 & -0.776255 & 8000 & 0.428571 & 8000 & 0.043827 \\
\hline
\end{tabular}




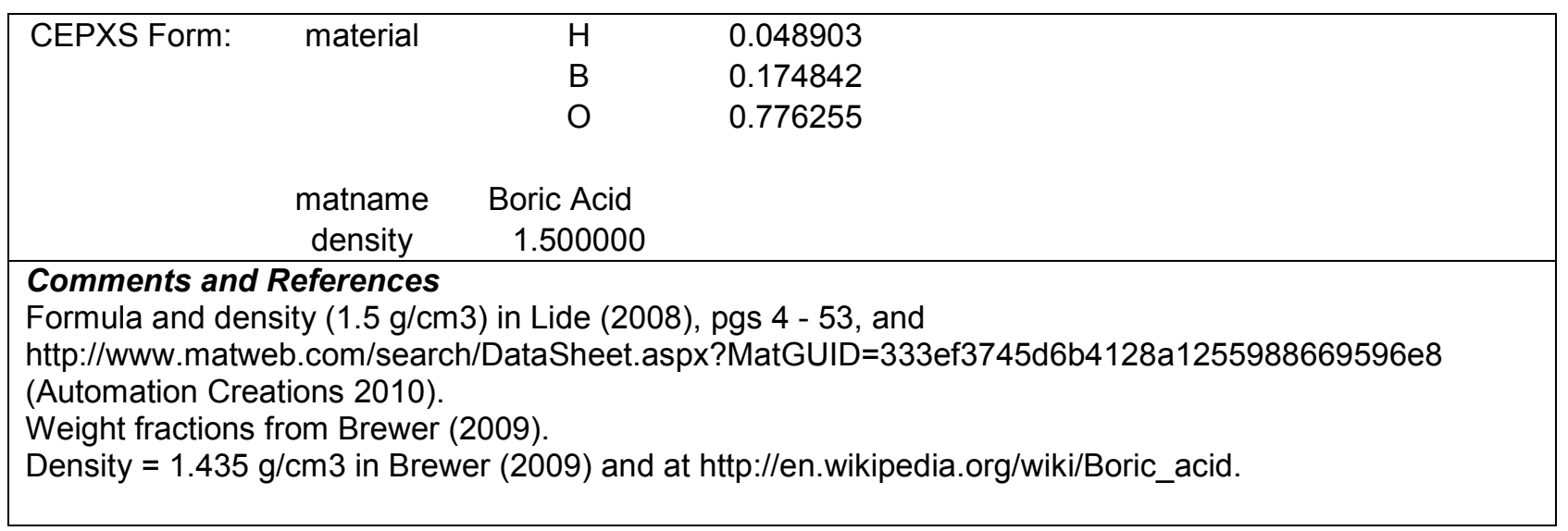

\begin{tabular}{|c|c|c|c|c|c|c|}
\hline \multicolumn{7}{|l|}{39 Boron } \\
\hline \multicolumn{7}{|c|}{$\begin{array}{l}\text { The above density is estimated to be accurate to } 3 \text { significant digits. Uncertainties are not addressed. } \\
\text { The following data was calculated from the input formula. }\end{array}$} \\
\hline$\frac{\text { Element }}{\mathrm{B}}$ & $\frac{\text { Neutron ZA }}{-}$ & $\frac{\text { Photon ZA }}{5000}$ & $\begin{array}{l}\text { Weight } \\
\frac{\text { Fraction }}{1.000000}\end{array}$ & $\begin{array}{c}\text { Atom } \\
\frac{\text { Fraction }}{1.000000}\end{array}$ & $\begin{array}{c}\text { Atom } \\
\text { Density } \\
0.13201\end{array}$ & \\
\hline Total & & & 1.000000 & 1.000000 & 0.13201 & \\
\hline MCNP Form & \multicolumn{2}{|c|}{ Weight Fractions } & \multicolumn{2}{|c|}{ Atom Fractions } & \multicolumn{2}{|c|}{ Atom Densities } \\
\hline Neutrons & - & -1.000000 & - & 1.000000 & - & 0.132018 \\
\hline Photons & 5000 & -1.000000 & 5000 & 1.000000 & 5000 & 0.132018 \\
\hline CEPXS Form: & $\begin{array}{l}\text { material } \\
\text { matname } \\
\text { density }\end{array}$ & $\begin{array}{c}\text { B } \\
\text { Boron } \\
2.370000\end{array}$ & 1.000000 & & & \\
\hline $\begin{array}{l}\text { Comments ano } \\
\text { Density from htt }\end{array}$ & $\begin{array}{l}\text { References } \\
: / / \text { physics.nis }\end{array}$ & t. . . & 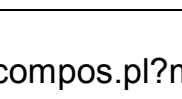 & -605e & 98). & \\
\hline
\end{tabular}

\section{Boron Carbide}

\begin{tabular}{llll}
\hline Formula $=$ & B4C & Molecular weight $(\mathrm{g} / \mathrm{mole})=$ & 55.2547 \\
Density $(\mathrm{g} / \mathrm{cm} 3)=$ & 2.520000 & Total atom density $($ atoms $/ \mathrm{b}-\mathrm{cm})=$ & $1.373 \mathrm{E}-01$
\end{tabular}

The above density is estimated to be accurate to 3 significant digits. Uncertainties are not addressed. The following data were calculated from the input weight fractions. 


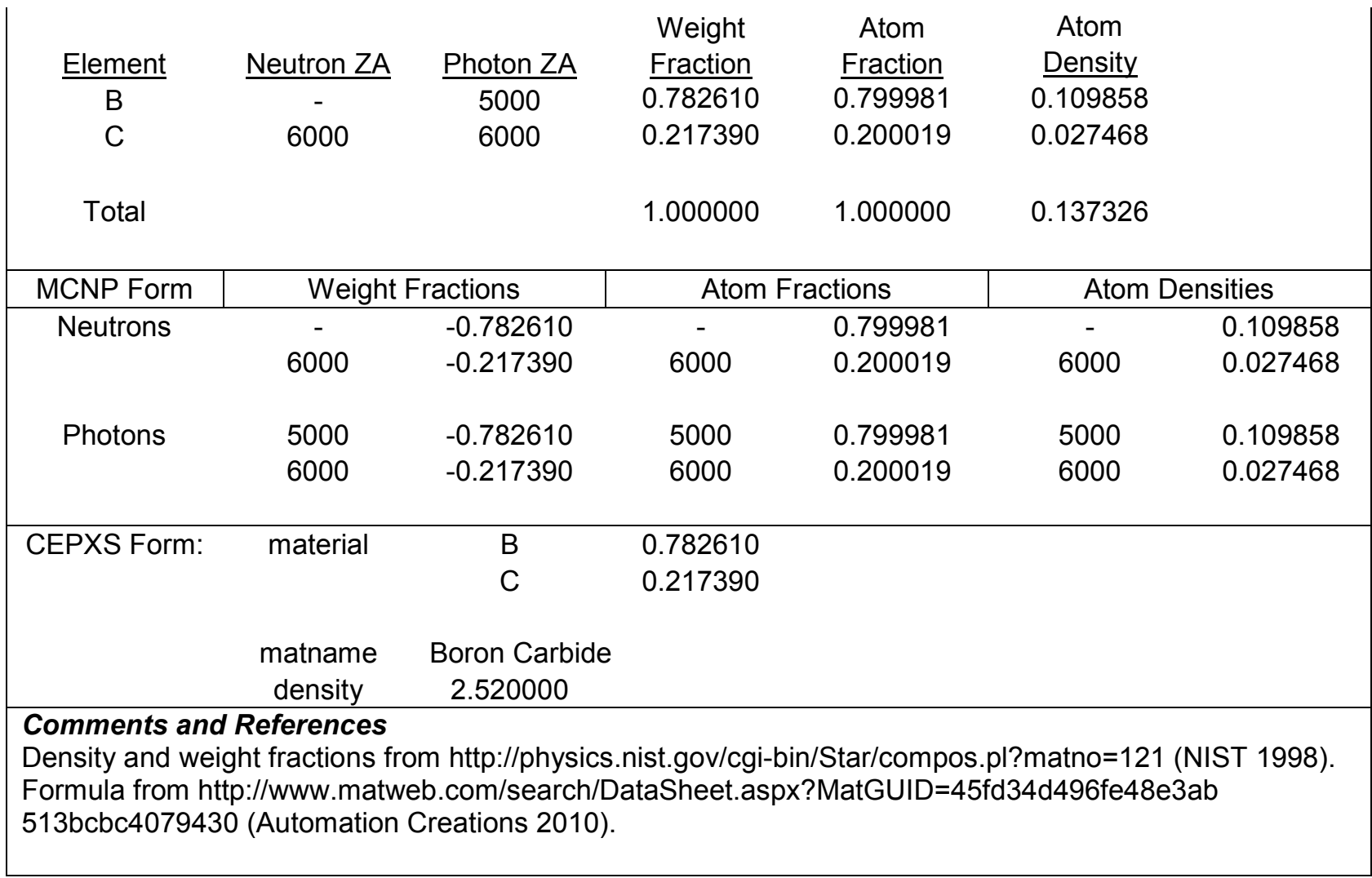

\section{Boron Fluoride (B2F4)}

\begin{tabular}{llll}
\hline Formula $=$ & B2F4 & Molecular weight $(\mathrm{g} / \mathrm{mole})=$ & 97.6156128 \\
Density $(\mathrm{g} / \mathrm{cm} 3)=$ & 0.004058 & Total atom density $($ atoms $/ \mathrm{b}-\mathrm{cm})=$ & $1.502 \mathrm{E}-04$
\end{tabular}

The above density is estimated to be accurate to 4 significant digits. Uncertainties are not addressed.

The following data was calculated from the input formula.

\begin{tabular}{|c|c|c|c|c|c|c|}
\hline $\begin{array}{c}\text { Element } \\
\mathrm{B} \\
\mathrm{F}\end{array}$ & $\begin{array}{c}\text { Neutron ZA } \\
- \\
9019\end{array}$ & $\begin{array}{c}\text { Photon ZA } \\
5000 \\
9000\end{array}$ & $\begin{array}{c}\text { Weight } \\
\text { Fraction } \\
0.221501 \\
0.778499\end{array}$ & $\begin{array}{c}\begin{array}{c}\text { Atom } \\
\text { Fraction }\end{array} \\
0.333333 \\
0.666667\end{array}$ & $\begin{array}{r}\begin{array}{c}\text { Atom } \\
\text { Density } \\
0.00005 \\
0.00010\end{array}\end{array}$ & \\
\hline Total & & & 1.000000 & 1.000000 & 0.00015 & \\
\hline MCNP Form & \multicolumn{2}{|c|}{ Weight Fractions } & \multicolumn{2}{|c|}{ Atom Fractions } & \multicolumn{2}{|c|}{ Atom Densities } \\
\hline Neutrons & $\begin{array}{c}- \\
9019\end{array}$ & $\begin{array}{l}-0.221501 \\
-0.778499\end{array}$ & $\begin{array}{c}- \\
9019\end{array}$ & $\begin{array}{l}0.333333 \\
0.666667\end{array}$ & $\begin{array}{c}- \\
9019\end{array}$ & $\begin{array}{l}0.000050 \\
0.000100\end{array}$ \\
\hline Photons & $\begin{array}{l}5000 \\
9000\end{array}$ & $\begin{array}{l}-0.221501 \\
-0.778499\end{array}$ & $\begin{array}{l}5000 \\
9000\end{array}$ & $\begin{array}{l}0.333333 \\
0.666667\end{array}$ & $\begin{array}{l}5000 \\
9000\end{array}$ & $\begin{array}{l}0.000050 \\
0.000100\end{array}$ \\
\hline CEPXS Form: & material & $\begin{array}{l}B \\
F\end{array}$ & $\begin{array}{l}0.221501 \\
0.778499\end{array}$ & & & \\
\hline
\end{tabular}


matname Boron Fluoride (B2F4)

density $\quad 0.004058$

Comments and References

The $0.004058 \mathrm{~g} / \mathrm{cm} 3$ density is calculated for $20^{\circ} \mathrm{C}$ and 1.0 atmosphere using the ideal gas law.

Density $=0.00399 \mathrm{~g} / \mathrm{cm} 3$ at

http://www.matweb.com/search/DataSheet.aspx?MatGUID=1505ad001ba3450db792e036eba3cc5d

(Automation Creations 2010) is evidently for $25^{\circ} \mathrm{C}$ and 1.0 atmosphere.

\section{Boron Fluoride (BF3)}

\begin{tabular}{|c|c|c|c|}
\hline Formula $=$ & BF3 & Molecular weight (g/mole) $=$ & 67.8062096 \\
\hline Density $(\mathrm{g} / \mathrm{cm} 3)=$ & 0.002831 & Total atom density $($ atoms $/ \mathrm{b}-\mathrm{cm})=$ & 1.006E-04 \\
\hline
\end{tabular}

\begin{tabular}{|c|c|c|c|c|c|c|}
\hline Element & Neutron ZA & Photon ZA & $\begin{array}{l}\text { Weight } \\
\text { Fraction }\end{array}$ & $\begin{array}{c}\text { Atom } \\
\text { Fraction }\end{array}$ & \multicolumn{2}{|l|}{$\begin{array}{l}\text { Atom } \\
\text { Density }\end{array}$} \\
\hline$B$ & - & 5000 & 0.159440 & 0.250000 & \multicolumn{2}{|l|}{0.000025} \\
\hline $\mathrm{F}$ & 9019 & 9000 & 0.840560 & 0.750000 & \multicolumn{2}{|l|}{0.000075} \\
\hline Total & & & 1.000000 & 1.000000 & \multicolumn{2}{|l|}{0.000101} \\
\hline MCNP Form & \multicolumn{2}{|c|}{ Weight Fractions } & \multicolumn{2}{|c|}{ Atom Fractions } & \multicolumn{2}{|c|}{ Atom Densities } \\
\hline Neutrons & $\begin{array}{c}- \\
9019\end{array}$ & $\begin{array}{l}-0.159440 \\
-0.840560\end{array}$ & $\begin{array}{c}- \\
9019\end{array}$ & $\begin{array}{l}0.250000 \\
0.750000\end{array}$ & $\begin{array}{c}- \\
9019\end{array}$ & $\begin{array}{l}0.000025 \\
0.000075\end{array}$ \\
\hline Photons & $\begin{array}{l}5000 \\
9000\end{array}$ & $\begin{array}{l}-0.159440 \\
-0.840560\end{array}$ & $\begin{array}{l}5000 \\
9000\end{array}$ & $\begin{array}{l}0.250000 \\
0.750000\end{array}$ & $\begin{array}{l}5000 \\
9000\end{array}$ & $\begin{array}{l}0.000025 \\
0.000075\end{array}$ \\
\hline CEPXS Form: & $\begin{array}{l}\text { matname } \\
\text { density }\end{array}$ & $\begin{array}{l}\mathrm{B} \\
\mathrm{F} \\
\text { Boron Fluoric } \\
0.002831\end{array}$ & $\begin{array}{l}0.159440 \\
0.840560 \\
\text { BF3) }\end{array}$ & $\begin{array}{l}\text { Boron Fluoride (BF3) } \\
0.002831\end{array}$ & & \\
\hline \multicolumn{7}{|c|}{$\begin{array}{l}\text { Comments and References } \\
\text { The density is calculated for } 20^{\circ} \mathrm{C} \text { and } 1.0 \text { atmosphere using a Van der Waals equation of state. } \\
\text { Density }=0.002771 \mathrm{~g} / \mathrm{cm} 3 \text { at } \\
\text { http://www.matweb.com } / \mathrm{search} / \text { DataSheet.aspx?MatGUID=d5db4876 db3f4107aa3340d0f3ceb633 } \\
\text { (Automation Creations } 2010 \text { ) is evidently for } 25^{\circ} \mathrm{C} \text { and } 1.0 \text { atmosphere. } \\
\text { Also called boron trifluoride. }\end{array}$} \\
\hline
\end{tabular}

\section{Boron Oxide}

\begin{tabular}{llll}
\hline Formula $=$ & B2O3 & Molecular weight $(\mathrm{g} / \mathrm{mole})=$ & 69.6202 \\
Density $(\mathrm{g} / \mathrm{cm} 3)=$ & 1.812000 & Total atom density $($ atoms $/ \mathrm{b}-\mathrm{cm})=$ & $7.837 \mathrm{E}-02$
\end{tabular}


The above density is estimated to be accurate to 4 significant digits. Uncertainties are not addressed. The following data were calculated from the input weight fractions.

\begin{tabular}{|c|c|c|c|c|c|}
\hline Element & Neutron ZA & Photon ZA & $\begin{array}{l}\text { Weight } \\
\text { Fraction }\end{array}$ & $\begin{array}{c}\text { Atom } \\
\text { Fraction }\end{array}$ & $\begin{array}{c}\text { Atom } \\
\text { Density }\end{array}$ \\
\hline$B$ & - & 5000 & $\overline{0.310551}$ & $\overline{0.399978}$ & 0.031346 \\
\hline O & 8016 & 8000 & 0.689449 & 0.600022 & 0.047023 \\
\hline Total & & & 1.000000 & 1.000000 & 0.078368 \\
\hline
\end{tabular}

\begin{tabular}{|c|c|c|c|c|c|c|}
\hline MCNP Form & \multicolumn{2}{|c|}{ Weight Fractions } & \multicolumn{2}{|c|}{ Atom Fractions } & \multicolumn{2}{|c|}{ Atom Densities } \\
\hline \multirow{2}{*}{ Neutrons } & - & -0.310551 & - & 0.399978 & - & 0.031346 \\
\hline & 8016 & -0.689449 & 8016 & 0.600022 & 8016 & 0.047023 \\
\hline \multirow[t]{2}{*}{ Photons } & 5000 & -0.310551 & 5000 & 0.399978 & 5000 & 0.031346 \\
\hline & 8000 & -0.689449 & 8000 & 0.600022 & 8000 & 0.047023 \\
\hline \multirow[t]{3}{*}{ CEPXS Form: } & material & $B$ & 0.310551 & & & \\
\hline & & O & 0.689449 & & & \\
\hline & $\begin{array}{l}\text { matname } \\
\text { density }\end{array}$ & $\begin{array}{c}\text { Boron Oxide } \\
1.812000\end{array}$ & & & & \\
\hline
\end{tabular}

Comments and References

Density and weight fractions from http://physics.nist.gov/cgi-bin/Star/compos.pl?matno=122 (NIST 1998).

Formula from Lide (2008), pgs 4 - 53.

Also called boron trioxide.

\section{Brain (ICRP)}

$\begin{array}{llll}\text { Formula }= & - & \text { Molecular weight }(\mathrm{g} / \mathrm{mole})= & - \\ \text { Density }(\mathrm{g} / \mathrm{cm} 3)= & 1.030000 & \text { Total atom density }(\text { atoms } / \mathrm{b}-\mathrm{cm})= & 1.040 \mathrm{E}-01\end{array}$

The above density is estimated to be accurate to 3 significant digits. Uncertainties are not addressed.

The following data were calculated from the input weight fractions.

\begin{tabular}{|c|c|c|c|c|c|}
\hline Element & Neutron ZA & Photon ZA & $\begin{array}{l}\text { Weight } \\
\text { Fraction }\end{array}$ & $\begin{array}{c}\text { Atom } \\
\text { Fraction }\end{array}$ & $\begin{array}{c}\text { Atom } \\
\text { Density }\end{array}$ \\
\hline $\mathrm{H}$ & 1001 & 1000 & $\overline{0.110667}$ & $\overline{0.654712}$ & 0.068104 \\
\hline C & 6000 & 6000 & 0.125420 & 0.062268 & 0.006477 \\
\hline $\mathrm{N}$ & 7014 & 7000 & 0.013280 & 0.005654 & 0.000588 \\
\hline O & 8016 & 8000 & 0.737723 & 0.274952 & 0.028601 \\
\hline $\mathrm{Na}$ & 11023 & 11000 & 0.001840 & 0.000477 & 0.000050 \\
\hline $\mathrm{Mg}$ & 12000 & 12000 & 0.000150 & 0.000037 & 0.000004 \\
\hline $\mathrm{P}$ & 15031 & 15000 & 0.003540 & 0.000682 & 0.000071 \\
\hline$S$ & 16000 & 16000 & 0.001770 & 0.000329 & 0.000034 \\
\hline $\mathrm{Cl}$ & 17000 & 17000 & 0.002360 & 0.000397 & 0.000041 \\
\hline $\mathrm{K}$ & 19000 & 19000 & 0.003100 & 0.000473 & 0.000049 \\
\hline $\mathrm{Ca}$ & 20000 & 20000 & 0.000090 & 0.000013 & 0.000001 \\
\hline $\mathrm{Fe}$ & 26000 & 26000 & 0.000050 & 0.000005 & 0.000001 \\
\hline
\end{tabular}


PIET-43741-TM-963

PNNL-15870 Rev. 1

\begin{tabular}{|c|c|c|c|c|c|c|}
\hline $\mathrm{Zn}$ & 30000 & 30000 & 0.000010 & 0.000001 & \multicolumn{2}{|c|}{0.000000} \\
\hline Total & & & 1.000000 & 1.000000 & 0.10402 & \\
\hline MCNP Form & \multicolumn{2}{|c|}{ Weight Fractions } & \multicolumn{2}{|c|}{ Atom Fractions } & \multicolumn{2}{|c|}{ Atom Densities } \\
\hline \multirow[t]{13}{*}{ Neutrons } & 1001 & -0.110667 & 1001 & 0.654712 & 1001 & 0.068104 \\
\hline & 6000 & -0.125420 & 6000 & 0.062268 & 6000 & 0.006477 \\
\hline & 7014 & -0.013280 & 7014 & 0.005654 & 7014 & 0.000588 \\
\hline & 8016 & -0.737723 & 8016 & 0.274952 & 8016 & 0.028601 \\
\hline & 11023 & -0.001840 & 11023 & 0.000477 & 11023 & 0.000050 \\
\hline & 12000 & -0.000150 & 12000 & 0.000037 & 12000 & 0.000004 \\
\hline & 15031 & -0.003540 & 15031 & 0.000682 & 15031 & 0.000071 \\
\hline & 16000 & -0.001770 & 16000 & 0.000329 & 16000 & 0.000034 \\
\hline & 17000 & -0.002360 & 17000 & 0.000397 & 17000 & 0.000041 \\
\hline & 19000 & -0.003100 & 19000 & 0.000473 & 19000 & 0.000049 \\
\hline & 20000 & -0.000090 & 20000 & 0.000013 & 20000 & 0.000001 \\
\hline & 26000 & -0.000050 & 26000 & 0.000005 & 26000 & 0.000001 \\
\hline & 30000 & -0.000010 & 30000 & 0.000001 & 30000 & 0.000000 \\
\hline \multirow[t]{13}{*}{ Photons } & 1000 & -0.110667 & 1000 & 0.654712 & 1000 & 0.068104 \\
\hline & 6000 & -0.125420 & 6000 & 0.062268 & 6000 & 0.006477 \\
\hline & 7000 & -0.013280 & 7000 & 0.005654 & 7000 & 0.000588 \\
\hline & 8000 & -0.737723 & 8000 & 0.274952 & 8000 & 0.028601 \\
\hline & 11000 & -0.001840 & 11000 & 0.000477 & 11000 & 0.000050 \\
\hline & 12000 & -0.000150 & 12000 & 0.000037 & 12000 & 0.000004 \\
\hline & 15000 & -0.003540 & 15000 & 0.000682 & 15000 & 0.000071 \\
\hline & 16000 & -0.001770 & 16000 & 0.000329 & 16000 & 0.000034 \\
\hline & 17000 & -0.002360 & 17000 & 0.000397 & 17000 & 0.000041 \\
\hline & 19000 & -0.003100 & 19000 & 0.000473 & 19000 & 0.000049 \\
\hline & 20000 & -0.000090 & 20000 & 0.000013 & 20000 & 0.000001 \\
\hline & 26000 & -0.000050 & 26000 & 0.000005 & 26000 & 0.000001 \\
\hline & 30000 & -0.000010 & 30000 & 0.000001 & 30000 & 0.000000 \\
\hline \multirow[t]{15}{*}{ CEPXS Form: } & material & $\mathrm{H}$ & 0.110667 & & & \\
\hline & & C & 0.125420 & & & \\
\hline & & $\mathrm{N}$ & 0.013280 & & & \\
\hline & & $\mathrm{O}$ & 0.737723 & & & \\
\hline & & $\mathrm{Na}$ & 0.001840 & & & \\
\hline & & $\mathrm{Mg}$ & 0.000150 & & & \\
\hline & & $P$ & 0.003540 & & & \\
\hline & & $S$ & 0.001770 & & & \\
\hline & & $\mathrm{Cl}$ & 0.002360 & & & \\
\hline & & $\mathrm{K}$ & 0.003100 & & & \\
\hline & & $\mathrm{Ca}$ & 0.000090 & & & \\
\hline & & $\mathrm{Fe}$ & 0.000050 & & & \\
\hline & & $\mathrm{Zn}$ & 0.000010 & & & \\
\hline & matname & Brain (ICRP) & & & & \\
\hline & density & 1.030000 & & & & \\
\hline
\end{tabular}


Density and weight fractions from http://physics.nist.gov/cgi-bin/Star/compos.pl?matno=123 (NIST 1998).

\begin{tabular}{|c|c|c|c|c|c|c|}
\hline \multicolumn{3}{|c|}{45 Brass (Typical Composition) } & & & & \\
\hline $\begin{array}{l}\text { Formula }= \\
\text { Density }(\mathrm{g} / \mathrm{cm} 3)\end{array}$ & \multicolumn{6}{|c|}{$\begin{array}{l}\text { The above density is estimated to be accurate to } 3 \text { significant digits. Uncertainties are not addressed. } \\
\text { The following data were calculated from the input weight fractions. }\end{array}$} \\
\hline Element & Neutron ZA & Photon ZA & $\begin{array}{l}\text { Weight } \\
\text { Fraction }\end{array}$ & $\begin{array}{c}\text { Atom } \\
\text { Fraction }\end{array}$ & Atom & \\
\hline $\mathrm{Fe}$ & 26000 & 26000 & 0.000868 & 0.001002 & 0.000076 & \\
\hline $\mathrm{Cu}$ & 29000 & 29000 & 0.665381 & 0.674918 & 0.050887 & \\
\hline $\mathrm{Zn}$ & 30000 & 30000 & 0.325697 & 0.320956 & 0.024199 & \\
\hline Sn & 50000 & 50000 & 0.002672 & 0.001451 & 0.000109 & \\
\hline $\mathrm{Pb}$ & 82000 & 82000 & 0.005377 & 0.001673 & 0.000126 & \\
\hline \multicolumn{2}{|l|}{ Total } & & 0.999996 & 1.000000 & 0.075397 & \\
\hline MCNP Form & \multicolumn{2}{|c|}{ Weight Fractions } & \multicolumn{2}{|c|}{ Atom Fractions } & \multicolumn{2}{|c|}{ Atom Densities } \\
\hline \multirow[t]{5}{*}{ Neutrons } & 26000 & -0.000868 & 26000 & 0.001002 & 26000 & 0.000076 \\
\hline & 29000 & -0.665381 & 29000 & 0.674918 & 29000 & 0.050887 \\
\hline & 30000 & -0.325697 & 30000 & 0.320956 & 30000 & 0.024199 \\
\hline & 50000 & -0.002672 & 50000 & 0.001451 & 50000 & 0.000109 \\
\hline & 82000 & -0.005377 & 82000 & 0.001673 & 82000 & 0.000126 \\
\hline \multirow[t]{5}{*}{ Photons } & 26000 & -0.000868 & 26000 & 0.001002 & 26000 & 0.000076 \\
\hline & 29000 & -0.665381 & 29000 & 0.674918 & 29000 & 0.050887 \\
\hline & 30000 & -0.325697 & 30000 & 0.320956 & 30000 & 0.024199 \\
\hline & 50000 & -0.002672 & 50000 & 0.001451 & 50000 & 0.000109 \\
\hline & 82000 & -0.005377 & 82000 & 0.001673 & 82000 & 0.000126 \\
\hline \multirow[t]{6}{*}{ CEPXS Form: } & material & $\mathrm{Fe}$ & 0.000868 & & & \\
\hline & & $\mathrm{Cu}$ & 0.665381 & & & \\
\hline & & $\mathrm{Zn}$ & 0.325697 & & & \\
\hline & & Sn & 0.002672 & & & \\
\hline & & $\mathrm{Pb}$ & 0.005377 & & & \\
\hline & $\begin{array}{c}\text { matname } \\
\text { density }\end{array}$ & $\begin{array}{c}\text { Brass (Typic } \\
8.070000\end{array}$ & Composition) & & & \\
\hline \multicolumn{7}{|c|}{$\begin{array}{l}\text { Comments and References } \\
\text { Weight fractions are adjusted so that they sum to unity, based on average values from } \\
\text { http://www.matweb.com/search/DataSheet.aspx?MatGUID=d3bd4617903543ada92f4c101c2a20e5 } \\
\text { (Automation Creations } 2010 \text { ). } \\
\text { Hundreds of types of brass are listed at this site. Caution: best to input your specific weight fractions. }\end{array}$} \\
\hline
\end{tabular}




\section{Brick, Common Silica}

\begin{tabular}{llll}
\hline Formula $=$ & - & Molecular weight $(\mathrm{g} / \mathrm{mole})=$ \\
Density $(\mathrm{g} / \mathrm{cm} 3)=$ & 1.800000 & Total atom density $($ atoms $/ \mathrm{b}-\mathrm{cm})=$ & - \\
\hline & $5.361 \mathrm{E}-02$
\end{tabular}

The above density is estimated to be accurate to 2 significant digits. Uncertainties are not addressed.

The following data were calculated from the input weight fractions.

\begin{tabular}{|c|c|c|c|c|c|}
\hline Element & Neutron ZA & Photon ZA & $\begin{array}{l}\text { Weight } \\
\text { Fraction }\end{array}$ & $\begin{array}{c}\text { Atom } \\
\text { Fraction }\end{array}$ & $\begin{array}{c}\text { Atom } \\
\text { Density }\end{array}$ \\
\hline 0 & 8016 & 8000 & $\overline{0.525000}$ & $\overline{0.663432}$ & 0.035570 \\
\hline$A L$ & 13027 & 13000 & 0.005000 & 0.003747 & 0.000201 \\
\hline $\mathrm{Si}$ & 14000 & 14000 & 0.449000 & 0.323225 & 0.017330 \\
\hline $\mathrm{Ca}$ & 20000 & 20000 & 0.014000 & 0.007063 & 0.000379 \\
\hline $\mathrm{Fe}$ & 26000 & 26000 & 0.007000 & 0.002534 & 0.000136 \\
\hline Total & & & 1.000000 & 1.000000 & 0.053615 \\
\hline
\end{tabular}

\begin{tabular}{|c|cc|cc|cr|}
\hline MCNP Form & \multicolumn{2}{c|}{ Weight Fractions } & \multicolumn{2}{c|}{ Atom Fractions } & \multicolumn{2}{c|}{ Atom Densities } \\
\hline Neutrons & 8016 & -0.525000 & 8016 & 0.663432 & 8016 & 0.035570 \\
& 13027 & -0.005000 & 13027 & 0.003747 & 13027 & 0.000201 \\
& 14000 & -0.449000 & 14000 & 0.323225 & 14000 & 0.017330 \\
& 20000 & -0.014000 & 20000 & 0.007063 & 20000 & 0.000379 \\
& 26000 & -0.007000 & 26000 & 0.002534 & 26000 & 0.000136 \\
Photons & & & & & & \\
& 8000 & -0.525000 & 8000 & 0.663432 & 8000 & 0.035570 \\
& 13000 & -0.005000 & 13000 & 0.003747 & 13000 & 0.000201 \\
& 14000 & -0.449000 & 14000 & 0.323225 & 14000 & 0.017330 \\
& 20000 & -0.014000 & 20000 & 0.007063 & 20000 & 0.000379 \\
& 26000 & -0.007000 & 26000 & 0.002534 & 26000 & 0.000136
\end{tabular}

\begin{tabular}{|c|c|c|c|}
\hline \multirow[t]{5}{*}{ CEPXS Form: } & material & $\mathrm{O}$ & 0.525000 \\
\hline & & $\mathrm{AL}$ & 0.005000 \\
\hline & & $\mathrm{Si}$ & 0.449000 \\
\hline & & $\mathrm{Ca}$ & 0.014000 \\
\hline & & $\mathrm{Fe}$ & 0.007000 \\
\hline
\end{tabular}

matname Brick, Common Silica density $\quad 1.800000$

\section{Comments and References}

Density and weight fractions from Brewer (2009), which were taken from Carter et al. (1968) pg II.F1-2. Density $=1.6$ to $2.0 \mathrm{~g} / \mathrm{cm} 3$ for medium brick in Table 6.1 .5 of Avallone and Baumeister III (1996).

\section{$47 \quad$ Brick, Fire}

\begin{tabular}{|llll}
\hline Formula $=$ & - & Molecular weight $(\mathrm{g} / \mathrm{mole})=$ \\
Density $(\mathrm{g} / \mathrm{cm} 3)=$ & 2.100000 & Total atom density $($ atoms $/ \mathrm{b}-\mathrm{cm})=$ & - \\
\hline
\end{tabular}


The above density is estimated to be accurate to 2 significant digits. Uncertainties are not addressed. The following data were calculated from the input weight fractions.

\begin{tabular}{|c|c|c|c|c|c|}
\hline Element & Neutron ZA & Photon ZA & $\begin{array}{l}\text { Weight } \\
\text { Fraction }\end{array}$ & $\begin{array}{c}\text { Atom } \\
\text { Fraction }\end{array}$ & $\begin{array}{l}\text { Atom } \\
\text { Density }\end{array}$ \\
\hline 0 & 8016 & 8000 & 0.497000 & 0.636337 & 0.039285 \\
\hline $\mathrm{Mg}$ & 12000 & 12000 & 0.006000 & 0.005057 & 0.000312 \\
\hline $\mathrm{Al}$ & 13027 & 13000 & 0.212000 & 0.160955 & 0.009937 \\
\hline $\mathrm{Si}$ & 14000 & 14000 & 0.252000 & 0.183803 & 0.011347 \\
\hline $\mathrm{Ca}$ & 20000 & 20000 & 0.007000 & 0.003578 & 0.000221 \\
\hline $\mathrm{Ti}$ & 22000 & 22000 & 0.012000 & 0.005135 & 0.000317 \\
\hline $\mathrm{Fe}$ & 26000 & 26000 & 0.014000 & 0.005135 & 0.000317 \\
\hline Total & & & 1.000000 & 1.000000 & 0.061736 \\
\hline
\end{tabular}

\begin{tabular}{|c|cc|cc|cr|}
\hline MCNP Form & \multicolumn{2}{c|}{ Weight Fractions } & \multicolumn{2}{c|}{ Atom Fractions } & \multicolumn{2}{c|}{ Atom Densities } \\
\hline Neutrons & 8016 & -0.497000 & 8016 & 0.636337 & 8016 & 0.039285 \\
& 12000 & -0.006000 & 12000 & 0.005057 & 12000 & 0.000312 \\
& 13027 & -0.212000 & 13027 & 0.160955 & 13027 & 0.009937 \\
& 14000 & -0.252000 & 14000 & 0.183803 & 14000 & 0.011347 \\
& 20000 & -0.007000 & 20000 & 0.003578 & 20000 & 0.000221 \\
& 22000 & -0.012000 & 22000 & 0.005135 & 22000 & 0.000317 \\
& 26000 & -0.014000 & 26000 & 0.005135 & 26000 & 0.000317 \\
& & & & & & \\
Photons & 8000 & -0.497000 & 8000 & 0.636337 & 8000 & 0.039285 \\
& 12000 & -0.006000 & 12000 & 0.005057 & 12000 & 0.000312 \\
& 13000 & -0.212000 & 13000 & 0.160955 & 13000 & 0.009937 \\
& 14000 & -0.252000 & 14000 & 0.183803 & 14000 & 0.011347 \\
& 20000 & -0.007000 & 20000 & 0.003578 & 20000 & 0.000221 \\
& 22000 & -0.012000 & 22000 & 0.005135 & 22000 & 0.000317 \\
& 26000 & -0.014000 & 26000 & 0.005135 & 26000 & 0.000317
\end{tabular}

\begin{tabular}{|c|c|c|c|}
\hline \multirow[t]{7}{*}{ CEPXS Form: } & material & $\mathrm{O}$ & 0.497000 \\
\hline & & $\mathrm{Mg}$ & 0.006000 \\
\hline & & $\mathrm{Al}$ & 0.212000 \\
\hline & & $\mathrm{Si}$ & 0.252000 \\
\hline & & $\mathrm{Ca}$ & 0.007000 \\
\hline & & $\mathrm{Ti}$ & 0.012000 \\
\hline & & $\mathrm{Fe}$ & 0.014000 \\
\hline
\end{tabular}

matname Brick, Fire density 2.100000

Comments and References

Density and weight fractions from Brewer (2009), which were taken from Carter et al. (1968), pg II.F1-2. 


\section{Brick, Kaolin (White)}

\begin{tabular}{llll}
\hline Formula $=$ & - & Molecular weight $(\mathrm{g} / \mathrm{mole})=$ \\
Density $(\mathrm{g} / \mathrm{cm} 3)=$ & 2.100000 & Total atom density $($ atoms $/ \mathrm{b}-\mathrm{cm})=$ & - \\
\hline & $6.221 \mathrm{E}-02$
\end{tabular}

The above density is estimated to be accurate to 2 significant digits. Uncertainties are not addressed.

The following data were calculated from the input weight fractions.

\begin{tabular}{|c|c|c|c|c|c|c|}
\hline Element & Neutron ZA & Photon ZA & $\begin{array}{l}\text { Weight } \\
\text { Fraction }\end{array}$ & $\begin{array}{c}\text { Atom } \\
\text { Fraction }\end{array}$ & $\begin{array}{l}\text { Atom } \\
\text { Density }\end{array}$ & \\
\hline 0 & 8016 & 8000 & $\overline{0.500318}$ & $\overline{0.635745}$ & 0.039547 & \\
\hline $\mathrm{Mg}$ & 12000 & 12000 & 0.001205 & 0.001008 & 0.000063 & \\
\hline $\mathrm{Al}$ & 13027 & 13000 & 0.240568 & 0.181264 & 0.011276 & \\
\hline $\mathrm{Si}$ & 14000 & 14000 & 0.242823 & 0.175771 & 0.010934 & \\
\hline $\mathrm{Ca}$ & 20000 & 20000 & 0.000714 & 0.000362 & 0.000023 & \\
\hline $\mathrm{Ti}$ & 22000 & 22000 & 0.010179 & 0.004323 & 0.000269 & \\
\hline $\mathrm{Fe}$ & 26000 & 26000 & 0.004192 & 0.001526 & 0.000095 & \\
\hline Total & & & 1.000000 & 1.000000 & 0.062206 & \\
\hline MCNP Form & \multicolumn{2}{|c|}{ Weight Fractions } & \multicolumn{2}{|c|}{ Atom Fractions } & \multicolumn{2}{|c|}{ Atom Densities } \\
\hline \multirow[t]{7}{*}{ Neutrons } & 8016 & -0.500318 & 8016 & 0.635745 & 8016 & 0.039547 \\
\hline & 12000 & -0.001205 & 12000 & 0.001008 & 12000 & 0.000063 \\
\hline & 13027 & -0.240568 & 13027 & 0.181264 & 13027 & 0.011276 \\
\hline & 14000 & -0.242823 & 14000 & 0.175771 & 14000 & 0.010934 \\
\hline & 20000 & -0.000714 & 20000 & 0.000362 & 20000 & 0.000023 \\
\hline & 22000 & -0.010179 & 22000 & 0.004323 & 22000 & 0.000269 \\
\hline & 26000 & -0.004192 & 26000 & 0.001526 & 26000 & 0.000095 \\
\hline \multirow[t]{7}{*}{ Photons } & 8000 & -0.500318 & 8000 & 0.635745 & 8000 & 0.039547 \\
\hline & 12000 & -0.001205 & 12000 & 0.001008 & 12000 & 0.000063 \\
\hline & 13000 & -0.240568 & 13000 & 0.181264 & 13000 & 0.011276 \\
\hline & 14000 & -0.242823 & 14000 & 0.175771 & 14000 & 0.010934 \\
\hline & 20000 & -0.000714 & 20000 & 0.000362 & 20000 & 0.000023 \\
\hline & 22000 & -0.010179 & 22000 & 0.004323 & 22000 & 0.000269 \\
\hline & 26000 & -0.004192 & 26000 & 0.001526 & 26000 & 0.000095 \\
\hline \multirow[t]{8}{*}{ CEPXS Form: } & material & $\mathrm{O}$ & 0.500318 & & & \\
\hline & & $\mathrm{Mg}$ & 0.001205 & & & \\
\hline & & $\mathrm{Al}$ & 0.240568 & & & \\
\hline & & $\mathrm{Si}$ & 0.242823 & & & \\
\hline & & $\mathrm{Ca}$ & 0.000714 & & & \\
\hline & & $\mathrm{Ti}$ & 0.010179 & & & \\
\hline & & $\mathrm{Fe}$ & 0.004192 & & & \\
\hline & $\begin{array}{l}\text { matname } \\
\text { density }\end{array}$ & \multicolumn{3}{|c|}{$\begin{array}{l}\text { Brick, Kaolin (White) } \\
2.100000\end{array}$} & & \\
\hline \multicolumn{7}{|c|}{$\begin{array}{l}\text { Comments and References } \\
\text { Density }=2.1 \mathrm{~g} / \mathrm{cm} 3 \text { and composition ( } 52 \mathrm{wt} \% \mathrm{SiO} 2,45.5 \% \mathrm{Al} 2 \mathrm{O} 3,0.6 \% \mathrm{Fe} 2 \mathrm{O} 3,1.7 \% \mathrm{TiO} 2,0.2 \% \mathrm{MgO} \text {, } \\
\text { and } 0.1 \% \mathrm{CaO} \text { ) from Tables } 51.67 \text { and } 51.68 \text { of Hungerford (1960). } \\
\text { See Tables } 12-6 \text { and } 12-7 \text { of Parker (1967) for other types of bricks. }\end{array}$} \\
\hline
\end{tabular}




\section{Bronze (Typical Composition)}

\begin{tabular}{|c|c|c|c|}
\hline Formula = & - & Molecular weight $(\mathrm{g} / \mathrm{mole})=$ & -1500 \\
\hline Density $(\mathrm{g} / \mathrm{cm} 3)=$ & 8.400000 & Total atom density $($ atoms $/ \mathrm{b}-\mathrm{cm})=$ & 8.152E-02 \\
\hline
\end{tabular}

The above density is estimated to be accurate to 2 significant digits. Uncertainties are not addressed.

The following data were calculated from the input weight fractions.

\begin{tabular}{|c|c|c|c|c|c|c|}
\hline Element & Neutron ZA & Photon ZA & $\begin{array}{l}\text { Weight } \\
\text { Fraction }\end{array}$ & $\begin{array}{c}\text { Atom } \\
\text { Fraction }\end{array}$ & $\begin{array}{l}\text { Atom } \\
\text { Density }\end{array}$ & \\
\hline $\mathrm{Al}$ & 13027 & 13000 & 0.028528 & 0.065613 & 0.005349 & \\
\hline $\mathrm{Si}$ & 14000 & 14000 & 0.003339 & 0.007378 & 0.000601 & \\
\hline $\mathrm{Mn}$ & 25055 & 25000 & 0.003555 & 0.004015 & 0.000327 & \\
\hline $\mathrm{Fe}$ & 26000 & 26000 & 0.010208 & 0.011344 & 0.000925 & \\
\hline $\mathrm{Ni}$ & 28000 & 28000 & 0.006718 & 0.007103 & 0.000579 & \\
\hline $\mathrm{Cu}$ & 29000 & 29000 & 0.874157 & 0.853667 & 0.069588 & \\
\hline $\mathrm{Zn}$ & 30000 & 30000 & 0.036037 & 0.034190 & 0.002787 & \\
\hline $\mathrm{Sn}$ & 50000 & 50000 & 0.024503 & 0.012809 & 0.001044 & \\
\hline $\mathrm{Pb}$ & 82000 & 82000 & 0.012957 & 0.003881 & 0.000316 & \\
\hline Total & & & 1.000002 & 1.000000 & 0.081516 & \\
\hline MCNP Form & \multicolumn{2}{|c|}{ Weight Fractions } & \multicolumn{2}{|c|}{ Atom Fractions } & \multicolumn{2}{|c|}{ Atom Densities } \\
\hline \multirow[t]{9}{*}{ Neutrons } & 13027 & -0.028528 & 13027 & 0.065613 & 13027 & 0.005349 \\
\hline & 14000 & -0.003339 & 14000 & 0.007378 & 14000 & 0.000601 \\
\hline & 25055 & -0.003555 & 25055 & 0.004015 & 25055 & 0.000327 \\
\hline & 26000 & -0.010208 & 26000 & 0.011344 & 26000 & 0.000925 \\
\hline & 28000 & -0.006718 & 28000 & 0.007103 & 28000 & 0.000579 \\
\hline & 29000 & -0.874157 & 29000 & 0.853667 & 29000 & 0.069588 \\
\hline & 30000 & -0.036037 & 30000 & 0.034190 & 30000 & 0.002787 \\
\hline & 50000 & -0.024503 & 50000 & 0.012809 & 50000 & 0.001044 \\
\hline & 82000 & -0.012957 & 82000 & 0.003881 & 82000 & 0.000316 \\
\hline \multirow[t]{9}{*}{ Photons } & 13000 & -0.028528 & 13000 & 0.065613 & 13000 & 0.005349 \\
\hline & 14000 & -0.003339 & 14000 & 0.007378 & 14000 & 0.000601 \\
\hline & 25000 & -0.003555 & 25000 & 0.004015 & 25000 & 0.000327 \\
\hline & 26000 & -0.010208 & 26000 & 0.011344 & 26000 & 0.000925 \\
\hline & 28000 & -0.006718 & 28000 & 0.007103 & 28000 & 0.000579 \\
\hline & 29000 & -0.874157 & 29000 & 0.853667 & 29000 & 0.069588 \\
\hline & 30000 & -0.036037 & 30000 & 0.034190 & 30000 & 0.002787 \\
\hline & 50000 & -0.024503 & 50000 & 0.012809 & 50000 & 0.001044 \\
\hline & 82000 & -0.012957 & 82000 & 0.003881 & 82000 & 0.000316 \\
\hline \multirow[t]{5}{*}{ CEPXS Form: } & material & $\mathrm{Al}$ & 0.028528 & & & \\
\hline & & $\mathrm{Si}$ & 0.003339 & & & \\
\hline & & $\mathrm{Mn}$ & 0.003555 & & & \\
\hline & & $\mathrm{Fe}$ & 0.010208 & & & \\
\hline & & $\mathrm{Ni}$ & 0.006718 & & & \\
\hline
\end{tabular}




\begin{tabular}{|ll}
$\mathrm{Cu}$ & 0.874157 \\
$\mathrm{Zn}$ & 0.036037 \\
$\mathrm{Sn}$ & 0.024503 \\
$\mathrm{~Pb}$ & 0.012957
\end{tabular}

matname Bronze (Typical Composition) density $\quad 8.400000$

\section{Comments and References}

Weight fractions are adjusted so that they sum to unity, based on average values from:

http://www.matweb.com/search/DataSheet.aspx?MatGUID=66575ff2cd5249c49d76df15b47dbca4 (Automation Creations 2010).

Hundreds of types of bronze are listed at this site. Caution: best to input your specific weight fractions.

\section{C-552 Air-Equivalent Plastic}

Formula $=$

Density $(\mathrm{g} / \mathrm{cm} 3)=1.760000$

The above density is estimated to be accurate to 3 significant digits. Uncertainties are not addressed.

The following data were calculated from the input weight fractions.

\begin{tabular}{|c|c|c|c|c|c|}
\hline Element & Neutron ZA & Photon ZA & $\begin{array}{l}\text { Weight } \\
\text { Fraction }\end{array}$ & $\begin{array}{c}\text { Atom } \\
\text { Fraction }\end{array}$ & $\begin{array}{l}\text { Atom } \\
\text { Density }\end{array}$ \\
\hline$\overline{\mathrm{H}}$ & 1001 & 1000 & $\overline{0.024680}$ & $\overline{0.268599}$ & 0.025952 \\
\hline C & 6000 & 6000 & 0.501610 & 0.458133 & 0.044265 \\
\hline $\mathrm{O}$ & 8016 & 8000 & 0.004527 & 0.003104 & 0.000300 \\
\hline $\mathrm{F}$ & 9019 & 9000 & 0.465209 & 0.268612 & 0.025953 \\
\hline $\mathrm{Si}$ & 14000 & 14000 & 0.003973 & 0.001552 & 0.000150 \\
\hline
\end{tabular}

Total

1.000000

0.096621

\begin{tabular}{|c|cccccc|}
\hline MCNP Form & \multicolumn{2}{c|}{ Weight Fractions } & \multicolumn{2}{c|}{ Atom Fractions } & \multicolumn{2}{c|}{ Atom Densities } \\
\hline Neutrons & 1001 & -0.024680 & 1001 & 0.268599 & 1001 & 0.025952 \\
& 6000 & -0.501610 & 6000 & 0.458133 & 6000 & 0.044265 \\
& 8016 & -0.004527 & 8016 & 0.003104 & 8016 & 0.000300 \\
& 9019 & -0.465209 & 9019 & 0.268612 & 9019 & 0.025953 \\
& 14000 & -0.003973 & 14000 & 0.001552 & 14000 & 0.000150 \\
Photons & 1000 & -0.024680 & 1000 & 0.268599 & 1000 & 0.025952 \\
& 6000 & -0.501610 & 6000 & 0.458133 & 6000 & 0.044265 \\
& 8000 & -0.004527 & 8000 & 0.003104 & 8000 & 0.000300 \\
& 9000 & -0.465209 & 9000 & 0.268612 & 9000 & 0.025953 \\
& 14000 & -0.003973 & 14000 & 0.001552 & 14000 & 0.000150 \\
& & & & & & \\
\hline CEPXS Form: & material & $\mathrm{H}$ & 0.024680 & & & \\
& & $\mathrm{C}$ & 0.501610 & & \\
& $\mathrm{O}$ & 0.004527 & & \\
& $\mathrm{~F}$ & 0.465209 & & \\
& & $\mathrm{Si}$ & 0.003973 & &
\end{tabular}


matname C-552 Air-Equivalent Plastic

density $\quad 1.760000$

Comments and References

Density and weight fractions from http://physics.nist.gov/cgi-bin/Star/compos.pl?matno=126 (NIST 1998).

\section{Cadmium}

\begin{tabular}{llll}
\hline Formula $=$ & Cd & Molecular weight $(\mathrm{g} / \mathrm{mole})=$ & 112.411 \\
Density $(\mathrm{g} / \mathrm{cm} 3)=$ & 8.650000 & Total atom density $($ atoms $/ \mathrm{b}-\mathrm{cm})=$ & $4.634 \mathrm{E}-02$
\end{tabular}

The above density is estimated to be accurate to 3 significant digits. Uncertainties are not addressed.

The following data was calculated from the input formula.

\begin{tabular}{|c|c|c|c|c|c|c|}
\hline$\frac{\text { Element }}{\mathrm{Cd}}$ & $\frac{\text { Neutron ZA }}{48000}$ & $\frac{\text { Photon ZA }}{48000}$ & $\begin{array}{c}\text { Weight } \\
\frac{\text { Fraction }}{1.000000}\end{array}$ & $\begin{array}{c}\text { Atom } \\
\frac{\text { Fraction }}{1.000000}\end{array}$ & $\begin{array}{c}\text { Atom } \\
\text { Density } \\
0.046340\end{array}$ & \\
\hline Total & & & 1.000000 & 1.000000 & 0.046340 & \\
\hline MCNP Form & \multicolumn{2}{|c|}{ Weight Fractions } & \multicolumn{2}{|c|}{ Atom Fractions } & \multicolumn{2}{|c|}{ Atom Densities } \\
\hline Neutrons & 48000 & -1.000000 & 48000 & 1.000000 & 48000 & 0.046340 \\
\hline Photons & 48000 & -1.000000 & 48000 & 1.000000 & 48000 & 0.046340 \\
\hline CEPXS Form: & $\begin{array}{l}\text { material } \\
\text { matname } \\
\text { density }\end{array}$ & $\begin{array}{c}\text { Cd } \\
\text { Cadmium } \\
8.650000\end{array}$ & 1.000000 & & & \\
\hline
\end{tabular}

\section{Cadmium Nitrate Tetrahydrate}

Formula $=\quad \mathrm{Cd}(\mathrm{NO} 3) 2-4(\mathrm{H} 2 \mathrm{O}) \quad$ Molecular weight $(\mathrm{g} / \mathrm{mole})=\quad 308.48192$

Density $(\mathrm{g} / \mathrm{cm} 3)=2.450000 \quad$ Total atom density $($ atoms $/ \mathrm{b}-\mathrm{cm})=1.004 \mathrm{E}-01$

The above density is estimated to be accurate to 3 significant digits. Uncertainties are not addressed.

The following data was calculated from the input formula.

\begin{tabular}{|c|c|c|c|c|c|}
\hline Element & Neutron ZA & Photon ZA & $\begin{array}{l}\text { Weight } \\
\text { Fraction }\end{array}$ & $\begin{array}{c}\text { Atom } \\
\text { Fraction }\end{array}$ & $\begin{array}{l}\text { Atom } \\
\text { Density }\end{array}$ \\
\hline $\mathrm{H}$ & 1001 & 1000 & $\overline{0.026139}$ & $\overline{0.380952}$ & 0.038263 \\
\hline $\mathrm{N}$ & 7014 & 7000 & 0.090811 & 0.095238 & 0.009566 \\
\hline 0 & 8016 & 8000 & 0.518650 & 0.476190 & 0.047829 \\
\hline $\mathrm{Cd}$ & 48000 & 48000 & 0.364401 & 0.047619 & 0.004783 \\
\hline
\end{tabular}


PIET-43741-TM-963

PNNL-15870 Rev. 1

\begin{tabular}{|c|c|c|c|c|c|c|}
\hline \multicolumn{3}{|l|}{ Total } & 1.000000 & 1.000000 & \multicolumn{2}{|c|}{0.100440} \\
\hline MCNP Form & \multicolumn{2}{|c|}{ Weight Fractions } & \multicolumn{2}{|c|}{ Atom Fractions } & \multicolumn{2}{|c|}{ Atom Densities } \\
\hline \multirow[t]{4}{*}{ Neutrons } & 1001 & -0.026139 & 1001 & 0.380952 & 1001 & 0.038263 \\
\hline & 7014 & -0.090811 & 7014 & 0.095238 & 7014 & 0.009566 \\
\hline & 8016 & -0.518650 & 8016 & 0.476190 & 8016 & 0.047829 \\
\hline & 48000 & -0.364401 & 48000 & 0.047619 & 48000 & 0.004783 \\
\hline \multirow[t]{4}{*}{ Photons } & 1000 & -0.026139 & 1000 & 0.380952 & 1000 & 0.038263 \\
\hline & 7000 & -0.090811 & 7000 & 0.095238 & 7000 & 0.009566 \\
\hline & 8000 & -0.518650 & 8000 & 0.476190 & 8000 & 0.047829 \\
\hline & 48000 & -0.364401 & 48000 & 0.047619 & 48000 & 0.004783 \\
\hline \multirow[t]{5}{*}{ CEPXS Form: } & material & $\mathrm{H}$ & 0.026139 & & & \\
\hline & & $\mathrm{N}$ & 0.090811 & & & \\
\hline & & 0 & 0.518650 & & & \\
\hline & & $\mathrm{Cd}$ & 0.364401 & & & \\
\hline & $\begin{array}{c}\text { matname } \\
\text { density }\end{array}$ & $\begin{array}{c}\text { Cadmium Ni } \\
2.450000\end{array}$ & Tetrahydr & & & \\
\hline \multicolumn{7}{|c|}{$\begin{array}{l}\text { Comments and References } \\
\text { Density and formula from } \\
\text { http://www.matweb.com/search/DataSheet.aspx?MatGUID=80e2491150724055982967256325061f } \\
\text { (Automation Creations 2010). }\end{array}$} \\
\hline
\end{tabular}

\section{Cadmium Telluride}

\begin{tabular}{llll}
\hline Formula $=$ & CdTe & Molecular weight $(\mathrm{g} / \mathrm{mole})=$ & 240.011 \\
Density $(\mathrm{g} / \mathrm{cm} 3)=$ & 6.200000 & Total atom density $($ atoms $/ \mathrm{b}-\mathrm{cm})=$ & $3.111 \mathrm{E}-02$
\end{tabular}

The above density is estimated to be accurate to 2 significant digits. Uncertainties are not addressed.

The following data were calculated from the input weight fractions.

\begin{tabular}{|c|c|c|c|c|c|c|}
\hline $\begin{array}{c}\text { Element } \\
\mathrm{Cd} \\
\mathrm{Te}\end{array}$ & $\frac{\text { Neutron ZA }}{48000}$ & $\begin{array}{c}\text { Photon ZA } \\
48000 \\
52000\end{array}$ & $\begin{array}{c}\text { Weight } \\
\text { Fraction } \\
0.468355 \\
0.531645\end{array}$ & $\begin{array}{c}\begin{array}{c}\text { Atom } \\
\text { Fraction }\end{array} \\
0.499997 \\
0.500003\end{array}$ & $\begin{array}{c}\text { Atom } \\
\text { Density } \\
0.015556 \\
0.015557\end{array}$ & \\
\hline Total & & & 1.000000 & 1.000000 & 0.031113 & \\
\hline MCNP Form & \multicolumn{2}{|c|}{ Weight Fractions } & \multicolumn{2}{|c|}{ Atom Fractions } & \multicolumn{2}{|c|}{ Atom Densities } \\
\hline Neutrons & $\begin{array}{c}48000 \\
-\end{array}$ & $\begin{array}{l}-0.468355 \\
-0.531645\end{array}$ & $\begin{array}{c}48000 \\
-\end{array}$ & $\begin{array}{l}0.499997 \\
0.500003\end{array}$ & $\begin{array}{c}48000 \\
-\end{array}$ & $\begin{array}{l}0.015556 \\
0.015557\end{array}$ \\
\hline Photons & $\begin{array}{l}48000 \\
52000\end{array}$ & $\begin{array}{l}-0.468355 \\
-0.531645\end{array}$ & $\begin{array}{l}48000 \\
52000\end{array}$ & $\begin{array}{l}0.499997 \\
0.500003\end{array}$ & $\begin{array}{l}48000 \\
52000\end{array}$ & $\begin{array}{l}0.015556 \\
0.015557\end{array}$ \\
\hline CEPXS Form: & material & $\begin{array}{l}\mathrm{Cd} \\
\mathrm{Te}\end{array}$ & $\begin{array}{l}0.468355 \\
0.531645\end{array}$ & & & \\
\hline
\end{tabular}


matname Cadmium Telluride density $\quad 6.200000$

\section{Comments and References}

Density and weight fractions from http://physics.nist.gov/cgi-bin/Star/compos.pl?matno=127 (NIST 1998).

Formula from Lide (2008), pgs 4 - 54.

\section{Cadmium Tungstate (CWO)}

\begin{tabular}{llll}
\hline Formula $=$ & CdWO4 & Molecular weight $(\mathrm{g} / \mathrm{mole})=$ & 360.2486 \\
Density $(\mathrm{g} / \mathrm{cm} 3)=$ & 7.900000 & Total atom density $($ atoms $/ \mathrm{b}-\mathrm{cm})=$ & $7.924 \mathrm{E}-02$ \\
The above density is estimated to be accurate to 2 significant digits. Uncertainties are not addressed. & \\
The following data were calculated from the input weight fractions.
\end{tabular}

\begin{tabular}{|c|c|c|c|c|c|}
\hline Element & Neutron ZA & Photon ZA & $\begin{array}{l}\text { Weight } \\
\text { Fraction }\end{array}$ & $\begin{array}{c}\text { Atom } \\
\text { Fraction }\end{array}$ & $\begin{array}{l}\text { Atom } \\
\text { Density }\end{array}$ \\
\hline 0 & 8016 & 8000 & $\overline{0.177644}$ & $\overline{0.666662}$ & 0.052823 \\
\hline $\mathrm{Cd}$ & 48000 & 48000 & 0.312027 & 0.166664 & 0.013206 \\
\hline W & 74000 & 74000 & 0.510329 & 0.166674 & 0.013207 \\
\hline Total & & & 1.000000 & 1.000000 & 0.079235 \\
\hline
\end{tabular}

\begin{tabular}{|c|cccccc|}
\hline MCNP Form & \multicolumn{2}{c|}{ Weight Fractions } & \multicolumn{2}{c|}{ Atom Fractions } & \multicolumn{2}{c|}{ Atom Densities } \\
\hline Neutrons & 8016 & -0.177644 & 8016 & 0.666662 & 8016 & 0.052823 \\
& 48000 & -0.312027 & 48000 & 0.166664 & 48000 & 0.013206 \\
& 74000 & -0.510329 & 74000 & 0.166674 & 74000 & 0.013207 \\
Photons & 8000 & -0.177644 & 8000 & 0.666662 & 8000 & 0.052823 \\
& 48000 & -0.312027 & 48000 & 0.166664 & 48000 & 0.013206 \\
& 74000 & -0.510329 & 74000 & 0.166674 & 74000 & 0.013207 \\
& & & & & & \\
\hline CEPXS Form: & material & $\mathrm{O}$ & 0.177644 & & \\
& & $\mathrm{Cd}$ & 0.312027 & & & \\
& & $\mathrm{~W}$ & 0.510329 & & &
\end{tabular}

matname Cadmium Tungstate (CWO)

density $\quad 7.900000$

\section{Comments and References}

Density $=7.9 \mathrm{~g} / \mathrm{cm} 3$ and weight fractions from http://physics.nist.gov/cgi-bin/Star/compos.pl?matno=128

(NIST 1998).

Formula and same density on pg 235 of Knoll (2000).

\section{Calcium Carbonate}

\begin{tabular}{|llll}
\hline Formula $=$ & CaCO3 & Molecular weight $(\mathrm{g} / \mathrm{mole})=$ & 100.0869 \\
Density $(\mathrm{g} / \mathrm{cm} 3)=$ & 2.800000 & Total atom density $($ atoms $/ \mathrm{b}-\mathrm{cm})=$ & $8.424 \mathrm{E}-02$
\end{tabular}


The above density is estimated to be accurate to 2 significant digits. Uncertainties are not addressed. The following data were calculated from the input weight fractions.

\begin{tabular}{|c|c|c|c|c|c|}
\hline Element & Neutron ZA & Photon ZA & $\begin{array}{l}\text { Weight } \\
\text { Fraction }\end{array}$ & $\begin{array}{l}\text { Atom } \\
\text { Fraction }\end{array}$ & $\begin{array}{l}\text { Atom } \\
\text { Density }\end{array}$ \\
\hline $\mathrm{C}$ & 6000 & 6000 & $\overline{0.120003}$ & $\overline{0.200002}$ & 0.016847 \\
\hline 0 & 8016 & 8000 & 0.479554 & 0.599991 & 0.050541 \\
\hline $\mathrm{Ca}$ & 20000 & 20000 & 0.400443 & 0.200007 & 0.016848 \\
\hline Total & & & 1.000000 & 1.000000 & 0.084236 \\
\hline
\end{tabular}

\begin{tabular}{|c|cccccc|}
\hline MCNP Form & \multicolumn{2}{c|}{ Weight Fractions } & \multicolumn{2}{c|}{ Atom Fractions } & \multicolumn{2}{c|}{ Atom Densities } \\
\hline Neutrons & 6000 & -0.120003 & 6000 & 0.200002 & 6000 & 0.016847 \\
& 8016 & -0.479554 & 8016 & 0.599991 & 8016 & 0.050541 \\
& 20000 & -0.400443 & 20000 & 0.200007 & 20000 & 0.016848 \\
Photons & 6000 & -0.120003 & 6000 & 0.200002 & 6000 & 0.016847 \\
& 8000 & -0.479554 & 8000 & 0.599991 & 8000 & 0.050541 \\
& 20000 & -0.400443 & 20000 & 0.200007 & 20000 & 0.016848 \\
& & & & & & \\
\hline CEPXS Form: & material & $\mathrm{C}$ & 0.120003 & & \\
& & $\mathrm{O}$ & 0.479554 & & & \\
& & $\mathrm{Ca}$ & 0.400443 & & & \\
\end{tabular}

matname Calcium Carbonate density $\quad 2.800000$

\section{Comments and References}

Density and weight fractions from http://physics.nist.gov/cgi-bin/Star/compos.pl?matno=129 (NIST 1998). Formula from Lide (2008), pgs 4 - 54.

Calcium carbonate is the mineral calcite, which is the main constituent of limestone, which is a sedimentary rock (http://en.wikipedia.org/wiki/Limestone).

\section{Calcium Fluoride}

\begin{tabular}{llll}
\hline Formula $=$ & CaF2 & Molecular weight $(\mathrm{g} / \mathrm{mole})=$ & 78.0748064 \\
Density $(\mathrm{g} / \mathrm{cm} 3)=$ & 3.180000 & Total atom density $($ atoms $/ \mathrm{b}-\mathrm{cm})=$ & $7.358 \mathrm{E}-02$
\end{tabular}

The above density is estimated to be accurate to 3 significant digits. Uncertainties are not addressed.

The following data were calculated from the input weight fractions.

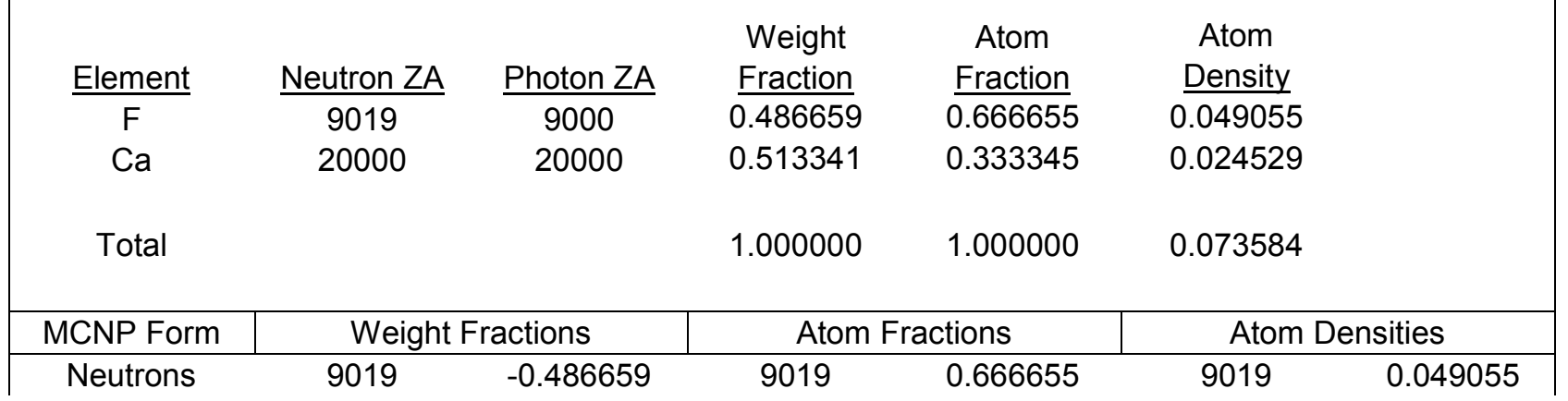


PIET-43741-TM-963

PNNL-15870 Rev. 1

\begin{tabular}{|c|c|c|c|c|c|c|}
\hline & 20000 & -0.513341 & 20000 & 0.333345 & 20000 & 0.024529 \\
\hline \multirow[t]{2}{*}{ Photons } & 9000 & -0.486659 & 9000 & 0.666655 & 9000 & 0.049055 \\
\hline & 20000 & -0.513341 & 20000 & 0.333345 & 20000 & 0.024529 \\
\hline \multirow[t]{3}{*}{ CEPXS Form: } & material & $F$ & 0.486659 & & & \\
\hline & & $\mathrm{Ca}$ & 0.513341 & & & \\
\hline & $\begin{array}{c}\text { matname } \\
\text { density }\end{array}$ & $\begin{array}{c}\text { Calcium Flu } \\
3.180000\end{array}$ & & & & \\
\hline \multicolumn{7}{|c|}{$\begin{array}{l}\text { Comments and References } \\
\text { Density }=3.18 \mathrm{~g} / \mathrm{cm} 3 \text { and weight fractions from http://physics.nist.gov/cgi-bin/Star/compos.pl?matno=130 } \\
\text { (NIST 1998). } \\
\text { Density }=3.19 \mathrm{~g} / \mathrm{cm} 3 \text { on pg } 235 \text { of Knoll (2000). }\end{array}$} \\
\hline
\end{tabular}

\section{Calcium Oxide}

\begin{tabular}{llll}
\hline Formula $=$ & $\mathrm{CaO}$ & Molecular weight $(\mathrm{g} / \mathrm{mole})=$ & 56.0774 \\
Density $(\mathrm{g} / \mathrm{cm} 3)=$ & 3.300000 & Total atom density $($ atoms $/ \mathrm{b}-\mathrm{cm})=$ & $7.088 \mathrm{E}-02$
\end{tabular}

The above density is estimated to be accurate to 2 significant digits. Uncertainties are not addressed.

The following data were calculated from the input weight fractions.

\begin{tabular}{|c|c|c|c|c|c|c|}
\hline $\begin{array}{c}\text { Element } \\
\mathrm{O} \\
\mathrm{Ca}\end{array}$ & $\begin{array}{c}\text { Neutron ZA } \\
8016 \\
20000\end{array}$ & $\begin{array}{c}\text { Photon ZA } \\
8000 \\
20000\end{array}$ & $\begin{array}{c}\text { Weight } \\
\text { Fraction } \\
0.285299 \\
0.714701\end{array}$ & $\begin{array}{c}\text { Atom } \\
\text { Fraction } \\
0.499987 \\
0.500013\end{array}$ & $\begin{array}{c}\text { Atom } \\
\text { Density } \\
0.035437 \\
0.035439\end{array}$ & \\
\hline Total & & & 1.000000 & 1.000000 & 0.070877 & \\
\hline MCNP Form & \multicolumn{2}{|c|}{ Weight Fractions } & \multicolumn{2}{|c|}{ Atom Fractions } & \multicolumn{2}{|c|}{ Atom Densities } \\
\hline Neutrons & $\begin{array}{c}8016 \\
20000\end{array}$ & $\begin{array}{l}-0.285299 \\
-0.714701\end{array}$ & $\begin{array}{c}8016 \\
20000\end{array}$ & $\begin{array}{l}0.499987 \\
0.500013\end{array}$ & $\begin{array}{c}8016 \\
20000\end{array}$ & $\begin{array}{l}0.035437 \\
0.035439\end{array}$ \\
\hline Photons & $\begin{array}{c}8000 \\
20000\end{array}$ & $\begin{array}{l}-0.285299 \\
-0.714701\end{array}$ & $\begin{array}{c}8000 \\
20000\end{array}$ & $\begin{array}{l}0.499987 \\
0.500013\end{array}$ & $\begin{array}{c}8000 \\
20000\end{array}$ & $\begin{array}{l}0.035437 \\
0.035439\end{array}$ \\
\hline CEPXS Form: & $\begin{array}{c}\text { matname } \\
\text { density }\end{array}$ & $\begin{array}{c}\text { O } \\
\text { Ca } \\
\text { Calcium Oxide } \\
3.300000\end{array}$ & $\begin{array}{l}0.285299 \\
0.714701\end{array}$ & & & \\
\hline $\begin{array}{l}\text { Comments an } \\
\text { Density and we } \\
\text { Formula from } L\end{array}$ & $\begin{array}{l}\text { Peferences } \\
\text { it fractions } \mathrm{f} \\
(2008), \mathrm{pgs}\end{array}$ & $\begin{array}{l}\text { http://physics } \\
-55 \text {. }\end{array}$ & st.gov/cgi & $\operatorname{tar} /$ comp & natno $=$ & ST 1998). \\
\hline
\end{tabular}




\section{Calcium Sulfate}

\begin{tabular}{llll}
\hline Formula $=$ & CaSO4 & Molecular weight $(\mathrm{g} / \mathrm{mole})=$ & 136.1406 \\
Density $(\mathrm{g} / \mathrm{cm} 3)=$ & 2.960000 & Total atom density $($ atoms $/ \mathrm{b}-\mathrm{cm})=$ & $7.856 \mathrm{E}-02$
\end{tabular}

The above density is estimated to be accurate to 3 significant digits. Uncertainties are not addressed. The following data were calculated from the input weight fractions.

$\begin{array}{ccccccc}\frac{\text { Element }}{\mathrm{O}} & \frac{\text { Neutron ZA }}{8016} & \frac{\text { Photon ZA }}{8000} & & \begin{array}{c}\text { Weight } \\ \text { Fraction }\end{array} & \begin{array}{c}\text { Atom } \\ \text { Fraction }\end{array} & \begin{array}{c}\text { Atom } \\ \text { Density }\end{array} \\ \mathrm{S} & 16000 & 16000 & 0.470095 & 0.666678 & 0.052375 \\ \mathrm{Ca} & 20000 & 20000 & 0.294408 & 0.166644 & 0.013092 \\ \text { Total } & & & & & & \\ & & & & 1.000000 & 1.000000 & 0.078561\end{array}$

\begin{tabular}{|c|cccccc|}
\hline MCNP Form & \multicolumn{2}{c|}{ Weight Fractions } & \multicolumn{2}{c|}{ Atom Fractions } & \multicolumn{2}{c|}{ Atom Densities } \\
\hline Neutrons & 8016 & -0.470095 & 8016 & 0.666678 & 8016 & 0.052375 \\
& 16000 & -0.235497 & 16000 & 0.166644 & 16000 & 0.013092 \\
& 20000 & -0.294408 & 20000 & 0.166678 & 20000 & 0.013094 \\
Photons & 8000 & -0.470095 & 8000 & 0.666678 & 8000 & 0.052375 \\
& 16000 & -0.235497 & 16000 & 0.166644 & 16000 & 0.013092 \\
& 20000 & -0.294408 & 20000 & 0.166678 & 20000 & 0.013094 \\
& & & & & & \\
\hline CEPXS Form: & material & $\mathrm{O}$ & 0.470095 & & \\
& & $\mathrm{~S}$ & 0.235497 & & & \\
& & $\mathrm{Ca}$ & 0.294408 & & &
\end{tabular}

matname Calcium Sulfate

density 2.960000

\section{Comments and References}

http://physics.nist.gov/cgi-bin/Star/compos.pl?matno=132 (NIST 1998).

Formula from Lide (2008), pgs 4 - 56.

\section{Carbon Dioxide}

Formula $=\quad$ CO2

Density $(\mathrm{g} / \mathrm{cm} 3)=\quad 0.001842 \quad$ Total atom density $($ atoms $/ \mathrm{b}-\mathrm{cm})=\quad 7.562 \mathrm{E}-05$

The above density is estimated to be accurate to 4 significant digits. Uncertainties are not addressed.

The following data was calculated from the input formula.

\begin{tabular}{|c|c|c|c|c|c|}
\hline Element & Neutron ZA & Photon ZA & $\begin{array}{l}\text { Weight } \\
\text { Fraction }\end{array}$ & $\begin{array}{c}\text { Atom } \\
\text { Fraction }\end{array}$ & $\begin{array}{l}\text { Atom } \\
\text { Density }\end{array}$ \\
\hline $\mathrm{C}$ & 6000 & 6000 & 0.272912 & 0.333333 & 0.000025 \\
\hline 0 & 8016 & 8000 & 0.727088 & 0.666667 & 0.000050 \\
\hline Total & & & 1.000000 & 1.000000 & 0.000076 \\
\hline
\end{tabular}


PIET-43741-TM-963

PNNL-15870 Rev. 1

\begin{tabular}{|c|c|c|c|c|c|c|}
\hline MCNP Form & \multicolumn{2}{|c|}{ Weight Fractions } & \multicolumn{2}{|c|}{ Atom Fractions } & \multicolumn{2}{|c|}{ Atom Densities } \\
\hline \multirow[t]{2}{*}{ Neutrons } & 6000 & -0.272912 & 6000 & 0.333333 & 6000 & 0.000025 \\
\hline & 8016 & -0.727088 & 8016 & 0.666667 & 8016 & 0.000050 \\
\hline \multirow[t]{2}{*}{ Photons } & 6000 & -0.272912 & 6000 & 0.333333 & 6000 & 0.000025 \\
\hline & 8000 & -0.727088 & 8000 & 0.666667 & 8000 & 0.000050 \\
\hline \multirow[t]{3}{*}{ CEPXS Form: } & material & C & 0.272912 & & & \\
\hline & & $\mathrm{O}$ & 0.727088 & & & \\
\hline & $\begin{array}{c}\text { matname } \\
\text { density }\end{array}$ & $\begin{array}{c}\text { Carbon Diox } \\
0.001842\end{array}$ & & & & \\
\hline \multicolumn{7}{|c|}{$\begin{array}{l}\text { Comments and References } \\
\text { Density from http://physics.nis }\end{array}$} \\
\hline
\end{tabular}

\section{Carbon Tetrachloride}

\begin{tabular}{llll}
\hline Formula $=$ & $\mathrm{CCl} 4$ & Molecular weight $(\mathrm{g} / \mathrm{mole})=$ & 153.8227 \\
Density $(\mathrm{g} / \mathrm{cm} 3)=$ & 1.594000 & Total atom density $($ atoms $/ \mathrm{b}-\mathrm{cm})=$ & $3.120 \mathrm{E}-02$
\end{tabular}

The above density is estimated to be accurate to 4 significant digits. Uncertainties are not addressed.

The following data were calculated from the input weight fractions.

\begin{tabular}{|c|c|c|c|c|c|c|}
\hline Element & Neutron ZA & Photon ZA & $\begin{array}{l}\text { Weight } \\
\text { Fraction }\end{array}$ & $\begin{array}{l}\text { Atom } \\
\text { Fraction }\end{array}$ & $\begin{array}{c}\text { Atom } \\
\text { Density }\end{array}$ & \\
\hline $\mathrm{C}$ & 6000 & 6000 & $\overline{0.078083}$ & 0.200003 & 0.006241 & \\
\hline $\mathrm{Cl}$ & 17000 & 17000 & 0.921917 & 0.799997 & 0.024962 & \\
\hline Total & & & 1.000000 & 1.000000 & 0.031203 & \\
\hline MCNP Form & \multicolumn{2}{|c|}{ Weight Fractions } & \multicolumn{2}{|c|}{ Atom Fractions } & \multicolumn{2}{|c|}{ Atom Densities } \\
\hline Neutrons & 6000 & -0.078083 & 6000 & 0.200003 & 6000 & 0.006241 \\
\hline & 17000 & -0.921917 & 17000 & 0.799997 & 17000 & 0.024962 \\
\hline \multirow[t]{2}{*}{ Photons } & 6000 & -0.078083 & 6000 & 0.200003 & 6000 & 0.006241 \\
\hline & 17000 & -0.921917 & 17000 & 0.799997 & 17000 & 0.024962 \\
\hline \multirow[t]{3}{*}{ CEPXS Form: } & material & C & 0.078083 & & & \\
\hline & & $\mathrm{Cl}$ & 0.921917 & & & \\
\hline & $\begin{array}{l}\text { matname } \\
\text { density }\end{array}$ & \multicolumn{2}{|c|}{$\begin{array}{l}\text { Carbon Tetrachloride } \\
1.594000\end{array}$} & & & \\
\hline
\end{tabular}




\section{Carbon, Activated}

\begin{tabular}{|c|c|c|c|}
\hline Formula $=$ & C & Molecular weight $(\mathrm{g} / \mathrm{mole})=$ & - \\
\hline Density $(\mathrm{g} / \mathrm{cm} 3)=$ & 0.320000 & Total atom density $($ atoms $/ \mathrm{b}-\mathrm{cm})=$ & 1.604E-02 \\
\hline
\end{tabular}

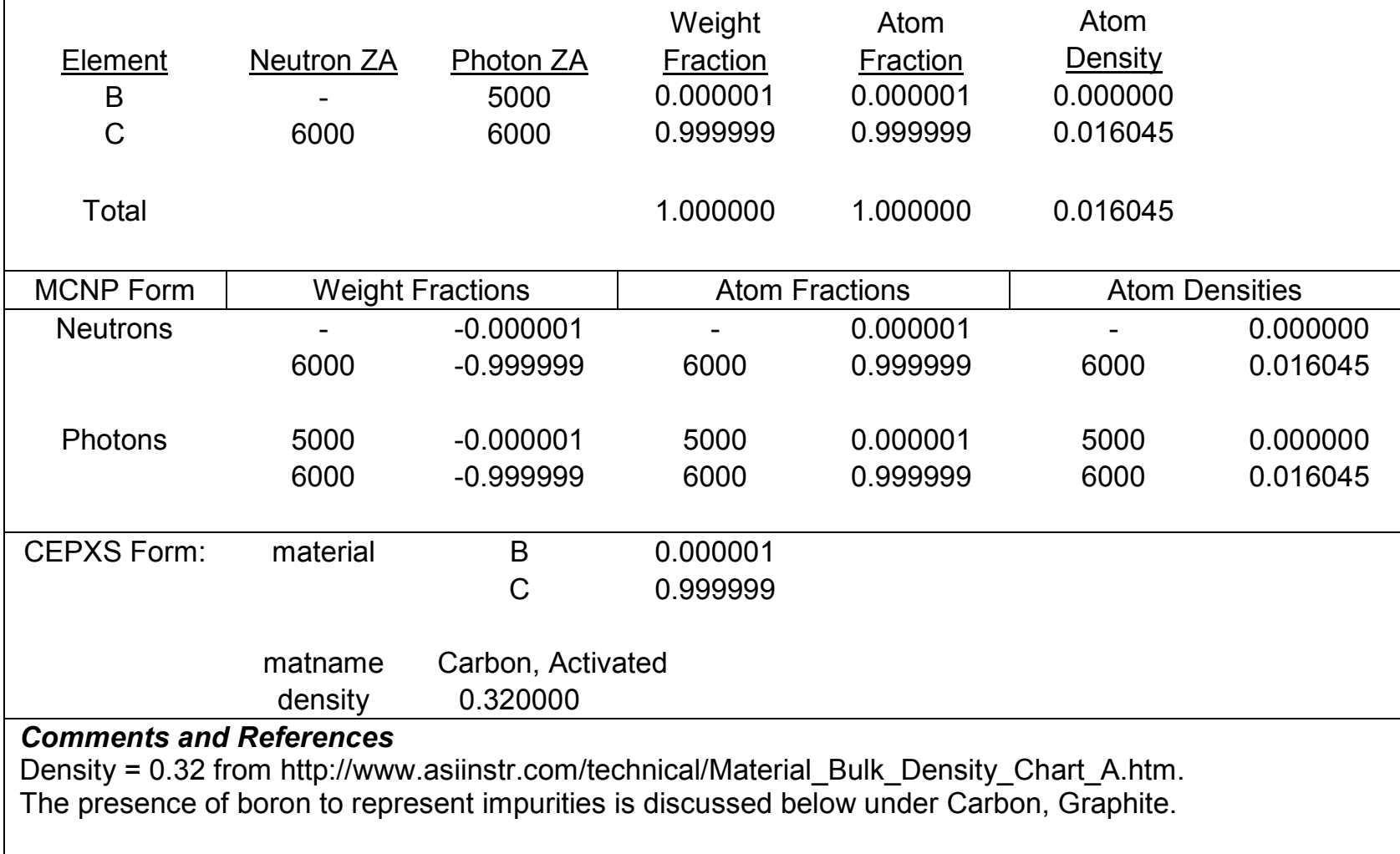

\section{Carbon, Amorphous}

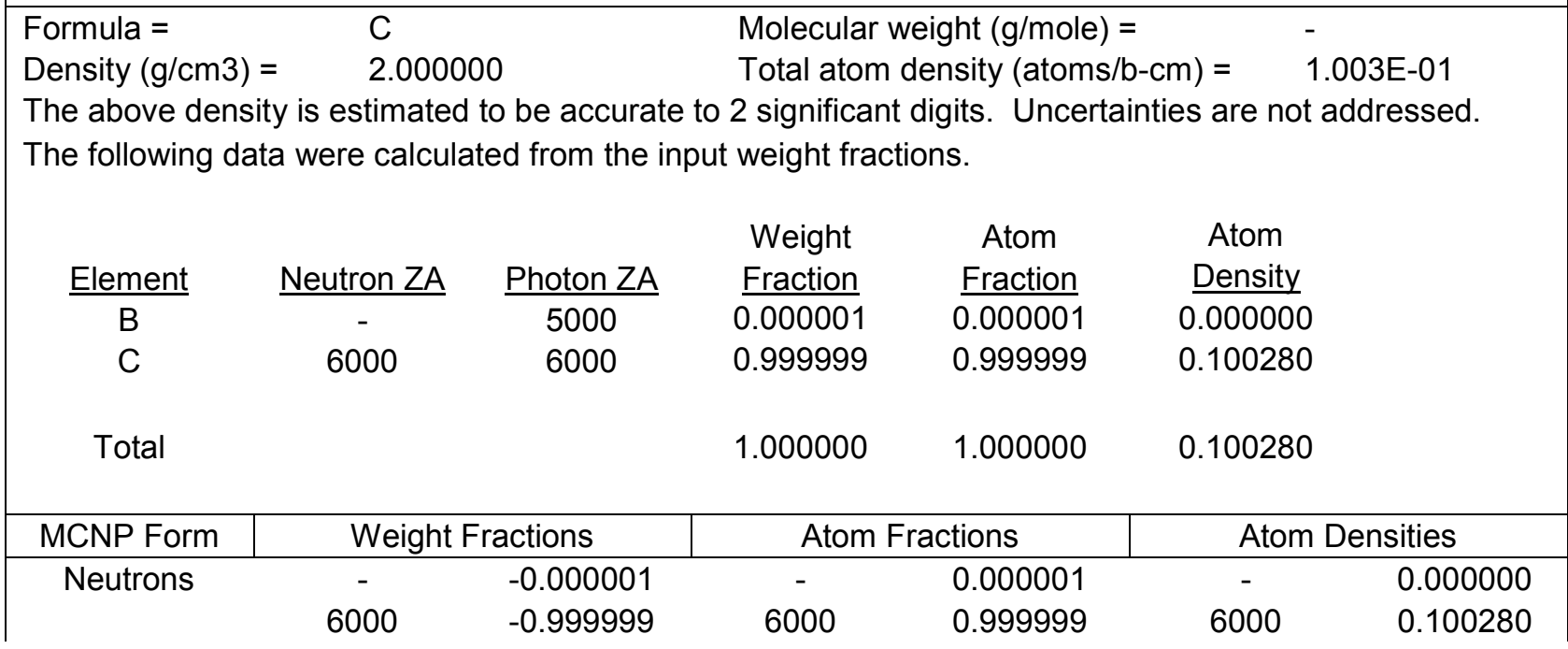


PIET-43741-TM-963

PNNL-15870 Rev. 1

\begin{tabular}{|c|c|c|c|c|c|c|}
\hline Photons & $\begin{array}{l}5000 \\
6000\end{array}$ & $\begin{array}{l}-0.000001 \\
-0.999999\end{array}$ & $\begin{array}{l}5000 \\
6000\end{array}$ & $\begin{array}{l}0.000001 \\
0.999999\end{array}$ & $\begin{array}{l}5000 \\
6000\end{array}$ & $\begin{array}{l}0.000000 \\
0.100280\end{array}$ \\
\hline CEPXS Form: & $\begin{array}{c}\text { matname } \\
\text { density }\end{array}$ & $\begin{array}{c}\text { B } \\
\text { C } \\
\text { Carbon, Am } \\
2.000000\end{array}$ & $\begin{array}{l}0.000001 \\
0.999999 \\
\text { hous }\end{array}$ & & & \\
\hline \multicolumn{7}{|c|}{$\begin{array}{l}\text { Comments and References } \\
\text { Density }=2.0 \mathrm{~g} / \mathrm{cm} 3 \text { from http://physics.nist.gov/cgi-bin/Star/compos.pl?matno=006 (NIST 1998). } \\
\text { The presence of boron to represent impurities is discussed below under "Carbon, Graphite." }\end{array}$} \\
\hline
\end{tabular}

\section{Carbon, Graphite (Reactor Grade)}

\begin{tabular}{lll}
\hline Formula $=$ & $\mathrm{C}$ & Molecular weight $(\mathrm{g} / \mathrm{mole})=$ \\
Density $(\mathrm{g} / \mathrm{cm} 3)=$ & 1.700000 & Total atom density $($ atoms $/ \mathrm{b}-\mathrm{cm})=\overline{-}$ \\
\hline
\end{tabular}

The above density is estimated to be accurate to 2 significant digits. Uncertainties are not addressed.

The following data were calculated from the input weight fractions.

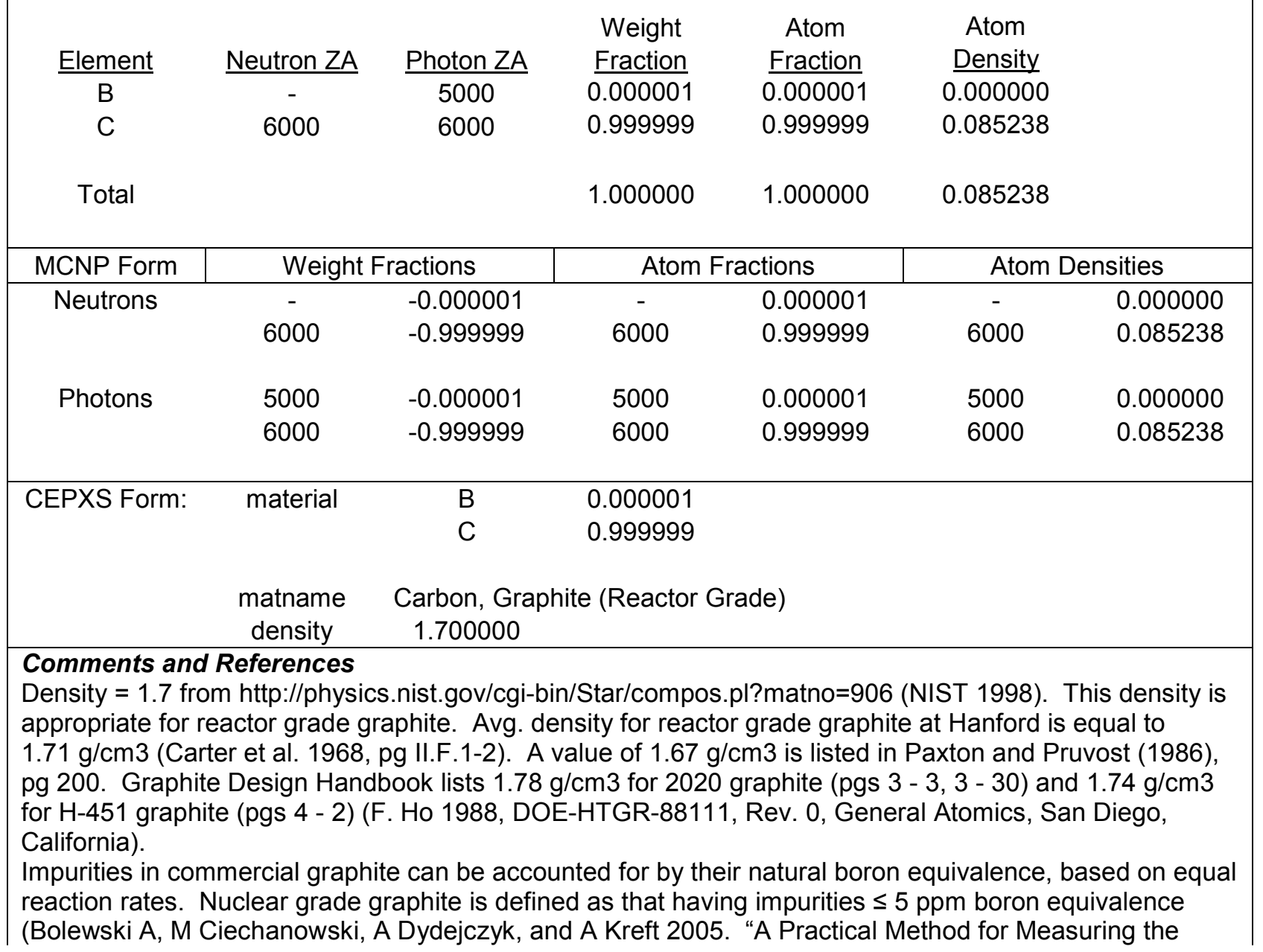


Boron Equivalent of Graphite Impurity." Nuclear Instruments and Methods in Physics Research, Section B 237(3-4):602-612). A boron equivalence of $1.0 \mathrm{ppm}$ was selected for nuclear graphite based on Evaluation of High Temperature Gas-Cooled Reactor Physics Experiments as VHTR Benchmark Problems, by T.A. Taiwo, et al., ANL-GenIV-059, Sept. 15, 2005. A detailed list of impurities in graphite is at http://www.graphite-eng.com/materials.html.

The density and boron equivalence of impurities can vary significantly for different types of graphite, and the boron equivalence of non-burnable impurities should be distinguished from the boron equivalence of burnable impurities in burnup calculations. The user should use values appropriate for his purpose.

\section{Cat Litter (Clumping)}

\begin{tabular}{llll}
\hline Formula $=$ & Na0.2Ca0.1Al2Si4O10 & Molecular weight $(\mathrm{g} / \mathrm{mole})=$ & - \\
Density $(\mathrm{g} / \mathrm{cm} 3)=$ & $(\mathrm{OH}) 2(\mathrm{H} 2 \mathrm{O}) 10$ & 1.100000 & Total atom density $($ atoms $/ \mathrm{b}-\mathrm{cm})=\quad 6.070 \mathrm{E}-02$
\end{tabular}

The above density is estimated to be accurate to 1 significant digits. Uncertainties are not addressed.

The following data were calculated from the input weight fractions.

\begin{tabular}{|c|c|c|c|c|c|c|}
\hline Element & Neutron ZA & Photon ZA & $\begin{array}{l}\text { Weight } \\
\text { Fraction }\end{array}$ & $\begin{array}{c}\text { Atom } \\
\text { Fraction }\end{array}$ & $\begin{array}{l}\text { Atom } \\
\text { Density }\end{array}$ & \\
\hline $\mathrm{H}$ & 1001 & 1000 & 0.040400 & 0.437442 & 0.026552 & \\
\hline 0 & 8016 & 8000 & 0.641100 & 0.437316 & 0.026544 & \\
\hline $\mathrm{Na}$ & 11023 & 11000 & 0.008400 & 0.003988 & 0.000242 & \\
\hline $\mathrm{Al}$ & 13027 & 13000 & 0.098300 & 0.039761 & 0.002413 & \\
\hline $\mathrm{Si}$ & 14000 & 14000 & 0.204600 & 0.079505 & 0.004826 & \\
\hline $\mathrm{Ca}$ & 20000 & 20000 & 0.007300 & 0.001988 & 0.000121 & \\
\hline Total & & & 1.000100 & 1.000000 & 0.060697 & \\
\hline MCNP Form & \multicolumn{2}{|c|}{ Weight Fractions } & \multicolumn{2}{|c|}{ Atom Fractions } & \multicolumn{2}{|c|}{ Atom Densities } \\
\hline \multirow[t]{6}{*}{ Neutrons } & 1001 & -0.040400 & 1001 & 0.437442 & 1001 & 0.026552 \\
\hline & 8016 & -0.641100 & 8016 & 0.437316 & 8016 & 0.026544 \\
\hline & 11023 & -0.008400 & 11023 & 0.003988 & 11023 & 0.000242 \\
\hline & 13027 & -0.098300 & 13027 & 0.039761 & 13027 & 0.002413 \\
\hline & 14000 & -0.204600 & 14000 & 0.079505 & 14000 & 0.004826 \\
\hline & 20000 & -0.007300 & 20000 & 0.001988 & 20000 & 0.000121 \\
\hline \multirow[t]{6}{*}{ Photons } & 1000 & -0.040400 & 1000 & 0.437442 & 1000 & 0.026552 \\
\hline & 8000 & -0.641100 & 8000 & 0.437316 & 8000 & 0.026544 \\
\hline & 11000 & -0.008400 & 11000 & 0.003988 & 11000 & 0.000242 \\
\hline & 13000 & -0.098300 & 13000 & 0.039761 & 13000 & 0.002413 \\
\hline & 14000 & -0.204600 & 14000 & 0.079505 & 14000 & 0.004826 \\
\hline & 20000 & -0.007300 & 20000 & 0.001988 & 20000 & 0.000121 \\
\hline \multirow[t]{5}{*}{ CEPXS Form: } & material & $\mathrm{H}$ & 0.040400 & & & \\
\hline & & 0 & 0.641100 & & & \\
\hline & & $\mathrm{Na}$ & 0.008400 & & & \\
\hline & & $\mathrm{Al}$ & 0.098300 & & & \\
\hline & & $\mathrm{Si}$ & 0.204600 & & & \\
\hline
\end{tabular}




\begin{tabular}{|l|} 
matname Ca Cat Litter (Clumping) \\
density 1.100000
\end{tabular}

\section{Cat Litter (Non-clumping)}

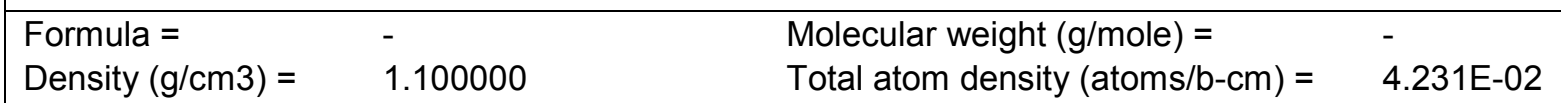

The above density is estimated to be accurate to 1 significant digits. Uncertainties are not addressed. The following data were calculated from the input weight fractions.

\begin{tabular}{|c|c|c|c|c|c|c|}
\hline Element & Neutron ZA & Photon ZA & $\begin{array}{l}\text { Weight } \\
\text { Fraction }\end{array}$ & $\begin{array}{l}\text { Atom } \\
\text { Fraction }\end{array}$ & $\begin{array}{c}\text { Atom } \\
\text { Density }\end{array}$ & \\
\hline $\mathrm{H}$ & 1001 & 1000 & $\overline{0.013732}$ & $\overline{0.213314}$ & 0.009025 & \\
\hline $\mathrm{O}$ & 8016 & 8000 & 0.539919 & 0.528366 & 0.022355 & \\
\hline $\mathrm{Na}$ & 11023 & 11000 & 0.043271 & 0.029469 & 0.001247 & \\
\hline $\mathrm{Mg}$ & 12000 & 12000 & 0.050466 & 0.032510 & 0.001375 & \\
\hline $\mathrm{Al}$ & 13027 & 13000 & 0.052132 & 0.030252 & 0.001280 & \\
\hline $\mathrm{Si}$ & 14000 & 14000 & 0.293185 & 0.163444 & 0.006915 & \\
\hline $\mathrm{K}$ & 19000 & 19000 & 0.003765 & 0.001508 & 0.000064 & \\
\hline $\mathrm{Ca}$ & 20000 & 20000 & 0.001341 & 0.000524 & 0.000022 & \\
\hline $\mathrm{Fe}$ & 26000 & 26000 & 0.002188 & 0.000613 & 0.000026 & \\
\hline Total & & & 1.000000 & 1.000000 & 0.042309 & \\
\hline MCNP Form & \multicolumn{2}{|c|}{ Weight Fractions } & \multicolumn{2}{|c|}{ Atom Fractions } & \multicolumn{2}{|c|}{ Atom Densities } \\
\hline \multirow[t]{7}{*}{ Neutrons } & 1001 & -0.013732 & 1001 & 0.213314 & 1001 & 0.009025 \\
\hline & 8016 & -0.539919 & 8016 & 0.528366 & 8016 & 0.022355 \\
\hline & 11023 & -0.043271 & 11023 & 0.029469 & 11023 & 0.001247 \\
\hline & 12000 & -0.050466 & 12000 & 0.032510 & 12000 & 0.001375 \\
\hline & 13027 & -0.052132 & 13027 & 0.030252 & 13027 & 0.001280 \\
\hline & 14000 & -0.293185 & 14000 & 0.163444 & 14000 & 0.006915 \\
\hline & 19000 & -0.003765 & 19000 & 0.001508 & 19000 & 0.000064 \\
\hline
\end{tabular}


PIET-43741-TM-963

PNNL-15870 Rev. 1

\begin{tabular}{|c|c|c|c|c|c|c|}
\hline & 20000 & -0.001341 & 20000 & 0.000524 & 20000 & 0.000022 \\
\hline & 26000 & -0.002188 & 26000 & 0.000613 & 26000 & 0.000026 \\
\hline Photons & 1000 & -0.013732 & 1000 & 0.213314 & 1000 & 0.009025 \\
\hline & 8000 & -0.539919 & 8000 & 0.528366 & 8000 & 0.022355 \\
\hline & 11000 & -0.043271 & 11000 & 0.029469 & 11000 & 0.001247 \\
\hline & 12000 & -0.050466 & 12000 & 0.032510 & 12000 & 0.001375 \\
\hline & 13000 & -0.052132 & 13000 & 0.030252 & 13000 & 0.001280 \\
\hline & 14000 & -0.293185 & 14000 & 0.163444 & 14000 & 0.006915 \\
\hline & 19000 & -0.003765 & 19000 & 0.001508 & 19000 & 0.000064 \\
\hline & 20000 & -0.001341 & 20000 & 0.000524 & 20000 & 0.000022 \\
\hline & 26000 & -0.002188 & 26000 & 0.000613 & 26000 & 0.000026 \\
\hline CEPXS Form: & material & $\mathrm{H}$ & 0.013732 & & & \\
\hline & & $\mathrm{O}$ & 0.539919 & & & \\
\hline & & $\mathrm{Na}$ & 0.043271 & & & \\
\hline & & $\mathrm{Mg}$ & 0.050466 & & & \\
\hline & & $\mathrm{Al}$ & 0.052132 & & & \\
\hline & & Si & 0.293185 & & & \\
\hline & & $\mathrm{K}$ & 0.003765 & & & \\
\hline & & $\mathrm{Ca}$ & 0.001341 & & & \\
\hline & & $\mathrm{Fe}$ & 0.002188 & & & \\
\hline & $\begin{array}{c}\text { matname } \\
\text { density }\end{array}$ & $\begin{array}{c}\text { Cat Litter (Nc } \\
1.100000\end{array}$ & lumping) & & & \\
\hline $\begin{array}{l}\text { Comments an } \\
\text { Cat litter can be } \\
\text { Non-clumping } \\
\text { (http://en.wikip } \\
\text { The cat litter sp } \\
\text { earth, } 33 \text { wt\% } \\
\text { from } 0.55 \text { to } 1 . \\
\text { The density wa } \\
\text { in some of the } \\
\text { Cat litter may a }\end{array}$ & $\begin{array}{l}\text { References } \\
\text { ther clump } \\
\text { litter is ofte } \\
\text { a.org/wiki/C } \\
\text { ified here is } \\
\text { iolite, and } 3 \\
\mathrm{~cm} 3 \text { at ww } \\
\text { hosen to bc } \\
\text { ace betwee } \\
\text { be called " }\end{array}$ & $\begin{array}{l}\text { or non-clump } \\
\text { nade of zeolit } \\
\text { litter). } \\
\text { ssumed to be } \\
\text { wt\% zeolite. } \\
\text { purapet.com/d } \\
\text { ne maximum } \\
\text { he diatoms in } \\
\text { ty litter." }\end{array}$ & $\begin{array}{l}\text { iatomaceo } \\
\text {-clumping } \\
\text { sity for vari } \\
\text { nload/produ } \\
\text { e of } 1.1 \mathrm{~g} / \mathrm{c} \\
\text { diatomace }\end{array}$ & $\begin{array}{l}\text { arth, and se } \\
\text { itter compo } \\
\text { types of no } \\
\text { ochure.pdf } \\
\text { assuming } \\
\text { earth. }\end{array}$ & $\begin{array}{l}34 \text { wt } \% \\
\text { nping ca } \\
\text { e sepioli }\end{array}$ & $\begin{array}{l}\text { maceous } \\
\text { range } \\
\text { d zeolite fill }\end{array}$ \\
\hline
\end{tabular}

\section{Cellulose Acetate}

\begin{tabular}{llll}
\hline Formula $=$ & - & Molecular weight $(\mathrm{g} / \mathrm{mole})=$ & - \\
Density $(\mathrm{g} / \mathrm{cm} 3)=$ & 1.420000 & Total atom density $($ atoms $/ \mathrm{b}-\mathrm{cm})=$ & $1.108 \mathrm{E}-01$
\end{tabular}

The above density is estimated to be accurate to 3 significant digits. Uncertainties are not addressed.

The following data were calculated from the input weight fractions.

\begin{tabular}{|c|c|c|c|c|c|}
\hline Element & Neutron ZA & Photon ZA & $\begin{array}{l}\text { Weight } \\
\text { Fraction }\end{array}$ & $\begin{array}{c}\text { Atom } \\
\text { Fraction }\end{array}$ & $\begin{array}{l}\text { Atom } \\
\text { Density }\end{array}$ \\
\hline $\mathrm{H}$ & 1001 & 1000 & $\overline{0.062162}$ & $\overline{0.476179}$ & 0.052739 \\
\hline C & 6000 & 6000 & 0.444462 & 0.285724 & 0.031645 \\
\hline 0 & 8016 & 8000 & 0.493376 & 0.238097 & 0.026370 \\
\hline
\end{tabular}


PIET-43741-TM-963

PNNL-15870 Rev. 1

\begin{tabular}{|c|c|c|c|c|c|c|}
\hline Total & & & 1.000000 & 1.000000 & 0.11075 & \\
\hline MCNP Form & Weigh & ractions & Atom & ctions & Ator & sities \\
\hline Neutrons & 1001 & -0.062162 & 1001 & 0.476179 & 1001 & 0.052739 \\
\hline & 6000 & -0.444462 & 6000 & 0.285724 & 6000 & 0.031645 \\
\hline & 8016 & -0.493376 & 8016 & 0.238097 & 8016 & 0.026370 \\
\hline Photons & 1000 & -0.062162 & 1000 & 0.476179 & 1000 & 0.052739 \\
\hline & 6000 & -0.444462 & 6000 & 0.285724 & 6000 & 0.031645 \\
\hline & 8000 & -0.493376 & 8000 & 0.238097 & 8000 & 0.026370 \\
\hline CEPXS Form: & material & $\mathrm{H}$ & 0.062162 & & & \\
\hline & & $\mathrm{C}$ & 0.444462 & & & \\
\hline & & $\mathrm{O}$ & 0.493376 & & & \\
\hline & $\begin{array}{c}\text { matname } \\
\text { density }\end{array}$ & $\begin{array}{c}\text { Cellulose Ac } \\
1.420000\end{array}$ & & & & \\
\hline $\begin{array}{l}\text { Comments anc } \\
\text { Density and we } \\
\text { Also called "cell }\end{array}$ & $\begin{array}{l}\text { eferences } \\
\text { t fractions } \mathrm{f} \\
\text { nane." }\end{array}$ & http://phy & st.gov/cg & $\mathrm{ar} / \mathrm{com}$ & $\operatorname{tno}=$ & ST 1998). \\
\hline
\end{tabular}

\section{Celotex}

\begin{tabular}{llll}
\hline Formula $=$ & $\mathrm{C} 6 \mathrm{H} 10 \mathrm{O} 5$ & Molecular weight $(\mathrm{g} / \mathrm{mole})=$ & 162.1406 \\
Density $(\mathrm{g} / \mathrm{cm} 3)=$ & 0.240000 & Total atom density $($ atoms $/ \mathrm{b}-\mathrm{cm})=$ & $1.872 \mathrm{E}-02$
\end{tabular}

The above density is estimated to be accurate to 2 significant digits. Uncertainties are not addressed.

The following data was calculated from the input formula.

\begin{tabular}{|c|c|c|c|c|c|c|}
\hline Element & Neutron ZA & Photon ZA & $\begin{array}{l}\text { Weight } \\
\text { Fraction }\end{array}$ & $\begin{array}{c}\text { Atom } \\
\text { Fraction }\end{array}$ & $\begin{array}{l}\text { Atom } \\
\text { Density }\end{array}$ & \\
\hline $\mathrm{H}$ & 1001 & 1000 & 0.062165 & 0.476190 & 0.00891 & \\
\hline $\mathrm{C}$ & 6000 & 6000 & 0.444455 & 0.285714 & 0.00534 & \\
\hline 0 & 8016 & 8000 & 0.493380 & 0.238095 & 0.00445 & \\
\hline Total & & & 1.000000 & 1.000000 & 0.01871 & \\
\hline MCNP Form & \multicolumn{2}{|c|}{ Weight Fractions } & \multicolumn{2}{|c|}{ Atom Fractions } & \multicolumn{2}{|c|}{ Atom Densities } \\
\hline \multirow[t]{3}{*}{ Neutrons } & 1001 & -0.062165 & 1001 & 0.476190 & 1001 & 0.008914 \\
\hline & 6000 & -0.444455 & 6000 & 0.285714 & 6000 & 0.005348 \\
\hline & 8016 & -0.493380 & 8016 & 0.238095 & 8016 & 0.004457 \\
\hline \multirow[t]{3}{*}{ Photons } & 1000 & -0.062165 & 1000 & 0.476190 & 1000 & 0.008914 \\
\hline & 6000 & -0.444455 & 6000 & 0.285714 & 6000 & 0.005348 \\
\hline & 8000 & -0.493380 & 8000 & 0.238095 & 8000 & 0.004457 \\
\hline \multirow[t]{2}{*}{ CEPXS Form: } & material & $\mathrm{H}$ & 0.062165 & & & \\
\hline & & C & 0.444455 & & & \\
\hline
\end{tabular}




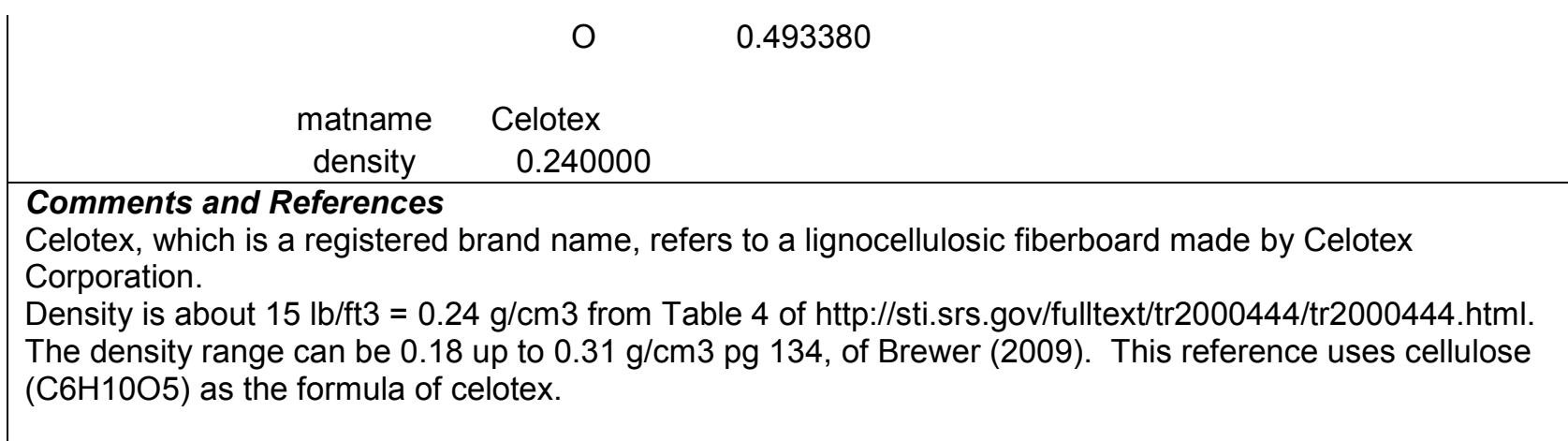

\section{Ceric Sulfate Dosimeter Solution}

\begin{tabular}{|c|c|c|c|}
\hline$a=$ & $\mathrm{H} 2 \mathrm{O}: \mathrm{Ce} 2 \mathrm{SO} 4$ & $\begin{array}{l}\text { Molecular weight }(\mathrm{g} / \mathrm{mole})= \\
\text { Total atom density }(\text { atoms } / \mathrm{b}-\mathrm{cm})=\end{array}$ & $1.005 \mathrm{E}-01$ \\
\hline
\end{tabular}

The above density is estimated to be accurate to 3 significant digits. Uncertainties are not addressed.

The following data were calculated from the input weight fractions.

\begin{tabular}{|c|c|c|c|c|c|c|}
\hline Element & Neutron ZA & Photon ZA & $\begin{array}{l}\text { Weight } \\
\text { Fraction }\end{array}$ & $\begin{array}{c}\text { Atom } \\
\text { Fraction }\end{array}$ & $\begin{array}{l}\text { Atom } \\
\text { Density }\end{array}$ & \\
\hline $\mathrm{H}$ & 1001 & 1000 & 0.107596 & 0.659087 & 0.066214 & \\
\hline $\mathrm{N}$ & 7014 & 7000 & 0.000800 & 0.000353 & 0.000035 & \\
\hline $\mathrm{O}$ & 8016 & 8000 & 0.874976 & 0.337656 & 0.033922 & \\
\hline$S$ & 16000 & 16000 & 0.014627 & 0.002816 & 0.000283 & \\
\hline $\mathrm{Ce}$ & - & 58000 & 0.002001 & 0.000088 & 0.000009 & \\
\hline Total & & & 1.000000 & 1.000000 & 0.100463 & \\
\hline MCNP Form & \multicolumn{2}{|c|}{ Weight Fractions } & \multicolumn{2}{|c|}{ Atom Fractions } & \multicolumn{2}{|c|}{ Atom Densities } \\
\hline \multirow[t]{5}{*}{ Neutrons } & 1001 & -0.107596 & 1001 & 0.659087 & 1001 & 0.066214 \\
\hline & 7014 & -0.000800 & 7014 & 0.000353 & 7014 & 0.000035 \\
\hline & 8016 & -0.874976 & 8016 & 0.337656 & 8016 & 0.033922 \\
\hline & 16000 & -0.014627 & 16000 & 0.002816 & 16000 & 0.000283 \\
\hline & - & -0.002001 & - & 0.000088 & - & 0.000009 \\
\hline \multirow[t]{5}{*}{ Photons } & 1000 & -0.107596 & 1000 & 0.659087 & 1000 & 0.066214 \\
\hline & 7000 & -0.000800 & 7000 & 0.000353 & 7000 & 0.000035 \\
\hline & 8000 & -0.874976 & 8000 & 0.337656 & 8000 & 0.033922 \\
\hline & 16000 & -0.014627 & 16000 & 0.002816 & 16000 & 0.000283 \\
\hline & 58000 & -0.002001 & 58000 & 0.000088 & 58000 & 0.000009 \\
\hline \multirow[t]{6}{*}{ CEPXS Form: } & material & $\mathrm{H}$ & 0.107596 & & & \\
\hline & & $\mathrm{N}$ & 0.000800 & & & \\
\hline & & $\mathrm{O}$ & 0.874976 & & & \\
\hline & & $S$ & 0.014627 & & & \\
\hline & & $\mathrm{Ce}$ & 0.002001 & & & \\
\hline & matname & \multicolumn{5}{|c|}{ Ceric Sulfate Dosimeter Solution } \\
\hline
\end{tabular}


density 1.030000

Comments and References

Density and weight fractions from http://physics.nist.gov/cgi-bin/Star/compos.pl?matno=139 (NIST 1998).

\section{Cerium Fluoride}

\begin{tabular}{llll}
\hline Formula $=$ & CeF3 & Molecular weight $(\mathrm{g} / \mathrm{mole})=$ & 197.1112096 \\
Density $(\mathrm{g} / \mathrm{cm} 3)=$ & 6.160000 & Total atom density $($ atoms $/ \mathrm{b}-\mathrm{cm})=$ & $7.528 \mathrm{E}-02$
\end{tabular}

The above density is estimated to be accurate to 3 significant digits. Uncertainties are not addressed. The following data was calculated from the input formula.

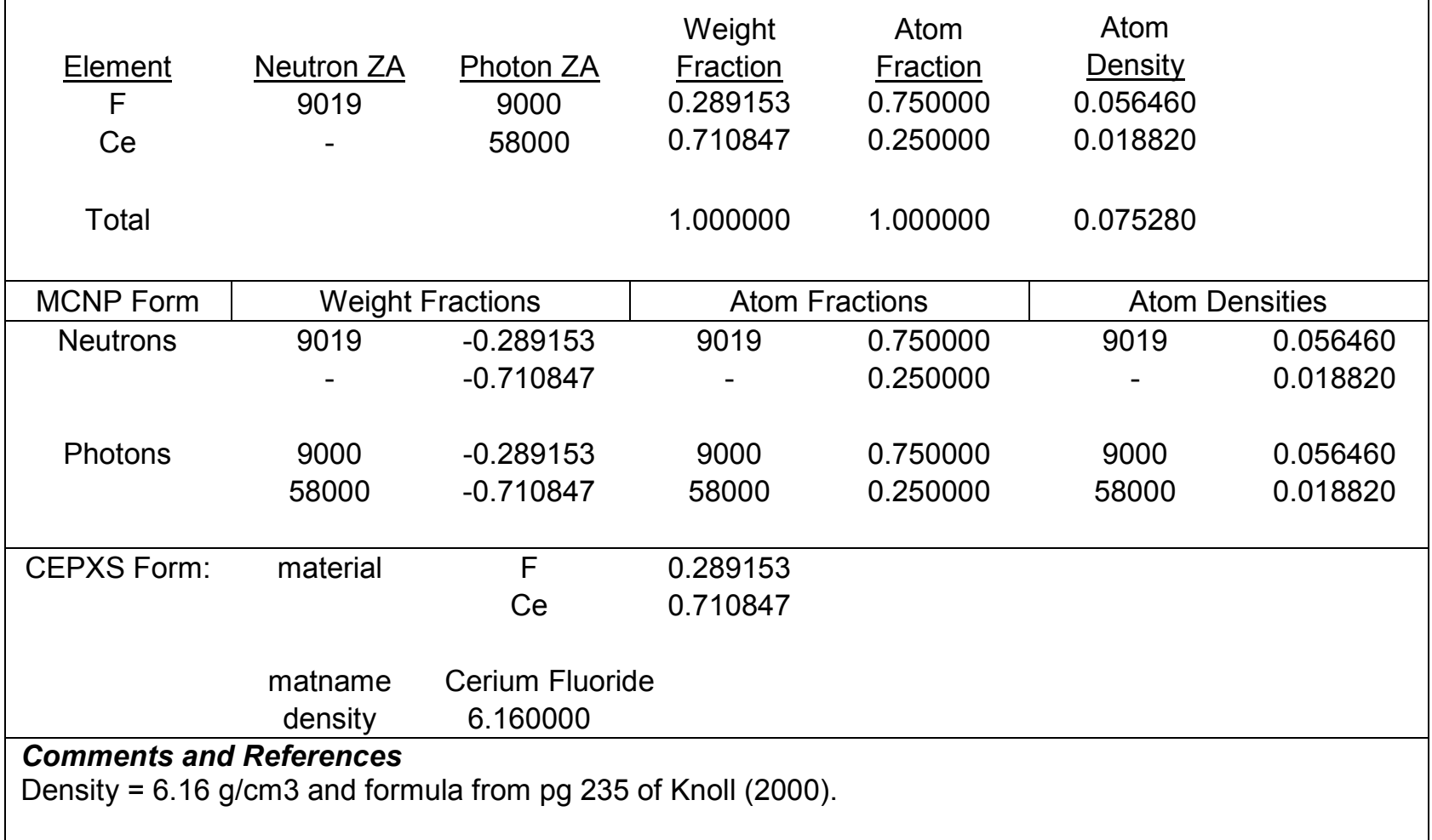

\section{Cesium lodide}

Formula $=\quad \mathrm{Csl}$

Density $(\mathrm{g} / \mathrm{cm} 3)=4.510000$

Molecular weight $(\mathrm{g} / \mathrm{mole})=$

259.80992

Total atom density $($ atoms $/ \mathrm{b}-\mathrm{cm})=2.091 \mathrm{E}-02$

The above density is estimated to be accurate to 3 significant digits. Uncertainties are not addressed.

The following data were calculated from the input weight fractions.

$\begin{array}{cccccc}\text { Element } & \text { Neutron ZA } & \text { Photon ZA } & \begin{array}{c}\text { Weight } \\ \text { Fraction }\end{array} & \begin{array}{c}\text { Atom } \\ \text { Fraction }\end{array} & \begin{array}{c}\text { Atom } \\ \text { Density }\end{array} \\ & 53127 & 53000 & 0.488451 & 0.500000 & 0.010454 \\ \text { Cs } & 55133 & 55000 & 0.511549 & 0.500000 & 0.010454\end{array}$


PIET-43741-TM-963

PNNL-15870 Rev. 1

\begin{tabular}{|c|c|c|c|c|c|c|}
\hline Total & & & 1.000000 & 1.000000 & 0.020907 & \\
\hline MCNP Form & \multicolumn{2}{|c|}{ Weight Fractions } & \multicolumn{2}{|c|}{ Atom Fractions } & \multicolumn{2}{|c|}{ Atom Densities } \\
\hline \multirow[t]{2}{*}{ Neutrons } & 53127 & -0.488451 & 53127 & 0.500000 & 53127 & 0.010454 \\
\hline & 55133 & -0.511549 & 55133 & 0.500000 & 55133 & 0.010454 \\
\hline \multirow[t]{2}{*}{ Photons } & 53000 & -0.488451 & 53000 & 0.500000 & 53000 & 0.010454 \\
\hline & 55000 & -0.511549 & 55000 & 0.500000 & 55000 & 0.010454 \\
\hline \multirow[t]{3}{*}{ CEPXS Form: } & material & $\mathrm{I}$ & 0.488451 & & & \\
\hline & & Cs & 0.511549 & & & \\
\hline & $\begin{array}{l}\text { matname } \\
\text { density }\end{array}$ & $\begin{array}{c}\text { Cesium lodide } \\
4.510000\end{array}$ & & & & \\
\hline
\end{tabular}

\section{Chromium}

\begin{tabular}{llll}
\hline Formula $=$ & $\mathrm{Cr}$ & Molecular weight $(\mathrm{g} / \mathrm{mole})=$ & 51.9961 \\
Density $(\mathrm{g} / \mathrm{cm} 3)=$ & 7.180000 & Total atom density $($ atoms $/ \mathrm{b}-\mathrm{cm})=$ & $8.316 \mathrm{E}-02$
\end{tabular}

The above density is estimated to be accurate to 3 significant digits. Uncertainties are not addressed.

The following data was calculated from the input formula.

\begin{tabular}{|c|c|c|c|c|c|c|}
\hline$\frac{\text { Element }}{\mathrm{Cr}}$ & $\frac{\text { Neutron ZA }}{24000}$ & $\frac{\text { Photon ZA }}{24000}$ & $\begin{array}{c}\text { Weight } \\
\text { Fraction } \\
1.000000\end{array}$ & $\begin{array}{c}\text { Atom } \\
\frac{\text { Fraction }}{1.000000}\end{array}$ & $\begin{array}{c}\text { Atom } \\
\text { Density } \\
0.083158\end{array}$ & \\
\hline Total & & & 1.000000 & 1.000000 & 0.083158 & \\
\hline MCNP Form & \multicolumn{2}{|c|}{ Weight Fractions } & \multicolumn{2}{|c|}{ Atom Fractions } & \multicolumn{2}{|c|}{ Atom Densities } \\
\hline Neutrons & 24000 & -1.000000 & 24000 & 1.000000 & 24000 & 0.083158 \\
\hline Photons & 24000 & -1.000000 & 24000 & 1.000000 & 24000 & 0.083158 \\
\hline CEPXS Form: & $\begin{array}{l}\text { material } \\
\text { matname } \\
\text { density }\end{array}$ & $\begin{array}{c}\mathrm{Cr} \\
\text { Chromium } \\
7.180000\end{array}$ & 1.000000 & & & \\
\hline
\end{tabular}




\section{Clay}

Formula $=$

Density $(\mathrm{g} / \mathrm{cm} 3)=2.200000$

Molecular weight $(\mathrm{g} / \mathrm{mole})=$

Total atom density $($ atoms $/ \mathrm{b}-\mathrm{cm})=6.333 \mathrm{E}-02$

The above density is estimated to be accurate to 3 significant digits. Uncertainties are not addressed.

The following data were calculated from the input weight fractions.

\begin{tabular}{|c|c|c|c|c|c|c|}
\hline Element & Neutron ZA & Photon ZA & $\begin{array}{l}\text { Weight } \\
\text { Fraction }\end{array}$ & $\begin{array}{c}\text { Atom } \\
\text { Fraction }\end{array}$ & $\begin{array}{c}\text { Atom } \\
\text { Density }\end{array}$ & \\
\hline 0 & 8016 & 8000 & 0.484345 & 0.633300 & 0.040107 & \\
\hline $\mathrm{Na}$ & 11023 & 11000 & 0.007608 & 0.006923 & 0.000438 & \\
\hline $\mathrm{Mg}$ & 12000 & 12000 & 0.010691 & 0.009202 & 0.000583 & \\
\hline $\mathrm{Al}$ & 13027 & 13000 & 0.122125 & 0.094689 & 0.005997 & \\
\hline $\mathrm{Si}$ & 14000 & 14000 & 0.294194 & 0.219134 & 0.013878 & \\
\hline $\mathrm{P}$ & 15031 & 15000 & 0.000113 & 0.000076 & 0.000005 & \\
\hline $\mathrm{K}$ & 19000 & 19000 & 0.020427 & 0.010930 & 0.000692 & \\
\hline $\mathrm{Ca}$ & 20000 & 20000 & 0.018957 & 0.009895 & 0.000627 & \\
\hline $\mathrm{Ti}$ & 22000 & 22000 & 0.004668 & 0.002040 & 0.000129 & \\
\hline $\mathrm{Mn}$ & 25055 & 25000 & 0.000064 & 0.000024 & 0.000002 & \\
\hline $\mathrm{Fe}$ & 26000 & 26000 & 0.036804 & 0.013787 & 0.000873 & \\
\hline Total & & & 0.999996 & 1.000000 & 0.063331 & \\
\hline MCNP Form & \multicolumn{2}{|c|}{ Weight Fractions } & \multicolumn{2}{|c|}{ Atom Fractions } & \multicolumn{2}{|c|}{ Atom Densities } \\
\hline \multirow[t]{11}{*}{ Neutrons } & 8016 & -0.484345 & 8016 & 0.633300 & 8016 & 0.040107 \\
\hline & 11023 & -0.007608 & 11023 & 0.006923 & 11023 & 0.000438 \\
\hline & 12000 & -0.010691 & 12000 & 0.009202 & 12000 & 0.000583 \\
\hline & 13027 & -0.122125 & 13027 & 0.094689 & 13027 & 0.005997 \\
\hline & 14000 & -0.294194 & 14000 & 0.219134 & 14000 & 0.013878 \\
\hline & 15031 & -0.000113 & 15031 & 0.000076 & 15031 & 0.000005 \\
\hline & 19000 & -0.020427 & 19000 & 0.010930 & 19000 & 0.000692 \\
\hline & 20000 & -0.018957 & 20000 & 0.009895 & 20000 & 0.000627 \\
\hline & 22000 & -0.004668 & 22000 & 0.002040 & 22000 & 0.000129 \\
\hline & 25055 & -0.000064 & 25055 & 0.000024 & 25055 & 0.000002 \\
\hline & 26000 & -0.036804 & 26000 & 0.013787 & 26000 & 0.000873 \\
\hline \multirow[t]{11}{*}{ Photons } & 8000 & -0.484345 & 8000 & 0.633300 & 8000 & 0.040107 \\
\hline & 11000 & -0.007608 & 11000 & 0.006923 & 11000 & 0.000438 \\
\hline & 12000 & -0.010691 & 12000 & 0.009202 & 12000 & 0.000583 \\
\hline & 13000 & -0.122125 & 13000 & 0.094689 & 13000 & 0.005997 \\
\hline & 14000 & -0.294194 & 14000 & 0.219134 & 14000 & 0.013878 \\
\hline & 15000 & -0.000113 & 15000 & 0.000076 & 15000 & 0.000005 \\
\hline & 19000 & -0.020427 & 19000 & 0.010930 & 19000 & 0.000692 \\
\hline & 20000 & -0.018957 & 20000 & 0.009895 & 20000 & 0.000627 \\
\hline & 22000 & -0.004668 & 22000 & 0.002040 & 22000 & 0.000129 \\
\hline & 25000 & -0.000064 & 25000 & 0.000024 & 25000 & 0.000002 \\
\hline & 26000 & -0.036804 & 26000 & 0.013787 & 26000 & 0.000873 \\
\hline
\end{tabular}


PIET-43741-TM-963

PNNL-15870 Rev. 1

\begin{tabular}{|ccc} 
CEPXS Form: $\quad$ material & $\mathrm{O}$ & 0.484345 \\
& $\mathrm{Na}$ & 0.007608 \\
$\mathrm{Mg}$ & 0.010691 \\
$\mathrm{Al}$ & 0.122125 \\
$\mathrm{Si}$ & 0.294194 \\
$\mathrm{P}$ & 0.000113 \\
$\mathrm{~K}$ & 0.020427 \\
$\mathrm{Ca}$ & 0.018957 \\
$\mathrm{Ti}$ & 0.004668 \\
$\mathrm{Mn}$ & 0.000064 \\
& $\mathrm{Fe}$ & 0.036804
\end{tabular}

$\begin{array}{cc}\text { matname } & \text { Clay } \\ \text { density } & 2.200000\end{array}$

\section{Comments and References}

The element weight fractions are calculated based on the listed weight fractions of compounds in 19 clays from 8 regions in the world. Data is from Applied Clay Science, pgs 461 - 473 of Vol. 4 (1989), pgs 379 - 395 of Vol. 5 (1991), pgs 247 - 266 and pgs 463 - 477 of Vol. 12 (1998), pgs 337 - 366 of Vol. 15 (1999). Also from http://www.springerlink.com/content/u692183538748146/fulltext.pdf and https://www.mri.psu.edu/conferences/sint03/pdf/Zanelli_1_1.pdf.

Density $=2.2 \mathrm{~g} / \mathrm{cm} 3$ from Table 51.14 of Hungerford $(1 \overline{9} 6 \overline{0})$. This is consistent with a density of 1.8 to $2.6 \mathrm{~g} / \mathrm{cm} 3$ listed under minerals for marl clay in Table 6.1 .5 of Avallone and Baumeister III (1996). There is a wide variation of densities for clay depending on the type of clay. For example, densities for clay from 1.07 to $1.83 \mathrm{~g} / \mathrm{cm} 3$ are listed at http://www.simetric.co.uk/si_materials.htm (Walker 2009); densities from 0.48 to $0.96 \mathrm{~g} / \mathrm{cm} 3$ are listed at http://www.powderandbulk.com/resources/bulk_density/material_bulk_density_chart_c.htm (Powder and Bulk Dot Com 2010), and densities from 1.0 to $\overline{2} .9$ are listed in Table $\overline{5} 1.59$ of Hungerford (1960).

\section{Coal, Anthracite}

\begin{tabular}{llll}
\hline Formula $=$ & - & Molecular weight $(\mathrm{g} / \mathrm{mole})=$ \\
Density $(\mathrm{g} / \mathrm{cm} 3)=$ & 0.840000 & Total atom density $($ atoms $/ \mathrm{b}-\mathrm{cm})=$ & - \\
The & $5.269 \mathrm{E}-02$
\end{tabular}

The above density is estimated to be accurate to 2 significant digits. Uncertainties are not addressed. The following data were calculated from the input weight fractions.

\begin{tabular}{|c|c|c|c|c|c|c|}
\hline Element & Neutron ZA & Photon ZA & $\begin{array}{l}\text { Weight } \\
\text { Fraction }\end{array}$ & $\begin{array}{l}\text { Atom } \\
\text { Fraction }\end{array}$ & $\begin{array}{l}\text { Atom } \\
\text { Density }\end{array}$ & \\
\hline $\mathrm{H}$ & 1001 & 1000 & $\overline{0.024000}$ & $\overline{0.228612}$ & 0.012045 & \\
\hline C & 6000 & 6000 & 0.937000 & 0.749020 & 0.039464 & \\
\hline $\mathrm{N}$ & 7014 & 7000 & 0.009000 & 0.006169 & 0.000325 & \\
\hline $\mathrm{O}$ & 8016 & 8000 & 0.024000 & 0.014402 & 0.000759 & \\
\hline$S$ & 16000 & 16000 & 0.006000 & 0.001797 & 0.000095 & \\
\hline Total & & & 1.000000 & 1.000000 & 0.052688 & \\
\hline MCNP Form & \multicolumn{2}{|c|}{ Weight Fractions } & \multicolumn{2}{|c|}{ Atom Fractions } & \multicolumn{2}{|c|}{ Atom Densities } \\
\hline \multirow[t]{3}{*}{ Neutrons } & 1001 & -0.024000 & 1001 & 0.228612 & 1001 & 0.012045 \\
\hline & 6000 & -0.937000 & 6000 & 0.749020 & 6000 & 0.039464 \\
\hline & 7014 & -0.009000 & 7014 & 0.006169 & 7014 & 0.000325 \\
\hline
\end{tabular}


PIET-43741-TM-963

PNNL-15870 Rev. 1

\begin{tabular}{|c|c|c|c|c|c|c|}
\hline & $\begin{array}{c}8016 \\
16000\end{array}$ & $\begin{array}{l}-0.024000 \\
-0.006000\end{array}$ & $\begin{array}{c}8016 \\
16000\end{array}$ & $\begin{array}{l}0.014402 \\
0.001797\end{array}$ & $\begin{array}{c}8016 \\
16000\end{array}$ & $\begin{array}{l}0.000759 \\
0.000095\end{array}$ \\
\hline Photons & $\begin{array}{c}1000 \\
6000 \\
7000 \\
8000 \\
16000\end{array}$ & $\begin{array}{l}-0.024000 \\
-0.937000 \\
-0.009000 \\
-0.024000 \\
-0.006000\end{array}$ & $\begin{array}{c}1000 \\
6000 \\
7000 \\
8000 \\
16000\end{array}$ & $\begin{array}{l}0.228612 \\
0.749020 \\
0.006169 \\
0.014402 \\
0.001797\end{array}$ & $\begin{array}{c}1000 \\
6000 \\
7000 \\
8000 \\
16000\end{array}$ & $\begin{array}{l}0.012045 \\
0.039464 \\
0.000325 \\
0.000759 \\
0.000095\end{array}$ \\
\hline CEPXS Form: & $\begin{array}{c}\text { matname } \\
\text { density }\end{array}$ & $\begin{array}{c}\mathrm{H} \\
\mathrm{C} \\
\mathrm{N} \\
\mathrm{O} \\
\mathrm{S} \\
\text { Coal, Anthra } \\
0.840000\end{array}$ & $\begin{array}{l}0.024000 \\
0.937000 \\
0.009000 \\
0.024000 \\
0.006000\end{array}$ & & & \\
\hline \multicolumn{7}{|c|}{$\begin{array}{l}\text { Comments and References } \\
\text { Weight fractions from Table } 4.1 \text { of (Speight } 2001) \text {. } \\
\text { Density }=1.4 \text { to } 1.8 \mathrm{~g} / \mathrm{cm} 3 \text { and bulk density }=0.75 \text { to } 0.93 \mathrm{~g} / \mathrm{cm} 3 \text { for piled coal in Table } 6.1 .5 \text { of Avallone } \\
\text { and Baumeister III ( } 1996) \text {. } \\
\text { Density }=1.105 \mathrm{~g} / \mathrm{cm} 3 \text { for broken coal and } 1.506 \text { for solid coal at } \\
\text { http://www.simetric.co.uk/si_materials.htm (Walker } 2009) . \\
\text { Density }=1.3 \text { to } 1.7 \mathrm{~g} / \mathrm{cm} 3 \text {, and bulk density }=0.75 \text { to } 0.93 \mathrm{~g} / \mathrm{cm} 3 \text {, in Table } 51.65 \text { of Hungerford (1960). }\end{array}$} \\
\hline
\end{tabular}

\section{Coal, Bituminous}

\begin{tabular}{|c|c|c|c|}
\hline la $=$ & - & Molecular weight $(\mathrm{g} / \mathrm{mole})=$ & $\begin{array}{l}- \\
5054 F_{-} 02\end{array}$ \\
\hline
\end{tabular}

The above density is estimated to be accurate to 2 significant digits. Uncertainties are not addressed.

The following data were calculated from the input weight fractions.

\begin{tabular}{|c|c|c|c|c|c|c|}
\hline Element & Neutron ZA & Photon ZA & $\begin{array}{l}\text { Weight } \\
\text { Fraction }\end{array}$ & $\begin{array}{c}\text { Atom } \\
\text { Fraction }\end{array}$ & $\begin{array}{l}\text { Atom } \\
\text { Density }\end{array}$ & \\
\hline $\mathrm{H}$ & 1001 & 1000 & 0.056000 & 0.421425 & 0.025094 & \\
\hline C & 6000 & 6000 & 0.845000 & 0.533649 & 0.031776 & \\
\hline $\mathrm{N}$ & 7014 & 7000 & 0.016000 & 0.008665 & 0.000516 & \\
\hline $\mathrm{O}$ & 8016 & 8000 & 0.070000 & 0.033186 & 0.001976 & \\
\hline$S$ & 16000 & 16000 & 0.013000 & 0.003075 & 0.000183 & \\
\hline Total & & & 1.000000 & 1.000000 & 0.059545 & \\
\hline MCNP Form & \multicolumn{2}{|c|}{ Weight Fractions } & \multicolumn{2}{|c|}{ Atom Fractions } & \multicolumn{2}{|c|}{ Atom Densities } \\
\hline \multirow[t]{5}{*}{ Neutrons } & 1001 & -0.056000 & 1001 & 0.421425 & 1001 & 0.025094 \\
\hline & 6000 & -0.845000 & 6000 & 0.533649 & 6000 & 0.031776 \\
\hline & 7014 & -0.016000 & 7014 & 0.008665 & 7014 & 0.000516 \\
\hline & 8016 & -0.070000 & 8016 & 0.033186 & 8016 & 0.001976 \\
\hline & 16000 & -0.013000 & 16000 & 0.003075 & 16000 & 0.000183 \\
\hline
\end{tabular}


PIET-43741-TM-963

PNNL-15870 Rev. 1

\begin{tabular}{|c|c|c|c|c|c|c|}
\hline \multirow[t]{5}{*}{ Photons } & 1000 & -0.056000 & 1000 & 0.421425 & 1000 & 0.025094 \\
\hline & 6000 & -0.845000 & 6000 & 0.533649 & 6000 & 0.031776 \\
\hline & 7000 & -0.016000 & 7000 & 0.008665 & 7000 & 0.000516 \\
\hline & 8000 & -0.070000 & 8000 & 0.033186 & 8000 & 0.001976 \\
\hline & 16000 & -0.013000 & 16000 & 0.003075 & 16000 & 0.000183 \\
\hline \multirow[t]{6}{*}{ CEPXS Form: } & material & $\mathrm{H}$ & 0.056000 & & & \\
\hline & & C & 0.845000 & & & \\
\hline & & $\mathrm{N}$ & 0.016000 & & & \\
\hline & & $\mathrm{O}$ & 0.070000 & & & \\
\hline & & $S$ & 0.013000 & & & \\
\hline & $\begin{array}{c}\text { matname } \\
\text { density }\end{array}$ & \multicolumn{5}{|c|}{$\begin{array}{l}\text { Coal, Bituminous } \\
0.750000\end{array}$} \\
\hline \multicolumn{7}{|c|}{$\begin{array}{l}\text { Comments and References } \\
\text { Weight fractions from Table } 4.1 \text { of Speight }(2001) \\
\text { Density }=1.2 \text { to } 1.5 \mathrm{~g} / \mathrm{cm} 3 \text { and bulk density }=0.64 \text { to } 0.87 \mathrm{~g} / \mathrm{cm} 3 \text { for piled coal in Table } 6.1 .5 \text { of Avallone } \\
\text { and Baumeister III ( } 1996) \text {. } \\
\text { Density }=0.833 \mathrm{~g} / \mathrm{cm} 3 \text { for broken coal and } 1.346 \text { for solid coal at } \\
\text { http://www.simetric.co.uk } / \mathrm{si} \text { materials.htm (Walker } 2009) . \\
\text { Density }=1.2 \text { to } 1.4 \mathrm{~g} / \mathrm{cm} 3 \text {, and bulk density }=0.70 \text { to } 0.86 \mathrm{~g} / \mathrm{cm} 3 \text {, in Table } 51.65 \text { of Hungerford (1960). }\end{array}$} \\
\hline
\end{tabular}

\section{Coal, Lignite}

Formula $=$

Density $(\mathrm{g} / \mathrm{cm} 3)=0.750000$
Molecular weight $(\mathrm{g} / \mathrm{mole})=$

Total atom density $($ atoms $/ \mathrm{b}-\mathrm{cm})=5.264 \mathrm{E}-02$

The above density is estimated to be accurate to 2 significant digits. Uncertainties are not addressed.

The following data were calculated from the input weight fractions.

\begin{tabular}{|c|c|c|c|c|c|c|}
\hline Element & Neutron ZA & Photon ZA & $\begin{array}{l}\text { Weight } \\
\text { Fraction }\end{array}$ & $\begin{array}{c}\text { Atom } \\
\text { Fraction }\end{array}$ & $\begin{array}{l}\text { Atom } \\
\text { Density }\end{array}$ & \\
\hline $\mathrm{H}$ & 1001 & 1000 & 0.042000 & 0.357505 & 0.018820 & \\
\hline C & 6000 & 6000 & 0.727000 & 0.519319 & 0.027339 & \\
\hline $\mathrm{N}$ & 7014 & 7000 & 0.012000 & 0.007350 & 0.000387 & \\
\hline $\mathrm{O}$ & 8016 & 8000 & 0.213000 & 0.114220 & 0.006013 & \\
\hline$S$ & 16000 & 16000 & 0.006000 & 0.001605 & 0.000085 & \\
\hline Total & & & 1.000000 & 1.000000 & 0.052643 & \\
\hline MCNP Form & \multicolumn{2}{|c|}{ Weight Fractions } & \multicolumn{2}{|c|}{ Atom Fractions } & \multicolumn{2}{|c|}{ Atom Densities } \\
\hline \multirow[t]{5}{*}{ Neutrons } & 1001 & -0.042000 & 1001 & 0.357505 & 1001 & 0.018820 \\
\hline & 6000 & -0.727000 & 6000 & 0.519319 & 6000 & 0.027339 \\
\hline & 7014 & -0.012000 & 7014 & 0.007350 & 7014 & 0.000387 \\
\hline & 8016 & -0.213000 & 8016 & 0.114220 & 8016 & 0.006013 \\
\hline & 16000 & -0.006000 & 16000 & 0.001605 & 16000 & 0.000085 \\
\hline
\end{tabular}


PIET-43741-TM-963

PNNL-15870 Rev. 1

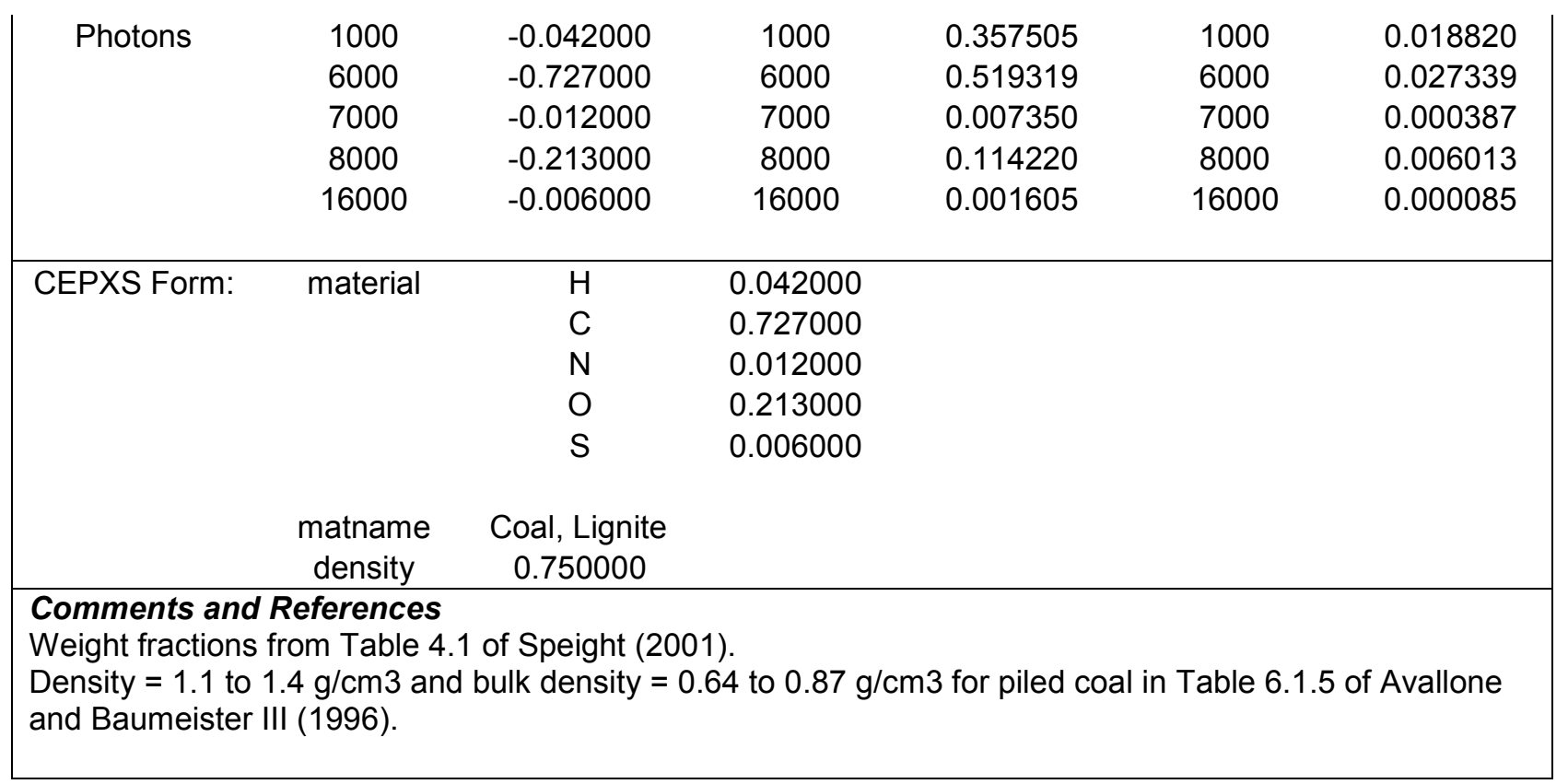

\section{Concrete, Barite (Type BA)}

\begin{tabular}{llll}
\hline Formula $=$ & - & Molecular weight $(\mathrm{g} / \mathrm{mole})=$ \\
Density $(\mathrm{g} / \mathrm{cm} 3)=$ & 3.350000 & Total atom density $($ atoms $/ \mathrm{b}-\mathrm{cm})=$ & - \\
\hline & $6.547 \mathrm{E}-02$
\end{tabular}

The above density is estimated to be accurate to 3 significant digits. Uncertainties are not addressed.

The following data were calculated from the input weight fractions.

\begin{tabular}{|c|c|c|c|c|c|c|}
\hline Element & Neutron ZA & Photon ZA & $\begin{array}{l}\text { Weight } \\
\text { Fraction }\end{array}$ & $\begin{array}{c}\text { Atom } \\
\text { Fraction }\end{array}$ & $\begin{array}{l}\text { Atom } \\
\text { Density }\end{array}$ & \\
\hline $\mathrm{H}$ & 1001 & 1000 & $\overline{0.003585}$ & $\overline{0.109602}$ & 0.007175 & \\
\hline $\mathrm{O}$ & 8016 & 8000 & 0.311622 & 0.600189 & 0.039293 & \\
\hline $\mathrm{Mg}$ & 12000 & 12000 & 0.001195 & 0.001515 & 0.000099 & \\
\hline $\mathrm{Al}$ & 13027 & 13000 & 0.004183 & 0.004777 & 0.000313 & \\
\hline Si & 14000 & 14000 & 0.010457 & 0.011473 & 0.000751 & \\
\hline$S$ & 16000 & 16000 & 0.107858 & 0.103654 & 0.006786 & \\
\hline $\mathrm{Ca}$ & 20000 & 20000 & 0.050194 & 0.038593 & 0.002527 & \\
\hline $\mathrm{Fe}$ & 26000 & 26000 & 0.047505 & 0.026213 & 0.001716 & \\
\hline $\mathrm{Ba}$ & - & 56000 & 0.463400 & 0.103983 & 0.006808 & \\
\hline Total & & & 0.999999 & 1.000000 & 0.065468 & \\
\hline MCNP Form & \multicolumn{2}{|c|}{ Weight Fractions } & \multicolumn{2}{|c|}{ Atom Fractions } & \multicolumn{2}{|c|}{ Atom Densities } \\
\hline \multirow[t]{7}{*}{ Neutrons } & 1001 & -0.003585 & 1001 & 0.109602 & 1001 & 0.007175 \\
\hline & 8016 & -0.311622 & 8016 & 0.600189 & 8016 & 0.039293 \\
\hline & 12000 & -0.001195 & 12000 & 0.001515 & 12000 & 0.000099 \\
\hline & 13027 & -0.004183 & 13027 & 0.004777 & 13027 & 0.000313 \\
\hline & 14000 & -0.010457 & 14000 & 0.011473 & 14000 & 0.000751 \\
\hline & 16000 & -0.107858 & 16000 & 0.103654 & 16000 & 0.006786 \\
\hline & 20000 & -0.050194 & 20000 & 0.038593 & 20000 & 0.002527 \\
\hline
\end{tabular}


PIET-43741-TM-963

PNNL-15870 Rev. 1

\begin{tabular}{|c|c|c|c|c|c|c|}
\hline & 26000 & $\begin{array}{l}-0.047505 \\
-0.463400\end{array}$ & 26000 & $\begin{array}{l}0.026213 \\
0.103983\end{array}$ & 26000 & $\begin{array}{l}0.001716 \\
0.006808\end{array}$ \\
\hline \multirow[t]{9}{*}{ Photons } & 1000 & -0.003585 & 1000 & 0.109602 & 1000 & 0.007175 \\
\hline & 8000 & -0.311622 & 8000 & 0.600189 & 8000 & 0.039293 \\
\hline & 12000 & -0.001195 & 12000 & 0.001515 & 12000 & 0.000099 \\
\hline & 13000 & -0.004183 & 13000 & 0.004777 & 13000 & 0.000313 \\
\hline & 14000 & -0.010457 & 14000 & 0.011473 & 14000 & 0.000751 \\
\hline & 16000 & -0.107858 & 16000 & 0.103654 & 16000 & 0.006786 \\
\hline & 20000 & -0.050194 & 20000 & 0.038593 & 20000 & 0.002527 \\
\hline & 26000 & -0.047505 & 26000 & 0.026213 & 26000 & 0.001716 \\
\hline & 56000 & -0.463400 & 56000 & 0.103983 & 56000 & 0.006808 \\
\hline \multirow[t]{10}{*}{ CEPXS Form: } & material & $\mathrm{H}$ & 0.003585 & & & \\
\hline & & $\mathrm{O}$ & 0.311622 & & & \\
\hline & & $\mathrm{Mg}$ & 0.001195 & & & \\
\hline & & $\mathrm{Al}$ & 0.004183 & & & \\
\hline & & $\mathrm{Si}$ & 0.010457 & & & \\
\hline & & $S$ & 0.107858 & & & \\
\hline & & $\mathrm{Ca}$ & 0.050194 & & & \\
\hline & & $\mathrm{Fe}$ & 0.047505 & & & \\
\hline & & $\mathrm{Ba}$ & 0.463400 & & & \\
\hline & $\begin{array}{l}\text { matname } \\
\text { density }\end{array}$ & \multicolumn{2}{|c|}{$\begin{array}{l}\text { Concrete, Barite (Type BA) } \\
3.350000\end{array}$} & & & \\
\hline \multicolumn{7}{|c|}{$\begin{array}{l}\text { Comments and References } \\
\text { Density and weight fractions from http://physics.nist.gov/PhysRefData/XrayMassCoef/tab2.html } \\
\text { (NIST 1996). See Table } 8.8 \text { of Shultis and Faw (1996), for a similar composition. } \\
\text { Data in this table are from ANSI/ANS-6.4-1985. } \\
\text { This concrete has barytes, a BaSO4 ore, as aggregate. }\end{array}$} \\
\hline
\end{tabular}

\section{Concrete, Barytes-limonite}

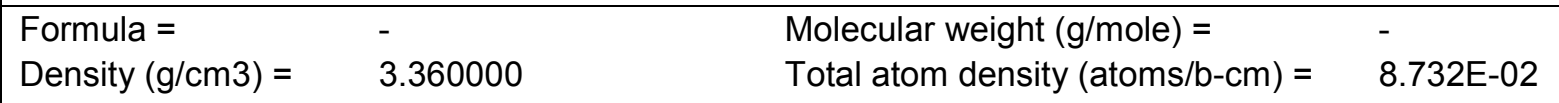

The above density is estimated to be accurate to 2 significant digits. Uncertainties are not addressed.

The following data were calculated from the input weight fractions.

\begin{tabular}{|c|c|c|c|c|c|}
\hline Element & Neutron ZA & Photon ZA & $\begin{array}{l}\text { Weight } \\
\text { Fraction }\end{array}$ & $\begin{array}{c}\text { Atom } \\
\text { Fraction }\end{array}$ & $\begin{array}{c}\text { Atom } \\
\text { Density }\end{array}$ \\
\hline $\mathrm{H}$ & 1001 & 1000 & $\overline{0.010240}$ & 0.235416 & 0.020557 \\
\hline 0 & 8016 & 8000 & 0.378476 & 0.548162 & 0.047866 \\
\hline $\mathrm{Na}$ & 11023 & 11000 & 0.000904 & 0.000911 & 0.000080 \\
\hline $\mathrm{Mg}$ & 12000 & 12000 & 0.002309 & 0.002201 & 0.000192 \\
\hline $\mathrm{Al}$ & 13027 & 13000 & 0.005020 & 0.004311 & 0.000376 \\
\hline $\mathrm{Si}$ & 14000 & 14000 & 0.013553 & 0.011182 & 0.000976 \\
\hline$S$ & 16000 & 16000 & 0.076097 & 0.054993 & 0.004802 \\
\hline $\mathrm{Ca}$ & 20000 & 20000 & 0.053910 & 0.031170 & 0.002722 \\
\hline
\end{tabular}


PIET-43741-TM-963

PNNL-15870 Rev. 1

\begin{tabular}{|c|c|c|c|c|c|c|}
\hline $\mathrm{Mn}$ & 25055 & 25000 & 0.001405 & 0.000593 & \multicolumn{2}{|l|}{0.000052} \\
\hline $\mathrm{Fe}$ & 26000 & 26000 & 0.137135 & 0.056903 & \multicolumn{2}{|c|}{0.004969} \\
\hline $\mathrm{Ba}$ & - & 56000 & 0.320952 & 0.054157 & \multicolumn{2}{|l|}{0.004729} \\
\hline Total & & & 1.000000 & 1.000000 & \multicolumn{2}{|l|}{0.087321} \\
\hline MCNP Form & \multicolumn{2}{|c|}{ Weight Fractions } & \multicolumn{2}{|c|}{ Atom Fractions } & \multicolumn{2}{|c|}{ Atom Densities } \\
\hline \multirow[t]{11}{*}{ Neutrons } & 1001 & -0.010240 & 1001 & 0.235416 & 1001 & 0.020557 \\
\hline & 8016 & -0.378476 & 8016 & 0.548162 & 8016 & 0.047866 \\
\hline & 11023 & -0.000904 & 11023 & 0.000911 & 11023 & 0.000080 \\
\hline & 12000 & -0.002309 & 12000 & 0.002201 & 12000 & 0.000192 \\
\hline & 13027 & -0.005020 & 13027 & 0.004311 & 13027 & 0.000376 \\
\hline & 14000 & -0.013553 & 14000 & 0.011182 & 14000 & 0.000976 \\
\hline & 16000 & -0.076097 & 16000 & 0.054993 & 16000 & 0.004802 \\
\hline & 20000 & -0.053910 & 20000 & 0.031170 & 20000 & 0.002722 \\
\hline & 25055 & -0.001405 & 25055 & 0.000593 & 25055 & 0.000052 \\
\hline & 26000 & -0.137135 & 26000 & 0.056903 & 26000 & 0.004969 \\
\hline & - & -0.320952 & - & 0.054157 & - & 0.004729 \\
\hline \multirow[t]{11}{*}{ Photons } & 1000 & -0.010240 & 1000 & 0.235416 & 1000 & 0.020557 \\
\hline & 8000 & -0.378476 & 8000 & 0.548162 & 8000 & 0.047866 \\
\hline & 11000 & -0.000904 & 11000 & 0.000911 & 11000 & 0.000080 \\
\hline & 12000 & -0.002309 & 12000 & 0.002201 & 12000 & 0.000192 \\
\hline & 13000 & -0.005020 & 13000 & 0.004311 & 13000 & 0.000376 \\
\hline & 14000 & -0.013553 & 14000 & 0.011182 & 14000 & 0.000976 \\
\hline & 16000 & -0.076097 & 16000 & 0.054993 & 16000 & 0.004802 \\
\hline & 20000 & -0.053910 & 20000 & 0.031170 & 20000 & 0.002722 \\
\hline & 25000 & -0.001405 & 25000 & 0.000593 & 25000 & 0.000052 \\
\hline & 26000 & -0.137135 & 26000 & 0.056903 & 26000 & 0.004969 \\
\hline & 56000 & -0.320952 & 56000 & 0.054157 & 56000 & 0.004729 \\
\hline \multirow[t]{12}{*}{ CEPXS Form: } & material & $\mathrm{H}$ & 0.010240 & & & \\
\hline & & 0 & 0.378476 & & & \\
\hline & & $\mathrm{Na}$ & 0.000904 & & & \\
\hline & & $\mathrm{Mg}$ & 0.002309 & & & \\
\hline & & $\mathrm{Al}$ & 0.005020 & & & \\
\hline & & $\mathrm{Si}$ & 0.013553 & & & \\
\hline & & $S$ & 0.076097 & & & \\
\hline & & $\mathrm{Ca}$ & 0.053910 & & & \\
\hline & & $\mathrm{Mn}$ & 0.001405 & & & \\
\hline & & $\mathrm{Fe}$ & 0.137135 & & & \\
\hline & & $\mathrm{Ba}$ & 0.320952 & & & \\
\hline & $\begin{array}{l}\text { matname } \\
\text { density }\end{array}$ & \multicolumn{3}{|c|}{$\begin{array}{l}\text { Concrete, Barytes-limonite } \\
3.360000\end{array}$} & & \\
\hline \multicolumn{7}{|c|}{$\begin{array}{l}\text { Comments and References } \\
\text { Density and weight fractions from Tables } 9.1 .12-55 \text { and } 77 \text { of Jaeger et al. (1975). } \\
\text { Weight fractions are adjusted so that they sum to unity. } \\
\text { Barytes (a BaSO4 ore) and Limonite (a hydrated Fe2O3 ore) as aggregate. }\end{array}$} \\
\hline
\end{tabular}




\section{Concrete, Boron Frits-baryte}

\begin{tabular}{|c|c|c|c|}
\hline Formula $=$ & - & Molecular weight $(\mathrm{g} / \mathrm{mole})=$ & - \\
\hline Density $(\mathrm{g} / \mathrm{cm} 3)=$ & 3.100000 & Total atom density $($ atoms $/ \mathrm{b}-\mathrm{cm})=$ & 7.064E-02 \\
\hline
\end{tabular}

\begin{tabular}{|c|c|c|c|c|c|c|}
\hline Element & Neutron ZA & Photon ZA & $\begin{array}{l}\text { Weight } \\
\text { Fraction }\end{array}$ & $\begin{array}{c}\text { Atom } \\
\text { Fraction }\end{array}$ & $\begin{array}{c}\text { Atom } \\
\text { Density }\end{array}$ & \\
\hline $\mathrm{H}$ & 1001 & 1000 & 0.005626 & 0.147522 & 0.010421 & \\
\hline$B$ & - & 5000 & 0.010449 & 0.025543 & 0.001804 & \\
\hline 0 & 8016 & 8000 & 0.339596 & 0.560939 & 0.039625 & \\
\hline $\mathrm{F}$ & 9019 & 9000 & 0.002311 & 0.003215 & 0.000227 & \\
\hline $\mathrm{Na}$ & 11023 & 11000 & 0.012157 & 0.013975 & 0.000987 & \\
\hline $\mathrm{Mg}$ & 12000 & 12000 & 0.002311 & 0.002513 & 0.000177 & \\
\hline $\mathrm{Al}$ & 13027 & 13000 & 0.006430 & 0.006298 & 0.000445 & \\
\hline $\mathrm{Si}$ & 14000 & 14000 & 0.033256 & 0.031293 & 0.002211 & \\
\hline$S$ & 16000 & 16000 & 0.091932 & 0.075769 & 0.005352 & \\
\hline $\mathrm{K}$ & 19000 & 19000 & 0.001005 & 0.000679 & 0.000048 & \\
\hline $\mathrm{Ca}$ & 20000 & 20000 & 0.062896 & 0.041474 & 0.002930 & \\
\hline $\mathrm{Mn}$ & 25055 & 25000 & 0.000201 & 0.000097 & 0.000007 & \\
\hline $\mathrm{Fe}$ & 26000 & 26000 & 0.022003 & 0.010413 & 0.000736 & \\
\hline $\mathrm{Zn}$ & 30000 & 30000 & 0.006631 & 0.002679 & 0.000189 & \\
\hline $\mathrm{Ba}$ & - & 56000 & 0.403195 & 0.077592 & 0.005481 & \\
\hline Total & & & 1.000000 & 1.000000 & 0.070641 & \\
\hline MCNP Form & \multicolumn{2}{|c|}{ Weight Fractions } & \multicolumn{2}{|c|}{ Atom Fractions } & \multicolumn{2}{|c|}{ Atom Densities } \\
\hline \multirow[t]{15}{*}{ Neutrons } & 1001 & -0.005626 & 1001 & 0.147522 & 1001 & 0.010421 \\
\hline & - & -0.010449 & - & 0.025543 & - & 0.001804 \\
\hline & 8016 & -0.339596 & 8016 & 0.560939 & 8016 & 0.039625 \\
\hline & 9019 & -0.002311 & 9019 & 0.003215 & 9019 & 0.000227 \\
\hline & 11023 & -0.012157 & 11023 & 0.013975 & 11023 & 0.000987 \\
\hline & 12000 & -0.002311 & 12000 & 0.002513 & 12000 & 0.000177 \\
\hline & 13027 & -0.006430 & 13027 & 0.006298 & 13027 & 0.000445 \\
\hline & 14000 & -0.033256 & 14000 & 0.031293 & 14000 & 0.002211 \\
\hline & 16000 & -0.091932 & 16000 & 0.075769 & 16000 & 0.005352 \\
\hline & 19000 & -0.001005 & 19000 & 0.000679 & 19000 & 0.000048 \\
\hline & 20000 & -0.062896 & 20000 & 0.041474 & 20000 & 0.002930 \\
\hline & 25055 & -0.000201 & 25055 & 0.000097 & 25055 & 0.000007 \\
\hline & 26000 & -0.022003 & 26000 & 0.010413 & 26000 & 0.000736 \\
\hline & 30000 & -0.006631 & 30000 & 0.002679 & 30000 & 0.000189 \\
\hline & - & -0.403195 & - & 0.077592 & - & 0.005481 \\
\hline \multirow[t]{6}{*}{ Photons } & 1000 & -0.005626 & 1000 & 0.147522 & 1000 & 0.010421 \\
\hline & 5000 & -0.010449 & 5000 & 0.025543 & 5000 & 0.001804 \\
\hline & 8000 & -0.339596 & 8000 & 0.560939 & 8000 & 0.039625 \\
\hline & 9000 & -0.002311 & 9000 & 0.003215 & 9000 & 0.000227 \\
\hline & 11000 & -0.012157 & 11000 & 0.013975 & 11000 & 0.000987 \\
\hline & 12000 & -0.002311 & 12000 & 0.002513 & 12000 & 0.000177 \\
\hline
\end{tabular}




\begin{tabular}{|c|c|c|c|c|c|c|}
\hline & 13000 & -0.006430 & 13000 & 0.006298 & 13000 & 0.000445 \\
\hline & 14000 & -0.033256 & 14000 & 0.031293 & 14000 & 0.002211 \\
\hline & 16000 & -0.091932 & 16000 & 0.075769 & 16000 & 0.005352 \\
\hline & 19000 & -0.001005 & 19000 & 0.000679 & 19000 & 0.000048 \\
\hline & 20000 & -0.062896 & 20000 & 0.041474 & 20000 & 0.002930 \\
\hline & 25000 & -0.000201 & 25000 & 0.000097 & 25000 & 0.000007 \\
\hline & 26000 & -0.022003 & 26000 & 0.010413 & 26000 & 0.000736 \\
\hline & 30000 & -0.006631 & 30000 & 0.002679 & 30000 & 0.000189 \\
\hline & 56000 & -0.403195 & 56000 & 0.077592 & 56000 & 0.005481 \\
\hline CEPXS Form: & material & $\mathrm{H}$ & 0.005626 & & & \\
\hline & & B & 0.010449 & & & \\
\hline & & 0 & 0.339596 & & & \\
\hline & & $\mathrm{F}$ & 0.002311 & & & \\
\hline & & $\mathrm{Na}$ & 0.012157 & & & \\
\hline & & $\mathrm{Mg}$ & 0.002311 & & & \\
\hline & & $\mathrm{Al}$ & 0.006430 & & & \\
\hline & & $\mathrm{Si}$ & 0.033256 & & & \\
\hline & & $S$ & 0.091932 & & & \\
\hline & & $\mathrm{K}$ & 0.001005 & & & \\
\hline & & $\mathrm{Ca}$ & 0.062896 & & & \\
\hline & & $\mathrm{Mn}$ & 0.000201 & & & \\
\hline & & $\mathrm{Fe}$ & 0.022003 & & & \\
\hline & & $\mathrm{Zn}$ & 0.006631 & & & \\
\hline & & $\mathrm{Ba}$ & 0.403195 & & & \\
\hline & $\begin{array}{c}\text { matname } \\
\text { density }\end{array}$ & $\begin{array}{c}\text { Concrete, B } \\
3.100000\end{array}$ & Frits-baryt & & & \\
\hline $\begin{array}{l}\text { Comments an } \\
\text { Density and we } \\
\text { adjusted so the }\end{array}$ & $\begin{array}{l}\text { eferences } \\
\text { t fractions } \\
\text { um to unity }\end{array}$ & Tables 5 & id 51.9 & Tu & & s are \\
\hline
\end{tabular}

\section{Concrete, Colemanite-baryte}

\begin{tabular}{|c|c|c|c|}
\hline$a=$ & - & Molecular weight $(\mathrm{g} / \mathrm{mole})=$ & $7845 \mathrm{E}-02$ \\
\hline
\end{tabular}

The above density is estimated to be accurate to 2 significant digits. Uncertainties are not addressed.

The following data were calculated from the input weight fractions.

\begin{tabular}{|c|c|c|c|c|c|}
\hline Element & Neutron ZA & Photon ZA & $\begin{array}{l}\text { Weight } \\
\text { Fraction }\end{array}$ & $\begin{array}{c}\text { Atom } \\
\text { Fraction }\end{array}$ & $\begin{array}{c}\text { Atom } \\
\text { Density }\end{array}$ \\
\hline $\mathrm{H}$ & 1001 & 1000 & 0.008564 & 0.208729 & 0.016374 \\
\hline B & - & 5000 & 0.009874 & 0.022437 & 0.001760 \\
\hline 0 & 8016 & 8000 & 0.351537 & 0.539754 & 0.042342 \\
\hline $\mathrm{Na}$ & 11023 & 11000 & 0.001108 & 0.001184 & 0.000093 \\
\hline $\mathrm{Mg}$ & 12000 & 12000 & 0.002217 & 0.002240 & 0.000176 \\
\hline $\mathrm{Al}$ & 13027 & 13000 & 0.006146 & 0.005596 & 0.000439 \\
\hline $\mathrm{Si}$ & 14000 & 14000 & 0.017733 & 0.015511 & 0.001217 \\
\hline
\end{tabular}


PIET-43741-TM-963

PNNL-15870 Rev. 1

\begin{tabular}{|c|c|c|c|c|c|c|}
\hline$S$ & 16000 & 16000 & 0.097028 & 0.074335 & \multicolumn{2}{|l|}{0.005831} \\
\hline $\mathrm{Ca}$ & 20000 & 20000 & 0.085239 & 0.052247 & \multicolumn{2}{|c|}{0.004099} \\
\hline $\mathrm{Mn}$ & 25055 & 25000 & 0.000101 & 0.000045 & \multicolumn{2}{|l|}{0.000004} \\
\hline $\mathrm{Fe}$ & 26000 & 26000 & 0.010378 & 0.004565 & \multicolumn{2}{|l|}{0.000358} \\
\hline $\mathrm{Ba}$ & - & 56000 & 0.410076 & 0.073356 & \multicolumn{2}{|l|}{0.005755} \\
\hline Total & & & 1.000000 & 1.000000 & \multicolumn{2}{|l|}{0.078446} \\
\hline MCNP Form & \multicolumn{2}{|c|}{ Weight Fractions } & \multicolumn{2}{|c|}{ Atom Fractions } & \multicolumn{2}{|c|}{ Atom Densities } \\
\hline \multirow[t]{12}{*}{ Neutrons } & 1001 & -0.008564 & 1001 & 0.208729 & 1001 & 0.016374 \\
\hline & - & -0.009874 & - & 0.022437 & - & 0.001760 \\
\hline & 8016 & -0.351537 & 8016 & 0.539754 & 8016 & 0.042342 \\
\hline & 11023 & -0.001108 & 11023 & 0.001184 & 11023 & 0.000093 \\
\hline & 12000 & -0.002217 & 12000 & 0.002240 & 12000 & 0.000176 \\
\hline & 13027 & -0.006146 & 13027 & 0.005596 & 13027 & 0.000439 \\
\hline & 14000 & -0.017733 & 14000 & 0.015511 & 14000 & 0.001217 \\
\hline & 16000 & -0.097028 & 16000 & 0.074335 & 16000 & 0.005831 \\
\hline & 20000 & -0.085239 & 20000 & 0.052247 & 20000 & 0.004099 \\
\hline & 25055 & -0.000101 & 25055 & 0.000045 & 25055 & 0.000004 \\
\hline & 26000 & -0.010378 & 26000 & 0.004565 & 26000 & 0.000358 \\
\hline & - & -0.410076 & - & 0.073356 & - & 0.005755 \\
\hline \multirow[t]{12}{*}{ Photons } & 1000 & -0.008564 & 1000 & 0.208729 & 1000 & 0.016374 \\
\hline & 5000 & -0.009874 & 5000 & 0.022437 & 5000 & 0.001760 \\
\hline & 8000 & -0.351537 & 8000 & 0.539754 & 8000 & 0.042342 \\
\hline & 11000 & -0.001108 & 11000 & 0.001184 & 11000 & 0.000093 \\
\hline & 12000 & -0.002217 & 12000 & 0.002240 & 12000 & 0.000176 \\
\hline & 13000 & -0.006146 & 13000 & 0.005596 & 13000 & 0.000439 \\
\hline & 14000 & -0.017733 & 14000 & 0.015511 & 14000 & 0.001217 \\
\hline & 16000 & -0.097028 & 16000 & 0.074335 & 16000 & 0.005831 \\
\hline & 20000 & -0.085239 & 20000 & 0.052247 & 20000 & 0.004099 \\
\hline & 25000 & -0.000101 & 25000 & 0.000045 & 25000 & 0.000004 \\
\hline & 26000 & -0.010378 & 26000 & 0.004565 & 26000 & 0.000358 \\
\hline & 56000 & -0.410076 & 56000 & 0.073356 & 56000 & 0.005755 \\
\hline \multirow[t]{13}{*}{ CEPXS Form: } & material & $\mathrm{H}$ & 0.008564 & & & \\
\hline & & B & 0.009874 & & & \\
\hline & & O & 0.351537 & & & \\
\hline & & $\mathrm{Na}$ & 0.001108 & & & \\
\hline & & $\mathrm{Mg}$ & 0.002217 & & & \\
\hline & & $\mathrm{Al}$ & 0.006146 & & & \\
\hline & & $\mathrm{Si}$ & 0.017733 & & & \\
\hline & & $S$ & 0.097028 & & & \\
\hline & & $\mathrm{Ca}$ & 0.085239 & & & \\
\hline & & $\mathrm{Mn}$ & 0.000101 & & & \\
\hline & & $\mathrm{Fe}$ & 0.010378 & & & \\
\hline & & $\mathrm{Ba}$ & 0.410076 & & & \\
\hline & $\begin{array}{l}\text { matname } \\
\text { density }\end{array}$ & $\begin{array}{c}\text { Concrete, C } \\
3.200000\end{array}$ & hanite-bary & & & \\
\hline
\end{tabular}


Comments and References

Density and weight fractions from Tables 51.84 and 51.95 of Hungerford (1960). Weight fractions are adjusted so they sum to unity.

\section{Concrete, Ferro-phosphorus}

\begin{tabular}{llll}
\hline Formula $=$ & - & Molecular weight $(\mathrm{g} / \mathrm{mole})=$ \\
Density $(\mathrm{g} / \mathrm{cm} 3)=$ & 4.800000 & Total atom density $($ atoms $/ \mathrm{b}-\mathrm{cm})=$ & - \\
\hline & $9.039 \mathrm{E}-02$
\end{tabular}

The above density is estimated to be accurate to 2 significant digits. Uncertainties are not addressed.

The following data were calculated from the input weight fractions.

\begin{tabular}{|c|c|c|c|c|c|c|}
\hline Element & Neutron ZA & Photon ZA & $\begin{array}{l}\text { Weight } \\
\text { Fraction }\end{array}$ & $\begin{array}{c}\text { Atom } \\
\text { Fraction }\end{array}$ & $\begin{array}{c}\text { Atom } \\
\text { Density }\end{array}$ & \\
\hline $\mathrm{H}$ & 1001 & 1000 & 0.005000 & $\overline{0.158643}$ & 0.014339 & \\
\hline $\mathrm{O}$ & 8016 & 8000 & 0.104000 & 0.207881 & 0.018790 & \\
\hline $\mathrm{Mg}$ & 12000 & 12000 & 0.002000 & 0.002632 & 0.000238 & \\
\hline $\mathrm{Al}$ & 13027 & 13000 & 0.004000 & 0.004741 & 0.000429 & \\
\hline $\mathrm{Si}$ & 14000 & 14000 & 0.034000 & 0.038715 & 0.003499 & \\
\hline$P$ & 15031 & 15000 & 0.197000 & 0.203403 & 0.018385 & \\
\hline $\mathrm{Ca}$ & 20000 & 20000 & 0.042000 & 0.033514 & 0.003029 & \\
\hline $\mathrm{Fe}$ & 26000 & 26000 & 0.612000 & 0.350471 & 0.031678 & \\
\hline Total & & & 1.000000 & 1.000000 & 0.090387 & \\
\hline MCNP Form & \multicolumn{2}{|c|}{ Weight Fractions } & \multicolumn{2}{|c|}{ Atom Fractions } & \multicolumn{2}{|c|}{ Atom Densities } \\
\hline \multirow[t]{8}{*}{ Neutrons } & 1001 & -0.005000 & 1001 & 0.158643 & 1001 & 0.014339 \\
\hline & 8016 & -0.104000 & 8016 & 0.207881 & 8016 & 0.018790 \\
\hline & 12000 & -0.002000 & 12000 & 0.002632 & 12000 & 0.000238 \\
\hline & 13027 & -0.004000 & 13027 & 0.004741 & 13027 & 0.000429 \\
\hline & 14000 & -0.034000 & 14000 & 0.038715 & 14000 & 0.003499 \\
\hline & 15031 & -0.197000 & 15031 & 0.203403 & 15031 & 0.018385 \\
\hline & 20000 & -0.042000 & 20000 & 0.033514 & 20000 & 0.003029 \\
\hline & 26000 & -0.612000 & 26000 & 0.350471 & 26000 & 0.031678 \\
\hline \multirow[t]{8}{*}{ Photons } & 1000 & -0.005000 & 1000 & 0.158643 & 1000 & 0.014339 \\
\hline & 8000 & -0.104000 & 8000 & 0.207881 & 8000 & 0.018790 \\
\hline & 12000 & -0.002000 & 12000 & 0.002632 & 12000 & 0.000238 \\
\hline & 13000 & -0.004000 & 13000 & 0.004741 & 13000 & 0.000429 \\
\hline & 14000 & -0.034000 & 14000 & 0.038715 & 14000 & 0.003499 \\
\hline & 15000 & -0.197000 & 15000 & 0.203403 & 15000 & 0.018385 \\
\hline & 20000 & -0.042000 & 20000 & 0.033514 & 20000 & 0.003029 \\
\hline & 26000 & -0.612000 & 26000 & 0.350471 & 26000 & 0.031678 \\
\hline \multirow[t]{5}{*}{ CEPXS Form: } & material & $\mathrm{H}$ & 0.005000 & & & \\
\hline & & 0 & 0.104000 & & & \\
\hline & & $\mathrm{Mg}$ & 0.002000 & & & \\
\hline & & $\mathrm{Al}$ & 0.004000 & & & \\
\hline & & $\mathrm{Si}$ & 0.034000 & & & \\
\hline
\end{tabular}




\begin{tabular}{|ccc|} 
& $\mathrm{P}$ & 0.197000 \\
& $\mathrm{Ca}$ & 0.042000 \\
& $\mathrm{Fe}$ & 0.612000 \\
& & \\
matname & Concrete, Ferro-phosphorus \\
density & 4.800000 \\
\hline Comments and References & \\
Density and weight fractions from pg 1081 and Tables 51.95 of Hungerford (1960). \\
\hline
\end{tabular}

\section{Concrete, Hanford Dry}

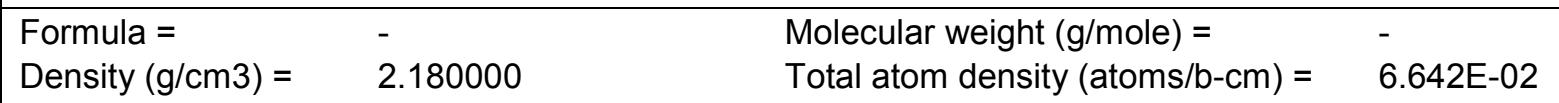

The above density is estimated to be accurate to 3 significant digits. Uncertainties are not addressed.

The following data were calculated from the input weight fractions.

\begin{tabular}{|c|c|c|c|c|c|c|}
\hline Element & Neutron ZA & Photon ZA & $\begin{array}{l}\text { Weight } \\
\text { Fraction }\end{array}$ & $\begin{array}{c}\text { Atom } \\
\text { Fraction }\end{array}$ & $\begin{array}{l}\text { Atom } \\
\text { Density }\end{array}$ & \\
\hline $\mathrm{H}$ & 1001 & 1000 & $\overline{0.004000}$ & $\overline{0.078440}$ & 0.005210 & \\
\hline 0 & 8016 & 8000 & 0.482102 & 0.595591 & 0.039559 & \\
\hline $\mathrm{Na}$ & 11023 & 11000 & 0.002168 & 0.001864 & 0.000124 & \\
\hline $\mathrm{Mg}$ & 12000 & 12000 & 0.014094 & 0.011462 & 0.000761 & \\
\hline $\mathrm{Al}$ & 13027 & 13000 & 0.069387 & 0.050831 & 0.003376 & \\
\hline $\mathrm{Si}$ & 14000 & 14000 & 0.277549 & 0.195330 & 0.012974 & \\
\hline $\mathrm{K}$ & 19000 & 19000 & 0.013010 & 0.006577 & 0.000437 & \\
\hline $\mathrm{Ca}$ & 20000 & 20000 & 0.080229 & 0.039567 & 0.002628 & \\
\hline $\mathrm{Fe}$ & 26000 & 26000 & 0.057461 & 0.020338 & 0.001351 & \\
\hline Total & & & 1.000000 & 1.000000 & 0.066419 & \\
\hline MCNP Form & \multicolumn{2}{|c|}{ Weight Fractions } & \multicolumn{2}{|c|}{ Atom Fractions } & \multicolumn{2}{|c|}{ Atom Densities } \\
\hline \multirow[t]{9}{*}{ Neutrons } & 1001 & -0.004000 & 1001 & 0.078440 & 1001 & 0.005210 \\
\hline & 8016 & -0.482102 & 8016 & 0.595591 & 8016 & 0.039559 \\
\hline & 11023 & -0.002168 & 11023 & 0.001864 & 11023 & 0.000124 \\
\hline & 12000 & -0.014094 & 12000 & 0.011462 & 12000 & 0.000761 \\
\hline & 13027 & -0.069387 & 13027 & 0.050831 & 13027 & 0.003376 \\
\hline & 14000 & -0.277549 & 14000 & 0.195330 & 14000 & 0.012974 \\
\hline & 19000 & -0.013010 & 19000 & 0.006577 & 19000 & 0.000437 \\
\hline & 20000 & -0.080229 & 20000 & 0.039567 & 20000 & 0.002628 \\
\hline & 26000 & -0.057461 & 26000 & 0.020338 & 26000 & 0.001351 \\
\hline \multirow[t]{7}{*}{ Photons } & 1000 & -0.004000 & 1000 & 0.078440 & 1000 & 0.005210 \\
\hline & 8000 & -0.482102 & 8000 & 0.595591 & 8000 & 0.039559 \\
\hline & 11000 & -0.002168 & 11000 & 0.001864 & 11000 & 0.000124 \\
\hline & 12000 & -0.014094 & 12000 & 0.011462 & 12000 & 0.000761 \\
\hline & 13000 & -0.069387 & 13000 & 0.050831 & 13000 & 0.003376 \\
\hline & 14000 & -0.277549 & 14000 & 0.195330 & 14000 & 0.012974 \\
\hline & 19000 & -0.013010 & 19000 & 0.006577 & 19000 & 0.000437 \\
\hline
\end{tabular}


PIET-43741-TM-963

PNNL-15870 Rev. 1

\begin{tabular}{|c|c|c|c|c|c|c|}
\hline & $\begin{array}{l}20000 \\
26000\end{array}$ & $\begin{array}{l}-0.080229 \\
-0.057461\end{array}$ & $\begin{array}{l}20000 \\
26000\end{array}$ & $\begin{array}{l}0.039567 \\
0.020338\end{array}$ & $\begin{array}{l}20000 \\
26000\end{array}$ & $\begin{array}{l}0.002628 \\
0.001351\end{array}$ \\
\hline CEPXS Form: & material & $\begin{array}{c}\mathrm{H} \\
\mathrm{O} \\
\mathrm{Na} \\
\mathrm{Mg} \\
\mathrm{Al} \\
\mathrm{Si} \\
\mathrm{K} \\
\mathrm{Ca} \\
\mathrm{Fe}\end{array}$ & $\begin{array}{l}0.004000 \\
0.482102 \\
0.002168 \\
0.014094 \\
0.069387 \\
0.277549 \\
0.013010 \\
0.080229 \\
0.057461\end{array}$ & & & \\
\hline & $\begin{array}{l}\text { matname } \\
\text { density }\end{array}$ & \multicolumn{2}{|c|}{$\begin{array}{l}\text { Concrete, Hanford Dry } \\
2.180000\end{array}$} & & & \\
\hline \multicolumn{7}{|c|}{$\begin{array}{l}\text { Comments and References } \\
\text { Data from Table } 1 \text { of Carter (1978). } \\
\text { Starting from the data in the reference for wet concrete, the water content was reduced to model drying for } \\
\text { decades in a dry environment. A reasonable minimum hydrogen content for old dry concrete is about } \\
0.4 \text { wt. } \% \text {. The change in the density due to drying from a hydrogen content of } 1.23 \text { to } 0.4 \text { wt. } \% \text {, assuming } \\
\text { that the concrete does not shrink as it dries, leads to a reduction in the concrete density from } 2.35 \mathrm{~g} / \mathrm{cm} 3 \\
\text { to } 2.169 \mathrm{~g} / \mathrm{cm} 3 \text {. Based on Table } 9.1 .12-7 \text { of Jaeger et al. }(1975) \text {, total concrete shrinkage due to drying } \\
\text { can be about } 1 \text { part in } 1000 \text {, so the density would only increase to about } 2.169 \times 1.001^{\wedge} 3=2.176 \mathrm{~g} / \mathrm{cm} 3 \text {. }\end{array}$} \\
\hline
\end{tabular}

\section{Concrete, Hanford Wet}

\begin{tabular}{|c|c|c|c|}
\hline $\begin{array}{l}= \\
(\mathrm{g} / \mathrm{cm} 3)=\end{array}$ & $\begin{array}{l}- \\
2.350000\end{array}$ & $\begin{array}{l}\text { Molecular weight }(\mathrm{g} / \mathrm{mole})= \\
\text { Total atom density }(\text { atoms } / \mathrm{b}-\mathrm{cm})=\end{array}$ & $\begin{array}{l}- \\
8.423 E-02\end{array}$ \\
\hline
\end{tabular}

The above density is estimated to be accurate to 3 significant digits. Uncertainties are not addressed.

The following data were calculated from the input weight fractions.

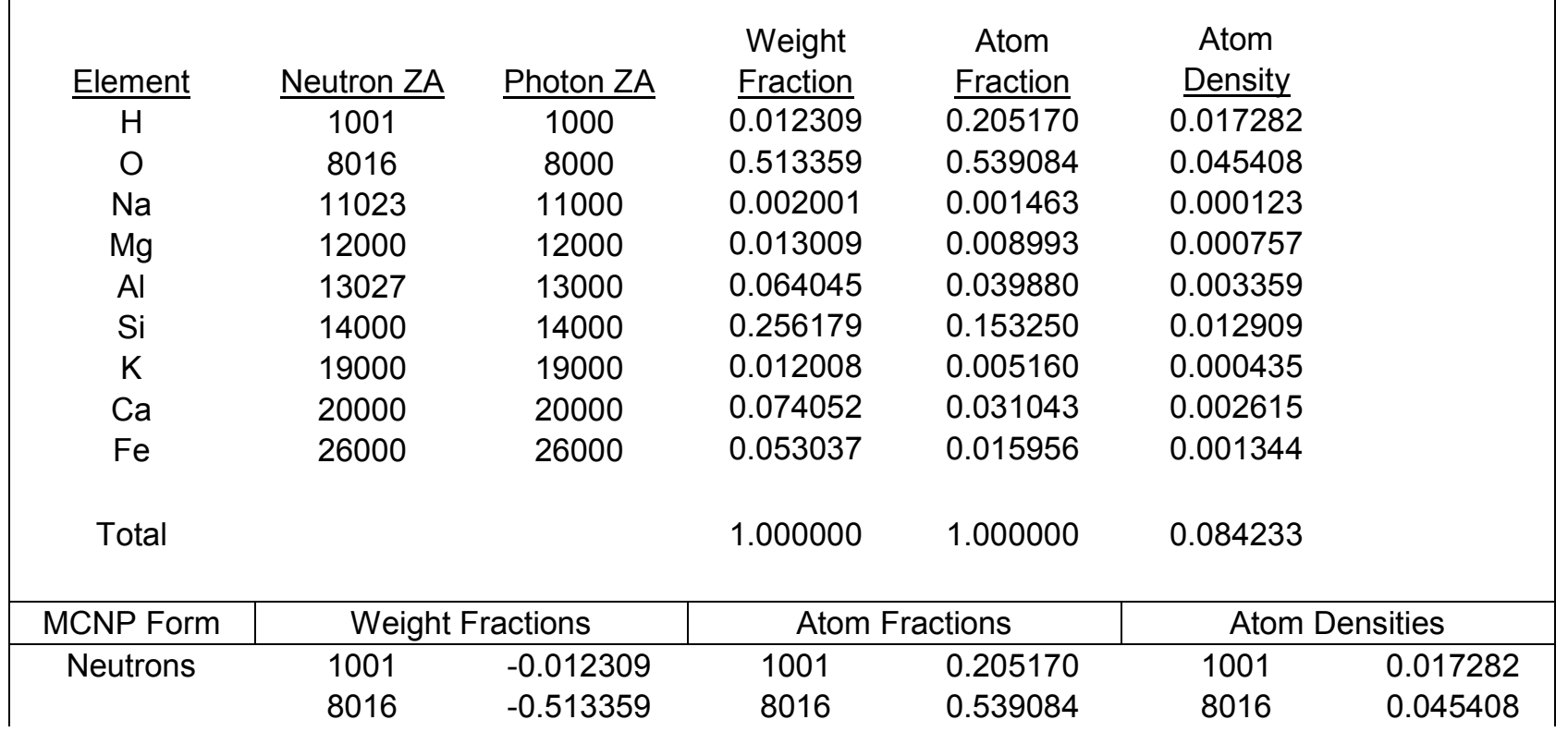


PIET-43741-TM-963

PNNL-15870 Rev. 1

\begin{tabular}{|c|c|c|c|c|c|c|}
\hline & 11023 & -0.002001 & 11023 & 0.001463 & 11023 & 0.000123 \\
\hline & 12000 & -0.013009 & 12000 & 0.008993 & 12000 & 0.000757 \\
\hline & 13027 & -0.064045 & 13027 & 0.039880 & 13027 & 0.003359 \\
\hline & 14000 & -0.256179 & 14000 & 0.153250 & 14000 & 0.012909 \\
\hline & 19000 & -0.012008 & 19000 & 0.005160 & 19000 & 0.000435 \\
\hline & 20000 & -0.074052 & 20000 & 0.031043 & 20000 & 0.002615 \\
\hline & 26000 & -0.053037 & 26000 & 0.015956 & 26000 & 0.001344 \\
\hline Photons & 1000 & -0.012309 & 1000 & 0.205170 & 1000 & 0.017282 \\
\hline & 8000 & -0.513359 & 8000 & 0.539084 & 8000 & 0.045408 \\
\hline & 11000 & -0.002001 & 11000 & 0.001463 & 11000 & 0.000123 \\
\hline & 12000 & -0.013009 & 12000 & 0.008993 & 12000 & 0.000757 \\
\hline & 13000 & -0.064045 & 13000 & 0.039880 & 13000 & 0.003359 \\
\hline & 14000 & -0.256179 & 14000 & 0.153250 & 14000 & 0.012909 \\
\hline & 19000 & -0.012008 & 19000 & 0.005160 & 19000 & 0.000435 \\
\hline & 20000 & -0.074052 & 20000 & 0.031043 & 20000 & 0.002615 \\
\hline & 26000 & -0.053037 & 26000 & 0.015956 & 26000 & 0.001344 \\
\hline CEPXS Form: & material & $\mathrm{H}$ & 0.012309 & & & \\
\hline & & 0 & 0.513359 & & & \\
\hline & & $\mathrm{Na}$ & 0.002001 & & & \\
\hline & & $\mathrm{Mg}$ & 0.013009 & & & \\
\hline & & $\mathrm{Al}$ & 0.064045 & & & \\
\hline & & $\mathrm{Si}$ & 0.256179 & & & \\
\hline & & $\mathrm{K}$ & 0.012008 & & & \\
\hline & & $\mathrm{Ca}$ & 0.074052 & & & \\
\hline & & $\mathrm{Fe}$ & 0.053037 & & & \\
\hline & $\begin{array}{c}\text { matname } \\
\text { density }\end{array}$ & $\begin{array}{c}\text { Concrete, } \mathrm{He} \\
2.350000\end{array}$ & ord Wet & & & \\
\hline $\begin{array}{l}\text { Comments anc } \\
\text { Data from Table } \\
\text { This concrete } \\
\text { time. The weig }\end{array}$ & $\begin{array}{l}\text { References } \\
\text { of Carter ( } \\
\text { tains } 1.23 \text { v } \\
\text { fractions ar }\end{array}$ & $\begin{array}{l}\text { 78). } \\
\% \text { hydrogen. } \\
\text { idjusted so th }\end{array}$ & $\begin{array}{l}\text { s is reason } \\
\text { sum to unit }\end{array}$ & for concret & has not & for a long \\
\hline
\end{tabular}

\section{Concrete, Iron-limonite}

\begin{tabular}{llll}
\hline Formula $=$ & - & Molecular weight $(\mathrm{g} / \mathrm{mole})=$ & - \\
Density $(\mathrm{g} / \mathrm{cm} 3)=$ & 4.400000 & Total atom density $($ atoms $/ \mathrm{b}-\mathrm{cm})=$ & $7.222 \mathrm{E}-02$
\end{tabular}

The above density is estimated to be accurate to 2 significant digits. Uncertainties are not addressed.

The following data were calculated from the input weight fractions.

\begin{tabular}{|c|c|c|c|c|c|}
\hline Element & Neutron ZA & Photon ZA & $\begin{array}{l}\text { Weight } \\
\text { Fraction }\end{array}$ & $\begin{array}{c}\text { Atom } \\
\text { Fraction }\end{array}$ & $\begin{array}{l}\text { Atom } \\
\text { Density }\end{array}$ \\
\hline $\mathrm{H}$ & 1001 & 1000 & $\overline{0.000500}$ & $\overline{0.018192}$ & 0.001314 \\
\hline O & 8016 & 8000 & 0.179910 & 0.412591 & 0.029796 \\
\hline $\mathrm{Mg}$ & 12000 & 12000 & 0.001999 & 0.003018 & 0.000218 \\
\hline $\mathrm{Al}$ & 13027 & 13000 & 0.004998 & 0.006796 & 0.000491 \\
\hline
\end{tabular}


PIET-43741-TM-963

PNNL-15870 Rev. 1

\begin{tabular}{|c|c|c|c|c|c|c|}
\hline $\mathrm{Si}$ & 14000 & 14000 & 0.013993 & 0.018281 & \multicolumn{2}{|l|}{0.001320} \\
\hline$S$ & 16000 & 16000 & 0.001000 & 0.001144 & \multicolumn{2}{|l|}{0.000083} \\
\hline $\mathrm{Ca}$ & 20000 & 20000 & 0.060970 & 0.055818 & \multicolumn{2}{|l|}{0.004031} \\
\hline $\mathrm{Mn}$ & 25055 & 25000 & 0.015992 & 0.010681 & \multicolumn{2}{|l|}{0.000771} \\
\hline $\mathrm{Fe}$ & 26000 & 26000 & 0.720640 & 0.473480 & \multicolumn{2}{|l|}{0.034193} \\
\hline Total & & & 1.000000 & 1.000000 & \multicolumn{2}{|l|}{0.072216} \\
\hline MCNP Form & \multicolumn{2}{|c|}{ Weight Fractions } & \multicolumn{2}{|c|}{ Atom Fractions } & \multicolumn{2}{|c|}{ Atom Densities } \\
\hline \multirow[t]{9}{*}{ Neutrons } & 1001 & -0.000500 & 1001 & 0.018192 & 1001 & 0.001314 \\
\hline & 8016 & -0.179910 & 8016 & 0.412591 & 8016 & 0.029796 \\
\hline & 12000 & -0.001999 & 12000 & 0.003018 & 12000 & 0.000218 \\
\hline & 13027 & -0.004998 & 13027 & 0.006796 & 13027 & 0.000491 \\
\hline & 14000 & -0.013993 & 14000 & 0.018281 & 14000 & 0.001320 \\
\hline & 16000 & -0.001000 & 16000 & 0.001144 & 16000 & 0.000083 \\
\hline & 20000 & -0.060970 & 20000 & 0.055818 & 20000 & 0.004031 \\
\hline & 25055 & -0.015992 & 25055 & 0.010681 & 25055 & 0.000771 \\
\hline & 26000 & -0.720640 & 26000 & 0.473480 & 26000 & 0.034193 \\
\hline \multirow[t]{9}{*}{ Photons } & 1000 & -0.000500 & 1000 & 0.018192 & 1000 & 0.001314 \\
\hline & 8000 & -0.179910 & 8000 & 0.412591 & 8000 & 0.029796 \\
\hline & 12000 & -0.001999 & 12000 & 0.003018 & 12000 & 0.000218 \\
\hline & 13000 & -0.004998 & 13000 & 0.006796 & 13000 & 0.000491 \\
\hline & 14000 & -0.013993 & 14000 & 0.018281 & 14000 & 0.001320 \\
\hline & 16000 & -0.001000 & 16000 & 0.001144 & 16000 & 0.000083 \\
\hline & 20000 & -0.060970 & 20000 & 0.055818 & 20000 & 0.004031 \\
\hline & 25000 & -0.015992 & 25000 & 0.010681 & 25000 & 0.000771 \\
\hline & 26000 & -0.720640 & 26000 & 0.473480 & 26000 & 0.034193 \\
\hline \multirow[t]{10}{*}{ CEPXS Form: } & material & $\mathrm{H}$ & 0.000500 & & & \\
\hline & & 0 & 0.179910 & & & \\
\hline & & $\mathrm{Mg}$ & 0.001999 & & & \\
\hline & & $\mathrm{Al}$ & 0.004998 & & & \\
\hline & & $\mathrm{Si}$ & 0.013993 & & & \\
\hline & & $S$ & 0.001000 & & & \\
\hline & & $\mathrm{Ca}$ & 0.060970 & & & \\
\hline & & $\mathrm{Mn}$ & 0.015992 & & & \\
\hline & & $\mathrm{Fe}$ & 0.720640 & & & \\
\hline & $\begin{array}{c}\text { matname } \\
\text { density }\end{array}$ & \multicolumn{3}{|c|}{$\begin{array}{l}\text { Concrete, Iron-limonite } \\
4.400000\end{array}$} & & \\
\hline \multicolumn{7}{|c|}{$\begin{array}{l}\text { Comments and References } \\
\text { Weight fractions from Tables } 51.95 \text { of Hungerford (1960). Weight fractions are adjusted so they sum to } \\
\text { unity. } \\
\text { Density }=4.27 \text { for wet concrete and } 4.3 \text { to } 4.5 \mathrm{~g} / \mathrm{cm} 3 \text { for hardened concrete (Table } 9.1 .12-40 \text { of Jaeger } \\
\text { et al. 1975). Also see "Concrete, Limonite and Steel." }\end{array}$} \\
\hline
\end{tabular}




\section{Concrete, Iron-Portland}

\begin{tabular}{|c|c|c|c|}
\hline Formula $=$ & - & Molecular weight $(\mathrm{g} / \mathrm{mole})=$ & - \\
\hline Density $(\mathrm{g} / \mathrm{cm} 3)=$ & 5.900000 & Total atom density $($ atoms $/ \mathrm{b}-\mathrm{cm})=$ & 8.633E-02 \\
\hline
\end{tabular}

\begin{tabular}{|c|c|c|c|c|c|c|}
\hline Element & Neutron ZA & Photon ZA & $\begin{array}{l}\text { Weight } \\
\text { Fraction }\end{array}$ & $\begin{array}{c}\text { Atom } \\
\text { Fraction }\end{array}$ & $\begin{array}{l}\text { Atom } \\
\text { Density }\end{array}$ & \\
\hline $\mathrm{H}$ & 1001 & 1000 & 0.003321 & 0.135585 & 0.011705 & \\
\hline O & 8016 & 8000 & 0.058563 & 0.150644 & 0.013005 & \\
\hline $\mathrm{Mg}$ & 12000 & 12000 & 0.001308 & 0.002215 & 0.000191 & \\
\hline $\mathrm{Al}$ & 13027 & 13000 & 0.003321 & 0.005065 & 0.000437 & \\
\hline $\mathrm{Si}$ & 14000 & 14000 & 0.009157 & 0.013418 & 0.001158 & \\
\hline$S$ & 16000 & 16000 & 0.000503 & 0.000646 & 0.000056 & \\
\hline $\mathrm{Ca}$ & 20000 & 20000 & 0.039847 & 0.040919 & 0.003533 & \\
\hline $\mathrm{Mn}$ & 25055 & 25000 & 0.003522 & 0.002638 & 0.000228 & \\
\hline $\mathrm{Fe}$ & 26000 & 26000 & 0.880459 & 0.648869 & 0.056018 & \\
\hline Total & & & 1.000000 & 1.000000 & 0.086332 & \\
\hline MCNP Form & \multicolumn{2}{|c|}{ Weight Fractions } & \multicolumn{2}{|c|}{ Atom Fractions } & \multicolumn{2}{|c|}{ Atom Densities } \\
\hline \multirow[t]{9}{*}{ Neutrons } & 1001 & -0.003321 & 1001 & 0.135585 & 1001 & 0.011705 \\
\hline & 8016 & -0.058563 & 8016 & 0.150644 & 8016 & 0.013005 \\
\hline & 12000 & -0.001308 & 12000 & 0.002215 & 12000 & 0.000191 \\
\hline & 13027 & -0.003321 & 13027 & 0.005065 & 13027 & 0.000437 \\
\hline & 14000 & -0.009157 & 14000 & 0.013418 & 14000 & 0.001158 \\
\hline & 16000 & -0.000503 & 16000 & 0.000646 & 16000 & 0.000056 \\
\hline & 20000 & -0.039847 & 20000 & 0.040919 & 20000 & 0.003533 \\
\hline & 25055 & -0.003522 & 25055 & 0.002638 & 25055 & 0.000228 \\
\hline & 26000 & -0.880459 & 26000 & 0.648869 & 26000 & 0.056018 \\
\hline \multirow[t]{9}{*}{ Photons } & 1000 & -0.003321 & 1000 & 0.135585 & 1000 & 0.011705 \\
\hline & 8000 & -0.058563 & 8000 & 0.150644 & 8000 & 0.013005 \\
\hline & 12000 & -0.001308 & 12000 & 0.002215 & 12000 & 0.000191 \\
\hline & 13000 & -0.003321 & 13000 & 0.005065 & 13000 & 0.000437 \\
\hline & 14000 & -0.009157 & 14000 & 0.013418 & 14000 & 0.001158 \\
\hline & 16000 & -0.000503 & 16000 & 0.000646 & 16000 & 0.000056 \\
\hline & 20000 & -0.039847 & 20000 & 0.040919 & 20000 & 0.003533 \\
\hline & 25000 & -0.003522 & 25000 & 0.002638 & 25000 & 0.000228 \\
\hline & 26000 & -0.880459 & 26000 & 0.648869 & 26000 & 0.056018 \\
\hline \multirow[t]{7}{*}{ CEPXS Form: } & material & $\mathrm{H}$ & 0.003321 & & & \\
\hline & & 0 & 0.058563 & & & \\
\hline & & $\mathrm{Mg}$ & 0.001308 & & & \\
\hline & & $\mathrm{Al}$ & 0.003321 & & & \\
\hline & & $\mathrm{Si}$ & 0.009157 & & & \\
\hline & & $S$ & 0.000503 & & & \\
\hline & & $\mathrm{Ca}$ & 0.039847 & & & \\
\hline
\end{tabular}




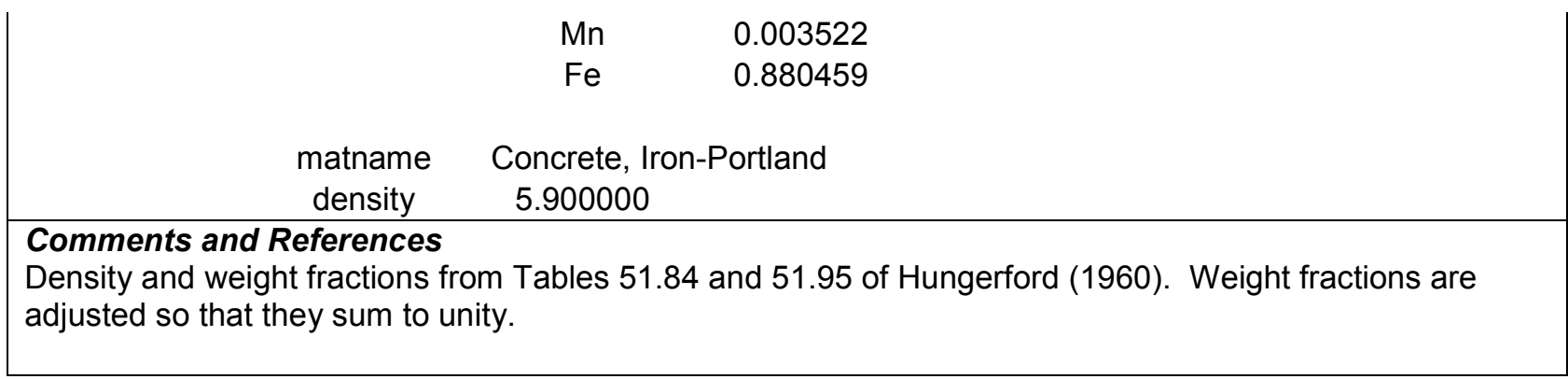

\section{Concrete, Limonite and Steel}

\begin{tabular}{llll}
\hline Formula $=$ & - & Molecular weight $(\mathrm{g} / \mathrm{mole})=$ \\
Density $(\mathrm{g} / \mathrm{cm} 3)=$ & 4.540000 & Total atom density $($ atoms $/ \mathrm{b}-\mathrm{cm})=$ & - \\
\hline
\end{tabular}

The above density is estimated to be accurate to 2 significant digits. Uncertainties are not addressed.

The following data were calculated from the input weight fractions.

\begin{tabular}{|c|c|c|c|c|c|c|}
\hline Element & Neutron ZA & Photon ZA & $\begin{array}{l}\text { Weight } \\
\text { Fraction }\end{array}$ & $\begin{array}{c}\text { Atom } \\
\text { Fraction }\end{array}$ & $\begin{array}{c}\text { Atom } \\
\text { Density }\end{array}$ & \\
\hline $\mathrm{H}$ & 1001 & 1000 & $\overline{0.006840}$ & $\overline{0.209640}$ & 0.018554 & \\
\hline $\mathrm{O}$ & 8016 & 8000 & 0.156222 & 0.301631 & 0.026696 & \\
\hline $\mathrm{Mg}$ & 12000 & 12000 & 0.001545 & 0.001963 & 0.000174 & \\
\hline $\mathrm{Al}$ & 13027 & 13000 & 0.006399 & 0.007326 & 0.000648 & \\
\hline $\mathrm{Si}$ & 14000 & 14000 & 0.014784 & 0.016261 & 0.001439 & \\
\hline $\mathrm{K}$ & 19000 & 19000 & 0.000883 & 0.000697 & 0.000062 & \\
\hline $\mathrm{Ca}$ & 20000 & 20000 & 0.057590 & 0.044390 & 0.003929 & \\
\hline $\mathrm{V}$ & 23000 & 23000 & 0.000883 & 0.000535 & 0.000047 & \\
\hline $\mathrm{Fe}$ & 26000 & 26000 & 0.754854 & 0.417557 & 0.036956 & \\
\hline Total & & & 1.000000 & 1.000000 & 0.088505 & \\
\hline MCNP Form & \multicolumn{2}{|c|}{ Weight Fractions } & \multicolumn{2}{|c|}{ Atom Fractions } & \multicolumn{2}{|c|}{ Atom Densities } \\
\hline \multirow[t]{9}{*}{ Neutrons } & 1001 & -0.006840 & 1001 & 0.209640 & 1001 & 0.018554 \\
\hline & 8016 & -0.156222 & 8016 & 0.301631 & 8016 & 0.026696 \\
\hline & 12000 & -0.001545 & 12000 & 0.001963 & 12000 & 0.000174 \\
\hline & 13027 & -0.006399 & 13027 & 0.007326 & 13027 & 0.000648 \\
\hline & 14000 & -0.014784 & 14000 & 0.016261 & 14000 & 0.001439 \\
\hline & 19000 & -0.000883 & 19000 & 0.000697 & 19000 & 0.000062 \\
\hline & 20000 & -0.057590 & 20000 & 0.044390 & 20000 & 0.003929 \\
\hline & 23000 & -0.000883 & 23000 & 0.000535 & 23000 & 0.000047 \\
\hline & 26000 & -0.754854 & 26000 & 0.417557 & 26000 & 0.036956 \\
\hline \multirow[t]{7}{*}{ Photons } & 1000 & -0.006840 & 1000 & 0.209640 & 1000 & 0.018554 \\
\hline & 8000 & -0.156222 & 8000 & 0.301631 & 8000 & 0.026696 \\
\hline & 12000 & -0.001545 & 12000 & 0.001963 & 12000 & 0.000174 \\
\hline & 13000 & -0.006399 & 13000 & 0.007326 & 13000 & 0.000648 \\
\hline & 14000 & -0.014784 & 14000 & 0.016261 & 14000 & 0.001439 \\
\hline & 19000 & -0.000883 & 19000 & 0.000697 & 19000 & 0.000062 \\
\hline & 20000 & -0.057590 & 20000 & 0.044390 & 20000 & 0.003929 \\
\hline
\end{tabular}


PIET-43741-TM-963

PNNL-15870 Rev. 1

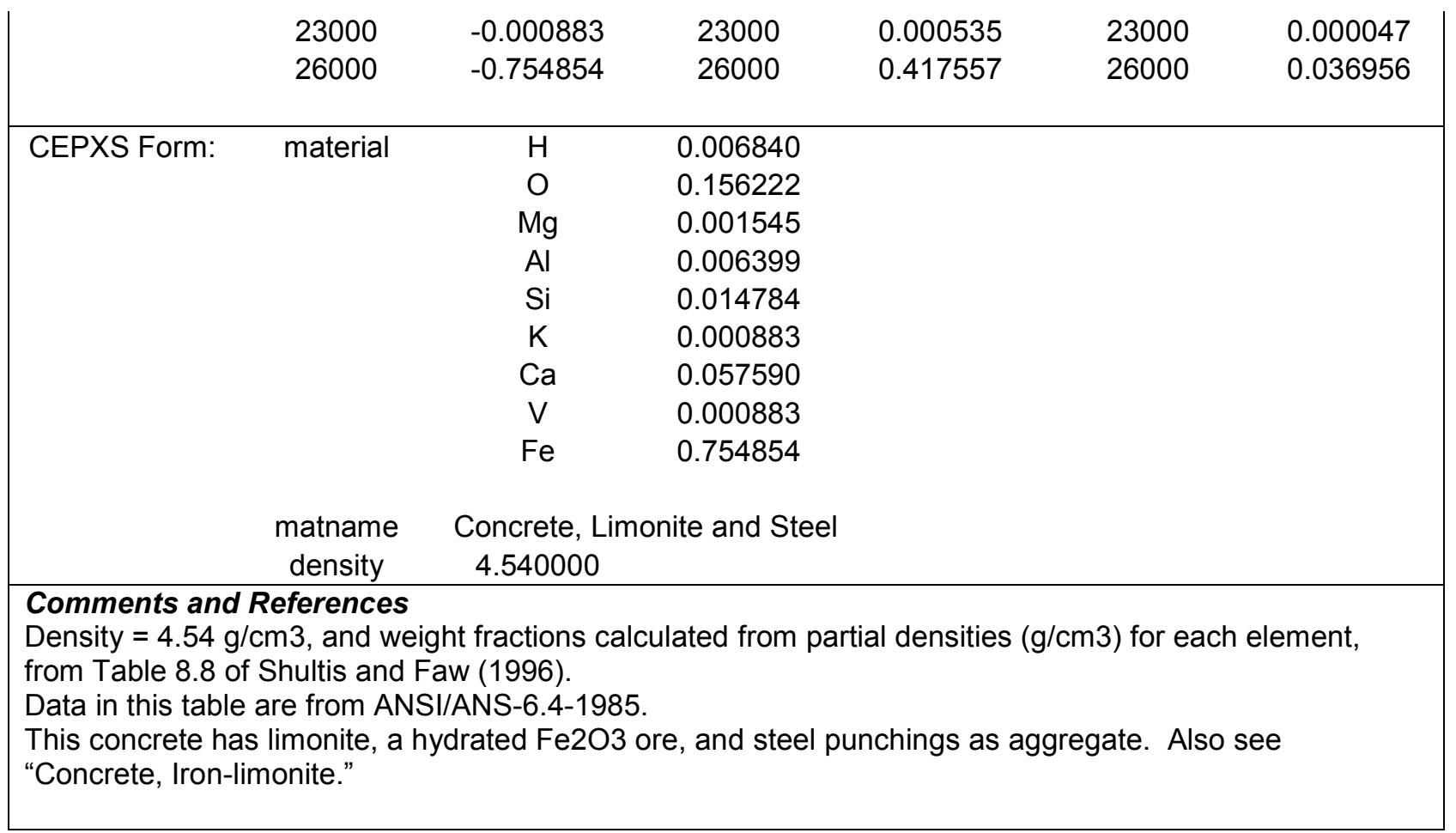

\section{Concrete, Los Alamos (MCNP)}

\begin{tabular}{llll}
\hline Formula $=$ & - & Molecular weight $(\mathrm{g} / \mathrm{mole})=$ & - \\
Density $(\mathrm{g} / \mathrm{cm} 3)=$ & 2.250000 & Total atom density $($ atoms $/ \mathrm{b}-\mathrm{cm})=$ & $7.186 \mathrm{E}-02$
\end{tabular}

The above density is estimated to be accurate to 3 significant digits. Uncertainties are not addressed.

The following data were calculated from the input weight fractions.

\begin{tabular}{|c|c|c|c|c|c|c|}
\hline Element & Neutron ZA & Photon ZA & $\begin{array}{l}\text { Weight } \\
\text { Fraction }\end{array}$ & $\begin{array}{c}\text { Atom } \\
\text { Fraction }\end{array}$ & $\begin{array}{l}\text { Atom } \\
\text { Density }\end{array}$ & \\
\hline $\mathrm{H}$ & 1001 & 1000 & 0.004530 & $\overline{0.084739}$ & 0.006090 & \\
\hline $\mathrm{O}$ & 8016 & 8000 & 0.512600 & 0.604079 & 0.043412 & \\
\hline $\mathrm{Na}$ & 11023 & 11000 & 0.015270 & 0.012523 & 0.000900 & \\
\hline $\mathrm{Al}$ & 13027 & 13000 & 0.035550 & 0.024842 & 0.001785 & \\
\hline $\mathrm{Si}$ & 14000 & 14000 & 0.360360 & 0.241921 & 0.017386 & \\
\hline $\mathrm{Ca}$ & 20000 & 20000 & 0.057910 & 0.027244 & 0.001958 & \\
\hline $\mathrm{Fe}$ & 26000 & 26000 & 0.013780 & 0.004652 & 0.000334 & \\
\hline Total & & & 1.000000 & 1.000000 & 0.071865 & \\
\hline MCNP Form & \multicolumn{2}{|c|}{ Weight Fractions } & \multicolumn{2}{|c|}{ Atom Fractions } & \multicolumn{2}{|c|}{ Atom Densities } \\
\hline \multirow[t]{6}{*}{ Neutrons } & 1001 & -0.004530 & 1001 & 0.084739 & 1001 & 0.006090 \\
\hline & 8016 & -0.512600 & 8016 & 0.604079 & 8016 & 0.043412 \\
\hline & 11023 & -0.015270 & 11023 & 0.012523 & 11023 & 0.000900 \\
\hline & 13027 & -0.035550 & 13027 & 0.024842 & 13027 & 0.001785 \\
\hline & 14000 & -0.360360 & 14000 & 0.241921 & 14000 & 0.017386 \\
\hline & 20000 & -0.057910 & 20000 & 0.027244 & 20000 & 0.001958 \\
\hline
\end{tabular}


PIET-43741-TM-963

PNNL-15870 Rev. 1

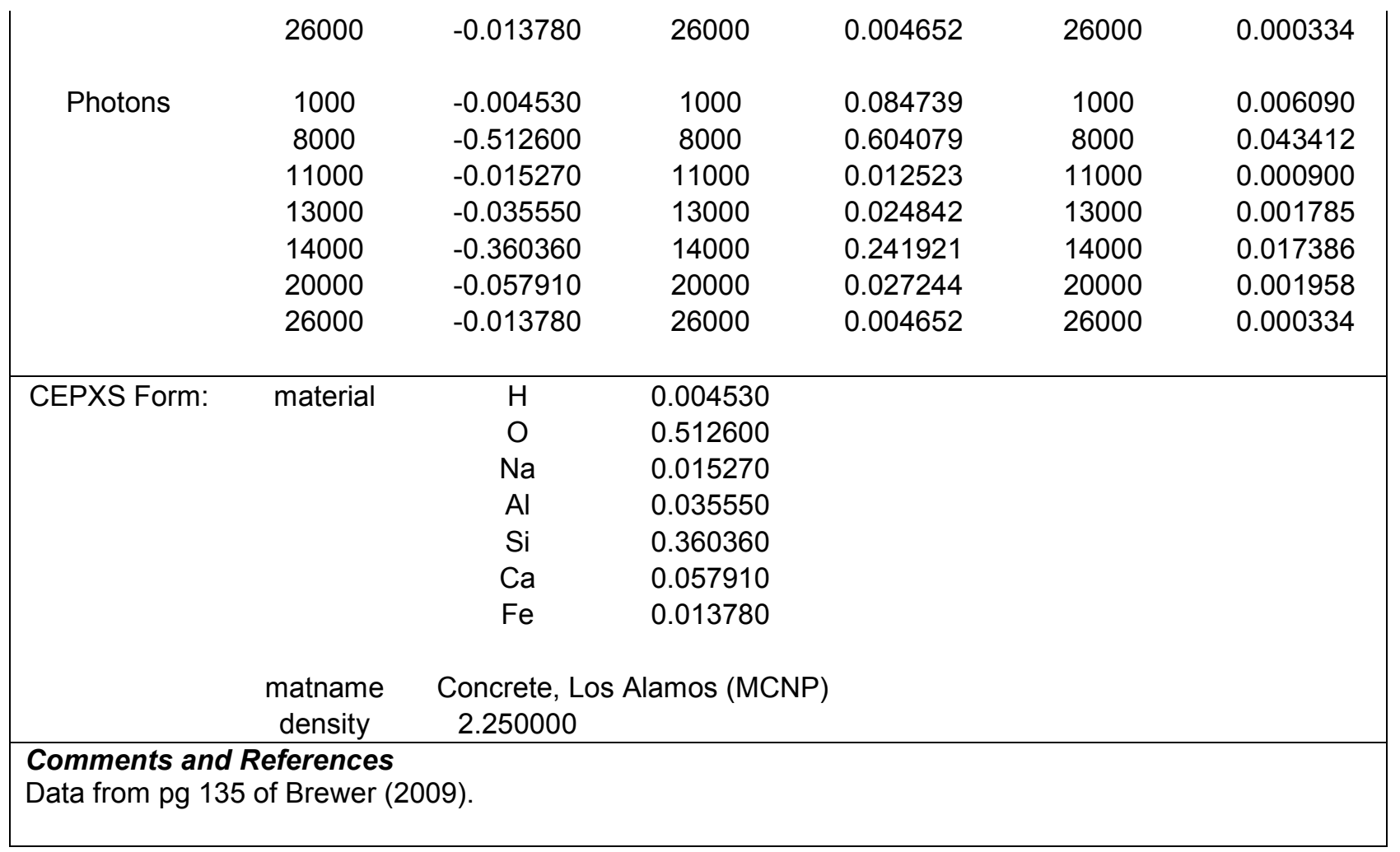

\section{Concrete, Luminite-colemanite-baryte}

\begin{tabular}{|c|c|c|c|}
\hline Formula $=$ & - & Molecular weight $(\mathrm{g} / \mathrm{mole})=$ & - \\
\hline Density $(\mathrm{g} / \mathrm{cm} 3)=$ & 3.100000 & Total atom density $($ atoms $/ \mathrm{b}-\mathrm{cm})=$ & 8.194E-02 \\
\hline
\end{tabular}

\begin{tabular}{|c|c|c|c|c|c|}
\hline Element & Neutron ZA & Photon ZA & $\begin{array}{l}\text { Weight } \\
\text { Fraction }\end{array}$ & $\begin{array}{c}\text { Atom } \\
\text { Fraction }\end{array}$ & $\begin{array}{c}\text { Atom } \\
\text { Density }\end{array}$ \\
\hline $\mathrm{H}$ & 1001 & 1000 & $\overline{0.010957}$ & 0.247678 & 0.020294 \\
\hline B & - & 5000 & 0.008846 & 0.018643 & 0.001528 \\
\hline 0 & 8016 & 8000 & 0.371431 & 0.528939 & 0.043340 \\
\hline $\mathrm{Na}$ & 11023 & 11000 & 0.001106 & 0.001096 & 0.000090 \\
\hline $\mathrm{Mg}$ & 12000 & 12000 & 0.001407 & 0.001319 & 0.000108 \\
\hline $\mathrm{Al}$ & 13027 & 13000 & 0.017692 & 0.014940 & 0.001224 \\
\hline $\mathrm{Si}$ & 14000 & 14000 & 0.009650 & 0.007829 & 0.000641 \\
\hline$S$ & 16000 & 16000 & 0.091074 & 0.064713 & 0.005302 \\
\hline $\mathrm{Ca}$ & 20000 & 20000 & 0.055086 & 0.031316 & 0.002566 \\
\hline $\mathrm{Ti}$ & 22000 & 22000 & 0.012766 & 0.006077 & 0.000498 \\
\hline $\mathrm{Mn}$ & 25055 & 25000 & 0.001206 & 0.000500 & 0.000041 \\
\hline $\mathrm{Fe}$ & 26000 & 26000 & 0.030860 & 0.012591 & 0.001032 \\
\hline $\mathrm{Ba}$ & - & 56000 & 0.387917 & 0.064360 & 0.005273 \\
\hline Total & & & 1.000000 & 1.000000 & 0.081937 \\
\hline
\end{tabular}


PIET-43741-TM-963

PNNL-15870 Rev. 1

\begin{tabular}{|c|c|c|c|c|c|c|}
\hline MCNP Form & \multicolumn{2}{|c|}{ Weight Fractions } & \multicolumn{2}{|c|}{ Atom Fractions } & \multicolumn{2}{|c|}{ Atom Densities } \\
\hline \multirow{13}{*}{ Neutrons } & 1001 & -0.010957 & 1001 & 0.247678 & 1001 & 0.020294 \\
\hline & - & -0.008846 & - & 0.018643 & - & 0.001528 \\
\hline & 8016 & -0.371431 & 8016 & 0.528939 & 8016 & 0.043340 \\
\hline & 11023 & -0.001106 & 11023 & 0.001096 & 11023 & 0.000090 \\
\hline & 12000 & -0.001407 & 12000 & 0.001319 & 12000 & 0.000108 \\
\hline & 13027 & -0.017692 & 13027 & 0.014940 & 13027 & 0.001224 \\
\hline & 14000 & -0.009650 & 14000 & 0.007829 & 14000 & 0.000641 \\
\hline & 16000 & -0.091074 & 16000 & 0.064713 & 16000 & 0.005302 \\
\hline & 20000 & -0.055086 & 20000 & 0.031316 & 20000 & 0.002566 \\
\hline & 22000 & -0.012766 & 22000 & 0.006077 & 22000 & 0.000498 \\
\hline & 25055 & -0.001206 & 25055 & 0.000500 & 25055 & 0.000041 \\
\hline & 26000 & -0.030860 & 26000 & 0.012591 & 26000 & 0.001032 \\
\hline & - & -0.387917 & - & 0.064360 & - & 0.005273 \\
\hline \multirow[t]{13}{*}{ Photons } & 1000 & -0.010957 & 1000 & 0.247678 & 1000 & 0.020294 \\
\hline & 5000 & -0.008846 & 5000 & 0.018643 & 5000 & 0.001528 \\
\hline & 8000 & -0.371431 & 8000 & 0.528939 & 8000 & 0.043340 \\
\hline & 11000 & -0.001106 & 11000 & 0.001096 & 11000 & 0.000090 \\
\hline & 12000 & -0.001407 & 12000 & 0.001319 & 12000 & 0.000108 \\
\hline & 13000 & -0.017692 & 13000 & 0.014940 & 13000 & 0.001224 \\
\hline & 14000 & -0.009650 & 14000 & 0.007829 & 14000 & 0.000641 \\
\hline & 16000 & -0.091074 & 16000 & 0.064713 & 16000 & 0.005302 \\
\hline & 20000 & -0.055086 & 20000 & 0.031316 & 20000 & 0.002566 \\
\hline & 22000 & -0.012766 & 22000 & 0.006077 & 22000 & 0.000498 \\
\hline & 25000 & -0.001206 & 25000 & 0.000500 & 25000 & 0.000041 \\
\hline & 26000 & -0.030860 & 26000 & 0.012591 & 26000 & 0.001032 \\
\hline & 56000 & -0.387917 & 56000 & 0.064360 & 56000 & 0.005273 \\
\hline \multirow[t]{14}{*}{ CEPXS Form: } & material & $\mathrm{H}$ & 0.010957 & & & \\
\hline & & B & 0.008846 & & & \\
\hline & & O & 0.371431 & & & \\
\hline & & $\mathrm{Na}$ & 0.001106 & & & \\
\hline & & $\mathrm{Mg}$ & 0.001407 & & & \\
\hline & & $\mathrm{Al}$ & 0.017692 & & & \\
\hline & & $\mathrm{Si}$ & 0.009650 & & & \\
\hline & & $S$ & 0.091074 & & & \\
\hline & & $\mathrm{Ca}$ & 0.055086 & & & \\
\hline & & $\mathrm{Ti}$ & 0.012766 & & & \\
\hline & & $\mathrm{Mn}$ & 0.001206 & & & \\
\hline & & $\mathrm{Fe}$ & 0.030860 & & & \\
\hline & & $\mathrm{Ba}$ & 0.387917 & & & \\
\hline & $\begin{array}{c}\text { matname } \\
\text { density }\end{array}$ & \multicolumn{3}{|c|}{$\begin{array}{l}\text { Concrete, Luminite-colemanite-baryte } \\
3.100000\end{array}$} & & \\
\hline \multicolumn{7}{|c|}{$\begin{array}{l}\text { Comments and References } \\
\text { Density and weight fractions from Tables } 51.84 \text { and } 51.95 \text { of Hungerford (1960). Weight fractions are } \\
\text { adjusted so they sum to unity. }\end{array}$} \\
\hline
\end{tabular}




\section{Concrete, Luminite-Portland-colemanite-baryte}

\begin{tabular}{|c|c|c|c|}
\hline Formula $=$ & - & Molecular weight $(\mathrm{g} / \mathrm{mole})=$ & - \\
\hline Density $(\mathrm{g} / \mathrm{cm} 3)=$ & 3.100000 & Total atom density $($ atoms $/ \mathrm{b}-\mathrm{cm})=$ & 8.300E-02 \\
\hline
\end{tabular}

\begin{tabular}{|c|c|c|c|c|c|c|}
\hline Element & Neutron ZA & Photon ZA & $\begin{array}{l}\text { Weight } \\
\text { Fraction }\end{array}$ & $\begin{array}{c}\text { Atom } \\
\text { Fraction }\end{array}$ & $\begin{array}{l}\text { Atom } \\
\text { Density }\end{array}$ & \\
\hline $\mathrm{H}$ & 1001 & 1000 & 0.011126 & 0.248270 & 0.020606 & \\
\hline $\mathrm{B}$ & - & 5000 & 0.010316 & 0.021464 & 0.001781 & \\
\hline 0 & 8016 & 8000 & 0.374023 & 0.525811 & 0.043642 & \\
\hline $\mathrm{Na}$ & 11023 & 11000 & 0.001113 & 0.001088 & 0.000090 & \\
\hline $\mathrm{Mg}$ & 12000 & 12000 & 0.002023 & 0.001872 & 0.000155 & \\
\hline $\mathrm{Al}$ & 13027 & 13000 & 0.013351 & 0.011129 & 0.000924 & \\
\hline $\mathrm{Si}$ & 14000 & 14000 & 0.015070 & 0.012069 & 0.001002 & \\
\hline$S$ & 16000 & 16000 & 0.090724 & 0.063640 & 0.005282 & \\
\hline $\mathrm{Ca}$ & 20000 & 20000 & 0.077576 & 0.043537 & 0.003614 & \\
\hline $\mathrm{Ti}$ & 22000 & 22000 & 0.000718 & 0.000337 & 0.000028 & \\
\hline $\mathrm{Mn}$ & 25055 & 25000 & 0.000405 & 0.000166 & 0.000014 & \\
\hline $\mathrm{Fe}$ & 26000 & 26000 & 0.018914 & 0.007618 & 0.000632 & \\
\hline $\mathrm{Ba}$ & - & 56000 & 0.384643 & 0.062999 & 0.005229 & \\
\hline Total & & & 1.000000 & 1.000000 & 0.083000 & \\
\hline MCNP Form & \multicolumn{2}{|c|}{ Weight Fractions } & \multicolumn{2}{|c|}{ Atom Fractions } & \multicolumn{2}{|c|}{ Atom Densities } \\
\hline \multirow[t]{13}{*}{ Neutrons } & 1001 & -0.011126 & 1001 & 0.248270 & 1001 & 0.020606 \\
\hline & - & -0.010316 & - & 0.021464 & - & 0.001781 \\
\hline & 8016 & -0.374023 & 8016 & 0.525811 & 8016 & 0.043642 \\
\hline & 11023 & -0.001113 & 11023 & 0.001088 & 11023 & 0.000090 \\
\hline & 12000 & -0.002023 & 12000 & 0.001872 & 12000 & 0.000155 \\
\hline & 13027 & -0.013351 & 13027 & 0.011129 & 13027 & 0.000924 \\
\hline & 14000 & -0.015070 & 14000 & 0.012069 & 14000 & 0.001002 \\
\hline & 16000 & -0.090724 & 16000 & 0.063640 & 16000 & 0.005282 \\
\hline & 20000 & -0.077576 & 20000 & 0.043537 & 20000 & 0.003614 \\
\hline & 22000 & -0.000718 & 22000 & 0.000337 & 22000 & 0.000028 \\
\hline & 25055 & -0.000405 & 25055 & 0.000166 & 25055 & 0.000014 \\
\hline & 26000 & -0.018914 & 26000 & 0.007618 & 26000 & 0.000632 \\
\hline & - & -0.384643 & - & 0.062999 & - & 0.005229 \\
\hline \multirow[t]{9}{*}{ Photons } & 1000 & -0.011126 & 1000 & 0.248270 & 1000 & 0.020606 \\
\hline & 5000 & -0.010316 & 5000 & 0.021464 & 5000 & 0.001781 \\
\hline & 8000 & -0.374023 & 8000 & 0.525811 & 8000 & 0.043642 \\
\hline & 11000 & -0.001113 & 11000 & 0.001088 & 11000 & 0.000090 \\
\hline & 12000 & -0.002023 & 12000 & 0.001872 & 12000 & 0.000155 \\
\hline & 13000 & -0.013351 & 13000 & 0.011129 & 13000 & 0.000924 \\
\hline & 14000 & -0.015070 & 14000 & 0.012069 & 14000 & 0.001002 \\
\hline & 16000 & -0.090724 & 16000 & 0.063640 & 16000 & 0.005282 \\
\hline & 20000 & -0.077576 & 20000 & 0.043537 & 20000 & 0.003614 \\
\hline
\end{tabular}


PIET-43741-TM-963

PNNL-15870 Rev. 1

\begin{tabular}{|c|c|c|c|c|c|c|}
\hline & 22000 & -0.000718 & 22000 & 0.000337 & 22000 & 0.000028 \\
\hline & 25000 & -0.000405 & 25000 & 0.000166 & 25000 & 0.000014 \\
\hline & 26000 & -0.018914 & 26000 & 0.007618 & 26000 & 0.000632 \\
\hline & 56000 & -0.384643 & 56000 & 0.062999 & 56000 & 0.005229 \\
\hline CEPXS Form: & material & $\mathrm{H}$ & 0.011126 & & & \\
\hline & & B & 0.010316 & & & \\
\hline & & 0 & 0.374023 & & & \\
\hline & & $\mathrm{Na}$ & 0.001113 & & & \\
\hline & & $\mathrm{Mg}$ & 0.002023 & & & \\
\hline & & $\mathrm{Al}$ & 0.013351 & & & \\
\hline & & $\mathrm{Si}$ & 0.015070 & & & \\
\hline & & $S$ & 0.090724 & & & \\
\hline & & $\mathrm{Ca}$ & 0.077576 & & & \\
\hline & & $\mathrm{Ti}$ & 0.000718 & & & \\
\hline & & $\mathrm{Mn}$ & 0.000405 & & & \\
\hline & & $\mathrm{Fe}$ & 0.018914 & & & \\
\hline & & $\mathrm{Ba}$ & 0.384643 & & & \\
\hline & $\begin{array}{c}\text { matname } \\
\text { density }\end{array}$ & $\begin{array}{c}\text { Concrete, L } \\
3.100000\end{array}$ & ite-Portlar & lemanite-ba & & \\
\hline $\begin{array}{l}\text { Comments an } \\
\text { Density and we } \\
\text { adjusted so the }\end{array}$ & $\begin{array}{l}\text { eferences } \\
\text { it fractions } \\
\text { um to unity }\end{array}$ & Tables 51 & nd 51.95 & gerford ( & Weig & ns are \\
\hline
\end{tabular}

\section{Concrete, $\mathrm{M}-1$}

Formula $=$

Density $(\mathrm{g} / \mathrm{cm} 3)=\quad 4.500000$
Molecular weight $(\mathrm{g} / \mathrm{mole})=$

Total atom density $($ atoms $/ \mathrm{b}-\mathrm{cm})=8.790 \mathrm{E}-02$

The above density is estimated to be accurate to 2 significant digits. Uncertainties are not addressed.

The following data were calculated from the input weight fractions.

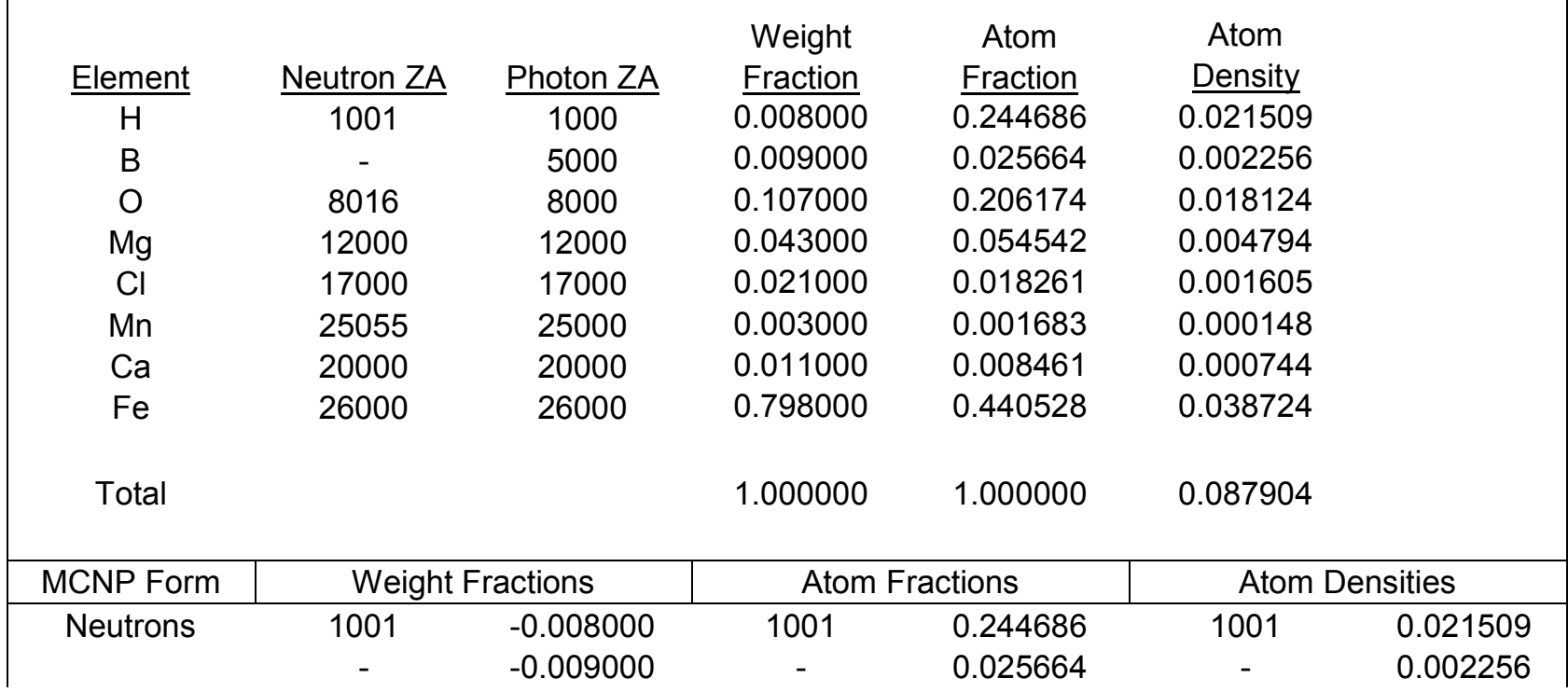




\begin{tabular}{|c|c|c|c|c|c|c|}
\hline & 8016 & -0.107000 & 8016 & 0.206174 & 8016 & 0.018124 \\
\hline & 12000 & -0.043000 & 12000 & 0.054542 & 12000 & 0.004794 \\
\hline & 17000 & -0.021000 & 17000 & 0.018261 & 17000 & 0.001605 \\
\hline & 25055 & -0.003000 & 25055 & 0.001683 & 25055 & 0.000148 \\
\hline & 20000 & -0.011000 & 20000 & 0.008461 & 20000 & 0.000744 \\
\hline & 26000 & -0.798000 & 26000 & 0.440528 & 26000 & 0.038724 \\
\hline Photons & 1000 & -0.008000 & 1000 & 0.244686 & 1000 & 0.021509 \\
\hline & 5000 & -0.009000 & 5000 & 0.025664 & 5000 & 0.002256 \\
\hline & 8000 & -0.107000 & 8000 & 0.206174 & 8000 & 0.018124 \\
\hline & 12000 & -0.043000 & 12000 & 0.054542 & 12000 & 0.004794 \\
\hline & 17000 & -0.021000 & 17000 & 0.018261 & 17000 & 0.001605 \\
\hline & 25000 & -0.003000 & 25000 & 0.001683 & 25000 & 0.000148 \\
\hline & 20000 & -0.011000 & 20000 & 0.008461 & 20000 & 0.000744 \\
\hline & 26000 & -0.798000 & 26000 & 0.440528 & 26000 & 0.038724 \\
\hline CEPXS Form: & material & $\mathrm{H}$ & 0.008000 & & & \\
\hline & & $B$ & 0.009000 & & & \\
\hline & & $\mathrm{O}$ & 0.107000 & & & \\
\hline & & $\mathrm{Mg}$ & 0.043000 & & & \\
\hline & & $\mathrm{Cl}$ & 0.021000 & & & \\
\hline & & $\mathrm{Mn}$ & 0.003000 & & & \\
\hline & & $\mathrm{Ca}$ & 0.011000 & & & \\
\hline & & $\mathrm{Fe}$ & 0.798000 & & & \\
\hline & $\begin{array}{c}\text { matname } \\
\text { density }\end{array}$ & $\begin{array}{c}\text { Concrete, } M-1 \\
4.500000\end{array}$ & & & & \\
\hline $\begin{array}{l}\text { Comments an } \\
\text { Density }=4.5 \mathrm{~g}\end{array}$ & $\begin{array}{l}\text { eferences } \\
3 \text { and weic }\end{array}$ & & & & & \\
\hline
\end{tabular}

\section{Concrete, Magnetite}

\begin{tabular}{llll}
\hline Formula $=$ & - & Molecular weight $(\mathrm{g} / \mathrm{mole})=$ & - \\
Density $(\mathrm{g} / \mathrm{cm} 3)=$ & 3.530000 & Total atom density $($ atoms $/ \mathrm{b}-\mathrm{cm})=$ & $7.970 \mathrm{E}-02$
\end{tabular}

The above density is estimated to be accurate to 2 significant digits. Uncertainties are not addressed. The following data were calculated from the input weight fractions.

\begin{tabular}{|c|c|c|c|c|c|}
\hline Element & Neutron ZA & Photon ZA & $\begin{array}{l}\text { Weight } \\
\text { Fraction }\end{array}$ & $\begin{array}{c}\text { Atom } \\
\text { Fraction }\end{array}$ & $\begin{array}{c}\text { Atom } \\
\text { Density }\end{array}$ \\
\hline $\mathrm{H}$ & 1001 & 1000 & $\overline{0.003113}$ & $\overline{0.082371}$ & 0.006565 \\
\hline $\mathrm{O}$ & 8016 & 8000 & 0.330504 & 0.551004 & 0.043914 \\
\hline $\mathrm{Mg}$ & 12000 & 12000 & 0.009338 & 0.010248 & 0.000817 \\
\hline $\mathrm{Al}$ & 13027 & 13000 & 0.023486 & 0.023218 & 0.001850 \\
\hline $\mathrm{Si}$ & 14000 & 14000 & 0.025750 & 0.024455 & 0.001949 \\
\hline$S$ & 16000 & 16000 & 0.001415 & 0.001177 & 0.000094 \\
\hline $\mathrm{Ca}$ & 20000 & 20000 & 0.071024 & 0.047270 & 0.003767 \\
\hline $\mathrm{Ti}$ & 22000 & 22000 & 0.054329 & 0.030275 & 0.002413 \\
\hline V & 23000 & 23000 & 0.003113 & 0.001630 & 0.000130 \\
\hline
\end{tabular}


PIET-43741-TM-963

PNNL-15870 Rev. 1

\begin{tabular}{|c|c|c|c|c|c|c|}
\hline $\mathrm{Cr}$ & 24000 & 24000 & 0.001698 & 0.000871 & 0.000069 & \\
\hline $\mathrm{Mn}$ & 25055 & 25000 & 0.001981 & 0.000962 & 0.000077 & \\
\hline $\mathrm{Fe}$ & 26000 & 26000 & 0.474250 & 0.226519 & 0.018053 & \\
\hline Total & & & 1.000000 & 1.000000 & 0.079697 & \\
\hline MCNP Form & Weigh & ractions & Atom & ctions & Atom & sities \\
\hline Neutrons & 1001 & -0.003113 & 1001 & 0.082371 & 1001 & 0.006565 \\
\hline & 8016 & -0.330504 & 8016 & 0.551004 & 8016 & 0.043914 \\
\hline & 12000 & -0.009338 & 12000 & 0.010248 & 12000 & 0.000817 \\
\hline & 13027 & -0.023486 & 13027 & 0.023218 & 13027 & 0.001850 \\
\hline & 14000 & -0.025750 & 14000 & 0.024455 & 14000 & 0.001949 \\
\hline & 16000 & -0.001415 & 16000 & 0.001177 & 16000 & 0.000094 \\
\hline & 20000 & -0.071024 & 20000 & 0.047270 & 20000 & 0.003767 \\
\hline & 22000 & -0.054329 & 22000 & 0.030275 & 22000 & 0.002413 \\
\hline & 23000 & -0.003113 & 23000 & 0.001630 & 23000 & 0.000130 \\
\hline & 24000 & -0.001698 & 24000 & 0.000871 & 24000 & 0.000069 \\
\hline & 25055 & -0.001981 & 25055 & 0.000962 & 25055 & 0.000077 \\
\hline & 26000 & -0.474250 & 26000 & 0.226519 & 26000 & 0.018053 \\
\hline Photons & 1000 & -0.003113 & 1000 & 0.082371 & 1000 & 0.006565 \\
\hline & 8000 & -0.330504 & 8000 & 0.551004 & 8000 & 0.043914 \\
\hline & 12000 & -0.009338 & 12000 & 0.010248 & 12000 & 0.000817 \\
\hline & 13000 & -0.023486 & 13000 & 0.023218 & 13000 & 0.001850 \\
\hline & 14000 & -0.025750 & 14000 & 0.024455 & 14000 & 0.001949 \\
\hline & 16000 & -0.001415 & 16000 & 0.001177 & 16000 & 0.000094 \\
\hline & 20000 & -0.071024 & 20000 & 0.047270 & 20000 & 0.003767 \\
\hline & 22000 & -0.054329 & 22000 & 0.030275 & 22000 & 0.002413 \\
\hline & 23000 & -0.003113 & 23000 & 0.001630 & 23000 & 0.000130 \\
\hline & 24000 & -0.001698 & 24000 & 0.000871 & 24000 & 0.000069 \\
\hline & 25000 & -0.001981 & 25000 & 0.000962 & 25000 & 0.000077 \\
\hline & 26000 & -0.474250 & 26000 & 0.226519 & 26000 & 0.018053 \\
\hline CEPXS Form: & material & $\mathrm{H}$ & 0.003113 & & & \\
\hline & & 0 & 0.330504 & & & \\
\hline & & $\mathrm{Mg}$ & 0.009338 & & & \\
\hline & & $\mathrm{Al}$ & 0.023486 & & & \\
\hline & & Si & 0.025750 & & & \\
\hline & & $S$ & 0.001415 & & & \\
\hline & & $\mathrm{Ca}$ & 0.071024 & & & \\
\hline & & $\mathrm{Ti}$ & 0.054329 & & & \\
\hline & & V & 0.003113 & & & \\
\hline & & $\mathrm{Cr}$ & 0.001698 & & & \\
\hline & & $\mathrm{Mn}$ & 0.001981 & & & \\
\hline & & $\mathrm{Fe}$ & 0.474250 & & & \\
\hline & $\begin{array}{c}\text { matname } \\
\text { density }\end{array}$ & $\begin{array}{c}\text { Concrete, } \mathrm{M} \\
3.530000\end{array}$ & etite & & & \\
\hline
\end{tabular}


from Table 8.8 of Shultis and Faw (1996).

Data in this table are from ANSI/ANS-6.4-1985.

This concrete has magnetite (FeO-Fe2O3) as aggregate.

Density $=3.45 \mathrm{~g} / \mathrm{cm} 3$ and a similar composition in Table 8.3 of Schaeffer (1973).

Density $=3.41 \mathrm{~g} / \mathrm{cm} 3$ for wet concrete (Table 9.1.12-40 of Jaeger et al. 1975).

\section{Concrete, Magnetite and Steel}

\begin{tabular}{llll}
\hline Formula $=$ & - & Molecular weight $(\mathrm{g} / \mathrm{mole})=$ & - \\
Density $(\mathrm{g} / \mathrm{cm} 3)=$ & 4.640000 & Total atom density $($ atoms $/ \mathrm{b}-\mathrm{cm})=$ & $7.646 \mathrm{E}-02$
\end{tabular}

The above density is estimated to be accurate to 2 significant digits. Uncertainties are not addressed.

The following data were calculated from the input weight fractions.

\begin{tabular}{|c|c|c|c|c|c|c|}
\hline Element & Neutron ZA & Photon ZA & $\begin{array}{l}\text { Weight } \\
\text { Fraction }\end{array}$ & $\begin{array}{c}\text { Atom } \\
\text { Fraction }\end{array}$ & $\begin{array}{c}\text { Atom } \\
\text { Density }\end{array}$ & \\
\hline $\mathrm{H}$ & 1001 & 1000 & $\overline{0.002374}$ & $\overline{0.086069}$ & 0.006581 & \\
\hline $\mathrm{O}$ & 8016 & 8000 & 0.137678 & 0.314488 & 0.024045 & \\
\hline $\mathrm{Mg}$ & 12000 & 12000 & 0.003669 & 0.005516 & 0.000422 & \\
\hline $\mathrm{Al}$ & 13027 & 13000 & 0.010358 & 0.014030 & 0.001073 & \\
\hline $\mathrm{Si}$ & 14000 & 14000 & 0.015753 & 0.020499 & 0.001567 & \\
\hline $\mathrm{Ca}$ & 20000 & 20000 & 0.055675 & 0.050769 & 0.003882 & \\
\hline $\mathrm{Ti}$ & 22000 & 22000 & 0.015969 & 0.012192 & 0.000932 & \\
\hline $\mathrm{V}$ & 23000 & 23000 & 0.000647 & 0.000464 & 0.000036 & \\
\hline $\mathrm{Fe}$ & 26000 & 26000 & 0.757877 & 0.495972 & 0.037921 & \\
\hline Total & & & 1.000000 & 1.000000 & 0.076458 & \\
\hline MCNP Form & \multicolumn{2}{|c|}{ Weight Fractions } & \multicolumn{2}{|c|}{ Atom Fractions } & \multicolumn{2}{|c|}{ Atom Densities } \\
\hline \multirow[t]{9}{*}{ Neutrons } & 1001 & -0.002374 & 1001 & 0.086069 & 1001 & 0.006581 \\
\hline & 8016 & -0.137678 & 8016 & 0.314488 & 8016 & 0.024045 \\
\hline & 12000 & -0.003669 & 12000 & 0.005516 & 12000 & 0.000422 \\
\hline & 13027 & -0.010358 & 13027 & 0.014030 & 13027 & 0.001073 \\
\hline & 14000 & -0.015753 & 14000 & 0.020499 & 14000 & 0.001567 \\
\hline & 20000 & -0.055675 & 20000 & 0.050769 & 20000 & 0.003882 \\
\hline & 22000 & -0.015969 & 22000 & 0.012192 & 22000 & 0.000932 \\
\hline & 23000 & -0.000647 & 23000 & 0.000464 & 23000 & 0.000036 \\
\hline & 26000 & -0.757877 & 26000 & 0.495972 & 26000 & 0.037921 \\
\hline \multirow[t]{9}{*}{ Photons } & 1000 & -0.002374 & 1000 & 0.086069 & 1000 & 0.006581 \\
\hline & 8000 & -0.137678 & 8000 & 0.314488 & 8000 & 0.024045 \\
\hline & 12000 & -0.003669 & 12000 & 0.005516 & 12000 & 0.000422 \\
\hline & 13000 & -0.010358 & 13000 & 0.014030 & 13000 & 0.001073 \\
\hline & 14000 & -0.015753 & 14000 & 0.020499 & 14000 & 0.001567 \\
\hline & 20000 & -0.055675 & 20000 & 0.050769 & 20000 & 0.003882 \\
\hline & 22000 & -0.015969 & 22000 & 0.012192 & 22000 & 0.000932 \\
\hline & 23000 & -0.000647 & 23000 & 0.000464 & 23000 & 0.000036 \\
\hline & 26000 & -0.757877 & 26000 & 0.495972 & 26000 & 0.037921 \\
\hline
\end{tabular}




\begin{tabular}{|ccc} 
CEPXS Form: $\quad$ material & $\mathrm{H}$ & 0.002374 \\
& $\mathrm{O}$ & 0.137678 \\
$\mathrm{Mg}$ & 0.003669 \\
& $\mathrm{Al}$ & 0.010358 \\
$\mathrm{Si}$ & 0.015753 \\
& $\mathrm{Ca}$ & 0.055675 \\
& $\mathrm{Ti}$ & 0.015969 \\
& $\mathrm{~V}$ & 0.000647 \\
& $\mathrm{Fe}$ & 0.757877
\end{tabular}

matname Concrete, Magnetite and Steel

density 4.640000

\section{Comments and References}

Density $=4.63 \mathrm{~g} / \mathrm{cm} 3$, and weight fractions calculated from partial densities $(\mathrm{g} / \mathrm{cm} 3)$ for each element, from Table 8.8 of Shultis and Faw (1996).

Data in this table are from ANSI/ANS-6.4-1985.

This concrete has magnetite (FeO-Fe2O3) and steel as aggregate.

\section{Concrete, Magnuson}

\begin{tabular}{llll}
\hline Formula $=$ & - & Molecular weight $(\mathrm{g} / \mathrm{mole})=$ & - \\
Density $(\mathrm{g} / \mathrm{cm} 3)=$ & 2.147000 & Total atom density $($ atoms $/ \mathrm{b}-\mathrm{cm})=$ & $7.128 \mathrm{E}-02$
\end{tabular}

The above density is estimated to be accurate to 2 significant digits. Uncertainties are not addressed.

The following data were calculated from the input weight fractions.

\begin{tabular}{|c|c|c|c|c|c|c|}
\hline Element & Neutron ZA & Photon ZA & $\begin{array}{l}\text { Weight } \\
\text { Fraction }\end{array}$ & $\begin{array}{c}\text { Atom } \\
\text { Fraction }\end{array}$ & $\begin{array}{l}\text { Atom } \\
\text { Density }\end{array}$ & \\
\hline $\mathrm{H}$ & 1001 & 1000 & $\overline{0.003319}$ & $\overline{0.059733}$ & 0.004257 & \\
\hline $\mathrm{C}$ & 6000 & 6000 & 0.105320 & 0.159071 & 0.011338 & \\
\hline O & 8016 & 8000 & 0.499428 & 0.566258 & 0.040360 & \\
\hline $\mathrm{Na}$ & 11023 & 11000 & 0.001411 & 0.001113 & 0.000079 & \\
\hline $\mathrm{Mg}$ & 12000 & 12000 & 0.094200 & 0.070307 & 0.005011 & \\
\hline $\mathrm{Al}$ & 13027 & 13000 & 0.007859 & 0.005284 & 0.000377 & \\
\hline $\mathrm{Si}$ & 14000 & 14000 & 0.042101 & 0.027193 & 0.001938 & \\
\hline$S$ & 16000 & 16000 & 0.002483 & 0.001405 & 0.000100 & \\
\hline $\mathrm{Cl}$ & 17000 & 17000 & 0.000523 & 0.000268 & 0.000019 & \\
\hline $\mathrm{K}$ & 19000 & 19000 & 0.009445 & 0.004382 & 0.000312 & \\
\hline $\mathrm{Ca}$ & 20000 & 20000 & 0.226317 & 0.102437 & 0.007301 & \\
\hline $\mathrm{Ti}$ & 22000 & 22000 & 0.001488 & 0.000564 & 0.000040 & \\
\hline $\mathrm{Mn}$ & 25055 & 25000 & 0.000512 & 0.000169 & 0.000012 & \\
\hline $\mathrm{Fe}$ & 26000 & 26000 & 0.005595 & 0.001817 & 0.000130 & \\
\hline Total & & & 1.000000 & 1.000000 & 0.071275 & \\
\hline MCNP Form & \multicolumn{2}{|c|}{ Weight Fractions } & \multicolumn{2}{|c|}{ Atom Fractions } & Atom & sities \\
\hline \multirow[t]{3}{*}{ Neutrons } & 1001 & -0.003319 & 1001 & 0.059733 & 1001 & 0.004257 \\
\hline & 6000 & -0.105320 & 6000 & 0.159071 & 6000 & 0.011338 \\
\hline & 8016 & -0.499428 & 8016 & 0.566258 & 8016 & 0.040360 \\
\hline
\end{tabular}


PIET-43741-TM-963

PNNL-15870 Rev. 1

\begin{tabular}{|c|c|c|c|c|c|c|}
\hline & 11023 & -0.001411 & 11023 & 0.001113 & 11023 & 0.000079 \\
\hline & 12000 & -0.094200 & 12000 & 0.070307 & 12000 & 0.005011 \\
\hline & 13027 & -0.007859 & 13027 & 0.005284 & 13027 & 0.000377 \\
\hline & 14000 & -0.042101 & 14000 & 0.027193 & 14000 & 0.001938 \\
\hline & 16000 & -0.002483 & 16000 & 0.001405 & 16000 & 0.000100 \\
\hline & 17000 & -0.000523 & 17000 & 0.000268 & 17000 & 0.000019 \\
\hline & 19000 & -0.009445 & 19000 & 0.004382 & 19000 & 0.000312 \\
\hline & 20000 & -0.226317 & 20000 & 0.102437 & 20000 & 0.007301 \\
\hline & 22000 & -0.001488 & 22000 & 0.000564 & 22000 & 0.000040 \\
\hline & 25055 & -0.000512 & 25055 & 0.000169 & 25055 & 0.000012 \\
\hline & 26000 & -0.005595 & 26000 & 0.001817 & 26000 & 0.000130 \\
\hline Photons & 1000 & -0.003319 & 1000 & 0.059733 & 1000 & 0.004257 \\
\hline & 6000 & -0.105320 & 6000 & 0.159071 & 6000 & 0.011338 \\
\hline & 8000 & -0.499428 & 8000 & 0.566258 & 8000 & 0.040360 \\
\hline & 11000 & -0.001411 & 11000 & 0.001113 & 11000 & 0.000079 \\
\hline & 12000 & -0.094200 & 12000 & 0.070307 & 12000 & 0.005011 \\
\hline & 13000 & -0.007859 & 13000 & 0.005284 & 13000 & 0.000377 \\
\hline & 14000 & -0.042101 & 14000 & 0.027193 & 14000 & 0.001938 \\
\hline & 16000 & -0.002483 & 16000 & 0.001405 & 16000 & 0.000100 \\
\hline & 17000 & -0.000523 & 17000 & 0.000268 & 17000 & 0.000019 \\
\hline & 19000 & -0.009445 & 19000 & 0.004382 & 19000 & 0.000312 \\
\hline & 20000 & -0.226317 & 20000 & 0.102437 & 20000 & 0.007301 \\
\hline & 22000 & -0.001488 & 22000 & 0.000564 & 22000 & 0.000040 \\
\hline & 25000 & -0.000512 & 25000 & 0.000169 & 25000 & 0.000012 \\
\hline & 26000 & -0.005595 & 26000 & 0.001817 & 26000 & 0.000130 \\
\hline CEPXS Form: & material & $\mathrm{H}$ & 0.003319 & & & \\
\hline & & C & 0.105320 & & & \\
\hline & & O & 0.499428 & & & \\
\hline & & $\mathrm{Na}$ & 0.001411 & & & \\
\hline & & $\mathrm{Mg}$ & 0.094200 & & & \\
\hline & & $\mathrm{Al}$ & 0.007859 & & & \\
\hline & & $\mathrm{Si}$ & 0.042101 & & & \\
\hline & & $\mathrm{S}$ & 0.002483 & & & \\
\hline & & $\mathrm{Cl}$ & 0.000523 & & & \\
\hline & & $\mathrm{K}$ & 0.009445 & & & \\
\hline & & $\mathrm{Ca}$ & 0.226317 & & & \\
\hline & & $\mathrm{Ti}$ & 0.001488 & & & \\
\hline & & $\mathrm{Mn}$ & 0.000512 & & & \\
\hline & & $\mathrm{Fe}$ & 0.005595 & & & \\
\hline & $\begin{array}{l}\text { matname } \\
\text { density }\end{array}$ & $\begin{array}{c}\text { Concrete, M } \\
2.147000\end{array}$ & Iuson & & & \\
\hline $\begin{array}{l}\text { Comments an } \\
\text { Data from Petri }\end{array}$ & $\begin{array}{l}\text { eferences } \\
\text { t al. (2000). }\end{array}$ & & & & & \\
\hline
\end{tabular}




\section{Concrete, MO}

$\begin{array}{llll}\text { Formula }= & - & \text { Molecular weight }(\mathrm{g} / \mathrm{mole})= & - \\ \text { Density }(\mathrm{g} / \mathrm{cm} 3)= & 5.500000 & \text { Total atom density }(\text { atoms } / \mathrm{b}-\mathrm{cm})= & 8.760 \mathrm{E}-02 \\ \text { The above density is estimated to be accurate to } 2 \text { significant digits. Uncertainties are not addressed. } \\ \text { The following data were calculated from the input weight fractions. }\end{array}$

\begin{tabular}{|c|c|c|c|c|c|}
\hline Element & Neutron ZA & Photon ZA & $\begin{array}{l}\text { Weight } \\
\text { Fraction }\end{array}$ & $\begin{array}{c}\text { Atom } \\
\text { Fraction }\end{array}$ & $\begin{array}{c}\text { Atom } \\
\text { Density }\end{array}$ \\
\hline $\mathrm{H}$ & 1001 & 1000 & $\overline{0.005000}$ & $\overline{0.187558}$ & 0.016430 \\
\hline 0 & 8016 & 8000 & 0.060000 & 0.141791 & 0.012421 \\
\hline $\mathrm{Mg}$ & 12000 & 12000 & 0.037000 & 0.057558 & 0.005042 \\
\hline $\mathrm{Mn}$ & 25055 & 25000 & 0.004000 & 0.002753 & 0.000241 \\
\hline $\mathrm{Cl}$ & 17000 & 17000 & 0.013000 & 0.013864 & 0.001215 \\
\hline $\mathrm{Fe}$ & 26000 & 26000 & 0.881000 & 0.596476 & 0.052252 \\
\hline Total & & & 1.000000 & 1.000000 & 0.087602 \\
\hline
\end{tabular}

\begin{tabular}{|c|cccccc|}
\hline MCNP Form & \multicolumn{2}{c|}{ Weight Fractions } & \multicolumn{2}{c|}{ Atom Fractions } & \multicolumn{2}{c|}{ Atom Densities } \\
\hline Neutrons & 1001 & -0.005000 & 1001 & 0.187558 & 1001 & 0.016430 \\
& 8016 & -0.060000 & 8016 & 0.141791 & 8016 & 0.012421 \\
& 12000 & -0.037000 & 12000 & 0.057558 & 12000 & 0.005042 \\
& 25055 & -0.004000 & 25055 & 0.002753 & 25055 & 0.000241 \\
& 17000 & -0.013000 & 17000 & 0.013864 & 17000 & 0.001215 \\
& 26000 & -0.881000 & 26000 & 0.596476 & 26000 & 0.052252 \\
Photons & & & & & & \\
& 1000 & -0.005000 & 1000 & 0.187558 & 1000 & 0.016430 \\
& 8000 & -0.060000 & 8000 & 0.141791 & 8000 & 0.012421 \\
& 12000 & -0.037000 & 12000 & 0.057558 & 12000 & 0.005042 \\
& 25000 & -0.004000 & 25000 & 0.002753 & 25000 & 0.000241 \\
& 17000 & -0.013000 & 17000 & 0.013864 & 17000 & 0.001215 \\
& 26000 & -0.881000 & 26000 & 0.596476 & 26000 & 0.052252
\end{tabular}

$\begin{array}{ccc}\text { CEPXS Form: material } & \mathrm{H} & 0.005000 \\ & \mathrm{O} & 0.060000 \\ \mathrm{Mg} & 0.037000 \\ \mathrm{Mn} & 0.004000 \\ & \mathrm{Cl} & 0.013000 \\ & \mathrm{Fe} & 0.881000\end{array}$

matname Concrete, $\mathrm{MO}$

density $\quad 5.500000$

Comments and References

Density $=5.2$ to $5.8 \mathrm{~g} / \mathrm{cm} 3$ and weight fractions from Tables 51.93 and 51.95 of Hungerford (1960). 


\section{Concrete, Oak Ridge (ORNL)}

\begin{tabular}{llll}
\hline Formula $=$ & - & Molecular weight $(\mathrm{g} / \mathrm{mole})=$ & - \\
Density $(\mathrm{g} / \mathrm{cm} 3)=$ & 2.300000 & Total atom density $($ atoms $/ \mathrm{b}-\mathrm{cm})=$ & $7.969 \mathrm{E}-02$
\end{tabular}

The above density is estimated to be accurate to 2 significant digits. Uncertainties are not addressed.

The following data were calculated from the input weight fractions.

\begin{tabular}{|c|c|c|c|c|c|c|}
\hline Element & Neutron ZA & Photon ZA & $\begin{array}{l}\text { Weight } \\
\text { Fraction }\end{array}$ & $\begin{array}{c}\text { Atom } \\
\text { Fraction }\end{array}$ & $\begin{array}{l}\text { Atom } \\
\text { Density }\end{array}$ & \\
\hline $\mathrm{H}$ & 1001 & 1000 & $\overline{0.006187}$ & $\overline{0.106691}$ & 0.008502 & \\
\hline C & 6000 & 6000 & 0.175193 & 0.253540 & 0.020204 & \\
\hline 0 & 8016 & 8000 & 0.410184 & 0.445629 & 0.035510 & \\
\hline $\mathrm{Na}$ & 11023 & 11000 & 0.000271 & 0.000205 & 0.000016 & \\
\hline $\mathrm{Mg}$ & 12000 & 12000 & 0.032649 & 0.023349 & 0.001861 & \\
\hline $\mathrm{Al}$ & 13027 & 13000 & 0.010830 & 0.006977 & 0.000556 & \\
\hline $\mathrm{Si}$ & 14000 & 14000 & 0.034479 & 0.021339 & 0.001700 & \\
\hline $\mathrm{K}$ & 19000 & 19000 & 0.001138 & 0.000506 & 0.000040 & \\
\hline $\mathrm{Ca}$ & 20000 & 20000 & 0.321287 & 0.139343 & 0.011104 & \\
\hline $\mathrm{Fe}$ & 26000 & 26000 & 0.007784 & 0.002423 & 0.000193 & \\
\hline Total & & & 1.000000 & 1.000000 & 0.079686 & \\
\hline MCNP Form & \multicolumn{2}{|c|}{ Weight Fractions } & \multicolumn{2}{|c|}{ Atom Fractions } & \multicolumn{2}{|c|}{ Atom Densities } \\
\hline \multirow[t]{10}{*}{ Neutrons } & 1001 & -0.006187 & 1001 & 0.106691 & 1001 & 0.008502 \\
\hline & 6000 & -0.175193 & 6000 & 0.253540 & 6000 & 0.020204 \\
\hline & 8016 & -0.410184 & 8016 & 0.445629 & 8016 & 0.035510 \\
\hline & 11023 & -0.000271 & 11023 & 0.000205 & 11023 & 0.000016 \\
\hline & 12000 & -0.032649 & 12000 & 0.023349 & 12000 & 0.001861 \\
\hline & 13027 & -0.010830 & 13027 & 0.006977 & 13027 & 0.000556 \\
\hline & 14000 & -0.034479 & 14000 & 0.021339 & 14000 & 0.001700 \\
\hline & 19000 & -0.001138 & 19000 & 0.000506 & 19000 & 0.000040 \\
\hline & 20000 & -0.321287 & 20000 & 0.139343 & 20000 & 0.011104 \\
\hline & 26000 & -0.007784 & 26000 & 0.002423 & 26000 & 0.000193 \\
\hline \multirow[t]{10}{*}{ Photons } & 1000 & -0.006187 & 1000 & 0.106691 & 1000 & 0.008502 \\
\hline & 6000 & -0.175193 & 6000 & 0.253540 & 6000 & 0.020204 \\
\hline & 8000 & -0.410184 & 8000 & 0.445629 & 8000 & 0.035510 \\
\hline & 11000 & -0.000271 & 11000 & 0.000205 & 11000 & 0.000016 \\
\hline & 12000 & -0.032649 & 12000 & 0.023349 & 12000 & 0.001861 \\
\hline & 13000 & -0.010830 & 13000 & 0.006977 & 13000 & 0.000556 \\
\hline & 14000 & -0.034479 & 14000 & 0.021339 & 14000 & 0.001700 \\
\hline & 19000 & -0.001138 & 19000 & 0.000506 & 19000 & 0.000040 \\
\hline & 20000 & -0.321287 & 20000 & 0.139343 & 20000 & 0.011104 \\
\hline & 26000 & -0.007784 & 26000 & 0.002423 & 26000 & 0.000193 \\
\hline \multirow[t]{4}{*}{ CEPXS Form: } & material & $\mathrm{H}$ & 0.006187 & & & \\
\hline & & C & 0.175193 & & & \\
\hline & & $\mathrm{O}$ & 0.410184 & & & \\
\hline & & $\mathrm{Na}$ & 0.000271 & & & \\
\hline
\end{tabular}




\begin{tabular}{|lcc|} 
& $\mathrm{Mg}$ & 0.032649 \\
$\mathrm{Al}$ & 0.010830 \\
$\mathrm{Si}$ & 0.034479 \\
& $\mathrm{~K}$ & 0.001138 \\
& $\mathrm{Ca}$ & 0.321287 \\
& $\mathrm{Fe}$ & 0.007784 \\
& & \\
matname & Concrete, Oak Ridge (ORNL) \\
density & 2.300000 \\
\hline Comments and References & \\
Data from Petrie et al. (2000). \\
Weight fractions are adjusted so they sum to unity. Also listed as ORNL concrete in Table 1 of Carter \\
(1978), with reference to Maerker and Muckenthaler (1966). \\
\hline
\end{tabular}

\section{Concrete, Ordinary (NBS 03)}

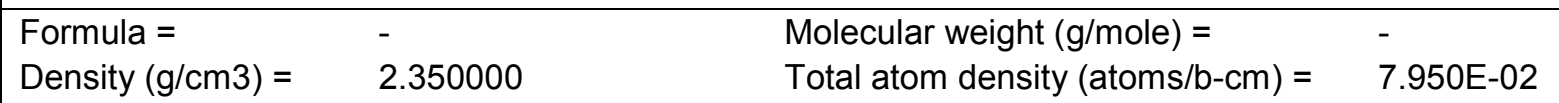

The above density is estimated to be accurate to 3 significant digits. Uncertainties are not addressed.

The following data were calculated from the input weight fractions.

\begin{tabular}{|c|c|c|c|c|c|c|}
\hline Element & Neutron ZA & Photon ZA & $\begin{array}{l}\text { Weight } \\
\text { Fraction }\end{array}$ & $\begin{array}{c}\text { Atom } \\
\text { Fraction }\end{array}$ & $\begin{array}{l}\text { Atom } \\
\text { Density }\end{array}$ & \\
\hline $\mathrm{H}$ & 1001 & 1000 & 0.008485 & 0.149867 & 0.011914 & \\
\hline $\mathrm{C}$ & 6000 & 6000 & 0.050064 & 0.074204 & 0.005899 & \\
\hline $\mathrm{O}$ & 8016 & 8000 & 0.473483 & 0.526832 & 0.041881 & \\
\hline $\mathrm{Mg}$ & 12000 & 12000 & 0.024183 & 0.017713 & 0.001408 & \\
\hline $\mathrm{Al}$ & 13027 & 13000 & 0.036063 & 0.023794 & 0.001892 & \\
\hline Si & 14000 & 14000 & 0.145100 & 0.091972 & 0.007311 & \\
\hline$S$ & 16000 & 16000 & 0.002970 & 0.001649 & 0.000131 & \\
\hline $\mathrm{K}$ & 19000 & 19000 & 0.001697 & 0.000773 & 0.000061 & \\
\hline $\mathrm{Ca}$ & 20000 & 20000 & 0.246924 & 0.109680 & 0.008719 & \\
\hline $\mathrm{Fe}$ & 26000 & 26000 & 0.011031 & 0.003516 & 0.000280 & \\
\hline Total & & & 1.000000 & 1.000000 & 0.079496 & \\
\hline MCNP Form & \multicolumn{2}{|c|}{ Weight Fractions } & \multicolumn{2}{|c|}{ Atom Fractions } & \multicolumn{2}{|c|}{ Atom Densities } \\
\hline \multirow[t]{10}{*}{ Neutrons } & 1001 & -0.008485 & 1001 & 0.149867 & 1001 & 0.011914 \\
\hline & 6000 & -0.050064 & 6000 & 0.074204 & 6000 & 0.005899 \\
\hline & 8016 & -0.473483 & 8016 & 0.526832 & 8016 & 0.041881 \\
\hline & 12000 & -0.024183 & 12000 & 0.017713 & 12000 & 0.001408 \\
\hline & 13027 & -0.036063 & 13027 & 0.023794 & 13027 & 0.001892 \\
\hline & 14000 & -0.145100 & 14000 & 0.091972 & 14000 & 0.007311 \\
\hline & 16000 & -0.002970 & 16000 & 0.001649 & 16000 & 0.000131 \\
\hline & 19000 & -0.001697 & 19000 & 0.000773 & 19000 & 0.000061 \\
\hline & 20000 & -0.246924 & 20000 & 0.109680 & 20000 & 0.008719 \\
\hline & 26000 & -0.011031 & 26000 & 0.003516 & 26000 & 0.000280 \\
\hline
\end{tabular}


PIET-43741-TM-963

PNNL-15870 Rev. 1

\begin{tabular}{|c|c|c|c|c|c|c|}
\hline \multirow{10}{*}{ Photons } & 1000 & -0.008485 & 1000 & 0.149867 & 1000 & 0.011914 \\
\hline & 6000 & -0.050064 & 6000 & 0.074204 & 6000 & 0.005899 \\
\hline & 8000 & -0.473483 & 8000 & 0.526832 & 8000 & 0.041881 \\
\hline & 12000 & -0.024183 & 12000 & 0.017713 & 12000 & 0.001408 \\
\hline & 13000 & -0.036063 & 13000 & 0.023794 & 13000 & 0.001892 \\
\hline & 14000 & -0.145100 & 14000 & 0.091972 & 14000 & 0.007311 \\
\hline & 16000 & -0.002970 & 16000 & 0.001649 & 16000 & 0.000131 \\
\hline & 19000 & -0.001697 & 19000 & 0.000773 & 19000 & 0.000061 \\
\hline & 20000 & -0.246924 & 20000 & 0.109680 & 20000 & 0.008719 \\
\hline & 26000 & -0.011031 & 26000 & 0.003516 & 26000 & 0.000280 \\
\hline \multirow[t]{11}{*}{ CEPXS Form: } & material & $\mathrm{H}$ & 0.008485 & & & \\
\hline & & C & 0.050064 & & & \\
\hline & & $\mathrm{O}$ & 0.473483 & & & \\
\hline & & $\mathrm{Mg}$ & 0.024183 & & & \\
\hline & & $\mathrm{Al}$ & 0.036063 & & & \\
\hline & & $\mathrm{Si}$ & 0.145100 & & & \\
\hline & & $S$ & 0.002970 & & & \\
\hline & & $\mathrm{K}$ & 0.001697 & & & \\
\hline & & $\mathrm{Ca}$ & 0.246924 & & & \\
\hline & & $\mathrm{Fe}$ & 0.011031 & & & \\
\hline & $\begin{array}{l}\text { matname } \\
\text { density }\end{array}$ & \multicolumn{2}{|c|}{$\begin{array}{l}\text { Concrete, Ordinary (NBS 03) } \\
2.350000\end{array}$} & & & \\
\hline \multicolumn{7}{|c|}{$\begin{array}{l}\text { Comments and References } \\
\text { Density }=2.35 \mathrm{~g} / \mathrm{cm} 3 \text {, and weight fractions calculated from partial densities }(\mathrm{g} / \mathrm{cm} 3) \text { listed for each } \\
\text { element in Table } 8.8 \text { of Shultis and Faw (1996), and extracted from ANSI/ANS-6.4-1985. }\end{array}$} \\
\hline
\end{tabular}

\section{Concrete, Ordinary (NBS 04)}

\begin{tabular}{|c|c|c|c|}
\hline Formula $=$ & - & Molecular weight $(\mathrm{g} / \mathrm{mole})=$ & - \\
\hline Density $(\mathrm{g} / \mathrm{cm} 3)=$ & 2.350000 & Total atom density $($ atoms $/ \mathrm{b}-\mathrm{cm})=$ & 7.533E-02 \\
\hline
\end{tabular}

\begin{tabular}{|c|c|c|c|c|c|}
\hline Element & Neutron ZA & Photon ZA & $\begin{array}{l}\text { Weight } \\
\text { Fraction }\end{array}$ & $\begin{array}{c}\text { Atom } \\
\text { Fraction }\end{array}$ & $\begin{array}{l}\text { Atom } \\
\text { Density }\end{array}$ \\
\hline $\mathrm{H}$ & 1001 & 1000 & $\overline{0.005558}$ & 0.103586 & 0.007804 \\
\hline $\mathrm{O}$ & 8016 & 8000 & 0.498076 & 0.584810 & 0.044057 \\
\hline $\mathrm{Na}$ & 11023 & 11000 & 0.017101 & 0.013974 & 0.001053 \\
\hline $\mathrm{Mg}$ & 12000 & 12000 & 0.002565 & 0.001983 & 0.000149 \\
\hline $\mathrm{Al}$ & 13027 & 13000 & 0.045746 & 0.031850 & 0.002399 \\
\hline $\mathrm{Si}$ & 14000 & 14000 & 0.315092 & 0.210755 & 0.015877 \\
\hline$S$ & 16000 & 16000 & 0.001283 & 0.000751 & 0.000057 \\
\hline $\mathrm{K}$ & 19000 & 19000 & 0.019239 & 0.009244 & 0.000696 \\
\hline $\mathrm{Ca}$ & 20000 & 20000 & 0.082941 & 0.038877 & 0.002929 \\
\hline $\mathrm{Fe}$ & 26000 & 26000 & 0.012398 & 0.004171 & 0.000314 \\
\hline
\end{tabular}


PIET-43741-TM-963

PNNL-15870 Rev. 1

\begin{tabular}{|c|c|c|c|c|c|c|}
\hline Total & & & 1.000000 & 1.000000 & 0.07533 & \\
\hline MCNP Form & Weigh & ractions & Atom & ctions & Aton & sities \\
\hline Neutrons & 1001 & -0.005558 & 1001 & 0.103586 & 1001 & 0.007804 \\
\hline & 8016 & -0.498076 & 8016 & 0.584810 & 8016 & 0.044057 \\
\hline & 11023 & -0.017101 & 11023 & 0.013974 & 11023 & 0.001053 \\
\hline & 12000 & -0.002565 & 12000 & 0.001983 & 12000 & 0.000149 \\
\hline & 13027 & -0.045746 & 13027 & 0.031850 & 13027 & 0.002399 \\
\hline & 14000 & -0.315092 & 14000 & 0.210755 & 14000 & 0.015877 \\
\hline & 16000 & -0.001283 & 16000 & 0.000751 & 16000 & 0.000057 \\
\hline & 19000 & -0.019239 & 19000 & 0.009244 & 19000 & 0.000696 \\
\hline & 20000 & -0.082941 & 20000 & 0.038877 & 20000 & 0.002929 \\
\hline & 26000 & -0.012398 & 26000 & 0.004171 & 26000 & 0.000314 \\
\hline Photons & 1000 & -0.005558 & 1000 & 0.103586 & 1000 & 0.007804 \\
\hline & 8000 & -0.498076 & 8000 & 0.584810 & 8000 & 0.044057 \\
\hline & 11000 & -0.017101 & 11000 & 0.013974 & 11000 & 0.001053 \\
\hline & 12000 & -0.002565 & 12000 & 0.001983 & 12000 & 0.000149 \\
\hline & 13000 & -0.045746 & 13000 & 0.031850 & 13000 & 0.002399 \\
\hline & 14000 & -0.315092 & 14000 & 0.210755 & 14000 & 0.015877 \\
\hline & 16000 & -0.001283 & 16000 & 0.000751 & 16000 & 0.000057 \\
\hline & 19000 & -0.019239 & 19000 & 0.009244 & 19000 & 0.000696 \\
\hline & 20000 & -0.082941 & 20000 & 0.038877 & 20000 & 0.002929 \\
\hline & 26000 & -0.012398 & 26000 & 0.004171 & 26000 & 0.000314 \\
\hline CEPXS Form: & material & $\mathrm{H}$ & 0.005558 & & & \\
\hline & & 0 & 0.498076 & & & \\
\hline & & $\mathrm{Na}$ & 0.017101 & & & \\
\hline & & $\mathrm{Mg}$ & 0.002565 & & & \\
\hline & & $\mathrm{Al}$ & 0.045746 & & & \\
\hline & & $\mathrm{Si}$ & 0.315092 & & & \\
\hline & & $S$ & 0.001283 & & & \\
\hline & & $\mathrm{K}$ & 0.019239 & & & \\
\hline & & $\mathrm{Ca}$ & 0.082941 & & & \\
\hline & & $\mathrm{Fe}$ & 0.012398 & & & \\
\hline & $\begin{array}{c}\text { matname } \\
\text { density }\end{array}$ & $\begin{array}{c}\text { Concrete, O } \\
2.350000\end{array}$ & Iary (NBS 0 & & & \\
\hline $\begin{array}{l}\text { Comments an } \\
\text { Density }=2.35 \\
\text { element in } \mathrm{Tab}\end{array}$ & $\begin{array}{l}\text { eferences } \\
\mathrm{m} 3 \text {, and we } \\
.8 \text { of Shult }\end{array}$ & $\begin{array}{l}\mathrm{t} \text { fractions } \\
\text { ld Faw (19 }\end{array}$ & - & $m \wedge N$ & $4-198$ & \\
\hline
\end{tabular}

\section{Concrete, Ordinary (NIST)}

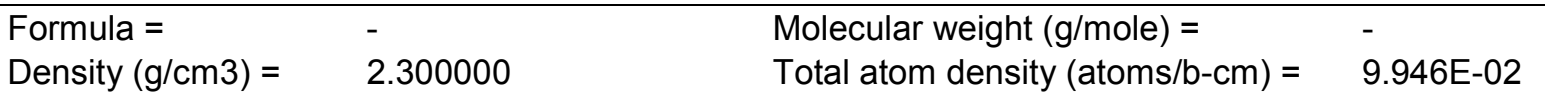

The above density is estimated to be accurate to 2 significant digits. Uncertainties are not addressed.

The following data were calculated from the input weight fractions. 
PIET-43741-TM-963

PNNL-15870 Rev. 1

\begin{tabular}{|c|c|c|c|c|c|c|}
\hline Element & Neutron ZA & Photon ZA & $\begin{array}{l}\text { Weight } \\
\text { Fraction }\end{array}$ & $\begin{array}{l}\text { Atom } \\
\text { Fraction }\end{array}$ & $\begin{array}{l}\text { Atom } \\
\text { Density }\end{array}$ & \\
\hline $\mathrm{H}$ & 1001 & 1000 & $\overline{0.022100}$ & $\overline{0.305330}$ & 0.030369 & \\
\hline $\mathrm{C}$ & 6000 & 6000 & 0.002484 & 0.002880 & 0.000286 & \\
\hline $\mathrm{O}$ & 8016 & 8000 & 0.574930 & 0.500407 & 0.049773 & \\
\hline $\mathrm{Na}$ & 11023 & 11000 & 0.015208 & 0.009212 & 0.000916 & \\
\hline $\mathrm{Mg}$ & 12000 & 12000 & 0.001266 & 0.000725 & 0.000072 & \\
\hline $\mathrm{Al}$ & 13027 & 13000 & 0.019953 & 0.010298 & 0.001024 & \\
\hline $\mathrm{Si}$ & 14000 & 14000 & 0.304627 & 0.151042 & 0.015023 & \\
\hline $\mathrm{K}$ & 19000 & 19000 & 0.010045 & 0.003578 & 0.000356 & \\
\hline $\mathrm{Ca}$ & 20000 & 20000 & 0.042951 & 0.014924 & 0.001484 & \\
\hline $\mathrm{Fe}$ & 26000 & 26000 & 0.006435 & 0.001605 & 0.000160 & \\
\hline Total & & & 0.999999 & 1.000000 & 0.099464 & \\
\hline MCNP Form & \multicolumn{2}{|c|}{ Weight Fractions } & \multicolumn{2}{|c|}{ Atom Fractions } & \multicolumn{2}{|c|}{ Atom Densities } \\
\hline \multirow[t]{10}{*}{ Neutrons } & 1001 & -0.022100 & 1001 & 0.305330 & 1001 & 0.030369 \\
\hline & 6000 & -0.002484 & 6000 & 0.002880 & 6000 & 0.000286 \\
\hline & 8016 & -0.574930 & 8016 & 0.500407 & 8016 & 0.049773 \\
\hline & 11023 & -0.015208 & 11023 & 0.009212 & 11023 & 0.000916 \\
\hline & 12000 & -0.001266 & 12000 & 0.000725 & 12000 & 0.000072 \\
\hline & 13027 & -0.019953 & 13027 & 0.010298 & 13027 & 0.001024 \\
\hline & 14000 & -0.304627 & 14000 & 0.151042 & 14000 & 0.015023 \\
\hline & 19000 & -0.010045 & 19000 & 0.003578 & 19000 & 0.000356 \\
\hline & 20000 & -0.042951 & 20000 & 0.014924 & 20000 & 0.001484 \\
\hline & 26000 & -0.006435 & 26000 & 0.001605 & 26000 & 0.000160 \\
\hline \multirow[t]{10}{*}{ Photons } & 1000 & -0.022100 & 1000 & 0.305330 & 1000 & 0.030369 \\
\hline & 6000 & -0.002484 & 6000 & 0.002880 & 6000 & 0.000286 \\
\hline & 8000 & -0.574930 & 8000 & 0.500407 & 8000 & 0.049773 \\
\hline & 11000 & -0.015208 & 11000 & 0.009212 & 11000 & 0.000916 \\
\hline & 12000 & -0.001266 & 12000 & 0.000725 & 12000 & 0.000072 \\
\hline & 13000 & -0.019953 & 13000 & 0.010298 & 13000 & 0.001024 \\
\hline & 14000 & -0.304627 & 14000 & 0.151042 & 14000 & 0.015023 \\
\hline & 19000 & -0.010045 & 19000 & 0.003578 & 19000 & 0.000356 \\
\hline & 20000 & -0.042951 & 20000 & 0.014924 & 20000 & 0.001484 \\
\hline & 26000 & -0.006435 & 26000 & 0.001605 & 26000 & 0.000160 \\
\hline \multirow[t]{10}{*}{ CEPXS Form: } & material & $\mathrm{H}$ & 0.022100 & & & \\
\hline & & $\mathrm{C}$ & 0.002484 & & & \\
\hline & & 0 & 0.574930 & & & \\
\hline & & $\mathrm{Na}$ & 0.015208 & & & \\
\hline & & $\mathrm{Mg}$ & 0.001266 & & & \\
\hline & & $\mathrm{Al}$ & 0.019953 & & & \\
\hline & & $\mathrm{Si}$ & 0.304627 & & & \\
\hline & & $\mathrm{K}$ & 0.010045 & & & \\
\hline & & $\mathrm{Ca}$ & 0.042951 & & & \\
\hline & & $\mathrm{Fe}$ & 0.006435 & & & \\
\hline
\end{tabular}


matname Concrete, Ordinary (NIST)

density 2.300000

Comments and References

Density and weight fractions from http://physics.nist.gov/PhysRefData/XrayMassCoef/tab2.html

(NIST 1996).

\section{Concrete, Portland}

\begin{tabular}{|c|c|c|c|}
\hline Formula $=$ & - & Molecular weight $(\mathrm{g} / \mathrm{mole})=$ & - \\
\hline Density $(\mathrm{g} / \mathrm{cm} 3)=$ & 2.300000 & Total atom density $($ atoms $/ \mathrm{b}-\mathrm{cm})=$ & 8.143E-02 \\
\hline
\end{tabular}

\begin{tabular}{|c|c|c|c|c|c|c|}
\hline Element & Neutron ZA & Photon ZA & $\begin{array}{l}\text { Weight } \\
\text { Fraction }\end{array}$ & $\begin{array}{c}\text { Atom } \\
\text { Fraction }\end{array}$ & $\begin{array}{l}\text { Atom } \\
\text { Density }\end{array}$ & \\
\hline $\mathrm{H}$ & 1001 & 1000 & 0.010000 & 0.168759 & 0.013742 & \\
\hline C & 6000 & 6000 & 0.001000 & 0.001416 & 0.000115 & \\
\hline $\mathrm{O}$ & 8016 & 8000 & 0.529107 & 0.562524 & 0.045806 & \\
\hline $\mathrm{Na}$ & 11023 & 11000 & 0.016000 & 0.011838 & 0.000964 & \\
\hline $\mathrm{Mg}$ & 12000 & 12000 & 0.002000 & 0.001400 & 0.000114 & \\
\hline $\mathrm{Al}$ & 13027 & 13000 & 0.033872 & 0.021354 & 0.001739 & \\
\hline $\mathrm{Si}$ & 14000 & 14000 & 0.337021 & 0.204115 & 0.016621 & \\
\hline $\mathrm{K}$ & 19000 & 19000 & 0.013000 & 0.005656 & 0.000461 & \\
\hline $\mathrm{Ca}$ & 20000 & 20000 & 0.044000 & 0.018674 & 0.001521 & \\
\hline $\mathrm{Fe}$ & 26000 & 26000 & 0.014000 & 0.004264 & 0.000347 & \\
\hline Total & & & 1.000000 & 1.000000 & 0.081429 & \\
\hline MCNP Form & \multicolumn{2}{|c|}{ Weight Fractions } & \multicolumn{2}{|c|}{ Atom Fractions } & \multicolumn{2}{|c|}{ Atom Densities } \\
\hline \multirow[t]{10}{*}{ Neutrons } & 1001 & -0.010000 & 1001 & 0.168759 & 1001 & 0.013742 \\
\hline & 6000 & -0.001000 & 6000 & 0.001416 & 6000 & 0.000115 \\
\hline & 8016 & -0.529107 & 8016 & 0.562524 & 8016 & 0.045806 \\
\hline & 11023 & -0.016000 & 11023 & 0.011838 & 11023 & 0.000964 \\
\hline & 12000 & -0.002000 & 12000 & 0.001400 & 12000 & 0.000114 \\
\hline & 13027 & -0.033872 & 13027 & 0.021354 & 13027 & 0.001739 \\
\hline & 14000 & -0.337021 & 14000 & 0.204115 & 14000 & 0.016621 \\
\hline & 19000 & -0.013000 & 19000 & 0.005656 & 19000 & 0.000461 \\
\hline & 20000 & -0.044000 & 20000 & 0.018674 & 20000 & 0.001521 \\
\hline & 26000 & -0.014000 & 26000 & 0.004264 & 26000 & 0.000347 \\
\hline \multirow[t]{8}{*}{ Photons } & 1000 & -0.010000 & 1000 & 0.168759 & 1000 & 0.013742 \\
\hline & 6000 & -0.001000 & 6000 & 0.001416 & 6000 & 0.000115 \\
\hline & 8000 & -0.529107 & 8000 & 0.562524 & 8000 & 0.045806 \\
\hline & 11000 & -0.016000 & 11000 & 0.011838 & 11000 & 0.000964 \\
\hline & 12000 & -0.002000 & 12000 & 0.001400 & 12000 & 0.000114 \\
\hline & 13000 & -0.033872 & 13000 & 0.021354 & 13000 & 0.001739 \\
\hline & 14000 & -0.337021 & 14000 & 0.204115 & 14000 & 0.016621 \\
\hline & 19000 & -0.013000 & 19000 & 0.005656 & 19000 & 0.000461 \\
\hline
\end{tabular}


PIET-43741-TM-963

PNNL-15870 Rev. 1

\begin{tabular}{|c|c|c|c|c|c|c|}
\hline & $\begin{array}{l}20000 \\
26000\end{array}$ & $\begin{array}{l}-0.044000 \\
-0.014000\end{array}$ & $\begin{array}{l}20000 \\
26000\end{array}$ & $\begin{array}{l}0.018674 \\
0.004264\end{array}$ & $\begin{array}{l}20000 \\
26000\end{array}$ & $\begin{array}{l}0.001521 \\
0.000347\end{array}$ \\
\hline CEPXS Form: & material & $\begin{array}{c}\mathrm{H} \\
\mathrm{C} \\
\mathrm{O} \\
\mathrm{Na} \\
\mathrm{Mg} \\
\mathrm{Al} \\
\mathrm{Si} \\
\mathrm{K} \\
\mathrm{Ca} \\
\mathrm{Fe}\end{array}$ & $\begin{array}{l}0.010000 \\
0.001000 \\
0.529107 \\
0.016000 \\
0.002000 \\
0.033872 \\
0.337021 \\
0.013000 \\
0.044000 \\
0.014000\end{array}$ & & & \\
\hline & $\begin{array}{c}\text { matname } \\
\text { density }\end{array}$ & \multicolumn{2}{|c|}{$\begin{array}{l}\text { Concrete, Portland } \\
2.300000\end{array}$} & & & \\
\hline
\end{tabular}

\section{Concrete, Regular}

\begin{tabular}{llll}
\hline Formula $=$ & - & Molecular weight $(\mathrm{g} / \mathrm{mole})=$ & - \\
Density $(\mathrm{g} / \mathrm{cm} 3)=$ & 2.300000 & Total atom density $($ atoms $/ \mathrm{b}-\mathrm{cm})=$ & $8.178 \mathrm{E}-02$
\end{tabular}

The above density is estimated to be accurate to 2 significant digits. Uncertainties are not addressed.

The following data were calculated from the input weight fractions.

\begin{tabular}{|c|c|c|c|c|c|c|}
\hline Element & Neutron ZA & Photon ZA & $\begin{array}{l}\text { Weight } \\
\text { Fraction }\end{array}$ & $\begin{array}{c}\text { Atom } \\
\text { Fraction }\end{array}$ & $\begin{array}{c}\text { Atom } \\
\text { Density }\end{array}$ & \\
\hline $\mathrm{H}$ & 1001 & 1000 & $\overline{0.010000}$ & 0.168038 & 0.013742 & \\
\hline 0 & 8016 & 8000 & 0.532000 & 0.563183 & 0.046056 & \\
\hline $\mathrm{Na}$ & 11023 & 11000 & 0.029000 & 0.021365 & 0.001747 & \\
\hline $\mathrm{Al}$ & 13027 & 13000 & 0.034000 & 0.021343 & 0.001745 & \\
\hline $\mathrm{Si}$ & 14000 & 14000 & 0.337000 & 0.203231 & 0.016620 & \\
\hline $\mathrm{Ca}$ & 20000 & 20000 & 0.044000 & 0.018595 & 0.001521 & \\
\hline $\mathrm{Fe}$ & 26000 & 26000 & 0.014000 & 0.004246 & 0.000347 & \\
\hline Total & & & 1.000000 & 1.000000 & 0.081778 & \\
\hline MCNP Form & \multicolumn{2}{|c|}{ Weight Fractions } & \multicolumn{2}{|c|}{ Atom Fractions } & \multicolumn{2}{|c|}{ Atom Densities } \\
\hline \multirow[t]{7}{*}{ Neutrons } & 1001 & -0.010000 & 1001 & 0.168038 & 1001 & 0.013742 \\
\hline & 8016 & -0.532000 & 8016 & 0.563183 & 8016 & 0.046056 \\
\hline & 11023 & -0.029000 & 11023 & 0.021365 & 11023 & 0.001747 \\
\hline & 13027 & -0.034000 & 13027 & 0.021343 & 13027 & 0.001745 \\
\hline & 14000 & -0.337000 & 14000 & 0.203231 & 14000 & 0.016620 \\
\hline & 20000 & -0.044000 & 20000 & 0.018595 & 20000 & 0.001521 \\
\hline & 26000 & -0.014000 & 26000 & 0.004246 & 26000 & 0.000347 \\
\hline
\end{tabular}


PIET-43741-TM-963

PNNL-15870 Rev. 1

\begin{tabular}{|c|c|c|c|c|c|c|}
\hline \multirow[t]{7}{*}{ Photons } & 1000 & -0.010000 & 1000 & 0.168038 & 1000 & 0.013742 \\
\hline & 8000 & -0.532000 & 8000 & 0.563183 & 8000 & 0.046056 \\
\hline & 11000 & -0.029000 & 11000 & 0.021365 & 11000 & 0.001747 \\
\hline & 13000 & -0.034000 & 13000 & 0.021343 & 13000 & 0.001745 \\
\hline & 14000 & -0.337000 & 14000 & 0.203231 & 14000 & 0.016620 \\
\hline & 20000 & -0.044000 & 20000 & 0.018595 & 20000 & 0.001521 \\
\hline & 26000 & -0.014000 & 26000 & 0.004246 & 26000 & 0.000347 \\
\hline \multirow[t]{8}{*}{ CEPXS Form: } & material & $\mathrm{H}$ & 0.010000 & & & \\
\hline & & 0 & 0.532000 & & & \\
\hline & & $\mathrm{Na}$ & 0.029000 & & & \\
\hline & & $\mathrm{Al}$ & 0.034000 & & & \\
\hline & & $\mathrm{Si}$ & 0.337000 & & & \\
\hline & & $\mathrm{Ca}$ & 0.044000 & & & \\
\hline & & $\mathrm{Fe}$ & 0.014000 & & & \\
\hline & $\begin{array}{c}\text { matname } \\
\text { density }\end{array}$ & \multicolumn{2}{|c|}{$\begin{array}{l}\text { Concrete, Regular } \\
2300000\end{array}$} & & & \\
\hline
\end{tabular}

\section{Concrete, Rocky Flats}

\begin{tabular}{|c|c|c|c|}
\hline Formula $=$ & - & Molecular weight $(\mathrm{g} / \mathrm{mole})=$ & - \\
\hline Density $(\mathrm{g} / \mathrm{cm} 3)=$ & 2.320000 & Total atom density $($ atoms $/ \mathrm{b}-\mathrm{cm})=$ & 7.799E-02 \\
\hline
\end{tabular}

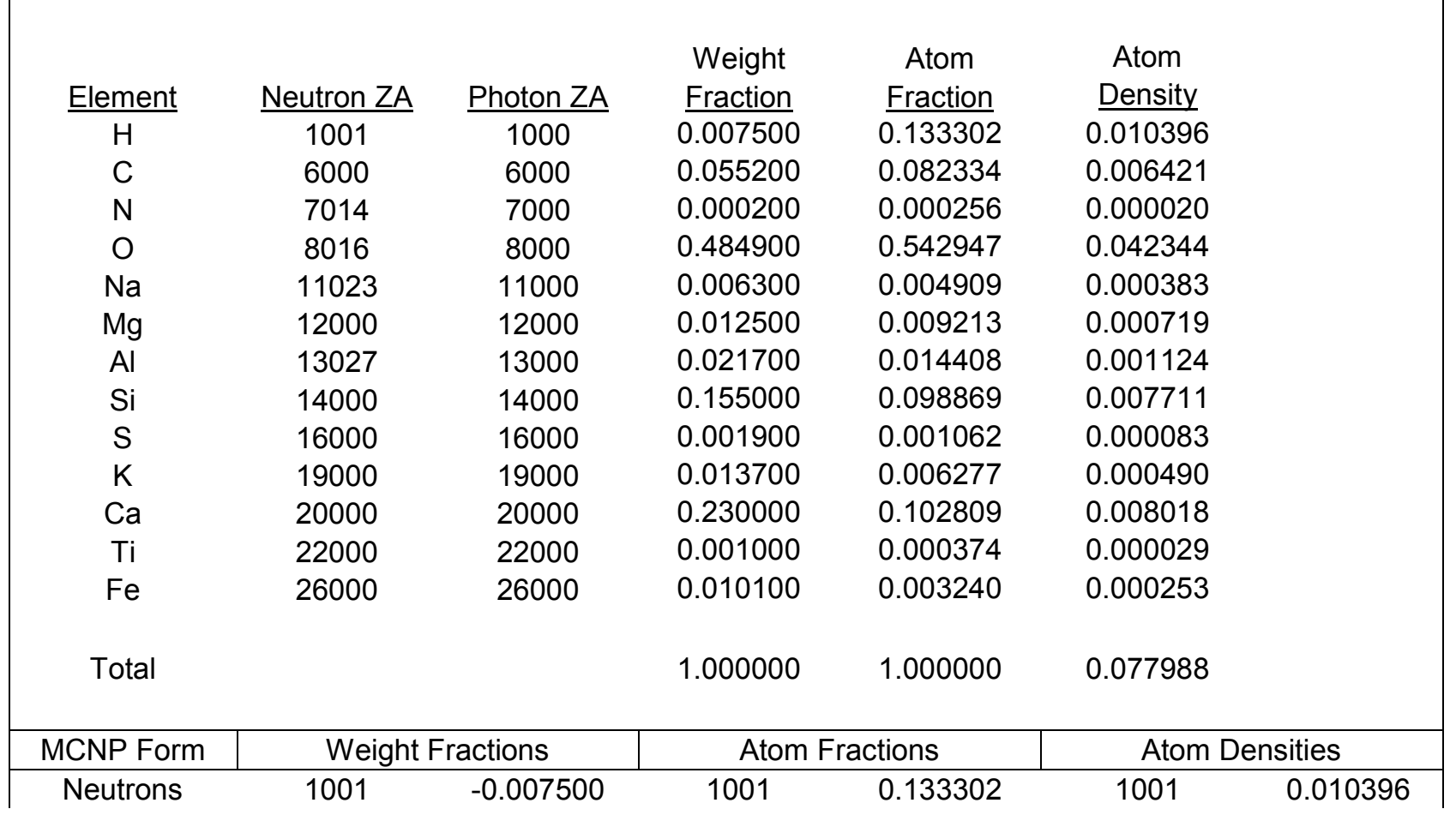


PIET-43741-TM-963

PNNL-15870 Rev. 1

\begin{tabular}{|c|c|c|c|c|c|c|}
\hline & 6000 & -0.055200 & 6000 & 0.082334 & 6000 & 0.006421 \\
\hline & 7014 & -0.000200 & 7014 & 0.000256 & 7014 & 0.000020 \\
\hline & 8016 & -0.484900 & 8016 & 0.542947 & 8016 & 0.042344 \\
\hline & 11023 & -0.006300 & 11023 & 0.004909 & 11023 & 0.000383 \\
\hline & 12000 & -0.012500 & 12000 & 0.009213 & 12000 & 0.000719 \\
\hline & 13027 & -0.021700 & 13027 & 0.014408 & 13027 & 0.001124 \\
\hline & 14000 & -0.155000 & 14000 & 0.098869 & 14000 & 0.007711 \\
\hline & 16000 & -0.001900 & 16000 & 0.001062 & 16000 & 0.000083 \\
\hline & 19000 & -0.013700 & 19000 & 0.006277 & 19000 & 0.000490 \\
\hline & 20000 & -0.230000 & 20000 & 0.102809 & 20000 & 0.008018 \\
\hline & 22000 & -0.001000 & 22000 & 0.000374 & 22000 & 0.000029 \\
\hline & 26000 & -0.010100 & 26000 & 0.003240 & 26000 & 0.000253 \\
\hline Photons & 1000 & -0.007500 & 1000 & 0.133302 & 1000 & 0.010396 \\
\hline & 6000 & -0.055200 & 6000 & 0.082334 & 6000 & 0.006421 \\
\hline & 7000 & -0.000200 & 7000 & 0.000256 & 7000 & 0.000020 \\
\hline & 8000 & -0.484900 & 8000 & 0.542947 & 8000 & 0.042344 \\
\hline & 11000 & -0.006300 & 11000 & 0.004909 & 11000 & 0.000383 \\
\hline & 12000 & -0.012500 & 12000 & 0.009213 & 12000 & 0.000719 \\
\hline & 13000 & -0.021700 & 13000 & 0.014408 & 13000 & 0.001124 \\
\hline & 14000 & -0.155000 & 14000 & 0.098869 & 14000 & 0.007711 \\
\hline & 16000 & -0.001900 & 16000 & 0.001062 & 16000 & 0.000083 \\
\hline & 19000 & -0.013700 & 19000 & 0.006277 & 19000 & 0.000490 \\
\hline & 20000 & -0.230000 & 20000 & 0.102809 & 20000 & 0.008018 \\
\hline & 22000 & -0.001000 & 22000 & 0.000374 & 22000 & 0.000029 \\
\hline & 26000 & -0.010100 & 26000 & 0.003240 & 26000 & 0.000253 \\
\hline CEPXS Form: & material & $\mathrm{H}$ & 0.007500 & & & \\
\hline & & C & 0.055200 & & & \\
\hline & & $\mathrm{N}$ & 0.000200 & & & \\
\hline & & 0 & 0.484900 & & & \\
\hline & & $\mathrm{Na}$ & 0.006300 & & & \\
\hline & & $\mathrm{Mg}$ & 0.012500 & & & \\
\hline & & $\mathrm{Al}$ & 0.021700 & & & \\
\hline & & $\mathrm{Si}$ & 0.155000 & & & \\
\hline & & $S$ & 0.001900 & & & \\
\hline & & $\mathrm{K}$ & 0.013700 & & & \\
\hline & & $\mathrm{Ca}$ & 0.230000 & & & \\
\hline & & $\mathrm{Ti}$ & 0.001000 & & & \\
\hline & & $\mathrm{Fe}$ & 0.010100 & & & \\
\hline & $\begin{array}{l}\text { matname } \\
\text { density }\end{array}$ & $\begin{array}{c}\text { Concrete, } \mathrm{Rc} \\
2.320000\end{array}$ & y Flats & & & \\
\hline $\begin{array}{l}\text { Comme } \\
\text { Data frol }\end{array}$ & $\begin{array}{l}\text { eferences } \\
t \text { al. (2000). }\end{array}$ & & & & & \\
\hline
\end{tabular}




\section{Concrete, Serpentine}

\begin{tabular}{llll}
\hline Formula $=$ & - & Molecular weight $(\mathrm{g} / \mathrm{mole})=$ \\
Density $(\mathrm{g} / \mathrm{cm} 3)=$ & 2.100000 & Total atom density $($ atoms $/ \mathrm{b}-\mathrm{cm})=$ & - \\
\hline & $8.108 \mathrm{E}-02$
\end{tabular}

The above density is estimated to be accurate to 3 significant digits. Uncertainties are not addressed.

The following data were calculated from the input weight fractions.

\begin{tabular}{|c|c|c|c|c|c|c|}
\hline Element & Neutron ZA & Photon ZA & $\begin{array}{l}\text { Weight } \\
\text { Fraction }\end{array}$ & $\begin{array}{c}\text { Atom } \\
\text { Fraction }\end{array}$ & $\begin{array}{c}\text { Atom } \\
\text { Density }\end{array}$ & \\
\hline $\mathrm{H}$ & 1001 & 1000 & $\overline{0.015909}$ & $\overline{0.246195}$ & 0.019961 & \\
\hline C & 6000 & 6000 & 0.000909 & 0.001181 & 0.000096 & \\
\hline O & 8016 & 8000 & 0.511818 & 0.498977 & 0.040456 & \\
\hline $\mathrm{Na}$ & 11023 & 11000 & 0.004091 & 0.002776 & 0.000225 & \\
\hline $\mathrm{Mg}$ & 12000 & 12000 & 0.135000 & 0.086638 & 0.007024 & \\
\hline $\mathrm{Al}$ & 13027 & 13000 & 0.019091 & 0.011036 & 0.000895 & \\
\hline $\mathrm{Si}$ & 14000 & 14000 & 0.209091 & 0.116124 & 0.009415 & \\
\hline $\mathrm{K}$ & 19000 & 19000 & 0.004091 & 0.001632 & 0.000132 & \\
\hline $\mathrm{Ca}$ & 20000 & 20000 & 0.068182 & 0.026536 & 0.002151 & \\
\hline $\mathrm{Cr}$ & 24000 & 24000 & 0.000909 & 0.000273 & 0.000022 & \\
\hline $\mathrm{Fe}$ & 26000 & 26000 & 0.030909 & 0.008633 & 0.000700 & \\
\hline Total & & & 1.000000 & 1.000000 & 0.081078 & \\
\hline MCNP Form & \multicolumn{2}{|c|}{ Weight Fractions } & \multicolumn{2}{|c|}{ Atom Fractions } & \multicolumn{2}{|c|}{ Atom Densities } \\
\hline \multirow[t]{11}{*}{ Neutrons } & 1001 & -0.015909 & 1001 & 0.246195 & 1001 & 0.019961 \\
\hline & 6000 & -0.000909 & 6000 & 0.001181 & 6000 & 0.000096 \\
\hline & 8016 & -0.511818 & 8016 & 0.498977 & 8016 & 0.040456 \\
\hline & 11023 & -0.004091 & 11023 & 0.002776 & 11023 & 0.000225 \\
\hline & 12000 & -0.135000 & 12000 & 0.086638 & 12000 & 0.007024 \\
\hline & 13027 & -0.019091 & 13027 & 0.011036 & 13027 & 0.000895 \\
\hline & 14000 & -0.209091 & 14000 & 0.116124 & 14000 & 0.009415 \\
\hline & 19000 & -0.004091 & 19000 & 0.001632 & 19000 & 0.000132 \\
\hline & 20000 & -0.068182 & 20000 & 0.026536 & 20000 & 0.002151 \\
\hline & 24000 & -0.000909 & 24000 & 0.000273 & 24000 & 0.000022 \\
\hline & 26000 & -0.030909 & 26000 & 0.008633 & 26000 & 0.000700 \\
\hline \multirow[t]{11}{*}{ Photons } & 1000 & -0.015909 & 1000 & 0.246195 & 1000 & 0.019961 \\
\hline & 6000 & -0.000909 & 6000 & 0.001181 & 6000 & 0.000096 \\
\hline & 8000 & -0.511818 & 8000 & 0.498977 & 8000 & 0.040456 \\
\hline & 11000 & -0.004091 & 11000 & 0.002776 & 11000 & 0.000225 \\
\hline & 12000 & -0.135000 & 12000 & 0.086638 & 12000 & 0.007024 \\
\hline & 13000 & -0.019091 & 13000 & 0.011036 & 13000 & 0.000895 \\
\hline & 14000 & -0.209091 & 14000 & 0.116124 & 14000 & 0.009415 \\
\hline & 19000 & -0.004091 & 19000 & 0.001632 & 19000 & 0.000132 \\
\hline & 20000 & -0.068182 & 20000 & 0.026536 & 20000 & 0.002151 \\
\hline & 24000 & -0.000909 & 24000 & 0.000273 & 24000 & 0.000022 \\
\hline & 26000 & -0.030909 & 26000 & 0.008633 & 26000 & 0.000700 \\
\hline
\end{tabular}




\begin{tabular}{|ccc} 
CEPXS Form: material & $\mathrm{H}$ & 0.015909 \\
& $\mathrm{C}$ & 0.000909 \\
$\mathrm{O}$ & 0.511818 \\
$\mathrm{Na}$ & 0.004091 \\
$\mathrm{Mg}$ & 0.135000 \\
& $\mathrm{Al}$ & 0.019091 \\
$\mathrm{Si}$ & 0.209091 \\
$\mathrm{~K}$ & 0.004091 \\
& $\mathrm{Ca}$ & 0.068182 \\
& $\mathrm{Cr}$ & 0.000909 \\
& $\mathrm{Fe}$ & 0.030909
\end{tabular}

matname Concrete, Serpentine density 2.100000

Comments and References

Density $=2.1 \mathrm{~g} / \mathrm{cm} 3$, and weight fractions calculated from partial densities $(\mathrm{g} / \mathrm{cm} 3)$ for each element, from Table 8.8 of Shultis and Faw (1996).

Data in this table are from ANSI/ANS-6.4-1985.

This concrete has serpentine $(3 \mathrm{MgO}-2 \mathrm{SiO} 2-2 \mathrm{H} 2 \mathrm{O})$ as aggregate.

Density $=2.13 \mathrm{~g} / \mathrm{cm} 3$ and a similar composition in Tables 9.1.12-33 and 34 of Jaeger et al. (1975).

\section{Copper}

Formula $=$ $\mathrm{Cu}$

8.960000 Molecular weight $(\mathrm{g} / \mathrm{mole})=$

Total atom density $($ atoms $/ \mathrm{b}-\mathrm{cm})=$

63.546

Density $(\mathrm{g} / \mathrm{cm} 3)=$

The above density is estimated to be accurate to 3 significant digits. Uncertainties are not addressed.

The following data was calculated from the input formula.

$\begin{array}{llllll}\frac{\text { Element }}{\mathrm{Cu}} & \frac{\text { Neutron ZA }}{29000} & \frac{\text { Photon ZA }}{29000} & \frac{\begin{array}{c}\text { Weight } \\ \text { Fraction }\end{array}}{1.000000} & \frac{1}{\text { Fraction }} & \begin{array}{c}\text { Atom } \\ \text { Density }\end{array} \\ \text { Total } & & & 1.000000 & 0.084912 \\ & & 1.000000 & 1.000000 & 0.084912\end{array}$

\begin{tabular}{|cccc}
\hline MCNP Form & \multicolumn{2}{|c|}{ Weight Fractions } & Atom \\
\hline Neutrons & 29000 & -1.000000 & 29000 \\
Photons & 29000 & -1.000000 & 29000 \\
& & $\mathrm{Cu}$ & 1.000000 \\
\hline CEPXS Form: & material & & \\
& $\begin{array}{c}\text { matname } \\
\text { density }\end{array}$ & $\begin{array}{c}\text { Copper } \\
8.960000\end{array}$ & \\
\hline
\end{tabular}

Comments and References

Density from http://physics.nist.gov/cgi-bin/Star/compos.pl?matno=029 (NIST 1998). 


\section{Diatomaceous Earth}

\begin{tabular}{llll}
\hline Formula $=$ & - & Molecular weight $(\mathrm{g} / \mathrm{mole})=$ \\
Density $(\mathrm{g} / \mathrm{cm} 3)=$ & 0.220000 & Total atom density $($ atoms $/ \mathrm{b}-\mathrm{cm})=$ & - \\
\hline & $7.780 \mathrm{E}-03$
\end{tabular}

The above density is estimated to be accurate to 2 significant digits. Uncertainties are not addressed.

The following data were calculated from the input weight fractions.

\begin{tabular}{|c|c|c|c|c|c|c|}
\hline Element & Neutron ZA & Photon ZA & $\begin{array}{l}\text { Weight } \\
\text { Fraction }\end{array}$ & $\begin{array}{c}\text { Atom } \\
\text { Fraction }\end{array}$ & $\begin{array}{c}\text { Atom } \\
\text { Density }\end{array}$ & \\
\hline $\mathrm{H}$ & 1001 & 1000 & $\overline{0.008956}$ & $\overline{0.151312}$ & 0.001177 & \\
\hline $\mathrm{O}$ & 8016 & 8000 & 0.546579 & 0.581761 & 0.004526 & \\
\hline $\mathrm{Na}$ & 11023 & 11000 & 0.009896 & 0.007330 & 0.000057 & \\
\hline $\mathrm{Mg}$ & 12000 & 12000 & 0.002774 & 0.001943 & 0.000015 & \\
\hline $\mathrm{Al}$ & 13027 & 13000 & 0.015581 & 0.009834 & 0.000077 & \\
\hline $\mathrm{Si}$ & 14000 & 14000 & 0.394761 & 0.239358 & 0.001862 & \\
\hline $\mathrm{K}$ & 19000 & 19000 & 0.011074 & 0.004823 & 0.000038 & \\
\hline $\mathrm{Ca}$ & 20000 & 20000 & 0.003945 & 0.001676 & 0.000013 & \\
\hline $\mathrm{Fe}$ & 26000 & 26000 & 0.006434 & 0.001962 & 0.000015 & \\
\hline Total & & & 1.000000 & 1.000000 & 0.007780 & \\
\hline MCNP Form & \multicolumn{2}{|c|}{ Weight Fractions } & \multicolumn{2}{|c|}{ Atom Fractions } & \multicolumn{2}{|c|}{ Atom Densities } \\
\hline \multirow[t]{9}{*}{ Neutrons } & 1001 & -0.008956 & 1001 & 0.151312 & 1001 & 0.001177 \\
\hline & 8016 & -0.546579 & 8016 & 0.581761 & 8016 & 0.004526 \\
\hline & 11023 & -0.009896 & 11023 & 0.007330 & 11023 & 0.000057 \\
\hline & 12000 & -0.002774 & 12000 & 0.001943 & 12000 & 0.000015 \\
\hline & 13027 & -0.015581 & 13027 & 0.009834 & 13027 & 0.000077 \\
\hline & 14000 & -0.394761 & 14000 & 0.239358 & 14000 & 0.001862 \\
\hline & 19000 & -0.011074 & 19000 & 0.004823 & 19000 & 0.000038 \\
\hline & 20000 & -0.003945 & 20000 & 0.001676 & 20000 & 0.000013 \\
\hline & 26000 & -0.006434 & 26000 & 0.001962 & 26000 & 0.000015 \\
\hline \multirow[t]{9}{*}{ Photons } & 1000 & -0.008956 & 1000 & 0.151312 & 1000 & 0.001177 \\
\hline & 8000 & -0.546579 & 8000 & 0.581761 & 8000 & 0.004526 \\
\hline & 11000 & -0.009896 & 11000 & 0.007330 & 11000 & 0.000057 \\
\hline & 12000 & -0.002774 & 12000 & 0.001943 & 12000 & 0.000015 \\
\hline & 13000 & -0.015581 & 13000 & 0.009834 & 13000 & 0.000077 \\
\hline & 14000 & -0.394761 & 14000 & 0.239358 & 14000 & 0.001862 \\
\hline & 19000 & -0.011074 & 19000 & 0.004823 & 19000 & 0.000038 \\
\hline & 20000 & -0.003945 & 20000 & 0.001676 & 20000 & 0.000013 \\
\hline & 26000 & -0.006434 & 26000 & 0.001962 & 26000 & 0.000015 \\
\hline \multirow[t]{7}{*}{ CEPXS Form: } & material & $\mathrm{H}$ & 0.008956 & & & \\
\hline & & 0 & 0.546579 & & & \\
\hline & & $\mathrm{Na}$ & 0.009896 & & & \\
\hline & & $\mathrm{Mg}$ & 0.002774 & & & \\
\hline & & $\mathrm{Al}$ & 0.015581 & & & \\
\hline & & $\mathrm{Si}$ & 0.394761 & & & \\
\hline & & $\mathrm{K}$ & 0.011074 & & & \\
\hline
\end{tabular}


$\begin{array}{lll}\mathrm{Ca} & 0.003945 \\ \mathrm{Fe} & 0.006434\end{array}$

matname Diatomaceous Earth

density $\quad 0.220000$

\section{Comments and References}

Also known as DE or diatomite. Diatomaceous earth is a naturally occurring soft sedimentary rock composed of fossilized remains of diatoms, a type of hard-shelled algae. It is usually a major component of cat litter.

Density $=0.22 \mathrm{~g} / \mathrm{cm} 3$ listed for diatomaceous earth product and for diatomite at

http://www.powderandbulk.com/resources/bulk_density/material_bulk_density_chart_d.htm (Powder and Bulk Dot Com 2010). Water content is typically 3 to $13 \mathrm{wt} \%$ based on http://www.mineengineer.com/mining/mineral/diatomaceous-earth.htm (Mine-Engineer.Com 2010). Based on this, the water content was assumed to be $8 \mathrm{wt} \%$. The dry weight fractions are based on http://www.matweb.com/search/DataSheet.aspx?MatGUID=3f468dd193804209b8f28e6bdfdbb379\&ckck= 1 (Automation Creations 2010).

\section{Earth, Typical Western U.S.}

\begin{tabular}{llll}
\hline Formula $=$ & - & Molecular weight $(\mathrm{g} / \mathrm{mole})=$ \\
Density $(\mathrm{g} / \mathrm{cm} 3)=$ & 1.520000 & Total atom density $($ atoms $/ \mathrm{b}-\mathrm{cm})=$ & - \\
& $6.831 \mathrm{E}-02$
\end{tabular}

The above density is estimated to be accurate to 2 significant digits. Uncertainties are not addressed.

The following data were calculated from the input weight fractions.

\begin{tabular}{|c|c|c|c|c|c|c|}
\hline Element & Neutron ZA & Photon ZA & $\begin{array}{l}\text { Weight } \\
\text { Fraction }\end{array}$ & $\begin{array}{c}\text { Atom } \\
\text { Fraction }\end{array}$ & $\begin{array}{c}\text { Atom } \\
\text { Density }\end{array}$ & \\
\hline $\mathrm{H}$ & 1001 & 1000 & 0.023834 & 0.316855 & 0.021645 & \\
\hline $\mathrm{O}$ & 8016 & 8000 & 0.598898 & 0.501581 & 0.034264 & \\
\hline $\mathrm{Al}$ & 13027 & 13000 & 0.080446 & 0.039951 & 0.002729 & \\
\hline $\mathrm{Si}$ & 14000 & 14000 & 0.296821 & 0.141613 & 0.009674 & \\
\hline Total & & & 1.000000 & 1.000000 & 0.068313 & \\
\hline MCNP Form & \multicolumn{2}{|c|}{ Weight Fractions } & \multicolumn{2}{|c|}{ Atom Fractions } & \multicolumn{2}{|c|}{ Atom Densities } \\
\hline \multirow[t]{4}{*}{ Neutrons } & 1001 & -0.023834 & 1001 & 0.316855 & 1001 & 0.021645 \\
\hline & 8016 & -0.598898 & 8016 & 0.501581 & 8016 & 0.034264 \\
\hline & 13027 & -0.080446 & 13027 & 0.039951 & 13027 & 0.002729 \\
\hline & 14000 & -0.296821 & 14000 & 0.141613 & 14000 & 0.009674 \\
\hline \multirow[t]{4}{*}{ Photons } & 1000 & -0.023834 & 1000 & 0.316855 & 1000 & 0.021645 \\
\hline & 8000 & -0.598898 & 8000 & 0.501581 & 8000 & 0.034264 \\
\hline & 13000 & -0.080446 & 13000 & 0.039951 & 13000 & 0.002729 \\
\hline & 14000 & -0.296821 & 14000 & 0.141613 & 14000 & 0.009674 \\
\hline \multirow[t]{4}{*}{ CEPXS Form: } & material & $\mathrm{H}$ & 0.023834 & & & \\
\hline & & 0 & 0.598898 & & & \\
\hline & & $\mathrm{Al}$ & 0.080446 & & & \\
\hline & & $\mathrm{Si}$ & 0.296821 & & & \\
\hline
\end{tabular}




$$
\text { matname Earth, Typical Western U.S. }
$$

density $\quad 1.520000$

\section{Comments and References}

Also called "soil" or "dirt." Composition $(63.5 \% \mathrm{SiO} 2,15.2 \% \mathrm{Al} 2 \mathrm{O} 3$, and $21.3 \% \mathrm{H} 2 \mathrm{O})$ is from pg 135 of Brewer (2009). Packed earth is $1.52 \mathrm{~g} / \mathrm{cm} 3$ and excavated earth is 1.25 to $1.60 \mathrm{~g} / \mathrm{cm} 3$, depending on water content, according to http://www.simetric.co.uk/si_materials.htm (Walker 2009). Many different densities for different types of earth are listed in Table 6.1.5 of Avallone and Baumeister III (1996), and in Table 51.60 of Hungerford (1960).

\section{Earth, U.S. Average}

\begin{tabular}{llll}
\hline Formula $=$ & - & Molecular weight $(\mathrm{g} / \mathrm{mole})=$ \\
Density $(\mathrm{g} / \mathrm{cm} 3)=$ & 1.520000 & Total atom density $($ atoms $/ \mathrm{b}-\mathrm{cm})=$ & - \\
The & 4.383E-02
\end{tabular}

The above density is estimated to be accurate to 2 significant digits. Uncertainties are not addressed. The following data were calculated from the input weight fractions.

\begin{tabular}{|c|c|c|c|c|c|c|}
\hline Element & Neutron ZA & Photon ZA & $\begin{array}{l}\text { Weight } \\
\text { Fraction }\end{array}$ & $\begin{array}{c}\text { Atom } \\
\text { Fraction }\end{array}$ & $\begin{array}{l}\text { Atom } \\
\text { Density }\end{array}$ & \\
\hline 0 & 8016 & 8000 & $\overline{0.513713}$ & $\overline{0.670604}$ & 0.029391 & \\
\hline $\mathrm{Na}$ & 11023 & 11000 & 0.006140 & 0.005578 & 0.000244 & \\
\hline $\mathrm{Mg}$ & 12000 & 12000 & 0.013303 & 0.011432 & 0.000501 & \\
\hline $\mathrm{Al}$ & 13027 & 13000 & 0.068563 & 0.053073 & 0.002326 & \\
\hline $\mathrm{Si}$ & 14000 & 14000 & 0.271183 & 0.201665 & 0.008838 & \\
\hline $\mathrm{K}$ & 19000 & 19000 & 0.014327 & 0.007653 & 0.000335 & \\
\hline $\mathrm{Ca}$ & 20000 & 20000 & 0.051167 & 0.026664 & 0.001169 & \\
\hline $\mathrm{Ti}$ & 22000 & 22000 & 0.004605 & 0.002009 & 0.000088 & \\
\hline $\mathrm{Mn}$ & 25055 & 25000 & 0.000716 & 0.000272 & 0.000012 & \\
\hline $\mathrm{Fe}$ & 26000 & 26000 & 0.056283 & 0.021050 & 0.000923 & \\
\hline Total & & & 1.000000 & 1.000000 & 0.043827 & \\
\hline MCNP Form & \multicolumn{2}{|c|}{ Weight Fractions } & \multicolumn{2}{|c|}{ Atom Fractions } & \multicolumn{2}{|c|}{ Atom Densities } \\
\hline \multirow[t]{10}{*}{ Neutrons } & 8016 & -0.513713 & 8016 & 0.670604 & 8016 & 0.029391 \\
\hline & 11023 & -0.006140 & 11023 & 0.005578 & 11023 & 0.000244 \\
\hline & 12000 & -0.013303 & 12000 & 0.011432 & 12000 & 0.000501 \\
\hline & 13027 & -0.068563 & 13027 & 0.053073 & 13027 & 0.002326 \\
\hline & 14000 & -0.271183 & 14000 & 0.201665 & 14000 & 0.008838 \\
\hline & 19000 & -0.014327 & 19000 & 0.007653 & 19000 & 0.000335 \\
\hline & 20000 & -0.051167 & 20000 & 0.026664 & 20000 & 0.001169 \\
\hline & 22000 & -0.004605 & 22000 & 0.002009 & 22000 & 0.000088 \\
\hline & 25055 & -0.000716 & 25055 & 0.000272 & 25055 & 0.000012 \\
\hline & 26000 & -0.056283 & 26000 & 0.021050 & 26000 & 0.000923 \\
\hline \multirow[t]{4}{*}{ Photons } & 8000 & -0.513713 & 8000 & 0.670604 & 8000 & 0.029391 \\
\hline & 11000 & -0.006140 & 11000 & 0.005578 & 11000 & 0.000244 \\
\hline & 12000 & -0.013303 & 12000 & 0.011432 & 12000 & 0.000501 \\
\hline & 13000 & -0.068563 & 13000 & 0.053073 & 13000 & 0.002326 \\
\hline
\end{tabular}


PIET-43741-TM-963

PNNL-15870 Rev. 1

\begin{tabular}{|c|c|c|c|c|c|c|}
\hline & 14000 & -0.271183 & 14000 & 0.201665 & 14000 & 0.008838 \\
\hline & 19000 & -0.014327 & 19000 & 0.007653 & 19000 & 0.000335 \\
\hline & 20000 & -0.051167 & 20000 & 0.026664 & 20000 & 0.001169 \\
\hline & 22000 & -0.004605 & 22000 & 0.002009 & 22000 & 0.000088 \\
\hline & 25000 & -0.000716 & 25000 & 0.000272 & 25000 & 0.000012 \\
\hline & 26000 & -0.056283 & 26000 & 0.021050 & 26000 & 0.000923 \\
\hline \multirow[t]{11}{*}{ CEPXS Form: } & material & $\mathrm{O}$ & 0.513713 & & & \\
\hline & & $\mathrm{Na}$ & 0.006140 & & & \\
\hline & & $\mathrm{Mg}$ & 0.013303 & & & \\
\hline & & $\mathrm{Al}$ & 0.068563 & & & \\
\hline & & $\mathrm{Si}$ & 0.271183 & & & \\
\hline & & $\mathrm{K}$ & 0.014327 & & & \\
\hline & & $\mathrm{Ca}$ & 0.051167 & & & \\
\hline & & $\mathrm{Ti}$ & 0.004605 & & & \\
\hline & & $\mathrm{Mn}$ & 0.000716 & & & \\
\hline & & $\mathrm{Fe}$ & 0.056283 & & & \\
\hline & $\begin{array}{l}\text { matname } \\
\text { density }\end{array}$ & \multicolumn{2}{|c|}{$\begin{array}{l}\text { Earth, U.S. Average } \\
1.520000\end{array}$} & & & \\
\hline \multicolumn{7}{|c|}{$\begin{array}{l}\text { Comments and References } \\
\text { Average of } 28 \text { soils (dried) from throughout the U.S. Weight fractions based on Table } 11.7 \text { of Chilton et al. } \\
\text { (1984). }\end{array}$} \\
\hline
\end{tabular}

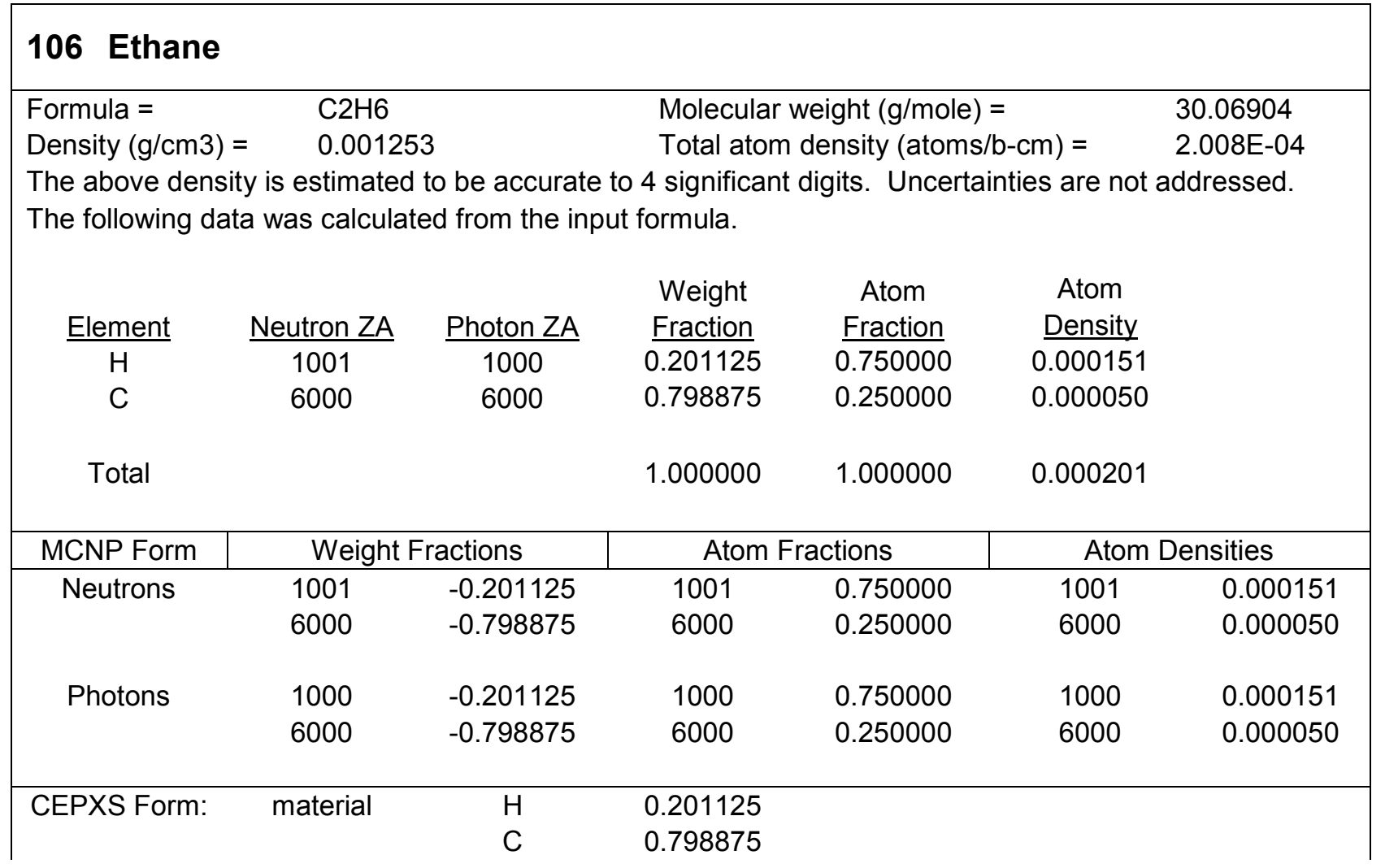




\begin{tabular}{|ll|}
\multicolumn{1}{|c|}{$\begin{array}{c}\text { matname } \\
\text { density }\end{array}$} & Ethane \\
0.001253 \\
\hline Comments and References & \\
Density from http://physics.nist.gov/cgi-bin/Star/compos.pl?matno=152 (NIST 1998). \\
\hline
\end{tabular}

\section{Ethyl Acetate}

\begin{tabular}{llll}
\hline Formula $=$ & $\mathrm{C} 4 \mathrm{H} 8 \mathrm{O} 2$ & Molecular weight $(\mathrm{g} / \mathrm{mole})=$ & 88.10512 \\
Density $(\mathrm{g} / \mathrm{cm} 3)=$ & 0.901000 & Total atom density $($ atoms $/ \mathrm{b}-\mathrm{cm})=$ & $8.622 \mathrm{E}-02$
\end{tabular}

The above density is estimated to be accurate to 3 significant digits. Uncertainties are not addressed. The following data was calculated from the input formula.

\begin{tabular}{|c|c|c|c|c|c|c|}
\hline Element & Neutron ZA & Photon ZA & $\begin{array}{l}\text { Weight } \\
\text { Fraction }\end{array}$ & $\begin{array}{c}\text { Atom } \\
\text { Fraction }\end{array}$ & $\begin{array}{l}\text { Atom } \\
\text { Density }\end{array}$ & \\
\hline $\mathrm{H}$ & 1001 & 1000 & 0.091522 & 0.571429 & 0.049268 & \\
\hline C & 6000 & 6000 & 0.545290 & 0.285714 & 0.024634 & \\
\hline $\mathrm{O}$ & 8016 & 8000 & 0.363189 & 0.142857 & 0.012317 & \\
\hline Total & & & 1.000000 & 1.000000 & 0.086219 & \\
\hline MCNP Form & \multicolumn{2}{|c|}{ Weight Fractions } & \multicolumn{2}{|c|}{ Atom Fractions } & \multicolumn{2}{|c|}{ Atom Densities } \\
\hline \multirow[t]{3}{*}{ Neutrons } & 1001 & -0.091522 & 1001 & 0.571429 & 1001 & 0.049268 \\
\hline & 6000 & -0.545290 & 6000 & 0.285714 & 6000 & 0.024634 \\
\hline & 8016 & -0.363189 & 8016 & 0.142857 & 8016 & 0.012317 \\
\hline \multirow[t]{3}{*}{ Photons } & 1000 & -0.091522 & 1000 & 0.571429 & 1000 & 0.049268 \\
\hline & 6000 & -0.545290 & 6000 & 0.285714 & 6000 & 0.024634 \\
\hline & 8000 & -0.363189 & 8000 & 0.142857 & 8000 & 0.012317 \\
\hline \multirow[t]{4}{*}{ CEPXS Form: } & material & $\mathrm{H}$ & 0.091522 & & & \\
\hline & & C & 0.545290 & & & \\
\hline & & $\mathrm{O}$ & 0.363189 & & & \\
\hline & $\begin{array}{l}\text { matname } \\
\text { density }\end{array}$ & $\begin{array}{c}\text { Ethyl Acetate } \\
0.901000\end{array}$ & & & & \\
\hline \multicolumn{7}{|c|}{$\begin{array}{l}\text { Comments and References } \\
\text { Formula and density }=0.901 \mathrm{~g} / \mathrm{cm} 3 \text { at } 20^{\circ} \mathrm{C} \text { from } \\
\text { http://www.matweb.com } / \mathrm{search} / \mathrm{DataSheet} . \mathrm{aspx} \text { ?MatGUID=c634566b56e04467bbfc09ffd3434ebb\&ckck= } \\
1 \text { (Automation Creations 2010). }\end{array}$} \\
\hline
\end{tabular}

\section{Ethyl Alcohol}

\begin{tabular}{llll}
\hline Formula $=$ & $\mathrm{C} 2 \mathrm{H} 6 \mathrm{O}$ & Molecular weight $(\mathrm{g} / \mathrm{mole})=$ & 46.06844 \\
Density $(\mathrm{g} / \mathrm{cm} 3)=$ & 0.789300 & Total atom density $($ atoms $/ \mathrm{b}-\mathrm{cm})=$ & $9.286 \mathrm{E}-02$ \\
The above density is estimated to be accurate to 3 significant digits. Uncertainties are not addressed
\end{tabular}


The following data were calculated from the input weight fractions.

\begin{tabular}{|c|c|c|c|c|c|}
\hline Element & Neutron ZA & Photon ZA & $\begin{array}{l}\text { Weight } \\
\text { Fraction }\end{array}$ & $\begin{array}{c}\text { Atom } \\
\text { Fraction }\end{array}$ & $\begin{array}{c}\text { Atom } \\
\text { Density }\end{array}$ \\
\hline $\mathrm{H}$ & 1001 & 1000 & $\overline{0.131269}$ & $\overline{0.666654}$ & 0.061904 \\
\hline C & 6000 & 6000 & 0.521438 & 0.222232 & 0.020636 \\
\hline O & 8016 & 8000 & 0.347294 & 0.111113 & 0.010318 \\
\hline Total & & & 1.000001 & 1.000000 & 0.092858 \\
\hline
\end{tabular}

\begin{tabular}{|c|cccccc|}
\hline MCNP Form & \multicolumn{2}{c|}{ Weight Fractions } & \multicolumn{2}{c|}{ Atom Fractions } & \multicolumn{2}{c|}{ Atom Densities } \\
\hline Neutrons & 1001 & -0.131269 & 1001 & 0.666654 & 1001 & 0.061904 \\
& 6000 & -0.521438 & 6000 & 0.222232 & 6000 & 0.020636 \\
& 8016 & -0.347294 & 8016 & 0.111113 & 8016 & 0.010318 \\
Photons & 1000 & -0.131269 & 1000 & 0.666654 & 1000 & 0.061904 \\
& 6000 & -0.521438 & 6000 & 0.222232 & 6000 & 0.020636 \\
& 8000 & -0.347294 & 8000 & 0.111113 & 8000 & 0.010318 \\
& & & & & & \\
\hline CEPXS Form: & material & $\mathrm{H}$ & 0.131269 & & & \\
& & $\mathrm{C}$ & 0.521438 & & & \\
& & $\mathrm{O}$ & 0.347294 & & &
\end{tabular}

\begin{tabular}{cc|} 
matname & Ethyl Alcohol \\
density & 0.789300 \\
\hline
\end{tabular}

\section{Comments and References}

Density and weight fractions from http://physics.nist.gov/cgi-bin/Star/compos.pl?matno=153 (NIST 1998). Formula from pgs 3 - 232 of Lide (2008) and Table 51.120 of Hungerford (1960).

Also called "Ethanol" (http://en.wikipedia.org/wiki/Ethanol).

\section{Ethylene}

\begin{tabular}{llll}
\hline Formula $=$ & $\mathrm{C} 2 \mathrm{H} 4$ & Molecular weight $(\mathrm{g} / \mathrm{mole})=$ & 28.05316 \\
Density $(\mathrm{g} / \mathrm{cm} 3)=$ & 0.001175 & Total atom density $($ atoms $/ \mathrm{b}-\mathrm{cm})=$ & $1.513 \mathrm{E}-04$
\end{tabular}

The above density is estimated to be accurate to 4 significant digits. Uncertainties are not addressed.

The following data were calculated from the input weight fractions.

\begin{tabular}{|c|c|c|c|c|c|c|}
\hline Element & Neutron ZA & Photon ZA & $\begin{array}{l}\text { Weight } \\
\text { Fraction }\end{array}$ & $\begin{array}{c}\text { Atom } \\
\text { Fraction }\end{array}$ & $\begin{array}{l}\text { Atom } \\
\text { Density }\end{array}$ & \\
\hline $\mathrm{H}$ & 1001 & 1000 & $\overline{0.143711}$ & $\overline{0.666653}$ & 0.000101 & \\
\hline C & 6000 & 6000 & 0.856289 & 0.333347 & 0.000050 & \\
\hline Total & & & 1.000000 & 1.000000 & 0.000151 & \\
\hline MCNP Form & \multicolumn{2}{|c|}{ Weight Fractions } & \multicolumn{2}{|c|}{ Atom Fractions } & \multicolumn{2}{|c|}{ Atom Densities } \\
\hline Neutrons & 1001 & -0.143711 & 1001 & 0.666653 & 1001 & 0.000101 \\
\hline & 6000 & -0.856289 & 6000 & 0.333347 & 6000 & 0.000050 \\
\hline
\end{tabular}


PIET-43741-TM-963

PNNL-15870 Rev. 1

\begin{tabular}{|c|c|c|c|c|c|c|}
\hline Photons & $\begin{array}{l}1000 \\
6000\end{array}$ & $\begin{array}{l}-0.143711 \\
-0.856289\end{array}$ & $\begin{array}{l}1000 \\
6000\end{array}$ & $\begin{array}{l}0.666653 \\
0.333347\end{array}$ & $\begin{array}{l}1000 \\
6000\end{array}$ & $\begin{array}{l}0.000101 \\
0.000050\end{array}$ \\
\hline CEPXS Form: & $\begin{array}{c}\text { matname } \\
\text { density }\end{array}$ & $\begin{array}{c}\mathrm{H} \\
\mathrm{C} \\
\text { Ethylene } \\
0.001175\end{array}$ & $\begin{array}{l}0.143711 \\
0.856289\end{array}$ & & & \\
\hline $\begin{array}{l}\text { Comments an } \\
\text { Density and we } \\
\text { Formula and } d\end{array}$ & $\begin{array}{l}\text { References } \\
\text { it fractions } \\
\text { ity }(0.5678\end{array}$ & $\begin{array}{l}\text { n http://physi } \\
\text { m3) in Lide ( }\end{array}$ & $\begin{array}{l}\text { ist.gov/cgi- } \\
\text { 8), pgs } 3 \text { - }\end{array}$ & Star/comp & $\operatorname{atno}=1$ & ST 1998). \\
\hline
\end{tabular}

\section{Ethylene Glycol}

\begin{tabular}{llll}
\hline Formula $=$ & $\mathrm{C} 2 \mathrm{H} 6 \mathrm{O} 2$ & Molecular weight $(\mathrm{g} / \mathrm{mole})=$ & 62.06784 \\
Density $(\mathrm{g} / \mathrm{cm} 3)=$ & 1.114000 & Total atom density $($ atoms $/ \mathrm{b}-\mathrm{cm})=$ & $1.081 \mathrm{E}-01$
\end{tabular}

The above density is estimated to be accurate to 4 significant digits. Uncertainties are not addressed.

The following data was calculated from the input formula.

\begin{tabular}{|c|c|c|c|c|c|c|}
\hline Element & Neutron ZA & Photon ZA & $\begin{array}{l}\text { Weight } \\
\text { Fraction }\end{array}$ & $\begin{array}{c}\text { Atom } \\
\text { Fraction }\end{array}$ & $\begin{array}{c}\text { Atom } \\
\text { Density }\end{array}$ & \\
\hline $\mathrm{H}$ & 1001 & 1000 & $\overline{0.097436}$ & $\overline{0.600000}$ & 0.064852 & \\
\hline C & 6000 & 6000 & 0.387018 & 0.200000 & 0.021617 & \\
\hline $\mathrm{O}$ & 8016 & 8000 & 0.515546 & 0.200000 & 0.021617 & \\
\hline Total & & & 1.000000 & 1.000000 & 0.108086 & \\
\hline MCNP Form & Weight & ractions & Atom & ictions & Atom & sities \\
\hline Neutrons & 1001 & -0.097436 & 1001 & 0.600000 & 1001 & 0.064852 \\
\hline & 6000 & -0.387018 & 6000 & 0.200000 & 6000 & 0.021617 \\
\hline & 8016 & -0.515546 & 8016 & 0.200000 & 8016 & 0.021617 \\
\hline Photons & 1000 & -0.097436 & 1000 & 0.600000 & 1000 & 0.064852 \\
\hline & 6000 & -0.387018 & 6000 & 0.200000 & 6000 & 0.021617 \\
\hline & 8000 & -0.515546 & 8000 & 0.200000 & 8000 & 0.021617 \\
\hline CEPXS Form: & material & $\mathrm{H}$ & 0.097436 & & & \\
\hline & & C & 0.387018 & & & \\
\hline & & $\mathrm{O}$ & 0.515546 & & & \\
\hline & $\begin{array}{l}\text { matname } \\
\text { density }\end{array}$ & $\begin{array}{c}\text { Ethylene Gly } \\
1.114000\end{array}$ & & & & \\
\hline $\begin{array}{l}\text { Comments an } \\
\text { Density = } 1.11 \\
\text { http://www.mat } \\
\text { (Automation Cr }\end{array}$ & $\begin{array}{l}\text { References } \\
\mathrm{g} / \mathrm{cm} 3 \text { at } 20^{\circ} \mathrm{C} \\
\text { eb.com/searcl } \\
\text { ations } 2010) \text {. }\end{array}$ & $\begin{array}{l}\text { and formula fr } \\
\text { /DataSheet.as } \\
\text { See also Table }\end{array}$ & $\begin{array}{l}\text { MatGUID }= \\
.120 \text { of } \mathrm{Hu}\end{array}$ & $\begin{array}{l}\text { 4ad885e9f } \\
\text { ford (1960) }\end{array}$ & a50146ae & 4801 \\
\hline
\end{tabular}




\section{Explosive Compound, AN}

\begin{tabular}{llll}
\hline Formula $=$ & NH4NO3 & Molecular weight $(\mathrm{g} / \mathrm{mole})=$ & 80.04336 \\
Density $(\mathrm{g} / \mathrm{cm} 3)=$ & 1.720000 & Total atom density $($ atoms $/ \mathrm{b}-\mathrm{cm})=$ & $1.165 \mathrm{E}-01$
\end{tabular}

The above density is estimated to be accurate to 3 significant digits. Uncertainties are not addressed. The following data was calculated from the input formula.

\begin{tabular}{|c|c|c|c|c|c|c|}
\hline Element & Neutron ZA & Photon ZA & $\begin{array}{l}\text { Weight } \\
\text { Fraction }\end{array}$ & $\begin{array}{l}\text { Atom } \\
\text { Fraction }\end{array}$ & $\begin{array}{l}\text { Atom } \\
\text { Density }\end{array}$ & \\
\hline $\mathrm{H}$ & 1001 & 1000 & 0.050370 & 0.444444 & 0.051762 & \\
\hline $\mathrm{N}$ & 7014 & 7000 & 0.349978 & 0.222222 & 0.025881 & \\
\hline $\mathrm{O}$ & 8016 & 8000 & 0.599652 & 0.333333 & 0.038822 & \\
\hline Total & & & 1.000000 & 1.000000 & 0.116465 & \\
\hline MCNP Form & \multicolumn{2}{|c|}{ Weight Fractions } & \multicolumn{2}{|c|}{ Atom Fractions } & \multicolumn{2}{|c|}{ Atom Densities } \\
\hline \multirow[t]{3}{*}{ Neutrons } & 1001 & -0.050370 & 1001 & 0.444444 & 1001 & 0.051762 \\
\hline & 7014 & -0.349978 & 7014 & 0.222222 & 7014 & 0.025881 \\
\hline & 8016 & -0.599652 & 8016 & 0.333333 & 8016 & 0.038822 \\
\hline \multirow[t]{3}{*}{ Photons } & 1000 & -0.050370 & 1000 & 0.444444 & 1000 & 0.051762 \\
\hline & 7000 & -0.349978 & 7000 & 0.222222 & 7000 & 0.025881 \\
\hline & 8000 & -0.599652 & 8000 & 0.333333 & 8000 & 0.038822 \\
\hline \multirow[t]{4}{*}{ CEPXS Form: } & material & $\mathrm{H}$ & 0.050370 & & & \\
\hline & & $\mathrm{N}$ & 0.349978 & & & \\
\hline & & $\mathrm{O}$ & 0.599652 & & & \\
\hline & $\begin{array}{l}\text { matname } \\
\text { density }\end{array}$ & \multicolumn{2}{|c|}{$\begin{array}{l}\text { Explosive Compound, AN } \\
1.720000\end{array}$} & & & \\
\hline \multicolumn{7}{|c|}{$\begin{array}{l}\text { Comments and References } \\
\text { Ammonium Nitrate (AN) abbreviation and formula from pg } 12 \text { of Yinon and Zitrin (1993). } \\
\text { Formula and density (1.72 } \mathrm{g} / \mathrm{cm} 3 \text { ) from Lide (2008), pgs } 4-47 . \\
\text { Also density }=1.72 \mathrm{~g} / \mathrm{cm} 3 \mathrm{at} \\
\text { http://www.matweb.com/search/DataSheet.aspx?MatGUID=646f6adaf15e46d48ff2d9a3a8311da0 } \\
\text { (Automation Creations 2010). Also see http://en.wikipedia.org/wiki/Ammonium_nitrate. }\end{array}$} \\
\hline
\end{tabular}

\section{Explosive Compound, EGDN}

\begin{tabular}{llll}
\hline Formula $=$ & C2H4N2O6 & Molecular weight $(\mathrm{g} / \mathrm{mole})=$ & 152.06296 \\
Density $(\mathrm{g} / \mathrm{cm} 3)=$ & 1.490000 & Total atom density $($ atoms $/ \mathrm{b}-\mathrm{cm})=$ & $8.261 \mathrm{E}-02$
\end{tabular}

The above density is estimated to be accurate to 3 significant digits. Uncertainties are not addressed.

The following data was calculated from the input formula. 
PIET-43741-TM-963

PNNL-15870 Rev. 1

\begin{tabular}{|c|c|c|c|c|c|c|}
\hline Element & Neutron ZA & Photon ZA & $\begin{array}{l}\text { Weight } \\
\text { Fraction }\end{array}$ & $\begin{array}{c}\text { Atom } \\
\text { Fraction }\end{array}$ & $\begin{array}{l}\text { Atom } \\
\text { Density }\end{array}$ & \\
\hline $\mathrm{H}$ & 1001 & 1000 & 0.026514 & 0.285714 & 0.023603 & \\
\hline $\mathrm{C}$ & 6000 & 6000 & 0.157970 & 0.142857 & 0.011802 & \\
\hline $\mathrm{N}$ & 7014 & 7000 & 0.184222 & 0.142857 & 0.011802 & \\
\hline O & 8016 & 8000 & 0.631294 & 0.428571 & 0.035405 & \\
\hline Total & & & 1.000000 & 1.000000 & 0.082612 & \\
\hline MCNP Form & \multicolumn{2}{|c|}{ Weight Fractions } & \multicolumn{2}{|c|}{ Atom Fractions } & \multicolumn{2}{|c|}{ Atom Densities } \\
\hline \multirow[t]{4}{*}{ Neutrons } & 1001 & -0.026514 & 1001 & 0.285714 & 1001 & 0.023603 \\
\hline & 6000 & -0.157970 & 6000 & 0.142857 & 6000 & 0.011802 \\
\hline & 7014 & -0.184222 & 7014 & 0.142857 & 7014 & 0.011802 \\
\hline & 8016 & -0.631294 & 8016 & 0.428571 & 8016 & 0.035405 \\
\hline \multirow[t]{4}{*}{ Photons } & 1000 & -0.026514 & 1000 & 0.285714 & 1000 & 0.023603 \\
\hline & 6000 & -0.157970 & 6000 & 0.142857 & 6000 & 0.011802 \\
\hline & 7000 & -0.184222 & 7000 & 0.142857 & 7000 & 0.011802 \\
\hline & 8000 & -0.631294 & 8000 & 0.428571 & 8000 & 0.035405 \\
\hline \multirow[t]{5}{*}{ CEPXS Form: } & material & $\mathrm{H}$ & 0.026514 & & & \\
\hline & & C & 0.157970 & & & \\
\hline & & $N$ & 0.184222 & & & \\
\hline & & $\mathrm{O}$ & 0.631294 & & & \\
\hline & $\begin{array}{c}\text { matname } \\
\text { density }\end{array}$ & $\begin{array}{c}\text { Explosive Cc } \\
1.490000\end{array}$ & ound, EGD & & & \\
\hline \multicolumn{7}{|c|}{$\begin{array}{l}\text { Comments and References } \\
\text { Ethylene Glycol Dinitrate (EGDN), or nitroglycol, abbreviation and formula from pg } 11 \text { of Yinon and Zitrin } \\
\text { (1993). } \\
\text { Formula and density from Lide (2008), pgs } 3-232 \text {. } \\
\text { Also see http://en.wikipedia.org/wiki/EGDN. }\end{array}$} \\
\hline
\end{tabular}

\section{Explosive Compound, HMX}

\begin{tabular}{llll}
\hline Formula $=$ & C4H8N8O8 & Molecular weight $(\mathrm{g} / \mathrm{mole})=$ & 296.15512 \\
Density $(\mathrm{g} / \mathrm{cm} 3)=$ & 1.890000 & Total atom density $($ atoms $/ \mathrm{b}-\mathrm{cm})=$ & $1.076 \mathrm{E}-01$
\end{tabular}

The above density is estimated to be accurate to 3 significant digits. Uncertainties are not addressed.

The following data was calculated from the input formula.

\begin{tabular}{|c|c|c|c|c|c|}
\hline Element & Neutron ZA & Photon ZA & $\begin{array}{l}\text { Weight } \\
\text { Fraction }\end{array}$ & $\begin{array}{c}\text { Atom } \\
\text { Fraction }\end{array}$ & $\begin{array}{l}\text { Atom } \\
\text { Density }\end{array}$ \\
\hline $\mathrm{H}$ & 1001 & 1000 & $\overline{0.027227}$ & $\overline{0.285714}$ & 0.030746 \\
\hline C & 6000 & 6000 & 0.162222 & 0.142857 & 0.015373 \\
\hline $\mathrm{N}$ & 7014 & 7000 & 0.378361 & 0.285714 & 0.030746 \\
\hline 0 & 8016 & 8000 & 0.432190 & 0.285714 & 0.030746 \\
\hline Total & & & 1.000000 & 1.000000 & 0.107610 \\
\hline
\end{tabular}


PIET-43741-TM-963

PNNL-15870 Rev. 1

\begin{tabular}{|c|c|c|c|c|c|c|}
\hline MCNP Form & \multicolumn{2}{|c|}{ Weight Fractions } & \multicolumn{2}{|c|}{ Atom Fractions } & \multicolumn{2}{|c|}{ Atom Densities } \\
\hline \multirow[t]{4}{*}{ Neutrons } & 1001 & -0.027227 & 1001 & 0.285714 & 1001 & 0.030746 \\
\hline & 6000 & -0.162222 & 6000 & 0.142857 & 6000 & 0.015373 \\
\hline & 7014 & -0.378361 & 7014 & 0.285714 & 7014 & 0.030746 \\
\hline & 8016 & -0.432190 & 8016 & 0.285714 & 8016 & 0.030746 \\
\hline \multirow[t]{4}{*}{ Photons } & 1000 & -0.027227 & 1000 & 0.285714 & 1000 & 0.030746 \\
\hline & 6000 & -0.162222 & 6000 & 0.142857 & 6000 & 0.015373 \\
\hline & 7000 & -0.378361 & 7000 & 0.285714 & 7000 & 0.030746 \\
\hline & 8000 & -0.432190 & 8000 & 0.285714 & 8000 & 0.030746 \\
\hline \multirow[t]{5}{*}{ CEPXS Form: } & material & $\mathrm{H}$ & 0.027227 & & & \\
\hline & & $\mathrm{C}$ & 0.162222 & & & \\
\hline & & $\mathrm{N}$ & 0.378361 & & & \\
\hline & & $\mathrm{O}$ & 0.432190 & & & \\
\hline & $\begin{array}{c}\text { matname } \\
\text { density }\end{array}$ & $\begin{array}{c}\text { Explosive Cc } \\
1.890000\end{array}$ & ound, HMX & & & \\
\hline \multicolumn{7}{|c|}{$\begin{array}{l}\text { Comments and References } \\
\text { Chemical names: Cyclotetramethylenetetranitramine or 1,3,5,7-Tetranitro-1,3,5,7-tetrazacyclooctane. } \\
\text { Abbreviation, names, and formula from p. } 6 \text { of Yinon and Zitrin (1993). } \\
\text { Density }=1.89 \mathrm{~g} / \mathrm{cm} 3 \text { from Table } 7.1 \text { of Zudas and Walters (2002). Also see } \\
\text { http://en.wikipedia.org/wiki/HMX. }\end{array}$} \\
\hline
\end{tabular}

\section{Explosive Compound, NC}

\begin{tabular}{llll}
\hline Formula $=$ & - & Molecular weight $(\mathrm{g} / \mathrm{mole})=$ & - \\
Density $(\mathrm{g} / \mathrm{cm} 3)=$ & 1.490000 & Total atom density $($ atoms $/ \mathrm{b}-\mathrm{cm})=$ & $8.647 \mathrm{E}-02$
\end{tabular}

The above density is estimated to be accurate to 3 significant digits. Uncertainties are not addressed.

The following data were calculated from the input weight fractions.

\begin{tabular}{|c|c|c|c|c|c|c|}
\hline Element & Neutron ZA & Photon ZA & $\begin{array}{l}\text { Weight } \\
\text { Fraction }\end{array}$ & $\begin{array}{c}\text { Atom } \\
\text { Fraction }\end{array}$ & $\begin{array}{c}\text { Atom } \\
\text { Density }\end{array}$ & \\
\hline $\mathrm{H}$ & 1001 & 1000 & 0.029216 & 0.300771 & 0.026009 & \\
\hline C & 6000 & 6000 & 0.271296 & 0.234383 & 0.020268 & \\
\hline $\mathrm{N}$ & 7014 & 7000 & 0.121276 & 0.089844 & 0.007769 & \\
\hline $\mathrm{O}$ & 8016 & 8000 & 0.578212 & 0.375002 & 0.032428 & \\
\hline Total & & & 1.000000 & 1.000000 & 0.086474 & \\
\hline MCNP Form & \multicolumn{2}{|c|}{ Weight Fractions } & \multicolumn{2}{|c|}{ Atom Fractions } & \multicolumn{2}{|c|}{ Atom Densities } \\
\hline \multirow[t]{4}{*}{ Neutrons } & 1001 & -0.029216 & 1001 & 0.300771 & 1001 & 0.026009 \\
\hline & 6000 & -0.271296 & 6000 & 0.234383 & 6000 & 0.020268 \\
\hline & 7014 & -0.121276 & 7014 & 0.089844 & 7014 & 0.007769 \\
\hline & 8016 & -0.578212 & 8016 & 0.375002 & 8016 & 0.032428 \\
\hline
\end{tabular}




\begin{tabular}{|c|c|c|c|c|c|c|}
\hline \multirow[t]{4}{*}{ Photons } & 1000 & -0.029216 & 1000 & 0.300771 & 1000 & 0.026009 \\
\hline & 6000 & -0.271296 & 6000 & 0.234383 & 6000 & 0.020268 \\
\hline & 7000 & -0.121276 & 7000 & 0.089844 & 7000 & 0.007769 \\
\hline & 8000 & -0.578212 & 8000 & 0.375002 & 8000 & 0.032428 \\
\hline \multirow[t]{5}{*}{ CEPXS Form: } & material & $\mathrm{H}$ & 0.029216 & & & \\
\hline & & C & 0.271296 & & & \\
\hline & & $\mathrm{N}$ & 0.121276 & & & \\
\hline & & $\mathrm{O}$ & 0.578212 & & & \\
\hline & $\begin{array}{l}\text { matname } \\
\text { density }\end{array}$ & \multicolumn{2}{|c|}{$\begin{array}{l}\text { Explosive Compound, NC } \\
1.490000\end{array}$} & & & \\
\hline \multicolumn{7}{|c|}{$\begin{array}{l}\text { Comments and References } \\
\text { Also called nitrocellulose or cellulose nitrate. The chemical formula is apparently uncertain due to the } \\
\text { complexity. } \\
\text { Density }=1.49 \mathrm{~g} / \mathrm{cm} 3 \text { and weight fractions from http://physics.nist.gov/cgi-bin/Star/compos.pl?matno=138 } \\
\text { (NIST 1998). } \\
\text { General reference: Yinon and Zitrin (1993). }\end{array}$} \\
\hline
\end{tabular}

\section{Explosive Compound, NG}

\begin{tabular}{lllc}
\hline Formula $=$ & C3H5N3O9 & Molecular weight $(\mathrm{g} / \mathrm{mole})=$ & 227.0865 \\
Density $(\mathrm{g} / \mathrm{cm} 3)=$ & 1.600000 & Total atom density $($ atoms $/ \mathrm{b}-\mathrm{cm})=$ & $8.486 \mathrm{E}-02$ \\
The above density is estimated to be accurate to 3 significant digits. Uncertainties are not addressed. & Un \\
The following data was calculated from the input formula.
\end{tabular}

\begin{tabular}{|c|c|c|c|c|c|c|}
\hline Element & Neutron ZA & Photon ZA & $\begin{array}{l}\text { Weight } \\
\text { Fraction }\end{array}$ & $\begin{array}{l}\text { Atom } \\
\text { Fraction }\end{array}$ & $\begin{array}{l}\text { Atom } \\
\text { Density }\end{array}$ & \\
\hline $\mathrm{H}$ & 1001 & 1000 & $\overline{0.022193}$ & $\overline{0.250000}$ & 0.021215 & \\
\hline C & 6000 & 6000 & 0.158671 & 0.150000 & 0.012729 & \\
\hline $\mathrm{N}$ & 7014 & 7000 & 0.185040 & 0.150000 & 0.012729 & \\
\hline $\mathrm{O}$ & 8016 & 8000 & 0.634096 & 0.450000 & 0.038188 & \\
\hline Total & & & 1.000000 & 1.000000 & 0.084861 & \\
\hline MCNP Form & \multicolumn{2}{|c|}{ Weight Fractions } & \multicolumn{2}{|c|}{ Atom Fractions } & \multicolumn{2}{|c|}{ Atom Densities } \\
\hline \multirow[t]{4}{*}{ Neutrons } & 1001 & -0.022193 & 1001 & 0.250000 & 1001 & 0.021215 \\
\hline & 6000 & -0.158671 & 6000 & 0.150000 & 6000 & 0.012729 \\
\hline & 7014 & -0.185040 & 7014 & 0.150000 & 7014 & 0.012729 \\
\hline & 8016 & -0.634096 & 8016 & 0.450000 & 8016 & 0.038188 \\
\hline \multirow[t]{4}{*}{ Photons } & 1000 & -0.022193 & 1000 & 0.250000 & 1000 & 0.021215 \\
\hline & 6000 & -0.158671 & 6000 & 0.150000 & 6000 & 0.012729 \\
\hline & 7000 & -0.185040 & 7000 & 0.150000 & 7000 & 0.012729 \\
\hline & 8000 & -0.634096 & 8000 & 0.450000 & 8000 & 0.038188 \\
\hline \multirow[t]{2}{*}{ CEPXS Form: } & material & $\mathrm{H}$ & 0.022193 & & & \\
\hline & & C & 0.158671 & & & \\
\hline
\end{tabular}




\begin{tabular}{|lcc|} 
& $\mathrm{N}$ & 0.185040 \\
& $\mathrm{O}$ & 0.634096 \\
matname & Explosive Compound, NG \\
density & 1.600000 \\
\hline Comments and References & & \\
Nitroglycerin, Trinitroglycerol, or Glycerol Trinitrate from http://en.wikipedia.org/wiki/Nitroglycerin. \\
Abbreviation and formula from pg 8 of Yinon and Zitrin (1993). \\
Density = 1.6 g/cm3 from Knovel (2008). \\
\hline
\end{tabular}

\section{Explosive Compound, PETN}

\begin{tabular}{llll}
\hline Formula $=$ & C5H8N4O12 & Molecular weight $(\mathrm{g} / \mathrm{mole})=$ & 316.13662 \\
Density $(\mathrm{g} / \mathrm{cm} 3)=$ & 1.770000 & Total atom density $($ atoms $/ \mathrm{b}-\mathrm{cm})=$ & $9.778 \mathrm{E}-02$
\end{tabular}

The above density is estimated to be accurate to 3 significant digits. Uncertainties are not addressed.

The following data was calculated from the input formula.

\begin{tabular}{|c|c|c|c|c|c|c|}
\hline Element & Neutron ZA & Photon ZA & $\begin{array}{l}\text { Weight } \\
\text { Fraction }\end{array}$ & $\begin{array}{l}\text { Atom } \\
\text { Fraction }\end{array}$ & $\begin{array}{l}\text { Atom } \\
\text { Density }\end{array}$ & \\
\hline $\mathrm{H}$ & 1001 & 1000 & 0.025506 & 0.275862 & 0.026974 & \\
\hline $\mathrm{C}$ & 6000 & 6000 & 0.189961 & 0.172414 & 0.016859 & \\
\hline $\mathrm{N}$ & 7014 & 7000 & 0.177223 & 0.137931 & 0.013487 & \\
\hline $\mathrm{O}$ & 8016 & 8000 & 0.607310 & 0.413793 & 0.040460 & \\
\hline Total & & & 1.000000 & 1.000000 & 0.097779 & \\
\hline MCNP Form & \multicolumn{2}{|c|}{ Weight Fractions } & \multicolumn{2}{|c|}{ Atom Fractions } & \multicolumn{2}{|c|}{ Atom Densities } \\
\hline \multirow[t]{4}{*}{ Neutrons } & 1001 & -0.025506 & 1001 & 0.275862 & 1001 & 0.026974 \\
\hline & 6000 & -0.189961 & 6000 & 0.172414 & 6000 & 0.016859 \\
\hline & 7014 & -0.177223 & 7014 & 0.137931 & 7014 & 0.013487 \\
\hline & 8016 & -0.607310 & 8016 & 0.413793 & 8016 & 0.040460 \\
\hline \multirow[t]{4}{*}{ Photons } & 1000 & -0.025506 & 1000 & 0.275862 & 1000 & 0.026974 \\
\hline & 6000 & -0.189961 & 6000 & 0.172414 & 6000 & 0.016859 \\
\hline & 7000 & -0.177223 & 7000 & 0.137931 & 7000 & 0.013487 \\
\hline & 8000 & -0.607310 & 8000 & 0.413793 & 8000 & 0.040460 \\
\hline \multirow[t]{5}{*}{ CEPXS Form: } & material & $\mathrm{H}$ & 0.025506 & & & \\
\hline & & C & 0.189961 & & & \\
\hline & & $\mathrm{N}$ & 0.177223 & & & \\
\hline & & O & 0.607310 & & & \\
\hline & $\begin{array}{c}\text { matname } \\
\text { density }\end{array}$ & $\begin{array}{c}\text { Explosive Co } \\
1.770000\end{array}$ & pound, PETN & & & \\
\hline \multicolumn{7}{|c|}{$\begin{array}{l}\text { Comments and References } \\
\text { Pentaerythritol tetranitrate, baritrate. Abbreviation and formula from pgs } 9-10 \text { of Yinon and Zitrin (1993). } \\
\text { Density }=1.773 \mathrm{~g} / \mathrm{cm} 3 \text { from Knovel (2008). } \\
\text { Density }=1.76 \mathrm{~g} / \mathrm{cm} 3 \text { in Table } 7.1 \text { of Zudas and Walters (2002). }\end{array}$} \\
\hline
\end{tabular}




\section{Explosive Compound, RDX}

\begin{tabular}{llll}
\hline Formula $=$ & C3H6N6O6 & Molecular weight $(\mathrm{g} / \mathrm{mole})=$ & 222.11634 \\
Density $(\mathrm{g} / \mathrm{cm} 3)=$ & 1.820000 & Total atom density $($ atoms $/ \mathrm{b}-\mathrm{cm})=$ & $1.036 \mathrm{E}-01$
\end{tabular}

The above density is estimated to be accurate to 3 significant digits. Uncertainties are not addressed.

The following data was calculated from the input formula.

$\begin{array}{ccccccc}\text { Element } & \text { Neutron ZA } & \frac{\text { Photon ZA }}{1000} & & \begin{array}{c}\text { Weight } \\ \text { Fraction }\end{array} & \begin{array}{c}\text { Atom } \\ \text { Fraction }\end{array} & \begin{array}{c}\text { Atom } \\ \text { Density }\end{array} \\ & 1001 & & 0.027227 & 0.285714 & 0.029607 \\ \mathrm{C} & 6000 & 6000 & 0.162222 & 0.142857 & 0.014803 \\ \mathrm{~N} & 7014 & 7000 & 0.378361 & 0.285714 & 0.029607 \\ \mathrm{O} & 8016 & 8000 & 0.432190 & 0.285714 & 0.029607 \\ \text { Total } & & & & & \\ & & & 1.000000 & 1.000000 & 0.103624\end{array}$

\begin{tabular}{|c|cc|cc|cr|}
\hline MCNP Form & \multicolumn{2}{|c|}{ Weight Fractions } & \multicolumn{2}{c|}{ Atom Fractions } & \multicolumn{2}{c|}{ Atom Densities } \\
\hline Neutrons & 1001 & -0.027227 & 1001 & 0.285714 & 1001 & 0.029607 \\
& 6000 & -0.162222 & 6000 & 0.142857 & 6000 & 0.014803 \\
& 7014 & -0.378361 & 7014 & 0.285714 & 7014 & 0.029607 \\
& 8016 & -0.432190 & 8016 & 0.285714 & 8016 & 0.029607 \\
Photons & & & & & & \\
& 1000 & -0.027227 & 1000 & 0.285714 & 1000 & 0.029607 \\
& 6000 & -0.162222 & 6000 & 0.142857 & 6000 & 0.014803 \\
& 7000 & -0.378361 & 7000 & 0.285714 & 7000 & 0.029607 \\
& 8000 & -0.432190 & 8000 & 0.285714 & 8000 & 0.029607
\end{tabular}

\begin{tabular}{|c|c|c|c|}
\hline \multirow[t]{4}{*}{ CEPXS Form: } & material & $\mathrm{H}$ & 0.027227 \\
\hline & & C & 0.162222 \\
\hline & & $\mathrm{N}$ & 0.378361 \\
\hline & & $\mathrm{O}$ & 0.432190 \\
\hline
\end{tabular}

matname Explosive Compound, RDX density $\quad 1.820000$

\section{Comments and References}

Commonly known as cyclonite, hexogen, or T4 (http://en.wikipedia.org/wiki/RDX).

Chemical name: Cyclotrimethylenetrinitramine or 1,3,5-Tinitro-1,3,5-triazacyclohexane.

Abbreviation and formula from p.5 of Yinon and Zitrin (1993). Density $=1.82 \mathrm{~g} / \mathrm{cm} 3$ from Yaws (2008).

Density $=1.77 \mathrm{~g} / \mathrm{cm} 3$ in Table 7.1 of Zudas and Walters (2002).

\section{Explosive Compound, TNT}

\begin{tabular}{llll}
\hline Formula $=$ & $\mathrm{C} 6 \mathrm{H} 2(\mathrm{NO} 2) 3 \mathrm{CH} 3$ & Molecular weight $(\mathrm{g} / \mathrm{mole})=$ & 227.1311 \\
Density $(\mathrm{g} / \mathrm{cm} 3)=$ & 1.650000 & Total atom density $($ atoms $/ \mathrm{b}-\mathrm{cm})=$ & $9.187 \mathrm{E}-02$
\end{tabular}

The above density is estimated to be accurate to 3 significant digits. Uncertainties are not addressed.

The following data was calculated from the input formula. 


\begin{tabular}{|c|c|c|c|c|c|c|}
\hline Element & Neutron ZA & Photon ZA & $\begin{array}{l}\text { Weight } \\
\text { Fraction }\end{array}$ & $\begin{array}{l}\text { Atom } \\
\text { Fraction }\end{array}$ & $\begin{array}{l}\text { Atom } \\
\text { Density }\end{array}$ & \\
\hline $\mathrm{H}$ & 1001 & 1000 & 0.022189 & $\overline{0.238095}$ & 0.021874 & \\
\hline C & 6000 & 6000 & 0.370160 & 0.333333 & 0.030624 & \\
\hline $\mathrm{N}$ & 7014 & 7000 & 0.185004 & 0.142857 & 0.013124 & \\
\hline O & 8016 & 8000 & 0.422648 & 0.285714 & 0.026249 & \\
\hline Total & & & 1.000000 & 1.000000 & 0.091871 & \\
\hline MCNP Form & \multicolumn{2}{|c|}{ Weight Fractions } & \multicolumn{2}{|c|}{ Atom Fractions } & \multicolumn{2}{|c|}{ Atom Densities } \\
\hline \multirow[t]{4}{*}{ Neutrons } & 1001 & -0.022189 & 1001 & 0.238095 & 1001 & 0.021874 \\
\hline & 6000 & -0.370160 & 6000 & 0.333333 & 6000 & 0.030624 \\
\hline & 7014 & -0.185004 & 7014 & 0.142857 & 7014 & 0.013124 \\
\hline & 8016 & -0.422648 & 8016 & 0.285714 & 8016 & 0.026249 \\
\hline \multirow[t]{4}{*}{ Photons } & 1000 & -0.022189 & 1000 & 0.238095 & 1000 & 0.021874 \\
\hline & 6000 & -0.370160 & 6000 & 0.333333 & 6000 & 0.030624 \\
\hline & 7000 & -0.185004 & 7000 & 0.142857 & 7000 & 0.013124 \\
\hline & 8000 & -0.422648 & 8000 & 0.285714 & 8000 & 0.026249 \\
\hline \multirow[t]{5}{*}{ CEPXS Form: } & material & $\mathrm{H}$ & 0.022189 & & & \\
\hline & & $\mathrm{C}$ & 0.370160 & & & \\
\hline & & $\mathrm{N}$ & 0.185004 & & & \\
\hline & & $\mathrm{O}$ & 0.422648 & & & \\
\hline & $\begin{array}{l}\text { matname } \\
\text { density }\end{array}$ & \multicolumn{2}{|c|}{$\begin{array}{l}\text { Explosive Compound, TNT } \\
1.650000\end{array}$} & & & \\
\hline \multicolumn{7}{|c|}{$\begin{array}{l}\text { Comments and References } \\
\text { Chemical name: } 2,4,6 \text {-trinitrotoluene (http://en.wikipedia.org/wiki/Trinitrotoluene). } \\
\text { Name and formula from pg } 3 \text { of Yinon and Zitrin (1993). } \\
\text { Density }=1.654 \mathrm{~g} / \mathrm{cm} 3 \text { from Knovel (2008). } \\
\text { Density }=1.63 \mathrm{~g} / \mathrm{cm} 3 \text { in Table } 7.1 \text { of Zudas and Walters (2002). }\end{array}$} \\
\hline
\end{tabular}

\section{Eye Lens (ICRP)}

\begin{tabular}{llll}
\hline Formula $=$ & - & Molecular weight $(\mathrm{g} / \mathrm{mole})=$ & - \\
Density $(\mathrm{g} / \mathrm{cm} 3)=$ & 1.100000 & Total atom density $($ atoms $/ \mathrm{b}-\mathrm{cm})=$ & $1.055 \mathrm{E}-01$
\end{tabular}

The above density is estimated to be accurate to 3 significant digits. Uncertainties are not addressed.

The following data were calculated from the input weight fractions.

\begin{tabular}{|c|c|c|c|c|c|}
\hline Element & Neutron ZA & Photon ZA & $\begin{array}{l}\text { Weight } \\
\text { Fraction }\end{array}$ & $\begin{array}{c}\text { Atom } \\
\text { Fraction }\end{array}$ & $\begin{array}{l}\text { Atom } \\
\text { Density }\end{array}$ \\
\hline$\overline{\mathrm{H}}$ & 1001 & 1000 & 0.099269 & 0.618329 & 0.065241 \\
\hline C & 6000 & 6000 & 0.193710 & 0.101257 & 0.010684 \\
\hline $\mathrm{N}$ & 7014 & 7000 & 0.053270 & 0.023877 & 0.002519 \\
\hline $\mathrm{O}$ & 8016 & 8000 & 0.653751 & 0.256537 & 0.027068 \\
\hline
\end{tabular}

Total

$1.000000 \quad 1.000000 \quad 0.105512$


PIET-43741-TM-963

PNNL-15870 Rev. 1

\begin{tabular}{|c|c|c|c|c|c|c|}
\hline MCNP Form & \multicolumn{2}{|c|}{ Weight Fractions } & \multicolumn{2}{|c|}{ Atom Fractions } & \multicolumn{2}{|c|}{ Atom Densities } \\
\hline \multirow{4}{*}{ Neutrons } & 1001 & -0.099269 & 1001 & 0.618329 & 1001 & 0.065241 \\
\hline & 6000 & -0.193710 & 6000 & 0.101257 & 6000 & 0.010684 \\
\hline & 7014 & -0.053270 & 7014 & 0.023877 & 7014 & 0.002519 \\
\hline & 8016 & -0.653751 & 8016 & 0.256537 & 8016 & 0.027068 \\
\hline \multirow[t]{4}{*}{ Photons } & 1000 & -0.099269 & 1000 & 0.618329 & 1000 & 0.065241 \\
\hline & 6000 & -0.193710 & 6000 & 0.101257 & 6000 & 0.010684 \\
\hline & 7000 & -0.053270 & 7000 & 0.023877 & 7000 & 0.002519 \\
\hline & 8000 & -0.653751 & 8000 & 0.256537 & 8000 & 0.027068 \\
\hline \multirow[t]{5}{*}{ CEPXS Form: } & material & $\mathrm{H}$ & 0.099269 & & & \\
\hline & & $\mathrm{C}$ & 0.193710 & & & \\
\hline & & $\mathrm{N}$ & 0.053270 & & & \\
\hline & & $\mathrm{O}$ & 0.653751 & & & \\
\hline & $\begin{array}{c}\text { matname } \\
\text { density }\end{array}$ & $\begin{array}{c}\text { Eye Lens (IC } \\
1.100000\end{array}$ & & & & \\
\hline
\end{tabular}

\section{Felt}

\begin{tabular}{llll}
\hline Formula $=$ & - & Molecular weight $(\mathrm{g} / \mathrm{mole})=$ & - \\
Density $(\mathrm{g} / \mathrm{cm} 3)=$ & 0.185000 & Total atom density $($ atoms $/ \mathrm{b}-\mathrm{cm})=$ & $1.272 \mathrm{E}-02$ \\
The above density is estimated to be accurate to 3 significant digits. Uncertainties are not addressed. & \\
The following data were calculated from the input weight fractions.
\end{tabular}

\begin{tabular}{|c|c|c|c|c|c|c|}
\hline Element & Neutron ZA & Photon ZA & $\begin{array}{l}\text { Weight } \\
\text { Fraction }\end{array}$ & $\begin{array}{c}\text { Atom } \\
\text { Fraction }\end{array}$ & \multicolumn{2}{|c|}{$\begin{array}{l}\text { Atom } \\
\text { Density }\end{array}$} \\
\hline $\mathrm{H}$ & 1001 & 1000 & 0.044200 & 0.384052 & \multicolumn{2}{|c|}{0.004886} \\
\hline C & 6000 & 6000 & 0.434600 & 0.316901 & \multicolumn{2}{|c|}{0.004031} \\
\hline $\mathrm{N}$ & 7014 & 7000 & 0.176500 & 0.110360 & \multicolumn{2}{|c|}{0.001404} \\
\hline $\mathrm{O}$ & 8016 & 8000 & 0.344700 & 0.188686 & \multicolumn{2}{|c|}{0.002400} \\
\hline Total & & & 1.000000 & 1.000000 & \multicolumn{2}{|c|}{0.012721} \\
\hline MCNP Form & \multicolumn{2}{|c|}{ Weight Fractions } & \multicolumn{2}{|c|}{ Atom Fractions } & \multicolumn{2}{|c|}{ Atom Densities } \\
\hline \multirow[t]{4}{*}{ Neutrons } & 1001 & -0.044200 & 1001 & 0.384052 & 1001 & 0.004886 \\
\hline & 6000 & -0.434600 & 6000 & 0.316901 & 6000 & 0.004031 \\
\hline & 7014 & -0.176500 & 7014 & 0.110360 & 7014 & 0.001404 \\
\hline & 8016 & -0.344700 & 8016 & 0.188686 & 8016 & 0.002400 \\
\hline \multirow[t]{4}{*}{ Photons } & 1000 & -0.044200 & 1000 & 0.384052 & 1000 & 0.004886 \\
\hline & 6000 & -0.434600 & 6000 & 0.316901 & 6000 & 0.004031 \\
\hline & 7000 & -0.176500 & 7000 & 0.110360 & 7000 & 0.001404 \\
\hline & 8000 & -0.344700 & 8000 & 0.188686 & 8000 & 0.002400 \\
\hline
\end{tabular}




\begin{tabular}{|lccc|} 
& & \\
\hline CEPXS Form: & material & $\mathrm{H}$ & 0.044200 \\
& $\mathrm{C}$ & 0.434600 \\
& $\mathrm{~N}$ & 0.176500 \\
& $\mathrm{O}$ & 0.344700 \\
& & \\
& & \\
matname & Felt & \\
density & 0.185000 \\
\hline Comments and References & & \\
Density and weight fractions from pg II.F.1-3 of Carter et al. (1968).
\end{tabular}

\section{Ferric Oxide}

\begin{tabular}{llll}
\hline Formula $=$ & Fe2O3 & Molecular weight $(\mathrm{g} / \mathrm{mole})=$ & 159.6882 \\
Density $(\mathrm{g} / \mathrm{cm} 3)=$ & 5.200000 & Total atom density $($ atoms $/ \mathrm{b}-\mathrm{cm})=$ & $9.805 \mathrm{E}-02$
\end{tabular}

The above density is estimated to be accurate to 3 significant digits. Uncertainties are not addressed.

The following data were calculated from the input weight fractions.

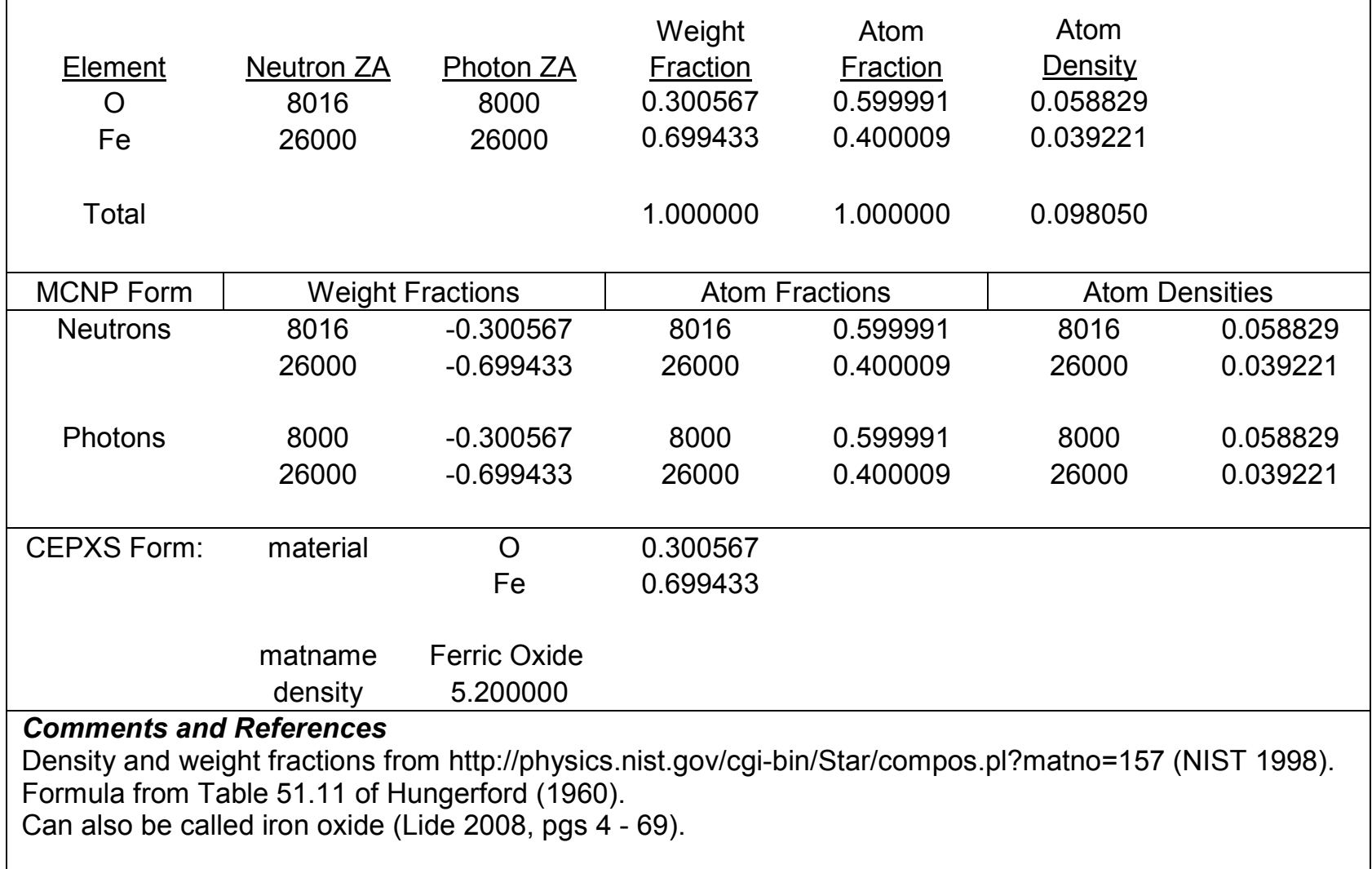




\section{Ferrous Sulfate Dosimeter Solution}

\begin{tabular}{llll}
\hline Formula $=$ & H2O:FeSO4 & Molecular weight $(\mathrm{g} / \mathrm{mole})=$ \\
Density $(\mathrm{g} / \mathrm{cm} 3)=$ & 1.024000 & Total atom density $($ atoms $/ \mathrm{b}-\mathrm{cm})=$ & - \\
\hline
\end{tabular}

The above density is estimated to be accurate to 4 significant digits. Uncertainties are not addressed.

The following data were calculated from the input weight fractions.

\begin{tabular}{|c|c|c|c|c|c|}
\hline Element & Neutron ZA & Photon ZA & $\begin{array}{l}\text { Weight } \\
\text { Fraction }\end{array}$ & $\begin{array}{c}\text { Atom } \\
\text { Fraction }\end{array}$ & $\begin{array}{c}\text { Atom } \\
\text { Density }\end{array}$ \\
\hline$\overline{\mathrm{H}}$ & 1001 & 1000 & 0.108259 & $\overline{0.660018}$ & 0.066234 \\
\hline $\mathrm{N}$ & 7014 & 7000 & 0.000027 & 0.000012 & 0.000001 \\
\hline 0 & 8016 & 8000 & 0.878636 & 0.337467 & 0.033865 \\
\hline $\mathrm{Na}$ & 11023 & 11000 & 0.000022 & 0.000006 & 0.000001 \\
\hline$S$ & 16000 & 16000 & 0.012968 & 0.002485 & 0.000249 \\
\hline $\mathrm{Cl}$ & 17000 & 17000 & 0.000034 & 0.000006 & 0.000001 \\
\hline $\mathrm{Fe}$ & 26000 & 26000 & 0.000054 & 0.000006 & 0.000001 \\
\hline Total & & & 1.000000 & 1.000000 & 0.100352 \\
\hline
\end{tabular}

\begin{tabular}{|c|cc|cc|cr|}
\hline MCNP Form & \multicolumn{2}{c|}{ Weight Fractions } & \multicolumn{2}{c|}{ Atom Fractions } & \multicolumn{2}{c|}{ Atom Densities } \\
\hline Neutrons & 1001 & -0.108259 & 1001 & 0.660018 & 1001 & 0.066234 \\
& 7014 & -0.000027 & 7014 & 0.000012 & 7014 & 0.000001 \\
& 8016 & -0.878636 & 8016 & 0.337467 & 8016 & 0.033865 \\
& 11023 & -0.000022 & 11023 & 0.000006 & 11023 & 0.000001 \\
& 16000 & -0.012968 & 16000 & 0.002485 & 16000 & 0.000249 \\
& 17000 & -0.000034 & 17000 & 0.000006 & 17000 & 0.000001 \\
& 26000 & -0.000054 & 26000 & 0.000006 & 26000 & 0.000001 \\
Photons & & & & & & \\
& 1000 & -0.108259 & 1000 & 0.660018 & 1000 & 0.066234 \\
& 7000 & -0.000027 & 7000 & 0.000012 & 7000 & 0.000001 \\
& 8000 & -0.878636 & 8000 & 0.337467 & 8000 & 0.033865 \\
& 11000 & -0.000022 & 11000 & 0.000006 & 11000 & 0.000001 \\
& 16000 & -0.012968 & 16000 & 0.002485 & 16000 & 0.000249 \\
& 17000 & -0.000034 & 17000 & 0.000006 & 17000 & 0.000001 \\
& 26000 & -0.000054 & 26000 & 0.000006 & 26000 & 0.000001
\end{tabular}

\begin{tabular}{|c|c|c|c|}
\hline \multirow[t]{7}{*}{ CEPXS Form: } & \multirow[t]{7}{*}{ material } & $\mathrm{H}$ & 0.108259 \\
\hline & & $\mathrm{N}$ & 0.000027 \\
\hline & & $\mathrm{O}$ & 0.878636 \\
\hline & & $\mathrm{Na}$ & 0.000022 \\
\hline & & $S$ & 0.012968 \\
\hline & & $\mathrm{Cl}$ & 0.000034 \\
\hline & & $\mathrm{Fe}$ & 0.000054 \\
\hline
\end{tabular}

$\begin{array}{cc}\begin{array}{c}\text { matname } \\ \text { density }\end{array} & \text { Ferrous Sulfate Dosimeter Solution } \\ & 1.024000\end{array}$

Comments and References

Density and weight fractions from http://physics.nist.gov/cgi-bin/Star/compos.pl?matno=160 (NIST 1998). Also called standard Fricke solution. 


\section{Fertilizer (Muriate of Potash)}

\begin{tabular}{|c|c|c|c|}
\hline Formula $=$ & - & Molecular weight $(\mathrm{g} / \mathrm{mole})=$ & - \\
\hline Density $(\mathrm{g} / \mathrm{cm} 3)=$ & 1.270000 & Total atom density $($ atoms $/ \mathrm{b}-\mathrm{cm})=$ & 2.070E-02 \\
\hline
\end{tabular}

\begin{tabular}{|c|c|c|c|c|c|c|}
\hline Element & Neutron ZA & Photon ZA & $\begin{array}{l}\text { Weight } \\
\text { Fraction }\end{array}$ & $\begin{array}{c}\text { Atom } \\
\text { Fraction }\end{array}$ & $\begin{array}{c}\text { Atom } \\
\text { Density }\end{array}$ & \\
\hline $\mathrm{H}$ & 1001 & 1000 & 0.000050 & 0.001849 & 0.000038 & \\
\hline O & 8016 & 8000 & 0.000718 & 0.001658 & 0.000034 & \\
\hline $\mathrm{Na}$ & 11023 & 11000 & 0.008487 & 0.013643 & 0.000282 & \\
\hline $\mathrm{Mg}$ & 12000 & 12000 & 0.000206 & 0.000313 & 0.000006 & \\
\hline$S$ & 16000 & 16000 & 0.000159 & 0.000183 & 0.000004 & \\
\hline $\mathrm{Cl}$ & 17000 & 17000 & 0.477922 & 0.498162 & 0.010310 & \\
\hline $\mathrm{K}$ & 19000 & 19000 & 0.511852 & 0.483786 & 0.010012 & \\
\hline $\mathrm{Ca}$ & 20000 & 20000 & 0.000276 & 0.000254 & 0.000005 & \\
\hline $\mathrm{Br}$ & - & 35000 & 0.000330 & 0.000153 & 0.000003 & \\
\hline Total & & & 1.000000 & 1.000000 & 0.020696 & \\
\hline MCNP Form & \multicolumn{2}{|c|}{ Weight Fractions } & \multicolumn{2}{|c|}{ Atom Fractions } & \multicolumn{2}{|c|}{ Atom Densities } \\
\hline \multirow[t]{9}{*}{ Neutrons } & 1001 & -0.000050 & 1001 & 0.001849 & 1001 & 0.000038 \\
\hline & 8016 & -0.000718 & 8016 & 0.001658 & 8016 & 0.000034 \\
\hline & 11023 & -0.008487 & 11023 & 0.013643 & 11023 & 0.000282 \\
\hline & 12000 & -0.000206 & 12000 & 0.000313 & 12000 & 0.000006 \\
\hline & 16000 & -0.000159 & 16000 & 0.000183 & 16000 & 0.000004 \\
\hline & 17000 & -0.477922 & 17000 & 0.498162 & 17000 & 0.010310 \\
\hline & 19000 & -0.511852 & 19000 & 0.483786 & 19000 & 0.010012 \\
\hline & 20000 & -0.000276 & 20000 & 0.000254 & 20000 & 0.000005 \\
\hline & - & -0.000330 & - & 0.000153 & - & 0.000003 \\
\hline \multirow[t]{9}{*}{ Photons } & 1000 & -0.000050 & 1000 & 0.001849 & 1000 & 0.000038 \\
\hline & 8000 & -0.000718 & 8000 & 0.001658 & 8000 & 0.000034 \\
\hline & 11000 & -0.008487 & 11000 & 0.013643 & 11000 & 0.000282 \\
\hline & 12000 & -0.000206 & 12000 & 0.000313 & 12000 & 0.000006 \\
\hline & 16000 & -0.000159 & 16000 & 0.000183 & 16000 & 0.000004 \\
\hline & 17000 & -0.477922 & 17000 & 0.498162 & 17000 & 0.010310 \\
\hline & 19000 & -0.511852 & 19000 & 0.483786 & 19000 & 0.010012 \\
\hline & 20000 & -0.000276 & 20000 & 0.000254 & 20000 & 0.000005 \\
\hline & 35000 & -0.000330 & 35000 & 0.000153 & 35000 & 0.000003 \\
\hline \multirow[t]{7}{*}{ CEPXS Form: } & material & $\mathrm{H}$ & 0.000050 & & & \\
\hline & & $\mathrm{O}$ & 0.000718 & & & \\
\hline & & $\mathrm{Na}$ & 0.008487 & & & \\
\hline & & $\mathrm{Mg}$ & 0.000206 & & & \\
\hline & & $S$ & 0.000159 & & & \\
\hline & & $\mathrm{Cl}$ & 0.477922 & & & \\
\hline & & $\mathrm{K}$ & 0.511852 & & & \\
\hline
\end{tabular}




\begin{tabular}{|c|c|}
\hline & $\begin{array}{ll}\mathrm{Ca} & 0.000276 \\
\mathrm{Br} & 0.000330\end{array}$ \\
\hline $\begin{array}{c}\text { matname } \\
\text { density }\end{array}$ & $\begin{array}{l}\text { Fertilizer (Muriate of Potash) } \\
1.270000\end{array}$ \\
\hline $\begin{array}{l}\text { Comments and References } \\
\text { Combination of "Evergro" and } \\
\text { Density }=1.27 \mathrm{~g} / \mathrm{cm} 3 \text { is for th } \\
\text { http://www.agrium.com/uploac } \\
\text { http://www.growercentral.com } \\
\text { (Evergro Canada 2001). The } \\
\text { they sum to unity. }\end{array}$ & $\begin{array}{l}\text { Agrium" } \\
\text { average bulk tap density from } \\
\text { Imuriate_potash_blender_coarse_grade_e.pdf (no longer available) and } \\
\text { PLOADS/PDFS/0-0-62\%20muriate } \% 20 \text { of } \% 20 \text { potash } \% 20 \text { fine } \% 20 \text { label.pdf } \\
\text { ose density is } 1.09 \text { to } 1.153 \mathrm{~g} / \mathrm{cm} 3 \text {. Weight fractions are adjusted so that }\end{array}$ \\
\hline
\end{tabular}

\section{Fiberglass, Type $C$}

\begin{tabular}{llll}
\hline Formula $=$ & - & Molecular weight $(\mathrm{g} / \mathrm{mole})=$ & - \\
Density $(\mathrm{g} / \mathrm{cm} 3)=$ & 2.490000 & Total atom density $($ atoms $/ \mathrm{b}-\mathrm{cm})=$ & $7.354 \mathrm{E}-02$
\end{tabular}

The above density is estimated to be accurate to 3 significant digits. Uncertainties are not addressed.

The following data were calculated from the input weight fractions.

\begin{tabular}{|c|c|c|c|c|c|c|}
\hline Element & Neutron ZA & Photon ZA & $\begin{array}{l}\text { Weight } \\
\text { Fraction }\end{array}$ & $\begin{array}{c}\text { Atom } \\
\text { Fraction }\end{array}$ & $\begin{array}{l}\text { Atom } \\
\text { Density }\end{array}$ & \\
\hline$B$ & - & 5000 & $\overline{0.018579}$ & 0.035039 & 0.002577 & \\
\hline $\mathrm{O}$ & 8016 & 8000 & 0.478631 & 0.609968 & 0.044859 & \\
\hline $\mathrm{Na}$ & 11023 & 11000 & 0.059171 & 0.052479 & 0.003859 & \\
\hline $\mathrm{Mg}$ & 12000 & 12000 & 0.018037 & 0.015131 & 0.001113 & \\
\hline $\mathrm{Al}$ & 13027 & 13000 & 0.021107 & 0.015950 & 0.001173 & \\
\hline $\mathrm{Si}$ & 14000 & 14000 & 0.302924 & 0.219918 & 0.016173 & \\
\hline$S$ & 16000 & 16000 & 0.000399 & 0.000254 & 0.000019 & \\
\hline $\mathrm{Ca}$ & 20000 & 20000 & 0.099757 & 0.050751 & 0.003732 & \\
\hline $\mathrm{Fe}$ & 26000 & 26000 & 0.001395 & 0.000509 & 0.000037 & \\
\hline Total & & & 1.000000 & 1.000000 & 0.073543 & \\
\hline MCNP Form & \multicolumn{2}{|c|}{ Weight Fractions } & \multicolumn{2}{|c|}{ Atom Fractions } & \multicolumn{2}{|c|}{ Atom Densities } \\
\hline \multirow[t]{9}{*}{ Neutrons } & - & -0.018579 & - & 0.035039 & - & 0.002577 \\
\hline & 8016 & -0.478631 & 8016 & 0.609968 & 8016 & 0.044859 \\
\hline & 11023 & -0.059171 & 11023 & 0.052479 & 11023 & 0.003859 \\
\hline & 12000 & -0.018037 & 12000 & 0.015131 & 12000 & 0.001113 \\
\hline & 13027 & -0.021107 & 13027 & 0.015950 & 13027 & 0.001173 \\
\hline & 14000 & -0.302924 & 14000 & 0.219918 & 14000 & 0.016173 \\
\hline & 16000 & -0.000399 & 16000 & 0.000254 & 16000 & 0.000019 \\
\hline & 20000 & -0.099757 & 20000 & 0.050751 & 20000 & 0.003732 \\
\hline & 26000 & -0.001395 & 26000 & 0.000509 & 26000 & 0.000037 \\
\hline \multirow[t]{3}{*}{ Photons } & 5000 & -0.018579 & 5000 & 0.035039 & 5000 & 0.002577 \\
\hline & 8000 & -0.478631 & 8000 & 0.609968 & 8000 & 0.044859 \\
\hline & 11000 & -0.059171 & 11000 & 0.052479 & 11000 & 0.003859 \\
\hline
\end{tabular}




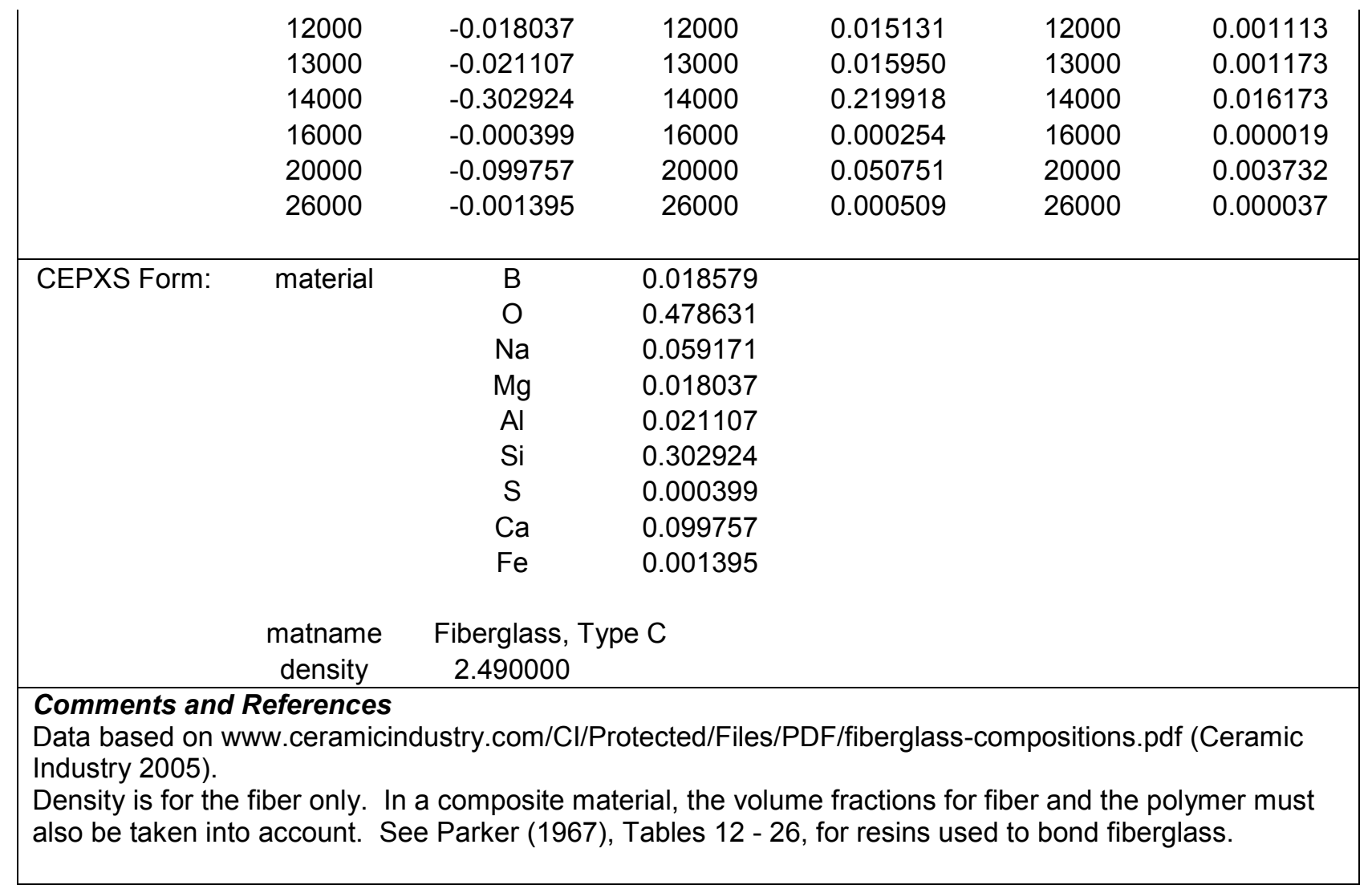

\section{Fiberglass, Type E}

\begin{tabular}{llll}
\hline Formula $=$ & - & Molecular weight $(\mathrm{g} / \mathrm{mole})=$ & - \\
Density $(\mathrm{g} / \mathrm{cm} 3)=$ & 2.565000 & Total atom density $($ atoms $/ \mathrm{b}-\mathrm{cm})=$ & $7.446 \mathrm{E}-02$
\end{tabular}

The above density is estimated to be accurate to 3 significant digits. Uncertainties are not addressed.

The following data were calculated from the input weight fractions.

$\begin{array}{cccccc}\text { Element } & \text { Neutron ZA } & \text { Photon ZA } & \begin{array}{c}\text { Weight } \\ \text { Fraction }\end{array} & \begin{array}{c}\text { Atom } \\ \text { Fraction }\end{array} & \begin{array}{c}\text { Atom } \\ \text { Density }\end{array} \\ \mathrm{B} & - & 5000 & 0.022803 & 0.043757 & 0.003258 \\ \mathrm{O} & 8016 & 8000 & 0.471950 & 0.611965 & 0.045565 \\ \mathrm{~F} & 9019 & 9000 & 0.004895 & 0.005345 & 0.000398 \\ \mathrm{Na} & 11023 & 11000 & 0.007262 & 0.006554 & 0.000488 \\ \mathrm{Mg} & 12000 & 12000 & 0.014759 & 0.012597 & 0.000938 \\ \mathrm{Al} & 13027 & 13000 & 0.072536 & 0.055772 & 0.004153 \\ \mathrm{Si} & 14000 & 14000 & 0.247102 & 0.182528 & 0.013590 \\ \mathrm{~K} & 19000 & 19000 & 0.008127 & 0.004312 & 0.000321 \\ \mathrm{Ca} & 20000 & 20000 & 0.143428 & 0.074244 & 0.005528 \\ \mathrm{Ti} & 22000 & 22000 & 0.004400 & 0.001907 & 0.000142 \\ \mathrm{Fe} & 26000 & 26000 & 0.002739 & 0.001017 & 0.000076 \\ \mathrm{Tin} & & & & & \\ \mathrm{T} & & & 1.000000 & 1.000000 & 0.074457\end{array}$


PIET-43741-TM-963

PNNL-15870 Rev. 1

\begin{tabular}{|c|c|c|c|c|c|c|}
\hline MCNP Form & \multicolumn{2}{|c|}{ Weight Fractions } & \multicolumn{2}{|c|}{ Atom Fractions } & \multicolumn{2}{|c|}{ Atom Densities } \\
\hline \multirow{11}{*}{ Neutrons } & - & -0.022803 & - & 0.043757 & - & 0.003258 \\
\hline & 8016 & -0.471950 & 8016 & 0.611965 & 8016 & 0.045565 \\
\hline & 9019 & -0.004895 & 9019 & 0.005345 & 9019 & 0.000398 \\
\hline & 11023 & -0.007262 & 11023 & 0.006554 & 11023 & 0.000488 \\
\hline & 12000 & -0.014759 & 12000 & 0.012597 & 12000 & 0.000938 \\
\hline & 13027 & -0.072536 & 13027 & 0.055772 & 13027 & 0.004153 \\
\hline & 14000 & -0.247102 & 14000 & 0.182528 & 14000 & 0.013590 \\
\hline & 19000 & -0.008127 & 19000 & 0.004312 & 19000 & 0.000321 \\
\hline & 20000 & -0.143428 & 20000 & 0.074244 & 20000 & 0.005528 \\
\hline & 22000 & -0.004400 & 22000 & 0.001907 & 22000 & 0.000142 \\
\hline & 26000 & -0.002739 & 26000 & 0.001017 & 26000 & 0.000076 \\
\hline \multirow[t]{11}{*}{ Photons } & 5000 & -0.022803 & 5000 & 0.043757 & 5000 & 0.003258 \\
\hline & 8000 & -0.471950 & 8000 & 0.611965 & 8000 & 0.045565 \\
\hline & 9000 & -0.004895 & 9000 & 0.005345 & 9000 & 0.000398 \\
\hline & 11000 & -0.007262 & 11000 & 0.006554 & 11000 & 0.000488 \\
\hline & 12000 & -0.014759 & 12000 & 0.012597 & 12000 & 0.000938 \\
\hline & 13000 & -0.072536 & 13000 & 0.055772 & 13000 & 0.004153 \\
\hline & 14000 & -0.247102 & 14000 & 0.182528 & 14000 & 0.013590 \\
\hline & 19000 & -0.008127 & 19000 & 0.004312 & 19000 & 0.000321 \\
\hline & 20000 & -0.143428 & 20000 & 0.074244 & 20000 & 0.005528 \\
\hline & 22000 & -0.004400 & 22000 & 0.001907 & 22000 & 0.000142 \\
\hline & 26000 & -0.002739 & 26000 & 0.001017 & 26000 & 0.000076 \\
\hline \multirow[t]{12}{*}{ CEPXS Form: } & material & $B$ & 0.022803 & & & \\
\hline & & $\mathrm{O}$ & 0.471950 & & & \\
\hline & & $\mathrm{F}$ & 0.004895 & & & \\
\hline & & $\mathrm{Na}$ & 0.007262 & & & \\
\hline & & $\mathrm{Mg}$ & 0.014759 & & & \\
\hline & & $\mathrm{Al}$ & 0.072536 & & & \\
\hline & & $\mathrm{Si}$ & 0.247102 & & & \\
\hline & & $\mathrm{K}$ & 0.008127 & & & \\
\hline & & $\mathrm{Ca}$ & 0.143428 & & & \\
\hline & & $\mathrm{Ti}$ & 0.004400 & & & \\
\hline & & $\mathrm{Fe}$ & 0.002739 & & & \\
\hline & $\begin{array}{c}\text { matname } \\
\text { density }\end{array}$ & $\begin{array}{c}\text { Fiberglass, } \mathrm{T} \\
2.565000\end{array}$ & & & & \\
\hline \multicolumn{7}{|c|}{$\begin{array}{l}\text { Comments and References } \\
\text { Data based on www.ceramicindustry.com/CI/Protected/Files/PDF/fiberglass-compositions.pdf (Ceramic } \\
\text { Industry 2005). } \\
\text { Density is for the fiber only. In a composite material, the volume fractions for fiber and the polymer must } \\
\text { also be taken into account. See Parker (1967), Tables } 12-26 \text {, for resins used to bond fiberglass. }\end{array}$} \\
\hline
\end{tabular}




\section{Fiberglass, Type $\mathbf{R}$}

\begin{tabular}{llll}
\hline Formula $=$ & - & Molecular weight $(\mathrm{g} / \mathrm{mole})=$ & - \\
Density $(\mathrm{g} / \mathrm{cm} 3)=$ & 2.550000 & Total atom density $($ atoms $/ \mathrm{b}-\mathrm{cm})=$ & $7.433 \mathrm{E}-02$
\end{tabular}

The above density is estimated to be accurate to 3 significant digits. Uncertainties are not addressed.

The following data were calculated from the input weight fractions.

\begin{tabular}{|c|c|c|c|c|c|}
\hline Element & Neutron ZA & Photon ZA & $\begin{array}{l}\text { Weight } \\
\text { Fraction }\end{array}$ & $\begin{array}{c}\text { Atom } \\
\text { Fraction }\end{array}$ & $\begin{array}{c}\text { Atom } \\
\text { Density }\end{array}$ \\
\hline 0 & 8016 & 8000 & $\overline{0.486722}$ & $\overline{0.628478}$ & 0.046716 \\
\hline $\mathrm{Mg}$ & 12000 & 12000 & 0.036182 & 0.030755 & 0.002286 \\
\hline $\mathrm{Al}$ & 13027 & 13000 & 0.132313 & 0.101309 & 0.007531 \\
\hline Si & 14000 & 14000 & 0.280461 & 0.206302 & 0.015335 \\
\hline $\mathrm{Ca}$ & 20000 & 20000 & 0.064322 & 0.033156 & 0.002465 \\
\hline Total & & & 1.000000 & 1.000000 & 0.074332 \\
\hline
\end{tabular}

\begin{tabular}{|c|cc|cc|cr|}
\hline MCNP Form & \multicolumn{2}{c|}{ Weight Fractions } & \multicolumn{2}{c|}{ Atom Fractions } & \multicolumn{2}{c|}{ Atom Densities } \\
\hline Neutrons & 8016 & -0.486722 & 8016 & 0.628478 & 8016 & 0.046716 \\
& 12000 & -0.036182 & 12000 & 0.030755 & 12000 & 0.002286 \\
& 13027 & -0.132313 & 13027 & 0.101309 & 13027 & 0.007531 \\
& 14000 & -0.280461 & 14000 & 0.206302 & 14000 & 0.015335 \\
& 20000 & -0.064322 & 20000 & 0.033156 & 20000 & 0.002465 \\
Photons & & & & & & \\
& 8000 & -0.486722 & 8000 & 0.628478 & 8000 & 0.046716 \\
& 12000 & -0.036182 & 12000 & 0.030755 & 12000 & 0.002286 \\
& 13000 & -0.132313 & 13000 & 0.101309 & 13000 & 0.007531 \\
& 14000 & -0.280461 & 14000 & 0.206302 & 14000 & 0.015335 \\
& 20000 & -0.064322 & 20000 & 0.033156 & 20000 & 0.002465
\end{tabular}

\begin{tabular}{|c|c|c|c|}
\hline \multirow[t]{5}{*}{ CEPXS Form: } & material & $\mathrm{O}$ & 0.486722 \\
\hline & & $\mathrm{Mg}$ & 0.036182 \\
\hline & & $\mathrm{Al}$ & 0.132313 \\
\hline & & $\mathrm{Si}$ & 0.280461 \\
\hline & & $\mathrm{Ca}$ & 0.064322 \\
\hline
\end{tabular}

matname Fiberglass, Type $\mathrm{R}$ density 2.550000

\section{Comments and References}

Data based on www.ceramicindustry.com/Cl/Protected/Files/PDF/fiberglass-compositions.pdf (Ceramic Industry 2005).

Density is for the fiber only. In a composite material, the volume fractions for fiber and the polymer must also be taken into account. See Parker (1967), Tables 12 - 26, for resins used to bond fiberglass. 


\section{Freon-12}

\begin{tabular}{|c|c|c|c|}
\hline ula $=$ & - & Molecular weight $(\mathrm{g} / \mathrm{mole})=$ & \\
\hline$(\mathrm{g} / \mathrm{cm} 3)=$ & 1.120000 & Total atom density $($ atoms $/ \mathrm{b}-\mathrm{cm})=$ & $2.789 \mathrm{E}-02$ \\
\hline
\end{tabular}

The above density is estimated to be accurate to 3 significant digits. Uncertainties are not addressed.

The following data were calculated from the input weight fractions.

\begin{tabular}{|c|c|c|c|c|c|}
\hline Element & Neutron ZA & Photon ZA & $\begin{array}{l}\text { Weight } \\
\text { Fraction }\end{array}$ & $\begin{array}{c}\text { Atom } \\
\text { Fraction }\end{array}$ & $\begin{array}{c}\text { Atom } \\
\text { Density }\end{array}$ \\
\hline $\mathrm{C}$ & 6000 & 6000 & $\overline{0.099335}$ & $\overline{0.200004}$ & 0.005578 \\
\hline$F$ & 9019 & 9000 & 0.314247 & 0.399998 & 0.011156 \\
\hline $\mathrm{Cl}$ & 17000 & 17000 & 0.586418 & 0.399998 & 0.011156 \\
\hline Total & & & 1.000000 & 1.000000 & 0.027891 \\
\hline
\end{tabular}

\begin{tabular}{|ccccccc|}
\hline MCNP Form & \multicolumn{2}{c|}{ Weight Fractions } & \multicolumn{2}{c|}{ Atom Fractions } & \multicolumn{2}{c|}{ Atom Densities } \\
\hline Neutrons & 6000 & -0.099335 & 6000 & 0.200004 & 6000 & 0.005578 \\
& 9019 & -0.314247 & 9019 & 0.399998 & 9019 & 0.011156 \\
& 17000 & -0.586418 & 17000 & 0.399998 & 17000 & 0.011156 \\
Photons & 6000 & -0.099335 & 6000 & 0.200004 & 6000 & 0.005578 \\
& 9000 & -0.314247 & 9000 & 0.399998 & 9000 & 0.011156 \\
& 17000 & -0.586418 & 17000 & 0.399998 & 17000 & 0.011156 \\
& & & & & & \\
\hline CEPXS Form: & material & $\mathrm{C}$ & 0.099335 & & & \\
& & $\mathrm{~F}$ & 0.314247 & & & \\
& & $\mathrm{Cl}$ & 0.586418 & & & \\
\end{tabular}

matname Freon-12

density $\quad 1.120000$

\section{Comments and References}

Density and weight fractions from http://physics.nist.gov/cgi-bin/Star/compos.pl?matno=161 (NIST 1998).

\section{Freon-12B2}

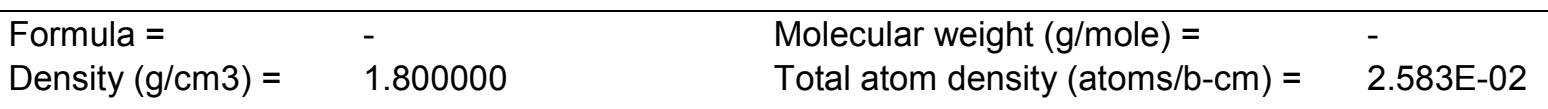

The above density is estimated to be accurate to 3 significant digits. Uncertainties are not addressed.

The following data were calculated from the input weight fractions.

\begin{tabular}{|c|c|c|c|c|c|}
\hline Element & Neutron ZA & Photon ZA & $\begin{array}{l}\text { Weight } \\
\text { Fraction }\end{array}$ & $\begin{array}{c}\text { Atom } \\
\text { Fraction }\end{array}$ & $\begin{array}{c}\text { Atom } \\
\text { Density }\end{array}$ \\
\hline $\mathrm{C}$ & 6000 & 6000 & 0.057245 & 0.200003 & 0.005166 \\
\hline $\mathrm{F}$ & 9019 & 9000 & 0.181096 & 0.399999 & 0.010333 \\
\hline $\mathrm{Br}$ & - & 35000 & 0.761659 & 0.399999 & 0.010333 \\
\hline Total & & & 1.000000 & 1.000000 & 0.025832 \\
\hline
\end{tabular}


PIET-43741-TM-963

PNNL-15870 Rev. 1

\begin{tabular}{|c|c|c|c|c|c|c|}
\hline MCNP Form & \multicolumn{2}{|c|}{ Weight Fractions } & \multicolumn{2}{|c|}{ Atom Fractions } & \multicolumn{2}{|c|}{ Atom Densities } \\
\hline \multirow[t]{3}{*}{ Neutrons } & 6000 & -0.057245 & 6000 & 0.200003 & 6000 & 0.005166 \\
\hline & 9019 & -0.181096 & 9019 & 0.399999 & 9019 & 0.010333 \\
\hline & - & -0.761659 & - & 0.399999 & - & 0.010333 \\
\hline \multirow[t]{3}{*}{ Photons } & 6000 & -0.057245 & 6000 & 0.200003 & 6000 & 0.005166 \\
\hline & 9000 & -0.181096 & 9000 & 0.399999 & 9000 & 0.010333 \\
\hline & 35000 & -0.761659 & 35000 & 0.399999 & 35000 & 0.010333 \\
\hline \multirow[t]{4}{*}{ CEPXS Form: } & material & C & 0.057245 & & & \\
\hline & & $\mathrm{F}$ & 0.181096 & & & \\
\hline & & $\mathrm{Br}$ & 0.761659 & & & \\
\hline & $\begin{array}{c}\text { matname } \\
\text { density }\end{array}$ & $\begin{array}{c}\text { Freon-12B2 } \\
1.800000\end{array}$ & & & & \\
\hline $\begin{array}{l}\text { Comments an } \\
\text { Density and we }\end{array}$ & $\begin{array}{l}\text { References } \\
\text { th fractions }\end{array}$ & ...0. & & & & 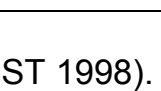 \\
\hline
\end{tabular}

\section{Freon-13}

Formula $=$

Density $(\mathrm{g} / \mathrm{cm} 3)=0.950000$
Molecular weight $(\mathrm{g} / \mathrm{mole})=$

Total atom density $($ atoms $/ \mathrm{b}-\mathrm{cm})=2.738 \mathrm{E}-02$

The above density is estimated to be accurate to 2 significant digits. Uncertainties are not addressed.

The following data were calculated from the input weight fractions.

\begin{tabular}{|c|c|c|c|c|c|c|}
\hline Element & Neutron ZA & Photon ZA & $\begin{array}{l}\text { Weight } \\
\text { Fraction }\end{array}$ & $\begin{array}{c}\text { Atom } \\
\text { Fraction }\end{array}$ & $\begin{array}{c}\text { Atom } \\
\text { Density }\end{array}$ & \\
\hline C & 6000 & 6000 & 0.114983 & 0.200004 & 0.005477 & \\
\hline $\mathrm{F}$ & 9019 & 9000 & 0.545622 & 0.599997 & 0.016430 & \\
\hline $\mathrm{Cl}$ & 17000 & 17000 & 0.339396 & 0.199999 & 0.005477 & \\
\hline Total & & & 1.000001 & 1.000000 & 0.027384 & \\
\hline MCNP Form & \multicolumn{2}{|c|}{ Weight Fractions } & \multicolumn{2}{|c|}{ Atom Fractions } & \multicolumn{2}{|c|}{ Atom Densities } \\
\hline \multirow[t]{3}{*}{ Neutrons } & 6000 & -0.114983 & 6000 & 0.200004 & 6000 & 0.005477 \\
\hline & 9019 & -0.545622 & 9019 & 0.599997 & 9019 & 0.016430 \\
\hline & 17000 & -0.339396 & 17000 & 0.199999 & 17000 & 0.005477 \\
\hline \multirow[t]{3}{*}{ Photons } & 6000 & -0.114983 & 6000 & 0.200004 & 6000 & 0.005477 \\
\hline & 9000 & -0.545622 & 9000 & 0.599997 & 9000 & 0.016430 \\
\hline & 17000 & -0.339396 & 17000 & 0.199999 & 17000 & 0.005477 \\
\hline \multirow[t]{3}{*}{ CEPXS Form: } & material & $\mathrm{C}$ & 0.114983 & & & \\
\hline & & $\mathrm{F}$ & 0.545622 & & & \\
\hline & & $\mathrm{Cl}$ & 0.339396 & & & \\
\hline
\end{tabular}




\begin{tabular}{|ll|}
\multicolumn{1}{|c|}{$\begin{array}{c}\text { matname } \\
\text { density }\end{array}$} & Freon-13 \\
0.950000 \\
\hline Comments and References \\
Density and weight fractions from http://physics.nist.gov/cgi-bin/Star/compos.pl?matno=163 (NIST 1998). \\
\hline
\end{tabular}

\section{Freon-13B1}

\begin{tabular}{|c|c|c|c|}
\hline Formula $=$ & - & Molecular weight $(\mathrm{g} / \mathrm{mole})=$ & \\
\hline Density $(\mathrm{g} / \mathrm{cm} 3)=$ & 1.500000 & Total atom density $($ atoms $/ \mathrm{b}-\mathrm{cm})=$ & 3.033E-02 \\
\hline
\end{tabular}

The above density is estimated to be accurate to 2 significant digits. Uncertainties are not addressed. The following data were calculated from the input weight fractions.

\begin{tabular}{|c|c|c|c|c|c|c|}
\hline Element & Neutron ZA & Photon ZA & $\begin{array}{l}\text { Weight } \\
\text { Fraction }\end{array}$ & $\begin{array}{c}\text { Atom } \\
\text { Fraction }\end{array}$ & $\begin{array}{l}\text { Atom } \\
\text { Density }\end{array}$ & \\
\hline $\mathrm{C}$ & 6000 & 6000 & 0.080659 & $\overline{0.200003}$ & 0.006066 & \\
\hline $\mathrm{F}$ & 9019 & 9000 & 0.382749 & 0.599998 & 0.018199 & \\
\hline $\mathrm{Br}$ & - & 35000 & 0.536592 & 0.199999 & 0.006066 & \\
\hline Total & & & 1.000000 & 1.000000 & 0.030331 & \\
\hline MCNP Form & \multicolumn{2}{|c|}{ Weight Fractions } & \multicolumn{2}{|c|}{ Atom Fractions } & \multicolumn{2}{|c|}{ Atom Densities } \\
\hline \multirow[t]{3}{*}{ Neutrons } & 6000 & -0.080659 & 6000 & 0.200003 & 6000 & 0.006066 \\
\hline & 9019 & -0.382749 & 9019 & 0.599998 & 9019 & 0.018199 \\
\hline & - & -0.536592 & - & 0.199999 & - & 0.006066 \\
\hline \multirow[t]{3}{*}{ Photons } & 6000 & -0.080659 & 6000 & 0.200003 & 6000 & 0.006066 \\
\hline & 9000 & -0.382749 & 9000 & 0.599998 & 9000 & 0.018199 \\
\hline & 35000 & -0.536592 & 35000 & 0.199999 & 35000 & 0.006066 \\
\hline \multirow[t]{4}{*}{ CEPXS Form: } & material & $\mathrm{C}$ & 0.080659 & & & \\
\hline & & $\mathrm{F}$ & 0.382749 & & & \\
\hline & & $\mathrm{Br}$ & 0.536592 & & & \\
\hline & $\begin{array}{l}\text { matname } \\
\text { density }\end{array}$ & $\begin{array}{c}\text { Freon-13B1 } \\
1.500000\end{array}$ & & & & \\
\hline $\begin{array}{l}\text { Comments an } \\
\text { Density and we }\end{array}$ & eferences & & & & & \\
\hline
\end{tabular}

\section{Freon-13|1}

Formula $=$ Molecular weight $(\mathrm{g} / \mathrm{mole})=$

Density $(\mathrm{g} / \mathrm{cm} 3)=\quad 1.800000 \quad$ Total atom density $($ atoms $/ \mathrm{b}-\mathrm{cm})=\quad 2.767 \mathrm{E}-02$
The above density is estimated to be accurate to 2 significant digits. Uncertainties are not addressed.

The following data were calculated from the input weight fractions. 
PIET-43741-TM-963

PNNL-15870 Rev. 1

\begin{tabular}{|c|c|c|c|c|c|c|}
\hline Element & Neutron ZA & Photon ZA & $\begin{array}{l}\text { Weight } \\
\text { Fraction }\end{array}$ & $\begin{array}{c}\text { Atom } \\
\text { Fraction } \\
\end{array}$ & $\begin{array}{c}\text { Atom } \\
\text { Density }\end{array}$ & \\
\hline C & 6000 & 6000 & $\overline{0.061309}$ & 0.200005 & 0.005533 & \\
\hline $\mathrm{F}$ & 9019 & 9000 & 0.290924 & 0.599996 & 0.016599 & \\
\hline I & 53127 & 53000 & 0.647767 & 0.199999 & 0.005533 & \\
\hline Total & & & 1.000000 & 1.000000 & 0.027665 & \\
\hline MCNP Form & \multicolumn{2}{|c|}{ Weight Fractions } & \multicolumn{2}{|c|}{ Atom Fractions } & \multicolumn{2}{|c|}{ Atom Densities } \\
\hline \multirow[t]{3}{*}{ Neutrons } & 6000 & -0.061309 & 6000 & 0.200005 & 6000 & 0.005533 \\
\hline & 9019 & -0.290924 & 9019 & 0.599996 & 9019 & 0.016599 \\
\hline & 53127 & -0.647767 & 53127 & 0.199999 & 53127 & 0.005533 \\
\hline \multirow[t]{3}{*}{ Photons } & 6000 & -0.061309 & 6000 & 0.200005 & 6000 & 0.005533 \\
\hline & 9000 & -0.290924 & 9000 & 0.599996 & 9000 & 0.016599 \\
\hline & 53000 & -0.647767 & 53000 & 0.199999 & 53000 & 0.005533 \\
\hline \multirow[t]{4}{*}{ CEPXS Form: } & material & $\mathrm{C}$ & 0.061309 & & & \\
\hline & & $\mathrm{F}$ & 0.290924 & & & \\
\hline & & I & 0.647767 & & & \\
\hline & $\begin{array}{l}\text { matname } \\
\text { density }\end{array}$ & $\begin{array}{r}\text { Freon-13I1 } \\
1.800000\end{array}$ & & & & \\
\hline $\begin{array}{l}\text { Comments ar } \\
\text { Density and } w\end{array}$ & $\begin{array}{l}\text { Peferences } \\
\text { t fractions fr }\end{array}$ & & & & & \\
\hline
\end{tabular}

\section{Gadolinium}

\begin{tabular}{llll}
\hline Formula $=$ & Gd & Molecular weight $(\mathrm{g} / \mathrm{mole})=$ & 157.25 \\
Density $(\mathrm{g} / \mathrm{cm} 3)=$ & 7.900400 & Total atom density $($ atoms $/ \mathrm{b}-\mathrm{cm})=$ & $3.026 \mathrm{E}-02$
\end{tabular}

The above density is estimated to be accurate to 4 significant digits. Uncertainties are not addressed.

The following data was calculated from the input formula.

\begin{tabular}{|c|c|c|c|c|c|c|}
\hline$\frac{\text { Element }}{\mathrm{Gd}}$ & $\frac{\text { Neutron ZA }}{64000}$ & $\frac{\text { Photon ZA }}{64000}$ & $\begin{array}{c}\text { Weight } \\
\frac{\text { Fraction }}{1.000000}\end{array}$ & $\begin{array}{c}\text { Atom } \\
\frac{\text { Fraction }}{1.000000}\end{array}$ & $\begin{array}{c}\text { Atom } \\
\text { Density } \\
0.030256\end{array}$ & \\
\hline Total & & & 1.000000 & 1.000000 & 0.030256 & \\
\hline MCNP Form & \multicolumn{2}{|c|}{ Weight Fractions } & \multicolumn{2}{|c|}{ Atom Fractions } & \multicolumn{2}{|c|}{ Atom Densities } \\
\hline Neutrons & 64000 & -1.000000 & 64000 & 1.000000 & 64000 & 0.030256 \\
\hline Photons & 64000 & -1.000000 & 64000 & 1.000000 & 64000 & 0.030256 \\
\hline CEPXS Form: & $\begin{array}{l}\text { material } \\
\text { matname } \\
\text { density }\end{array}$ & $\begin{array}{c}\mathrm{Gd} \\
\text { Gadolinium } \\
7.900400\end{array}$ & 1.000000 & & & \\
\hline
\end{tabular}


Comments and References

Density from http://physics.nist.gov/cgi-bin/Star/compos.pl?matno=064 (NIST 1998).

\section{Gadolinium Oxysulfide}

\begin{tabular}{|c|c|c|c|}
\hline Formula $=$ & $\mathrm{Gd} 2 \mathrm{O} 2 \mathrm{~S}$ & Molecular weight $(\mathrm{g} / \mathrm{mole})=$ & 378.5638 \\
\hline Density $(\mathrm{g} / \mathrm{cm} 3)=$ & 7.440000 & Total atom density $($ atoms $/ \mathrm{b}-\mathrm{cm})=$ & $5.918 \mathrm{E}-02$ \\
\hline
\end{tabular}

\begin{tabular}{|c|c|c|c|c|c|}
\hline Element & Neutron ZA & Photon ZA & $\begin{array}{l}\text { Weight } \\
\text { Fraction }\end{array}$ & $\begin{array}{c}\text { Atom } \\
\text { Fraction }\end{array}$ & $\begin{array}{c}\text { Atom } \\
\text { Density }\end{array}$ \\
\hline 0 & 8016 & 8000 & 0.084528 & 0.400012 & 0.023671 \\
\hline$S$ & 16000 & 16000 & 0.084690 & 0.199976 & 0.011834 \\
\hline $\mathrm{Gd}$ & 64000 & 64000 & 0.830782 & 0.400012 & 0.023671 \\
\hline Total & & & 1.000000 & 1.000000 & 0.059176 \\
\hline
\end{tabular}

\begin{tabular}{|c|cccccc|}
\hline MCNP Form & \multicolumn{2}{c}{ Weight Fractions } & \multicolumn{2}{c|}{ Atom Fractions } & \multicolumn{2}{c|}{ Atom Densities } \\
\hline Neutrons & 8016 & -0.084528 & 8016 & 0.400012 & 8016 & 0.023671 \\
& 16000 & -0.084690 & 16000 & 0.199976 & 16000 & 0.011834 \\
& 64000 & -0.830782 & 64000 & 0.400012 & 64000 & 0.023671 \\
Photons & 8000 & -0.084528 & 8000 & 0.400012 & 8000 & 0.023671 \\
& 16000 & -0.084690 & 16000 & 0.199976 & 16000 & 0.011834 \\
& 64000 & -0.830782 & 64000 & 0.400012 & 64000 & 0.023671 \\
& & & & & & \\
CEPXS Form: & material & $\mathrm{O}$ & 0.084528 & & \\
& & $\mathrm{~S}$ & 0.084690 & & & \\
& & $\mathrm{Gd}$ & 0.830782 & & &
\end{tabular}

matname Gadolinium Oxysulfide density $\quad 7.440000$

\section{Comments and References}

Density and weight fractions from http://physics.nist.gov/cgi-bin/Star/compos.pl?matno=166 (NIST 1998). Formula from Table 1A of Greskovich and Duclos (1997).

Also called gadolinium sulfoxylate or GOS (http://en.wikipedia.org/wiki/Gadolinium_oxysulfide).

\section{Gadolinium Silicate (GSO)}

\begin{tabular}{llll}
\hline Formula $=$ & Gd2SiO5 & Molecular weight $(\mathrm{g} / \mathrm{mole})=$ & 422.5825 \\
Density $(\mathrm{g} / \mathrm{cm} 3)=$ & 6.710000 & Total atom density $($ atoms $/ \mathrm{b}-\mathrm{cm})=$ & $7.650 \mathrm{E}-02$
\end{tabular}

The above density is estimated to be accurate to 3 significant digits. Uncertainties are not addressed.

The following data was calculated from the input formula. 


\begin{tabular}{|c|c|c|c|c|c|c|}
\hline Element & Neutron ZA & $\underline{\text { Photon ZA }}$ & $\begin{array}{l}\text { Weight } \\
\text { Fraction }\end{array}$ & $\begin{array}{c}\text { Atom } \\
\text { Fraction }\end{array}$ & $\begin{array}{l}\text { Atom } \\
\text { Density }\end{array}$ & \\
\hline 0 & 8016 & 8000 & 0.189305 & 0.625000 & 0.047811 & \\
\hline $\mathrm{Si}$ & 14000 & 14000 & 0.066462 & 0.125000 & 0.009562 & \\
\hline $\mathrm{Gd}$ & 64000 & 64000 & 0.744233 & 0.250000 & 0.019125 & \\
\hline Total & & & 1.000000 & 1.000000 & 0.076498 & \\
\hline MCNP Form & \multicolumn{2}{|c|}{ Weight Fractions } & \multicolumn{2}{|c|}{ Atom Fractions } & \multicolumn{2}{|c|}{ Atom Densities } \\
\hline \multirow[t]{3}{*}{ Neutrons } & 8016 & -0.189305 & 8016 & 0.625000 & 8016 & 0.047811 \\
\hline & 14000 & -0.066462 & 14000 & 0.125000 & 14000 & 0.009562 \\
\hline & 64000 & -0.744233 & 64000 & 0.250000 & 64000 & 0.019125 \\
\hline \multirow[t]{3}{*}{ Photons } & 8000 & -0.189305 & 8000 & 0.625000 & 8000 & 0.047811 \\
\hline & 14000 & -0.066462 & 14000 & 0.125000 & 14000 & 0.009562 \\
\hline & 64000 & -0.744233 & 64000 & 0.250000 & 64000 & 0.019125 \\
\hline \multirow[t]{4}{*}{ CEPXS Form: } & material & $\mathrm{O}$ & 0.189305 & & & \\
\hline & & $\mathrm{Si}$ & 0.066462 & & & \\
\hline & & $\mathrm{Gd}$ & 0.744233 & & & \\
\hline & $\begin{array}{l}\text { matname } \\
\text { density }\end{array}$ & \multicolumn{2}{|c|}{$\begin{array}{l}\text { Gadolinium Silicate (GSO) } \\
\quad 6.710000\end{array}$} & & & \\
\hline \multicolumn{7}{|c|}{$\begin{array}{l}\text { Comments and References } \\
\text { Density }=6.71 \mathrm{~g} / \mathrm{cm} 3 \text { for GSO from pg } 235 \text { of Knoll (2000). Formula from Tanaka et al. (1998) at } \\
\text { http://citeseerx.ist.psu.edu/viewdoc/summary?doi=10.1.1.30.4620. The same formula and density are } \\
\text { given at http://www.apace-science.com/misc/crystalj.htm (APACE 2009). }\end{array}$} \\
\hline
\end{tabular}

\section{Gafchromic Sensor (GS)}

\begin{tabular}{llll}
\hline Formula $=$ & - & Molecular weight $(\mathrm{g} / \mathrm{mole})=$ \\
Density $(\mathrm{g} / \mathrm{cm} 3)=$ & 1.300000 & Total atom density $($ atoms $/ \mathrm{b}-\mathrm{cm})=$ & $\overline{1.248 \mathrm{E}-01}$
\end{tabular}

The above density is estimated to be accurate to 2 significant digits. Uncertainties are not addressed.

The following data were calculated from the input weight fractions.

\begin{tabular}{|c|c|c|c|c|c|c|}
\hline Element & Neutron ZA & Photon ZA & $\begin{array}{l}\text { Weight } \\
\text { Fraction }\end{array}$ & $\begin{array}{l}\text { Atom } \\
\text { Fraction }\end{array}$ & $\begin{array}{l}\text { Atom } \\
\text { Density }\end{array}$ & \\
\hline $\mathrm{H}$ & 1001 & 1000 & 0.089700 & 0.558087 & 0.069671 & \\
\hline $\mathrm{C}$ & 6000 & 6000 & 0.605800 & 0.316304 & 0.039487 & \\
\hline $\mathrm{N}$ & 7014 & 7000 & 0.112200 & 0.050234 & 0.006271 & \\
\hline $\mathrm{O}$ & 8016 & 8000 & 0.192300 & 0.075374 & 0.009410 & \\
\hline Total & & & 1.000000 & 1.000000 & 0.124839 & \\
\hline MCNP Form & \multicolumn{2}{|c|}{ Weight Fractions } & \multicolumn{2}{|c|}{ Atom Fractions } & \multicolumn{2}{|c|}{ Atom Densities } \\
\hline Neutrons & 1001 & -0.089700 & 1001 & 0.558087 & 1001 & 0.069671 \\
\hline & 6000 & -0.605800 & 6000 & 0.316304 & 6000 & 0.039487 \\
\hline
\end{tabular}


PIET-43741-TM-963

PNNL-15870 Rev. 1

\begin{tabular}{|c|c|c|c|c|c|c|}
\hline & 7014 & -0.112200 & 7014 & 0.050234 & 7014 & 0.006271 \\
\hline & 8016 & -0.192300 & 8016 & 0.075374 & 8016 & 0.009410 \\
\hline Photons & 1000 & -0.089700 & 1000 & 0.558087 & 1000 & 0.069671 \\
\hline & 6000 & -0.605800 & 6000 & 0.316304 & 6000 & 0.039487 \\
\hline & 7000 & -0.112200 & 7000 & 0.050234 & 7000 & 0.006271 \\
\hline & 8000 & -0.192300 & 8000 & 0.075374 & 8000 & 0.009410 \\
\hline CEPXS Form: & material & $\mathrm{H}$ & 0.089700 & & & \\
\hline & & C & 0.605800 & & & \\
\hline & & $\mathrm{N}$ & 0.112200 & & & \\
\hline & & $\mathrm{O}$ & 0.192300 & & & \\
\hline & $\begin{array}{c}\text { matname } \\
\text { density }\end{array}$ & $\begin{array}{c}\text { Gafchromic } \\
1.300000\end{array}$ & sor (GS) & & & \\
\hline $\begin{array}{l}\text { Comments an } \\
\text { http://physics.n }\end{array}$ & eferences & 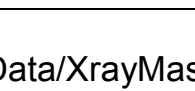 & 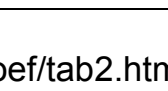 & TT 1996). & & \\
\hline
\end{tabular}

\section{Gallium Arsenide}

\begin{tabular}{llll}
\hline Formula $=$ & GaAs & Molecular weight $(\mathrm{g} / \mathrm{mole})=$ & 144.6446 \\
Density $(\mathrm{g} / \mathrm{cm} 3)=$ & 5.310000 & Total atom density $($ atoms $/ \mathrm{b}-\mathrm{cm})=$ & $4.422 \mathrm{E}-02$
\end{tabular}

The above density is estimated to be accurate to 3 significant digits. Uncertainties are not addressed.

The following data were calculated from the input weight fractions.

\begin{tabular}{|c|c|c|c|c|c|c|}
\hline Element & Neutron ZA & Photon ZA & $\begin{array}{l}\text { Weight } \\
\text { Fraction }\end{array}$ & $\begin{array}{c}\text { Atom } \\
\text { Fraction }\end{array}$ & $\begin{array}{c}\text { Atom } \\
\text { Density }\end{array}$ & \\
\hline $\mathrm{Ga}$ & 31000 & 31000 & 0.482030 & 0.500000 & $0.02210 \varepsilon$ & \\
\hline As & 33075 & 33000 & 0.517970 & 0.500000 & $0.02210 \varepsilon$ & \\
\hline Total & & & 1.000000 & 1.000000 & 0.044215 & \\
\hline MCNP Form & \multicolumn{2}{|c|}{ Weight Fractions } & \multicolumn{2}{|c|}{ Atom Fractions } & \multicolumn{2}{|c|}{ Atom Densities } \\
\hline \multirow[t]{2}{*}{ Neutrons } & 31000 & -0.482030 & 31000 & 0.500000 & 31000 & 0.022108 \\
\hline & 33075 & -0.517970 & 33075 & 0.500000 & 33075 & 0.022108 \\
\hline \multirow[t]{2}{*}{ Photons } & 31000 & -0.482030 & 31000 & 0.500000 & 31000 & 0.022108 \\
\hline & 33000 & -0.517970 & 33000 & 0.500000 & 33000 & 0.022108 \\
\hline \multirow[t]{3}{*}{ CEPXS Form: } & material & Ga & 0.482030 & & & \\
\hline & & As & 0.517970 & & & \\
\hline & $\begin{array}{c}\text { matname } \\
\text { density }\end{array}$ & \multicolumn{3}{|c|}{$\begin{array}{l}\text { Gallium Arsenide } \\
5.310000\end{array}$} & & \\
\hline
\end{tabular}




\section{Gasoline}

\begin{tabular}{llll}
\hline Formula $=$ & $\mathrm{C} 8 \mathrm{H} 18$ & Molecular weight $(\mathrm{g} / \mathrm{mole})=$ & 114.22852 \\
Density $(\mathrm{g} / \mathrm{cm} 3)=$ & 0.721000 & Total atom density $($ atoms $/ \mathrm{b}-\mathrm{cm})=$ & $9.811 \mathrm{E}-02$
\end{tabular}

The above density is estimated to be accurate to 2 significant digits. Uncertainties are not addressed. The following data were calculated from the input weight fractions.

$\begin{array}{ccccccc}\frac{\text { Element }}{\mathrm{H}} & \frac{\text { Neutron ZA }}{1001} & \frac{\text { Photon ZA }}{1000} & \frac{\begin{array}{c}\text { Weight } \\ \text { Fraction }\end{array}}{0.157000} & \begin{array}{c}\text { Atom } \\ \text { Fraction }\end{array} & \begin{array}{c}\text { Atom } \\ \text { Density }\end{array} \\ \mathrm{C} & 6000 & 6000 & 0.843000 & 0.3106368 & 0.067632 \\ \text { Total } & & & & & & 0.030475 \\ & & & 1.000000 & 1.000000 & 0.098107\end{array}$

\begin{tabular}{|c|cccccc|}
\hline MCNP Form & \multicolumn{2}{c|}{ Weight Fractions } & \multicolumn{2}{c|}{ Atom Fractions } & \multicolumn{2}{c|}{ Atom Densities } \\
\hline Neutrons & 1001 & -0.157000 & 1001 & 0.689368 & 1001 & 0.067632 \\
& 6000 & -0.843000 & 6000 & 0.310632 & 6000 & 0.030475 \\
Photons & 1000 & -0.157000 & 1000 & 0.689368 & 1000 & 0.067632 \\
& 6000 & -0.843000 & 6000 & 0.310632 & 6000 & 0.030475 \\
& & & & & & \\
\hline CEPXS Form: & material & $\mathrm{H}$ & 0.157000 & & \\
& & $\mathrm{C}$ & 0.843000 & &
\end{tabular}

$\begin{array}{cc}\text { matname } & \text { Gasoline } \\ \text { density } & 0.721000\end{array}$

\section{Comments and References}

Gasoline is sometimes called petrol. It is a mixture consisting almost exclusively of hydrocarbons (compounds with only $\mathrm{C}$ and $\mathrm{H}$ ) in four series: paraffins $(\mathrm{CnH} 2 \mathrm{n}+2)$, olefins $(\mathrm{CnH} 2 n)$, cycloparaffins $(\mathrm{CnH} 2 \mathrm{n})$, and aromatics $(\mathrm{CnH} 2 \mathrm{n}-6)$. There are probably several hundred such compounds in any one gasoline. The paraffins in gasoline have 4 to 12 carbon atoms (Guthrie 1960) with an average of C8H18 (Table 51.101 of Hungerford 1960).

Density $=0.721 \mathrm{~g} / \mathrm{cm} 3$ from Table 6.1 .5 of Avallone and Baumeister III (1996). Density $=0.737 \mathrm{~g} / \mathrm{cm} 3$ at http://www.simetric.co.uk/si_liquids.htm (Walker 2009), and http://www.engineeringtoolbox.com/liquidsdensities-d_743.html (Engineering Toolbox n.d.). Density $=0.70$ to $0.77 \mathrm{~g} / \mathrm{cm} 3$ in Table 7.4 of Speight (2001). Density $=0.70$ to 0.74 in Table 51.102 of Hungerford (1960).

Weight fractions from Table 7.1.8 of Avallone and Baumeister III (1996).

\section{Germanium, High Purity}

\begin{tabular}{llll}
\hline Formula $=$ & Ge & Molecular weight $(\mathrm{g} / \mathrm{mole})=$ & 72.64 \\
Density $(\mathrm{g} / \mathrm{cm} 3)=$ & 5.323000 & Total atom density $($ atoms $/ \mathrm{b}-\mathrm{cm})=$ & $4.413 \mathrm{E}-02$
\end{tabular}

The above density is estimated to be accurate to 4 significant digits. Uncertainties are not addressed.

The following data was calculated from the input formula.

\begin{tabular}{|c|c|c|c|c|c|}
\hline lement & Neutron ZA & Photon ZA & $\begin{array}{l}\text { Weight } \\
\text { Fraction }\end{array}$ & $\begin{array}{c}\text { Atom } \\
\text { Fraction }\end{array}$ & $\begin{array}{c}\text { Atom } \\
\text { Density }\end{array}$ \\
\hline $\mathrm{Ge}$ & - & 32000 & $\overline{1.000000}$ & $\overline{1.000000}$ & 0.044130 \\
\hline
\end{tabular}


PIET-43741-TM-963

PNNL-15870 Rev. 1

\begin{tabular}{|c|c|c|c|c|c|c|}
\hline Total & & & 1.000000 & 1.000000 & \multicolumn{2}{|c|}{0.044130} \\
\hline MCNP Form & \multicolumn{2}{|c|}{ Weight Fractions } & \multicolumn{2}{|c|}{ Atom Fractions } & \multicolumn{2}{|c|}{ Atom Densities } \\
\hline Neutrons & - & -1.000000 & - & 1.000000 & - & 0.044130 \\
\hline Photons & 32000 & -1.000000 & 32000 & 1.000000 & 32000 & 0.044130 \\
\hline CEPXS Form: & $\begin{array}{l}\text { material } \\
\text { matname } \\
\text { density }\end{array}$ & \multicolumn{3}{|c|}{$\begin{array}{l}\text { Germanium, High Purity } \\
5.323000\end{array}$} & & \\
\hline $\begin{array}{l}\text { Comments an } \\
\text { Density from ht }\end{array}$ & $\begin{array}{l}\text { eferences } \\
\text { /physics.ni }\end{array}$ & $\mathrm{V} / \mathrm{cgi}$-bin/S & -5 & חמרח & & \\
\hline
\end{tabular}

\section{Glass Scintillator, Li Doped (GS1, GS2, GS3)}

\begin{tabular}{|c|c|c|c|}
\hline Formula $=$ & - & Molecular weight $(\mathrm{g} / \mathrm{mole})=$ & - \\
\hline
\end{tabular}

The above density is estimated to be accurate to 3 significant digits. Uncertainties are not addressed.

The following data were calculated from the input weight fractions.

\begin{tabular}{|c|c|c|c|c|c|c|}
\hline Element & Neutron ZA & Photon ZA & $\begin{array}{l}\text { Weight } \\
\text { Fraction }\end{array}$ & $\begin{array}{c}\text { Atom } \\
\text { Fraction }\end{array}$ & $\begin{array}{l}\text { Atom } \\
\text { Density }\end{array}$ & \\
\hline$\overline{\mathrm{Li}}$ & - & 3000 & $\overline{0.027874}$ & 0.078133 & 0.006433 & \\
\hline $\mathrm{O}$ & 8016 & 8000 & 0.477940 & 0.581195 & 0.047852 & \\
\hline $\mathrm{Mg}$ & 12000 & 12000 & 0.144729 & 0.115854 & 0.009539 & \\
\hline $\mathrm{Al}$ & 13027 & 13000 & 0.058218 & 0.041980 & 0.003456 & \\
\hline $\mathrm{Si}$ & 14000 & 14000 & 0.257089 & 0.178096 & 0.014663 & \\
\hline $\mathrm{Ce}$ & - & 58000 & 0.034151 & 0.004742 & 0.000390 & \\
\hline Total & & & 1.000000 & 1.000000 & 0.082334 & \\
\hline MCNP Form & \multicolumn{2}{|c|}{ Weight Fractions } & \multicolumn{2}{|c|}{ Atom Fractions } & \multicolumn{2}{|c|}{ Atom Densities } \\
\hline \multirow[t]{6}{*}{ Neutrons } & - & -0.027874 & - & 0.078133 & - & 0.006433 \\
\hline & 8016 & -0.477940 & 8016 & 0.581195 & 8016 & 0.047852 \\
\hline & 12000 & -0.144729 & 12000 & 0.115854 & 12000 & 0.009539 \\
\hline & 13027 & -0.058218 & 13027 & 0.041980 & 13027 & 0.003456 \\
\hline & 14000 & -0.257089 & 14000 & 0.178096 & 14000 & 0.014663 \\
\hline & - & -0.034151 & - & 0.004742 & - & 0.000390 \\
\hline \multirow[t]{6}{*}{ Photons } & 3000 & -0.027874 & 3000 & 0.078133 & 3000 & 0.006433 \\
\hline & 8000 & -0.477940 & 8000 & 0.581195 & 8000 & 0.047852 \\
\hline & 12000 & -0.144729 & 12000 & 0.115854 & 12000 & 0.009539 \\
\hline & 13000 & -0.058218 & 13000 & 0.041980 & 13000 & 0.003456 \\
\hline & 14000 & -0.257089 & 14000 & 0.178096 & 14000 & 0.014663 \\
\hline & 58000 & -0.034151 & 58000 & 0.004742 & 58000 & 0.000390 \\
\hline
\end{tabular}




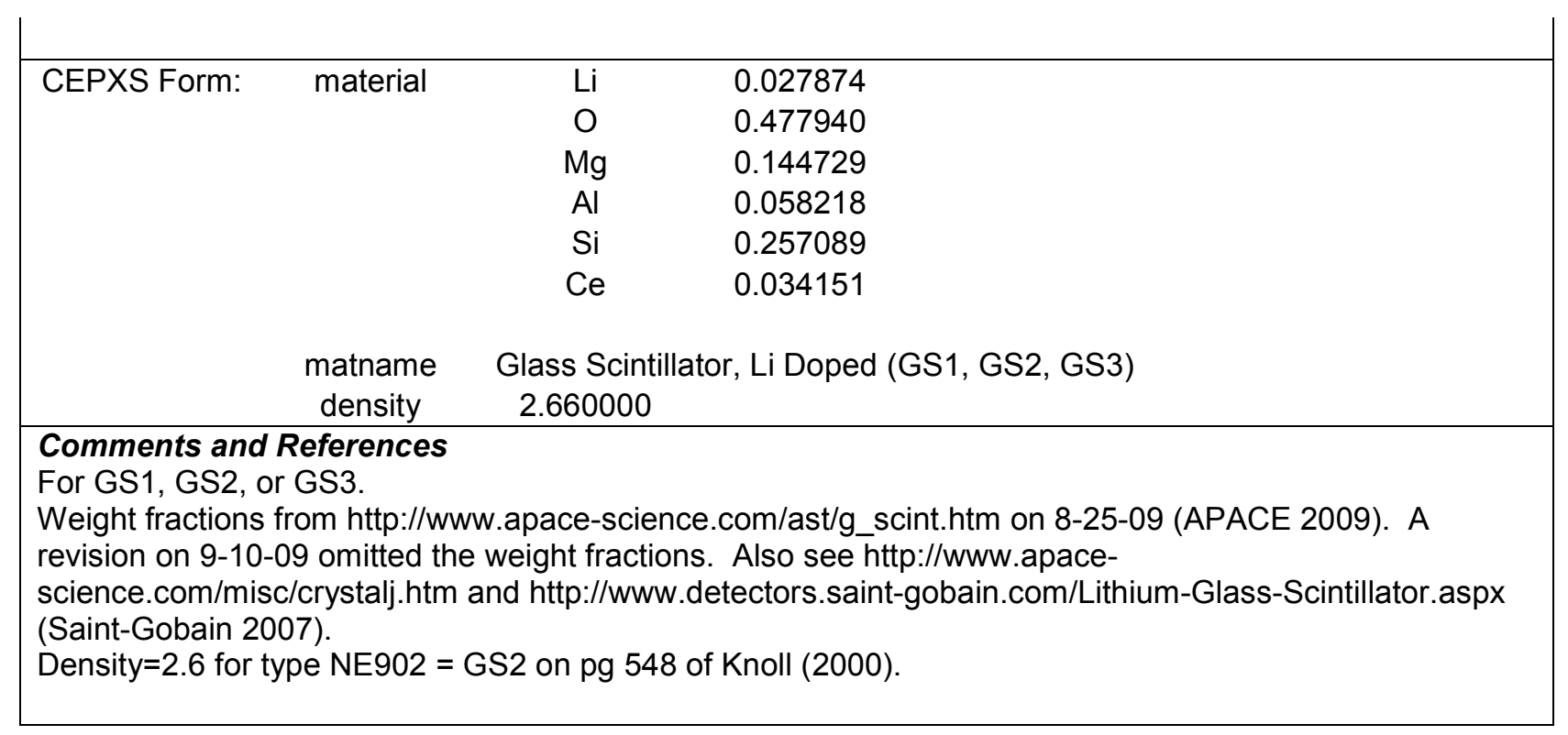

\section{Glass Scintillator, Li Doped (GS10, GS20, GS30)}

\begin{tabular}{llll}
\hline Formula $=$ & - & Molecular weight $(\mathrm{g} / \mathrm{mole})=$ \\
Density $(\mathrm{g} / \mathrm{cm} 3)=$ & 2.500000 & Total atom density $($ atoms $/ \mathrm{b}-\mathrm{cm})=$ & - \\
\hline
\end{tabular}

The above density is estimated to be accurate to 3 significant digits. Uncertainties are not addressed. The following data were calculated from the input weight fractions.

\begin{tabular}{|c|c|c|c|c|c|c|}
\hline Element & Neutron ZA & Photon ZA & $\begin{array}{l}\text { Weight } \\
\text { Fraction }\end{array}$ & $\begin{array}{c}\text { Atom } \\
\text { Fraction }\end{array}$ & $\begin{array}{l}\text { Atom } \\
\text { Density }\end{array}$ & \\
\hline $\mathrm{Li}$ & - & 3000 & 0.083623 & 0.209694 & 0.018138 & \\
\hline 0 & 8016 & 8000 & 0.501077 & 0.545112 & 0.047151 & \\
\hline $\mathrm{Mg}$ & 12000 & 12000 & 0.024121 & 0.017274 & 0.001494 & \\
\hline $\mathrm{Al}$ & 13027 & 13000 & 0.095265 & 0.061454 & 0.005316 & \\
\hline $\mathrm{Si}$ & 14000 & 14000 & 0.261764 & 0.162223 & 0.014032 & \\
\hline $\mathrm{Ce}$ & - & 58000 & 0.034151 & 0.004242 & 0.000367 & \\
\hline Total & & & 1.000000 & 1.000000 & 0.086498 & \\
\hline MCNP Form & \multicolumn{2}{|c|}{ Weight Fractions } & \multicolumn{2}{|c|}{ Atom Fractions } & \multicolumn{2}{|c|}{ Atom Densities } \\
\hline \multirow[t]{6}{*}{ Neutrons } & - & -0.083623 & - & 0.209694 & - & 0.018138 \\
\hline & 8016 & -0.501077 & 8016 & 0.545112 & 8016 & 0.047151 \\
\hline & 12000 & -0.024121 & 12000 & 0.017274 & 12000 & 0.001494 \\
\hline & 13027 & -0.095265 & 13027 & 0.061454 & 13027 & 0.005316 \\
\hline & 14000 & -0.261764 & 14000 & 0.162223 & 14000 & 0.014032 \\
\hline & - & -0.034151 & - & 0.004242 & - & 0.000367 \\
\hline \multirow[t]{4}{*}{ Photons } & 3000 & -0.083623 & 3000 & 0.209694 & 3000 & 0.018138 \\
\hline & 8000 & -0.501077 & 8000 & 0.545112 & 8000 & 0.047151 \\
\hline & 12000 & -0.024121 & 12000 & 0.017274 & 12000 & 0.001494 \\
\hline & 13000 & -0.095265 & 13000 & 0.061454 & 13000 & 0.005316 \\
\hline
\end{tabular}


PIET-43741-TM-963

PNNL-15870 Rev. 1

\begin{tabular}{|c|c|c|c|c|c|c|}
\hline & $\begin{array}{l}14000 \\
58000\end{array}$ & $\begin{array}{l}-0.261764 \\
-0.034151\end{array}$ & $\begin{array}{l}14000 \\
58000\end{array}$ & $\begin{array}{l}0.162223 \\
0.004242\end{array}$ & $\begin{array}{l}14000 \\
58000\end{array}$ & $\begin{array}{l}0.014032 \\
0.000367\end{array}$ \\
\hline CEPXS Form: & material & $\begin{array}{c}\mathrm{Li} \\
\mathrm{O} \\
\mathrm{Mg} \\
\mathrm{Al} \\
\mathrm{Si} \\
\mathrm{Ce}\end{array}$ & $\begin{array}{l}0.083623 \\
0.501077 \\
0.024121 \\
0.095265 \\
0.261764 \\
0.034151\end{array}$ & . & & \\
\hline & $\begin{array}{l}\text { matname } \\
\text { density }\end{array}$ & \multicolumn{5}{|c|}{$\begin{array}{l}\text { Glass Scintillator, Li Doped (GS10, GS20, GS30) } \\
2.500000\end{array}$} \\
\hline \multicolumn{7}{|c|}{$\begin{array}{l}\text { Comments and References } \\
\text { For GS10, GS20, or GS30. } \\
\text { Weight fractions from http://www.apace-science.com/ast/g_scint.htm on 8-25-09 (APACE 2009). A } \\
\text { revision on 9-10-09 omitted the weight fractions. Also see http://www.apace- } \\
\text { science.com/misc/crystalj.htm and http://www.detectors.saint-gobain.com/Lithium-Glass-Scintillator.aspx } \\
\text { (Saint-Gobain 2007). } \\
\text { Density=2.48 for type NE905 = GS20 on pg } 548 \text { of Knoll (2000). }\end{array}$} \\
\hline
\end{tabular}

\section{Glass Scintillator, Li Doped (GSF1)}

Formula $=$

Density $(\mathrm{g} / \mathrm{cm} 3)=2.420000$

The above density is estimated to be accurate to 3 significant digits. Uncertainties are not addressed.

The following data were calculated from the input weight fractions.

\begin{tabular}{|c|c|c|c|c|c|c|}
\hline Element & Neutron ZA & Photon ZA & $\begin{array}{l}\text { Weight } \\
\text { Fraction }\end{array}$ & $\begin{array}{c}\text { Atom } \\
\text { Fraction }\end{array}$ & $\begin{array}{c}\text { Atom } \\
\text { Density }\end{array}$ & \\
\hline $\mathrm{Li}$ & - & 3000 & 0.046550 & 0.124299 & 0.009774 & \\
\hline $\mathrm{O}$ & 8016 & 8000 & 0.505813 & 0.585945 & 0.046074 & \\
\hline $\mathrm{Na}$ & 11023 & 11000 & 0.017840 & 0.014383 & 0.001131 & \\
\hline $\mathrm{Al}$ & 13027 & 13000 & 0.095456 & 0.065570 & 0.005156 & \\
\hline $\mathrm{Si}$ & 14000 & 14000 & 0.313809 & 0.207087 & 0.016284 & \\
\hline $\mathrm{Ce}$ & - & 58000 & 0.020531 & 0.002716 & 0.000214 & \\
\hline Total & & & 1.000000 & 1.000000 & 0.078631 & \\
\hline MCNP Form & \multicolumn{2}{|c|}{ Weight Fractions } & \multicolumn{2}{|c|}{ Atom Fractions } & \multicolumn{2}{|c|}{ Atom Densities } \\
\hline \multirow[t]{6}{*}{ Neutrons } & - & -0.046550 & - & 0.124299 & - & 0.009774 \\
\hline & 8016 & -0.505813 & 8016 & 0.585945 & 8016 & 0.046074 \\
\hline & 11023 & -0.017840 & 11023 & 0.014383 & 11023 & 0.001131 \\
\hline & 13027 & -0.095456 & 13027 & 0.065570 & 13027 & 0.005156 \\
\hline & 14000 & -0.313809 & 14000 & 0.207087 & 14000 & 0.016284 \\
\hline & - & -0.020531 & - & 0.002716 & - & 0.000214 \\
\hline \multirow[t]{2}{*}{ Photons } & 3000 & -0.046550 & 3000 & 0.124299 & 3000 & 0.009774 \\
\hline & 8000 & -0.505813 & 8000 & 0.585945 & 8000 & 0.046074 \\
\hline
\end{tabular}


PIET-43741-TM-963

PNNL-15870 Rev. 1

\begin{tabular}{|c|c|c|c|c|c|c|}
\hline & 11000 & -0.017840 & 11000 & 0.014383 & 11000 & 0.001131 \\
\hline & 13000 & -0.095456 & 13000 & 0.065570 & 13000 & 0.005156 \\
\hline & 14000 & -0.313809 & 14000 & 0.207087 & 14000 & 0.016284 \\
\hline & 58000 & -0.020531 & 58000 & 0.002716 & 58000 & 0.000214 \\
\hline CEPXS Form: & material & $\mathrm{Li}$ & 0.046550 & & & \\
\hline & & $\mathrm{O}$ & 0.505813 & & & \\
\hline & & $\mathrm{Na}$ & 0.017840 & & & \\
\hline & & $\mathrm{Al}$ & 0.095456 & & & \\
\hline & & $\mathrm{Si}$ & 0.313809 & & & \\
\hline & & $\mathrm{Ce}$ & 0.020531 & & & \\
\hline & $\begin{array}{c}\text { matname } \\
\text { density }\end{array}$ & $\begin{array}{c}\text { Glass Scinti } \\
2.420000\end{array}$ & r, Li Doped & F1) & & \\
\hline $\begin{array}{l}\text { Comments an } \\
\text { For GSF1. } \\
\text { Weight fraction } \\
\text { revision on } 9-1 \\
\text { science.com } / \mathrm{m} \\
\text { (Saint-Gobain } \\
\text { Weight fraction }\end{array}$ & $\begin{array}{l}\text { References } \\
\text { om http://w } \\
9 \text { omitted t } \\
\text { /crystalj.htn } \\
7 \text { ). } \\
\text { re adjusted }\end{array}$ & $\begin{array}{l}\text { v.apace-scie } \\
\text { weight fracti } \\
\text { ind http://ww } \\
\text { that they su }\end{array}$ & $\begin{array}{l}\text { com/ast/g } \\
\text { Also see } \\
\text { tectors.sair } \\
\text { unity. }\end{array}$ & $\begin{array}{l}\text { thtm on 8-2 } \\
\text { /www.apac } \\
\text { bain.com/L }\end{array}$ & $\begin{array}{l}\text { APACE } \\
\text {-Glass- }\end{array}$ & $\begin{array}{l}A \\
\text { lator.aspx }\end{array}$ \\
\hline
\end{tabular}

\section{Glass Scintillator, Li Doped (KG1, KG2, KG3)}

\begin{tabular}{lll}
\hline Formula $=$ & - & Molecular weight $(\mathrm{g} / \mathrm{mole})=$ \\
Density $(\mathrm{g} / \mathrm{cm} 3)=$ & 2.420000 & Total atom density $($ atoms $/ \mathrm{b}-\mathrm{cm})=$ \\
\hline
\end{tabular}

The above density is estimated to be accurate to 3 significant digits. Uncertainties are not addressed.

The following data were calculated from the input weight fractions.

\begin{tabular}{|c|c|c|c|c|c|c|}
\hline Element & Neutron ZA & Photon ZA & $\begin{array}{l}\text { Weight } \\
\text { Fraction }\end{array}$ & $\begin{array}{c}\text { Atom } \\
\text { Fraction }\end{array}$ & $\begin{array}{l}\text { Atom } \\
\text { Density }\end{array}$ & \\
\hline$\overline{\mathrm{Li}}$ & - & 3000 & 0.097560 & 0.239068 & 0.020484 & \\
\hline $\mathrm{O}$ & 8016 & 8000 & 0.513850 & 0.546269 & 0.046806 & \\
\hline $\mathrm{Si}$ & 14000 & 14000 & 0.345902 & 0.209481 & 0.017949 & \\
\hline $\mathrm{Ce}$ & - & 58000 & 0.042688 & 0.005182 & 0.000444 & \\
\hline Total & & & 1.000000 & 1.000000 & 0.085683 & \\
\hline MCNP Form & \multicolumn{2}{|c|}{ Weight Fractions } & \multicolumn{2}{|c|}{ Atom Fractions } & \multicolumn{2}{|c|}{ Atom Densities } \\
\hline \multirow[t]{4}{*}{ Neutrons } & - & -0.097560 & - & 0.239068 & - & 0.020484 \\
\hline & 8016 & -0.513850 & 8016 & 0.546269 & 8016 & 0.046806 \\
\hline & 14000 & -0.345902 & 14000 & 0.209481 & 14000 & 0.017949 \\
\hline & - & -0.042688 & - & 0.005182 & - & 0.000444 \\
\hline \multirow[t]{4}{*}{ Photons } & 3000 & -0.097560 & 3000 & 0.239068 & 3000 & 0.020484 \\
\hline & 8000 & -0.513850 & 8000 & 0.546269 & 8000 & 0.046806 \\
\hline & 14000 & -0.345902 & 14000 & 0.209481 & 14000 & 0.017949 \\
\hline & 58000 & -0.042688 & 58000 & 0.005182 & 58000 & 0.000444 \\
\hline
\end{tabular}




\begin{tabular}{|c|c|c|c|}
\hline \multirow[t]{2}{*}{ CEPXS Form: } & material & $\begin{array}{l}\mathrm{Li} \\
\mathrm{O} \\
\mathrm{Si} \\
\mathrm{Ce}\end{array}$ & $\begin{array}{l}0.097560 \\
0.513850 \\
0.345902 \\
0.042688\end{array}$ \\
\hline & $\begin{array}{l}\text { matname } \\
\text { density }\end{array}$ & $\begin{array}{r}\text { Glass S } \\
2.4200\end{array}$ & r, Li Doped (KG1, KG2, KG3) \\
\hline \multicolumn{4}{|c|}{$\begin{array}{l}\text { Comments and References } \\
\text { For KG1, KG2, or KG3. } \\
\text { Weight fractions from http://www.apace-science.com/ast/g_scint.htm on 8-25-09 (APACE 2009). A } \\
\text { revision on 9-10-09 omitted the weight fractions. Also see http://www.apace- } \\
\text { science.com/misc/crystalj.htm and http://www.detectors.saint-gobain.com/Lithium-Glass-Scintillator.aspx } \\
\text { (Saint-Gobain 2007). } \\
\text { Density=2.674 for type NE908 = KG2 on pg } 548 \text { of Knoll (2000). }\end{array}$} \\
\hline
\end{tabular}

\section{Glass, Borosilicate (Pyrex Glass)}

\begin{tabular}{llll}
\hline Formula $=$ & - & Molecular weight $(\mathrm{g} / \mathrm{mole})=$ & - \\
Density $(\mathrm{g} / \mathrm{cm} 3)=$ & 2.230000 & Total atom density $($ atoms $/ \mathrm{b}-\mathrm{cm})=$ & $7.064 \mathrm{E}-02$
\end{tabular}

The above density is estimated to be accurate to 3 significant digits. Uncertainties are not addressed.

The following data were calculated from the input weight fractions.

\begin{tabular}{|c|c|c|c|c|c|c|}
\hline Element & Neutron ZA & Photon ZA & $\begin{array}{l}\text { Weight } \\
\text { Fraction }\end{array}$ & $\begin{array}{l}\text { Atom } \\
\text { Fraction }\end{array}$ & $\begin{array}{l}\text { Atom } \\
\text { Density }\end{array}$ & \\
\hline$B$ & - & 5000 & 0.040064 & 0.070449 & 0.004977 & \\
\hline O & 8016 & 8000 & 0.539562 & 0.641095 & 0.045289 & \\
\hline $\mathrm{Na}$ & 11023 & 11000 & 0.028191 & 0.023311 & 0.001647 & \\
\hline $\mathrm{Al}$ & 13027 & 13000 & 0.011644 & 0.008204 & 0.000580 & \\
\hline $\mathrm{Si}$ & 14000 & 14000 & 0.377220 & 0.255327 & 0.018037 & \\
\hline $\mathrm{K}$ & 19000 & 19000 & 0.003321 & 0.001615 & 0.000114 & \\
\hline Total & & & 1.000002 & 1.000000 & 0.070643 & \\
\hline MCNP Form & \multicolumn{2}{|c|}{ Weight Fractions } & \multicolumn{2}{|c|}{ Atom Fractions } & \multicolumn{2}{|c|}{ Atom Densities } \\
\hline \multirow[t]{6}{*}{ Neutrons } & - & -0.040064 & - & 0.070449 & - & 0.004977 \\
\hline & 8016 & -0.539562 & 8016 & 0.641095 & 8016 & 0.045289 \\
\hline & 11023 & -0.028191 & 11023 & 0.023311 & 11023 & 0.001647 \\
\hline & 13027 & -0.011644 & 13027 & 0.008204 & 13027 & 0.000580 \\
\hline & 14000 & -0.377220 & 14000 & 0.255327 & 14000 & 0.018037 \\
\hline & 19000 & -0.003321 & 19000 & 0.001615 & 19000 & 0.000114 \\
\hline \multirow[t]{6}{*}{ Photons } & 5000 & -0.040064 & 5000 & 0.070449 & 5000 & 0.004977 \\
\hline & 8000 & -0.539562 & 8000 & 0.641095 & 8000 & 0.045289 \\
\hline & 11000 & -0.028191 & 11000 & 0.023311 & 11000 & 0.001647 \\
\hline & 13000 & -0.011644 & 13000 & 0.008204 & 13000 & 0.000580 \\
\hline & 14000 & -0.377220 & 14000 & 0.255327 & 14000 & 0.018037 \\
\hline & 19000 & -0.003321 & 19000 & 0.001615 & 19000 & 0.000114 \\
\hline
\end{tabular}




\begin{tabular}{|c|c|c|c|}
\hline \multirow[t]{7}{*}{ CEPXS Form: } & material & B & 0.040064 \\
\hline & & $\mathrm{O}$ & 0.539562 \\
\hline & & $\mathrm{Na}$ & 0.028191 \\
\hline & & $\mathrm{Al}$ & 0.011644 \\
\hline & & Si & 0.377220 \\
\hline & & $\mathrm{K}$ & 0.003321 \\
\hline & $\begin{array}{c}\text { matname } \\
\text { density }\end{array}$ & $\begin{array}{r}\text { Glass, B } \\
2.2300\end{array}$ & ate (Pyrex Glass) \\
\hline
\end{tabular}

\section{Glass, Foam}

\begin{tabular}{|c|c|c|c|}
\hline $\begin{array}{l}a= \\
(a / c m 3)=\end{array}$ & $\begin{array}{l}- \\
0.128000\end{array}$ & $\begin{array}{l}\text { Molecular weight }(\mathrm{g} / \mathrm{mole})= \\
\text { Total atom density }(\text { atoms } / \mathrm{b}-\mathrm{cm})=\end{array}$ & 4.086E-03 \\
\hline
\end{tabular}

The above density is estimated to be accurate to 3 significant digits. Uncertainties are not addressed.

The following data were calculated from the input weight fractions.

\begin{tabular}{|c|c|c|c|c|c|c|}
\hline Element & Neutron ZA & Photon ZA & $\begin{array}{l}\text { Weight } \\
\text { Fraction }\end{array}$ & $\begin{array}{c}\text { Atom } \\
\text { Fraction }\end{array}$ & $\begin{array}{l}\text { Atom } \\
\text { Density }\end{array}$ & \\
\hline $\mathrm{H}$ & 1001 & 1000 & 0.001000 & 0.018718 & 0.000076 & \\
\hline B & - & 5000 & 0.015000 & 0.026176 & 0.000107 & \\
\hline O & 8016 & 8000 & 0.534000 & 0.629684 & 0.002573 & \\
\hline $\mathrm{Na}$ & 11023 & 11000 & 0.161000 & 0.132122 & 0.000540 & \\
\hline $\mathrm{Si}$ & 14000 & 14000 & 0.279000 & 0.187416 & 0.000766 & \\
\hline$S$ & 16000 & 16000 & 0.010000 & 0.005884 & 0.000024 & \\
\hline Total & & & 1.000000 & 1.000000 & 0.004086 & \\
\hline MCNP Form & \multicolumn{2}{|c|}{ Weight Fractions } & \multicolumn{2}{|c|}{ Atom Fractions } & \multicolumn{2}{|c|}{ Atom Densities } \\
\hline \multirow[t]{6}{*}{ Neutrons } & 1001 & -0.001000 & 1001 & 0.018718 & 1001 & 0.000076 \\
\hline & - & -0.015000 & - & 0.026176 & - & 0.000107 \\
\hline & 8016 & -0.534000 & 8016 & 0.629684 & 8016 & 0.002573 \\
\hline & 11023 & -0.161000 & 11023 & 0.132122 & 11023 & 0.000540 \\
\hline & 14000 & -0.279000 & 14000 & 0.187416 & 14000 & 0.000766 \\
\hline & 16000 & -0.010000 & 16000 & 0.005884 & 16000 & 0.000024 \\
\hline \multirow[t]{6}{*}{ Photons } & 1000 & -0.001000 & 1000 & 0.018718 & 1000 & 0.000076 \\
\hline & 5000 & -0.015000 & 5000 & 0.026176 & 5000 & 0.000107 \\
\hline & 8000 & -0.534000 & 8000 & 0.629684 & 8000 & 0.002573 \\
\hline & 11000 & -0.161000 & 11000 & 0.132122 & 11000 & 0.000540 \\
\hline & 14000 & -0.279000 & 14000 & 0.187416 & 14000 & 0.000766 \\
\hline & 16000 & -0.010000 & 16000 & 0.005884 & 16000 & 0.000024 \\
\hline CEPXS Form: & material & $\begin{array}{l}\mathrm{H} \\
\mathrm{B}\end{array}$ & $\begin{array}{l}0.001000 \\
0.015000\end{array}$ & & & \\
\hline
\end{tabular}




\begin{tabular}{|ccc|} 
& $\mathrm{O}$ & 0.534000 \\
& $\mathrm{Na}$ & 0.161000 \\
& $\mathrm{Si}$ & 0.279000 \\
& $\mathrm{~S}$ & 0.010000 \\
& & \\
matname & Glass, Foam \\
$\quad$ density & 0.128000 \\
\hline Comments and References & \\
Pg II.F.1-3 of Carter et al. (1968). & \\
\hline
\end{tabular}

\section{Glass, Lead}

\begin{tabular}{llll}
\hline Formula $=$ & - & Molecular weight $(\mathrm{g} / \mathrm{mole})=$ \\
Density $(\mathrm{g} / \mathrm{cm} 3)=$ & 6.220000 & Total atom density $($ atoms $/ \mathrm{b}-\mathrm{cm})=$ & - \\
The.177E-02
\end{tabular}

The above density is estimated to be accurate to 3 significant digits. Uncertainties are not addressed.

The following data were calculated from the input weight fractions.

\begin{tabular}{|c|c|c|c|c|c|c|}
\hline Element & Neutron ZA & Photon ZA & $\begin{array}{l}\text { Weight } \\
\text { Fraction }\end{array}$ & $\begin{array}{c}\text { Atom } \\
\text { Fraction }\end{array}$ & $\begin{array}{l}\text { Atom } \\
\text { Density }\end{array}$ & \\
\hline 0 & 8016 & 8000 & $\overline{0.156453}$ & $\overline{0.592955}$ & 0.036629 & \\
\hline Si & 14000 & 14000 & 0.080866 & 0.174592 & 0.010785 & \\
\hline $\mathrm{Ti}$ & 22000 & 22000 & 0.008092 & 0.010251 & 0.000633 & \\
\hline As & 33075 & 33000 & 0.002651 & 0.002146 & 0.000133 & \\
\hline $\mathrm{Pb}$ & 82000 & 82000 & 0.751938 & 0.220056 & 0.013594 & \\
\hline Total & & & 1.000000 & 1.000000 & 0.061773 & \\
\hline MCNP Form & \multicolumn{2}{|c|}{ Weight Fractions } & \multicolumn{2}{|c|}{ Atom Fractions } & \multicolumn{2}{|c|}{ Atom Densities } \\
\hline \multirow[t]{5}{*}{ Neutrons } & 8016 & -0.156453 & 8016 & 0.592955 & 8016 & 0.036629 \\
\hline & 14000 & -0.080866 & 14000 & 0.174592 & 14000 & 0.010785 \\
\hline & 22000 & -0.008092 & 22000 & 0.010251 & 22000 & 0.000633 \\
\hline & 33075 & -0.002651 & 33075 & 0.002146 & 33075 & 0.000133 \\
\hline & 82000 & -0.751938 & 82000 & 0.220056 & 82000 & 0.013594 \\
\hline \multirow[t]{5}{*}{ Photons } & 8000 & -0.156453 & 8000 & 0.592955 & 8000 & 0.036629 \\
\hline & 14000 & -0.080866 & 14000 & 0.174592 & 14000 & 0.010785 \\
\hline & 22000 & -0.008092 & 22000 & 0.010251 & 22000 & 0.000633 \\
\hline & 33000 & -0.002651 & 33000 & 0.002146 & 33000 & 0.000133 \\
\hline & 82000 & -0.751938 & 82000 & 0.220056 & 82000 & 0.013594 \\
\hline \multirow[t]{6}{*}{ CEPXS Form: } & material & $\mathrm{O}$ & 0.156453 & & & \\
\hline & & $\mathrm{Si}$ & 0.080866 & & & \\
\hline & & $\mathrm{Ti}$ & 0.008092 & & & \\
\hline & & As & 0.002651 & & & \\
\hline & & $\mathrm{Pb}$ & 0.751938 & & & \\
\hline & $\begin{array}{c}\text { matname } \\
\text { density }\end{array}$ & $\begin{array}{c}\text { Glass, Lead } \\
6.220000\end{array}$ & & & & \\
\hline
\end{tabular}




\section{Glass, Plate}

\begin{tabular}{|c|c|c|c|}
\hline Formula $=$ & - & Molecular weight $(\mathrm{g} / \mathrm{mole})=$ & - \\
\hline Density $(\mathrm{g} / \mathrm{cm} 3)=$ & 2.400000 & Total atom density $($ atoms $/ \mathrm{b}-\mathrm{cm})=$ & $6.878 \mathrm{E}-02$ \\
\hline
\end{tabular}

\begin{tabular}{|c|c|c|c|c|c|}
\hline Element & Neutron ZA & Photon ZA & $\begin{array}{l}\text { Weight } \\
\text { Fraction }\end{array}$ & $\begin{array}{c}\text { Atom } \\
\text { Fraction }\end{array}$ & $\begin{array}{c}\text { Atom } \\
\text { Density }\end{array}$ \\
\hline 0 & 8016 & 8000 & 0.459800 & 0.603858 & 0.041536 \\
\hline $\mathrm{Na}$ & 11023 & 11000 & 0.096441 & 0.088145 & 0.006063 \\
\hline $\mathrm{Si}$ & 14000 & 14000 & 0.336553 & 0.251791 & 0.017319 \\
\hline $\mathrm{Ca}$ & 20000 & 20000 & 0.107205 & 0.056205 & 0.003866 \\
\hline Total & & & 0.999999 & 1.000000 & 0.068785 \\
\hline
\end{tabular}

\begin{tabular}{|c|cc|cc|cr|}
\hline MCNP Form & \multicolumn{2}{c}{ Weight Fractions } & \multicolumn{2}{c|}{ Atom Fractions } & \multicolumn{2}{c|}{ Atom Densities } \\
\hline Neutrons & 8016 & -0.459800 & 8016 & 0.603858 & 8016 & 0.041536 \\
& 11023 & -0.096441 & 11023 & 0.088145 & 11023 & 0.006063 \\
& 14000 & -0.336553 & 14000 & 0.251791 & 14000 & 0.017319 \\
& 20000 & -0.107205 & 20000 & 0.056205 & 20000 & 0.003866 \\
Photons & & & & & & \\
& 8000 & -0.459800 & 8000 & 0.603858 & 8000 & 0.041536 \\
& 11000 & -0.096441 & 11000 & 0.088145 & 11000 & 0.006063 \\
& 14000 & -0.336553 & 14000 & 0.251791 & 14000 & 0.017319 \\
& 20000 & -0.107205 & 20000 & 0.056205 & 20000 & 0.003866
\end{tabular}

\begin{tabular}{ccc}
\hline CEPXS Form: $\quad$ material & $\mathrm{O}$ & 0.459800 \\
& $\mathrm{Na}$ & 0.096441 \\
& $\mathrm{Si}$ & 0.336553 \\
& $\mathrm{Ca}$ & 0.107205
\end{tabular}

$\begin{array}{cc}\text { matname } & \text { Glass, Plate } \\ \text { density } & 2.400000\end{array}$

Comments and References

Density = $2.40 \mathrm{~g} / \mathrm{cm} 3$ and weight fractions from http://physics.nist.gov/cgi-bin/Star/compos.pl?matno=171 (NIST 1998).

See Tables 12 - 16 and 12 - 17 of Parker (1967) for other types of glass.

Density $=2.56 \mathrm{~g} / \mathrm{cm} 3$ in Table 7.5 of Shultis and Faw (1996). 


\section{Glycerol}

\begin{tabular}{llll}
\hline Formula $=$ & $\mathrm{C} 3 \mathrm{H} 8 \mathrm{O} 3$ & Molecular weight $(\mathrm{g} / \mathrm{mole})=$ & 92.09382 \\
Density $(\mathrm{g} / \mathrm{cm} 3)=$ & 1.261300 & Total atom density $($ atoms $/ \mathrm{b}-\mathrm{cm})=$ & $1.155 \mathrm{E}-01$
\end{tabular}

The above density is estimated to be accurate to 4 significant digits. Uncertainties are not addressed. The following data were calculated from the input weight fractions.

$\begin{array}{ccccccc}\text { Element } & \text { Neutron ZA } & \text { Photon ZA } & \begin{array}{c}\text { Weight } \\ \text { Fraction }\end{array} & \begin{array}{c}\text { Atom } \\ \text { Fraction }\end{array} & \begin{array}{c}\text { Atom } \\ \text { Density }\end{array} \\ & 1001 & & 1000 & 0.087554 & 0.571417 & 0.065980 \\ \mathrm{O} & 6000 & 6000 & 0.391262 & 0.214294 & 0.024744 \\ \text { Total } & 8016 & 8000 & 0.521185 & 0.214289 & 0.024743 \\ & & & & & & \\ & & & & 1.000001 & 1.000000 & 0.115467\end{array}$

\begin{tabular}{|c|c|c|c|c|c|c|}
\hline MCNP Form & \multicolumn{2}{|c|}{ Weight Fractions } & \multicolumn{2}{|c|}{ Atom Fractions } & \multicolumn{2}{|c|}{ Atom Densities } \\
\hline \multirow[t]{3}{*}{ Neutrons } & 1001 & -0.087554 & 1001 & 0.571417 & 1001 & 0.065980 \\
\hline & 6000 & -0.391262 & 6000 & 0.214294 & 6000 & 0.024744 \\
\hline & 8016 & -0.521185 & 8016 & 0.214289 & 8016 & 0.024743 \\
\hline \multirow[t]{3}{*}{ Photons } & 1000 & -0.087554 & 1000 & 0.571417 & 1000 & 0.065980 \\
\hline & 6000 & -0.391262 & 6000 & 0.214294 & 6000 & 0.024744 \\
\hline & 8000 & -0.521185 & 8000 & 0.214289 & 8000 & 0.024743 \\
\hline \multirow[t]{4}{*}{ CEPXS Form: } & material & $\mathrm{H}$ & 0.087554 & & & \\
\hline & & C & 0.391262 & & & \\
\hline & & $\mathrm{O}$ & 0.521185 & & & \\
\hline & $\begin{array}{c}\text { matname } \\
\text { density }\end{array}$ & $\begin{array}{l}\text { Glycerol } \\
1.261300 \\
\end{array}$ & & & & \\
\hline \multicolumn{7}{|c|}{$\begin{array}{l}\text { Comments and References } \\
\text { Density }=1.2613 \mathrm{~g} / \mathrm{cm} 3 \text { and weight fractions from http://physics.nist.gov/cgi- } \\
\text { bin/Star/compos.pl?matno=174 (NIST 1998). } \\
\text { Density }=1.2613 \mathrm{~g} / \mathrm{cm} 3 \text { at } 20^{\circ} \mathrm{C} \text { from } \\
\text { http://www.matweb.com/search/DataSheet.aspx?MatGUID=015b4c540c454ad7b944980dfa9438c8 } \\
\text { (Automation Creations } 2010 \text { ). Also called glycerin (http://en.wikipedia.org/wiki/Glycerin). } \\
\text { Formula from pgs } 3-268 \text { of Lide (2008) and Table } 51.120 \text { of Hungerford (1960). }\end{array}$} \\
\hline
\end{tabular}

\section{Gold}

\begin{tabular}{llll}
\hline Formula $=$ & $\mathrm{Au}$ & Molecular weight $(\mathrm{g} / \mathrm{mole})=$ & 196.96655 \\
Density $(\mathrm{g} / \mathrm{cm} 3)=$ & 19.320000 & Total atom density $($ atoms $/ \mathrm{b}-\mathrm{cm})=$ & $5.907 \mathrm{E}-02$
\end{tabular}

The above density is estimated to be accurate to 4 significant digits. Uncertainties are not addressed. The following data was calculated from the input formula.

$\begin{array}{llllll}\text { Element } & \frac{\text { Neutron ZA }}{\mathrm{Au}} & \frac{\text { Photon ZA }}{79197} & \frac{1}{\text { Fraction }} & \begin{array}{c}\text { Atom } \\ \text { Fraction }\end{array} & \begin{array}{c}\text { Atom } \\ \text { Density }\end{array} \\ 1.000000 & & \frac{1.000000}{0.059070} & \end{array}$


PIET-43741-TM-963

PNNL-15870 Rev. 1

\begin{tabular}{|c|c|c|c|c|c|c|}
\hline \multicolumn{3}{|l|}{ Total } & 1.000000 & 1.000000 & \multicolumn{2}{|c|}{0.059070} \\
\hline MCNP Form & \multicolumn{2}{|c|}{ Weight Fractions } & \multicolumn{2}{|c|}{ Atom Fractions } & \multicolumn{2}{|c|}{ Atom Densities } \\
\hline Neutrons & 79197 & -1.000000 & 79197 & 1.000000 & 79197 & 0.059070 \\
\hline Photons & 79000 & -1.000000 & 79000 & 1.000000 & 79000 & 0.059070 \\
\hline \multirow[t]{2}{*}{ CEPXS Form: } & material & $\mathrm{Au}$ & 1.000000 & & & \\
\hline & $\begin{array}{l}\text { matname } \\
\text { density }\end{array}$ & $\begin{array}{l}\text { Gold } \\
19.320000\end{array}$ & & & & \\
\hline $\begin{array}{l}\text { Comments an } \\
\text { Density from ht }\end{array}$ & $\begin{array}{l}\text { eferences } \\
\text { physics.ni }\end{array}$ & & & & & \\
\hline
\end{tabular}

\section{Gypsum (Plaster of Paris)}

\begin{tabular}{llll}
\hline Formula $=$ & CaSO4-2(H2O) & Molecular weight $(\mathrm{g} / \mathrm{mole})=$ & 172.17116 \\
Density $(\mathrm{g} / \mathrm{cm} 3)=$ & 2.320000 & Total atom density $($ atoms $/ \mathrm{b}-\mathrm{cm})=$ & $9.738 \mathrm{E}-02$
\end{tabular}

The above density is estimated to be accurate to 3 significant digits. Uncertainties are not addressed.

The following data were calculated from the input weight fractions.

\begin{tabular}{|c|c|c|c|c|c|c|}
\hline Element & Neutron ZA & Photon ZA & $\begin{array}{l}\text { Weight } \\
\text { Fraction }\end{array}$ & $\begin{array}{c}\text { Atom } \\
\text { Fraction }\end{array}$ & $\begin{array}{l}\text { Atom } \\
\text { Density }\end{array}$ & \\
\hline $\mathrm{H}$ & 1001 & 1000 & $\overline{0.023416}$ & $\overline{0.333321}$ & 0.032458 & \\
\hline $\mathrm{O}$ & 8016 & 8000 & 0.557572 & 0.500014 & 0.048690 & \\
\hline$S$ & 16000 & 16000 & 0.186215 & 0.083324 & 0.008114 & \\
\hline $\mathrm{Ca}$ & 20000 & 20000 & 0.232797 & 0.083341 & 0.008115 & \\
\hline Total & & & 1.000000 & 1.000000 & 0.097376 & \\
\hline MCNP Form & \multicolumn{2}{|c|}{ Weight Fractions } & \multicolumn{2}{|c|}{ Atom Fractions } & \multicolumn{2}{|c|}{ Atom Densities } \\
\hline Neutrons & 1001 & -0.023416 & 1001 & 0.333321 & 1001 & 0.032458 \\
\hline & 8016 & -0.557572 & 8016 & 0.500014 & 8016 & 0.048690 \\
\hline & 16000 & -0.186215 & 16000 & 0.083324 & 16000 & 0.008114 \\
\hline & 20000 & -0.232797 & 20000 & 0.083341 & 20000 & 0.008115 \\
\hline \multirow[t]{4}{*}{ Photons } & 1000 & -0.023416 & 1000 & 0.333321 & 1000 & 0.032458 \\
\hline & 8000 & -0.557572 & 8000 & 0.500014 & 8000 & 0.048690 \\
\hline & 16000 & -0.186215 & 16000 & 0.083324 & 16000 & 0.008114 \\
\hline & 20000 & -0.232797 & 20000 & 0.083341 & 20000 & 0.008115 \\
\hline \multirow[t]{4}{*}{ CEPXS Form: } & material & $\mathrm{H}$ & 0.023416 & & & \\
\hline & & $\mathrm{O}$ & 0.557572 & & & \\
\hline & & $S$ & 0.186215 & & & \\
\hline & & $\mathrm{Ca}$ & 0.232797 & & & \\
\hline
\end{tabular}




\begin{tabular}{|c|c|}
\hline $\begin{array}{c}\text { matname } \\
\text { density }\end{array}$ & $\begin{array}{l}\text { Gypsum (Plaster of Paris) } \\
2.320000\end{array}$ \\
\hline $\begin{array}{l}\text { Comments and References } \\
\text { Density }=2.32 \mathrm{~g} / \mathrm{cm} 3 \text { and we } \\
\text { (NIST 1998). } \\
\text { Density }=2.32 \mathrm{~g} / \mathrm{cm} 3 \text { and for } \\
\text { http://www.matweb.com/sear } \\
1 \text { (Automation Creations } 201( \\
\text { For example, density = } 0.67 \mathrm{t} \\
\text { http://www.powderandbulk.co } \\
\text { Bulk Dot Com 2010), and der } \\
\text { 2009). Gypsum is the primar } \\
0.69 \mathrm{~g} / \mathrm{cm} 3 \text { in Mantell (1958), }\end{array}$ & $\begin{array}{l}\text { ht fractions from http://physics.nist.gov/cgi-bin/Star/compos.pl?matno=176 } \\
\text { ula at } \\
\text { /DataSheet.aspx?MatGUID=fdac5563c7f2472a825d6cc0f16e2785\&ckck= } \\
\text { A significant variation in densities is listed for different types of gypsum. } \\
0.88 \mathrm{~g} / \mathrm{cm} 3 \text { at } \\
\text { /resources/bulk_density/material_bulk_density_chart_g.htm (Powder and } \\
\text { ty }=1.12 \text { to } 2.79 \text { at http://www.simetric.co.uk/si_materials.htm (Walker } \\
\text { component of wallboard or drywall. Density of wallboard = } 43 \mathrm{lb} / \mathrm{ft} 3= \\
\text { able } 35-1 \text {. Density }=0.75 \mathrm{~g} / \mathrm{cm} 3 \text { in Table } 7.5 \text { of Shultis and Faw (1996). }\end{array}$ \\
\hline
\end{tabular}

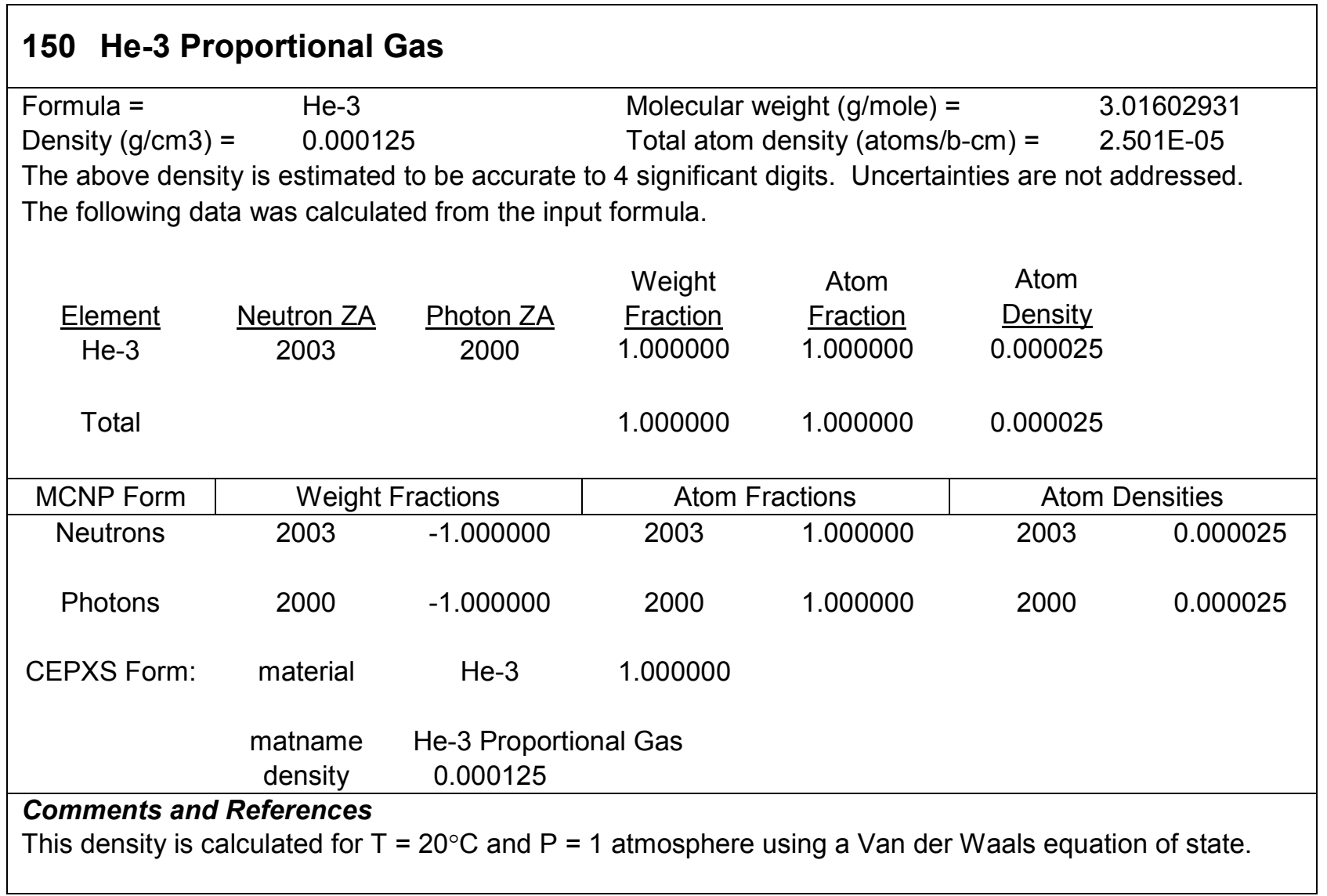

\section{Helium, Natural}

\begin{tabular}{llll}
\hline Formula $=$ & $\mathrm{He}$ & Molecular weight $(\mathrm{g} / \mathrm{mole})=$ & 4.002602 \\
Density $(\mathrm{g} / \mathrm{cm} 3)=$ & 0.000166 & Total atom density $($ atoms $/ \mathrm{b}-\mathrm{cm})=$ & $2.502 \mathrm{E}-05$
\end{tabular}

The above density is estimated to be accurate to 4 significant digits. Uncertainties are not addressed. The following data was calculated from the input formula. 


\begin{tabular}{|c|c|c|c|c|c|c|}
\hline$\frac{\text { Element }}{\mathrm{He}}$ & $\frac{\text { Neutron ZA }}{2004}$ & $\frac{\text { Photon ZA }}{2000}$ & $\begin{array}{c}\text { Weight } \\
\text { Fraction } \\
1.000000\end{array}$ & $\begin{array}{c}\begin{array}{c}\text { Atom } \\
\text { Fraction }\end{array} \\
1.000000\end{array}$ & $\begin{array}{c}\text { Atom } \\
\text { Density } \\
0.000025\end{array}$ & \\
\hline Total & & & 1.000000 & 1.000000 & 0.000025 & \\
\hline MCNP Form & \multicolumn{2}{|c|}{ Weight Fractions } & \multicolumn{2}{|c|}{ Atom Fractions } & \multicolumn{2}{|c|}{ Atom Densities } \\
\hline Neutrons & 2004 & -1.000000 & 2004 & 1.000000 & 2004 & 0.000025 \\
\hline Photons & 2000 & -1.000000 & 2000 & 1.000000 & 2000 & 0.000025 \\
\hline CEPXS Form: & $\begin{array}{l}\text { material } \\
\text { matname } \\
\text { density }\end{array}$ & \multicolumn{4}{|l|}{$\begin{array}{c}\text { Helium, Natural } \\
0.000166\end{array}$} & \\
\hline $\begin{array}{l}\text { Comments an } \\
\text { Density from } \mathrm{h}\end{array}$ & $\begin{array}{l}\text { eferences } \\
\text { physics.nis }\end{array}$ & 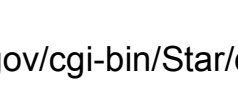 & & & & \\
\hline
\end{tabular}

\section{Hydrogen}

\begin{tabular}{llll}
\hline Formula $=$ & $\mathrm{H} 2$ & Molecular weight $(\mathrm{g} / \mathrm{mole})=$ & 2.01588 \\
Density $(\mathrm{g} / \mathrm{cm} 3)=$ & 0.000084 & Total atom density $($ atoms $/ \mathrm{b}-\mathrm{cm})=$ & $5.004 \mathrm{E}-05$
\end{tabular}

The above density is estimated to be accurate to 4 significant digits. Uncertainties are not addressed.

The following data was calculated from the input formula.

\begin{tabular}{|c|c|c|c|c|c|c|}
\hline$\frac{\text { Element }}{\mathrm{H}}$ & $\frac{\text { Neutron ZA }}{1001}$ & $\frac{\text { Photon ZA }}{1000}$ & $\begin{array}{c}\text { Weight } \\
\text { Fraction } \\
1.000000\end{array}$ & $\begin{array}{c}\begin{array}{c}\text { Atom } \\
\text { Fraction }\end{array} \\
1.000000\end{array}$ & $\begin{array}{c}\text { Atom } \\
\text { Density } \\
0.00005\end{array}$ & \\
\hline Total & & & 1.000000 & 1.000000 & 0.00005 & \\
\hline MCNP Form & \multicolumn{2}{|c|}{ Weight Fractions } & \multicolumn{2}{|c|}{ Atom Fractions } & \multicolumn{2}{|c|}{ Atom Densities } \\
\hline Neutrons & 1001 & -1.000000 & 1001 & 1.000000 & 1001 & 0.000050 \\
\hline Photons & 1000 & -1.000000 & 1000 & 1.000000 & 1000 & 0.000050 \\
\hline CEPXS Form: & $\begin{array}{l}\text { material } \\
\text { matname } \\
\text { density }\end{array}$ & $\begin{array}{c}\mathrm{H} \\
\text { Hydrogen } \\
0.000084\end{array}$ & 1.000000 & & & \\
\hline
\end{tabular}




\section{Incoloy-800}

\begin{tabular}{llll}
\hline Formula $=$ & - & Molecular weight $(\mathrm{g} / \mathrm{mole})=$ \\
Density $(\mathrm{g} / \mathrm{cm} 3)=$ & 7.940000 & Total atom density $($ atoms $/ \mathrm{b}-\mathrm{cm})=$ & - \\
\hline & $8.672 \mathrm{E}-02$
\end{tabular}

The above density is estimated to be accurate to 3 significant digits. Uncertainties are not addressed.

The following data were calculated from the input weight fractions.

\begin{tabular}{|c|c|c|c|c|c|c|}
\hline Element & Neutron ZA & Photon ZA & $\begin{array}{l}\text { Weight } \\
\text { Fraction }\end{array}$ & $\begin{array}{l}\text { Atom } \\
\text { Fraction }\end{array}$ & $\begin{array}{l}\text { Atom } \\
\text { Density }\end{array}$ & \\
\hline $\mathrm{C}$ & 6000 & 6000 & 0.000650 & 0.002984 & 0.000259 & \\
\hline $\mathrm{Al}$ & 13027 & 13000 & 0.003750 & 0.007663 & 0.000665 & \\
\hline $\mathrm{Si}$ & 14000 & 14000 & 0.006500 & 0.012760 & 0.001107 & \\
\hline$S$ & 16000 & 16000 & 0.000100 & 0.000172 & 0.000015 & \\
\hline $\mathrm{Ti}$ & 22000 & 22000 & 0.003750 & 0.004319 & 0.000375 & \\
\hline $\mathrm{Cr}$ & 24000 & 24000 & 0.210000 & 0.222681 & 0.019312 & \\
\hline $\mathrm{Mn}$ & 25055 & 25000 & 0.009750 & 0.009785 & 0.000849 & \\
\hline $\mathrm{Fe}$ & 26000 & 26000 & 0.435630 & 0.430099 & 0.037300 & \\
\hline $\mathrm{Ni}$ & 28000 & 28000 & 0.325000 & 0.305302 & 0.026477 & \\
\hline $\mathrm{Cu}$ & 29000 & 29000 & 0.004880 & 0.004234 & 0.000367 & \\
\hline Total & & & 1.000010 & 1.000000 & 0.086723 & \\
\hline MCNP Form & \multicolumn{2}{|c|}{ Weight Fractions } & \multicolumn{2}{|c|}{ Atom Fractions } & \multicolumn{2}{|c|}{ Atom Densities } \\
\hline \multirow[t]{10}{*}{ Neutrons } & 6000 & -0.000650 & 6000 & 0.002984 & 6000 & 0.000259 \\
\hline & 13027 & -0.003750 & 13027 & 0.007663 & 13027 & 0.000665 \\
\hline & 14000 & -0.006500 & 14000 & 0.012760 & 14000 & 0.001107 \\
\hline & 16000 & -0.000100 & 16000 & 0.000172 & 16000 & 0.000015 \\
\hline & 22000 & -0.003750 & 22000 & 0.004319 & 22000 & 0.000375 \\
\hline & 24000 & -0.210000 & 24000 & 0.222681 & 24000 & 0.019312 \\
\hline & 25055 & -0.009750 & 25055 & 0.009785 & 25055 & 0.000849 \\
\hline & 26000 & -0.435630 & 26000 & 0.430099 & 26000 & 0.037300 \\
\hline & 28000 & -0.325000 & 28000 & 0.305302 & 28000 & 0.026477 \\
\hline & 29000 & -0.004880 & 29000 & 0.004234 & 29000 & 0.000367 \\
\hline \multirow[t]{10}{*}{ Photons } & 6000 & -0.000650 & 6000 & 0.002984 & 6000 & 0.000259 \\
\hline & 13000 & -0.003750 & 13000 & 0.007663 & 13000 & 0.000665 \\
\hline & 14000 & -0.006500 & 14000 & 0.012760 & 14000 & 0.001107 \\
\hline & 16000 & -0.000100 & 16000 & 0.000172 & 16000 & 0.000015 \\
\hline & 22000 & -0.003750 & 22000 & 0.004319 & 22000 & 0.000375 \\
\hline & 24000 & -0.210000 & 24000 & 0.222681 & 24000 & 0.019312 \\
\hline & 25000 & -0.009750 & 25000 & 0.009785 & 25000 & 0.000849 \\
\hline & 26000 & -0.435630 & 26000 & 0.430099 & 26000 & 0.037300 \\
\hline & 28000 & -0.325000 & 28000 & 0.305302 & 28000 & 0.026477 \\
\hline & 29000 & -0.004880 & 29000 & 0.004234 & 29000 & 0.000367 \\
\hline \multirow[t]{4}{*}{ CEPXS Form: } & material & $\mathrm{C}$ & 0.000650 & & & \\
\hline & & $\mathrm{Al}$ & 0.003750 & & & \\
\hline & & $\mathrm{Si}$ & 0.006500 & & & \\
\hline & & $S$ & 0.000100 & & & \\
\hline
\end{tabular}




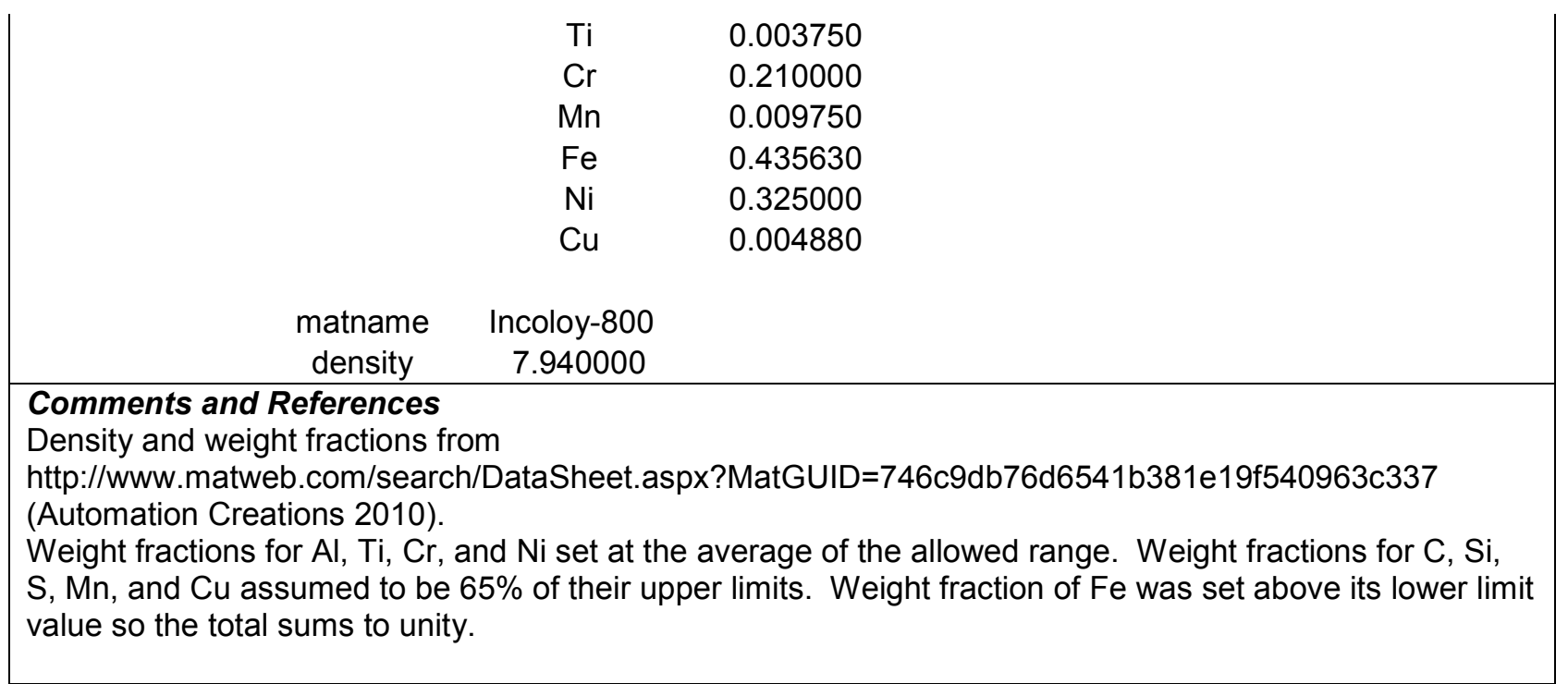

\section{Inconel-600}

\begin{tabular}{llll}
\hline Formula $=$ & - & Molecular weight $(\mathrm{g} / \mathrm{mole})=$ \\
Density $(\mathrm{g} / \mathrm{cm} 3)=$ & 8.470000 & Total atom density $($ atoms $/ \mathrm{b}-\mathrm{cm})=$ & - \\
\hline & & $3.966 \mathrm{E}-02$
\end{tabular}

The above density is estimated to be accurate to 3 significant digits. Uncertainties are not addressed.

The following data were calculated from the input weight fractions.

\begin{tabular}{|c|c|c|c|c|c|c|}
\hline Element & Neutron ZA & Photon ZA & $\begin{array}{l}\text { Weight } \\
\text { Fraction }\end{array}$ & $\begin{array}{l}\text { Atom } \\
\text { Fraction }\end{array}$ & $\begin{array}{l}\text { Atom } \\
\text { Density }\end{array}$ & \\
\hline $\mathrm{C}$ & 6000 & 6000 & 0.000980 & 0.004642 & 0.000416 & \\
\hline $\mathrm{Si}$ & 14000 & 14000 & 0.003250 & 0.006583 & 0.000590 & \\
\hline$S$ & 16000 & 16000 & 0.000100 & 0.000177 & 0.000016 & \\
\hline $\mathrm{Cr}$ & 24000 & 24000 & 0.155000 & 0.169591 & 0.015205 & \\
\hline $\mathrm{Mn}$ & 25055 & 25000 & 0.006500 & 0.006731 & 0.000603 & \\
\hline $\mathrm{Fe}$ & 26000 & 26000 & 0.080000 & 0.081498 & 0.007307 & \\
\hline $\mathrm{Ni}$ & 28000 & 28000 & 0.750930 & 0.727867 & 0.065260 & \\
\hline $\mathrm{Cu}$ & 29000 & 29000 & 0.003250 & 0.002910 & 0.000261 & \\
\hline Total & & & 1.000010 & 1.000000 & 0.089659 & \\
\hline MCNP Form & \multicolumn{2}{|c|}{ Weight Fractions } & \multicolumn{2}{|c|}{ Atom Fractions } & \multicolumn{2}{|c|}{ Atom Densities } \\
\hline \multirow[t]{8}{*}{ Neutrons } & 6000 & -0.000980 & 6000 & 0.004642 & 6000 & 0.000416 \\
\hline & 14000 & -0.003250 & 14000 & 0.006583 & 14000 & 0.000590 \\
\hline & 16000 & -0.000100 & 16000 & 0.000177 & 16000 & 0.000016 \\
\hline & 24000 & -0.155000 & 24000 & 0.169591 & 24000 & 0.015205 \\
\hline & 25055 & -0.006500 & 25055 & 0.006731 & 25055 & 0.000603 \\
\hline & 26000 & -0.080000 & 26000 & 0.081498 & 26000 & 0.007307 \\
\hline & 28000 & -0.750930 & 28000 & 0.727867 & 28000 & 0.065260 \\
\hline & 29000 & -0.003250 & 29000 & 0.002910 & 29000 & 0.000261 \\
\hline
\end{tabular}


PIET-43741-TM-963

PNNL-15870 Rev. 1

\begin{tabular}{|c|c|c|c|c|c|c|}
\hline \multirow[t]{8}{*}{ Photons } & 6000 & -0.000980 & 6000 & 0.004642 & 6000 & 0.000416 \\
\hline & 14000 & -0.003250 & 14000 & 0.006583 & 14000 & 0.000590 \\
\hline & 16000 & -0.000100 & 16000 & 0.000177 & 16000 & 0.000016 \\
\hline & 24000 & -0.155000 & 24000 & 0.169591 & 24000 & 0.015205 \\
\hline & 25000 & -0.006500 & 25000 & 0.006731 & 25000 & 0.000603 \\
\hline & 26000 & -0.080000 & 26000 & 0.081498 & 26000 & 0.007307 \\
\hline & 28000 & -0.750930 & 28000 & 0.727867 & 28000 & 0.065260 \\
\hline & 29000 & -0.003250 & 29000 & 0.002910 & 29000 & 0.000261 \\
\hline \multirow[t]{9}{*}{ CEPXS Form: } & material & $\mathrm{C}$ & 0.000980 & & & \\
\hline & & $\mathrm{Si}$ & 0.003250 & & & \\
\hline & & $S$ & 0.000100 & & & \\
\hline & & $\mathrm{Cr}$ & 0.155000 & & & \\
\hline & & $\mathrm{Mn}$ & 0.006500 & & & \\
\hline & & $\mathrm{Fe}$ & 0.080000 & & & \\
\hline & & $\mathrm{Ni}$ & 0.750930 & & & \\
\hline & & $\mathrm{Cu}$ & 0.003250 & & & \\
\hline & $\begin{array}{c}\text { matname } \\
\text { density }\end{array}$ & $\begin{array}{c}\text { Inconel-600 } \\
8.470000\end{array}$ & & & & \\
\hline \multicolumn{7}{|c|}{$\begin{array}{l}\text { Comments and References } \\
\text { http://www.matweb.com/search/DataSheet.aspx?MatGUID=029d44b293ee41a1926d8de74e6369bc } \\
\text { (Automation Creations 2010). } \\
\text { http://www.espi-metals.com/tech/Tech-\%20Inconel\%20600\%20-\%20Alloy\%20Composition.htm. } \\
\text { Weight fractions for Cr and Fe set at the average of the allowed range. Weight fractions for C, Si, S, Mn, } \\
\text { and Cu assumed to be } 65 \% \text { of their upper limits. Weight fraction of Ni was set above its lower limit value } \\
\text { so the total sums to unity. }\end{array}$} \\
\hline
\end{tabular}

\section{Inconel-625}

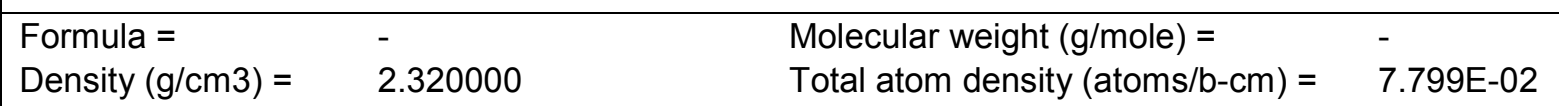

The above density is estimated to be accurate to 3 significant digits. Uncertainties are not addressed.

The following data were calculated from the input weight fractions.

\begin{tabular}{|c|c|c|c|c|c|}
\hline Element & Neutron ZA & Photon ZA & $\begin{array}{l}\text { Weight } \\
\text { Fraction }\end{array}$ & $\begin{array}{c}\text { Atom } \\
\text { Fraction }\end{array}$ & $\begin{array}{c}\text { Atom } \\
\text { Density }\end{array}$ \\
\hline $\mathrm{H}$ & 1001 & 1000 & $\overline{0.007500}$ & $\overline{0.133302}$ & 0.010396 \\
\hline C & 6000 & 6000 & 0.055200 & 0.082334 & 0.006421 \\
\hline $\mathrm{N}$ & 7014 & 7000 & 0.000200 & 0.000256 & 0.000020 \\
\hline $\mathrm{O}$ & 8016 & 8000 & 0.484900 & 0.542947 & 0.042344 \\
\hline $\mathrm{Na}$ & 11023 & 11000 & 0.006300 & 0.004909 & 0.000383 \\
\hline $\mathrm{Mg}$ & 12000 & 12000 & 0.012500 & 0.009213 & 0.000719 \\
\hline $\mathrm{Al}$ & 13027 & 13000 & 0.021700 & 0.014408 & 0.001124 \\
\hline $\mathrm{Si}$ & 14000 & 14000 & 0.155000 & 0.098869 & 0.007711 \\
\hline$S$ & 16000 & 16000 & 0.001900 & 0.001062 & 0.000083 \\
\hline $\mathrm{K}$ & 19000 & 19000 & 0.013700 & 0.006277 & 0.000490 \\
\hline $\mathrm{Ca}$ & 20000 & 20000 & 0.230000 & 0.102809 & 0.008018 \\
\hline
\end{tabular}


PIET-43741-TM-963

PNNL-15870 Rev. 1

\begin{tabular}{|c|c|c|c|c|c|c|}
\hline $\mathrm{Ti}$ & 22000 & 22000 & 0.001000 & 0.000374 & \multicolumn{2}{|c|}{0.000029} \\
\hline $\mathrm{Fe}$ & 26000 & 26000 & 0.010100 & 0.003240 & \multicolumn{2}{|c|}{0.000253} \\
\hline Total & & & 1.000000 & 1.000000 & \multicolumn{2}{|c|}{0.077988} \\
\hline MCNP Form & \multicolumn{2}{|c|}{ Weight Fractions } & \multicolumn{2}{|c|}{ Atom Fractions } & \multicolumn{2}{|c|}{ Atom Densities } \\
\hline \multirow[t]{13}{*}{ Neutrons } & 1001 & -0.007500 & 1001 & 0.133302 & 1001 & 0.010396 \\
\hline & 6000 & -0.055200 & 6000 & 0.082334 & 6000 & 0.006421 \\
\hline & 7014 & -0.000200 & 7014 & 0.000256 & 7014 & 0.000020 \\
\hline & 8016 & -0.484900 & 8016 & 0.542947 & 8016 & 0.042344 \\
\hline & 11023 & -0.006300 & 11023 & 0.004909 & 11023 & 0.000383 \\
\hline & 12000 & -0.012500 & 12000 & 0.009213 & 12000 & 0.000719 \\
\hline & 13027 & -0.021700 & 13027 & 0.014408 & 13027 & 0.001124 \\
\hline & 14000 & -0.155000 & 14000 & 0.098869 & 14000 & 0.007711 \\
\hline & 16000 & -0.001900 & 16000 & 0.001062 & 16000 & 0.000083 \\
\hline & 19000 & -0.013700 & 19000 & 0.006277 & 19000 & 0.000490 \\
\hline & 20000 & -0.230000 & 20000 & 0.102809 & 20000 & 0.008018 \\
\hline & 22000 & -0.001000 & 22000 & 0.000374 & 22000 & 0.000029 \\
\hline & 26000 & -0.010100 & 26000 & 0.003240 & 26000 & 0.000253 \\
\hline \multirow[t]{13}{*}{ Photons } & 1000 & -0.007500 & 1000 & 0.133302 & 1000 & 0.010396 \\
\hline & 6000 & -0.055200 & 6000 & 0.082334 & 6000 & 0.006421 \\
\hline & 7000 & -0.000200 & 7000 & 0.000256 & 7000 & 0.000020 \\
\hline & 8000 & -0.484900 & 8000 & 0.542947 & 8000 & 0.042344 \\
\hline & 11000 & -0.006300 & 11000 & 0.004909 & 11000 & 0.000383 \\
\hline & 12000 & -0.012500 & 12000 & 0.009213 & 12000 & 0.000719 \\
\hline & 13000 & -0.021700 & 13000 & 0.014408 & 13000 & 0.001124 \\
\hline & 14000 & -0.155000 & 14000 & 0.098869 & 14000 & 0.007711 \\
\hline & 16000 & -0.001900 & 16000 & 0.001062 & 16000 & 0.000083 \\
\hline & 19000 & -0.013700 & 19000 & 0.006277 & 19000 & 0.000490 \\
\hline & 20000 & -0.230000 & 20000 & 0.102809 & 20000 & 0.008018 \\
\hline & 22000 & -0.001000 & 22000 & 0.000374 & 22000 & 0.000029 \\
\hline & 26000 & -0.010100 & 26000 & 0.003240 & 26000 & 0.000253 \\
\hline \multirow[t]{14}{*}{ CEPXS Form: } & material & $\mathrm{H}$ & 0.007500 & & & \\
\hline & & C & 0.055200 & & & \\
\hline & & $\mathrm{N}$ & 0.000200 & & & \\
\hline & & $\mathrm{O}$ & 0.484900 & & & \\
\hline & & $\mathrm{Na}$ & 0.006300 & & & \\
\hline & & $\mathrm{Mg}$ & 0.012500 & & & \\
\hline & & $\mathrm{Al}$ & 0.021700 & & & \\
\hline & & Si & 0.155000 & & & \\
\hline & & $S$ & 0.001900 & & & \\
\hline & & $\mathrm{K}$ & 0.013700 & & & \\
\hline & & $\mathrm{Ca}$ & 0.230000 & & & \\
\hline & & $\mathrm{Ti}$ & 0.001000 & & & \\
\hline & & $\mathrm{Fe}$ & 0.010100 & & & \\
\hline & $\begin{array}{c}\text { matname } \\
\text { density }\end{array}$ & $\begin{array}{c}\text { Inconel-625 } \\
2.320000\end{array}$ & & & & \\
\hline
\end{tabular}


Comments and References

Density and weight fractions from

http://www.matweb.com/search/DataSheet.aspx?MatGUID=4a194f59f35a427dbc5009f043349cb5

(Automation Creations 2010).

Same weight fractions also in the technical bulletin from

http://www.specialmetals.com/products/inconelalloy625.php.

Weight fractions for $\mathrm{Cr}, \mathrm{Nb}$, and $\mathrm{Mo}$ set at the average of the allowed range. Weight fraction for Ni set at the minimum value of 0.58 . Weight fractions for C, Al, Si, P, S, TI, Mn, Fe, and Co set to be $99 \%$ of their upper limits so all weight fractions sum to unity.

\section{Inconel-718}

\begin{tabular}{|c|c|c|c|}
\hline Formula $=$ & - & Molecular weight $(\mathrm{g} / \mathrm{mole})=$ & - \\
\hline Density $(\mathrm{g} / \mathrm{cm} 3)=$ & 8.190000 & Total atom density $($ atoms $/ \mathrm{b}-\mathrm{cm})=$ & 8.547E-02 \\
\hline
\end{tabular}

\begin{tabular}{|c|c|c|c|c|c|c|}
\hline Element & Neutron ZA & Photon ZA & $\begin{array}{l}\text { Weight } \\
\text { Fraction }\end{array}$ & $\begin{array}{c}\text { Atom } \\
\text { Fraction }\end{array}$ & $\begin{array}{l}\text { Atom } \\
\text { Density }\end{array}$ & \\
\hline $\mathrm{B}$ & - & 5000 & 0.000050 & 0.000267 & 0.000023 & \\
\hline C & 6000 & 6000 & 0.000730 & 0.003507 & 0.000300 & \\
\hline $\mathrm{Al}$ & 13027 & 13000 & 0.005000 & 0.010694 & 0.000914 & \\
\hline $\mathrm{Si}$ & 14000 & 14000 & 0.003180 & 0.006534 & 0.000558 & \\
\hline $\mathrm{P}$ & 15031 & 15000 & 0.000140 & 0.000261 & 0.000022 & \\
\hline S & 16000 & 16000 & 0.000140 & 0.000252 & 0.000022 & \\
\hline $\mathrm{Ti}$ & 22000 & 22000 & 0.009000 & 0.010850 & 0.000927 & \\
\hline $\mathrm{Cr}$ & 24000 & 24000 & 0.190000 & 0.210871 & 0.018023 & \\
\hline $\mathrm{Mn}$ & 25055 & 25000 & 0.003180 & 0.003340 & 0.000285 & \\
\hline $\mathrm{Fe}$ & 26000 & 26000 & 0.170000 & 0.175671 & 0.015014 & \\
\hline $\mathrm{Ni}$ & 28000 & 28000 & 0.525000 & 0.516184 & 0.044117 & \\
\hline Co & 27059 & 27000 & 0.009100 & 0.008911 & 0.000762 & \\
\hline $\mathrm{Cu}$ & 29000 & 29000 & 0.002730 & 0.002479 & 0.000212 & \\
\hline $\mathrm{Nb}$ & 41093 & 41000 & 0.051250 & 0.031833 & 0.002721 & \\
\hline Mo & 42000 & 42000 & 0.030500 & 0.018346 & 0.001568 & \\
\hline Total & & & 1.000000 & 1.000000 & 0.085467 & \\
\hline MCNP Form & \multicolumn{2}{|c|}{ Weight Fractions } & \multicolumn{2}{|c|}{ Atom Fractions } & \multicolumn{2}{|c|}{ Atom Densities } \\
\hline \multirow[t]{10}{*}{ Neutrons } & - & -0.000050 & - & 0.000267 & - & 0.000023 \\
\hline & 6000 & -0.000730 & 6000 & 0.003507 & 6000 & 0.000300 \\
\hline & 13027 & -0.005000 & 13027 & 0.010694 & 13027 & 0.000914 \\
\hline & 14000 & -0.003180 & 14000 & 0.006534 & 14000 & 0.000558 \\
\hline & 15031 & -0.000140 & 15031 & 0.000261 & 15031 & 0.000022 \\
\hline & 16000 & -0.000140 & 16000 & 0.000252 & 16000 & 0.000022 \\
\hline & 22000 & -0.009000 & 22000 & 0.010850 & 22000 & 0.000927 \\
\hline & 24000 & -0.190000 & 24000 & 0.210871 & 24000 & 0.018023 \\
\hline & 25055 & -0.003180 & 25055 & 0.003340 & 25055 & 0.000285 \\
\hline & 26000 & -0.170000 & 26000 & 0.175671 & 26000 & 0.015014 \\
\hline
\end{tabular}


PIET-43741-TM-963

PNNL-15870 Rev. 1

\begin{tabular}{|c|c|c|c|c|c|c|}
\hline & 28000 & -0.525000 & 28000 & 0.516184 & 28000 & 0.044117 \\
\hline & 27059 & -0.009100 & 27059 & 0.008911 & 27059 & 0.000762 \\
\hline & 29000 & -0.002730 & 29000 & 0.002479 & 29000 & 0.000212 \\
\hline & 41093 & -0.051250 & 41093 & 0.031833 & 41093 & 0.002721 \\
\hline & 42000 & -0.030500 & 42000 & 0.018346 & 42000 & 0.001568 \\
\hline Photons & 5000 & -0.000050 & 5000 & 0.000267 & 5000 & 0.000023 \\
\hline & 6000 & -0.000730 & 6000 & 0.003507 & 6000 & 0.000300 \\
\hline & 13000 & -0.005000 & 13000 & 0.010694 & 13000 & 0.000914 \\
\hline & 14000 & -0.003180 & 14000 & 0.006534 & 14000 & 0.000558 \\
\hline & 15000 & -0.000140 & 15000 & 0.000261 & 15000 & 0.000022 \\
\hline & 16000 & -0.000140 & 16000 & 0.000252 & 16000 & 0.000022 \\
\hline & 22000 & -0.009000 & 22000 & 0.010850 & 22000 & 0.000927 \\
\hline & 24000 & -0.190000 & 24000 & 0.210871 & 24000 & 0.018023 \\
\hline & 25000 & -0.003180 & 25000 & 0.003340 & 25000 & 0.000285 \\
\hline & 26000 & -0.170000 & 26000 & 0.175671 & 26000 & 0.015014 \\
\hline & 28000 & -0.525000 & 28000 & 0.516184 & 28000 & 0.044117 \\
\hline & 27000 & -0.009100 & 27000 & 0.008911 & 27000 & 0.000762 \\
\hline & 29000 & -0.002730 & 29000 & 0.002479 & 29000 & 0.000212 \\
\hline & 41000 & -0.051250 & 41000 & 0.031833 & 41000 & 0.002721 \\
\hline & 42000 & -0.030500 & 42000 & 0.018346 & 42000 & 0.001568 \\
\hline CEPXS Form: & material & B & 0.000050 & & & \\
\hline & & $\mathrm{C}$ & 0.000730 & & & \\
\hline & & $\mathrm{Al}$ & 0.005000 & & & \\
\hline & & $\mathrm{Si}$ & 0.003180 & & & \\
\hline & & $\mathrm{P}$ & 0.000140 & & & \\
\hline & & $S$ & 0.000140 & & & \\
\hline & & $\mathrm{Ti}$ & 0.009000 & & & \\
\hline & & $\mathrm{Cr}$ & 0.190000 & & & \\
\hline & & $\mathrm{Mn}$ & 0.003180 & & & \\
\hline & & $\mathrm{Fe}$ & 0.170000 & & & \\
\hline & & $\mathrm{Ni}$ & 0.525000 & & & \\
\hline & & Co & 0.009100 & & & \\
\hline & & $\mathrm{Cu}$ & 0.002730 & & & \\
\hline & & $\mathrm{Nb}$ & 0.051250 & & & \\
\hline & & Mo & 0.030500 & & & \\
\hline & $\begin{array}{c}\text { matname } \\
\text { density }\end{array}$ & $\begin{array}{c}\text { Inconel-718 } \\
8.190000\end{array}$ & & & & \\
\hline $\begin{array}{l}\text { Comments anc } \\
\text { Density and we } \\
\text { http://www.matv } \\
\text { (Automation Cr } \\
\text { Same weight fra } \\
\text { http://www.spec } \\
\text { Weight fractions } \\
\text { fractions for B, } \\
\text { to unity. }\end{array}$ & $\begin{array}{l}\text { References } \\
\text { t fractions } \\
\text { b.com/sear } \\
\text { tions 2010) } \\
\text { ions also in } \\
\text { Imetals.con } \\
\text { or Al, Ti, Cr } \\
\text { Si, P, S, M }\end{array}$ & $\begin{array}{l}\text { D } \\
\text { DataSheet.as } \\
\text { e technical bu } \\
\text { roducts/incon } \\
\text { e, } \mathrm{Ni}, \mathrm{Nb} \text {, and } \\
\mathrm{Co} \text {, and } \mathrm{Cu} \text { se }\end{array}$ & $\begin{array}{l}\text { ?atGUID } \\
\text { in from } \\
\text { loy } 718 . p h p \\
\text { set at the } \\
\text { be } 91 \% \text { of }\end{array}$ & $\begin{array}{l}50 a 2 d 2090 \\
\text { age of the a } \\
r \text { upper limit }\end{array}$ & $\begin{array}{l}\text { d range. } \\
\text { all weigh }\end{array}$ & $\begin{array}{l}36134 \\
\text { ght } \\
\text { ions sum }\end{array}$ \\
\hline
\end{tabular}




\section{Indium}

\begin{tabular}{llll}
\hline Formula $=$ & In & Molecular weight $(\mathrm{g} / \mathrm{mole})=$ & 114.818 \\
Density $(\mathrm{g} / \mathrm{cm} 3)=$ & 7.310000 & Total atom density $($ atoms $/ \mathrm{b}-\mathrm{cm})=$ & $3.834 \mathrm{E}-02$
\end{tabular}

The above density is estimated to be accurate to 3 significant digits. Uncertainties are not addressed.

The following data was calculated from the input formula.

\begin{tabular}{|c|c|c|c|c|c|c|}
\hline$\frac{\text { Element }}{\ln }$ & $\frac{\text { Neutron ZA }}{49000}$ & $\frac{\text { Photon ZA }}{49000}$ & $\begin{array}{l}\text { Weight } \\
\text { Fraction } \\
1.000000\end{array}$ & $\begin{array}{c}\text { Atom } \\
\frac{\text { Fraction }}{1.000000}\end{array}$ & $\begin{array}{c}\text { Atom } \\
\text { Density } \\
0.038341\end{array}$ & \\
\hline Total & & & 1.000000 & 1.000000 & 0.038341 & \\
\hline MCNP Form & \multicolumn{2}{|c|}{ Weight Fractions } & \multicolumn{2}{|c|}{ Atom Fractions } & \multicolumn{2}{|c|}{ Atom Densities } \\
\hline Neutrons & 49000 & -1.000000 & 49000 & 1.000000 & 49000 & 0.038341 \\
\hline Photons & 49000 & -1.000000 & 49000 & 1.000000 & 49000 & 0.038341 \\
\hline CEPXS Form: & $\begin{array}{l}\text { material } \\
\text { matname } \\
\text { density }\end{array}$ & $\begin{array}{c}\text { In } \\
\text { Indium } \\
7.310000\end{array}$ & 1.000000 & & & \\
\hline
\end{tabular}

\section{Iron}

\begin{tabular}{llll}
\hline Formula $=$ & Fe & Molecular weight $(\mathrm{g} / \mathrm{mole})=$ & 55.845 \\
Density $(\mathrm{g} / \mathrm{cm} 3)=$ & 7.874000 & Total atom density $($ atoms $/ \mathrm{b}-\mathrm{cm})=$ & $8.491 \mathrm{E}-02$
\end{tabular}

The above density is estimated to be accurate to 3 significant digits. Uncertainties are not addressed.

The following data was calculated from the input formula.

\begin{tabular}{|c|c|c|c|c|c|c|}
\hline$\frac{\text { Element }}{\mathrm{Fe}}$ & $\frac{\text { Neutron ZA }}{26000}$ & $\frac{\text { Photon ZA }}{26000}$ & $\begin{array}{l}\text { Weight } \\
\text { Fraction } \\
1.000000\end{array}$ & $\begin{array}{c}\text { Atom } \\
\text { Fraction } \\
1.000000\end{array}$ & $\begin{array}{c}\text { Atom } \\
\text { Density } \\
0.08491\end{array}$ & \\
\hline Total & & & 1.000000 & 1.000000 & 0.08491 & \\
\hline MCNP Form & \multicolumn{2}{|c|}{ Weight Fractions } & \multicolumn{2}{|c|}{ Atom Fractions } & \multicolumn{2}{|c|}{ Atom Densities } \\
\hline Neutrons & 26000 & -1.000000 & 26000 & 1.000000 & 26000 & 0.084911 \\
\hline Photons & 26000 & -1.000000 & 26000 & 1.000000 & 26000 & 0.084911 \\
\hline CEPXS Form: & $\begin{array}{l}\text { material } \\
\text { matname } \\
\text { density }\end{array}$ & \begin{tabular}{l}
\multicolumn{1}{c}{$\mathrm{Fe}$} \\
Iron \\
7.874000
\end{tabular} & 1.000000 & & & \\
\hline
\end{tabular}


Comments and References

Density from http://physics.nist.gov/cgi-bin/Star/compos.pl?matno=026 (NIST 1998).

\section{Iron Boride (Fe2B)}

\begin{tabular}{lllc}
\hline Formula $=$ & Fe2B & Molecular weight $(\mathrm{g} / \mathrm{mole})=$ & 122.501 \\
Density $(\mathrm{g} / \mathrm{cm} 3)=$ & 7.300000 & Total atom density $($ atoms $/ \mathrm{b}-\mathrm{cm})=$ & $1.077 \mathrm{E}-01$ \\
The above density is estimated to be accurate to & 3 significant digits. Uncertainties are not addressed. \\
The following data was calculated from the input formula.
\end{tabular}

\begin{tabular}{|c|c|c|c|c|c|}
\hline Element & Neutron ZA & Photon ZA & $\begin{array}{l}\text { Weight } \\
\text { Fraction }\end{array}$ & $\begin{array}{c}\text { Atom } \\
\text { Fraction }\end{array}$ & $\begin{array}{l}\text { Atom } \\
\text { Density }\end{array}$ \\
\hline$B$ & - & 5000 & $\overline{0.088252}$ & $\overline{0.333333}$ & 0.035887 \\
\hline $\mathrm{Fe}$ & 26000 & 26000 & 0.911748 & 0.666667 & 0.071774 \\
\hline Total & & & 1.000000 & 1.000000 & 0.107660 \\
\hline
\end{tabular}

\begin{tabular}{|ccccccc|}
\hline MCNP Form & \multicolumn{2}{c|}{ Weight Fractions } & \multicolumn{2}{c|}{ Atom Fractions } & \multicolumn{2}{c|}{ Atom Densities } \\
\hline Neutrons & - & -0.088252 & - & 0.333333 & - & 0.035887 \\
& 26000 & -0.911748 & 26000 & 0.666667 & 26000 & 0.071774 \\
Photons & & & & & & \\
& 5000 & -0.088252 & 5000 & 0.333333 & 5000 & 0.035887 \\
& 26000 & -0.911748 & 26000 & 0.666667 & 26000 & 0.071774 \\
\hline CEPXS Form: & material & B & & & & \\
& & Fe & 0.088252 & & & \\
& & & & & &
\end{tabular}

matname Iron Boride (Fe2B)

density $\quad 7.300000$

Comments and References

Formula for iron boride can be FeB or Fe2B. See "Iron boride (FeB)" for naming conventions.

Density for $\mathrm{Fe} 2 \mathrm{~B}=7.30 \mathrm{~g} / \mathrm{cm} 3$ from

http://www.matweb.com/search/DataSheet.aspx?MatGUID=b9dbb726fb444cf4b6fcde21039e98bd (Automation Creations 2010), and from pgs 4 - 68 of Lide (2008).

\section{Iron Boride (FeB)}

\begin{tabular}{llll}
\hline Formula $=$ & FeB & Molecular weight $(\mathrm{g} / \mathrm{mole})=$ & 66.656 \\
Density $(\mathrm{g} / \mathrm{cm} 3)=$ & 7.150000 & Total atom density $($ atoms $/ \mathrm{b}-\mathrm{cm})=$ & $1.292 \mathrm{E}-01$
\end{tabular}

The above density is estimated to be accurate to 3 significant digits. Uncertainties are not addressed.

The following data were calculated from the input weight fractions.

\begin{tabular}{|c|c|c|c|c|c|}
\hline Element & Neutron ZA & Photon ZA & $\begin{array}{l}\text { Weight } \\
\text { Fraction }\end{array}$ & $\begin{array}{c}\text { Atom } \\
\text { Fraction }\end{array}$ & $\begin{array}{c}\text { Atom } \\
\text { Density }\end{array}$ \\
\hline$B$ & & 5000 & $\overline{0.162174}$ & $\overline{0.499969}$ & 0.064591 \\
\hline $\mathrm{Fe}$ & 26000 & 26000 & 0.837826 & 0.500031 & 0.064599 \\
\hline
\end{tabular}


PIET-43741-TM-963

PNNL-15870 Rev. 1

\begin{tabular}{|c|c|c|c|c|c|c|}
\hline \multicolumn{3}{|l|}{ Total } & 1.000000 & 1.000000 & \multicolumn{2}{|c|}{0.129190} \\
\hline MCNP Form & \multicolumn{2}{|c|}{ Weight Fractions } & \multicolumn{2}{|c|}{ Atom Fractions } & \multicolumn{2}{|c|}{ Atom Densities } \\
\hline \multirow{2}{*}{ Neutrons } & - & -0.162174 & - & 0.499969 & - & 0.064591 \\
\hline & 26000 & -0.837826 & 26000 & 0.500031 & 26000 & 0.064599 \\
\hline \multirow[t]{2}{*}{ Photons } & 5000 & -0.162174 & 5000 & 0.499969 & 5000 & 0.064591 \\
\hline & 26000 & -0.837826 & 26000 & 0.500031 & 26000 & 0.064599 \\
\hline \multirow[t]{3}{*}{ CEPXS Form: } & material & $B$ & 0.162174 & & & \\
\hline & & $\mathrm{Fe}$ & 0.837826 & & & \\
\hline & $\begin{array}{c}\text { matname } \\
\text { density }\end{array}$ & $\begin{array}{c}\text { Iron Boride } \\
7.150000\end{array}$ & & & & \\
\hline \multicolumn{7}{|c|}{$\begin{array}{l}\text { Comments and References } \\
\text { FeB may be called ferroboride (http://physics.nist.gov/cgi-bin/Star/compos.pl?matno=158 at NIST 1998); } \\
\text { ferro boron (Table } 51.11 \text { of Hungerford 1960); or iron boride, but iron boride may also refer to Fe2B (see } \\
\text { Automation Creations [2010] and Lide [2008]). } \\
\text { Weight fractions are from http://physics.nist.gov/cgi-bin/Star/compos.pl?matno=158 (NIST 1998). These } \\
\text { weight fractions agree with a composition of FeB. } \\
\text { Density of FeB }=7.15 \mathrm{~g} / \mathrm{cm} 3 \text { is from http://physics.nist.gov/cgi-bin/Star/compos.pl?matno=158 } \\
\text { (NIST 1998). Density of FeB } 7 \mathrm{~g} / \mathrm{cm} 3 \text { on pgs } 4-68 \text { of Lide (2008). Density of FeB }=7.00 \text { at } \\
\text { http://www.matweb.com/search/DataSheet.aspx?MatGUID=89e73550b5174b00b7cc66d117501ec8\&ckck } \\
=1 \text { (Automation Creations 2010). }\end{array}$} \\
\hline
\end{tabular}

\section{Iron, Armco Ingot}

\begin{tabular}{lll}
\hline Formula $=$ & - & Molecular weight $(\mathrm{g} / \mathrm{mole})=$ \\
Density $(\mathrm{g} / \mathrm{cm} 3)=$ & 7.866000 & Total atom density $($ atoms $/ \mathrm{b}-\mathrm{cm})=$ \\
\hline
\end{tabular}

The above density is estimated to be accurate to 3 significant digits. Uncertainties are not addressed.

The following data were calculated from the input weight fractions.

\begin{tabular}{|c|c|c|c|c|c|c|}
\hline Element & Neutron ZA & Photon ZA & $\begin{array}{l}\text { Weight } \\
\text { Fraction }\end{array}$ & $\begin{array}{c}\text { Atom } \\
\text { Fraction }\end{array}$ & $\begin{array}{l}\text { Atom } \\
\text { Density }\end{array}$ & \\
\hline $\mathrm{C}$ & 6000 & 6000 & 0.000120 & $\overline{0.000556}$ & 0.000047 & \\
\hline 0 & 8016 & 8000 & 0.001100 & 0.003826 & 0.000326 & \\
\hline$P$ & 15031 & 15000 & 0.000050 & 0.000090 & 0.000008 & \\
\hline$S$ & 16000 & 16000 & 0.000250 & 0.000434 & 0.000037 & \\
\hline $\mathrm{Mn}$ & 25055 & 25000 & 0.000170 & 0.000172 & 0.000015 & \\
\hline $\mathrm{Fe}$ & 26000 & 26000 & 0.998310 & 0.994921 & 0.084681 & \\
\hline Total & & & 1.000000 & 1.000000 & 0.085113 & \\
\hline MCNP Form & \multicolumn{2}{|c|}{ Weight Fractions } & \multicolumn{2}{|c|}{ Atom Fractions } & \multicolumn{2}{|c|}{ Atom Densities } \\
\hline \multirow[t]{3}{*}{ Neutrons } & 6000 & -0.000120 & 6000 & 0.000556 & 6000 & 0.000047 \\
\hline & 8016 & -0.001100 & 8016 & 0.003826 & 8016 & 0.000326 \\
\hline & 15031 & -0.000050 & 15031 & 0.000090 & 15031 & 0.000008 \\
\hline
\end{tabular}


PIET-43741-TM-963

PNNL-15870 Rev. 1

\begin{tabular}{|c|c|c|c|c|c|c|}
\hline & 16000 & -0.000250 & 16000 & 0.000434 & 16000 & 0.000037 \\
\hline & 25055 & -0.000170 & 25055 & 0.000172 & 25055 & 0.000015 \\
\hline & 26000 & -0.998310 & 26000 & 0.994921 & 26000 & 0.084681 \\
\hline Photons & 6000 & -0.000120 & 6000 & 0.000556 & 6000 & 0.000047 \\
\hline & 8000 & -0.001100 & 8000 & 0.003826 & 8000 & 0.000326 \\
\hline & 15000 & -0.000050 & 15000 & 0.000090 & 15000 & 0.000008 \\
\hline & 16000 & -0.000250 & 16000 & 0.000434 & 16000 & 0.000037 \\
\hline & 25000 & -0.000170 & 25000 & 0.000172 & 25000 & 0.000015 \\
\hline & 26000 & -0.998310 & 26000 & 0.994921 & 26000 & 0.084681 \\
\hline CEPXS Form: & material & $\mathrm{C}$ & 0.000120 & & & \\
\hline & & $\mathrm{O}$ & 0.001100 & & & \\
\hline & & $P$ & 0.000050 & & & \\
\hline & & $S$ & 0.000250 & & & \\
\hline & & $\mathrm{Mn}$ & 0.000170 & & & \\
\hline & & $\mathrm{Fe}$ & 0.998310 & & & \\
\hline & $\begin{array}{c}\text { matname } \\
\text { density }\end{array}$ & $\begin{array}{c}\text { Iron, Armco } \\
7.866000 \\
\end{array}$ & & & & \\
\hline $\begin{array}{l}\text { Comments an } \\
\text { Weight fraction }\end{array}$ & $\begin{array}{l}\text { eferences } \\
\text { om Table } 5\end{array}$ & & & & & \\
\hline
\end{tabular}

\section{Iron, Cast (Gray)}

\begin{tabular}{|c|c|c|c|}
\hline Formula $=$ & - & Molecular weight $(\mathrm{g} / \mathrm{mole})=$ & - \\
\hline Density $(\mathrm{g} / \mathrm{cm} 3)=$ & 7.150000 & Total atom density $($ atoms $/ \mathrm{b}-\mathrm{cm})=$ & 8.890E-02 \\
\hline
\end{tabular}

\begin{tabular}{|c|c|c|c|c|c|c|}
\hline Element & Neutron ZA & Photon ZA & $\begin{array}{l}\text { Weight } \\
\text { Fraction }\end{array}$ & $\begin{array}{c}\text { Atom } \\
\text { Fraction }\end{array}$ & $\begin{array}{l}\text { Atom } \\
\text { Density }\end{array}$ & \\
\hline C & 6000 & 6000 & 0.034000 & 0.137104 & 0.012189 & \\
\hline $\mathrm{Si}$ & 14000 & 14000 & 0.026000 & 0.044836 & 0.003986 & \\
\hline $\mathrm{P}$ & 15031 & 15000 & 0.003000 & 0.004691 & 0.000417 & \\
\hline$S$ & 16000 & 16000 & 0.001000 & 0.001510 & 0.000134 & \\
\hline $\mathrm{Mn}$ & 25055 & 25000 & 0.006500 & 0.005730 & 0.000509 & \\
\hline $\mathrm{Fe}$ & 26000 & 26000 & 0.929500 & 0.806128 & 0.071667 & \\
\hline Total & & & 1.000000 & 1.000000 & 0.088903 & \\
\hline MCNP Form & \multicolumn{2}{|c|}{ Weight Fractions } & \multicolumn{2}{|c|}{ Atom Fractions } & \multicolumn{2}{|c|}{ Atom Densities } \\
\hline \multirow[t]{6}{*}{ Neutrons } & 6000 & -0.034000 & 6000 & 0.137104 & 6000 & 0.012189 \\
\hline & 14000 & -0.026000 & 14000 & 0.044836 & 14000 & 0.003986 \\
\hline & 15031 & -0.003000 & 15031 & 0.004691 & 15031 & 0.000417 \\
\hline & 16000 & -0.001000 & 16000 & 0.001510 & 16000 & 0.000134 \\
\hline & 25055 & -0.006500 & 25055 & 0.005730 & 25055 & 0.000509 \\
\hline & 26000 & -0.929500 & 26000 & 0.806128 & 26000 & 0.071667 \\
\hline
\end{tabular}


PIET-43741-TM-963

PNNL-15870 Rev. 1

\begin{tabular}{|c|c|c|c|c|c|c|}
\hline \multirow[t]{6}{*}{ Photons } & 6000 & -0.034000 & 6000 & 0.137104 & 6000 & 0.012189 \\
\hline & 14000 & -0.026000 & 14000 & 0.044836 & 14000 & 0.003986 \\
\hline & 15000 & -0.003000 & 15000 & 0.004691 & 15000 & 0.000417 \\
\hline & 16000 & -0.001000 & 16000 & 0.001510 & 16000 & 0.000134 \\
\hline & 25000 & -0.006500 & 25000 & 0.005730 & 25000 & 0.000509 \\
\hline & 26000 & -0.929500 & 26000 & 0.806128 & 26000 & 0.071667 \\
\hline \multirow[t]{7}{*}{ CEPXS Form: } & material & $\mathrm{C}$ & 0.034000 & & & \\
\hline & & $\mathrm{Si}$ & 0.026000 & & & \\
\hline & & $\mathrm{P}$ & 0.003000 & & & \\
\hline & & $S$ & 0.001000 & & & \\
\hline & & $\mathrm{Mn}$ & 0.006500 & & & \\
\hline & & $\mathrm{Fe}$ & 0.929500 & & & \\
\hline & $\begin{array}{l}\text { matname } \\
\text { density }\end{array}$ & \multicolumn{5}{|c|}{ Iron, Cast (Gray) } \\
\hline $\begin{array}{l}\text { Comments and References } \\
\text { Weight fractions from Table } 51.40 \text {, and density from Table } 51.41 \text { of Hungerford (1960). }\end{array}$ & \multicolumn{6}{|c|}{ Comments and References } \\
\hline
\end{tabular}

\section{Iron, Wrought (Byers No. 1)}

\begin{tabular}{llll}
\hline Formula $=$ & - & Molecular weight $(\mathrm{g} / \mathrm{mole})=$ \\
Density $(\mathrm{g} / \mathrm{cm} 3)=$ & 7.700000 & Total atom density $($ atoms $/ \mathrm{b}-\mathrm{cm})=$ & - \\
\hline & $3.346 \mathrm{E}-02$
\end{tabular}

The above density is estimated to be accurate to 3 significant digits. Uncertainties are not addressed.

The following data were calculated from the input weight fractions.

\begin{tabular}{|cccccccc} 
Element & Neutron ZA & Photon ZA & $\begin{array}{c}\text { Weight } \\
\text { Fraction }\end{array}$ & $\begin{array}{c}\text { Atom } \\
\text { Fraction }\end{array}$ & $\begin{array}{c}\text { Atom } \\
\text { Density }\end{array}$ & \\
C & 6000 & 6000 & 0.000810 & 0.003746 & 0.000313 & \\
$\mathrm{Si}$ & 14000 & 14000 & 0.001599 & 0.003164 & 0.000264 & \\
$\mathrm{P}$ & 15031 & 15000 & 0.000628 & 0.001126 & 0.000094 & \\
$\mathrm{~S}$ & 16000 & 16000 & 0.000101 & 0.000175 & 0.000015 & \\
$\mathrm{Mn}$ & 25055 & 25000 & 0.000152 & 0.000154 & 0.000013 & \\
$\mathrm{Fe}$ & 26000 & 26000 & 0.996711 & 0.991636 & 0.082761 & \\
& & & & & & \\
Total & & & & & & \\
& & & & & & & \\
MCNP Form & Weight Fractions & Atom Fractions & Atom Densities \\
\hline Neutrons & 6000 & -0.000810 & 6000 & 0.003746 & 6000 & 0.000313 \\
& 14000 & -0.001599 & 14000 & 0.003164 & 14000 & 0.000264 \\
& 15031 & -0.000628 & 15031 & 0.001126 & 15031 & 0.000094 \\
& 16000 & -0.000101 & 16000 & 0.000175 & 16000 & 0.000015 \\
& 25055 & -0.000152 & 25055 & 0.000154 & 25055 & 0.000013 \\
& 26000 & -0.996711 & 26000 & 0.991636 & 26000 & 0.082761 \\
Photons & 6000 & -0.000810 & 6000 & 0.003746 & 6000 & 0.000313 \\
& 14000 & -0.001599 & 14000 & 0.003164 & 14000 & 0.000264
\end{tabular}


PIET-43741-TM-963

PNNL-15870 Rev. 1

\begin{tabular}{|c|c|c|c|c|c|c|}
\hline & $\begin{array}{l}15000 \\
16000 \\
25000 \\
26000\end{array}$ & $\begin{array}{l}-0.000628 \\
-0.000101 \\
-0.000152 \\
-0.996711\end{array}$ & $\begin{array}{l}15000 \\
16000 \\
25000 \\
26000\end{array}$ & $\begin{array}{l}0.001126 \\
0.000175 \\
0.000154 \\
0.991636\end{array}$ & $\begin{array}{l}15000 \\
16000 \\
25000 \\
26000\end{array}$ & $\begin{array}{l}0.000094 \\
0.000015 \\
0.000013 \\
0.082761\end{array}$ \\
\hline CEPXS Form: & $\begin{array}{c}\text { matname } \\
\text { density }\end{array}$ & $\begin{array}{c}\mathrm{C} \\
\mathrm{Si} \\
\mathrm{P} \\
\mathrm{S} \\
\mathrm{Mn} \\
\mathrm{Fe} \\
\\
\text { Iron, Wrough } \\
7.700000\end{array}$ & $\begin{array}{l}0.000810 \\
0.001599 \\
0.000628 \\
0.000101 \\
0.000152 \\
0.996711 \\
\end{array}$ & & & \\
\hline \multicolumn{7}{|c|}{$\begin{array}{l}\text { Comments and References } \\
\text { Weight fractions from Table } 51.40 \text {, and density from Table } 51.41 \text { of Hungerford (1960). Table } 51.40 \text { lists } \\
1.2 \text { wt. \% as slag. This was omitted since it is not specified what elements are in slag. The weight } \\
\text { fractions in the table were then divided by } 0.988 \text { so the weight fractions would sum to unity. }\end{array}$} \\
\hline
\end{tabular}

\section{Kaowool}

\begin{tabular}{llll}
\hline Formula $=$ & - & Molecular weight $(\mathrm{g} / \mathrm{mole})=$ \\
Density $(\mathrm{g} / \mathrm{cm} 3)=$ & 0.096000 & Total atom density $($ atoms $/ \mathrm{b}-\mathrm{cm})=$ & - \\
\hline
\end{tabular}

The above density is estimated to be accurate to 3 significant digits. Uncertainties are not addressed.

The following data were calculated from the input weight fractions.

\begin{tabular}{|c|c|c|c|c|c|c|}
\hline Element & Neutron ZA & Photon ZA & $\begin{array}{l}\text { Weight } \\
\text { Fraction }\end{array}$ & $\begin{array}{c}\text { Atom } \\
\text { Fraction }\end{array}$ & $\begin{array}{c}\text { Atom } \\
\text { Density }\end{array}$ & \\
\hline $\mathrm{B}$ & - & 5000 & 0.000248 & 0.000468 & 0.000001 & \\
\hline $\mathrm{O}$ & 8016 & 8000 & 0.500064 & 0.636102 & 0.001807 & \\
\hline $\mathrm{Al}$ & 13027 & 13000 & 0.238163 & 0.179644 & 0.000510 & \\
\hline $\mathrm{Si}$ & 14000 & 14000 & 0.243627 & 0.176542 & 0.000501 & \\
\hline $\mathrm{Ca}$ & 20000 & 20000 & 0.000715 & 0.000363 & 0.000001 & \\
\hline $\mathrm{Ti}$ & 22000 & 22000 & 0.010189 & 0.004332 & 0.000012 & \\
\hline $\mathrm{Fe}$ & 26000 & 26000 & 0.006994 & 0.002549 & 0.000007 & \\
\hline Total & & & 1.000000 & 1.000000 & 0.002841 & \\
\hline MCNP Form & \multicolumn{2}{|c|}{ Weight Fractions } & \multicolumn{2}{|c|}{ Atom Fractions } & \multicolumn{2}{|c|}{ Atom Densities } \\
\hline \multirow[t]{7}{*}{ Neutrons } & - & -0.000248 & - & 0.000468 & - & 0.000001 \\
\hline & 8016 & -0.500064 & 8016 & 0.636102 & 8016 & 0.001807 \\
\hline & 13027 & -0.238163 & 13027 & 0.179644 & 13027 & 0.000510 \\
\hline & 14000 & -0.243627 & 14000 & 0.176542 & 14000 & 0.000501 \\
\hline & 20000 & -0.000715 & 20000 & 0.000363 & 20000 & 0.000001 \\
\hline & 22000 & -0.010189 & 22000 & 0.004332 & 22000 & 0.000012 \\
\hline & 26000 & -0.006994 & 26000 & 0.002549 & 26000 & 0.000007 \\
\hline
\end{tabular}


PIET-43741-TM-963

PNNL-15870 Rev. 1

\begin{tabular}{|c|c|c|c|c|c|c|}
\hline \multirow[t]{7}{*}{ Photons } & 5000 & -0.000248 & 5000 & 0.000468 & 5000 & 0.000001 \\
\hline & 8000 & -0.500064 & 8000 & 0.636102 & 8000 & 0.001807 \\
\hline & 13000 & -0.238163 & 13000 & 0.179644 & 13000 & 0.000510 \\
\hline & 14000 & -0.243627 & 14000 & 0.176542 & 14000 & 0.000501 \\
\hline & 20000 & -0.000715 & 20000 & 0.000363 & 20000 & 0.000001 \\
\hline & 22000 & -0.010189 & 22000 & 0.004332 & 22000 & 0.000012 \\
\hline & 26000 & -0.006994 & 26000 & 0.002549 & 26000 & 0.000007 \\
\hline \multirow[t]{8}{*}{ CEPXS Form: } & material & $B$ & 0.000248 & & & \\
\hline & & O & 0.500064 & & & \\
\hline & & $\mathrm{Al}$ & 0.238163 & & & \\
\hline & & $\mathrm{Si}$ & 0.243627 & & & \\
\hline & & $\mathrm{Ca}$ & 0.000715 & & & \\
\hline & & $\mathrm{Ti}$ & 0.010189 & & & \\
\hline & & $\mathrm{Fe}$ & 0.006994 & & & \\
\hline & $\begin{array}{c}\text { matname } \\
\text { density }\end{array}$ & $\begin{array}{l}\text { Kaowool } \\
0.096000\end{array}$ & & & & \\
\hline \multicolumn{7}{|c|}{$\begin{array}{l}\text { Comments and References } \\
\text { Fibers in a Kaowool insulating blanket are made from Kaolinite, which is a naturally occurring clay mineral. } \\
\text { The density of the Kaolinite fibers is } 2.65 \mathrm{~g} / \mathrm{cm} 3 \text { on pg II.F.1-4 of Carter et al. (1968). The density of the } \\
\text { mineral is given as } 2.16-2.68 \mathrm{~g} / \mathrm{cm} 3 \text { at http://en.wikipedia.org/wiki/Kaolin, and } 2.6 \mathrm{at} \\
\text { http://www.galleries.com/Minerals/By_Name.htm. The density of the fibers }(2.65 \mathrm{~g} / \mathrm{cm} 3) \text { must be } \\
\text { multiplied by the volume fraction of the fibers to get the bulk density of the blanket. Bulk densities range } \\
\text { from } 0.048 \text { to } 0.192 \mathrm{~g} / \mathrm{cm} 3 \text { for five examples at } \\
\text { http://www.matweb.com/search/DataSheet.aspx?MatGUID=cb830e74bc69422aa560a7b57494955a } \\
\text { (Automation Creations } 2010) \text {. } \\
\text { Density }=0.096 \mathrm{~g} / \mathrm{cm} 3 \text { for one example of a Kaowool blanket at this reference, and this value is the } \\
\text { closest to the average of the five values. This reference also gives the composition for a Kaowool blanket. }\end{array}$} \\
\hline
\end{tabular}

\section{Kapton Polyimide Film}

\begin{tabular}{llll}
\hline Formula $=$ & - & Molecular weight $(\mathrm{g} / \mathrm{mole})=$ \\
Density $(\mathrm{g} / \mathrm{cm} 3)=$ & 1.420000 & Total atom density $($ atoms $/ \mathrm{b}-\mathrm{cm})=$ & - \\
\hline & $8.723 \mathrm{E}-02$
\end{tabular}

The above density is estimated to be accurate to 3 significant digits. Uncertainties are not addressed.

The following data were calculated from the input weight fractions.

\begin{tabular}{|c|c|c|c|c|c|c|}
\hline Element & Neutron ZA & Photon ZA & $\begin{array}{l}\text { Weight } \\
\text { Fraction }\end{array}$ & $\begin{array}{c}\text { Atom } \\
\text { Fraction }\end{array}$ & $\begin{array}{l}\text { Atom } \\
\text { Density }\end{array}$ & \\
\hline $\mathrm{H}$ & 1001 & 1000 & $\overline{0.026362}$ & $\overline{0.256399}$ & 0.022366 & \\
\hline C & 6000 & 6000 & 0.691133 & 0.564114 & 0.049208 & \\
\hline $\mathrm{N}$ & 7014 & 7000 & 0.073270 & 0.051282 & 0.004473 & \\
\hline $\mathrm{O}$ & 8016 & 8000 & 0.209235 & 0.128205 & 0.011183 & \\
\hline Total & & & 1.000000 & 1.000000 & 0.087230 & \\
\hline MCNP Form & \multicolumn{2}{|c|}{ Weight Fractions } & \multicolumn{2}{|c|}{ Atom Fractions } & Atom & sities \\
\hline Neutrons & 1001 & -0.026362 & 1001 & 0.256399 & 1001 & 0.022366 \\
\hline
\end{tabular}


PIET-43741-TM-963

PNNL-15870 Rev. 1

\begin{tabular}{|c|c|c|c|c|c|c|}
\hline & 7014 & -0.073270 & 7014 & 0.051282 & 7014 & 0.004473 \\
\hline & 8016 & -0.209235 & 8016 & 0.128205 & 8016 & 0.011183 \\
\hline Photons & 1000 & -0.026362 & 1000 & 0.256399 & 1000 & 0.022366 \\
\hline & 6000 & -0.691133 & 6000 & 0.564114 & 6000 & 0.049208 \\
\hline & 7000 & -0.073270 & 7000 & 0.051282 & 7000 & 0.004473 \\
\hline & 8000 & -0.209235 & 8000 & 0.128205 & 8000 & 0.011183 \\
\hline CEPXS Form: & material & $\mathrm{H}$ & 0.026362 & & & \\
\hline & & C & 0.691133 & & & \\
\hline & & $\mathrm{N}$ & 0.073270 & & & \\
\hline & & $\mathrm{O}$ & 0.209235 & & & \\
\hline & $\begin{array}{c}\text { matname } \\
\text { density }\end{array}$ & $\begin{array}{c}\text { Kapton Poly } \\
1.420000\end{array}$ & de Film & & & \\
\hline $\begin{array}{l}\text { Comments an } \\
\text { Density and } w\end{array}$ & $\begin{array}{l}\text { eferences } \\
\text { t fractions }\end{array}$ & & & & & 'Т 1998). \\
\hline
\end{tabular}

\section{Kennertium}

Formula $=$

Density $(\mathrm{g} / \mathrm{cm} 3)=\quad 16.800000 \quad$ Total atom density $($ atoms $/ \mathrm{b}-\mathrm{cm})=8.122 \mathrm{E}-02$

The above density is estimated to be accurate to 3 significant digits. Uncertainties are not addressed.

The following data were calculated from the input weight fractions.

\begin{tabular}{|c|c|c|c|c|c|c|}
\hline Element & Neutron ZA & Photon ZA & $\begin{array}{l}\text { Weight } \\
\text { Fraction }\end{array}$ & $\begin{array}{c}\text { Atom } \\
\text { Fraction }\end{array}$ & $\begin{array}{c}\text { Atom } \\
\text { Density }\end{array}$ & \\
\hline $\mathrm{Ni}$ & 28000 & 28000 & $\overline{0.090000}$ & $\overline{0.191007}$ & 0.015514 & \\
\hline $\mathrm{Cu}$ & 29000 & 29000 & 0.150000 & 0.294036 & 0.023882 & \\
\hline W & 74000 & 74000 & 0.760000 & 0.514957 & 0.041825 & \\
\hline Total & & & 1.000000 & 1.000000 & 0.081220 & \\
\hline MCNP Form & \multicolumn{2}{|c|}{ Weight Fractions } & \multicolumn{2}{|c|}{ Atom Fractions } & \multicolumn{2}{|c|}{ Atom Densities } \\
\hline \multirow[t]{3}{*}{ Neutrons } & 28000 & -0.090000 & 28000 & 0.191007 & 28000 & 0.015514 \\
\hline & 29000 & -0.150000 & 29000 & 0.294036 & 29000 & 0.023882 \\
\hline & 74000 & -0.760000 & 74000 & 0.514957 & 74000 & 0.041825 \\
\hline \multirow[t]{3}{*}{ Photons } & 28000 & -0.090000 & 28000 & 0.191007 & 28000 & 0.015514 \\
\hline & 29000 & -0.150000 & 29000 & 0.294036 & 29000 & 0.023882 \\
\hline & 74000 & -0.760000 & 74000 & 0.514957 & 74000 & 0.041825 \\
\hline \multirow[t]{4}{*}{ CEPXS Form: } & material & $\mathrm{Ni}$ & 0.090000 & & & \\
\hline & & $\mathrm{Cu}$ & 0.150000 & & & \\
\hline & & W & 0.760000 & & & \\
\hline & $\begin{array}{c}\text { matname } \\
\text { density }\end{array}$ & $\begin{array}{r}\text { Kennertium } \\
16.800000\end{array}$ & & & & \\
\hline
\end{tabular}


Comments and References

Kennertium is a high density tungsten alloy for shielding.

Data from pg 137 of Brewer (2009).

\section{Kernite}

\begin{tabular}{llll}
\hline Formula $=$ & Na2B4O7-4 $(\mathrm{H} 2 \mathrm{O})$ & Molecular weight $(\mathrm{g} / \mathrm{mole})=$ & 273.28046 \\
Density $(\mathrm{g} / \mathrm{cm} 3)=$ & 1.950000 & Total atom density $($ atoms $/ \mathrm{b}-\mathrm{cm})=$ & $1.074 \mathrm{E}-01$
\end{tabular}

The above density is estimated to be accurate to 3 significant digits. Uncertainties are not addressed.

The following data was calculated from the input formula.

\begin{tabular}{|c|c|c|c|c|c|}
\hline Element & Neutron ZA & Photon ZA & $\begin{array}{l}\text { Weight } \\
\text { Fraction }\end{array}$ & $\begin{array}{c}\text { Atom } \\
\text { Fraction }\end{array}$ & $\begin{array}{c}\text { Atom } \\
\text { Density }\end{array}$ \\
\hline $\mathrm{H}$ & 1001 & 1000 & $\overline{0.029506}$ & 0.320000 & 0.034377 \\
\hline B & - & 5000 & 0.158240 & 0.160000 & 0.017188 \\
\hline 0 & 8016 & 8000 & 0.644003 & 0.440000 & 0.047268 \\
\hline $\mathrm{Na}$ & 11023 & 11000 & 0.168250 & 0.080000 & 0.008594 \\
\hline Total & & & 1.000000 & 1.000000 & 0.107428 \\
\hline
\end{tabular}

\begin{tabular}{|c|c|c|c|c|c|c|}
\hline MCNP Form & \multicolumn{2}{|c|}{ Weight Fractions } & \multicolumn{2}{|c|}{ Atom Fractions } & \multicolumn{2}{|c|}{ Atom Densities } \\
\hline \multirow{4}{*}{ Neutrons } & 1001 & -0.029506 & 1001 & 0.320000 & 1001 & 0.034377 \\
\hline & - & -0.158240 & - & 0.160000 & - & 0.017188 \\
\hline & 8016 & -0.644003 & 8016 & 0.440000 & 8016 & 0.047268 \\
\hline & 11023 & -0.168250 & 11023 & 0.080000 & 11023 & 0.008594 \\
\hline \multirow[t]{4}{*}{ Photons } & 1000 & -0.029506 & 1000 & 0.320000 & 1000 & 0.034377 \\
\hline & 5000 & -0.158240 & 5000 & 0.160000 & 5000 & 0.017188 \\
\hline & 8000 & -0.644003 & 8000 & 0.440000 & 8000 & 0.047268 \\
\hline & 11000 & -0.168250 & 11000 & 0.080000 & 11000 & 0.008594 \\
\hline \multirow[t]{5}{*}{ CEPXS Form: } & material & $\mathrm{H}$ & 0.029506 & & & \\
\hline & & B & 0.158240 & & & \\
\hline & & O & 0.644003 & & & \\
\hline & & $\mathrm{Na}$ & 0.168250 & & & \\
\hline & $\begin{array}{c}\text { matname } \\
\text { density }\end{array}$ & $\begin{array}{l}\text { Kernite } \\
1.950000\end{array}$ & & & & \\
\hline $\begin{array}{l}\text { Comments an } \\
\text { Density and for } \\
\text { http://www.mat } \\
\text { (Automation Cr }\end{array}$ & $\begin{array}{l}\text { eferences } \\
\text { la from pg } \\
\text {.com/sear } \\
\text { ions 2010). }\end{array}$ & $\begin{array}{l}\text { 1-4 of Cart } \\
\text { DataSheet.a }\end{array}$ & $\begin{array}{l}\text { l. (1968). } \\
\text { MatGUID= }\end{array}$ & $\begin{array}{l}\text { sity }=1.9 \\
\text { b152b80f }\end{array}$ & $\begin{array}{l}\text { also at } \\
15157\end{array}$ & $\mathrm{fdO}$ \\
\hline
\end{tabular}




\section{Kerosene}

\begin{tabular}{llll}
\hline Formula $=$ & $\mathrm{C} 14 \mathrm{H} 30$ & Molecular weight $(\mathrm{g} / \mathrm{mole})=$ & 198.388 \\
Density $(\mathrm{g} / \mathrm{cm} 3)=$ & 0.819000 & Total atom density $($ atoms $/ \mathrm{b}-\mathrm{cm})=$ & $1.128 \mathrm{E}-01$
\end{tabular}

The above density is estimated to be accurate to 3 significant digits. Uncertainties are not addressed. The following data were calculated from the input weight fractions.

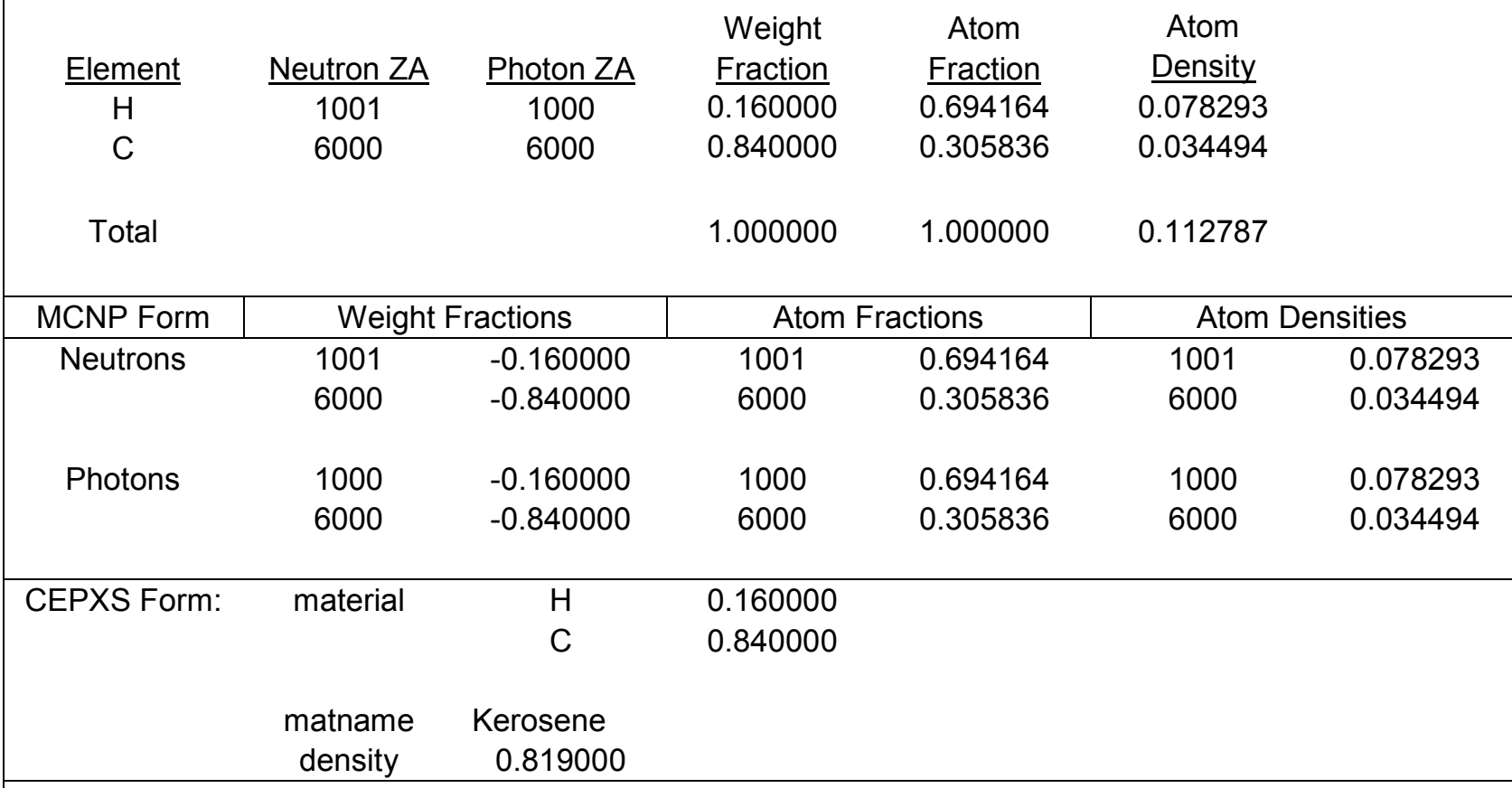

\section{Comments and References}

Kerosene is sometimes spelled kerosine. It is usually called paraffin (sometimes paraffin oil) in the United Kingdom, Southeast Asia, and South Africa. Kerosene is a clear liquid consisting of a mixture of hydrocarbons containing between 6 and 16 carbon atoms per molecule (Collins C. 2007. "Implementing Phytoremediation of Petroleum Hydrocarbons" in Methods in Biotechnology 23:99-108).

Average composition (near $\mathrm{C} 14 \mathrm{H} 30)$, weight fractions, and density $\left(0.819 \mathrm{~g} / \mathrm{cm} 3\right.$ at $\left.16^{\circ} \mathrm{C}\right)$ from Table 51.104 of Hungerford (1960). Density also $0.819 \mathrm{~g} / \mathrm{cm} 3$ in Table 7.1.8 of Avallone and Baumeister III (1996). Density = $0.817 \mathrm{~g} / \mathrm{cm} 3$ at http://www.simetric.co.uk/si_liquids.htm (Walker 2009). Density = 0.820 at http://www.engineeringtoolbox.com/liquids-densities-d_ $743 . \mathrm{html}$ and $0.810 \mathrm{~g} / \mathrm{cm} 3$ at http://physics.info/density/. Density $=0.77$ to $0.82 \mathrm{~g} / \mathrm{cm} 3$ in Table 7.4 of Speight (2001). Density $=0.775$ to $0.840 \mathrm{~g} / \mathrm{cm} 3$ for jet kerosene at http://www.matweb.com/search/DataSheet.aspx?MatGUID=a4d612fd553c4bcb94b117f5d4302d28 (Automation Creations 2010).

\section{Krypton}

$\begin{array}{llll}\text { Formula }= & \mathrm{Kr} & \text { Molecular weight }(\mathrm{g} / \mathrm{mole})= & 83.798 \\ \text { Density }(\mathrm{g} / \mathrm{cm} 3)= & 0.003478 & \text { Total atom density }(\text { atoms } / \mathrm{b}-\mathrm{cm})= & 2.500 \mathrm{E}-05\end{array}$

The above density is estimated to be accurate to 4 significant digits. Uncertainties are not addressed. The following data was calculated from the input formula. 


\begin{tabular}{|c|c|c|c|c|c|c|}
\hline$\frac{\text { Element }}{\mathrm{Kr}}$ & $\frac{\text { Neutron ZA }}{-}$ & $\frac{\text { Photon ZA }}{36000}$ & $\begin{array}{l}\text { Weight } \\
\text { Fraction } \\
1.000000\end{array}$ & $\begin{array}{c}\text { Atom } \\
\frac{\text { Fraction }}{1.000000}\end{array}$ & $\begin{array}{c}\text { Atom } \\
\text { Density } \\
0.000025\end{array}$ & \\
\hline Total & & & 1.000000 & 1.000000 & 0.000025 & \\
\hline MCNP Form & \multicolumn{2}{|c|}{ Weight Fractions } & \multicolumn{2}{|c|}{ Atom Fractions } & \multicolumn{2}{|c|}{ Atom Densities } \\
\hline Neutrons & - & -1.000000 & - & 1.000000 & - & 0.000025 \\
\hline Photons & 36000 & -1.000000 & 36000 & 1.000000 & 36000 & 0.000025 \\
\hline CEPXS Form: & $\begin{array}{l}\text { material } \\
\text { matname } \\
\text { density }\end{array}$ & $\begin{array}{c}\mathrm{Kr} \\
\text { Krypton } \\
0.003478\end{array}$ & 1.000000 & & & \\
\hline
\end{tabular}

\section{Kynar}

\begin{tabular}{llll}
\hline Formula $=$ & H2C2F2 & Molecular weight $(\mathrm{g} / \mathrm{mole})=$ & 64.0340864 \\
Density $(\mathrm{g} / \mathrm{cm} 3)=$ & 1.790000 & Total atom density $($ atoms $/ \mathrm{b}-\mathrm{cm})=$ & $1.010 \mathrm{E}-01$
\end{tabular}

The above density is estimated to be accurate to 3 significant digits. Uncertainties are not addressed.

The following data was calculated from the input formula.

\begin{tabular}{|c|c|c|c|c|c|c|}
\hline Element & Neutron ZA & Photon ZA & $\begin{array}{l}\text { Weight } \\
\text { Fraction }\end{array}$ & $\begin{array}{c}\text { Atom } \\
\text { Fraction }\end{array}$ & $\begin{array}{c}\text { Atom } \\
\text { Density }\end{array}$ & \\
\hline $\mathrm{H}$ & 1001 & 1000 & 0.031481 & 0.333333 & 0.03366 & \\
\hline C & 6000 & 6000 & 0.375135 & 0.333333 & 0.03366 & \\
\hline $\mathrm{F}$ & 9019 & 9000 & 0.593384 & 0.333333 & 0.03366 & \\
\hline Total & & & 1.000000 & 1.000000 & 0.10100 & \\
\hline MCNP Form & \multicolumn{2}{|c|}{ Weight Fractions } & \multicolumn{2}{|c|}{ Atom Fractions } & \multicolumn{2}{|c|}{ Atom Densities } \\
\hline \multirow[t]{3}{*}{ Neutrons } & 1001 & -0.031481 & 1001 & 0.333333 & 1001 & 0.033668 \\
\hline & 6000 & -0.375135 & 6000 & 0.333333 & 6000 & 0.033668 \\
\hline & 9019 & -0.593384 & 9019 & 0.333333 & 9019 & 0.033668 \\
\hline \multirow[t]{3}{*}{ Photons } & 1000 & -0.031481 & 1000 & 0.333333 & 1000 & 0.033668 \\
\hline & 6000 & -0.375135 & 6000 & 0.333333 & 6000 & 0.033668 \\
\hline & 9000 & -0.593384 & 9000 & 0.333333 & 9000 & 0.033668 \\
\hline \multirow[t]{4}{*}{ CEPXS Form: } & material & $\mathrm{H}$ & 0.031481 & & & \\
\hline & & C & 0.375135 & & & \\
\hline & & $\mathrm{F}$ & 0.593384 & & & \\
\hline & $\begin{array}{l}\text { matname } \\
\text { density }\end{array}$ & $\begin{array}{l}\text { Kynar } \\
1.790000\end{array}$ & & & & \\
\hline
\end{tabular}


Comments and References

Density $=1.78$ to 1.80 at

http://www.matweb.com/search/DataSheet.aspx?MatGUID=8144c044f8a347739734597e0025a723

(Automation Creations 2010). Trade name for Polyvinylidene Fluoride, abbreviated PVDF

(http://en.wikipedia.org/wiki/Kynar).

Formula $=\mathrm{H} 2 \mathrm{C} 2 \mathrm{~F} 2$ from Brandrup et al. (2005).

\section{Lead}

$\begin{array}{llll}\text { Formula }= & \mathrm{Pb} & \text { Molecular weight }(\mathrm{g} / \mathrm{mole})= & 207.2 \\ \text { Density }(\mathrm{g} / \mathrm{cm} 3)= & 11.350000 & \text { Total atom density }(\text { atoms } / \mathrm{b}-\mathrm{cm})= & 3.299 \mathrm{E}-02\end{array}$

The above density is estimated to be accurate to 4 significant digits. Uncertainties are not addressed.

The following data was calculated from the input formula.

\begin{tabular}{|c|c|c|c|c|c|c|}
\hline$\frac{\text { Element }}{\mathrm{Pb}}$ & $\frac{\text { Neutron ZA }}{82000}$ & $\frac{\text { Photon ZA }}{82000}$ & $\begin{array}{l}\text { Weight } \\
\frac{\text { Fraction }}{1.000000}\end{array}$ & $\begin{array}{c}\text { Atom } \\
\frac{\text { Fraction }}{1.000000}\end{array}$ & $\begin{array}{c}\text { Atom } \\
\text { Density } \\
0.032988\end{array}$ & \\
\hline Total & & & 1.000000 & 1.000000 & 0.032988 & \\
\hline MCNP Form & \multicolumn{2}{|c|}{ Weight Fractions } & \multicolumn{2}{|c|}{ Atom Fractions } & \multicolumn{2}{|c|}{ Atom Densities } \\
\hline Neutrons & 82000 & -1.000000 & 82000 & 1.000000 & 82000 & 0.032988 \\
\hline Photons & 82000 & -1.000000 & 82000 & 1.000000 & 82000 & 0.032988 \\
\hline CEPXS Form: & $\begin{array}{l}\text { material } \\
\text { matname } \\
\text { density }\end{array}$ & $\begin{array}{l}\qquad \mathrm{Pb} \\
\text { Lead } \\
11.350000\end{array}$ & 1.000000 & & & \\
\hline Comments ar & eferences & & & & & \\
\hline
\end{tabular}

\section{Lead Tungstate (PWO)}

\begin{tabular}{llll}
\hline Formula $=$ & PbWO4 & Molecular weight $(\mathrm{g} / \mathrm{mole})=$ & 455.0376 \\
Density $(\mathrm{g} / \mathrm{cm} 3)=$ & 8.240000 & Total atom density $($ atoms $/ \mathrm{b}-\mathrm{cm})=$ & $6.543 \mathrm{E}-02$
\end{tabular}

The above density is estimated to be accurate to 3 significant digits. Uncertainties are not addressed.

The following data was calculated from the input formula.

\begin{tabular}{|c|c|c|c|c|c|}
\hline Element & Neutron ZA & Photon ZA & $\begin{array}{l}\text { Weight } \\
\text { Fraction }\end{array}$ & $\begin{array}{c}\text { Atom } \\
\text { Fraction }\end{array}$ & $\begin{array}{l}\text { Atom } \\
\text { Density }\end{array}$ \\
\hline 0 & 8016 & 8000 & $\overline{0.140642}$ & $\overline{0.666667}$ & 0.043621 \\
\hline W & 74000 & 74000 & 0.404011 & 0.166667 & 0.010905 \\
\hline $\mathrm{Pb}$ & 82000 & 82000 & 0.455347 & 0.166667 & 0.010905 \\
\hline
\end{tabular}


PIET-43741-TM-963

PNNL-15870 Rev. 1

\begin{tabular}{|c|c|c|c|c|c|c|}
\hline Total & & & 1.000000 & 1.000000 & 0.065431 & \\
\hline MCNP Form & \multicolumn{2}{|c|}{ Weight Fractions } & \multicolumn{2}{|c|}{ Atom Fractions } & \multicolumn{2}{|c|}{ Atom Densities } \\
\hline \multirow[t]{3}{*}{ Neutrons } & 8016 & -0.140642 & 8016 & 0.666667 & 8016 & 0.043621 \\
\hline & 74000 & -0.404011 & 74000 & 0.166667 & 74000 & 0.010905 \\
\hline & 82000 & -0.455347 & 82000 & 0.166667 & 82000 & 0.010905 \\
\hline \multirow[t]{3}{*}{ Photons } & 8000 & -0.140642 & 8000 & 0.666667 & 8000 & 0.043621 \\
\hline & 74000 & -0.404011 & 74000 & 0.166667 & 74000 & 0.010905 \\
\hline & 82000 & -0.455347 & 82000 & 0.166667 & 82000 & 0.010905 \\
\hline \multirow[t]{4}{*}{ CEPXS Form: } & material & $\mathrm{O}$ & 0.140642 & & & \\
\hline & & W & 0.404011 & & & \\
\hline & & $\mathrm{Pb}$ & 0.455347 & & & \\
\hline & $\begin{array}{c}\text { matname } \\
\text { density }\end{array}$ & \multicolumn{2}{|c|}{ Lead Tungstate (PWO) } & & & \\
\hline \multicolumn{7}{|c|}{$\begin{array}{l}\text { Comments and References } \\
\text { http://www.matweb.com/search/DataSheet.aspx?MatGUID=f0dffd70a17946ceb8032738f311aa8e } \\
\text { (Automation Creations 2010). }\end{array}$} \\
\hline
\end{tabular}

\section{Lithium}

\begin{tabular}{llll}
\hline Formula $=$ & $\mathrm{Li}$ & Molecular weight $(\mathrm{g} / \mathrm{mole})=$ & 6.941 \\
Density $(\mathrm{g} / \mathrm{cm} 3)=$ & 0.534000 & Total atom density $($ atoms $/ \mathrm{b}-\mathrm{cm})=$ & $4.633 \mathrm{E}-02$
\end{tabular}

The above density is estimated to be accurate to 3 significant digits. Uncertainties are not addressed.

The following data was calculated from the input formula.

\begin{tabular}{|c|c|c|c|c|c|c|}
\hline$\frac{\text { Element }}{\mathrm{Li}}$ & $\frac{\text { Neutron ZA }}{-}$ & $\frac{\text { Photon ZA }}{3000}$ & $\begin{array}{l}\text { Weight } \\
\frac{\text { Fraction }}{1.000000}\end{array}$ & $\begin{array}{c}\text { Atom } \\
\frac{\text { Fraction }}{1.000000}\end{array}$ & $\begin{array}{c}\text { Atom } \\
\text { Density } \\
0.046331\end{array}$ & \\
\hline Total & & & 1.000000 & 1.000000 & 0.046331 & \\
\hline MCNP Form & \multicolumn{2}{|c|}{ Weight Fractions } & \multicolumn{2}{|c|}{ Atom Fractions } & \multicolumn{2}{|c|}{ Atom Densities } \\
\hline Neutrons & - & -1.000000 & - & 1.000000 & - & 0.046331 \\
\hline Photons & 3000 & -1.000000 & 3000 & 1.000000 & 3000 & 0.046331 \\
\hline CEPXS Form: & $\begin{array}{l}\text { material } \\
\text { matname } \\
\text { density }\end{array}$ & $\begin{array}{c}\qquad \mathrm{Li} \\
\text { Lithium } \\
0.534000\end{array}$ & 1.000000 & & & \\
\hline
\end{tabular}




\section{Lithium Amide}

\begin{tabular}{llll}
\hline Formula $=$ & LiNH2 & Molecular weight $(\mathrm{g} / \mathrm{mole})=$ & 22.96358 \\
Density $(\mathrm{g} / \mathrm{cm} 3)=$ & 1.178000 & Total atom density $($ atoms $/ \mathrm{b}-\mathrm{cm})=$ & $1.236 \mathrm{E}-01$
\end{tabular}

The above density is estimated to be accurate to 4 significant digits. Uncertainties are not addressed.

The following data were calculated from the input weight fractions.

$\begin{array}{ccccccc}\text { Element } & \text { Neutron ZA } & \text { Photon ZA } & \begin{array}{c}\text { Weight } \\ \text { Fraction }\end{array} & \begin{array}{c}\text { Atom } \\ \text { Fraction }\end{array} & \begin{array}{c}\text { Atom } \\ \text { Density }\end{array} \\ & 1001 & & 1000 & 0.087783 & 0.499991 & 0.061783 \\ \mathrm{Li} & - & 3000 & 0.302262 & 0.250004 & 0.030893 \\ \mathrm{~N} & 7014 & 7000 & 0.609955 & 0.250005 & 0.030893 \\ \text { Total } & & & & & & \\ & & & & 1.000000 & 1.000000 & 0.123569\end{array}$

\begin{tabular}{|c|cccccc|}
\hline MCNP Form & \multicolumn{2}{c|}{ Weight Fractions } & \multicolumn{2}{c|}{ Atom Fractions } & \multicolumn{2}{c|}{ Atom Densities } \\
\hline Neutrons & 1001 & -0.087783 & 1001 & 0.499991 & 1001 & 0.061783 \\
& - & -0.302262 & - & 0.250004 & - & 0.030893 \\
& 7014 & -0.609955 & 7014 & 0.250005 & 7014 & 0.030893 \\
Photons & 1000 & -0.087783 & 1000 & 0.499991 & 1000 & 0.061783 \\
& 3000 & -0.302262 & 3000 & 0.250004 & 3000 & 0.030893 \\
& 7000 & -0.609955 & 7000 & 0.250005 & 7000 & 0.030893 \\
& & & & & & \\
\hline CEPXS Form: & material & $\mathrm{H}$ & 0.087783 & & & \\
& & $\mathrm{Li}$ & 0.302262 & & & \\
& & $\mathrm{~N}$ & 0.609955 & & &
\end{tabular}

matname Lithium Amide

density $\quad 1.178000$

\section{Comments and References}

Density and weight fractions from http://physics.nist.gov/cgi-bin/Star/compos.pl?matno=183 (NIST 1998).

Formula from Lide (2008), pgs 4 - 71.

\section{Lithium Fluoride}

\begin{tabular}{llll}
\hline Formula $=$ & LiF & Molecular weight $(\mathrm{g} / \mathrm{mole})=$ & 25.9394032 \\
Density $(\mathrm{g} / \mathrm{cm} 3)=$ & 2.635000 & Total atom density $($ atoms $/ \mathrm{b}-\mathrm{cm})=$ & $1.223 \mathrm{E}-01$
\end{tabular}

The above density is estimated to be accurate to 4 significant digits. Uncertainties are not addressed.

The following data were calculated from the input weight fractions.

\begin{tabular}{|c|c|c|c|c|c|}
\hline Element & Neutron ZA & Photon ZA & $\begin{array}{l}\text { Weight } \\
\text { Fraction }\end{array}$ & $\begin{array}{c}\text { Atom } \\
\text { Fraction }\end{array}$ & $\begin{array}{c}\text { Atom } \\
\text { Density }\end{array}$ \\
\hline$\overline{\mathrm{Li}}$ & - & 3000 & $\overline{0.267585}$ & $\overline{0.500000}$ & 0.061175 \\
\hline $\mathrm{F}$ & 9019 & 9000 & 0.732415 & 0.500000 & 0.061175 \\
\hline Total & & & 1.000000 & 1.000000 & 0.122349 \\
\hline
\end{tabular}


PIET-43741-TM-963

PNNL-15870 Rev. 1

\begin{tabular}{|c|c|c|c|c|c|c|}
\hline MCNP Form & \multicolumn{2}{|c|}{ Weight Fractions } & \multicolumn{2}{|c|}{ Atom Fractions } & \multicolumn{2}{|c|}{ Atom Densities } \\
\hline \multirow{2}{*}{ Neutrons } & - & -0.267585 & - & 0.500000 & - & 0.061175 \\
\hline & 9019 & -0.732415 & 9019 & 0.500000 & 9019 & 0.061175 \\
\hline \multirow[t]{2}{*}{ Photons } & 3000 & -0.267585 & 3000 & 0.500000 & 3000 & 0.061175 \\
\hline & 9000 & -0.732415 & 9000 & 0.500000 & 9000 & 0.061175 \\
\hline \multirow[t]{3}{*}{ CEPXS Form: } & material & $\mathrm{Li}$ & 0.267585 & & & \\
\hline & & $\mathrm{F}$ & 0.732415 & & & \\
\hline & $\begin{array}{c}\text { matname } \\
\text { density }\end{array}$ & $\begin{array}{c}\text { Lithium Fluo } \\
2.635000\end{array}$ & & & & \\
\hline \multicolumn{7}{|c|}{$\begin{array}{l}\text { Comments and References } \\
\text { Density and weight fractions from http://physics.nist.gov/cgi-bin/Star/compos.pl?matno=185 (NIST 1998). } \\
\text { Formula from Lide (2008), pgs } 4 \text { - 72. }\end{array}$} \\
\hline
\end{tabular}

\section{Lithium Gadrium Borate (LGB)}

\begin{tabular}{llll}
\hline Formula $=$ & Li6Gd $(\mathrm{BO} 3) 3$ & Molecular weight $(\mathrm{g} / \mathrm{mole})=$ & 367.3741448 \\
Density $(\mathrm{g} / \mathrm{cm} 3)=$ & 3.500000 & Total atom density $($ atoms $/ \mathrm{b}-\mathrm{cm})=$ & $1.090 \mathrm{E}-01$
\end{tabular}

The above density is estimated to be accurate to 3 significant digits. Uncertainties are not addressed.

The following data was calculated from the input formula.

\begin{tabular}{|c|c|c|c|c|c|c|}
\hline Element & Neutron ZA & Photon ZA & $\begin{array}{l}\text { Weight } \\
\text { Fraction }\end{array}$ & $\begin{array}{l}\text { Atom } \\
\text { Fraction }\end{array}$ & $\begin{array}{l}\text { Atom } \\
\text { Density }\end{array}$ & \\
\hline$\overline{L i-6}$ & 3006 & 3000 & $\overline{0.098240}$ & $\overline{0.315789}$ & 0.034424 & \\
\hline B-10 & 5010 & 5000 & 0.081766 & 0.157895 & 0.017212 & \\
\hline 0 & 8016 & 8000 & 0.391956 & 0.473684 & 0.051636 & \\
\hline $\mathrm{Gd}$ & 64000 & 64000 & 0.428038 & 0.052632 & 0.005737 & \\
\hline Total & & & 1.000000 & 1.000000 & 0.109009 & \\
\hline MCNP Form & \multicolumn{2}{|c|}{ Weight Fractions } & \multicolumn{2}{|c|}{ Atom Fractions } & \multicolumn{2}{|c|}{ Atom Densities } \\
\hline \multirow[t]{4}{*}{ Neutrons } & 3006 & -0.098240 & 3006 & 0.315789 & 3006 & 0.034424 \\
\hline & 5010 & -0.081766 & 5010 & 0.157895 & 5010 & 0.017212 \\
\hline & 8016 & -0.391956 & 8016 & 0.473684 & 8016 & 0.051636 \\
\hline & 64000 & -0.428038 & 64000 & 0.052632 & 64000 & 0.005737 \\
\hline \multirow[t]{4}{*}{ Photons } & 3000 & -0.098240 & 3000 & 0.315789 & 3000 & 0.034424 \\
\hline & 5000 & -0.081766 & 5000 & 0.157895 & 5000 & 0.017212 \\
\hline & 8000 & -0.391956 & 8000 & 0.473684 & 8000 & 0.051636 \\
\hline & 64000 & -0.428038 & 64000 & 0.052632 & 64000 & 0.005737 \\
\hline \multirow[t]{4}{*}{ CEPXS Form: } & material & $\mathrm{Li}-6$ & 0.098240 & & & \\
\hline & & B-10 & 0.081766 & & & \\
\hline & & 0 & 0.391956 & & & \\
\hline & & $\mathrm{Gd}$ & 0.428038 & & & \\
\hline
\end{tabular}




$$
\text { matname Lithium Gadrium Borate (LGB) }
$$

density $\quad 3.500000$

Comments and References

$\mathrm{Li}$ is $\mathrm{Li}-6, \mathrm{~B}$ is $\mathrm{B}-10, \mathrm{Gd}$, and $\mathrm{O}$ are natural. Formula and density from http://www.apace-

science.com/photogen/index.htm and http://www.apace-science.com/misc/crystalj.htm (APACE 2009).

\section{Lithium Hydride}

\begin{tabular}{llll}
\hline Formula $=$ & $\mathrm{LiH}$ & Molecular weight $(\mathrm{g} / \mathrm{mole})=$ & 7.94894 \\
Density $(\mathrm{g} / \mathrm{cm} 3)=$ & 0.820000 & Total atom density $($ atoms $/ \mathrm{b}-\mathrm{cm})=$ & $1.242 \mathrm{E}-01$ \\
The above density is estimated to be accurate to & 2 significant digits. Uncertainties are not addressed. \\
The following data were calculated from the input weight fractions.
\end{tabular}

\begin{tabular}{|c|c|c|c|c|c|}
\hline Element & Neutron ZA & Photon ZA & $\begin{array}{l}\text { Weight } \\
\text { Fraction }\end{array}$ & $\begin{array}{c}\text { Atom } \\
\text { Fraction }\end{array}$ & $\begin{array}{c}\text { Atom } \\
\text { Density }\end{array}$ \\
\hline $\mathrm{H}$ & 1001 & 1000 & 0.126797 & $\overline{0.499989}$ & 0.062121 \\
\hline $\mathrm{Li}$ & - & 3000 & 0.873203 & 0.500011 & 0.062124 \\
\hline Total & & & 1.000000 & 1.000000 & 0.124245 \\
\hline
\end{tabular}

\begin{tabular}{|c|cc|cc|cr|}
\hline MCNP Form & \multicolumn{2}{c|}{ Weight Fractions } & \multicolumn{2}{c|}{ Atom Fractions } & \multicolumn{2}{c|}{ Atom Densities } \\
\hline Neutrons & 1001 & -0.126797 & 1001 & 0.499989 & 1001 & 0.062121 \\
& - & -0.873203 & - & 0.500011 & - & 0.062124 \\
Photons & 1000 & -0.126797 & 1000 & 0.499989 & 1000 & 0.062121 \\
& 3000 & -0.873203 & 3000 & 0.500011 & 3000 & 0.062124
\end{tabular}

\begin{tabular}{|c|c|c|c|}
\hline EPXS Form: & material & $\begin{array}{l}\mathrm{H} \\
\mathrm{Li}\end{array}$ & $\begin{array}{l}0.126797 \\
0.873203\end{array}$ \\
\hline
\end{tabular}

$\begin{array}{cc}\text { matname } & \text { Lithium Hydride } \\ \text { density } & 0.820000\end{array}$

\section{Comments and References}

Density and weight fractions from http://physics.nist.gov/cgi-bin/Star/compos.pl?matno=186 (NIST 1998).

Formula from Lide (2008), pgs 4 - 72.

Density $=0.70 \mathrm{~g} / \mathrm{cm} 3$ for pressed power (Table 51.14 of Hungerford 1960).

\section{Lithium lodide (High Density)}

\begin{tabular}{llll}
\hline Formula $=$ & Lil & Molecular weight $(\mathrm{g} / \mathrm{mole})=$ & 133.84547 \\
Density $(\mathrm{g} / \mathrm{cm} 3)=$ & 4.080000 & Total atom density $($ atoms $/ \mathrm{b}-\mathrm{cm})=$ & $3.671 \mathrm{E}-02$
\end{tabular}

The above density is estimated to be accurate to 3 significant digits. Uncertainties are not addressed.

The following data were calculated from the input weight fractions. 


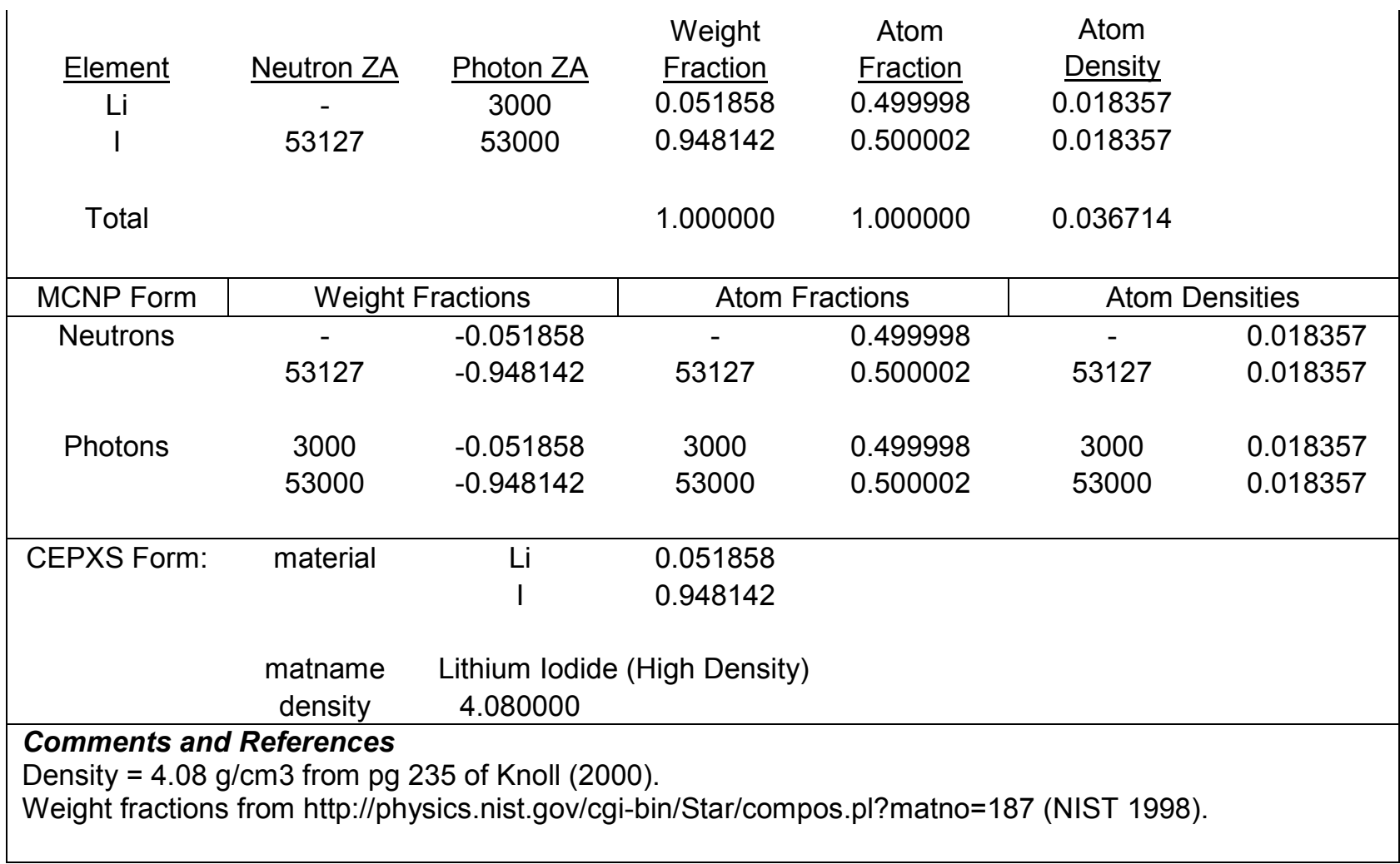

\section{Lithium lodide (Low Density)}

\begin{tabular}{llll}
\hline Formula $=$ & Lil & Molecular weight $(\mathrm{g} / \mathrm{mole})=$ & 133.84547 \\
Density $(\mathrm{g} / \mathrm{cm} 3)=$ & 3.494000 & Total atom density $($ atoms $/ \mathrm{b}-\mathrm{cm})=$ & $3.144 \mathrm{E}-02$
\end{tabular}

The above density is estimated to be accurate to 3 significant digits. Uncertainties are not addressed.

The following data were calculated from the input weight fractions.

\begin{tabular}{|c|c|c|c|c|c|c|}
\hline $\begin{array}{c}\text { Element } \\
\mathrm{Li} \\
\mathrm{I}\end{array}$ & $\begin{array}{c}\text { Neutron ZA } \\
- \\
53127\end{array}$ & 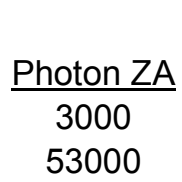 & $\begin{array}{c}\text { Weight } \\
\text { Fraction } \\
0.051858 \\
0.948142\end{array}$ & $\begin{array}{c}\text { Atom } \\
\text { Fraction } \\
0.499998 \\
0.500002\end{array}$ & $\begin{array}{c}\text { Atom } \\
\text { Density } \\
0.01572 \\
0.01572\end{array}$ & \\
\hline Total & & & 1.000000 & 1.000000 & 0.03144 & \\
\hline MCNP Form & \multicolumn{2}{|c|}{ Weight Fractions } & \multicolumn{2}{|c|}{ Atom Fractions } & \multicolumn{2}{|c|}{ Atom Densities } \\
\hline Neutrons & $\begin{array}{c}- \\
53127\end{array}$ & $\begin{array}{l}-0.051858 \\
-0.948142\end{array}$ & $\begin{array}{c}- \\
53127\end{array}$ & $\begin{array}{l}0.499998 \\
0.500002\end{array}$ & $\begin{array}{c}- \\
53127\end{array}$ & $\begin{array}{l}0.015721 \\
0.015721\end{array}$ \\
\hline Photons & $\begin{array}{c}3000 \\
53000\end{array}$ & $\begin{array}{l}-0.051858 \\
-0.948142\end{array}$ & $\begin{array}{c}3000 \\
53000\end{array}$ & $\begin{array}{l}0.499998 \\
0.500002\end{array}$ & $\begin{array}{c}3000 \\
53000\end{array}$ & $\begin{array}{l}0.015721 \\
0.015721\end{array}$ \\
\hline CEPXS Form: & material & $\begin{array}{c}\mathrm{Li} \\
\mathrm{I}\end{array}$ & $\begin{array}{l}0.051858 \\
0.948142\end{array}$ & & & \\
\hline
\end{tabular}




\begin{tabular}{|cc} 
matname & Lithium lodide (Low Density) \\
density & 3.494000 \\
\hline
\end{tabular}

Comments and References

Density $=3.494 \mathrm{~g} / \mathrm{cm} 3$ and weight fractions from http://physics.nist.gov/cgi-bin/Star/compos.pl?matno=187 (NIST 1998).

\section{Lithium Oxide}

\begin{tabular}{llll}
\hline Formula $=$ & $\mathrm{Li} 2 \mathrm{O}$ & Molecular weight $(\mathrm{g} / \mathrm{mole})=$ & 29.8814 \\
Density $(\mathrm{g} / \mathrm{cm} 3)=$ & 2.013000 & Total atom density $($ atoms $/ \mathrm{b}-\mathrm{cm})=$ & $1.217 \mathrm{E}-01$
\end{tabular}

The above density is estimated to be accurate to 4 significant digits. Uncertainties are not addressed.

The following data were calculated from the input weight fractions.

$\begin{array}{ccccccc}\frac{\text { Element }}{\mathrm{Li}} & \frac{\text { Neutron ZA }}{-} & \frac{\text { Photon ZA }}{3000} & \frac{\begin{array}{c}\text { Weight } \\ \text { Fraction }\end{array}}{0.464570} & \begin{array}{c}\text { Atom } \\ \text { Fraction }\end{array} & \begin{array}{c}\text { Atom } \\ \text { Density }\end{array} \\ \mathrm{O} & 8016 & 8000 & 0.535430 & 0.333333 & 0.081138 \\ \text { Total } & & & & & & \\ & & & & 1.000000 & 1.000000 & 0.121707\end{array}$

\begin{tabular}{|c|cccccc|}
\hline MCNP Form & \multicolumn{2}{c|}{ Weight Fractions } & \multicolumn{2}{c|}{ Atom Fractions } & \multicolumn{2}{c|}{ Atom Densities } \\
\hline Neutrons & - & -0.464570 & - & 0.666667 & - & 0.081138 \\
& 8016 & -0.535430 & 8016 & 0.333333 & 8016 & 0.040569 \\
Photons & & & & & & \\
& 3000 & -0.464570 & 3000 & 0.666667 & 3000 & 0.081138 \\
& 8000 & -0.535430 & 8000 & 0.333333 & 8000 & 0.040569 \\
\hline CEPXS Form: & material & $\mathrm{Li}$ & 0.464570 & & & \\
& & $\mathrm{O}$ & 0.535430 & & & \\
& & & & & &
\end{tabular}

$\begin{array}{cc}\text { matname } & \text { Lithium Oxide } \\ \text { density } & 2.013000\end{array}$

Comments and References

Density and weight fractions from http://physics.nist.gov/cgi-bin/Star/compos.pl?matno=188 (NIST 1998).

Formula from Lide (2008), pgs 4 - 72.

\section{Lithium Tetraborate}

\begin{tabular}{|c|c|c|c|}
\hline Formula $=$ & Li2B4O7 & Molecular weight $(\mathrm{g} / \mathrm{mole})=$ & 169.1218 \\
\hline Density $(\mathrm{g} / \mathrm{cm} 3)=$ & 2.440000 & Total atom density $($ atoms $/ \mathrm{b}-\mathrm{cm})=$ & 1.129E-01 \\
\hline
\end{tabular}

\begin{tabular}{llllll} 
Element & Neutron ZA & $\frac{\text { Photon ZA }}{3000}$ & $\frac{\begin{array}{c}\text { Weight } \\
\text { Fraction }\end{array}}{0.082085}$ & $\frac{1}{\text { Fraction }}$ & $\begin{array}{c}\text { Atom } \\
\text { Density }\end{array}$ \\
\hline & -153851 & 0.017377
\end{tabular}


PIET-43741-TM-963

PNNL-15870 Rev. 1

\begin{tabular}{|c|c|c|c|c|c|c|}
\hline B & - & 5000 & 0.255680 & 0.307673 & 0.03475 & \\
\hline $\mathrm{O}$ & 8016 & 8000 & 0.662235 & 0.538476 & 0.06082 & \\
\hline Total & & & 1.000000 & 1.000000 & 0.11294 & \\
\hline MCNP Form & Weigh & actions & Atom & ctions & Ato & sities \\
\hline Neutrons & - & -0.082085 & - & 0.153851 & - & 0.017377 \\
\hline & - & -0.255680 & - & 0.307673 & - & 0.034751 \\
\hline & 8016 & -0.662235 & 8016 & 0.538476 & 8016 & 0.060820 \\
\hline Photons & 3000 & -0.082085 & 3000 & 0.153851 & 3000 & 0.017377 \\
\hline & 5000 & -0.255680 & 5000 & 0.307673 & 5000 & 0.034751 \\
\hline & 8000 & -0.662235 & 8000 & 0.538476 & 8000 & 0.060820 \\
\hline CEPXS Form: & material & $\mathrm{Li}$ & 0.082085 & & & \\
\hline & & B & 0.255680 & & & \\
\hline & & O & 0.662235 & & & \\
\hline & $\begin{array}{c}\text { matname } \\
\text { density }\end{array}$ & $\begin{array}{c}\text { Lithium Tetr } \\
2.440000\end{array}$ & ate & & & \\
\hline $\begin{array}{l}\text { Comments an } \\
\text { Density and we } \\
\text { Formula from L }\end{array}$ & $\begin{array}{l}\text { eferences } \\
\text { t fractions } \\
\text { (2008), pg }\end{array}$ & $\begin{array}{l}\text { http://phy } \\
72 \text {. }\end{array}$ & t.gov/cs & ar/com & (1) & ST 1998 \\
\hline
\end{tabular}

\section{Lucite}

\begin{tabular}{llll}
\hline Formula $=$ & C5O2H8 & Molecular weight $(\mathrm{g} / \mathrm{mole})=$ & 100.11582 \\
Density $(\mathrm{g} / \mathrm{cm} 3)=$ & 1.190000 & Total atom density $($ atoms $/ \mathrm{b}-\mathrm{cm})=$ & $1.074 \mathrm{E}-01$
\end{tabular}

The above density is estimated to be accurate to 3 significant digits. Uncertainties are not addressed.

The following data were calculated from the input weight fractions.

\begin{tabular}{|c|c|c|c|c|c|c|}
\hline Element & Neutron ZA & Photon ZA & $\begin{array}{l}\text { Weight } \\
\text { Fraction }\end{array}$ & $\begin{array}{c}\text { Atom } \\
\text { Fraction }\end{array}$ & $\begin{array}{c}\text { Atom } \\
\text { Density }\end{array}$ & \\
\hline $\mathrm{H}$ & 1001 & 1000 & 0.080538 & 0.533320 & 0.05726 & \\
\hline C & 6000 & 6000 & 0.599848 & 0.333345 & 0.03579 & \\
\hline $\mathrm{O}$ & 8016 & 8000 & 0.319614 & 0.133335 & 0.01431 & \\
\hline Total & & & 1.000000 & 1.000000 & 0.10736 & \\
\hline MCNP Form & \multicolumn{2}{|c|}{ Weight Fractions } & \multicolumn{2}{|c|}{ Atom Fractions } & \multicolumn{2}{|c|}{ Atom Densities } \\
\hline \multirow[t]{3}{*}{ Neutrons } & 1001 & -0.080538 & 1001 & 0.533320 & 1001 & 0.057262 \\
\hline & 6000 & -0.599848 & 6000 & 0.333345 & 6000 & 0.035791 \\
\hline & 8016 & -0.319614 & 8016 & 0.133335 & 8016 & 0.014316 \\
\hline \multirow[t]{3}{*}{ Photons } & 1000 & -0.080538 & 1000 & 0.533320 & 1000 & 0.057262 \\
\hline & 6000 & -0.599848 & 6000 & 0.333345 & 6000 & 0.035791 \\
\hline & 8000 & -0.319614 & 8000 & 0.133335 & 8000 & 0.014316 \\
\hline
\end{tabular}




\begin{tabular}{|lccc|} 
& & \\
\hline CEPXS Form: & material & $\mathrm{H}$ & 0.080538 \\
& $\mathrm{C}$ & 0.599848 \\
& $\mathrm{O}$ & 0.319614 \\
& & \\
& matname & Lucite \\
& density & 1.190000 \\
\hline Comments and References & & \\
Also called polymethyl methacrylate (PMMA), plexiglas, perspex, acrylite, acrylic glass, or acrylic. \\
Density = 1.19 g/cm3 and weight fractions from http://physics.nist.gov/cgi-bin/Star/compos.pl?matno=223 \\
(NIST 1998).
\end{tabular}

\section{Lutetium Aluminum Garnet (LuAG)}

\begin{tabular}{llll}
\hline Formula $=$ & Al5Lu3O12 & Molecular weight $(\mathrm{g} / \mathrm{mole})=$ & 851.80149 \\
Density $(\mathrm{g} / \mathrm{cm} 3)=$ & 6.730000 & Total atom density $($ atoms $/ \mathrm{b}-\mathrm{cm})=$ & $9.516 \mathrm{E}-02$
\end{tabular}

The above density is estimated to be accurate to 3 significant digits. Uncertainties are not addressed. The following data was calculated from the input formula.

\begin{tabular}{|c|c|c|c|c|c|c|}
\hline Element & Neutron ZA & Photon ZA & $\begin{array}{l}\text { Weight } \\
\text { Fraction }\end{array}$ & $\begin{array}{l}\text { Atom } \\
\text { Fraction }\end{array}$ & $\begin{array}{l}\text { Atom } \\
\text { Density }\end{array}$ & \\
\hline 0 & 8016 & 8000 & $\overline{0.225396}$ & $\overline{0.600000}$ & \multicolumn{2}{|l|}{$\frac{\text { Density }}{0.057096}$} \\
\hline $\mathrm{Al}$ & 13027 & 13000 & 0.158379 & 0.250000 & \multicolumn{2}{|l|}{0.023790} \\
\hline Lu & - & 71000 & 0.616225 & 0.150000 & \multicolumn{2}{|l|}{0.014274} \\
\hline Total & & & 1.000000 & 1.000000 & \multicolumn{2}{|l|}{0.095161} \\
\hline MCNP Form & \multicolumn{2}{|c|}{ Weight Fractions } & \multicolumn{2}{|c|}{ Atom Fractions } & \multicolumn{2}{|c|}{ Atom Densities } \\
\hline \multirow[t]{3}{*}{ Neutrons } & 8016 & -0.225396 & 8016 & 0.600000 & 8016 & 0.057096 \\
\hline & 13027 & -0.158379 & 13027 & 0.250000 & 13027 & 0.023790 \\
\hline & - & -0.616225 & - & 0.150000 & - & 0.014274 \\
\hline \multirow[t]{3}{*}{ Photons } & 8000 & -0.225396 & 8000 & 0.600000 & 8000 & 0.057096 \\
\hline & 13000 & -0.158379 & 13000 & 0.250000 & 13000 & 0.023790 \\
\hline & 71000 & -0.616225 & 71000 & 0.150000 & 71000 & 0.014274 \\
\hline \multirow[t]{4}{*}{ CEPXS Form: } & material & $\mathrm{O}$ & \multicolumn{4}{|l|}{0.225396} \\
\hline & & $\mathrm{Al}$ & \multicolumn{4}{|l|}{0.158379} \\
\hline & & Lu & \multicolumn{4}{|l|}{0.616225} \\
\hline & $\begin{array}{l}\text { matname } \\
\text { density }\end{array}$ & \multicolumn{5}{|c|}{$\begin{array}{l}\text { Lutetium Aluminum Garnet (LuAG) } \\
\quad 6.730000\end{array}$} \\
\hline \multicolumn{7}{|c|}{$\begin{array}{l}\text { Comments and References } \\
\text { The formula is listed as Al5Lu3012 at http://en.wikipedia.org/wiki/LuAG and at "Preparation and } \\
\text { characterization of nanoscale lutetium aluminium garnet (LuAG) powders doped by Eu3+" by Dominik } \\
\text { Uhlicha, et al. and at } \\
\text { http://www.diracdelta.co.uk/science/source///u/lutetium\%20aluminium\%20garnet/source.html. }\end{array}$} \\
\hline
\end{tabular}




\begin{tabular}{|c|c|c|c|c|c|c|}
\hline \multicolumn{3}{|c|}{ Formula $=\quad$ LuAlO3 } & \multicolumn{3}{|c|}{ Molecular weight $(\mathrm{g} / \mathrm{mole})=$} & 249.946738 \\
\hline \multirow{2}{*}{\multicolumn{7}{|c|}{ 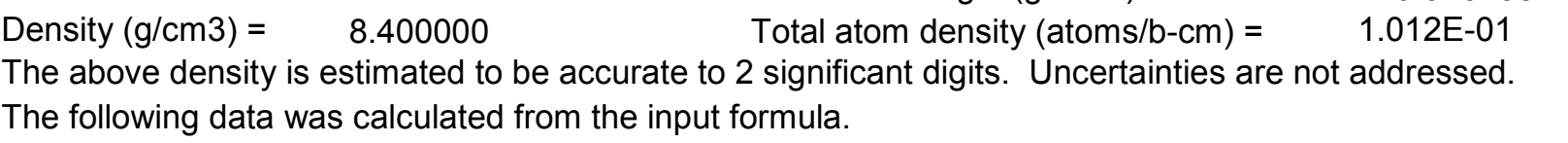 }} \\
\hline & & & & & & \\
\hline & & & Weight & Atom & Atom & \\
\hline Element & Neutron ZA & Photon ZA & Fraction & Fraction & Density & \\
\hline 0 & 8016 & 8000 & 0.192034 & 0.600000 & 0.060716 & \\
\hline $\mathrm{Al}$ & 13027 & 13000 & 0.107949 & 0.200000 & 0.020239 & \\
\hline Lu & - & 71000 & 0.700017 & 0.200000 & 0.020239 & \\
\hline Total & & & 1.000000 & 1.000000 & 0.101194 & \\
\hline MCNP Form & \multicolumn{2}{|c|}{ Weight Fractions } & \multicolumn{2}{|c|}{ Atom Fractions } & \multicolumn{2}{|c|}{ Atom Densities } \\
\hline \multirow{3}{*}{ Neutrons } & 8016 & -0.192034 & 8016 & 0.600000 & 8016 & 0.060716 \\
\hline & 13027 & -0.107949 & 13027 & 0.200000 & 13027 & 0.020239 \\
\hline & - & -0.700017 & - & 0.200000 & - & 0.020239 \\
\hline \multirow[t]{3}{*}{ Photons } & 8000 & -0.192034 & 8000 & 0.600000 & 8000 & 0.060716 \\
\hline & 13000 & -0.107949 & 13000 & 0.200000 & 13000 & 0.020239 \\
\hline & 71000 & -0.700017 & 71000 & 0.200000 & 71000 & 0.020239 \\
\hline \multirow[t]{4}{*}{ CEPXS Form: } & material & $\mathrm{O}$ & 0.192034 & & & \\
\hline & & $\mathrm{Al}$ & 0.107949 & & & \\
\hline & & $\mathrm{Lu}$ & 0.700017 & & & \\
\hline & $\begin{array}{c}\text { matname } \\
\text { density }\end{array}$ & $\begin{array}{c}\text { Lutetium Ort } \\
8.400000\end{array}$ & aluminate (L & & & \\
\hline \multicolumn{7}{|c|}{$\begin{array}{l}\text { Comments and References } \\
\text { Density from pg } 235 \text { of Knoll (2000). Formula and density at http://www.apace- } \\
\text { science.com/misc/crystalj.htm (APACE 2009). }\end{array}$} \\
\hline
\end{tabular}

\section{Lutetium Oxyorthosilicate (LSO)}

\begin{tabular}{llll}
\hline Formula $=$ & Lu2SiO5 & Molecular weight $(\mathrm{g} / \mathrm{mole})=$ & 458.0165 \\
Density $(\mathrm{g} / \mathrm{cm} 3)=$ & 7.400000 & Total atom density $($ atoms $/ \mathrm{b}-\mathrm{cm})=$ & $7.784 \mathrm{E}-02$ \\
The above density is estimated to be accurate to & 2 significant digits. Uncertainties are not addressed. \\
The following data was calculated from the input formula.
\end{tabular}

$\begin{array}{llllll}\text { Element } & \frac{\text { Neutron ZA }}{\mathrm{O}} & \frac{\text { Photon ZA }}{8016} & \frac{\begin{array}{c}\text { Weight } \\ \text { Fraction }\end{array}}{0.174660} & \frac{\begin{array}{c}\text { Atom } \\ \text { Fraction }\end{array}}{0.625000} & \begin{array}{c}\text { Atom } \\ \text { Density }\end{array} \\ 0.048649\end{array}$




\begin{tabular}{|c|c|c|c|c|c|c|}
\hline $\mathrm{Si}$ & 14000 & 14000 & 0.061320 & 0.125000 & 0.009730 & \\
\hline Lu & - & 71000 & 0.764021 & 0.250000 & 0.019459 & \\
\hline Total & & & 1.000000 & 1.000000 & 0.077838 & \\
\hline MCNP Form & Weigh & ractions & Atom & tions & Atom & sities \\
\hline Neutrons & 8016 & -0.174660 & 8016 & 0.625000 & 8016 & 0.048649 \\
\hline & 14000 & -0.061320 & 14000 & 0.125000 & 14000 & 0.009730 \\
\hline & - & -0.764021 & - & 0.250000 & - & 0.019459 \\
\hline Photons & 8000 & -0.174660 & 8000 & 0.625000 & 8000 & 0.048649 \\
\hline & 14000 & -0.061320 & 14000 & 0.125000 & 14000 & 0.009730 \\
\hline & 71000 & -0.764021 & 71000 & 0.250000 & 71000 & 0.019459 \\
\hline CEPXS Form: & material & $\mathrm{O}$ & 0.174660 & & & \\
\hline & & $\mathrm{Si}$ & 0.061320 & & & \\
\hline & & Lu & 0.764021 & & & \\
\hline & $\begin{array}{c}\text { matname } \\
\text { density }\end{array}$ & $\begin{array}{c}\text { Lutetium Ox } \\
7.400000\end{array}$ & nosilicate ( & & & \\
\hline $\begin{array}{l}\text { Comments an } \\
\text { Density from pg } \\
\text { Formula and de } \\
\text { (Lu2SiO5:Ce) } \\
\text { http://www.apa }\end{array}$ & $\begin{array}{l}\text { Peferences } \\
35 \text { of Knoll } \\
\text { ity in Guoh } \\
\text { stals Dopec } \\
\text { science.cor }\end{array}$ & $\begin{array}{l}\text { O0). } \\
\text { Ren et al., "s } \\
\text { ith Cerium Io } \\
\text { nisc/crystalj.l }\end{array}$ & $\begin{array}{l}\text { illation Cha } \\
\text { at www.sci } \\
\text { APACE } 20\end{array}$ & $\begin{array}{l}\text { diristics of L } \\
\text { direct.com } \\
\text { Ce atoms }\end{array}$ & $\begin{array}{l}\text { um Oxyor } \\
\text { mula and } \\
\text { gnored. }\end{array}$ & $\begin{array}{l}\text { icate } \\
\text { ity also at }\end{array}$ \\
\hline
\end{tabular}

\section{Lutetium Yttrium OxyorthoSilicate (LYSO)}

\begin{tabular}{llll}
\hline Formula $=$ & Lu2Y2SiO5 & Molecular weight $(\mathrm{g} / \mathrm{mole})=$ & 635.8282 \\
Density $(\mathrm{g} / \mathrm{cm} 3)=$ & 7.300000 & Total atom density $($ atoms $/ \mathrm{b}-\mathrm{cm})=$ & $6.914 \mathrm{E}-02$
\end{tabular}

The above density is estimated to be accurate to 2 significant digits. Uncertainties are not addressed.

The following data was calculated from the input formula.

\begin{tabular}{|c|c|c|c|c|c|c|}
\hline Element & Neutron ZA & Photon ZA & $\begin{array}{l}\text { Weight } \\
\text { Fraction }\end{array}$ & $\begin{array}{c}\text { Atom } \\
\text { Fraction }\end{array}$ & $\begin{array}{l}\text { Atom } \\
\text { Density }\end{array}$ & \\
\hline 0 & 8016 & 8000 & 0.125815 & 0.500000 & 0.034570 & \\
\hline Si & 14000 & 14000 & 0.044172 & 0.100000 & 0.006914 & \\
\hline $\mathrm{Y}$ & 39089 & 39000 & 0.279654 & 0.200000 & 0.013828 & \\
\hline Lu & - & 71000 & 0.550359 & 0.200000 & 0.013828 & \\
\hline Total & & & 1.000000 & 1.000000 & 0.069141 & \\
\hline MCNP Form & \multicolumn{2}{|c|}{ Weight Fractions } & \multicolumn{2}{|c|}{ Atom Fractions } & \multicolumn{2}{|c|}{ Atom Densities } \\
\hline \multirow[t]{4}{*}{ Neutrons } & 8016 & -0.125815 & 8016 & 0.500000 & 8016 & 0.034570 \\
\hline & 14000 & -0.044172 & 14000 & 0.100000 & 14000 & 0.006914 \\
\hline & 39089 & -0.279654 & 39089 & 0.200000 & 39089 & 0.013828 \\
\hline & - & -0.550359 & - & 0.200000 & - & 0.013828 \\
\hline
\end{tabular}


PIET-43741-TM-963

PNNL-15870 Rev. 1

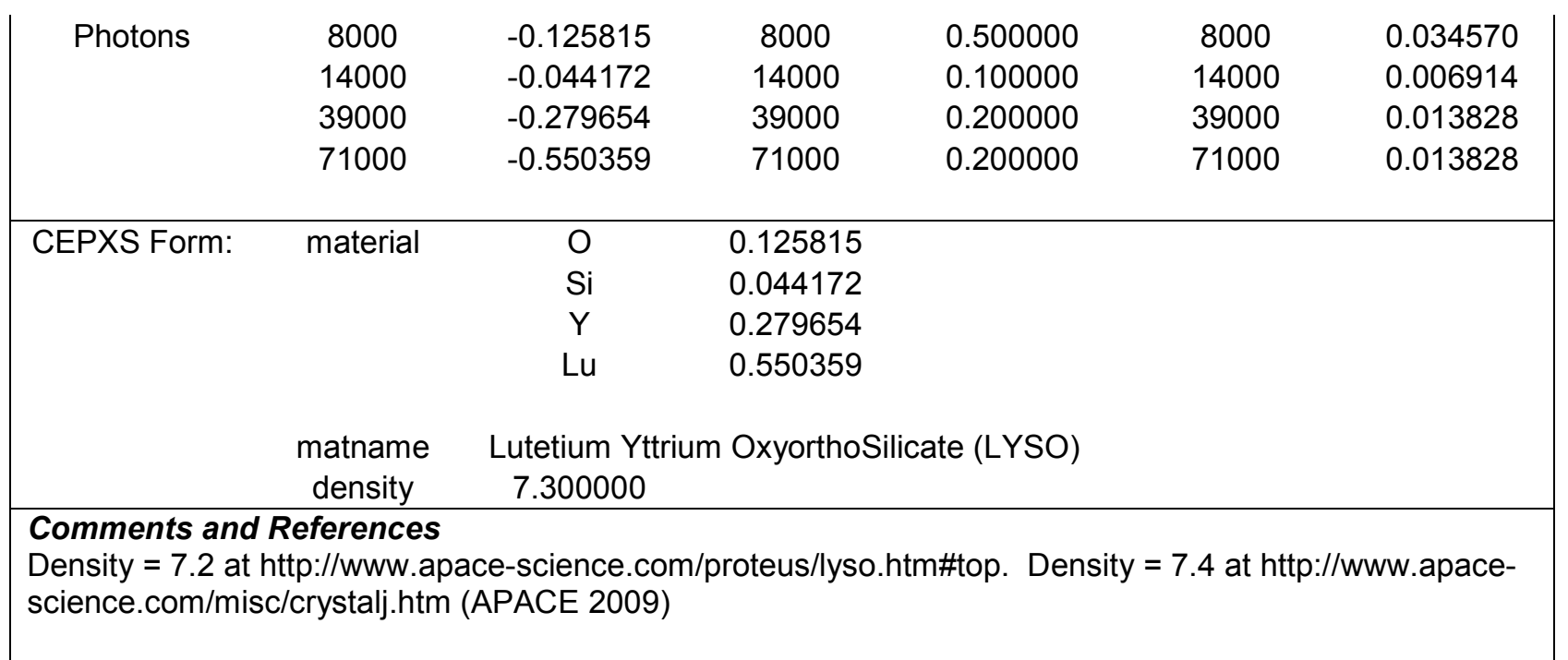

\section{Magnesium}

\begin{tabular}{llll}
\hline Formula $=$ & $\mathrm{Mg}$ & Molecular weight $(\mathrm{g} / \mathrm{mole})=$ & 24.305 \\
Density $(\mathrm{g} / \mathrm{cm} 3)=$ & 1.740000 & Total atom density $($ atoms $/ \mathrm{b}-\mathrm{cm})=$ & $4.311 \mathrm{E}-02$
\end{tabular}

The above density is estimated to be accurate to 3 significant digits. Uncertainties are not addressed.

The following data was calculated from the input formula.

\begin{tabular}{|c|c|c|c|c|c|c|}
\hline$\frac{\text { Element }}{\mathrm{Mg}}$ & $\frac{\text { Neutron ZA }}{12000}$ & $\frac{\text { Photon ZA }}{12000}$ & $\begin{array}{c}\text { Weight } \\
\text { Fraction } \\
1.000000\end{array}$ & $\begin{array}{c}\begin{array}{c}\text { Atom } \\
\text { Fraction }\end{array} \\
1.000000\end{array}$ & $\begin{array}{c}\text { Atom } \\
\text { Density } \\
0.043113\end{array}$ & \\
\hline Total & & & 1.000000 & 1.000000 & 0.043113 & \\
\hline MCNP Form & \multicolumn{2}{|c|}{ Weight Fractions } & \multicolumn{2}{|c|}{ Atom Fractions } & \multicolumn{2}{|c|}{ Atom Densities } \\
\hline Neutrons & 12000 & -1.000000 & 12000 & 1.000000 & 12000 & 0.043113 \\
\hline Photons & 12000 & -1.000000 & 12000 & 1.000000 & 12000 & 0.043113 \\
\hline CEPXS Form: & material & $\mathrm{Mg}$ & 1.000000 & & & \\
\hline & $\begin{array}{l}\text { matname } \\
\text { density }\end{array}$ & $\begin{array}{c}\text { Magnesium } \\
1.740000\end{array}$ & & & & \\
\hline
\end{tabular}

\section{Magnesium Oxide}

\begin{tabular}{|llll}
\hline Formula $=$ & $\mathrm{MgO}$ & Molecular weight $(\mathrm{g} / \mathrm{mole})=$ & 40.3044 \\
Density $(\mathrm{g} / \mathrm{cm} 3)=$ & 3.580000 & Total atom density $($ atoms $/ \mathrm{b}-\mathrm{cm})=$ & $1.070 \mathrm{E}-01$
\end{tabular}


The above density is estimated to be accurate to 3 significant digits. Uncertainties are not addressed. The following data were calculated from the input weight fractions.

\begin{tabular}{|c|c|c|c|c|c|}
\hline Element & Neutron ZA & Photon ZA & $\begin{array}{l}\text { Weight } \\
\text { Fraction }\end{array}$ & $\begin{array}{c}\text { Atom } \\
\text { Fraction }\end{array}$ & $\begin{array}{l}\text { Atom } \\
\text { Density }\end{array}$ \\
\hline 0 & 8016 & 8000 & $\overline{0.396964}$ & $\overline{0.500000}$ & 0.053491 \\
\hline $\mathrm{Mg}$ & 12000 & 12000 & 0.603036 & 0.500000 & 0.053491 \\
\hline Total & & & 1.000000 & 1.000000 & 0.106982 \\
\hline
\end{tabular}

\begin{tabular}{|c|cccccc|}
\hline MCNP Form & \multicolumn{2}{c}{ Weight Fractions } & \multicolumn{2}{c|}{ Atom Fractions } & \multicolumn{2}{c|}{ Atom Densities } \\
\hline Neutrons & 8016 & -0.396964 & 8016 & 0.500000 & 8016 & 0.053491 \\
& 12000 & -0.603036 & 12000 & 0.500000 & 12000 & 0.053491 \\
Photons & 8000 & -0.396964 & 8000 & 0.500000 & 8000 & 0.053491 \\
& 12000 & -0.603036 & 12000 & 0.500000 & 12000 & 0.053491 \\
& & & & & & \\
\hline CEPXS Form: & material & $\mathrm{O}$ & 0.396964 & & \\
& & $\mathrm{Mg}$ & 0.603036 & &
\end{tabular}

matname Magnesium Oxide density $\quad 3.580000$

Comments and References

Density and weight fractions from http://physics.nist.gov/cgi-bin/Star/compos.pl?matno=194 (NIST 1998).

\section{Magnesium Tetraborate}

\begin{tabular}{llll}
\hline Formula $=$ & MgB4O7 & Molecular weight $(\mathrm{g} / \mathrm{mole})=$ & 179.5448 \\
Density $(\mathrm{g} / \mathrm{cm} 3)=$ & 2.530000 & Total atom density $($ atoms $/ \mathrm{b}-\mathrm{cm})=$ & $1.018 \mathrm{E}-01$
\end{tabular}

The above density is estimated to be accurate to 3 significant digits. Uncertainties are not addressed.

The following data were calculated from the input weight fractions.

\begin{tabular}{|c|c|c|c|c|c|c|}
\hline Element & Neutron ZA & Photon ZA & $\begin{array}{l}\text { Weight } \\
\text { Fraction }\end{array}$ & $\begin{array}{c}\text { Atom } \\
\text { Fraction }\end{array}$ & $\begin{array}{c}\text { Atom } \\
\text { Density }\end{array}$ & \\
\hline B & - & 5000 & 0.240837 & $\overline{0.333313}$ & 0.03394 & \\
\hline $\mathrm{O}$ & 8016 & 8000 & 0.623790 & 0.583351 & 0.05940 & \\
\hline $\mathrm{Mg}$ & 12000 & 12000 & 0.135373 & 0.083336 & 0.00848 & \\
\hline Total & & & 1.000000 & 1.000000 & 0.10183 & \\
\hline MCNP Form & \multicolumn{2}{|c|}{ Weight Fractions } & \multicolumn{2}{|c|}{ Atom Fractions } & \multicolumn{2}{|c|}{ Atom Densities } \\
\hline \multirow[t]{3}{*}{ Neutrons } & - & -0.240837 & - & 0.333313 & - & 0.033941 \\
\hline & 8016 & -0.623790 & 8016 & 0.583351 & 8016 & 0.059403 \\
\hline & 12000 & -0.135373 & 12000 & 0.083336 & 12000 & 0.008486 \\
\hline \multirow[t]{2}{*}{ Photons } & 5000 & -0.240837 & 5000 & 0.333313 & 5000 & 0.033941 \\
\hline & 8000 & -0.623790 & 8000 & 0.583351 & 8000 & 0.059403 \\
\hline
\end{tabular}




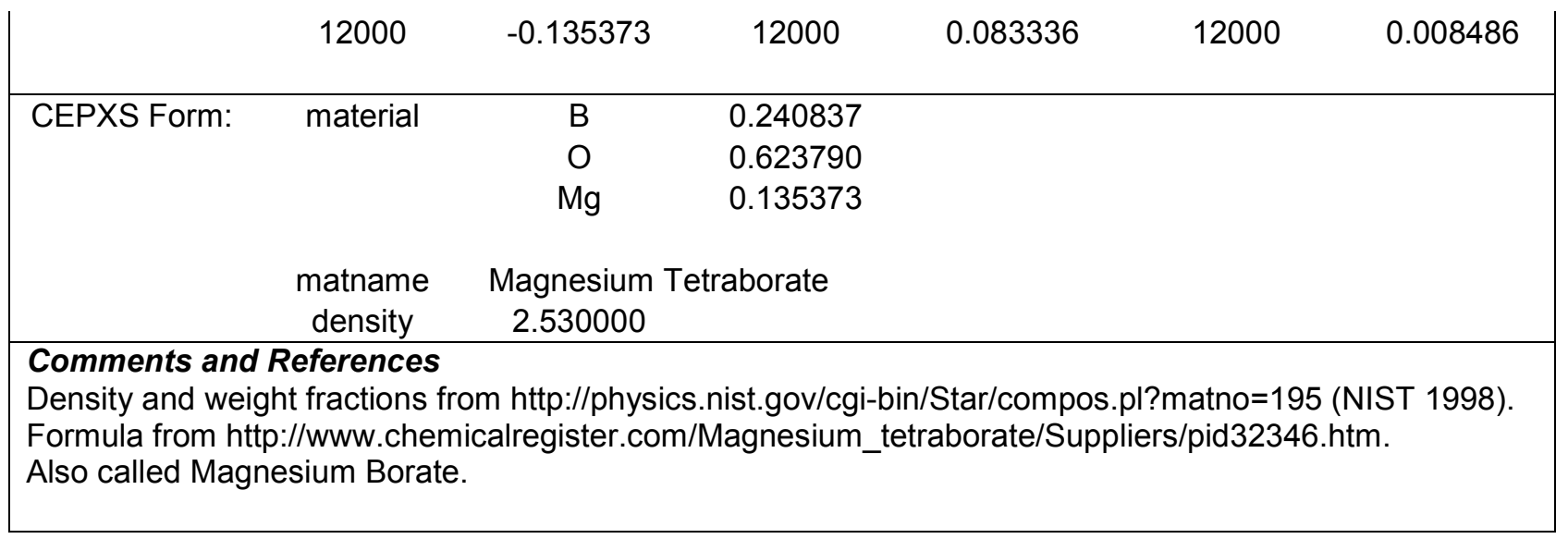

\begin{tabular}{|c|c|c|c|c|c|c|}
\hline \multicolumn{7}{|c|}{190 Masonite } \\
\hline Density $(\mathrm{g} / \mathrm{cm} 3)$ & \multicolumn{2}{|c|}{1.300000} & \multicolumn{3}{|c|}{ Total atom density $($ atoms $/ \mathrm{b}-\mathrm{cm})=$} & 1.014E-01 \\
\hline \multicolumn{7}{|c|}{$\begin{array}{l}\text { The above density is estimated to be accurate to } 2 \text { significant digits. Uncertainties are not addressed. } \\
\text { The following data was calculated from the input formula. }\end{array}$} \\
\hline Element & Neutron ZA & Photon ZA & $\begin{array}{l}\text { Weight } \\
\text { Fraction }\end{array}$ & $\begin{array}{l}\text { Atom } \\
\text { Fraction }\end{array}$ & \multicolumn{2}{|l|}{ Atom } \\
\hline $\mathrm{H}$ & 1001 & 1000 & 0.062165 & 0.476190 & \multicolumn{2}{|c|}{0.048284} \\
\hline C & 6000 & 6000 & 0.444455 & 0.285714 & \multicolumn{2}{|c|}{0.028970} \\
\hline $\mathrm{O}$ & 8016 & 8000 & 0.493380 & 0.238095 & \multicolumn{2}{|c|}{0.024142} \\
\hline \multicolumn{3}{|l|}{ Total } & 1.000000 & 1.000000 & \multicolumn{2}{|c|}{0.101396} \\
\hline MCNP Form & \multicolumn{2}{|c|}{ Weight Fractions } & \multicolumn{2}{|c|}{ Atom Fractions } & \multicolumn{2}{|c|}{ Atom Densities } \\
\hline \multirow[t]{3}{*}{ Neutrons } & 1001 & -0.062165 & 1001 & 0.476190 & 1001 & 0.048284 \\
\hline & 6000 & -0.444455 & 6000 & 0.285714 & 6000 & 0.028970 \\
\hline & 8016 & -0.493380 & 8016 & 0.238095 & 8016 & 0.024142 \\
\hline \multirow[t]{3}{*}{ Photons } & 1000 & -0.062165 & 1000 & 0.476190 & 1000 & 0.048284 \\
\hline & 6000 & -0.444455 & 6000 & 0.285714 & 6000 & 0.028970 \\
\hline & 8000 & -0.493380 & 8000 & 0.238095 & 8000 & 0.024142 \\
\hline \multirow[t]{4}{*}{ CEPXS Form: } & material & $\mathrm{H}$ & 0.062165 & & & \\
\hline & & C & 0.444455 & & & \\
\hline & & 0 & 0.493380 & & & \\
\hline & $\begin{array}{l}\text { matname } \\
\text { density }\end{array}$ & $\begin{array}{l}\text { Masonite } \\
1.300000\end{array}$ & & & & \\
\hline
\end{tabular}


Comments and References

Masonite is a type of hardboard (http://en.wikipedia.org/wiki/Masonite) produced from by-product wood chips reduced to cellulose fibers by high-pressure steam (Table 51.114 of Hungerford 1960) so cellulose $(\mathrm{C} 6 \mathrm{H} 10 \mathrm{O} 5)$ is used for the formula.

Density $=1.3 \mathrm{~g} / \mathrm{cm} 3$ from this ref., and from pg II.F.1-5 of Carter et al. (1968). Average density of Masonite is listed at http://www.hudsonhighland.com/fiberboardchart.htm as about 79 to $80 \mathrm{lb} / \mathrm{ft} 3$, which averages to $1.27 \mathrm{~g} / \mathrm{cm} 3$.

\section{Melamine}

\begin{tabular}{llll}
\hline Formula $=$ & C5H7N6 & Molecular weight $(\mathrm{g} / \mathrm{mole})=$ & 151.14928 \\
Density $(\mathrm{g} / \mathrm{cm} 3)=$ & 1.350000 & Total atom density $($ atoms $/ \mathrm{b}-\mathrm{cm})=$ & $9.682 \mathrm{E}-02$
\end{tabular}

The above density is estimated to be accurate to 3 significant digits. Uncertainties are not addressed. The following data was calculated from the input formula.

\begin{tabular}{|c|c|c|c|c|c|}
\hline Element & Neutron ZA & Photon ZA & $\begin{array}{l}\text { Weight } \\
\text { Fraction }\end{array}$ & $\begin{array}{c}\text { Atom } \\
\text { Fraction }\end{array}$ & $\begin{array}{l}\text { Atom } \\
\text { Density }\end{array}$ \\
\hline $\mathrm{H}$ & 1001 & 1000 & $\overline{0.046680}$ & 0.388889 & 0.037651 \\
\hline C & 6000 & 6000 & 0.397313 & 0.277778 & 0.026894 \\
\hline $\mathrm{N}$ & 7014 & 7000 & 0.556008 & 0.333333 & 0.032272 \\
\hline Total & & & 1.000000 & 1.000000 & 0.096817 \\
\hline
\end{tabular}

\begin{tabular}{|c|cc|cc|cr|}
\hline MCNP Form & \multicolumn{2}{c|}{ Weight Fractions } & \multicolumn{2}{c|}{ Atom Fractions } & \multicolumn{2}{c|}{ Atom Densities } \\
\hline Neutrons & 1001 & -0.046680 & 1001 & 0.388889 & 1001 & 0.037651 \\
& 6000 & -0.397313 & 6000 & 0.277778 & 6000 & 0.026894 \\
& 7014 & -0.556008 & 7014 & 0.333333 & 7014 & 0.032272 \\
Photons & & & & & & \\
& 1000 & -0.046680 & 1000 & 0.388889 & 1000 & 0.037651 \\
& 6000 & -0.397313 & 6000 & 0.277778 & 6000 & 0.026894 \\
& 7000 & -0.556008 & 7000 & 0.333333 & 7000 & 0.032272
\end{tabular}

\begin{tabular}{cccc}
\hline CEPXS Form: & material & $\mathrm{H}$ & 0.046680 \\
& & $\mathrm{C}$ & 0.397313 \\
& & $\mathrm{~N}$ & 0.556008
\end{tabular}

$\begin{array}{cc}\text { matname } & \text { Melamine } \\ \text { density } & 1.350000\end{array}$

Comments and References

Also called melamine resin or melamine formaldehyde. It is a hard thermosetting plastic often used for kitchen utensils and plates (Melmac), and is the main constituent of Formica and laminate flooring (http://en.wikipedia.org/wiki/Melamine_resin). Density and formula from Table 51.2 of Hungerford (1960). 


\section{Mercury}

\begin{tabular}{llll}
\hline Formula $=$ & $\mathrm{Hg}$ & Molecular weight $(\mathrm{g} / \mathrm{mole})=$ & 200.59 \\
Density $(\mathrm{g} / \mathrm{cm} 3)=$ & 13.546000 & Total atom density $($ atoms $/ \mathrm{b}-\mathrm{cm})=$ & $4.067 \mathrm{E}-02$
\end{tabular}

The above density is estimated to be accurate to 4 significant digits. Uncertainties are not addressed.

The following data was calculated from the input formula.

\begin{tabular}{|c|c|c|c|c|c|c|}
\hline$\frac{\text { Element }}{\mathrm{Hg}}$ & $\frac{\text { Neutron ZA }}{80000}$ & $\frac{\text { Photon ZA }}{80000}$ & $\begin{array}{c}\text { Weight } \\
\frac{\text { Fraction }}{1.000000}\end{array}$ & $\begin{array}{c}\text { Atom } \\
\frac{\text { Fraction }}{1.000000}\end{array}$ & $\begin{array}{c}\text { Atom } \\
\text { Density } \\
0.040668\end{array}$ & \\
\hline Total & & & 1.000000 & 1.000000 & 0.040668 & \\
\hline MCNP Form & \multicolumn{2}{|c|}{ Weight Fractions } & \multicolumn{2}{|c|}{ Atom Fractions } & \multicolumn{2}{|c|}{ Atom Densities } \\
\hline Neutrons & 80000 & -1.000000 & 80000 & 1.000000 & 80000 & 0.040668 \\
\hline Photons & 80000 & -1.000000 & 80000 & 1.000000 & 80000 & 0.040668 \\
\hline CEPXS Form: & $\begin{array}{l}\text { material } \\
\text { matname } \\
\text { density }\end{array}$ & $\begin{array}{c}\mathrm{Hg} \\
\text { Mercury } \\
13.546000\end{array}$ & 1.000000 & & & \\
\hline
\end{tabular}

\section{Mercury lodide}

\begin{tabular}{llll}
\hline Formula $=$ & $\mathrm{Hgl} 2$ & Molecular weight $(\mathrm{g} / \mathrm{mole})=$ & 454.39894 \\
Density $(\mathrm{g} / \mathrm{cm} 3)=$ & 6.360000 & Total atom density $($ atoms $/ \mathrm{b}-\mathrm{cm})=$ & $2.529 \mathrm{E}-02$
\end{tabular}

The above density is estimated to be accurate to 3 significant digits. Uncertainties are not addressed. The following data were calculated from the input weight fractions.

\begin{tabular}{|c|c|c|c|c|c|c|}
\hline Element & Neutron ZA & Photon ZA & $\begin{array}{l}\text { Weight } \\
\text { Fraction }\end{array}$ & $\begin{array}{c}\text { Atom } \\
\text { Fraction }\end{array}$ & $\begin{array}{c}\text { Atom } \\
\text { Density }\end{array}$ & \\
\hline 1 & 53127 & 53000 & 0.558560 & 0.666667 & 0.016858 & \\
\hline $\mathrm{Hg}$ & 80000 & 80000 & 0.441440 & 0.333333 & 0.008429 & \\
\hline Total & & & 1.000000 & 1.000000 & 0.025287 & \\
\hline MCNP Form & \multicolumn{2}{|c|}{ Weight Fractions } & \multicolumn{2}{|c|}{ Atom Fractions } & \multicolumn{2}{|c|}{ Atom Densities } \\
\hline \multirow[t]{2}{*}{ Neutrons } & 53127 & -0.558560 & 53127 & 0.666667 & 53127 & 0.016858 \\
\hline & 80000 & -0.441440 & 80000 & 0.333333 & 80000 & 0.008429 \\
\hline \multirow[t]{2}{*}{ Photons } & 53000 & -0.558560 & 53000 & 0.666667 & 53000 & 0.016858 \\
\hline & 80000 & -0.441440 & 80000 & 0.333333 & 80000 & 0.008429 \\
\hline
\end{tabular}




\begin{tabular}{|cccc} 
CEPXS Form: & material & $\mathrm{I}$ & 0.558560 \\
& & $\mathrm{Hg}$ & 0.441440 \\
& & & \\
& matname & Mercury lodide & \\
& density & 6.360000 & \\
\hline
\end{tabular}

\section{Comments and References}

Density and weight fractions from http://physics.nist.gov/cgi-bin/Star/compos.pl?matno=196 (NIST 1998).

Formula from Lide (2008), pgs 4 - 76, for Mercury (II) lodide.

\section{Methane}

\begin{tabular}{llll}
\hline Formula $=$ & $\mathrm{CH} 4$ & Molecular weight $(\mathrm{g} / \mathrm{mole})=$ & 16.04246 \\
Density $(\mathrm{g} / \mathrm{cm} 3)=$ & 0.000667 & Total atom density $($ atoms $/ \mathrm{b}-\mathrm{cm})=$ & $1.252 \mathrm{E}-04$
\end{tabular}

The above density is estimated to be accurate to 5 significant digits. Uncertainties are not addressed.

The following data was calculated from the input formula.

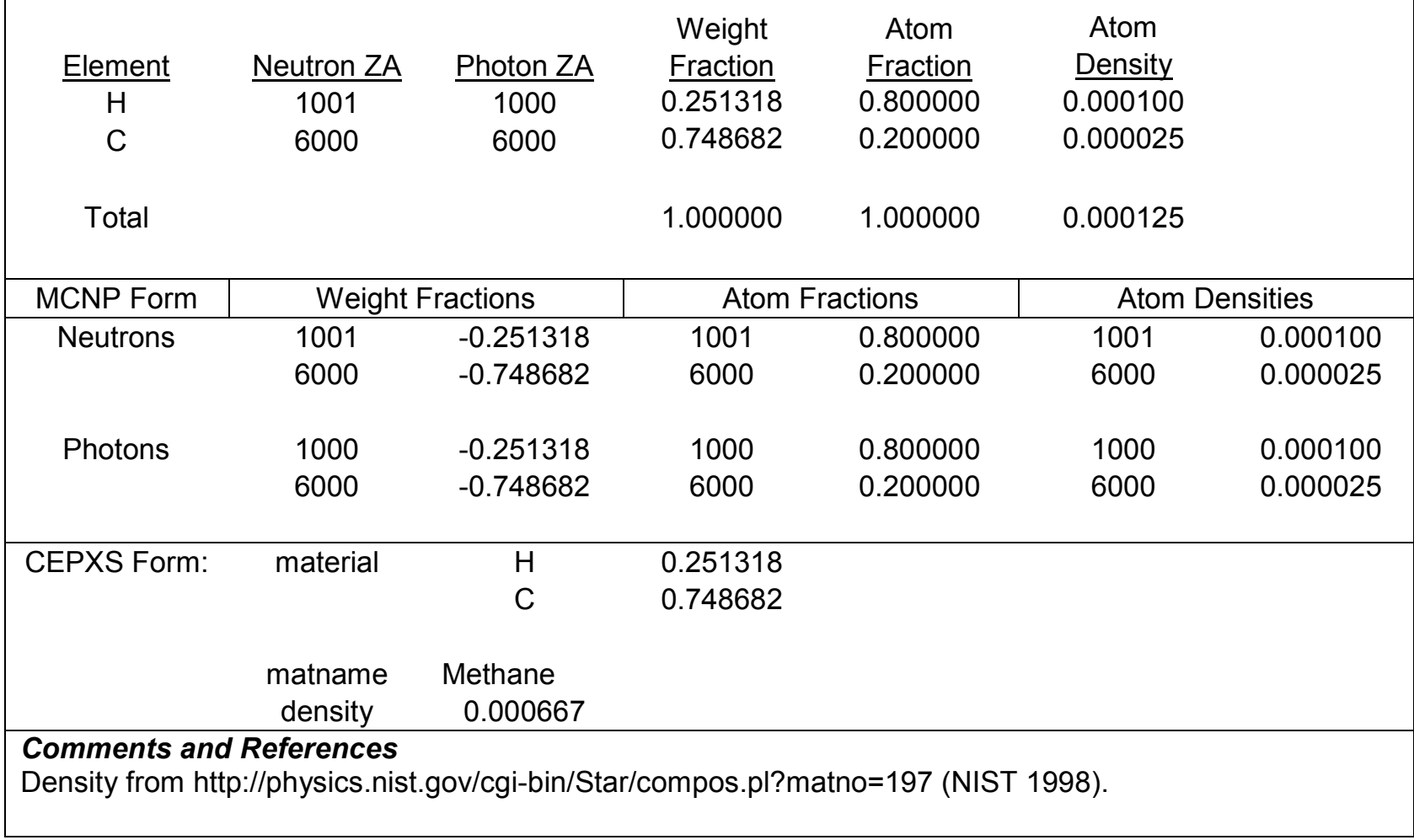

\section{Methanol}

Formula $=$

$\mathrm{CH} 4 \mathrm{O}$

0.791400

Molecular weight $(\mathrm{g} / \mathrm{mole})=$

Total atom density $($ atoms $/ \mathrm{b}-\mathrm{cm})=$

32.04186

8.924E-02

The above density is estimated to be accurate to 4 significant digits. Uncertainties are not addressed.

The following data were calculated from the input weight fractions. 


\begin{tabular}{|c|c|c|c|c|c|c|}
\hline Element & Neutron ZA & Photon ZA & $\begin{array}{l}\text { Weight } \\
\text { Fraction }\end{array}$ & $\begin{array}{c}\text { Atom } \\
\text { Fraction }\end{array}$ & $\begin{array}{l}\text { Atom } \\
\text { Density }\end{array}$ & \\
\hline $\mathrm{H}$ & 1001 & 1000 & 0.125822 & $\overline{0.666654}$ & 0.059493 & \\
\hline $\mathrm{C}$ & 6000 & 6000 & 0.374852 & 0.166675 & 0.014874 & \\
\hline $\mathrm{O}$ & 8016 & 8000 & 0.499326 & 0.166671 & 0.014874 & \\
\hline Total & & & 1.000000 & 1.000000 & 0.089242 & \\
\hline MCNP Form & \multicolumn{2}{|c|}{ Weight Fractions } & \multicolumn{2}{|c|}{ Atom Fractions } & \multicolumn{2}{|c|}{ Atom Densities } \\
\hline \multirow[t]{3}{*}{ Neutrons } & 1001 & -0.125822 & 1001 & 0.666654 & 1001 & 0.059493 \\
\hline & 6000 & -0.374852 & 6000 & 0.166675 & 6000 & 0.014874 \\
\hline & 8016 & -0.499326 & 8016 & 0.166671 & 8016 & 0.014874 \\
\hline \multirow[t]{3}{*}{ Photons } & 1000 & -0.125822 & 1000 & 0.666654 & 1000 & 0.059493 \\
\hline & 6000 & -0.374852 & 6000 & 0.166675 & 6000 & 0.014874 \\
\hline & 8000 & -0.499326 & 8000 & 0.166671 & 8000 & 0.014874 \\
\hline \multirow[t]{4}{*}{ CEPXS Form: } & material & $\mathrm{H}$ & 0.125822 & & & \\
\hline & & C & 0.374852 & & & \\
\hline & & $\mathrm{O}$ & 0.499326 & & & \\
\hline & $\begin{array}{l}\text { matname } \\
\text { density }\end{array}$ & $\begin{array}{l}\text { Methanol } \\
0.791400\end{array}$ & & & & \\
\hline \multicolumn{7}{|c|}{$\begin{array}{l}\text { Comments and References } \\
\text { Density and weight fractions from http://physics.nist.gov/cgi-bin/Star/compos.pl?matno=198 (NIST 1998). } \\
\text { Formula from Lide (2008), pgs } 3-326 \text {. } \\
\text { Also called methyl alcohol (Table } 51.120 \text { of Hungerford 1960). }\end{array}$} \\
\hline
\end{tabular}

\section{Methylene Chloride}

\begin{tabular}{llll}
\hline Formula $=$ & $\mathrm{CH} 2 \mathrm{Cl} 2$ & Molecular weight $(\mathrm{g} / \mathrm{mole})=$ & 84.93258 \\
Density $(\mathrm{g} / \mathrm{cm} 3)=$ & 1.326600 & Total atom density $($ atoms $/ \mathrm{b}-\mathrm{cm})=$ & $4.703 \mathrm{E}-02$
\end{tabular}

The above density is estimated to be accurate to 4 significant digits. Uncertainties are not addressed.

The following data was calculated from the input formula.

\begin{tabular}{|c|c|c|c|c|c|c|}
\hline Element & Neutron ZA & Photon ZA & $\begin{array}{l}\text { Weight } \\
\text { Fraction }\end{array}$ & $\begin{array}{l}\text { Atom } \\
\text { Fraction }\end{array}$ & $\begin{array}{l}\text { Atom } \\
\text { Density }\end{array}$ & \\
\hline$\overline{\mathrm{H}}$ & 1001 & 1000 & $\overline{0.023735}$ & $\overline{0.400000}$ & 0.018813 & \\
\hline C & 6000 & 6000 & 0.141415 & 0.200000 & 0.009406 & \\
\hline $\mathrm{Cl}$ & 17000 & 17000 & 0.834850 & 0.400000 & 0.018813 & \\
\hline Total & & & 1.000000 & 1.000000 & 0.047031 & \\
\hline MCNP Form & \multicolumn{2}{|c|}{ Weight Fractions } & \multicolumn{2}{|c|}{ Atom Fractions } & \multicolumn{2}{|c|}{ Atom Densities } \\
\hline \multirow{3}{*}{ Neutrons } & 1001 & -0.023735 & 1001 & 0.400000 & 1001 & 0.018813 \\
\hline & 6000 & -0.141415 & 6000 & 0.200000 & 6000 & 0.009406 \\
\hline & 17000 & -0.834850 & 17000 & 0.400000 & 17000 & 0.018813 \\
\hline
\end{tabular}




\begin{tabular}{|c|c|c|c|c|c|c|}
\hline Photons & $\begin{array}{c}1000 \\
6000 \\
17000\end{array}$ & $\begin{array}{l}-0.023735 \\
-0.141415 \\
-0.834850\end{array}$ & $\begin{array}{c}1000 \\
6000 \\
17000\end{array}$ & $\begin{array}{l}0.400000 \\
0.200000 \\
0.400000\end{array}$ & $\begin{array}{c}1000 \\
6000 \\
17000\end{array}$ & $\begin{array}{l}0.018813 \\
0.009406 \\
0.018813\end{array}$ \\
\hline CEPXS Form: & $\begin{array}{c}\text { matname } \\
\text { density }\end{array}$ & $\begin{array}{l}\mathrm{H} \\
\mathrm{C} \\
\mathrm{Cl} \\
\text { Methylene C } \\
1.326600\end{array}$ & $\begin{array}{l}0.023735 \\
0.141415 \\
0.834850 \\
\text { ride }\end{array}$ & & & \\
\hline \multicolumn{7}{|c|}{$\begin{array}{l}\text { Comments and References } \\
\text { Formula and density at } \\
\text { http://www.matweb.com/search/DataSheet.aspx?MatGUID=ce197b29a2644022be281b606729a1e7 } \\
\text { (Automation Creations 2010). Formula and density also in Lide (2008), pgs 3-156. } \\
\text { Also called dichloromethane. }\end{array}$} \\
\hline
\end{tabular}

\section{Molybdenum}

\begin{tabular}{llll}
\hline Formula $=$ & Mo & Molecular weight $(\mathrm{g} / \mathrm{mole})=$ & 95.94 \\
Density $(\mathrm{g} / \mathrm{cm} 3)=$ & 10.220000 & Total atom density $($ atoms $/ \mathrm{b}-\mathrm{cm})=$ & $6.415 \mathrm{E}-02$
\end{tabular}

The above density is estimated to be accurate to 4 significant digits. Uncertainties are not addressed.

The following data was calculated from the input formula.

\begin{tabular}{|c|c|c|c|c|c|c|}
\hline$\frac{\text { Element }}{\text { Mo }}$ & $\frac{\text { Neutron ZA }}{42000}$ & $\frac{\text { Photon ZA }}{42000}$ & $\begin{array}{l}\text { Weight } \\
\text { Fraction } \\
1.000000\end{array}$ & $\begin{array}{c}\text { Atom } \\
\frac{\text { Fraction }}{1.000000}\end{array}$ & $\begin{array}{c}\text { Atom } \\
\text { Density } \\
0.064151\end{array}$ & \\
\hline Total & & & 1.000000 & 1.000000 & 0.064151 & \\
\hline MCNP Form & \multicolumn{2}{|c|}{ Weight Fractions } & \multicolumn{2}{|c|}{ Atom Fractions } & \multicolumn{2}{|c|}{ Atom Densities } \\
\hline Neutrons & 42000 & -1.000000 & 42000 & 1.000000 & 42000 & 0.064151 \\
\hline Photons & 42000 & -1.000000 & 42000 & 1.000000 & 42000 & 0.064151 \\
\hline CEPXS Form: & $\begin{array}{l}\text { material } \\
\text { matname } \\
\text { density }\end{array}$ & $\begin{array}{c}\text { Mo } \\
\text { Molybdenum } \\
10.220000\end{array}$ & 1.000000 & & & \\
\hline $\begin{array}{l}\text { Comments ar } \\
\text { Density from }\end{array}$ & $\begin{array}{l}\text { References } \\
\text { //physics.nis }\end{array}$ & 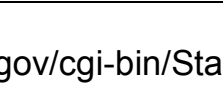 & & & & \\
\hline
\end{tabular}

\section{Monosodium Titanate, MST}

\begin{tabular}{|llll}
\hline Formula $=$ & NaTi2O5H & Molecular weight $(\mathrm{g} / \mathrm{mole})=$ & 199.72871 \\
Density $(\mathrm{g} / \mathrm{cm} 3)=$ & 1.000000 & Total atom density $($ atoms $/ \mathrm{b}-\mathrm{cm})=$ & $2.714 \mathrm{E}-02$
\end{tabular}


The above density is estimated to be accurate to 2 significant digits. Uncertainties are not addressed. The following data was calculated from the input formula.

\begin{tabular}{|c|c|c|c|c|c|}
\hline Element & Neutron ZA & Photon ZA & $\begin{array}{l}\text { Weight } \\
\text { Fraction }\end{array}$ & $\begin{array}{c}\text { Atom } \\
\text { Fraction }\end{array}$ & $\begin{array}{l}\text { Atom } \\
\text { Density }\end{array}$ \\
\hline $\mathrm{H}$ & 1001 & 1000 & $\overline{0.005047}$ & $\overline{0.111111}$ & 0.003015 \\
\hline $\mathrm{O}$ & 8016 & 8000 & 0.400528 & 0.555556 & 0.015076 \\
\hline $\mathrm{Na}$ & 11023 & 11000 & 0.115105 & 0.111111 & 0.003015 \\
\hline $\mathrm{Ti}$ & 22000 & 22000 & 0.479320 & 0.222222 & 0.006030 \\
\hline Total & & & 1.000000 & 1.000000 & 0.027136 \\
\hline
\end{tabular}

\begin{tabular}{|c|c|c|c|c|c|c|}
\hline MCNP Form & \multicolumn{2}{|c|}{ Weight Fractions } & \multicolumn{2}{|c|}{ Atom Fractions } & \multicolumn{2}{|c|}{ Atom Densities } \\
\hline \multirow{4}{*}{ Neutrons } & 1001 & -0.005047 & 1001 & 0.111111 & 1001 & 0.003015 \\
\hline & 8016 & -0.400528 & 8016 & 0.555556 & 8016 & 0.015076 \\
\hline & 11023 & -0.115105 & 11023 & 0.111111 & 11023 & 0.003015 \\
\hline & 22000 & -0.479320 & 22000 & 0.222222 & 22000 & 0.006030 \\
\hline \multirow[t]{4}{*}{ Photons } & 1000 & -0.005047 & 1000 & 0.111111 & 1000 & 0.003015 \\
\hline & 8000 & -0.400528 & 8000 & 0.555556 & 8000 & 0.015076 \\
\hline & 11000 & -0.115105 & 11000 & 0.111111 & 11000 & 0.003015 \\
\hline & 22000 & -0.479320 & 22000 & 0.222222 & 22000 & 0.006030 \\
\hline \multirow[t]{4}{*}{ CEPXS Form: } & material & $\bar{H}$ & 0.005047 & & & \\
\hline & & 0 & 0.400528 & & & \\
\hline & & $\mathrm{Na}$ & 0.115105 & & & \\
\hline & & $\mathrm{Ti}$ & 0.479320 & & & \\
\hline
\end{tabular}

matname Monosodium Titanate, MST density $\quad 1.000000$

\section{Comments and References}

Formula from http://www.osti.gov/bridge/purl.cover.jsp?purl=/881358-Y9ExpG/.

MST is normally used in a solution (Hobbs DT, MS Blume, and HL Thacker. 2000. Phase V Simulant Testing of Monosodium Titanate Adsorption Kinetics, WSRC-TR-2000-00142, Rev. 0, Westinghouse Savannah River Company).

Since it is not used as a solid and a reference for the density as a solid could not be located, $1.0 \mathrm{~g} / \mathrm{cm} 3$ was assumed.

\section{Muscle Equivalent-Liquid, with Sucrose}

\begin{tabular}{|c|c|c|c|}
\hline nula $=$ & - & Molecular weight $(\mathrm{g} / \mathrm{mole})=$ & - \\
\hline$(\mathrm{g} / \mathrm{cm} 3)=$ & 1.110000 & Total atom density $($ atoms $/ \mathrm{b}-\mathrm{cm})=$ & 1.052E-01 \\
\hline
\end{tabular}

The above density is estimated to be accurate to 3 significant digits. Uncertainties are not addressed.

The following data were calculated from the input weight fractions.

\begin{tabular}{|c|c|c|c|c|c|}
\hline ement & Neutron ZA & Photon ZA & $\begin{array}{l}\text { Weight } \\
\text { Fraction }\end{array}$ & $\begin{array}{c}\text { Atom } \\
\text { Fraction }\end{array}$ & $\begin{array}{l}\text { Atom } \\
\text { Density }\end{array}$ \\
\hline $\mathrm{H}$ & 1001 & 1000 & 0.098234 & 0.619265 & 0.065148 \\
\hline
\end{tabular}


PIET-43741-TM-963

PNNL-15870 Rev. 1

\begin{tabular}{|c|c|c|c|c|c|c|}
\hline C & 6000 & 6000 & 0.156214 & 0.082642 & \multicolumn{2}{|c|}{0.008694} \\
\hline $\mathrm{N}$ & 7014 & 7000 & 0.035451 & 0.016082 & \multicolumn{2}{|c|}{0.001692} \\
\hline $\mathrm{O}$ & 8016 & 8000 & 0.710101 & 0.282011 & \multicolumn{2}{|c|}{0.029668} \\
\hline Total & & & 1.000000 & 1.000000 & \multicolumn{2}{|c|}{0.105202} \\
\hline MCNP Form & \multicolumn{2}{|c|}{ Weight Fractions } & \multicolumn{2}{|c|}{ Atom Fractions } & \multicolumn{2}{|c|}{ Atom Densities } \\
\hline \multirow[t]{4}{*}{ Neutrons } & 1001 & -0.098234 & 1001 & 0.619265 & 1001 & 0.065148 \\
\hline & 6000 & -0.156214 & 6000 & 0.082642 & 6000 & 0.008694 \\
\hline & 7014 & -0.035451 & 7014 & 0.016082 & 7014 & 0.001692 \\
\hline & 8016 & -0.710101 & 8016 & 0.282011 & 8016 & 0.029668 \\
\hline \multirow[t]{4}{*}{ Photons } & 1000 & -0.098234 & 1000 & 0.619265 & 1000 & 0.065148 \\
\hline & 6000 & -0.156214 & 6000 & 0.082642 & 6000 & 0.008694 \\
\hline & 7000 & -0.035451 & 7000 & 0.016082 & 7000 & 0.001692 \\
\hline & 8000 & -0.710101 & 8000 & 0.282011 & 8000 & 0.029668 \\
\hline \multirow[t]{5}{*}{ CEPXS Form: } & material & $\mathrm{H}$ & 0.098234 & & & \\
\hline & & C & 0.156214 & & & \\
\hline & & $\mathrm{N}$ & 0.035451 & & & \\
\hline & & $\mathrm{O}$ & 0.710101 & & & \\
\hline & $\begin{array}{l}\text { matname } \\
\text { density }\end{array}$ & \multicolumn{5}{|c|}{$\begin{array}{l}\text { Muscle Equivalent-Liquid, with Sucrose } \\
1.110000\end{array}$} \\
\hline
\end{tabular}

\section{Muscle Equivalent-Liquid, without Sucrose}

\begin{tabular}{llll}
\hline Formula $=$ & - & Molecular weight $(\mathrm{g} / \mathrm{mole})=$ & - \\
Density $(\mathrm{g} / \mathrm{cm} 3)=$ & 1.070000 & Total atom density $($ atoms $/ \mathrm{b}-\mathrm{cm})=$ & $1.032 \mathrm{E}-01$
\end{tabular}

The above density is estimated to be accurate to 3 significant digits. Uncertainties are not addressed.

The following data were calculated from the input weight fractions.

\begin{tabular}{|c|c|c|c|c|c|c|}
\hline Element & Neutron ZA & Photon ZA & $\begin{array}{l}\text { Weight } \\
\text { Fraction }\end{array}$ & $\begin{array}{c}\text { Atom } \\
\text { Fraction }\end{array}$ & $\begin{array}{l}\text { Atom } \\
\text { Density }\end{array}$ & \\
\hline $\mathrm{H}$ & 1001 & 1000 & $\overline{0.101969}$ & $\overline{0.631883}$ & 0.065188 & \\
\hline C & 6000 & 6000 & 0.120058 & 0.062435 & 0.006441 & \\
\hline $\mathrm{N}$ & 7014 & 7000 & 0.035451 & 0.015809 & 0.001631 & \\
\hline $\mathrm{O}$ & 8016 & 8000 & 0.742522 & 0.289874 & 0.029905 & \\
\hline Total & & & 1.000000 & 1.000000 & 0.103165 & \\
\hline MCNP Form & \multicolumn{2}{|c|}{ Weight Fractions } & \multicolumn{2}{|c|}{ Atom Fractions } & \multicolumn{2}{|c|}{ Atom Densities } \\
\hline \multirow[t]{3}{*}{ Neutrons } & 1001 & -0.101969 & 1001 & 0.631883 & 1001 & 0.065188 \\
\hline & 6000 & -0.120058 & 6000 & 0.062435 & 6000 & 0.006441 \\
\hline & 7014 & -0.035451 & 7014 & 0.015809 & 7014 & 0.001631 \\
\hline
\end{tabular}


PIET-43741-TM-963

PNNL-15870 Rev. 1

\begin{tabular}{|c|c|c|c|c|c|c|}
\hline & 8016 & -0.742522 & 8016 & 0.289874 & 8016 & 0.029905 \\
\hline \multirow[t]{4}{*}{ Photons } & 1000 & -0.101969 & 1000 & 0.631883 & 1000 & 0.065188 \\
\hline & 6000 & -0.120058 & 6000 & 0.062435 & 6000 & 0.006441 \\
\hline & 7000 & -0.035451 & 7000 & 0.015809 & 7000 & 0.001631 \\
\hline & 8000 & -0.742522 & 8000 & 0.289874 & 8000 & 0.029905 \\
\hline \multirow[t]{5}{*}{ CEPXS Form: } & material & $\mathrm{H}$ & 0.101969 & & & \\
\hline & & C & 0.120058 & & & \\
\hline & & $\mathrm{N}$ & 0.035451 & & & \\
\hline & & O & 0.742522 & & & \\
\hline & $\begin{array}{l}\text { matname } \\
\text { density }\end{array}$ & \multicolumn{3}{|c|}{$\begin{array}{l}\text { Muscle Equivalent-Liquid, without Sucrose } \\
1.070000\end{array}$} & & \\
\hline
\end{tabular}

\section{Muscle, Skeletal (ICRP)}

\begin{tabular}{llll}
\hline Formula $=$ & - & Molecular weight $(\mathrm{g} / \mathrm{mole})=$ & - \\
Density $(\mathrm{g} / \mathrm{cm} 3)=$ & 1.040000 & Total atom density $($ atoms $/ \mathrm{b}-\mathrm{cm})=$ & $9.911 \mathrm{E}-02$
\end{tabular}

The above density is estimated to be accurate to 3 significant digits. Uncertainties are not addressed.

The following data were calculated from the input weight fractions.

\begin{tabular}{|c|c|c|c|c|c|c|}
\hline Element & Neutron ZA & Photon ZA & $\begin{array}{l}\text { Weight } \\
\text { Fraction }\end{array}$ & $\begin{array}{c}\text { Atom } \\
\text { Fraction }\end{array}$ & $\begin{array}{l}\text { Atom } \\
\text { Density }\end{array}$ & \\
\hline $\mathrm{H}$ & 1001 & 1000 & 0.100637 & 0.630932 & 0.062533 & \\
\hline C & 6000 & 6000 & 0.107830 & 0.056732 & 0.005623 & \\
\hline $\mathrm{N}$ & 7014 & 7000 & 0.027680 & 0.012488 & 0.001238 & \\
\hline 0 & 8016 & 8000 & 0.754773 & 0.298107 & 0.029546 & \\
\hline $\mathrm{Na}$ & 11023 & 11000 & 0.000750 & 0.000206 & 0.000020 & \\
\hline $\mathrm{Mg}$ & 12000 & 12000 & 0.000190 & 0.000049 & 0.000005 & \\
\hline $\mathrm{P}$ & 15031 & 15000 & 0.001800 & 0.000367 & 0.000036 & \\
\hline$S$ & 16000 & 16000 & 0.002410 & 0.000475 & 0.000047 & \\
\hline $\mathrm{Cl}$ & 17000 & 17000 & 0.000790 & 0.000141 & 0.000014 & \\
\hline $\mathrm{K}$ & 19000 & 19000 & 0.003020 & 0.000488 & 0.000048 & \\
\hline $\mathrm{Ca}$ & 20000 & 20000 & 0.000030 & 0.000005 & 0.000000 & \\
\hline $\mathrm{Fe}$ & 26000 & 26000 & 0.000040 & 0.000005 & 0.000000 & \\
\hline $\mathrm{Zn}$ & 30000 & 30000 & 0.000050 & 0.000005 & 0.000000 & \\
\hline Total & & & 1.000000 & 1.000000 & 0.099112 & \\
\hline MCNP Form & \multicolumn{2}{|c|}{ Weight Fractions } & \multicolumn{2}{|c|}{ Atom Fractions } & \multicolumn{2}{|c|}{ Atom Densities } \\
\hline \multirow[t]{5}{*}{ Neutrons } & 1001 & -0.100637 & 1001 & 0.630932 & 1001 & 0.062533 \\
\hline & 6000 & -0.107830 & 6000 & 0.056732 & 6000 & 0.005623 \\
\hline & 7014 & -0.027680 & 7014 & 0.012488 & 7014 & 0.001238 \\
\hline & 8016 & -0.754773 & 8016 & 0.298107 & 8016 & 0.029546 \\
\hline & 11023 & -0.000750 & 11023 & 0.000206 & 11023 & 0.000020 \\
\hline
\end{tabular}


PIET-43741-TM-963

PNNL-15870 Rev. 1

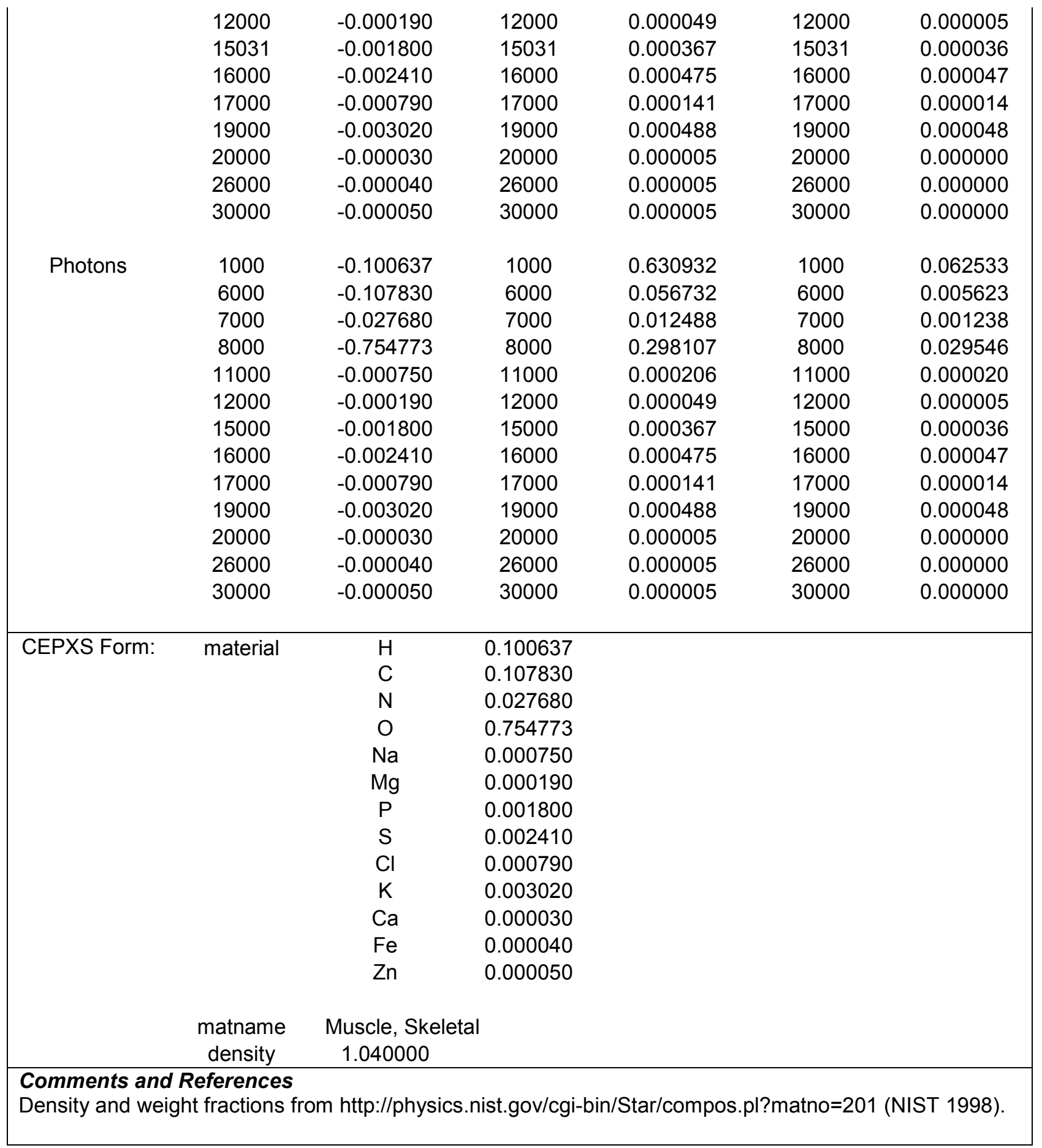

\section{Muscle, Striated (ICRU)}

Formula $=$

Density $(\mathrm{g} / \mathrm{cm} 3)=1.040000$

The above density is estimated to be accurate to 3 significant digits. Uncertainties are not addressed.

The following data were calculated from the input weight fractions. 
PIET-43741-TM-963

PNNL-15870 Rev. 1

\begin{tabular}{|c|c|c|c|c|c|c|}
\hline Element & Neutron ZA & Photon ZA & $\begin{array}{l}\text { Weight } \\
\text { Fraction }\end{array}$ & $\begin{array}{c}\text { Atom } \\
\text { Fraction }\end{array}$ & $\begin{array}{l}\text { Atom } \\
\text { Density }\end{array}$ & \\
\hline $\mathrm{H}$ & 1001 & 1000 & 0.101997 & 0.633101 & 0.063378 & \\
\hline $\mathrm{C}$ & 6000 & 6000 & 0.123000 & 0.064070 & 0.006414 & \\
\hline $\mathrm{N}$ & 7014 & 7000 & 0.035000 & 0.015633 & 0.001565 & \\
\hline 0 & 8016 & 8000 & 0.729003 & 0.285066 & 0.028537 & \\
\hline $\mathrm{Na}$ & 11023 & 11000 & 0.000800 & 0.000218 & 0.000022 & \\
\hline $\mathrm{Mg}$ & 12000 & 12000 & 0.000200 & 0.000051 & 0.000005 & \\
\hline$P$ & 15031 & 15000 & 0.002000 & 0.000404 & 0.000040 & \\
\hline$S$ & 16000 & 16000 & 0.005000 & 0.000976 & 0.000098 & \\
\hline $\mathrm{K}$ & 19000 & 19000 & 0.003000 & 0.000480 & 0.000048 & \\
\hline Total & & & 1.000000 & 1.000000 & 0.100107 & \\
\hline MCNP Form & \multicolumn{2}{|c|}{ Weight Fractions } & \multicolumn{2}{|c|}{ Atom Fractions } & \multicolumn{2}{|c|}{ Atom Densities } \\
\hline \multirow{9}{*}{ Neutrons } & 1001 & -0.101997 & 1001 & 0.633101 & 1001 & 0.063378 \\
\hline & 6000 & -0.123000 & 6000 & 0.064070 & 6000 & 0.006414 \\
\hline & 7014 & -0.035000 & 7014 & 0.015633 & 7014 & 0.001565 \\
\hline & 8016 & -0.729003 & 8016 & 0.285066 & 8016 & 0.028537 \\
\hline & 11023 & -0.000800 & 11023 & 0.000218 & 11023 & 0.000022 \\
\hline & 12000 & -0.000200 & 12000 & 0.000051 & 12000 & 0.000005 \\
\hline & 15031 & -0.002000 & 15031 & 0.000404 & 15031 & 0.000040 \\
\hline & 16000 & -0.005000 & 16000 & 0.000976 & 16000 & 0.000098 \\
\hline & 19000 & -0.003000 & 19000 & 0.000480 & 19000 & 0.000048 \\
\hline \multirow[t]{9}{*}{ Photons } & 1000 & -0.101997 & 1000 & 0.633101 & 1000 & 0.063378 \\
\hline & 6000 & -0.123000 & 6000 & 0.064070 & 6000 & 0.006414 \\
\hline & 7000 & -0.035000 & 7000 & 0.015633 & 7000 & 0.001565 \\
\hline & 8000 & -0.729003 & 8000 & 0.285066 & 8000 & 0.028537 \\
\hline & 11000 & -0.000800 & 11000 & 0.000218 & 11000 & 0.000022 \\
\hline & 12000 & -0.000200 & 12000 & 0.000051 & 12000 & 0.000005 \\
\hline & 15000 & -0.002000 & 15000 & 0.000404 & 15000 & 0.000040 \\
\hline & 16000 & -0.005000 & 16000 & 0.000976 & 16000 & 0.000098 \\
\hline & 19000 & -0.003000 & 19000 & 0.000480 & 19000 & 0.000048 \\
\hline \multirow[t]{10}{*}{ CEPXS Form: } & material & $\mathrm{H}$ & 0.101997 & & & \\
\hline & & C & 0.123000 & & & \\
\hline & & $\mathrm{N}$ & 0.035000 & & & \\
\hline & & 0 & 0.729003 & & & \\
\hline & & $\mathrm{Na}$ & 0.000800 & & & \\
\hline & & $\mathrm{Mg}$ & 0.000200 & & & \\
\hline & & $\mathrm{P}$ & 0.002000 & & & \\
\hline & & $S$ & 0.005000 & & & \\
\hline & & $\mathrm{K}$ & 0.003000 & & & \\
\hline & $\begin{array}{l}\text { matname } \\
\text { density }\end{array}$ & \multicolumn{3}{|c|}{$\begin{array}{l}\text { Muscle, Striated } \\
1.040000\end{array}$} & & \\
\hline
\end{tabular}




\section{Neon}

\begin{tabular}{llll}
\hline Formula $=$ & $\mathrm{Ne}$ & Molecular weight $(\mathrm{g} / \mathrm{mole})=$ & 20.1797 \\
Density $(\mathrm{g} / \mathrm{cm} 3)=$ & 0.000839 & Total atom density $($ atoms $/ \mathrm{b}-\mathrm{cm})=$ & $2.502 \mathrm{E}-05$
\end{tabular}

The above density is estimated to be accurate to 5 significant digits. Uncertainties are not addressed.

The following data was calculated from the input formula.

\begin{tabular}{|c|c|c|c|c|c|c|}
\hline$\frac{\text { Element }}{\mathrm{Ne}}$ & $\frac{\text { Neutron ZA }}{10020}$ & $\frac{\text { Photon ZA }}{10000}$ & $\begin{array}{c}\text { Weight } \\
\text { Fraction } \\
1.000000\end{array}$ & $\begin{array}{c}\text { Atom } \\
\frac{\text { Fraction }}{1.000000}\end{array}$ & $\begin{array}{c}\begin{array}{c}\text { Atom } \\
\text { Density }\end{array} \\
0.000025\end{array}$ & \\
\hline Total & & & 1.000000 & 1.000000 & 0.000025 & \\
\hline MCNP Form & \multicolumn{2}{|c|}{ Weight Fractions } & \multicolumn{2}{|c|}{ Atom Fractions } & \multicolumn{2}{|c|}{ Atom Densities } \\
\hline Neutrons & 10020 & -1.000000 & 10020 & 1.000000 & 10020 & 0.000025 \\
\hline Photons & 10000 & -1.000000 & 10000 & 1.000000 & 10000 & 0.000025 \\
\hline CEPXS Form: & $\begin{array}{l}\text { material } \\
\text { matname } \\
\text { density }\end{array}$ & $\begin{array}{c}\mathrm{Ne} \\
\text { Neon } \\
0.000839\end{array}$ & 1.000000 & & & \\
\hline
\end{tabular}

\section{Nickel}

\begin{tabular}{llll}
\hline Formula $=$ & $\mathrm{Ni}$ & Molecular weight $(\mathrm{g} / \mathrm{mole})=$ & 58.6934 \\
Density $(\mathrm{g} / \mathrm{cm} 3)=$ & 8.902000 & Total atom density $($ atoms $/ \mathrm{b}-\mathrm{cm})=$ & $9.134 \mathrm{E}-02$
\end{tabular}

The above density is estimated to be accurate to 4 significant digits. Uncertainties are not addressed.

The following data was calculated from the input formula.

\begin{tabular}{|c|c|c|c|c|c|c|}
\hline$\frac{\text { Element }}{\mathrm{Ni}}$ & $\frac{\text { Neutron ZA }}{28000}$ & $\frac{\text { Photon ZA }}{28000}$ & $\begin{array}{c}\text { Weight } \\
\text { Fraction } \\
1.000000\end{array}$ & $\begin{array}{c}\text { Atom } \\
\frac{\text { Fraction }}{1.000000}\end{array}$ & $\begin{array}{c}\text { Atom } \\
\text { Density } \\
0.091338\end{array}$ & \\
\hline Total & & & 1.000000 & 1.000000 & 0.091338 & \\
\hline MCNP Form & \multicolumn{2}{|c|}{ Weight Fractions } & \multicolumn{2}{|c|}{ Atom Fractions } & \multicolumn{2}{|c|}{ Atom Densities } \\
\hline Neutrons & 28000 & -1.000000 & 28000 & 1.000000 & 28000 & 0.091338 \\
\hline Photons & 28000 & -1.000000 & 28000 & 1.000000 & 28000 & 0.091338 \\
\hline CEPXS Form: & $\begin{array}{l}\text { material } \\
\text { matname } \\
\text { density }\end{array}$ & $\begin{array}{c}\mathrm{Ni} \\
\text { Nickel } \\
8.902000\end{array}$ & 1.000000 & & & \\
\hline
\end{tabular}


Comments and References

Density from http://physics.nist.gov/cgi-bin/Star/compos.pl?matno=028 (NIST 1998).

\section{Niobium}

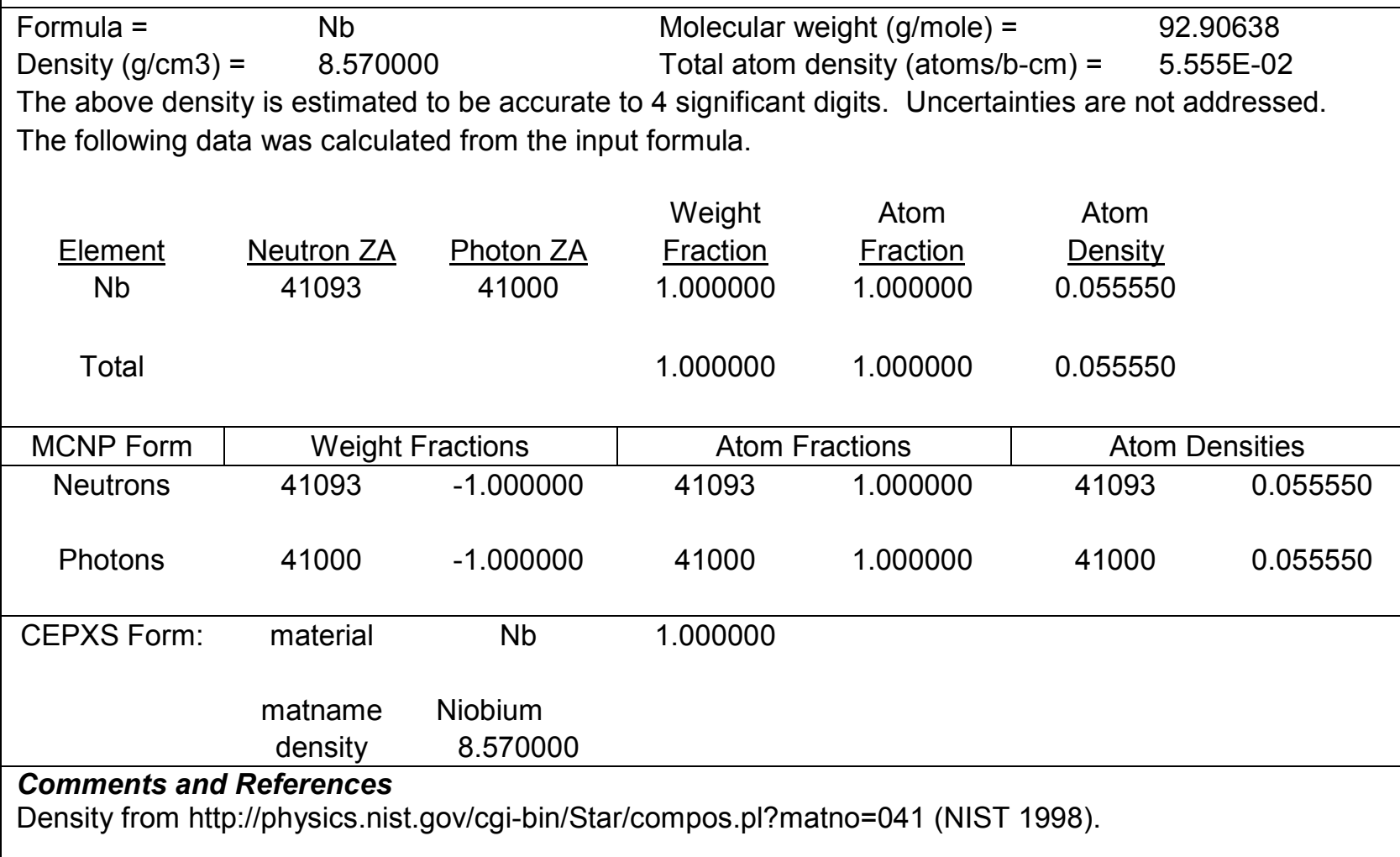

\section{Nitrogen}

\begin{tabular}{llll}
\hline Formula $=$ & $\mathrm{N} 2$ & Molecular weight $(\mathrm{g} / \mathrm{mole})=$ & 28.0134 \\
Density $(\mathrm{g} / \mathrm{cm} 3)=$ & 0.001165 & Total atom density $($ atoms $/ \mathrm{b}-\mathrm{cm})=$ & $5.010 \mathrm{E}-05$
\end{tabular}

The above density is estimated to be accurate to 4 significant digits. Uncertainties are not addressed.

The following data was calculated from the input formula.

\begin{tabular}{|c|c|c|c|c|c|c|}
\hline$\frac{\text { Element }}{\mathrm{N}}$ & $\frac{\text { Neutron ZA }}{7014}$ & $\frac{\text { Photon ZA }}{7000}$ & $\begin{array}{l}\text { Weight } \\
\text { Fraction } \\
1.000000\end{array}$ & $\begin{array}{c}\begin{array}{c}\text { Atom } \\
\text { Fraction }\end{array} \\
\frac{1.000000}{}\end{array}$ & $\begin{array}{c}\text { Atom } \\
\text { Density } \\
0.00005\end{array}$ & \\
\hline Total & & & 1.000000 & 1.000000 & 0.00005 & \\
\hline MCNP Form & \multicolumn{2}{|c|}{ Weight Fractions } & \multicolumn{2}{|c|}{ Atom Fractions } & \multicolumn{2}{|c|}{ Atom Densities } \\
\hline Neutrons & 7014 & -1.000000 & 7014 & 1.000000 & 7014 & 0.000050 \\
\hline Photons & 7000 & -1.000000 & 7000 & 1.000000 & 7000 & 0.000050 \\
\hline
\end{tabular}




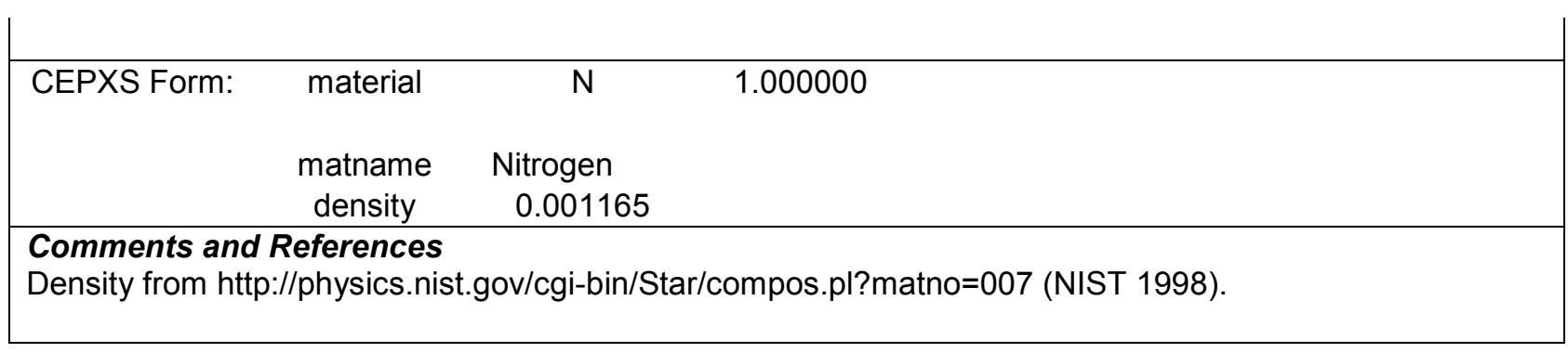

\section{Nylon, Dupont ELVAmide 8062}

\begin{tabular}{llll}
\hline Formula $=$ & - & Molecular weight $(\mathrm{g} / \mathrm{mole})=$ \\
Density $(\mathrm{g} / \mathrm{cm} 3)=$ & 1.080000 & Total atom density $($ atoms $/ \mathrm{b}-\mathrm{cm})=$ & - \\
\hline
\end{tabular}

The above density is estimated to be accurate to 3 significant digits. Uncertainties are not addressed.

The following data were calculated from the input weight fractions.

\begin{tabular}{|c|c|c|c|c|c|c|}
\hline Element & Neutron ZA & Photon ZA & $\begin{array}{l}\text { Weight } \\
\text { Fraction }\end{array}$ & $\begin{array}{l}\text { Atom } \\
\text { Fraction }\end{array}$ & $\begin{array}{l}\text { Atom } \\
\text { Density }\end{array}$ & \\
\hline $\mathrm{H}$ & 1001 & 1000 & $\overline{0.103509}$ & $\overline{0.593363}$ & 0.06679 & \\
\hline $\mathrm{C}$ & 6000 & 6000 & 0.648416 & 0.311934 & 0.03511 & \\
\hline $\mathrm{N}$ & 7014 & 7000 & 0.099536 & 0.041060 & 0.00462 & \\
\hline $\mathrm{O}$ & 8016 & 8000 & 0.148539 & 0.053643 & 0.00603 & \\
\hline Total & & & 1.000000 & 1.000000 & 0.11256 & \\
\hline MCNP Form & \multicolumn{2}{|c|}{ Weight Fractions } & \multicolumn{2}{|c|}{ Atom Fractions } & \multicolumn{2}{|c|}{ Atom Densities } \\
\hline \multirow[t]{4}{*}{ Neutrons } & 1001 & -0.103509 & 1001 & 0.593363 & 1001 & 0.066791 \\
\hline & 6000 & -0.648416 & 6000 & 0.311934 & 6000 & 0.035112 \\
\hline & 7014 & -0.099536 & 7014 & 0.041060 & 7014 & 0.004622 \\
\hline & 8016 & -0.148539 & 8016 & 0.053643 & 8016 & 0.006038 \\
\hline \multirow[t]{4}{*}{ Photons } & 1000 & -0.103509 & 1000 & 0.593363 & 1000 & 0.066791 \\
\hline & 6000 & -0.648416 & 6000 & 0.311934 & 6000 & 0.035112 \\
\hline & 7000 & -0.099536 & 7000 & 0.041060 & 7000 & 0.004622 \\
\hline & 8000 & -0.148539 & 8000 & 0.053643 & 8000 & 0.006038 \\
\hline \multirow[t]{5}{*}{ CEPXS Form: } & material & $\mathrm{H}$ & 0.103509 & & & \\
\hline & & C & 0.648416 & & & \\
\hline & & $\mathrm{N}$ & 0.099536 & & & \\
\hline & & 0 & 0.148539 & & & \\
\hline & $\begin{array}{l}\text { matname } \\
\text { density }\end{array}$ & \multicolumn{5}{|c|}{$\begin{array}{l}\text { Nylon, Dupont ELVAmide } 8062 \\
1.080000\end{array}$} \\
\hline
\end{tabular}




\section{Nylon, Type 11 (Rilsan)}

\begin{tabular}{|c|c|c|c|}
\hline Formula = & - & Molecular weight $(\mathrm{g} / \mathrm{mole})=$ & \\
\hline Density $(\mathrm{g} / \mathrm{cm} 3)=$ & 1.425000 & Total atom density $($ atoms $/ \mathrm{b}-\mathrm{cm})=$ & 1.592E-01 \\
\hline
\end{tabular}

The above density is estimated to be accurate to 3 significant digits. Uncertainties are not addressed.

The following data were calculated from the input weight fractions.

\begin{tabular}{|c|c|c|c|c|c|c|}
\hline Element & Neutron ZA & Photon ZA & $\begin{array}{l}\text { Weight } \\
\text { Fraction }\end{array}$ & $\begin{array}{l}\text { Atom } \\
\text { Fraction }\end{array}$ & $\begin{array}{l}\text { Atom } \\
\text { Density }\end{array}$ & \\
\hline $\mathrm{H}$ & 1001 & 1000 & 0.115476 & 0.617633 & 0.098316 & \\
\hline C & 6000 & 6000 & 0.720819 & 0.323542 & 0.051502 & \\
\hline $\mathrm{N}$ & 7014 & 7000 & 0.076417 & 0.029412 & 0.004682 & \\
\hline $\mathrm{O}$ & 8016 & 8000 & 0.087289 & 0.029412 & 0.004682 & \\
\hline Total & & & 1.000001 & 1.000000 & 0.159181 & \\
\hline MCNP Form & \multicolumn{2}{|c|}{ Weight Fractions } & \multicolumn{2}{|c|}{ Atom Fractions } & \multicolumn{2}{|c|}{ Atom Densities } \\
\hline Neutrons & 1001 & -0.115476 & 1001 & 0.617633 & 1001 & 0.098316 \\
\hline & 6000 & -0.720819 & 6000 & 0.323542 & 6000 & 0.051502 \\
\hline & 7014 & -0.076417 & 7014 & 0.029412 & 7014 & 0.004682 \\
\hline & 8016 & -0.087289 & 8016 & 0.029412 & 8016 & 0.004682 \\
\hline \multirow[t]{4}{*}{ Photons } & 1000 & -0.115476 & 1000 & 0.617633 & 1000 & 0.098316 \\
\hline & 6000 & -0.720819 & 6000 & 0.323542 & 6000 & 0.051502 \\
\hline & 7000 & -0.076417 & 7000 & 0.029412 & 7000 & 0.004682 \\
\hline & 8000 & -0.087289 & 8000 & 0.029412 & 8000 & 0.004682 \\
\hline \multirow[t]{5}{*}{ CEPXS Form: } & material & $\mathrm{H}$ & 0.115476 & & & \\
\hline & & C & 0.720819 & & & \\
\hline & & $\mathrm{N}$ & 0.076417 & & & \\
\hline & & $\mathrm{O}$ & 0.087289 & & & \\
\hline & $\begin{array}{l}\text { matname } \\
\text { density }\end{array}$ & \multicolumn{2}{|c|}{$\begin{array}{l}\text { Nylon, Type } 11 \text { (Rilsan) } \\
1.425000\end{array}$} & & & \\
\hline
\end{tabular}

\section{Nylon, Type 6 and Type 6/6}

\begin{tabular}{llll}
\hline Formula $=$ & $\mathrm{C} 12 \mathrm{H} 22 \mathrm{~N} 2 \mathrm{O} 2$ & Molecular weight $(\mathrm{g} / \mathrm{mole})=$ & 226.31528 \\
Density $(\mathrm{g} / \mathrm{cm} 3)=$ & 1.140000 & Total atom density $($ atoms $/ \mathrm{b}-\mathrm{cm})=$ & $1.153 \mathrm{E}-01$
\end{tabular}

The above density is estimated to be accurate to 3 significant digits. Uncertainties are not addressed.

The following data were calculated from the input weight fractions.

\begin{tabular}{|c|c|c|c|c|c|}
\hline ement & Neutron ZA & Photon ZA & $\begin{array}{l}\text { Weight } \\
\text { Fraction }\end{array}$ & $\begin{array}{c}\text { Atom } \\
\text { Fraction }\end{array}$ & $\begin{array}{c}\text { Atom } \\
\text { Density }\end{array}$ \\
\hline $\mathrm{H}$ & 1001 & 1000 & 0.097976 & 0.578932 & 0.066733 \\
\hline
\end{tabular}




\begin{tabular}{|c|c|c|c|c|c|c|}
\hline C & 6000 & 6000 & 0.636856 & 0.315803 & 0.036402 & \\
\hline $\mathrm{N}$ & 7014 & 7000 & 0.123779 & 0.052632 & 0.006067 & \\
\hline $\mathrm{O}$ & 8016 & 8000 & 0.141389 & 0.052633 & 0.006067 & \\
\hline Total & & & 1.000000 & 1.000000 & 0.115269 & \\
\hline MCNP Form & Weigh & ractions & Atom & ctions & Atom & sities \\
\hline Neutrons & 1001 & -0.097976 & 1001 & 0.578932 & 1001 & 0.066733 \\
\hline & 6000 & -0.636856 & 6000 & 0.315803 & 6000 & 0.036402 \\
\hline & 7014 & -0.123779 & 7014 & 0.052632 & 7014 & 0.006067 \\
\hline & 8016 & -0.141389 & 8016 & 0.052633 & 8016 & 0.006067 \\
\hline Photons & 1000 & -0.097976 & 1000 & 0.578932 & 1000 & 0.066733 \\
\hline & 6000 & -0.636856 & 6000 & 0.315803 & 6000 & 0.036402 \\
\hline & 7000 & -0.123779 & 7000 & 0.052632 & 7000 & 0.006067 \\
\hline & 8000 & -0.141389 & 8000 & 0.052633 & 8000 & 0.006067 \\
\hline CEPXS Form: & material & $\mathrm{H}$ & 0.097976 & & & \\
\hline & & C & 0.636856 & & & \\
\hline & & $\mathrm{N}$ & 0.123779 & & & \\
\hline & & $\mathrm{O}$ & 0.141389 & & & \\
\hline & $\begin{array}{c}\text { matname } \\
\text { density }\end{array}$ & $\begin{array}{c}\text { Nylon, Type } \\
1.140000\end{array}$ & nd Type 6/6 & & & \\
\hline $\begin{array}{l}\text { Comments an } \\
\text { Density and we } \\
\text { Formula from p } \\
\text { This nylon is ar } \\
(\mathrm{C} 6 \mathrm{H} 11 \mathrm{ON}) \mathrm{n} \text { o }\end{array}$ & $\begin{array}{l}\text { References } \\
\text { t fractions } \\
38 \text { of Brew } \\
\text { xample of a } \\
\text { g II.F.1-6 o }\end{array}$ & $\begin{array}{l}\text { http://phys } \\
\text { 2009). } \\
\text { lyamide, wh } \\
\text { arter et al. (1 }\end{array}$ & $\begin{array}{l}\text { ist.gov/cgi-k } \\
\text { sometimes }\end{array}$ & $\begin{array}{l}\text { Star/compc } \\
\text { breviated F }\end{array}$ & $\begin{array}{l}\text { matno }=20 \\
\text { d has the }\end{array}$ & $\begin{array}{l}\text { ST 1998). } \\
\text { ula }\end{array}$ \\
\hline
\end{tabular}

\section{Nylon, Type 6/10}

\begin{tabular}{llll}
\hline Formula $=$ & - & Molecular weight $(\mathrm{g} / \mathrm{mole})=$ \\
Density $(\mathrm{g} / \mathrm{cm} 3)=$ & 1.140000 & Total atom density $($ atoms $/ \mathrm{b}-\mathrm{cm})=$ & - \\
The & $1.215 \mathrm{E}-01$
\end{tabular}

The above density is estimated to be accurate to 3 significant digits. Uncertainties are not addressed.

The following data were calculated from the input weight fractions.

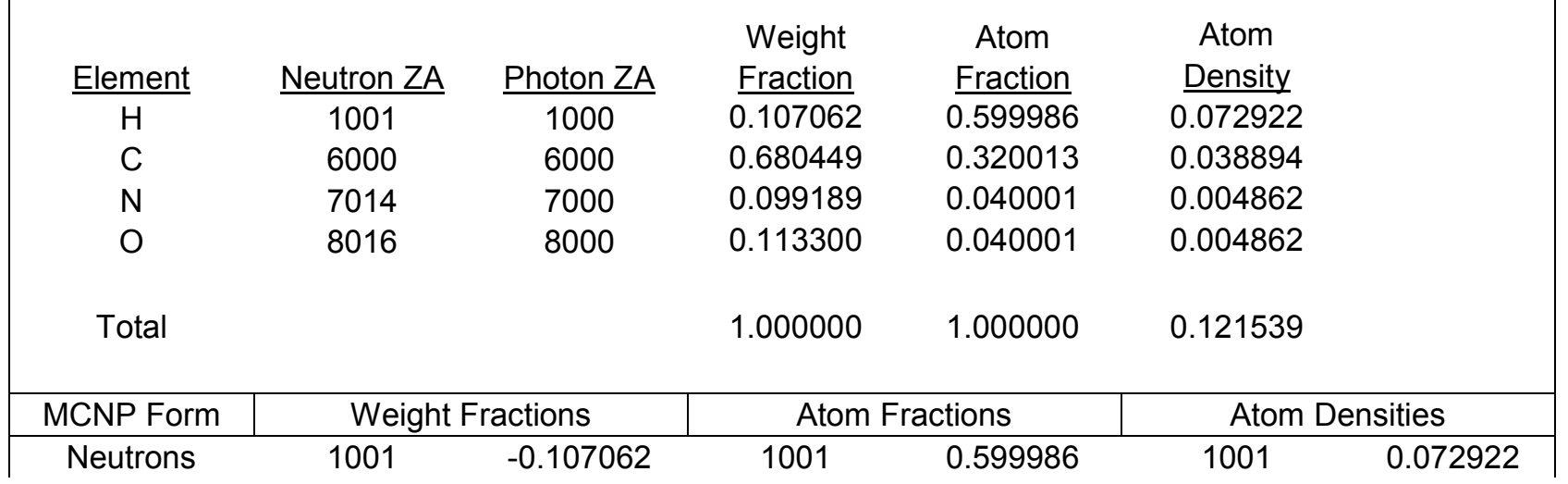


PIET-43741-TM-963

PNNL-15870 Rev. 1

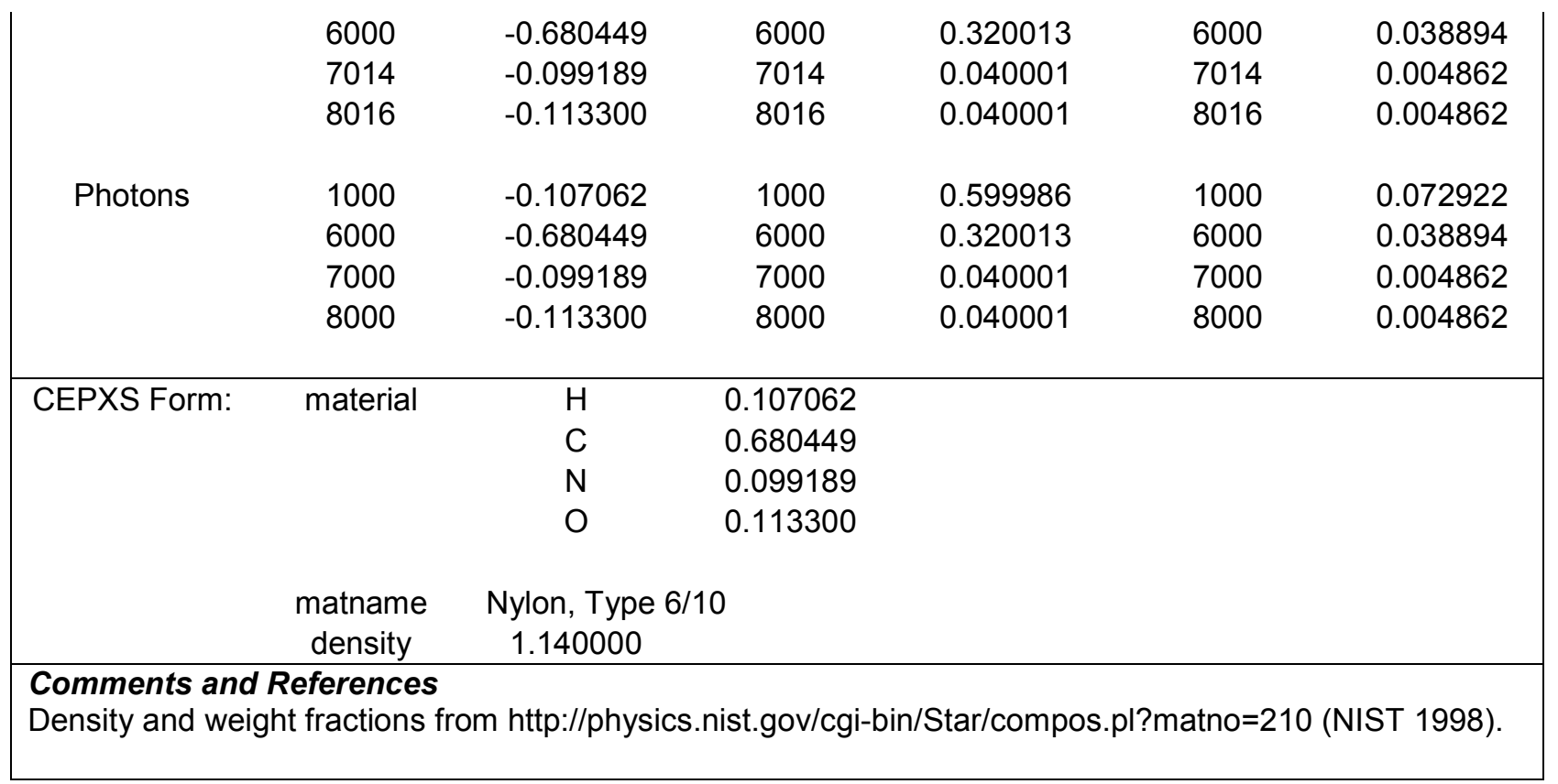

\section{Oil, Crude (Heavy, Cold Lake, Canada)}

\begin{tabular}{|c|c|c|c|}
\hline Formula $=$ & - & Molecular weight $(\mathrm{g} / \mathrm{mole})=$ & - \\
\hline
\end{tabular}

The above density is estimated to be accurate to 2 significant digits. Uncertainties are not addressed.

The following data were calculated from the input weight fractions.

\begin{tabular}{|c|c|c|c|c|c|c|}
\hline Element & Neutron ZA & Photon ZA & $\begin{array}{l}\text { Weight } \\
\text { Fraction }\end{array}$ & $\begin{array}{c}\text { Atom } \\
\text { Fraction }\end{array}$ & $\begin{array}{l}\text { Atom } \\
\text { Density }\end{array}$ & \\
\hline $\mathrm{H}$ & 1001 & 1000 & 0.104000 & $\overline{0.588884}$ & 0.060273 & \\
\hline C & 6000 & 6000 & 0.837000 & 0.397730 & 0.040708 & \\
\hline $\mathrm{N}$ & 7014 & 7000 & 0.004000 & 0.001630 & 0.000167 & \\
\hline $\mathrm{O}$ & 8016 & 8000 & 0.011000 & 0.003924 & 0.000402 & \\
\hline$S$ & 16000 & 16000 & 0.044000 & 0.007832 & 0.000802 & \\
\hline Total & & & 1.000000 & 1.000000 & 0.102351 & \\
\hline MCNP Form & \multicolumn{2}{|c|}{ Weight Fractions } & \multicolumn{2}{|c|}{ Atom Fractions } & \multicolumn{2}{|c|}{ Atom Densities } \\
\hline \multirow[t]{5}{*}{ Neutrons } & 1001 & -0.104000 & 1001 & 0.588884 & 1001 & 0.060273 \\
\hline & 6000 & -0.837000 & 6000 & 0.397730 & 6000 & 0.040708 \\
\hline & 7014 & -0.004000 & 7014 & 0.001630 & 7014 & 0.000167 \\
\hline & 8016 & -0.011000 & 8016 & 0.003924 & 8016 & 0.000402 \\
\hline & 16000 & -0.044000 & 16000 & 0.007832 & 16000 & 0.000802 \\
\hline \multirow[t]{5}{*}{ Photons } & 1000 & -0.104000 & 1000 & 0.588884 & 1000 & 0.060273 \\
\hline & 6000 & -0.837000 & 6000 & 0.397730 & 6000 & 0.040708 \\
\hline & 7000 & -0.004000 & 7000 & 0.001630 & 7000 & 0.000167 \\
\hline & 8000 & -0.011000 & 8000 & 0.003924 & 8000 & 0.000402 \\
\hline & 16000 & -0.044000 & 16000 & 0.007832 & 16000 & 0.000802 \\
\hline
\end{tabular}




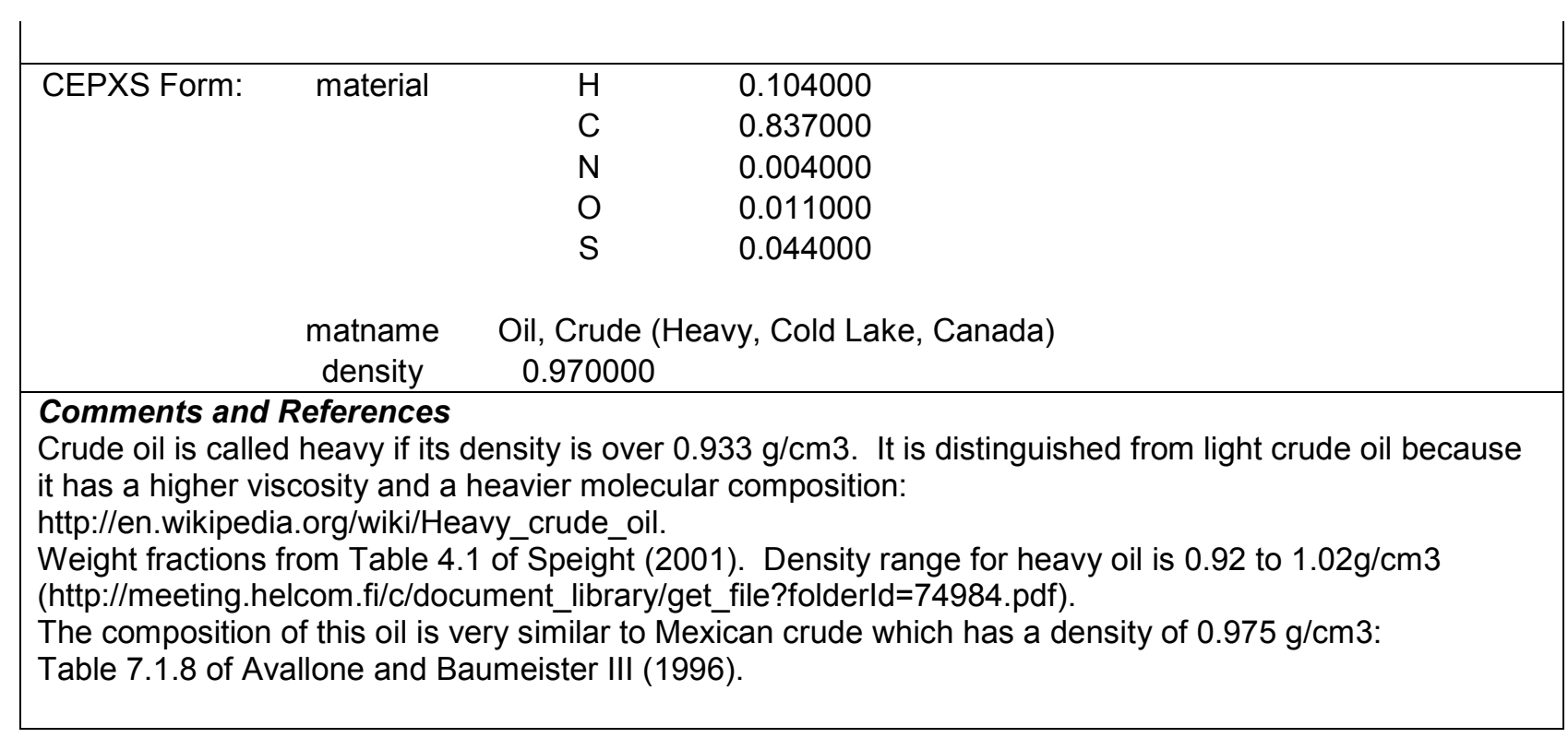

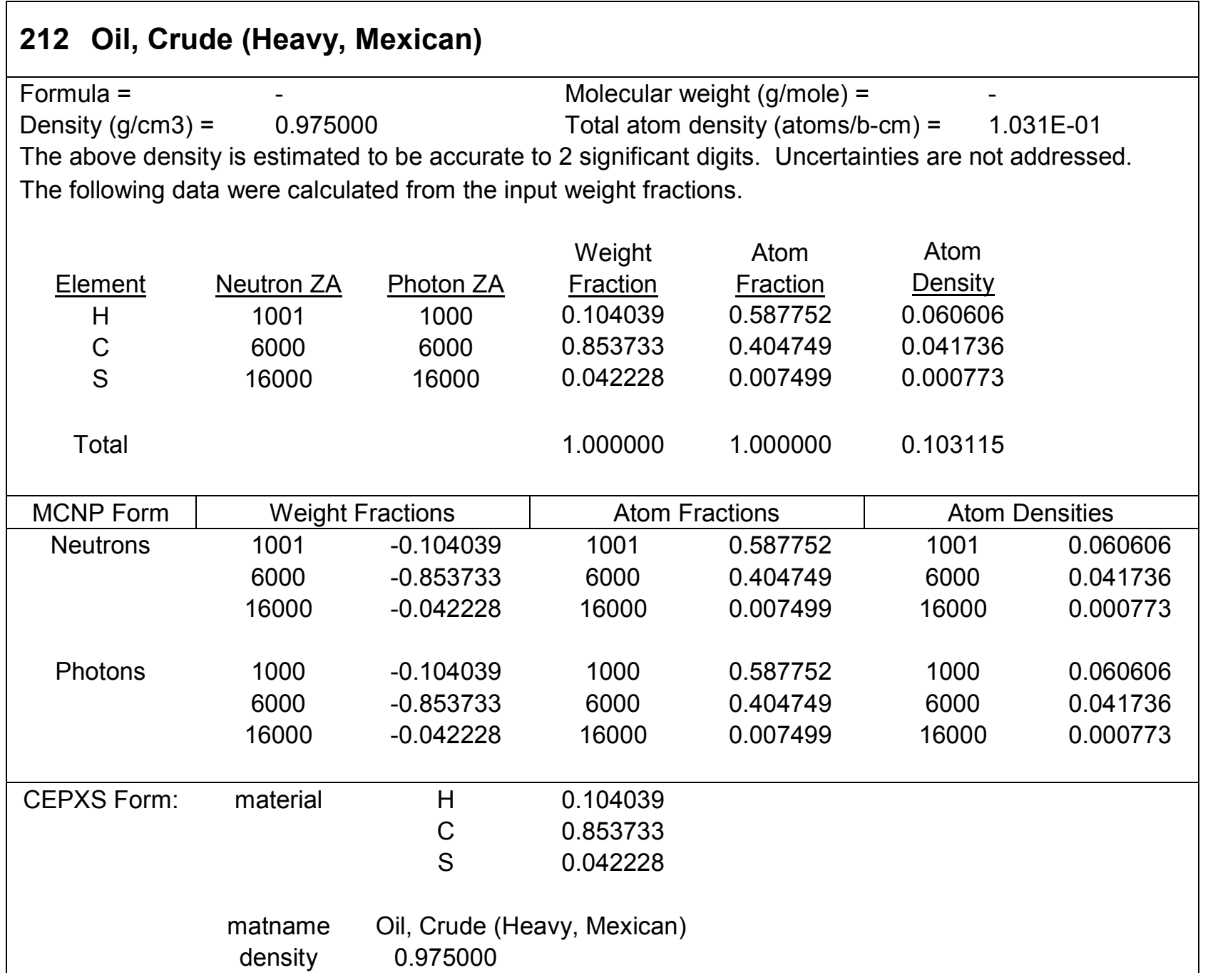


Comments and References

Density and weight fractions from Table 7.1.8 of Avallone and Baumeister III (1996). Weight fractions adjusted so elements sum to unity.

Other types of fuel oil are in Table 51.99 of Hungerford (1960).

\section{Oil, Crude (Heavy, Qayarah, Iraq)}

\begin{tabular}{llll}
\hline Formula $=$ & - & Molecular weight $(\mathrm{g} / \mathrm{mole})=$ \\
Density $(\mathrm{g} / \mathrm{cm} 3)=$ & 0.970000 & Total atom density $($ atoms $/ \mathrm{b}-\mathrm{cm})=$ & - \\
$1.002 \mathrm{E}-01$
\end{tabular}

The above density is estimated to be accurate to 2 significant digits. Uncertainties are not addressed.

The following data were calculated from the input weight fractions.

\begin{tabular}{|c|c|c|c|c|c|c|}
\hline Element & Neutron ZA & Photon ZA & $\begin{array}{l}\text { Weight } \\
\text { Fraction }\end{array}$ & $\begin{array}{l}\text { Atom } \\
\text { Fraction }\end{array}$ & $\begin{array}{l}\text { Atom } \\
\text { Density }\end{array}$ & \\
\hline $\mathrm{H}$ & 1001 & 1000 & 0.102000 & 0.590046 & 0.059114 & \\
\hline C & 6000 & 6000 & 0.807000 & 0.391765 & 0.039249 & \\
\hline $\mathrm{N}$ & 7014 & 7000 & 0.007000 & 0.002914 & 0.000292 & \\
\hline$S$ & 16000 & 16000 & 0.084000 & 0.015275 & 0.001530 & \\
\hline Total & & & 1.000000 & 1.000000 & 0.100185 & \\
\hline MCNP Form & \multicolumn{2}{|c|}{ Weight Fractions } & \multicolumn{2}{|c|}{ Atom Fractions } & \multicolumn{2}{|c|}{ Atom Densities } \\
\hline \multirow[t]{4}{*}{ Neutrons } & 1001 & -0.102000 & 1001 & 0.590046 & 1001 & 0.059114 \\
\hline & 6000 & -0.807000 & 6000 & 0.391765 & 6000 & 0.039249 \\
\hline & 7014 & -0.007000 & 7014 & 0.002914 & 7014 & 0.000292 \\
\hline & 16000 & -0.084000 & 16000 & 0.015275 & 16000 & 0.001530 \\
\hline \multirow[t]{4}{*}{ Photons } & 1000 & -0.102000 & 1000 & 0.590046 & 1000 & 0.059114 \\
\hline & 6000 & -0.807000 & 6000 & 0.391765 & 6000 & 0.039249 \\
\hline & 7000 & -0.007000 & 7000 & 0.002914 & 7000 & 0.000292 \\
\hline & 16000 & -0.084000 & 16000 & 0.015275 & 16000 & 0.001530 \\
\hline \multirow[t]{5}{*}{ CEPXS Form: } & material & $\mathrm{H}$ & 0.102000 & & & \\
\hline & & C & 0.807000 & & & \\
\hline & & $\mathrm{N}$ & 0.007000 & & & \\
\hline & & $S$ & 0.084000 & & & \\
\hline & $\begin{array}{l}\text { matname } \\
\text { density }\end{array}$ & \multicolumn{5}{|c|}{$\begin{array}{l}\text { Oil, Crude (Heavy, Qayarah, Iraq) } \\
0.970000\end{array}$} \\
\hline \multicolumn{7}{|c|}{$\begin{array}{l}\text { Comments and References } \\
\text { Crude oil is called heavy if its density is over } 0.933 \mathrm{~g} / \mathrm{cm} 3 \text {. It is distinguished from light crude oil because } \\
\text { it has a higher viscosity and a heavier molecular composition } \\
\text { (http://en.wikipedia.org/wiki/Heavy_crude_oil). } \\
\text { Weight fractions from Table } 4.1 \text { of Speight (2001). Density range for heavy oil is } 0.92 \text { to } 1.02 \mathrm{~g} / \mathrm{cm} 3 \\
\text { (http://meeting.helcom.fi/c/document library/get file?folderld=74984.pdf). }\end{array}$} \\
\hline
\end{tabular}




\section{Oil, Crude (Light, Texas)}

\begin{tabular}{llll}
\hline Formula $=$ & - & Molecular weight $(\mathrm{g} / \mathrm{mole})=$ \\
Density $(\mathrm{g} / \mathrm{cm} 3)=$ & 0.875000 & Total atom density $($ atoms $/ \mathrm{b}-\mathrm{cm})=$ & - \\
\hline & $1.024 \mathrm{E}-01$
\end{tabular}

The above density is estimated to be accurate to 2 significant digits. Uncertainties are not addressed.

The following data were calculated from the input weight fractions.

\begin{tabular}{|c|c|c|c|c|c|c|}
\hline Element & Neutron ZA & Photon ZA & $\begin{array}{l}\text { Weight } \\
\text { Fraction }\end{array}$ & $\begin{array}{c}\text { Atom } \\
\text { Fraction }\end{array}$ & $\begin{array}{c}\text { Atom } \\
\text { Density }\end{array}$ & \\
\hline $\mathrm{H}$ & 1001 & 1000 & 0.123246 & 0.629388 & 0.064432 & \\
\hline C & 6000 & 6000 & 0.852204 & 0.365220 & 0.037388 & \\
\hline $\mathrm{N}$ & 7014 & 7000 & 0.007014 & 0.002578 & 0.000264 & \\
\hline$S$ & 16000 & 16000 & 0.017535 & 0.002815 & 0.000288 & \\
\hline Total & & & 1.000000 & 1.000000 & 0.102372 & \\
\hline MCNP Form & \multicolumn{2}{|c|}{ Weight Fractions } & \multicolumn{2}{|c|}{ Atom Fractions } & \multicolumn{2}{|c|}{ Atom Densities } \\
\hline \multirow[t]{4}{*}{ Neutrons } & 1001 & -0.123246 & 1001 & 0.629388 & 1001 & 0.064432 \\
\hline & 6000 & -0.852204 & 6000 & 0.365220 & 6000 & 0.037388 \\
\hline & 7014 & -0.007014 & 7014 & 0.002578 & 7014 & 0.000264 \\
\hline & 16000 & -0.017535 & 16000 & 0.002815 & 16000 & 0.000288 \\
\hline \multirow[t]{4}{*}{ Photons } & 1000 & -0.123246 & 1000 & 0.629388 & 1000 & 0.064432 \\
\hline & 6000 & -0.852204 & 6000 & 0.365220 & 6000 & 0.037388 \\
\hline & 7000 & -0.007014 & 7000 & 0.002578 & 7000 & 0.000264 \\
\hline & 16000 & -0.017535 & 16000 & 0.002815 & 16000 & 0.000288 \\
\hline \multirow[t]{5}{*}{ CEPXS Form: } & material & $\mathrm{H}$ & 0.123246 & & & \\
\hline & & C & 0.852204 & & & \\
\hline & & $\mathrm{N}$ & 0.007014 & & & \\
\hline & & $S$ & 0.017535 & & & \\
\hline & $\begin{array}{l}\text { matname } \\
\text { density }\end{array}$ & $\begin{array}{c}\text { Oil, Crude (L } \\
0.875000\end{array}$ & t, Texas) & & & \\
\hline \multicolumn{7}{|c|}{$\begin{array}{l}\text { Comments and References } \\
\text { Density and weight fractions from Table } 7.1 .8 \text { of Avallone and Baumeister III (1996). Weight fractions } \\
\text { adjusted so elements sum to unity. } \\
\text { Density and composition for other domestic sources of crude oil also listed in Table } 7.1 .8 \text { of Avallone and } \\
\text { in Table } 51.97 \text { of Hungerford (1960). }\end{array}$} \\
\hline
\end{tabular}

\section{Oil, Fuel (California)}

\begin{tabular}{llll}
\hline Formula $=$ & - & Molecular weight $(\mathrm{g} / \mathrm{mole})=$ & - \\
Density $(\mathrm{g} / \mathrm{cm} 3)=$ & 0.955000 & Total atom density $($ atoms $/ \mathrm{b}-\mathrm{cm})=$ & $1.133 \mathrm{E}-01$
\end{tabular}

The above density is estimated to be accurate to 2 significant digits. Uncertainties are not addressed.

The following data were calculated from the input weight fractions. 


\begin{tabular}{|c|c|c|c|c|c|c|}
\hline Element & Neutron ZA & Photon ZA & $\begin{array}{l}\text { Weight } \\
\text { Fraction }\end{array}$ & $\begin{array}{c}\text { Atom } \\
\text { Fraction }\end{array}$ & $\begin{array}{l}\text { Atom } \\
\text { Density }\end{array}$ & \\
\hline $\mathrm{H}$ & 1001 & 1000 & 0.125878 & 0.633782 & 0.071824 & \\
\hline $\mathrm{C}$ & 6000 & 6000 & 0.862308 & 0.364349 & 0.041290 & \\
\hline$S$ & 16000 & 16000 & 0.011814 & 0.001870 & 0.000212 & \\
\hline Total & & & 1.000000 & 1.000000 & 0.113326 & \\
\hline MCNP Form & \multicolumn{2}{|c|}{ Weight Fractions } & \multicolumn{2}{|c|}{ Atom Fractions } & \multicolumn{2}{|c|}{ Atom Densities } \\
\hline \multirow[t]{3}{*}{ Neutrons } & 1001 & -0.125878 & 1001 & 0.633782 & 1001 & 0.071824 \\
\hline & 6000 & -0.862308 & 6000 & 0.364349 & 6000 & 0.041290 \\
\hline & 16000 & -0.011814 & 16000 & 0.001870 & 16000 & 0.000212 \\
\hline \multirow[t]{3}{*}{ Photons } & 1000 & -0.125878 & 1000 & 0.633782 & 1000 & 0.071824 \\
\hline & 6000 & -0.862308 & 6000 & 0.364349 & 6000 & 0.041290 \\
\hline & 16000 & -0.011814 & 16000 & 0.001870 & 16000 & 0.000212 \\
\hline \multirow[t]{4}{*}{ CEPXS Form: } & material & $\mathrm{H}$ & 0.125878 & & & \\
\hline & & C & 0.862308 & & & \\
\hline & & $S$ & 0.011814 & & & \\
\hline & $\begin{array}{l}\text { matname } \\
\text { density }\end{array}$ & \multicolumn{2}{|c|}{$\begin{array}{l}\text { Oil, Fuel (California) } \\
0.955000\end{array}$} & & & \\
\hline \multicolumn{7}{|c|}{$\begin{array}{l}\text { Comments and References } \\
\text { Density and weight fractions from Table } 7.1 .8 \text { of Avallone and Baumeister III (1996). Weight fractions } \\
\text { adjusted so elements sum to unity. } \\
\text { Other types of fuel oil are in Table } 51.99 \text { of Hungerford (1960). }\end{array}$} \\
\hline
\end{tabular}

\section{Oil, Hydraulic}

\begin{tabular}{llll}
\hline Formula $=$ & $\mathrm{C} 40 \mathrm{H} 33 \mathrm{O} 4 \mathrm{Cl}$ 6P & Molecular weight $(\mathrm{g} / \mathrm{mole})=$ & 821.379381 \\
Density $(\mathrm{g} / \mathrm{cm} 3)=$ & 0.871000 & Total atom density $($ atoms $/ \mathrm{b}-\mathrm{cm})=$ & $5.364 \mathrm{E}-02$
\end{tabular}

The above density is estimated to be accurate to 2 significant digits. Uncertainties are not addressed.

The following data was calculated from the input formula.

\begin{tabular}{|c|c|c|c|c|c|}
\hline Element & Neutron ZA & Photon ZA & $\begin{array}{l}\text { Weight } \\
\text { Fraction }\end{array}$ & $\begin{array}{c}\text { Atom } \\
\text { Fraction }\end{array}$ & $\begin{array}{l}\text { Atom } \\
\text { Density }\end{array}$ \\
\hline $\mathrm{H}$ & 1001 & 1000 & $\overline{0.040495}$ & 0.392857 & 0.021074 \\
\hline C & 6000 & 6000 & 0.584904 & 0.476190 & 0.025544 \\
\hline $\mathrm{O}$ & 8016 & 8000 & 0.077915 & 0.047619 & 0.002554 \\
\hline $\mathrm{P}$ & 15031 & 15000 & 0.037709 & 0.011905 & 0.000639 \\
\hline $\mathrm{Cl}$ & 17000 & 17000 & 0.258977 & 0.071429 & 0.003832 \\
\hline Total & & & 1.000000 & 1.000000 & 0.053642 \\
\hline MCNP Form & \multicolumn{2}{|c|}{ Weight Fractions } & \multicolumn{2}{|c|}{ Atom Fractions } & Atom \\
\hline Neutrons & 1001 & -0.040495 & 1001 & 0.392857 & 1001 \\
\hline
\end{tabular}


PIET-43741-TM-963

PNNL-15870 Rev. 1

\begin{tabular}{|c|c|c|c|c|c|c|}
\hline & 6000 & -0.584904 & 6000 & 0.476190 & 6000 & 0.025544 \\
\hline & 8016 & -0.077915 & 8016 & 0.047619 & 8016 & 0.002554 \\
\hline & 15031 & -0.037709 & 15031 & 0.011905 & 15031 & 0.000639 \\
\hline & 17000 & -0.258977 & 17000 & 0.071429 & 17000 & 0.003832 \\
\hline Photons & 1000 & -0.040495 & 1000 & 0.392857 & 1000 & 0.021074 \\
\hline & 6000 & -0.584904 & 6000 & 0.476190 & 6000 & 0.025544 \\
\hline & 8000 & -0.077915 & 8000 & 0.047619 & 8000 & 0.002554 \\
\hline & 15000 & -0.037709 & 15000 & 0.011905 & 15000 & 0.000639 \\
\hline & 17000 & -0.258977 & 17000 & 0.071429 & 17000 & 0.003832 \\
\hline CEPXS Form: & material & $\mathrm{H}$ & 0.040495 & & & \\
\hline & & $\mathrm{C}$ & 0.584904 & & & \\
\hline & & $\mathrm{O}$ & 0.077915 & & & \\
\hline & & $P$ & 0.037709 & & & \\
\hline & & $\mathrm{Cl}$ & 0.258977 & & & \\
\hline & $\begin{array}{c}\text { matname } \\
\text { density }\end{array}$ & $\begin{array}{c}\text { Oil, Hydraulic } \\
0.871000\end{array}$ & & & & \\
\hline $\begin{array}{l}\text { Comments an } \\
\text { Density = 0.87 } \\
\text { http://www.mat } \\
\text { (Automation Cr } \\
1.28 \mathrm{~g} / \mathrm{cm} 3, \text { wh } \\
\text { Density = } 0.89 \\
\text { (http://www.dee } \\
\text { g/cm3 for ESS } \\
\text { (www.imperialo }\end{array}$ & $\begin{array}{l}\text { eferences } \\
\mathrm{cm} 3 \text { at } \\
\text { o.com/sear } \\
\text { ions 2010) } \\
\text { seems hig } \\
\text { m3 for Hy- } \\
\text { com/en_U } \\
\text { rade } 46 \text { an } \\
\text { a/.../IOCAE }\end{array}$ & $\begin{array}{l}\text { DataSheet.as } \\
\text { ormula from } p \\
\text { rd hydraulic/tr } \\
\text { arts/partsinfo/ } \\
\text { vear hydraulic } \\
\text { NDESHydraul }\end{array}$ & $\begin{array}{l}\text { ?MatGUID= } \\
\text { I.F.1-5 of C } \\
\text { mission oil } \\
\text { _and_lubri } \\
\text { Oil_AW.pd }\end{array}$ & $\begin{array}{l}\text { 1f02f8ce4k } \\
\text { ret al. (196 } \\
\text { sineral oil is }\end{array}$ & $\begin{array}{l}\text { 5ba078 } \\
\text { t its dens } \\
\mathrm{ml}) \text {. Der } \\
\text { ally arou }\end{array}$ & $\begin{array}{l}=0.873 \\
870 \mathrm{~g} / \mathrm{cm} 3 .\end{array}$ \\
\hline
\end{tabular}

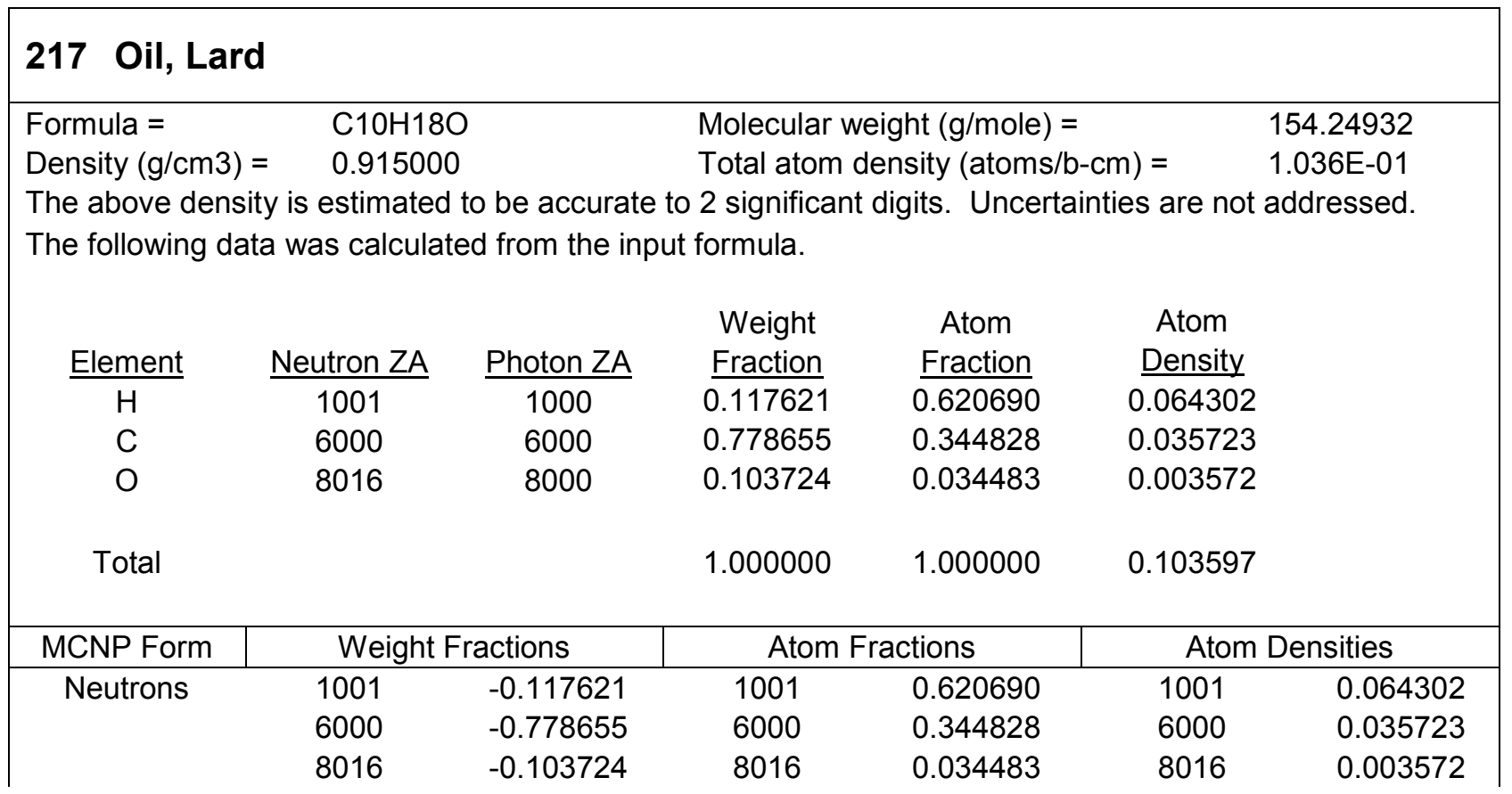


PIET-43741-TM-963

PNNL-15870 Rev. 1

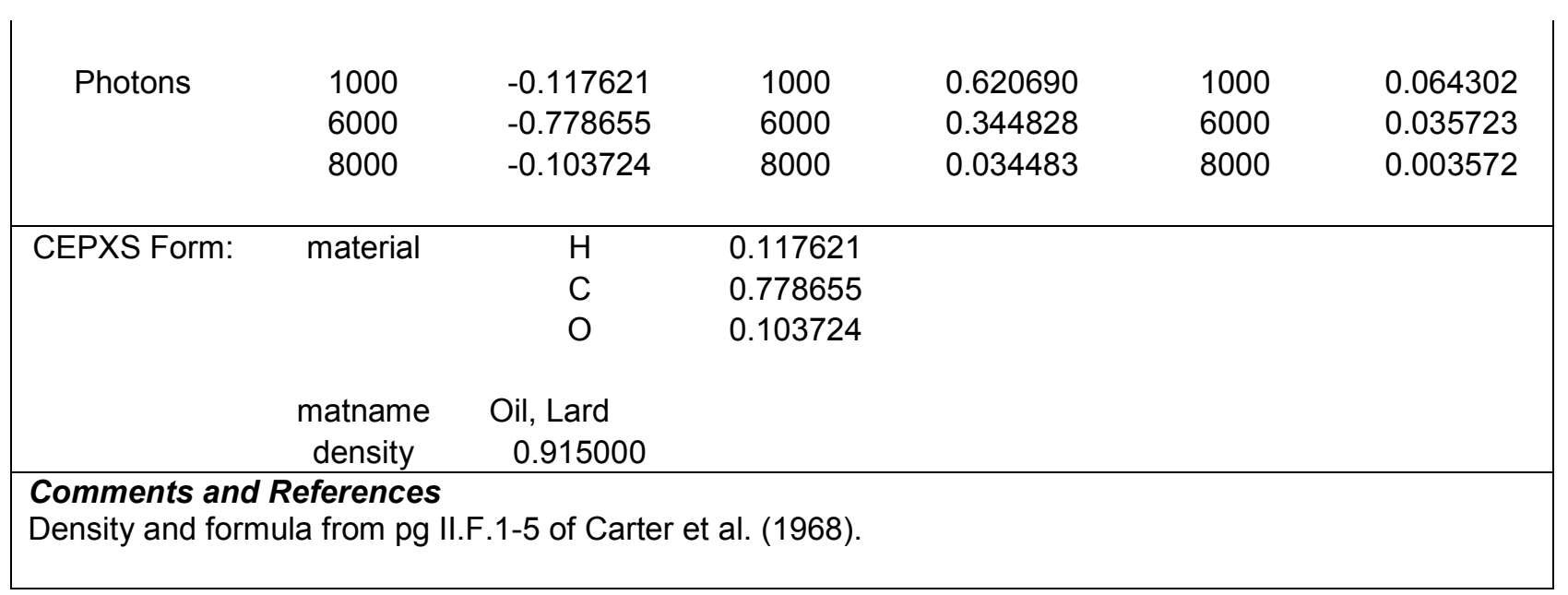

\section{Oxygen}

\begin{tabular}{llll}
\hline Formula $=$ & O2 & Molecular weight $(\mathrm{g} / \mathrm{mole})=$ & 31.9988 \\
Density $(\mathrm{g} / \mathrm{cm} 3)=$ & 0.001332 & Total atom density $($ atoms $/ \mathrm{b}-\mathrm{cm})=$ & $5.012 \mathrm{E}-05$
\end{tabular}

The above density is estimated to be accurate to 4 significant digits. Uncertainties are not addressed.

The following data was calculated from the input formula.

\begin{tabular}{|c|c|c|c|c|c|c|}
\hline$\frac{\text { Element }}{\mathrm{O}}$ & $\frac{\text { Neutron ZA }}{8016}$ & $\frac{\text { Photon ZA }}{8000}$ & $\begin{array}{l}\text { Weight } \\
\frac{\text { Fraction }}{1.000000}\end{array}$ & $\begin{array}{c}\text { Atom } \\
\text { Fraction } \\
1.000000\end{array}$ & $\begin{array}{c}\text { Atom } \\
\text { Density } \\
0.000050\end{array}$ & \\
\hline Total & & & 1.000000 & 1.000000 & 0.000050 & \\
\hline MCNP Form & \multicolumn{2}{|c|}{ Weight Fractions } & \multicolumn{2}{|c|}{ Atom Fractions } & \multicolumn{2}{|c|}{ Atom Densities } \\
\hline Neutrons & 8016 & -1.000000 & 8016 & 1.000000 & 8016 & 0.000050 \\
\hline Photons & 8000 & -1.000000 & 8000 & 1.000000 & 8000 & 0.000050 \\
\hline CEPXS Form: & material & $\mathrm{O}$ & 1.000000 & & & \\
\hline & $\begin{array}{c}\text { matname } \\
\text { density }\end{array}$ & $\begin{array}{l}\text { Oxygen } \\
0.001332\end{array}$ & & & & \\
\hline
\end{tabular}

\section{P-10 Gas}

Formula $=\quad 90 \% \mathrm{Ar}, 10 \% \mathrm{CH} 4 \quad$ Molecular weight $(\mathrm{g} / \mathrm{mole})=\quad 37.557446$

Density $(\mathrm{g} / \mathrm{cm} 3)=\quad 0.001561 \quad$ Total atom density $($ atoms $/ \mathrm{b}-\mathrm{cm})=3.505 \mathrm{E}-05$

The above density is estimated to be accurate to 4 significant digits. Uncertainties are not addressed.

The following data was calculated from the input formula. 


\begin{tabular}{|c|c|c|c|c|c|c|}
\hline Element & Neutron ZA & Photon ZA & $\begin{array}{l}\text { Weight } \\
\text { Fraction }\end{array}$ & $\begin{array}{c}\text { Atom } \\
\text { Fraction }\end{array}$ & $\begin{array}{l}\text { Atom } \\
\text { Density }\end{array}$ & \\
\hline $\mathrm{H}$ & 1001 & 1000 & 0.010735 & 0.285714 & 0.000010 & \\
\hline $\mathrm{C}$ & 6000 & 6000 & 0.031980 & 0.071429 & 0.000003 & \\
\hline $\mathrm{Ar}$ & 18000 & 18000 & 0.957286 & 0.642857 & 0.000023 & \\
\hline Total & & & 1.000000 & 1.000000 & 0.000035 & \\
\hline MCNP Form & \multicolumn{2}{|c|}{ Weight Fractions } & \multicolumn{2}{|c|}{ Atom Fractions } & \multicolumn{2}{|c|}{ Atom Densities } \\
\hline \multirow[t]{3}{*}{ Neutrons } & 1001 & -0.010735 & 1001 & 0.285714 & 1001 & 0.000010 \\
\hline & 6000 & -0.031980 & 6000 & 0.071429 & 6000 & 0.000003 \\
\hline & 18000 & -0.957286 & 18000 & 0.642857 & 18000 & 0.000023 \\
\hline \multirow[t]{3}{*}{ Photons } & 1000 & -0.010735 & 1000 & 0.285714 & 1000 & 0.000010 \\
\hline & 6000 & -0.031980 & 6000 & 0.071429 & 6000 & 0.000003 \\
\hline & 18000 & -0.957286 & 18000 & 0.642857 & 18000 & 0.000023 \\
\hline \multirow[t]{4}{*}{ CEPXS Form: } & material & $\mathrm{H}$ & 0.010735 & & & \\
\hline & & $\mathrm{C}$ & 0.031980 & & & \\
\hline & & $\mathrm{Ar}$ & 0.957286 & & & \\
\hline & $\begin{array}{c}\text { matname } \\
\text { density }\end{array}$ & $\begin{array}{l}\mathrm{P}-10 \text { Gas } \\
0.001561\end{array}$ & & & & \\
\hline $\begin{array}{l}\text { Comments an } \\
\text { This density is } \\
\text { Formula from } p\end{array}$ & $\begin{array}{l}\text { References } \\
\text { Iculated for T } \\
171 \text { of Knoll }\end{array}$ & $\begin{array}{l}=20^{\circ} \mathrm{C} \text { and } \mathrm{P} \\
000) . \mathrm{P}-10 \text { is }\end{array}$ & $\begin{array}{l}\text { atmosphe } \\
\% \text { Ar and }\end{array}$ & $\begin{array}{l}\text { ng the ide } \\
\text { H4. This }\end{array}$ & $\begin{array}{l}\text { law. } \\
\text { umed }\end{array}$ & olume $\%$. \\
\hline
\end{tabular}

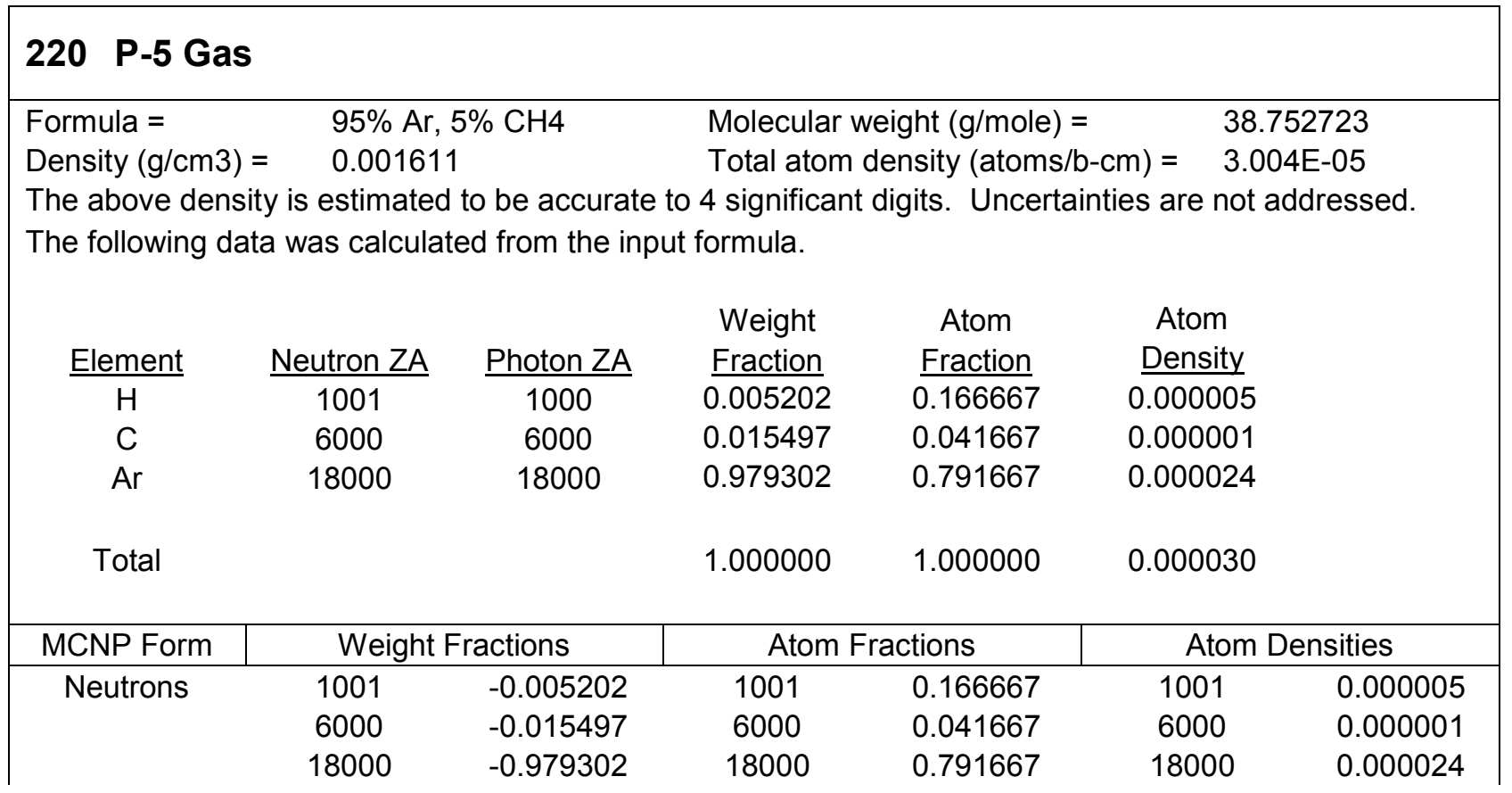


PIET-43741-TM-963

PNNL-15870 Rev. 1

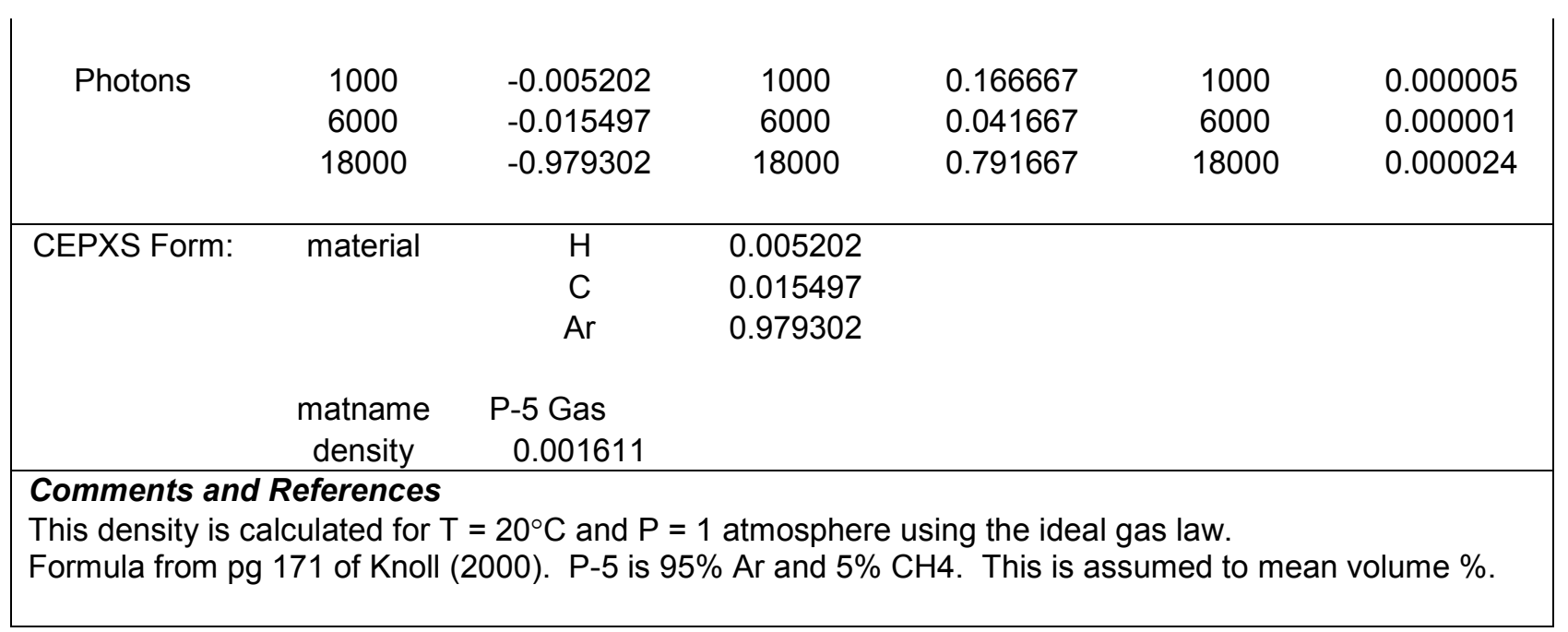

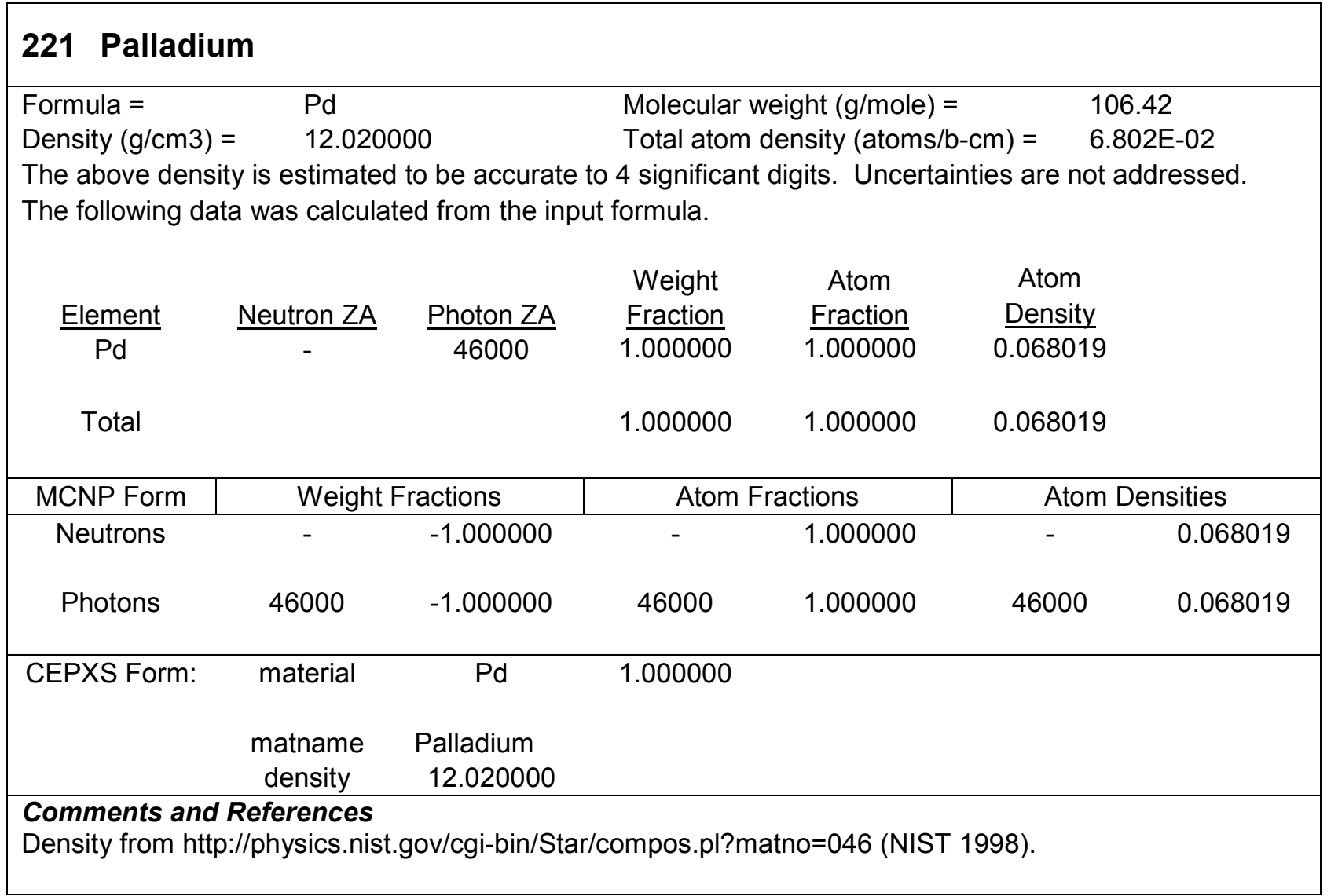

\section{Photographic Emulsion, Gel in}

\begin{tabular}{lll}
\hline Formula $=$ & - & Molecular weight $(\mathrm{g} / \mathrm{mole})=$ \\
Density $(\mathrm{g} / \mathrm{cm} 3)=$ & 1.291400 & Total atom density $($ atoms $/ \mathrm{b}-\mathrm{cm})=\overline{1.145 \mathrm{E}-01}$
\end{tabular}

The above density is estimated to be accurate to 3 significant digits. Uncertainties are not addressed. 
The following data were calculated from the input weight fractions.

\begin{tabular}{|c|c|c|c|c|c|}
\hline Element & Neutron ZA & Photon ZA & $\begin{array}{l}\text { Weight } \\
\text { Fraction }\end{array}$ & $\begin{array}{c}\text { Atom } \\
\text { Fraction }\end{array}$ & $\begin{array}{c}\text { Atom } \\
\text { Density }\end{array}$ \\
\hline $\mathrm{H}$ & 1001 & 1000 & 0.081180 & 0.546952 & 0.062636 \\
\hline C & 6000 & 6000 & 0.416060 & 0.235246 & 0.026940 \\
\hline $\mathrm{N}$ & 7014 & 7000 & 0.111240 & 0.053934 & 0.006176 \\
\hline 0 & 8016 & 8000 & 0.380640 & 0.161564 & 0.018502 \\
\hline$S$ & 16000 & 16000 & 0.010880 & 0.002304 & 0.000264 \\
\hline Total & & & 1.000000 & 1.000000 & 0.114519 \\
\hline
\end{tabular}

\begin{tabular}{|c|cc|cc|cr|}
\hline MCNP Form & \multicolumn{2}{c|}{ Weight Fractions } & \multicolumn{2}{c|}{ Atom Fractions } & \multicolumn{2}{c|}{ Atom Densities } \\
\hline Neutrons & 1001 & -0.081180 & 1001 & 0.546952 & 1001 & 0.062636 \\
& 6000 & -0.416060 & 6000 & 0.235246 & 6000 & 0.026940 \\
& 7014 & -0.111240 & 7014 & 0.053934 & 7014 & 0.006176 \\
& 8016 & -0.380640 & 8016 & 0.161564 & 8016 & 0.018502 \\
& 16000 & -0.010880 & 16000 & 0.002304 & 16000 & 0.000264 \\
Photons & & & & & & \\
& 1000 & -0.081180 & 1000 & 0.546952 & 1000 & 0.062636 \\
& 6000 & -0.416060 & 6000 & 0.235246 & 6000 & 0.026940 \\
& 7000 & -0.111240 & 7000 & 0.053934 & 7000 & 0.006176 \\
& 8000 & -0.380640 & 8000 & 0.161564 & 8000 & 0.018502 \\
& 16000 & -0.010880 & 16000 & 0.002304 & 16000 & 0.000264
\end{tabular}

\begin{tabular}{|c|c|c|c|}
\hline \multirow[t]{5}{*}{ CEPXS Form: } & material & $\mathrm{H}$ & 0.081180 \\
\hline & & C & 0.416060 \\
\hline & & $\mathrm{N}$ & 0.111240 \\
\hline & & $\mathrm{O}$ & 0.380640 \\
\hline & & $S$ & 0.010880 \\
\hline
\end{tabular}

matname Photographic Emulsion, Gel in density $\quad 1.291400$

Comments and References

Density and weight fractions from http://physics.nist.gov/cgi-bin/Star/compos.pl?matno=168 (NIST 1998).

\section{Photographic Emulsion, Kodak Type AA}

\begin{tabular}{|c|c|}
\hline Formula $=$ & - \\
\hline Density $(\mathrm{g} / \mathrm{cm} 3)=$ & 2.200000 \\
\hline
\end{tabular}

The above density is estimated to be accurate to 3 significant digits. Uncertainties are not addressed.

The following data were calculated from the input weight fractions.

\begin{tabular}{|c|c|c|c|c|c|}
\hline Element & Neutron ZA & Photon ZA & $\begin{array}{l}\text { Weight } \\
\text { Fraction }\end{array}$ & $\begin{array}{c}\text { Atom } \\
\text { Fraction }\end{array}$ & $\begin{array}{c}\text { Atom } \\
\text { Density }\end{array}$ \\
\hline $\mathrm{H}$ & 1001 & 1000 & $\overline{0.030500}$ & $\overline{0.440293}$ & 0.040090 \\
\hline C & 6000 & 6000 & 0.210700 & 0.255254 & 0.023242 \\
\hline $\mathrm{N}$ & 7014 & 7000 & 0.072100 & 0.074899 & 0.006820 \\
\hline
\end{tabular}


PIET-43741-TM-963

PNNL-15870 Rev. 1

\begin{tabular}{|c|c|c|c|c|c|c|}
\hline $\mathrm{O}$ & 8016 & 8000 & 0.163200 & 0.148420 & \multicolumn{2}{|l|}{0.013514} \\
\hline $\mathrm{Br}$ & - & 35000 & 0.222800 & 0.040572 & \multicolumn{2}{|l|}{0.003694} \\
\hline $\mathrm{Ag}$ & 47000 & 47000 & 0.300700 & 0.040562 & \multicolumn{2}{|l|}{0.003693} \\
\hline Total & & & 1.000000 & 1.000000 & \multicolumn{2}{|l|}{0.091054} \\
\hline MCNP Form & \multicolumn{2}{|c|}{ Weight Fractions } & \multicolumn{2}{|c|}{ Atom Fractions } & \multicolumn{2}{|c|}{ Atom Densities } \\
\hline \multirow[t]{6}{*}{ Neutrons } & 1001 & -0.030500 & 1001 & 0.440293 & 1001 & 0.040090 \\
\hline & 6000 & -0.210700 & 6000 & 0.255254 & 6000 & 0.023242 \\
\hline & 7014 & -0.072100 & 7014 & 0.074899 & 7014 & 0.006820 \\
\hline & 8016 & -0.163200 & 8016 & 0.148420 & 8016 & 0.013514 \\
\hline & - & -0.222800 & - & 0.040572 & - & 0.003694 \\
\hline & 47000 & -0.300700 & 47000 & 0.040562 & 47000 & 0.003693 \\
\hline \multirow[t]{6}{*}{ Photons } & 1000 & -0.030500 & 1000 & 0.440293 & 1000 & 0.040090 \\
\hline & 6000 & -0.210700 & 6000 & 0.255254 & 6000 & 0.023242 \\
\hline & 7000 & -0.072100 & 7000 & 0.074899 & 7000 & 0.006820 \\
\hline & 8000 & -0.163200 & 8000 & 0.148420 & 8000 & 0.013514 \\
\hline & 35000 & -0.222800 & 35000 & 0.040572 & 35000 & 0.003694 \\
\hline & 47000 & -0.300700 & 47000 & 0.040562 & 47000 & 0.003693 \\
\hline \multirow[t]{7}{*}{ CEPXS Form: } & material & $\mathrm{H}$ & 0.030500 & & & \\
\hline & & C & 0.210700 & & & \\
\hline & & $\mathrm{N}$ & 0.072100 & & & \\
\hline & & O & 0.163200 & & & \\
\hline & & $\mathrm{Br}$ & 0.222800 & & & \\
\hline & & $\mathrm{Ag}$ & 0.300700 & & & \\
\hline & $\begin{array}{l}\text { matname } \\
\text { density }\end{array}$ & \multicolumn{3}{|c|}{$\begin{array}{l}\text { Photographic Emulsion, Kodak Type AA } \\
2.200000\end{array}$} & & \\
\hline
\end{tabular}

\section{Photographic Emulsion, Standard Nuclear}

\begin{tabular}{|c|c|c|c|}
\hline Formula $=$ & - & Molecular weight $(\mathrm{g} / \mathrm{mole})=$ & - \\
\hline Density $(\mathrm{g} / \mathrm{cm} 3)=$ & 3.815000 & Total atom density $($ atoms $/ \mathrm{b}-\mathrm{cm})=$ & 7.895E-02 \\
\hline
\end{tabular}

\begin{tabular}{|c|c|c|c|c|c|}
\hline Element & Neutron ZA & Photon ZA & $\begin{array}{l}\text { Weight } \\
\text { Fraction }\end{array}$ & $\begin{array}{c}\text { Atom } \\
\text { Fraction }\end{array}$ & $\begin{array}{c}\text { Atom } \\
\text { Density }\end{array}$ \\
\hline $\mathrm{H}$ & 1001 & 1000 & $\overline{0.014100}$ & $\overline{0.407082}$ & 0.032139 \\
\hline C & 6000 & 6000 & 0.072261 & 0.175079 & 0.013822 \\
\hline $\mathrm{N}$ & 7014 & 7000 & 0.019320 & 0.040139 & 0.003169 \\
\hline $\mathrm{O}$ & 8016 & 8000 & 0.066101 & 0.120227 & 0.009492 \\
\hline$S$ & 16000 & 16000 & 0.001890 & 0.001715 & 0.000135 \\
\hline $\mathrm{Br}$ & - & 35000 & 0.349104 & 0.127140 & 0.010038 \\
\hline
\end{tabular}


PIET-43741-TM-963

PNNL-15870 Rev. 1

\begin{tabular}{|c|c|c|c|c|c|c|}
\hline $\mathrm{Ag}$ & 47000 & 47000 & 0.474105 & 0.127902 & \multicolumn{2}{|l|}{0.010098} \\
\hline 1 & 53127 & 53000 & 0.003120 & 0.000715 & \multicolumn{2}{|l|}{0.000056} \\
\hline Total & & & 1.000001 & 1.000000 & \multicolumn{2}{|c|}{0.078949} \\
\hline MCNP Form & \multicolumn{2}{|c|}{ Weight Fractions } & \multicolumn{2}{|c|}{ Atom Fractions } & \multicolumn{2}{|c|}{ Atom Densities } \\
\hline \multirow[t]{8}{*}{ Neutrons } & 1001 & -0.014100 & 1001 & 0.407082 & 1001 & 0.032139 \\
\hline & 6000 & -0.072261 & 6000 & 0.175079 & 6000 & 0.013822 \\
\hline & 7014 & -0.019320 & 7014 & 0.040139 & 7014 & 0.003169 \\
\hline & 8016 & -0.066101 & 8016 & 0.120227 & 8016 & 0.009492 \\
\hline & 16000 & -0.001890 & 16000 & 0.001715 & 16000 & 0.000135 \\
\hline & - & -0.349104 & - & 0.127140 & - & 0.010038 \\
\hline & 47000 & -0.474105 & 47000 & 0.127902 & 47000 & 0.010098 \\
\hline & 53127 & -0.003120 & 53127 & 0.000715 & 53127 & 0.000056 \\
\hline \multirow[t]{8}{*}{ Photons } & 1000 & -0.014100 & 1000 & 0.407082 & 1000 & 0.032139 \\
\hline & 6000 & -0.072261 & 6000 & 0.175079 & 6000 & 0.013822 \\
\hline & 7000 & -0.019320 & 7000 & 0.040139 & 7000 & 0.003169 \\
\hline & 8000 & -0.066101 & 8000 & 0.120227 & 8000 & 0.009492 \\
\hline & 16000 & -0.001890 & 16000 & 0.001715 & 16000 & 0.000135 \\
\hline & 35000 & -0.349104 & 35000 & 0.127140 & 35000 & 0.010038 \\
\hline & 47000 & -0.474105 & 47000 & 0.127902 & 47000 & 0.010098 \\
\hline & 53000 & -0.003120 & 53000 & 0.000715 & 53000 & 0.000056 \\
\hline \multirow[t]{9}{*}{ CEPXS Form: } & material & $\mathrm{H}$ & 0.014100 & & & \\
\hline & & $\mathrm{C}$ & 0.072261 & & & \\
\hline & & $\mathrm{N}$ & 0.019320 & & & \\
\hline & & $\mathrm{O}$ & 0.066101 & & & \\
\hline & & $S$ & 0.001890 & & & \\
\hline & & $\mathrm{Br}$ & 0.349104 & & & \\
\hline & & $\mathrm{Ag}$ & 0.474105 & & & \\
\hline & & 1 & 0.003120 & & & \\
\hline & $\begin{array}{l}\text { matname } \\
\text { density }\end{array}$ & \multicolumn{3}{|c|}{$\begin{array}{l}\text { Photographic Emulsion, Standard Nuclear } \\
3.815000\end{array}$} & & \\
\hline
\end{tabular}

\section{Platinum}

\begin{tabular}{llll}
\hline Formula $=$ & $\mathrm{Pt}$ & Molecular weight $(\mathrm{g} / \mathrm{mole})=$ & 195.078 \\
Density $(\mathrm{g} / \mathrm{cm} 3)=$ & 21.450000 & Total atom density $($ atoms $/ \mathrm{b}-\mathrm{cm})=$ & $6.622 \mathrm{E}-02$
\end{tabular}

The above density is estimated to be accurate to 4 significant digits. Uncertainties are not addressed.

The following data was calculated from the input formula.

\begin{tabular}{llllll} 
Element & Neutron ZA & $\frac{\text { Whoton ZA }}{\mathrm{Pt}}$ & $\frac{\begin{array}{c}\text { Weight } \\
\text { Fraction }\end{array}}{78000}$ & $\frac{1}{\text { Fraction }}$ & $\begin{array}{c}\text { Atom } \\
\text { Density }\end{array}$ \\
\hline 1.000000 & 1.000000 & 0.066217
\end{tabular}


PIET-43741-TM-963

PNNL-15870 Rev. 1

\begin{tabular}{|c|c|c|c|c|c|c|}
\hline Total & & & 1.000000 & 1.000000 & \multicolumn{2}{|c|}{0.066217} \\
\hline MCNP Form & \multicolumn{2}{|c|}{ Weight Fractions } & \multicolumn{2}{|c|}{ Atom Fractions } & \multicolumn{2}{|c|}{ Atom Densities } \\
\hline Neutrons & 78000 & -1.000000 & 78000 & 1.000000 & 78000 & 0.066217 \\
\hline Photons & 78000 & -1.000000 & 78000 & 1.000000 & 78000 & 0.066217 \\
\hline CEPXS Form: & $\begin{array}{l}\text { material } \\
\text { matname } \\
\text { density }\end{array}$ & $\begin{array}{c}\qquad \mathrm{Pt} \\
\text { Platinum } \\
21.450000\end{array}$ & 1.000000 & & & \\
\hline $\begin{array}{l}\text { Comments an } \\
\text { Density from h }\end{array}$ & $\begin{array}{l}\text { eferences } \\
\text { /physics.ni }\end{array}$ & v/cgi-bin/ & 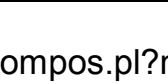 & -070 a & & \\
\hline
\end{tabular}

\section{Plutonium Bromide}

\begin{tabular}{llll}
\hline Formula $=$ & PuBr3 & Molecular weight $(\mathrm{g} / \mathrm{mole})=$ & 478.8329579 \\
Density $(\mathrm{g} / \mathrm{cm} 3)=$ & 6.750000 & Total atom density $($ atoms $/ \mathrm{b}-\mathrm{cm})=$ & $3.396 \mathrm{E}-02$
\end{tabular}

The above density is estimated to be accurate to 3 significant digits. Uncertainties are not addressed.

The following data was calculated from the input formula.

\begin{tabular}{|c|c|c|c|c|c|c|}
\hline Element & Neutron ZA & Photon ZA & $\begin{array}{l}\text { Weight } \\
\text { Fraction }\end{array}$ & $\begin{array}{c}\text { Atom } \\
\text { Fraction }\end{array}$ & $\begin{array}{l}\text { Atom } \\
\text { Density }\end{array}$ & \\
\hline $\mathrm{Br}$ & - & 35000 & 0.500617 & 0.750000 & 0.025468 & \\
\hline Pu-238 & 94238 & 94000 & 0.000250 & 0.000126 & 0.000004 & \\
\hline Pu-239 & 94239 & 94000 & 0.466923 & 0.233817 & 0.007940 & \\
\hline Pu-240 & 94240 & 94000 & 0.029963 & 0.014942 & 0.000507 & \\
\hline Pu-241 & 94241 & 94000 & 0.001998 & 0.000992 & 0.000034 & \\
\hline Pu-242 & 94242 & 94000 & 0.000250 & 0.000123 & 0.000004 & \\
\hline Am-241 & 95241 & 95000 & 0.000000 & 0.000000 & 0.000000 & \\
\hline Total & & & 1.000000 & 1.000000 & 0.033957 & \\
\hline MCNP Form & \multicolumn{2}{|c|}{ Weight Fractions } & \multicolumn{2}{|c|}{ Atom Fractions } & \multicolumn{2}{|c|}{ Atom Densities } \\
\hline \multirow[t]{7}{*}{ Neutrons } & - & -0.500617 & - & 0.750000 & - & 0.025468 \\
\hline & 94238 & -0.000250 & 94238 & 0.000126 & 94238 & 0.000004 \\
\hline & 94239 & -0.466923 & 94239 & 0.233817 & 94239 & 0.007940 \\
\hline & 94240 & -0.029963 & 94240 & 0.014942 & 94240 & 0.000507 \\
\hline & 94241 & -0.001998 & 94241 & 0.000992 & 94241 & 0.000034 \\
\hline & 94242 & -0.000250 & 94242 & 0.000123 & 94242 & 0.000004 \\
\hline & 95241 & 0.000000 & 95241 & 0.000000 & 95241 & 0.000000 \\
\hline \multirow[t]{4}{*}{ Photons } & 35000 & -0.500617 & 35000 & 0.750000 & 35000 & 0.025468 \\
\hline & 94000 & -0.000250 & 94000 & 0.000126 & 94000 & 0.000004 \\
\hline & 94000 & -0.466923 & 94000 & 0.233817 & 94000 & 0.007940 \\
\hline & 94000 & -0.029963 & 94000 & 0.014942 & 94000 & 0.000507 \\
\hline
\end{tabular}


PIET-43741-TM-963

PNNL-15870 Rev. 1

\begin{tabular}{|c|c|c|c|c|c|c|}
\hline & $\begin{array}{l}94000 \\
94000 \\
95000\end{array}$ & $\begin{array}{c}-0.001998 \\
-0.000250 \\
0.000000\end{array}$ & $\begin{array}{l}94000 \\
94000 \\
95000\end{array}$ & $\begin{array}{l}0.000992 \\
0.000123 \\
0.000000\end{array}$ & $\begin{array}{l}94000 \\
94000 \\
95000\end{array}$ & $\begin{array}{l}0.000034 \\
0.000004 \\
0.000000\end{array}$ \\
\hline CEPXS Form: & material & $\begin{array}{c}\mathrm{Br} \\
\mathrm{Pu}-238 \\
\mathrm{Pu}-239 \\
\mathrm{Pu}-240 \\
\mathrm{Pu}-241 \\
\mathrm{Pu}-242 \\
\mathrm{Am}-241\end{array}$ & $\begin{array}{l}0.500617 \\
0.000250 \\
0.466923 \\
0.029963 \\
0.001998 \\
0.000250 \\
0.000000\end{array}$ & & & \\
\hline & $\begin{array}{c}\text { matname } \\
\text { density }\end{array}$ & \multicolumn{2}{|c|}{$\begin{array}{l}\text { Plutonium Bromide } \\
6.750000\end{array}$} & & & \\
\hline \multicolumn{7}{|c|}{$\begin{array}{l}\text { Comments and References } \\
\text { Density and formula from Lide (2008), pgs } 4-81 \text {. } \\
\text { Plutonium isotopics assumed for DOE } 3013 \mathrm{WGPu}: \mathrm{Wt} \% \text { Pu238/239/240/241/242=0.05/93.5/6.0/0.4/0.05. } \\
\text { Pu atoms per molecule taken from atom fractions calculated for DOE } 3013 \mathrm{WGPu} \text {. }\end{array}$} \\
\hline
\end{tabular}

\section{Plutonium Carbide}

\begin{tabular}{llll}
\hline Formula $=$ & PuC & Molecular weight $(\mathrm{g} / \mathrm{mole})=$ & 251.1316579 \\
Density $(\mathrm{g} / \mathrm{cm} 3)=$ & 13.600000 & Total atom density $($ atoms $/ \mathrm{b}-\mathrm{cm})=$ & $6.523 \mathrm{E}-02$
\end{tabular}

The above density is estimated to be accurate to 4 significant digits. Uncertainties are not addressed.

The following data was calculated from the input formula.

\begin{tabular}{|cccccccc} 
Element & Neutron ZA & Photon ZA & $\begin{array}{c}\text { Weight } \\
\text { Fraction }\end{array}$ & $\begin{array}{c}\text { Atom } \\
\text { Fraction }\end{array}$ & $\begin{array}{c}\text { Atom } \\
\text { Density }\end{array}$ \\
C & 6000 & 6000 & 0.047826 & 0.500000 & 0.032613 & \\
Pu-238 & 94238 & 94000 & 0.000476 & 0.000251 & 0.000016 \\
Pu-239 & 94239 & 94000 & 0.890282 & 0.467635 & 0.030502 \\
Pu-240 & 94240 & 94000 & 0.057130 & 0.029883 & 0.001949 \\
Pu-241 & 94241 & 94000 & 0.003809 & 0.001984 & 0.000129 & \\
Pu-242 & 94242 & 94000 & 0.000476 & 0.000247 & 0.000016 & \\
Am-241 & 95241 & 95000 & 0.000000 & 0.000000 & 0.000000 & \\
& & & & & & \\
Total & & & & & & & \\
& & & & & & & \\
MCNP Form & Weight Fractions & & Atom Fractions & Atom Densities \\
\hline Neutrons & 6000 & -0.047826 & 6000 & 0.500000 & 6000 & 0.032613 \\
& 94238 & -0.000476 & 94238 & 0.000251 & 94238 & 0.000016 \\
& 94239 & -0.890282 & 94239 & 0.467635 & 94239 & 0.030502 \\
& 94240 & -0.057130 & 94240 & 0.029883 & 94240 & 0.001949 \\
& 94241 & -0.003809 & 94241 & 0.001984 & 94241 & 0.000129 \\
& 94242 & -0.000476 & 94242 & 0.000247 & 94242 & 0.000016 \\
& 95241 & 0.000000 & 95241 & 0.000000 & 95241 & 0.000000 \\
& & & & & &
\end{tabular}


PIET-43741-TM-963

PNNL-15870 Rev. 1

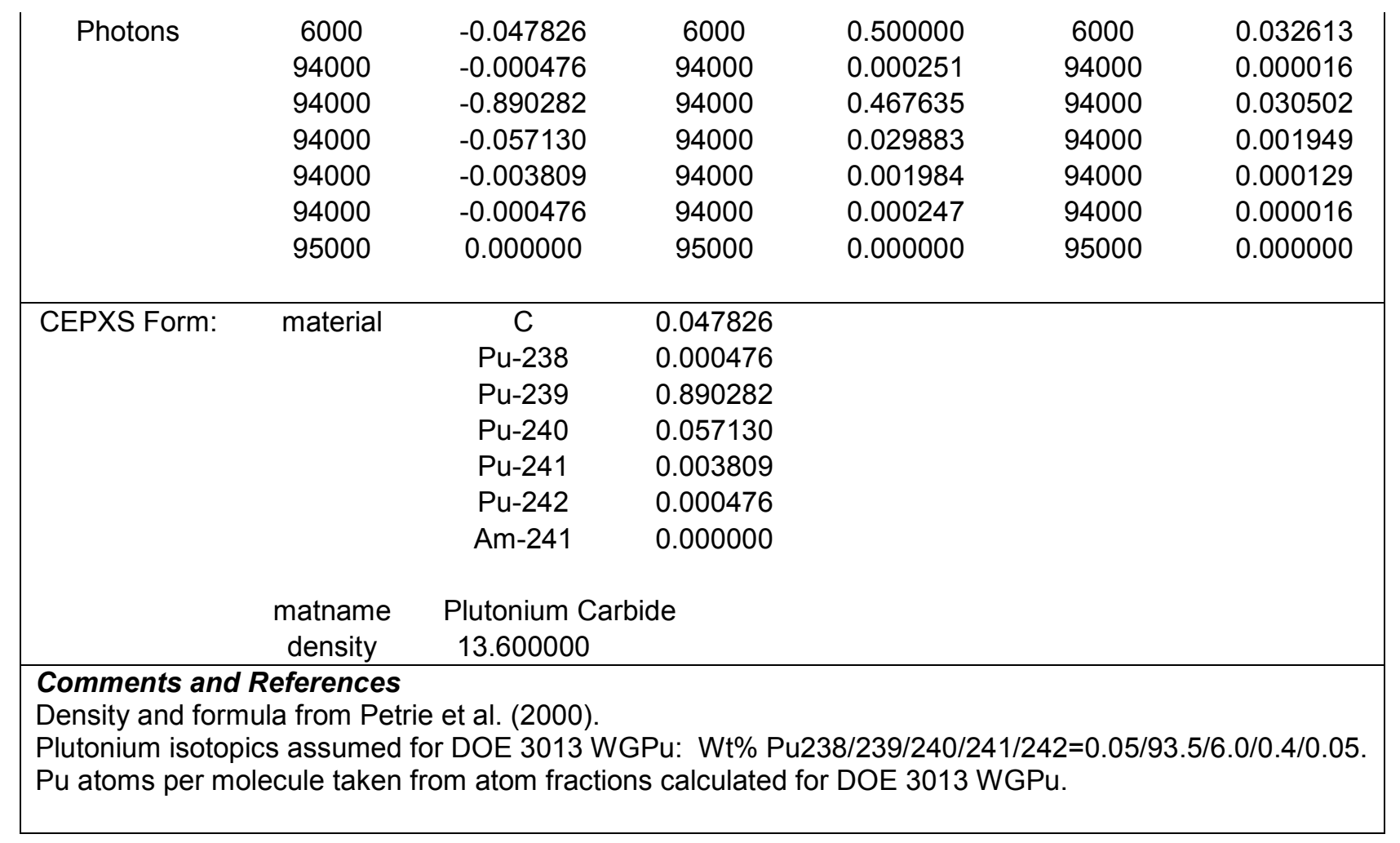

\begin{tabular}{|c|c|c|c|c|c|c|c|}
\hline \multicolumn{8}{|c|}{228 Plutonium Chloride } \\
\hline $\begin{array}{l}\text { Formula }= \\
\text { Density }(\mathrm{g} / \mathrm{cm} 3)\end{array}$ & \multicolumn{7}{|c|}{$\begin{array}{l}\text { The above density is estimated to be accurate to } 3 \text { significant digits. Uncertainties are not addressed. } \\
\text { The following data was calculated from the input formula. }\end{array}$} \\
\hline Element & Neutron ZA & Photon ZA & $\begin{array}{l}\text { Weight } \\
\text { Fraction }\end{array}$ & $\begin{array}{l}\text { Atom } \\
\text { Fraction }\end{array}$ & \multicolumn{2}{|c|}{ Atom } & \\
\hline $\mathrm{Cl}$ & 17000 & 17000 & 0.307859 & 0.750000 & \multicolumn{2}{|c|}{0.029860} & \\
\hline Pu-238 & 94238 & 94000 & 0.000346 & 0.000126 & \multicolumn{2}{|c|}{0.000005} & \\
\hline Pu-239 & 94239 & 94000 & 0.647152 & 0.233817 & \multicolumn{2}{|c|}{0.009309} & \\
\hline Pu-240 & 94240 & 94000 & 0.041528 & 0.014942 & \multicolumn{2}{|c|}{0.000595} & \\
\hline Pu-241 & 94241 & 94000 & 0.002769 & 0.000992 & \multicolumn{2}{|c|}{0.000039} & \\
\hline Pu-242 & 94242 & 94000 & 0.000346 & 0.000123 & \multicolumn{2}{|c|}{0.000005} & \\
\hline Am-241 & 95241 & 95000 & 0.000000 & 0.000000 & \multicolumn{2}{|c|}{0.000000} & \\
\hline \multicolumn{2}{|l|}{ Total } & & 1.000000 & 1.000000 & \multicolumn{2}{|c|}{0.039813} & \\
\hline MCNP Form & \multicolumn{2}{|c|}{ Weight Fractions } & \multicolumn{2}{|c|}{ Atom Fractions } & \multicolumn{3}{|c|}{ Atom Densities } \\
\hline \multirow[t]{5}{*}{ Neutrons } & 17000 & -0.307859 & 17000 & 0.750000 & & & 0.029860 \\
\hline & 94238 & -0.000346 & 94238 & 0.000126 & & & 0.000005 \\
\hline & 94239 & -0.647152 & 94239 & 0.233817 & & & 0.009309 \\
\hline & 94240 & -0.041528 & 94240 & 0.014942 & & & 0.000595 \\
\hline & 94241 & -0.002769 & 94241 & 0.000992 & & & 0.000039 \\
\hline
\end{tabular}


PIET-43741-TM-963

PNNL-15870 Rev. 1

\begin{tabular}{|c|c|c|c|c|c|c|}
\hline & 94242 & -0.000346 & 94242 & 0.000123 & 94242 & 0.000005 \\
\hline & 95241 & 0.000000 & 95241 & 0.000000 & 95241 & 0.000000 \\
\hline Photons & 17000 & -0.307859 & 17000 & 0.750000 & 17000 & 0.029860 \\
\hline & 94000 & -0.000346 & 94000 & 0.000126 & 94000 & 0.000005 \\
\hline & 94000 & -0.647152 & 94000 & 0.233817 & 94000 & 0.009309 \\
\hline & 94000 & -0.041528 & 94000 & 0.014942 & 94000 & 0.000595 \\
\hline & 94000 & -0.002769 & 94000 & 0.000992 & 94000 & 0.000039 \\
\hline & 94000 & -0.000346 & 94000 & 0.000123 & 94000 & 0.000005 \\
\hline & 95000 & 0.000000 & 95000 & 0.000000 & 95000 & 0.000000 \\
\hline CEPXS Form: & material & $\mathrm{Cl}$ & 0.307859 & & & \\
\hline & & Pu-238 & 0.000346 & & & \\
\hline & & Pu-239 & 0.647152 & & & \\
\hline & & Pu-240 & 0.041528 & & & \\
\hline & & Pu-241 & 0.002769 & & & \\
\hline & & Pu-242 & 0.000346 & & & \\
\hline & & Am-241 & 0.000000 & & & \\
\hline & $\begin{array}{c}\text { matname } \\
\text { density }\end{array}$ & $\begin{array}{c}\text { Plutonium C } \\
5.710000\end{array}$ & ide & & & \\
\hline $\begin{array}{l}\text { Comments an } \\
\text { Density and for } \\
\text { Plutonium isoto } \\
\text { Pu atoms per }\end{array}$ & $\begin{array}{l}\text { eferences } \\
\text { la from Lid } \\
\text { s assumed } \\
\text { ecule taken }\end{array}$ & $\begin{array}{l}\text { 2008), pgs } 4 \\
\text { DOE } 3013 \text { ' } \\
\text { m atom fract }\end{array}$ & $\begin{array}{l}\text { ut } W \mathrm{t} \% \\
\text { calculate }\end{array}$ & $\begin{array}{l}\text { /239/240 } \\
\text { DOE } 301\end{array}$ & $=0.05$ & $.0 / 0.4 / 0.05$ \\
\hline
\end{tabular}

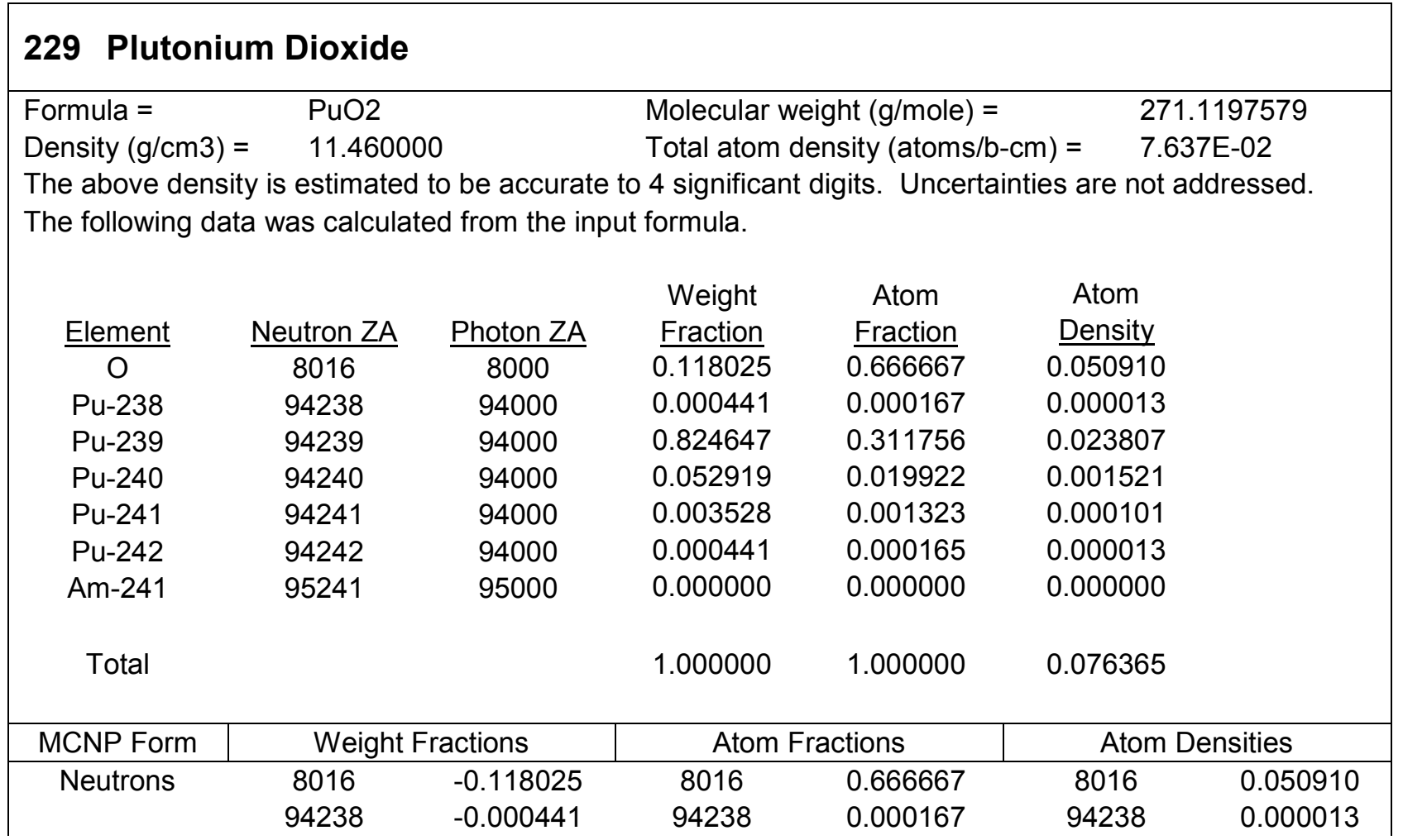


PIET-43741-TM-963

PNNL-15870 Rev. 1

\begin{tabular}{|c|c|c|c|c|c|c|}
\hline & 94239 & -0.824647 & 94239 & 0.311756 & 94239 & 0.023807 \\
\hline & 94240 & -0.052919 & 94240 & 0.019922 & 94240 & 0.001521 \\
\hline & 94241 & -0.003528 & 94241 & 0.001323 & 94241 & 0.000101 \\
\hline & 94242 & -0.000441 & 94242 & 0.000165 & 94242 & 0.000013 \\
\hline & 95241 & 0.000000 & 95241 & 0.000000 & 95241 & 0.000000 \\
\hline Photons & 8000 & -0.118025 & 8000 & 0.666667 & 8000 & 0.050910 \\
\hline & 94000 & -0.000441 & 94000 & 0.000167 & 94000 & 0.000013 \\
\hline & 94000 & -0.824647 & 94000 & 0.311756 & 94000 & 0.023807 \\
\hline & 94000 & -0.052919 & 94000 & 0.019922 & 94000 & 0.001521 \\
\hline & 94000 & -0.003528 & 94000 & 0.001323 & 94000 & 0.000101 \\
\hline & 94000 & -0.000441 & 94000 & 0.000165 & 94000 & 0.000013 \\
\hline & 95000 & 0.000000 & 95000 & 0.000000 & 95000 & 0.000000 \\
\hline CEPXS Form: & material & 0 & 0.118025 & & & \\
\hline & & Pu-238 & 0.000441 & & & \\
\hline & & Pu-239 & 0.824647 & & & \\
\hline & & Pu-240 & 0.052919 & & & \\
\hline & & Pu-241 & 0.003528 & & & \\
\hline & & Pu-242 & 0.000441 & & & \\
\hline & & Am-241 & 0.000000 & & & \\
\hline & $\begin{array}{c}\text { matname } \\
\text { density }\end{array}$ & $\begin{array}{c}\text { Plutonium Di } \\
11.460000\end{array}$ & & & & \\
\hline $\begin{array}{l}\text { Comments anc } \\
\text { Density and for } \\
\text { Plutonium isoto } \\
\text { Pu atoms per }\end{array}$ & $\begin{array}{l}\text { References } \\
\text { lla from Pet } \\
\text { s assumed } \\
\text { ecule taken }\end{array}$ & $\begin{array}{l}\text { et al. (2000). } \\
\text { r DOE } 3013 \mathrm{~V} \\
\text { om atom fract }\end{array}$ & $\begin{array}{l}\mathrm{Pu}: \mathrm{Wt} \% \mathrm{P} \\
\text { s calculatec }\end{array}$ & $\begin{array}{l}\text { 3/239/240/2 } \\
\text { DOE } 3013\end{array}$ & $2=0.05$ & $.0 / 0.4 / 0.05$ \\
\hline
\end{tabular}

\section{Plutonium Fluoride (PuF3)}

\begin{tabular}{llll}
\hline Formula $=$ & PuF3 & Molecular weight $(\mathrm{g} / \mathrm{mole})=$ & 296.1161675 \\
Density $(\mathrm{g} / \mathrm{cm} 3)=$ & 9.330000 & Total atom density $($ atoms $/ \mathrm{b}-\mathrm{cm})=$ & $7.590 \mathrm{E}-02$
\end{tabular}

The above density is estimated to be accurate to 3 significant digits. Uncertainties are not addressed.

The following data was calculated from the input formula.

\begin{tabular}{|c|c|c|c|c|c|}
\hline Element & Neutron ZA & Photon ZA & $\begin{array}{l}\text { Weight } \\
\text { Fraction }\end{array}$ & $\begin{array}{c}\text { Atom } \\
\text { Fraction }\end{array}$ & $\begin{array}{c}\text { Atom } \\
\text { Density }\end{array}$ \\
\hline $\mathrm{F}$ & 9019 & 9000 & 0.192476 & 0.750000 & 0.056924 \\
\hline Pu-238 & 94238 & 94000 & 0.000404 & 0.000126 & 0.000010 \\
\hline Pu-239 & 94239 & 94000 & 0.755035 & 0.233817 & 0.017746 \\
\hline Pu-240 & 94240 & 94000 & 0.048451 & 0.014942 & 0.001134 \\
\hline Pu-241 & 94241 & 94000 & 0.003230 & 0.000992 & 0.000075 \\
\hline Pu-242 & 94242 & 94000 & 0.000404 & 0.000123 & 0.000009 \\
\hline Am-241 & 95241 & 95000 & 0.000000 & 0.000000 & 0.000000 \\
\hline Total & & & 1.000000 & 1.000000 & 0.075898 \\
\hline
\end{tabular}


PIET-43741-TM-963

PNNL-15870 Rev. 1

\begin{tabular}{|c|c|c|c|c|c|c|}
\hline MCNP Form & \multicolumn{2}{|c|}{ Weight Fractions } & \multicolumn{2}{|c|}{ Atom Fractions } & \multicolumn{2}{|c|}{ Atom Densities } \\
\hline \multirow{7}{*}{ Neutrons } & 9019 & -0.192476 & 9019 & 0.750000 & 9019 & 0.056924 \\
\hline & 94238 & -0.000404 & 94238 & 0.000126 & 94238 & 0.000010 \\
\hline & 94239 & -0.755035 & 94239 & 0.233817 & 94239 & 0.017746 \\
\hline & 94240 & -0.048451 & 94240 & 0.014942 & 94240 & 0.001134 \\
\hline & 94241 & -0.003230 & 94241 & 0.000992 & 94241 & 0.000075 \\
\hline & 94242 & -0.000404 & 94242 & 0.000123 & 94242 & 0.000009 \\
\hline & 95241 & 0.000000 & 95241 & 0.000000 & 95241 & 0.000000 \\
\hline \multirow[t]{7}{*}{ Photons } & 9000 & -0.192476 & 9000 & 0.750000 & 9000 & 0.056924 \\
\hline & 94000 & -0.000404 & 94000 & 0.000126 & 94000 & 0.000010 \\
\hline & 94000 & -0.755035 & 94000 & 0.233817 & 94000 & 0.017746 \\
\hline & 94000 & -0.048451 & 94000 & 0.014942 & 94000 & 0.001134 \\
\hline & 94000 & -0.003230 & 94000 & 0.000992 & 94000 & 0.000075 \\
\hline & 94000 & -0.000404 & 94000 & 0.000123 & 94000 & 0.000009 \\
\hline & 95000 & 0.000000 & 95000 & 0.000000 & 95000 & 0.000000 \\
\hline \multirow[t]{8}{*}{ CEPXS Form: } & material & $\mathrm{F}$ & 0.192476 & & & \\
\hline & & Pu-238 & 0.000404 & & & \\
\hline & & Pu-239 & 0.755035 & & & \\
\hline & & Pu-240 & 0.048451 & & & \\
\hline & & Pu-241 & 0.003230 & & & \\
\hline & & Pu-242 & 0.000404 & & & \\
\hline & & Am-241 & 0.000000 & & & \\
\hline & $\begin{array}{l}\text { matname } \\
\text { density }\end{array}$ & $\begin{array}{c}\text { Plutonium F } \\
9.330000\end{array}$ & ide (PuF3) & & & \\
\hline \multicolumn{7}{|c|}{$\begin{array}{l}\text { Comments and References } \\
\text { Density and formula from Lide (2008), pgs } 4-81 \text {. } \\
\text { Plutonium isotopics assumed for DOE } 3013 \text { WGPu: Wt\% Pu238/239/240/241/242=0.05/93.5/6.0/0.4/0.05. } \\
\text { Pu atoms per molecule taken from atom fractions calculated for DOE } 3013 \mathrm{WGPu} \text {. }\end{array}$} \\
\hline
\end{tabular}

\section{Plutonium Fluoride (PuF4)}

\begin{tabular}{llll}
\hline Formula $=$ & PuF4 & Molecular weight $(\mathrm{g} / \mathrm{mole})=$ & 315.1145707 \\
Density $(\mathrm{g} / \mathrm{cm} 3)=$ & 7.000000 & Total atom density $($ atoms $/ \mathrm{b}-\mathrm{cm})=$ & $6.689 \mathrm{E}-02$
\end{tabular}

The above density is estimated to be accurate to 2 significant digits. Uncertainties are not addressed.

The following data was calculated from the input formula.

\begin{tabular}{|c|c|c|c|c|c|}
\hline Element & Neutron ZA & Photon ZA & $\begin{array}{l}\text { Weight } \\
\text { Fraction }\end{array}$ & $\begin{array}{c}\text { Atom } \\
\text { Fraction }\end{array}$ & $\begin{array}{l}\text { Atom } \\
\text { Density }\end{array}$ \\
\hline $\mathrm{F}$ & 9019 & 9000 & 0.241162 & 0.800000 & 0.053511 \\
\hline Pu-238 & 94238 & 94000 & 0.000379 & 0.000100 & 0.000007 \\
\hline Pu-239 & 94239 & 94000 & 0.709514 & 0.187054 & 0.012512 \\
\hline Pu-240 & 94240 & 94000 & 0.045530 & 0.011953 & 0.000800 \\
\hline Pu-241 & 94241 & 94000 & 0.003035 & 0.000794 & 0.000053 \\
\hline Pu-242 & 94242 & 94000 & 0.000379 & 0.000099 & 0.000007 \\
\hline Am-241 & 95241 & 95000 & 0.000000 & 0.000000 & 0.000000 \\
\hline
\end{tabular}


PIET-43741-TM-963

PNNL-15870 Rev. 1

\begin{tabular}{|c|c|c|c|c|c|c|}
\hline \multicolumn{3}{|l|}{ Total } & 1.000000 & 1.000000 & \multicolumn{2}{|c|}{0.066888} \\
\hline MCNP Form & \multicolumn{2}{|c|}{ Weight Fractions } & \multicolumn{2}{|c|}{ Atom Fractions } & \multicolumn{2}{|c|}{ Atom Densities } \\
\hline \multirow[t]{7}{*}{ Neutrons } & 9019 & -0.241162 & 9019 & 0.800000 & 9019 & 0.053511 \\
\hline & 94238 & -0.000379 & 94238 & 0.000100 & 94238 & 0.000007 \\
\hline & 94239 & -0.709514 & 94239 & 0.187054 & 94239 & 0.012512 \\
\hline & 94240 & -0.045530 & 94240 & 0.011953 & 94240 & 0.000800 \\
\hline & 94241 & -0.003035 & 94241 & 0.000794 & 94241 & 0.000053 \\
\hline & 94242 & -0.000379 & 94242 & 0.000099 & 94242 & 0.000007 \\
\hline & 95241 & 0.000000 & 95241 & 0.000000 & 95241 & 0.000000 \\
\hline \multirow[t]{7}{*}{ Photons } & 9000 & -0.241162 & 9000 & 0.800000 & 9000 & 0.053511 \\
\hline & 94000 & -0.000379 & 94000 & 0.000100 & 94000 & 0.000007 \\
\hline & 94000 & -0.709514 & 94000 & 0.187054 & 94000 & 0.012512 \\
\hline & 94000 & -0.045530 & 94000 & 0.011953 & 94000 & 0.000800 \\
\hline & 94000 & -0.003035 & 94000 & 0.000794 & 94000 & 0.000053 \\
\hline & 94000 & -0.000379 & 94000 & 0.000099 & 94000 & 0.000007 \\
\hline & 95000 & 0.000000 & 95000 & 0.000000 & 95000 & 0.000000 \\
\hline \multirow[t]{8}{*}{ CEPXS Form: } & material & $\mathrm{F}$ & 0.241162 & & & \\
\hline & & Pu-238 & 0.000379 & & & \\
\hline & & Pu-239 & 0.709514 & & & \\
\hline & & Pu-240 & 0.045530 & & & \\
\hline & & Pu-241 & 0.003035 & & & \\
\hline & & Pu-242 & 0.000379 & & & \\
\hline & & Am-241 & 0.000000 & & & \\
\hline & $\begin{array}{l}\text { matname } \\
\text { density }\end{array}$ & $\begin{array}{c}\text { Plutonium F } \\
7.000000\end{array}$ & ide (PuF4) & & & \\
\hline \multicolumn{7}{|c|}{$\begin{array}{l}\text { Comments and References } \\
\text { Density and formula from Petrie et al. (2000). } \\
\text { Plutonium isotopics assumed for DOE } 3013 \text { WGPu: Wt\% Pu238/239/240/241/242=0.05/93.5/6.0/0.4/0.05. } \\
\text { Pu atoms per molecule taken from atom fractions calculated for DOE 3013 WGPu. }\end{array}$} \\
\hline
\end{tabular}

\section{Plutonium Fluoride (PuF6)}

\begin{tabular}{llll}
\hline Formula $=$ & PuF6 & Molecular weight $(\mathrm{g} / \mathrm{mole})=$ & 353.1113771 \\
Density $(\mathrm{g} / \mathrm{cm} 3)=$ & 5.080000 & Total atom density $($ atoms $/ \mathrm{b}-\mathrm{cm})=$ & $6.065 \mathrm{E}-02$
\end{tabular}

The above density is estimated to be accurate to 3 significant digits. Uncertainties are not addressed.

The following data was calculated from the input formula.

\begin{tabular}{|c|c|c|c|c|c|}
\hline Element & Neutron ZA & Photon ZA & $\begin{array}{l}\text { Weight } \\
\text { Fraction }\end{array}$ & $\begin{array}{c}\text { Atom } \\
\text { Fraction }\end{array}$ & $\begin{array}{l}\text { Atom } \\
\text { Density }\end{array}$ \\
\hline $\mathrm{F}$ & 9019 & 9000 & $\overline{0.322817}$ & $\overline{0.857143}$ & 0.051982 \\
\hline Pu-238 & 94238 & 94000 & 0.000339 & 0.000072 & 0.000004 \\
\hline Pu-239 & 94239 & 94000 & 0.633166 & 0.133610 & 0.008103 \\
\hline Pu-240 & 94240 & 94000 & 0.040631 & 0.008538 & 0.000518 \\
\hline
\end{tabular}


PIET-43741-TM-963

PNNL-15870 Rev. 1

\begin{tabular}{|c|c|c|c|c|c|c|}
\hline Pu-241 & 94241 & 94000 & 0.002709 & 0.000567 & \multicolumn{2}{|l|}{0.000034} \\
\hline Pu-242 & 94242 & 94000 & 0.000339 & 0.000071 & \multicolumn{2}{|l|}{0.000004} \\
\hline Am-241 & 95241 & 95000 & 0.000000 & 0.000000 & \multicolumn{2}{|l|}{0.000000} \\
\hline Total & & & 1.000000 & 1.000000 & \multicolumn{2}{|l|}{0.060646} \\
\hline MCNP Form & \multicolumn{2}{|c|}{ Weight Fractions } & \multicolumn{2}{|c|}{ Atom Fractions } & \multicolumn{2}{|c|}{ Atom Densities } \\
\hline \multirow[t]{7}{*}{ Neutrons } & 9019 & -0.322817 & 9019 & 0.857143 & 9019 & 0.051982 \\
\hline & 94238 & -0.000339 & 94238 & 0.000072 & 94238 & 0.000004 \\
\hline & 94239 & -0.633166 & 94239 & 0.133610 & 94239 & 0.008103 \\
\hline & 94240 & -0.040631 & 94240 & 0.008538 & 94240 & 0.000518 \\
\hline & 94241 & -0.002709 & 94241 & 0.000567 & 94241 & 0.000034 \\
\hline & 94242 & -0.000339 & 94242 & 0.000071 & 94242 & 0.000004 \\
\hline & 95241 & 0.000000 & 95241 & 0.000000 & 95241 & 0.000000 \\
\hline \multirow[t]{7}{*}{ Photons } & 9000 & -0.322817 & 9000 & 0.857143 & 9000 & 0.051982 \\
\hline & 94000 & -0.000339 & 94000 & 0.000072 & 94000 & 0.000004 \\
\hline & 94000 & -0.633166 & 94000 & 0.133610 & 94000 & 0.008103 \\
\hline & 94000 & -0.040631 & 94000 & 0.008538 & 94000 & 0.000518 \\
\hline & 94000 & -0.002709 & 94000 & 0.000567 & 94000 & 0.000034 \\
\hline & 94000 & -0.000339 & 94000 & 0.000071 & 94000 & 0.000004 \\
\hline & 95000 & 0.000000 & 95000 & 0.000000 & 95000 & 0.000000 \\
\hline \multirow[t]{8}{*}{ CEPXS Form: } & material & $\mathrm{F}$ & 0.322817 & & & \\
\hline & & Pu-238 & 0.000339 & & & \\
\hline & & Pu-239 & 0.633166 & & & \\
\hline & & Pu-240 & 0.040631 & & & \\
\hline & & Pu-241 & 0.002709 & & & \\
\hline & & Pu-242 & 0.000339 & & & \\
\hline & & Am-241 & 0.000000 & & & \\
\hline & $\begin{array}{l}\text { matname } \\
\text { density }\end{array}$ & \multicolumn{2}{|c|}{$\begin{array}{l}\text { Plutonium Fluoride (PuF6) } \\
5.080000\end{array}$} & & & \\
\hline \multicolumn{7}{|c|}{$\begin{array}{l}\text { Comments and References } \\
\text { Density and formula from Lide (2008), pgs } 4-81 \text {. } \\
\text { Plutonium isotopics assumed for DOE } 3013 \text { WGPu: Wt\% Pu238/239/240/241/242=0.05/93.5/6.0/0.4/0.05. } \\
\text { Pu atoms per molecule taken from atom fractions calculated for DOE } 3013 \mathrm{WGPu} \text {. }\end{array}$} \\
\hline
\end{tabular}

\section{Plutonium lodide}

\begin{tabular}{llll}
\hline Formula $=$ & Pul3 & Molecular weight $(\mathrm{g} / \mathrm{mole})=$ & 619.8343679 \\
Density $(\mathrm{g} / \mathrm{cm} 3)=$ & 6.920000 & Total atom density $($ atoms $/ \mathrm{b}-\mathrm{cm})=$ & $2.689 \mathrm{E}-02$
\end{tabular}

The above density is estimated to be accurate to 3 significant digits. Uncertainties are not addressed.

The following data was calculated from the input formula.

$\begin{array}{llllll}\text { Element } & \frac{\text { Neutron ZA }}{5} & \frac{\text { Photon ZA }}{53000} & \begin{array}{c}\text { Weight } \\ \text { Fraction }\end{array} & \begin{array}{c}\text { Atom } \\ \text { Fraction }\end{array} & \begin{array}{c}\text { Atom } \\ \text { Density }\end{array} \\ & \frac{0.614218}{53127} & & 0.750000 & 0.020170\end{array}$




\begin{tabular}{|c|c|c|c|c|c|c|}
\hline Pu-238 & 94238 & 94000 & 0.000193 & 0.000126 & 0.000003 & \\
\hline Pu-239 & 94239 & 94000 & 0.360706 & 0.233817 & 0.006288 & \\
\hline Pu-240 & 94240 & 94000 & 0.023147 & 0.014942 & 0.000402 & \\
\hline Pu-241 & 94241 & 94000 & 0.001543 & 0.000992 & 0.000027 & \\
\hline Pu-242 & 94242 & 94000 & 0.000193 & 0.000123 & 0.000003 & \\
\hline Am-241 & 95241 & 95000 & 0.000000 & 0.000000 & 0.000000 & \\
\hline Total & & & 1.000000 & 1.000000 & 0.026893 & \\
\hline MCNP Form & \multicolumn{2}{|c|}{ Weight Fractions } & \multicolumn{2}{|c|}{ Atom Fractions } & \multicolumn{2}{|c|}{ Atom Densities } \\
\hline \multirow{7}{*}{ Neutrons } & 53127 & -0.614218 & 53127 & 0.750000 & 53127 & 0.020170 \\
\hline & 94238 & -0.000193 & 94238 & 0.000126 & 94238 & 0.000003 \\
\hline & 94239 & -0.360706 & 94239 & 0.233817 & 94239 & 0.006288 \\
\hline & 94240 & -0.023147 & 94240 & 0.014942 & 94240 & 0.000402 \\
\hline & 94241 & -0.001543 & 94241 & 0.000992 & 94241 & 0.000027 \\
\hline & 94242 & -0.000193 & 94242 & 0.000123 & 94242 & 0.000003 \\
\hline & 95241 & 0.000000 & 95241 & 0.000000 & 95241 & 0.000000 \\
\hline \multirow[t]{7}{*}{ Photons } & 53000 & -0.614218 & 53000 & 0.750000 & 53000 & 0.020170 \\
\hline & 94000 & -0.000193 & 94000 & 0.000126 & 94000 & 0.000003 \\
\hline & 94000 & -0.360706 & 94000 & 0.233817 & 94000 & 0.006288 \\
\hline & 94000 & -0.023147 & 94000 & 0.014942 & 94000 & 0.000402 \\
\hline & 94000 & -0.001543 & 94000 & 0.000992 & 94000 & 0.000027 \\
\hline & 94000 & -0.000193 & 94000 & 0.000123 & 94000 & 0.000003 \\
\hline & 95000 & 0.000000 & 95000 & 0.000000 & 95000 & 0.000000 \\
\hline \multirow[t]{8}{*}{ CEPXS Form: } & material & 1 & 0.614218 & & & \\
\hline & & Pu-238 & 0.000193 & & & \\
\hline & & Pu-239 & 0.360706 & & & \\
\hline & & Pu-240 & 0.023147 & & & \\
\hline & & Pu-241 & 0.001543 & & & \\
\hline & & Pu-242 & 0.000193 & & & \\
\hline & & Am-241 & 0.000000 & & & \\
\hline & $\begin{array}{c}\text { matname } \\
\text { density }\end{array}$ & $\begin{array}{c}\text { Plutonium Ic } \\
6.920000\end{array}$ & & & & \\
\hline \multicolumn{7}{|c|}{$\begin{array}{l}\text { Comments and References } \\
\text { Density and formula from Lide (2008), pgs } 4-81 \text {. } \\
\text { Plutonium isotopics assumed for DOE } 3013 \text { WGPu: Wt\% Pu238/239/240/241/242=0.05/93.5/6.0/0.4/0.05. } \\
\text { Pu atoms per molecule taken from atom fractions calculated for DOE } 3013 \mathrm{WGPu} \text {. }\end{array}$} \\
\hline
\end{tabular}

\section{Plutonium Nitrate}

\begin{tabular}{llll}
\hline Formula $=$ & Pu(NO3)4 & Molecular weight $(\mathrm{g} / \mathrm{mole})=$ & 487.1405579 \\
Density $(\mathrm{g} / \mathrm{cm} 3)=$ & 2.447000 & Total atom density $($ atoms $/ \mathrm{b}-\mathrm{cm})=$ & $5.143 \mathrm{E}-02$ \\
The above density is estimated to be accurate to 4 significant digits. Uncertainties are not addressed. & \\
The following data was calculated from the input formula.
\end{tabular}


PIET-43741-TM-963

PNNL-15870 Rev. 1

\begin{tabular}{|c|c|c|c|c|c|c|}
\hline Element & Neutron ZA & Photon ZA & $\begin{array}{l}\text { Weight } \\
\text { Fraction }\end{array}$ & $\begin{array}{l}\text { Atom } \\
\text { Fraction }\end{array}$ & $\begin{array}{l}\text { Atom } \\
\text { Density }\end{array}$ & \\
\hline $\mathrm{N}$ & 7014 & 7000 & 0.115012 & 0.235294 & 0.012100 & \\
\hline 0 & 8016 & 8000 & 0.394122 & 0.705882 & 0.036300 & \\
\hline Pu-238 & 94238 & 94000 & 0.000245 & 0.000030 & 0.000002 & \\
\hline Pu-239 & 94239 & 94000 & 0.458960 & 0.055016 & 0.002829 & \\
\hline Pu-240 & 94240 & 94000 & 0.029452 & 0.003516 & 0.000181 & \\
\hline Pu-241 & 94241 & 94000 & 0.001963 & 0.000233 & 0.000012 & \\
\hline Pu-242 & 94242 & 94000 & 0.000245 & 0.000029 & 0.000001 & \\
\hline Am-241 & 95241 & 95000 & 0.000000 & 0.000000 & 0.000000 & \\
\hline Total & & & 1.000000 & 1.000000 & 0.051426 & \\
\hline MCNP Form & Weight & ractions & Atom & ctions & Atom & sities \\
\hline Neutrons & 7014 & -0.115012 & 7014 & 0.235294 & 7014 & 0.012100 \\
\hline & 8016 & -0.394122 & 8016 & 0.705882 & 8016 & 0.036300 \\
\hline & 94238 & -0.000245 & 94238 & 0.000030 & 94238 & 0.000002 \\
\hline & 94239 & -0.458960 & 94239 & 0.055016 & 94239 & 0.002829 \\
\hline & 94240 & -0.029452 & 94240 & 0.003516 & 94240 & 0.000181 \\
\hline & 94241 & -0.001963 & 94241 & 0.000233 & 94241 & 0.000012 \\
\hline & 94242 & -0.000245 & 94242 & 0.000029 & 94242 & 0.000001 \\
\hline & 95241 & 0.000000 & 95241 & 0.000000 & 95241 & 0.000000 \\
\hline Photons & 7000 & -0.115012 & 7000 & 0.235294 & 7000 & 0.012100 \\
\hline & 8000 & -0.394122 & 8000 & 0.705882 & 8000 & 0.036300 \\
\hline & 94000 & -0.000245 & 94000 & 0.000030 & 94000 & 0.000002 \\
\hline & 94000 & -0.458960 & 94000 & 0.055016 & 94000 & 0.002829 \\
\hline & 94000 & -0.029452 & 94000 & 0.003516 & 94000 & 0.000181 \\
\hline & 94000 & -0.001963 & 94000 & 0.000233 & 94000 & 0.000012 \\
\hline & 94000 & -0.000245 & 94000 & 0.000029 & 94000 & 0.000001 \\
\hline & 95000 & 0.000000 & 95000 & 0.000000 & 95000 & 0.000000 \\
\hline CEPXS Form: & material & $\mathrm{N}$ & 0.115012 & & & \\
\hline & & 0 & 0.394122 & & & \\
\hline & & Pu-238 & 0.000245 & & & \\
\hline & & Pu-239 & 0.458960 & & & \\
\hline & & Pu-240 & 0.029452 & & & \\
\hline & & Pu-241 & 0.001963 & & & \\
\hline & & Pu-242 & 0.000245 & & & \\
\hline & & Am-241 & 0.000000 & & & \\
\hline & $\begin{array}{l}\text { matname } \\
\text { density }\end{array}$ & \multicolumn{3}{|c|}{$\begin{array}{l}\text { Plutonium Nitrate } \\
2.447000\end{array}$} & & \\
\hline \multicolumn{7}{|c|}{$\begin{array}{l}\text { Comments and References } \\
\text { Density and formula from Petrie et al. (2000). } \\
\text { Plutonium isotopics assumed for DOE } 3013 \mathrm{WGPu}: \mathrm{Wt} \% \text { Pu238/239/240/241/242=0.05/93.5/6.0/0.4/0.05 } \\
\text { Pu atoms per molecule taken from atom fractions calculated for DOE } 3013 \mathrm{WGPu} \text {. }\end{array}$} \\
\hline
\end{tabular}




\section{Plutonium Nitride}

\begin{tabular}{llll}
\hline Formula $=$ & PuN & Molecular weight $(\mathrm{g} / \mathrm{mole})=$ & 253.1276579 \\
Density $(\mathrm{g} / \mathrm{cm} 3)=$ & 14.250000 & Total atom density $($ atoms $/ \mathrm{b}-\mathrm{cm})=$ & $6.780 \mathrm{E}-02$
\end{tabular}

The above density is estimated to be accurate to 4 significant digits. Uncertainties are not addressed.

The following data was calculated from the input formula.

\begin{tabular}{|c|c|c|c|c|c|c|}
\hline Element & Neutron ZA & Photon ZA & $\begin{array}{l}\text { Weight } \\
\text { Fraction }\end{array}$ & $\begin{array}{l}\text { Atom } \\
\text { Fraction }\end{array}$ & $\begin{array}{l}\text { Atom } \\
\text { Density }\end{array}$ & \\
\hline $\mathrm{N}$ & 7014 & 7000 & 0.055335 & 0.500000 & 0.033902 & \\
\hline Pu-238 & 94238 & 94000 & 0.000472 & 0.000251 & 0.000017 & \\
\hline Pu-239 & 94239 & 94000 & 0.883262 & 0.467635 & 0.031708 & \\
\hline Pu-240 & 94240 & 94000 & 0.056680 & 0.029883 & 0.002026 & \\
\hline Pu-241 & 94241 & 94000 & 0.003779 & 0.001984 & 0.000135 & \\
\hline Pu-242 & 94242 & 94000 & 0.000472 & 0.000247 & 0.000017 & \\
\hline Am-241 & 95241 & 95000 & 0.000000 & 0.000000 & 0.000000 & \\
\hline Total & & & 1.000000 & 1.000000 & 0.067804 & \\
\hline MCNP Form & \multicolumn{2}{|c|}{ Weight Fractions } & \multicolumn{2}{|c|}{ Atom Fractions } & \multicolumn{2}{|c|}{ Atom Densities } \\
\hline \multirow[t]{7}{*}{ Neutrons } & 7014 & -0.055335 & 7014 & 0.500000 & 7014 & 0.033902 \\
\hline & 94238 & -0.000472 & 94238 & 0.000251 & 94238 & 0.000017 \\
\hline & 94239 & -0.883262 & 94239 & 0.467635 & 94239 & 0.031708 \\
\hline & 94240 & -0.056680 & 94240 & 0.029883 & 94240 & 0.002026 \\
\hline & 94241 & -0.003779 & 94241 & 0.001984 & 94241 & 0.000135 \\
\hline & 94242 & -0.000472 & 94242 & 0.000247 & 94242 & 0.000017 \\
\hline & 95241 & 0.000000 & 95241 & 0.000000 & 95241 & 0.000000 \\
\hline \multirow[t]{7}{*}{ Photons } & 7000 & -0.055335 & 7000 & 0.500000 & 7000 & 0.033902 \\
\hline & 94000 & -0.000472 & 94000 & 0.000251 & 94000 & 0.000017 \\
\hline & 94000 & -0.883262 & 94000 & 0.467635 & 94000 & 0.031708 \\
\hline & 94000 & -0.056680 & 94000 & 0.029883 & 94000 & 0.002026 \\
\hline & 94000 & -0.003779 & 94000 & 0.001984 & 94000 & 0.000135 \\
\hline & 94000 & -0.000472 & 94000 & 0.000247 & 94000 & 0.000017 \\
\hline & 95000 & 0.000000 & 95000 & 0.000000 & 95000 & 0.000000 \\
\hline \multirow[t]{8}{*}{ CEPXS Form: } & material & $\mathrm{N}$ & 0.055335 & & & \\
\hline & & Pu-238 & 0.000472 & & & \\
\hline & & Pu-239 & 0.883262 & & & \\
\hline & & Pu-240 & 0.056680 & & & \\
\hline & & Pu-241 & 0.003779 & & & \\
\hline & & Pu-242 & 0.000472 & & & \\
\hline & & Am-241 & 0.000000 & & & \\
\hline & $\begin{array}{c}\text { matname } \\
\text { density }\end{array}$ & \multicolumn{2}{|c|}{$\begin{array}{l}\text { Plutonium Nitride } \\
14.250000\end{array}$} & & & \\
\hline \multicolumn{7}{|c|}{$\begin{array}{l}\text { Comments and References } \\
\text { Density and formula from Petrie et al. (2000). } \\
\text { Plutonium isotopics assumed for DOE } 3013 \text { WGPu: Wt\% Pu238/239/240/241/242=0.05/93.5/6.0/0.4/0.05. } \\
\text { Pu atoms per molecule taken from atom fractions calculated for DOE 3013 WGPu. }\end{array}$} \\
\hline
\end{tabular}




\section{Plutonium Oxide (Pu2O3)}

\begin{tabular}{llll}
\hline Formula $=$ & Pu2O3 & Molecular weight $(\mathrm{g} / \mathrm{mole})=$ & 526.2401157 \\
Density $(\mathrm{g} / \mathrm{cm} 3)=$ & 10.500000 & Total atom density $($ atoms $/ \mathrm{b}-\mathrm{cm})=$ & $6.008 \mathrm{E}-02$
\end{tabular}

The above density is estimated to be accurate to 3 significant digits. Uncertainties are not addressed.

The following data was calculated from the input formula.

\begin{tabular}{|c|c|c|c|c|c|c|}
\hline Element & Neutron ZA & Photon ZA & $\begin{array}{l}\text { Weight } \\
\text { Fraction }\end{array}$ & $\begin{array}{l}\text { Atom } \\
\text { Fraction }\end{array}$ & $\begin{array}{c}\text { Atom } \\
\text { Density }\end{array}$ & \\
\hline 0 & 8016 & 8000 & $\overline{0.091210}$ & $\overline{0.600000}$ & 0.036048 & \\
\hline Pu-238 & 94238 & 94000 & 0.000454 & 0.000201 & 0.000012 & \\
\hline Pu-239 & 94239 & 94000 & 0.849719 & 0.374108 & 0.022476 & \\
\hline Pu-240 & 94240 & 94000 & 0.054527 & 0.023907 & 0.001436 & \\
\hline Pu-241 & 94241 & 94000 & 0.003635 & 0.001587 & 0.000095 & \\
\hline Pu-242 & 94242 & 94000 & 0.000454 & 0.000198 & 0.000012 & \\
\hline Am-241 & 95241 & 95000 & 0.000000 & 0.000000 & 0.000000 & \\
\hline Total & & & 1.000000 & 1.000000 & 0.060080 & \\
\hline MCNP Form & \multicolumn{2}{|c|}{ Weight Fractions } & \multicolumn{2}{|c|}{ Atom Fractions } & \multicolumn{2}{|c|}{ Atom Densities } \\
\hline \multirow[t]{7}{*}{ Neutrons } & 8016 & -0.091210 & 8016 & 0.600000 & 8016 & 0.036048 \\
\hline & 94238 & -0.000454 & 94238 & 0.000201 & 94238 & 0.000012 \\
\hline & 94239 & -0.849719 & 94239 & 0.374108 & 94239 & 0.022476 \\
\hline & 94240 & -0.054527 & 94240 & 0.023907 & 94240 & 0.001436 \\
\hline & 94241 & -0.003635 & 94241 & 0.001587 & 94241 & 0.000095 \\
\hline & 94242 & -0.000454 & 94242 & 0.000198 & 94242 & 0.000012 \\
\hline & 95241 & 0.000000 & 95241 & 0.000000 & 95241 & 0.000000 \\
\hline \multirow[t]{7}{*}{ Photons } & 8000 & -0.091210 & 8000 & 0.600000 & 8000 & 0.036048 \\
\hline & 94000 & -0.000454 & 94000 & 0.000201 & 94000 & 0.000012 \\
\hline & 94000 & -0.849719 & 94000 & 0.374108 & 94000 & 0.022476 \\
\hline & 94000 & -0.054527 & 94000 & 0.023907 & 94000 & 0.001436 \\
\hline & 94000 & -0.003635 & 94000 & 0.001587 & 94000 & 0.000095 \\
\hline & 94000 & -0.000454 & 94000 & 0.000198 & 94000 & 0.000012 \\
\hline & 95000 & 0.000000 & 95000 & 0.000000 & 95000 & 0.000000 \\
\hline \multirow[t]{8}{*}{ CEPXS Form: } & material & 0 & 0.091210 & & & \\
\hline & & $\mathrm{Pu}-238$ & 0.000454 & & & \\
\hline & & Pu-239 & 0.849719 & & & \\
\hline & & Pu-240 & 0.054527 & & & \\
\hline & & Pu-241 & 0.003635 & & & \\
\hline & & Pu-242 & 0.000454 & & & \\
\hline & & Am-241 & 0.000000 & & & \\
\hline & $\begin{array}{c}\text { matname } \\
\text { density }\end{array}$ & \multicolumn{3}{|c|}{$\begin{array}{l}\text { Plutonium Oxide (Pu2O3) } \\
10.500000\end{array}$} & & \\
\hline
\end{tabular}


Comments and References

Density and formula from Lide (2008), pgs 4 - 81.

Plutonium isotopics assumed for DOE 3013 WGPu: Wt\% Pu238/239/240/241/242=0.05/93.5/6.0/0.4/0.05.

$\mathrm{Pu}$ atoms per molecule are twice (2 Pu atoms/molecule) the atom fractions calculated for DOE 3013

WGPu.

\section{Plutonium Oxide (PuO)}

\begin{tabular}{llll}
\hline Formula $=$ & $\mathrm{PuO}$ & Molecular weight $(\mathrm{g} / \mathrm{mole})=$ & 255.1203579 \\
Density $(\mathrm{g} / \mathrm{cm} 3)=$ & 14.000000 & Total atom density $($ atoms $/ \mathrm{b}-\mathrm{cm})=$ & $6.609 \mathrm{E}-02$
\end{tabular}

The above density is estimated to be accurate to 3 significant digits. Uncertainties are not addressed.

The following data was calculated from the input formula.

\begin{tabular}{|c|c|c|c|c|c|c|}
\hline Element & Neutron ZA & Photon ZA & $\begin{array}{l}\text { Weight } \\
\text { Fraction }\end{array}$ & $\begin{array}{c}\text { Atom } \\
\text { Fraction }\end{array}$ & $\begin{array}{c}\text { Atom } \\
\text { Density }\end{array}$ & \\
\hline 0 & 8016 & 8000 & $\overline{0.062713}$ & $\overline{0.500000}$ & 0.033047 & \\
\hline Pu-238 & 94238 & 94000 & 0.000469 & 0.000251 & 0.000017 & \\
\hline Pu-239 & 94239 & 94000 & 0.876363 & 0.467635 & 0.030908 & \\
\hline Pu-240 & 94240 & 94000 & 0.056237 & 0.029883 & 0.001975 & \\
\hline Pu-241 & 94241 & 94000 & 0.003749 & 0.001984 & 0.000131 & \\
\hline Pu-242 & 94242 & 94000 & 0.000469 & 0.000247 & 0.000016 & \\
\hline Am-241 & 95241 & 95000 & 0.000000 & 0.000000 & 0.000000 & \\
\hline Total & & & 1.000000 & 1.000000 & 0.066094 & \\
\hline MCNP Form & \multicolumn{2}{|c|}{ Weight Fractions } & \multicolumn{2}{|c|}{ Atom Fractions } & \multicolumn{2}{|c|}{ Atom Densities } \\
\hline \multirow[t]{7}{*}{ Neutrons } & 8016 & -0.062713 & 8016 & 0.500000 & 8016 & 0.033047 \\
\hline & 94238 & -0.000469 & 94238 & 0.000251 & 94238 & 0.000017 \\
\hline & 94239 & -0.876363 & 94239 & 0.467635 & 94239 & 0.030908 \\
\hline & 94240 & -0.056237 & 94240 & 0.029883 & 94240 & 0.001975 \\
\hline & 94241 & -0.003749 & 94241 & 0.001984 & 94241 & 0.000131 \\
\hline & 94242 & -0.000469 & 94242 & 0.000247 & 94242 & 0.000016 \\
\hline & 95241 & 0.000000 & 95241 & 0.000000 & 95241 & 0.000000 \\
\hline \multirow[t]{7}{*}{ Photons } & 8000 & -0.062713 & 8000 & 0.500000 & 8000 & 0.033047 \\
\hline & 94000 & -0.000469 & 94000 & 0.000251 & 94000 & 0.000017 \\
\hline & 94000 & -0.876363 & 94000 & 0.467635 & 94000 & 0.030908 \\
\hline & 94000 & -0.056237 & 94000 & 0.029883 & 94000 & 0.001975 \\
\hline & 94000 & -0.003749 & 94000 & 0.001984 & 94000 & 0.000131 \\
\hline & 94000 & -0.000469 & 94000 & 0.000247 & 94000 & 0.000016 \\
\hline & 95000 & 0.000000 & 95000 & 0.000000 & 95000 & 0.000000 \\
\hline \multirow[t]{6}{*}{ CEPXS Form: } & material & 0 & 0.062713 & & & \\
\hline & & Pu-238 & 0.000469 & & & \\
\hline & & Pu-239 & 0.876363 & & & \\
\hline & & Pu-240 & 0.056237 & & & \\
\hline & & Pu-241 & 0.003749 & & & \\
\hline & & Pu-242 & 0.000469 & & & \\
\hline
\end{tabular}




\section{Am-241 $\quad 0.000000$}

matname Plutonium Oxide $(\mathrm{PuO})$

density $\quad 14.000000$

\section{Comments and References}

Density and formula from Lide (2008), pgs 4 - 81.

Plutonium isotopics assumed for DOE 3013 WGPu: Wt\% Pu238/239/240/241/242=0.05/93.5/6.0/0.4/0.05.

$\mathrm{Pu}$ atoms per molecule taken from atom fractions calculated for DOE $3013 \mathrm{WGPu}$.

\section{Plutonium, Aged WGPu (A: 4-7\% Pu-240)}

Formula $=$

Density $(\mathrm{g} / \mathrm{cm} 3)=19.840000$

The above density is estimated to be accurate to 4 signicant digits. Uncerainties are not addressed.

The following data were calculated from the input weight fractions.

\begin{tabular}{|c|c|c|c|c|c|c|}
\hline Element & Neutron ZA & Photon ZA & $\begin{array}{l}\text { Weight } \\
\text { Fraction }\end{array}$ & $\begin{array}{c}\text { Atom } \\
\text { Fraction }\end{array}$ & $\begin{array}{c}\text { Atom } \\
\text { Density }\end{array}$ & \\
\hline$\overline{P u-238}$ & 94238 & 94000 & $\overline{0.000100}$ & $\overline{0.000100}$ & 0.000005 & \\
\hline Pu-239 & 94239 & 94000 & 0.936296 & 0.936559 & 0.046796 & \\
\hline Pu-240 & 94240 & 94000 & 0.059910 & 0.059677 & 0.002982 & \\
\hline Pu-241 & 94241 & 94000 & 0.001997 & 0.001981 & 0.000099 & \\
\hline Pu-242 & 94242 & 94000 & 0.000300 & 0.000296 & 0.000015 & \\
\hline Am-241 & 95241 & 95000 & 0.001398 & 0.001387 & 0.000069 & \\
\hline Total & & & 1.000000 & 1.000000 & 0.049966 & \\
\hline MCNP Form & \multicolumn{2}{|c|}{ Weight Fractions } & \multicolumn{2}{|c|}{ Atom Fractions } & \multicolumn{2}{|c|}{ Atom Densities } \\
\hline \multirow[t]{6}{*}{ Neutrons } & 94238 & -0.000100 & 94238 & 0.000100 & 94238 & 0.000005 \\
\hline & 94239 & -0.936296 & 94239 & 0.936559 & 94239 & 0.046796 \\
\hline & 94240 & -0.059910 & 94240 & 0.059677 & 94240 & 0.002982 \\
\hline & 94241 & -0.001997 & 94241 & 0.001981 & 94241 & 0.000099 \\
\hline & 94242 & -0.000300 & 94242 & 0.000296 & 94242 & 0.000015 \\
\hline & 95241 & -0.001398 & 95241 & 0.001387 & 95241 & 0.000069 \\
\hline \multirow[t]{6}{*}{ Photons } & 94000 & -0.000100 & 94000 & 0.000100 & 94000 & 0.000005 \\
\hline & 94000 & -0.936296 & 94000 & 0.936559 & 94000 & 0.046796 \\
\hline & 94000 & -0.059910 & 94000 & 0.059677 & 94000 & 0.002982 \\
\hline & 94000 & -0.001997 & 94000 & 0.001981 & 94000 & 0.000099 \\
\hline & 94000 & -0.000300 & 94000 & 0.000296 & 94000 & 0.000015 \\
\hline & 95000 & -0.001398 & 95000 & 0.001387 & 95000 & 0.000069 \\
\hline \multirow[t]{6}{*}{ CEPXS Form: } & material & Pu-238 & 0.000100 & & & \\
\hline & & Pu-239 & 0.936296 & & & \\
\hline & & Pu-240 & 0.059910 & & & \\
\hline & & Pu-241 & 0.001997 & & & \\
\hline & & Pu-242 & 0.000300 & & & \\
\hline & & Am-241 & 0.001398 & & & \\
\hline
\end{tabular}




\begin{tabular}{|c|c|}
\hline $\begin{array}{l}\text { matname } \\
\text { density }\end{array}$ & $\begin{array}{l}\text { Plutonium, Aged WGPu (A: 4-7\% Pu-240) } \\
19.840000\end{array}$ \\
\hline $\begin{array}{l}\text { Comments and References } \\
\text { Table B-6 of DOE-STD-3013- } \\
\text { Density }=19.84 \mathrm{~g} / \mathrm{cm} 3 \text { for alph } \\
\text { (NIST 1998). } \\
\text { There are six different phases } \\
\text { densities (Söderlind } 2001 \text { at } \mathrm{h} \\
16.63 \mathrm{~g} / \mathrm{cm} 3 \text { for molten Pu (po } \\
\text { Weight fractions are adjusted }\end{array}$ & $\begin{array}{l}\text { plutonium from http://physics.nist.gov/cgi-bin/Star/compos.pl?matno=094 } \\
\text { or solid Pu at different temperatures. These have significantly different } \\
\text { p://www.iop.org/EJ/article/0295-5075/55/4/525/6673.html). Density = } \\
4-141 \text { of Lide 2008). } \\
\text { o they sum to unity. }\end{array}$ \\
\hline
\end{tabular}

\section{Plutonium, Aged WGPu (B: 10-13\% Pu-240)}

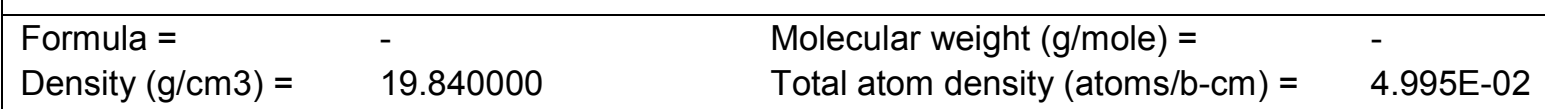

The above density is estimated to be accurate to 4 significant digits. Uncertainties are not addressed. The following data were calculated from the input weight fractions.

\begin{tabular}{|c|c|c|c|c|c|c|}
\hline Element & Neutron ZA & Photon ZA & $\begin{array}{l}\text { Weight } \\
\text { Fraction }\end{array}$ & $\begin{array}{c}\text { Atom } \\
\text { Fraction }\end{array}$ & $\begin{array}{l}\text { Atom } \\
\text { Density }\end{array}$ & \\
\hline$\overline{P u-238}$ & 94238 & 94000 & $\overline{0.000892}$ & $\overline{0.000897}$ & 0.000045 & \\
\hline Pu-239 & 94239 & 94000 & 0.861901 & 0.862470 & 0.043078 & \\
\hline Pu-240 & 94240 & 94000 & 0.117081 & 0.116670 & 0.005827 & \\
\hline Pu-241 & 94241 & 94000 & 0.009914 & 0.009838 & 0.000491 & \\
\hline Pu-242 & 94242 & 94000 & 0.001685 & 0.001666 & 0.000083 & \\
\hline Am-241 & 95241 & 95000 & 0.008526 & 0.008460 & 0.000423 & \\
\hline Total & & & 1.000000 & 1.000000 & 0.049947 & \\
\hline MCNP Form & \multicolumn{2}{|c|}{ Weight Fractions } & \multicolumn{2}{|c|}{ Atom Fractions } & \multicolumn{2}{|c|}{ Atom Densities } \\
\hline \multirow{6}{*}{ Neutrons } & 94238 & -0.000892 & 94238 & 0.000897 & 94238 & 0.000045 \\
\hline & 94239 & -0.861901 & 94239 & 0.862470 & 94239 & 0.043078 \\
\hline & 94240 & -0.117081 & 94240 & 0.116670 & 94240 & 0.005827 \\
\hline & 94241 & -0.009914 & 94241 & 0.009838 & 94241 & 0.000491 \\
\hline & 94242 & -0.001685 & 94242 & 0.001666 & 94242 & 0.000083 \\
\hline & 95241 & -0.008526 & 95241 & 0.008460 & 95241 & 0.000423 \\
\hline \multirow[t]{6}{*}{ Photons } & 94000 & -0.000892 & 94000 & 0.000897 & 94000 & 0.000045 \\
\hline & 94000 & -0.861901 & 94000 & 0.862470 & 94000 & 0.043078 \\
\hline & 94000 & -0.117081 & 94000 & 0.116670 & 94000 & 0.005827 \\
\hline & 94000 & -0.009914 & 94000 & 0.009838 & 94000 & 0.000491 \\
\hline & 94000 & -0.001685 & 94000 & 0.001666 & 94000 & 0.000083 \\
\hline & 95000 & -0.008526 & 95000 & 0.008460 & 95000 & 0.000423 \\
\hline \multirow[t]{3}{*}{ CEPXS Form: } & material & Pu-238 & 0.000892 & & & \\
\hline & & Pu-239 & 0.861901 & & & \\
\hline & & Pu-240 & 0.117081 & & & \\
\hline
\end{tabular}




\begin{tabular}{|lr} 
Pu-241 & 0.009914 \\
Pu-242 & 0.001685 \\
Am-241 & 0.008526
\end{tabular}

matname Plutonium, Aged WGPu (B: 10-13\% Pu-240)

density $\quad 19.840000$

\section{Comments and References}

Table B-6 of DOE-STD-3013-2000.

Density = $19.84 \mathrm{~g} / \mathrm{cm} 3$ for alpha plutonium from http://physics.nist.gov/cgi-bin/Star/compos.pl?matno=094 (NIST 1998).

There are six different phases for solid Pu at different temperatures. These have significantly different densities (Söderlind 2001 at http://www.iop.org/EJ/article/0295-5075/55/4/525/6673.html). Density = $16.63 \mathrm{~g} / \mathrm{cm} 3$ for molten Pu (pgs 4 - 141 of Lide 2008).

Weight fractions are adjusted so they sum to unity.

\section{Plutonium, Aged WGPu (C: $16-19 \%$ Pu-240)}

\begin{tabular}{llll}
\hline Formula $=$ & - & Molecular weight $(\mathrm{g} / \mathrm{mole})=$ \\
Density $(\mathrm{g} / \mathrm{cm} 3)=$ & 19.840000 & Total atom density $($ atoms $/ \mathrm{b}-\mathrm{cm})=$ & - \\
\hline
\end{tabular}

The above density is estimated to be accurate to 4 significant digits. Uncertainties are not addressed.

The following data were calculated from the input weight fractions.

\begin{tabular}{|c|c|c|c|c|c|c|}
\hline Element & Neutron ZA & Photon ZA & $\begin{array}{l}\text { Weight } \\
\text { Fraction }\end{array}$ & $\begin{array}{c}\text { Atom } \\
\text { Fraction }\end{array}$ & $\begin{array}{l}\text { Atom } \\
\text { Density }\end{array}$ & \\
\hline$\overline{\mathrm{Pu}-238}$ & 94238 & 94000 & $\overline{0.002334}$ & $\overline{0.002347}$ & 0.000117 & \\
\hline Pu-239 & 94239 & 94000 & 0.784554 & 0.785422 & 0.039212 & \\
\hline Pu-240 & 94240 & 94000 & 0.165159 & 0.164652 & 0.008220 & \\
\hline Pu-241 & 94241 & 94000 & 0.014006 & 0.013905 & 0.000694 & \\
\hline Pu-242 & 94242 & 94000 & 0.006711 & 0.006635 & 0.000331 & \\
\hline Am-241 & 95241 & 95000 & 0.027235 & 0.027038 & 0.001350 & \\
\hline Total & & & 1.000000 & 1.000000 & 0.049925 & \\
\hline MCNP Form & \multicolumn{2}{|c|}{ Weight Fractions } & \multicolumn{2}{|c|}{ Atom Fractions } & \multicolumn{2}{|c|}{ Atom Densities } \\
\hline \multirow[t]{6}{*}{ Neutrons } & 94238 & -0.002334 & 94238 & 0.002347 & 94238 & 0.000117 \\
\hline & 94239 & -0.784554 & 94239 & 0.785422 & 94239 & 0.039212 \\
\hline & 94240 & -0.165159 & 94240 & 0.164652 & 94240 & 0.008220 \\
\hline & 94241 & -0.014006 & 94241 & 0.013905 & 94241 & 0.000694 \\
\hline & 94242 & -0.006711 & 94242 & 0.006635 & 94242 & 0.000331 \\
\hline & 95241 & -0.027235 & 95241 & 0.027038 & 95241 & 0.001350 \\
\hline \multirow[t]{6}{*}{ Photons } & 94000 & -0.002334 & 94000 & 0.002347 & 94000 & 0.000117 \\
\hline & 94000 & -0.784554 & 94000 & 0.785422 & 94000 & 0.039212 \\
\hline & 94000 & -0.165159 & 94000 & 0.164652 & 94000 & 0.008220 \\
\hline & 94000 & -0.014006 & 94000 & 0.013905 & 94000 & 0.000694 \\
\hline & 94000 & -0.006711 & 94000 & 0.006635 & 94000 & 0.000331 \\
\hline & 95000 & -0.027235 & 95000 & 0.027038 & 95000 & 0.001350 \\
\hline
\end{tabular}




$\begin{array}{|lll|}\text { CEPXS Form: } \quad \text { material } & \text { Pu-238 } & 0.002334 \\ & \text { Pu-239 } & 0.784554 \\ & \text { Pu-240 } & 0.165159 \\ & \mathrm{Pu}-241 & 0.014006 \\ & \mathrm{Pu}-242 & 0.006711 \\ & \mathrm{Am}-241 & 0.027235\end{array}$

matname Plutonium, Aged WGPu (C: 16-19\% Pu-240)

density $\quad 19.840000$

\section{Comments and References}

Table B-6 of DOE-STD-3013-2000.

Density = $19.84 \mathrm{~g} / \mathrm{cm} 3$ for alpha plutonium from http://physics.nist.gov/cgi-bin/Star/compos.pl?matno=094 (NIST 1998).

There are six different phases for solid $\mathrm{Pu}$ at different temperatures. These have significantly different densities (Söderlind 2001 at http://www.iop.org/EJ/article/0295-5075/55/4/525/6673.html).

Density $=16.63 \mathrm{~g} / \mathrm{cm} 3$ for molten Pu (pgs $4-141$ of Lide 2008).

Weight fractions are adjusted so they sum to unity.

\section{Plutonium, DOE 3013 WGPu}

\begin{tabular}{llll}
\hline Formula $=$ & - & Molecular weight $(\mathrm{g} / \mathrm{mole})=$ & - \\
Density $(\mathrm{g} / \mathrm{cm} 3)=$ & 19.840000 & Total atom density $($ atoms $/ \mathrm{b}-\mathrm{cm})=$ & $4.997 \mathrm{E}-02$
\end{tabular}

The above density is estimated to be accurate to 4 significant digits. Uncertainties are not addressed.

The following data were calculated from the input weight fractions.

\begin{tabular}{|c|c|c|c|c|c|c|}
\hline Element & Neutron ZA & Photon ZA & $\begin{array}{l}\text { Weight } \\
\text { Fraction }\end{array}$ & $\begin{array}{c}\text { Atom } \\
\text { Fraction }\end{array}$ & $\begin{array}{l}\text { Atom } \\
\text { Density }\end{array}$ & \\
\hline $\mathrm{Pu}-238$ & 94238 & 94000 & 0.000500 & 0.000502 & 0.000025 & \\
\hline Pu-239 & 94239 & 94000 & 0.935000 & 0.935269 & 0.046732 & \\
\hline Pu-240 & 94240 & 94000 & 0.060000 & 0.059767 & 0.002986 & \\
\hline Pu-241 & 94241 & 94000 & 0.004000 & 0.003968 & 0.000198 & \\
\hline Pu-242 & 94242 & 94000 & 0.000500 & 0.000494 & 0.000025 & \\
\hline Total & & & 1.000000 & 1.000000 & 0.049966 & \\
\hline MCNP Form & \multicolumn{2}{|c|}{ Weight Fractions } & \multicolumn{2}{|c|}{ Atom Fractions } & \multicolumn{2}{|c|}{ Atom Densities } \\
\hline \multirow[t]{5}{*}{ Neutrons } & 94238 & -0.000500 & 94238 & 0.000502 & 94238 & 0.000025 \\
\hline & 94239 & -0.935000 & 94239 & 0.935269 & 94239 & 0.046732 \\
\hline & 94240 & -0.060000 & 94240 & 0.059767 & 94240 & 0.002986 \\
\hline & 94241 & -0.004000 & 94241 & 0.003968 & 94241 & 0.000198 \\
\hline & 94242 & -0.000500 & 94242 & 0.000494 & 94242 & 0.000025 \\
\hline \multirow[t]{5}{*}{ Photons } & 94000 & -0.000500 & 94000 & 0.000502 & 94000 & 0.000025 \\
\hline & 94000 & -0.935000 & 94000 & 0.935269 & 94000 & 0.046732 \\
\hline & 94000 & -0.060000 & 94000 & 0.059767 & 94000 & 0.002986 \\
\hline & 94000 & -0.004000 & 94000 & 0.003968 & 94000 & 0.000198 \\
\hline & 94000 & -0.000500 & 94000 & 0.000494 & 94000 & 0.000025 \\
\hline
\end{tabular}




\begin{tabular}{|c|c|c|c|}
\hline \multirow[t]{5}{*}{ CEPXS Form: } & \multirow[t]{5}{*}{ material } & Pu-238 & 0.000500 \\
\hline & & Pu-239 & 0.935000 \\
\hline & & Pu-240 & 0.06000 \\
\hline & & Pu-241 & 0.00400 \\
\hline & & Pu-242 & 0.00050 \\
\hline
\end{tabular}

matname Plutonium, DOE 3013 WGPu

density $\quad 19.840000$

\section{Comments and References}

Table B-6 of DOE-STD-3013-2000.

Density $=19.84 \mathrm{~g} / \mathrm{cm} 3$ for alpha plutonium from http://physics.nist.gov/cgi-bin/Star/compos.pl?matno=094 (NIST 1998).

There are six different phases for solid Pu at different temperatures. These have significantly different densities (Söderlind 2001 at http://www.iop.org/EJ/article/0295-5075/55/4/525/6673.html).

Density $=16.63 \mathrm{~g} / \mathrm{cm} 3$ for molten Pu (pgs $4-141$ of Lide 2008).

Weight fractions are adjusted so they sum to unity.

\section{Plutonium, Fuel Grade}

\begin{tabular}{llll}
\hline Formula $=$ & - & Molecular weight $(\mathrm{g} / \mathrm{mole})=$ \\
Density $(\mathrm{g} / \mathrm{cm} 3)=$ & 19.840000 & Total atom density $($ atoms $/ \mathrm{b}-\mathrm{cm})=$ & - \\
The & $4.995 \mathrm{E}-02$
\end{tabular}

The above density is estimated to be accurate to 4 significant digits. Uncertainties are not addressed.

The following data were calculated from the input weight fractions.

\begin{tabular}{|c|c|c|c|c|c|c|}
\hline Element & Neutron ZA & Photon ZA & $\begin{array}{l}\text { Weight } \\
\text { Fraction }\end{array}$ & $\begin{array}{c}\text { Atom } \\
\text { Fraction }\end{array}$ & $\begin{array}{c}\text { Atom } \\
\text { Density }\end{array}$ & \\
\hline Pu-238 & 94238 & 94000 & 0.001000 & 0.001005 & 0.000050 & \\
\hline Pu-239 & 94239 & 94000 & 0.861000 & 0.861564 & 0.043033 & \\
\hline Pu-240 & 94240 & 94000 & 0.120000 & 0.119578 & 0.005973 & \\
\hline Pu-241 & 94241 & 94000 & 0.016000 & 0.015877 & 0.000793 & \\
\hline Pu-242 & 94242 & 94000 & 0.002000 & 0.001976 & 0.000099 & \\
\hline Total & & & 1.000000 & 1.000000 & 0.049948 & \\
\hline MCNP Form & \multicolumn{2}{|c|}{ Weight Fractions } & \multicolumn{2}{|c|}{ Atom Fractions } & \multicolumn{2}{|c|}{ Atom Densities } \\
\hline \multirow[t]{5}{*}{ Neutrons } & 94238 & -0.001000 & 94238 & 0.001005 & 94238 & 0.000050 \\
\hline & 94239 & -0.861000 & 94239 & 0.861564 & 94239 & 0.043033 \\
\hline & 94240 & -0.120000 & 94240 & 0.119578 & 94240 & 0.005973 \\
\hline & 94241 & -0.016000 & 94241 & 0.015877 & 94241 & 0.000793 \\
\hline & 94242 & -0.002000 & 94242 & 0.001976 & 94242 & 0.000099 \\
\hline \multirow[t]{5}{*}{ Photons } & 94000 & -0.001000 & 94000 & 0.001005 & 94000 & 0.000050 \\
\hline & 94000 & -0.861000 & 94000 & 0.861564 & 94000 & 0.043033 \\
\hline & 94000 & -0.120000 & 94000 & 0.119578 & 94000 & 0.005973 \\
\hline & 94000 & -0.016000 & 94000 & 0.015877 & 94000 & 0.000793 \\
\hline & 94000 & -0.002000 & 94000 & 0.001976 & 94000 & 0.000099 \\
\hline
\end{tabular}




\begin{tabular}{|c|c|c|c|}
\hline \multirow[t]{5}{*}{ CEPXS Form: } & \multirow[t]{5}{*}{ material } & Pu-238 & 0.001000 \\
\hline & & Pu-239 & 0.861000 \\
\hline & & Pu-240 & 0.12000 \\
\hline & & Pu-241 & 0.01600 \\
\hline & & Pu-242 & 0.00200 \\
\hline
\end{tabular}

matname Plutonium, Fuel Grade

density $\quad 19.840000$

\section{Comments and References}

Table B-6 of DOE-STD-3013-2000.

Density $=19.84 \mathrm{~g} / \mathrm{cm} 3$ for alpha plutonium from http://physics.nist.gov/cgi-bin/Star/compos.pl?matno=094 (NIST 1998).

There are six different phases for solid Pu at different temperatures. These have significantly different densities (Söderlind 2001 at http://www.iop.org/EJ/article/0295-5075/55/4/525/6673.html).

Density $=16.63 \mathrm{~g} / \mathrm{cm} 3$ for molten Pu (pgs $4-141$ of Lide 2008).

Weight fractions are adjusted so they sum to unity.

\section{Plutonium, Power Grade}

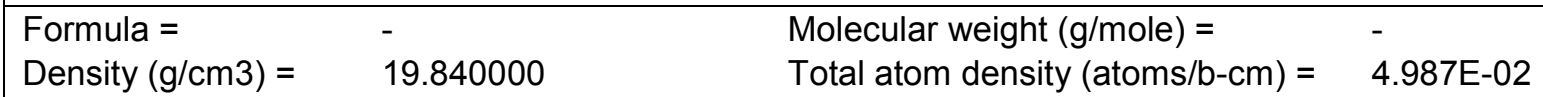

The above density is estimated to be accurate to 4 significant digits. Uncertainties are not addressed.

The following data were calculated from the input weight fractions.

\begin{tabular}{|c|c|c|c|c|c|c|}
\hline Element & Neutron ZA & Photon ZA & $\begin{array}{l}\text { Weight } \\
\text { Fraction }\end{array}$ & $\begin{array}{c}\text { Atom } \\
\text { Fraction }\end{array}$ & $\begin{array}{l}\text { Atom } \\
\text { Density }\end{array}$ & \\
\hline Pu-238 & 94238 & 94000 & 0.009901 & 0.009965 & 0.000497 & \\
\hline Pu-239 & 94239 & 94000 & 0.623762 & 0.625153 & 0.031176 & \\
\hline Pu-240 & 94240 & 94000 & 0.217822 & 0.217396 & 0.010841 & \\
\hline Pu-241 & 94241 & 94000 & 0.118812 & 0.118086 & 0.005889 & \\
\hline Pu-242 & 94242 & 94000 & 0.029703 & 0.029399 & 0.001466 & \\
\hline Total & & & 1.000000 & 1.000000 & 0.049869 & \\
\hline MCNP Form & \multicolumn{2}{|c|}{ Weight Fractions } & \multicolumn{2}{|c|}{ Atom Fractions } & \multicolumn{2}{|c|}{ Atom Densities } \\
\hline \multirow[t]{5}{*}{ Neutrons } & 94238 & -0.009901 & 94238 & 0.009965 & 94238 & 0.000497 \\
\hline & 94239 & -0.623762 & 94239 & 0.625153 & 94239 & 0.031176 \\
\hline & 94240 & -0.217822 & 94240 & 0.217396 & 94240 & 0.010841 \\
\hline & 94241 & -0.118812 & 94241 & 0.118086 & 94241 & 0.005889 \\
\hline & 94242 & -0.029703 & 94242 & 0.029399 & 94242 & 0.001466 \\
\hline \multirow[t]{5}{*}{ Photons } & 94000 & -0.009901 & 94000 & 0.009965 & 94000 & 0.000497 \\
\hline & 94000 & -0.623762 & 94000 & 0.625153 & 94000 & 0.031176 \\
\hline & 94000 & -0.217822 & 94000 & 0.217396 & 94000 & 0.010841 \\
\hline & 94000 & -0.118812 & 94000 & 0.118086 & 94000 & 0.005889 \\
\hline & 94000 & -0.029703 & 94000 & 0.029399 & 94000 & 0.001466 \\
\hline
\end{tabular}




\begin{tabular}{|c|c|c|c|}
\hline \multirow[t]{5}{*}{ CEPXS Form: } & \multirow[t]{5}{*}{ material } & Pu-238 & 0.009901 \\
\hline & & Pu-239 & 0.623762 \\
\hline & & Pu-240 & 0.217822 \\
\hline & & Pu-241 & 0.118812 \\
\hline & & Pu-242 & 0.029703 \\
\hline
\end{tabular}

matname Plutonium, Power Grade density $\quad 19.840000$

\section{Comments and References}

Table B-6 of DOE-STD-3013-2000.

Density $=19.84 \mathrm{~g} / \mathrm{cm} 3$ for alpha plutonium from http://physics.nist.gov/cgi-bin/Star/compos.pl?matno=094 (NIST 1998).

There are six different phases for solid Pu at different temperatures. These have significantly different densities (Söderlind 2001 at http://www.iop.org/EJ/article/0295-5075/55/4/525/6673.html).

Density $=16.63 \mathrm{~g} / \mathrm{cm} 3$ for molten Pu (pgs $4-141$ of Lide 2008).

Weight fractions are adjusted so they sum to unity.

\section{Plutonium, Shefelbine WGPu}

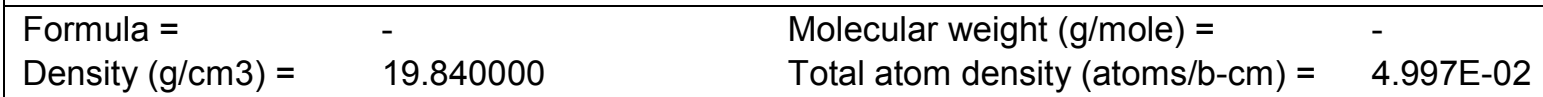

The above density is estimated to be accurate to 4 significant digits. Uncertainties are not addressed.

The following data were calculated from the input weight fractions.

\begin{tabular}{|c|c|c|c|c|c|c|}
\hline Element & Neutron ZA & Photon ZA & $\begin{array}{l}\text { Weight } \\
\text { Fraction }\end{array}$ & $\begin{array}{c}\text { Atom } \\
\text { Fraction }\end{array}$ & $\begin{array}{l}\text { Atom } \\
\text { Density }\end{array}$ & \\
\hline$\overline{\mathrm{Pu}-238}$ & 94238 & 94000 & $\overline{0.000300}$ & $\overline{0.000301}$ & 0.000015 & \\
\hline Pu-239 & 94239 & 94000 & 0.939200 & 0.939451 & 0.046942 & \\
\hline Pu-240 & 94240 & 94000 & 0.057000 & 0.056777 & 0.002837 & \\
\hline Pu-241 & 94241 & 94000 & 0.003000 & 0.002976 & 0.000149 & \\
\hline Pu-242 & 94242 & 94000 & 0.000300 & 0.000296 & 0.000015 & \\
\hline Am-241 & 95241 & 95000 & 0.000200 & 0.000198 & 0.000010 & \\
\hline Total & & & 1.000000 & 1.000000 & 0.049967 & \\
\hline MCNP Form & \multicolumn{2}{|c|}{ Weight Fractions } & \multicolumn{2}{|c|}{ Atom Fractions } & \multicolumn{2}{|c|}{ Atom Densities } \\
\hline \multirow[t]{6}{*}{ Neutrons } & 94238 & -0.000300 & 94238 & 0.000301 & 94238 & 0.000015 \\
\hline & 94239 & -0.939200 & 94239 & 0.939451 & 94239 & 0.046942 \\
\hline & 94240 & -0.057000 & 94240 & 0.056777 & 94240 & 0.002837 \\
\hline & 94241 & -0.003000 & 94241 & 0.002976 & 94241 & 0.000149 \\
\hline & 94242 & -0.000300 & 94242 & 0.000296 & 94242 & 0.000015 \\
\hline & 95241 & -0.000200 & 95241 & 0.000198 & 95241 & 0.000010 \\
\hline \multirow[t]{6}{*}{ Photons } & 94000 & -0.000300 & 94000 & 0.000301 & 94000 & 0.000015 \\
\hline & 94000 & -0.939200 & 94000 & 0.939451 & 94000 & 0.046942 \\
\hline & 94000 & -0.057000 & 94000 & 0.056777 & 94000 & 0.002837 \\
\hline & 94000 & -0.003000 & 94000 & 0.002976 & 94000 & 0.000149 \\
\hline & 94000 & -0.000300 & 94000 & 0.000296 & 94000 & 0.000015 \\
\hline & 95000 & -0.000200 & 95000 & 0.000198 & 95000 & 0.000010 \\
\hline
\end{tabular}




\begin{tabular}{|c|c|c|c|}
\hline \multirow[t]{2}{*}{ CEPXS Form: } & material & $\begin{array}{l}\text { Pu-238 } \\
\text { Pu-239 } \\
\text { Pu-240 } \\
\text { Pu-241 } \\
\text { Pu-242 } \\
\text { Am-241 }\end{array}$ & $\begin{array}{l}0.000300 \\
0.939200 \\
0.057000 \\
0.003000 \\
0.000300 \\
0.000200\end{array}$ \\
\hline & $\begin{array}{l}\text { matname } \\
\text { density }\end{array}$ & $\begin{array}{l}\text { Plutonium, } \\
19.840000\end{array}$ & elbine WGPu \\
\hline \multicolumn{4}{|c|}{$\begin{array}{l}\text { Comments and References } \\
\text { Table } 4 \text { of Preliminary Evaluation of the Characteristics of Defense Transuranic Wastes, SAND78-1850, } \\
\text { Sandia National Laboratory, November } 1978 \text {. } \\
\text { Density }=19.84 \mathrm{~g} / \mathrm{cm} 3 \text { for alpha plutonium from http://physics.nist.gov/cgi-bin/Star/compos.pl?matno=094 } \\
\text { (NIST 1998). } \\
\text { There are six different phases for solid Pu at different temperatures. These have significantly different } \\
\text { densities (Söderlind } 2001 \text { at http://www.iop.org/EJ/article/0295-5075/55/4/525/6673.html). } \\
\text { Density }=16.63 \mathrm{~g} / \mathrm{cm} 3 \text { for molten Pu (pgs } 4-141 \text { of Lide } 2008 \text { ). } \\
\text { Weight fractions are adjusted so they sum to unity. }\end{array}$} \\
\hline
\end{tabular}

\section{Polycarbonate}

\begin{tabular}{llll}
\hline Formula $=$ & $\mathrm{C} 16 \mathrm{H} 14 \mathrm{O} 3$ & Molecular weight $(\mathrm{g} / \mathrm{mole})=$ & 254.28056 \\
Density $(\mathrm{g} / \mathrm{cm} 3)=$ & 1.200000 & Total atom density $($ atoms $/ \mathrm{b}-\mathrm{cm})=$ & $9.378 \mathrm{E}-02$
\end{tabular}

The above density is estimated to be accurate to 3 significant digits. Uncertainties are not addressed.

The following data were calculated from the input weight fractions.

\begin{tabular}{|c|c|c|c|c|c|c|}
\hline Element & Neutron ZA & Photon ZA & $\begin{array}{l}\text { Weight } \\
\text { Fraction }\end{array}$ & $\begin{array}{c}\text { Atom } \\
\text { Fraction }\end{array}$ & $\begin{array}{c}\text { Atom } \\
\text { Density }\end{array}$ & \\
\hline $\mathrm{H}$ & 1001 & 1000 & 0.055491 & 0.424226 & 0.03978 & \\
\hline C & 6000 & 6000 & 0.755751 & 0.484864 & 0.04547 & \\
\hline $\mathrm{O}$ & 8016 & 8000 & 0.188758 & 0.090910 & 0.00852 & \\
\hline Total & & & 1.000000 & 1.000000 & 0.09378 & \\
\hline MCNP Form & \multicolumn{2}{|c|}{ Weight Fractions } & \multicolumn{2}{|c|}{ Atom Fractions } & \multicolumn{2}{|c|}{ Atom Densities } \\
\hline \multirow[t]{3}{*}{ Neutrons } & 1001 & -0.055491 & 1001 & 0.424226 & 1001 & 0.039785 \\
\hline & 6000 & -0.755751 & 6000 & 0.484864 & 6000 & 0.045472 \\
\hline & 8016 & -0.188758 & 8016 & 0.090910 & 8016 & 0.008526 \\
\hline \multirow[t]{3}{*}{ Photons } & 1000 & -0.055491 & 1000 & 0.424226 & 1000 & 0.039785 \\
\hline & 6000 & -0.755751 & 6000 & 0.484864 & 6000 & 0.045472 \\
\hline & 8000 & -0.188758 & 8000 & 0.090910 & 8000 & 0.008526 \\
\hline \multirow[t]{3}{*}{ CEPXS Form: } & material & $\mathrm{H}$ & 0.055491 & & & \\
\hline & & C & 0.755751 & & & \\
\hline & & $\mathrm{O}$ & 0.188758 & & & \\
\hline
\end{tabular}




\begin{tabular}{|c|c|}
\hline $\begin{array}{c}\text { matname } \\
\text { density }\end{array}$ & $\begin{array}{l}\text { Polycarbonate } \\
1.200000\end{array}$ \\
\hline $\begin{array}{l}\text { Comments and References } \\
\text { Also called Makrolon or Lexar } \\
\text { Density and weight fractions } f \\
\text { Formula from pg } 137 \text { of Brew }\end{array}$ & $\begin{array}{l}\text { http://en.wikipedia.org/wiki/Polycarbonate). } \\
\text { m http://physics.nist.gov/cgi-bin/Star/compos.pl?matno=219 (NIST 1998) } \\
\text { (2009). }\end{array}$ \\
\hline
\end{tabular}

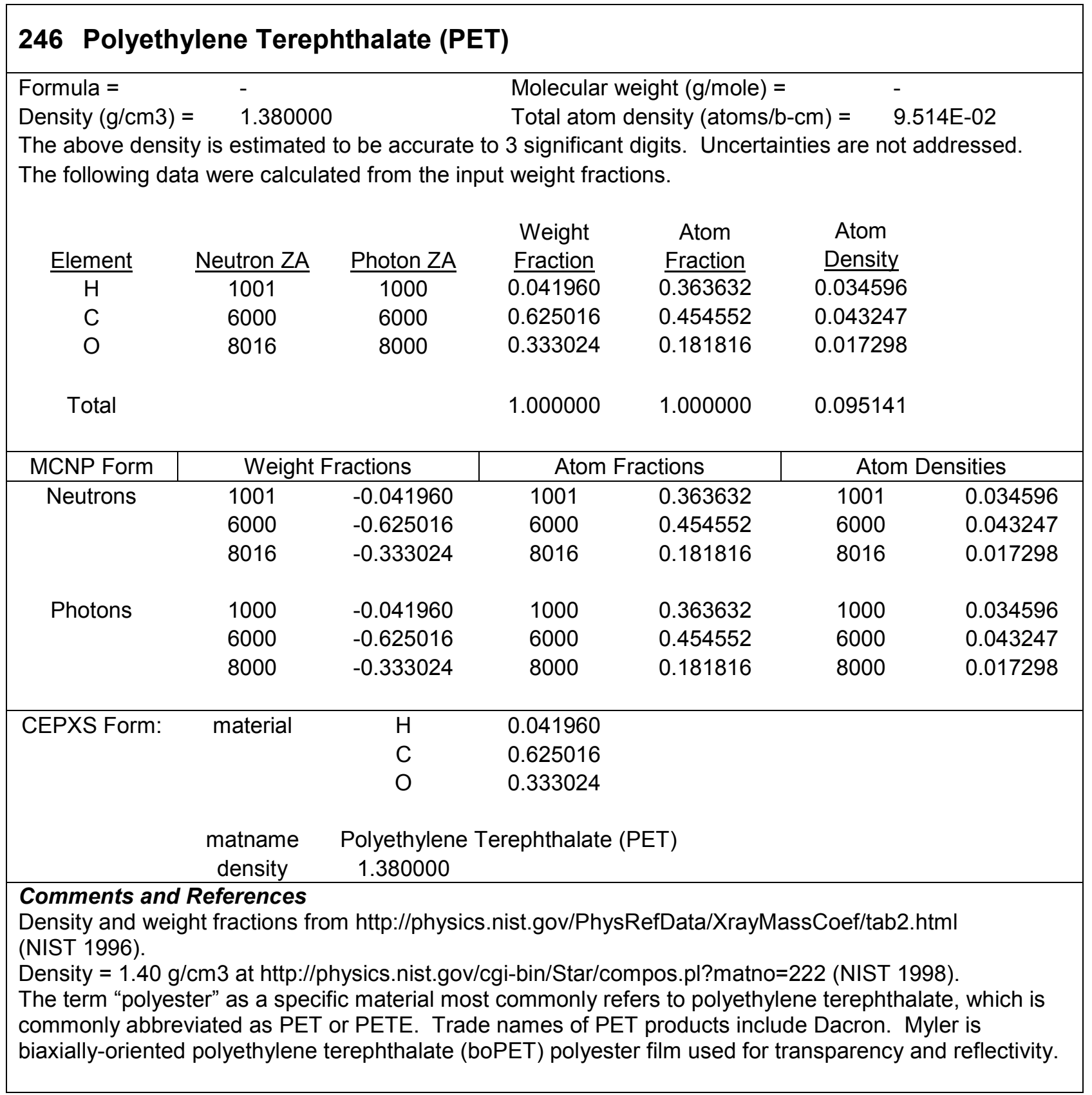




\section{Polyethylene, Borated}

\begin{tabular}{|c|c|c|c|}
\hline$a=$ & $\mathrm{B} 4 \mathrm{C}$ in $\mathrm{C} 2 \mathrm{H} 4$ & Molecular weight $(\mathrm{g} / \mathrm{mole})=$ & \\
\hline Density $(\mathrm{g} / \mathrm{cm} 3)=$ & 1.000000 & Total atom density $($ atoms $/ \mathrm{b}-\mathrm{cm})=$ & 1.193E-01 \\
\hline
\end{tabular}

The above density is estimated to be accurate to 3 significant digits. Uncertainties are not addressed.

The following data were calculated from the input weight fractions.

\begin{tabular}{|c|c|c|c|c|c|c|}
\hline Element & Neutron ZA & Photon ZA & $\begin{array}{l}\text { Weight } \\
\text { Fraction }\end{array}$ & $\begin{array}{c}\text { Atom } \\
\text { Fraction }\end{array}$ & $\begin{array}{l}\text { Atom } \\
\text { Density }\end{array}$ & \\
\hline $\mathrm{H}$ & 1001 & 1000 & $\overline{0.125355}$ & $\overline{0.627759}$ & 0.074896 & \\
\hline $\mathrm{B}$ & - & 5000 & 0.100000 & 0.046690 & 0.005570 & \\
\hline $\mathrm{C}$ & 6000 & 6000 & 0.774645 & 0.325552 & 0.038841 & \\
\hline Total & & & 1.000000 & 1.000000 & 0.119307 & \\
\hline MCNP Form & \multicolumn{2}{|c|}{ Weight Fractions } & \multicolumn{2}{|c|}{ Atom Fractions } & \multicolumn{2}{|c|}{ Atom Densities } \\
\hline \multirow[t]{3}{*}{ Neutrons } & 1001 & -0.125355 & 1001 & 0.627759 & 1001 & 0.074896 \\
\hline & - & -0.100000 & - & 0.046690 & - & 0.005570 \\
\hline & 6000 & -0.774645 & 6000 & 0.325552 & 6000 & 0.038841 \\
\hline \multirow[t]{3}{*}{ Photons } & 1000 & -0.125355 & 1000 & 0.627759 & 1000 & 0.074896 \\
\hline & 5000 & -0.100000 & 5000 & 0.046690 & 5000 & 0.005570 \\
\hline & 6000 & -0.774645 & 6000 & 0.325552 & 6000 & 0.038841 \\
\hline \multirow[t]{4}{*}{ CEPXS Form: } & material & $\mathrm{H}$ & 0.125355 & & & \\
\hline & & B & 0.100000 & & & \\
\hline & & C & 0.774645 & & & \\
\hline & $\begin{array}{l}\text { matname } \\
\text { density }\end{array}$ & \multicolumn{2}{|c|}{$\begin{array}{l}\text { Polyethylene, Borated } \\
1.000000\end{array}$} & & & \\
\hline
\end{tabular}

\section{Polyethylene, Non-borated}

\begin{tabular}{llll}
\hline Formula $=$ & $\mathrm{C} 2 \mathrm{H} 4$ & Molecular weight $(\mathrm{g} / \mathrm{mole})=$ & 28.05316 \\
Density $(\mathrm{g} / \mathrm{cm} 3)=$ & 0.930000 & Total atom density $($ atoms $/ \mathrm{b}-\mathrm{cm})=$ & $1.198 \mathrm{E}-01$
\end{tabular}

The above density is estimated to be accurate to 2 significant digits. Uncertainties are not addressed.

The following data were calculated from the input weight fractions.

\begin{tabular}{|c|c|c|c|c|c|}
\hline Element & Neutron ZA & Photon ZA & $\begin{array}{l}\text { Weight } \\
\text { Fraction }\end{array}$ & $\begin{array}{c}\text { Atom } \\
\text { Fraction }\end{array}$ & $\begin{array}{l}\text { Atom } \\
\text { Density }\end{array}$ \\
\hline $\mathrm{H}$ & 1001 & 1000 & $\overline{0.143716}$ & $\overline{0.666662}$ & 0.079855 \\
\hline C & 6000 & 6000 & 0.856284 & 0.333338 & 0.039929 \\
\hline Total & & & 1.000000 & 1.000000 & 0.119784 \\
\hline
\end{tabular}


PIET-43741-TM-963

PNNL-15870 Rev. 1

\begin{tabular}{|c|c|c|c|c|c|c|}
\hline MCNP Form & \multicolumn{2}{|c|}{ Weight Fractions } & \multicolumn{2}{|c|}{ Atom Fractions } & \multicolumn{2}{|c|}{ Atom Densities } \\
\hline \multirow[t]{2}{*}{ Neutrons } & 1001 & -0.143716 & 1001 & 0.666662 & 1001 & 0.079855 \\
\hline & 6000 & -0.856284 & 6000 & 0.333338 & 6000 & 0.039929 \\
\hline \multirow[t]{2}{*}{ Photons } & 1000 & -0.143716 & 1000 & 0.666662 & 1000 & 0.079855 \\
\hline & 6000 & -0.856284 & 6000 & 0.333338 & 6000 & 0.039929 \\
\hline \multirow[t]{3}{*}{ CEPXS Form: } & material & $\mathrm{H}$ & 0.143716 & & & \\
\hline & & C & 0.856284 & & & \\
\hline & $\begin{array}{c}\text { matname } \\
\text { density }\end{array}$ & $\begin{array}{c}\text { Polyethylene } \\
0.930000\end{array}$ & n-borated & & & \\
\hline \multicolumn{7}{|c|}{$\begin{array}{l}\text { Comments and References } \\
\text { Density }=0.93 \mathrm{~g} / \mathrm{cm} 3 \text { and weight fractions from } \\
\text { http://physics.nist.gov/PhysRefData/XrayMassCoef/tab2.html (NIST 1996). } \\
\text { High density polyethylene (HDPE) is } 0.944 \text { to } 0.965 \mathrm{~g} / \mathrm{cm} 3 \\
\text { (http://www.bpf.co.uk/Plastipedia/Polymers/HDPE.aspx). Low density polyethylene (LDPE) is } 0.917 \text { to } \\
0.930 \mathrm{~g} / \mathrm{cm} 3 \text { (http://www.bpf.co.uk/Plastipedia/Polymers/LDPE.aspx). Automation Creations (2010) at } \\
\text { http://www.matweb.com/search/QuickText.aspx has molded HDPE }=0.918-1.05 \mathrm{~g} / \mathrm{cm} 3 \text { and MDPE = } \\
0.926-0.95 . \text { Density }=0.94 \mathrm{~g} / \mathrm{cm} 3 \text { at http://physics.nist.gov/cgi-bin/Star/compos.pl?matno=221 } \\
\text { (NIST 1998). } \\
\text { Density }=0.92 \mathrm{~g} / \mathrm{cm} 3 \text { on pg } 138 \text { of Brewer (2009). The range of density values is discussed further at } \\
\text { http://en.wikipedia.org/wiki/Polyethylene. }\end{array}$} \\
\hline
\end{tabular}

\section{Polyisocyanurate (PIR)}

\begin{tabular}{llll}
\hline Formula $=$ & $\mathrm{C} 15 \mathrm{H} 10 \mathrm{~N} 2 \mathrm{O} 2$ & Molecular weight $(\mathrm{g} / \mathrm{mole})=$ & 250.2521 \\
Density $(\mathrm{g} / \mathrm{cm} 3)=$ & 0.048200 & Total atom density $($ atoms $/ \mathrm{b}-\mathrm{cm})=$ & $3.364 \mathrm{E}-03$
\end{tabular}

The above density is estimated to be accurate to 3 significant digits. Uncertainties are not addressed. The following data was calculated from the input formula.

\begin{tabular}{|c|c|c|c|c|c|c|}
\hline Element & Neutron ZA & Photon ZA & $\begin{array}{l}\text { Weight } \\
\text { Fraction }\end{array}$ & $\begin{array}{c}\text { Atom } \\
\text { Fraction }\end{array}$ & $\begin{array}{l}\text { Atom } \\
\text { Density }\end{array}$ & \\
\hline $\mathrm{H}$ & 1001 & 1000 & 0.040277 & 0.344828 & 0.00116 & \\
\hline $\mathrm{C}$ & 6000 & 6000 & 0.719916 & 0.517241 & 0.00174 & \\
\hline $\mathrm{N}$ & 7014 & 7000 & 0.111941 & 0.068966 & 0.00023 & \\
\hline $\mathrm{O}$ & 8016 & 8000 & 0.127866 & 0.068966 & 0.00023 & \\
\hline Total & & & 1.000000 & 1.000000 & 0.00336 & \\
\hline MCNP Form & \multicolumn{2}{|c|}{ Weight Fractions } & \multicolumn{2}{|c|}{ Atom Fractions } & \multicolumn{2}{|c|}{ Atom Densities } \\
\hline \multirow[t]{4}{*}{ Neutrons } & 1001 & -0.040277 & 1001 & 0.344828 & 1001 & 0.001160 \\
\hline & 6000 & -0.719916 & 6000 & 0.517241 & 6000 & 0.001740 \\
\hline & 7014 & -0.111941 & 7014 & 0.068966 & 7014 & 0.000232 \\
\hline & 8016 & -0.127866 & 8016 & 0.068966 & 8016 & 0.000232 \\
\hline \multirow[t]{2}{*}{ Photons } & 1000 & -0.040277 & 1000 & 0.344828 & 1000 & 0.001160 \\
\hline & 6000 & -0.719916 & 6000 & 0.517241 & 6000 & 0.001740 \\
\hline
\end{tabular}


PIET-43741-TM-963

PNNL-15870 Rev. 1

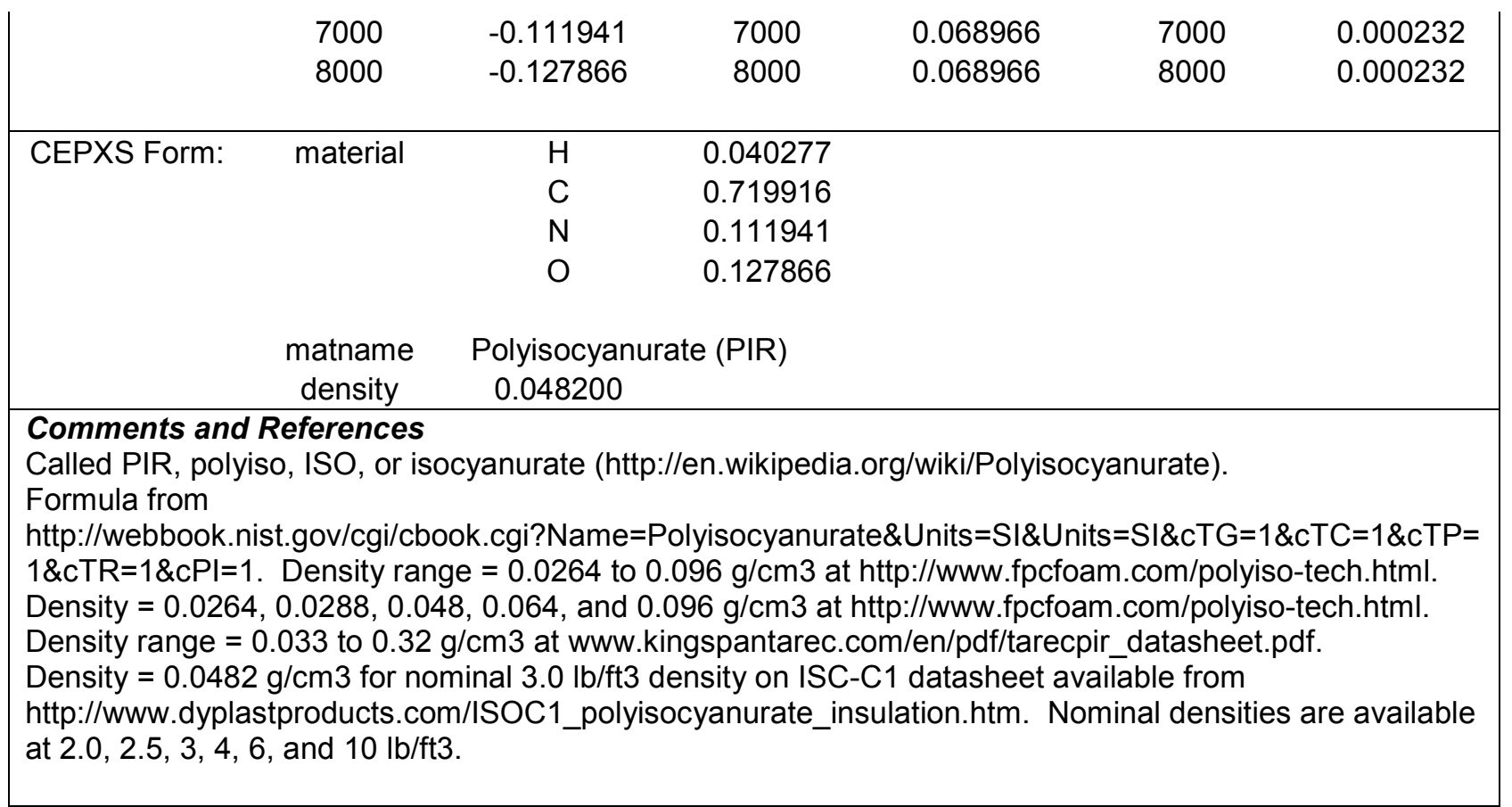

\section{Polypropylene (PP)}

\begin{tabular}{llll}
\hline Formula $=$ & $\mathrm{C} 3 \mathrm{H} 6$ & Molecular weight $(\mathrm{g} / \mathrm{mole})=$ & 42.07974 \\
Density $(\mathrm{g} / \mathrm{cm} 3)=$ & 0.900000 & Total atom density $($ atoms $/ \mathrm{b}-\mathrm{cm})=$ & $1.159 \mathrm{E}-01$
\end{tabular}

The above density is estimated to be accurate to 2 significant digits. Uncertainties are not addressed.

The following data were calculated from the input weight fractions.

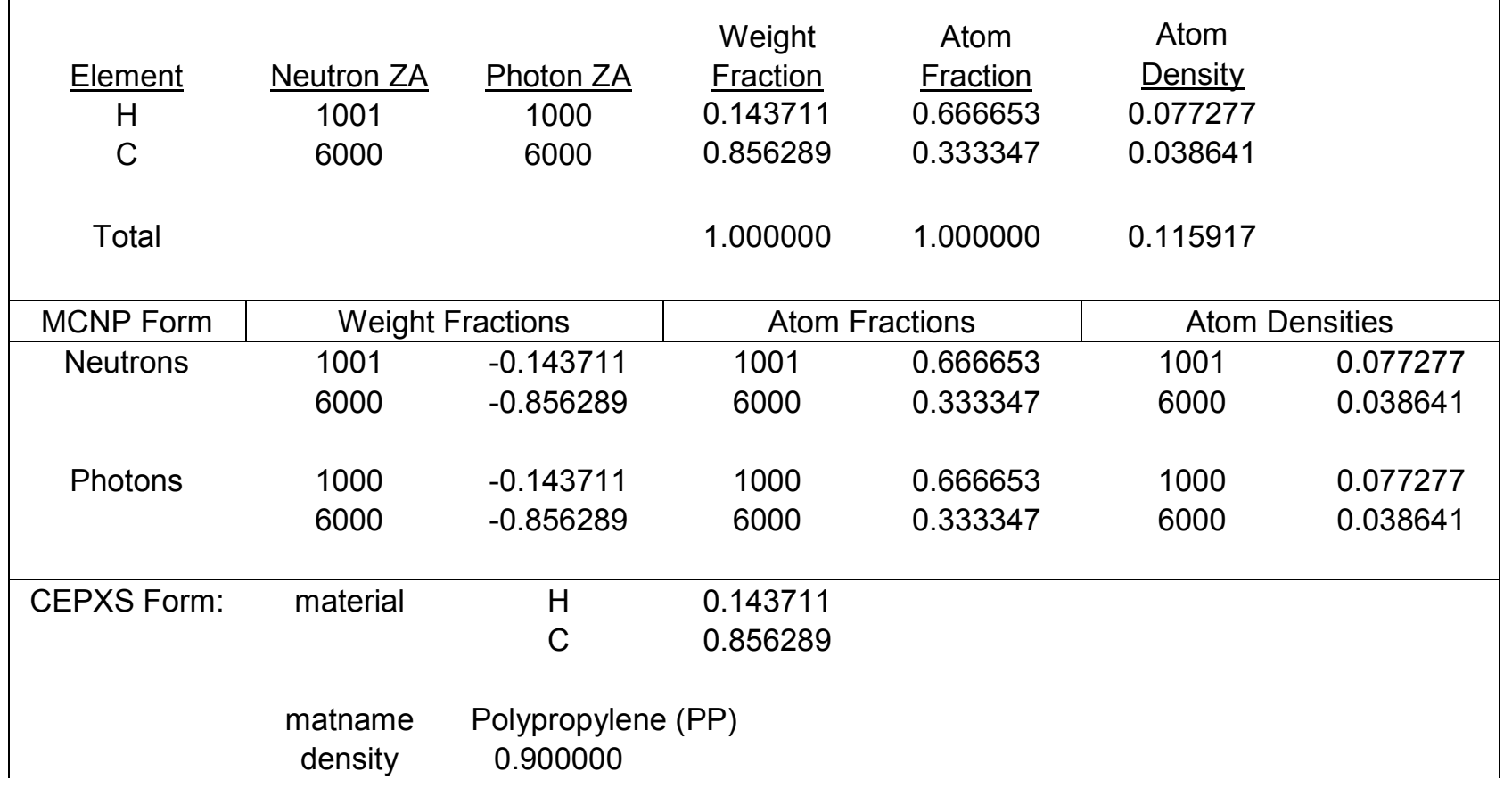


Comments and References

Density and weight fractions from http://physics.nist.gov/cgi-bin/Star/compos.pl?matno=225 (NIST 1998).

Formula $=\mathrm{C} 3 \mathrm{H} 6$ from Brandrup et al. (2005).

\section{Polystyrene (PS)}

\begin{tabular}{lllr}
\hline Formula $=$ & $\mathrm{C} 8 \mathrm{H} 8$ & Molecular weight $(\mathrm{g} / \mathrm{mole})=$ & 104.14912 \\
Density $(\mathrm{g} / \mathrm{cm} 3)=$ & 1.060000 & Total atom density $($ atoms $/ \mathrm{b}-\mathrm{cm})=$ & $9.807 \mathrm{E}-02$ \\
The above density is estimated to be accurate to 3 significant digits. Uncertainties are not addressed. & \\
The following data were calculated from the input weight fractions. &
\end{tabular}

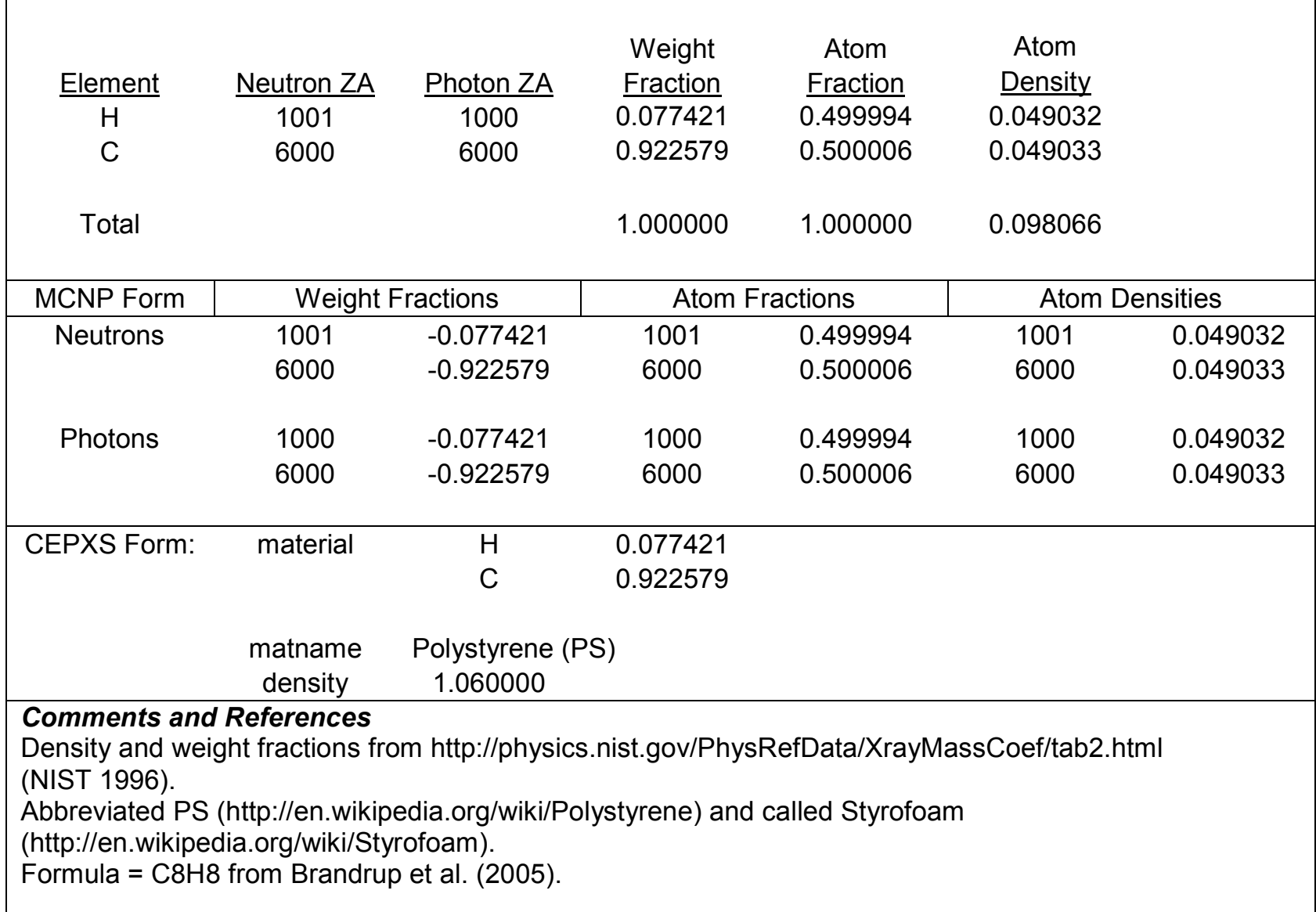

\section{Polytetrafluoroethylene (PTFE)}

\begin{tabular}{llll}
\hline Formula $=$ & $\mathrm{C} 2 \mathrm{~F} 4$ & Molecular weight $(\mathrm{g} / \mathrm{mole})=$ & 100.0150128 \\
Density $(\mathrm{g} / \mathrm{cm} 3)=$ & 2.250000 & Total atom density $($ atoms $/ \mathrm{b}-\mathrm{cm})=$ & $8.129 \mathrm{E}-02$ \\
The above density is estimated to be accurate to 3 significant digits. Uncertainties are not addressed. & \\
The following data were calculated from the input weight fractions.
\end{tabular}




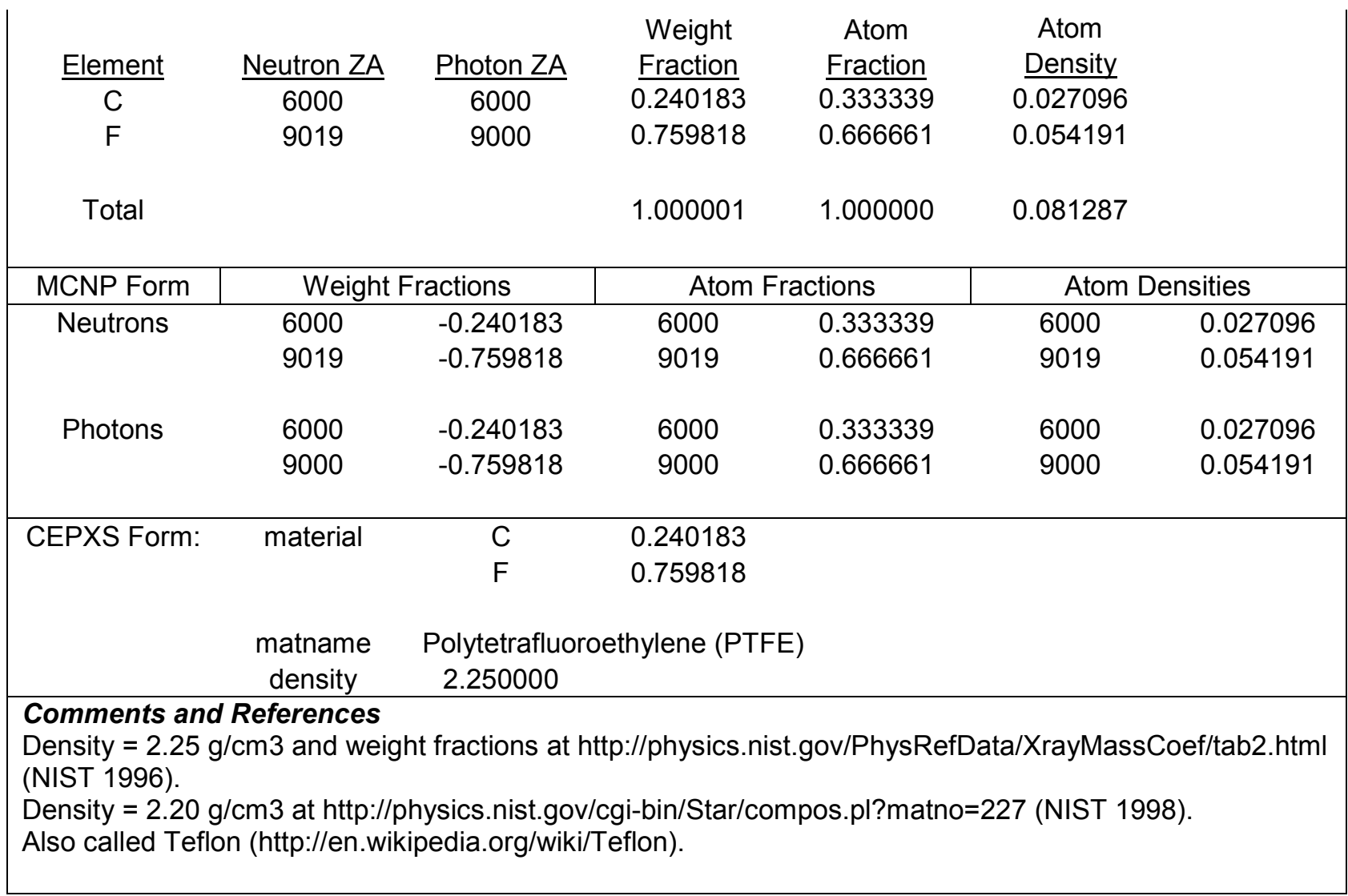

\section{Polyurethane Foam (PUR)}

\begin{tabular}{llll}
\hline Formula $=$ & - & Molecular weight $(\mathrm{g} / \mathrm{mole})=$ \\
Density $(\mathrm{g} / \mathrm{cm} 3)=$ & 0.021000 & Total atom density $($ atoms $/ \mathrm{b}-\mathrm{cm})=$ & - \\
\hline & $1.429 \mathrm{E}-03$
\end{tabular}

The above density is estimated to be accurate to 2 significant digits. Uncertainties are not addressed.

The following data were calculated from the input weight fractions.

\begin{tabular}{|c|c|c|c|c|c|c|}
\hline Element & Neutron ZA & Photon ZA & $\begin{array}{l}\text { Weight } \\
\text { Fraction }\end{array}$ & $\begin{array}{c}\text { Atom } \\
\text { Fraction }\end{array}$ & $\begin{array}{l}\text { Atom } \\
\text { Density }\end{array}$ & \\
\hline $\mathrm{H}$ & 1001 & 1000 & $\overline{0.041000}$ & $\overline{0.360023}$ & 0.000514 & \\
\hline C & 6000 & 6000 & 0.544000 & 0.400878 & 0.000573 & \\
\hline $\mathrm{N}$ & 7014 & 7000 & 0.121000 & 0.076459 & 0.000109 & \\
\hline $\mathrm{O}$ & 8016 & 8000 & 0.294000 & 0.162639 & 0.000232 & \\
\hline Total & & & 1.000000 & 1.000000 & 0.001429 & \\
\hline MCNP Form & \multicolumn{2}{|c|}{ Weight Fractions } & \multicolumn{2}{|c|}{ Atom Fractions } & \multicolumn{2}{|c|}{ Atom Densities } \\
\hline \multirow[t]{4}{*}{ Neutrons } & 1001 & -0.041000 & 1001 & 0.360023 & 1001 & 0.000514 \\
\hline & 6000 & -0.544000 & 6000 & 0.400878 & 6000 & 0.000573 \\
\hline & 7014 & -0.121000 & 7014 & 0.076459 & 7014 & 0.000109 \\
\hline & 8016 & -0.294000 & 8016 & 0.162639 & 8016 & 0.000232 \\
\hline
\end{tabular}


PIET-43741-TM-963

PNNL-15870 Rev. 1

\begin{tabular}{|c|c|c|c|c|c|c|}
\hline Photons & $\begin{array}{l}1000 \\
6000 \\
7000 \\
8000\end{array}$ & $\begin{array}{l}-0.041000 \\
-0.544000 \\
-0.121000 \\
-0.294000\end{array}$ & $\begin{array}{l}1000 \\
6000 \\
7000 \\
8000\end{array}$ & $\begin{array}{l}0.360023 \\
0.400878 \\
0.076459 \\
0.162639\end{array}$ & $\begin{array}{l}1000 \\
6000 \\
7000 \\
8000\end{array}$ & $\begin{array}{l}0.000514 \\
0.000573 \\
0.000109 \\
0.000232\end{array}$ \\
\hline CEPXS Form: & $\begin{array}{c}\text { matname } \\
\text { density }\end{array}$ & $\begin{array}{c}\mathrm{H} \\
\mathrm{C} \\
\mathrm{N} \\
\mathrm{O} \\
\text { Polyurethane } \\
0.021000\end{array}$ & $\begin{array}{l}0.041000 \\
0.544000 \\
0.121000 \\
0.294000\end{array}$ & & & \\
\hline \multicolumn{7}{|c|}{ 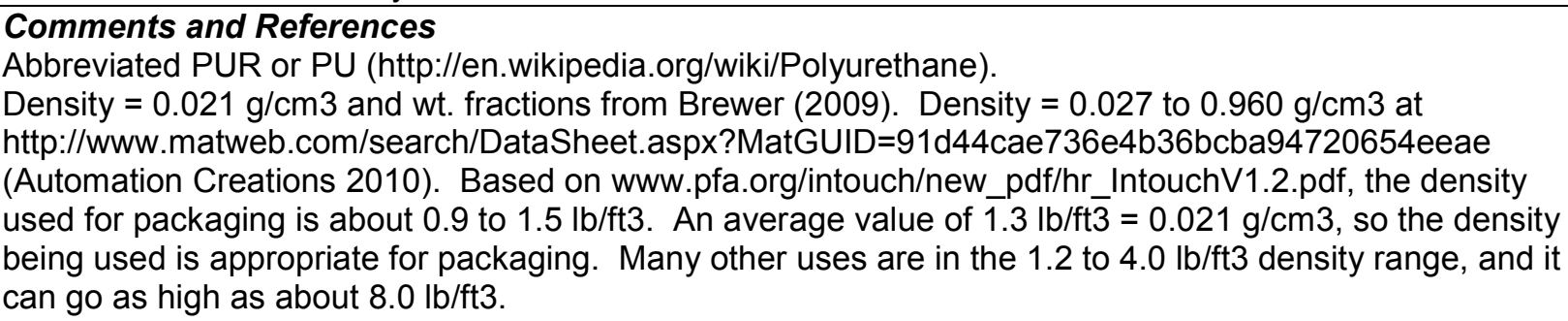 } \\
\hline
\end{tabular}

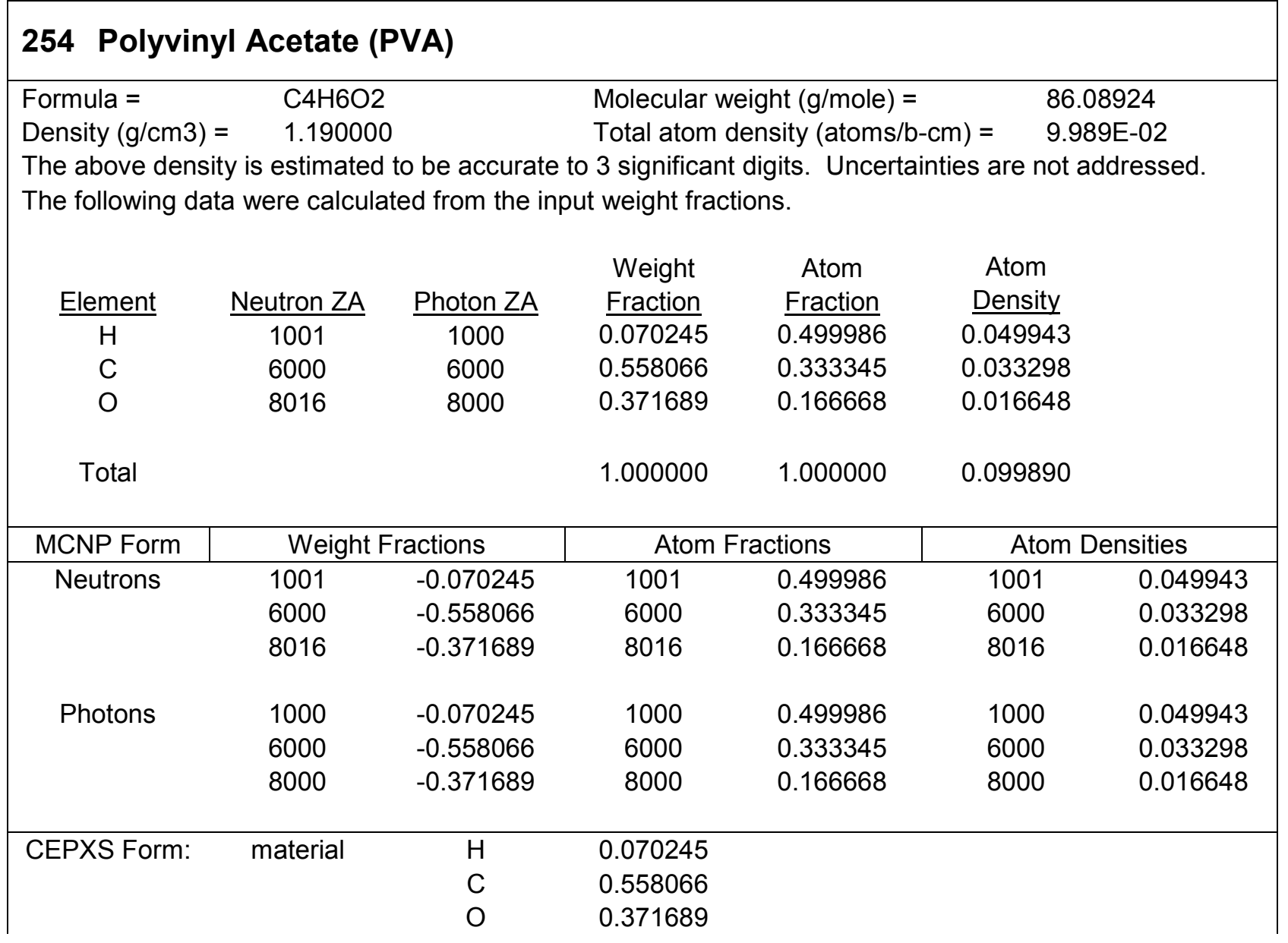


matname Polyvinyl Acetate (PVA)

density $\quad 1.190000$

Comments and References

Density and weight fractions from http://physics.nist.gov/cgi-bin/Star/compos.pl?matno=229.

\section{Polyvinyl Chloride (PVC)}

\begin{tabular}{llll}
\hline Formula $=$ & $\mathrm{C} 2 \mathrm{H} 3 \mathrm{Cl}$ & Molecular weight $(\mathrm{g} / \mathrm{mole})=$ & 62.49822 \\
Density $(\mathrm{g} / \mathrm{cm} 3)=$ & 1.406000 & Total atom density $($ atoms $/ \mathrm{b}-\mathrm{cm})=$ & $8.129 \mathrm{E}-02$
\end{tabular}

The above density is estimated to be accurate to 3 significant digits. Uncertainties are not addressed. The following data were calculated from the input weight fractions.

\begin{tabular}{ccccccc} 
Element & Neutron ZA & Photon ZA & & $\begin{array}{c}\text { Weight } \\
\text { Fraction }\end{array}$ & $\begin{array}{c}\text { Atom } \\
\text { Fraction }\end{array}$ & $\begin{array}{c}\text { Atom } \\
\text { Density }\end{array}$ \\
\cline { 3 - 3 } & 1001 & & 1000 & 0.048382 & 0.499995 & 0.040643 \\
$\mathrm{C}$ & 6000 & 6000 & 0.384361 & 0.333340 & 0.027096 \\
$\mathrm{Cl}$ & 17000 & 17000 & 0.567257 & 0.166665 & 0.013548 \\
Total & & & & & & \\
& & & & 1.000000 & 1.000000 & 0.081287
\end{tabular}

\begin{tabular}{|ccccccc|}
\hline MCNP Form & \multicolumn{2}{c|}{ Weight Fractions } & \multicolumn{2}{c|}{ Atom Fractions } & \multicolumn{2}{c|}{ Atom Densities } \\
\hline Neutrons & 1001 & -0.048382 & 1001 & 0.499995 & 1001 & 0.040643 \\
& 6000 & -0.384361 & 6000 & 0.333340 & 6000 & 0.027096 \\
& 17000 & -0.567257 & 17000 & 0.166665 & 17000 & 0.013548 \\
Photons & & & & & & \\
& 1000 & -0.048382 & 1000 & 0.499995 & 1000 & 0.040643 \\
& 6000 & -0.384361 & 6000 & 0.333340 & 6000 & 0.027096 \\
& 17000 & -0.567257 & 17000 & 0.166665 & 17000 & 0.013548
\end{tabular}

$\begin{array}{llll}\text { CEPXS Form: } & \text { material } & \mathrm{H} & 0.048382 \\ & & \mathrm{C} & 0.384361 \\ & \mathrm{Cl} & 0.567257\end{array}$

matname Polyvinyl Chloride (PVC)

density $\quad 1.406000$

\section{Comments and References}

Density $=1.406 \mathrm{~g} / \mathrm{cm} 3$ and weight fractions from

http://physics.nist.gov/PhysRefData/XrayMassCoef/tab2.html (NIST 1996); $1.38 \mathrm{~g} / \mathrm{cm} 3$ at

http://www.bpf.co.uk/Plastipedia/Polymers/PVC.aspx; 1.35 at

http://www.matweb.com/search/DataSheet.aspx?MatGUID=0fc1831d51e447879a5ae9ee7f3dc0bb\&

ckck=1 (Automation Creations 2010); and $1.30 \mathrm{~g} / \mathrm{cm} 3$ at http://physics.nist.gov/cgi-

bin/Star/compos.pl?matno=232 (NIST 1998).

Density $=1.39 \mathrm{~g} / \mathrm{cm} 3$ in http://en.wikipedia.org/wiki/Polyvinyl_chloride. 


\section{Polyvinyl Toluene (PVT)}

\begin{tabular}{llll}
\hline Formula $=$ & - & Molecular weight $(\mathrm{g} / \mathrm{mole})=$ \\
Density $(\mathrm{g} / \mathrm{cm} 3)=$ & 1.032000 & Total atom density $($ atoms $/ \mathrm{b}-\mathrm{cm})=$ & - \\
\hline & $9.976 \mathrm{E}-02$
\end{tabular}

The above density is estimated to be accurate to 3 significant digits. Uncertainties are not addressed.

The following data were calculated from the input weight fractions.

\begin{tabular}{|c|c|c|c|c|c|c|}
\hline $\begin{array}{c}\text { Element } \\
\mathrm{H} \\
\mathrm{C}\end{array}$ & $\begin{array}{c}\text { Neutron ZA } \\
1001 \\
6000\end{array}$ & 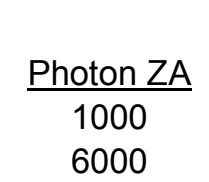 & $\begin{array}{c}\text { Weight } \\
\text { Fraction } \\
0.085000 \\
0.915000\end{array}$ & $\begin{array}{c}\begin{array}{c}\text { Atom } \\
\text { Fraction }\end{array} \\
0.525382 \\
0.474618\end{array}$ & $\begin{array}{c}\text { Atom } \\
\text { Density } \\
0.052410 \\
0.047346\end{array}$ & \\
\hline Total & & & 1.000000 & 1.000000 & 0.099756 & \\
\hline MCNP Form & \multicolumn{2}{|c|}{ Weight Fractions } & \multicolumn{2}{|c|}{ Atom Fractions } & \multicolumn{2}{|c|}{ Atom Densities } \\
\hline Neutrons & $\begin{array}{l}1001 \\
6000\end{array}$ & $\begin{array}{l}-0.085000 \\
-0.915000\end{array}$ & $\begin{array}{l}1001 \\
6000\end{array}$ & $\begin{array}{l}0.525382 \\
0.474618\end{array}$ & $\begin{array}{l}1001 \\
6000\end{array}$ & $\begin{array}{l}0.052410 \\
0.047346\end{array}$ \\
\hline Photons & $\begin{array}{l}1000 \\
6000\end{array}$ & $\begin{array}{l}-0.085000 \\
-0.915000\end{array}$ & $\begin{array}{l}1000 \\
6000\end{array}$ & $\begin{array}{l}0.525382 \\
0.474618\end{array}$ & $\begin{array}{l}1000 \\
6000\end{array}$ & $\begin{array}{l}0.052410 \\
0.047346\end{array}$ \\
\hline CEPXS Form: & $\begin{array}{c}\text { matname } \\
\text { density }\end{array}$ & $\begin{array}{c}\mathrm{H} \\
\mathrm{C} \\
\text { Polyvinyl Tol } \\
1.032000\end{array}$ & $\begin{array}{l}0.085000 \\
0.915000 \\
\text { e (PVT) }\end{array}$ & & & \\
\hline $\begin{array}{l}\text { Comments an } \\
\text { Plastic scintilla } \\
\text { form a solid. S } \\
\text { widely used pla } \\
\text { (http://en.wikip } \\
\text { Polyvinyl Tolue } \\
\text { For polyvinyl to } \\
\text { http://physics.n } \\
\text { Vinyltoluene." }\end{array}$ & $\begin{array}{l}\text { References } \\
\text { s are solution } \\
\text { ne of the com } \\
\text { ic solvents an } \\
\text { ia.org/wiki/Pl } \\
\text { e, or polyvinyl } \\
\text { ene (PVT), de } \\
\text { t.gov/PhysRe }\end{array}$ & $\begin{array}{l}\text { of organic sc } \\
\text { on solutes ar } \\
\text { polyvinyl tolu } \\
\text { tic_scintillator } \\
\text { luene, is abbr } \\
\text { sity }=1.032 \mathrm{~g} \\
\text { lata/XrayMas }\end{array}$ & $\begin{array}{l}\text { Ilators in a s } \\
\text {-Terphenyl, } \\
\text { and polyst } \\
\text { out many oth } \\
\text { iated PVT ( } \\
\text { n3 and weig } \\
\text { jef/tab2.htm }\end{array}$ & $\begin{array}{l}\text { ent which is } \\
D, b-P B D, \\
\text { ee } \\
\text { materials c } \\
\text { //en.wikipe } \\
\text { ractions fro } \\
\text { IIST 1996) }\end{array}$ & $\begin{array}{l}\text { sequently } \\
\text { POPOP. } \\
\text { so be used } \\
\text { g/wiki/Pol. } \\
\text { lastic Scin }\end{array}$ & $\begin{array}{l}\text { nerized to } \\
\text { most } \\
\text { or, }\end{array}$ \\
\hline
\end{tabular}

\section{Polyvinylidene Chloride (PVDC)}

\begin{tabular}{llll}
\hline Formula $=$ & $\mathrm{C} 2 \mathrm{H} 2 \mathrm{Cl} 2$ & Molecular weight $(\mathrm{g} / \mathrm{mole})=$ & 96.94328 \\
Density $(\mathrm{g} / \mathrm{cm} 3)=$ & 1.700000 & Total atom density $($ atoms $/ \mathrm{b}-\mathrm{cm})=$ & $6.336 \mathrm{E}-02$
\end{tabular}

The above density is estimated to be accurate to 3 significant digits. Uncertainties are not addressed.

The following data were calculated from the input weight fractions.

\begin{tabular}{|c|c|c|c|c|c|}
\hline Element & Neutron ZA & Photon ZA & $\begin{array}{l}\text { Weight } \\
\text { Fraction }\end{array}$ & $\begin{array}{l}\text { Atom } \\
\text { Fraction }\end{array}$ & $\begin{array}{l}\text { Atom } \\
\text { Density }\end{array}$ \\
\hline$\overline{\mathrm{H}}$ & 1001 & 1000 & $\overline{0.020793}$ & $\overline{0.333317}$ & 0.021119 \\
\hline C & 6000 & 6000 & 0.247793 & 0.333346 & 0.021121 \\
\hline $\mathrm{Cl}$ & 17000 & 17000 & 0.731413 & 0.333337 & 0.021121 \\
\hline
\end{tabular}




\begin{tabular}{|c|c|c|c|c|c|c|}
\hline \multicolumn{3}{|l|}{ Total } & 0.999999 & 1.000000 & \multicolumn{2}{|c|}{0.063361} \\
\hline MCNP Form & \multicolumn{2}{|c|}{ Weight Fractions } & \multicolumn{2}{|c|}{ Atom Fractions } & \multicolumn{2}{|c|}{ Atom Densities } \\
\hline \multirow[t]{3}{*}{ Neutrons } & 1001 & -0.020793 & 1001 & 0.333317 & 1001 & 0.021119 \\
\hline & 6000 & -0.247793 & 6000 & 0.333346 & 6000 & 0.021121 \\
\hline & 17000 & -0.731413 & 17000 & 0.333337 & 17000 & 0.021121 \\
\hline \multirow[t]{3}{*}{ Photons } & 1000 & -0.020793 & 1000 & 0.333317 & 1000 & 0.021119 \\
\hline & 6000 & -0.247793 & 6000 & 0.333346 & 6000 & 0.021121 \\
\hline & 17000 & -0.731413 & 17000 & 0.333337 & 17000 & 0.021121 \\
\hline \multirow[t]{4}{*}{ CEPXS Form: } & material & $\mathrm{H}$ & 0.020793 & & & \\
\hline & & C & 0.247793 & & & \\
\hline & & $\mathrm{Cl}$ & 0.731413 & & & \\
\hline & $\begin{array}{c}\text { matname } \\
\text { density }\end{array}$ & $\begin{array}{c}\text { Polyvinylide } \\
1.700000\end{array}$ & Shloride (P) & & & \\
\hline \multicolumn{7}{|c|}{$\begin{array}{l}\text { Comments and References } \\
\text { Abbreviated as PVDC and also called "saran" (http://en.wikipedia.org/wiki/Plastics). } \\
\text { Density }=1.70 \mathrm{~g} / \mathrm{cm} 3 \text { and weight fractions from http://physics.nist.gov/cgi-bin/Star/compos.pl?matno=233 } \\
\text { (NIST 1998). }\end{array}$} \\
\hline
\end{tabular}

\section{Potassium Aluminum Silicate}

$\begin{array}{llll}\text { Formula }= & \text { KAISi3O8 } & \text { Molecular weight }(\mathrm{g} / \mathrm{mole})= & 278.331538 \\ \text { Density }(\mathrm{g} / \mathrm{cm} 3)= & 1.100000 & \text { Total atom density }(\text { atoms } / \mathrm{b}-\mathrm{cm})= & 3.094 \mathrm{E}-02\end{array}$

The above density is estimated to be accurate to 2 significant digits. Uncertainties are not addressed. The following data was calculated from the input formula.

\begin{tabular}{|c|c|c|c|c|c|c|}
\hline Element & Neutron ZA & Photon ZA & $\begin{array}{l}\text { Weight } \\
\text { Fraction }\end{array}$ & $\begin{array}{c}\text { Atom } \\
\text { Fraction }\end{array}$ & $\begin{array}{c}\text { Atom } \\
\text { Density }\end{array}$ & \\
\hline $\mathrm{O}$ & 8016 & 8000 & $\overline{0.459866}$ & $\overline{0.615385}$ & 0.01904 & \\
\hline $\mathrm{Al}$ & 13027 & 13000 & 0.096940 & 0.076923 & 0.00238 & \\
\hline $\mathrm{Si}$ & 14000 & 14000 & 0.302720 & 0.230769 & 0.00714 & \\
\hline $\mathrm{K}$ & 19000 & 19000 & 0.140474 & 0.076923 & 0.00238 & \\
\hline Total & & & 1.000000 & 1.000000 & 0.03094 & \\
\hline MCNP Form & \multicolumn{2}{|c|}{ Weight Fractions } & \multicolumn{2}{|c|}{ Atom Fractions } & \multicolumn{2}{|c|}{ Atom Densities } \\
\hline \multirow[t]{4}{*}{ Neutrons } & 8016 & -0.459866 & 8016 & 0.615385 & 8016 & 0.019040 \\
\hline & 13027 & -0.096940 & 13027 & 0.076923 & 13027 & 0.002380 \\
\hline & 14000 & -0.302720 & 14000 & 0.230769 & 14000 & 0.007140 \\
\hline & 19000 & -0.140474 & 19000 & 0.076923 & 19000 & 0.002380 \\
\hline \multirow[t]{2}{*}{ Photons } & 8000 & -0.459866 & 8000 & 0.615385 & 8000 & 0.019040 \\
\hline & 13000 & -0.096940 & 13000 & 0.076923 & 13000 & 0.002380 \\
\hline
\end{tabular}


PIET-43741-TM-963

PNNL-15870 Rev. 1

\begin{tabular}{|c|c|c|c|c|c|c|}
\hline & $\begin{array}{l}14000 \\
19000\end{array}$ & $\begin{array}{l}-0.302720 \\
-0.140474\end{array}$ & $\begin{array}{l}14000 \\
19000\end{array}$ & $\begin{array}{l}0.230769 \\
0.076923\end{array}$ & $\begin{array}{l}14000 \\
19000\end{array}$ & $\begin{array}{l}0.007140 \\
0.002380\end{array}$ \\
\hline CEPXS Form: & material & $\begin{array}{l}\mathrm{O} \\
\mathrm{Al} \\
\mathrm{Si} \\
\mathrm{K}\end{array}$ & $\begin{array}{l}0.459866 \\
0.096940 \\
0.302720 \\
0.140474\end{array}$ & & & \\
\hline & $\begin{array}{c}\text { matname } \\
\text { density }\end{array}$ & $\begin{array}{c}\text { Potassium } \\
1.100000\end{array}$ & inum Silic & & & \\
\hline $\begin{array}{l}\text { Comments an } \\
\text { Formula from L } \\
\text { The mineral fo } \\
\text { http://webmine } \\
\text { The density = } \\
\text { See "cat litter } \\
\text { material (NOR }\end{array}$ & $\begin{array}{l}\text { References } \\
(2008), \mathrm{ps} \\
\text { of this com } \\
\text { com/data/ } \\
\mathrm{g} / \mathrm{cm} 3 \text { was } \\
\text { mping)." T } \\
\text { It is also }\end{array}$ & $\begin{array}{l}\text { - } 82 \text {. This } r \\
\text { und is called } \\
\text { rocline.shtml. } \\
\text { osen based } \\
\text { material is } \mathrm{s} \\
\text { ed Aluminum }\end{array}$ & $\begin{array}{l}\text { ence lists } \\
\text { ocline. Th } \\
\text { te maximu } \\
\text { times usec } \\
\text { assium Sil }\end{array}$ & $\begin{array}{l}\text { ensity }=2.5 \\
\text { nsity for this } \\
\text { lue for the } \\
\text { ound natur }\end{array}$ & $\begin{array}{l}\text { lal } \\
\text { ral }=2.5 \\
y \text { for clun } \\
\text { curring } r\end{array}$ & $\begin{array}{l}\text { n3 at } \\
\text { cat litter. } \\
\text { ctive }\end{array}$ \\
\hline
\end{tabular}

\section{Potassium lodide}

\begin{tabular}{llll}
\hline Formula $=$ & $\mathrm{Kl}$ & Molecular weight $(\mathrm{g} / \mathrm{mole})=$ & 166.00277 \\
Density $(\mathrm{g} / \mathrm{cm} 3)=$ & 3.130000 & Total atom density $($ atoms $/ \mathrm{b}-\mathrm{cm})=$ & $2.271 \mathrm{E}-02$
\end{tabular}

The above density is estimated to be accurate to 3 significant digits. Uncertainties are not addressed.

The following data were calculated from the input weight fractions.

\begin{tabular}{|c|c|c|c|c|c|c|}
\hline Element & Neutron ZA & Photon ZA & $\begin{array}{l}\text { Weight } \\
\text { Fraction }\end{array}$ & $\begin{array}{l}\text { Atom } \\
\text { Fraction }\end{array}$ & $\begin{array}{l}\text { Atom } \\
\text { Density }\end{array}$ & \\
\hline $\mathrm{K}$ & 19000 & 19000 & 0.235528 & 0.500000 & 0.011355 & \\
\hline I & 53127 & 53000 & 0.764472 & 0.500000 & 0.011355 & \\
\hline Total & & & 1.000000 & 1.000000 & 0.022710 & \\
\hline MCNP Form & \multicolumn{2}{|c|}{ Weight Fractions } & \multicolumn{2}{|c|}{ Atom Fractions } & \multicolumn{2}{|c|}{ Atom Densities } \\
\hline \multirow[t]{2}{*}{ Neutrons } & 19000 & -0.235528 & 19000 & 0.500000 & 19000 & 0.011355 \\
\hline & 53127 & -0.764472 & 53127 & 0.500000 & 53127 & 0.011355 \\
\hline \multirow[t]{2}{*}{ Photons } & 19000 & -0.235528 & 19000 & 0.500000 & 19000 & 0.011355 \\
\hline & 53000 & -0.764472 & 53000 & 0.500000 & 53000 & 0.011355 \\
\hline \multirow[t]{3}{*}{ CEPXS Form: } & material & $\mathrm{K}$ & 0.235528 & & & \\
\hline & & 1 & 0.764472 & & & \\
\hline & $\begin{array}{l}\text { matname } \\
\text { density }\end{array}$ & \multicolumn{3}{|c|}{$\begin{array}{l}\text { Potassium lodide } \\
3.130000\end{array}$} & & \\
\hline \multicolumn{7}{|c|}{$\begin{array}{l}\text { Comments and References } \\
\text { Density and weight fractions from http://physics.nist.gov/cgi-bin/Star/compos.pl?matno=236 (NIST 1998). } \\
\text { Formula from Lide (2008), pgs } 4 \text { - 83. }\end{array}$} \\
\hline
\end{tabular}




\section{Potassium Oxide}

\begin{tabular}{llll}
\hline Formula $=$ & K2O & Molecular weight $(\mathrm{g} / \mathrm{mole})=$ & 94.196 \\
Density $(\mathrm{g} / \mathrm{cm} 3)=$ & 2.320000 & Total atom density $($ atoms $/ \mathrm{b}-\mathrm{cm})=$ & $4.450 \mathrm{E}-02$
\end{tabular}

The above density is estimated to be accurate to 3 significant digits. Uncertainties are not addressed.

The following data were calculated from the input weight fractions.

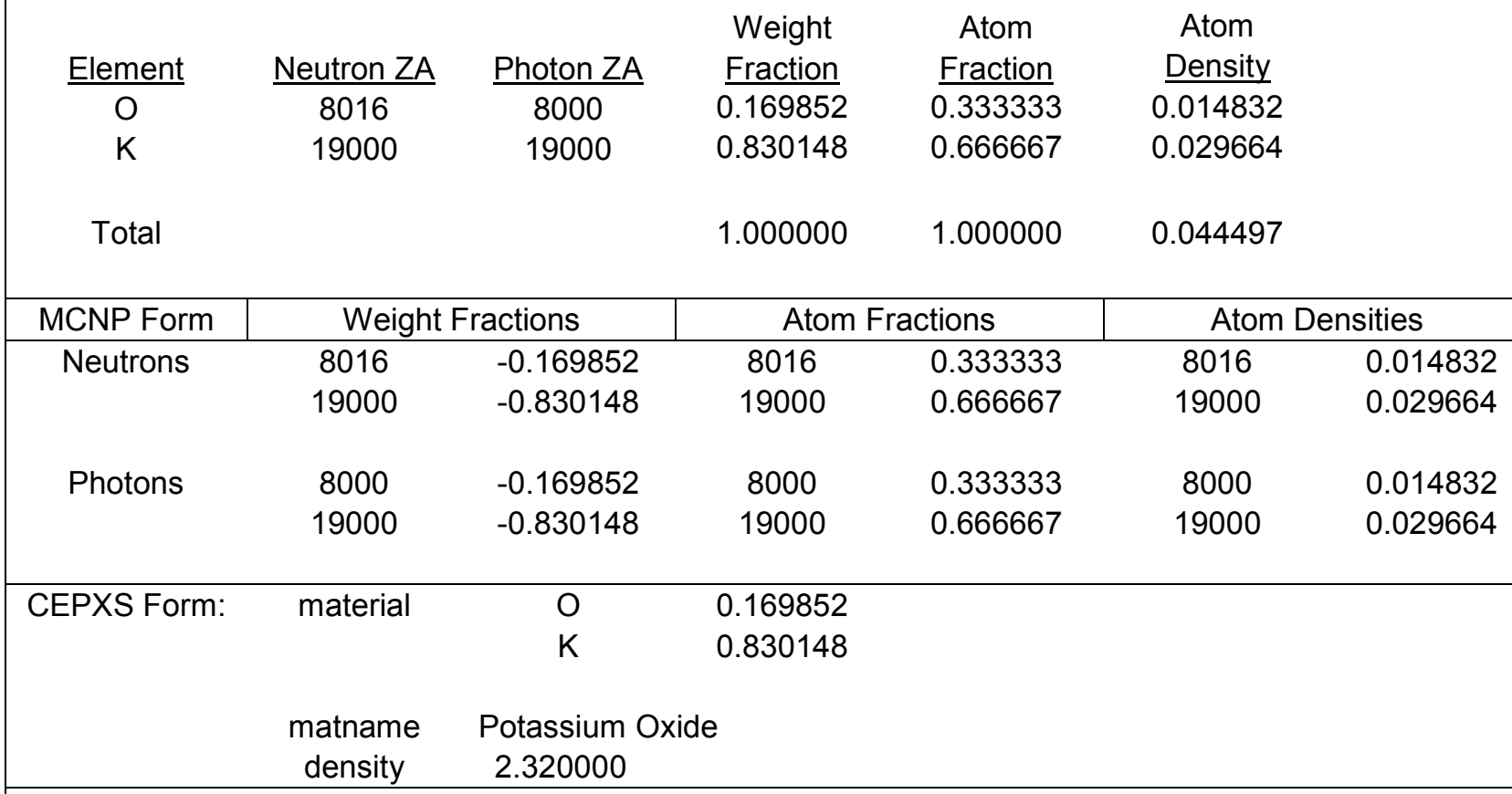

\section{Comments and References}

Density and weight fractions from http://physics.nist.gov/cgi-bin/Star/compos.pl?matno=237 (NIST 1998).

Formula from Lide (2008), pgs 4 - 83.

\section{Propane (Gas)}

\begin{tabular}{llll}
\hline Formula $=$ & $\mathrm{C} 3 \mathrm{H} 8$ & Molecular weight $(\mathrm{g} / \mathrm{mole})=$ & 44.09562 \\
Density $(\mathrm{g} / \mathrm{cm} 3)=$ & 0.001879 & Total atom density $($ atoms $/ \mathrm{b}-\mathrm{cm})=$ & $2.823 \mathrm{E}-04$
\end{tabular}

The above density is estimated to be accurate to 4 significant digits. Uncertainties are not addressed.

The following data were calculated from the input weight fractions.

\begin{tabular}{|c|c|c|c|c|c|c|}
\hline Element & Neutron ZA & Photon ZA & $\begin{array}{l}\text { Weight } \\
\text { Fraction }\end{array}$ & $\begin{array}{c}\text { Atom } \\
\text { Fraction }\end{array}$ & $\begin{array}{c}\text { Atom } \\
\text { Density }\end{array}$ & \\
\hline $\mathrm{H}$ & 1001 & 1000 & $\overline{0.182855}$ & $\overline{0.727260}$ & 0.000205 & \\
\hline C & 6000 & 6000 & 0.817145 & 0.272740 & 0.000077 & \\
\hline Total & & & 1.000000 & 1.000000 & 0.000282 & \\
\hline MCNP Form & \multicolumn{2}{|c|}{ Weight Fractions } & \multicolumn{2}{|c|}{ Atom Fractions } & \multicolumn{2}{|c|}{ Atom Densities } \\
\hline \multirow[t]{2}{*}{ Neutrons } & 1001 & -0.182855 & 1001 & 0.727260 & 1001 & 0.000205 \\
\hline & 6000 & -0.817145 & 6000 & 0.272740 & 6000 & 0.000077 \\
\hline
\end{tabular}


PIET-43741-TM-963

PNNL-15870 Rev. 1

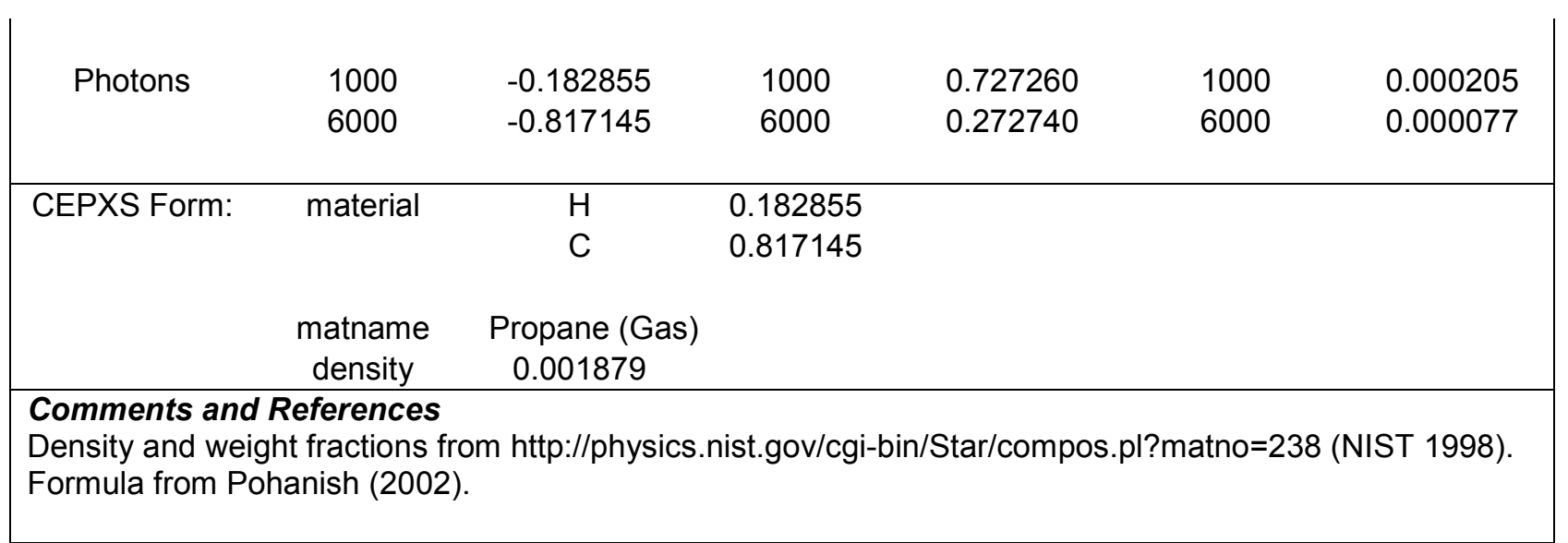

\section{Propane (Liquid)}

\begin{tabular}{llll}
\hline Formula $=$ & C3H8 & Molecular weight $(\mathrm{g} / \mathrm{mole})=$ & 44.09562 \\
Density $(\mathrm{g} / \mathrm{cm} 3)=$ & 0.430000 & Total atom density $($ atoms $/ \mathrm{b}-\mathrm{cm})=$ & $6.460 \mathrm{E}-02$
\end{tabular}

The above density is estimated to be accurate to 4 significant digits. Uncertainties are not addressed.

The following data were calculated from the input weight fractions.

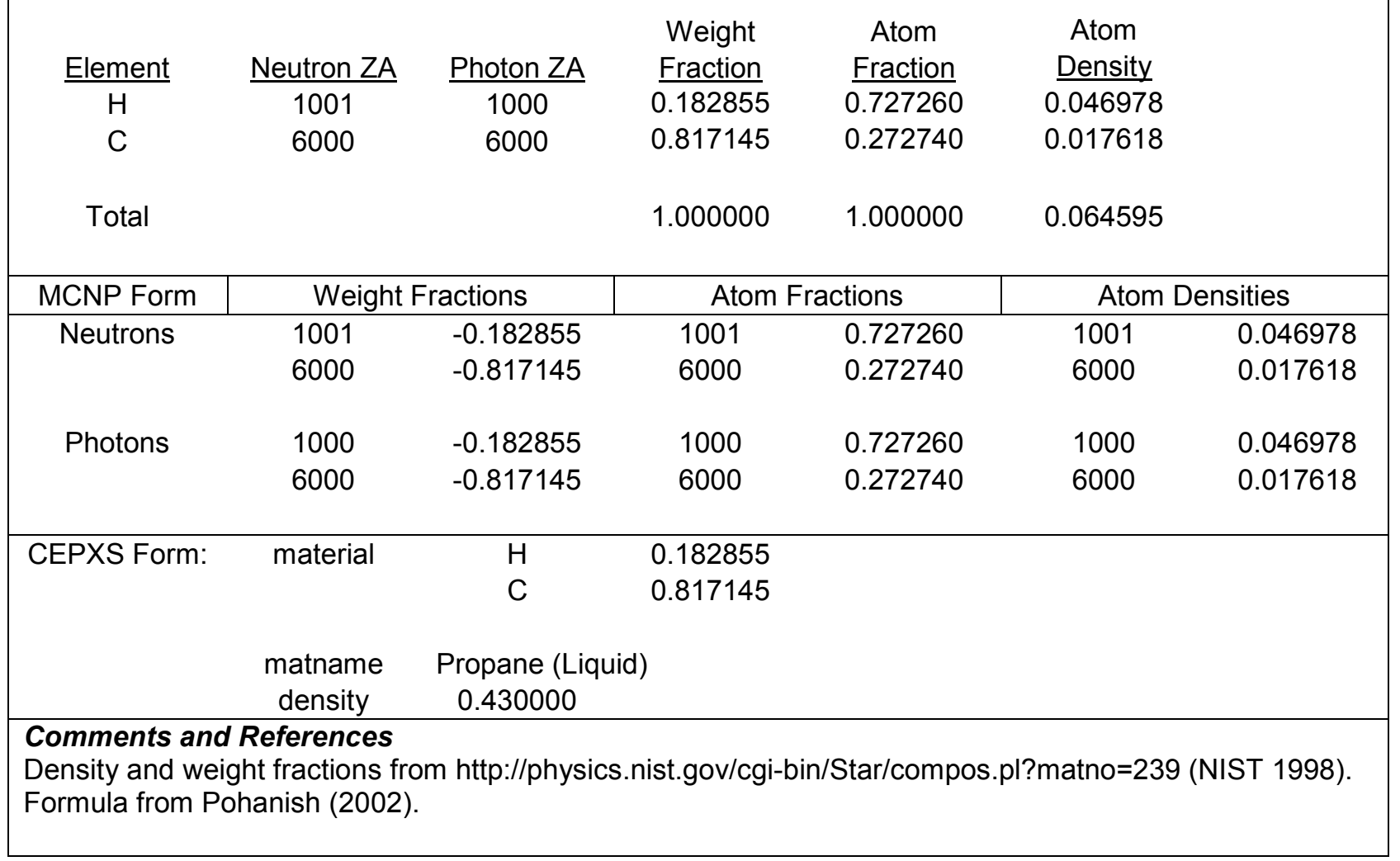




\section{P-terphenyl}

$\begin{array}{llll}\text { Formula }= & \mathrm{C} 14 \mathrm{H} 10 & \text { Molecular weight }(\mathrm{g} / \mathrm{mole})= & 178.2292 \\ \text { Density }(\mathrm{g} / \mathrm{cm} 3)= & 1.230000 & \text { Total atom density }(\text { atoms } / \mathrm{b}-\mathrm{cm})= & 9.974 \mathrm{E}-02\end{array}$

The above density is estimated to be accurate to 3 significant digits. Uncertainties are not addressed.

The following data was calculated from the input formula.

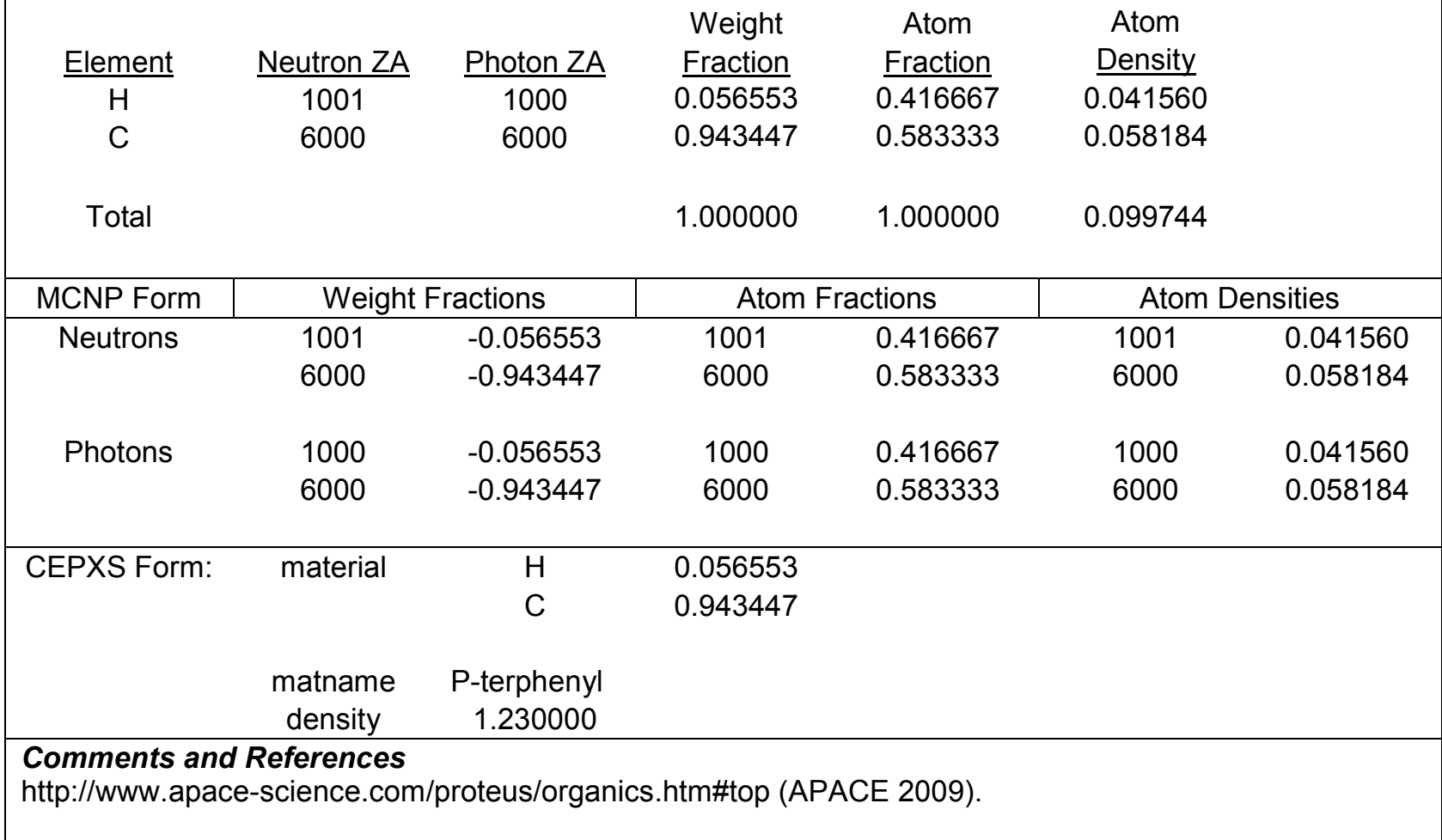

\section{Radiochromic Dye Film, Nylon Base (RDF: NB)}

\begin{tabular}{|c|c|c|c|}
\hline & - & Molecular weight $(\mathrm{g} / \mathrm{mole})=$ & \\
\hline Density $(\mathrm{g} / \mathrm{cm} 3)=$ & 1.080000 & Total atom density $($ atoms $/ \mathrm{b}-\mathrm{cm})=$ & 1.117E-01 \\
\hline
\end{tabular}

The above density is estimated to be accurate to 3 significant digits. Uncertainties are not addressed.

The following data were calculated from the input weight fractions.

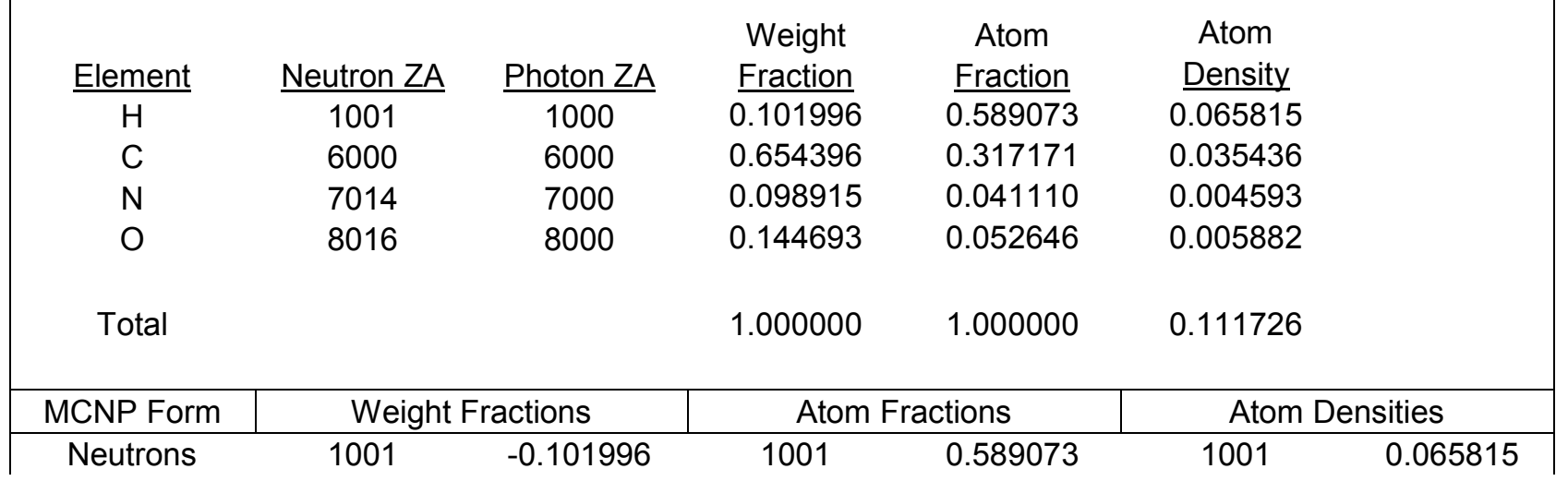


PIET-43741-TM-963

PNNL-15870 Rev. 1

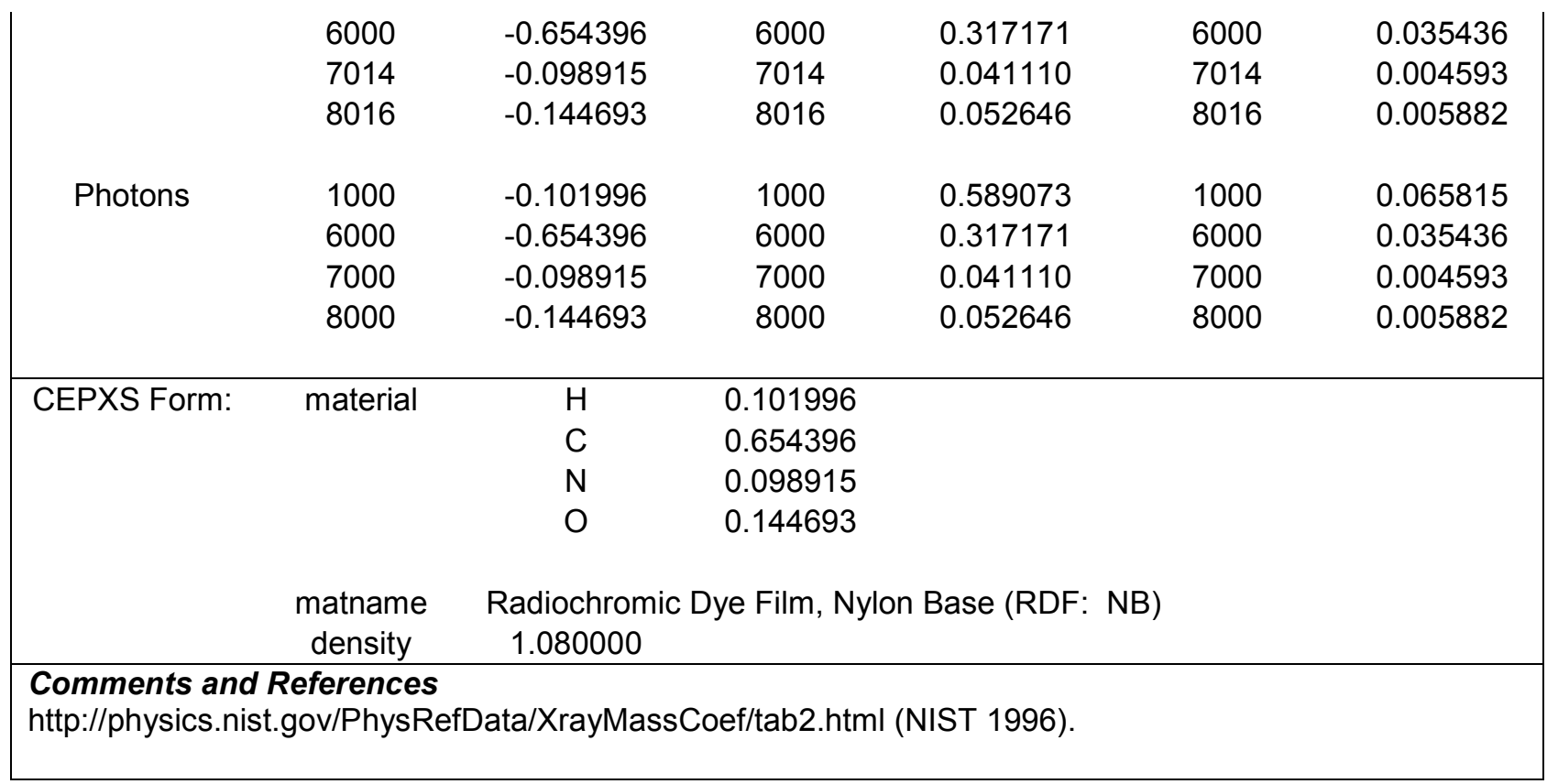

\section{Rock (Average of 5 Types)}

\begin{tabular}{llll}
\hline Formula $=$ & - & Molecular weight $(\mathrm{g} / \mathrm{mole})=$ & - \\
Density $(\mathrm{g} / \mathrm{cm} 3)=$ & 2.662000 & Total atom density $($ atoms $/ \mathrm{b}-\mathrm{cm})=$ & $8.028 \mathrm{E}-02$
\end{tabular}

The above density is estimated to be accurate to 3 significant digits. Uncertainties are not addressed.

The following data were calculated from the input weight fractions.

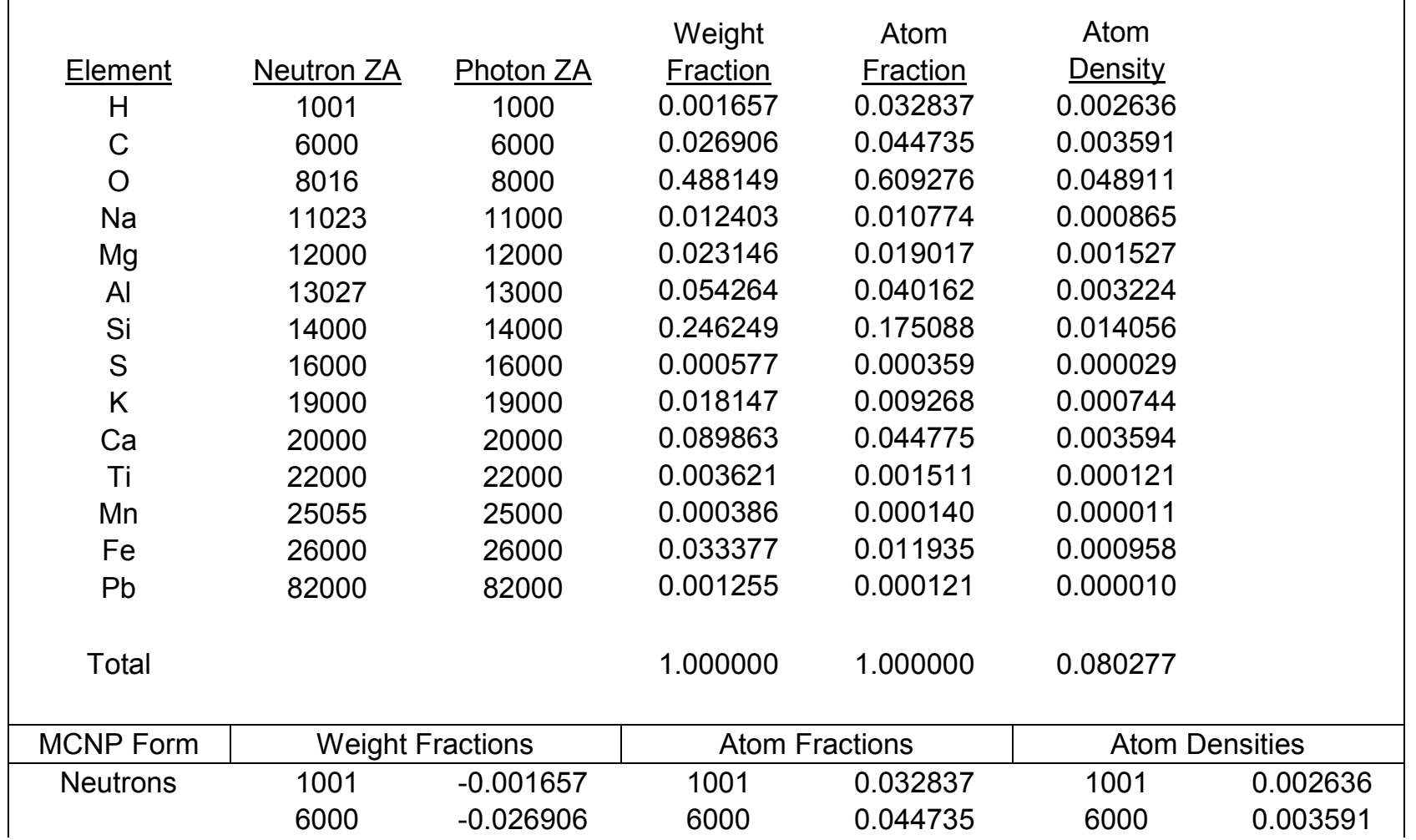


PIET-43741-TM-963

PNNL-15870 Rev. 1

\begin{tabular}{|c|c|c|c|c|c|c|}
\hline & 8016 & -0.488149 & 8016 & 0.609276 & 8016 & 0.048911 \\
\hline & 11023 & -0.012403 & 11023 & 0.010774 & 11023 & 0.000865 \\
\hline & 12000 & -0.023146 & 12000 & 0.019017 & 12000 & 0.001527 \\
\hline & 13027 & -0.054264 & 13027 & 0.040162 & 13027 & 0.003224 \\
\hline & 14000 & -0.246249 & 14000 & 0.175088 & 14000 & 0.014056 \\
\hline & 16000 & -0.000577 & 16000 & 0.000359 & 16000 & 0.000029 \\
\hline & 19000 & -0.018147 & 19000 & 0.009268 & 19000 & 0.000744 \\
\hline & 20000 & -0.089863 & 20000 & 0.044775 & 20000 & 0.003594 \\
\hline & 22000 & -0.003621 & 22000 & 0.001511 & 22000 & 0.000121 \\
\hline & 25055 & -0.000386 & 25055 & 0.000140 & 25055 & 0.000011 \\
\hline & 26000 & -0.033377 & 26000 & 0.011935 & 26000 & 0.000958 \\
\hline & 82000 & -0.001255 & 82000 & 0.000121 & 82000 & 0.000010 \\
\hline Photons & 1000 & -0.001657 & 1000 & 0.032837 & 1000 & 0.002636 \\
\hline & 6000 & -0.026906 & 6000 & 0.044735 & 6000 & 0.003591 \\
\hline & 8000 & -0.488149 & 8000 & 0.609276 & 8000 & 0.048911 \\
\hline & 11000 & -0.012403 & 11000 & 0.010774 & 11000 & 0.000865 \\
\hline & 12000 & -0.023146 & 12000 & 0.019017 & 12000 & 0.001527 \\
\hline & 13000 & -0.054264 & 13000 & 0.040162 & 13000 & 0.003224 \\
\hline & 14000 & -0.246249 & 14000 & 0.175088 & 14000 & 0.014056 \\
\hline & 16000 & -0.000577 & 16000 & 0.000359 & 16000 & 0.000029 \\
\hline & 19000 & -0.018147 & 19000 & 0.009268 & 19000 & 0.000744 \\
\hline & 20000 & -0.089863 & 20000 & 0.044775 & 20000 & 0.003594 \\
\hline & 22000 & -0.003621 & 22000 & 0.001511 & 22000 & 0.000121 \\
\hline & 25000 & -0.000386 & 25000 & 0.000140 & 25000 & 0.000011 \\
\hline & 26000 & -0.033377 & 26000 & 0.011935 & 26000 & 0.000958 \\
\hline & 82000 & -0.001255 & 82000 & 0.000121 & 82000 & 0.000010 \\
\hline CEPXS Form: & material & $\mathrm{H}$ & 0.001657 & & & \\
\hline & & C & 0.026906 & & & \\
\hline & & 0 & 0.488149 & & & \\
\hline & & $\mathrm{Na}$ & 0.012403 & & & \\
\hline & & $\mathrm{Mg}$ & 0.023146 & & & \\
\hline & & $\mathrm{Al}$ & 0.054264 & & & \\
\hline & & $\mathrm{Si}$ & 0.246249 & & & \\
\hline & & $S$ & 0.000577 & & & \\
\hline & & $\mathrm{K}$ & 0.018147 & & & \\
\hline & & $\mathrm{Ca}$ & 0.089863 & & & \\
\hline & & $\mathrm{Ti}$ & 0.003621 & & & \\
\hline & & $\mathrm{Mn}$ & 0.000386 & & & \\
\hline & & $\mathrm{Fe}$ & 0.033377 & & & \\
\hline & & $\mathrm{Pb}$ & 0.001255 & & & \\
\hline & $\begin{array}{c}\text { matname } \\
\text { density }\end{array}$ & $\begin{array}{c}\text { Rock (Avera } \\
2.662000\end{array}$ & ff 5 types) & & & \\
\hline $\begin{array}{l}\text { Comments an } \\
\text { Average densit } \\
\text { each of the foll } \\
\text { used for the ag } \\
\text { may also be us } \\
\text { Bulk density of }\end{array}$ & $\begin{array}{l}\text { eferences } \\
\text { nd weight } f \\
\text { ng: basalt, }\end{array}$ & tions for the & saristur & account fo & divturo & $\begin{array}{l}0 \text { wt. \% of } \\
\text { ypes is } \\
\text { ck types } \\
\text { s. }\end{array}$ \\
\hline
\end{tabular}


$2.00 \mathrm{~g} / \mathrm{cm} 3$ for wet gravel (1/4 to 2 inch) at http://www.simetric.co.uk/si_materials.htm (Walker 2009).

Density of gravel $=1.76 \mathrm{~g} / \mathrm{cm} 3$ at

http://www.powderandbulk.com/resources/bulk_density/material_bulk_density_chart_g.htm (Powder and Bulk Dot Com 2010). Density of gravel $=1.44$ to $1.92 \mathrm{~g} / \mathrm{cm} 3$ in Hungerford (1960).

Weight fractions for each of the 5 types of rock from Tables 3-4 and 7.1 of Blatt et al. (2006).

\section{Rock, Basalt}

\begin{tabular}{llll}
\hline Formula $=$ & - & Molecular weight $(\mathrm{g} / \mathrm{mole})=$ \\
Density $(\mathrm{g} / \mathrm{cm} 3)=$ & 3.010000 & Total atom density $($ atoms $/ \mathrm{b}-\mathrm{cm})=$ & - \\
\hline
\end{tabular}

The above density is estimated to be accurate to 3 significant digits. Uncertainties are not addressed.

The following data were calculated from the input weight fractions.

\begin{tabular}{|c|c|c|c|c|c|c|}
\hline Element & Neutron ZA & Photon ZA & $\begin{array}{l}\text { Weight } \\
\text { Fraction }\end{array}$ & $\begin{array}{c}\text { Atom } \\
\text { Fraction }\end{array}$ & $\begin{array}{l}\text { Atom } \\
\text { Density }\end{array}$ & \\
\hline 0 & 8016 & 8000 & $\overline{0.441115}$ & $\overline{0.607469}$ & 0.049976 & \\
\hline $\mathrm{Na}$ & 11023 & 11000 & 0.021700 & 0.020797 & 0.001711 & \\
\hline $\mathrm{Mg}$ & 12000 & 12000 & 0.041878 & 0.037964 & 0.003123 & \\
\hline $\mathrm{Al}$ & 13027 & 13000 & 0.083934 & 0.068541 & 0.005639 & \\
\hline $\mathrm{Si}$ & 14000 & 14000 & 0.232811 & 0.182640 & 0.015026 & \\
\hline $\mathrm{K}$ & 19000 & 19000 & 0.008920 & 0.005027 & 0.000414 & \\
\hline $\mathrm{Ca}$ & 20000 & 20000 & 0.068973 & 0.037918 & 0.003120 & \\
\hline $\mathrm{Ti}$ & 22000 & 22000 & 0.011151 & 0.005133 & 0.000422 & \\
\hline $\mathrm{Mn}$ & 25055 & 25000 & 0.001541 & 0.000618 & 0.000051 & \\
\hline $\mathrm{Fe}$ & 26000 & 26000 & 0.085141 & 0.033592 & 0.002764 & \\
\hline $\mathrm{Pb}$ & 82000 & 82000 & 0.002835 & 0.000302 & 0.000025 & \\
\hline Total & & & 1.000000 & 1.000000 & 0.082270 & \\
\hline MCNP Form & \multicolumn{2}{|c|}{ Weight Fractions } & \multicolumn{2}{|c|}{ Atom Fractions } & \multicolumn{2}{|c|}{ Atom Densities } \\
\hline \multirow[t]{11}{*}{ Neutrons } & 8016 & -0.441115 & 8016 & 0.607469 & 8016 & 0.049976 \\
\hline & 11023 & -0.021700 & 11023 & 0.020797 & 11023 & 0.001711 \\
\hline & 12000 & -0.041878 & 12000 & 0.037964 & 12000 & 0.003123 \\
\hline & 13027 & -0.083934 & 13027 & 0.068541 & 13027 & 0.005639 \\
\hline & 14000 & -0.232811 & 14000 & 0.182640 & 14000 & 0.015026 \\
\hline & 19000 & -0.008920 & 19000 & 0.005027 & 19000 & 0.000414 \\
\hline & 20000 & -0.068973 & 20000 & 0.037918 & 20000 & 0.003120 \\
\hline & 22000 & -0.011151 & 22000 & 0.005133 & 22000 & 0.000422 \\
\hline & 25055 & -0.001541 & 25055 & 0.000618 & 25055 & 0.000051 \\
\hline & 26000 & -0.085141 & 26000 & 0.033592 & 26000 & 0.002764 \\
\hline & 82000 & -0.002835 & 82000 & 0.000302 & 82000 & 0.000025 \\
\hline \multirow{6}{*}{ Photons } & 8000 & -0.441115 & 8000 & 0.607469 & 8000 & 0.049976 \\
\hline & 11000 & -0.021700 & 11000 & 0.020797 & 11000 & 0.001711 \\
\hline & 12000 & -0.041878 & 12000 & 0.037964 & 12000 & 0.003123 \\
\hline & 13000 & -0.083934 & 13000 & 0.068541 & 13000 & 0.005639 \\
\hline & 14000 & -0.232811 & 14000 & 0.182640 & 14000 & 0.015026 \\
\hline & 19000 & -0.008920 & 19000 & 0.005027 & 19000 & 0.000414 \\
\hline
\end{tabular}


PIET-43741-TM-963

PNNL-15870 Rev. 1

\begin{tabular}{|c|c|c|c|c|c|c|}
\hline & $\begin{array}{l}20000 \\
22000 \\
25000 \\
26000 \\
82000\end{array}$ & $\begin{array}{l}-0.068973 \\
-0.011151 \\
-0.001541 \\
-0.085141 \\
-0.002835\end{array}$ & $\begin{array}{l}20000 \\
22000 \\
25000 \\
26000 \\
82000\end{array}$ & $\begin{array}{l}0.037918 \\
0.005133 \\
0.000618 \\
0.033592 \\
0.000302\end{array}$ & $\begin{array}{l}20000 \\
22000 \\
25000 \\
26000 \\
82000\end{array}$ & $\begin{array}{l}0.003120 \\
0.000422 \\
0.000051 \\
0.002764 \\
0.000025\end{array}$ \\
\hline CEPXS Form: & material & $\begin{array}{c}\mathrm{O} \\
\mathrm{Na} \\
\mathrm{Mg} \\
\mathrm{Al} \\
\mathrm{Si} \\
\mathrm{K} \\
\mathrm{Ca} \\
\mathrm{Ti} \\
\mathrm{Mn} \\
\mathrm{Fe} \\
\mathrm{Pb}\end{array}$ & $\begin{array}{l}0.441115 \\
0.021700 \\
0.041878 \\
0.083934 \\
0.232811 \\
0.008920 \\
0.068973 \\
0.011151 \\
0.001541 \\
0.085141 \\
0.002835\end{array}$ & & & \\
\hline & $\begin{array}{l}\text { matname } \\
\text { density }\end{array}$ & $\begin{array}{c}\text { Rock, Basalt } \\
3.010000\end{array}$ & & & & \\
\hline \multicolumn{7}{|c|}{$\begin{array}{l}\text { Comments and References } \\
\text { The weight fractions are calculated based on the } 11 \text { compounds in basalt listed in Table } 3-4 \text { of Blatt et al. } \\
\text { (2006). } \\
\text { Average density of basalt }=2.95 \mathrm{~g} / \mathrm{cm} 3 \text { in Avallone and Baumeister III (1996). Density }=3.01 \mathrm{~g} / \mathrm{cm} 3 \text { for } \\
\text { solid basalt and } 1.95 \mathrm{~g} / \mathrm{cm} 3 \text { for broken basalt at http://www.simetric.co.uk/si_materials.htm (Walker 2009). } \\
\text { Density for solid basalt (an average of basalt densities from } 3 \text { regions) }=3.09 \mathrm{~g} / \mathrm{cm} 3 \mathrm{from} \text { pg } 52 \text { of } \\
\text { Washburn (2003). The Knovel online version is available at } \\
\text { http://totem.pnl.gov: } 2067 / \mathrm{web} / \text { portal/browse } / \text { display?_EXT_KNOVEL_DISPLAY_bookid=735\&VerticallD= } \\
\text { 0. Density }=2.7 \text { to } 3.2 \mathrm{~g} / \mathrm{cm} 3 \text {, and bulk density }=1.58 \mathrm{~g} / \mathrm{cm} 3 \text {, in Table } 6.1 .5 \text { of Avallone and Baumeister } \\
\text { III (1996). }\end{array}$} \\
\hline
\end{tabular}

\section{Rock, Granite}

\begin{tabular}{llll}
\hline Formula $=$ & - & Molecular weight $(\mathrm{g} / \mathrm{mole})=$ & - \\
Density $(\mathrm{g} / \mathrm{cm} 3)=$ & 2.690000 & Total atom density $($ atoms $/ \mathrm{b}-\mathrm{cm})=$ & $7.784 \mathrm{E}-02$
\end{tabular}

The above density is estimated to be accurate to 3 significant digits. Uncertainties are not addressed.

The following data were calculated from the input weight fractions.

\begin{tabular}{|c|c|c|c|c|c|}
\hline Element & Neutron ZA & Photon ZA & $\begin{array}{l}\text { Weight } \\
\text { Fraction }\end{array}$ & $\begin{array}{c}\text { Atom } \\
\text { Fraction }\end{array}$ & $\begin{array}{l}\text { Atom } \\
\text { Density }\end{array}$ \\
\hline 0 & 8016 & 8000 & $\overline{0.484170}$ & $\overline{0.629769}$ & 0.049023 \\
\hline $\mathrm{Na}$ & 11023 & 11000 & 0.027328 & 0.024738 & 0.001926 \\
\hline $\mathrm{Mg}$ & 12000 & 12000 & 0.004274 & 0.003660 & 0.000285 \\
\hline $\mathrm{Al}$ & 13027 & 13000 & 0.076188 & 0.058764 & 0.004574 \\
\hline $\mathrm{Si}$ & 14000 & 14000 & 0.336169 & 0.249093 & 0.019390 \\
\hline $\mathrm{K}$ & 19000 & 19000 & 0.034144 & 0.018174 & 0.001415 \\
\hline $\mathrm{Ca}$ & 20000 & 20000 & 0.012985 & 0.006743 & 0.000525 \\
\hline $\mathrm{Ti}$ & 22000 & 22000 & 0.001795 & 0.000780 & 0.000061 \\
\hline $\mathrm{Mn}$ & 25055 & 25000 & 0.000387 & 0.000146 & 0.000011 \\
\hline
\end{tabular}


PIET-43741-TM-963

PNNL-15870 Rev. 1

\begin{tabular}{|c|c|c|c|c|c|c|}
\hline $\mathrm{Fe}$ & 26000 & 26000 & 0.021555 & 0.008033 & \multicolumn{2}{|l|}{0.000625} \\
\hline $\mathrm{Pb}$ & 82000 & 82000 & 0.001004 & 0.000101 & \multicolumn{2}{|l|}{0.000008} \\
\hline Total & & & 1.000000 & 1.000000 & \multicolumn{2}{|c|}{0.077842} \\
\hline MCNP Form & \multicolumn{2}{|c|}{ Weight Fractions } & \multicolumn{2}{|c|}{ Atom Fractions } & \multicolumn{2}{|c|}{ Atom Densities } \\
\hline \multirow[t]{11}{*}{ Neutrons } & 8016 & -0.484170 & 8016 & 0.629769 & 8016 & 0.049023 \\
\hline & 11023 & -0.027328 & 11023 & 0.024738 & 11023 & 0.001926 \\
\hline & 12000 & -0.004274 & 12000 & 0.003660 & 12000 & 0.000285 \\
\hline & 13027 & -0.076188 & 13027 & 0.058764 & 13027 & 0.004574 \\
\hline & 14000 & -0.336169 & 14000 & 0.249093 & 14000 & 0.019390 \\
\hline & 19000 & -0.034144 & 19000 & 0.018174 & 19000 & 0.001415 \\
\hline & 20000 & -0.012985 & 20000 & 0.006743 & 20000 & 0.000525 \\
\hline & 22000 & -0.001795 & 22000 & 0.000780 & 22000 & 0.000061 \\
\hline & 25055 & -0.000387 & 25055 & 0.000146 & 25055 & 0.000011 \\
\hline & 26000 & -0.021555 & 26000 & 0.008033 & 26000 & 0.000625 \\
\hline & 82000 & -0.001004 & 82000 & 0.000101 & 82000 & 0.000008 \\
\hline \multirow[t]{11}{*}{ Photons } & 8000 & -0.484170 & 8000 & 0.629769 & 8000 & 0.049023 \\
\hline & 11000 & -0.027328 & 11000 & 0.024738 & 11000 & 0.001926 \\
\hline & 12000 & -0.004274 & 12000 & 0.003660 & 12000 & 0.000285 \\
\hline & 13000 & -0.076188 & 13000 & 0.058764 & 13000 & 0.004574 \\
\hline & 14000 & -0.336169 & 14000 & 0.249093 & 14000 & 0.019390 \\
\hline & 19000 & -0.034144 & 19000 & 0.018174 & 19000 & 0.001415 \\
\hline & 20000 & -0.012985 & 20000 & 0.006743 & 20000 & 0.000525 \\
\hline & 22000 & -0.001795 & 22000 & 0.000780 & 22000 & 0.000061 \\
\hline & 25000 & -0.000387 & 25000 & 0.000146 & 25000 & 0.000011 \\
\hline & 26000 & -0.021555 & 26000 & 0.008033 & 26000 & 0.000625 \\
\hline & 82000 & -0.001004 & 82000 & 0.000101 & 82000 & 0.000008 \\
\hline \multirow[t]{12}{*}{ CEPXS Form: } & material & 0 & 0.484170 & & & \\
\hline & & $\mathrm{Na}$ & 0.027328 & & & \\
\hline & & $\mathrm{Mg}$ & 0.004274 & & & \\
\hline & & $\mathrm{Al}$ & 0.076188 & & & \\
\hline & & $\mathrm{Si}$ & 0.336169 & & & \\
\hline & & $\mathrm{K}$ & 0.034144 & & & \\
\hline & & $\mathrm{Ca}$ & 0.012985 & & & \\
\hline & & $\mathrm{Ti}$ & 0.001795 & & & \\
\hline & & $\mathrm{Mn}$ & 0.000387 & & & \\
\hline & & $\mathrm{Fe}$ & 0.021555 & & & \\
\hline & & $\mathrm{Pb}$ & 0.001004 & & & \\
\hline & $\begin{array}{l}\text { matname } \\
\text { density }\end{array}$ & $\begin{array}{c}\text { Rock, Granite } \\
2.690000\end{array}$ & & & & \\
\hline
\end{tabular}

The weight fractions are calculated based on the 11 compounds in basalt listed in Tables 3-4 of Blatt et al. (2006).

Average density of granite $=2.64 \mathrm{~g} / \mathrm{cm} 3$ in Table 6.1 .5 of Avallone and Baumeister III (1996). Density = $2.69 \mathrm{~g} / \mathrm{cm} 3$ for solid granite and $1.65 \mathrm{~g} / \mathrm{cm} 3$ for broken granite at http://www.simetric.co.uk/si_materials.htm (Walker 2009). Bulk density of crushed granite $=1.55 \mathrm{~g} / \mathrm{cm} 3$ at http://www.powderandbulk.com/resources/bulk_density/material_bulk_density_chart_g.htm (Powder and 
Bulk Dot Com 2010). Density for solid granite (an average of granite densities from 8 regions) = $3.09 \mathrm{~g} / \mathrm{cm} 3$ from pg 53 of Washburn (2003). Knovel online version is available at http://totem.pnl.gov:2067/web/portal/browse/display?_EXT_KNOVEL_DISPLAY_bookid=735\&VerticallD= 0 . Density $=2.65 \mathrm{~g} / \mathrm{cm} 3$ in Table 51.64 of Hungerford (1960).

\section{Rock, Limestone}

\begin{tabular}{llll}
\hline Formula $=$ & - & Molecular weight $(\mathrm{g} / \mathrm{mole})=$ & - \\
Density $(\mathrm{g} / \mathrm{cm} 3)=$ & 2.610000 & Total atom density $($ atoms $/ \mathrm{b}-\mathrm{cm})=$ & $8.206 \mathrm{E}-02$
\end{tabular}

The above density is estimated to be accurate to 3 significant digits. Uncertainties are not addressed.

The following data were calculated from the input weight fractions.

\begin{tabular}{|c|c|c|c|c|c|c|}
\hline Element & Neutron ZA & Photon ZA & $\begin{array}{l}\text { Weight } \\
\text { Fraction }\end{array}$ & $\begin{array}{c}\text { Atom } \\
\text { Fraction }\end{array}$ & $\begin{array}{l}\text { Atom } \\
\text { Density }\end{array}$ & \\
\hline $\mathrm{H}$ & 1001 & 1000 & 0.000899 & 0.017089 & 0.001402 & \\
\hline C & 6000 & 6000 & 0.113782 & 0.181445 & 0.014890 & \\
\hline 0 & 8016 & 8000 & 0.497802 & 0.595930 & 0.048904 & \\
\hline $\mathrm{Na}$ & 11023 & 11000 & 0.000373 & 0.000310 & 0.000025 & \\
\hline $\mathrm{Mg}$ & 12000 & 12000 & 0.047860 & 0.037715 & 0.003095 & \\
\hline $\mathrm{Al}$ & 13027 & 13000 & 0.004254 & 0.003019 & 0.000248 & \\
\hline $\mathrm{Si}$ & 14000 & 14000 & 0.024419 & 0.016653 & 0.001367 & \\
\hline$S$ & 16000 & 16000 & 0.000201 & 0.000120 & 0.000010 & \\
\hline $\mathrm{K}$ & 19000 & 19000 & 0.000334 & 0.000163 & 0.000013 & \\
\hline $\mathrm{Ca}$ & 20000 & 20000 & 0.305865 & 0.146173 & 0.011995 & \\
\hline $\mathrm{Ti}$ & 22000 & 22000 & 0.000361 & 0.000145 & 0.000012 & \\
\hline $\mathrm{Fe}$ & 26000 & 26000 & 0.003513 & 0.001205 & 0.000099 & \\
\hline $\mathrm{Pb}$ & 82000 & 82000 & 0.000337 & 0.000031 & 0.000003 & \\
\hline Total & & & 1.000000 & 1.000000 & 0.082063 & \\
\hline MCNP Form & \multicolumn{2}{|c|}{ Weight Fractions } & \multicolumn{2}{|c|}{ Atom Fractions } & \multicolumn{2}{|c|}{ Atom Densities } \\
\hline \multirow[t]{13}{*}{ Neutrons } & 1001 & -0.000899 & 1001 & 0.017089 & 1001 & 0.001402 \\
\hline & 6000 & -0.113782 & 6000 & 0.181445 & 6000 & 0.014890 \\
\hline & 8016 & -0.497802 & 8016 & 0.595930 & 8016 & 0.048904 \\
\hline & 11023 & -0.000373 & 11023 & 0.000310 & 11023 & 0.000025 \\
\hline & 12000 & -0.047860 & 12000 & 0.037715 & 12000 & 0.003095 \\
\hline & 13027 & -0.004254 & 13027 & 0.003019 & 13027 & 0.000248 \\
\hline & 14000 & -0.024419 & 14000 & 0.016653 & 14000 & 0.001367 \\
\hline & 16000 & -0.000201 & 16000 & 0.000120 & 16000 & 0.000010 \\
\hline & 19000 & -0.000334 & 19000 & 0.000163 & 19000 & 0.000013 \\
\hline & 20000 & -0.305865 & 20000 & 0.146173 & 20000 & 0.011995 \\
\hline & 22000 & -0.000361 & 22000 & 0.000145 & 22000 & 0.000012 \\
\hline & 26000 & -0.003513 & 26000 & 0.001205 & 26000 & 0.000099 \\
\hline & 82000 & -0.000337 & 82000 & 0.000031 & 82000 & 0.000003 \\
\hline \multirow[t]{3}{*}{ Photons } & 1000 & -0.000899 & 1000 & 0.017089 & 1000 & 0.001402 \\
\hline & 6000 & -0.113782 & 6000 & 0.181445 & 6000 & 0.014890 \\
\hline & 8000 & -0.497802 & 8000 & 0.595930 & 8000 & 0.048904 \\
\hline
\end{tabular}


PIET-43741-TM-963

PNNL-15870 Rev. 1

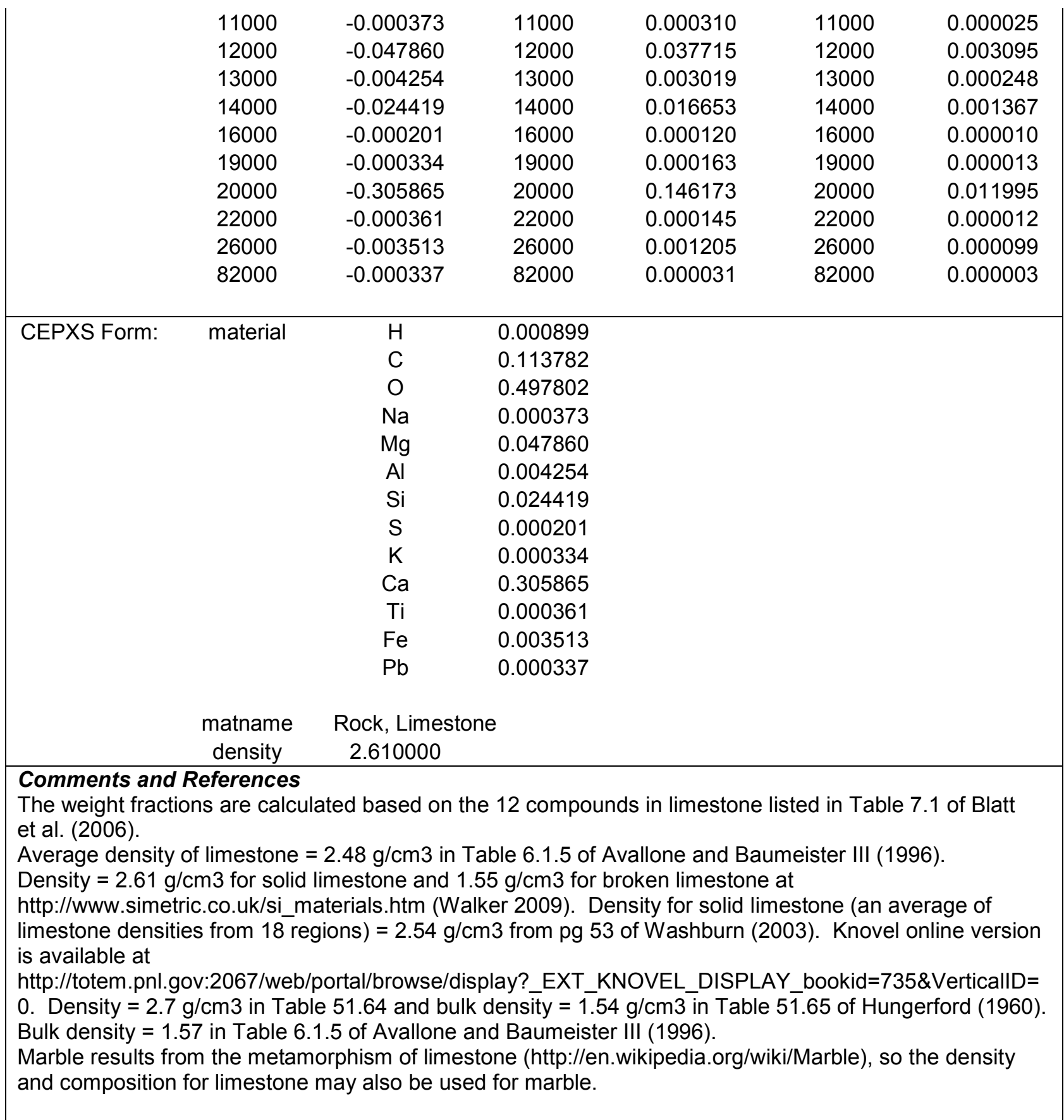

\section{Rock, Sandstone}

\begin{tabular}{llll}
\hline Formula $=$ & - & Molecular weight $(\mathrm{g} / \mathrm{mole})=$ \\
Density $(\mathrm{g} / \mathrm{cm} 3)=$ & 2.320000 & Total atom density $($ atoms $/ \mathrm{b}-\mathrm{cm})=$ & - \\
\hline
\end{tabular}

The above density is estimated to be accurate to 3 significant digits. Uncertainties are not addressed.

The following data were calculated from the input weight fractions. 


\begin{tabular}{|c|c|c|c|c|c|c|}
\hline Element & Neutron ZA & Photon ZA & $\begin{array}{l}\text { Weight } \\
\text { Fraction }\end{array}$ & $\begin{array}{c}\text { Atom } \\
\text { Fraction }\end{array}$ & $\begin{array}{l}\text { Atom } \\
\text { Density }\end{array}$ & \\
\hline $\mathrm{H}$ & 1001 & 1000 & 0.001791 & 0.034647 & 0.002483 & \\
\hline C & 6000 & 6000 & 0.013652 & 0.022161 & 0.001588 & \\
\hline $\mathrm{O}$ & 8016 & 8000 & 0.519609 & 0.633160 & 0.045375 & \\
\hline $\mathrm{Na}$ & 11023 & 11000 & 0.002969 & 0.002518 & 0.000180 & \\
\hline $\mathrm{Mg}$ & 12000 & 12000 & 0.007240 & 0.005807 & 0.000416 & \\
\hline $\mathrm{Al}$ & 13027 & 13000 & 0.025417 & 0.018365 & 0.001316 & \\
\hline $\mathrm{Si}$ & 14000 & 14000 & 0.366185 & 0.254190 & 0.018216 & \\
\hline$S$ & 16000 & 16000 & 0.000280 & 0.000171 & 0.000012 & \\
\hline $\mathrm{K}$ & 19000 & 19000 & 0.011628 & 0.005798 & 0.000416 & \\
\hline $\mathrm{Ca}$ & 20000 & 20000 & 0.039328 & 0.019131 & 0.001371 & \\
\hline $\mathrm{Ti}$ & 22000 & 22000 & 0.001199 & 0.000488 & 0.000035 & \\
\hline $\mathrm{Fe}$ & 26000 & 26000 & 0.010031 & 0.003502 & 0.000251 & \\
\hline $\mathrm{Pb}$ & 82000 & 82000 & 0.000671 & 0.000063 & 0.000005 & \\
\hline Total & & & 1.000000 & 1.000000 & 0.071664 & \\
\hline MCNP Form & \multicolumn{2}{|c|}{ Weight Fractions } & \multicolumn{2}{|c|}{ Atom Fractions } & \multicolumn{2}{|c|}{ Atom Densities } \\
\hline \multirow[t]{13}{*}{ Neutrons } & 1001 & -0.001791 & 1001 & 0.034647 & 1001 & 0.002483 \\
\hline & 6000 & -0.013652 & 6000 & 0.022161 & 6000 & 0.001588 \\
\hline & 8016 & -0.519609 & 8016 & 0.633160 & 8016 & 0.045375 \\
\hline & 11023 & -0.002969 & 11023 & 0.002518 & 11023 & 0.000180 \\
\hline & 12000 & -0.007240 & 12000 & 0.005807 & 12000 & 0.000416 \\
\hline & 13027 & -0.025417 & 13027 & 0.018365 & 13027 & 0.001316 \\
\hline & 14000 & -0.366185 & 14000 & 0.254190 & 14000 & 0.018216 \\
\hline & 16000 & -0.000280 & 16000 & 0.000171 & 16000 & 0.000012 \\
\hline & 19000 & -0.011628 & 19000 & 0.005798 & 19000 & 0.000416 \\
\hline & 20000 & -0.039328 & 20000 & 0.019131 & 20000 & 0.001371 \\
\hline & 22000 & -0.001199 & 22000 & 0.000488 & 22000 & 0.000035 \\
\hline & 26000 & -0.010031 & 26000 & 0.003502 & 26000 & 0.000251 \\
\hline & 82000 & -0.000671 & 82000 & 0.000063 & 82000 & 0.000005 \\
\hline \multirow[t]{13}{*}{ Photons } & 1000 & -0.001791 & 1000 & 0.034647 & 1000 & 0.002483 \\
\hline & 6000 & -0.013652 & 6000 & 0.022161 & 6000 & 0.001588 \\
\hline & 8000 & -0.519609 & 8000 & 0.633160 & 8000 & 0.045375 \\
\hline & 11000 & -0.002969 & 11000 & 0.002518 & 11000 & 0.000180 \\
\hline & 12000 & -0.007240 & 12000 & 0.005807 & 12000 & 0.000416 \\
\hline & 13000 & -0.025417 & 13000 & 0.018365 & 13000 & 0.001316 \\
\hline & 14000 & -0.366185 & 14000 & 0.254190 & 14000 & 0.018216 \\
\hline & 16000 & -0.000280 & 16000 & 0.000171 & 16000 & 0.000012 \\
\hline & 19000 & -0.011628 & 19000 & 0.005798 & 19000 & 0.000416 \\
\hline & 20000 & -0.039328 & 20000 & 0.019131 & 20000 & 0.001371 \\
\hline & 22000 & -0.001199 & 22000 & 0.000488 & 22000 & 0.000035 \\
\hline & 26000 & -0.010031 & 26000 & 0.003502 & 26000 & 0.000251 \\
\hline & 82000 & -0.000671 & 82000 & 0.000063 & 82000 & 0.000005 \\
\hline \multirow[t]{3}{*}{ CEPXS Form: } & material & $\mathrm{H}$ & 0.001791 & & & \\
\hline & & C & 0.013652 & & & \\
\hline & & 0 & 0.519609 & & & \\
\hline
\end{tabular}




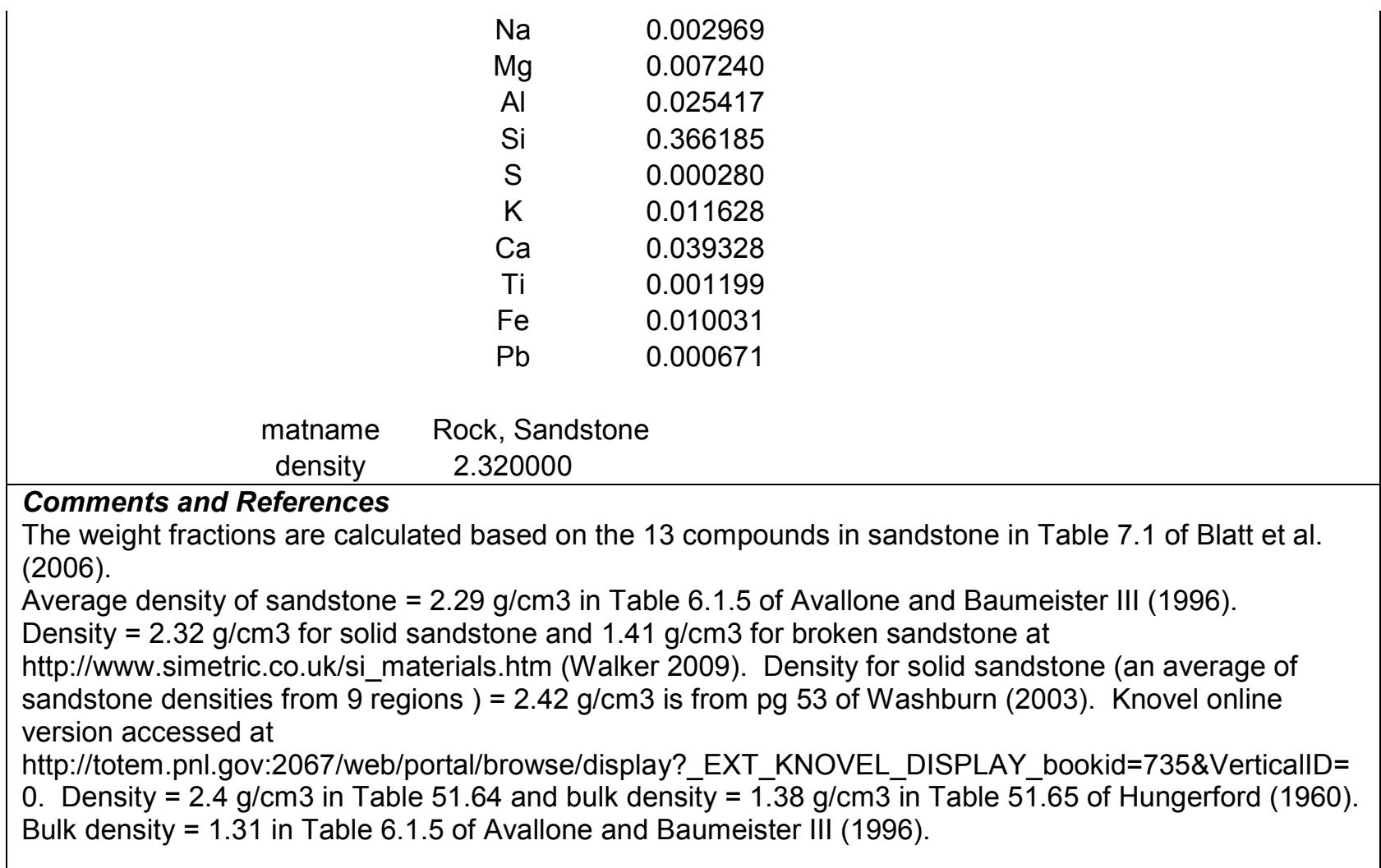

\section{Rock, Shale}

\begin{tabular}{|c|c|c|c|}
\hline $\begin{array}{l}\text { Formula }= \\
\text { Density }(\mathrm{q} / \mathrm{cm} 3)=\end{array}$ & $-\overline{2.680000}$ & $\begin{array}{l}\text { Molecular weight }(\mathrm{g} / \mathrm{mole})= \\
\text { Total atom density }(\text { atoms } / \mathrm{b}-\mathrm{cm})=\end{array}$ & $\begin{array}{l}- \\
8.625 E-02\end{array}$ \\
\hline
\end{tabular}

The above density is estimated to be accurate to 3 significant digits. Uncertainties are not addressed.

The following data were calculated from the input weight fractions.

\begin{tabular}{|c|c|c|c|c|c|}
\hline Element & Neutron ZA & Photon ZA & $\begin{array}{l}\text { Weight } \\
\text { Fraction }\end{array}$ & $\begin{array}{c}\text { Atom } \\
\text { Fraction }\end{array}$ & $\begin{array}{c}\text { Atom } \\
\text { Density }\end{array}$ \\
\hline $\mathrm{H}$ & 1001 & 1000 & $\overline{0.005597}$ & $\overline{0.103900}$ & 0.008961 \\
\hline C & 6000 & 6000 & 0.007098 & 0.011058 & 0.000954 \\
\hline $\mathrm{O}$ & 8016 & 8000 & 0.498049 & 0.582500 & 0.050241 \\
\hline $\mathrm{Na}$ & 11023 & 11000 & 0.009647 & 0.007852 & 0.000677 \\
\hline $\mathrm{Mg}$ & 12000 & 12000 & 0.014477 & 0.011146 & 0.000961 \\
\hline $\mathrm{Al}$ & 13027 & 13000 & 0.081529 & 0.056542 & 0.004877 \\
\hline $\mathrm{Si}$ & 14000 & 14000 & 0.271661 & 0.180997 & 0.015611 \\
\hline $\mathrm{S}$ & 16000 & 16000 & 0.002404 & 0.001403 & 0.000121 \\
\hline $\mathrm{K}$ & 19000 & 19000 & 0.035707 & 0.017089 & 0.001474 \\
\hline $\mathrm{Ca}$ & 20000 & 20000 & 0.022162 & 0.010347 & 0.000892 \\
\hline $\mathrm{Ti}$ & 22000 & 22000 & 0.003597 & 0.001406 & 0.000121 \\
\hline $\mathrm{Fe}$ & 26000 & 26000 & 0.046646 & 0.015630 & 0.001348 \\
\hline $\mathrm{Pb}$ & 82000 & 82000 & 0.001425 & 0.000129 & 0.000011 \\
\hline Total & & & 1.000000 & 1.000000 & 0.086250 \\
\hline
\end{tabular}


PIET-43741-TM-963

PNNL-15870 Rev. 1

\begin{tabular}{|c|c|c|c|c|c|c|}
\hline MCNP Form & \multicolumn{2}{|c|}{ Weight Fractions } & \multicolumn{2}{|c|}{ Atom Fractions } & \multicolumn{2}{|c|}{ Atom Densities } \\
\hline \multirow[t]{13}{*}{ Neutrons } & 1001 & -0.005597 & 1001 & 0.103900 & 1001 & 0.008961 \\
\hline & 6000 & -0.007098 & 6000 & 0.011058 & 6000 & 0.000954 \\
\hline & 8016 & -0.498049 & 8016 & 0.582500 & 8016 & 0.050241 \\
\hline & 11023 & -0.009647 & 11023 & 0.007852 & 11023 & 0.000677 \\
\hline & 12000 & -0.014477 & 12000 & 0.011146 & 12000 & 0.000961 \\
\hline & 13027 & -0.081529 & 13027 & 0.056542 & 13027 & 0.004877 \\
\hline & 14000 & -0.271661 & 14000 & 0.180997 & 14000 & 0.015611 \\
\hline & 16000 & -0.002404 & 16000 & 0.001403 & 16000 & 0.000121 \\
\hline & 19000 & -0.035707 & 19000 & 0.017089 & 19000 & 0.001474 \\
\hline & 20000 & -0.022162 & 20000 & 0.010347 & 20000 & 0.000892 \\
\hline & 22000 & -0.003597 & 22000 & 0.001406 & 22000 & 0.000121 \\
\hline & 26000 & -0.046646 & 26000 & 0.015630 & 26000 & 0.001348 \\
\hline & 82000 & -0.001425 & 82000 & 0.000129 & 82000 & 0.000011 \\
\hline \multirow[t]{13}{*}{ Photons } & 1000 & -0.005597 & 1000 & 0.103900 & 1000 & 0.008961 \\
\hline & 6000 & -0.007098 & 6000 & 0.011058 & 6000 & 0.000954 \\
\hline & 8000 & -0.498049 & 8000 & 0.582500 & 8000 & 0.050241 \\
\hline & 11000 & -0.009647 & 11000 & 0.007852 & 11000 & 0.000677 \\
\hline & 12000 & -0.014477 & 12000 & 0.011146 & 12000 & 0.000961 \\
\hline & 13000 & -0.081529 & 13000 & 0.056542 & 13000 & 0.004877 \\
\hline & 14000 & -0.271661 & 14000 & 0.180997 & 14000 & 0.015611 \\
\hline & 16000 & -0.002404 & 16000 & 0.001403 & 16000 & 0.000121 \\
\hline & 19000 & -0.035707 & 19000 & 0.017089 & 19000 & 0.001474 \\
\hline & 20000 & -0.022162 & 20000 & 0.010347 & 20000 & 0.000892 \\
\hline & 22000 & -0.003597 & 22000 & 0.001406 & 22000 & 0.000121 \\
\hline & 26000 & -0.046646 & 26000 & 0.015630 & 26000 & 0.001348 \\
\hline & 82000 & -0.001425 & 82000 & 0.000129 & 82000 & 0.000011 \\
\hline \multirow[t]{14}{*}{ CEPXS Form: } & material & $\mathrm{H}$ & 0.005597 & & & \\
\hline & & C & 0.007098 & & & \\
\hline & & 0 & 0.498049 & & & \\
\hline & & $\mathrm{Na}$ & 0.009647 & & & \\
\hline & & $\mathrm{Mg}$ & 0.014477 & & & \\
\hline & & $\mathrm{Al}$ & 0.081529 & & & \\
\hline & & $\mathrm{Si}$ & 0.271661 & & & \\
\hline & & $S$ & 0.002404 & & & \\
\hline & & $\mathrm{K}$ & 0.035707 & & & \\
\hline & & $\mathrm{Ca}$ & 0.022162 & & & \\
\hline & & $\mathrm{Ti}$ & 0.003597 & & & \\
\hline & & $\mathrm{Fe}$ & 0.046646 & & & \\
\hline & & $\mathrm{Pb}$ & 0.001425 & & & \\
\hline & $\begin{array}{c}\text { matname } \\
\text { density }\end{array}$ & $\begin{array}{c}\text { Rock, Shale } \\
2.680000\end{array}$ & & & & \\
\hline \multicolumn{7}{|c|}{$\begin{array}{l}\text { Comments and References } \\
\text { Weight fractions are calculated based on the } 13 \text { compounds in shale in Table } 7.1 \text { of Blatt et al. (2006). } \\
\text { Average density of shale }=2.76 \mathrm{~g} / \mathrm{cm} 3 \text { in Table } 6.1 .5 \text { of Avallone and Baumeister III (1996). Density }= \\
2.68 \mathrm{~g} / \mathrm{cm} 3 \text { for solid shale and } 1.59 \mathrm{~g} / \mathrm{cm} 3 \text { for broken shale at http://www.simetric.co.uk } / \mathrm{si} \text { materials.htm } \\
\text { (Walker } 2009 \text { ). Density }=2.4 \text { to } 2.8 \mathrm{~g} / \mathrm{cm} 3 \text {, and bulk density }=1.47 \mathrm{~g} / \mathrm{cm} 3 \text {, in Table } 51.65 \text { of Hungerford } \\
\text { (1960). Bulk density }=1.47 \text { in Table } 6.1 .5 \text { of Avallone and Baumeister III (1996). }\end{array}$} \\
\hline
\end{tabular}




\section{Rubber, Butyl}

\begin{tabular}{llll}
\hline Formula $=$ & - & Molecular weight $(\mathrm{g} / \mathrm{mole})=$ \\
Density $(\mathrm{g} / \mathrm{cm} 3)=$ & 0.920000 & Total atom density $($ atoms $/ \mathrm{b}-\mathrm{cm})=$ & - \\
$1.185 \mathrm{E}-01$
\end{tabular}

The above density is estimated to be accurate to 2 significant digits. Uncertainties are not addressed.

The following data were calculated from the input weight fractions.

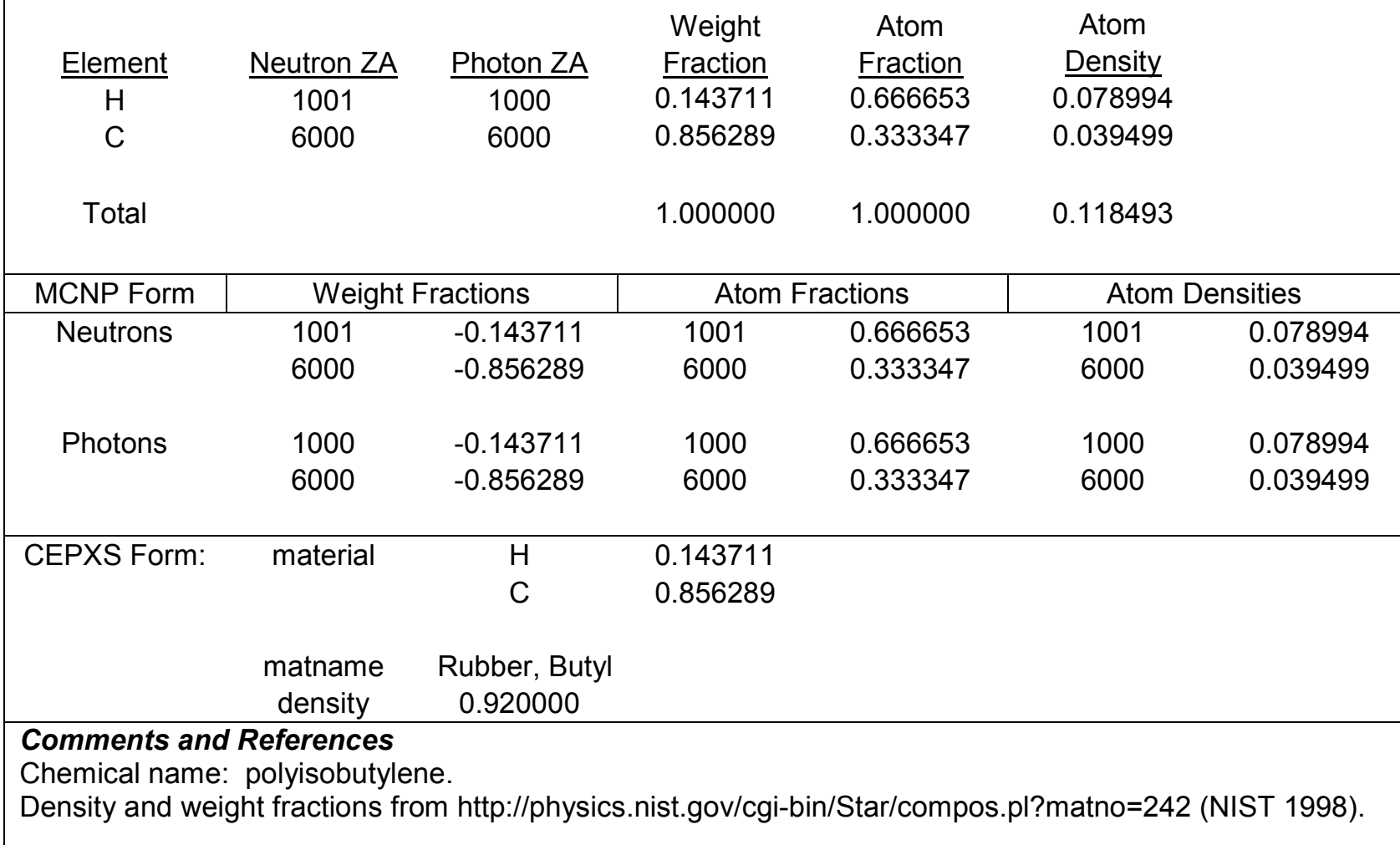

\section{Rubber, Natural}

Density $(\mathrm{g} / \mathrm{cm} 3)=\quad 0.920000 \quad$ Total atom density $($ atoms $/ \mathrm{b}-\mathrm{cm})=\begin{array}{r}1.057 \mathrm{E}-01 \\ \text {. }\end{array}$

The above density is estimated to be accurate to 2 significant digits. Uncertainties are not addressed.

The following data were calculated from the input weight fractions.

$\begin{array}{cccccc}\text { Element } & \text { Neutron ZA } & \text { Photon ZA } & \begin{array}{c}\text { Weight } \\ \text { Fraction }\end{array} & \begin{array}{c}\text { Atom } \\ \text { Fraction }\end{array} & \begin{array}{c}\text { Atom } \\ \text { Density }\end{array} \\ \mathrm{H} & 1001 & 1000 & 0.118371 & 0.615370 & 0.065065 \\ \text { Total } & 6000 & 6000 & 0.881629 & 0.384630 & 0.040668 \\ & & & & & \\ \end{array}$


PIET-43741-TM-963

PNNL-15870 Rev. 1

\begin{tabular}{|c|c|c|c|c|c|c|}
\hline MCNP Form & \multicolumn{2}{|c|}{ Weight Fractions } & \multicolumn{2}{|c|}{ Atom Fractions } & \multicolumn{2}{|c|}{ Atom Densities } \\
\hline \multirow[t]{2}{*}{ Neutrons } & 1001 & -0.118371 & 1001 & 0.615370 & 1001 & 0.065065 \\
\hline & 6000 & -0.881629 & 6000 & 0.384630 & 6000 & 0.040668 \\
\hline \multirow[t]{2}{*}{ Photons } & 1000 & -0.118371 & 1000 & 0.615370 & 1000 & 0.065065 \\
\hline & 6000 & -0.881629 & 6000 & 0.384630 & 6000 & 0.040668 \\
\hline \multirow[t]{3}{*}{ CEPXS Form: } & material & $\mathrm{H}$ & 0.118371 & & & \\
\hline & & C & 0.881629 & & & \\
\hline & $\begin{array}{c}\text { matname } \\
\text { density }\end{array}$ & $\begin{array}{c}\text { Rubber, Nat } \\
0.920000\end{array}$ & & & & \\
\hline \multicolumn{7}{|c|}{$\begin{array}{l}\text { Comments and References } \\
\text { Density and weight fractions from http://physics.nist.gov/cgi-bin/Star/compos.pl?matno=243 (NIST 1998). } \\
\text { Formula from Table } 51.11 \text { of Hungerford (1960). }\end{array}$} \\
\hline
\end{tabular}

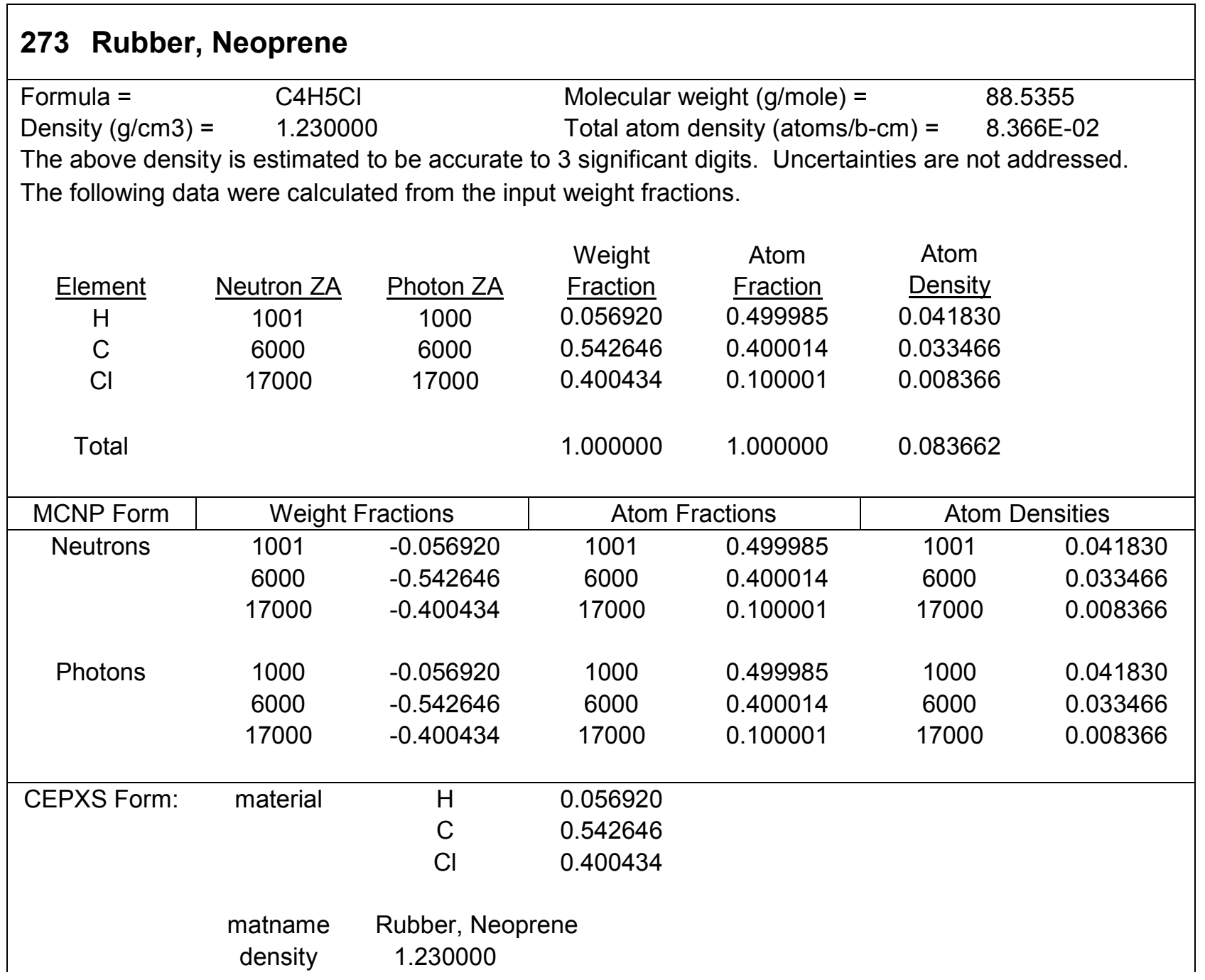


Comments and References

Chemical name: polychloroprene.

Density and weight fractions from http://physics.nist.gov/cgi-bin/Star/compos.pl?matno=244 (NIST 1998).

Density $=1.23 \mathrm{~g} / \mathrm{cm} 3$ for polychloroprene rubber from

http://www.matweb.com/search/DataSheet.aspx?MatGUID=f3743816df954959b10cad28927578f0

(Automation Creations 2010).

\section{Rubber, Silicon}

\begin{tabular}{llll}
\hline Formula $=$ & - & Molecular weight $(\mathrm{g} / \mathrm{mole})=$ \\
Density $(\mathrm{g} / \mathrm{cm} 3)=$ & 1.018500 & Total atom density $($ atoms $/ \mathrm{b}-\mathrm{cm})=$ & - \\
\hline & $8.227 \mathrm{E}-02$
\end{tabular}

The above density is estimated to be accurate to 3 significant digits. Uncertainties are not addressed.

The following data were calculated from the input weight fractions.

\begin{tabular}{|c|c|c|c|c|c|c|}
\hline Element & Neutron ZA & Photon ZA & $\begin{array}{l}\text { Weight } \\
\text { Fraction }\end{array}$ & $\begin{array}{c}\text { Atom } \\
\text { Fraction }\end{array}$ & $\begin{array}{l}\text { Atom } \\
\text { Density }\end{array}$ & \\
\hline $\mathrm{H}$ & 1001 & 1000 & $\overline{0.080716}$ & $\overline{0.597039}$ & 0.049118 & \\
\hline $\mathrm{C}$ & 6000 & 6000 & 0.321164 & 0.199359 & 0.016401 & \\
\hline $\mathrm{O}$ & 8016 & 8000 & 0.223545 & 0.104169 & 0.008570 & \\
\hline $\mathrm{Si}$ & 14000 & 14000 & 0.374575 & 0.099434 & 0.008180 & \\
\hline Total & & & 1.000000 & 1.000000 & 0.082269 & \\
\hline MCNP Form & \multicolumn{2}{|c|}{ Weight Fractions } & \multicolumn{2}{|c|}{ Atom Fractions } & \multicolumn{2}{|c|}{ Atom Densities } \\
\hline \multirow[t]{4}{*}{ Neutrons } & 1001 & -0.080716 & 1001 & 0.597039 & 1001 & 0.049118 \\
\hline & 6000 & -0.321164 & 6000 & 0.199359 & 6000 & 0.016401 \\
\hline & 8016 & -0.223545 & 8016 & 0.104169 & 8016 & 0.008570 \\
\hline & 14000 & -0.374575 & 14000 & 0.099434 & 14000 & 0.008180 \\
\hline \multirow[t]{4}{*}{ Photons } & 1000 & -0.080716 & 1000 & 0.597039 & 1000 & 0.049118 \\
\hline & 6000 & -0.321164 & 6000 & 0.199359 & 6000 & 0.016401 \\
\hline & 8000 & -0.223545 & 8000 & 0.104169 & 8000 & 0.008570 \\
\hline & 14000 & -0.374575 & 14000 & 0.099434 & 14000 & 0.008180 \\
\hline \multirow[t]{5}{*}{ CEPXS Form: } & material & $\mathrm{H}$ & 0.080716 & & & \\
\hline & & C & 0.321164 & & & \\
\hline & & $\mathrm{O}$ & 0.223545 & & & \\
\hline & & $\mathrm{Si}$ & 0.374575 & & & \\
\hline & $\begin{array}{l}\text { matname } \\
\text { density }\end{array}$ & $\begin{array}{c}\text { Rubber, Silicon } \\
1.018500\end{array}$ & & & & \\
\hline
\end{tabular}




\section{Salt Water $\left(\mathrm{T}=0^{\circ} \mathrm{C}\right)$}

\begin{tabular}{llll}
\hline Formula $=$ & $\mathrm{H} 2 \mathrm{O}: \mathrm{NaCl}$ & Molecular weight $(\mathrm{g} / \mathrm{mole})=$ \\
Density $(\mathrm{g} / \mathrm{cm} 3)=$ & 1.209865 & Total atom density $($ atoms $/ \mathrm{b}-\mathrm{cm})=$ & - \\
\hline & $9.600 \mathrm{E}-02$
\end{tabular}

The above density is estimated to be accurate to 4 significant digits. Uncertainties are not addressed.

The following data were calculated from the input weight fractions.

\begin{tabular}{|c|c|c|c|c|c|c|}
\hline Element & Neutron ZA & Photon ZA & $\begin{array}{l}\text { Weight } \\
\text { Fraction }\end{array}$ & $\begin{array}{l}\text { Atom } \\
\text { Fraction }\end{array}$ & $\begin{array}{l}\text { Atom } \\
\text { Density }\end{array}$ & \\
\hline $\mathrm{H}$ & 1001 & 1000 & $\overline{0.082491}$ & $\overline{0.621161}$ & 0.059630 & \\
\hline $\mathrm{O}$ & 8016 & 8000 & 0.654709 & 0.310581 & 0.029815 & \\
\hline $\mathrm{Na}$ & 11023 & 11000 & 0.103378 & 0.034129 & 0.003276 & \\
\hline $\mathrm{Cl}$ & 17000 & 17000 & 0.159422 & 0.034129 & 0.003276 & \\
\hline Total & & & 1.000000 & 1.000000 & 0.095997 & \\
\hline MCNP Form & \multicolumn{2}{|c|}{ Weight Fractions } & \multicolumn{2}{|c|}{ Atom Fractions } & \multicolumn{2}{|c|}{ Atom Densities } \\
\hline \multirow{4}{*}{ Neutrons } & 1001 & -0.082491 & 1001 & 0.621161 & 1001 & 0.059630 \\
\hline & 8016 & -0.654709 & 8016 & 0.310581 & 8016 & 0.029815 \\
\hline & 11023 & -0.103378 & 11023 & 0.034129 & 11023 & 0.003276 \\
\hline & 17000 & -0.159422 & 17000 & 0.034129 & 17000 & 0.003276 \\
\hline \multirow[t]{4}{*}{ Photons } & 1000 & -0.082491 & 1000 & 0.621161 & 1000 & 0.059630 \\
\hline & 8000 & -0.654709 & 8000 & 0.310581 & 8000 & 0.029815 \\
\hline & 11000 & -0.103378 & 11000 & 0.034129 & 11000 & 0.003276 \\
\hline & 17000 & -0.159422 & 17000 & 0.034129 & 17000 & 0.003276 \\
\hline \multirow[t]{5}{*}{ CEPXS Form: } & material & $\mathrm{H}$ & 0.082491 & & & \\
\hline & & 0 & 0.654709 & & & \\
\hline & & $\mathrm{Na}$ & 0.103378 & & & \\
\hline & & $\mathrm{Cl}$ & 0.159422 & & & \\
\hline & $\begin{array}{l}\text { matname } \\
\text { density }\end{array}$ & \multicolumn{3}{|c|}{$\begin{array}{l}\text { Salt Water }\left(T=0^{\circ} \mathrm{C}\right) \\
1.209865\end{array}$} & & \\
\hline Con & eferences & & & & & \\
\hline
\end{tabular}

\section{Salt Water $\left(T=20^{\circ} \mathrm{C}\right)$}

\begin{tabular}{|c|c|c|c|}
\hline & $\mathrm{H} 2 \mathrm{O}: \mathrm{NaCl}$ & Molecular weight $(\mathrm{g} / \mathrm{mole})=$ & - \\
\hline Density $(\mathrm{g} / \mathrm{cm} 3)=$ & 1.022394 & Total atom density $($ atoms $/ \mathrm{b}-\mathrm{cm})=$ & 9.978E-02 \\
\hline
\end{tabular}

The above density is estimated to be accurate to 4 significant digits. Uncertainties are not addressed.

The following data were calculated from the input weight fractions.

$\begin{array}{llllll}\text { Element } & \text { Neutron ZA } & \frac{1}{\text { Weight }} & \begin{array}{c}\text { Atom } \\ \text { Photon ZA }\end{array} & \frac{\begin{array}{c}\text { Fraction } \\ \text { Fraction }\end{array}}{0.108114} & \frac{\text { Atom }}{\text { Density }} \\ 0.661906 & 0.066042\end{array}$


PIET-43741-TM-963

PNNL-15870 Rev. 1

\begin{tabular}{|c|c|c|c|c|c|c|}
\hline $\mathrm{O}$ & 8016 & 8000 & 0.858069 & 0.330953 & \multicolumn{2}{|l|}{0.033021} \\
\hline $\mathrm{Na}$ & 11023 & 11000 & 0.013302 & 0.003571 & \multicolumn{2}{|l|}{0.000356} \\
\hline $\mathrm{Cl}$ & 17000 & 17000 & 0.020514 & 0.003571 & \multicolumn{2}{|c|}{0.000356} \\
\hline Total & & & 1.000000 & 1.000000 & \multicolumn{2}{|l|}{0.099775} \\
\hline MCNP Form & \multicolumn{2}{|c|}{ Weight Fractions } & \multicolumn{2}{|c|}{ Atom Fractions } & \multicolumn{2}{|c|}{ Atom Densities } \\
\hline \multirow[t]{4}{*}{ Neutrons } & 1001 & -0.108114 & 1001 & 0.661906 & 1001 & 0.066042 \\
\hline & 8016 & -0.858069 & 8016 & 0.330953 & 8016 & 0.033021 \\
\hline & 11023 & -0.013302 & 11023 & 0.003571 & 11023 & 0.000356 \\
\hline & 17000 & -0.020514 & 17000 & 0.003571 & 17000 & 0.000356 \\
\hline \multirow[t]{4}{*}{ Photons } & 1000 & -0.108114 & 1000 & 0.661906 & 1000 & 0.066042 \\
\hline & 8000 & -0.858069 & 8000 & 0.330953 & 8000 & 0.033021 \\
\hline & 11000 & -0.013302 & 11000 & 0.003571 & 11000 & 0.000356 \\
\hline & 17000 & -0.020514 & 17000 & 0.003571 & 17000 & 0.000356 \\
\hline \multirow[t]{5}{*}{ CEPXS Form: } & material & $\mathrm{H}$ & 0.108114 & & & \\
\hline & & $\mathrm{O}$ & 0.858069 & & & \\
\hline & & $\mathrm{Na}$ & 0.013302 & & & \\
\hline & & $\mathrm{Cl}$ & 0.020514 & & & \\
\hline & $\begin{array}{l}\text { matname } \\
\text { density }\end{array}$ & \multicolumn{2}{|c|}{$\begin{array}{l}\text { Salt Water }\left(T=20^{\circ} \mathrm{C}\right) \\
1.022394\end{array}$} & & & \\
\hline \multicolumn{5}{|c|}{ Comments and References } & & \\
\hline
\end{tabular}

\section{Sand}

Formula $=$ Molecular weight $(\mathrm{g} / \mathrm{mole})=$

Density $(\mathrm{g} / \mathrm{cm} 3)=1.700000 \quad$ Total atom density $($ atoms $/ \mathrm{b}-\mathrm{cm})=5.876 \mathrm{E}-02$

The above density is estimated to be accurate to 2 significant digits. Uncertainties are not addressed.

The following data were calculated from the input weight fractions.

\begin{tabular}{|c|c|c|c|c|c|}
\hline Element & Neutron ZA & Photon ZA & $\begin{array}{l}\text { Weight } \\
\text { Fraction }\end{array}$ & $\begin{array}{c}\text { Atom } \\
\text { Fraction }\end{array}$ & $\begin{array}{c}\text { Atom } \\
\text { Density }\end{array}$ \\
\hline $\mathrm{H}$ & 1001 & 1000 & $\overline{0.007833}$ & 0.135405 & 0.007956 \\
\hline C & 6000 & 6000 & 0.003360 & 0.004874 & 0.000286 \\
\hline O & 8016 & 8000 & 0.536153 & 0.583890 & 0.034307 \\
\hline $\mathrm{Na}$ & 11023 & 11000 & 0.017063 & 0.012932 & 0.000760 \\
\hline $\mathrm{Al}$ & 13027 & 13000 & 0.034401 & 0.022215 & 0.001305 \\
\hline $\mathrm{Si}$ & 14000 & 14000 & 0.365067 & 0.226483 & 0.013307 \\
\hline $\mathrm{K}$ & 19000 & 19000 & 0.011622 & 0.005179 & 0.000304 \\
\hline $\mathrm{Ca}$ & 20000 & 20000 & 0.011212 & 0.004874 & 0.000286 \\
\hline $\mathrm{Fe}$ & 26000 & 26000 & 0.013289 & 0.004146 & 0.000244 \\
\hline Total & & & 1.000000 & 1.000000 & 0.058756 \\
\hline
\end{tabular}


PIET-43741-TM-963

PNNL-15870 Rev. 1

\begin{tabular}{|c|c|c|c|c|c|c|}
\hline MCNP Form & \multicolumn{2}{|c|}{ Weight Fractions } & \multicolumn{2}{|c|}{ Atom Fractions } & \multicolumn{2}{|c|}{ Atom Densities } \\
\hline \multirow[t]{9}{*}{ Neutrons } & 1001 & -0.007833 & 1001 & 0.135405 & 1001 & 0.007956 \\
\hline & 6000 & -0.003360 & 6000 & 0.004874 & 6000 & 0.000286 \\
\hline & 8016 & -0.536153 & 8016 & 0.583890 & 8016 & 0.034307 \\
\hline & 11023 & -0.017063 & 11023 & 0.012932 & 11023 & 0.000760 \\
\hline & 13027 & -0.034401 & 13027 & 0.022215 & 13027 & 0.001305 \\
\hline & 14000 & -0.365067 & 14000 & 0.226483 & 14000 & 0.013307 \\
\hline & 19000 & -0.011622 & 19000 & 0.005179 & 19000 & 0.000304 \\
\hline & 20000 & -0.011212 & 20000 & 0.004874 & 20000 & 0.000286 \\
\hline & 26000 & -0.013289 & 26000 & 0.004146 & 26000 & 0.000244 \\
\hline \multirow[t]{9}{*}{ Photons } & 1000 & -0.007833 & 1000 & 0.135405 & 1000 & 0.007956 \\
\hline & 6000 & -0.003360 & 6000 & 0.004874 & 6000 & 0.000286 \\
\hline & 8000 & -0.536153 & 8000 & 0.583890 & 8000 & 0.034307 \\
\hline & 11000 & -0.017063 & 11000 & 0.012932 & 11000 & 0.000760 \\
\hline & 13000 & -0.034401 & 13000 & 0.022215 & 13000 & 0.001305 \\
\hline & 14000 & -0.365067 & 14000 & 0.226483 & 14000 & 0.013307 \\
\hline & 19000 & -0.011622 & 19000 & 0.005179 & 19000 & 0.000304 \\
\hline & 20000 & -0.011212 & 20000 & 0.004874 & 20000 & 0.000286 \\
\hline & 26000 & -0.013289 & 26000 & 0.004146 & 26000 & 0.000244 \\
\hline \multirow[t]{10}{*}{ CEPXS Form: } & material & $\mathrm{H}$ & 0.007833 & & & \\
\hline & & $\mathrm{C}$ & 0.003360 & & & \\
\hline & & $\mathrm{O}$ & 0.536153 & & & \\
\hline & & $\mathrm{Na}$ & 0.017063 & & & \\
\hline & & $\mathrm{Al}$ & 0.034401 & & & \\
\hline & & $\mathrm{Si}$ & 0.365067 & & & \\
\hline & & $\mathrm{K}$ & 0.011622 & & & \\
\hline & & $\mathrm{Ca}$ & 0.011212 & & & \\
\hline & & $\mathrm{Fe}$ & 0.013289 & & & \\
\hline & $\begin{array}{c}\text { matname } \\
\text { density }\end{array}$ & $\begin{array}{l}\text { Sand } \\
1.700000\end{array}$ & & & & \\
\hline \multicolumn{7}{|c|}{$\begin{array}{l}\text { Comments and References } \\
\text { Element weight fractions calculated based on } 78.1 \mathrm{wt} \% \mathrm{SiO} 2,6.5 \% \mathrm{Al} 2 \mathrm{O} 3,1.9 \% \mathrm{Fe} 2 \mathrm{O} 3,2.8 \% \mathrm{CaCO} \text {, } \\
2.3 \% \mathrm{Na} 2 \mathrm{O}, 1.4 \% \mathrm{~K} 2 \mathrm{O} \text {, and } 7.0 \% \mathrm{H} 2 \mathrm{O} \text { from Table } 51.62 \text { of Hungerford (1960). Density }=1.7 \mathrm{~g} / \mathrm{cm} 3 \mathrm{for} \\
\text { normal sand (4 to } 23 \mathrm{wt} \% \text { water) at } \\
\text { http://www.matweb.com/search/DataSheet.aspx?MatGUID=ce6e6b2274534e35b6a14945e778e391 and } \\
1.65 \mathrm{~g} / \mathrm{cm} 3 \text { for totally dry sand at } \\
\text { http://www.matweb.com/search/DataSheet.aspx?MatGUID=18a1e365613b478f880e5506d6fb2ec1 } \\
\text { (Automation Creations } 2010 \text { ). Data for sand density is at http://www.simetric.co.uk/si_materials.htm } \\
\text { (Walker 2009), http://www.powderandbulk.com/resources/bulk_density/material_bulk_density_chart_s.htm } \\
\text { (Powder and Bulk Dot Com 2010), and in Hungerford (1960). }\end{array}$} \\
\hline
\end{tabular}

\section{Sea Water, Simple Artificial}

\begin{tabular}{|llll}
\hline Formula $=$ & - & Molecular weight $(\mathrm{g} / \mathrm{mole})=$ \\
Density $(\mathrm{g} / \mathrm{cm} 3)=$ & 1.023343 & Total atom density $($ atoms $/ \mathrm{b}-\mathrm{cm})=$ & - \\
$9.979 \mathrm{E}-02$
\end{tabular}


The above density is estimated to be accurate to 4 significant digits. Uncertainties are not addressed. The following data were calculated from the input weight fractions.

\begin{tabular}{|c|c|c|c|c|c|}
\hline Element & Neutron ZA & Photon ZA & $\begin{array}{l}\text { Weight } \\
\text { Fraction }\end{array}$ & $\begin{array}{c}\text { Atom } \\
\text { Fraction }\end{array}$ & $\begin{array}{c}\text { Atom } \\
\text { Density }\end{array}$ \\
\hline$\overline{\mathrm{H}}$ & 1001 & 1000 & $\overline{0.107974}$ & $\overline{0.661590}$ & 0.066017 \\
\hline 0 & 8016 & 8000 & 0.858765 & 0.331493 & 0.033078 \\
\hline $\mathrm{Na}$ & 11023 & 11000 & 0.010785 & 0.002897 & 0.000289 \\
\hline $\mathrm{Mg}$ & 12000 & 12000 & 0.001284 & 0.000326 & 0.000033 \\
\hline$S$ & 16000 & 16000 & 0.000906 & 0.000174 & 0.000017 \\
\hline $\mathrm{Cl}$ & 17000 & 17000 & 0.019472 & 0.003392 & 0.000338 \\
\hline $\mathrm{K}$ & 19000 & 19000 & 0.000399 & 0.000063 & 0.000006 \\
\hline $\mathrm{Ca}$ & 20000 & 20000 & 0.000415 & 0.000064 & 0.000006 \\
\hline
\end{tabular}

Total

$1.000000 \quad 1.000000 \quad 0.099786$

\begin{tabular}{|c|c|c|c|c|c|c|}
\hline MCNP Form & \multicolumn{2}{|c|}{ Weight Fractions } & \multicolumn{2}{|c|}{ Atom Fractions } & \multicolumn{2}{|c|}{ Atom Densities } \\
\hline \multirow{8}{*}{ Neutrons } & 1001 & -0.107974 & 1001 & 0.661590 & 1001 & 0.066017 \\
\hline & 8016 & -0.858765 & 8016 & 0.331493 & 8016 & 0.033078 \\
\hline & 11023 & -0.010785 & 11023 & 0.002897 & 11023 & 0.000289 \\
\hline & 12000 & -0.001284 & 12000 & 0.000326 & 12000 & 0.000033 \\
\hline & 16000 & -0.000906 & 16000 & 0.000174 & 16000 & 0.000017 \\
\hline & 17000 & -0.019472 & 17000 & 0.003392 & 17000 & 0.000338 \\
\hline & 19000 & -0.000399 & 19000 & 0.000063 & 19000 & 0.000006 \\
\hline & 20000 & -0.000415 & 20000 & 0.000064 & 20000 & 0.000006 \\
\hline \multirow[t]{8}{*}{ Photons } & 1000 & -0.107974 & 1000 & 0.661590 & 1000 & 0.066017 \\
\hline & 8000 & -0.858765 & 8000 & 0.331493 & 8000 & 0.033078 \\
\hline & 11000 & -0.010785 & 11000 & 0.002897 & 11000 & 0.000289 \\
\hline & 12000 & -0.001284 & 12000 & 0.000326 & 12000 & 0.000033 \\
\hline & 16000 & -0.000906 & 16000 & 0.000174 & 16000 & 0.000017 \\
\hline & 17000 & -0.019472 & 17000 & 0.003392 & 17000 & 0.000338 \\
\hline & 19000 & -0.000399 & 19000 & 0.000063 & 19000 & 0.000006 \\
\hline & 20000 & -0.000415 & 20000 & 0.000064 & 20000 & 0.000006 \\
\hline \multirow[t]{9}{*}{ CEPXS Form: } & material & $\mathrm{H}$ & 0.107974 & & & \\
\hline & & $\mathrm{O}$ & 0.858765 & & & \\
\hline & & $\mathrm{Na}$ & 0.010785 & & & \\
\hline & & $\mathrm{Mg}$ & 0.001284 & & & \\
\hline & & $S$ & 0.000906 & & & \\
\hline & & $\mathrm{Cl}$ & 0.019472 & & & \\
\hline & & $\mathrm{K}$ & 0.000399 & & & \\
\hline & & $\mathrm{Ca}$ & 0.000415 & & & \\
\hline & $\begin{array}{l}\text { matname } \\
\text { density }\end{array}$ & \multicolumn{3}{|c|}{$\begin{array}{l}\text { Sea Water, Simple Artificial } \\
1.023343\end{array}$} & & \\
\hline \multicolumn{5}{|c|}{ Comments and References } & & \\
\hline
\end{tabular}




\section{Sea Water, Standard}

\begin{tabular}{|c|c|c|c|}
\hline Formula $=$ & - & Molecular weight ( $\mathrm{g} / \mathrm{mole})=$ & - \\
\hline Density $(\mathrm{g} / \mathrm{cm} 3)=$ & 1.023343 & Total atom density $($ atoms $/ \mathrm{b}-\mathrm{cm})=$ & 9.979E-02 \\
\hline
\end{tabular}

\begin{tabular}{|c|c|c|c|c|c|c|}
\hline Element & Neutron ZA & Photon ZA & $\begin{array}{l}\text { Weight } \\
\text { Fraction }\end{array}$ & $\begin{array}{c}\text { Atom } \\
\text { Fraction }\end{array}$ & $\begin{array}{l}\text { Atom } \\
\text { Density }\end{array}$ & \\
\hline $\mathrm{H}$ & 1001 & 1000 & 0.107979 & 0.661599 & 0.066020 & \\
\hline$B$ & - & 5000 & 0.000005 & 0.000003 & 0.000000 & \\
\hline $\mathrm{O}$ & 8016 & 8000 & 0.858803 & 0.331497 & 0.033080 & \\
\hline $\mathrm{F}$ & 9019 & 9000 & 0.000001 & 0.000000 & 0.000000 & \\
\hline $\mathrm{Na}$ & 11023 & 11000 & 0.010784 & 0.002897 & 0.000289 & \\
\hline $\mathrm{Mg}$ & 12000 & 12000 & 0.001284 & 0.000326 & 0.000033 & \\
\hline $\mathrm{S}$ & 16000 & 16000 & 0.000905 & 0.000174 & 0.000017 & \\
\hline $\mathrm{Cl}$ & 17000 & 17000 & 0.019352 & 0.003371 & 0.000336 & \\
\hline $\mathrm{K}$ & 19000 & 19000 & 0.000399 & 0.000063 & 0.000006 & \\
\hline $\mathrm{Ca}$ & 20000 & 20000 & 0.000412 & 0.000064 & 0.000006 & \\
\hline $\mathrm{Br}$ & - & 35000 & 0.000067 & 0.000005 & 0.000001 & \\
\hline $\mathrm{Sr}$ & - & 38000 & 0.000008 & 0.000001 & 0.000000 & \\
\hline Total & & & 1.000000 & 1.000000 & 0.099789 & \\
\hline MCNP Form & \multicolumn{2}{|c|}{ Weight Fractions } & \multicolumn{2}{|c|}{ Atom Fractions } & \multicolumn{2}{|c|}{ Atom Densities } \\
\hline \multirow[t]{12}{*}{ Neutrons } & 1001 & -0.107979 & 1001 & 0.661599 & 1001 & 0.066020 \\
\hline & - & -0.000005 & - & 0.000003 & - & 0.000000 \\
\hline & 8016 & -0.858803 & 8016 & 0.331497 & 8016 & 0.033080 \\
\hline & 9019 & -0.000001 & 9019 & 0.000000 & 9019 & 0.000000 \\
\hline & 11023 & -0.010784 & 11023 & 0.002897 & 11023 & 0.000289 \\
\hline & 12000 & -0.001284 & 12000 & 0.000326 & 12000 & 0.000033 \\
\hline & 16000 & -0.000905 & 16000 & 0.000174 & 16000 & 0.000017 \\
\hline & 17000 & -0.019352 & 17000 & 0.003371 & 17000 & 0.000336 \\
\hline & 19000 & -0.000399 & 19000 & 0.000063 & 19000 & 0.000006 \\
\hline & 20000 & -0.000412 & 20000 & 0.000064 & 20000 & 0.000006 \\
\hline & - & -0.000067 & - & 0.000005 & - & 0.000001 \\
\hline & - & -0.000008 & - & 0.000001 & - & 0.000000 \\
\hline \multirow[t]{12}{*}{ Photons } & 1000 & -0.107979 & 1000 & 0.661599 & 1000 & 0.066020 \\
\hline & 5000 & -0.000005 & 5000 & 0.000003 & 5000 & 0.000000 \\
\hline & 8000 & -0.858803 & 8000 & 0.331497 & 8000 & 0.033080 \\
\hline & 9000 & -0.000001 & 9000 & 0.000000 & 9000 & 0.000000 \\
\hline & 11000 & -0.010784 & 11000 & 0.002897 & 11000 & 0.000289 \\
\hline & 12000 & -0.001284 & 12000 & 0.000326 & 12000 & 0.000033 \\
\hline & 16000 & -0.000905 & 16000 & 0.000174 & 16000 & 0.000017 \\
\hline & 17000 & -0.019352 & 17000 & 0.003371 & 17000 & 0.000336 \\
\hline & 19000 & -0.000399 & 19000 & 0.000063 & 19000 & 0.000006 \\
\hline & 20000 & -0.000412 & 20000 & 0.000064 & 20000 & 0.000006 \\
\hline & 35000 & -0.000067 & 35000 & 0.000005 & 35000 & 0.000001 \\
\hline & 38000 & -0.000008 & 38000 & 0.000001 & 38000 & 0.000000 \\
\hline
\end{tabular}




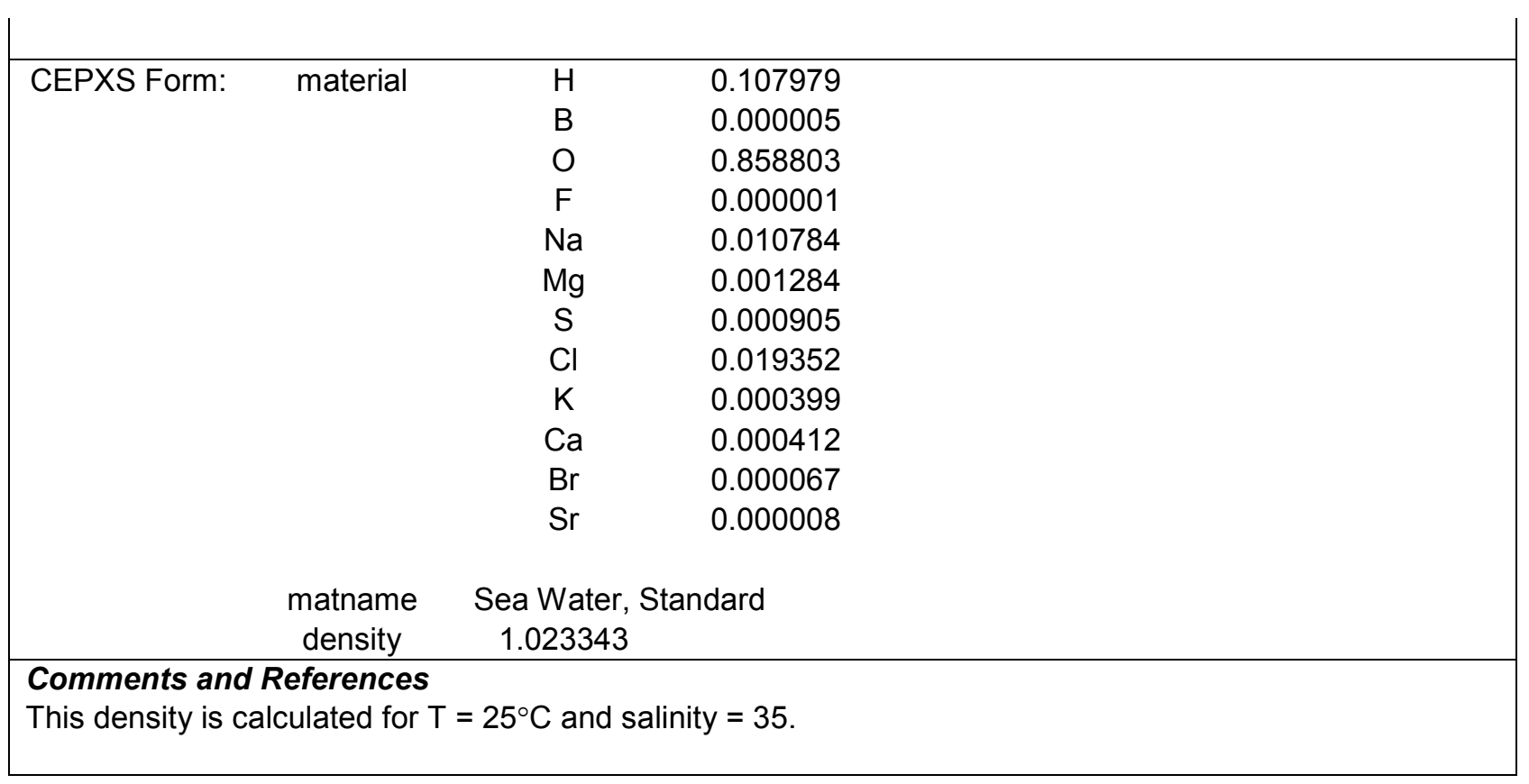

\section{Sepiolite}

\begin{tabular}{|c|c|c|c|}
\hline Formula $=$ & $\begin{array}{l}\text { Mg4Si6O15(OH)2- } \\
\text { 6(H2O) }\end{array}$ & Molecular weight $(\mathrm{g} / \mathrm{mole})=$ & 647.83036 \\
\hline Density $(\mathrm{g} / \mathrm{cm} 3)=$ & 2.140000 & Total atom density $($ atoms $/ \mathrm{b}-\mathrm{cm})=$ & 9.350E-02 \\
\hline
\end{tabular}

The above density is estimated to be accurate to 3 significant digits. Uncertainties are not addressed.

The following data was calculated from the input formula.

\begin{tabular}{|c|c|c|c|c|c|c|}
\hline Element & Neutron ZA & Photon ZA & $\begin{array}{l}\text { Weight } \\
\text { Fraction }\end{array}$ & $\begin{array}{l}\text { Atom } \\
\text { Fraction }\end{array}$ & $\begin{array}{l}\text { Atom } \\
\text { Density }\end{array}$ & \\
\hline $\mathrm{H}$ & 1001 & 1000 & 0.021782 & 0.297872 & 0.027850 & \\
\hline $\mathrm{O}$ & 8016 & 8000 & 0.568029 & 0.489362 & 0.045754 & \\
\hline $\mathrm{Mg}$ & 12000 & 12000 & 0.150070 & 0.085106 & 0.007957 & \\
\hline $\mathrm{Si}$ & 14000 & 14000 & 0.260119 & 0.127660 & 0.011936 & \\
\hline Total & & & 1.000000 & 1.000000 & 0.093498 & \\
\hline MCNP Form & \multicolumn{2}{|c|}{ Weight Fractions } & \multicolumn{2}{|c|}{ Atom Fractions } & \multicolumn{2}{|c|}{ Atom Densities } \\
\hline \multirow[t]{4}{*}{ Neutrons } & 1001 & -0.021782 & 1001 & 0.297872 & 1001 & 0.027850 \\
\hline & 8016 & -0.568029 & 8016 & 0.489362 & 8016 & 0.045754 \\
\hline & 12000 & -0.150070 & 12000 & 0.085106 & 12000 & 0.007957 \\
\hline & 14000 & -0.260119 & 14000 & 0.127660 & 14000 & 0.011936 \\
\hline \multirow[t]{4}{*}{ Photons } & 1000 & -0.021782 & 1000 & 0.297872 & 1000 & 0.027850 \\
\hline & 8000 & -0.568029 & 8000 & 0.489362 & 8000 & 0.045754 \\
\hline & 12000 & -0.150070 & 12000 & 0.085106 & 12000 & 0.007957 \\
\hline & 14000 & -0.260119 & 14000 & 0.127660 & 14000 & 0.011936 \\
\hline
\end{tabular}




\begin{tabular}{|c|c|c|c|}
\hline \multirow[t]{4}{*}{ CEPXS Form: } & \multirow[t]{4}{*}{ material } & $\mathrm{H}$ & 0.021782 \\
\hline & & $\mathrm{O}$ & 0.568029 \\
\hline & & $\mathrm{Mg}$ & 0.150070 \\
\hline & & $\mathrm{Si}$ & 0.260119 \\
\hline
\end{tabular}

matname Sepiolite

density $\quad 2.140000$

\section{Comments and References}

Non-clumping cat litter is often made of zeolite, diatomaceous earth, and/or sepiolite.

Formula from http://webmineral.com/data/Sepiolite.shtml and

http://rpd.oxfordjournals.org/content/131/3/390.full.

Density $=2.14$ and $2.18 \mathrm{~g} / \mathrm{cm} 3$ at http://webmineral.com $/$ data/Sepiolite.$s h t m l$. Density $=2.08 \mathrm{~g} / \mathrm{cm} 3$ at www.minersoc.org/pages/Archive-CM/Volume_34/34-4-647.pdf.

\section{Silicon}

\begin{tabular}{llll}
\hline Formula $=$ & $\mathrm{Si}$ & Molecular weight $(\mathrm{g} / \mathrm{mole})=$ & 28.0855 \\
Density $(\mathrm{g} / \mathrm{cm} 3)=$ & 2.330000 & Total atom density $($ atoms $/ \mathrm{b}-\mathrm{cm})=$ & $4.996 \mathrm{E}-02$
\end{tabular}

The above density is estimated to be accurate to 3 significant digits. Uncertainties are not addressed.

The following data was calculated from the input formula.

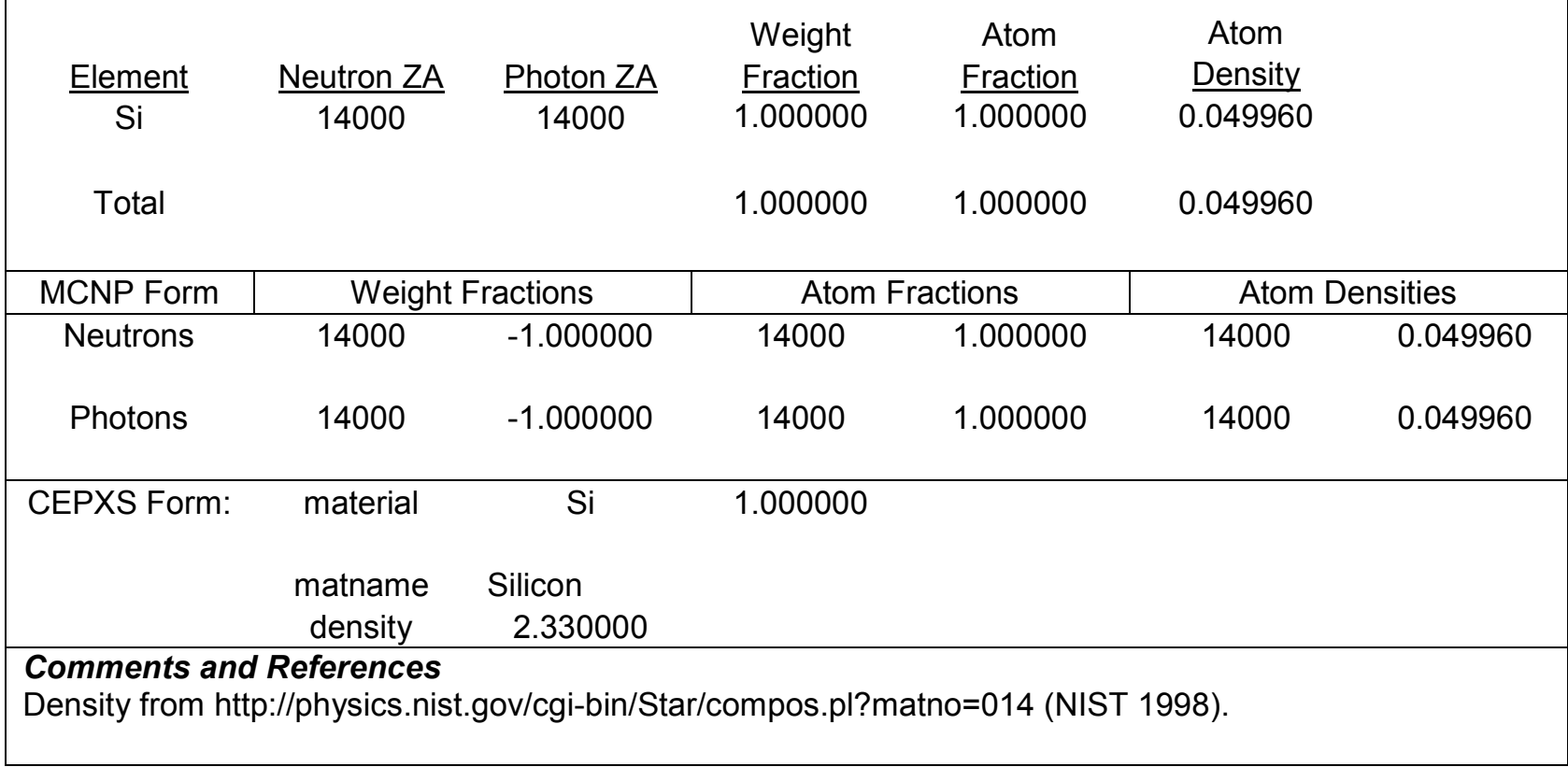

\section{Silicon Carbide (Hexagonal)}

\begin{tabular}{llll}
\hline Formula $=$ & SiC & Molecular weight $(\mathrm{g} / \mathrm{mole})=$ & 40.0962 \\
Density $(\mathrm{g} / \mathrm{cm} 3)=$ & 3.210000 & Total atom density $($ atoms $/ \mathrm{b}-\mathrm{cm})=$ & $9.642 \mathrm{E}-02$
\end{tabular}

The above density is estimated to be accurate to 3 significant digits. Uncertainties are not addressed.

The following data was calculated from the input formula. 


\begin{tabular}{|c|c|c|c|c|c|c|}
\hline Element & Neutron ZA & Photon ZA & $\begin{array}{l}\text { Weight } \\
\text { Fraction }\end{array}$ & $\begin{array}{c}\text { Atom } \\
\text { Fraction }\end{array}$ & $\begin{array}{c}\text { Atom } \\
\text { Density }\end{array}$ & \\
\hline C & 6000 & 6000 & 0.299547 & 0.500000 & 0.048212 & \\
\hline Si & 14000 & 14000 & 0.700453 & 0.500000 & 0.048212 & \\
\hline Total & & & 1.000000 & 1.000000 & 0.096423 & \\
\hline MCNP Form & \multicolumn{2}{|c|}{ Weight Fractions } & \multicolumn{2}{|c|}{ Atom Fractions } & \multicolumn{2}{|c|}{ Atom Densities } \\
\hline Neutrons & $\begin{array}{c}6000 \\
14000\end{array}$ & $\begin{array}{l}-0.299547 \\
-0.700453\end{array}$ & $\begin{array}{l}6000 \\
14000\end{array}$ & $\begin{array}{l}0.500000 \\
0.500000\end{array}$ & $\begin{array}{c}6000 \\
14000\end{array}$ & $\begin{array}{l}0.048212 \\
0.048212\end{array}$ \\
\hline Photons & $\begin{array}{c}6000 \\
14000\end{array}$ & $\begin{array}{l}-0.299547 \\
-0.700453\end{array}$ & $\begin{array}{c}6000 \\
14000\end{array}$ & $\begin{array}{l}0.500000 \\
0.500000\end{array}$ & $\begin{array}{c}6000 \\
14000\end{array}$ & $\begin{array}{l}0.048212 \\
0.048212\end{array}$ \\
\hline CEPXS Form: & $\begin{array}{l}\text { matname } \\
\text { density }\end{array}$ & $\begin{array}{c}\text { C } \\
\text { Si } \\
\text { Silicon Carbic } \\
3.210000\end{array}$ & $\begin{array}{l}0.299547 \\
0.700453 \\
\\
\text { (Hexagonal) }\end{array}$ & & & \\
\hline $\begin{array}{l}\text { Comments an } \\
\text { Density and for } \\
\text { W Alexander, } 3 \\
\text { Also known as }\end{array}$ & $\begin{array}{l}\text { References } \\
\text { ula from CRC } \\
\text { ed., CRC Pr } \\
\text { rborundum. }\end{array}$ & $\begin{array}{l}\text { Materials Sci } \\
\text { s, } 2001 .\end{array}$ & and Engine & landbook & Shack & \\
\hline
\end{tabular}

\section{Silicon Dioxide (Alpha-quartz)}

\begin{tabular}{llll}
\hline Formula $=$ & $\mathrm{SiO} 2$ & Molecular weight $(\mathrm{g} / \mathrm{mole})=$ & 60.0843 \\
Density $(\mathrm{g} / \mathrm{cm} 3)=$ & 2.648000 & Total atom density $($ atoms $/ \mathrm{b}-\mathrm{cm})=$ & $7.962 \mathrm{E}-02$
\end{tabular}

The above density is estimated to be accurate to 3 significant digits. Uncertainties are not addressed.

The following data was calculated from the input formula.

\begin{tabular}{|c|c|c|c|c|c|c|}
\hline $\begin{array}{c}\text { Element } \\
\mathrm{O} \\
\mathrm{Si}\end{array}$ & $\begin{array}{c}\text { Neutron ZA } \\
8016 \\
14000\end{array}$ & $\begin{array}{c}\text { Photon ZA } \\
8000 \\
14000\end{array}$ & $\begin{array}{c}\text { Weight } \\
\text { Fraction } \\
0.532565 \\
0.467435\end{array}$ & $\begin{array}{c}\text { Atom } \\
\text { Fraction } \\
0.666667 \\
0.333333\end{array}$ & $\begin{array}{c}\text { Atom } \\
\text { Density } \\
0.05308 \\
0.02654\end{array}$ & \\
\hline Total & & & 1.000000 & 1.000000 & 0.07962 & \\
\hline MCNP Form & \multicolumn{2}{|c|}{ Weight Fractions } & \multicolumn{2}{|c|}{ Atom Fractions } & \multicolumn{2}{|c|}{ Atom Densities } \\
\hline Neutrons & $\begin{array}{c}8016 \\
14000\end{array}$ & $\begin{array}{l}-0.532565 \\
-0.467435\end{array}$ & $\begin{array}{c}8016 \\
14000\end{array}$ & $\begin{array}{l}0.666667 \\
0.333333\end{array}$ & $\begin{array}{c}8016 \\
14000\end{array}$ & $\begin{array}{l}0.053081 \\
0.026540\end{array}$ \\
\hline Photons & $\begin{array}{c}8000 \\
14000\end{array}$ & $\begin{array}{l}-0.532565 \\
-0.467435\end{array}$ & $\begin{array}{c}8000 \\
14000\end{array}$ & $\begin{array}{l}0.666667 \\
0.333333\end{array}$ & $\begin{array}{c}8000 \\
14000\end{array}$ & $\begin{array}{l}0.053081 \\
0.026540\end{array}$ \\
\hline CEPXS Form: & material & $\begin{array}{l}\mathrm{O} \\
\mathrm{Si}\end{array}$ & $\begin{array}{l}0.532565 \\
0.467435\end{array}$ & & & \\
\hline
\end{tabular}


matname Silicon Dioxide (Alpha-quartz)

density 2.648000

Comments and References

Density of SiO2 for alpha-quartz $=2.648 \mathrm{~g} / \mathrm{cm} 3$ in Lide (2008), pgs $4-88$, and at

http://www.matweb.com/search/DataSheet.aspx?MatGUID=d5c906beded84f18a394afec8735c2a4

(Automation Creations 2010).

\section{Silicon Dioxide (Silica)}

\begin{tabular}{llll}
\hline Formula $=$ & $\mathrm{SiO} 2$ & Molecular weight $(\mathrm{g} / \mathrm{mole})=$ & 60.0843 \\
Density $(\mathrm{g} / \mathrm{cm} 3)=$ & 2.320000 & Total atom density $($ atoms $/ \mathrm{b}-\mathrm{cm})=$ & $6.976 \mathrm{E}-02$
\end{tabular}

The above density is estimated to be accurate to 3 significant digits. Uncertainties are not addressed.

The following data were calculated from the input weight fractions.

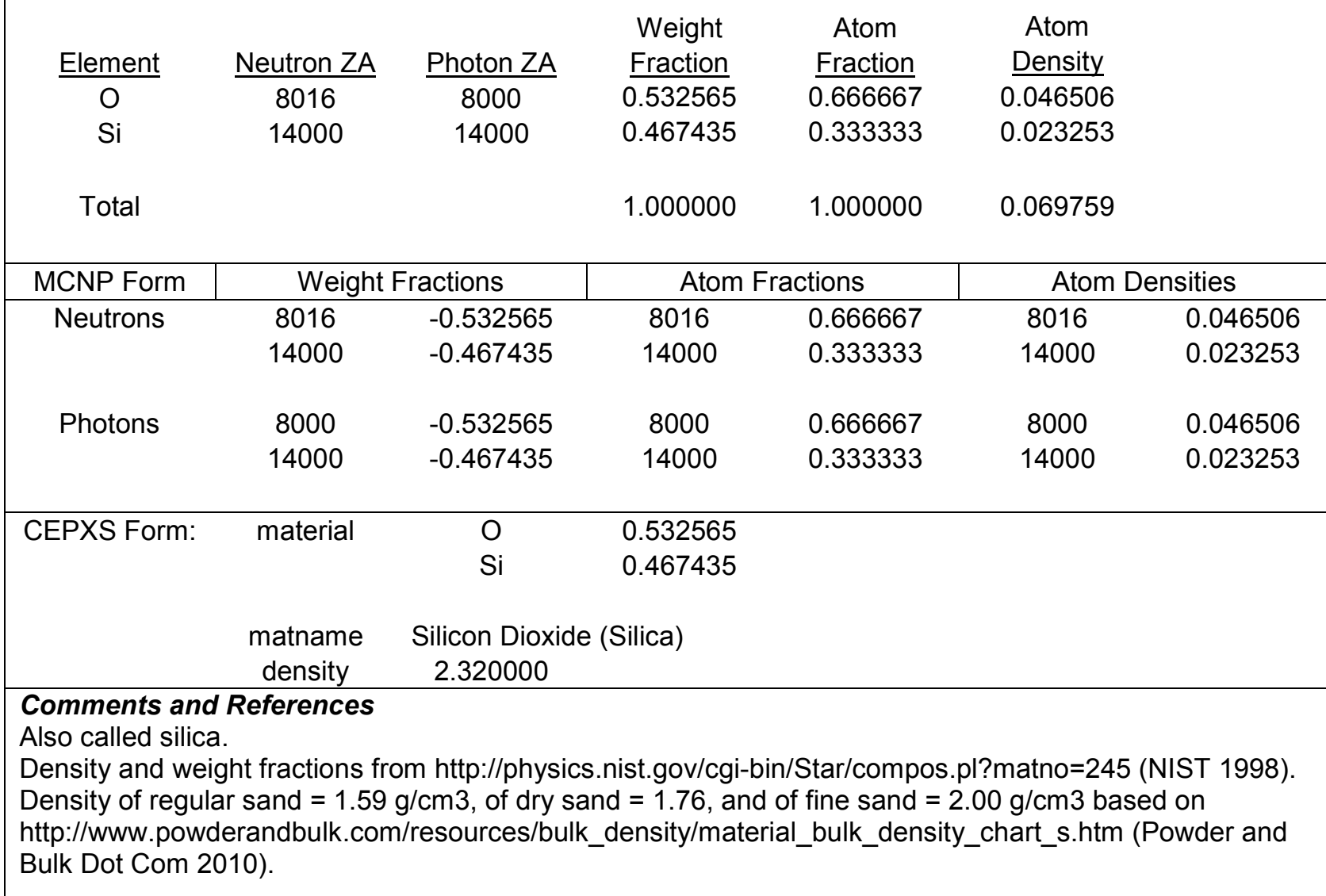

\section{Silver}

Formula $=$

Density $(\mathrm{g} / \mathrm{cm} 3)=10.500000$

The above density is estimated to be accurate to 3 significant digits. Uncertainties are not addressed. 
The following data was calculated from the input formula.

\begin{tabular}{|c|c|c|c|c|c|c|}
\hline$\frac{\text { Element }}{\mathrm{Ag}}$ & $\frac{\text { Neutron ZA }}{47000}$ & $\frac{\text { Photon ZA }}{47000}$ & $\begin{array}{l}\text { Weight } \\
\text { Fraction } \\
1.000000\end{array}$ & $\begin{array}{c}\begin{array}{c}\text { Atom } \\
\text { Fraction }\end{array} \\
1.000000\end{array}$ & $\begin{array}{c}\text { Atom } \\
\text { Density } \\
0.058620\end{array}$ & \\
\hline Total & & & 1.000000 & 1.000000 & 0.058620 & \\
\hline MCNP Form & \multicolumn{2}{|c|}{ Weight Fractions } & \multicolumn{2}{|c|}{ Atom Fractions } & \multicolumn{2}{|c|}{ Atom Densities } \\
\hline Neutrons & 47000 & -1.000000 & 47000 & 1.000000 & 47000 & 0.058620 \\
\hline Photons & 47000 & -1.000000 & 47000 & 1.000000 & 47000 & 0.058620 \\
\hline CEPXS Form: & $\begin{array}{l}\text { material } \\
\text { matname } \\
\text { density }\end{array}$ & $\begin{array}{c}\mathrm{Ag} \\
\text { Silver } \\
10.500000\end{array}$ & 1.000000 & & & \\
\hline $\begin{array}{l}\text { Comments ar } \\
\text { Density from } \mathrm{h}\end{array}$ & $\begin{array}{l}\text { References } \\
\text { /physics.nis }\end{array}$ & & & & & \\
\hline
\end{tabular}

\section{Skin (ICRP)}

\begin{tabular}{llll}
\hline Formula $=$ & - & Molecular weight $(\mathrm{g} / \mathrm{mole})=$ \\
Density $(\mathrm{g} / \mathrm{cm} 3)=$ & 1.100000 & Total atom density $($ atoms $/ \mathrm{b}-\mathrm{cm})=$ & - \\
The & $1.066 \mathrm{E}-01$
\end{tabular}

The above density is estimated to be accurate to 3 significant digits. Uncertainties are not addressed.

The following data were calculated from the input weight fractions.

\begin{tabular}{|c|c|c|c|c|c|}
\hline Flement & Neutron $7 \mathrm{~A}$ & Photon 7A & Weight & Atom & $\begin{array}{l}\text { Atom } \\
\text { Density }\end{array}$ \\
\hline $\mathrm{H}$ & 1001 & 1000 & 0.100588 & 0.619966 & 0.066108 \\
\hline C & 6000 & 6000 & 0.228250 & 0.118059 & 0.012589 \\
\hline $\mathrm{N}$ & 7014 & 7000 & 0.046420 & 0.020589 & 0.002195 \\
\hline 0 & 8016 & 8000 & 0.619002 & 0.240350 & 0.025629 \\
\hline $\mathrm{Na}$ & 11023 & 11000 & 0.000070 & 0.000019 & 0.000002 \\
\hline $\mathrm{Mg}$ & 12000 & 12000 & 0.000060 & 0.000015 & 0.000002 \\
\hline $\mathrm{P}$ & 15031 & 15000 & 0.000330 & 0.000066 & 0.000007 \\
\hline S & 16000 & 16000 & 0.001590 & 0.000308 & 0.000033 \\
\hline $\mathrm{Cl}$ & 17000 & 17000 & 0.002670 & 0.000468 & 0.000050 \\
\hline $\mathrm{K}$ & 19000 & 19000 & 0.000850 & 0.000135 & 0.000014 \\
\hline $\mathrm{Ca}$ & 20000 & 20000 & 0.000150 & 0.000023 & 0.000002 \\
\hline $\mathrm{Fe}$ & 26000 & 26000 & 0.000010 & 0.000001 & 0.000000 \\
\hline $\mathrm{Zn}$ & 30000 & 30000 & 0.000010 & 0.000001 & 0.000000 \\
\hline Total & & & 1.000000 & 1.000000 & 0.106632 \\
\hline
\end{tabular}


PIET-43741-TM-963

PNNL-15870 Rev. 1

\begin{tabular}{|c|c|c|c|c|c|c|}
\hline MCNP Form & \multicolumn{2}{|c|}{ Weight Fractions } & \multicolumn{2}{|c|}{ Atom Fractions } & \multicolumn{2}{|c|}{ Atom Densities } \\
\hline \multirow[t]{13}{*}{ Neutrons } & 1001 & -0.100588 & 1001 & 0.619966 & 1001 & 0.066108 \\
\hline & 6000 & -0.228250 & 6000 & 0.118059 & 6000 & 0.012589 \\
\hline & 7014 & -0.046420 & 7014 & 0.020589 & 7014 & 0.002195 \\
\hline & 8016 & -0.619002 & 8016 & 0.240350 & 8016 & 0.025629 \\
\hline & 11023 & -0.000070 & 11023 & 0.000019 & 11023 & 0.000002 \\
\hline & 12000 & -0.000060 & 12000 & 0.000015 & 12000 & 0.000002 \\
\hline & 15031 & -0.000330 & 15031 & 0.000066 & 15031 & 0.000007 \\
\hline & 16000 & -0.001590 & 16000 & 0.000308 & 16000 & 0.000033 \\
\hline & 17000 & -0.002670 & 17000 & 0.000468 & 17000 & 0.000050 \\
\hline & 19000 & -0.000850 & 19000 & 0.000135 & 19000 & 0.000014 \\
\hline & 20000 & -0.000150 & 20000 & 0.000023 & 20000 & 0.000002 \\
\hline & 26000 & -0.000010 & 26000 & 0.000001 & 26000 & 0.000000 \\
\hline & 30000 & -0.000010 & 30000 & 0.000001 & 30000 & 0.000000 \\
\hline \multirow[t]{13}{*}{ Photons } & 1000 & -0.100588 & 1000 & 0.619966 & 1000 & 0.066108 \\
\hline & 6000 & -0.228250 & 6000 & 0.118059 & 6000 & 0.012589 \\
\hline & 7000 & -0.046420 & 7000 & 0.020589 & 7000 & 0.002195 \\
\hline & 8000 & -0.619002 & 8000 & 0.240350 & 8000 & 0.025629 \\
\hline & 11000 & -0.000070 & 11000 & 0.000019 & 11000 & 0.000002 \\
\hline & 12000 & -0.000060 & 12000 & 0.000015 & 12000 & 0.000002 \\
\hline & 15000 & -0.000330 & 15000 & 0.000066 & 15000 & 0.000007 \\
\hline & 16000 & -0.001590 & 16000 & 0.000308 & 16000 & 0.000033 \\
\hline & 17000 & -0.002670 & 17000 & 0.000468 & 17000 & 0.000050 \\
\hline & 19000 & -0.000850 & 19000 & 0.000135 & 19000 & 0.000014 \\
\hline & 20000 & -0.000150 & 20000 & 0.000023 & 20000 & 0.000002 \\
\hline & 26000 & -0.000010 & 26000 & 0.000001 & 26000 & 0.000000 \\
\hline & 30000 & -0.000010 & 30000 & 0.000001 & 30000 & 0.000000 \\
\hline \multirow[t]{15}{*}{ CEPXS Form: } & material & $\mathrm{H}$ & 0.100588 & & & \\
\hline & & C & 0.228250 & & & \\
\hline & & $\mathrm{N}$ & 0.046420 & & & \\
\hline & & $\mathrm{O}$ & 0.619002 & & & \\
\hline & & $\mathrm{Na}$ & 0.000070 & & & \\
\hline & & $\mathrm{Mg}$ & 0.000060 & & & \\
\hline & & $\mathrm{P}$ & 0.000330 & & & \\
\hline & & $S$ & 0.001590 & & & \\
\hline & & $\mathrm{Cl}$ & 0.002670 & & & \\
\hline & & $\mathrm{K}$ & 0.000850 & & & \\
\hline & & $\mathrm{Ca}$ & 0.000150 & & & \\
\hline & & $\mathrm{Fe}$ & 0.000010 & & & \\
\hline & & $\mathrm{Zn}$ & 0.000010 & & & \\
\hline & matname & Skin (ICRP) & & & & \\
\hline & density & 1.100000 & & & & \\
\hline \multicolumn{7}{|c|}{$\begin{array}{l}\text { Comments and References } \\
\text { Densities and weight fractions from http://physics.nist.gov/cgi-bin/Star/compos.pl?matno=250 } \\
\text { (NIST 1998). }\end{array}$} \\
\hline
\end{tabular}




\section{Sodium}

\begin{tabular}{llll}
\hline Formula $=$ & $\mathrm{Na}$ & Molecular weight $(\mathrm{g} / \mathrm{mole})=$ & 22.98977 \\
Density $(\mathrm{g} / \mathrm{cm} 3)=$ & 0.971000 & Total atom density $($ atoms $/ \mathrm{b}-\mathrm{cm})=$ & $2.544 \mathrm{E}-02$
\end{tabular}

The above density is estimated to be accurate to 3 significant digits. Uncertainties are not addressed.

The following data was calculated from the input formula.

\begin{tabular}{|c|c|c|c|c|c|c|}
\hline$\frac{\text { Element }}{\mathrm{Na}}$ & $\frac{\text { Neutron ZA }}{11023}$ & $\frac{\text { Photon ZA }}{11000}$ & $\begin{array}{l}\text { Weight } \\
\text { Fraction } \\
1.000000\end{array}$ & $\begin{array}{c}\text { Atom } \\
\frac{\text { Fraction }}{1.000000}\end{array}$ & $\begin{array}{c}\text { Atom } \\
\text { Density } \\
0.025435\end{array}$ & \\
\hline Total & & & 1.000000 & 1.000000 & 0.025435 & \\
\hline MCNP Form & \multicolumn{2}{|c|}{ Weight Fractions } & \multicolumn{2}{|c|}{ Atom Fractions } & \multicolumn{2}{|c|}{ Atom Densities } \\
\hline Neutrons & 11023 & -1.000000 & 11023 & 1.000000 & 11023 & 0.025435 \\
\hline Photons & 11000 & -1.000000 & 11000 & 1.000000 & 11000 & 0.025435 \\
\hline CEPXS Form: & $\begin{array}{l}\text { material } \\
\text { matname } \\
\text { density }\end{array}$ & $\begin{array}{c}\mathrm{Na} \\
\text { Sodium } \\
0.971000\end{array}$ & 1.000000 & & & \\
\hline
\end{tabular}

\section{Sodium Bismuth Tungstate (NBWO)}

\begin{tabular}{llll}
\hline Formula $=$ & $\mathrm{NaBi}(\mathrm{WO} 4) 2$ & Molecular weight $(\mathrm{g} / \mathrm{mole})=$ & 727.64535 \\
Density $(\mathrm{g} / \mathrm{cm} 3)=$ & 7.570000 & Total atom density $($ atoms $/ \mathrm{b}-\mathrm{cm})=$ & $7.518 \mathrm{E}-02$
\end{tabular}

The above density is estimated to be accurate to 3 significant digits. Uncertainties are not addressed.

The following data was calculated from the input formula.

\begin{tabular}{|c|c|c|c|c|c|c|}
\hline Element & Neutron ZA & Photon ZA & $\begin{array}{l}\text { Weight } \\
\text { Fraction }\end{array}$ & $\begin{array}{c}\text { Atom } \\
\text { Fraction }\end{array}$ & $\begin{array}{l}\text { Atom } \\
\text { Density }\end{array}$ & \\
\hline 0 & 8016 & 8000 & 0.175903 & 0.666667 & 0.050121 & \\
\hline $\mathrm{Na}$ & 11023 & 11000 & 0.031595 & 0.083333 & 0.006265 & \\
\hline W & 74000 & 74000 & 0.505301 & 0.166667 & 0.012530 & \\
\hline $\mathrm{Bi}$ & 83209 & 83000 & 0.287201 & 0.083333 & 0.006265 & \\
\hline Total & & & 1.000000 & 1.000000 & 0.075181 & \\
\hline MCNP Form & \multicolumn{2}{|c|}{ Weight Fractions } & \multicolumn{2}{|c|}{ Atom Fractions } & \multicolumn{2}{|c|}{ Atom Densities } \\
\hline \multirow[t]{4}{*}{ Neutrons } & 8016 & -0.175903 & 8016 & 0.666667 & 8016 & 0.050121 \\
\hline & 11023 & -0.031595 & 11023 & 0.083333 & 11023 & 0.006265 \\
\hline & 74000 & -0.505301 & 74000 & 0.166667 & 74000 & 0.012530 \\
\hline & 83209 & -0.287201 & 83209 & 0.083333 & 83209 & 0.006265 \\
\hline
\end{tabular}


PIET-43741-TM-963

PNNL-15870 Rev. 1

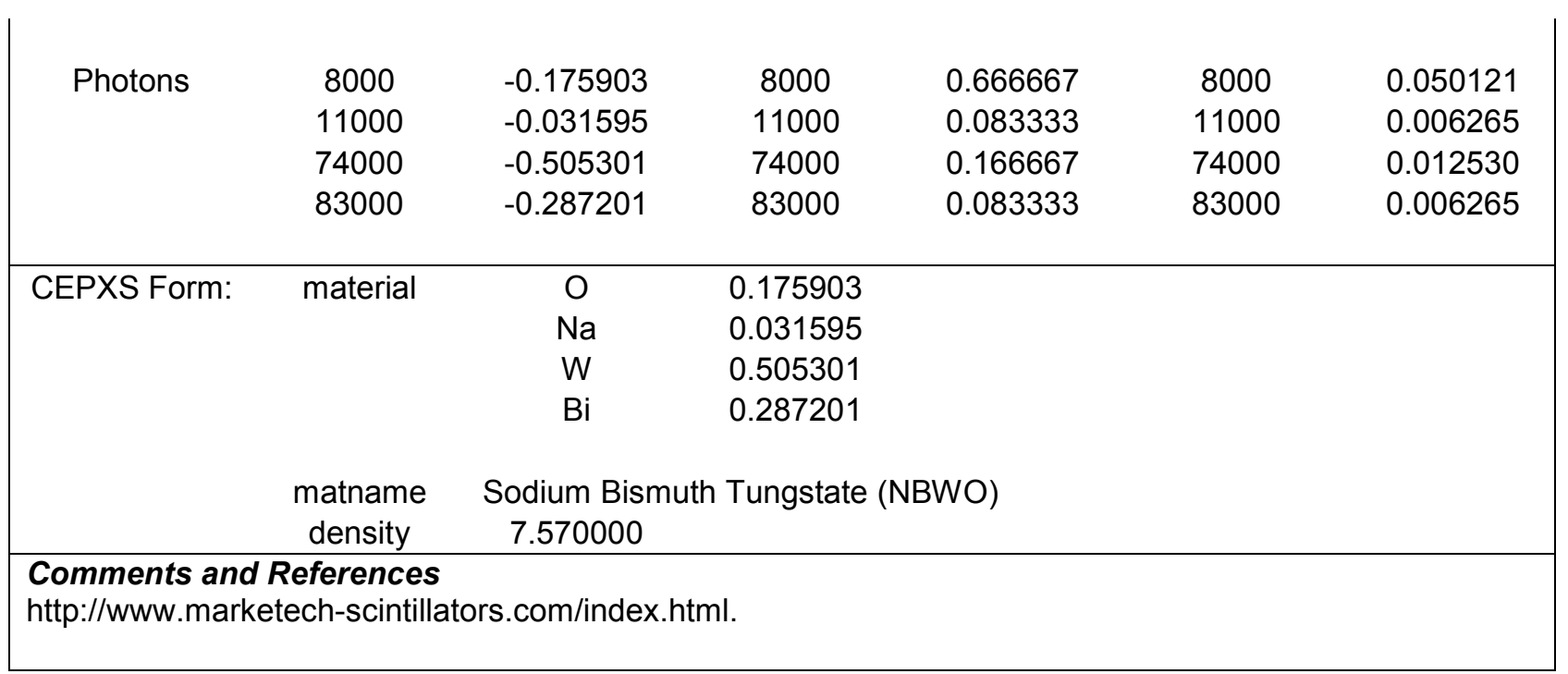

\section{Sodium Chloride}

\begin{tabular}{llll}
\hline Formula $=$ & $\mathrm{NaCl}$ & Molecular weight $(\mathrm{g} / \mathrm{mole})=$ & 58.44277 \\
Density $(\mathrm{g} / \mathrm{cm} 3)=$ & 2.170000 & Total atom density $($ atoms $/ \mathrm{b}-\mathrm{cm})=$ & $4.472 \mathrm{E}-02$
\end{tabular}

The above density is estimated to be accurate to 3 significant digits. Uncertainties are not addressed.

The following data was calculated from the input formula.

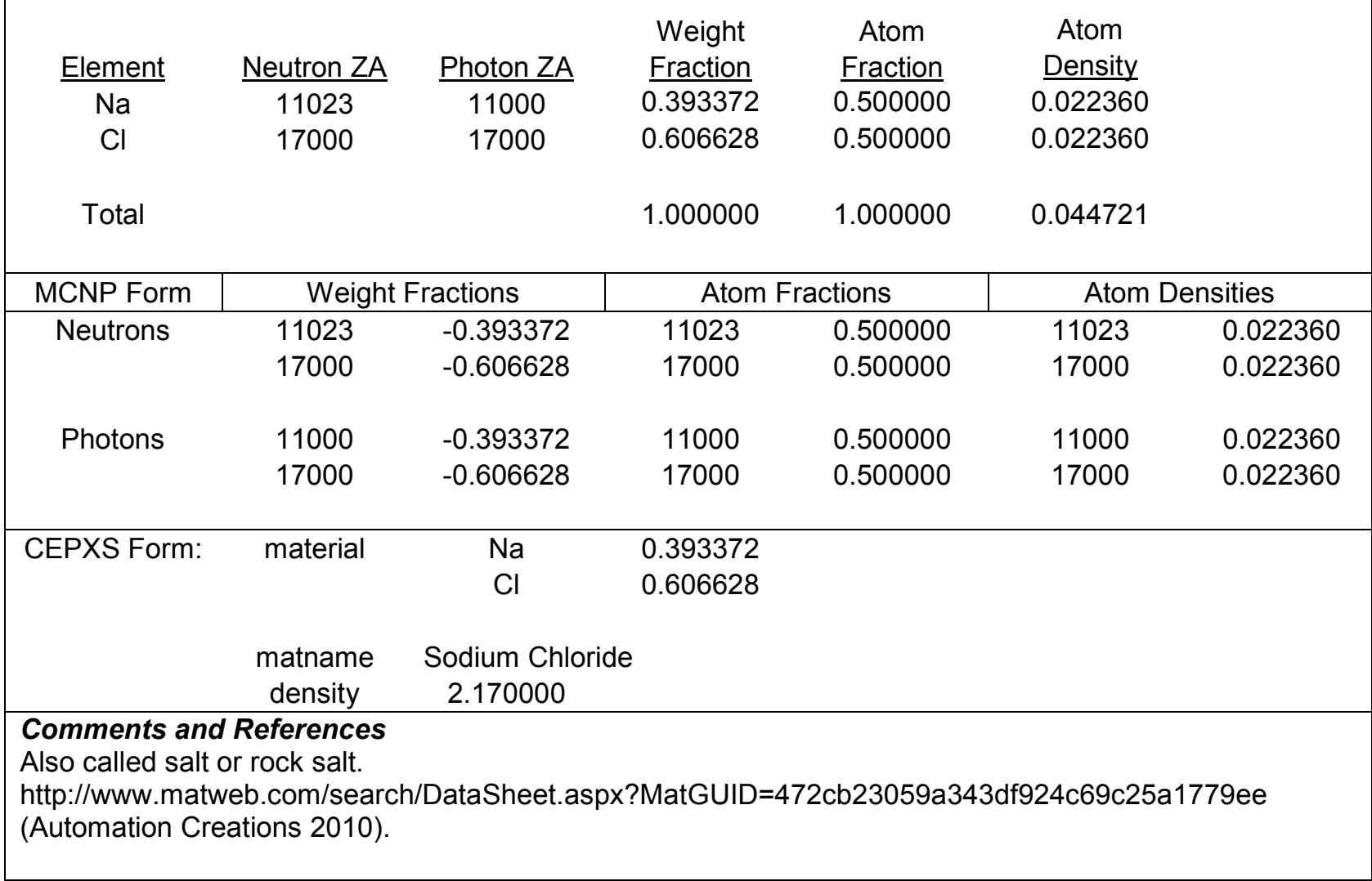




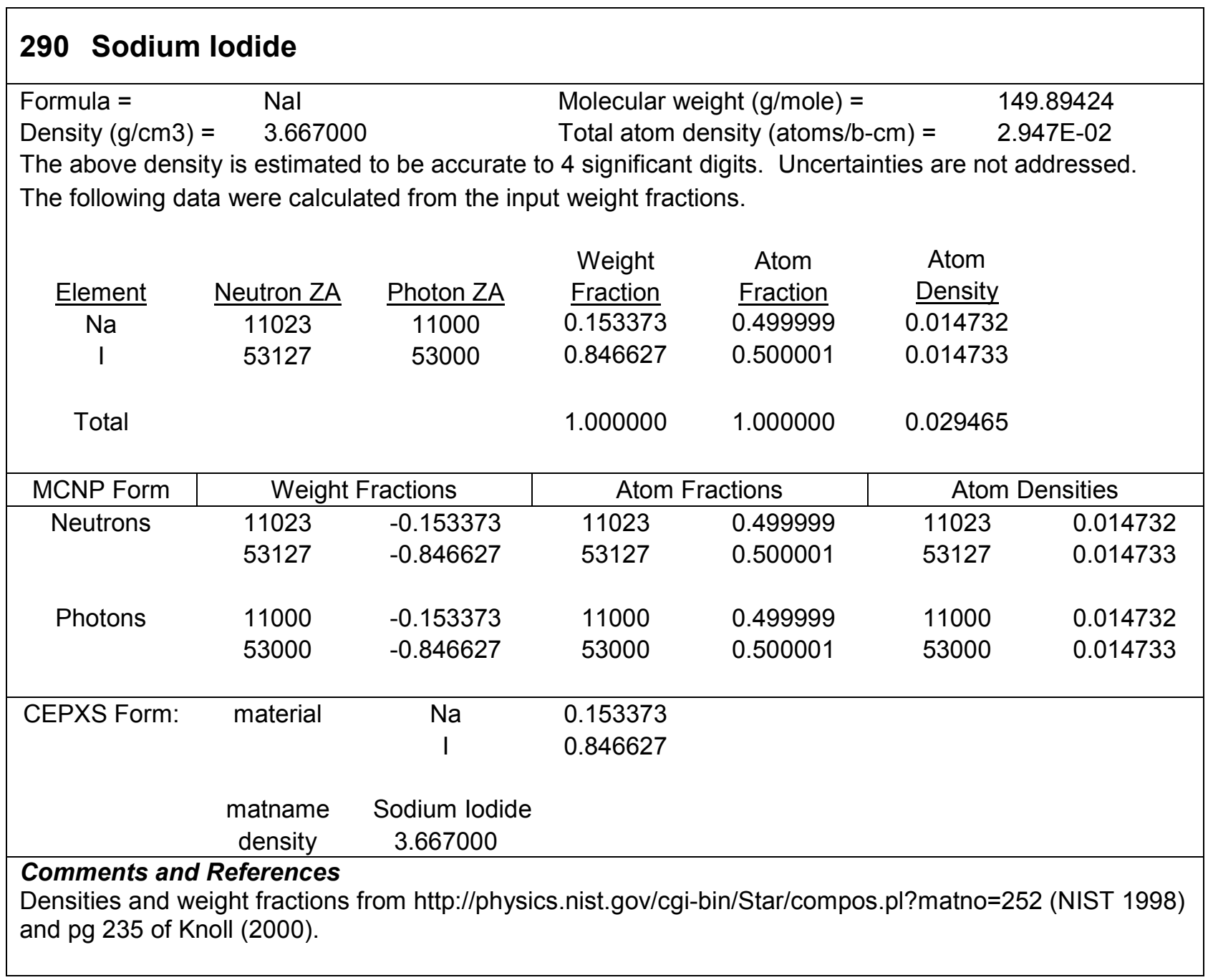

\section{Sodium Nitrate}

\begin{tabular}{llll}
\hline Formula $=$ & NaNO3 & Molecular weight $(\mathrm{g} / \mathrm{mole})=$ & 84.99467 \\
Density $(\mathrm{g} / \mathrm{cm} 3)=$ & 2.261000 & Total atom density $($ atoms $/ \mathrm{b}-\mathrm{cm})=$ & $8.010 \mathrm{E}-02$
\end{tabular}

The above density is estimated to be accurate to 4 significant digits. Uncertainties are not addressed.

The following data were calculated from the input weight fractions.

\begin{tabular}{|c|c|c|c|c|c|}
\hline Element & Neutron ZA & Photon ZA & $\begin{array}{l}\text { Weight } \\
\text { Fraction }\end{array}$ & $\begin{array}{c}\text { Atom } \\
\text { Fraction }\end{array}$ & $\begin{array}{l}\text { Atom } \\
\text { Density }\end{array}$ \\
\hline $\mathrm{N}$ & 7014 & 7000 & 0.164795 & 0.200000 & 0.016020 \\
\hline $\mathrm{O}$ & 8016 & 8000 & 0.564720 & 0.600000 & 0.048060 \\
\hline $\mathrm{Na}$ & 11023 & 11000 & 0.270485 & 0.200000 & 0.016020 \\
\hline Total & & & 1.000000 & 1.000000 & 0.080100 \\
\hline
\end{tabular}


PIET-43741-TM-963

PNNL-15870 Rev. 1

\begin{tabular}{|c|c|c|c|c|c|c|}
\hline MCNP Form & \multicolumn{2}{|c|}{ Weight Fractions } & \multicolumn{2}{|c|}{ Atom Fractions } & \multicolumn{2}{|c|}{ Atom Densities } \\
\hline \multirow{3}{*}{ Neutrons } & 7014 & -0.164795 & 7014 & 0.200000 & 7014 & 0.016020 \\
\hline & 8016 & -0.564720 & 8016 & 0.600000 & 8016 & 0.048060 \\
\hline & 11023 & -0.270485 & 11023 & 0.200000 & 11023 & 0.016020 \\
\hline \multirow[t]{3}{*}{ Photons } & 7000 & -0.164795 & 7000 & 0.200000 & 7000 & 0.016020 \\
\hline & 8000 & -0.564720 & 8000 & 0.600000 & 8000 & 0.048060 \\
\hline & 11000 & -0.270485 & 11000 & 0.200000 & 11000 & 0.016020 \\
\hline \multirow[t]{4}{*}{ CEPXS Form: } & material & $\mathrm{N}$ & 0.164795 & & & \\
\hline & & 0 & 0.564720 & & & \\
\hline & & $\mathrm{Na}$ & 0.270485 & & & \\
\hline & $\begin{array}{c}\text { matname } \\
\text { density }\end{array}$ & $\begin{array}{c}\text { Sodium Nitra } \\
2.261000\end{array}$ & & & & \\
\hline \multicolumn{7}{|c|}{$\begin{array}{l}\text { Comments and References } \\
\text { Theoretical density }=2.261 \mathrm{~g} / \mathrm{cm} 3 \text { and weight fractions from http://physics.nist.gov/cgi- } \\
\text { bin/Star/compos.pl?matno=254 (NIST 1998). Bulk density }=1.35 \mathrm{~g} / \mathrm{cm} 3 \text { at } \\
\text { http://www.powderandbulk.com/resources/bulk_density/material_bulk_density_chart_s.htm (Powder and } \\
\text { Bulk Dot Com 2010). } \\
\text { Formula from Lide (2008), pgs } 4 \text { - } 90 .\end{array}$} \\
\hline
\end{tabular}

\section{Sodium Oxide}

\begin{tabular}{llll}
\hline Formula $=$ & Na2O & Molecular weight $(\mathrm{g} / \mathrm{mole})=$ & 61.97894 \\
Density $(\mathrm{g} / \mathrm{cm} 3)=$ & 2.270000 & Total atom density $($ atoms $/ \mathrm{b}-\mathrm{cm})=$ & $6.617 \mathrm{E}-02$
\end{tabular}

The above density is estimated to be accurate to 4 significant digits. Uncertainties are not addressed.

The following data was calculated from the input formula.

\begin{tabular}{|c|c|c|c|c|c|c|}
\hline Element & Neutron ZA & Photon ZA & $\begin{array}{l}\text { Weight } \\
\text { Fraction }\end{array}$ & $\begin{array}{c}\text { Atom } \\
\text { Fraction }\end{array}$ & $\begin{array}{l}\text { Atom } \\
\text { Density }\end{array}$ & \\
\hline 0 & 8016 & 8000 & $\overline{0.258143}$ & 0.333333 & 0.022056 & \\
\hline $\mathrm{Na}$ & 11023 & 11000 & 0.741857 & 0.666667 & 0.044113 & \\
\hline Total & & & 1.000000 & 1.000000 & 0.066169 & \\
\hline MCNP Form & \multicolumn{2}{|c|}{ Weight Fractions } & \multicolumn{2}{|c|}{ Atom Fractions } & \multicolumn{2}{|c|}{ Atom Densities } \\
\hline \multirow[t]{2}{*}{ Neutrons } & 8016 & -0.258143 & 8016 & 0.333333 & 8016 & 0.022056 \\
\hline & 11023 & -0.741857 & 11023 & 0.666667 & 11023 & 0.044113 \\
\hline \multirow[t]{2}{*}{ Photons } & 8000 & -0.258143 & 8000 & 0.333333 & 8000 & 0.022056 \\
\hline & 11000 & -0.741857 & 11000 & 0.666667 & 11000 & 0.044113 \\
\hline \multirow[t]{3}{*}{ CEPXS Form: } & material & $\mathrm{O}$ & 0.258143 & & & \\
\hline & & $\mathrm{Na}$ & 0.741857 & & & \\
\hline & $\begin{array}{l}\text { matname } \\
\text { density }\end{array}$ & $\begin{array}{c}\text { Sodium Oxide } \\
2.270000\end{array}$ & & & & \\
\hline
\end{tabular}


Comments and References

Formula and density from Lide (2008), pgs 4 - 91, and from Table 51.11 of Hungerford (1960).

\section{Steel, Boron Stainless}

\begin{tabular}{|c|c|c|c|}
\hline Formula $=$ & - & Molecular weight $(\mathrm{g} / \mathrm{mole})=$ & - \\
\hline Density $(\mathrm{g} / \mathrm{cm} 3)=$ & 7.870000 & Total atom density $($ atoms $/ \mathrm{b}-\mathrm{cm})=$ & 8.978E-02 \\
\hline
\end{tabular}

\begin{tabular}{|c|c|c|c|c|c|c|}
\hline Element & Neutron ZA & Photon ZA & $\begin{array}{l}\text { Weight } \\
\text { Fraction }\end{array}$ & $\begin{array}{c}\text { Atom } \\
\text { Fraction }\end{array}$ & $\begin{array}{c}\text { Atom } \\
\text { Density }\end{array}$ & \\
\hline B & - & 5000 & 0.010000 & 0.048827 & 0.004384 & \\
\hline $\mathrm{C}$ & 6000 & 6000 & 0.000396 & 0.001740 & 0.000156 & \\
\hline $\mathrm{Si}$ & 14000 & 14000 & 0.004950 & 0.009304 & 0.000835 & \\
\hline$P$ & 15031 & 15000 & 0.000228 & 0.000388 & 0.000035 & \\
\hline$S$ & 16000 & 16000 & 0.000149 & 0.000244 & 0.000022 & \\
\hline $\mathrm{Cr}$ & 24000 & 24000 & 0.188100 & 0.190960 & 0.017145 & \\
\hline $\mathrm{Mn}$ & 25055 & 25000 & 0.009900 & 0.009512 & 0.000854 & \\
\hline $\mathrm{Fe}$ & 26000 & 26000 & 0.694713 & 0.656666 & 0.058959 & \\
\hline $\mathrm{Ni}$ & 28000 & 28000 & 0.091575 & 0.082359 & 0.007395 & \\
\hline Total & & & 1.000010 & 1.000000 & 0.089785 & \\
\hline MCNP Form & \multicolumn{2}{|c|}{ Weight Fractions } & \multicolumn{2}{|c|}{ Atom Fractions } & \multicolumn{2}{|c|}{ Atom Densities } \\
\hline \multirow{9}{*}{ Neutrons } & - & -0.010000 & - & 0.048827 & - & 0.004384 \\
\hline & 6000 & -0.000396 & 6000 & 0.001740 & 6000 & 0.000156 \\
\hline & 14000 & -0.004950 & 14000 & 0.009304 & 14000 & 0.000835 \\
\hline & 15031 & -0.000228 & 15031 & 0.000388 & 15031 & 0.000035 \\
\hline & 16000 & -0.000149 & 16000 & 0.000244 & 16000 & 0.000022 \\
\hline & 24000 & -0.188100 & 24000 & 0.190960 & 24000 & 0.017145 \\
\hline & 25055 & -0.009900 & 25055 & 0.009512 & 25055 & 0.000854 \\
\hline & 26000 & -0.694713 & 26000 & 0.656666 & 26000 & 0.058959 \\
\hline & 28000 & -0.091575 & 28000 & 0.082359 & 28000 & 0.007395 \\
\hline \multirow[t]{9}{*}{ Photons } & 5000 & -0.010000 & 5000 & 0.048827 & 5000 & 0.004384 \\
\hline & 6000 & -0.000396 & 6000 & 0.001740 & 6000 & 0.000156 \\
\hline & 14000 & -0.004950 & 14000 & 0.009304 & 14000 & 0.000835 \\
\hline & 15000 & -0.000228 & 15000 & 0.000388 & 15000 & 0.000035 \\
\hline & 16000 & -0.000149 & 16000 & 0.000244 & 16000 & 0.000022 \\
\hline & 24000 & -0.188100 & 24000 & 0.190960 & 24000 & 0.017145 \\
\hline & 25000 & -0.009900 & 25000 & 0.009512 & 25000 & 0.000854 \\
\hline & 26000 & -0.694713 & 26000 & 0.656666 & 26000 & 0.058959 \\
\hline & 28000 & -0.091575 & 28000 & 0.082359 & 28000 & 0.007395 \\
\hline \multirow[t]{3}{*}{ CEPXS Form: } & material & B & 0.010000 & & & \\
\hline & & C & 0.000396 & & & \\
\hline & & Si & 0.004950 & & & \\
\hline
\end{tabular}




\begin{tabular}{|c|cc|} 
& $\mathrm{P}$ & 0.000228 \\
$\mathrm{~S}$ & 0.000149 \\
$\mathrm{Cr}$ & 0.188100 \\
$\mathrm{Mn}$ & 0.009900 \\
& $\mathrm{Fe}$ & 0.694713 \\
& $\mathrm{Ni}$ & 0.091575 \\
& \\
matname & Steel, Boron Stainless \\
density & 7.870000 \\
\hline Comments and References \\
1.0 wt\% boron in the 304 stainless steel specified below. \\
Density from pg II.F.1-2 of Carter et al. (1968). \\
\hline
\end{tabular}

\section{Steel, Carbon}

$\begin{array}{llll}\text { Formula }= & - & \text { Molecular weight }(\mathrm{g} / \mathrm{mole})= & - \\ \text { Density }(\mathrm{g} / \mathrm{cm} 3)= & 7.820000 & \text { Total atom density }(\text { atoms } / \mathrm{b}-\mathrm{cm})= & 8.587 \mathrm{E}-02\end{array}$

The above density is estimated to be accurate to 3 significant digits. Uncertainties are not addressed.

The following data were calculated from the input weight fractions.

\begin{tabular}{|c|c|c|c|c|c|c|}
\hline $\begin{array}{c}\text { Element } \\
\mathrm{C} \\
\mathrm{Fe}\end{array}$ & $\begin{array}{c}\text { Neutron ZA } \\
6000 \\
26000\end{array}$ & $\begin{array}{c}\text { Photon ZA } \\
6000 \\
26000\end{array}$ & $\begin{array}{c}\text { Weight } \\
\text { Fraction } \\
0.005000 \\
0.995000\end{array}$ & $\begin{array}{c}\text { Atom } \\
\text { Fraction } \\
0.022831 \\
0.977169\end{array}$ & $\begin{array}{c}\text { Atom } \\
\text { Density } \\
\underline{0.00196} \\
0.08390\end{array}$ & \\
\hline Total & & & 1.000000 & 1.000000 & 0.08586 & \\
\hline MCNP Form & \multicolumn{2}{|c|}{ Weight Fractions } & \multicolumn{2}{|c|}{ Atom Fractions } & \multicolumn{2}{|c|}{ Atom Densities } \\
\hline \multirow[t]{2}{*}{ Neutrons } & 6000 & -0.005000 & 6000 & 0.022831 & 6000 & 0.001960 \\
\hline & 26000 & -0.995000 & 26000 & 0.977169 & 26000 & 0.083907 \\
\hline \multirow[t]{2}{*}{ Photons } & 6000 & -0.005000 & 6000 & 0.022831 & 6000 & 0.001960 \\
\hline & 26000 & -0.995000 & 26000 & 0.977169 & 26000 & 0.083907 \\
\hline \multirow[t]{3}{*}{ CEPXS Form: } & material & C & 0.005000 & & & \\
\hline & & $\mathrm{Fe}$ & 0.995000 & & & \\
\hline & $\begin{array}{l}\text { matname } \\
\text { density }\end{array}$ & $\begin{array}{c}\text { Steel, Carbon } \\
7.820000\end{array}$ & & & & \\
\hline Comments ar & $\begin{array}{l}\text { References } \\
\text { ). }\end{array}$ & & & & & \\
\hline
\end{tabular}




\section{Steel, HT9 Stainless}

\begin{tabular}{|c|c|c|c|}
\hline Formula $=$ & - & Molecular weight $(\mathrm{g} / \mathrm{mole})=$ & - \\
\hline Density $(\mathrm{g} / \mathrm{cm} 3)=$ & 7.874000 & Total atom density $($ atoms $/ \mathrm{b}-\mathrm{cm})=$ & 8.598E-02 \\
\hline
\end{tabular}

\begin{tabular}{|c|c|c|c|c|c|c|}
\hline Element & Neutron ZA & Photon ZA & $\begin{array}{l}\text { Weight } \\
\text { Fraction }\end{array}$ & $\begin{array}{c}\text { Atom } \\
\text { Fraction }\end{array}$ & $\begin{array}{l}\text { Atom } \\
\text { Density }\end{array}$ & \\
\hline $\mathrm{C}$ & 6000 & 6000 & 0.002000 & 0.009183 & 0.000790 & \\
\hline Si & 14000 & 14000 & 0.004000 & 0.007854 & 0.000675 & \\
\hline $\mathrm{P}$ & 15031 & 15000 & 0.000300 & 0.000534 & 0.000046 & \\
\hline$S$ & 16000 & 16000 & 0.000200 & 0.000344 & 0.000030 & \\
\hline $\mathrm{V}$ & 23000 & 23000 & 0.003000 & 0.003248 & 0.000279 & \\
\hline $\mathrm{Cr}$ & 24000 & 24000 & 0.115000 & 0.121971 & 0.010488 & \\
\hline $\mathrm{Mn}$ & 25055 & 25000 & 0.006000 & 0.006023 & 0.000518 & \\
\hline $\mathrm{Fe}$ & 26000 & 26000 & 0.849500 & 0.838897 & 0.072132 & \\
\hline $\mathrm{Ni}$ & 28000 & 28000 & 0.005000 & 0.004698 & 0.000404 & \\
\hline Mo & 42000 & 42000 & 0.010000 & 0.005748 & 0.000494 & \\
\hline W & 74000 & 74000 & 0.005000 & 0.001500 & 0.000129 & \\
\hline Total & & & 1.000000 & 1.000000 & 0.085984 & \\
\hline MCNP Form & \multicolumn{2}{|c|}{ Weight Fractions } & \multicolumn{2}{|c|}{ Atom Fractions } & \multicolumn{2}{|c|}{ Atom Densities } \\
\hline \multirow[t]{11}{*}{ Neutrons } & 6000 & -0.002000 & 6000 & 0.009183 & 6000 & 0.000790 \\
\hline & 14000 & -0.004000 & 14000 & 0.007854 & 14000 & 0.000675 \\
\hline & 15031 & -0.000300 & 15031 & 0.000534 & 15031 & 0.000046 \\
\hline & 16000 & -0.000200 & 16000 & 0.000344 & 16000 & 0.000030 \\
\hline & 23000 & -0.003000 & 23000 & 0.003248 & 23000 & 0.000279 \\
\hline & 24000 & -0.115000 & 24000 & 0.121971 & 24000 & 0.010488 \\
\hline & 25055 & -0.006000 & 25055 & 0.006023 & 25055 & 0.000518 \\
\hline & 26000 & -0.849500 & 26000 & 0.838897 & 26000 & 0.072132 \\
\hline & 28000 & -0.005000 & 28000 & 0.004698 & 28000 & 0.000404 \\
\hline & 42000 & -0.010000 & 42000 & 0.005748 & 42000 & 0.000494 \\
\hline & 74000 & -0.005000 & 74000 & 0.001500 & 74000 & 0.000129 \\
\hline \multirow[t]{11}{*}{ Photons } & 6000 & -0.002000 & 6000 & 0.009183 & 6000 & 0.000790 \\
\hline & 14000 & -0.004000 & 14000 & 0.007854 & 14000 & 0.000675 \\
\hline & 15000 & -0.000300 & 15000 & 0.000534 & 15000 & 0.000046 \\
\hline & 16000 & -0.000200 & 16000 & 0.000344 & 16000 & 0.000030 \\
\hline & 23000 & -0.003000 & 23000 & 0.003248 & 23000 & 0.000279 \\
\hline & 24000 & -0.115000 & 24000 & 0.121971 & 24000 & 0.010488 \\
\hline & 25000 & -0.006000 & 25000 & 0.006023 & 25000 & 0.000518 \\
\hline & 26000 & -0.849500 & 26000 & 0.838897 & 26000 & 0.072132 \\
\hline & 28000 & -0.005000 & 28000 & 0.004698 & 28000 & 0.000404 \\
\hline & 42000 & -0.010000 & 42000 & 0.005748 & 42000 & 0.000494 \\
\hline & 74000 & -0.005000 & 74000 & 0.001500 & 74000 & 0.000129 \\
\hline
\end{tabular}




\begin{tabular}{|ccc} 
CEPXS Form: $\quad$ material & $\mathrm{C}$ & 0.002000 \\
& $\mathrm{Si}$ & 0.004000 \\
$\mathrm{P}$ & 0.000300 \\
$\mathrm{~S}$ & 0.000200 \\
$\mathrm{~V}$ & 0.003000 \\
$\mathrm{Cr}$ & 0.115000 \\
$\mathrm{Mn}$ & 0.006000 \\
& $\mathrm{Fe}$ & 0.849500 \\
$\mathrm{Ni}$ & 0.005000 \\
& $\mathrm{Mo}$ & 0.010000 \\
& $\mathrm{~W}$ & 0.005000
\end{tabular}

matname Steel, HT9 Stainless

density

7.874000

Comments and References

Advanced Fuel Cycle Initiative (AFCl) Materials Handbook, Materials Data for Particle Accelerator

Applications, LA-CP-03-0868, Rev. 4, pgs 18 - 5, Los Alamos National Laboratory, 2003.

\section{Steel, Stainless 202}

\begin{tabular}{llll}
\hline Formula $=$ & - & Molecular weight $(\mathrm{g} / \mathrm{mole})=$ & - \\
Density $(\mathrm{g} / \mathrm{cm} 3)=$ & 7.860000 & Total atom density $($ atoms $/ \mathrm{b}-\mathrm{cm})=$ & $8.680 \mathrm{E}-02$
\end{tabular}

The above density is estimated to be accurate to 3 significant digits. Uncertainties are not addressed.

The following data were calculated from the input weight fractions.

\begin{tabular}{|c|c|c|c|c|c|c|}
\hline Element & Neutron ZA & Photon ZA & $\begin{array}{l}\text { Weight } \\
\text { Fraction }\end{array}$ & $\begin{array}{c}\text { Atom } \\
\text { Fraction }\end{array}$ & $\begin{array}{c}\text { Atom } \\
\text { Density }\end{array}$ & \\
\hline $\mathrm{C}$ & 6000 & 6000 & 0.000750 & 0.003405 & 0.000296 & \\
\hline $\mathrm{N}$ & 7014 & 7000 & 0.001250 & 0.004866 & 0.000422 & \\
\hline $\mathrm{Si}$ & 14000 & 14000 & 0.005000 & 0.009708 & 0.000843 & \\
\hline$P$ & 15031 & 15000 & 0.000300 & 0.000528 & 0.000046 & \\
\hline$S$ & 16000 & 16000 & 0.000150 & 0.000255 & 0.000022 & \\
\hline $\mathrm{Cr}$ & 24000 & 24000 & 0.180000 & 0.188773 & 0.016386 & \\
\hline $\mathrm{Mn}$ & 25055 & 25000 & 0.087500 & 0.086851 & 0.007539 & \\
\hline $\mathrm{Fe}$ & 26000 & 26000 & 0.675050 & 0.659160 & 0.057217 & \\
\hline $\mathrm{Ni}$ & 28000 & 28000 & 0.050000 & 0.046454 & 0.004032 & \\
\hline Total & & & 1.000000 & 1.000000 & 0.086803 & \\
\hline MCNP Form & \multicolumn{2}{|c|}{ Weight Fractions } & \multicolumn{2}{|c|}{ Atom Fractions } & \multicolumn{2}{|c|}{ Atom Densities } \\
\hline \multirow[t]{8}{*}{ Neutrons } & 6000 & -0.000750 & 6000 & 0.003405 & 6000 & 0.000296 \\
\hline & 7014 & -0.001250 & 7014 & 0.004866 & 7014 & 0.000422 \\
\hline & 14000 & -0.005000 & 14000 & 0.009708 & 14000 & 0.000843 \\
\hline & 15031 & -0.000300 & 15031 & 0.000528 & 15031 & 0.000046 \\
\hline & 16000 & -0.000150 & 16000 & 0.000255 & 16000 & 0.000022 \\
\hline & 24000 & -0.180000 & 24000 & 0.188773 & 24000 & 0.016386 \\
\hline & 25055 & -0.087500 & 25055 & 0.086851 & 25055 & 0.007539 \\
\hline & 26000 & -0.675050 & 26000 & 0.659160 & 26000 & 0.057217 \\
\hline
\end{tabular}


PIET-43741-TM-963

PNNL-15870 Rev. 1

\begin{tabular}{|c|c|c|c|c|c|c|}
\hline & 28000 & -0.050000 & 28000 & 0.046454 & 28000 & 0.004032 \\
\hline \multirow[t]{9}{*}{ Photons } & 6000 & -0.000750 & 6000 & 0.003405 & 6000 & 0.000296 \\
\hline & 7000 & -0.001250 & 7000 & 0.004866 & 7000 & 0.000422 \\
\hline & 14000 & -0.005000 & 14000 & 0.009708 & 14000 & 0.000843 \\
\hline & 15000 & -0.000300 & 15000 & 0.000528 & 15000 & 0.000046 \\
\hline & 16000 & -0.000150 & 16000 & 0.000255 & 16000 & 0.000022 \\
\hline & 24000 & -0.180000 & 24000 & 0.188773 & 24000 & 0.016386 \\
\hline & 25000 & -0.087500 & 25000 & 0.086851 & 25000 & 0.007539 \\
\hline & 26000 & -0.675050 & 26000 & 0.659160 & 26000 & 0.057217 \\
\hline & 28000 & -0.050000 & 28000 & 0.046454 & 28000 & 0.004032 \\
\hline \multirow[t]{10}{*}{ CEPXS Form: } & material & $\mathrm{C}$ & 0.000750 & & & \\
\hline & & $\mathrm{N}$ & 0.001250 & & & \\
\hline & & $\mathrm{Si}$ & 0.005000 & & & \\
\hline & & $\mathrm{P}$ & 0.000300 & & & \\
\hline & & $S$ & 0.000150 & & & \\
\hline & & $\mathrm{Cr}$ & 0.180000 & & & \\
\hline & & $\mathrm{Mn}$ & 0.087500 & & & \\
\hline & & $\mathrm{Fe}$ & 0.675050 & & & \\
\hline & & $\mathrm{Ni}$ & 0.050000 & & & \\
\hline & $\begin{array}{c}\text { matname } \\
\text { density }\end{array}$ & $\begin{array}{c}\text { Steel, Stainl } \\
7.860000\end{array}$ & 202 & & & \\
\hline \multicolumn{7}{|c|}{$\begin{array}{l}\text { Comments and References } \\
\text { Density }=7.86 \mathrm{~g} / \mathrm{cm} 3 \text { and weight fractions from } \\
\text { http://www.matweb.com/search/DataSheet.aspx?MatGUID=043ff1a4b83944d197421017d8f95fab } \\
\text { (Automation Creations } 2010 \text { ). } \\
\text { Weight fractions for } \mathrm{Cr}, \mathrm{Mn} \text {, and Fe set at the average of the allowed range. Weight fractions for C, N, Si, } \\
\mathrm{P} \text {, and S assumed to be } 50 \% \text { of their upper limits. Weight fractions of Fe set so they round to the } \\
\text { specified value of } 68 \% \text { and so that the total sums to unity. }\end{array}$} \\
\hline
\end{tabular}

\section{Steel, Stainless 302}

\begin{tabular}{llll}
\hline Formula $=$ & - & Molecular weight $(\mathrm{g} / \mathrm{mole})=$ \\
Density $(\mathrm{g} / \mathrm{cm} 3)=$ & 7.860000 & Total atom density $($ atoms $/ \mathrm{b}-\mathrm{cm})=$ & - \\
\hline
\end{tabular}

The above density is estimated to be accurate to 3 significant digits. Uncertainties are not addressed.

The following data were calculated from the input weight fractions.

\begin{tabular}{|c|c|c|c|c|c|}
\hline Element & Neutron ZA & Photon ZA & $\begin{array}{l}\text { Weight } \\
\text { Fraction }\end{array}$ & $\begin{array}{c}\text { Atom } \\
\text { Fraction }\end{array}$ & $\begin{array}{c}\text { Atom } \\
\text { Density }\end{array}$ \\
\hline C & 6000 & 6000 & $\overline{0.001400}$ & $\overline{0.006356}$ & 0.000552 \\
\hline Si & 14000 & 14000 & 0.009300 & 0.018057 & 0.001567 \\
\hline $\mathrm{P}$ & 15031 & 15000 & 0.000420 & 0.000739 & 0.000064 \\
\hline$S$ & 16000 & 16000 & 0.000280 & 0.000476 & 0.000041 \\
\hline $\mathrm{Cr}$ & 24000 & 24000 & 0.180000 & 0.188773 & 0.016386 \\
\hline $\mathrm{Mn}$ & 25055 & 25000 & 0.018600 & 0.018462 & 0.001603 \\
\hline $\mathrm{Fe}$ & 26000 & 26000 & 0.700000 & 0.683520 & 0.059332 \\
\hline
\end{tabular}


PIET-43741-TM-963

PNNL-15870 Rev. 1

\begin{tabular}{|c|c|c|c|c|c|c|}
\hline $\mathrm{Ni}$ & 28000 & 28000 & 0.090000 & 0.083616 & \multicolumn{2}{|l|}{0.007258} \\
\hline \multicolumn{3}{|l|}{ Total } & 1.000000 & 1.000000 & \multicolumn{2}{|l|}{0.086803} \\
\hline MCNP Form & \multicolumn{2}{|c|}{ Weight Fractions } & \multicolumn{2}{|c|}{ Atom Fractions } & \multicolumn{2}{|c|}{ Atom Densities } \\
\hline \multirow[t]{8}{*}{ Neutrons } & 6000 & -0.001400 & 6000 & 0.006356 & 6000 & 0.000552 \\
\hline & 14000 & -0.009300 & 14000 & 0.018057 & 14000 & 0.001567 \\
\hline & 15031 & -0.000420 & 15031 & 0.000739 & 15031 & 0.000064 \\
\hline & 16000 & -0.000280 & 16000 & 0.000476 & 16000 & 0.000041 \\
\hline & 24000 & -0.180000 & 24000 & 0.188773 & 24000 & 0.016386 \\
\hline & 25055 & -0.018600 & 25055 & 0.018462 & 25055 & 0.001603 \\
\hline & 26000 & -0.700000 & 26000 & 0.683520 & 26000 & 0.059332 \\
\hline & 28000 & -0.090000 & 28000 & 0.083616 & 28000 & 0.007258 \\
\hline \multirow[t]{8}{*}{ Photons } & 6000 & -0.001400 & 6000 & 0.006356 & 6000 & 0.000552 \\
\hline & 14000 & -0.009300 & 14000 & 0.018057 & 14000 & 0.001567 \\
\hline & 15000 & -0.000420 & 15000 & 0.000739 & 15000 & 0.000064 \\
\hline & 16000 & -0.000280 & 16000 & 0.000476 & 16000 & 0.000041 \\
\hline & 24000 & -0.180000 & 24000 & 0.188773 & 24000 & 0.016386 \\
\hline & 25000 & -0.018600 & 25000 & 0.018462 & 25000 & 0.001603 \\
\hline & 26000 & -0.700000 & 26000 & 0.683520 & 26000 & 0.059332 \\
\hline & 28000 & -0.090000 & 28000 & 0.083616 & 28000 & 0.007258 \\
\hline \multirow[t]{9}{*}{ CEPXS Form: } & material & $\mathrm{C}$ & 0.001400 & & & \\
\hline & & $\mathrm{Si}$ & 0.009300 & & & \\
\hline & & $P$ & 0.000420 & & & \\
\hline & & $S$ & 0.000280 & & & \\
\hline & & $\mathrm{Cr}$ & 0.180000 & & & \\
\hline & & $\mathrm{Mn}$ & 0.018600 & & & \\
\hline & & $\mathrm{Fe}$ & 0.700000 & & & \\
\hline & & $\mathrm{Ni}$ & 0.090000 & & & \\
\hline & $\begin{array}{c}\text { matname } \\
\text { density }\end{array}$ & $\begin{array}{c}\text { Steel, Stainl } \\
7.860000\end{array}$ & 302 & & & \\
\hline \multicolumn{7}{|c|}{$\begin{array}{l}\text { Comments and References } \\
\text { Density }=7.86 \mathrm{~g} / \mathrm{cm} 3 \text { and weight fractions from } \\
\text { http://www.matweb.com/search/DataSheet.aspx?MatGUID=05efb28c10154f2796f4bf033363880a } \\
\text { (Automation Creations } 2010 \text { ). } \\
\text { Weight fractions for } \mathrm{Cr}, \mathrm{Fe} \text {, and Ni set at the value specified in the reference. Weight fractions for C, Si, P, } \\
\mathrm{S} \text {, and } \mathrm{Mn} \text { were set at } 93.0 \% \text { of their upper limits to allow the total to sum to unity. }\end{array}$} \\
\hline
\end{tabular}

\section{Steel, Stainless 304}

\begin{tabular}{llll}
\hline Formula $=$ & - & Molecular weight $(\mathrm{g} / \mathrm{mole})=$ & - \\
Density $(\mathrm{g} / \mathrm{cm} 3)=$ & 8.000000 & Total atom density $($ atoms $/ \mathrm{b}-\mathrm{cm})=$ & $8.769 \mathrm{E}-02$
\end{tabular}

The above density is estimated to be accurate to 3 significant digits. Uncertainties are not addressed.

The following data were calculated from the input weight fractions. 


\begin{tabular}{|c|c|c|c|c|c|c|}
\hline Element & Neutron ZA & Photon ZA & $\begin{array}{l}\text { Weight } \\
\text { Fraction }\end{array}$ & $\begin{array}{c}\text { Atom } \\
\text { Fraction }\end{array}$ & $\begin{array}{l}\text { Atom } \\
\text { Density }\end{array}$ & \\
\hline $\mathrm{C}$ & 6000 & 6000 & 0.000400 & 0.001830 & 0.000160 & \\
\hline $\mathrm{Si}$ & 14000 & 14000 & 0.005000 & 0.009781 & 0.000858 & \\
\hline$P$ & 15031 & 15000 & 0.000230 & 0.000408 & 0.000036 & \\
\hline$S$ & 16000 & 16000 & 0.000150 & 0.000257 & 0.000023 & \\
\hline $\mathrm{Cr}$ & 24000 & 24000 & 0.190000 & 0.200762 & 0.017605 & \\
\hline $\mathrm{Mn}$ & 25055 & 25000 & 0.010000 & 0.010001 & 0.000877 & \\
\hline $\mathrm{Fe}$ & 26000 & 26000 & 0.701730 & 0.690375 & 0.060538 & \\
\hline $\mathrm{Ni}$ & 28000 & 28000 & 0.092500 & 0.086587 & 0.007593 & \\
\hline Total & & & 1.000010 & 1.000000 & 0.087688 & \\
\hline MCNP Form & \multicolumn{2}{|c|}{ Weight Fractions } & \multicolumn{2}{|c|}{ Atom Fractions } & \multicolumn{2}{|c|}{ Atom Densities } \\
\hline \multirow[t]{8}{*}{ Neutrons } & 6000 & -0.000400 & 6000 & 0.001830 & 6000 & 0.000160 \\
\hline & 14000 & -0.005000 & 14000 & 0.009781 & 14000 & 0.000858 \\
\hline & 15031 & -0.000230 & 15031 & 0.000408 & 15031 & 0.000036 \\
\hline & 16000 & -0.000150 & 16000 & 0.000257 & 16000 & 0.000023 \\
\hline & 24000 & -0.190000 & 24000 & 0.200762 & 24000 & 0.017605 \\
\hline & 25055 & -0.010000 & 25055 & 0.010001 & 25055 & 0.000877 \\
\hline & 26000 & -0.701730 & 26000 & 0.690375 & 26000 & 0.060538 \\
\hline & 28000 & -0.092500 & 28000 & 0.086587 & 28000 & 0.007593 \\
\hline \multirow[t]{8}{*}{ Photons } & 6000 & -0.000400 & 6000 & 0.001830 & 6000 & 0.000160 \\
\hline & 14000 & -0.005000 & 14000 & 0.009781 & 14000 & 0.000858 \\
\hline & 15000 & -0.000230 & 15000 & 0.000408 & 15000 & 0.000036 \\
\hline & 16000 & -0.000150 & 16000 & 0.000257 & 16000 & 0.000023 \\
\hline & 24000 & -0.190000 & 24000 & 0.200762 & 24000 & 0.017605 \\
\hline & 25000 & -0.010000 & 25000 & 0.010001 & 25000 & 0.000877 \\
\hline & 26000 & -0.701730 & 26000 & 0.690375 & 26000 & 0.060538 \\
\hline & 28000 & -0.092500 & 28000 & 0.086587 & 28000 & 0.007593 \\
\hline \multirow[t]{9}{*}{ CEPXS Form: } & material & $\mathrm{C}$ & 0.000400 & & & \\
\hline & & $\mathrm{Si}$ & 0.005000 & & & \\
\hline & & $P$ & 0.000230 & & & \\
\hline & & $S$ & 0.000150 & & & \\
\hline & & $\mathrm{Cr}$ & 0.190000 & & & \\
\hline & & $\mathrm{Mn}$ & 0.010000 & & & \\
\hline & & $\mathrm{Fe}$ & 0.701730 & & & \\
\hline & & $\mathrm{Ni}$ & 0.092500 & & & \\
\hline & $\begin{array}{l}\text { matname } \\
\text { density }\end{array}$ & $\begin{array}{c}\text { Steel, Stainle } \\
8.000000\end{array}$ & 304 & & & \\
\hline \multicolumn{7}{|c|}{ 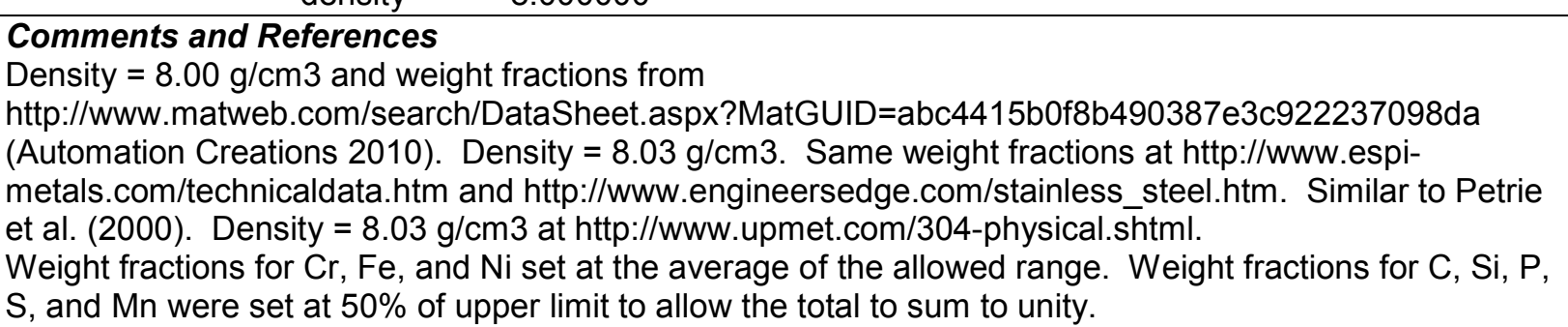 } \\
\hline
\end{tabular}




\section{Steel, Stainless 304L}

\begin{tabular}{|c|c|c|c|}
\hline Formula $=$ & - & Molecular weight $(\mathrm{g} / \mathrm{mole})=$ & - \\
\hline Density $(\mathrm{g} / \mathrm{cm} 3)=$ & 8.000000 & Total atom density $($ atoms $/ \mathrm{b}-\mathrm{cm})=$ & 8.758E-02 \\
\hline
\end{tabular}

following data were cal

\begin{tabular}{|c|c|c|c|c|c|c|}
\hline Element & Neutron ZA & Photon ZA & $\begin{array}{l}\text { Weight } \\
\text { Fraction }\end{array}$ & $\begin{array}{c}\text { Atom } \\
\text { Fraction }\end{array}$ & $\begin{array}{l}\text { Atom } \\
\text { Density }\end{array}$ & \\
\hline $\mathrm{C}$ & 6000 & 6000 & 0.000150 & 0.000687 & 0.000060 & \\
\hline $\mathrm{Si}$ & 14000 & 14000 & 0.005000 & 0.009793 & 0.000858 & \\
\hline $\mathrm{P}$ & 15031 & 15000 & 0.000230 & 0.000408 & 0.000036 & \\
\hline$S$ & 16000 & 16000 & 0.000150 & 0.000257 & 0.000023 & \\
\hline $\mathrm{Cr}$ & 24000 & 24000 & 0.190000 & 0.201015 & 0.017605 & \\
\hline $\mathrm{Mn}$ & 25055 & 25000 & 0.010000 & 0.010013 & 0.000877 & \\
\hline $\mathrm{Fe}$ & 26000 & 26000 & 0.694480 & 0.684101 & 0.059912 & \\
\hline $\mathrm{Ni}$ & 28000 & 28000 & 0.100000 & 0.093725 & 0.008208 & \\
\hline Total & & & 1.000010 & 1.000000 & 0.087578 & \\
\hline MCNP Form & \multicolumn{2}{|c|}{ Weight Fractions } & \multicolumn{2}{|c|}{ Atom Fractions } & \multicolumn{2}{|c|}{ Atom Densities } \\
\hline \multirow[t]{8}{*}{ Neutrons } & 6000 & -0.000150 & 6000 & 0.000687 & 6000 & 0.000060 \\
\hline & 14000 & -0.005000 & 14000 & 0.009793 & 14000 & 0.000858 \\
\hline & 15031 & -0.000230 & 15031 & 0.000408 & 15031 & 0.000036 \\
\hline & 16000 & -0.000150 & 16000 & 0.000257 & 16000 & 0.000023 \\
\hline & 24000 & -0.190000 & 24000 & 0.201015 & 24000 & 0.017605 \\
\hline & 25055 & -0.010000 & 25055 & 0.010013 & 25055 & 0.000877 \\
\hline & 26000 & -0.694480 & 26000 & 0.684101 & 26000 & 0.059912 \\
\hline & 28000 & -0.100000 & 28000 & 0.093725 & 28000 & 0.008208 \\
\hline \multirow[t]{8}{*}{ Photons } & 6000 & -0.000150 & 6000 & 0.000687 & 6000 & 0.000060 \\
\hline & 14000 & -0.005000 & 14000 & 0.009793 & 14000 & 0.000858 \\
\hline & 15000 & -0.000230 & 15000 & 0.000408 & 15000 & 0.000036 \\
\hline & 16000 & -0.000150 & 16000 & 0.000257 & 16000 & 0.000023 \\
\hline & 24000 & -0.190000 & 24000 & 0.201015 & 24000 & 0.017605 \\
\hline & 25000 & -0.010000 & 25000 & 0.010013 & 25000 & 0.000877 \\
\hline & 26000 & -0.694480 & 26000 & 0.684101 & 26000 & 0.059912 \\
\hline & 28000 & -0.100000 & 28000 & 0.093725 & 28000 & 0.008208 \\
\hline \multirow[t]{8}{*}{ CEPXS Form: } & material & $\mathrm{C}$ & 0.000150 & & & \\
\hline & & $\mathrm{Si}$ & 0.005000 & & & \\
\hline & & $\mathrm{P}$ & 0.000230 & & & \\
\hline & & $S$ & 0.000150 & & & \\
\hline & & $\mathrm{Cr}$ & 0.190000 & & & \\
\hline & & $\mathrm{Mn}$ & 0.010000 & & & \\
\hline & & $\mathrm{Fe}$ & 0.694480 & & & \\
\hline & & $\mathrm{Ni}$ & 0.100000 & & & \\
\hline
\end{tabular}




\begin{tabular}{|c|c|}
\hline $\begin{array}{c}\text { matname } \\
\text { density }\end{array}$ & $\begin{array}{l}\text { Steel, Stainless 304L } \\
8.000000\end{array}$ \\
\hline $\begin{array}{l}\text { Comments and References } \\
\text { Density }=8.00 \mathrm{~g} / \mathrm{cm} 3 \text { and weic } \\
\text { http://www.matweb.com } / \text { searc } \\
\text { (Automation Creations } 2010 \text { ). } \\
\text { Weight fractions for } \mathrm{Cr} \text { and } \mathrm{Ni} \\
\text { and Mn assumed to be } 50 \% \text { o }\end{array}$ & $\begin{array}{l}\text { ht fractions from } \\
\text { /DataSheet.aspx?MatGUID }=e 2147 \mathrm{~b} 8 \mathrm{f} 727343 \mathrm{~b} 0 \mathrm{~b} 0 \mathrm{~d} 51 \text { efe } 02 \mathrm{a} 6127 \mathrm{e} \\
\text { et at the average of the allowed range. Weight fractions for } \mathrm{C}, \mathrm{Si}, \mathrm{P}, \mathrm{S} \text {, } \\
\text { their upper limits. Weight fraction of Fe set so the total sums to unity. }\end{array}$ \\
\hline
\end{tabular}

\section{Steel, Stainless 316}

\begin{tabular}{llll}
\hline Formula $=$ & - & Molecular weight $(\mathrm{g} / \mathrm{mole})=$ & - \\
Density $(\mathrm{g} / \mathrm{cm} 3)=$ & 8.000000 & Total atom density $($ atoms $/ \mathrm{b}-\mathrm{cm})=$ & $8.655 \mathrm{E}-02$
\end{tabular}

The above density is estimated to be accurate to 3 significant digits. Uncertainties are not addressed.

The following data were calculated from the input weight fractions.

\begin{tabular}{|c|c|c|c|c|c|c|}
\hline Element & Neutron ZA & Photon ZA & $\begin{array}{l}\text { Weight } \\
\text { Fraction }\end{array}$ & $\begin{array}{c}\text { Atom } \\
\text { Fraction }\end{array}$ & $\begin{array}{l}\text { Atom } \\
\text { Density }\end{array}$ & \\
\hline $\mathrm{C}$ & 6000 & 6000 & $\overline{0.000410}$ & $\overline{0.001900}$ & 0.000164 & \\
\hline $\mathrm{Si}$ & 14000 & 14000 & 0.005070 & 0.010048 & 0.000870 & \\
\hline $\mathrm{P}$ & 15031 & 15000 & 0.000230 & 0.000413 & 0.000036 & \\
\hline$S$ & 16000 & 16000 & 0.000150 & 0.000260 & 0.000023 & \\
\hline $\mathrm{Cr}$ & 24000 & 24000 & 0.170000 & 0.181986 & 0.015751 & \\
\hline $\mathrm{Mn}$ & 25055 & 25000 & 0.010140 & 0.010274 & 0.000889 & \\
\hline $\mathrm{Fe}$ & 26000 & 26000 & 0.669000 & 0.666811 & 0.057714 & \\
\hline $\mathrm{Ni}$ & 28000 & 28000 & 0.120000 & 0.113803 & 0.009850 & \\
\hline Mo & 42000 & 42000 & 0.025000 & 0.014504 & 0.001255 & \\
\hline Total & & & 1.000000 & 1.000000 & 0.086553 & \\
\hline MCNP Form & \multicolumn{2}{|c|}{ Weight Fractions } & \multicolumn{2}{|c|}{ Atom Fractions } & \multicolumn{2}{|c|}{ Atom Densities } \\
\hline \multirow[t]{9}{*}{ Neutrons } & 6000 & -0.000410 & 6000 & 0.001900 & 6000 & 0.000164 \\
\hline & 14000 & -0.005070 & 14000 & 0.010048 & 14000 & 0.000870 \\
\hline & 15031 & -0.000230 & 15031 & 0.000413 & 15031 & 0.000036 \\
\hline & 16000 & -0.000150 & 16000 & 0.000260 & 16000 & 0.000023 \\
\hline & 24000 & -0.170000 & 24000 & 0.181986 & 24000 & 0.015751 \\
\hline & 25055 & -0.010140 & 25055 & 0.010274 & 25055 & 0.000889 \\
\hline & 26000 & -0.669000 & 26000 & 0.666811 & 26000 & 0.057714 \\
\hline & 28000 & -0.120000 & 28000 & 0.113803 & 28000 & 0.009850 \\
\hline & 42000 & -0.025000 & 42000 & 0.014504 & 42000 & 0.001255 \\
\hline \multirow[t]{7}{*}{ Photons } & 6000 & -0.000410 & 6000 & 0.001900 & 6000 & 0.000164 \\
\hline & 14000 & -0.005070 & 14000 & 0.010048 & 14000 & 0.000870 \\
\hline & 15000 & -0.000230 & 15000 & 0.000413 & 15000 & 0.000036 \\
\hline & 16000 & -0.000150 & 16000 & 0.000260 & 16000 & 0.000023 \\
\hline & 24000 & -0.170000 & 24000 & 0.181986 & 24000 & 0.015751 \\
\hline & 25000 & -0.010140 & 25000 & 0.010274 & 25000 & 0.000889 \\
\hline & 26000 & -0.669000 & 26000 & 0.666811 & 26000 & 0.057714 \\
\hline
\end{tabular}


PIET-43741-TM-963

PNNL-15870 Rev. 1

\begin{tabular}{|c|c|c|c|c|c|c|}
\hline & $\begin{array}{l}28000 \\
42000\end{array}$ & $\begin{array}{l}-0.120000 \\
-0.025000\end{array}$ & $\begin{array}{l}28000 \\
42000\end{array}$ & $\begin{array}{l}0.113803 \\
0.014504\end{array}$ & $\begin{array}{l}28000 \\
42000\end{array}$ & $\begin{array}{l}0.009850 \\
0.001255\end{array}$ \\
\hline CEPXS Form: & material & $\begin{array}{l}\mathrm{C} \\
\mathrm{Si} \\
\mathrm{P} \\
\mathrm{S} \\
\mathrm{Cr} \\
\mathrm{Mn} \\
\mathrm{Fe} \\
\mathrm{Ni} \\
\mathrm{Mo}\end{array}$ & $\begin{array}{l}0.000410 \\
0.005070 \\
0.000230 \\
0.000150 \\
0.170000 \\
0.010140 \\
0.669000 \\
0.120000 \\
0.025000\end{array}$ & & & \\
\hline & $\begin{array}{l}\text { matname } \\
\text { density }\end{array}$ & \multicolumn{2}{|c|}{$\begin{array}{l}\text { Steel, Stainless } 316 \\
8.000000\end{array}$} & & & \\
\hline \multicolumn{7}{|c|}{$\begin{array}{l}\text { Comments and References } \\
\text { Density }=8.00 \mathrm{~g} / \mathrm{cm} 3 \text { and weight fractions from } \\
\text { http://www.matweb.com/search/DataSheet.aspx?MatGUID=50f320bd1daf4fa7965448c30d3114ad\&ckck= } \\
1 \text { (Automation Creations } 2010) \text {. Density }=8.03 \mathrm{~g} / \mathrm{cm} 3 \text { and same weight fractions at http://www.espi- } \\
\text { metals.com/technicaldata.htm. Same weight fractions at } \\
\text { http://www.engineersedge.com/stainless_steel.htm. } \\
\text { Similar to Petrie et al. }(2000) \text {. } \\
\text { Density }=8.027 \mathrm{~g} / \mathrm{cm} 3 \text { at http://www.upmet.com } / 304-p h y s i c a l . s h t m l . \\
\text { Weight fractions for Cr, Fe, Ni, and Mo set at the average of the allowed range. Weight fractions for C, Si, } \\
\mathrm{P}, \mathrm{S} \text {, and Mn set at } 50.7 \% \text { of their upper limits to allow the total to sum to unity. }\end{array}$} \\
\hline
\end{tabular}

\section{Steel, Stainless 316L}

\begin{tabular}{llll}
\hline Formula $=$ & - & Molecular weight $(\mathrm{g} / \mathrm{mole})=$ \\
Density $(\mathrm{g} / \mathrm{cm} 3)=$ & 8.000000 & Total atom density $($ atoms $/ \mathrm{b}-\mathrm{cm})=$ & - \\
\hline & $8.698 \mathrm{E}-02$
\end{tabular}

The above density is estimated to be accurate to 3 significant digits. Uncertainties are not addressed.

The following data were calculated from the input weight fractions.

\begin{tabular}{|c|c|c|c|c|c|}
\hline Element & Neutron ZA & Photon ZA & $\begin{array}{l}\text { Weight } \\
\text { Fraction }\end{array}$ & $\begin{array}{c}\text { Atom } \\
\text { Fraction }\end{array}$ & $\begin{array}{l}\text { Atom } \\
\text { Density }\end{array}$ \\
\hline $\mathrm{C}$ & 6000 & 6000 & 0.000300 & 0.001384 & 0.000120 \\
\hline $\mathrm{Si}$ & 14000 & 14000 & 0.010000 & 0.019722 & 0.001715 \\
\hline$P$ & 15031 & 15000 & 0.000450 & 0.000805 & 0.000070 \\
\hline$S$ & 16000 & 16000 & 0.000300 & 0.000518 & 0.000045 \\
\hline $\mathrm{Cr}$ & 24000 & 24000 & 0.170000 & 0.181098 & 0.015751 \\
\hline $\mathrm{Mn}$ & 25055 & 25000 & 0.020000 & 0.020165 & 0.001754 \\
\hline $\mathrm{Fe}$ & 26000 & 26000 & 0.653950 & 0.648628 & 0.056416 \\
\hline $\mathrm{Ni}$ & 28000 & 28000 & 0.120000 & 0.113247 & 0.009850 \\
\hline Mo & 42000 & 42000 & 0.025000 & 0.014434 & 0.001255 \\
\hline Total & & & 1.000000 & 1.000000 & 0.086977 \\
\hline
\end{tabular}


PIET-43741-TM-963

PNNL-15870 Rev. 1

\begin{tabular}{|c|c|c|c|c|c|c|}
\hline MCNP Form & \multicolumn{2}{|c|}{ Weight Fractions } & \multicolumn{2}{|c|}{ Atom Fractions } & \multicolumn{2}{|c|}{ Atom Densities } \\
\hline \multirow[t]{9}{*}{ Neutrons } & 6000 & -0.000300 & 6000 & 0.001384 & 6000 & 0.000120 \\
\hline & 14000 & -0.010000 & 14000 & 0.019722 & 14000 & 0.001715 \\
\hline & 15031 & -0.000450 & 15031 & 0.000805 & 15031 & 0.000070 \\
\hline & 16000 & -0.000300 & 16000 & 0.000518 & 16000 & 0.000045 \\
\hline & 24000 & -0.170000 & 24000 & 0.181098 & 24000 & 0.015751 \\
\hline & 25055 & -0.020000 & 25055 & 0.020165 & 25055 & 0.001754 \\
\hline & 26000 & -0.653950 & 26000 & 0.648628 & 26000 & 0.056416 \\
\hline & 28000 & -0.120000 & 28000 & 0.113247 & 28000 & 0.009850 \\
\hline & 42000 & -0.025000 & 42000 & 0.014434 & 42000 & 0.001255 \\
\hline \multirow[t]{9}{*}{ Photons } & 6000 & -0.000300 & 6000 & 0.001384 & 6000 & 0.000120 \\
\hline & 14000 & -0.010000 & 14000 & 0.019722 & 14000 & 0.001715 \\
\hline & 15000 & -0.000450 & 15000 & 0.000805 & 15000 & 0.000070 \\
\hline & 16000 & -0.000300 & 16000 & 0.000518 & 16000 & 0.000045 \\
\hline & 24000 & -0.170000 & 24000 & 0.181098 & 24000 & 0.015751 \\
\hline & 25000 & -0.020000 & 25000 & 0.020165 & 25000 & 0.001754 \\
\hline & 26000 & -0.653950 & 26000 & 0.648628 & 26000 & 0.056416 \\
\hline & 28000 & -0.120000 & 28000 & 0.113247 & 28000 & 0.009850 \\
\hline & 42000 & -0.025000 & 42000 & 0.014434 & 42000 & 0.001255 \\
\hline \multirow[t]{10}{*}{ CEPXS Form: } & material & $\mathrm{C}$ & 0.000300 & & & \\
\hline & & Si & 0.010000 & & & \\
\hline & & $\mathrm{P}$ & 0.000450 & & & \\
\hline & & $S$ & 0.000300 & & & \\
\hline & & $\mathrm{Cr}$ & 0.170000 & & & \\
\hline & & $\mathrm{Mn}$ & 0.020000 & & & \\
\hline & & $\mathrm{Fe}$ & 0.653950 & & & \\
\hline & & $\mathrm{Ni}$ & 0.120000 & & & \\
\hline & & Mo & 0.025000 & & & \\
\hline & $\begin{array}{c}\text { matname } \\
\text { density }\end{array}$ & $\begin{array}{c}\text { Steel, Stainl } \\
8.000000\end{array}$ & $316 \mathrm{~L}$ & & & \\
\hline \multicolumn{7}{|c|}{$\begin{array}{l}\text { Comments and References } \\
\text { Density }=8.00 \mathrm{~g} / \mathrm{cm} 3 \text { and weight fractions from } \\
\text { http://www.matweb.com/search/DataSheet.aspx?MatGUID=530144e2752b47709a58ca8fe0849969 } \\
\text { (Automation Creations } 2010 \text { ). } \\
\text { Fe calculated so the elements sum to unity. } \\
\text { Weight fractions for all elements set at specified value, except weight fraction for Fe increased by } 0.00395 \\
\text { so weight fractions sum to unity. }\end{array}$} \\
\hline
\end{tabular}

\section{Steel, Stainless 321}

Formula $=\quad-\quad$ Molecular weight $(\mathrm{g} / \mathrm{mole})=$

Density $(\mathrm{g} / \mathrm{cm} 3)=\quad 8.000000 \quad$ Total atom density $($ atoms $/ \mathrm{b}-\mathrm{cm})=8.816 \mathrm{E}-02$

The above density is estimated to be accurate to 3 significant digits. Uncertainties are not addressed.

The following data were calculated from the input weight fractions. 
PIET-43741-TM-963

PNNL-15870 Rev. 1

\begin{tabular}{|c|c|c|c|c|c|c|}
\hline Element & Neutron ZA & Photon ZA & $\begin{array}{l}\text { Weight } \\
\text { Fraction }\end{array}$ & $\begin{array}{l}\text { Atom } \\
\text { Fraction }\end{array}$ & $\begin{array}{l}\text { Atom } \\
\text { Density }\end{array}$ & \\
\hline $\mathrm{C}$ & 6000 & 6000 & 0.000800 & 0.003640 & 0.000321 & \\
\hline $\mathrm{Si}$ & 14000 & 14000 & 0.010000 & 0.019457 & 0.001715 & \\
\hline$P$ & 15031 & 15000 & 0.000450 & 0.000794 & 0.000070 & \\
\hline$S$ & 16000 & 16000 & 0.000300 & 0.000511 & 0.000045 & \\
\hline $\mathrm{Ti}$ & 22000 & 22000 & 0.001500 & 0.001712 & 0.000151 & \\
\hline $\mathrm{Cr}$ & 24000 & 24000 & 0.180000 & 0.189171 & 0.016678 & \\
\hline $\mathrm{Mn}$ & 25055 & 25000 & 0.020000 & 0.019893 & 0.001754 & \\
\hline $\mathrm{Fe}$ & 26000 & 26000 & 0.676950 & 0.662408 & 0.058400 & \\
\hline $\mathrm{Ni}$ & 28000 & 28000 & 0.110000 & 0.102413 & 0.009029 & \\
\hline Total & & & 1.000000 & 1.000000 & 0.088163 & \\
\hline MCNP Form & \multicolumn{2}{|c|}{ Weight Fractions } & \multicolumn{2}{|c|}{ Atom Fractions } & \multicolumn{2}{|c|}{ Atom Densities } \\
\hline \multirow[t]{9}{*}{ Neutrons } & 6000 & -0.000800 & 6000 & 0.003640 & 6000 & 0.000321 \\
\hline & 14000 & -0.010000 & 14000 & 0.019457 & 14000 & 0.001715 \\
\hline & 15031 & -0.000450 & 15031 & 0.000794 & 15031 & 0.000070 \\
\hline & 16000 & -0.000300 & 16000 & 0.000511 & 16000 & 0.000045 \\
\hline & 22000 & -0.001500 & 22000 & 0.001712 & 22000 & 0.000151 \\
\hline & 24000 & -0.180000 & 24000 & 0.189171 & 24000 & 0.016678 \\
\hline & 25055 & -0.020000 & 25055 & 0.019893 & 25055 & 0.001754 \\
\hline & 26000 & -0.676950 & 26000 & 0.662408 & 26000 & 0.058400 \\
\hline & 28000 & -0.110000 & 28000 & 0.102413 & 28000 & 0.009029 \\
\hline \multirow[t]{9}{*}{ Photons } & 6000 & -0.000800 & 6000 & 0.003640 & 6000 & 0.000321 \\
\hline & 14000 & -0.010000 & 14000 & 0.019457 & 14000 & 0.001715 \\
\hline & 15000 & -0.000450 & 15000 & 0.000794 & 15000 & 0.000070 \\
\hline & 16000 & -0.000300 & 16000 & 0.000511 & 16000 & 0.000045 \\
\hline & 22000 & -0.001500 & 22000 & 0.001712 & 22000 & 0.000151 \\
\hline & 24000 & -0.180000 & 24000 & 0.189171 & 24000 & 0.016678 \\
\hline & 25000 & -0.020000 & 25000 & 0.019893 & 25000 & 0.001754 \\
\hline & 26000 & -0.676950 & 26000 & 0.662408 & 26000 & 0.058400 \\
\hline & 28000 & -0.110000 & 28000 & 0.102413 & 28000 & 0.009029 \\
\hline \multirow[t]{10}{*}{ CEPXS Form: } & material & $\mathrm{C}$ & 0.000800 & & & \\
\hline & & $\mathrm{Si}$ & 0.010000 & & & \\
\hline & & $P$ & 0.000450 & & & \\
\hline & & $S$ & 0.000300 & & & \\
\hline & & $\mathrm{Ti}$ & 0.001500 & & & \\
\hline & & $\mathrm{Cr}$ & 0.180000 & & & \\
\hline & & $\mathrm{Mn}$ & 0.020000 & & & \\
\hline & & $\mathrm{Fe}$ & 0.676950 & & & \\
\hline & & $\mathrm{Ni}$ & 0.110000 & & & \\
\hline & $\begin{array}{l}\text { matname } \\
\text { density }\end{array}$ & \multicolumn{3}{|c|}{$\begin{array}{l}\text { Steel, Stainless } 321 \\
8.000000\end{array}$} & & \\
\hline
\end{tabular}


Fe calculated so elements sum to unity. Weight fractions for all elements set at specified value, except weight fraction for Fe decreased by 0.00305 so weight fractions sum to unity.

\section{Steel, Stainless 347}

\begin{tabular}{|c|c|c|c|}
\hline Formula $=$ & - & Molecular weight $(\mathrm{g} / \mathrm{mole})=$ & - \\
\hline Density $(\mathrm{g} / \mathrm{cm} 3)=$ & 8.000000 & Total atom density $($ atoms $/ \mathrm{b}-\mathrm{cm})=$ & 8.770E-02 \\
\hline
\end{tabular}

\begin{tabular}{|c|c|c|c|c|c|c|}
\hline Element & Neutron ZA & Photon ZA & $\begin{array}{l}\text { Weight } \\
\text { Fraction }\end{array}$ & $\begin{array}{c}\text { Atom } \\
\text { Fraction }\end{array}$ & $\begin{array}{c}\text { Atom } \\
\text { Density }\end{array}$ & \\
\hline $\mathrm{C}$ & 6000 & 6000 & $\overline{0.000800}$ & $\overline{0.003659}$ & 0.000321 & \\
\hline $\mathrm{Si}$ & 14000 & 14000 & 0.010000 & 0.019559 & 0.001715 & \\
\hline$P$ & 15031 & 15000 & 0.000450 & 0.000798 & 0.000070 & \\
\hline$S$ & 16000 & 16000 & 0.000300 & 0.000514 & 0.000045 & \\
\hline $\mathrm{Cr}$ & 24000 & 24000 & 0.170000 & 0.179602 & 0.015751 & \\
\hline $\mathrm{Mn}$ & 25055 & 25000 & 0.020000 & 0.019998 & 0.001754 & \\
\hline $\mathrm{Fe}$ & 26000 & 26000 & 0.680450 & 0.669338 & 0.058702 & \\
\hline $\mathrm{Ni}$ & 28000 & 28000 & 0.110000 & 0.102952 & 0.009029 & \\
\hline $\mathrm{Nb}$ & 41093 & 41000 & 0.004000 & 0.002365 & 0.000207 & \\
\hline $\mathrm{Ta}$ & 73181 & 73000 & 0.004000 & 0.001214 & 0.000106 & \\
\hline Total & & & 1.000000 & 1.000000 & 0.087702 & \\
\hline MCNP Form & \multicolumn{2}{|c|}{ Weight Fractions } & \multicolumn{2}{|c|}{ Atom Fractions } & \multicolumn{2}{|c|}{ Atom Densities } \\
\hline \multirow[t]{10}{*}{ Neutrons } & 6000 & -0.000800 & 6000 & 0.003659 & 6000 & 0.000321 \\
\hline & 14000 & -0.010000 & 14000 & 0.019559 & 14000 & 0.001715 \\
\hline & 15031 & -0.000450 & 15031 & 0.000798 & 15031 & 0.000070 \\
\hline & 16000 & -0.000300 & 16000 & 0.000514 & 16000 & 0.000045 \\
\hline & 24000 & -0.170000 & 24000 & 0.179602 & 24000 & 0.015751 \\
\hline & 25055 & -0.020000 & 25055 & 0.019998 & 25055 & 0.001754 \\
\hline & 26000 & -0.680450 & 26000 & 0.669338 & 26000 & 0.058702 \\
\hline & 28000 & -0.110000 & 28000 & 0.102952 & 28000 & 0.009029 \\
\hline & 41093 & -0.004000 & 41093 & 0.002365 & 41093 & 0.000207 \\
\hline & 73181 & -0.004000 & 73181 & 0.001214 & 73181 & 0.000106 \\
\hline \multirow[t]{10}{*}{ Photons } & 6000 & -0.000800 & 6000 & 0.003659 & 6000 & 0.000321 \\
\hline & 14000 & -0.010000 & 14000 & 0.019559 & 14000 & 0.001715 \\
\hline & 15000 & -0.000450 & 15000 & 0.000798 & 15000 & 0.000070 \\
\hline & 16000 & -0.000300 & 16000 & 0.000514 & 16000 & 0.000045 \\
\hline & 24000 & -0.170000 & 24000 & 0.179602 & 24000 & 0.015751 \\
\hline & 25000 & -0.020000 & 25000 & 0.019998 & 25000 & 0.001754 \\
\hline & 26000 & -0.680450 & 26000 & 0.669338 & 26000 & 0.058702 \\
\hline & 28000 & -0.110000 & 28000 & 0.102952 & 28000 & 0.009029 \\
\hline & 41000 & -0.004000 & 41000 & 0.002365 & 41000 & 0.000207 \\
\hline & 73000 & -0.004000 & 73000 & 0.001214 & 73000 & 0.000106 \\
\hline
\end{tabular}




\begin{tabular}{|ccc} 
CEPXS Form: material & $\mathrm{C}$ & 0.000800 \\
& $\mathrm{Si}$ & 0.010000 \\
$\mathrm{P}$ & 0.000450 \\
$\mathrm{~S}$ & 0.000300 \\
$\mathrm{Cr}$ & 0.170000 \\
$\mathrm{Mn}$ & 0.020000 \\
$\mathrm{Fe}$ & 0.680450 \\
$\mathrm{Ni}$ & 0.110000 \\
$\mathrm{Nb}$ & 0.004000 \\
& $\mathrm{Ta}$ & 0.004000
\end{tabular}

matname Steel, Stainless 347

density 8.000000

\section{Comments and References}

Density $=8.00 \mathrm{~g} / \mathrm{cm} 3$ and weight fractions from

http://www.matweb.com/search/DataSheet.aspx?MatGUID=cecb69a2b862447f9c748c2e22cc0210 (Automation Creations 2010).

Weight fractions for $\mathrm{Cr}$ and $\mathrm{Ni}$ set at the specified value. Weight fraction of Fe increased by 0.045 above its specified value to allow the total to sum to unity. Weight fractions for $\mathrm{C}, \mathrm{Si}, \mathrm{P}, \mathrm{S}$, and $\mathrm{Mn}$ set at upper limits. Weight fractions for $\mathrm{Nb}$ and $\mathrm{Ta}$ each set at half of the combined upper limit for $\mathrm{Nb}+\mathrm{Ta}$.

\section{Steel, Stainless 409}

\begin{tabular}{llll}
\hline Formula $=$ & - & Molecular weight $(\mathrm{g} / \mathrm{mole})=$ & - \\
Density $(\mathrm{g} / \mathrm{cm} 3)=$ & 7.800000 & Total atom density $($ atoms $/ \mathrm{b}-\mathrm{cm})=$ & $8.604 \mathrm{E}-02$
\end{tabular}

The above density is estimated to be accurate to 3 significant digits. Uncertainties are not addressed.

The following data were calculated from the input weight fractions.

\begin{tabular}{|c|c|c|c|c|c|c|}
\hline Element & Neutron ZA & Photon ZA & $\begin{array}{l}\text { Weight } \\
\text { Fraction }\end{array}$ & $\begin{array}{c}\text { Atom } \\
\text { Fraction }\end{array}$ & $\begin{array}{l}\text { Atom } \\
\text { Density }\end{array}$ & \\
\hline $\mathrm{C}$ & 6000 & 6000 & $\overline{0.000790}$ & $\overline{0.003591}$ & 0.000309 & \\
\hline $\mathrm{Si}$ & 14000 & 14000 & 0.009830 & 0.019108 & 0.001644 & \\
\hline $\mathrm{P}$ & 15031 & 15000 & 0.000440 & 0.000776 & 0.000067 & \\
\hline$S$ & 16000 & 16000 & 0.000440 & 0.000749 & 0.000064 & \\
\hline $\mathrm{Ti}$ & 22000 & 22000 & 0.007370 & 0.008406 & 0.000723 & \\
\hline $\mathrm{Cr}$ & 24000 & 24000 & 0.111300 & 0.116862 & 0.010055 & \\
\hline $\mathrm{Mn}$ & 25055 & 25000 & 0.009830 & 0.009769 & 0.000840 & \\
\hline $\mathrm{Fe}$ & 26000 & 26000 & 0.860000 & 0.840740 & 0.072337 & \\
\hline Total & & & 1.000000 & 1.000000 & 0.086040 & \\
\hline MCNP Form & \multicolumn{2}{|c|}{ Weight Fractions } & \multicolumn{2}{|c|}{ Atom Fractions } & \multicolumn{2}{|c|}{ Atom Densities } \\
\hline \multirow[t]{6}{*}{ Neutrons } & 6000 & -0.000790 & 6000 & 0.003591 & 6000 & 0.000309 \\
\hline & 14000 & -0.009830 & 14000 & 0.019108 & 14000 & 0.001644 \\
\hline & 15031 & -0.000440 & 15031 & 0.000776 & 15031 & 0.000067 \\
\hline & 16000 & -0.000440 & 16000 & 0.000749 & 16000 & 0.000064 \\
\hline & 22000 & -0.007370 & 22000 & 0.008406 & 22000 & 0.000723 \\
\hline & 24000 & -0.111300 & 24000 & 0.116862 & 24000 & 0.010055 \\
\hline
\end{tabular}


PIET-43741-TM-963

PNNL-15870 Rev. 1

\begin{tabular}{|c|c|c|c|c|c|c|}
\hline & 25055 & -0.009830 & 25055 & 0.009769 & 25055 & 0.000840 \\
\hline & 26000 & -0.860000 & 26000 & 0.840740 & 26000 & 0.072337 \\
\hline Photons & 6000 & -0.000790 & 6000 & 0.003591 & 6000 & 0.000309 \\
\hline & 14000 & -0.009830 & 14000 & 0.019108 & 14000 & 0.001644 \\
\hline & 15000 & -0.000440 & 15000 & 0.000776 & 15000 & 0.000067 \\
\hline & 16000 & -0.000440 & 16000 & 0.000749 & 16000 & 0.000064 \\
\hline & 22000 & -0.007370 & 22000 & 0.008406 & 22000 & 0.000723 \\
\hline & 24000 & -0.111300 & 24000 & 0.116862 & 24000 & 0.010055 \\
\hline & 25000 & -0.009830 & 25000 & 0.009769 & 25000 & 0.000840 \\
\hline & 26000 & -0.860000 & 26000 & 0.840740 & 26000 & 0.072337 \\
\hline CEPXS Form: & material & $\mathrm{C}$ & 0.000790 & & & \\
\hline & & $\mathrm{Si}$ & 0.009830 & & & \\
\hline & & $P$ & 0.000440 & & & \\
\hline & & $S$ & 0.000440 & & & \\
\hline & & $\mathrm{Ti}$ & 0.007370 & & & \\
\hline & & $\mathrm{Cr}$ & 0.111300 & & & \\
\hline & & $\mathrm{Mn}$ & 0.009830 & & & \\
\hline & & $\mathrm{Fe}$ & 0.860000 & & & \\
\hline & $\begin{array}{c}\text { matname } \\
\text { density }\end{array}$ & $\begin{array}{c}\text { Steel, Stainl } \\
7.800000\end{array}$ & 409 & & & \\
\hline $\begin{array}{l}\text { Comments an } \\
\text { Density = } 7.80 \\
\text { http://www.mat } \\
\text { (Automation Cr } \\
\text { Weight fraction } \\
98.3 \% \text { of their }\end{array}$ & $\begin{array}{l}\text { References } \\
\text { m3 and we } \\
\text { b.com/sear } \\
\text { tions 2010) } \\
\text { or Cr and F } \\
\text { er limits to }\end{array}$ & $\begin{array}{l}\text { t fractions fr } \\
\text { DataSheet.c } \\
\text { et at the spe } \\
\text { w the total }\end{array}$ & $\begin{array}{l}\text { MatGUID } \\
\text { value. W }\end{array}$ & $\begin{array}{l}\text { db56864e } \\
\text { fractions }\end{array}$ & is & $\begin{array}{l}\text { a5db } \\
\text { d Mn set at }\end{array}$ \\
\hline
\end{tabular}

\section{Steel, Stainless 440}

\begin{tabular}{|c|c|c|c|}
\hline $\begin{array}{l}= \\
(\mathrm{g} / \mathrm{cm} 3)=\end{array}$ & $-\overline{7} 800000$ & $\begin{array}{l}\text { Molecular weight }(\mathrm{g} / \mathrm{mole})= \\
\text { Total atom density }(\text { atoms } / \mathrm{b}-\mathrm{cm})=\end{array}$ & \\
\hline
\end{tabular}

The above density is estimated to be accurate to 3 significant digits. Uncertainties are not addressed.

The following data were calculated from the input weight fractions.

\begin{tabular}{|c|c|c|c|c|c|}
\hline Element & Neutron ZA & Photon ZA & $\begin{array}{l}\text { Weight } \\
\text { Fraction }\end{array}$ & $\begin{array}{c}\text { Atom } \\
\text { Fraction }\end{array}$ & $\begin{array}{l}\text { Atom } \\
\text { Density }\end{array}$ \\
\hline C & 6000 & 6000 & $\overline{0.006750}$ & $\overline{0.030406}$ & 0.002640 \\
\hline $\mathrm{Si}$ & 14000 & 14000 & 0.006500 & 0.012521 & 0.001087 \\
\hline$P$ & 15031 & 15000 & 0.000260 & 0.000454 & 0.000039 \\
\hline$S$ & 16000 & 16000 & 0.000200 & 0.000337 & 0.000029 \\
\hline $\mathrm{Cr}$ & 24000 & 24000 & 0.170000 & 0.176887 & 0.015358 \\
\hline $\mathrm{Mn}$ & 25055 & 25000 & 0.006500 & 0.006401 & 0.000556 \\
\hline $\mathrm{Fe}$ & 26000 & 26000 & 0.795050 & 0.770242 & 0.066874 \\
\hline Mo & 42000 & 42000 & 0.004880 & 0.002752 & 0.000239 \\
\hline
\end{tabular}


PIET-43741-TM-963

PNNL-15870 Rev. 1

\begin{tabular}{|c|c|c|c|c|c|c|}
\hline Total & & & 0.990140 & 1.000000 & 0.086822 & \\
\hline MCNP Form & Weigh & ractions & Atom & ctions & Atom & sities \\
\hline Neutrons & 6000 & -0.006750 & 6000 & 0.030406 & 6000 & 0.002640 \\
\hline & 14000 & -0.006500 & 14000 & 0.012521 & 14000 & 0.001087 \\
\hline & 15031 & -0.000260 & 15031 & 0.000454 & 15031 & 0.000039 \\
\hline & 16000 & -0.000200 & 16000 & 0.000337 & 16000 & 0.000029 \\
\hline & 24000 & -0.170000 & 24000 & 0.176887 & 24000 & 0.015358 \\
\hline & 25055 & -0.006500 & 25055 & 0.006401 & 25055 & 0.000556 \\
\hline & 26000 & -0.795050 & 26000 & 0.770242 & 26000 & 0.066874 \\
\hline & 42000 & -0.004880 & 42000 & 0.002752 & 42000 & 0.000239 \\
\hline Photons & 6000 & -0.006750 & 6000 & 0.030406 & 6000 & 0.002640 \\
\hline & 14000 & -0.006500 & 14000 & 0.012521 & 14000 & 0.001087 \\
\hline & 15000 & -0.000260 & 15000 & 0.000454 & 15000 & 0.000039 \\
\hline & 16000 & -0.000200 & 16000 & 0.000337 & 16000 & 0.000029 \\
\hline & 24000 & -0.170000 & 24000 & 0.176887 & 24000 & 0.015358 \\
\hline & 25000 & -0.006500 & 25000 & 0.006401 & 25000 & 0.000556 \\
\hline & 26000 & -0.795050 & 26000 & 0.770242 & 26000 & 0.066874 \\
\hline & 42000 & -0.004880 & 42000 & 0.002752 & 42000 & 0.000239 \\
\hline CEPXS Form: & material & $\mathrm{C}$ & 0.006750 & & & \\
\hline & & $\mathrm{Si}$ & 0.006500 & & & \\
\hline & & $P$ & 0.000260 & & & \\
\hline & & $S$ & 0.000200 & & & \\
\hline & & $\mathrm{Cr}$ & 0.170000 & & & \\
\hline & & $\mathrm{Mn}$ & 0.006500 & & & \\
\hline & & $\mathrm{Fe}$ & 0.795050 & & & \\
\hline & & Mo & 0.004880 & & & \\
\hline & $\begin{array}{l}\text { matname } \\
\text { density }\end{array}$ & $\begin{array}{c}\text { Steel, Stainl } \\
7.800000\end{array}$ & 440 & & & \\
\hline $\begin{array}{l}\text { Comments an } \\
\text { Density = } 7.80 \\
\text { http://www.mat } \\
1 \text { (Automation } \\
\text { Weight fraction } \\
\text { set at average }\end{array}$ & $\begin{array}{l}\text { References } \\
\mathrm{m} 3 \text { and we } \\
\text { b.com/sear } \\
\text { ations } 201 \\
\text { r Si, P, S, } \\
\text { ues of allov }\end{array}$ & $\begin{array}{l}\text { th fractions frc } \\
\text { /DataSheet.a } \\
\text {, and Mo set } \\
\text { d range. Fe c }\end{array}$ & $\begin{array}{l}\text { MatGUID= } \\
5 \% \text { of their } \\
\text { lated so the }\end{array}$ & $\begin{array}{l}\text { ebd5797b9 } \\
\text { er limits. } \\
\text { ments sum }\end{array}$ & $\begin{array}{l}8 f 5 \mathrm{cf} 3926 \\
\text { fractions } \\
\text { ity. }\end{array}$ & $\begin{array}{l}\text { ce0\&ckck= } \\
\text { and } \mathrm{Cr}\end{array}$ \\
\hline
\end{tabular}

\section{Sterotex}

\begin{tabular}{llll}
\hline Formula $=$ & $(\mathrm{C} 17 \mathrm{H} 35 \mathrm{CO} 2) 3-\mathrm{C} 3 \mathrm{H} 5$ & Molecular weight $(\mathrm{g} / \mathrm{mole})=$ & 891.4797 \\
Density $(\mathrm{g} / \mathrm{cm} 3)=$ & 0.862000 & Total atom density $($ atoms $/ \mathrm{b}-\mathrm{cm})=$ & $1.007 \mathrm{E}-01$
\end{tabular}

The above density is estimated to be accurate to 3 significant digits. Uncertainties are not addressed.

The following data was calculated from the input formula. 
PIET-43741-TM-963

PNNL-15870 Rev. 1

\begin{tabular}{|c|c|c|c|c|c|c|}
\hline Element & Neutron ZA & Photon ZA & $\begin{array}{l}\text { Weight } \\
\text { Fraction }\end{array}$ & $\begin{array}{l}\text { Atom } \\
\text { Fraction }\end{array}$ & $\begin{array}{l}\text { Atom } \\
\text { Density }\end{array}$ & \\
\hline $\mathrm{H}$ & 1001 & 1000 & 0.124370 & 0.635838 & 0.064053 & \\
\hline $\mathrm{C}$ & 6000 & 6000 & 0.767948 & 0.329480 & 0.033191 & \\
\hline O & 8016 & 8000 & 0.107682 & 0.034682 & 0.003494 & \\
\hline Total & & & 1.000000 & 1.000000 & 0.100738 & \\
\hline MCNP Form & \multicolumn{2}{|c|}{ Weight Fractions } & \multicolumn{2}{|c|}{ Atom Fractions } & \multicolumn{2}{|c|}{ Atom Densities } \\
\hline \multirow[t]{3}{*}{ Neutrons } & 1001 & -0.124370 & 1001 & 0.635838 & 1001 & 0.064053 \\
\hline & 6000 & -0.767948 & 6000 & 0.329480 & 6000 & 0.033191 \\
\hline & 8016 & -0.107682 & 8016 & 0.034682 & 8016 & 0.003494 \\
\hline \multirow[t]{3}{*}{ Photons } & 1000 & -0.124370 & 1000 & 0.635838 & 1000 & 0.064053 \\
\hline & 6000 & -0.767948 & 6000 & 0.329480 & 6000 & 0.033191 \\
\hline & 8000 & -0.107682 & 8000 & 0.034682 & 8000 & 0.003494 \\
\hline \multirow[t]{4}{*}{ CEPXS Form: } & material & $\mathrm{H}$ & 0.124370 & & & \\
\hline & & C & 0.767948 & & & \\
\hline & & O & 0.107682 & & & \\
\hline & $\begin{array}{l}\text { matname } \\
\text { density }\end{array}$ & $\begin{array}{l}\text { Sterotex } \\
0.862000\end{array}$ & & & & \\
\hline $\begin{array}{l}\text { Comments an } \\
\text { Paxton and Pru }\end{array}$ & $\begin{array}{l}\text { References } \\
\text { st (1986) re }\end{array}$ & 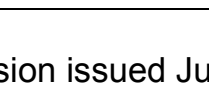 & & & & \\
\hline
\end{tabular}

\section{Stilbene (Trans-stilbene Isomer)}

\begin{tabular}{llll}
\hline Formula $=$ & $\mathrm{C} 14 \mathrm{H} 10$ & Molecular weight $(\mathrm{g} / \mathrm{mole})=$ & 178.2292 \\
Density $(\mathrm{g} / \mathrm{cm} 3)=$ & 1.220000 & Total atom density $($ atoms $/ \mathrm{b}-\mathrm{cm})=$ & $9.893 \mathrm{E}-02$
\end{tabular}

The above density is estimated to be accurate to 3 significant digits. Uncertainties are not addressed.

The following data was calculated from the input formula.

\begin{tabular}{|c|c|c|c|c|c|c|}
\hline Element & Neutron ZA & Photon ZA & $\begin{array}{l}\text { Weight } \\
\text { Fraction }\end{array}$ & $\begin{array}{c}\text { Atom } \\
\text { Fraction }\end{array}$ & $\begin{array}{c}\text { Atom } \\
\text { Density }\end{array}$ & \\
\hline $\mathrm{H}$ & 1001 & 1000 & $\overline{0.056553}$ & $\overline{0.416667}$ & 0.041222 & \\
\hline C & 6000 & 6000 & 0.943447 & 0.583333 & 0.05771 & \\
\hline Total & & & 1.000000 & 1.000000 & 0.098933 & \\
\hline MCNP Form & \multicolumn{2}{|c|}{ Weight Fractions } & \multicolumn{2}{|c|}{ Atom Fractions } & \multicolumn{2}{|c|}{ Atom Densities } \\
\hline \multirow[t]{2}{*}{ Neutrons } & 1001 & -0.056553 & 1001 & 0.416667 & 1001 & 0.041222 \\
\hline & 6000 & -0.943447 & 6000 & 0.583333 & 6000 & 0.057711 \\
\hline \multirow[t]{2}{*}{ Photons } & 1000 & -0.056553 & 1000 & 0.416667 & 1000 & 0.041222 \\
\hline & 6000 & -0.943447 & 6000 & 0.583333 & 6000 & 0.057711 \\
\hline
\end{tabular}




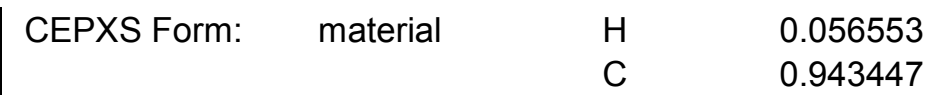

matname Stilbene (Trans-stilbene Isomer)

density $\quad 1.220000$

\section{Comments and References}

Density and formula from http://www.apace-science.com/proteus/organics.htm\#top (APACE 2009).

\section{Sulphur}

\begin{tabular}{llll}
\hline Formula $=$ & $\mathrm{S}$ & Molecular weight $(\mathrm{g} / \mathrm{mole})=$ & 32.065 \\
Density $(\mathrm{g} / \mathrm{cm} 3)=$ & 2.000000 & Total atom density $($ atoms $/ \mathrm{b}-\mathrm{cm})=$ & $3.756 \mathrm{E}-02$
\end{tabular}

The above density is estimated to be accurate to 2 significant digits. Uncertainties are not addressed.

The following data was calculated from the input formula.

\begin{tabular}{|c|c|c|c|c|c|c|}
\hline$\frac{\text { Element }}{\mathrm{S}}$ & $\frac{\text { Neutron ZA }}{16000}$ & $\frac{\text { Photon ZA }}{16000}$ & $\begin{array}{l}\text { Weight } \\
\text { Fraction } \\
1.000000\end{array}$ & $\begin{array}{c}\text { Atom } \\
\frac{\text { Fraction }}{1.000000}\end{array}$ & $\begin{array}{c}\text { Atom } \\
\text { Density } \\
0.037562\end{array}$ & \\
\hline Total & & & 1.000000 & 1.000000 & 0.037562 & \\
\hline MCNP Form & \multicolumn{2}{|c|}{ Weight Fractions } & \multicolumn{2}{|c|}{ Atom Fractions } & \multicolumn{2}{|c|}{ Atom Densities } \\
\hline Neutrons & 16000 & -1.000000 & 16000 & 1.000000 & 16000 & 0.037562 \\
\hline Photons & 16000 & -1.000000 & 16000 & 1.000000 & 16000 & 0.037562 \\
\hline CEPXS Form: & $\begin{array}{l}\text { material } \\
\text { matname } \\
\text { density }\end{array}$ & $\begin{array}{c}\text { S } \\
\text { Sulphur } \\
2.000000\end{array}$ & 1.000000 & & & \\
\hline $\begin{array}{l}\text { Comments an } \\
\text { Density }=2.00 \\
\text { Density }=2.07 \\
\text { Lide (2008) }\end{array}$ & $\begin{array}{l}\text { References } \\
\text { m3 from htt } \\
\text { m3 for rhom }\end{array}$ & $\begin{array}{l}\text { physics.nist } \\
\text { sulphur an }\end{array}$ & $\begin{array}{l}\mathrm{cgi}-\mathrm{bin} / \mathrm{S} \\
0 \mathrm{~g} / \mathrm{cm} 3\end{array}$ & $\begin{array}{l}\text { npos.pl?n } \\
\text { noclinic s }\end{array}$ & $\begin{array}{l}=016 \text { (NIS } \\
\text { on pgs } 4\end{array}$ & \\
\hline
\end{tabular}

\section{Tantalum}

$\begin{array}{llll}\text { Formula }= & \text { Ta } & \text { Molecular weight }(\mathrm{g} / \mathrm{mole})= & 180.9479 \\ \text { Density }(\mathrm{g} / \mathrm{cm} 3)= & 16.654000 & \text { Total atom density }(\text { atoms } / \mathrm{b}-\mathrm{cm})= & 5.543 \mathrm{E}-02\end{array}$

The above density is estimated to be accurate to 4 significant digits. Uncertainties are not addressed.

The following data was calculated from the input formula.

$$
\begin{array}{cccccc}
\text { Element } & \frac{\text { Neutron ZA }}{73181} & \frac{\text { Weight }}{73000} & \frac{\text { Atom }}{1.000000} & \frac{\text { Fraction }}{1.000000} & \frac{\text { Atom }}{\text { Density }} \\
0.055426
\end{array}
$$


PIET-43741-TM-963

PNNL-15870 Rev. 1

\begin{tabular}{|c|c|c|c|c|c|c|}
\hline Total & & & 1.000000 & 1.000000 & 0.055426 & \\
\hline MCNP Form & \multicolumn{2}{|c|}{ Weight Fractions } & \multicolumn{2}{|c|}{ Atom Fractions } & \multicolumn{2}{|c|}{ Atom Densities } \\
\hline Neutrons & 73181 & -1.000000 & 73181 & 1.000000 & 73181 & 0.055426 \\
\hline Photons & 73000 & -1.000000 & 73000 & 1.000000 & 73000 & 0.055426 \\
\hline CEPXS Form: & material & $\mathrm{Ta}$ & 1.000000 & & & \\
\hline & $\begin{array}{c}\text { matname } \\
\text { density }\end{array}$ & $\begin{array}{l}\text { Tantalum } \\
16.654000\end{array}$ & & & & \\
\hline
\end{tabular}

\section{Thorium}

\begin{tabular}{llll}
\hline Formula $=$ & Th & Molecular weight $(\mathrm{g} / \mathrm{mole})=$ & 232.0381 \\
Density $(\mathrm{g} / \mathrm{cm} 3)=$ & 11.720000 & Total atom density $($ atoms $/ \mathrm{b}-\mathrm{cm})=$ & $3.042 \mathrm{E}-02$
\end{tabular}

The above density is estimated to be accurate to 4 significant digits. Uncertainties are not addressed.

The following data was calculated from the input formula.

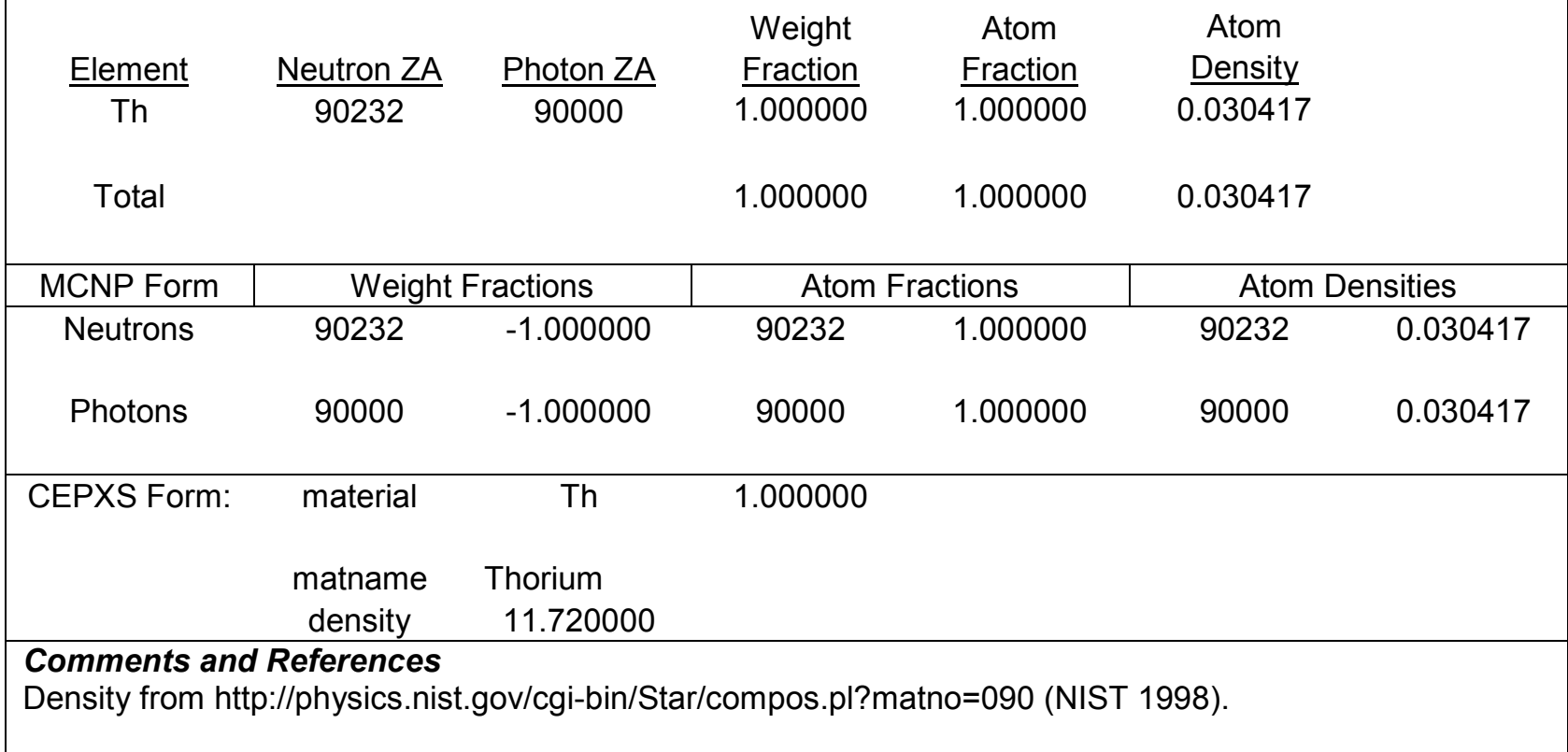

\section{Thorium Dioxide}

Formula $=\quad$ ThO2

Molecular weight $(\mathrm{g} / \mathrm{mole})=$

264.0369

Density $(\mathrm{g} / \mathrm{cm} 3)=10.000000$

Total atom density $($ atoms $/ \mathrm{b}-\mathrm{cm})=6.842 \mathrm{E}-02$

The above density is estimated to be accurate to 3 significant digits. Uncertainties are not addressed.

The following data was calculated from the input formula. 


\begin{tabular}{|c|c|c|c|c|c|c|}
\hline Element & Neutron ZA & Photon ZA & $\begin{array}{l}\text { Weight } \\
\text { Fraction }\end{array}$ & $\begin{array}{l}\text { Atom } \\
\text { Fraction }\end{array}$ & \multicolumn{2}{|l|}{ Atom } \\
\hline 0 & 8016 & 8000 & 0.121191 & 0.666667 & \multicolumn{2}{|l|}{0.045616} \\
\hline Th & 90232 & 90000 & 0.878809 & 0.333333 & \multicolumn{2}{|l|}{0.022808} \\
\hline Total & & & 1.000000 & 1.000000 & \multicolumn{2}{|l|}{0.068424} \\
\hline MCNP Form & \multicolumn{2}{|c|}{ Weight Fractions } & \multicolumn{2}{|c|}{ Atom Fractions } & \multicolumn{2}{|c|}{ Atom Densities } \\
\hline \multirow[t]{2}{*}{ Neutrons } & 8016 & -0.121191 & 8016 & 0.666667 & 8016 & 0.045616 \\
\hline & 90232 & -0.878809 & 90232 & 0.333333 & 90232 & 0.022808 \\
\hline \multirow[t]{2}{*}{ Photons } & 8000 & -0.121191 & 8000 & 0.666667 & 8000 & 0.045616 \\
\hline & 90000 & -0.878809 & 90000 & 0.333333 & 90000 & 0.022808 \\
\hline \multirow[t]{3}{*}{ CEPXS Form: } & material & $\mathrm{O}$ & 0.121191 & & & \\
\hline & & Th & 0.878809 & & & \\
\hline & $\begin{array}{l}\text { matname } \\
\text { density }\end{array}$ & $\begin{array}{c}\text { Thorium Diox } \\
10.000000\end{array}$ & & & & \\
\hline \multicolumn{7}{|c|}{$\begin{array}{l}\text { Comments and References } \\
\text { Density = } 10.0 \text { on pgs } 4-95 \text { of Lide (2008), and at } \\
\text { http://www.matweb.com/search/DataSheet.aspx?MatGUID=db32b396093d446aa4206468f0681736 } \\
\text { (Automation Creations } 2010 \text { ). } \\
\text { Density = } 10.03 \text { on pg II.F.1-7 of Carter et al. (1968). }\end{array}$} \\
\hline
\end{tabular}

\section{Tin}

\begin{tabular}{llll}
\hline Formula $=$ & $\mathrm{Sn}$ & Molecular weight $(\mathrm{g} / \mathrm{mole})=$ & 118.71 \\
Density $(\mathrm{g} / \mathrm{cm} 3)=$ & 7.310000 & Total atom density $($ atoms $/ \mathrm{b}-\mathrm{cm})=$ & $3.708 \mathrm{E}-02$
\end{tabular}

The above density is estimated to be accurate to 3 significant digits. Uncertainties are not addressed.

The following data was calculated from the input formula.

\begin{tabular}{|c|c|c|c|c|c|c|}
\hline$\frac{\text { Element }}{\mathrm{Sn}}$ & $\frac{\text { Neutron ZA }}{50000}$ & $\frac{\text { Photon ZA }}{50000}$ & $\begin{array}{l}\text { Weight } \\
\text { Fraction } \\
1.000000\end{array}$ & $\begin{array}{c}\begin{array}{c}\text { Atom } \\
\text { Fraction }\end{array} \\
1.000000\end{array}$ & $\begin{array}{c}\text { Atom } \\
\text { Density } \\
0.037084\end{array}$ & \\
\hline Total & & & 1.000000 & 1.000000 & 0.037084 & \\
\hline MCNP Form & \multicolumn{2}{|c|}{ Weight Fractions } & \multicolumn{2}{|c|}{ Atom Fractions } & \multicolumn{2}{|c|}{ Atom Densities } \\
\hline Neutrons & 50000 & -1.000000 & 50000 & 1.000000 & 50000 & 0.037084 \\
\hline Photons & 50000 & -1.000000 & 50000 & 1.000000 & 50000 & 0.037084 \\
\hline CEPXS Form: & $\begin{array}{l}\text { material } \\
\text { matname } \\
\text { density }\end{array}$ & $\begin{array}{c}\text { Sn } \\
\text { Tin } \\
\quad 7.310000\end{array}$ & 1.000000 & & & \\
\hline
\end{tabular}


Comments and References

Density from http://physics.nist.gov/cgi-bin/Star/compos.pl?matno=050 (NIST 1998).

\section{Tissue Equivalent, MS20}

\begin{tabular}{|c|c|c|c|}
\hline Formula $=$ & - & Molecular weight $(\mathrm{g} / \mathrm{mole})=$ & - \\
\hline Density $(\mathrm{g} / \mathrm{cm} 3)=$ & 1.000000 & Total atom density $($ atoms $/ \mathrm{b}-\mathrm{cm})=$ & 8.879E-02 \\
\hline
\end{tabular}

$\begin{array}{ccccccc}\text { Element } & \text { Neutron ZA } & \frac{\text { Photon ZA }}{1000} & & \begin{array}{c}\text { Weight } \\ \text { Fraction }\end{array} & \begin{array}{c}\text { Atom } \\ \text { Fraction }\end{array} & \begin{array}{c}\text { Atom } \\ \text { Density }\end{array} \\ \mathrm{H} & 1001 & & 0.081192 & 0.546359 & 0.048510 \\ \mathrm{C} & 6000 & 6000 & 0.583442 & 0.329480 & 0.029254 \\ \mathrm{O} & 7014 & 7000 & 0.017798 & 0.008619 & 0.000765 \\ \mathrm{Mg} & 8016 & 8000 & 0.186381 & 0.079013 & 0.007015 \\ \mathrm{Cl} & 12000 & 12000 & 0.130287 & 0.036358 & 0.003228 \\ & 17000 & 17000 & 0.000900 & 0.000172 & 0.000015\end{array}$

Total

$\begin{array}{lll}1.000000 & 1.000000 & 0.088787\end{array}$

\begin{tabular}{|c|cc|cccr|}
\hline MCNP Form & \multicolumn{2}{c|}{ Weight Fractions } & \multicolumn{2}{c|}{ Atom Fractions } & \multicolumn{2}{c|}{ Atom Densities } \\
\hline Neutrons & 1001 & -0.081192 & 1001 & 0.546359 & 1001 & 0.048510 \\
& 6000 & -0.583442 & 6000 & 0.329480 & 6000 & 0.029254 \\
& 7014 & -0.017798 & 7014 & 0.008619 & 7014 & 0.000765 \\
& 8016 & -0.186381 & 8016 & 0.079013 & 8016 & 0.007015 \\
& 12000 & -0.130287 & 12000 & 0.036358 & 12000 & 0.003228 \\
& 17000 & -0.000900 & 17000 & 0.000172 & 17000 & 0.000015 \\
Photons & & & & & & \\
& 1000 & -0.081192 & 1000 & 0.546359 & 1000 & 0.048510 \\
& 6000 & -0.583442 & 6000 & 0.329480 & 6000 & 0.029254 \\
& 7000 & -0.017798 & 7000 & 0.008619 & 7000 & 0.000765 \\
& 8000 & -0.186381 & 8000 & 0.079013 & 8000 & 0.007015 \\
& 12000 & -0.130287 & 12000 & 0.036358 & 12000 & 0.003228 \\
& 17000 & -0.000900 & 17000 & 0.000172 & 17000 & 0.000015
\end{tabular}

\begin{tabular}{|c|c|c|c|}
\hline \multirow[t]{6}{*}{ CEPXS Form: } & material & $\mathrm{H}$ & 0.081192 \\
\hline & & C & 0.583442 \\
\hline & & $\mathrm{N}$ & 0.017798 \\
\hline & & $\mathrm{O}$ & 0.186381 \\
\hline & & $\mathrm{Mg}$ & 0.130287 \\
\hline & & $\mathrm{Cl}$ & 0.000900 \\
\hline
\end{tabular}

matname Tissue Equivalent, MS20

density $\quad 1.000000$

\section{Comments and References}

Density and weight fractions from http://physics.nist.gov/cgi-bin/Star/compos.pl?matno=200 (NIST 1998). 


\section{Tissue Equivalent-Gas, Methane Based (TEG: MB)}

\begin{tabular}{|c|c|c|c|}
\hline Formula $=$ & - & Molecular weight $(\mathrm{g} / \mathrm{mole})=$ & - \\
\hline Density $(\mathrm{g} / \mathrm{cm} 3)=$ & 0.001064 & Total atom density $($ atoms $/ \mathrm{b}-\mathrm{cm})=$ & 1.070E-04 \\
\hline
\end{tabular}

The above density is estimated to be accurate to 4 significant digits. Uncertainties are not addressed.

The following data were calculated from the input weight fractions.

\begin{tabular}{|c|c|c|c|c|c|c|}
\hline Element & Neutron ZA & Photon ZA & $\begin{array}{l}\text { Weight } \\
\text { Fraction }\end{array}$ & $\begin{array}{l}\text { Atom } \\
\text { Fraction }\end{array}$ & $\begin{array}{l}\text { Atom } \\
\text { Density }\end{array}$ & \\
\hline $\mathrm{H}$ & 1001 & 1000 & 0.101869 & $\overline{0.605249}$ & \multicolumn{2}{|c|}{0.000065} \\
\hline C & 6000 & 6000 & 0.456179 & 0.227454 & \multicolumn{2}{|c|}{0.000024} \\
\hline $\mathrm{N}$ & 7014 & 7000 & 0.035172 & 0.015038 & \multicolumn{2}{|c|}{0.000002} \\
\hline $\mathrm{O}$ & 8016 & 8000 & 0.406780 & 0.152259 & \multicolumn{2}{|c|}{0.000016} \\
\hline Total & & & 1.000000 & 1.000000 & \multicolumn{2}{|c|}{0.000107} \\
\hline MCNP Form & \multicolumn{2}{|c|}{ Weight Fractions } & \multicolumn{2}{|c|}{ Atom Fractions } & \multicolumn{2}{|c|}{ Atom Densities } \\
\hline \multirow[t]{4}{*}{ Neutrons } & 1001 & -0.101869 & 1001 & 0.605249 & 1001 & 0.000065 \\
\hline & 6000 & -0.456179 & 6000 & 0.227454 & 6000 & 0.000024 \\
\hline & 7014 & -0.035172 & 7014 & 0.015038 & 7014 & 0.000002 \\
\hline & 8016 & -0.406780 & 8016 & 0.152259 & 8016 & 0.000016 \\
\hline \multirow[t]{4}{*}{ Photons } & 1000 & -0.101869 & 1000 & 0.605249 & 1000 & 0.000065 \\
\hline & 6000 & -0.456179 & 6000 & 0.227454 & 6000 & 0.000024 \\
\hline & 7000 & -0.035172 & 7000 & 0.015038 & 7000 & 0.000002 \\
\hline & 8000 & -0.406780 & 8000 & 0.152259 & 8000 & 0.000016 \\
\hline \multirow[t]{5}{*}{ CEPXS Form: } & material & $\mathrm{H}$ & 0.101869 & & & \\
\hline & & C & 0.456179 & & & \\
\hline & & $\mathrm{N}$ & 0.035172 & & & \\
\hline & & 0 & 0.406780 & & & \\
\hline & $\begin{array}{l}\text { matname } \\
\text { density }\end{array}$ & \multicolumn{5}{|c|}{$\begin{array}{l}\text { Tissue Equivalent-Gas, Methane Based (TEG: MB) } \\
0.001064\end{array}$} \\
\hline
\end{tabular}

\section{Tissue Equivalent-Gas, Propane Based (TEG: PB)}

\begin{tabular}{|c|c|c|c|}
\hline Formula $=$ & - & Molecular weight $(\mathrm{g} / \mathrm{mole})=$ & - \\
\hline Density $(\mathrm{g} / \mathrm{cm} 3)=$ & 0.001826 & Total atom density $($ atoms $/ \mathrm{b}-\mathrm{cm})=$ & 1.870E-04 \\
\hline
\end{tabular}

The above density is estimated to be accurate to 4 significant digits. Uncertainties are not addressed.

The following data were calculated from the input weight fractions. 


\begin{tabular}{|c|c|c|c|c|c|c|}
\hline Element & Neutron ZA & Photon ZA & $\begin{array}{l}\text { Weight } \\
\text { Fraction }\end{array}$ & $\begin{array}{l}\text { Atom } \\
\text { Fraction }\end{array}$ & $\begin{array}{l}\text { Atom } \\
\text { Density }\end{array}$ & \\
\hline $\mathrm{H}$ & 1001 & 1000 & 0.102672 & 0.598952 & 0.000112 & \\
\hline C & 6000 & 6000 & 0.568940 & 0.278531 & 0.000052 & \\
\hline $\mathrm{N}$ & 7014 & 7000 & 0.035022 & 0.014702 & 0.000003 & \\
\hline O & 8016 & 8000 & 0.293366 & 0.107815 & 0.000020 & \\
\hline Total & & & 1.000000 & 1.000000 & 0.000187 & \\
\hline MCNP Form & \multicolumn{2}{|c|}{ Weight Fractions } & \multicolumn{2}{|c|}{ Atom Fractions } & \multicolumn{2}{|c|}{ Atom Densities } \\
\hline \multirow[t]{4}{*}{ Neutrons } & 1001 & -0.102672 & 1001 & 0.598952 & 1001 & 0.000112 \\
\hline & 6000 & -0.568940 & 6000 & 0.278531 & 6000 & 0.000052 \\
\hline & 7014 & -0.035022 & 7014 & 0.014702 & 7014 & 0.000003 \\
\hline & 8016 & -0.293366 & 8016 & 0.107815 & 8016 & 0.000020 \\
\hline \multirow[t]{4}{*}{ Photons } & 1000 & -0.102672 & 1000 & 0.598952 & 1000 & 0.000112 \\
\hline & 6000 & -0.568940 & 6000 & 0.278531 & 6000 & 0.000052 \\
\hline & 7000 & -0.035022 & 7000 & 0.014702 & 7000 & 0.000003 \\
\hline & 8000 & -0.293366 & 8000 & 0.107815 & 8000 & 0.000020 \\
\hline \multirow[t]{5}{*}{ CEPXS Form: } & material & $\mathrm{H}$ & 0.102672 & & & \\
\hline & & $\mathrm{C}$ & 0.568940 & & & \\
\hline & & $N$ & 0.035022 & & & \\
\hline & & $\mathrm{O}$ & 0.293366 & & & \\
\hline & $\begin{array}{l}\text { matname } \\
\text { density }\end{array}$ & \multicolumn{5}{|c|}{$\begin{array}{l}\text { Tissue Equivalent-Gas, Propane Based (TEG: PB) } \\
0.001826\end{array}$} \\
\hline
\end{tabular}

\section{Tissue, Adipose (ICRP)}

\begin{tabular}{llll}
\hline Formula $=$ & - & Molecular weight $(\mathrm{g} / \mathrm{mole})=$ & - \\
Density $(\mathrm{g} / \mathrm{cm} 3)=$ & 0.920000 & Total atom density $($ atoms $/ \mathrm{b}-\mathrm{cm})=$ & $1.035 \mathrm{E}-01$
\end{tabular}

The above density is estimated to be accurate to 2 significant digits. Uncertainties are not addressed.

The following data were calculated from the input weight fractions.

\begin{tabular}{|c|c|c|c|c|c|}
\hline Element & Neutron ZA & Photon ZA & $\begin{array}{l}\text { Weight } \\
\text { Fraction }\end{array}$ & $\begin{array}{c}\text { Atom } \\
\text { Fraction }\end{array}$ & $\begin{array}{c}\text { Atom } \\
\text { Density }\end{array}$ \\
\hline $\mathrm{H}$ & 1001 & 1000 & 0.119477 & $\overline{0.634643}$ & 0.065673 \\
\hline C & 6000 & 6000 & 0.637240 & 0.284063 & 0.029395 \\
\hline $\mathrm{N}$ & 7014 & 7000 & 0.007970 & 0.003047 & 0.000315 \\
\hline 0 & 8016 & 8000 & 0.232333 & 0.077748 & 0.008045 \\
\hline $\mathrm{Na}$ & 11023 & 11000 & 0.000500 & 0.000116 & 0.000012 \\
\hline $\mathrm{Mg}$ & 12000 & 12000 & 0.000020 & 0.000004 & 0.000000 \\
\hline$P$ & 15031 & 15000 & 0.000160 & 0.000028 & 0.000003 \\
\hline$S$ & 16000 & 16000 & 0.000730 & 0.000122 & 0.000013 \\
\hline $\mathrm{Cl}$ & 17000 & 17000 & 0.001190 & 0.000180 & 0.000019 \\
\hline
\end{tabular}


PIET-43741-TM-963

PNNL-15870 Rev. 1

\begin{tabular}{|c|c|c|c|c|c|c|}
\hline $\mathrm{K}$ & 19000 & 19000 & 0.000320 & 0.000044 & \multicolumn{2}{|c|}{0.000005} \\
\hline $\mathrm{Ca}$ & 20000 & 20000 & 0.000020 & 0.000003 & \multicolumn{2}{|c|}{0.000000} \\
\hline $\mathrm{Fe}$ & 26000 & 26000 & 0.000020 & 0.000002 & \multicolumn{2}{|c|}{0.000000} \\
\hline $\mathrm{Zn}$ & 30000 & 30000 & 0.000020 & 0.000002 & \multicolumn{2}{|c|}{0.000000} \\
\hline Total & & & 1.000000 & 1.000000 & \multicolumn{2}{|c|}{0.103481} \\
\hline MCNP Form & \multicolumn{2}{|c|}{ Weight Fractions } & \multicolumn{2}{|c|}{ Atom Fractions } & \multicolumn{2}{|c|}{ Atom Densities } \\
\hline \multirow[t]{13}{*}{ Neutrons } & 1001 & -0.119477 & 1001 & 0.634643 & 1001 & 0.065673 \\
\hline & 6000 & -0.637240 & 6000 & 0.284063 & 6000 & 0.029395 \\
\hline & 7014 & -0.007970 & 7014 & 0.003047 & 7014 & 0.000315 \\
\hline & 8016 & -0.232333 & 8016 & 0.077748 & 8016 & 0.008045 \\
\hline & 11023 & -0.000500 & 11023 & 0.000116 & 11023 & 0.000012 \\
\hline & 12000 & -0.000020 & 12000 & 0.000004 & 12000 & 0.000000 \\
\hline & 15031 & -0.000160 & 15031 & 0.000028 & 15031 & 0.000003 \\
\hline & 16000 & -0.000730 & 16000 & 0.000122 & 16000 & 0.000013 \\
\hline & 17000 & -0.001190 & 17000 & 0.000180 & 17000 & 0.000019 \\
\hline & 19000 & -0.000320 & 19000 & 0.000044 & 19000 & 0.000005 \\
\hline & 20000 & -0.000020 & 20000 & 0.000003 & 20000 & 0.000000 \\
\hline & 26000 & -0.000020 & 26000 & 0.000002 & 26000 & 0.000000 \\
\hline & 30000 & -0.000020 & 30000 & 0.000002 & 30000 & 0.000000 \\
\hline \multirow[t]{13}{*}{ Photons } & 1000 & -0.119477 & 1000 & 0.634643 & 1000 & 0.065673 \\
\hline & 6000 & -0.637240 & 6000 & 0.284063 & 6000 & 0.029395 \\
\hline & 7000 & -0.007970 & 7000 & 0.003047 & 7000 & 0.000315 \\
\hline & 8000 & -0.232333 & 8000 & 0.077748 & 8000 & 0.008045 \\
\hline & 11000 & -0.000500 & 11000 & 0.000116 & 11000 & 0.000012 \\
\hline & 12000 & -0.000020 & 12000 & 0.000004 & 12000 & 0.000000 \\
\hline & 15000 & -0.000160 & 15000 & 0.000028 & 15000 & 0.000003 \\
\hline & 16000 & -0.000730 & 16000 & 0.000122 & 16000 & 0.000013 \\
\hline & 17000 & -0.001190 & 17000 & 0.000180 & 17000 & 0.000019 \\
\hline & 19000 & -0.000320 & 19000 & 0.000044 & 19000 & 0.000005 \\
\hline & 20000 & -0.000020 & 20000 & 0.000003 & 20000 & 0.000000 \\
\hline & 26000 & -0.000020 & 26000 & 0.000002 & 26000 & 0.000000 \\
\hline & 30000 & -0.000020 & 30000 & 0.000002 & 30000 & 0.000000 \\
\hline \multirow[t]{13}{*}{ CEPXS Form: } & material & $\mathrm{H}$ & 0.119477 & & & \\
\hline & & C & 0.637240 & & & \\
\hline & & $N$ & 0.007970 & & & \\
\hline & & $\mathrm{O}$ & 0.232333 & & & \\
\hline & & $\mathrm{Na}$ & 0.000500 & & & \\
\hline & & $\mathrm{Mg}$ & 0.000020 & & & \\
\hline & & $P$ & 0.000160 & & & \\
\hline & & $S$ & 0.000730 & & & \\
\hline & & $\mathrm{Cl}$ & 0.001190 & & & \\
\hline & & $\mathrm{K}$ & 0.000320 & & & \\
\hline & & $\mathrm{Ca}$ & 0.000020 & & & \\
\hline & & $\mathrm{Fe}$ & 0.000020 & & & \\
\hline & & $\mathrm{Zn}$ & 0.000020 & & & \\
\hline
\end{tabular}


matname Tissue, Adipose (ICRP) density $\quad 0.920000$

Comments and References

Density and weight fractions from http://physics.nist.gov/cgi-bin/Star/compos.pl?matno=103 (NIST 1998).

\section{Tissue, Breast}

$\begin{array}{llll}\text { Formula }= & - & \text { Molecular weight }(\mathrm{g} / \mathrm{mole})= & - \\ \text { Density }(\mathrm{g} / \mathrm{cm} 3)= & 1.020000 & \text { Total atom density }(\text { atoms } / \mathrm{b}-\mathrm{cm})= & 1.032 \mathrm{E}-01\end{array}$

The above density is estimated to be accurate to 3 significant digits. Uncertainties are not addressed.

The following data were calculated from the input weight fractions.

\begin{tabular}{|c|c|c|c|c|c|c|}
\hline Element & Neutron ZA & Photon ZA & $\begin{array}{l}\text { Weight } \\
\text { Fraction }\end{array}$ & $\begin{array}{l}\text { Atom } \\
\text { Fraction }\end{array}$ & $\begin{array}{l}\text { Atom } \\
\text { Density }\end{array}$ & \\
\hline $\mathrm{H}$ & 1001 & 1000 & 0.106000 & 0.625781 & 0.064598 & \\
\hline C & 6000 & 6000 & 0.332000 & 0.164483 & 0.016979 & \\
\hline $\mathrm{N}$ & 7014 & 7000 & 0.030000 & 0.012745 & 0.001316 & \\
\hline 0 & 8016 & 8000 & 0.527000 & 0.196001 & 0.020233 & \\
\hline $\mathrm{Na}$ & 11023 & 11000 & 0.001000 & 0.000259 & 0.000027 & \\
\hline$P$ & 15031 & 15000 & 0.001000 & 0.000192 & 0.000020 & \\
\hline$S$ & 16000 & 16000 & 0.002000 & 0.000371 & 0.000038 & \\
\hline $\mathrm{Cl}$ & 17000 & 17000 & 0.001000 & 0.000168 & 0.000017 & \\
\hline Total & & & 1.000000 & 1.000000 & 0.103229 & \\
\hline MCNP Form & \multicolumn{2}{|c|}{ Weight Fractions } & \multicolumn{2}{|c|}{ Atom Fractions } & \multicolumn{2}{|c|}{ Atom Densities } \\
\hline \multirow[t]{8}{*}{ Neutrons } & 1001 & -0.106000 & 1001 & 0.625781 & 1001 & 0.064598 \\
\hline & 6000 & -0.332000 & 6000 & 0.164483 & 6000 & 0.016979 \\
\hline & 7014 & -0.030000 & 7014 & 0.012745 & 7014 & 0.001316 \\
\hline & 8016 & -0.527000 & 8016 & 0.196001 & 8016 & 0.020233 \\
\hline & 11023 & -0.001000 & 11023 & 0.000259 & 11023 & 0.000027 \\
\hline & 15031 & -0.001000 & 15031 & 0.000192 & 15031 & 0.000020 \\
\hline & 16000 & -0.002000 & 16000 & 0.000371 & 16000 & 0.000038 \\
\hline & 17000 & -0.001000 & 17000 & 0.000168 & 17000 & 0.000017 \\
\hline \multirow[t]{8}{*}{ Photons } & 1000 & -0.106000 & 1000 & 0.625781 & 1000 & 0.064598 \\
\hline & 6000 & -0.332000 & 6000 & 0.164483 & 6000 & 0.016979 \\
\hline & 7000 & -0.030000 & 7000 & 0.012745 & 7000 & 0.001316 \\
\hline & 8000 & -0.527000 & 8000 & 0.196001 & 8000 & 0.020233 \\
\hline & 11000 & -0.001000 & 11000 & 0.000259 & 11000 & 0.000027 \\
\hline & 15000 & -0.001000 & 15000 & 0.000192 & 15000 & 0.000020 \\
\hline & 16000 & -0.002000 & 16000 & 0.000371 & 16000 & 0.000038 \\
\hline & 17000 & -0.001000 & 17000 & 0.000168 & 17000 & 0.000017 \\
\hline \multirow[t]{4}{*}{ CEPXS Form: } & material & $\mathrm{H}$ & 0.106000 & & & \\
\hline & & $\mathrm{C}$ & 0.332000 & & & \\
\hline & & $\mathrm{N}$ & 0.030000 & & & \\
\hline & & 0 & 0.527000 & & & \\
\hline
\end{tabular}




\begin{tabular}{|ccc|} 
& $\mathrm{Na}$ & 0.001000 \\
$\mathrm{P}$ & 0.001000 \\
& $\mathrm{~S}$ & 0.002000 \\
& $\mathrm{Cl}$ & 0.001000 \\
& & \\
matname & Tissue, Breast \\
density & 1.020000 \\
\hline Comments and References & \\
http://physics.nist.gov/PhysRefData/XrayMassCoef/tab2.html (NIST 1996). \\
\hline
\end{tabular}

\section{Tissue, Lung (ICRP)}

\begin{tabular}{llll}
\hline Formula $=$ & - & Molecular weight $(\mathrm{g} / \mathrm{mole})=$ & - \\
Density $(\mathrm{g} / \mathrm{cm} 3)=$ & 1.050000 & Total atom density $($ atoms $/ \mathrm{b}-\mathrm{cm})=$ & $1.004 \mathrm{E}-01$
\end{tabular}

The above density is estimated to be accurate to 3 significant digits. Uncertainties are not addressed. The following data were calculated from the input weight fractions.

\begin{tabular}{|c|c|c|c|c|c|c|}
\hline Element & Neutron ZA & Photon ZA & $\begin{array}{l}\text { Weight } \\
\text { Fraction }\end{array}$ & $\begin{array}{c}\text { Atom } \\
\text { Fraction }\end{array}$ & $\begin{array}{l}\text { Atom } \\
\text { Density }\end{array}$ & \\
\hline $\mathrm{H}$ & 1001 & 1000 & $\overline{0.101278}$ & $\overline{0.633136}$ & 0.063536 & \\
\hline C & 6000 & 6000 & 0.102310 & 0.053674 & 0.005386 & \\
\hline $\mathrm{N}$ & 7014 & 7000 & 0.028650 & 0.012889 & 0.001293 & \\
\hline $\mathrm{O}$ & 8016 & 8000 & 0.757072 & 0.298160 & 0.029921 & \\
\hline $\mathrm{Na}$ & 11023 & 11000 & 0.001840 & 0.000504 & 0.000051 & \\
\hline $\mathrm{Mg}$ & 12000 & 12000 & 0.000730 & 0.000189 & 0.000019 & \\
\hline $\mathrm{P}$ & 15031 & 15000 & 0.000800 & 0.000163 & 0.000016 & \\
\hline$S$ & 16000 & 16000 & 0.002250 & 0.000442 & 0.000044 & \\
\hline $\mathrm{Cl}$ & 17000 & 17000 & 0.002660 & 0.000473 & 0.000047 & \\
\hline $\mathrm{K}$ & 19000 & 19000 & 0.001940 & 0.000313 & 0.000031 & \\
\hline $\mathrm{Ca}$ & 20000 & 20000 & 0.000090 & 0.000014 & 0.000001 & \\
\hline $\mathrm{Fe}$ & 26000 & 26000 & 0.000370 & 0.000042 & 0.000004 & \\
\hline $\mathrm{Zn}$ & 30000 & 30000 & 0.000010 & 0.000001 & 0.000000 & \\
\hline Total & & & 1.000000 & 1.000000 & 0.100351 & \\
\hline MCNP Form & \multicolumn{2}{|c|}{ Weight Fractions } & \multicolumn{2}{|c|}{ Atom Fractions } & \multicolumn{2}{|c|}{ Atom Densities } \\
\hline \multirow[t]{12}{*}{ Neutrons } & 1001 & -0.101278 & 1001 & 0.633136 & 1001 & 0.063536 \\
\hline & 6000 & -0.102310 & 6000 & 0.053674 & 6000 & 0.005386 \\
\hline & 7014 & -0.028650 & 7014 & 0.012889 & 7014 & 0.001293 \\
\hline & 8016 & -0.757072 & 8016 & 0.298160 & 8016 & 0.029921 \\
\hline & 11023 & -0.001840 & 11023 & 0.000504 & 11023 & 0.000051 \\
\hline & 12000 & -0.000730 & 12000 & 0.000189 & 12000 & 0.000019 \\
\hline & 15031 & -0.000800 & 15031 & 0.000163 & 15031 & 0.000016 \\
\hline & 16000 & -0.002250 & 16000 & 0.000442 & 16000 & 0.000044 \\
\hline & 17000 & -0.002660 & 17000 & 0.000473 & 17000 & 0.000047 \\
\hline & 19000 & -0.001940 & 19000 & 0.000313 & 19000 & 0.000031 \\
\hline & 20000 & -0.000090 & 20000 & 0.000014 & 20000 & 0.000001 \\
\hline & 26000 & -0.000370 & 26000 & 0.000042 & 26000 & 0.000004 \\
\hline
\end{tabular}


PIET-43741-TM-963

PNNL-15870 Rev. 1

\begin{tabular}{|c|c|c|c|c|c|c|}
\hline & 30000 & -0.000010 & 30000 & 0.000001 & 30000 & 0.000000 \\
\hline \multirow[t]{13}{*}{ Photons } & 1000 & -0.101278 & 1000 & 0.633136 & 1000 & 0.063536 \\
\hline & 6000 & -0.102310 & 6000 & 0.053674 & 6000 & 0.005386 \\
\hline & 7000 & -0.028650 & 7000 & 0.012889 & 7000 & 0.001293 \\
\hline & 8000 & -0.757072 & 8000 & 0.298160 & 8000 & 0.029921 \\
\hline & 11000 & -0.001840 & 11000 & 0.000504 & 11000 & 0.000051 \\
\hline & 12000 & -0.000730 & 12000 & 0.000189 & 12000 & 0.000019 \\
\hline & 15000 & -0.000800 & 15000 & 0.000163 & 15000 & 0.000016 \\
\hline & 16000 & -0.002250 & 16000 & 0.000442 & 16000 & 0.000044 \\
\hline & 17000 & -0.002660 & 17000 & 0.000473 & 17000 & 0.000047 \\
\hline & 19000 & -0.001940 & 19000 & 0.000313 & 19000 & 0.000031 \\
\hline & 20000 & -0.000090 & 20000 & 0.000014 & 20000 & 0.000001 \\
\hline & 26000 & -0.000370 & 26000 & 0.000042 & 26000 & 0.000004 \\
\hline & 30000 & -0.000010 & 30000 & 0.000001 & 30000 & 0.000000 \\
\hline \multirow[t]{14}{*}{ CEPXS Form: } & material & $\mathrm{H}$ & 0.101278 & & & \\
\hline & & C & 0.102310 & & & \\
\hline & & $\mathrm{N}$ & 0.028650 & & & \\
\hline & & $\mathrm{O}$ & 0.757072 & & & \\
\hline & & $\mathrm{Na}$ & 0.001840 & & & \\
\hline & & $\mathrm{Mg}$ & 0.000730 & & & \\
\hline & & $\mathrm{P}$ & 0.000800 & & & \\
\hline & & $S$ & 0.002250 & & & \\
\hline & & $\mathrm{Cl}$ & 0.002660 & & & \\
\hline & & $\mathrm{K}$ & 0.001940 & & & \\
\hline & & $\mathrm{Ca}$ & 0.000090 & & & \\
\hline & & $\mathrm{Fe}$ & 0.000370 & & & \\
\hline & & $\mathrm{Zn}$ & 0.000010 & & & \\
\hline & $\begin{array}{l}\text { matname } \\
\text { density }\end{array}$ & \multicolumn{2}{|c|}{$\begin{array}{l}\text { Tissue, Lung (ICRP) } \\
1.050000\end{array}$} & & & \\
\hline
\end{tabular}

\section{Tissue, Ovary}

\begin{tabular}{llll}
\hline Formula $=$ & - & Molecular weight $(\mathrm{g} / \mathrm{mole})=$ \\
Density $(\mathrm{g} / \mathrm{cm} 3)=$ & 1.050000 & Total atom density $($ atoms $/ \mathrm{b}-\mathrm{cm})=$ & - \\
The & 1.024E-01
\end{tabular}

The above density is estimated to be accurate to 3 significant digits. Uncertainties are not addressed.

The following data were calculated from the input weight fractions.

\begin{tabular}{|c|c|c|c|c|c|}
\hline Element & Neutron ZA & Photon ZA & $\begin{array}{l}\text { Weight } \\
\text { Fraction }\end{array}$ & $\begin{array}{c}\text { Atom } \\
\text { Fraction }\end{array}$ & $\begin{array}{c}\text { Atom } \\
\text { Density }\end{array}$ \\
\hline $\mathrm{H}$ & 1001 & 1000 & 0.105000 & 0.643230 & 0.065871 \\
\hline C & 6000 & 6000 & 0.093000 & 0.047811 & 0.004896 \\
\hline $\mathrm{N}$ & 7014 & 7000 & 0.024000 & 0.010580 & 0.001083 \\
\hline $\mathrm{O}$ & 8016 & 8000 & 0.768000 & 0.296394 & 0.030353 \\
\hline
\end{tabular}


PIET-43741-TM-963

PNNL-15870 Rev. 1

\begin{tabular}{|c|c|c|c|c|c|c|}
\hline $\mathrm{Na}$ & 11023 & 11000 & 0.002000 & 0.000537 & 0.000055 & \\
\hline $\mathrm{P}$ & 15031 & 15000 & 0.002000 & 0.000399 & 0.000041 & \\
\hline$S$ & 16000 & 16000 & 0.002000 & 0.000385 & 0.000039 & \\
\hline $\mathrm{Cl}$ & 17000 & 17000 & 0.002000 & 0.000348 & 0.000036 & \\
\hline $\mathrm{K}$ & 19000 & 19000 & 0.002000 & 0.000316 & 0.000032 & \\
\hline Total & & & 1.000000 & 1.000000 & 0.102407 & \\
\hline MCNP Form & \multicolumn{2}{|c|}{ Weight Fractions } & \multicolumn{2}{|c|}{ Atom Fractions } & \multicolumn{2}{|c|}{ Atom Densities } \\
\hline \multirow[t]{9}{*}{ Neutrons } & 1001 & -0.105000 & 1001 & 0.643230 & 1001 & 0.065871 \\
\hline & 6000 & -0.093000 & 6000 & 0.047811 & 6000 & 0.004896 \\
\hline & 7014 & -0.024000 & 7014 & 0.010580 & 7014 & 0.001083 \\
\hline & 8016 & -0.768000 & 8016 & 0.296394 & 8016 & 0.030353 \\
\hline & 11023 & -0.002000 & 11023 & 0.000537 & 11023 & 0.000055 \\
\hline & 15031 & -0.002000 & 15031 & 0.000399 & 15031 & 0.000041 \\
\hline & 16000 & -0.002000 & 16000 & 0.000385 & 16000 & 0.000039 \\
\hline & 17000 & -0.002000 & 17000 & 0.000348 & 17000 & 0.000036 \\
\hline & 19000 & -0.002000 & 19000 & 0.000316 & 19000 & 0.000032 \\
\hline \multirow[t]{9}{*}{ Photons } & 1000 & -0.105000 & 1000 & 0.643230 & 1000 & 0.065871 \\
\hline & 6000 & -0.093000 & 6000 & 0.047811 & 6000 & 0.004896 \\
\hline & 7000 & -0.024000 & 7000 & 0.010580 & 7000 & 0.001083 \\
\hline & 8000 & -0.768000 & 8000 & 0.296394 & 8000 & 0.030353 \\
\hline & 11000 & -0.002000 & 11000 & 0.000537 & 11000 & 0.000055 \\
\hline & 15000 & -0.002000 & 15000 & 0.000399 & 15000 & 0.000041 \\
\hline & 16000 & -0.002000 & 16000 & 0.000385 & 16000 & 0.000039 \\
\hline & 17000 & -0.002000 & 17000 & 0.000348 & 17000 & 0.000036 \\
\hline & 19000 & -0.002000 & 19000 & 0.000316 & 19000 & 0.000032 \\
\hline \multirow[t]{10}{*}{ CEPXS Form: } & material & $\mathrm{H}$ & 0.105000 & & & \\
\hline & & C & 0.093000 & & & \\
\hline & & $\mathrm{N}$ & 0.024000 & & & \\
\hline & & $\mathrm{O}$ & 0.768000 & & & \\
\hline & & $\mathrm{Na}$ & 0.002000 & & & \\
\hline & & $\mathrm{P}$ & 0.002000 & & & \\
\hline & & $S$ & 0.002000 & & & \\
\hline & & $\mathrm{Cl}$ & 0.002000 & & & \\
\hline & & $\mathrm{K}$ & 0.002000 & & & \\
\hline & $\begin{array}{c}\text { matname } \\
\text { density }\end{array}$ & $\begin{array}{c}\text { Tissue, Ovary } \\
1.050000\end{array}$ & & & & \\
\hline $\begin{array}{l}\text { Comments an } \\
\text { http://physics.n }\end{array}$ & eferences & 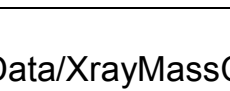 & & 996). & & \\
\hline
\end{tabular}

\section{Tissue, Soft (ICRP)}

\begin{tabular}{|llll|}
\hline Formula $=$ & - & Molecular weight $(\mathrm{g} / \mathrm{mole})=$ \\
Density $(\mathrm{g} / \mathrm{cm} 3)=$ & 1.000000 & Total atom density $($ atoms $/ \mathrm{b}-\mathrm{cm})=$ & - \\
\hline
\end{tabular}


The above density is estimated to be accurate to 3 significant digits. Uncertainties are not addressed. The following data were calculated from the input weight fractions.

\begin{tabular}{|c|c|c|c|c|c|c|}
\hline Element & Neutron ZA & Photon ZA & $\begin{array}{l}\text { Weight } \\
\text { Fraction }\end{array}$ & $\begin{array}{c}\text { Atom } \\
\text { Fraction }\end{array}$ & $\begin{array}{l}\text { Atom } \\
\text { Density }\end{array}$ & \\
\hline $\mathrm{H}$ & 1001 & 1000 & 0.104472 & 0.630454 & 0.062419 & \\
\hline $\mathrm{C}$ & 6000 & 6000 & 0.232190 & 0.117588 & 0.011642 & \\
\hline $\mathrm{N}$ & 7014 & 7000 & 0.024880 & 0.010804 & 0.001070 & \\
\hline $\mathrm{O}$ & 8016 & 8000 & 0.630238 & 0.239601 & 0.023722 & \\
\hline $\mathrm{Na}$ & 11023 & 11000 & 0.001130 & 0.000299 & 0.000030 & \\
\hline $\mathrm{Mg}$ & 12000 & 12000 & 0.000130 & 0.000033 & 0.000003 & \\
\hline $\mathrm{P}$ & 15031 & 15000 & 0.001330 & 0.000261 & 0.000026 & \\
\hline$S$ & 16000 & 16000 & 0.001990 & 0.000377 & 0.000037 & \\
\hline $\mathrm{Cl}$ & 17000 & 17000 & 0.001340 & 0.000230 & 0.000023 & \\
\hline $\mathrm{K}$ & 19000 & 19000 & 0.001990 & 0.000310 & 0.000031 & \\
\hline $\mathrm{Ca}$ & 20000 & 20000 & 0.000230 & 0.000035 & 0.000003 & \\
\hline $\mathrm{Fe}$ & 26000 & 26000 & 0.000050 & 0.000005 & 0.000001 & \\
\hline $\mathrm{Zn}$ & 30000 & 30000 & 0.000030 & 0.000003 & 0.000000 & \\
\hline Total & & & 1.000000 & 1.000000 & 0.099006 & \\
\hline MCNP Form & \multicolumn{2}{|c|}{ Weight Fractions } & \multicolumn{2}{|c|}{ Atom Fractions } & \multicolumn{2}{|c|}{ Atom Densities } \\
\hline \multirow[t]{13}{*}{ Neutrons } & 1001 & -0.104472 & 1001 & 0.630454 & 1001 & 0.062419 \\
\hline & 6000 & -0.232190 & 6000 & 0.117588 & 6000 & 0.011642 \\
\hline & 7014 & -0.024880 & 7014 & 0.010804 & 7014 & 0.001070 \\
\hline & 8016 & -0.630238 & 8016 & 0.239601 & 8016 & 0.023722 \\
\hline & 11023 & -0.001130 & 11023 & 0.000299 & 11023 & 0.000030 \\
\hline & 12000 & -0.000130 & 12000 & 0.000033 & 12000 & 0.000003 \\
\hline & 15031 & -0.001330 & 15031 & 0.000261 & 15031 & 0.000026 \\
\hline & 16000 & -0.001990 & 16000 & 0.000377 & 16000 & 0.000037 \\
\hline & 17000 & -0.001340 & 17000 & 0.000230 & 17000 & 0.000023 \\
\hline & 19000 & -0.001990 & 19000 & 0.000310 & 19000 & 0.000031 \\
\hline & 20000 & -0.000230 & 20000 & 0.000035 & 20000 & 0.000003 \\
\hline & 26000 & -0.000050 & 26000 & 0.000005 & 26000 & 0.000001 \\
\hline & 30000 & -0.000030 & 30000 & 0.000003 & 30000 & 0.000000 \\
\hline \multirow[t]{13}{*}{ Photons } & 1000 & -0.104472 & 1000 & 0.630454 & 1000 & 0.062419 \\
\hline & 6000 & -0.232190 & 6000 & 0.117588 & 6000 & 0.011642 \\
\hline & 7000 & -0.024880 & 7000 & 0.010804 & 7000 & 0.001070 \\
\hline & 8000 & -0.630238 & 8000 & 0.239601 & 8000 & 0.023722 \\
\hline & 11000 & -0.001130 & 11000 & 0.000299 & 11000 & 0.000030 \\
\hline & 12000 & -0.000130 & 12000 & 0.000033 & 12000 & 0.000003 \\
\hline & 15000 & -0.001330 & 15000 & 0.000261 & 15000 & 0.000026 \\
\hline & 16000 & -0.001990 & 16000 & 0.000377 & 16000 & 0.000037 \\
\hline & 17000 & -0.001340 & 17000 & 0.000230 & 17000 & 0.000023 \\
\hline & 19000 & -0.001990 & 19000 & 0.000310 & 19000 & 0.000031 \\
\hline & 20000 & -0.000230 & 20000 & 0.000035 & 20000 & 0.000003 \\
\hline & 26000 & -0.000050 & 26000 & 0.000005 & 26000 & 0.000001 \\
\hline & 30000 & -0.000030 & 30000 & 0.000003 & 30000 & 0.000000 \\
\hline
\end{tabular}




\begin{tabular}{|ccc} 
CEPXS Form: material & $\mathrm{H}$ & 0.104472 \\
& $\mathrm{C}$ & 0.232190 \\
$\mathrm{~N}$ & 0.024880 \\
$\mathrm{O}$ & 0.630238 \\
$\mathrm{Na}$ & 0.001130 \\
$\mathrm{Mg}$ & 0.000130 \\
$\mathrm{P}$ & 0.001330 \\
$\mathrm{~S}$ & 0.001990 \\
$\mathrm{Cl}$ & 0.001340 \\
$\mathrm{~K}$ & 0.001990 \\
$\mathrm{Ca}$ & 0.000230 \\
$\mathrm{Fe}$ & 0.000050 \\
$\mathrm{Zn}$ & 0.000030
\end{tabular}

matname Tissue, Soft (ICRP) density $\quad 1.000000$

Comments and References

Density and weight fractions from http://physics.nist.gov/cgi-bin/Star/compos.pl?matno=261 (NIST 1998).

\section{Tissue, Soft (ICRU Four Component)}

\begin{tabular}{|c|c|c|c|}
\hline$a=$ & - & Molecular weight $(\mathrm{g} / \mathrm{mole})=$ & $9581 \mathrm{~F}-02$ \\
\hline
\end{tabular}

The above density is estimated to be accurate to 3 significant digits. Uncertainties are not addressed.

The following data were calculated from the input weight fractions.

\begin{tabular}{|c|c|c|c|c|c|c|}
\hline Element & Neutron ZA & Photon ZA & $\begin{array}{l}\text { Weight } \\
\text { Fraction }\end{array}$ & $\begin{array}{l}\text { Atom } \\
\text { Fraction }\end{array}$ & $\begin{array}{l}\text { Atom } \\
\text { Density }\end{array}$ & \\
\hline $\mathrm{H}$ & 1001 & 1000 & 0.101172 & 0.630936 & 0.060447 & \\
\hline C & 6000 & 6000 & 0.111000 & 0.058092 & 0.005566 & \\
\hline $\mathrm{N}$ & 7014 & 7000 & 0.026000 & 0.011668 & 0.001118 & \\
\hline $\mathrm{O}$ & 8016 & 8000 & 0.761828 & 0.299304 & 0.028675 & \\
\hline Total & & & 1.000000 & 1.000000 & 0.095806 & \\
\hline MCNP Form & \multicolumn{2}{|c|}{ Weight Fractions } & \multicolumn{2}{|c|}{ Atom Fractions } & \multicolumn{2}{|c|}{ Atom Densities } \\
\hline \multirow[t]{4}{*}{ Neutrons } & 1001 & -0.101172 & 1001 & 0.630936 & 1001 & 0.060447 \\
\hline & 6000 & -0.111000 & 6000 & 0.058092 & 6000 & 0.005566 \\
\hline & 7014 & -0.026000 & 7014 & 0.011668 & 7014 & 0.001118 \\
\hline & 8016 & -0.761828 & 8016 & 0.299304 & 8016 & 0.028675 \\
\hline \multirow[t]{4}{*}{ Photons } & 1000 & -0.101172 & 1000 & 0.630936 & 1000 & 0.060447 \\
\hline & 6000 & -0.111000 & 6000 & 0.058092 & 6000 & 0.005566 \\
\hline & 7000 & -0.026000 & 7000 & 0.011668 & 7000 & 0.001118 \\
\hline & 8000 & -0.761828 & 8000 & 0.299304 & 8000 & 0.028675 \\
\hline CEPXS Form: & material & $\mathrm{H}$ & $\begin{array}{l}0.101172 \\
011100 \Omega\end{array}$ & & & \\
\hline
\end{tabular}




\begin{tabular}{|ccc|} 
& $\mathrm{N}$ & 0.026000 \\
& $\mathrm{O}$ & 0.761828 \\
& & \\
matname & Tissue, Soft (ICRU Four Component) \\
density & 1.000000 \\
\hline Comments and References & & \\
Density and weight fractions from http://physics.nist.gov/cgi-bin/Star/compos.pl?matno=262 (NIST 1998). \\
\hline
\end{tabular}

\section{Tissue, Testes (ICRP)}

\begin{tabular}{llll}
\hline Formula $=$ & - & Molecular weight $(\mathrm{g} / \mathrm{mole})=$ & - \\
Density $(\mathrm{g} / \mathrm{cm} 3)=$ & 1.040000 & Total atom density $($ atoms $/ \mathrm{b}-\mathrm{cm})=$ & $1.009 \mathrm{E}-01$ \\
The above density is estimated to be accurate to & 3 significant digits. Uncertainties are not addressed. \\
The following data were calculated from the input weight fractions.
\end{tabular}

\begin{tabular}{|c|c|c|c|c|c|c|}
\hline Element & Neutron ZA & Photon ZA & $\begin{array}{l}\text { Weight } \\
\text { Fraction }\end{array}$ & $\begin{array}{c}\text { Atom } \\
\text { Fraction }\end{array}$ & $\begin{array}{l}\text { Atom } \\
\text { Density }\end{array}$ & \\
\hline $\mathrm{H}$ & 1001 & 1000 & 0.104166 & 0.641360 & 0.064726 & \\
\hline C & 6000 & 6000 & 0.092270 & 0.047676 & 0.004811 & \\
\hline $\mathrm{N}$ & 7014 & 7000 & 0.019940 & 0.008835 & 0.000892 & \\
\hline $\mathrm{O}$ & 8016 & 8000 & 0.773884 & 0.300181 & 0.030294 & \\
\hline $\mathrm{Na}$ & 11023 & 11000 & 0.002260 & 0.000610 & 0.000062 & \\
\hline $\mathrm{Mg}$ & 12000 & 12000 & 0.000110 & 0.000028 & 0.000003 & \\
\hline$P$ & 15031 & 15000 & 0.001250 & 0.000250 & 0.000025 & \\
\hline$S$ & 16000 & 16000 & 0.001460 & 0.000283 & 0.000029 & \\
\hline $\mathrm{Cl}$ & 17000 & 17000 & 0.002440 & 0.000427 & 0.000043 & \\
\hline $\mathrm{K}$ & 19000 & 19000 & 0.002080 & 0.000330 & 0.000033 & \\
\hline $\mathrm{Ca}$ & 20000 & 20000 & 0.000100 & 0.000015 & 0.000002 & \\
\hline $\mathrm{Fe}$ & 26000 & 26000 & 0.000020 & 0.000002 & 0.000000 & \\
\hline $\mathrm{Zn}$ & 30000 & 30000 & 0.000020 & 0.000002 & 0.000000 & \\
\hline Total & & & 1.000000 & 1.000000 & 0.100919 & \\
\hline MCNP Form & \multicolumn{2}{|c|}{ Weight Fractions } & \multicolumn{2}{|c|}{ Atom Fractions } & \multicolumn{2}{|c|}{ Atom Densities } \\
\hline \multirow[t]{13}{*}{ Neutrons } & 1001 & -0.104166 & 1001 & 0.641360 & 1001 & 0.064726 \\
\hline & 6000 & -0.092270 & 6000 & 0.047676 & 6000 & 0.004811 \\
\hline & 7014 & -0.019940 & 7014 & 0.008835 & 7014 & 0.000892 \\
\hline & 8016 & -0.773884 & 8016 & 0.300181 & 8016 & 0.030294 \\
\hline & 11023 & -0.002260 & 11023 & 0.000610 & 11023 & 0.000062 \\
\hline & 12000 & -0.000110 & 12000 & 0.000028 & 12000 & 0.000003 \\
\hline & 15031 & -0.001250 & 15031 & 0.000250 & 15031 & 0.000025 \\
\hline & 16000 & -0.001460 & 16000 & 0.000283 & 16000 & 0.000029 \\
\hline & 17000 & -0.002440 & 17000 & 0.000427 & 17000 & 0.000043 \\
\hline & 19000 & -0.002080 & 19000 & 0.000330 & 19000 & 0.000033 \\
\hline & 20000 & -0.000100 & 20000 & 0.000015 & 20000 & 0.000002 \\
\hline & 26000 & -0.000020 & 26000 & 0.000002 & 26000 & 0.000000 \\
\hline & 30000 & -0.000020 & 30000 & 0.000002 & 30000 & 0.000000 \\
\hline
\end{tabular}


PIET-43741-TM-963

PNNL-15870 Rev. 1

\begin{tabular}{|c|c|c|c|c|c|c|}
\hline \multirow[t]{13}{*}{ Photons } & 1000 & -0.104166 & 1000 & 0.641360 & 1000 & 0.064726 \\
\hline & 6000 & -0.092270 & 6000 & 0.047676 & 6000 & 0.004811 \\
\hline & 7000 & -0.019940 & 7000 & 0.008835 & 7000 & 0.000892 \\
\hline & 8000 & -0.773884 & 8000 & 0.300181 & 8000 & 0.030294 \\
\hline & 11000 & -0.002260 & 11000 & 0.000610 & 11000 & 0.000062 \\
\hline & 12000 & -0.000110 & 12000 & 0.000028 & 12000 & 0.000003 \\
\hline & 15000 & -0.001250 & 15000 & 0.000250 & 15000 & 0.000025 \\
\hline & 16000 & -0.001460 & 16000 & 0.000283 & 16000 & 0.000029 \\
\hline & 17000 & -0.002440 & 17000 & 0.000427 & 17000 & 0.000043 \\
\hline & 19000 & -0.002080 & 19000 & 0.000330 & 19000 & 0.000033 \\
\hline & 20000 & -0.000100 & 20000 & 0.000015 & 20000 & 0.000002 \\
\hline & 26000 & -0.000020 & 26000 & 0.000002 & 26000 & 0.000000 \\
\hline & 30000 & -0.000020 & 30000 & 0.000002 & 30000 & 0.000000 \\
\hline \multirow[t]{14}{*}{ CEPXS Form: } & material & $\mathrm{H}$ & 0.104166 & & & \\
\hline & & C & 0.092270 & & & \\
\hline & & $\mathrm{N}$ & 0.019940 & & & \\
\hline & & 0 & 0.773884 & & & \\
\hline & & $\mathrm{Na}$ & 0.002260 & & & \\
\hline & & $\mathrm{Mg}$ & 0.000110 & & & \\
\hline & & $P$ & 0.001250 & & & \\
\hline & & $\mathrm{s}$ & 0.001460 & & & \\
\hline & & $\mathrm{Cl}$ & 0.002440 & & & \\
\hline & & $\mathrm{K}$ & 0.002080 & & & \\
\hline & & $\mathrm{Ca}$ & 0.000100 & & & \\
\hline & & $\mathrm{Fe}$ & 0.000020 & & & \\
\hline & & $\mathrm{Zn}$ & 0.000020 & & & \\
\hline & $\begin{array}{l}\text { matname } \\
\text { density }\end{array}$ & \multicolumn{2}{|c|}{$\begin{array}{l}\text { Tissue, Testes (ICRP) } \\
1.040000\end{array}$} & & & \\
\hline
\end{tabular}

\section{Tissue, Testis (ICRU)}

\begin{tabular}{llll}
\hline Formula $=$ & - & Molecular weight $(\mathrm{g} / \mathrm{mole})=$ \\
Density $(\mathrm{g} / \mathrm{cm} 3)=$ & 1.040000 & Total atom density $($ atoms $/ \mathrm{b}-\mathrm{cm})=$ & - \\
& & & $1.021 \mathrm{E}-01$
\end{tabular}

The above density is estimated to be accurate to 3 significant digits. Uncertainties are not addressed.

The following data were calculated from the input weight fractions.

$\begin{array}{cccccc}\text { Element } & \text { Neutron ZA } & \text { Photon ZA } & \begin{array}{c}\text { Weight } \\ \text { Fraction }\end{array} & \begin{array}{c}\text { Atom } \\ \text { Fraction }\end{array} & \begin{array}{c}\text { Atom } \\ \text { Density }\end{array} \\ \mathrm{H} & 1001 & 1000 & 0.106000 & 0.645178 & 0.065865 \\ \mathrm{C} & 6000 & 6000 & 0.099000 & 0.050568 & 0.005162 \\ \mathrm{~N} & 7014 & 7000 & 0.020000 & 0.008760 & 0.000894 \\ \mathrm{O} & 8016 & 8000 & 0.766000 & 0.293720 & 0.029985 \\ \mathrm{Na} & 11023 & 11000 & 0.002000 & 0.000534 & 0.000054 \\ \mathrm{P} & 15031 & 15000 & 0.001000 & 0.000198 & 0.000020\end{array}$


PIET-43741-TM-963

PNNL-15870 Rev. 1

\begin{tabular}{|c|c|c|c|c|c|c|}
\hline$S$ & 16000 & 16000 & 0.002000 & 0.000383 & 0.000039 & \\
\hline $\mathrm{Cl}$ & 17000 & 17000 & 0.002000 & 0.000346 & 0.000035 & \\
\hline $\mathrm{K}$ & 19000 & 19000 & 0.002000 & 0.000314 & 0.000032 & \\
\hline Total & & & 1.000000 & 1.000000 & 0.102088 & \\
\hline MCNP Form & Weigh & ractions & Atom & ctions & Atom & sities \\
\hline Neutrons & 1001 & -0.106000 & 1001 & 0.645178 & 1001 & 0.065865 \\
\hline & 6000 & -0.099000 & 6000 & 0.050568 & 6000 & 0.005162 \\
\hline & 7014 & -0.020000 & 7014 & 0.008760 & 7014 & 0.000894 \\
\hline & 8016 & -0.766000 & 8016 & 0.293720 & 8016 & 0.029985 \\
\hline & 11023 & -0.002000 & 11023 & 0.000534 & 11023 & 0.000054 \\
\hline & 15031 & -0.001000 & 15031 & 0.000198 & 15031 & 0.000020 \\
\hline & 16000 & -0.002000 & 16000 & 0.000383 & 16000 & 0.000039 \\
\hline & 17000 & -0.002000 & 17000 & 0.000346 & 17000 & 0.000035 \\
\hline & 19000 & -0.002000 & 19000 & 0.000314 & 19000 & 0.000032 \\
\hline Photons & 1000 & -0.106000 & 1000 & 0.645178 & 1000 & 0.065865 \\
\hline & 6000 & -0.099000 & 6000 & 0.050568 & 6000 & 0.005162 \\
\hline & 7000 & -0.020000 & 7000 & 0.008760 & 7000 & 0.000894 \\
\hline & 8000 & -0.766000 & 8000 & 0.293720 & 8000 & 0.029985 \\
\hline & 11000 & -0.002000 & 11000 & 0.000534 & 11000 & 0.000054 \\
\hline & 15000 & -0.001000 & 15000 & 0.000198 & 15000 & 0.000020 \\
\hline & 16000 & -0.002000 & 16000 & 0.000383 & 16000 & 0.000039 \\
\hline & 17000 & -0.002000 & 17000 & 0.000346 & 17000 & 0.000035 \\
\hline & 19000 & -0.002000 & 19000 & 0.000314 & 19000 & 0.000032 \\
\hline CEPXS Form: & material & $\mathrm{H}$ & 0.106000 & & & \\
\hline & & C & 0.099000 & & & \\
\hline & & $\mathrm{N}$ & 0.020000 & & & \\
\hline & & 0 & 0.766000 & & & \\
\hline & & $\mathrm{Na}$ & 0.002000 & & & \\
\hline & & $P$ & 0.001000 & & & \\
\hline & & $S$ & 0.002000 & & & \\
\hline & & $\mathrm{Cl}$ & 0.002000 & & & \\
\hline & & $\mathrm{K}$ & 0.002000 & & & \\
\hline & $\begin{array}{c}\text { matname } \\
\text { density }\end{array}$ & $\begin{array}{c}\text { Tissue, Test } \\
1.040000\end{array}$ & ICRU) & & & \\
\hline
\end{tabular}

\section{Titanium}

\begin{tabular}{llll}
\hline Formula $=$ & $\mathrm{Ti}$ & Molecular weight $(\mathrm{g} / \mathrm{mole})=$ & 47.867 \\
Density $(\mathrm{g} / \mathrm{cm} 3)=$ & 4.540000 & Total atom density $($ atoms $/ \mathrm{b}-\mathrm{cm})=$ & $5.712 \mathrm{E}-02$
\end{tabular}

The above density is estimated to be accurate to 3 significant digits. Uncertainties are not addressed.

The following data was calculated from the input formula. 


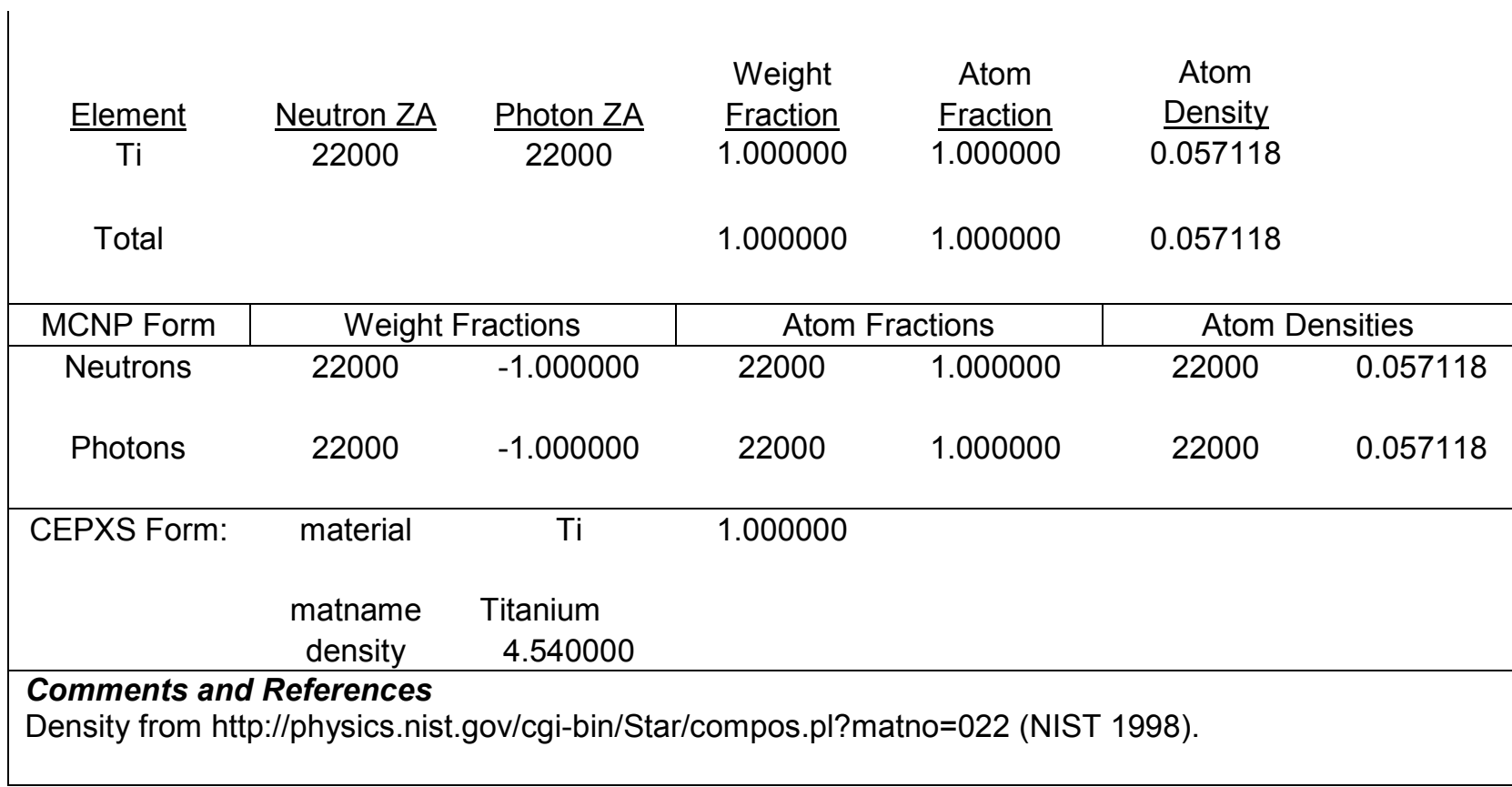

\section{Titanium Alloy, Grade 5}

\begin{tabular}{llll}
\hline Formula $=$ & - & Molecular weight $(\mathrm{g} / \mathrm{mole})=$ & - \\
Density $(\mathrm{g} / \mathrm{cm} 3)=$ & 4.430000 & Total atom density $($ atoms $/ \mathrm{b}-\mathrm{cm})=$ & $5.878 \mathrm{E}-02$
\end{tabular}

The above density is estimated to be accurate to 3 significant digits. Uncertainties are not addressed.

The following data were calculated from the input weight fractions.

\begin{tabular}{|c|c|c|c|c|c|c|}
\hline Element & Neutron ZA & Photon ZA & $\begin{array}{l}\text { Weight } \\
\text { Fraction }\end{array}$ & $\begin{array}{c}\text { Atom } \\
\text { Fraction }\end{array}$ & $\begin{array}{c}\text { Atom } \\
\text { Density }\end{array}$ & \\
\hline $\mathrm{H}$ & 1001 & 1000 & $\overline{0.000110}$ & 0.004953 & 0.000291 & \\
\hline C & 6000 & 6000 & 0.000570 & 0.002154 & 0.000127 & \\
\hline $\mathrm{N}$ & 7014 & 7000 & 0.000210 & 0.000680 & 0.000040 & \\
\hline $\mathrm{O}$ & 8016 & 8000 & 0.001410 & 0.004000 & 0.000235 & \\
\hline $\mathrm{Al}$ & 13027 & 13000 & 0.061250 & 0.103023 & 0.006056 & \\
\hline $\mathrm{Ti}$ & 22000 & 22000 & 0.893630 & 0.847256 & 0.049805 & \\
\hline $\mathrm{V}$ & 23000 & 23000 & 0.040000 & 0.035635 & 0.002095 & \\
\hline $\mathrm{Fe}$ & 26000 & 26000 & 0.002830 & 0.002300 & 0.000135 & \\
\hline Total & & & 1.000010 & 1.000000 & 0.058784 & \\
\hline MCNP Form & \multicolumn{2}{|c|}{ Weight Fractions } & \multicolumn{2}{|c|}{ Atom Fractions } & \multicolumn{2}{|c|}{ Atom Densities } \\
\hline \multirow[t]{7}{*}{ Neutrons } & 1001 & -0.000110 & 1001 & 0.004953 & 1001 & 0.000291 \\
\hline & 6000 & -0.000570 & 6000 & 0.002154 & 6000 & 0.000127 \\
\hline & 7014 & -0.000210 & 7014 & 0.000680 & 7014 & 0.000040 \\
\hline & 8016 & -0.001410 & 8016 & 0.004000 & 8016 & 0.000235 \\
\hline & 13027 & -0.061250 & 13027 & 0.103023 & 13027 & 0.006056 \\
\hline & 22000 & -0.893630 & 22000 & 0.847256 & 22000 & 0.049805 \\
\hline & 23000 & -0.040000 & 23000 & 0.035635 & 23000 & 0.002095 \\
\hline
\end{tabular}


PIET-43741-TM-963

PNNL-15870 Rev. 1

\begin{tabular}{|c|c|c|c|c|c|c|}
\hline & 26000 & -0.002830 & 26000 & 0.002300 & 26000 & 0.000135 \\
\hline \multirow[t]{8}{*}{ Photons } & 1000 & -0.000110 & 1000 & 0.004953 & 1000 & 0.000291 \\
\hline & 6000 & -0.000570 & 6000 & 0.002154 & 6000 & 0.000127 \\
\hline & 7000 & -0.000210 & 7000 & 0.000680 & 7000 & 0.000040 \\
\hline & 8000 & -0.001410 & 8000 & 0.004000 & 8000 & 0.000235 \\
\hline & 13000 & -0.061250 & 13000 & 0.103023 & 13000 & 0.006056 \\
\hline & 22000 & -0.893630 & 22000 & 0.847256 & 22000 & 0.049805 \\
\hline & 23000 & -0.040000 & 23000 & 0.035635 & 23000 & 0.002095 \\
\hline & 26000 & -0.002830 & 26000 & 0.002300 & 26000 & 0.000135 \\
\hline \multirow[t]{9}{*}{ CEPXS Form: } & material & $\mathrm{H}$ & 0.000110 & & & \\
\hline & & C & 0.000570 & & & \\
\hline & & $\mathrm{N}$ & 0.000210 & & & \\
\hline & & $\mathrm{O}$ & 0.001410 & & & \\
\hline & & $\mathrm{Al}$ & 0.061250 & & & \\
\hline & & $\mathrm{Ti}$ & 0.893630 & & & \\
\hline & & $\mathrm{V}$ & 0.040000 & & & \\
\hline & & $\mathrm{Fe}$ & 0.002830 & & & \\
\hline & $\begin{array}{l}\text { matname } \\
\text { density }\end{array}$ & \multicolumn{2}{|c|}{$\begin{array}{l}\text { Titanium Alloy, Grade } 5 \\
4.430000\end{array}$} & & & \\
\hline \multicolumn{7}{|c|}{$\begin{array}{l}\text { Comments and References } \\
\text { ASTM International defines many grades of titanium alloy such as grade 5, which is the most common } \\
\text { titanium alloy (http://en.wikipedia.org/wiki/Titanium_alloy). } \\
\text { Density }=4.43 \mathrm{~g} / \mathrm{cm} 3 \text { and weight fractions from } \\
\text { http://www.matweb.com/search/DataSheet.aspx?MatGUID=b350a789eda946c6b86a3e4d3c577b39 } \\
\text { (Automation Creations } 2010) \text {. } \\
\text { Weight fractions for Al, Ti, and V set at average values of allowed range. Weight fractions for H, C, N, O, } \\
\text { and Fe set at } 70.7 \% \text { of their upper limit so all weight fractions sum to unity. }\end{array}$} \\
\hline
\end{tabular}

\section{Titanium Dioxide}

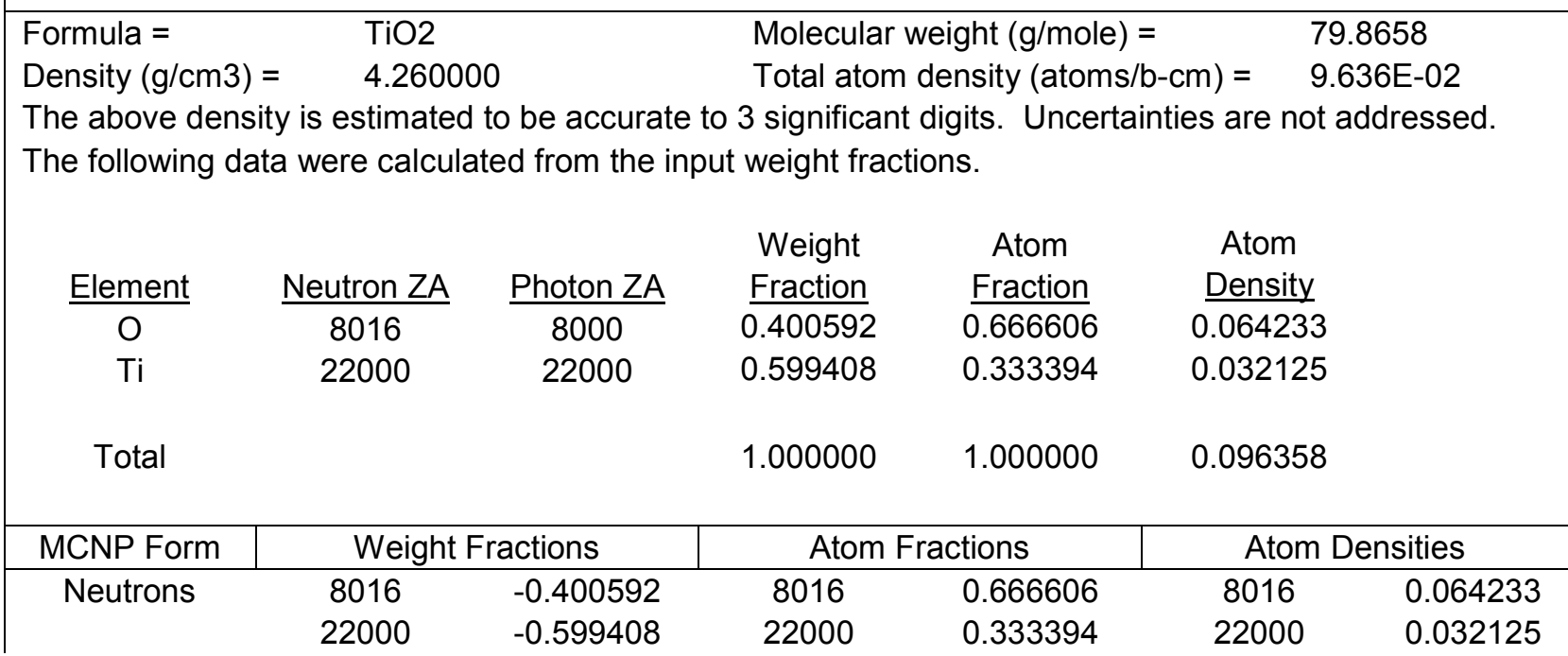


PIET-43741-TM-963

PNNL-15870 Rev. 1

\begin{tabular}{|c|c|c|c|c|c|c|}
\hline Photons & $\begin{array}{c}8000 \\
22000\end{array}$ & $\begin{array}{l}-0.400592 \\
-0.599408\end{array}$ & $\begin{array}{c}8000 \\
22000\end{array}$ & $\begin{array}{l}0.666606 \\
0.333394\end{array}$ & $\begin{array}{c}8000 \\
22000\end{array}$ & $\begin{array}{l}0.064233 \\
0.032125\end{array}$ \\
\hline CEPXS Form: & $\begin{array}{c}\text { matname } \\
\text { density }\end{array}$ & $\begin{array}{c}\mathrm{O} \\
\mathrm{Ti} \\
\text { Titanium Dio } \\
4.260000\end{array}$ & $\begin{array}{l}0.400592 \\
0.599408\end{array}$ & & & \\
\hline $\begin{array}{l}\text { Comments an } \\
\text { Density and we } \\
\text { Also see Lide }\end{array}$ & $\begin{array}{l}\text { References } \\
\text { it fractions } \\
\text { 08). }\end{array}$ & m http://physi & ist.gov/cgi- & tar/compc & atno $=2$ & IIST 1998). \\
\hline
\end{tabular}

\section{Titanium Hydride}

\begin{tabular}{llll}
\hline Formula $=$ & TiH2 & Molecular weight $(\mathrm{g} / \mathrm{mole})=$ & 49.88288 \\
Density $(\mathrm{g} / \mathrm{cm} 3)=$ & 3.750000 & Total atom density $($ atoms $/ \mathrm{b}-\mathrm{cm})=$ & $1.358 \mathrm{E}-01$
\end{tabular}

The above density is estimated to be accurate to 3 significant digits. Uncertainties are not addressed.

The following data was calculated from the input formula.

\begin{tabular}{|c|c|c|c|c|c|c|}
\hline Element & Neutron ZA & Photon ZA & $\begin{array}{l}\text { Weight } \\
\text { Fraction }\end{array}$ & $\begin{array}{c}\text { Atom } \\
\text { Fraction }\end{array}$ & $\begin{array}{c}\text { Atom } \\
\text { Density }\end{array}$ & \\
\hline $\mathrm{H}$ & 1001 & 1000 & 0.040412 & 0.666667 & 0.090544 & \\
\hline $\mathrm{Ti}$ & 22000 & 22000 & 0.959588 & 0.333333 & 0.045272 & \\
\hline Total & & & 1.000000 & 1.000000 & 0.135816 & \\
\hline MCNP Form & \multicolumn{2}{|c|}{ Weight Fractions } & \multicolumn{2}{|c|}{ Atom Fractions } & \multicolumn{2}{|c|}{ Atom Densities } \\
\hline Neutrons & $\begin{array}{c}1001 \\
22000\end{array}$ & $\begin{array}{l}-0.040412 \\
-0.959588\end{array}$ & $\begin{array}{c}1001 \\
22000\end{array}$ & $\begin{array}{l}0.666667 \\
0.333333\end{array}$ & $\begin{array}{c}1001 \\
22000\end{array}$ & $\begin{array}{l}0.090544 \\
0.045272\end{array}$ \\
\hline Photons & $\begin{array}{c}1000 \\
22000\end{array}$ & $\begin{array}{l}-0.040412 \\
-0.959588\end{array}$ & $\begin{array}{c}1000 \\
22000\end{array}$ & $\begin{array}{l}0.666667 \\
0.333333\end{array}$ & $\begin{array}{c}1000 \\
22000\end{array}$ & $\begin{array}{l}0.090544 \\
0.045272\end{array}$ \\
\hline CEPXS Form: & $\begin{array}{l}\text { matname } \\
\text { density }\end{array}$ & $\begin{array}{c}\mathrm{H} \\
\mathrm{Ti} \\
\text { Titanium Hyd } \\
3.750000\end{array}$ & $\begin{array}{l}0.040412 \\
0.959588\end{array}$ & & & \\
\hline $\begin{array}{l}\text { Comments an } \\
\text { Density = 3.75 } \\
\text { http://www.mat } \\
k=1 \text { (Automatio } \\
\text { from http://www } \\
\text { (Automation Cr }\end{array}$ & $\begin{array}{l}\text { References } \\
\text { cm3 and form } \\
\text { b.com/searc } \\
\text { Creations } 20 \\
\text { latweb.com/s } \\
\text { tions 2010). }\end{array}$ & $\begin{array}{l}\text { la from } \\
\text { DataSheet.as } \\
\text { and from pg } \\
\text { arch/DataShe } \\
\text { ensity = } 3.90\end{array}$ & $\begin{array}{l}\text { MatGUID= } \\
-96 \text { of Lid } \\
\text { ispx?MatG } \\
\text { n3 in Tabl }\end{array}$ & $\begin{array}{l}\text { 4b82a7d6d } \\
008) . \text { Dens } \\
=\text { bbc565cf } \\
5 \text { of Schaef }\end{array}$ & $\begin{array}{l}\text { b688180 } \\
3.901 \mathrm{~g} / \mathrm{c} \\
41 \mathrm{e} 0 \mathrm{a} 9 \mathrm{ec} \\
973) .\end{array}$ & $\begin{array}{l}\text { 70d8\&ckc } \\
\text { r powder } \\
40199 \mathrm{~b} 70\end{array}$ \\
\hline
\end{tabular}




\section{Toluene}

\begin{tabular}{llll}
\hline Formula $=$ & $\mathrm{C} 7 \mathrm{H} 8$ & Molecular weight $(\mathrm{g} / \mathrm{mole})=$ & 92.13842 \\
Density $(\mathrm{g} / \mathrm{cm} 3)=$ & 0.866900 & Total atom density $($ atoms $/ \mathrm{b}-\mathrm{cm})=$ & $8.499 \mathrm{E}-02$
\end{tabular}

The above density is estimated to be accurate to 4 significant digits. Uncertainties are not addressed.

The following data were calculated from the input weight fractions.

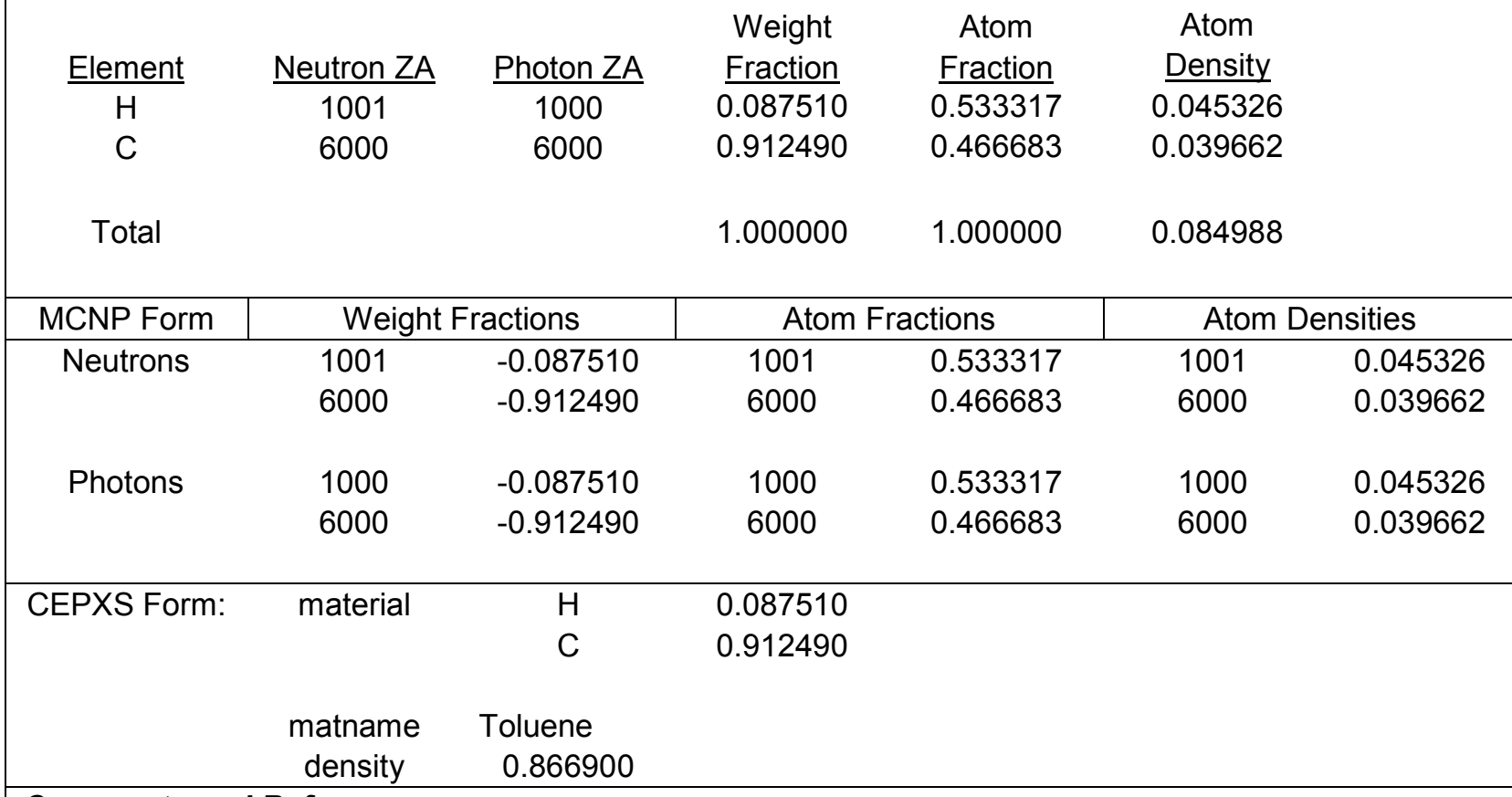

\section{Comments and References}

Also called F1063.

Density and weight fractions from http://physics.nist.gov/cgi-bin/Star/compos.pl?matno=266 (NIST 1998).

Formula and density $=0.8623$ from pgs $3-486$ of Lide (2008). Formula $=\mathrm{C} 6 \mathrm{H} 5 \mathrm{CH} 3$ and density $=$ 0.8669 at

http://www.matweb.com/search/DataSheet.aspx?MatGUID=d9cd9f172f4d4753be619931978c1670 (Automation Creations 2010).

\section{Tributyl Borate}

\begin{tabular}{llll}
\hline Formula $=$ & $\mathrm{B}(\mathrm{OC} 4 \mathrm{H} 9) 3$ & Molecular weight $(\mathrm{g} / \mathrm{mole})=$ & 230.15198 \\
Density $(\mathrm{g} / \mathrm{cm} 3)=$ & 0.864000 & Total atom density $($ atoms $/ \mathrm{b}-\mathrm{cm})=$ & $9.721 \mathrm{E}-02$
\end{tabular}

The above density is estimated to be accurate to 4 significant digits. Uncertainties are not addressed.

The following data was calculated from the input formula.

\begin{tabular}{|c|c|c|c|c|c|}
\hline Element & Neutron ZA & Photon ZA & $\begin{array}{l}\text { Weight } \\
\text { Fraction }\end{array}$ & $\begin{array}{l}\text { Atom } \\
\text { Fraction }\end{array}$ & $\begin{array}{c}\text { Atom } \\
\text { Density }\end{array}$ \\
\hline $\mathrm{H}$ & 1001 & 1000 & $\overline{0.118245}$ & $\overline{0.627907}$ & 0.061040 \\
\hline$B$ & - & 5000 & 0.046973 & 0.023256 & 0.002261 \\
\hline C & 6000 & 6000 & 0.626231 & 0.279070 & 0.027129 \\
\hline $\mathrm{O}$ & 8016 & 8000 & 0.208550 & 0.069767 & 0.006782 \\
\hline
\end{tabular}


PIET-43741-TM-963

PNNL-15870 Rev. 1

\begin{tabular}{|c|c|c|c|c|c|c|}
\hline Total & & & 1.000000 & 1.000000 & 0.09721 & \\
\hline MCNP Form & \multicolumn{2}{|c|}{ Weight Fractions } & \multicolumn{2}{|c|}{ Atom Fractions } & \multicolumn{2}{|c|}{ Atom Densities } \\
\hline \multirow[t]{4}{*}{ Neutrons } & 1001 & -0.118245 & 1001 & 0.627907 & 1001 & 0.061040 \\
\hline & - & -0.046973 & - & 0.023256 & - & 0.002261 \\
\hline & 6000 & -0.626231 & 6000 & 0.279070 & 6000 & 0.027129 \\
\hline & 8016 & -0.208550 & 8016 & 0.069767 & 8016 & 0.006782 \\
\hline \multirow[t]{4}{*}{ Photons } & 1000 & -0.118245 & 1000 & 0.627907 & 1000 & 0.061040 \\
\hline & 5000 & -0.046973 & 5000 & 0.023256 & 5000 & 0.002261 \\
\hline & 6000 & -0.626231 & 6000 & 0.279070 & 6000 & 0.027129 \\
\hline & 8000 & -0.208550 & 8000 & 0.069767 & 8000 & 0.006782 \\
\hline \multirow[t]{5}{*}{ CEPXS Form: } & material & $\mathrm{H}$ & 0.118245 & & & \\
\hline & & B & 0.046973 & & & \\
\hline & & $\mathrm{C}$ & 0.626231 & & & \\
\hline & & $\mathrm{O}$ & 0.208550 & & & \\
\hline & $\begin{array}{l}\text { matname } \\
\text { density }\end{array}$ & $\begin{array}{l}\text { Tributyl Borate } \\
0.864000\end{array}$ & & & & \\
\hline \multicolumn{7}{|c|}{$\begin{array}{l}\text { Comments and References } \\
\text { Density }=0.864 \mathrm{~g} / \mathrm{cm} 3 \text { at } 20^{\circ} \mathrm{C}\end{array}$} \\
\hline
\end{tabular}

\section{Tributyl Phosphate (TBP)}

\begin{tabular}{llll}
\hline Formula $=$ & $(\mathrm{C} 4 \mathrm{H} 9) 3 \mathrm{PO} 4$ & Molecular weight $(\mathrm{g} / \mathrm{mole})=$ & 266.314141 \\
Density $(\mathrm{g} / \mathrm{cm} 3)=$ & 0.972400 & Total atom density $($ atoms $/ \mathrm{b}-\mathrm{cm})=$ & $9.675 \mathrm{E}-02$
\end{tabular}

The above density is estimated to be accurate to 4 significant digits. Uncertainties are not addressed.

The following data was calculated from the input formula.

\begin{tabular}{|c|c|c|c|c|c|c|}
\hline Element & Neutron ZA & Photon ZA & $\begin{array}{l}\text { Weight } \\
\text { Fraction }\end{array}$ & $\begin{array}{l}\text { Atom } \\
\text { Fraction }\end{array}$ & $\begin{array}{l}\text { Atom } \\
\text { Density }\end{array}$ & \\
\hline $\mathrm{H}$ & 1001 & 1000 & $\overline{0.102189}$ & $\overline{0.613636}$ & 0.059370 & \\
\hline C & 6000 & 6000 & 0.541197 & 0.272727 & 0.026387 & \\
\hline $\mathrm{O}$ & 8016 & 8000 & 0.240309 & 0.090909 & 0.008796 & \\
\hline $\mathrm{P}$ & 15031 & 15000 & 0.116305 & 0.022727 & 0.002199 & \\
\hline Total & & & 1.000000 & 1.000000 & 0.096751 & \\
\hline MCNP Form & \multicolumn{2}{|c|}{ Weight Fractions } & \multicolumn{2}{|c|}{ Atom Fractions } & \multicolumn{2}{|c|}{ Atom Densities } \\
\hline \multirow[t]{4}{*}{ Neutrons } & 1001 & -0.102189 & 1001 & 0.613636 & 1001 & 0.059370 \\
\hline & 6000 & -0.541197 & 6000 & 0.272727 & 6000 & 0.026387 \\
\hline & 8016 & -0.240309 & 8016 & 0.090909 & 8016 & 0.008796 \\
\hline & 15031 & -0.116305 & 15031 & 0.022727 & 15031 & 0.002199 \\
\hline \multirow[t]{2}{*}{ Photons } & 1000 & -0.102189 & 1000 & 0.613636 & 1000 & 0.059370 \\
\hline & 6000 & -0.541197 & 6000 & 0.272727 & 6000 & 0.026387 \\
\hline
\end{tabular}


PIET-43741-TM-963

PNNL-15870 Rev. 1

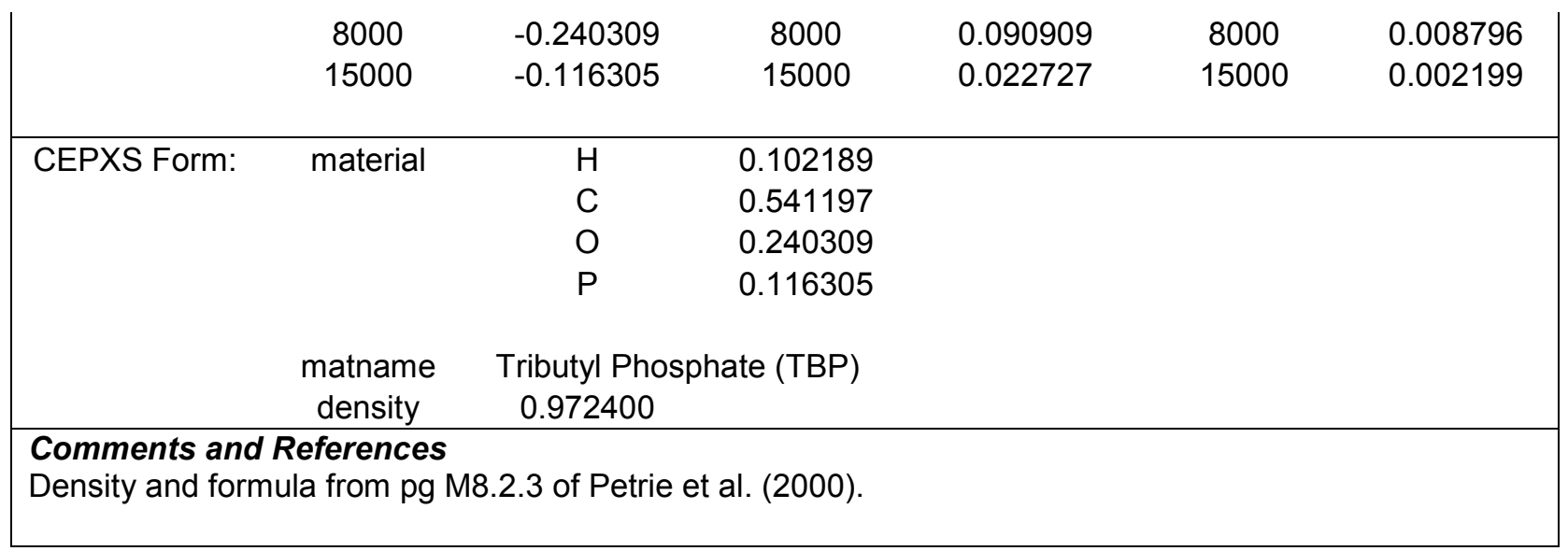

\section{Tungsten}

\begin{tabular}{llll}
\hline Formula $=$ & W & Molecular weight $(\mathrm{g} / \mathrm{mole})=$ & 183.84 \\
Density $(\mathrm{g} / \mathrm{cm} 3)=$ & 19.300000 & Total atom density $($ atoms $/ \mathrm{b}-\mathrm{cm})=$ & $6.322 \mathrm{E}-02$
\end{tabular}

The above density is estimated to be accurate to 3 significant digits. Uncertainties are not addressed.

The following data was calculated from the input formula.

\begin{tabular}{|c|c|c|c|c|c|c|}
\hline$\frac{\text { Element }}{\mathrm{W}}$ & $\frac{\text { Neutron ZA }}{74000}$ & $\frac{\text { Photon ZA }}{74000}$ & $\begin{array}{l}\text { Weight } \\
\frac{\text { Fraction }}{1.000000}\end{array}$ & $\begin{array}{c}\begin{array}{c}\text { Atom } \\
\text { Fraction }\end{array} \\
1.000000\end{array}$ & $\begin{array}{c}\text { Atom } \\
\text { Density } \\
0.063222\end{array}$ & \\
\hline Total & & & 1.000000 & 1.000000 & 0.063222 & \\
\hline MCNP Form & \multicolumn{2}{|c|}{ Weight Fractions } & \multicolumn{2}{|c|}{ Atom Fractions } & \multicolumn{2}{|c|}{ Atom Densities } \\
\hline Neutrons & 74000 & -1.000000 & 74000 & 1.000000 & 74000 & 0.063222 \\
\hline Photons & 74000 & -1.000000 & 74000 & 1.000000 & 74000 & 0.063222 \\
\hline CEPXS Form: & $\begin{array}{l}\text { material } \\
\text { matname } \\
\text { density }\end{array}$ & $\begin{array}{c}\text { W } \\
\text { Tungsten } \\
19.300000\end{array}$ & 1.000000 & & & \\
\hline $\begin{array}{l}\text { Comments an } \\
\text { Density from } \mathrm{h}\end{array}$ & $\begin{array}{l}\text { eferences } \\
\text { /physics.nis }\end{array}$ & 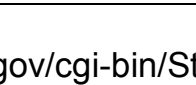 & & & & \\
\hline
\end{tabular}

\section{Uranium Carbide}

Formula $=\quad \mathrm{UC}$

Density $(\mathrm{g} / \mathrm{cm} 3)=13.630000$

The above density is estimated to be accurate to 3 significant digits. Uncertainties are not addressed.

The following data was calculated from the input formula. 


\begin{tabular}{|c|c|c|c|c|c|c|}
\hline Element & Neutron ZA & Photon ZA & $\begin{array}{l}\text { Weight } \\
\text { Fraction }\end{array}$ & $\begin{array}{l}\text { Atom } \\
\text { Fraction }\end{array}$ & $\begin{array}{l}\text { Atom } \\
\text { Density }\end{array}$ & \\
\hline C & 6000 & 6000 & 0.048049 & 0.500000 & 0.032837 & \\
\hline U-234 & 92234 & 92000 & 0.000254 & 0.000136 & 0.000009 & \\
\hline U-235 & 92235 & 92000 & 0.028559 & 0.015186 & 0.000997 & \\
\hline U-236 & 92236 & 92000 & 0.000131 & 0.000070 & 0.000005 & \\
\hline U-238 & 92238 & 92000 & 0.923007 & 0.484609 & 0.031826 & \\
\hline Total & & & 1.000000 & 1.000000 & 0.065674 & \\
\hline MCNP Form & \multicolumn{2}{|c|}{ Weight Fractions } & \multicolumn{2}{|c|}{ Atom Fractions } & \multicolumn{2}{|c|}{ Atom Densities } \\
\hline \multirow[t]{5}{*}{ Neutrons } & 6000 & -0.048049 & 6000 & 0.500000 & 6000 & 0.032837 \\
\hline & 92234 & -0.000254 & 92234 & 0.000136 & 92234 & 0.000009 \\
\hline & 92235 & -0.028559 & 92235 & 0.015186 & 92235 & 0.000997 \\
\hline & 92236 & -0.000131 & 92236 & 0.000070 & 92236 & 0.000005 \\
\hline & 92238 & -0.923007 & 92238 & 0.484609 & 92238 & 0.031826 \\
\hline \multirow[t]{5}{*}{ Photons } & 6000 & -0.048049 & 6000 & 0.500000 & 6000 & 0.032837 \\
\hline & 92000 & -0.000254 & 92000 & 0.000136 & 92000 & 0.000009 \\
\hline & 92000 & -0.028559 & 92000 & 0.015186 & 92000 & 0.000997 \\
\hline & 92000 & -0.000131 & 92000 & 0.000070 & 92000 & 0.000005 \\
\hline & 92000 & -0.923007 & 92000 & 0.484609 & 92000 & 0.031826 \\
\hline \multirow[t]{6}{*}{ CEPXS Form: } & material & $\mathrm{C}$ & 0.048049 & & & \\
\hline & & U-234 & 0.000254 & & & \\
\hline & & U-235 & 0.028559 & & & \\
\hline & & U-236 & 0.000131 & & & \\
\hline & & U-238 & 0.923007 & & & \\
\hline & $\begin{array}{c}\text { matname } \\
\text { density }\end{array}$ & \multicolumn{3}{|c|}{$\begin{array}{l}\text { Uranium Carbide } \\
13.630000\end{array}$} & & \\
\hline \multicolumn{7}{|c|}{$\begin{array}{l}\text { Comments and References } \\
\text { Density from http://physics.nist.gov/cgi-bin/Star/compos.pl?matno=271 (NIST 1998). } \\
\text { Formula from pg M8.2.3 of Petrie et al. (2000). } \\
\text { Uranium isotopics assumed for LEU: Wt\% U234/235/236/238 = 0.0267/3.0/0.0138/96.9595. }\end{array}$} \\
\hline
\end{tabular}

\section{Uranium Dicarbide}

\begin{tabular}{llll}
\hline Formula $=$ & UC2 & Molecular weight $(\mathrm{g} / \mathrm{mole})=$ & 261.9794909 \\
Density $(\mathrm{g} / \mathrm{cm} 3)=$ & 11.280000 & Total atom density $($ atoms $/ \mathrm{b}-\mathrm{cm})=$ & $7.779 \mathrm{E}-02$
\end{tabular}

The above density is estimated to be accurate to 3 significant digits. Uncertainties are not addressed.

The following data was calculated from the input formula.

\begin{tabular}{|c|c|c|c|c|c|}
\hline Element & Neutron ZA & Photon ZA & $\begin{array}{l}\text { Weight } \\
\text { Fraction }\end{array}$ & $\begin{array}{c}\text { Atom } \\
\text { Fraction }\end{array}$ & $\begin{array}{l}\text { Atom } \\
\text { Density }\end{array}$ \\
\hline$C$ & 6000 & 6000 & $\overline{0.091692}$ & $\overline{0.666667}$ & 0.051859 \\
\hline U-234 & 92234 & 92000 & 0.000243 & 0.000090 & 0.000007 \\
\hline U-235 & 92235 & 92000 & 0.027249 & 0.010124 & 0.000788 \\
\hline
\end{tabular}


PIET-43741-TM-963

PNNL-15870 Rev. 1

\begin{tabular}{|c|c|c|c|c|c|c|}
\hline U-236 & 92236 & 92000 & 0.000125 & 0.000046 & 0.000004 & \\
\hline U-238 & 92238 & 92000 & 0.880691 & 0.323072 & 0.025131 & \\
\hline Total & & & 1.000000 & 1.000000 & 0.077788 & \\
\hline MCNP Form & Weigh & ractions & Atom & ctions & Atom & sities \\
\hline Neutrons & 6000 & -0.091692 & 6000 & 0.666667 & 6000 & 0.051859 \\
\hline & 92234 & -0.000243 & 92234 & 0.000090 & 92234 & 0.000007 \\
\hline & 92235 & -0.027249 & 92235 & 0.010124 & 92235 & 0.000788 \\
\hline & 92236 & -0.000125 & 92236 & 0.000046 & 92236 & 0.000004 \\
\hline & 92238 & -0.880691 & 92238 & 0.323072 & 92238 & 0.025131 \\
\hline Photons & 6000 & -0.091692 & 6000 & 0.666667 & 6000 & 0.051859 \\
\hline & 92000 & -0.000243 & 92000 & 0.000090 & 92000 & 0.000007 \\
\hline & 92000 & -0.027249 & 92000 & 0.010124 & 92000 & 0.000788 \\
\hline & 92000 & -0.000125 & 92000 & 0.000046 & 92000 & 0.000004 \\
\hline & 92000 & -0.880691 & 92000 & 0.323072 & 92000 & 0.025131 \\
\hline CEPXS Form: & material & C & 0.091692 & & & \\
\hline & & U-234 & 0.000243 & & & \\
\hline & & U-235 & 0.027249 & & & \\
\hline & & U-236 & 0.000125 & & & \\
\hline & & U-238 & 0.880691 & & & \\
\hline & $\begin{array}{c}\text { matname } \\
\text { density }\end{array}$ & $\begin{array}{c}\text { Uranium Dic } \\
11.280000\end{array}$ & & & & \\
\hline $\begin{array}{l}\text { Comments an } \\
\text { Density from ht } \\
\text { Formula from } \\
\text { Uranium isotop }\end{array}$ & $\begin{array}{l}\text { eferences } \\
\text { /physics.ni } \\
4-97 \text { of Li } \\
\text { assumed f }\end{array}$ & $\begin{array}{l}\text { Jov/cgi-bin/St } \\
\text { (2008). } \\
\text { EU: Wt\% U }\end{array}$ & $\begin{array}{l}\text { mpos.pl? } \\
\text { 235/236/2 }\end{array}$ & $\begin{array}{l}D=270 \text { (NIS } \\
0.0267 / 3.0\end{array}$ & 98). & \\
\hline
\end{tabular}

\section{Uranium Dioxide}

\begin{tabular}{llll}
\hline Formula $=$ & UO2 & Molecular weight $(\mathrm{g} / \mathrm{mole})=$ & 269.9568909 \\
Density $(\mathrm{g} / \mathrm{cm} 3)=$ & 10.960000 & Total atom density $($ atoms $/ \mathrm{b}-\mathrm{cm})=$ & $7.335 \mathrm{E}-02$
\end{tabular}

The above density is estimated to be accurate to 3 significant digits. Uncertainties are not addressed.

The following data was calculated from the input formula.

\begin{tabular}{|c|c|c|c|c|c|}
\hline Element & Neutron ZA & Photon ZA & $\begin{array}{l}\text { Weight } \\
\text { Fraction }\end{array}$ & $\begin{array}{c}\text { Atom } \\
\text { Fraction }\end{array}$ & $\begin{array}{c}\text { Atom } \\
\text { Density }\end{array}$ \\
\hline 0 & 8016 & 8000 & 0.118533 & 0.666667 & 0.048899 \\
\hline U-234 & 92234 & 92000 & 0.000235 & 0.000090 & 0.000007 \\
\hline U-235 & 92235 & 92000 & 0.026444 & 0.010124 & 0.000743 \\
\hline U-236 & 92236 & 92000 & 0.000122 & 0.000046 & 0.000003 \\
\hline U-238 & 92238 & 92000 & 0.854666 & 0.323072 & 0.023697 \\
\hline Total & & & 1.000000 & 1.000000 & 0.073348 \\
\hline
\end{tabular}


PIET-43741-TM-963

PNNL-15870 Rev. 1

\begin{tabular}{|c|c|c|c|c|c|c|}
\hline MCNP Form & \multicolumn{2}{|c|}{ Weight Fractions } & \multicolumn{2}{|c|}{ Atom Fractions } & \multicolumn{2}{|c|}{ Atom Densities } \\
\hline \multirow[t]{5}{*}{ Neutrons } & 8016 & -0.118533 & 8016 & 0.666667 & 8016 & 0.048899 \\
\hline & 92234 & -0.000235 & 92234 & 0.000090 & 92234 & 0.000007 \\
\hline & 92235 & -0.026444 & 92235 & 0.010124 & 92235 & 0.000743 \\
\hline & 92236 & -0.000122 & 92236 & 0.000046 & 92236 & 0.000003 \\
\hline & 92238 & -0.854666 & 92238 & 0.323072 & 92238 & 0.023697 \\
\hline \multirow[t]{5}{*}{ Photons } & 8000 & -0.118533 & 8000 & 0.666667 & 8000 & 0.048899 \\
\hline & 92000 & -0.000235 & 92000 & 0.000090 & 92000 & 0.000007 \\
\hline & 92000 & -0.026444 & 92000 & 0.010124 & 92000 & 0.000743 \\
\hline & 92000 & -0.000122 & 92000 & 0.000046 & 92000 & 0.000003 \\
\hline & 92000 & -0.854666 & 92000 & 0.323072 & 92000 & 0.023697 \\
\hline \multirow[t]{6}{*}{ CEPXS Form: } & material & $\mathrm{O}$ & 0.118533 & & & \\
\hline & & $U-234$ & 0.000235 & & & \\
\hline & & U-235 & 0.026444 & & & \\
\hline & & U-236 & 0.000122 & & & \\
\hline & & $\mathrm{U}-238$ & 0.854666 & & & \\
\hline & $\begin{array}{c}\text { matname } \\
\text { density }\end{array}$ & $\begin{array}{c}\text { Uranium Dio } \\
10.960000\end{array}$ & & & & \\
\hline \multicolumn{7}{|c|}{$\begin{array}{l}\text { Comments and References } \\
\text { Density from http://physics.nist.gov/cgi-bin/Star/compos.pl?matno=272 (NIST 1998). } \\
\text { Also called uranium dioxide. } \\
\text { Paxton and Pruvost (1986) appears to have weight fractions appropriate for UO3 instead of UO2. } \\
\text { Density and formula also from pg M8.2.4 of Petrie et al. (2000). } \\
\text { Uranium isotopics assumed for LEU: Wt\% U234/235/236/238 }=0.0267 / 3.0 / 0.0138 / 96.9595 .\end{array}$} \\
\hline
\end{tabular}

\section{Uranium Hexafluoride}

\begin{tabular}{llll}
\hline Formula $=$ & UF6 & Molecular weight $(\mathrm{g} / \mathrm{mole})=$ & 351.9485101 \\
Density $(\mathrm{g} / \mathrm{cm} 3)=$ & 4.680000 & Total atom density $($ atoms $/ \mathrm{b}-\mathrm{cm})=$ & $5.606 \mathrm{E}-02$
\end{tabular}

The above density is estimated to be accurate to 3 significant digits. Uncertainties are not addressed.

The following data was calculated from the input formula.

\begin{tabular}{|c|c|c|c|c|c|c|}
\hline Element & Neutron ZA & Photon ZA & $\begin{array}{l}\text { Weight } \\
\text { Fraction }\end{array}$ & $\begin{array}{c}\text { Atom } \\
\text { Fraction }\end{array}$ & $\begin{array}{l}\text { Atom } \\
\text { Density }\end{array}$ & \\
\hline$F$ & 9019 & 9000 & $\overline{0.323884}$ & $\overline{0.857143}$ & 0.048047 & \\
\hline U-234 & 92234 & 92000 & 0.000181 & 0.000039 & 0.000002 & \\
\hline U-235 & 92235 & 92000 & 0.020283 & 0.004339 & 0.000243 & \\
\hline U-236 & 92236 & 92000 & 0.000093 & 0.000020 & 0.000001 & \\
\hline U-238 & 92238 & 92000 & 0.655559 & 0.138460 & 0.007761 & \\
\hline Total & & & 1.000000 & 1.000000 & 0.056055 & \\
\hline MCNP Form & \multicolumn{2}{|c|}{ Weight Fractions } & \multicolumn{2}{|c|}{ Atom Fractions } & Atom & Isities \\
\hline \multirow[t]{2}{*}{ Neutrons } & 9019 & -0.323884 & 9019 & 0.857143 & 9019 & 0.048047 \\
\hline & 92234 & -0.000181 & 92234 & 0.000039 & 92234 & 0.000002 \\
\hline
\end{tabular}


PIET-43741-TM-963

PNNL-15870 Rev. 1

\begin{tabular}{|c|c|c|c|c|c|c|}
\hline & 92235 & -0.020283 & 92235 & 0.004339 & 92235 & 0.000243 \\
\hline & 92236 & -0.000093 & 92236 & 0.000020 & 92236 & 0.000001 \\
\hline & 92238 & -0.655559 & 92238 & 0.138460 & 92238 & 0.007761 \\
\hline Photons & 9000 & -0.323884 & 9000 & 0.857143 & 9000 & 0.048047 \\
\hline & 92000 & -0.000181 & 92000 & 0.000039 & 92000 & 0.000002 \\
\hline & 92000 & -0.020283 & 92000 & 0.004339 & 92000 & 0.000243 \\
\hline & 92000 & -0.000093 & 92000 & 0.000020 & 92000 & 0.000001 \\
\hline & 92000 & -0.655559 & 92000 & 0.138460 & 92000 & 0.007761 \\
\hline CEPXS Form: & material & $\mathrm{F}$ & 0.323884 & & & \\
\hline & & U-234 & 0.000181 & & & \\
\hline & & U-235 & 0.020283 & & & \\
\hline & & U-236 & 0.000093 & & & \\
\hline & & U-238 & 0.655559 & & & \\
\hline & $\begin{array}{c}\text { matname } \\
\text { density }\end{array}$ & $\begin{array}{c}\text { Uranium He } \\
4.680000\end{array}$ & coride & & & \\
\hline $\begin{array}{l}\text { Comments an } \\
\text { Density = } 4.68 \\
\text { HF6 at an elev } \\
5.09 \mathrm{~g} / \mathrm{cm} 3 \text { for } \\
\text { http://en.wikipe } \\
\text { The phase diag } \\
\text { Uranium isotop }\end{array}$ & $\begin{array}{l}\text { References } \\
\text { m3 and for } \\
d \text { temp. on } \\
\text { id UF6 bas } \\
\text { org/wiki/U } \\
\text { m for UF6 i } \\
\text { assumed } f\end{array}$ & $\begin{array}{l}\text { la from pg M } \\
201 \text { of Paxto } \\
\text { on pgs } 4-97 \\
\text { ium_hexafluc } \\
\text { t http://en.wik } \\
\text { LEU: Wt } \% \text { U }\end{array}$ & $\begin{array}{l}3 \text { of Petrie } \\
\text { d Pruvost } \\
\text { ide (2008) } \\
\text { lia.org/wiki/ } \\
\text { 235/236/2 }\end{array}$ & $\begin{array}{l}(2000) .4 \\
6) \text {, revision } \\
\text { nium_hexa } \\
0.0267 / 3.0\end{array}$ & $\begin{array}{l}\mathrm{cm} 3 \text { is lis } \\
\text { d July } 1 \\
\text { le. } \\
38 / 96.95\end{array}$ & $\begin{array}{l}\text { or liquid } \\
\text { Density = }\end{array}$ \\
\hline
\end{tabular}

\section{Uranium Hydride}

\begin{tabular}{llll}
\hline Formula $=$ & $\mathrm{UH} 3$ & Molecular weight $(\mathrm{g} / \mathrm{mole})=$ & 240.9819109 \\
Density $(\mathrm{g} / \mathrm{cm} 3)=$ & 11.100000 & Total atom density $($ atoms $/ \mathrm{b}-\mathrm{cm})=$ & $1.110 \mathrm{E}-01$
\end{tabular}

The above density is estimated to be accurate to 3 significant digits. Uncertainties are not addressed.

The following data was calculated from the input formula.

\begin{tabular}{|c|c|c|c|c|c|c|}
\hline Element & Neutron ZA & Photon ZA & $\begin{array}{l}\text { Weight } \\
\text { Fraction }\end{array}$ & $\begin{array}{c}\text { Atom } \\
\text { Fraction }\end{array}$ & $\begin{array}{l}\text { Atom } \\
\text { Density }\end{array}$ & \\
\hline $\mathrm{H}$ & 1001 & 1000 & $\overline{0.012548}$ & $\overline{0.750000}$ & 0.083217 & \\
\hline U-234 & 92234 & 92000 & 0.000264 & 0.000068 & 0.000008 & \\
\hline U-235 & 92235 & 92000 & 0.029624 & 0.007593 & 0.000842 & \\
\hline U-236 & 92236 & 92000 & 0.000136 & 0.000035 & 0.000004 & \\
\hline U-238 & 92238 & 92000 & 0.957429 & 0.242304 & 0.026885 & \\
\hline Total & & & 1.000000 & 1.000000 & 0.110956 & \\
\hline MCNP Form & \multicolumn{2}{|c|}{ Weight Fractions } & \multicolumn{2}{|c|}{ Atom Fractions } & \multicolumn{2}{|c|}{ Atom Densities } \\
\hline \multirow[t]{4}{*}{ Neutrons } & 1001 & -0.012548 & 1001 & 0.750000 & 1001 & 0.083217 \\
\hline & 92234 & -0.000264 & 92234 & 0.000068 & 92234 & 0.000008 \\
\hline & 92235 & -0.029624 & 92235 & 0.007593 & 92235 & 0.000842 \\
\hline & 92236 & -0.000136 & 92236 & 0.000035 & 92236 & 0.000004 \\
\hline
\end{tabular}


PIET-43741-TM-963

PNNL-15870 Rev. 1

\begin{tabular}{|c|c|c|c|c|c|c|}
\hline & 92238 & -0.957429 & 92238 & 0.242304 & 92238 & 0.026885 \\
\hline \multirow[t]{5}{*}{ Photons } & 1000 & -0.012548 & 1000 & 0.750000 & 1000 & 0.083217 \\
\hline & 92000 & -0.000264 & 92000 & 0.000068 & 92000 & 0.000008 \\
\hline & 92000 & -0.029624 & 92000 & 0.007593 & 92000 & 0.000842 \\
\hline & 92000 & -0.000136 & 92000 & 0.000035 & 92000 & 0.000004 \\
\hline & 92000 & -0.957429 & 92000 & 0.242304 & 92000 & 0.026885 \\
\hline \multirow[t]{6}{*}{ CEPXS Form: } & material & $\mathrm{H}$ & 0.012548 & & & \\
\hline & & U-234 & 0.000264 & & & \\
\hline & & U-235 & 0.029624 & & & \\
\hline & & U-236 & 0.000136 & & & \\
\hline & & U-238 & 0.957429 & & & \\
\hline & $\begin{array}{l}\text { matname } \\
\text { density }\end{array}$ & \multicolumn{2}{|c|}{$\begin{array}{l}\text { Uranium Hydride } \\
11.100000\end{array}$} & & & \\
\hline \multicolumn{7}{|c|}{$\begin{array}{l}\text { Comments and References } \\
\text { Formula and density from pgs } 4-97 \text { of Lide }(2008) \text {. } \\
\text { Density }=11.5 \mathrm{~g} / \mathrm{cm} 3 \text { in Table } 51.14 \text { of Hungerford }(1960) \\
\text { Uranium isotopics assumed for LEU: Wt\% U234/235/236/238 = 0.0267/3.0/0.0138/96.9595. }\end{array}$} \\
\hline
\end{tabular}

\section{Uranium Nitride}

\begin{tabular}{llll}
\hline Formula $=$ & UN & Molecular weight $(\mathrm{g} / \mathrm{mole})=$ & 251.9647909 \\
Density $(\mathrm{g} / \mathrm{cm} 3)=$ & 14.310000 & Total atom density $($ atoms $/ \mathrm{b}-\mathrm{cm})=$ & $6.840 \mathrm{E}-02$
\end{tabular}

The above density is estimated to be accurate to 3 significant digits. Uncertainties are not addressed.

The following data was calculated from the input formula.

\begin{tabular}{|c|c|c|c|c|c|c|}
\hline Element & Neutron ZA & Photon ZA & $\begin{array}{l}\text { Weight } \\
\text { Fraction }\end{array}$ & $\begin{array}{c}\text { Atom } \\
\text { Fraction }\end{array}$ & $\begin{array}{c}\text { Atom } \\
\text { Density }\end{array}$ & \\
\hline $\mathrm{N}$ & 7014 & 7000 & 0.055590 & 0.500000 & 0.034202 & \\
\hline$U-234$ & 92234 & 92000 & 0.000252 & 0.000136 & 0.000009 & \\
\hline U-235 & 92235 & 92000 & 0.028332 & 0.015186 & 0.001039 & \\
\hline $\mathrm{U}-236$ & 92236 & 92000 & 0.000130 & 0.000070 & 0.000005 & \\
\hline$U-238$ & 92238 & 92000 & 0.915695 & 0.484609 & 0.033149 & \\
\hline Total & & & 1.000000 & 1.000000 & 0.068404 & \\
\hline MCNP Form & \multicolumn{2}{|c|}{ Weight Fractions } & \multicolumn{2}{|c|}{ Atom Fractions } & \multicolumn{2}{|c|}{ Atom Densities } \\
\hline \multirow[t]{5}{*}{ Neutrons } & 7014 & -0.055590 & 7014 & 0.500000 & 7014 & 0.034202 \\
\hline & 92234 & -0.000252 & 92234 & 0.000136 & 92234 & 0.000009 \\
\hline & 92235 & -0.028332 & 92235 & 0.015186 & 92235 & 0.001039 \\
\hline & 92236 & -0.000130 & 92236 & 0.000070 & 92236 & 0.000005 \\
\hline & 92238 & -0.915695 & 92238 & 0.484609 & 92238 & 0.033149 \\
\hline \multirow[t]{3}{*}{ Photons } & 7000 & -0.055590 & 7000 & 0.500000 & 7000 & 0.034202 \\
\hline & 92000 & -0.000252 & 92000 & 0.000136 & 92000 & 0.000009 \\
\hline & 92000 & -0.028332 & 92000 & 0.015186 & 92000 & 0.001039 \\
\hline
\end{tabular}


PIET-43741-TM-963

PNNL-15870 Rev. 1

\begin{tabular}{|c|c|c|c|c|c|c|}
\hline & $\begin{array}{l}92000 \\
92000\end{array}$ & $\begin{array}{l}-0.000130 \\
-0.915695\end{array}$ & $\begin{array}{l}92000 \\
92000\end{array}$ & $\begin{array}{l}0.000070 \\
0.484609\end{array}$ & $\begin{array}{l}92000 \\
92000\end{array}$ & $\begin{array}{l}0.000005 \\
0.033149\end{array}$ \\
\hline \multirow[t]{2}{*}{ CEPXS Form: } & material & $\begin{array}{c}N \\
U-234 \\
U-235 \\
U-236 \\
U-238\end{array}$ & $\begin{array}{l}0.055590 \\
0.000252 \\
0.028332 \\
0.000130 \\
0.915695\end{array}$ & & & \\
\hline & $\begin{array}{c}\text { matname } \\
\text { density }\end{array}$ & $\begin{array}{r}\text { Uranium Nit } \\
14.310000\end{array}$ & & & & \\
\hline \multicolumn{7}{|c|}{$\begin{array}{l}\text { Comments and References } \\
\text { Density and formula from pg M8.2.3 of Petrie et al. }(2000) \text {. } \\
\text { Uranium isotopics assumed for LEU: Wt\% U234/235/236/238 = 0.0267/3.0/0.0138/96.9595. }\end{array}$} \\
\hline
\end{tabular}

\section{Uranium Oxide}

\begin{tabular}{llll}
\hline Formula $=$ & U3O8 & Molecular weight $(\mathrm{g} / \mathrm{mole})=$ & 841.8694727 \\
Density $(\mathrm{g} / \mathrm{cm} 3)=$ & 8.300000 & Total atom density $($ atoms $/ \mathrm{b}-\mathrm{cm})=$ & $6.531 \mathrm{E}-02$
\end{tabular}

The above density is estimated to be accurate to 3 significant digits. Uncertainties are not addressed.

The following data was calculated from the input formula.

\begin{tabular}{|c|c|c|c|c|c|c|}
\hline Element & Neutron ZA & Photon ZA & $\begin{array}{l}\text { Weight } \\
\text { Fraction }\end{array}$ & $\begin{array}{c}\text { Atom } \\
\text { Fraction }\end{array}$ & $\begin{array}{l}\text { Atom } \\
\text { Density }\end{array}$ & \\
\hline 0 & 8016 & 8000 & 0.152037 & 0.727273 & 0.047498 & \\
\hline U-234 & 92234 & 92000 & 0.000226 & 0.000074 & 0.000005 & \\
\hline U-235 & 92235 & 92000 & 0.025439 & 0.008283 & 0.000541 & \\
\hline U-236 & 92236 & 92000 & 0.000117 & 0.000038 & 0.000002 & \\
\hline$U-238$ & 92238 & 92000 & 0.822181 & 0.264332 & 0.017263 & \\
\hline Total & & & 1.000000 & 1.000000 & 0.065310 & \\
\hline MCNP Form & \multicolumn{2}{|c|}{ Weight Fractions } & \multicolumn{2}{|c|}{ Atom Fractions } & \multicolumn{2}{|c|}{ Atom Densities } \\
\hline \multirow[t]{5}{*}{ Neutrons } & 8016 & -0.152037 & 8016 & 0.727273 & 8016 & 0.047498 \\
\hline & 92234 & -0.000226 & 92234 & 0.000074 & 92234 & 0.000005 \\
\hline & 92235 & -0.025439 & 92235 & 0.008283 & 92235 & 0.000541 \\
\hline & 92236 & -0.000117 & 92236 & 0.000038 & 92236 & 0.000002 \\
\hline & 92238 & -0.822181 & 92238 & 0.264332 & 92238 & 0.017263 \\
\hline \multirow[t]{5}{*}{ Photons } & 8000 & -0.152037 & 8000 & 0.727273 & 8000 & 0.047498 \\
\hline & 92000 & -0.000226 & 92000 & 0.000074 & 92000 & 0.000005 \\
\hline & 92000 & -0.025439 & 92000 & 0.008283 & 92000 & 0.000541 \\
\hline & 92000 & -0.000117 & 92000 & 0.000038 & 92000 & 0.000002 \\
\hline & 92000 & -0.822181 & 92000 & 0.264332 & 92000 & 0.017263 \\
\hline \multirow[t]{3}{*}{ CEPXS Form: } & material & $\mathrm{O}$ & 0.152037 & & & \\
\hline & & U-234 & 0.000226 & & & \\
\hline & & U-235 & 0.025439 & & & \\
\hline
\end{tabular}




\begin{tabular}{|l|cc|} 
& U-236 & 0.000117 \\
U-238 & 0.822181 \\
matname & Uranium Oxide \\
density & 8.300000 \\
\hline Comments and References & \\
Density and formula from pg M8.2.3 of Petrie et al. (2000). \\
Also called yellowcake. \\
Uranium isotopics assumed for LEU: Wt\% U234/235/236/238 $=0.0267 / 3.0 / 0.0138 / 96.9595$. \\
\hline
\end{tabular}

\section{Uranium Tetrafluoride}

\begin{tabular}{llll}
\hline Formula $=$ & UF4 & Molecular weight $(\mathrm{g} / \mathrm{mole})=$ & 313.9517037 \\
Density $(\mathrm{g} / \mathrm{cm} 3)=$ & 6.700000 & Total atom density $($ atoms $/ \mathrm{b}-\mathrm{cm})=$ & $6.426 \mathrm{E}-02$ \\
The above density is estimated to be accurate to 3 significant digits. Uncertainties are not addressed. & \\
The following data was calculated from the input formula.
\end{tabular}

\begin{tabular}{|c|c|c|c|c|c|c|}
\hline Element & Neutron ZA & Photon ZA & $\begin{array}{l}\text { Weight } \\
\text { Fraction }\end{array}$ & $\begin{array}{l}\text { Atom } \\
\text { Fraction }\end{array}$ & $\begin{array}{l}\text { Atom } \\
\text { Density }\end{array}$ & \\
\hline $\mathrm{F}$ & 9019 & 9000 & 0.242055 & 0.800000 & 0.051407 & \\
\hline$U-234$ & 92234 & 92000 & 0.000202 & 0.000054 & 0.000003 & \\
\hline U-235 & 92235 & 92000 & 0.022738 & 0.006074 & 0.000390 & \\
\hline U-236 & 92236 & 92000 & 0.000105 & 0.000028 & 0.000002 & \\
\hline U-238 & 92238 & 92000 & 0.734900 & 0.193843 & 0.012456 & \\
\hline Total & & & 1.000000 & 1.000000 & 0.064259 & \\
\hline MCNP Form & \multicolumn{2}{|c|}{ Weight Fractions } & \multicolumn{2}{|c|}{ Atom Fractions } & \multicolumn{2}{|c|}{ Atom Densities } \\
\hline \multirow[t]{5}{*}{ Neutrons } & 9019 & -0.242055 & 9019 & 0.800000 & 9019 & 0.051407 \\
\hline & 92234 & -0.000202 & 92234 & 0.000054 & 92234 & 0.000003 \\
\hline & 92235 & -0.022738 & 92235 & 0.006074 & 92235 & 0.000390 \\
\hline & 92236 & -0.000105 & 92236 & 0.000028 & 92236 & 0.000002 \\
\hline & 92238 & -0.734900 & 92238 & 0.193843 & 92238 & 0.012456 \\
\hline \multirow[t]{5}{*}{ Photons } & 9000 & -0.242055 & 9000 & 0.800000 & 9000 & 0.051407 \\
\hline & 92000 & -0.000202 & 92000 & 0.000054 & 92000 & 0.000003 \\
\hline & 92000 & -0.022738 & 92000 & 0.006074 & 92000 & 0.000390 \\
\hline & 92000 & -0.000105 & 92000 & 0.000028 & 92000 & 0.000002 \\
\hline & 92000 & -0.734900 & 92000 & 0.193843 & 92000 & 0.012456 \\
\hline \multirow[t]{6}{*}{ CEPXS Form: } & material & $F$ & 0.242055 & & & \\
\hline & & U-234 & 0.000202 & & & \\
\hline & & U-235 & 0.022738 & & & \\
\hline & & U-236 & 0.000105 & & & \\
\hline & & U-238 & 0.734900 & & & \\
\hline & $\begin{array}{l}\text { matname } \\
\text { density }\end{array}$ & \multicolumn{3}{|c|}{$\begin{array}{l}\text { Uranium Tetrafluoride } \\
6.700000\end{array}$} & & \\
\hline
\end{tabular}


Comments and References

Density and formula from pg M8.2.3 of Petrie et al. (2000). Density $=6.7 \mathrm{~g} / \mathrm{cm} 3$ also on pgs $4-97$ of Lide (2008), at http://en.wikipedia.org/wiki/Uranium_hexafluoride, and pg 201 of Paxton and Pruvost (1986) revision issued July 1987.

Uranium isotopics assumed for LEU: Wt\% U234/235/236/238 $=0.0267 / 3.0 / 0.0138 / 96.9595$.

\section{Uranium Trioxide}

\begin{tabular}{llll}
\hline Formula $=$ & UO3 & Molecular weight $(\mathrm{g} / \mathrm{mole})=$ & 285.9562909 \\
Density $(\mathrm{g} / \mathrm{cm} 3)=$ & 7.290000 & Total atom density $($ atoms $/ \mathrm{b}-\mathrm{cm})=$ & $6.141 \mathrm{E}-02$
\end{tabular}

The above density is estimated to be accurate to 3 significant digits. Uncertainties are not addressed.

The following data was calculated from the input formula.

\begin{tabular}{|c|c|c|c|c|c|c|}
\hline Element & Neutron ZA & Photon ZA & $\begin{array}{l}\text { Weight } \\
\text { Fraction }\end{array}$ & $\begin{array}{c}\text { Atom } \\
\text { Fraction }\end{array}$ & $\begin{array}{l}\text { Atom } \\
\text { Density }\end{array}$ & \\
\hline 0 & 8016 & 8000 & $\overline{0.167852}$ & $\overline{0.750000}$ & 0.046057 & \\
\hline$U-234$ & 92234 & 92000 & 0.000222 & 0.000068 & 0.000004 & \\
\hline U-235 & 92235 & 92000 & 0.024964 & 0.007593 & 0.000466 & \\
\hline U-236 & 92236 & 92000 & 0.000115 & 0.000035 & 0.000002 & \\
\hline U-238 & 92238 & 92000 & 0.806847 & 0.242304 & 0.014880 & \\
\hline Total & & & 1.000000 & 1.000000 & 0.061410 & \\
\hline MCNP Form & \multicolumn{2}{|c|}{ Weight Fractions } & \multicolumn{2}{|c|}{ Atom Fractions } & \multicolumn{2}{|c|}{ Atom Densities } \\
\hline \multirow[t]{5}{*}{ Neutrons } & 8016 & -0.167852 & 8016 & 0.750000 & 8016 & 0.046057 \\
\hline & 92234 & -0.000222 & 92234 & 0.000068 & 92234 & 0.000004 \\
\hline & 92235 & -0.024964 & 92235 & 0.007593 & 92235 & 0.000466 \\
\hline & 92236 & -0.000115 & 92236 & 0.000035 & 92236 & 0.000002 \\
\hline & 92238 & -0.806847 & 92238 & 0.242304 & 92238 & 0.014880 \\
\hline \multirow[t]{5}{*}{ Photons } & 8000 & -0.167852 & 8000 & 0.750000 & 8000 & 0.046057 \\
\hline & 92000 & -0.000222 & 92000 & 0.000068 & 92000 & 0.000004 \\
\hline & 92000 & -0.024964 & 92000 & 0.007593 & 92000 & 0.000466 \\
\hline & 92000 & -0.000115 & 92000 & 0.000035 & 92000 & 0.000002 \\
\hline & 92000 & -0.806847 & 92000 & 0.242304 & 92000 & 0.014880 \\
\hline \multirow[t]{6}{*}{ CEPXS Form: } & material & 0 & 0.167852 & & & \\
\hline & & U-234 & 0.000222 & & & \\
\hline & & U-235 & 0.024964 & & & \\
\hline & & U-236 & 0.000115 & & & \\
\hline & & U-238 & 0.806847 & & & \\
\hline & $\begin{array}{l}\text { matname } \\
\text { density }\end{array}$ & \multicolumn{3}{|c|}{$\begin{array}{l}\text { Uranium Trioxide } \\
7.290000\end{array}$} & & \\
\hline
\end{tabular}

Comments and References

Density and formula from pg M8.2.4 of Petrie et al. (2000).

Uranium isotopics assumed for LEU: Wt\% U234/235/236/238 $=0.0267 / 3.0 / 0.0138 / 96.9595$. 


\section{Uranium, Depleted, Typical}

\begin{tabular}{|c|c|c|c|}
\hline ula $=$ & - & Molecular weight $(\mathrm{g} / \mathrm{mole})=$ & \\
\hline$(\mathrm{g} / \mathrm{cm} 3)=$ & 18.951157 & Total atom density $($ atoms $/ \mathrm{b}-\mathrm{cm})=$ & 4.794E-02 \\
\hline
\end{tabular}

The above density is estimated to be accurate to 3 significant digits. Uncertainties are not addressed.

The following data were calculated from the input weight fractions.

\begin{tabular}{|c|c|c|c|c|c|c|}
\hline Element & Neutron ZA & Photon ZA & $\begin{array}{l}\text { Weight } \\
\text { Fraction }\end{array}$ & $\begin{array}{l}\text { Atom } \\
\text { Fraction }\end{array}$ & $\begin{array}{l}\text { Atom } \\
\text { Density }\end{array}$ & \\
\hline$U-234$ & 92234 & 92000 & $\overline{0.000005}$ & $\overline{0.000005}$ & 0.000000 & \\
\hline $\mathrm{U}-235$ & 92235 & 92000 & 0.002500 & 0.002532 & 0.000121 & \\
\hline $\mathrm{U}-238$ & 92238 & 92000 & 0.997495 & 0.997463 & 0.047822 & \\
\hline Total & & & 1.000000 & 1.000000 & 0.047944 & \\
\hline MCNP Form & \multicolumn{2}{|c|}{ Weight Fractions } & \multicolumn{2}{|c|}{ Atom Fractions } & \multicolumn{2}{|c|}{ Atom Densities } \\
\hline \multirow{3}{*}{ Neutrons } & 92234 & -0.000005 & 92234 & 0.000005 & 92234 & 0.000000 \\
\hline & 92235 & -0.002500 & 92235 & 0.002532 & 92235 & 0.000121 \\
\hline & 92238 & -0.997495 & 92238 & 0.997463 & 92238 & 0.047822 \\
\hline \multirow[t]{3}{*}{ Photons } & 92000 & -0.000005 & 92000 & 0.000005 & 92000 & 0.000000 \\
\hline & 92000 & -0.002500 & 92000 & 0.002532 & 92000 & 0.000121 \\
\hline & 92000 & -0.997495 & 92000 & 0.997463 & 92000 & 0.047822 \\
\hline \multirow[t]{4}{*}{ CEPXS Form: } & material & U-234 & 0.000005 & & & \\
\hline & & U-235 & 0.002500 & & & \\
\hline & & $\mathrm{U}-238$ & 0.997495 & & & \\
\hline & $\begin{array}{c}\text { matname } \\
\text { density }\end{array}$ & \multicolumn{2}{|c|}{$\begin{array}{l}\text { Uranium, Depleted, Typical } \\
18.951157\end{array}$} & & & \\
\hline \multicolumn{7}{|c|}{$\begin{array}{l}\text { Comments and References } \\
\text { See pg } 286 \text { of Shleien (1992). } \\
\text { Density adjusted from } 18.95 \mathrm{~g} / \mathrm{cm} 3 \text { to maintain same total atoms as for natural uranium based on } \\
\text { http://physics.nist.gov/cgi-bin/Star/compos.pl?matno=092 (NIST 1998). }\end{array}$} \\
\hline
\end{tabular}

\section{Uranium, Enriched, Typical Commercial}

Formula $=$

Density $(\mathrm{g} / \mathrm{cm} 3)=18.944492$

The above density is estimated to be accurate to 3 significant digits. Uncertainties are not addressed.

The following data were calculated from the input weight fractions.

\begin{tabular}{|c|c|c|c|c|c|}
\hline lement & Neutron ZA & Photon ZA & $\begin{array}{l}\text { Weight } \\
\text { Fraction }\end{array}$ & $\begin{array}{c}\text { Atom } \\
\text { Fraction }\end{array}$ & $\begin{array}{l}\text { Atom } \\
\text { Density }\end{array}$ \\
\hline U-234 & 92234 & 92000 & 0.000305 & 0.000310 & 0.000015 \\
\hline
\end{tabular}


PIET-43741-TM-963

PNNL-15870 Rev. 1

\begin{tabular}{|c|c|c|c|c|c|c|}
\hline U-235 & 92235 & 92000 & 0.029600 & 0.029967 & 0.001437 & \\
\hline U-238 & 92238 & 92000 & 0.970095 & 0.969723 & 0.046492 & \\
\hline Total & & & 1.000000 & 1.000000 & 0.047944 & \\
\hline MCNP Form & Weigh & ractions & Atom & ctions & Atom & nsities \\
\hline Neutrons & 92234 & -0.000305 & 92234 & 0.000310 & 92234 & 0.000015 \\
\hline & 92235 & -0.029600 & 92235 & 0.029967 & 92235 & 0.001437 \\
\hline & 92238 & -0.970095 & 92238 & 0.969723 & 92238 & 0.046492 \\
\hline Photons & 92000 & -0.000305 & 92000 & 0.000310 & 92000 & 0.000015 \\
\hline & 92000 & -0.029600 & 92000 & 0.029967 & 92000 & 0.001437 \\
\hline & 92000 & -0.970095 & 92000 & 0.969723 & 92000 & 0.046492 \\
\hline CEPXS Form: & material & U-234 & 0.000305 & & & \\
\hline & & U-235 & 0.029600 & & & \\
\hline & & $\mathrm{U}-238$ & 0.970095 & & & \\
\hline & $\begin{array}{c}\text { matname } \\
\text { density }\end{array}$ & $\begin{array}{r}\text { Uranium, Er } \\
18.944492\end{array}$ & ed, Typical & nmercial & & \\
\hline $\begin{array}{l}\text { Comments an } \\
\text { See pg } 286 \text { of } \\
\text { Density adjuste } \\
\text { http://physics.n }\end{array}$ & $\begin{array}{l}\text { eferences } \\
\text { eien (1992 } \\
\text { rom } 18.95 \\
\text { gov/cgi-bin }\end{array}$ & $\begin{array}{l}\text { m3 to mainta } \\
\text { ar/compos.pl }\end{array}$ & $\begin{array}{l}\text { ame total a } \\
\text { tno }=092(\end{array}$ & $\begin{array}{l}\text { as for nat } \\
\text { 1998). }\end{array}$ & Iranium b & on \\
\hline
\end{tabular}

\section{Uranium, HEU, Health Physics Society}

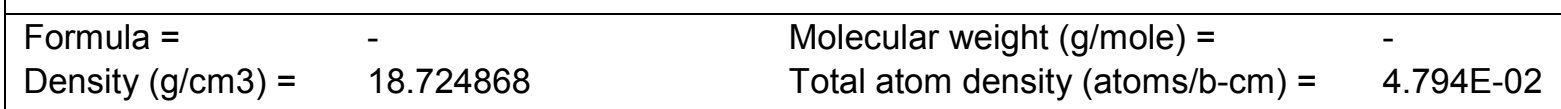

The above density is estimated to be accurate to 3 significant digits. Uncertainties are not addressed.

The following data were calculated from the input weight fractions.

\begin{tabular}{|c|c|c|c|c|c|c|}
\hline Element & Neutron ZA & Photon ZA & $\begin{array}{l}\text { Weight } \\
\text { Fraction }\end{array}$ & $\begin{array}{c}\text { Atom } \\
\text { Fraction }\end{array}$ & $\begin{array}{c}\text { Atom } \\
\text { Density }\end{array}$ & \\
\hline U-234 & 92234 & 92000 & 0.010530 & 0.010582 & 0.000507 & \\
\hline $\mathrm{U}-235$ & 92235 & 92000 & 0.931740 & 0.932362 & 0.044701 & \\
\hline U-236 & 92236 & 92000 & 0.002060 & 0.002053 & 0.000098 & \\
\hline U-238 & 92238 & 92000 & 0.055670 & 0.055003 & 0.002637 & \\
\hline Total & & & 1.000000 & 1.000000 & 0.047944 & \\
\hline MCNP Form & \multicolumn{2}{|c|}{ Weight Fractions } & \multicolumn{2}{|c|}{ Atom Fractions } & \multicolumn{2}{|c|}{ Atom Densities } \\
\hline \multirow[t]{4}{*}{ Neutrons } & 92234 & -0.010530 & 92234 & 0.010582 & 92234 & 0.000507 \\
\hline & 92235 & -0.931740 & 92235 & 0.932362 & 92235 & 0.044701 \\
\hline & 92236 & -0.002060 & 92236 & 0.002053 & 92236 & 0.000098 \\
\hline & 92238 & -0.055670 & 92238 & 0.055003 & 92238 & 0.002637 \\
\hline
\end{tabular}


PIET-43741-TM-963

PNNL-15870 Rev. 1

\begin{tabular}{|c|c|c|c|c|c|c|}
\hline \multirow[t]{4}{*}{ Photons } & 92000 & -0.010530 & 92000 & 0.010582 & 92000 & 0.000507 \\
\hline & 92000 & -0.931740 & 92000 & 0.932362 & 92000 & 0.044701 \\
\hline & 92000 & -0.002060 & 92000 & 0.002053 & 92000 & 0.000098 \\
\hline & 92000 & -0.055670 & 92000 & 0.055003 & 92000 & 0.002637 \\
\hline \multirow[t]{5}{*}{ CEPXS Form: } & material & U-234 & 0.010530 & & & \\
\hline & & U-235 & 0.931740 & & & \\
\hline & & U-236 & 0.002060 & & & \\
\hline & & U-238 & 0.055670 & & & \\
\hline & $\begin{array}{l}\text { matname } \\
\text { density }\end{array}$ & \multicolumn{5}{|c|}{$\begin{array}{l}\text { Uranium, HEU, Health Physics Society } \\
18.724868\end{array}$} \\
\hline \multicolumn{7}{|c|}{$\begin{array}{l}\text { Comments and References } \\
\text { Bioassay Programs for Uranium, HPS 13.22-1995, American National Standards Institute, Inc., } \\
\text { Oct. } 1995 . \\
\text { Density adjusted from } 18.95 \mathrm{~g} / \mathrm{cm} 3 \text { to maintain same total atoms as for natural uranium based on } \\
\text { http://physics.nist.gov/cgi-bin/Star/compos.pl?matno=092 (NIST 1998). }\end{array}$} \\
\hline
\end{tabular}

\section{Uranium, HEU, Russian Average}

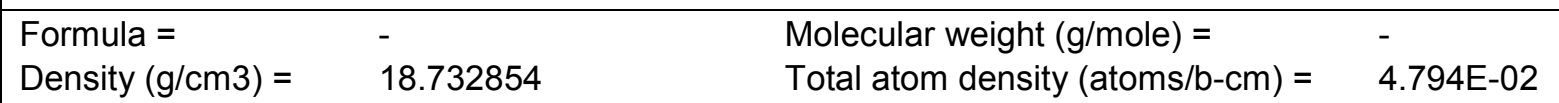

The above density is estimated to be accurate to 3 significant digits. Uncertainties are not addressed.

The following data were calculated from the input weight fractions.

\begin{tabular}{|c|c|c|c|c|c|c|}
\hline Element & Neutron ZA & Photon ZA & $\begin{array}{l}\text { Weight } \\
\text { Fraction }\end{array}$ & $\begin{array}{c}\text { Atom } \\
\text { Fraction }\end{array}$ & $\begin{array}{c}\text { Atom } \\
\text { Density }\end{array}$ & \\
\hline U-234 & 92234 & 92000 & 0.009670 & 0.009722 & 0.000466 & \\
\hline U-235 & 92235 & 92000 & 0.898000 & 0.898982 & 0.043100 & \\
\hline U-236 & 92236 & 92000 & 0.003810 & 0.003798 & 0.000182 & \\
\hline U-238 & 92238 & 92000 & 0.088520 & 0.087498 & 0.004195 & \\
\hline Total & & & 1.000000 & 1.000000 & 0.047944 & \\
\hline MCNP Form & \multicolumn{2}{|c|}{ Weight Fractions } & \multicolumn{2}{|c|}{ Atom Fractions } & \multicolumn{2}{|c|}{ Atom Densities } \\
\hline \multirow[t]{4}{*}{ Neutrons } & 92234 & -0.009670 & 92234 & 0.009722 & 92234 & 0.000466 \\
\hline & 92235 & -0.898000 & 92235 & 0.898982 & 92235 & 0.043100 \\
\hline & 92236 & -0.003810 & 92236 & 0.003798 & 92236 & 0.000182 \\
\hline & 92238 & -0.088520 & 92238 & 0.087498 & 92238 & 0.004195 \\
\hline \multirow[t]{4}{*}{ Photons } & 92000 & -0.009670 & 92000 & 0.009722 & 92000 & 0.000466 \\
\hline & 92000 & -0.898000 & 92000 & 0.898982 & 92000 & 0.043100 \\
\hline & 92000 & -0.003810 & 92000 & 0.003798 & 92000 & 0.000182 \\
\hline & 92000 & -0.088520 & 92000 & 0.087498 & 92000 & 0.004195 \\
\hline \multirow[t]{3}{*}{ CEPXS Form: } & material & U-234 & 0.009670 & & & \\
\hline & & $U-235$ & 0.898000 & & & \\
\hline & & U-236 & 0.003810 & & & \\
\hline
\end{tabular}




$$
\text { U-238 } \quad 0.088520
$$

matname Uranium, HEU, Russian Average

density $\quad 18.732854$

\section{Comments and References}

Personal communication with Andy Luksic based on Y-12 information.

Density adjusted from $18.95 \mathrm{~g} / \mathrm{cm} 3$ to maintain same total atoms as for natural uranium based on http://physics.nist.gov/cgi-bin/Star/compos.pl?matno=092 (NIST 1998).

\section{Uranium, HEU, U.S. Average}

\begin{tabular}{|c|c|c|c|}
\hline Formula $=$ & - & Molecular weight $(\mathrm{g} / \mathrm{mole})=$ & - \\
\hline Density $(\mathrm{g} / \mathrm{cm} 3)=$ & 18.724760 & Total atom density $($ atoms $/ \mathrm{b}-\mathrm{cm})=$ & 4.794E-02 \\
\hline
\end{tabular}

\begin{tabular}{|c|c|c|c|c|c|c|}
\hline Element & Neutron ZA & Photon ZA & $\begin{array}{l}\text { Weight } \\
\text { Fraction }\end{array}$ & $\begin{array}{l}\text { Atom } \\
\text { Fraction }\end{array}$ & $\begin{array}{l}\text { Atom } \\
\text { Density }\end{array}$ & \\
\hline$\overline{U-234}$ & 92234 & 92000 & 0.009800 & $\overline{0.009849}$ & 0.000472 & \\
\hline U-235 & 92235 & 92000 & 0.931550 & 0.932166 & 0.044691 & \\
\hline $\mathrm{U}-236$ & 92236 & 92000 & 0.004500 & 0.004484 & 0.000215 & \\
\hline $\mathrm{U}-238$ & 92238 & 92000 & 0.054150 & 0.053501 & 0.002565 & \\
\hline Total & & & 1.000000 & 1.000000 & 0.047944 & \\
\hline MCNP Form & \multicolumn{2}{|c|}{ Weight Fractions } & \multicolumn{2}{|c|}{ Atom Fractions } & \multicolumn{2}{|c|}{ Atom Densities } \\
\hline \multirow[t]{4}{*}{ Neutrons } & 92234 & -0.009800 & 92234 & 0.009849 & 92234 & 0.000472 \\
\hline & 92235 & -0.931550 & 92235 & 0.932166 & 92235 & 0.044691 \\
\hline & 92236 & -0.004500 & 92236 & 0.004484 & 92236 & 0.000215 \\
\hline & 92238 & -0.054150 & 92238 & 0.053501 & 92238 & 0.002565 \\
\hline \multirow[t]{4}{*}{ Photons } & 92000 & -0.009800 & 92000 & 0.009849 & 92000 & 0.000472 \\
\hline & 92000 & -0.931550 & 92000 & 0.932166 & 92000 & 0.044691 \\
\hline & 92000 & -0.004500 & 92000 & 0.004484 & 92000 & 0.000215 \\
\hline & 92000 & -0.054150 & 92000 & 0.053501 & 92000 & 0.002565 \\
\hline \multirow[t]{5}{*}{ CEPXS Form: } & material & U-234 & 0.009800 & & & \\
\hline & & U-235 & 0.931550 & & & \\
\hline & & U-236 & 0.004500 & & & \\
\hline & & $U-238$ & 0.054150 & & & \\
\hline & $\begin{array}{l}\text { matname } \\
\text { density }\end{array}$ & $\begin{array}{c}\text { Uranium, HE } \\
18.724760\end{array}$ & U.S. Averas & & & \\
\hline \multicolumn{7}{|c|}{$\begin{array}{l}\text { Comments and References } \\
\text { Personal communication with Andy Luksic based on Y-12 information. } \\
\text { Density adjusted from } 18.95 \mathrm{~g} / \mathrm{cm} 3 \text { to maintain same total atoms as for natural uranium based on } \\
\text { http://physics.nist.gov/cgi-bin/Star/compos.pl?matno=092 (NIST 1998). }\end{array}$} \\
\hline
\end{tabular}




\section{Uranium, Low Enriched (LEU)}

\begin{tabular}{|c|c|c|c|}
\hline & $U$ & Molecular weight $(\mathrm{g} / \mathrm{mole})=$ & \\
\hline Density $(\mathrm{g} / \mathrm{cm} 3)=$ & 18.944386 & Total atom density $($ atoms $/ \mathrm{b}-\mathrm{cm})=$ & 4.794E-02 \\
\hline
\end{tabular}

The above density is estimated to be accurate to 3 significant digits. Uncertainties are not addressed.

The following data were calculated from the input weight fractions.

\begin{tabular}{|c|c|c|c|c|c|c|}
\hline Element & Neutron ZA & Photon ZA & $\begin{array}{l}\text { Weight } \\
\text { Fraction }\end{array}$ & $\begin{array}{l}\text { Atom } \\
\text { Fraction }\end{array}$ & $\begin{array}{l}\text { Atom } \\
\text { Density }\end{array}$ & \\
\hline $\mathrm{U}-234$ & 92234 & 92000 & 0.000267 & 0.000271 & 0.000013 & \\
\hline U-235 & 92235 & 92000 & 0.030000 & 0.030372 & 0.001456 & \\
\hline $\mathrm{U}-236$ & 92236 & 92000 & 0.000138 & 0.000139 & 0.000007 & \\
\hline$U-238$ & 92238 & 92000 & 0.969595 & 0.969217 & 0.046468 & \\
\hline Total & & & 1.000000 & 1.000000 & 0.047944 & \\
\hline MCNP Form & \multicolumn{2}{|c|}{ Weight Fractions } & \multicolumn{2}{|c|}{ Atom Fractions } & \multicolumn{2}{|c|}{ Atom Densities } \\
\hline \multirow[t]{4}{*}{ Neutrons } & 92234 & -0.000267 & 92234 & 0.000271 & 92234 & 0.000013 \\
\hline & 92235 & -0.030000 & 92235 & 0.030372 & 92235 & 0.001456 \\
\hline & 92236 & -0.000138 & 92236 & 0.000139 & 92236 & 0.000007 \\
\hline & 92238 & -0.969595 & 92238 & 0.969217 & 92238 & 0.046468 \\
\hline \multirow[t]{4}{*}{ Photons } & 92000 & -0.000267 & 92000 & 0.000271 & 92000 & 0.000013 \\
\hline & 92000 & -0.030000 & 92000 & 0.030372 & 92000 & 0.001456 \\
\hline & 92000 & -0.000138 & 92000 & 0.000139 & 92000 & 0.000007 \\
\hline & 92000 & -0.969595 & 92000 & 0.969217 & 92000 & 0.046468 \\
\hline \multirow[t]{5}{*}{ CEPXS Form: } & material & U-234 & 0.000267 & & & \\
\hline & & U-235 & 0.030000 & & & \\
\hline & & $U-236$ & 0.000138 & & & \\
\hline & & $\mathrm{U}-238$ & 0.969595 & & & \\
\hline & $\begin{array}{c}\text { matname } \\
\text { density }\end{array}$ & $\begin{array}{c}\text { Uranium, Lo } \\
18.944386\end{array}$ & Enriched (LE & & & \\
\hline \multicolumn{7}{|c|}{$\begin{array}{l}\text { Comments and References } \\
\text { Density adjusted from } 18.95 \mathrm{~g} / \mathrm{cm} 3 \text { to maintain same total atoms as for natural uranium based on } \\
\text { http://physics.nist.gov/cgi-bin/Star/compos.pl?matno=092 (NIST 1998). } \\
\text { Weight fractions from "A Nondestructive Method for Discriminating MOX Fuel from LEU Fuel for } \\
\text { Safeguards Purposes," [Willman C, A Håkansson, O Osifo, A Bäcklin, and S J Svärd. 2006. Annals of } \\
\text { Nuclear Energy, 33(9): } 766-773] \text {. Accessed at } \\
\text { http://www.sciencedirect.com/science?_ob=ArticleURL\&_udi=B6V1R-4K4PSWP_ } \\
\text { 2\&_user=2741876\&_rdoc=1\&_fmt=\&_orig=search\&_sort=d\&view=c\&_acct=C000058656\&_version=1\&_} \\
\text { urIVersion=0\&_userid=2741876\&md5=57b9a508b0289ea60aa9587f39303309\#bbib5. }\end{array}$} \\
\hline
\end{tabular}

\section{Uranium, Natural (NU)}

\begin{tabular}{|llll}
\hline Formula $=$ & - & Molecular weight $(\mathrm{g} / \mathrm{mole})=$ \\
Density $(\mathrm{g} / \mathrm{cm} 3)=$ & 18.950000 & Total atom density $($ atoms $/ \mathrm{b}-\mathrm{cm})=$ & - \\
$4.794 \mathrm{E}-02$
\end{tabular}


The above density is estimated to be accurate to 3 significant digits. Uncertainties are not addressed. The following data were calculated from the input weight fractions.

\begin{tabular}{|c|c|c|c|c|c|c|}
\hline Element & Neutron ZA & Photon ZA & $\begin{array}{l}\text { Weight } \\
\text { Fraction }\end{array}$ & $\begin{array}{l}\text { Atom } \\
\text { Fraction }\end{array}$ & $\begin{array}{l}\text { Atom } \\
\text { Density }\end{array}$ & \\
\hline $\mathrm{U}-234$ & 92234 & 92000 & 0.000057 & $\overline{0.000058}$ & 0.000003 & \\
\hline$U-235$ & 92235 & 92000 & 0.007204 & 0.007295 & 0.000350 & \\
\hline U-238 & 92238 & 92000 & 0.992739 & 0.992647 & 0.047591 & \\
\hline Total & & & 1.000000 & 1.000000 & 0.047944 & \\
\hline MCNP Form & \multicolumn{2}{|c|}{ Weight Fractions } & \multicolumn{2}{|c|}{ Atom Fractions } & \multicolumn{2}{|c|}{ Atom Densities } \\
\hline \multirow{3}{*}{ Neutrons } & 92234 & -0.000057 & 92234 & 0.000058 & 92234 & 0.000003 \\
\hline & 92235 & -0.007204 & 92235 & 0.007295 & 92235 & 0.000350 \\
\hline & 92238 & -0.992739 & 92238 & 0.992647 & 92238 & 0.047591 \\
\hline \multirow[t]{3}{*}{ Photons } & 92000 & -0.000057 & 92000 & 0.000058 & 92000 & 0.000003 \\
\hline & 92000 & -0.007204 & 92000 & 0.007295 & 92000 & 0.000350 \\
\hline & 92000 & -0.992739 & 92000 & 0.992647 & 92000 & 0.047591 \\
\hline \multirow[t]{4}{*}{ CEPXS Form: } & material & U-234 & 0.000057 & & & \\
\hline & & U-235 & 0.007204 & & & \\
\hline & & $\mathrm{U}-238$ & 0.992739 & & & \\
\hline & $\begin{array}{l}\text { matname } \\
\text { density }\end{array}$ & \multicolumn{2}{|c|}{$\begin{array}{l}\text { Uranium, Natural (NU) } \\
18.950000\end{array}$} & & & \\
\hline \multicolumn{7}{|c|}{$\begin{array}{l}\text { Comments and References } \\
\text { See pg } 286 \text { of Shleien }(1992) \text {. } \\
\text { Density for natural uranium }=18.95 \mathrm{~g} / \mathrm{cm} 3 \text {, http://physics.nist.gov/cgi-bin/Star/compos.pl?matno=092 } \\
\text { (NIST 1998). }\end{array}$} \\
\hline
\end{tabular}

\section{Uranium-Plutonium, Mixed Oxide (MOX)}

\begin{tabular}{llll}
\hline Formula $=$ & - & Molecular weight $(\mathrm{g} / \mathrm{mole})=$ \\
Density $(\mathrm{g} / \mathrm{cm} 3)=$ & 11.000000 & Total atom density $($ atoms $/ \mathrm{b}-\mathrm{cm})=$ & $\overline{7}$ \\
\hline & &
\end{tabular}

The above density is estimated to be accurate to 3 significant digits. Uncertainties are not addressed.

The following data were calculated from the input weight fractions.

\begin{tabular}{|c|c|c|c|c|c|}
\hline Element & Neutron ZA & Photon ZA & $\begin{array}{l}\text { Weight } \\
\text { Fraction }\end{array}$ & $\begin{array}{c}\text { Atom } \\
\text { Fraction }\end{array}$ & $\begin{array}{c}\text { Atom } \\
\text { Density }\end{array}$ \\
\hline 0 & 8016 & 8000 & 0.118462 & $\overline{0.666666}$ & 0.049048 \\
\hline U-234 & 92234 & 92000 & 0.000010 & 0.000004 & 0.000000 \\
\hline U-235 & 92235 & 92000 & 0.002101 & 0.000805 & 0.000059 \\
\hline U-236 & 92236 & 92000 & 0.000000 & 0.000000 & 0.000000 \\
\hline U-238 & 92238 & 92000 & 0.838236 & 0.317052 & 0.023326 \\
\hline Pu-238 & 94238 & 94000 & 0.001030 & 0.000390 & 0.000029 \\
\hline Pu-239 & 94239 & 94000 & 0.022532 & 0.008487 & 0.000624 \\
\hline Pu-240 & 94240 & 94000 & 0.010751 & 0.004032 & 0.000297 \\
\hline
\end{tabular}


PIET-43741-TM-963

PNNL-15870 Rev. 1

\begin{tabular}{|c|c|c|c|c|c|c|}
\hline Pu-241 & 94241 & 94000 & 0.003913 & 0.001462 & \multicolumn{2}{|c|}{0.000108} \\
\hline Pu-242 & 94242 & 94000 & 0.002966 & 0.001103 & \multicolumn{2}{|c|}{0.000081} \\
\hline Total & & & 1.000000 & 1.000000 & \multicolumn{2}{|c|}{0.073572} \\
\hline MCNP Form & \multicolumn{2}{|c|}{ Weight Fractions } & \multicolumn{2}{|c|}{ Atom Fractions } & \multicolumn{2}{|c|}{ Atom Densities } \\
\hline \multirow[t]{10}{*}{ Neutrons } & 8016 & -0.118462 & 8016 & 0.666666 & 8016 & 0.049048 \\
\hline & 92234 & -0.000010 & 92234 & 0.000004 & 92234 & 0.000000 \\
\hline & 92235 & -0.002101 & 92235 & 0.000805 & 92235 & 0.000059 \\
\hline & 92236 & 0.000000 & 92236 & 0.000000 & 92236 & 0.000000 \\
\hline & 92238 & -0.838236 & 92238 & 0.317052 & 92238 & 0.023326 \\
\hline & 94238 & -0.001030 & 94238 & 0.000390 & 94238 & 0.000029 \\
\hline & 94239 & -0.022532 & 94239 & 0.008487 & 94239 & 0.000624 \\
\hline & 94240 & -0.010751 & 94240 & 0.004032 & 94240 & 0.000297 \\
\hline & 94241 & -0.003913 & 94241 & 0.001462 & 94241 & 0.000108 \\
\hline & 94242 & -0.002966 & 94242 & 0.001103 & 94242 & 0.000081 \\
\hline \multirow[t]{10}{*}{ Photons } & 8000 & -0.118462 & 8000 & 0.666666 & 8000 & 0.049048 \\
\hline & 92000 & -0.000010 & 92000 & 0.000004 & 92000 & 0.000000 \\
\hline & 92000 & -0.002101 & 92000 & 0.000805 & 92000 & 0.000059 \\
\hline & 92000 & 0.000000 & 92000 & 0.000000 & 92000 & 0.000000 \\
\hline & 92000 & -0.838236 & 92000 & 0.317052 & 92000 & 0.023326 \\
\hline & 94000 & -0.001030 & 94000 & 0.000390 & 94000 & 0.000029 \\
\hline & 94000 & -0.022532 & 94000 & 0.008487 & 94000 & 0.000624 \\
\hline & 94000 & -0.010751 & 94000 & 0.004032 & 94000 & 0.000297 \\
\hline & 94000 & -0.003913 & 94000 & 0.001462 & 94000 & 0.000108 \\
\hline & 94000 & -0.002966 & 94000 & 0.001103 & 94000 & 0.000081 \\
\hline \multirow[t]{11}{*}{ CEPXS Form: } & material & $\mathrm{O}$ & 0.118462 & & & \\
\hline & & U-234 & 0.000010 & & & \\
\hline & & $U-235$ & 0.002101 & & & \\
\hline & & $U-236$ & 0.000000 & & & \\
\hline & & U-238 & 0.838236 & & & \\
\hline & & Pu-238 & 0.001030 & & & \\
\hline & & Pu-239 & 0.022532 & & & \\
\hline & & Pu-240 & 0.010751 & & & \\
\hline & & Pu-241 & 0.003913 & & & \\
\hline & & Pu-242 & 0.002966 & & & \\
\hline & $\begin{array}{c}\text { matname } \\
\text { density }\end{array}$ & \multicolumn{3}{|c|}{$\begin{array}{l}\text { Uranium-Plutonium, Mixed Oxide (MOX) } \\
11.000000\end{array}$} & & \\
\hline
\end{tabular}

\section{Comments and References}

Density (4 wt\% PuO2, 96 wt\% UO2) MOX from American Nuclear Society Light Water Reactor Mixed Oxide Benchmark I (Gemin JC and RT Primm, III. 1997. Oak Ridge National Laboratory, Oak Ridge Tennessee.) Accessed at http://local.ans.org/oakridge/pdf/benchmark.pdf.

Weight fractions based on MOX with $3.0 \mathrm{wt} \%$ fissile Pu in heavy metal from "A Nondestructive Method for Discriminating MOX Fuel from LEU Fuel for Safeguards Purposes" [Willman C, A Håkansson, O Osifo, A Bäcklin and S J Svärd. 2006. Annals of Nuclear Energy, 33(9): 766-773]. Accessed at http://www.sciencedirect.com/science?_ob=ArticleURL\&_udi=B6V1R-4K4PSWP-

$2 \&$ user $=2741876 \&$ rdoc $=1 \&$ fmt $=$ \&_orig=search\&_sort $=\mathrm{d} \&$ view $=\mathrm{c} \&$ acct $=\mathrm{C} 000058656 \&$ version $=1 \&$ urlVersion=0\&_userid $=27418 \overline{76} \& \mathrm{md} \overline{5}=57 \mathrm{~b} 9 \mathrm{a} 508 \mathrm{~b} 0289 \mathrm{ea} 60 \mathrm{aa} 9587 \mathrm{f} 39303309 \mathrm{\# bbib5}$. 


\section{Uranyl Fluoride}

\begin{tabular}{llll}
\hline Formula $=$ & UO2F2 & Molecular weight $(\mathrm{g} / \mathrm{mole})=$ & 307.9536973 \\
Density $(\mathrm{g} / \mathrm{cm} 3)=$ & 6.370000 & Total atom density $($ atoms $/ \mathrm{b}-\mathrm{cm})=$ & $6.228 \mathrm{E}-02$
\end{tabular}

The above density is estimated to be accurate to 3 significant digits. Uncertainties are not addressed.

The following data was calculated from the input formula.

\begin{tabular}{|c|c|c|c|c|c|c|}
\hline Element & Neutron ZA & Photon ZA & $\begin{array}{l}\text { Weight } \\
\text { Fraction }\end{array}$ & $\begin{array}{l}\text { Atom } \\
\text { Fraction }\end{array}$ & $\begin{array}{l}\text { Atom } \\
\text { Density }\end{array}$ & \\
\hline 0 & 8016 & 8000 & $\overline{0.103908}$ & $\overline{0.400000}$ & 0.024914 & \\
\hline $\mathrm{F}$ & 9019 & 9000 & 0.123385 & 0.400000 & 0.024914 & \\
\hline $\mathrm{U}-234$ & 92234 & 92000 & 0.000206 & 0.000054 & 0.000003 & \\
\hline U-235 & 92235 & 92000 & 0.023181 & 0.006074 & $0.00037 \varepsilon$ & \\
\hline $\mathrm{U}-236$ & 92236 & 92000 & 0.000107 & 0.000028 & 0.000002 & \\
\hline $\mathrm{U}-238$ & 92238 & 92000 & 0.749213 & 0.193843 & 0.012073 & \\
\hline Total & & & 1.000000 & 1.000000 & 0.062284 & \\
\hline MCNP Form & \multicolumn{2}{|c|}{ Weight Fractions } & \multicolumn{2}{|c|}{ Atom Fractions } & \multicolumn{2}{|c|}{ Atom Densities } \\
\hline \multirow[t]{6}{*}{ Neutrons } & 8016 & -0.103908 & 8016 & 0.400000 & 8016 & 0.024914 \\
\hline & 9019 & -0.123385 & 9019 & 0.400000 & 9019 & 0.024914 \\
\hline & 92234 & -0.000206 & 92234 & 0.000054 & 92234 & 0.000003 \\
\hline & 92235 & -0.023181 & 92235 & 0.006074 & 92235 & 0.000378 \\
\hline & 92236 & -0.000107 & 92236 & 0.000028 & 92236 & 0.000002 \\
\hline & 92238 & -0.749213 & 92238 & 0.193843 & 92238 & 0.012073 \\
\hline \multirow[t]{6}{*}{ Photons } & 8000 & -0.103908 & 8000 & 0.400000 & 8000 & 0.024914 \\
\hline & 9000 & -0.123385 & 9000 & 0.400000 & 9000 & 0.024914 \\
\hline & 92000 & -0.000206 & 92000 & 0.000054 & 92000 & 0.000003 \\
\hline & 92000 & -0.023181 & 92000 & 0.006074 & 92000 & 0.000378 \\
\hline & 92000 & -0.000107 & 92000 & 0.000028 & 92000 & 0.000002 \\
\hline & 92000 & -0.749213 & 92000 & 0.193843 & 92000 & 0.012073 \\
\hline \multirow[t]{7}{*}{ CEPXS Form: } & material & $\mathrm{O}$ & 0.103908 & & & \\
\hline & & $\mathrm{F}$ & 0.123385 & & & \\
\hline & & U-234 & 0.000206 & & & \\
\hline & & U-235 & 0.023181 & & & \\
\hline & & U-236 & 0.000107 & & & \\
\hline & & $\mathrm{U}-238$ & 0.749213 & & & \\
\hline & $\begin{array}{l}\text { matname } \\
\text { density }\end{array}$ & $\begin{array}{c}\text { Uranyl Fluoride } \\
6.370000\end{array}$ & & & & \\
\hline \multicolumn{7}{|c|}{$\begin{array}{l}\text { Comments and References } \\
\text { Density and formula from pg M8.2.4 of Petrie et al. (2000). Also from pg } 201 \text { of Paxton and Pruvost } \\
\text { (1986) revision, issued July } 1987 \text {. } \\
\text { Uranium isotopics assumed for LEU: Wt\% U234/235/236/238 = 0.0267/3.0/0.0138/96.9595. }\end{array}$} \\
\hline
\end{tabular}




\section{Uranyl Nitrate}

\begin{tabular}{llll}
\hline Formula $=$ & UO2 $(\mathrm{NO} 3) 2$ & Molecular weight $(\mathrm{g} / \mathrm{mole})=$ & 393.9666909 \\
Density $(\mathrm{g} / \mathrm{cm} 3)=$ & 2.203000 & Total atom density $($ atoms $/ \mathrm{b}-\mathrm{cm})=$ & $3.704 \mathrm{E}-02$
\end{tabular}

The above density is estimated to be accurate to 3 significant digits. Uncertainties are not addressed.

The following data was calculated from the input formula.

\begin{tabular}{|c|c|c|c|c|c|}
\hline Element & Neutron ZA & Photon ZA & $\begin{array}{l}\text { Weight } \\
\text { Fraction }\end{array}$ & $\begin{array}{c}\text { Atom } \\
\text { Fraction }\end{array}$ & $\begin{array}{c}\text { Atom } \\
\text { Density }\end{array}$ \\
\hline $\mathrm{N}$ & 7014 & 7000 & $\overline{0.071106}$ & $\overline{0.181818}$ & 0.006735 \\
\hline $\mathrm{O}$ & 8016 & 8000 & 0.324888 & 0.727273 & 0.026940 \\
\hline U-234 & 92234 & 92000 & 0.000161 & 0.000025 & 0.000001 \\
\hline U-235 & 92235 & 92000 & 0.018120 & 0.002761 & 0.000102 \\
\hline U-236 & 92236 & 92000 & 0.000083 & 0.000013 & 0.000000 \\
\hline U-238 & 92238 & 92000 & 0.585641 & 0.088111 & 0.003264 \\
\hline Total & & & 1.000000 & 1.000000 & 0.037042 \\
\hline
\end{tabular}

\begin{tabular}{|c|c|c|c|c|c|c|}
\hline MCNP Form & \multicolumn{2}{|c|}{ Weight Fractions } & \multicolumn{2}{|c|}{ Atom Fractions } & \multicolumn{2}{|c|}{ Atom Densities } \\
\hline \multirow[t]{6}{*}{ Neutrons } & 7014 & -0.071106 & 7014 & 0.181818 & 7014 & 0.006735 \\
\hline & 8016 & -0.324888 & 8016 & 0.727273 & 8016 & 0.026940 \\
\hline & 92234 & -0.000161 & 92234 & 0.000025 & 92234 & 0.000001 \\
\hline & 92235 & -0.018120 & 92235 & 0.002761 & 92235 & 0.000102 \\
\hline & 92236 & -0.000083 & 92236 & 0.000013 & 92236 & 0.000000 \\
\hline & 92238 & -0.585641 & 92238 & 0.088111 & 92238 & 0.003264 \\
\hline \multirow[t]{6}{*}{ Photons } & 7000 & -0.071106 & 7000 & 0.181818 & 7000 & 0.006735 \\
\hline & 8000 & -0.324888 & 8000 & 0.727273 & 8000 & 0.026940 \\
\hline & 92000 & -0.000161 & 92000 & 0.000025 & 92000 & 0.000001 \\
\hline & 92000 & -0.018120 & 92000 & 0.002761 & 92000 & 0.000102 \\
\hline & 92000 & -0.000083 & 92000 & 0.000013 & 92000 & 0.000000 \\
\hline & 92000 & -0.585641 & 92000 & 0.088111 & 92000 & 0.003264 \\
\hline \multirow[t]{6}{*}{ CEPXS Form: } & material & $\mathrm{N}$ & 0.071106 & & & \\
\hline & & 0 & 0.324888 & & & \\
\hline & & U-234 & 0.000161 & & & \\
\hline & & U-235 & 0.018120 & & & \\
\hline & & U-236 & 0.000083 & & & \\
\hline & & U-238 & 0.585641 & & & \\
\hline
\end{tabular}

matname Uranyl Nitrate

density $\quad 2.203000$

\section{Comments and References}

Density and formula from pg M8.2.4 of Petrie et al. (2000). Also from pg 201 of Paxton and Pruvost (1986) revision, issued July 1987.

Uranium isotopics assumed for LEU: Wt\% U234/235/236/238 $=0.0267 / 3.0 / 0.0138 / 96.9595$. 


\section{Vermiculite, Exfoliated}

\begin{tabular}{|c|c|c|c|}
\hline Formula $=$ & - & Molecular weight $(\mathrm{g} / \mathrm{mole})=$ & - \\
\hline Density $(\mathrm{g} / \mathrm{cm} 3)=$ & 0.085000 & Total atom density $($ atoms $/ \mathrm{b}-\mathrm{cm})=$ & $3.048 \mathrm{E}-03$ \\
\hline
\end{tabular}

\begin{tabular}{|c|c|c|c|c|c|c|}
\hline Element & Neutron ZA & Photon ZA & $\begin{array}{l}\text { Weight } \\
\text { Fraction }\end{array}$ & $\begin{array}{l}\text { Atom } \\
\text { Fraction }\end{array}$ & $\begin{array}{l}\text { Atom } \\
\text { Density }\end{array}$ & \\
\hline $\mathrm{H}$ & 1001 & 1000 & $\overline{0.011835}$ & $\overline{0.197175}$ & 0.000601 & \\
\hline $\mathrm{O}$ & 8016 & 8000 & 0.496356 & 0.520978 & 0.001588 & \\
\hline $\mathrm{Mg}$ & 12000 & 12000 & 0.133383 & 0.092159 & 0.000281 & \\
\hline $\mathrm{Al}$ & 13027 & 13000 & 0.063151 & 0.039305 & 0.000120 & \\
\hline $\mathrm{Si}$ & 14000 & 14000 & 0.189668 & 0.113407 & 0.000346 & \\
\hline $\mathrm{K}$ & 19000 & 19000 & 0.021668 & 0.009307 & 0.000028 & \\
\hline $\mathrm{Ca}$ & 20000 & 20000 & 0.016353 & 0.006852 & 0.000021 & \\
\hline $\mathrm{Ti}$ & 22000 & 22000 & 0.009854 & 0.003457 & 0.000011 & \\
\hline $\mathrm{Fe}$ & 26000 & 26000 & 0.057732 & 0.017361 & 0.000053 & \\
\hline Total & & & 1.000000 & 1.000000 & 0.003048 & \\
\hline MCNP Form & \multicolumn{2}{|c|}{ Weight Fractions } & \multicolumn{2}{|c|}{ Atom Fractions } & \multicolumn{2}{|c|}{ Atom Densities } \\
\hline \multirow[t]{9}{*}{ Neutrons } & 1001 & -0.011835 & 1001 & 0.197175 & 1001 & 0.000601 \\
\hline & 8016 & -0.496356 & 8016 & 0.520978 & 8016 & 0.001588 \\
\hline & 12000 & -0.133383 & 12000 & 0.092159 & 12000 & 0.000281 \\
\hline & 13027 & -0.063151 & 13027 & 0.039305 & 13027 & 0.000120 \\
\hline & 14000 & -0.189668 & 14000 & 0.113407 & 14000 & 0.000346 \\
\hline & 19000 & -0.021668 & 19000 & 0.009307 & 19000 & 0.000028 \\
\hline & 20000 & -0.016353 & 20000 & 0.006852 & 20000 & 0.000021 \\
\hline & 22000 & -0.009854 & 22000 & 0.003457 & 22000 & 0.000011 \\
\hline & 26000 & -0.057732 & 26000 & 0.017361 & 26000 & 0.000053 \\
\hline \multirow[t]{9}{*}{ Photons } & 1000 & -0.011835 & 1000 & 0.197175 & 1000 & 0.000601 \\
\hline & 8000 & -0.496356 & 8000 & 0.520978 & 8000 & 0.001588 \\
\hline & 12000 & -0.133383 & 12000 & 0.092159 & 12000 & 0.000281 \\
\hline & 13000 & -0.063151 & 13000 & 0.039305 & 13000 & 0.000120 \\
\hline & 14000 & -0.189668 & 14000 & 0.113407 & 14000 & 0.000346 \\
\hline & 19000 & -0.021668 & 19000 & 0.009307 & 19000 & 0.000028 \\
\hline & 20000 & -0.016353 & 20000 & 0.006852 & 20000 & 0.000021 \\
\hline & 22000 & -0.009854 & 22000 & 0.003457 & 22000 & 0.000011 \\
\hline & 26000 & -0.057732 & 26000 & 0.017361 & 26000 & 0.000053 \\
\hline \multirow[t]{6}{*}{ CEPXS Form: } & material & $\mathrm{H}$ & 0.011835 & & & \\
\hline & & 0 & 0.496356 & & & \\
\hline & & $\mathrm{Mg}$ & 0.133383 & & & \\
\hline & & $\mathrm{Al}$ & 0.063151 & & & \\
\hline & & $\mathrm{Si}$ & 0.189668 & & & \\
\hline & & $\mathrm{K}$ & 0.021668 & & & \\
\hline
\end{tabular}




\begin{tabular}{|l} 
Ca \\
matname \\
density \\
$\mathrm{Fe}$
\end{tabular}

\section{Viton Fluoroelastomer}

\begin{tabular}{llll}
\hline Formula $=$ & - & Molecular weight $(\mathrm{g} / \mathrm{mole})=$ & - \\
Density $(\mathrm{g} / \mathrm{cm} 3)=$ & Total atom density $($ atoms $/ \mathrm{b}-\mathrm{cm})=$ & $7.596 \mathrm{E}-02$ \\
The above density is estimated to be accurate to 3 significant digits. Uncertainties are not addressed. \\
The following data were calculated from the input weight fractions.
\end{tabular}

\begin{tabular}{|c|c|c|c|c|c|c|}
\hline Element & Neutron ZA & Photon ZA & $\begin{array}{l}\text { Weight } \\
\text { Fraction }\end{array}$ & $\begin{array}{l}\text { Atom } \\
\text { Fraction }\end{array}$ & \multicolumn{2}{|c|}{$\begin{array}{l}\text { Atom } \\
\text { Density }\end{array}$} \\
\hline $\mathrm{H}$ & 1001 & 1000 & 0.009417 & 0.133327 & \multicolumn{2}{|c|}{$\frac{\text { Density }}{0.010127}$} \\
\hline $\mathrm{C}$ & 6000 & 6000 & 0.280555 & 0.333341 & \multicolumn{2}{|c|}{0.025321} \\
\hline $\mathrm{F}$ & 9019 & 9000 & 0.710028 & 0.533332 & \multicolumn{2}{|c|}{0.040512} \\
\hline Total & & & 1.000000 & 1.000000 & \multicolumn{2}{|c|}{0.075960} \\
\hline MCNP Form & \multicolumn{2}{|c|}{ Weight Fractions } & \multicolumn{2}{|c|}{ Atom Fractions } & \multicolumn{2}{|c|}{ Atom Densities } \\
\hline \multirow[t]{3}{*}{ Neutrons } & 1001 & -0.009417 & 1001 & 0.133327 & 1001 & 0.010127 \\
\hline & 6000 & -0.280555 & 6000 & 0.333341 & 6000 & 0.025321 \\
\hline & 9019 & -0.710028 & 9019 & 0.533332 & 9019 & 0.040512 \\
\hline \multirow[t]{3}{*}{ Photons } & 1000 & -0.009417 & 1000 & 0.133327 & 1000 & 0.010127 \\
\hline & 6000 & -0.280555 & 6000 & 0.333341 & 6000 & 0.025321 \\
\hline & 9000 & -0.710028 & 9000 & 0.533332 & 9000 & 0.040512 \\
\hline \multirow[t]{4}{*}{ CEPXS Form: } & material & $\mathrm{H}$ & 0.009417 & & & \\
\hline & & C & 0.280555 & & & \\
\hline & & $\mathrm{F}$ & 0.710028 & & & \\
\hline & $\begin{array}{l}\text { matname } \\
\text { density }\end{array}$ & \multicolumn{5}{|c|}{$\begin{array}{l}\text { Viton Fluoroelastomer } \\
1.800000\end{array}$} \\
\hline
\end{tabular}


Comments and References

Density and weight fractions from http://physics.nist.gov/cgi-bin/Star/compos.pl?matno=275

(NIST 1998).

\section{Water, Heavy}

\begin{tabular}{llll}
\hline Formula $=$ & D2O & Molecular weight $(\mathrm{g} / \mathrm{mole})=$ & 20.02760356 \\
Density $(\mathrm{g} / \mathrm{cm} 3)=$ & 1.105340 & Total atom density $($ atoms $/ \mathrm{b}-\mathrm{cm})=$ & $9.971 \mathrm{E}-02$
\end{tabular}

The above density is estimated to be accurate to 4 significant digits. Uncertainties are not addressed.

The following data was calculated from the input formula.

\begin{tabular}{|c|c|c|c|c|c|c|}
\hline Element & Neutron ZA & Photon ZA & $\begin{array}{l}\text { Weight } \\
\text { Fraction }\end{array}$ & $\begin{array}{l}\text { Atom } \\
\text { Fraction }\end{array}$ & $\begin{array}{c}\text { Atom } \\
\text { Density }\end{array}$ & \\
\hline $\mathrm{H}-2$ & 1002 & 1000 & $\overline{0.201133}$ & 0.666667 & 0.066473 & \\
\hline 0 & 8016 & 8000 & 0.798867 & 0.333333 & 0.033237 & \\
\hline Total & & & 1.000000 & 1.000000 & 0.099710 & \\
\hline MCNP Form & \multicolumn{2}{|c|}{ Weight Fractions } & \multicolumn{2}{|c|}{ Atom Fractions } & \multicolumn{2}{|c|}{ Atom Densities } \\
\hline \multirow[t]{2}{*}{ Neutrons } & 1002 & -0.201133 & 1002 & 0.666667 & 1002 & 0.066473 \\
\hline & 8016 & -0.798867 & 8016 & 0.333333 & 8016 & 0.033237 \\
\hline \multirow[t]{2}{*}{ Photons } & 1000 & -0.201133 & 1000 & 0.666667 & 1000 & 0.066473 \\
\hline & 8000 & -0.798867 & 8000 & 0.333333 & 8000 & 0.033237 \\
\hline \multirow[t]{3}{*}{ CEPXS Form: } & material & $\mathrm{H}-2$ & 0.201133 & & & \\
\hline & & 0 & 0.798867 & & & \\
\hline & $\begin{array}{c}\text { matname } \\
\text { density }\end{array}$ & $\begin{array}{c}\text { Water, Heavy } \\
1.105340\end{array}$ & & & & \\
\hline \multicolumn{7}{|c|}{$\begin{array}{l}\text { Comments and References } \\
\text { The National Physical Laboratory, which is the national measurement standards laboratory for the } \\
\text { United Kingdom, lists the density for } \mathrm{D} 2 \mathrm{O} \text { for } \mathrm{T}=0^{\circ} \text { to } 100^{\circ} \mathrm{C} \\
\left.\text { (http://www.kayelaby.npl.co.uk/general_physics } / 2 \_2 / 2 \_2 \_1 . \mathrm{html}\right) \text {. At } \mathrm{T}=20^{\circ} \mathrm{C} \text {, the density }=1.10534 \\
\mathrm{~g} / \mathrm{cm} 3 \text { at } 101.325 \mathrm{kPa}(1.0 \mathrm{~atm}) \text { from measurements in } 1949,1951 \text {, and } 1963 \text {. } \\
\text { Density }=1.1054 \mathrm{~g} / \mathrm{cm} 3 \text { is from Petrie et al. }(2000) \text {. Density }=1.1056 \mathrm{~g} / \mathrm{cm} 3 \text { at STP }\left(20^{\circ} \mathrm{C}\right) \text { is listed at } \\
\text { http://en.wikipedia.org/wiki/Heavy_water. } \\
\text { Also call deuterium oxide. }\end{array}$} \\
\hline
\end{tabular}

\section{Water, Liquid}

$\begin{array}{llll}\text { Formula }= & \mathrm{H} 2 \mathrm{O} & \text { Molecular weight }(\mathrm{g} / \mathrm{mole})= & 18.01528 \\ \text { Density }(\mathrm{g} / \mathrm{cm} 3)= & 0.998207 & \text { Total atom density }(\text { atoms } / \mathrm{b}-\mathrm{cm})= & 1.001 \mathrm{E}-01\end{array}$

The above density is estimated to be accurate to 5 significant digits. Uncertainties are not addressed.

The following data were calculated from the input weight fractions. 


\begin{tabular}{|c|c|c|c|c|c|c|}
\hline 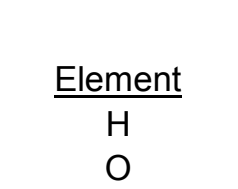 & $\begin{array}{c}\text { Neutron ZA } \\
1001 \\
8016\end{array}$ & 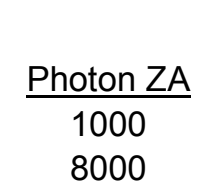 & $\begin{array}{c}\text { Weight } \\
\text { Fraction } \\
0.111894 \\
0.888106\end{array}$ & $\begin{array}{c}\text { Atom } \\
\text { Fraction } \\
0.666657 \\
0.333343\end{array}$ & $\begin{array}{c}\text { Atom } \\
\text { Density } \\
0.066733 \\
0.033368\end{array}$ & \\
\hline Total & & & 1.000000 & 1.000000 & 0.100102 & \\
\hline MCNP Form & \multicolumn{2}{|c|}{ Weight Fractions } & \multicolumn{2}{|c|}{ Atom Fractions } & \multicolumn{2}{|c|}{ Atom Densities } \\
\hline Neutrons & $\begin{array}{l}1001 \\
8016\end{array}$ & $\begin{array}{l}-0.111894 \\
-0.888106\end{array}$ & $\begin{array}{l}1001 \\
8016\end{array}$ & $\begin{array}{l}0.666657 \\
0.333343\end{array}$ & $\begin{array}{l}1001 \\
8016\end{array}$ & $\begin{array}{l}0.066733 \\
0.033368\end{array}$ \\
\hline Photons & $\begin{array}{l}1000 \\
8000\end{array}$ & $\begin{array}{l}-0.111894 \\
-0.888106\end{array}$ & $\begin{array}{l}1000 \\
8000\end{array}$ & $\begin{array}{l}0.666657 \\
0.333343\end{array}$ & $\begin{array}{l}1000 \\
8000\end{array}$ & $\begin{array}{l}0.066733 \\
0.033368\end{array}$ \\
\hline CEPXS Form: & $\begin{array}{l}\text { matname } \\
\text { density }\end{array}$ & $\begin{array}{c}\mathrm{H} \\
\mathrm{O} \\
\text { Water, Liquid } \\
0.998207\end{array}$ & $\begin{array}{l}0.111894 \\
0.888106\end{array}$ & & & \\
\hline $\begin{array}{l}\text { Comments an } \\
\text { Weight fraction } \\
\text { Density }=0.998 \\
\text { Standard Mean } \\
\text { Water Between } \\
\text { A Peuto, and N } \\
\text { density as } 0.83 \\
40^{\circ} \mathrm{C} . \\
\text { Density }=0.998 \\
\text { Density }=1.000\end{array}$ & $\begin{array}{l}\text { References } \\
\text { from http://phy } \\
067 \mathrm{~g} / \mathrm{cm} 3 \text { for } \\
\text { cean Water. } \\
{ }^{\circ} \mathrm{C} \text { and } 40^{\circ} \mathrm{C} \\
\text { ignell. } 2001 . \\
06 \mathrm{~g} / \mathrm{cm} 3 . \mathrm{Ta} \\
063 \mathrm{~g} / \mathrm{cm} 3 \text { at } \\
0 \mathrm{~g} / \mathrm{cm} 3 \text { at htt }\end{array}$ & $\begin{array}{l}\text { ics.nist.gov/cg } \\
\text { de-aerated wat } \\
\text { This value is gi } \\
\text { ased on Recer } \\
\text { Metrologia, } 38 \text { : } \\
\text { le } 1 \text { also gives } \\
=20^{\circ} \mathrm{C} \text { and } \mathrm{P}= \\
: / / \text { physics.nist. }\end{array}$ & $\begin{array}{l}\text { in/Star/com } \\
\text { at one atm } \\
\text { in Table } 1 \\
\text { Experiment } \\
1-309] \text {. Th } \\
\text { e water de } \\
\text { atm from p } \\
\text { //cgi-bin/St }\end{array}$ & $\begin{array}{l}\text { pl?matno= } \\
\text { ere (10132 } \\
\text { Recommen } \\
\text { eports" [Tar } \\
\text { ference list } \\
\text { for temper } \\
\text { - } 4 \text { of Lide } \\
\text { mpos.pl?m }\end{array}$ & $\begin{array}{l}\text { (NIST } 199 \\
\text { ) with the } \\
\text { Table for } \\
\text { M, G Gira } \\
\text { uncertain } \\
\text { es betwee } \\
\text { 8). } \\
=276 \text { (NIS }\end{array}$ & $\begin{array}{l}\text { topics of } \\
\text { Density of } \\
\text { R Davis, } \\
\text { or this } \\
\text { C and } \\
\text { 1998). }\end{array}$ \\
\hline
\end{tabular}

\section{Water, Vapor}

\begin{tabular}{llll}
\hline Formula $=$ & $\mathrm{H} 2 \mathrm{O}$ & Molecular weight $(\mathrm{g} / \mathrm{mole})=$ & 18.01528 \\
Density $(\mathrm{g} / \mathrm{cm} 3)=$ & 0.000756 & Total atom density $($ atoms $/ \mathrm{b}-\mathrm{cm})=$ & $7.583 \mathrm{E}-05$
\end{tabular}

The above density is estimated to be accurate to 4 significant digits. Uncertainties are not addressed.

The following data were calculated from the input weight fractions.

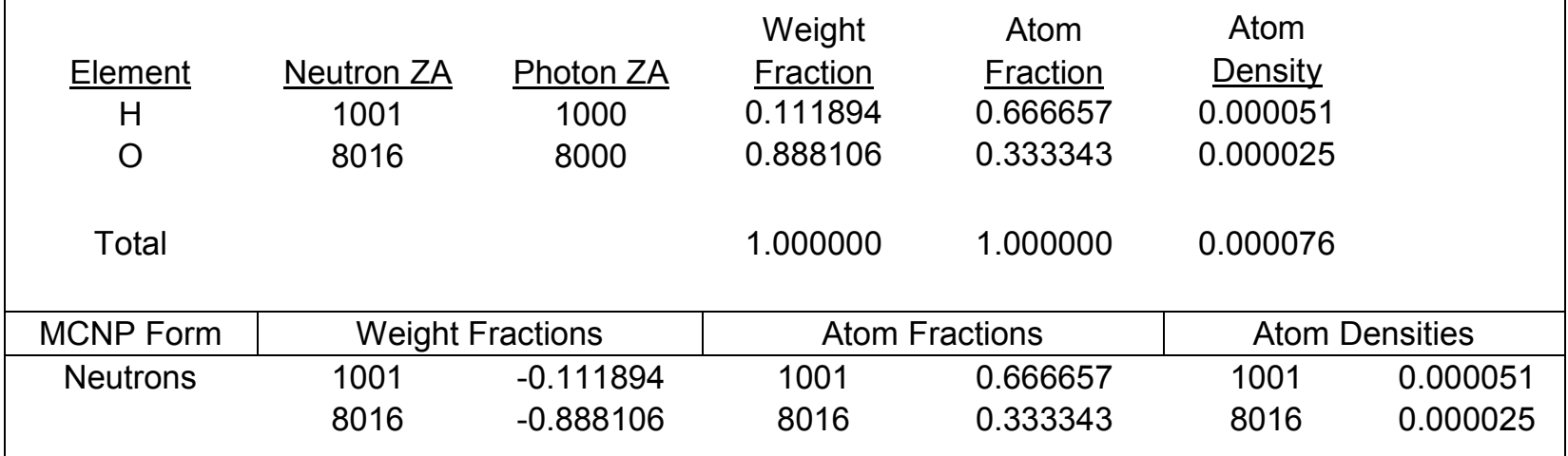


PIET-43741-TM-963

PNNL-15870 Rev. 1

\begin{tabular}{|c|c|c|c|c|c|c|}
\hline Photons & $\begin{array}{l}1000 \\
8000\end{array}$ & $\begin{array}{l}-0.111894 \\
-0.888106\end{array}$ & $\begin{array}{l}1000 \\
8000\end{array}$ & $\begin{array}{l}0.666657 \\
0.333343\end{array}$ & $\begin{array}{l}1000 \\
8000\end{array}$ & $\begin{array}{l}0.000051 \\
0.000025\end{array}$ \\
\hline CEPXS Form: & $\begin{array}{c}\text { matname } \\
\text { density }\end{array}$ & $\begin{array}{c}\mathrm{H} \\
\mathrm{O} \\
\text { Water, Vapor } \\
0.000756\end{array}$ & $\begin{array}{l}0.111894 \\
0.888106\end{array}$ & & & \\
\hline $\begin{array}{l}\text { Comments an } \\
\text { Density }=7.56 \\
\text { bin/Star/compc }\end{array}$ & $\begin{array}{l}\text { References } \\
\text { E-04 } \mathrm{g} / \mathrm{cm} \\
\text { ?matno=2 }\end{array}$ & $\begin{array}{l}\text { d weight fract } \\
\text { (NIST 1998). }\end{array}$ & $\mathrm{s}$ from htt & ysics.nist.g & & \\
\hline
\end{tabular}

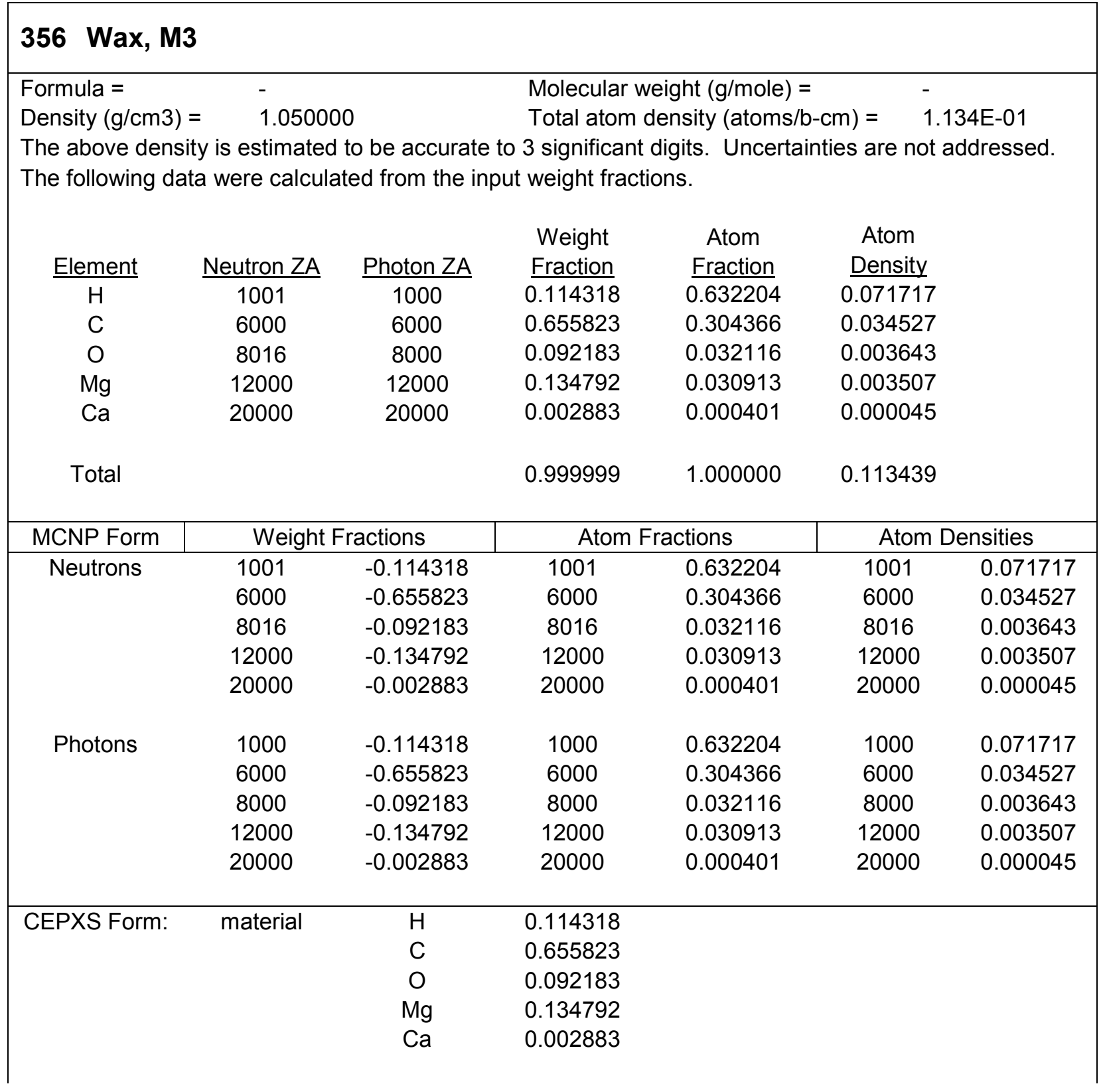




\begin{tabular}{|ll|}
\multicolumn{1}{|c|}{$\begin{array}{l}\text { matname } \\
\text { density }\end{array}$} & Wax, M3 \\
\multicolumn{1}{|c|}{1.050000} \\
\hline $\begin{array}{l}\text { Comments and References } \\
\text { Density and weight fractions from http://physics.nist.gov/cgi-bin/Star/compos.pl?matno=191 } \\
\text { (NIST 1998). }\end{array}$ \\
\hline
\end{tabular}

\section{Wax, Mix D}

\begin{tabular}{|c|c|c|c|}
\hline Formula $=$ & - & Molecular weight $(\mathrm{g} / \mathrm{mole})=$ & - \\
\hline Density $(\mathrm{g} / \mathrm{cm} 3)=$ & 0.990000 & Total atom density $($ atoms $/ \mathrm{b}-\mathrm{cm})=$ & $1.203 E-01$ \\
\hline
\end{tabular}

\begin{tabular}{|c|c|c|c|c|c|c|}
\hline Element & Neutron ZA & Photon ZA & $\begin{array}{l}\text { Weight } \\
\text { Fraction }\end{array}$ & $\begin{array}{c}\text { Atom } \\
\text { Fraction }\end{array}$ & $\begin{array}{l}\text { Atom } \\
\text { Density }\end{array}$ & \\
\hline $\mathrm{H}$ & 1001 & 1000 & 0.134040 & 0.658880 & 0.079284 & \\
\hline C & 6000 & 6000 & 0.777960 & 0.320919 & 0.038617 & \\
\hline $\mathrm{O}$ & 8016 & 8000 & 0.035020 & 0.010845 & 0.001305 & \\
\hline $\mathrm{Mg}$ & 12000 & 12000 & 0.038594 & 0.007867 & 0.000947 & \\
\hline $\mathrm{Ti}$ & 22000 & 22000 & 0.014386 & 0.001489 & 0.000179 & \\
\hline Total & & & 1.000000 & 1.000000 & 0.120332 & \\
\hline MCNP Form & \multicolumn{2}{|c|}{ Weight Fractions } & \multicolumn{2}{|c|}{ Atom Fractions } & \multicolumn{2}{|c|}{ Atom Densities } \\
\hline \multirow[t]{5}{*}{ Neutrons } & 1001 & -0.134040 & 1001 & 0.658880 & 1001 & 0.079284 \\
\hline & 6000 & -0.777960 & 6000 & 0.320919 & 6000 & 0.038617 \\
\hline & 8016 & -0.035020 & 8016 & 0.010845 & 8016 & 0.001305 \\
\hline & 12000 & -0.038594 & 12000 & 0.007867 & 12000 & 0.000947 \\
\hline & 22000 & -0.014386 & 22000 & 0.001489 & 22000 & 0.000179 \\
\hline \multirow[t]{5}{*}{ Photons } & 1000 & -0.134040 & 1000 & 0.658880 & 1000 & 0.079284 \\
\hline & 6000 & -0.777960 & 6000 & 0.320919 & 6000 & 0.038617 \\
\hline & 8000 & -0.035020 & 8000 & 0.010845 & 8000 & 0.001305 \\
\hline & 12000 & -0.038594 & 12000 & 0.007867 & 12000 & 0.000947 \\
\hline & 22000 & -0.014386 & 22000 & 0.001489 & 22000 & 0.000179 \\
\hline \multirow[t]{6}{*}{ CEPXS Form: } & material & $\mathrm{H}$ & 0.134040 & & & \\
\hline & & C & 0.777960 & & & \\
\hline & & 0 & 0.035020 & & & \\
\hline & & $\mathrm{Mg}$ & 0.038594 & & & \\
\hline & & $\mathrm{Ti}$ & 0.014386 & & & \\
\hline & $\begin{array}{c}\text { matname } \\
\text { density }\end{array}$ & $\begin{array}{r}\text { Wax, Mix D } \\
0.990000\end{array}$ & & & & \\
\hline $\begin{array}{l}\text { Comments an } \\
\text { Density and we } \\
\text { (NIST 1998). }\end{array}$ & $\begin{array}{l}\text { References } \\
\text { ht fractions fr }\end{array}$ & nttp://phy & & & tis & \\
\hline
\end{tabular}




\section{Wax, Paraffin}

\begin{tabular}{llll}
\hline Formula $=$ & $\mathrm{C} 25 \mathrm{H} 52$ & Molecular weight $(\mathrm{g} / \mathrm{mole})=$ & 352.68038 \\
Density $(\mathrm{g} / \mathrm{cm} 3)=$ & 0.930000 & Total atom density $($ atoms $/ \mathrm{b}-\mathrm{cm})=$ & $1.223 \mathrm{E}-01$
\end{tabular}

The above density is estimated to be accurate to 2 significant digits. Uncertainties are not addressed. The following data were calculated from the input weight fractions.

\begin{tabular}{|c|c|c|c|c|c|c|}
\hline 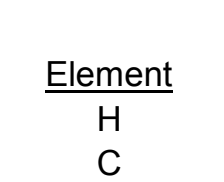 & $\begin{array}{c}\text { Neutron ZA } \\
1001 \\
6000\end{array}$ & $\begin{array}{c}\text { Photon ZA } \\
1000 \\
6000\end{array}$ & $\begin{array}{c}\text { Weight } \\
\text { Fraction } \\
0.148605 \\
0.851395\end{array}$ & $\begin{array}{c}\text { Atom } \\
\text { Fraction } \\
0.675311 \\
0.324689\end{array}$ & $\begin{array}{c}\text { Atom } \\
\text { Density } \\
0.08257 \\
0.03970\end{array}$ & \\
\hline Total & & & 1.000000 & 1.000000 & 0.12227 & \\
\hline MCNP Form & \multicolumn{2}{|c|}{ Weight Fractions } & \multicolumn{2}{|c|}{ Atom Fractions } & \multicolumn{2}{|c|}{ Atom Densities } \\
\hline Neutrons & $\begin{array}{l}1001 \\
6000\end{array}$ & $\begin{array}{l}-0.148605 \\
-0.851395\end{array}$ & $\begin{array}{l}1001 \\
6000\end{array}$ & $\begin{array}{l}0.675311 \\
0.324689\end{array}$ & $\begin{array}{l}1001 \\
6000\end{array}$ & $\begin{array}{l}0.082572 \\
0.039701\end{array}$ \\
\hline Photons & $\begin{array}{l}1000 \\
6000\end{array}$ & $\begin{array}{l}-0.148605 \\
-0.851395\end{array}$ & $\begin{array}{l}1000 \\
6000\end{array}$ & $\begin{array}{l}0.675311 \\
0.324689\end{array}$ & $\begin{array}{l}1000 \\
6000\end{array}$ & $\begin{array}{l}0.082572 \\
0.039701\end{array}$ \\
\hline CEPXS Form: & $\begin{array}{l}\text { matname } \\
\text { density }\end{array}$ & $\begin{array}{c}\mathrm{H} \\
\mathrm{C} \\
\text { Wax, Paraffin } \\
0.930000\end{array}$ & $\begin{array}{l}0.148605 \\
0.851395\end{array}$ & & & \\
\hline
\end{tabular}

\section{Comments and References}

Paraffin wax is a solid mixture of hydrocarbons with an approximation of $\mathrm{C} 25 \mathrm{H} 52$ (pentacosane).

Density and weight fractions from http://physics.nist.gov/cgi-bin/Star/compos.pl?matno=213

(NIST 1998).

This reference is consistent with an assumed formula of $\mathrm{C} 25 \mathrm{H} 52$, which is also used on pg 138 of Brewer (2009), and on pg M8.2.3 of Petrie et al. (2000).

\section{Wood (Southern Pine)}

\begin{tabular}{|c|c|c|c|}
\hline Formula = & - & Molecular weight $(\mathrm{g} / \mathrm{mole})=$ & \\
\hline$(\mathrm{g} / \mathrm{cm} 3)=$ & 0.640000 & Total atom density $($ atoms $/ \mathrm{b}-\mathrm{cm})=$ & 4.932E-02 \\
\hline
\end{tabular}

The above density is estimated to be accurate to 2 significant digits. Uncertainties are not addressed. The following data were calculated from the input weight fractions.

\begin{tabular}{|c|c|c|c|c|c|}
\hline Element & Neutron ZA & Photon ZA & $\begin{array}{l}\text { Weight } \\
\text { Fraction }\end{array}$ & $\begin{array}{c}\text { Atom } \\
\text { Fraction }\end{array}$ & $\begin{array}{c}\text { Atom } \\
\text { Density }\end{array}$ \\
\hline $\mathrm{H}$ & 1001 & 1000 & 0.059642 & 0.462423 & 0.022806 \\
\hline C & 6000 & 6000 & 0.497018 & 0.323389 & 0.015949 \\
\hline $\mathrm{N}$ & 7014 & 7000 & 0.004970 & 0.002773 & 0.000137 \\
\hline $\mathrm{O}$ & 8016 & 8000 & 0.427435 & 0.208779 & 0.010297 \\
\hline
\end{tabular}


PIET-43741-TM-963

PNNL-15870 Rev. 1

\begin{tabular}{|c|c|c|c|c|c|c|}
\hline $\mathrm{Mg}$ & 12000 & 12000 & 0.001988 & 0.000639 & \multicolumn{2}{|l|}{0.000032} \\
\hline$S$ & 16000 & 16000 & 0.004970 & 0.001211 & \multicolumn{2}{|l|}{0.000060} \\
\hline $\mathrm{K}$ & 19000 & 19000 & 0.001988 & 0.000397 & \multicolumn{2}{|l|}{0.000020} \\
\hline $\mathrm{Ca}$ & 20000 & 20000 & 0.001988 & 0.000388 & \multicolumn{2}{|l|}{0.000019} \\
\hline Total & & & 1.000000 & 1.000000 & \multicolumn{2}{|l|}{0.049319} \\
\hline MCNP Form & \multicolumn{2}{|c|}{ Weight Fractions } & \multicolumn{2}{|c|}{ Atom Fractions } & \multicolumn{2}{|c|}{ Atom Densities } \\
\hline \multirow[t]{8}{*}{ Neutrons } & 1001 & -0.059642 & 1001 & 0.462423 & 1001 & 0.022806 \\
\hline & 6000 & -0.497018 & 6000 & 0.323389 & 6000 & 0.015949 \\
\hline & 7014 & -0.004970 & 7014 & 0.002773 & 7014 & 0.000137 \\
\hline & 8016 & -0.427435 & 8016 & 0.208779 & 8016 & 0.010297 \\
\hline & 12000 & -0.001988 & 12000 & 0.000639 & 12000 & 0.000032 \\
\hline & 16000 & -0.004970 & 16000 & 0.001211 & 16000 & 0.000060 \\
\hline & 19000 & -0.001988 & 19000 & 0.000397 & 19000 & 0.000020 \\
\hline & 20000 & -0.001988 & 20000 & 0.000388 & 20000 & 0.000019 \\
\hline \multirow[t]{8}{*}{ Photons } & 1000 & -0.059642 & 1000 & 0.462423 & 1000 & 0.022806 \\
\hline & 6000 & -0.497018 & 6000 & 0.323389 & 6000 & 0.015949 \\
\hline & 7000 & -0.004970 & 7000 & 0.002773 & 7000 & 0.000137 \\
\hline & 8000 & -0.427435 & 8000 & 0.208779 & 8000 & 0.010297 \\
\hline & 12000 & -0.001988 & 12000 & 0.000639 & 12000 & 0.000032 \\
\hline & 16000 & -0.004970 & 16000 & 0.001211 & 16000 & 0.000060 \\
\hline & 19000 & -0.001988 & 19000 & 0.000397 & 19000 & 0.000020 \\
\hline & 20000 & -0.001988 & 20000 & 0.000388 & 20000 & 0.000019 \\
\hline \multirow[t]{9}{*}{ CEPXS Form: } & material & $\mathrm{H}$ & 0.059642 & & & \\
\hline & & C & 0.497018 & & & \\
\hline & & $\mathrm{N}$ & 0.004970 & & & \\
\hline & & $\mathrm{O}$ & 0.427435 & & & \\
\hline & & $\mathrm{Mg}$ & 0.001988 & & & \\
\hline & & $S$ & 0.004970 & & & \\
\hline & & $\mathrm{K}$ & 0.001988 & & & \\
\hline & & $\mathrm{Ca}$ & 0.001988 & & & \\
\hline & $\begin{array}{l}\text { matname } \\
\text { density }\end{array}$ & \multicolumn{3}{|c|}{$\begin{array}{l}\text { Wood (Southern Pine) } \\
0.640000\end{array}$} & & \\
\hline
\end{tabular}

Comments and References

Density $=0.64 \mathrm{~g} / \mathrm{cm} 3$ is average for southern pine, density for ash (black) $=0.55$, ash (white) $=0.67$, balsa $=0.125$, birch $=0.71$, ceder $=0.35$, cherry $=0.43$, fir (douglas) $=0.51$, elm $=0.56$, hickory $=0.77$, mahogany $=0.70$, maple (sugar) $=0.68$, maple $($ white $)=0.53$, oak (black or red) $=0.67$, oak $($ white $)=$ 0.77 , pine $($ white $)=0.43$, pine (yellow) $=0.71$, poplar $=0.43$, redwood $=0.42$, spruce $=0.45$, walnut $=$ 0.59 based on Table 6.1.5 of Avallone and Baumeister III (1996).

Density of course sawdust $=0.29 \mathrm{~g} / \mathrm{cm} 3$, of fine sawdust $=0.40 \mathrm{~g} / \mathrm{cm} 3$ based on

http://www.powderandbulk.com/resources/bulk_density/material_bulk_density_chart_s.htm (Powder and Bulk Dot Com 2010).

Weight fractions are from Mechanical Engineer's Reference Book, [Smith EH. 1998. Elsevier, 12th ed.]. It is assumed that the ash is composed of equal weight fractions of $\mathrm{Mg}, \mathrm{K}$, and $\mathrm{Ca}$. Weight fractions are normalized so they sum to unity.

Compositions for 6 different types of wood are given in Table 51.116 of Hungerford (1960).

Plywood density $=0.58 \mathrm{~g} / \mathrm{cm} 3$ based on $3.0 \mathrm{lb} / \mathrm{ft} 2$ for 1 inch thick plywood (Table 6.7 .13 of Avallone and 
Baumeister III 1996). Plywood density $=0.42$ to $0.68 \mathrm{~g} / \mathrm{cm} 3$ for 9 types of plywood in Table 51.115 of Hungerford (1960).

See Table 11-15 of Parker (1967) for green and dry densities.

\section{Xenon}

\begin{tabular}{llll}
\hline Formula $=$ & Xe & Molecular weight $(\mathrm{g} / \mathrm{mole})=$ & 131.293 \\
Density $(\mathrm{g} / \mathrm{cm} 3)=$ & 0.005485 & Total atom density $($ atoms $/ \mathrm{b}-\mathrm{cm})=$ & $2.516 \mathrm{E}-05$
\end{tabular}

The above density is estimated to be accurate to 4 significant digits. Uncertainties are not addressed.

The following data was calculated from the input formula.

\begin{tabular}{|c|c|c|c|c|c|c|}
\hline$\frac{\text { Element }}{\mathrm{Xe}}$ & $\frac{\text { Neutron ZA }}{54000}$ & $\frac{\text { Photon ZA }}{54000}$ & $\begin{array}{c}\text { Weight } \\
\text { Fraction } \\
1.000000\end{array}$ & $\begin{array}{c}\text { Atom } \\
\frac{\text { Fraction }}{1.000000}\end{array}$ & $\begin{array}{c}\text { Atom } \\
\text { Density } \\
0.00002\end{array}$ & \\
\hline Total & & & 1.000000 & 1.000000 & 0.00002 & \\
\hline MCNP Form & \multicolumn{2}{|c|}{ Weight Fractions } & \multicolumn{2}{|c|}{ Atom Fractions } & \multicolumn{2}{|c|}{ Atom Densities } \\
\hline Neutrons & 54000 & -1.000000 & 54000 & 1.000000 & 54000 & 0.000025 \\
\hline Photons & 54000 & -1.000000 & 54000 & 1.000000 & 54000 & 0.000025 \\
\hline CEPXS Form: & $\begin{array}{l}\text { material } \\
\text { matname } \\
\text { density }\end{array}$ & $\begin{array}{c}\text { Xe } \\
\text { Xenon } \\
0.005485\end{array}$ & 1.000000 & & & \\
\hline
\end{tabular}

Comments and References

Density from http://physics.nist.gov/cgi-bin/Star/compos.pl?matno=054 (NIST 1998).

\section{Yttrium Aluminum Garnet (YAG)}

\begin{tabular}{llll}
\hline Formula $=$ & Y3Al5O12 & Molecular weight $(\mathrm{g} / \mathrm{mole})=$ & 593.61804 \\
Density $(\mathrm{g} / \mathrm{cm} 3)=$ & 4.560000 & Total atom density $($ atoms $/ \mathrm{b}-\mathrm{cm})=$ & $9.252 \mathrm{E}-02$
\end{tabular}

The above density is estimated to be accurate to 3 significant digits. Uncertainties are not addressed.

The following data was calculated from the input formula.

\begin{tabular}{|c|c|c|c|c|c|}
\hline Element & Neutron ZA & Photon ZA & $\begin{array}{l}\text { Weight } \\
\text { Fraction }\end{array}$ & $\begin{array}{l}\text { Atom } \\
\text { Fraction }\end{array}$ & $\begin{array}{l}\text { Atom } \\
\text { Density }\end{array}$ \\
\hline 0 & 8016 & 8000 & $\overline{0.323428}$ & $\overline{0.600000}$ & 0.055512 \\
\hline $\mathrm{Al}$ & 13027 & 13000 & 0.227263 & 0.250000 & 0.023130 \\
\hline$Y$ & 39089 & 39000 & 0.449308 & 0.150000 & 0.013878 \\
\hline Total & & & 1.000000 & 1.000000 & 0.092521 \\
\hline
\end{tabular}


PIET-43741-TM-963

PNNL-15870 Rev. 1

\begin{tabular}{|c|c|c|c|c|c|c|}
\hline MCNP Form & \multicolumn{2}{|c|}{ Weight Fractions } & \multicolumn{2}{|c|}{ Atom Fractions } & \multicolumn{2}{|c|}{ Atom Densities } \\
\hline \multirow[t]{3}{*}{ Neutrons } & 8016 & -0.323428 & 8016 & 0.600000 & 8016 & 0.055512 \\
\hline & 13027 & -0.227263 & 13027 & 0.250000 & 13027 & 0.023130 \\
\hline & 39089 & -0.449308 & 39089 & 0.150000 & 39089 & 0.013878 \\
\hline \multirow[t]{3}{*}{ Photons } & 8000 & -0.323428 & 8000 & 0.600000 & 8000 & 0.055512 \\
\hline & 13000 & -0.227263 & 13000 & 0.250000 & 13000 & 0.023130 \\
\hline & 39000 & -0.449308 & 39000 & 0.150000 & 39000 & 0.013878 \\
\hline \multirow[t]{4}{*}{ CEPXS Form: } & material & $\mathrm{O}$ & 0.323428 & & & \\
\hline & & $\mathrm{Al}$ & 0.227263 & & & \\
\hline & & $\mathrm{Y}$ & 0.449308 & & & \\
\hline & $\begin{array}{c}\text { matname } \\
\text { density }\end{array}$ & $\begin{array}{c}\text { Yttrium Alum } \\
4.560000 \\
\end{array}$ & m Garnet (' & & & \\
\hline \multicolumn{7}{|c|}{$\begin{array}{l}\text { Comments and References } \\
\text { Density from pg } 235 \text { of Knoll (2000). } \\
\text { Formula from pgs } 4 \text { - } 99 \text { of Lide (2008). } \\
\text { Density and formula also at http://www.apace-science.com/misc/crystalj.htm (APACE 2009). } \\
\text { Also called Yttrium Aluminum Oxide. }\end{array}$} \\
\hline
\end{tabular}

\section{Yttrium Aluminum Perovskite (YAP)}

\begin{tabular}{llll}
\hline Formula $=$ & YAlO3 & Molecular weight $(\mathrm{g} / \mathrm{mole})=$ & 163.885588 \\
Density $(\mathrm{g} / \mathrm{cm} 3)=$ & 5.370000 & Total atom density $($ atoms $/ \mathrm{b}-\mathrm{cm})=$ & $9.866 \mathrm{E}-02$
\end{tabular}

The above density is estimated to be accurate to 3 significant digits. Uncertainties are not addressed.

The following data was calculated from the input formula.

\begin{tabular}{|c|c|c|c|c|c|c|}
\hline Element & Neutron ZA & Photon ZA & $\begin{array}{l}\text { Weight } \\
\text { Fraction }\end{array}$ & $\begin{array}{c}\text { Atom } \\
\text { Fraction }\end{array}$ & \multicolumn{2}{|l|}{ Atom } \\
\hline 0 & 8016 & 8000 & 0.292876 & 0.600000 & \multicolumn{2}{|l|}{0.059198} \\
\hline $\mathrm{Al}$ & 13027 & 13000 & 0.164636 & 0.200000 & \multicolumn{2}{|l|}{0.019733} \\
\hline $\mathrm{Y}$ & 39089 & 39000 & 0.542487 & 0.200000 & \multicolumn{2}{|l|}{0.019733} \\
\hline Total & & & 1.000000 & 1.000000 & \multicolumn{2}{|l|}{0.098663} \\
\hline MCNP Form & \multicolumn{2}{|c|}{ Weight Fractions } & \multicolumn{2}{|c|}{ Atom Fractions } & \multicolumn{2}{|c|}{ Atom Densities } \\
\hline \multirow[t]{3}{*}{ Neutrons } & 8016 & -0.292876 & 8016 & 0.600000 & 8016 & 0.059198 \\
\hline & 13027 & -0.164636 & 13027 & 0.200000 & 13027 & 0.019733 \\
\hline & 39089 & -0.542487 & 39089 & 0.200000 & 39089 & 0.019733 \\
\hline \multirow[t]{3}{*}{ Photons } & 8000 & -0.292876 & 8000 & 0.600000 & 8000 & 0.059198 \\
\hline & 13000 & -0.164636 & 13000 & 0.200000 & 13000 & 0.019733 \\
\hline & 39000 & -0.542487 & 39000 & 0.200000 & 39000 & 0.019733 \\
\hline \multirow[t]{3}{*}{ CEPXS Form: } & material & $\mathrm{O}$ & 0.292876 & & & \\
\hline & & $\mathrm{Al}$ & 0.164636 & & & \\
\hline & & Y & 0.542487 & & & \\
\hline
\end{tabular}


matname Yttrium Aluminum Perovskite (YAP)

density $\quad 5.370000$

Comments and References

Density from pg 235 of Knoll (2000).

Formula and same density given in http://www.apace-science.com/misc/crystalj.htm (APACE 2009).

\section{Yttrium OxyorthoSilicate (YSO)}

\begin{tabular}{llll}
\hline Formula $=$ & Y2SiO5 & Molecular weight $(\mathrm{g} / \mathrm{mole})=$ & 285.8942 \\
Density $(\mathrm{g} / \mathrm{cm} 3)=$ & 4.450000 & Total atom density $($ atoms $/ \mathrm{b}-\mathrm{cm})=$ & $7.499 \mathrm{E}-02$
\end{tabular}

The above density is estimated to be accurate to 3 significant digits. Uncertainties are not addressed.

The following data was calculated from the input formula.

$\begin{array}{ccccccc}\text { Element } & \text { Neutron ZA } & \text { Photon ZA } & & \begin{array}{c}\text { Weight } \\ \text { Fraction }\end{array} & \begin{array}{c}\text { Atom } \\ \text { Fraction }\end{array} & \begin{array}{c}\text { Atom } \\ \text { Density }\end{array} \\ & 8016 & 8000 & & 0.279813 & 0.625000 & 0.046868 \\ \mathrm{Si} & 14000 & 14000 & 0.098237 & 0.125000 & 0.009374 \\ \mathrm{Y} & 39089 & 39000 & 0.621949 & 0.250000 & 0.018747 \\ \text { Total } & & & & & & \\ & & & & 1.000000 & 1.000000 & 0.074989\end{array}$

\begin{tabular}{|c|cc|cccc|}
\hline MCNP Form & \multicolumn{2}{c|}{ Weight Fractions } & \multicolumn{2}{c|}{ Atom Fractions } & \multicolumn{2}{c|}{ Atom Densities } \\
\hline Neutrons & 8016 & -0.279813 & 8016 & 0.625000 & 8016 & 0.046868 \\
& 14000 & -0.098237 & 14000 & 0.125000 & 14000 & 0.009374 \\
& 39089 & -0.621949 & 39089 & 0.250000 & 39089 & 0.018747 \\
Photons & & & & & & \\
& 8000 & -0.279813 & 8000 & 0.625000 & 8000 & 0.046868 \\
& 14000 & -0.098237 & 14000 & 0.125000 & 14000 & 0.009374 \\
& 39000 & -0.621949 & 39000 & 0.250000 & 39000 & 0.018747 \\
\hline CEPXS Form: & material & $\mathrm{O}$ & 0.279813 & & & \\
& & $\mathrm{Si}$ & 0.098237 & & & \\
& & $\mathrm{Y}$ & 0.621949 & & & \\
& & & & & & \\
\end{tabular}

matname Yttrium OxyorthoSilicate (YSO)

density $\quad 4.450000$

\section{Comments and References}

See http://www.apace-science.com/proteus/yso.htm\#top and http://www.apacescience.com/misc/crystalj.htm (APACE 2009).

\section{Zeolite (Natrolite)}

\begin{tabular}{llll}
\hline Formula $=$ & NA2Al2Si3O10-2 $(\mathrm{H} 2 \mathrm{O})$ & Molecular weight $(\mathrm{g} / \mathrm{mole})=$ & 380.223676 \\
Density $(\mathrm{g} / \mathrm{cm} 3)=$ & 2.250000 & Total atom density $($ atoms $/ \mathrm{b}-\mathrm{cm})=$ & $8.196 \mathrm{E}-02$
\end{tabular}

The above density is estimated to be accurate to 3 significant digits. Uncertainties are not addressed. 
The following data was calculated from the input formula.

\begin{tabular}{|c|c|c|c|c|c|}
\hline Element & Neutron ZA & Photon ZA & $\begin{array}{l}\text { Weight } \\
\text { Fraction }\end{array}$ & $\begin{array}{c}\text { Atom } \\
\text { Fraction }\end{array}$ & $\begin{array}{c}\text { Atom } \\
\text { Density }\end{array}$ \\
\hline $\mathrm{H}$ & 1001 & 1000 & $\overline{0.010604}$ & $\overline{0.173913}$ & 0.014255 \\
\hline O & 8016 & 8000 & 0.504947 & 0.521739 & 0.042764 \\
\hline $\mathrm{Na}$ & 11023 & 11000 & 0.120928 & 0.086957 & 0.007127 \\
\hline $\mathrm{Al}$ & 13027 & 13000 & 0.141925 & 0.086957 & 0.007127 \\
\hline $\mathrm{Si}$ & 14000 & 14000 & 0.221597 & 0.130435 & 0.010691 \\
\hline Total & & & 1.000000 & 1.000000 & 0.081964 \\
\hline
\end{tabular}

\begin{tabular}{|c|cc|cccc|}
\hline MCNP Form & \multicolumn{2}{c|}{ Weight Fractions } & \multicolumn{2}{c|}{ Atom Fractions } & \multicolumn{2}{c|}{ Atom Densities } \\
\hline Neutrons & 1001 & -0.010604 & 1001 & 0.173913 & 1001 & 0.014255 \\
& 8016 & -0.504947 & 8016 & 0.521739 & 8016 & 0.042764 \\
& 11023 & -0.120928 & 11023 & 0.086957 & 11023 & 0.007127 \\
& 13027 & -0.141925 & 13027 & 0.086957 & 13027 & 0.007127 \\
& 14000 & -0.221597 & 14000 & 0.130435 & 14000 & 0.010691 \\
Photons & & & & & & \\
& 1000 & -0.010604 & 1000 & 0.173913 & 1000 & 0.014255 \\
& 8000 & -0.504947 & 8000 & 0.521739 & 8000 & 0.042764 \\
& 11000 & -0.120928 & 11000 & 0.086957 & 11000 & 0.007127 \\
& 13000 & -0.141925 & 13000 & 0.086957 & 13000 & 0.007127 \\
& 14000 & -0.221597 & 14000 & 0.130435 & 14000 & 0.010691
\end{tabular}

\begin{tabular}{|c|c|c|c|}
\hline \multirow{5}{*}{ CEPXS Form: } & material & $\mathrm{H}$ & 0.010604 \\
\hline & & $\mathrm{O}$ & 0.504947 \\
\hline & & $\mathrm{Na}$ & 0.120928 \\
\hline & & $\mathrm{Al}$ & 0.141925 \\
\hline & & $\mathrm{Si}$ & 0.221597 \\
\hline
\end{tabular}

matname Zeolite (Natrolite)

density 2.250000

\section{Comments and References}

Density of natrolite $=2.25 \mathrm{~g} / \mathrm{cm} 3$ and formula from http://webmineral.com $/$ data/Natrolite.shtml.

Non-clumping cat litter is often made of zeolite, diatomaceous earth, and/or sepiolite. The formula is for natrolite (http://www.galleries.com/Minerals/By_Name.htm) which is one form of the mineral group called zeolite (http://en.wikipedia.org/wiki/Zeolite).

\section{Zinc}

Formula $=$

Density $(\mathrm{g} / \mathrm{cm} 3)=7.133000$

The above density is estimated to be accurate to 4 significant digits. Uncertainties are not addressed.

The following data was calculated from the input formula. 


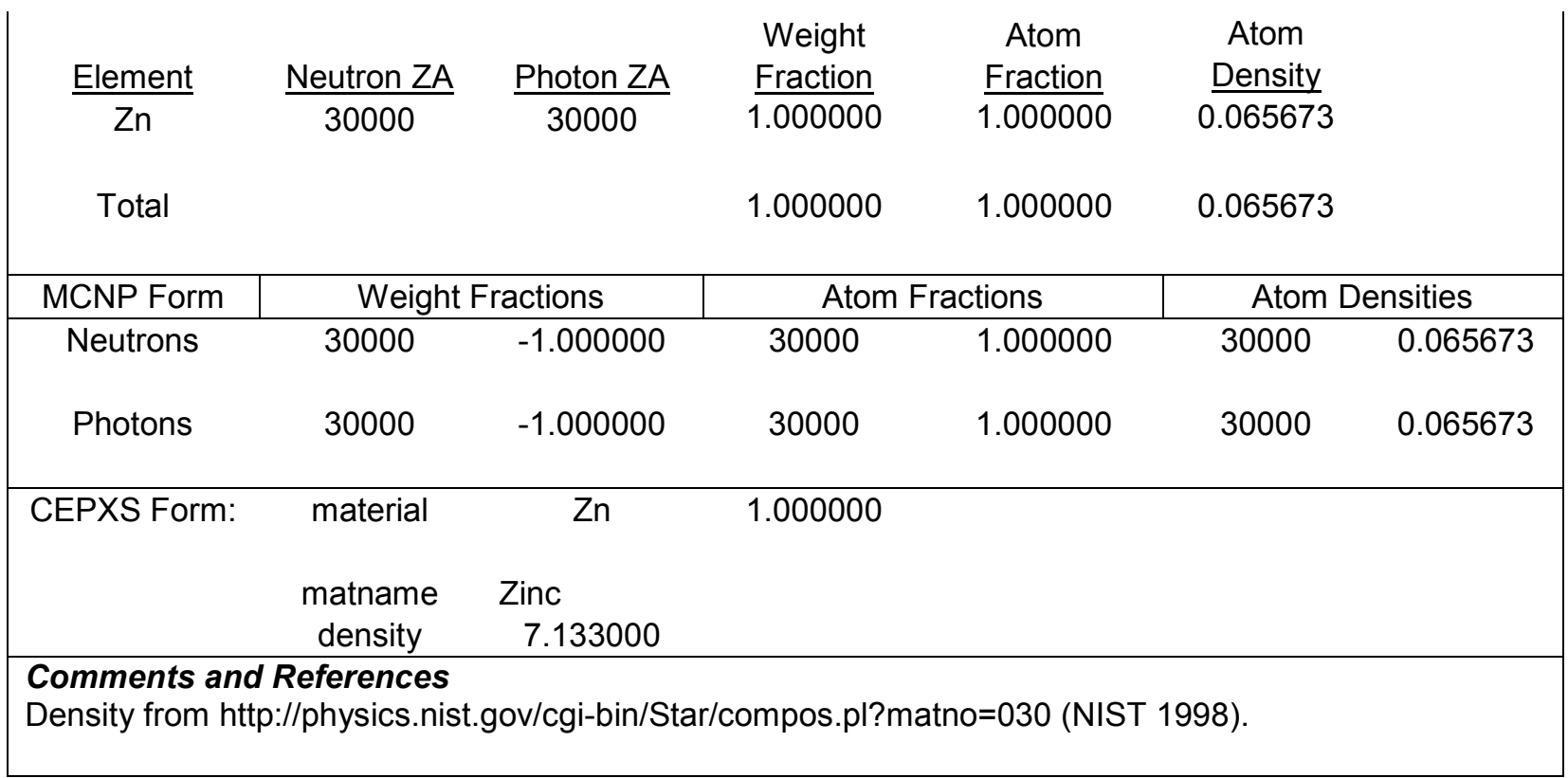

\section{Zinc Selenide}

\begin{tabular}{llll}
\hline Formula $=$ & ZnSe & Molecular weight $(\mathrm{g} / \mathrm{mole})=$ & 144.369 \\
Density $(\mathrm{g} / \mathrm{cm} 3)=$ & 5.420000 & Total atom density $($ atoms $/ \mathrm{b}-\mathrm{cm})=$ & $4.522 \mathrm{E}-02$
\end{tabular}

The above density is estimated to be accurate to 3 significant digits. Uncertainties are not addressed.

The following data was calculated from the input formula.

\begin{tabular}{|c|c|c|c|c|c|c|}
\hline Element & Neutron ZA & Photon ZA & $\begin{array}{l}\text { Weight } \\
\text { Fraction }\end{array}$ & $\begin{array}{l}\text { Atom } \\
\text { Fraction }\end{array}$ & $\begin{array}{l}\text { Atom } \\
\text { Density }\end{array}$ & \\
\hline $\mathrm{Zn}$ & 30000 & 30000 & $\overline{0.453068}$ & $\overline{0.500000}$ & 0.022609 & \\
\hline $\mathrm{Se}$ & - & 34000 & 0.546932 & 0.500000 & 0.022609 & \\
\hline Total & & & 1.000000 & 1.000000 & 0.045217 & \\
\hline MCNP Form & \multicolumn{2}{|c|}{ Weight Fractions } & \multicolumn{2}{|c|}{ Atom Fractions } & \multicolumn{2}{|c|}{ Atom Densities } \\
\hline \multirow[t]{2}{*}{ Neutrons } & 30000 & -0.453068 & 30000 & 0.500000 & 30000 & 0.022609 \\
\hline & - & -0.546932 & - & 0.500000 & - & 0.022609 \\
\hline \multirow[t]{2}{*}{ Photons } & 30000 & -0.453068 & 30000 & 0.500000 & 30000 & 0.022609 \\
\hline & 34000 & -0.546932 & 34000 & 0.500000 & 34000 & 0.022609 \\
\hline \multirow[t]{3}{*}{ CEPXS Form: } & material & $\mathrm{Zn}$ & 0.453068 & & & \\
\hline & & $\mathrm{Se}$ & 0.546932 & & & \\
\hline & $\begin{array}{l}\text { matname } \\
\text { density }\end{array}$ & $\begin{array}{c}\text { Zinc Selenide } \\
5.420000\end{array}$ & & & & \\
\hline
\end{tabular}




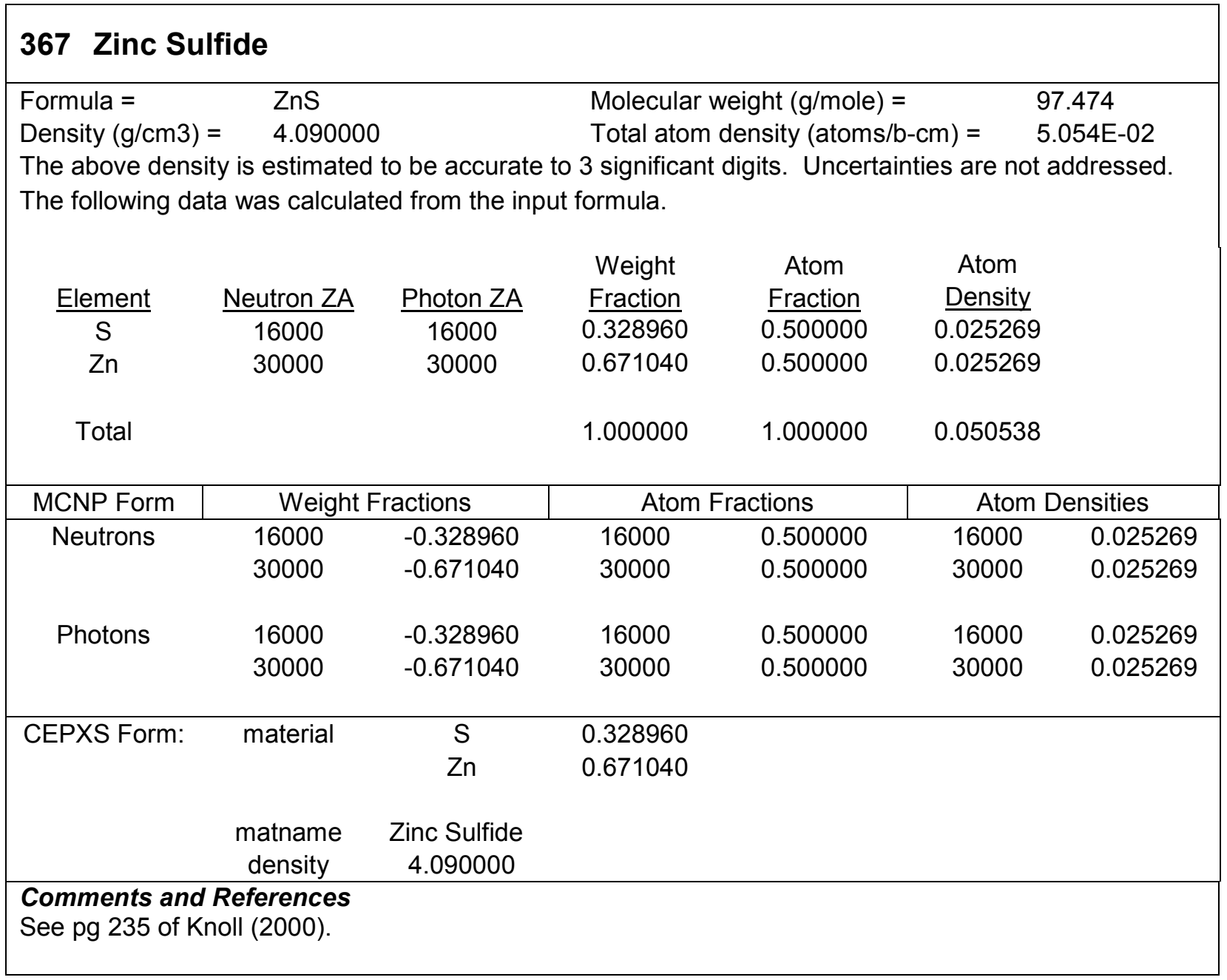

\section{Zircaloy-2}

\begin{tabular}{llll}
\hline Formula $=$ & - & Molecular weight $(\mathrm{g} / \mathrm{mole})=$ \\
Density $(\mathrm{g} / \mathrm{cm} 3)=$ & 6.560000 & Total atom density $($ atoms $/ \mathrm{b}-\mathrm{cm})=$ & - \\
\hline & $4.348 \mathrm{E}-02$
\end{tabular}

The above density is estimated to be accurate to 3 significant digits. Uncertainties are not addressed. The following data were calculated from the input weight fractions.

$\begin{array}{cccccc}\text { Element } & \text { Neutron ZA } & \text { Photon ZA } & \begin{array}{c}\text { Weight } \\ \text { Fraction }\end{array} & \begin{array}{c}\text { Atom } \\ \text { Fraction }\end{array} & \begin{array}{c}\text { Atom } \\ \text { Density }\end{array} \\ \mathrm{Cr} & 8016 & 8000 & 0.001197 & 0.006796 & 0.000296 \\ \mathrm{Fe} & 24000 & 24000 & 0.000997 & 0.001743 & 0.000076 \\ \mathrm{Ni} & 26000 & 26000 & 0.000997 & 0.001623 & 0.000071 \\ \mathrm{Zr} & 28000 & 28000 & 0.000499 & 0.000772 & 0.000034 \\ \mathrm{Sn} & 40000 & 40000 & 0.982348 & 0.978381 & 0.042541 \\ & 50000 & 50000 & 0.013962 & 0.010686 & 0.000465\end{array}$


PIET-43741-TM-963

PNNL-15870 Rev. 1

\begin{tabular}{|c|c|c|c|c|c|c|}
\hline Total & & & 1.000000 & 1.000000 & 0.04348 & \\
\hline MCNP Form & Weigh & ractions & Atom & ctions & Atom & nsities \\
\hline Neutrons & 8016 & -0.001197 & 8016 & 0.006796 & 8016 & 0.000296 \\
\hline & 24000 & -0.000997 & 24000 & 0.001743 & 24000 & 0.000076 \\
\hline & 26000 & -0.000997 & 26000 & 0.001623 & 26000 & 0.000071 \\
\hline & 28000 & -0.000499 & 28000 & 0.000772 & 28000 & 0.000034 \\
\hline & 40000 & -0.982348 & 40000 & 0.978381 & 40000 & 0.042541 \\
\hline & 50000 & -0.013962 & 50000 & 0.010686 & 50000 & 0.000465 \\
\hline Photons & 8000 & -0.001197 & 8000 & 0.006796 & 8000 & 0.000296 \\
\hline & 24000 & -0.000997 & 24000 & 0.001743 & 24000 & 0.000076 \\
\hline & 26000 & -0.000997 & 26000 & 0.001623 & 26000 & 0.000071 \\
\hline & 28000 & -0.000499 & 28000 & 0.000772 & 28000 & 0.000034 \\
\hline & 40000 & -0.982348 & 40000 & 0.978381 & 40000 & 0.042541 \\
\hline & 50000 & -0.013962 & 50000 & 0.010686 & 50000 & 0.000465 \\
\hline CEPXS Form: & material & $\mathrm{O}$ & 0.001197 & & & \\
\hline & & $\mathrm{Cr}$ & 0.000997 & & & \\
\hline & & $\mathrm{Fe}$ & 0.000997 & & & \\
\hline & & $\mathrm{Ni}$ & 0.000499 & & & \\
\hline & & $\mathrm{Zr}$ & 0.982348 & & & \\
\hline & & Sn & 0.013962 & & & \\
\hline & $\begin{array}{c}\text { matname } \\
\text { density }\end{array}$ & $\begin{array}{r}\text { Zircaloy-2 } \\
6.560000\end{array}$ & & & & \\
\hline $\begin{array}{l}\text { Comments an } \\
\text { See } \\
\text { http://www.mat } \\
\text { k=1 (Automatio } \\
\text { Weight fraction }\end{array}$ & $\begin{array}{l}\text { References } \\
\text { b.com/sear } \\
\text { Sreations } 2 \\
\text { ormalized }\end{array}$ & $\begin{array}{l}\text { DataSheet.a } \\
\text { D) and pg } 20 \\
\text { 1.0. }\end{array}$ & $\begin{array}{l}\text { MatGUID= } \\
\text { Paxton and }\end{array}$ & $\begin{array}{l}\text { dad5ce1ac } \\
\text { vost (1986 }\end{array}$ & $\begin{array}{l}\text { e92f86d } \\
\text { sion issu }\end{array}$ & $\begin{array}{l}4740 d \& c k c \\
\text { uly } 1987 .\end{array}$ \\
\hline
\end{tabular}

\section{Zircaloy-4}

\begin{tabular}{llll}
\hline Formula $=$ & - & Molecular weight $(\mathrm{g} / \mathrm{mole})=$ \\
Density $(\mathrm{g} / \mathrm{cm} 3)=$ & 6.560000 & Total atom density $($ atoms $/ \mathrm{b}-\mathrm{cm})=$ & - \\
\hline
\end{tabular}

The above density is estimated to be accurate to 3 significant digits. Uncertainties are not addressed.

The following data were calculated from the input weight fractions.

\begin{tabular}{|c|c|c|c|c|c|}
\hline Element & Neutron ZA & Photon ZA & $\begin{array}{l}\text { Weight } \\
\text { Fraction }\end{array}$ & $\begin{array}{c}\text { Atom } \\
\text { Fraction }\end{array}$ & $\begin{array}{c}\text { Atom } \\
\text { Density }\end{array}$ \\
\hline 0 & 8016 & 8000 & $\overline{0.001196}$ & $\overline{0.006790}$ & 0.000295 \\
\hline $\mathrm{Cr}$ & 24000 & 24000 & 0.000997 & 0.001741 & 0.000076 \\
\hline $\mathrm{Fe}$ & 26000 & 26000 & 0.001994 & 0.003242 & 0.000141 \\
\hline $\mathrm{Zr}$ & 40000 & 40000 & 0.981858 & 0.977549 & 0.042520 \\
\hline Sn & 50000 & 50000 & 0.013955 & 0.010677 & 0.000464 \\
\hline Total & & & 1.000000 & 1.000000 & 0.043497 \\
\hline
\end{tabular}


PIET-43741-TM-963

PNNL-15870 Rev. 1

\begin{tabular}{|c|c|c|c|c|c|c|}
\hline MCNP Form & \multicolumn{2}{|c|}{ Weight Fractions } & \multicolumn{2}{|c|}{ Atom Fractions } & \multicolumn{2}{|c|}{ Atom Densities } \\
\hline \multirow[t]{5}{*}{ Neutrons } & 8016 & -0.001196 & 8016 & 0.006790 & 8016 & 0.000295 \\
\hline & 24000 & -0.000997 & 24000 & 0.001741 & 24000 & 0.000076 \\
\hline & 26000 & -0.001994 & 26000 & 0.003242 & 26000 & 0.000141 \\
\hline & 40000 & -0.981858 & 40000 & 0.977549 & 40000 & 0.042520 \\
\hline & 50000 & -0.013955 & 50000 & 0.010677 & 50000 & 0.000464 \\
\hline \multirow[t]{5}{*}{ Photons } & 8000 & -0.001196 & 8000 & 0.006790 & 8000 & 0.000295 \\
\hline & 24000 & -0.000997 & 24000 & 0.001741 & 24000 & 0.000076 \\
\hline & 26000 & -0.001994 & 26000 & 0.003242 & 26000 & 0.000141 \\
\hline & 40000 & -0.981858 & 40000 & 0.977549 & 40000 & 0.042520 \\
\hline & 50000 & -0.013955 & 50000 & 0.010677 & 50000 & 0.000464 \\
\hline \multirow[t]{6}{*}{ CEPXS Form: } & material & $\mathrm{O}$ & 0.001196 & & & \\
\hline & & $\mathrm{Cr}$ & 0.000997 & & & \\
\hline & & $\mathrm{Fe}$ & 0.001994 & & & \\
\hline & & $\mathrm{Zr}$ & 0.981858 & & & \\
\hline & & Sn & 0.013955 & & & \\
\hline & $\begin{array}{c}\text { matname } \\
\text { density }\end{array}$ & $\begin{array}{l}\text { Zircaloy-4 } \\
6.560000\end{array}$ & & & & \\
\hline \multicolumn{7}{|c|}{$\begin{array}{l}\text { Comments and References } \\
\text { See http://www.matweb.com/search/DataSheet.aspx?MatGUID=e36a9590eb5945de94d89a35097b7faa } \\
\text { (Automation Creations 2010). } \\
\text { Weight fractions normalized to } 1.0 \text {. }\end{array}$} \\
\hline
\end{tabular}

\section{Zirconium}

\begin{tabular}{llll}
\hline Formula $=$ & $\mathrm{Zr}$ & Molecular weight $(\mathrm{g} / \mathrm{mole})=$ & 91.224 \\
Density $(\mathrm{g} / \mathrm{cm} 3)=$ & 6.506000 & Total atom density $($ atoms $/ \mathrm{b}-\mathrm{cm})=$ & $4.295 \mathrm{E}-02$ \\
The
\end{tabular}

The above density is estimated to be accurate to 4 significant digits. Uncertainties are not addressed.

The following data was calculated from the input formula.

\begin{tabular}{|c|c|c|c|c|c|c|}
\hline$\frac{\text { Element }}{\mathrm{Zr}}$ & $\frac{\text { Neutron ZA }}{40000}$ & $\frac{\text { Photon ZA }}{40000}$ & $\begin{array}{l}\text { Weight } \\
\frac{\text { Fraction }}{1.000000}\end{array}$ & $\begin{array}{c}\begin{array}{c}\text { Atom } \\
\text { Fraction }\end{array} \\
1.000000\end{array}$ & $\begin{array}{r}\begin{array}{c}\text { Atom } \\
\text { Density }\end{array} \\
0.04294\end{array}$ & \\
\hline Total & & & 1.000000 & 1.000000 & 0.04294 & \\
\hline MCNP Form & \multicolumn{2}{|c|}{ Weight Fractions } & \multicolumn{2}{|c|}{ Atom Fractions } & \multicolumn{2}{|c|}{ Atom Densities } \\
\hline Neutrons & 40000 & -1.000000 & 40000 & 1.000000 & 40000 & 0.042949 \\
\hline Photons & 40000 & -1.000000 & 40000 & 1.000000 & 40000 & 0.042949 \\
\hline CEPXS Form: & $\begin{array}{l}\text { material } \\
\text { matname }\end{array}$ & $\begin{array}{c}\text { Zr } \\
\text { Zirconium }\end{array}$ & 1.000000 & & & \\
\hline
\end{tabular}


density 6.506000

Comments and References

Density from http://physics.nist.gov/cgi-bin/Star/compos.pl?matno=040 (NIST 1998).

\section{Zirconium Hydride (Zr5H8)}

\begin{tabular}{llll}
\hline Formula $=$ & Zr5H8 & Molecular weight $(\mathrm{g} / \mathrm{mole})=$ & 464.18352 \\
Density $(\mathrm{g} / \mathrm{cm} 3)=$ & 5.610000 & Total atom density $($ atoms $/ \mathrm{b}-\mathrm{cm})=$ & $9.462 \mathrm{E}-02$
\end{tabular}

The above density is estimated to be accurate to 3 significant digits. Uncertainties are not addressed.

The following data was calculated from the input formula.

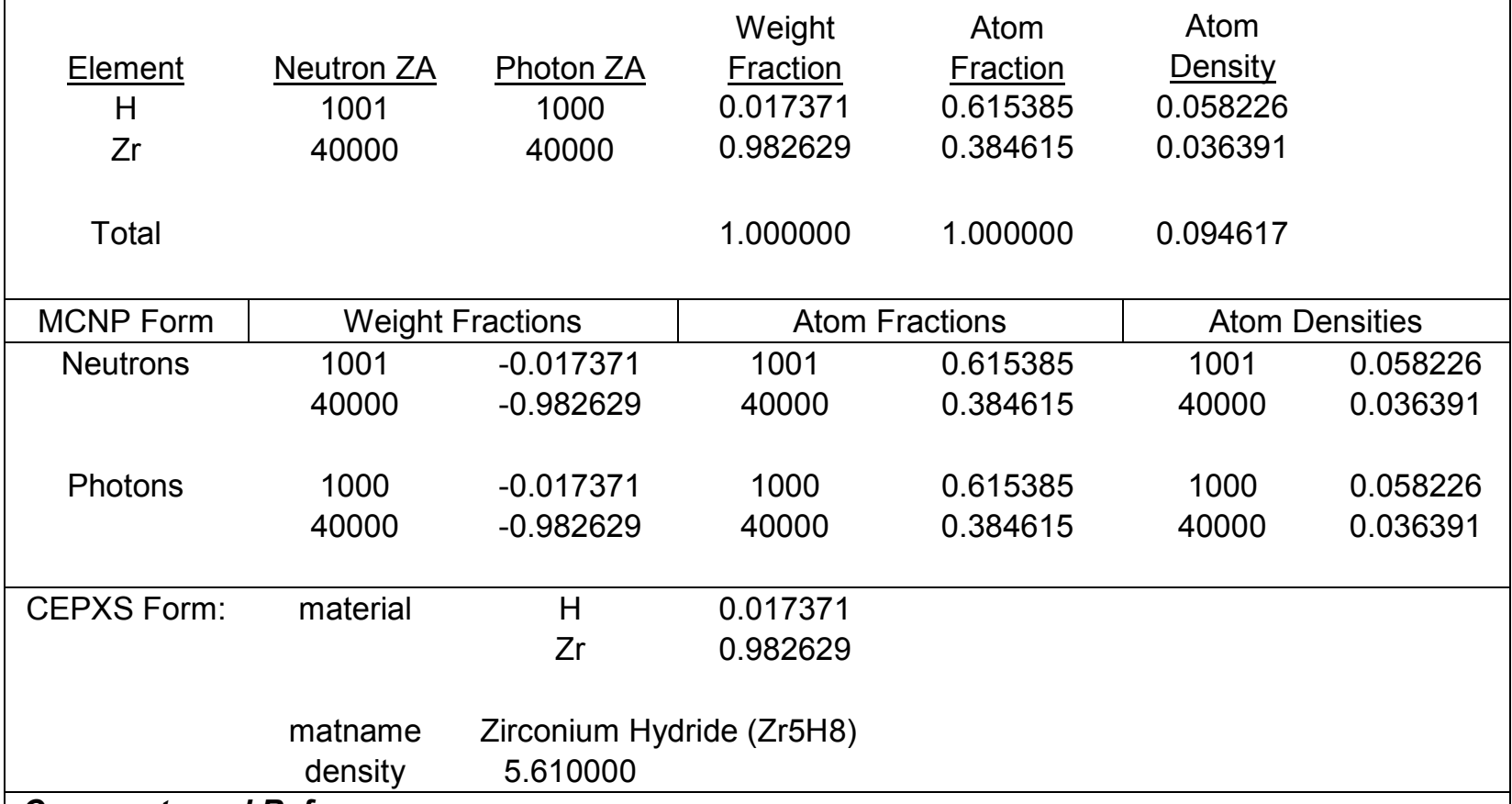

Comments and References

Density and formula from pg M8.2.4 of Petrie et al. (2000).

\section{Zirconium Hydride (ZrH2)}

\begin{tabular}{llll}
\hline Formula $=$ & $\mathrm{ZrH} 2$ & Molecular weight $(\mathrm{g} / \mathrm{mole})=$ & 93.23988 \\
Density $(\mathrm{g} / \mathrm{cm} 3)=$ & 5.610000 & Total atom density $($ atoms $/ \mathrm{b}-\mathrm{cm})=$ & $1.087 \mathrm{E}-01$
\end{tabular}

The above density is estimated to be accurate to 3 significant digits. Uncertainties are not addressed.

The following data was calculated from the input formula.

$\begin{array}{cccccc}\text { Element } & \text { Neutron ZA } & \text { Photon ZA } & \begin{array}{c}\text { Weight } \\ \text { Fraction }\end{array} & \begin{array}{c}\text { Atom } \\ \text { Fraction }\end{array} & \begin{array}{c}\text { Atom } \\ \text { Density }\end{array} \\ \mathrm{Zr} & 1001 & 1000 & 0.021620 & 0.666667 & 0.072467 \\ & 40000 & 40000 & 0.978380 & 0.333333 & 0.036234\end{array}$


PIET-43741-TM-963

PNNL-15870 Rev. 1

\begin{tabular}{|c|c|c|c|c|c|c|}
\hline Total & & & 1.000000 & 1.000000 & 0.10870 & \\
\hline MCNP Form & Weigl & ractions & Atom & ctions & Atom & nsities \\
\hline Neutrons & 1001 & -0.021620 & 1001 & 0.666667 & 1001 & 0.072467 \\
\hline & 40000 & -0.978380 & 40000 & 0.333333 & 40000 & 0.036234 \\
\hline Photons & 1000 & -0.021620 & 1000 & 0.666667 & 1000 & 0.072467 \\
\hline & 40000 & -0.978380 & 40000 & 0.333333 & 40000 & 0.036234 \\
\hline CEPXS Form: & material & $\mathrm{H}$ & 0.021620 & & & \\
\hline & & $\mathrm{Zr}$ & 0.978380 & & & \\
\hline & $\begin{array}{c}\text { matname } \\
\text { density }\end{array}$ & $\begin{array}{c}\text { Zirconium H } \\
5.610000\end{array}$ & de $(\mathrm{ZrH} 2)$ & & & \\
\hline $\begin{array}{l}\text { Comments an } \\
\text { Density and for }\end{array}$ & $\begin{array}{l}\text { eferences } \\
\text { la from pg }\end{array}$ & 1 of Detr & $(2000)$ & & & \\
\hline
\end{tabular}


PIET-43741-TM-963

PNNL-15870 Rev. 1

\section{Major References}

Alcoa Mill Products. n.d. “Alloy 2090-T83 Sheet, Technical Data," accessed October 14, 2010, at http://www.alcoa.com/mill_products/catalog/pdf/alloy2090-t83techsheet.pdf.

ANSI/ANS-6.4-1985. Guidelines on the Nuclear Analysis and Design of Concrete Radiation Shielding for Nuclear Power Plants. American National Standards Institute, New York; American Nuclear Society, La Grange Park, Illinois.

APACE Science. 2009. Product data accessed August 25, 2009, at http://www.apace-science.com (NOTE: A revision of September 10, 2009, omitted weight fractions.)

Automation Creations. 2010. "MatWeb Material Property Database." Automation Creations, Blacksburg, Virginia. Accessed September 28, 2010, at http://www.matweb.com/search/search.aspx.

Avallone EA and T Baumeister III, eds. 1996. Mark's Standard Handbook for Mechanical Engineers. $10^{\text {th }}$ ed., McGraw-Hill.

Blatt H, RJ Tracy, and BE Owens. 2006. Petrology: Igneous, Sedimentary, and Metamorphic. $3^{\text {rd }}$ ed., WH Freeman and Company, New York.

Brandrup J, EH Immergut, EA Grulke, A Abe, DR Bloch, and R Daniel. 2005. Polymer Handbook. $4^{\text {th }}$ ed., John Wiley \& Sons.

Brewer R. 2009. Criticality Calculations with MCNP5: A Primer. LA-UR-09-00380, Los Alamos National Laboratory, Los Alamos, New Mexico.

Carter RD, GR Kiel, and KR Ridgway. 1968. Criticality Handbook. ARH-600, Vol. 1, Atlantic Richfield Company, Hanford, Washington.

Carter RD. 1978. Concrete and Criticality. RHO-SA-30, Rockwell Hanford Operations, Richland, Washington.

Ceramic Industry. 2005. "Fiberglass Compositions and Their Comparative Properties." From Handbook of Reinforcement for Plastics, JV Milewski and HS Katz, 1987. Accessed November 11, 2010, on Ceramic Industry website at www.ceramicindustry.com/CI/Protected/Files/PDF/fiberglasscompositions.pdf.

Chilton AB, JK Shultis, and RE Faw. 1984. Principles of Radiation Shielding. Prentice Hall, Englewood Cliffs, New Jersey.

DOE-STD-3013-2000. DOE Standard: Stabilization, Packaging, and Storage of Plutonium-Bearing Materials. U.S. Department of Energy, Washington DC.

Engineering Toolbox. n.d. "Liquids - Densities." Accessed November 15, 2010, at http://www.engineeringtoolbox.com/liquids-densities-d_743.html.

Evergro Canada. 2001. Muriate of Potash product data. Evergro Canada, Delta, British Columbia. Accessed November 11, 2010, at http:/www.growercentral.com/UPLOADS/PDFS/0-0$62 \% 20$ muriate $\% 20 \mathrm{of} \% 20$ potash\%20fine\%20label.pdf (last updated April 2001).

Green DW and RH Perry. 2008. Perry's Chemical Engineers' Handbook. $8^{\text {th }}$ ed., McGraw-Hill. 
PIET-43741-TM-963

PNNL-15870 Rev. 1

Greskovich C and S Duclos. 1997. "Ceramic Scintillators." Annual Review of Material Science, 27:69-88.

Guthrie VB, ed. 1960. Petroleum Products Handbook. McGraw-Hill.

Hungerford, HE. 1960. "Materials.” In Reactor Handbook, $2^{\text {nd }}$ ed., Vol. 1, ed. CR Tipton, Jr., Interscience Publishers, New York.

Jaeger RG, EP Blizard, and AB Chilton, eds. 1975. "Shielding Materials.” In Engineering Compendium on Radiation Shielding, Vol. 2, Springer-Verlag, New York.

Knoll GF. 2000. Radiation Detection and Measurement. $3^{\text {rd }}$ ed., John Wiley \& Sons.

Knovel. 2008. Knovel Critical Tables. $2^{\text {nd }}$ ed. Compiled by Knovel Corporation. Online version accessed November 11, 2010, at:

http://knovel.com/web/portal/browse/display?_EXT_KNOVEL_DISPLAY_bookid=761\&VerticalID=0.

Lide DR, ed. 2008. CRC Handbook of Chemistry and Physics. $89^{\text {th }}$ ed., CRC Press, Taylor \& Francis Group.

Lide DR, ed. 2009. CRC Handbook of Chemistry and Physics. $90^{\text {th }}$ ed., CRC Press, Taylor \& Francis Group.

Maerker RE and FJ Muckenthaler. 1966. "Measurements and Single Velocity Calculations of Differential Angular Thermal-Neutron Albedos for Concrete." Nuclear Science and Engineering, 26(3):340.

Mantell CL, ed. 1958. Engineering Materials Handbook. McGraw-Hill.

Mine-Engineer.Com. 2010. "Minerals," accessed November 11, 2010, at http://www.mine-engineer.com (last updated October 15, 2010).

NCRP - National Council on Radiation Protection and Measurements. 2003. Radiation Protection for Particle Accelerator Facilities. NCRP Report No. 144, National Council on Radiation Protection and Measurements, Washington, DC.

NIST - National Institute of Standards and Technology. 1996. NIST Standard Reference Database 126, Tables of X-Ray Mass Attenuation Coefficients and Mass Energy-Absorption Coefficients. Table 2, XRay Mass Attenuation Coefficients, accessed September 28, 2010, at http://physics.nist.gov/PhysRefData/XrayMassCoef/tab2.html (last updated July 2004).

NIST - National Institute of Standards and Technology. 1998. NIST Standard Reference Database 124, Stopping-Power and Range Tables for Electrons, Protons, and Helium Ions. "PSTAR and ASTAR: for Protons and Helium Ions (alpha particles)," Table 2, List of Materials for PSTAR and ASTAR at http://physics.nist.gov/PhysRefData/Star/Text/table2.html, and Compositions of Materials data at http://physics.nist.gov/cgi-bin/Star/compos.pl?matno. Accessed September 28, 2010 (last updated August 2005).

Parker ER. 1967. Materials Data Book. McGraw-Hill.

Paxton HC and NL Pruvost. 1986. Critical Dimensions of Systems Containing ${ }^{235} U,{ }^{239} \mathrm{Pu}$, and ${ }^{233} \mathrm{U}$ : 1986 Revision. LA-10860-MS, Los Alamos National Laboratory, Los Alamos, New Mexico. 
PIET-43741-TM-963

PNNL-15870 Rev. 1

Petrie LM, PB Fox, and K Lucius. 2000. "Standard Composition Library." Section M8 of SCALE Ver 4.4: A Modular Code System for Performing Standardized Computer Analyses for Licensing Evaluation-- Functional Models F9 - F11. NUREG/CR-0200, Rev. 6, Vol. 3, prepared by Oak Ridge National Laboratory for the U.S. Nuclear Regulatory Commission, Washington, DC.

Pohanish RP. 2002. Sittig's Handbook of Toxic and Hazardous Chemicals and Carcinogens. $4^{\text {th }}$ ed., William Andrew Publishing/Noyes.

Powder and Bulk Dot Com. 2010. "Engineering Resources - Bulk Density Chart." Camber Southeast, Jacksonville Beach, Florida. Accessed September 28, 2010, at www.powderandbulk.com/resources/bulk_density/material_bulk_density_chart_a.htm.

Saint-Gobain. 2007. Lithium Glass Scintillators Product Data Sheet. Saint-Gobain Ceramics \& Plastics, Northboro, Massachusetts. Accessed November 30, 2010, at http://www.detectors.saintgobain.com/Lithium-Glass-Scintillator.aspx.

Schaeffer NM, ed. 1973. Reactor Shielding for Nuclear Engineers. TID-25951, prepared by Radiation Research Associates for the U.S. Atomic Energy Commission Office of Information Services, Oak Ridge, Tennessee.

Shleien B, ed. 1992. Health Physics and Radiological Health Handbook. Scinta, Inc., Silver Spring, Maryland.

Shultis JK and RE Faw. 1996. Radiation Shielding. Prentice Hall, Englewood Cliffs, New Jersey.

Söderlind P. 2001. "Ambient pressure phase diagram of plutonium: A unified theory for $\alpha-\mathrm{Pu}$ and

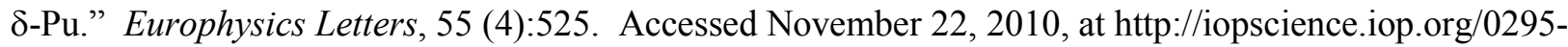
5075/55/4/525/fulltext.

Speight JG. 2001. Handbook of Petroleum Analysis. John Wiley \& Sons.

Spokas JJ and DR White. 1982. “A Conducting Plastic Simulating Cortical Bone.” Physics in Medicine and Biology 27(1):115.

Tanaka M, K Hara, S Kim, K Kondo, H Takano, M Kobayashi, H Ishibashi, K Kurashige, K Susa, and M Ishii. 1998. "Applications of Cerium-Doped Gadolinium Silicate $\mathrm{Gd}_{2} \mathrm{SiO}_{5}$ :Ce Scintillator to Calorimeters in High Radiation Environment." Nuclear Instruments and Methods in Physics Research, Section A: Accelerators, Spectrometers, Detectors and Associated Equipment, 404(2):283-294. Accessed November 11, 2010, at http://citeseerx.ist.psu.edu/viewdoc/summary?doi=10.1.1.30.4620.

Walker R. 2009. "Si Metric Density of Bulk Materials" chart. Accessed September 28, 2010, at http://www.simetric.co.uk/si_materials.htm (last updated April 4, 2009).

Washburn EW, ed. 2003; originally published 1926 - 1930. International Critical Tables of Numerical

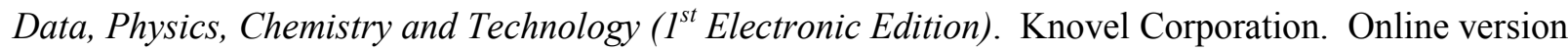
accessed November 30, 2010, at http://www.knovel.com/web/portal/basic_search/display?_EXT_KNOVEL_DISPLAY_bookid=735. Wess JA, LD Olsen, and MH Sweeney. 2004. Concise International Chemical Assessment Document 59, Asphalt (Bitumen). International Programme on Chemical Safety (IPCS), Geneva, Switzerland.

Williams III RG, CJ Gesh, and RT Pagh. 2006. Compendium of Material Composition Data for Radiation Transport Modeling. PNNL-15870, Rev. 0, Pacific Northwest National Laboratory, Richland, Washington. 
X-5 Monte Carlo Team. 2003. MCNP - A General Monte Carlo N-Particle Transport Code, Version 5 - Volume II: Users Guide. LA-CP-03-0245, Los Alamos National Laboratory, Los Alamos, New Mexico.

X-5 Monte Carlo Team. 2005. MCNP - A General Monte Carlo N-Particle Transport Code, Version 5, Volume I: Overview and Theory. LA-UR-03-1987 (issued April 24, 2003; revised October 3, 2005), Los Alamos National Laboratory, Los Alamos, New Mexico.

Yaws CL. 2008. Yaws' Handbook of Physical Properties for Hydrocarbons and Chemicals. Knovel Corporation. Online version accessed November 11, 2010, at:

http://knovel.com/web/portal/browse/display?_EXT_KNOVEL_DISPLAY_bookid=2147\&VerticalID=0. Yinon J and S Zitrin. 1993. Modern Methods and Applications in Analysis of Explosives. John Wiley \& Sons.

Zudas JA and WP Walters, eds. 2002. Explosive Effects and Applications. Springer-Verlag, New York. 


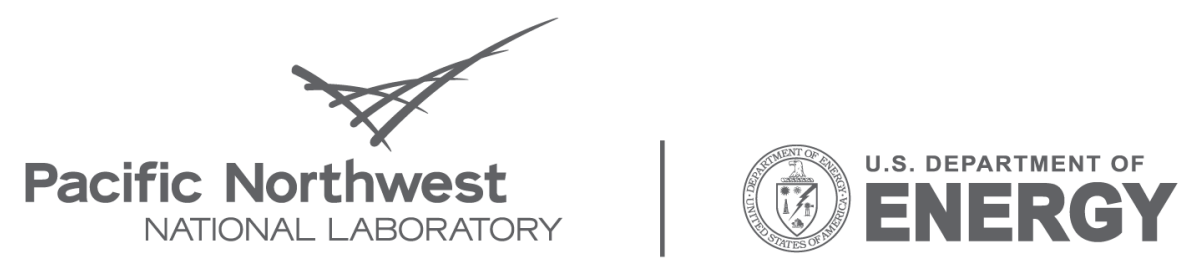

Proudly Operated by Battelle Since 1965

902 Battelle Boulevard

P.O. Box 999

Richland, WA 99352

1-888-375-PNNL (7665)

www.pnl.gov 